\title{
Interim Storage Report
}

\author{
John K. Rawlins
}

Published February 1998

\section{Idaho National Engineering and Environmental Laboratory High-Level Radioactive Waste (HLW) Programs Department Lockheed Martin Idaho Technologies Company Idaho Falls, Idaho 83415}

Prepared for the

U.S. Department of Energy

Assistant Secretary for Environmental Management

Under DOE Idaho Operations Office

Contract DE-AC07-94ID13223 


\begin{abstract}
High-level radioactive waste (HLW) stored at the Idaho Chemical Processing Plant (ICPP) in the form of calcine and liquid and liquid sodium-bearing waste (SBW) will be processed to provide a stable waste form and prepare the waste to be transported to a permanent repository. Because a permanent repository will not be available when the waste is processed, the waste must be stored at ICPP in an Interim Storage Facility (ISF). This report documents consideration of an ISF for each of the waste processing options under consideration.
\end{abstract}

\title{
DISCLAIMER
}

This report was prepared as an account of work sponsored by an agency of the United States Government. Neither the United States Government nor any agency thereof, nor any of their employees, makes any warranty, express or implied, or assumes any legal liability or responsibility for the accuracy, completeness, or usefulness of any information, apparatus, product, or process disclosed, or represents that its use would not infringe privately owned rights. Reference herein to any specific commercial product, process, or service by trade name, trademark, manufacturer, or otherwise does not necessarily constitute or imply its endorsement, recommendation, or favoring by the United States Government or any agency thereof. The views and opinions of authors expressed herein do not necessarily state or reflect those of the United States Government or any agency thereof. 


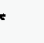




\section{ACKNOWLEDGMENTS}

The following people made significant contributions of their time and expertise to this study. Their work is reflected throughout this report and its appendices.

Rick D. Adams

Byron L. Blakely

Steve W. Hills

David E. Keller

Stephen S. Lortie

Jennifer Quigley and Rod R. Kimmitt

Robert J. Turk

Karen Williams
Cost Estimates

New facility design

FPR building modifications

FPR material handling

Technical Editing

Project Data Sheets

Life-Cycle Costs

Project Lead 


\section{CONTENTS}

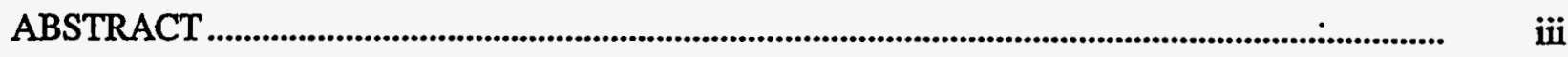

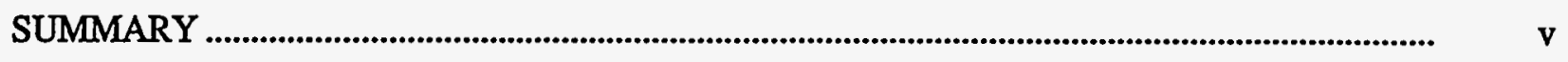

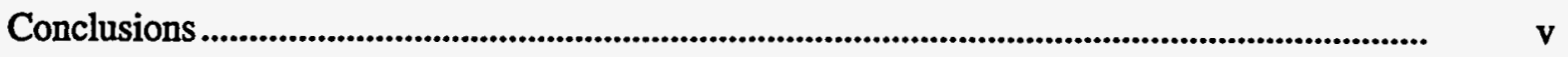

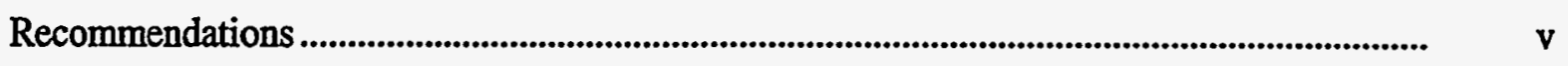

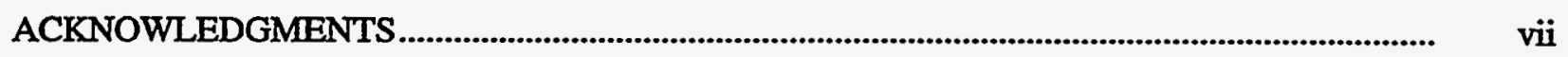

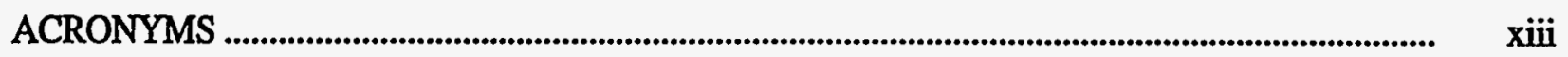

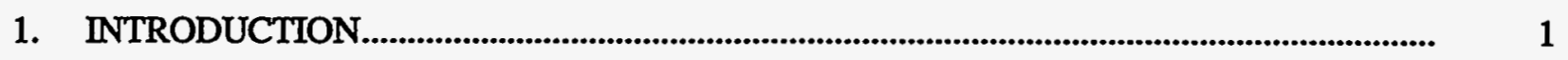

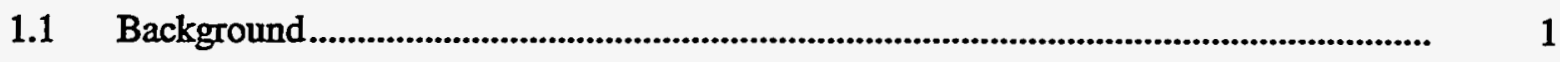

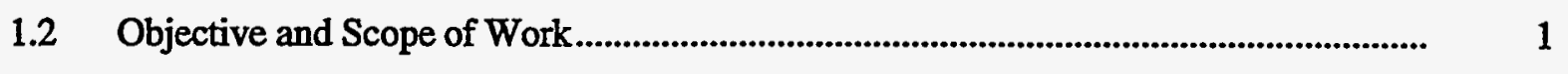

2. DESIGN BASIS, KEY ASSUMPTIONS, AND REQUIREMENTS................................... 4

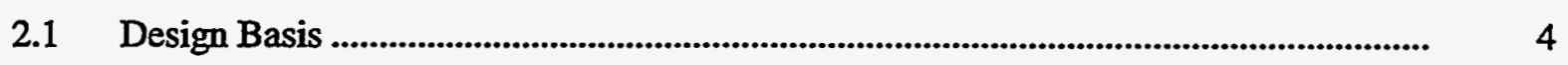

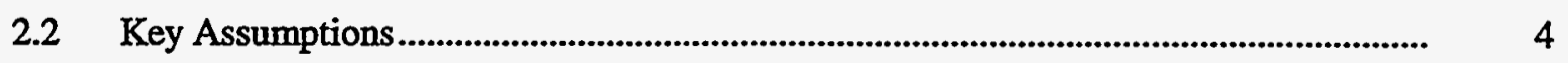

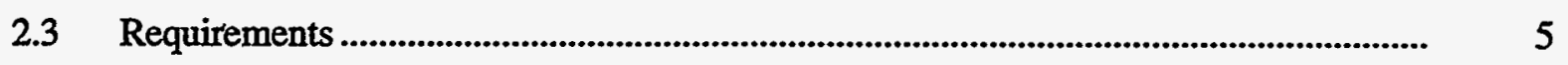

2.3.1 Regulations ..................................................................................... 5

2.3.2 HLW Repository WAC ................................................................................. 5

2.3.3 Applicable Codes and Standards .................................................................... 5

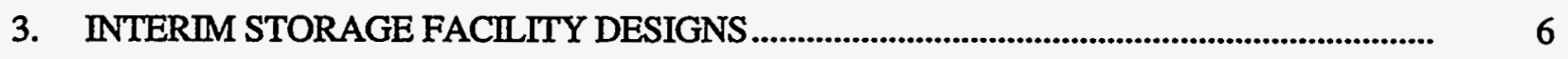

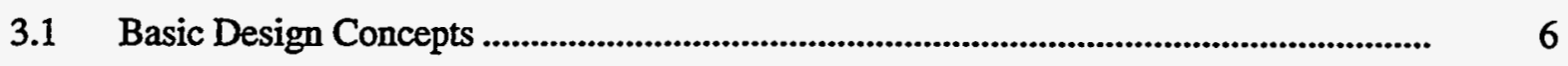

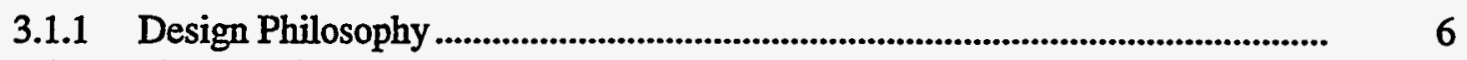

3.1.2 New Interim Storage Facility............................................................................ 6

3.1.3 Modified Existing Interim Storage Facility ..................................................... 9

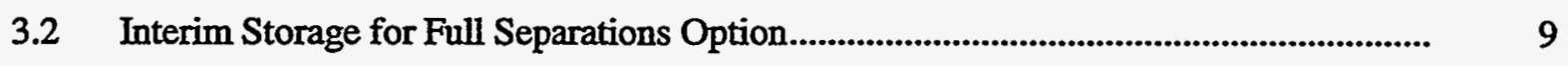

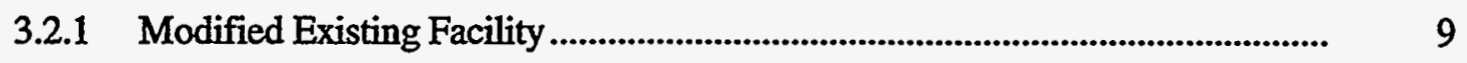

Interim Storage for TRU/Class A Separations Option ............................................... 10

3.3.1 New Storage Facility ............................................................................... 10

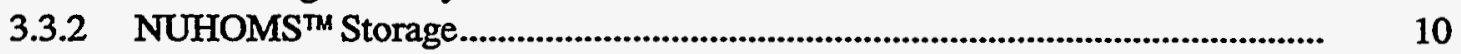

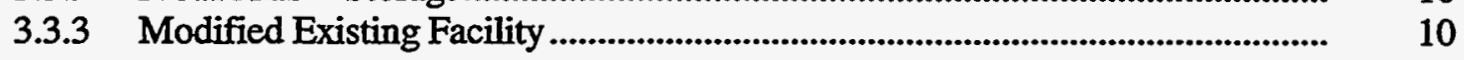

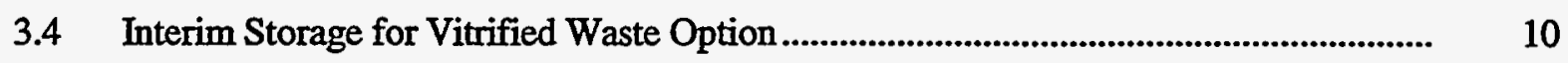


3.4.1 New Storage Facility .....................................................................................

3.4.2 Modified Existing Facility .................................................................................. 11

3.5 Interim Storage for Direct Cementitious Waste Option .................................................... 11

3.5.1 New Storage Facility ....................................................................................

3.5.2 Modified Existing Facility .......................................................................... 11

3.5.3 New Storage Facility for the HIP Preliminary Plan............................................. 11

3.6 Interim Storage for Cementitious Waste Option ............................................................ 11

3.6.1 New Storage Facility ......................................................................................

3.6.2 Modified Existing Facility .............................................................................. 12

3.6.3 New Storage Facility for the HIP Preliminary Plan............................................. 12

3.7 Interim Storage for Hot Isostatic Press Waste Option ..................................................... 12

3.7.1 New Storage Facility ………......................................................................

3.7.2 Modified Existing Facility ................................................................................. 12

4. TOTAL PROJECT, LIFE-CYCLE, AND D\&D COSTS ...........................................................

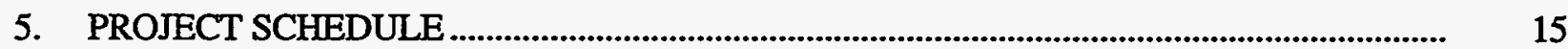

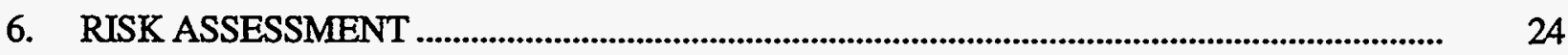

7. CONSTRUCTION AND OPERATIONAL ENVIRONMENTAL IMPACTS............................ 25

8. PROJECT-SPECIFIC OPTIONS ..................................................................................... 58

9. POTENTIAL IMPACTS OF NRC LICENSING ................................................................... 58

10. RECOMMENDATIONS ................................................................................................... 59

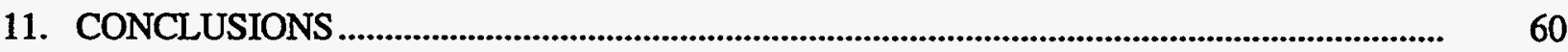

12. REFERENCES.................................................................................................................... 61

Appendix A-Engineering Design Files

\section{FIGURES}

1. Flow Diagram for the Storage of High-Level Waste in Interim Storage ...................................... 8

2. Project Schedules ..................................................................................................................... 16

TABLES

1. Waste Processing Options and Study Data ................................................................................... 2

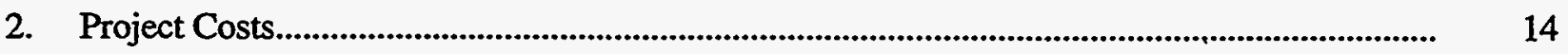

3. Project data sheet for Interim Storage ..................................................................................... 26 


\section{ACRONYMS}

CFR Code of Federal Regulations

CWO Cementitious Waste Option

D\&D decontamination and decommissioning

DOE U.S. Department of Energy

DOE-ID DOE Idaho Operations Office

DOT Department of Transportation

DPC Dual Purpose Canister

DTC Dry Transfer Cell

DCWO Direct Cementitious Waste Option

EDF Engineering Design File

EIS Environmental Impact Study

FPR Fuel Processing Restoration Facility

HAW high-activity waste

HIP Hot Isostatic Press

HLW high-level radioactive waste

HLW\&FD High-Level Waste and Facilities Disposition

HWO HIIP Waste Option

ICPP Idaho Chemical Processing Plant

IFSF Irradiated Fuel Storage Facility

INEEL Idaho National Engineering and Environmental Laboratory

ISF Interim Storage Facility-Facility for the storage of HLW in canisters at ICPP

LAW low-activity waste

LMITCO Lockheed Martin Idaho Technologies Company

MP-187 Transport/shipping cask commercially available through VECTRA Technologies, soon to be purchased by Chem Nuclear, Inc. 
NRC Nuclear Regulatory Commission

NUHOMS Trade mark for concrete storage modules commercially available through VECTRA Technologies, soon to be purchased by Chem Nuclear, Inc.

RCRA Resource Conservation and Recovery Act

SBW sodium-bearing waste

SNF spent nuclear fuel

SRS Savannah River Site

TRU transuranic

VWO Vitirified Waste Option

WAC Waste Acceptance Criteria

WIPP Waste Isolation Pilot Plant • 


\section{Interim Storage Report}

\section{INTRODUCTION}

\subsection{Background}

The Idaho Chemical Processing Plant (ICPP) located at the Idaho National Engineering and Environmental Laboratory (INEEL) has been used for reprocessing spent nuclear fuel (SNF) since 1953. SNF was reprocessed to recover fissile uranium; however, changing world events have diminished the need to recover and recycle this material. In April 1992, the Department of Energy (DOE) discontinued reprocessing SNF and shifted its focus toward continued management and disposal of waste accumulated from previous reprocessing activities. ICPP waste treatment activities produce a granular solid waste (calcine) that contains dissolved fission products and fuel cladding material. Decontamination activities at the ICPP produce liquid radioactive, sodium-bearing waste (SBW) and ongoing operations at the ICPP produce liquid waste that contains low levels of radioactivity. The treatment of radioactive wastes for transfer out of Idaho is mandated under a "Settlement Agreement" between DOE and the State of Idaho. The Settlement Agreement requires that the existing inventory of liquid high-level radioactive waste (HLW) be calcined by June 30,1998, and SBW by the end of 2012, and the target date for all waste to be ready to ship from the INEEL is 2035 .

The Department of Energy Idaho Operations Office (DOE-DD) is preparing the High-Level Waste and Facilities Disposition Environmental Impact Statement (HLW\&FD EIS), in accordance with the National Environmental Policy Act, to support the decision-making process regarding HLW management at the INEEL. The Fluor Daniel Corporation and the INEEL operating contractor, Lockheed Martin Idaho Technologies Company (LMITCO), have been tasked with performing studies that provide the basis for portions of the EIS. Waste treatment options include dissolving the calcine; both the dissolved calcine and the liquid SBW would then be separated into high-activity waste (HAW) and low-activity waste (LAW). The HAW would be mixed with a glass matrix and poured into canisters. These are termed the "Separations Options." Alternative options that do not involve a separation step are termed the "Nonseparations Options." The Waste Treatment Studies consider the several alternative processes for preparing the calcine and liquid waste for ultimate removal from the INEEL: Separations [(Full and transuranic (TRU)] and Nonseparations (Vitrified, Hot Isostatic Press, Cementitious, and Direct Cementitious). Fluor Daniel Corporation is studying the Full Separations alternative and the others are being studied by LMTTCO. The product of each of the processes will be waste in a storage canister that is ready for shipment to a permanent repository. Because the repositories will not be capable to receive the canisters at the time they are produced, interim storage will be required at the INEEL. This report documents cost, schedule, and environmental impact for interim storage of the waste generated with the above processes in an Interim Storage Facility (ISF). A new ISF for the Full Separations alternative is included in the Fluor Daniel Corporation Study. This study includes a modified existing facility study for interim storage for the Full Separations Option.

\subsection{Objective and Scope of Work}

The objective of this study is to explore alternatives for interim storage of the waste in canisters that result from SBW and HLW processing of the calcine and liquid waste at ICPP. Table 1 gives the processing options and associated data that are included in this study. 
Table 1. Waste processing options and study data.

\begin{tabular}{|c|c|c|c|c|c|}
\hline & stopge repe & 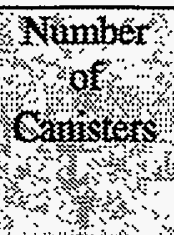 & 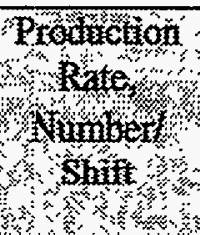 & 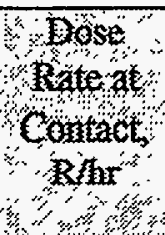 & $\begin{array}{l}\text { Oeatit } \\
\text { Waits } \\
\text { Cunister }\end{array}$ \\
\hline \multirow{2}{*}{$\begin{array}{c}\text { Separations Options } \\
\text { Full Separations }\end{array}$} & & & & & \\
\hline & $\begin{array}{l}\text { Modified } \\
\text { Existing Facility } \\
\end{array}$ & 780 & & $2.4-2500$ & $25-220$ \\
\hline \multirow[t]{3}{*}{ TRU/Class A Separations } & New Facility & 170 & $1 /$ month & 160,000 & 12,350 \\
\hline & NUHOMS ${ }^{T M}$ & 170 & $1 /$ month & 160,000 & 12,350 \\
\hline & $\begin{array}{l}\text { Modified } \\
\text { Existing Facility }\end{array}$ & 170 & $1 /$ month & 160,000 & 12,350 \\
\hline \multirow{3}{*}{$\begin{array}{c}\text { Nonseparations Options: } \\
\text { Vitrified }\end{array}$} & & & & & \\
\hline & New Facility & 14,000 & 6 & $6.1-172$ & $0.6-14.7$ \\
\hline & $\begin{array}{l}\text { Modified } \\
\text { Existing Facility }\end{array}$ & 14,000 & 6 & $6.1-172$ & $0.6-14.7$ \\
\hline \multirow[t]{2}{*}{ Direct Cementitious } & New Facility & 18,000 & 35 & $6.9-287$ & $0.4-16.4$ \\
\hline & $\begin{array}{l}\text { Modified } \\
\text { Existing Facility }\end{array}$ & 18,000 & 35 & $6.9-287$ & $0.4-16.4$ \\
\hline \multirow[t]{3}{*}{ Cementitious } & New Facility & 16,000 & 32 & 140 & 8.9 \\
\hline & \begin{tabular}{|l|} 
Modified \\
Existing Facility \\
\end{tabular} & 16,000 & 32 & 140 & 8.9 \\
\hline & $\begin{array}{l}\text { HIP Preliminary } \\
\text { Plan }\end{array}$ & $\begin{array}{l}31,000 \\
\text { drums }\end{array}$ & 32 & 140 & 8.9 \\
\hline \multirow[t]{2}{*}{ Hot Isostatic Press } & New Facility & 5,700 & 9 & $14-580$ & $1.7-61$ \\
\hline & $\begin{array}{l}\text { Modified } \\
\text { Existing Facility }\end{array}$ & 5,700 & 9 & $14-580$ & $1.7-61$ \\
\hline
\end{tabular}

The objective of this study was to determine the cost, schedule, and environmental impacts for the storage of the waste canisters that result from each of the processing options in Table 1. A new facility was studied that incorporates appropriate design features from the Fluor Daniel Corporation design for interim storage presented in their document, Idaho Chemical Processing Plant, Waste Treatment Facilities, Feasibility Study Report, December 12, 1997 (see Reference 2). Modification of existing facilities at ICPP for storage of the waste was considered. The Fuel Processing Restoration (FPR) facility is a large unfinished and unoccupied building that has large cells that can be used for waste storage. This facility was considered the most attractive existing facility at ICPP for interim storage. The Irradiated Fuel Storage Facility (IFSF) is another facility that was considered for modification for interim storage, but space limitations and current use made it impractical for interim storage of HLW. The storage of canisters resulting from the Full Separations Option in NUHOMS ${ }^{\mathrm{TM}}$ modules was the subject of report, DPC Loading Feasibility Study Report, INEEL/EXT-97-01251, November 1997 (see Reference 3). This study considers storage of the canisters that result from the TRU/Class A Separations Option in the NUHOMS ${ }^{\mathrm{TM}}$ module. The TRU/Class A Separations option would generate three waste streams designated as LLW: LAW, HAW, and TRU waste. All of these waste streams are actually considered HLW. It is expected that the LAW and TRU meet the definition of Nuclear Regulatory Commission (NRC) LLW Class A and TRU, respectively. However, these wastes do not conform to the existing classification scheme for radioactive waste. Therefore, they will need the evaluation and concurrence of 
appropriate government authorities (NRC and DOE) for reclassification. Only the HAW will require interim storage. The LAW is assumed to go directly to a Class A waste disposal site and the TRU waste to go directly to the Waste Isolation Pilot Plant (WIPP). The TRU/Class C Separations Option does not require interim storage.

A preliminary plan for the Cementitious and Direct Cementitious Options is under consideration. The resulting grout would be placed into drums within a 5-year period and placed into the ISF. Over a 20-year period, the drums would be subjected to a Hot Isostatic Press (HIP) process that would further reduce the volume. This alternative was included in this study. 


\section{DESIGN BASIS, KEY ASSUMPTIONS, AND REQUIREMENTS}

\subsection{Design Basis}

A facility is required near the processing facility to store the HLW from the time it leaves the processing facility until it can be moved to a permanent waste repository. The quantities of canisters and their characteristics given in Table 1 result from the studies for the processing alternatives. The design life for the facilities is from 40 to 60 years.

The Savanna River Site (SRS) HLW and the West Valley Demonstration Project HLW canisters meet the repository Waste Acceptance Criteria (WAC) for dimensions, although there are significant differences in these canisters' physical makeup. The SRS canister is used as the baseline for this study because it was selected in the ICPP Waste Treatment Facilities Feasibility Study Report. ${ }^{2}$ However, the DOE Hanford Facility has adopted a 4.5-m (15-ft) long canister for containing immobilized $\mathrm{HLW}$. This longer canister is apparently acceptable for co-disposal at the repository, and therefore should be considered before finalizing the canister size. Consideration of the $4.5-\mathrm{m}$ (15-ft) canister is addressed in the recommendations in Section 9. Some cost increase would result because the head height for handling the longer canisters is greater, and depth of excavation, equipment capacities, etc. increase. Cost increases would also occur because of the increase in the ISF transport tunnel height, canister handling machine size and capacity, and the ceiling height. However, smaller ISFs may be adequate with fewer canisters, and handling, shipping, and final disposal operations with fewer canisters would offset the cost increases if the longer canisters were adopted. The net result of the cost changes is unknown.

Transport of the waste to the ISF is included in this study, however transport from the facility is not included. Equipment for handling the canisters for movement into and from the facility is included.

A passive cooling system is desired. The additional safety issues and operating costs of an active system would thus be avoided.

\subsection{Key Assumptions}

The following assumptions formed the framework, i.e., and the directed charter, for this study. Therefore, although some of these assumptions are likely to change, and such changes may have a significant impact on the results of this study, it is beyond the scope of this study to predict such changes or evaluate the associated risk.

1. The quantities and characteristics of waste to be stored are tabulated in Table 1 .

2. The waste will be stored in the SRS canister described in the Engineering Design File, EDF-DPC001, Appendix E of DPC Loading Feasibility Study Report, INEEL/EXT-97-01251, November 1997.

3. The processed waste is not Resource Conservation and Recovery Act (RCRA) hazardous waste as a result of delisting before processing and by virtue of processing when all "listed" hazardous and characteristic constituents are treated to the Land Disposal Regulation standards, then the waste is no longer classed as RCRA waste.

4. The LAW and TRU waste will be reclassified as LLW Class A and TRU, respectively. 
5. The facility will be licensed by the NRC in accordance with the requirements of 10 CFR Part 72 . Existing facilities may not have been built to these requirements and replacement or upgrading of the components may not be possible. In these cases, it is assumed that the NRC will consider equivalence or alternative approaches and issue the license.

6. The processing facilities will not have lag storage. If the ISF cannot handle the canisters at the rate produced by the facility as given in Table 1, the ISF must have the capability to store them in a lag storage area. This lag storage would only be used if the processing facility releases the canisters in a batch or for maintenance of the canister handling equipment in the ISF.

7. The handling equipment will interface with an MP-187 cask for canister shipment from the ISF.

8. Physical security requirements are enveloped by those for SNF.

9. Dual Purpose Canisters (DPCs) will be acceptable for storage under 10 CFR Part 72.

10. The maximum weight of a canister is $5,300 \mathrm{lb}$.

\subsection{Requirements}

\subsubsection{Regulations}

The NRC in accordance with 10 CFR Part 72 will license the storage facility, however the additional cost for licensing is not included in the cost estimates. It is assumed that the canisters, as received from the processing facility, will meet Department of Transportation (DOT), NRC, and/or HLW Repository WAC requirements and require no further conditioning, testing, treatment, marking, etc. This requires that the waste will be excluded from RCRA hazardous waste management requirements at the INEEL due to successful delisting and/or treatment.

The ISF will be licensed to meet 10 CFR Part 72 for HLW; therefore the receipt, handling, packaging, and storage of canisters of waste must also be governed under 10 CFR Part 72. Changes required to the facility license must be evaluated through a 10 CFR 72.48 evaluation, issuance of a Safety Analysis Report, and licensing documentation amendments. It is assumed that the Department of Energy Idaho Operations Office (DOE-ID) will continue to implement the quality assurance program described in DOE-RW-0333P, Quality Assurance Requirements and Description ${ }^{4}$ and will apply it to this effort. The impact of implementing DOE-RW-0333P is reflected in the capital and operating cost estimates generated by this feasibility study.

\subsubsection{HLW Repository WAC}

The requirements of the potential future repository waste acceptance will be satisfied by the processing facility before the canisters being released to the ISF. The ISF will not provide any capability to process, modify, or change the waste or canisters.

\subsubsection{Applicable Codes and Standards}

The NRC licenses and regulates waste storage facilities in accordance with 10 CFR Part 72. DOE requirements that are included in 10 CFR Part 72 will be superseded by them, however any DOE requirements that are not covered by 10 CFR Part 72 will also apply. 


\section{INTERIM STORAGE FACILITY DESIGNS}

\subsection{Basic Design Concepts}

\subsubsection{Design Philosophy}

The Waste Treatment Facilities Studies that this report supports are discussed in reports covering the various treatment options. This study provides information about the interim storage required for each of the treatment options. A new facility has been studied that can be built near the processing facility. The design features of the Fluor Daniel Corporation interim storage facility ${ }^{2}$ have been incorporated into the new facility discussed here to the extent that the features were applicable. This allows comparison and use of common cost estimates. The modification of an existing facility at ICPP for this storage is also presented. Features of the Fluor Daniel Corporation design ${ }^{2}$ are used for this facility also where applicable. The flow of $H L W$ from the processing facility through the storage period and until it is shipped from INEEL to a permanent repository is shown in Figure 1.

\subsubsection{New Interim Storage Facility}

EDF-IS-001, New Facility for Interim Storage of HLW, Appendix A, documents the study for an ISF at ICPP to store the waste that results from the various processing alternatives. The number of canisters produced from the processing options range from 170 to 18,000 . The layout for an ISF has been arranged to accommodate modular sizing so that the core facility containing the receiving and shipping area, canister handling equipment, lag storage, and office and support space is centrally located. The alternatives discussed in EDF-IS-001 contain the required number of modules of storage vaults. Each module contains 630 storage tubes. Each tube can hold three canisters stacked vertically with space for cushioning material at the bottom and between each canister. Tests have been performed on the canisters to verify that they can be dropped from a height of 9 meters without rupture. Anytime the canisters are handled at a height that would allow a drop distance in excess of 9 meters $(30 \mathrm{ft}$ ), cushioning material must be provided to limit the impact to the equivalent impact for the 9 meters ( $30 \mathrm{ft}$ ). The ISF will be provided with cushioning material to satisfy this requirement. Storage of the canisters three high allows for a more compact facility than a two high arrangement would require. Cushioning can be provided to allow the handling of the canisters at this height.

An office area is provided similar to the one provided in the Fluor Daniel Corporation design. ${ }^{2}$ The gantry crane is also like the one included in the Fluor Daniel Corporation design. ${ }^{2}$ A transfer tunnel is included for transport of the canisters from the processing facility to the storage facility. The tunnel will be belowgrade to have the benefit of the soil for shielding. A load out-bay is provided to load the canisters into a shipping cask similar to the MP-187 cask that is mounted on a railroad car. The plot plan shows the railroad spur to the facility, however, the cost of the spur in not included in the cost estimate. It is included in a cost estimate that covers the infrastructure for the ICPP for all of the alternatives. This cost estimate is found in Utilities and Infrastructure Report, NEEL/EXT-98-01398, February 1998 (see Reference 5).

The handling crane is sized to handle the largest expected load, which is $\mathbf{5 0}$ tons. It is mounted in a gantry arrangement to allow it to locate the handling machine accurately over the storage positions. In some of the designs, a crane maintenance area is provided at the ends of the building. In the alternatives involving fewer canisters, the crane maintenance will occur over the central module. 
The new ISF facilities presented in this study have been used in the processing option studies as the baseline storage facility. This has been done to be consistent so that a direct cost comparison can be made. 


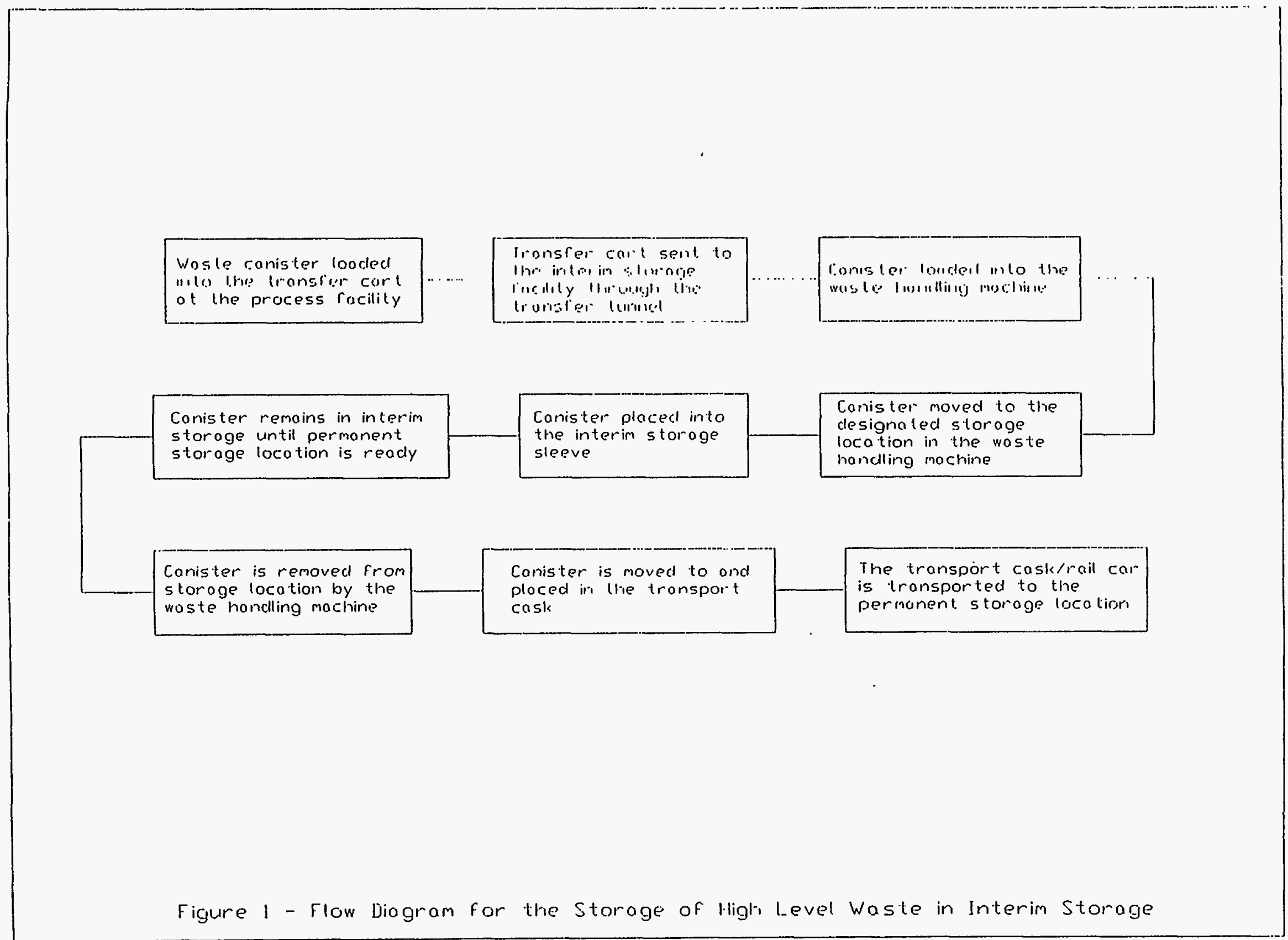




\subsubsection{Modified Existing Interim Storage Facility}

EDF-IS-002, Modification of FPR for Interim Storage of HLW (see Appendix A) describes the modification of the FPR Facility at ICPP. This facility was investigated for storage of the HLW.

The FPR Facility is a large unfinished and unoccupied building that was in the final stages of construction when stopped. The outside of the building is complete and the concrete and structure of the interior is in place. The lighting and life support systems were not installed. The facility has shielded cells that were investigated to determine suitability for interim storage. Modifications to the facility are required to interface with a transfer tunnel from the processing facility, a gantry crane to handle the canisters, pipe sleeves to store the canisters, and a load-out facility to transfer the canisters to a shipping cask for shipment to a repository.

The maximum number of canisters that can be stored in FPR was determined, assuming that the inner concrete walls are removed to form one cell of all cells 1 through 12 and the pump and valve corridor. It is also assumed that the storage sleeves can be located next to each other without the need for a cooling air path through the array. The maximum number of $66-\mathrm{cm}$ (26-inch) diameter storage sleeves that can be placed in the cell is 2,336 . With four canisters in each sleeve, a maximum of 9,344 canisters can be stored. This quick look at the facility reduced the subsequent investigation for the various processing options. The options with greater than 9,344 canisters are out of the question for storage in FPR. The options with fewer than 9,344 can conceivably be stored in FPR. Therefore, only the Full and TRU/Class A Separations Options and the HIP Waste Option (HWO) were investigated further.

The IFSF (CPP-603) was investigated for suitability for storage of HLW for the processing options that have small numbers of canisters. This facility was determined to not be suitable for the following reasons:

1. The facility is partly loaded with Fort St. Vrain fuel that will not be removed from IFSF until a permanent repository is available.

2. The storage configuration at IFSF is for canisters made from $46-\mathrm{cm}$ (18-inch) diameter pipe.

3. Expansion of the storage area to provide space suitable for the HLW canisters was an attractive storage alternative until a recent modification of the facility added concrete walls that make this unattractive. The walls were added because of seismic concerns with the temporary walls.

The HLW canisters cannot be stored in the current configuration. The storage configuration cannot be modified with the Fort St. Vrain fuel in storage. Expansion to provide space designed for HLW but able to use the basic facility features is no longer an attractive storage alternative. Therefore, the use of IFSF for HLW interim storage was not investigated further.

\subsection{Interim Storage for Full Separations Option}

A new facility for storage of waste processed by the full separation option is included in the Fluor Daniel Corporation report ${ }^{2}$ and therefore, is not part of this study.

\subsubsection{Modified Existing Facility}

The 780 canisters of waste material that result from the Full Separations Option can be stored in the FPR as shown in the drawings in Appendix 2 of EDF-IS-002. The drawings show the building 
modifications required for handling of the canisters and receiving and removal of the canisters. The building must be completed to include such items as lighting, rest rooms, alarms, etc., that are included in the cost estimate as "life safety" equipment.

\subsection{Interim Storage for TRU/Class A Separations Option}

The discussion on the TRU/Class A Separations Waste Option (TRU/Class A) is documented in the report, TRU Separations Option Study Report, INEEL/EXT-01428, February 1998 (see Reference 6).

\subsubsection{New Storage Facility}

A new storage facility has been studied to store the 170 canisters of $\mathrm{HLW}$, the only waste stream that requires interim storage, from the TRU/Class A Separations processing option. The facility is discussed in EDF-IS-001 and the drawings are in Appendix 2 of that EDF. Because of the small number of canisters (170), the basic facility design is modified to only have one canister in each storage sleeve. The design studied was one vault of the modular design that can store 630 canisters. The additional capacity would be available for other storage.

\subsubsection{NUHOMSTM Storage}

Assuming space for 75 DPCs is available on the pad for the ISFSI/NUHOMS storage area, the 170 canisters produced in the TRU/Class A Separations Option can be placed into 43 DPCs and stored in NUHOM ${ }^{\mathrm{TM}}$ storage casks on the existing pad. This scheme for storage is addressed in detail in the report, DPC Loading Feasibility Study Report, INEEL/EXT-97-01251 (see Reference 3). Assuming the modifications at the Dry Transfer Cell (DTC) would be required or additions at the processing facility having a similar cost are needed and the portion of the DPC/NUHOMS ${ }^{\mathrm{TM}}$ Procurement for 43 casks instead of 195 , the estimated cost for a storage facility would be $\$ 73,400,000$.

\subsubsection{Modified Existing Facility}

The 170 canisters of waste material that result from the Full Separations Option can be stored in the FPR as shown in the drawings in Appendix 2 of EDF-IS-002. The drawings show the building modifications required for handling of the canisters and receiving and removal of the canisters. The building must be completed to include such items as lighting, rest rooms, alarms, etc., that are included in the cost estimate as "life safety" equipment.

\subsection{Interim Storage for Vitrified Waste Option}

The discussion on the Vitrified Waste Option (VWO) is documented in the report, Vitrified Waste Option Study Report, INEEL/EXT-01389, February 1998 (see Reference 7).

\subsubsection{New Storage Facility}

A new storage facility has been studied to store the 14,000 canisters that require interim storage with this processing option. The facility is discussed in EDF-IS-001 and the drawings in Appendix B of that EDF. The facility consists of eight vaults capable of storing 15,120 canisters with them stacked three high. 


\subsubsection{Modified Existing Facility}

The FPR Facility can be modified to contain a maximum of 9,344 and, therefore, cannot store the 14,000 canisters produced by this processing option. A more realistic storage capability for FPR is 5,896 as discussed in Section 3.7.2 for the HIP processing option. In addition to modification of FPR, a new storage facility would be required to store the additional 8,104 canisters. This would require a five-vault size facility.

\subsection{Interim Storage for Direct Cementitious Waste Option}

The discussion on the Direct Cementitious Waste Option (DCWO) is documented in the report, Direct Cementitious Waste Option Study Report, INEEL/EXT-01399, February 1998 (see Reference 8).

\subsubsection{New Storage Facility}

A new storage facility has been studied to store the 18,000 canisters that require interim storage with this processing option. The facility is discussed in EDF-IS-001 and the drawings in Appendix 2 of that EDF. The facility consists of 10 vaults capable of storing 18,900 canisters with them stacked three high.

\subsubsection{Modified Existing Facility}

The FPR Facility can be modified to contain a maximum of 9,344 and, therefore, cannot store the 18,000 canisters produced by this processing option. A more realistic storage capability is 5,896 as discussed in Section 3.7.2 for the HIP processing option. In addition to modification of FPR, a new storage facility would be required to store the additional 12,104 canisters. This would require a sevenvault size facility.

\subsubsection{New Storage Facility for the HIP Preliminary Plan}

A preliminary plan is being considered for the DCWO that adds another process, HIP, that further reduces the waste volume. The number of canisters that results is estimated to be 11,700. An ISF with seven modules would be required to store the waste for this plan.

\subsection{Interim Storage for Cementitious Waste Option}

The discussion on the Cementitious Waste Option (CWO) is documented in the report, Cementitious Waste Option Study Report, INEEL/EXT-01389, February 1998 (see Reference 9).

\subsubsection{New Storage Facility}

A new storage facility has been studied to store the 16,000 canisters that require interim storage with this processing option. The facility is discussed in EDF-IS-001 and the drawings in Appendix 2 of that EDF. The facility consists of nine vaults capable of storing 18,900 canisters with them stacked three high. 


\subsubsection{Modified Existing Facility}

The FPR Facility can be modified to contain a maximum of 9,344 and, therefore, cannot store the 16,000 canisters produced by this processing option. A more realistic storage capability is 5,896 as discussed in Section 3.7.2 for the HIP processing option. In addition to modification of FPR, a new storage facility would be required to store the additional 10,104 canisters. This would require a six-vault size facility.

\subsubsection{New Storage Facility for the HIP Preliminary Plan}

A preliminary plan is being considered for the CWO that allows the waste to be processed in a 5-year period and stored in drums later to be subjected to a HIP process that would further reduce the volume of waste. The later process would be performed over a 20 -year period. Interim storage of the drums is required until processed and for the canisters that the HIPed drums are placed in after processing. The estimated number of drums is 31,000. For information about the drums and this process, refer to EDF-CWO-001 in Appendix E of the report Cementitious Waste Option Study Report, INEEL/EXT-01389 (see Reference 9). The drums can be stored in the same storage sleeves that are planned for the canisters. A special handling grapple would be required to handle the drums in addition to the handling equipment for canisters. The grapple must be capable of lowering the drums into the storage sleeves and releasing from the drum. The grapple must also be capable of the reverse operation to remove the drums for processing. The unprocessed drums are $142-\mathrm{cm}$ (56 inches) long, therefore, six can be stored in each storage sleeve. The maximum storage requirement will be at the end of 5 years of operation when 7,750 drums have been HIPed into 2,583 canisters. The facility will be required, at the end of the $5^{\text {th }}$ year of operation, to also store 23,250 drums. The canisters and drums will require 4,736 storage sleeves or eight modules, the same size as is needed for the VIT Option, which is one module smaller than required for the CWO option in Section 3.6.1. The cost for the facility is, therefore, the same as for the VIT Option with the additional cost for the handling equipment for the drums.

\subsection{Interim Storage for Hot Isostatic Press Waste Option}

The discussion on the HIP Option is documented in the report, Hot Isostatic Press Waste Option Report, INEEL/EXT-01392, February 1998 (see Reference 10).

\subsubsection{New Storage Facility}

A new storage facility has been studied to store the 5,700 canisters that require interim storage with this processing option. The facility is discussed in EDF-IS-001 and the drawings in Appendix 2 of that EDF. The facility consists of four vaults capable of storing 7,560 canisters with them stacked three high.

\subsubsection{Modified Existing Facility}

The 5,700 canisters of waste material that result from the HIP Option can be stored in the FPR as shown in the drawings in Appendix 2 of EDF-IS-002. The drawings show the building modifications required for handling of the canisters and receiving and removal of the canisters. The building must be completed to include such items as lighting, rest rooms, alarms, etc., that are included in the cost estimate as "life safety" equipment. 


\section{TOTAL PROJECT, LIFE-CYCLE, AND D\&D COSTS}

Cost estimates for the interim storage alternatives are in Appendix 1 of the respective EDFs (EDFIS-001 and EDF-IS-002). These EDFs are in Appendix A of this report. The costs are summarized in Table 2. 
Table 2. Project Costs

\begin{tabular}{|c|c|c|c|c|c|}
\hline \multirow[t]{4}{*}{ Cost item. } & \multicolumn{4}{|c|}{ Separations Options } & \multirow{3}{*}{$\begin{array}{l}\text { Vitrifie } \\
\text { New } \\
\text { Facili }\end{array}$} \\
\hline & Eull Séparations & \multicolumn{3}{|c|}{ TRU/Glass A Separations } & \\
\hline & $\begin{array}{c}\text { Módified Existing } \\
\text { Facility }\end{array}$ & $\begin{array}{c}\text { New } \\
\text { Faclilty }\end{array}$ & NUHOMS & $\begin{array}{l}\text { Modified Existing } \\
\text { Facility }\end{array}$ & \\
\hline & $\$$ & $\$$ & S: & $\$$ & $\$$ \\
\hline OPC (unescalated) & $13,941,000$ & $15,228,000$ & Not & $13,168,000$ & 16,2 \\
\hline OPC Escalation & $4,469,000$ & $4,118,000$ & Calculated & $4,747,000$ & 4,15 \\
\hline OPC Mgmt Reserve & 0 & 0 & & 0 & \\
\hline OPC Contingency & $4,590,000$ & $4,854,000$ & & $4,484,000$ & 5,11 \\
\hline Total OPC & $23,000,000$ & $24,200,000$ & & $22,400,000$ & $25,5 C$ \\
\hline TEC (unescalated) & $89,245,000$ & $66,308,000$ & Not & $77,219,000$ & $382,7 \varepsilon$ \\
\hline TEC Escalation & $44,166,000$ & $30,078,000$ & Calculated & $34,353,000$ & 138,31 \\
\hline TEC Mgmt Reserve & $9,188,000$ & $6,647,000$ & & $7,686,000$ & 40,06 \\
\hline TEC Contingency & $19,901,000$ & $14,267,000$ & & $16,942,000$ & $65,0<$ \\
\hline Total TEC & $162,500,000$ & $117,300,000$ & & $136,200,000$ & $626,2 C$ \\
\hline TPC (unescalated) & $103,186,000$ & $81,536,000$ & - Not & $90,387,000$ & 399,01 \\
\hline TPC Escalation & $48,634,000$ & $34,195,000$ & Calculated & $39,100,000$ & $142,4 \epsilon$ \\
\hline TPC Mgmt Reserve & $9,188,000$ & $6,647,000$ & & $7,686,000$ & 40,06 \\
\hline TPC Contingency & $24,491,000$ & $19,121,000$ & & $21,426,000$ & 70,16 \\
\hline Total TPC & $185,500,000$ & $141,500,000$ & $73,400,000$ & $158,600,000$ & 651,70 \\
\hline Operations (unescalated) & $45,526,000$ & $41,433,000$ & Not & $45,431,000$ & 149,75 \\
\hline Operations Escalation & $90,825,000$ & $80,511,000$ & Calculated & $88,164,000$ & 172,52 \\
\hline Operations Contingency & $40,905,000$ & $36,583,000$ & & $40,079,000$ & 96,68 \\
\hline Total Operations & $177,257,000$ & $158,527,000$ & & $173,674,000$ & 418,96 \\
\hline Post Operations (unescalated) & $29,049,000$ & $22,749,000$ & Not & $25,399,000$ & 106,26 \\
\hline Post Operations Escalation & $182,326,000$ & $142,784,000$ & Calculated & $159,413,000$ & 666,95 \\
\hline Post Operations Contingency & $63,413,000$ & $49,660,000$ & & $55,444,000$ & 231,96 \\
\hline Total Post Operations & $274,788,000$ & $215,193,000$ & & $240,256,000$ & $1,005,18$ \\
\hline Total Cost (unescalated) & $177,761,000$ & $145,718,000$ & Not & $161,217,000$ & 65 \\
\hline $\begin{array}{l}\text { Total Cost (w/escalation, mgt reserve, } \\
\text { and contingency) }\end{array}$ & $637,545,000$ & $515,220,000$ & Calculated & $572,529,000$ & $2,075,84$ \\
\hline Discounted Cost (escalated) & $105,247,000$ & $89,476,000$ & & $97,808,000$ & 426,04 \\
\hline
\end{tabular}




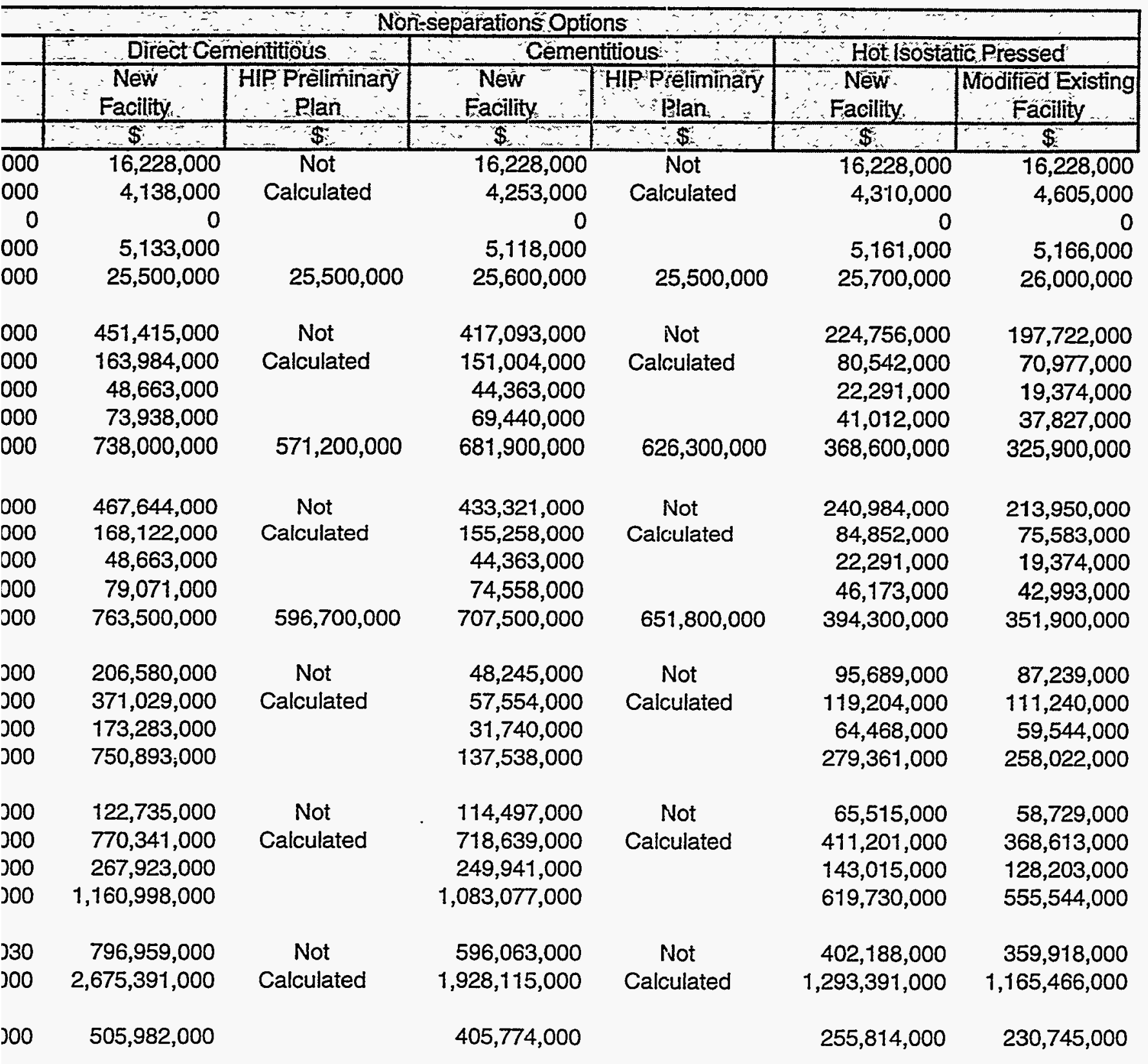




\section{PROJECT SCHEDULE}

Figure 2 for each processing option and interim storage alternative show the schedules for the installation or modification of the storage facilities. 
Figure 2. Project Schedule.

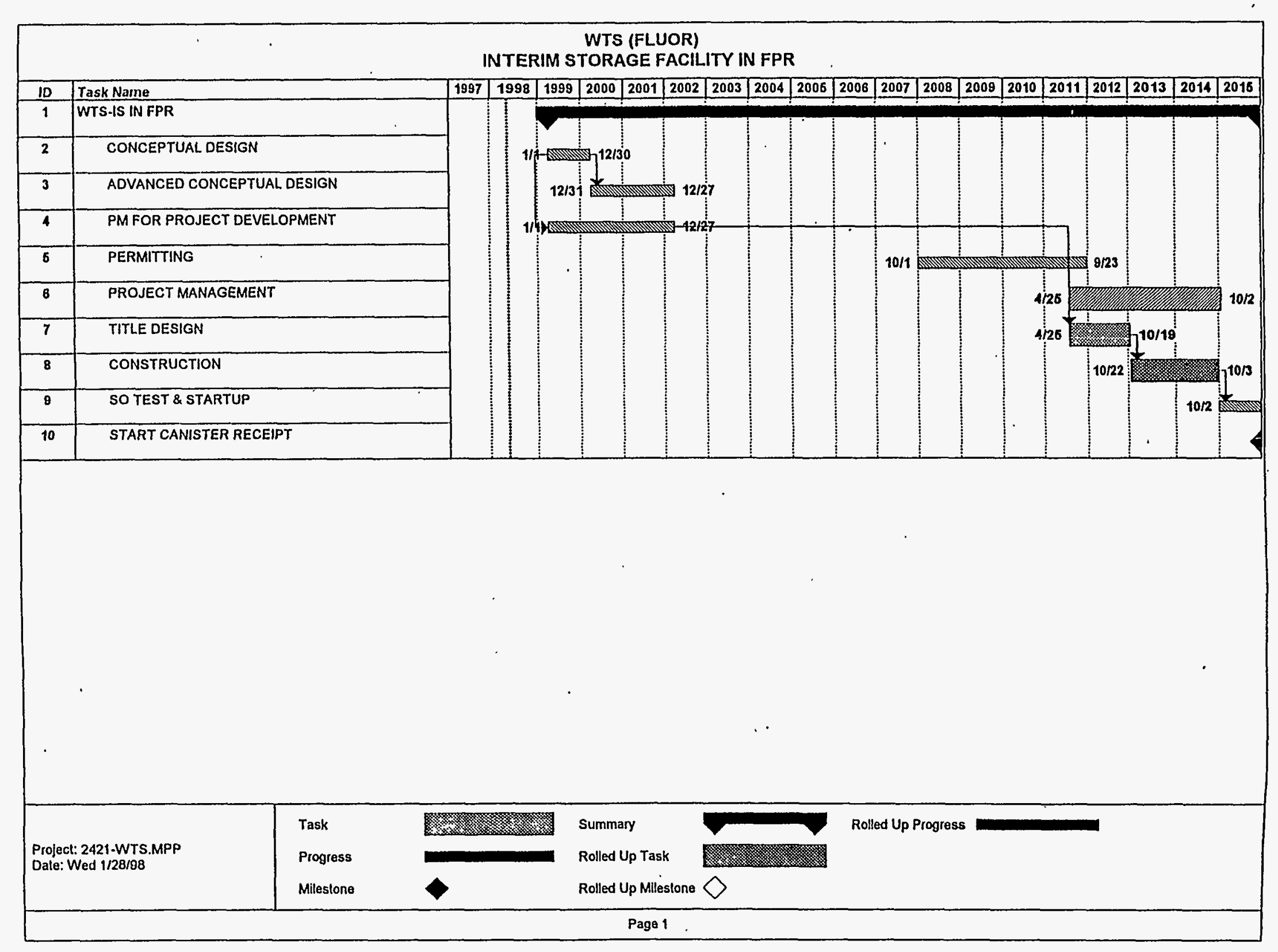


Figure 2. (continued).

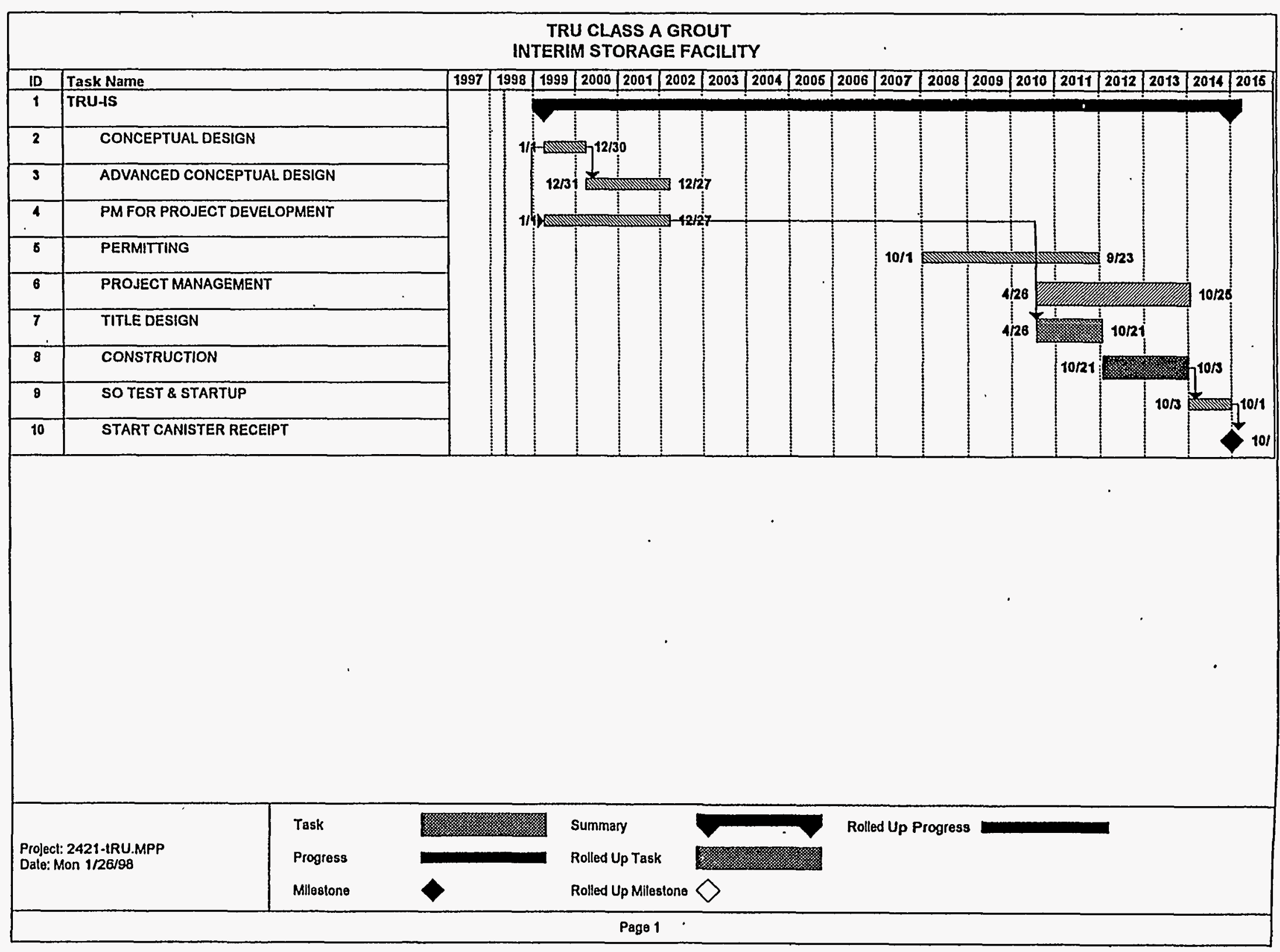


Figure 2. (continued).

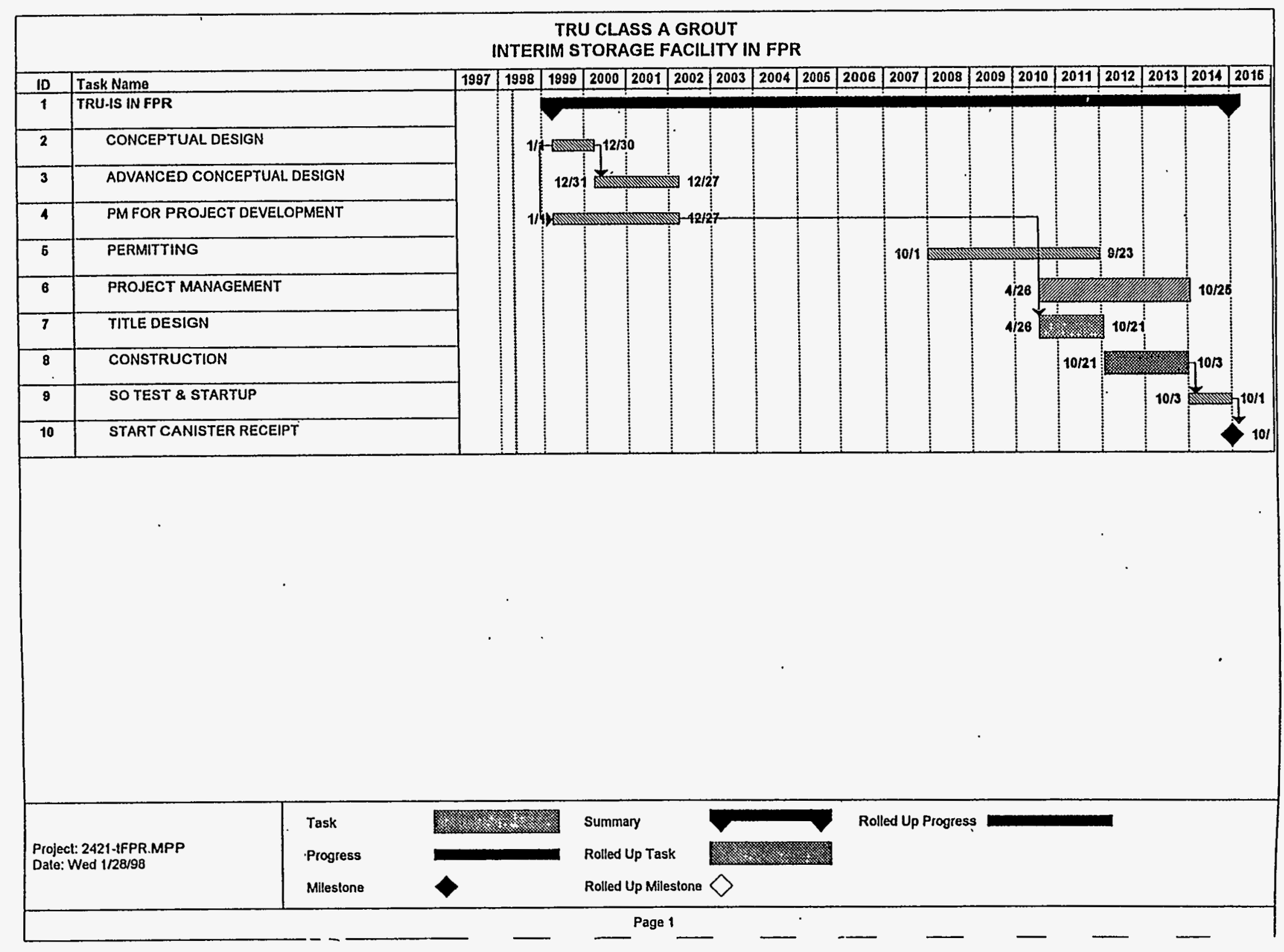




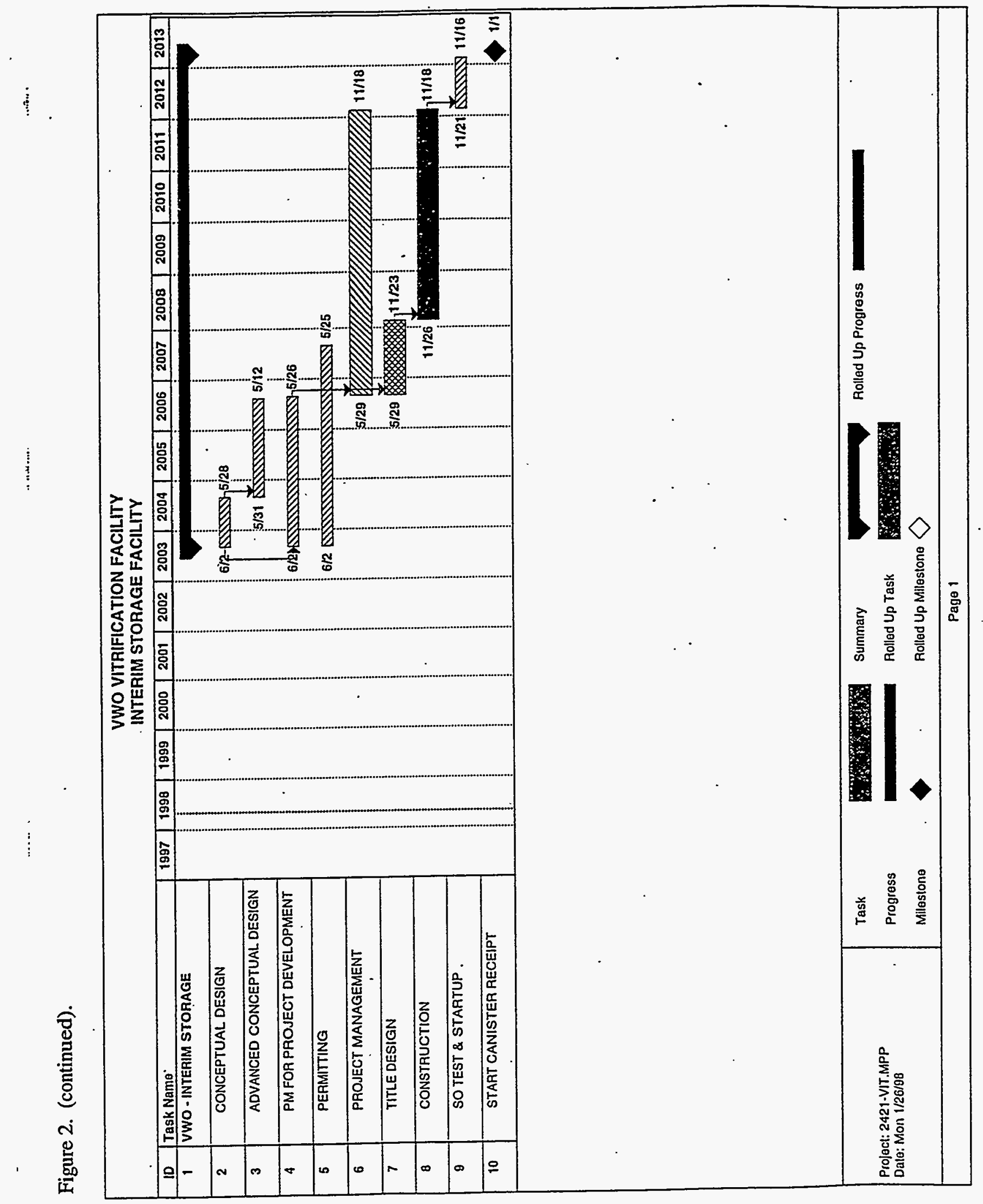


Figure 2. (continued).

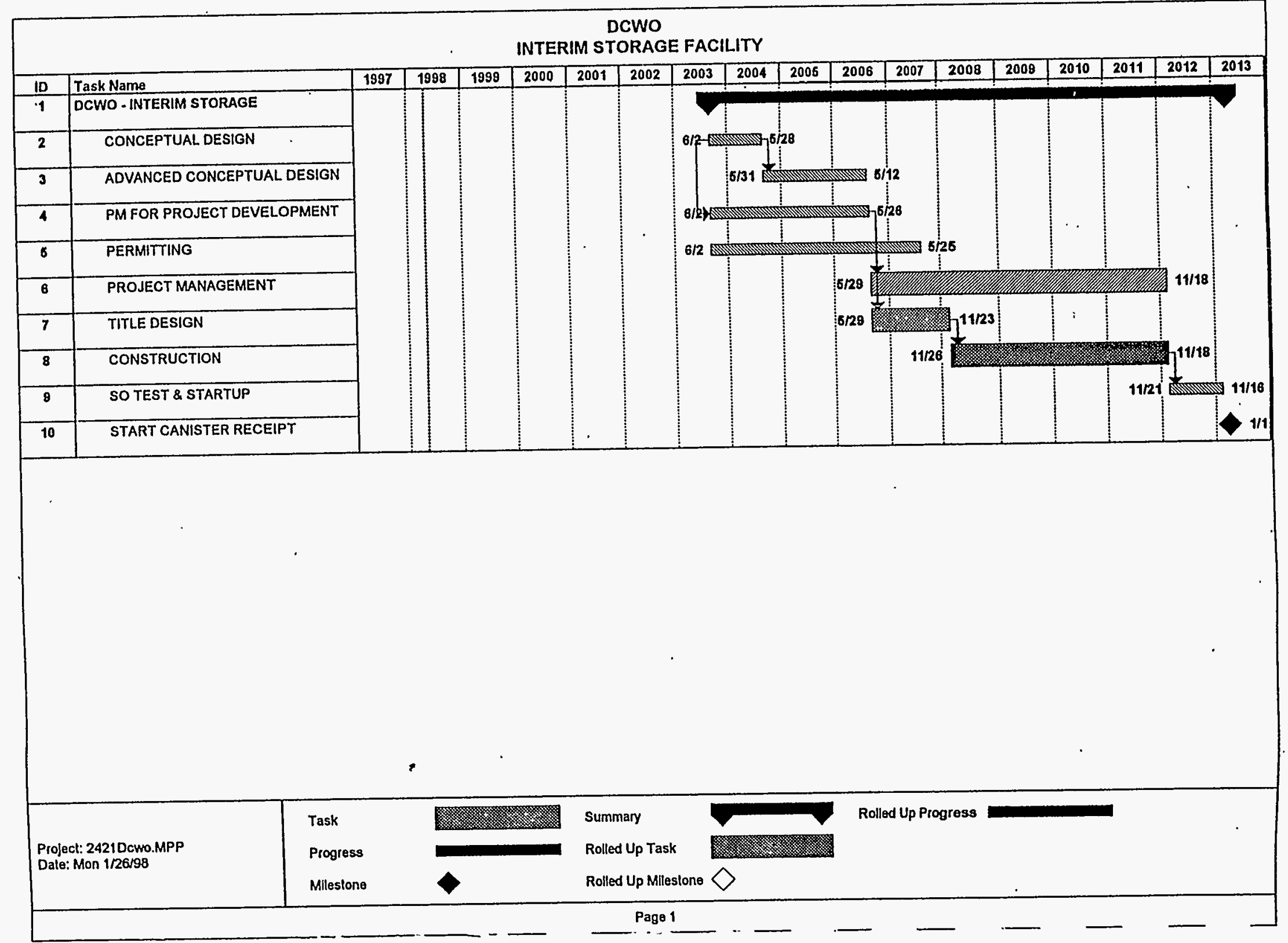


Figure 2. (continued).

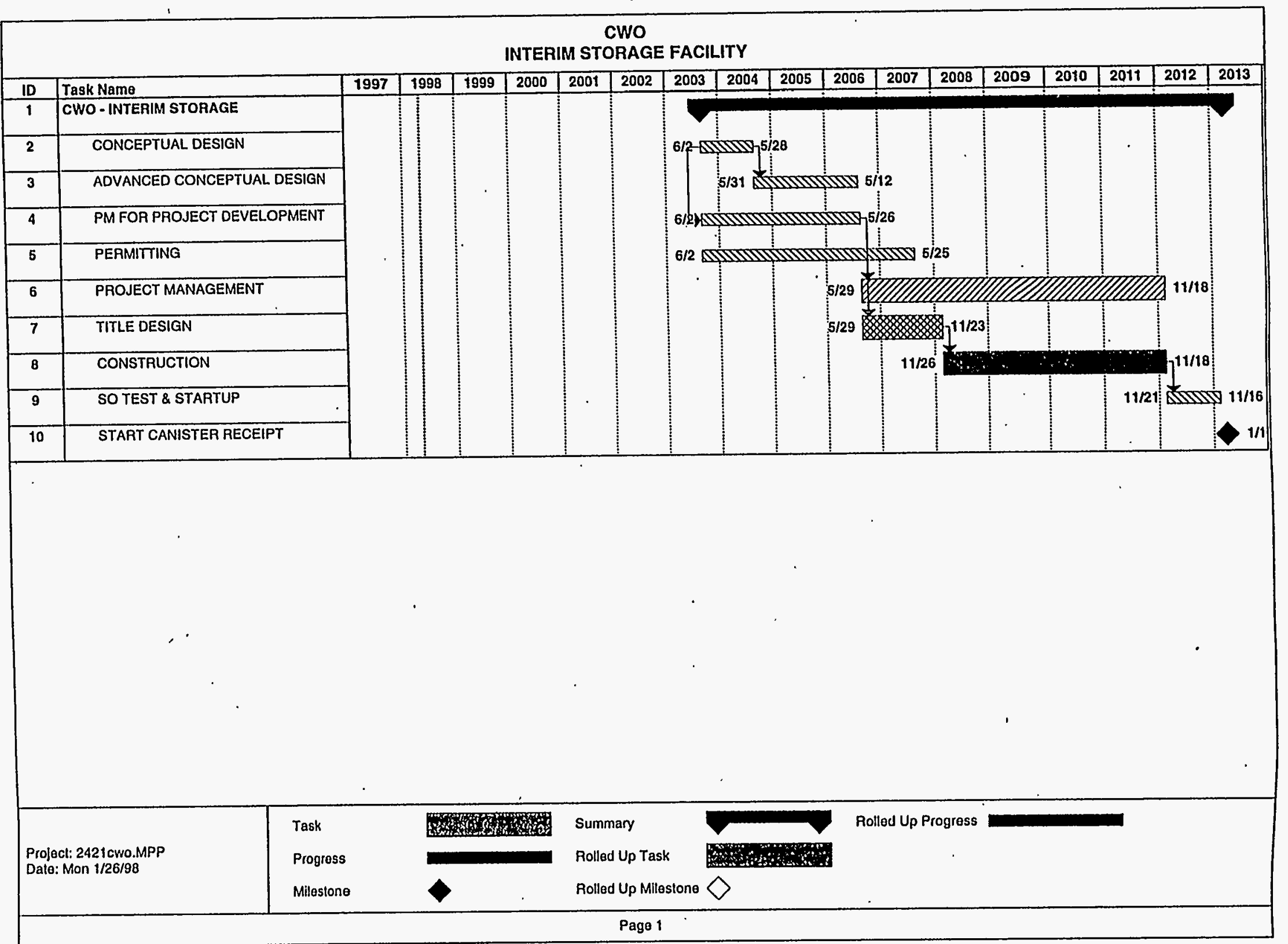


Figure 2. (continued).

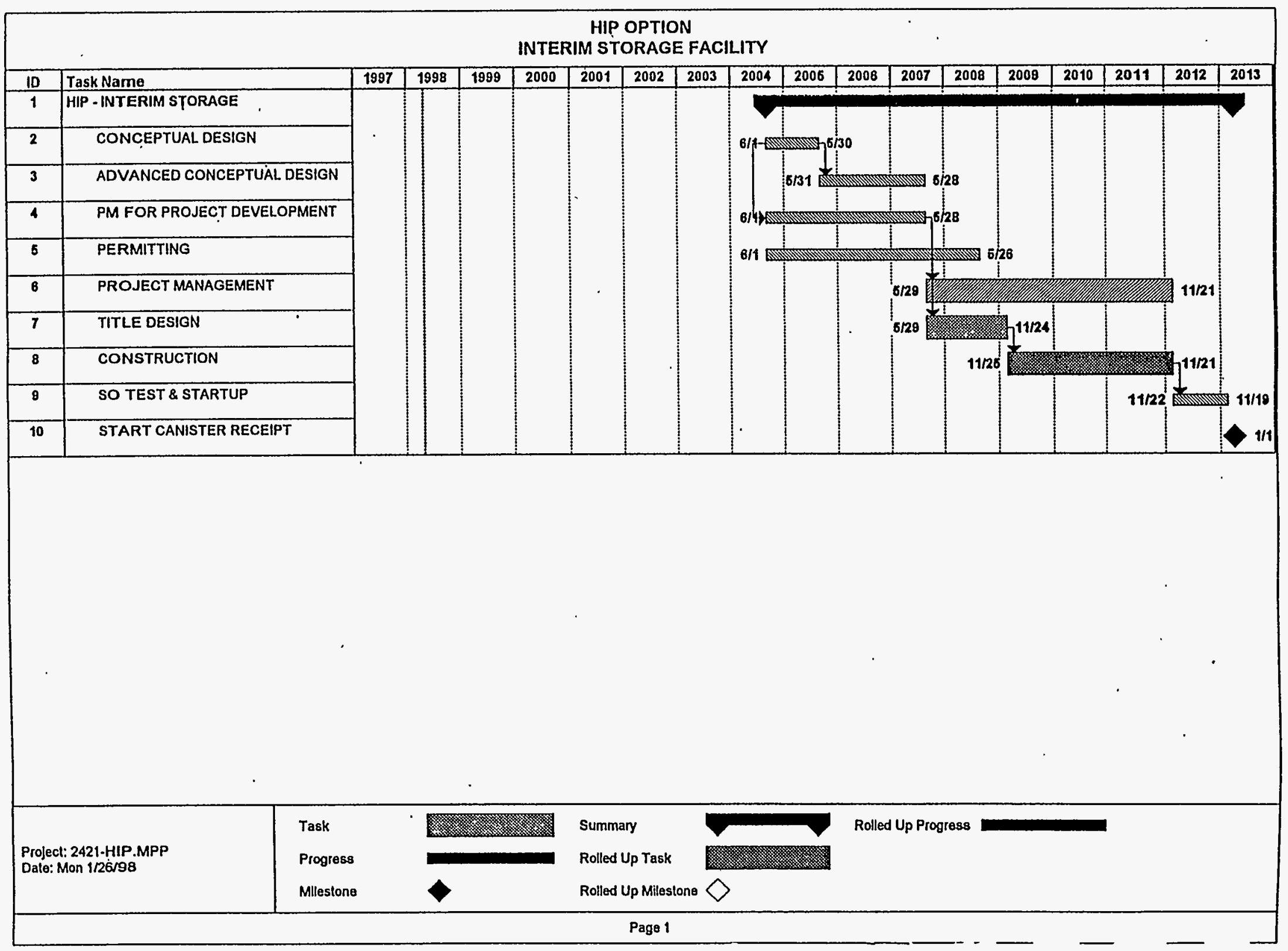




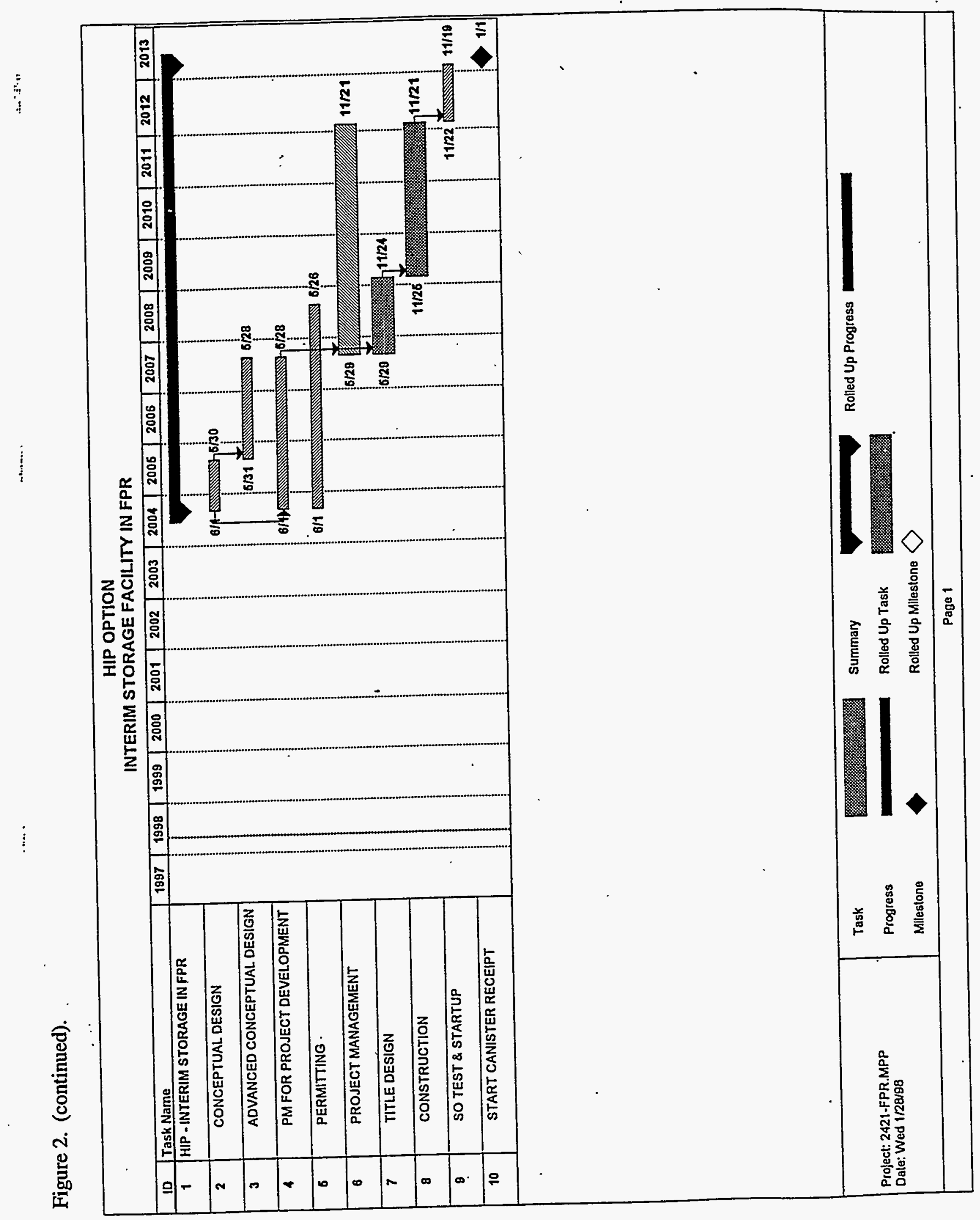




\section{RISK ASSESSMENT}

High-level risks identified in this study are categorized below. The assumptions in Section 2.2 provide the framework for this list. The highest risk is rated at 4. Data Sheets for all the identified risks and explanation of the risk rating calculation are included in Appendix 4 of the respective EDFs (EDF-IS001 and EDF-IS-002). These EDFs are in Appendix A of this report.

1. The quantities and characteristics of the waste are different than assumed in the study. Risk $=3$ (Probability = 3, Impact $=1$ ).

2. Waste will not be stored in the SRS canister. Risk $=2$ (Probability $=2$, Impact $=1$ ).

3. The facility would have to be redesigned if the waste is classified as a RCRA waste after processing. Risk $=3$ (Probability $=1$, Impact $=3$ ).

4. The obtaining of the NRC license for the FPR would be more difficult than assumed. Risk $=4$ (Probability $=2$, Impact $=2$ ).

5. A storage canister will be mishandled or dropped during waste transfer to, from, or in the facility. Risk $=2$ (Probability $=1$, Impact $=2$ ).

6. A catastrophic flood could flood the FPR Facility. Risk $=2$ (Probability $=1$, Impact $=2$ ).

7. The facility might not be operational when needed. Risk $=2$ (Probability $=1$, Impact $=2$ ).

Many risks including environmental, safety, and health risks were not identified in this study. 


\section{CONSTRUCTION AND OPERATIONAL ENVIRONMENTAL IMPACTS}

The Product Data Sheets that summarize the construction and operational environmental impacts for the interim storage facilities that support the waste processing options are in Table 3. Data are presented for the construction, operation, and decommissioning of the project. Estimates are included for the following parameters:

- Cost

- Schedule

- Air emissions

- Liquid effluents

- Solid wastes

- Utilities used

- Manpower requirements

- Regulatory requirements.

Because this project is at a very preliminary stage of design, much of the information presented in the Project Data Sheets is based on representative literature values, existing laboratory data, and engineering judgment. The cost data in Table 3 were obtained from the cost estimates in Appendix 1 and the supporting information for the remainder of the data is in Appendix 5 of the respective EDFs (EDF-IS-001 and EDF-IS-002) in Appendix A of this report.

The Product Data Sheets are presented in the following order in Table 3. Product Data Sheets were not prepared for the TRU/Class A Separations NUHOMS ${ }^{\top M}$ Option, and the HIP Preliminary Plan for the DCWO and CWO.

\begin{tabular}{|l|l|}
\hline Full Separations - IS in the FPR & I \\
\hline TRU/Class A Separations - New ISF & PDS for Storage at FPR for WFS \\
\hline TRU/Class A Separations - IS in the FPR & PDS for Interim Storage Facility for TRU \\
\hline Vitrified & PDS for Storage at FPR for TRU \\
\hline Direct Cementitious & PDS for Interim Storage Facility for VWO \\
\hline Cementitious & PDS for Interim Storage Facility for DCWO - 20 Yr \\
\hline Hot Isostatic Press - New ISF & PDS for Interim Storage Facility for CWO \\
\hline Hot Isostatic Press - IS in the FPR & PDS for Interim Storage Facility for HIP \\
\hline
\end{tabular}


Table 3. Project data sheet for Interim Storage.

\section{Project Data Sheet for Interim Storage Facility for TRU}

Generic Information

Description/function

EIS Alternatives (A, B, etc.)

Project type or waste stream

Action type

Structure type

Size $\left(\mathrm{m}^{2}\right)$

Other features (e.g. pits, ponds, power/water/sewer lines)

Location

Inside/outside of fence

Inside/outside of building

Candidate for privatization?
Provide Long-term storage for road-ready containers awaiting shipment to a permanent repository.

TRU Separations Waste Option

Treated HLW calcine.

New

$3,350 \mathrm{~m}^{2}$

None

Inside ICPP fence

Inside TRU Separation Facility

Yes

\section{Construction Information}

Cost (\$): Pre-Operations (Other Project Costs) with escalation an

Conceptual Design

Management for Project Development

Permitting and Documentation

SO Test \& Start-Up

Contingency

Total Preconstruction

Cost (\$): Construction with escalation and contingency.

ED\&I

Management (PM/CM)

Construction

G\&APIF

Procurement Fees, Management Reserve and Contingency

Total Construction (TEC)

Schedule start/end: Preconstruction

Schedule start/end: Construction

Schedule start/end: SO Test \& Start-up

Number of workers each vear of construction (new/existing)

Nonradiation

Number of radiation workers (construction)

Average annual worker radiation dose (rem/yr)

\section{Heavy equipment}

Equipment used

Trips (construction materials delivery)

Hours of operation

Acres disturbed and duration of disturbance

New

Previous
$\$ 4,240,000$

$\$ 4,520,000$

$\$ 3,656,000$

$\$ 6,930,000$

$\$ 4,854,000$

$\$ 24,200,000$

$\$ 10,601,000$

$\$ 10,177,000$

$\$ 42,405,000$

$\$ 2,701,000$

$\$ 15,116,000$

$\$ 81,000,000$

January 1999 through December 2001

January 2010 through December 2013

January 2014 through December 2014

\author{
58 New workers/yr \\ None \\ None
}

Excavator, dump trucks, cranes, cement trucks,grader, material delivery

1,156

5,287 hours (total)

January 2010 through December 2013

None

3.0 acres 
Table 3. (continued).

Revegetated

Air emissions

Dust

Major gases $\left(\mathrm{CO}_{2}, \mathrm{H}_{2} \mathrm{O}, \mathrm{O}_{2}, \mathrm{~N}_{2}\right)$ from diesel exhaust

Contaminants (Particulates, $\mathrm{CO}, \mathrm{NO}_{\mathrm{x}} \mathrm{SO}_{2}$, hydrocarbons) from diesel exhaust.

Total air emissions

Effluents

Sanitary wastewater

Lube Oil

Solid wastes

Type - construction trash

Radioactive wastes

Hazardous/toxic chemicals and wastes (type)

Storage/inventory

Hazardous waste (construction)

Pits/Ponds created $\left(\mathrm{m}^{2}\right)$

Water usage:

Dust control

Domestic water

Energy requirements

Electrical (MWh/yr)

Fossil fuel (liters)

Permits needed for construction

\section{Project Data Sheet for Interim Storage Facility for TRU \\ None}

86 tons (total)

4,814 tons (total)

28 tons (total)

4,928 tons (total)

$2,469,713$ liters (total) 1,001 liters (total)

$1,375 \mathrm{~m}^{3}$ (total)

None

\author{
$13 \mathrm{~m}^{3}$ \\ $217 \mathrm{~m}^{3}$ (total) \\ None
}

616,810 liters (total)

$2,469,713$ liters (total)

\author{
$156 \mathrm{MWh} / \mathrm{yr}$ \\ 0 liters (total)
}

NEPA documentation (prior to start of Title II construction); New stationary sources/PTC/NOC/PSD for non-rad air emissions; HAP's and TAP's and RCRA (part $A A, B B$, and CC for air) for hazardous air emissions; air operating permit; NESHAP's, NPDES, NESHAP's subpart $H$ for rad air emissions; approval of Engineering Plans; Cross Connection Control Plans; report and specifications for drinking water supply;RCRA Part A and Part B permits.

\section{Operational Information}

Cost (\$): Operations (unescalated)

Facility/Administration

$\$ 7,007,000$

Operations/Process Facility

$\$ 17,581,000$

$\$ 16,845,000$

Procurement, materials, utilities, maintenance

$\$ 41,433,000$

Schedule start/end

January 2014 through December 2068

Number of workers each year of operation (new/existing) 
Tāble 3. (continued).

Operations

Maintenance

Support

Total

Number of radiation workers

Average annual work radiation dose (rem/yr)

Heavy Equipment

Air Emissions

Radioactive off-gas

Effluents

Sanitary Wastewater

Solid Wastes

Sanitary/Industrial Trash

Radioactive wastes (canisters)

Hazardous/toxic chemicals and wastes

Pits/Ponds used $\left(\mathrm{m}^{2}\right)$

Water usage:

Domestic water

Energy Requirements:

Electrical (MWh/yr)

Fossil fuel (liters/yr)

Permits needed (for facility operations)

\section{Project Data Sheet for Interim Storage Facility for TRU}

3

0.5

3

6.5

2.5 (included in above total)

$0.19 \mathrm{rem} / \mathrm{yr}$ perworker

None

None

224,498 liters/yr

$$
36 \mathrm{~m}^{3} / \mathrm{yr}
$$$$
122 \mathrm{~m}^{3} / \mathrm{yr}
$$

None

224,498 liters/yr

\section{2,533 MWh/yr}

None

HAP's and TAP's and RCRA (part AA,BB, and CC for air) for hazardous air emissions; air operating permit; NESHAP's, NPDES, NESHAP's subpart $H$ for rad air emissions; approval of Engineering Plans; Cross Connection Control Plans; report and specifications for drinking water supply;RCRA Part A and Part B permits.
Decontamination \& Decommissioning (D\&D) Information

Cost (\$): D\&D (unescalated)

Decommission

Decontamination

Demolition

Total D\&D

Schedule start/end: D\&D

Number of workers each year of D\&D (new/existing)

Number of radiation workers (D\&D)

Average annual worker radiation dose (rem/yr)
$\$ 3,180,000$

$\$ 0$

$\$ 19,569,000$

$\$ 22,749,000$

January 2069 through December 2071

53 New workers/yr

5 New workers/yr

$0.19 \mathrm{rem} / \mathrm{yr}$ per worker

Mobile Cranes, Roll-off trucks, Dozers, Loaders

Heavy equipment:

Equipment used 
Table 3. (continued).

Trips

Project Data Sheet for Interim Storage Facility for TRU

Hours of operation (all heavy equipment)

Acres disturbed and duration of disturbance

New

Previous

Revegetated

3 per day

9,480 Hours

January 2069 through December 2071

None

3.0 acres

None

\title{
Air emissions
}

non-radioactive Fuel combustion gases $\left(\mathrm{CO}_{2}, \mathrm{H}_{2} \mathrm{O}, \mathrm{O}_{2}, \mathrm{~N}_{2}\right)$

non-radioactive Fuel combustion contaminants ( $\mathrm{CO}$,

radioactive HEPA filtered off-gas

5,510 tons (total)

32 tons (total)

None tons (total)

\section{Effluents}

radioactive Spent decontamination solution

non-radioactive Sanitary wastewater

non-radioactive Lube oil

None liters (total)

$3,414,175$ liters (total)

1,794 liters (total)

Solid wastes:

radioactive

Non-radioactive (industrial)

Hazardous

$$
\begin{array}{r}
10,663 \mathrm{~m}^{3} \\
2 \mathrm{~m}^{3} \\
23 \mathrm{~m}^{3}
\end{array}
$$

Hazardous/toxic chemicals and wastes (type)

Storage/inventory

Pits/Ponds created $\left(\mathrm{m}^{2}\right)$

Hazardous Waste

None $\mathrm{m}^{3}$ (total)

None

$909 \mathrm{~m}^{3}$ (total)

Water usage:

Domestic water

Source of water

$3,414,175$ liters (total)

ICPP site wells

Energy requirements:

Electrical (MWh/yr)

Fossil fuel (liters)

Permits needed (e.g. for facility closures, physical characteristics

and quantities of radioactive and hazardous materials remaining

after closure)

\author{
$156 \mathrm{MWh} / \mathrm{yr}$ \\ 215,291 liters (total)
}

Work will be done under closure provisions of existing permits. 
Table 3. (continued).

\section{Project Data Sheet for Interim Storage Facility for VWO}

\section{Generic Information}

Description/function

EIS Alternatives ( $A, B$, etc.)

Project type or waste stream

Action type

Structure type

Size $\left(\mathrm{m}^{2}\right)$

Other features (e.g. pits, ponds, power/water/sewer lines)

Location

Inside/outside of fence

Inside/outside of building

Candidate for privatization?
Provide Long-term storage for road-ready containers awaiting shipment to a permanent repository.

Vitrified Waste Option

Treated HLW calcine.

New

$$
\begin{aligned}
& 13,493 \mathrm{~m}^{2} \\
& \text { None }
\end{aligned}
$$

Inside ICPP fence

Inside Vitrification Facility

\section{Construction Information}

Cost (\$): Pre-Operations (Other Project Costs) with escalation and contingency.

Conceptual Design

Management for Project Development

Permitting and Documentation

SO Test \& Start-Up

Contingency

Total Preconstruction

Cost (\$): Construction with escalation and contingency.

\section{ED\&!}

Management (PM/CM)

Construction

G\&APIF

Procurement Fees, Management Reserve and Contingency

Total Construction (TEC)

Schedule start/end: Preconstruction

Schedule start/end: Construction

Schedule start/end: SO Test \& Start-up

Number of workers each year of construction (new/existing)

Nonradiation

Number of radiation workers (construction)

Average annual worker radiation dose (rem/yr)

\section{Heavy equipment}

Equipment used

Trips (construction materiais delivery)

Hours of operation

Acres disturbed and duration of disturbance

New

Previous
$\$ 5,000,000$

$\$ 4,000,000$

$\$ 2,728,432$

$\$ 4,500,000$

$\$ 7,571,568$

$\$ 23,800,000$

$\$ 35,000,000$

$\$ 55,337,700$

$\$ 273,376,540$

$\$ 16,334,715$

$\$ 79,451,045$

$\$ 459,500,000$

January 2003 through December 2007

January 2006 through December 2011

January 2012 through December 2012

114 New workers/yr

None

None

Excavator, dump trucks, cranes, cement trucks,grader, material delivery

$$
2,191
$$

13,683 hours (total)

January 2006 through December 2011

None

5 acres 
Table 3. (contịnued).

Revegetated

\section{Project Data Sheet for Interim Storage Facility for WWO \\ None}

\section{Air emissions}

Dust

Major gases $\left(\mathrm{CO}_{2}, \mathrm{H}_{2} \mathrm{O}, \mathrm{O}_{2}, \mathrm{~N}_{2}\right)$ from diesel exhaust

Contaminants (Particulates, $\mathrm{CO}, \mathrm{NO}_{\mathrm{x}}, \mathrm{SO}_{2}$, hydrocarbons) from diesel exhaust.

Total air emissions

Effluents

Sanitary wastewater

Lube Oil

\section{Solid wastes}

Type - construction trash

Radioactive wastes

Hazardous/toxic chemicals and wastes (type)

Storage/inventory

Hazardous waste (construction)

Pits/Ponds created $\left(\mathrm{m}^{2}\right)$

Water usage:

Dust control

Domestic water

Energy requirements

Electrical (MWh/yr)

Fossil fuel (liters)

Permits needed for construction
288 tons (total)

13,189 tons (total)

77 tons (total)

13,554 tons (total)

$9,708,525$ liters (total)

2,590 liters (total)

$5,406 \mathrm{~m}^{3}$ (total)
None

$13 \mathrm{~m}^{3}$

$217 \mathrm{~m}^{3}$ (total)

None

$2,056,032$ liters (total)

$9,708,525$ liters (total)

$156 \mathrm{MWh} / \mathrm{yr}$

0 liters (total)

NEPA documentation (prior to start of Title II construction); New stationary

sources/PTC/NOC/PSD for non-rad air emissions;

HAP's and TAP's and RCRA (part AA,BB, and CC for air) for hazardous air emissions; air operating permit; NESHAP's, NPDES, NESHAP's subpart $H$ for rad air emissions; approval of Engineering Plans; Cross Connection Control Plans; report and specifications for drinking water supply;RCRA Part A and Part B permits.
Operational Information

Cost (\$): Operations (unescalated)

Facility/Administration

Operations/Process Facility

Procurement, materials, utilities, maintenance

Total operations

Schedule start/end
$\$ 7,293,000$

$\$ 18,299,000$

$\$ 124,165,000$

$\$ 149,757,000$

January 2012 through December 2068

Number of workers each year of operation (new/existing) 
Táble 3. (continued).

Operations

Maintenance

Support

Total

Number of radiation workers

Average annual work radiation dose (rem/yr)

Heavy Equipment

Air Emissions

Radioactive off-gas

Effluents

Sanitary Wastewater

Solid Wastes

Sanitary/Industrial Trash

Radioactive wastes (canisters)

Hazardous/toxic chemicals and wastes

Pits/Ponds used $\left(\mathrm{m}^{2}\right)$

Water usage:

Domestic water

Enerav Requirements:

Electrical (MWh/yr)

Fossil fuel (liters/yr)

Permits needed (for facility operations)

\section{Project Data Sheet for Interim Storage Facility for WWO}

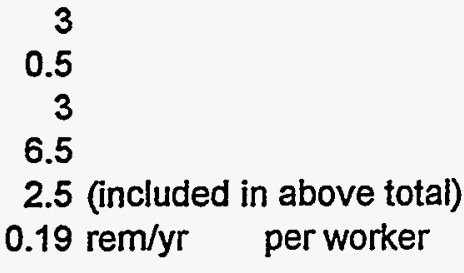

None

None

224,498 liters/yr

$$
\begin{array}{r}
36 \mathrm{~m}^{3} / \mathrm{yr} \\
10,584 \mathrm{~m}^{3} / \mathrm{yr}
\end{array}
$$

None

224,498 liters/yr

4,368 MWh/yr
None

HAP's and TAP's and RCRA (part AA,BB, and CC for air) for hazardous air emissions; air operating permit; NESHAP's, NPDES, NESHAP's subpart H for rad air emissions; approval of Engineering Plans; Cross Connection Control Plans; report and specifications for drinking water supply;RCRA Part A and Part B permits.
Decontamination \& Decommissioning (D\&D) Information

Cost (\$): D\&D (unescalated)

Decommission

Decontamination

Demolition

Total D\&D

Schedule start/end: D\&D

Number of workers each year of D\&D (new/existing)

Number of radiation workers (D\&D)

Average annual worker radiation dose (rem/yr)
$\$ 10,500,000$

$\$ 0$

$\$ 95,783,000$

$\$ 106,283,000$

January 2069 through December 2071

249 New workers/yr

25 New workers/yr

$0.19 \mathrm{rem} / \mathrm{yr}$ perworker

Mobile Cranes, Roll-off trucks, Dozers, Loaders

Heavy equipment:

Equipment used 
Table 3. (continued).

Project Data Sheet for Interim Storage Facility for VWO

Trips Roll-off trucks

Hours of operation (all heavy equipment)

9 per day

50,220 Hours

Acres disturbed and duration of disturbance

New

Previous

Revegetated

January 2069 through December 2071

None

5.0 acres

None

Air emissions

non-radioactive Fuel combustion gases $\left(\mathrm{CO}_{2}, \mathrm{H}_{2} \mathrm{O}, \mathrm{O}_{2}, \mathrm{~N}_{2}\right)$

29,189 tons (total)

Fuel combustion contaminants $(\mathrm{CO}$,

non-radioactive particulates, $\mathrm{NO}_{x}, \mathrm{SO}_{2}$, hydrocarbons)

170 tons (total)

radioactive HEPA filtered off-gas

None tons (total)

\section{Effluents}

radioactive Spent decontamination solution

non-radioactive Sanitary wastewater

non-radioactive Lube oil

None liters (total)

$15,898,593$ liters (total)

9,504 liters (total)

Solid wastes:

radioactive

Non-radioactive (industrial)

Hazardous

$$
\begin{aligned}
\text { None } \mathrm{m}^{3} \\
42,946 \mathrm{~m}^{3} \\
8 \mathrm{~m}^{3}
\end{aligned}
$$

Hazardous/toxic chemicals and wastes (type)

Storage/inventory

Pits/Ponds created $\left(\mathrm{m}^{2}\right)$

Hazardous waste

$$
\begin{aligned}
& \text { None } \mathrm{m}^{3} \text { (total) } \\
& \text { None } \\
& 909 \mathrm{~m}^{3} \text { (total) }
\end{aligned}
$$

Water usage:

Domestic water

Source of water

$15,898,593$ liters (total)

ICPP site wells

Energy requirements:

Electrical (MWh/yr)

Fossil fuel (liters)

Permits needed (e.g. for facility closures, physical characteristics

and quantities of radioactive and hazardous materials remaining after closure)

$$
\begin{gathered}
156 \mathrm{MWh} / \mathrm{yr} \\
1,140,496 \text { liters (total) }
\end{gathered}
$$

Work will be done under closure provisions of existing permits. 
Table 3. (continued).

\section{Project Data Sheet for Interim Storage Facility for DCWO - 20 yr}

\section{Generic Information}

Description/function

EIS Alternatives (A, B, etc.)

Project type or waste stream

Action type

Structure type

Size $\left(\mathrm{m}^{2}\right)$

Other features (e.g. pits, ponds, power/water/sewer lines)

Location

Inside/outside of fence

Inside/outside of building

Candidate for privatization?
Provide Long-term storage for road-ready containers awaiting shipment to a permanent repository.

Direct Cementitious Waste Option

Treated HLW calcine.

New

$$
\begin{aligned}
& 15,967 \mathrm{~m}^{2} \\
& \text { None }
\end{aligned}
$$

Inside ICPP fence

Inside Grouting Facility

Yes

Construction information

Cost (\$): Pre-Operations (Other Project Costs) with escalation an

Conceptual Design

Management for Project Development

Permitting and Documentation

So Test \& Start-Up

Contingency

Total Preconstruction

Cost (\$): Construction with escalation and contingency.

\section{ED\&I}

Management (PM/CM)

Construction

G\&AVIF

Procurement Fees, Management Reserve and Contingency

Total Construction (TEC)

Schedule start/end: Preconstruction

Schedule start/end: Construction

Schedule start/end: SO Test \& Start-up

Number of workers each year of construction (new/existing)

Nonradiation

Number of radiation workers (construction)

Average annual worker radiation dose (rem/yr)

Heavy equipment

Equipment used

Trips (construction materials delivery)

Hours of operation

Acres disturbed and duration of disturbance

New

Previous
$\$ 5,850,000$

$\$ 4,700,000$

$\$ 3,247,000$

$\$ 6,570,000$

$\$ 5,133,000$

$\$ 25,500,000$

$\$ 45,750,000$

$\$ 83,023,000$

$\$ 455,030,000$

$\$ 27,046,000$

$\$ 127,151,000$

$\$ 738,000,000$

January 2003 through December 2007

January 2006 through December 2011

January 2012 through December 2013

134 New workers/yr

None

None

Excavator, dump trucks, cranes, cement trucks,grader, material delivery

2,482

15,642 hours (total)

January 2006 through December 2011

None

9 acres 
Table 3. (continued).

Revegetated

\section{Project Data Sheet for Interim Storage Facility for DCWO - 20 yr \\ None}

Air emissions

Dust

Major gases $\left(\mathrm{CO}_{2}, \mathrm{H}_{2} \mathrm{O}, \mathrm{O}_{2}, \mathrm{~N}_{2}\right)$ from diesel exhaust

Contaminants (Particulates, $\mathrm{CO}, \mathrm{NO}_{x}, \mathrm{SO}_{2}$, hydrocarbons) from diesel exhaust.

Total air emissions

\section{Effluents}

Sanitary wastewater

Lube Oil

\section{Solid wastes}

Type - construction trash

Radioactive wastes

Hazardous/toxic chemicals and wastes (type)

Storage/inventory

Hazardous waste (construction)

Pits/Ponds created $\left(\mathrm{m}^{2}\right)$

Water usage:

Dust control

Domestic water

Energy requirements

Electrical (MWh/yr)

Fossil fuel (liters)

Permits needed for construction
518 tons (total)

15,023 tons (total)

88 tons (total)

15,629 tons (total)

$11,411,775$ liters (total)

2,960 liters (total)

$6,355 \mathrm{~m}^{3}$ (total)

None

$$
\begin{aligned}
& 13 \mathrm{~m}^{3} \\
& 217 \mathrm{~m}^{3} \text { (total) } \\
& \text { None }
\end{aligned}
$$

$3,700,858$ liters (total)

$11,411,775$ liters (total)

\author{
$156 \mathrm{MWh} / \mathrm{yr}$ \\ 586,966 liters (total)
}

NEPA documentation (prior to start of Title II construction); New stationary sources/PTC/NOC/PSD for non-rad air emissions; HAP'S and TAP's and RCRA (part AA,BB, and CC for air) for hazardous air emissions; air operating permit; NESHAP's, NPDES, NESHAP's subpart H for rad air emissions; approval of Engineering Plans; Cross Connection Control Plans; report and specifications for drinking water supply;RCRA Part A and Part B permits.
Operational Information

Cost (\$): Operations (unescalated)

Facility/Administration

Operations/Process Facility

Procurement, materials, utilities, maintenance

Total operations

Schedule start/end
$\$ 7,293,000$

$\$ 18,299,000$

$\$ 180,989,000$

$\$ 206,581,000$

January 2012 through December 2068

Number of workers each year of operation (new/existing) 
Table 3. (continued).

Operations

Maintenance

Support

Total

Number of radiation workers

Average annual work radiation dose (rem/yr)

Heavy Equipment

Air Emissions

Radioactive off-gas

Effluents

Sanitary Wastewater

Solid Wastes

Sanitary/Industrial Trash

Radioactive wastes (canisters)

Hazardous/toxic chemicals and wastes

Pits/Ponds used $\left(\mathrm{m}^{2}\right)$

Water usage:

Domestic water

Energy Requirements:

Electrical (MWh/yr)

Fossil fuel (liters/yr)

Permits needed (for facility operations)
4

0.5

4

8.5

2.5 (included in above total)

$0.19 \mathrm{rem} / \mathrm{yr}$ perworker

None

None

293,574 liters/yr

\author{
$47 \mathrm{~m}^{3} / \mathrm{yr}$ \\ $12,096 \mathrm{~m}^{3} / \mathrm{yr}$
}

None

293,574 liters/yr

\section{4,586 MWh/yr \\ None}

HAP's and TAP's and RCRA (part AA,BB, and CC for air) for hazardous air emissions; air operating permit; NESHAP's, NPDES, NESHAP's subpart H for rad air emissions; approval of Engineering Plans; Cross Connection Control Plans; report and specifications for drinking water supply;RCRA Part $A$ and Part B permits.

\section{Decontamination \& Decommissioning (D\&D) Information}

\section{Cost (\$): D\&D}

Decommission

Decontamination

Demolition

Total D\&D

Schedule startend: D\&D

Number of workers each year of D\&D (new/existing)

Number of radiation workers (D\&D)

Average annual worker radiation dose (rem/yr)

Heavy equipment:

Equipment used

$$
\begin{array}{r}
\$ 10,500,000 \\
\$ 0 \\
\$ 112,235,000 \\
\$ 122,735,000
\end{array}
$$

January 2069 through December 2071

287 New workers/yr

29 New workers/yr

$0.19 \mathrm{rem} / \mathrm{yr} \quad$ perworker

Mobile Cranes, Roll-off trucks, Dozers, Loaders 
Table 3. (continued).

\section{Project Data Sheet for Interim Storage Facility for DCWO - 20 yr}

Trips Roll-off trucks

Hours of operation (all heavy equipment)

12 per day

Acres disturbed and duration of disturbance

New

Previous

Revegetated

62,100 Hours

\section{Air emissions}

non-radioactive Fuel combustion gases $\left(\mathrm{CO}_{2}, \mathrm{H}_{2} \mathrm{O}, \mathrm{O}_{2}, \mathrm{~N}_{2}\right)$

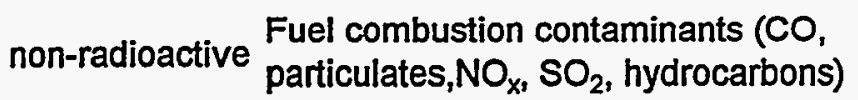

radioactive HEPA filtered off-gas

January 2073 through December 2075

None

9.0 acres

None

\section{Effluents}

radioactive Spent decontamination solution

non-radioactive Sanitary wastewater

non-radioactive Lube oil

36,094 tons (total)

211 tons (total)

None tons (total)

Solid wastes:

$5,109,750$ liters (total)

$18,346,756$ liters (total)

11,752 liters (total)

radioactive

Non-radioactive (industrial)

Hazardous

Hazardous/toxic chemicals and wastes (type)

Storage/inventory

Pits/Ponds created $\left(\mathrm{m}^{2}\right)$

radioactive (mixed waste)

$$
\begin{aligned}
50,817 & \mathrm{~m}^{3} \\
10 & \mathrm{~m}^{3} \\
108 & \mathrm{~m}^{3}
\end{aligned}
$$

Water usage:

Domestic water

Source of water

\section{Energy requirements:}

Electrical (MWh/yr)

Fossil fuel (liters)

Permits needed (e.g. for facility closures, physical characteristics and quantities of radioactive and hazardous materials remaining after closure)

$$
\begin{aligned}
& \text { None } \mathrm{m}^{3} \text { (total) } \\
& \text { None } \\
& 909 \mathrm{~m}^{3} \text { (total) }
\end{aligned}
$$

$18,346,756$ liters (total)

ICPP site wells

$\mathrm{Ci}$

$$
\begin{gathered}
156 \mathrm{MWh} / \mathrm{yr} \\
1,410,291 \text { liters (total) }
\end{gathered}
$$

Work will be done under closure provisions of existing permits. 
Table 3. (continued).

\section{Project Data Sheet for Interim Storage Facility for CWO}

\section{Generic Information}

Description/function

EIS Altematives (A, $B$, etc.)

Project type or waste stream

Action type

Structure type

Size $\left(\mathrm{m}^{2}\right)$

Other features (e.g. pits, ponds, power/water/sewer lines)

Location

inside/outside of fence

Inside/outside of building

Candidate for privatization?
Provide Long-term storage for road-ready containers awaiting shipment to a permanent repository.

Calcined Waste Option

Treated HLW calcine.

New

$14,725 \mathrm{~m}^{2}$

None

Inside ICPP fence

Inside Grouting Facility

\section{Construction Information}

Cost (\$): Pre-Operations (Other Project Costs) with escalation an

Conceptual Design

Management for Project Development

Permitting and Documentation

SO Test \& Start-Up

Contingency

Total Preconstruction

Cost (\$): Construction with escalation and contingency.

\section{ED\&l}

Management (PM/CM)

Construction

G\&AVIF

Procurement Fees, Management Reserve and Contingency

Total Construction (TEC)

Schedule start/end: Preconstruction

Schedule start/end: Construction

Schedule start/end: SO Test \& Start-up

Number of workers each year of construction (new/existing)

Nonradiation

Number of radiation workers (construction)

Average annual worker radiation dose (rem/yr)

Heavy equipment

Equipment used

Trips (construction materials delivery)

Hours of operation

Acres disturbed and duration of disturbance

New

Previous
$\$ 5,000,000$
$\$ 4,000,000$
$\$ 2,728,000$
$\$ 4,500,000$
$\$ 4,072,000$
$\$ 20,300,000$

$\$ 35,000,000$

$\$ 58,275,000$

$\$ 302,752,000$

$\$ 18,038,000$

$\$ 85,935,000$

$\$ 500,000,000$

January 2003 through December 2006

January 2006 through December 2011

January 2012 through December 2013

124 New workers/yr

None

None

Excavator, dump trucks, cranes, cement trucks,grader, material delivery

2,337

14,637 hours (total)

January 2006 through December 2011

None

9 acres 
Table 3. (continued).

Revegetated

Air emissions

Dust

Major gases $\left(\mathrm{CO}_{2}, \mathrm{H}_{2} \mathrm{O}, \mathrm{O}_{2}, \mathrm{~N}_{2}\right)$ from diesel exhaust

Contaminants (Particulates, $\mathrm{CO}, \mathrm{NO}_{x}, \mathrm{SO}_{2}$, hydrocarbons) from

diesel exhaust.

Total air emissions

\section{Effluents}

Sanitary wastewater

Lube Oil

Solid wastes

Type - construction trash

Radioactive wastes

Hazardous/toxic chemicals and wastes (type)

Storage/inventory

Hazardous waste (construction)

Pits/Ponds created $\left(\mathrm{m}^{2}\right)$

Water usage:

Dust control

Domestic water

Energy requirements

Electrical (MWh/yr)

Fossil fuel (liters)

Permits needed for construction
518 tons (total)

14,091 tons (total)

83 tons (total)

14,692 tons (total)

$10,560,150$ liters (total)

2,770 liters (total)

$5,880 \mathrm{~m}^{3}$ (total)
None

$$
\begin{aligned}
& 13 \mathrm{~m}^{3} \\
& 217 \mathrm{~m}^{3} \text { (total) } \\
& \text { None }
\end{aligned}
$$

$3,700,858$ liters (total)

$10,560,150$ liters (total)

\author{
$156 \mathrm{MWh} / \mathrm{yr}$ \\ 0 liters (total)
}

NEPA documentation (prior to start of Title II construction); New stationary sources/PTC/NOC/PSD for non-rad air emissions; HAP's and TAP's and RCRA (part AA,BB, and CC for air) for hazardous air emissions; air operating permit; NESHAP's, NPDES, NESHAP's subpart H for rad air emissions; approval of Engineering Plans; Cross Connection Control Plans; report and specifications for drinking water supply;RCRA Part A and Part B permits.
Operational Information

Cost (\$): Operations (unescalated)

Facility/Administration

Operations/Process Facility

Procurement, materials, utilities, maintenance

Total operations

Schedule start/end

$$
\begin{array}{r}
\$ 3,003,000 \\
\$ 7,535,000 \\
\$ 37,708,000 \\
\$ 48,246,000
\end{array}
$$

January 2012 through December 2068

Number of workers each year of operation (new/existing) 
Table 3. (continued).

Operations

Maintenance

Support

Total

Number of radiation workers

Average annual work radiation dose (rem/yr)

Heavy Equipment

Air Emissions

Radioactive off-gas

Effluents

Sanitary Wastewater

\section{Solid Wastes}

Sanitary/Industrial Trash

Radioactive wastes (canisters)

Hazardous/toxic chemicals and wastes

Pits/Ponds used $\left(\mathrm{m}^{2}\right)$

\section{Water usage:}

Domestic water

Energy Requirements:

Electrical (MWh/yr)

Fossil fuel (liters/yr)

Permits needed (for facility operations)

\section{Project Data Sheet for Interim Storage Facility for CWO}

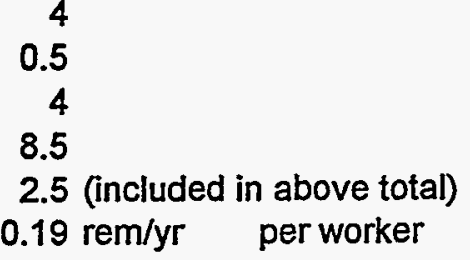

None

None

293,574 liters/yr

\author{
$47 \mathrm{~m}^{3} / \mathrm{yr}$ \\ $3,456 \mathrm{~m}^{3} / \mathrm{yr}$
}

None

293,574 liters/yr

\section{4,368 MWh/yr None}

HAP's and TAP's and RCRA (part $A A, B B$, and $C C$ for air) for hazardous air emissions; air operating permit; NESHAP's, NPDES, NESHAP's subpart H for rad air emissions; approval of Engineering Plans; Cross Connection Control Plans; report and specifications for drinking water supply;RCRA Part A and Part B permits.
Decontamination \& Decommissioning (D\&D) Information

\section{Cost (\$): D\&D (unescalated)}

Decommission

Decontamination

Demolition

Total D\&D

Schedule start/end: $D \& D$

Number of workers each year of D\&D (new/existing)

Number of radiation workers (D\&D)

Average annual worker radiation dose (rem/yr)

Heavy equipment:

Equipment used

$$
\begin{array}{r}
\$ 10,500,000 \\
\$ 0 \\
\$ 103,997,000 \\
\$ 114,497,000
\end{array}
$$

January 2069 through December 2071

268 New workers/yr

14 New workers/yr

$0.19 \mathrm{rem} / \mathrm{yr}$ perworker

Mobile Cranes, Roll-off trucks, Dozers, Loaders 
Table 3. (continued)

\section{Project Data Sheet for Interim Storage Facility for CWO}

Trips Roll-off trucks

Hours of operation (all heavy equipment)

Acres disturbed and duration of disturbance

New

Previous

Revegetated

Air emissions

non-radioactive Fuel combustion gases $\left(\mathrm{CO}_{2}, \mathrm{H}_{2} \mathrm{O}, \mathrm{O}_{2}, \mathrm{~N}_{2}\right)$

non-radioactive Fuel combustion contaminants ( $\mathrm{CO}$,

particulates, $\mathrm{NO}_{\mathrm{x}}, \mathrm{SO}_{2}$, hydrocarbons)

radioactive HEPA filtered off-gas

Effluents

radioactive Spent decontamination solution

non-radioactive Sanitary wastewater

non-radioactive Lube oil

Solid wastes:

radioactive

Non-radioactive (industrial)

Hazardous

Hazardous/toxic chemicals and wastes (type)

Storage/inventory

Pits/Ponds created $\left(\mathrm{m}^{2}\right)$

radioactive (mixed waste)

Water usage:

Domestic water

Source of water

Energy requirements:

Electrical (MWh/yr)

Fossil fuel (liters)

Permits needed (e.g. for facility closures, physical characteristics

and quantities of radioactive and hazardous materials remaining after closure)
12 per day

67,500 Hours

January 2069 through December 2071
None
9.0 acres
None

39,233 tons (total)

229 tons (total)

None tons (total)

None liters (total)

$17,111,456$ liters (total)

12,774 liters (total)

$46,864 \mathrm{~m}^{3}$

$9 \mathrm{~m}^{3}$

$100 \mathrm{~m}^{3}$

None $\mathrm{m}^{3}$ (total)

None

None $\mathrm{m}^{3}$ (total)

$17,111,456$ liters (total)

ICPP site wells

$156 \mathrm{MWh} / \mathrm{yr}$

$1,532,925$ liters (total)

Work will be done under closure provisions of existing permits. 
Table 3. (continued):-

\section{Project Data Sheet for Interim Storage Facility for HIP}

Generic Information

Description/function

EIS Alternatives (A, $B$, etc.)

Project type or waste stream

Action type

Provide Long-term storage for road-ready containers awaiting shipment to a permanent

Structure type

Size $\left(\mathrm{m}^{2}\right)$

Other features (e.g. pits, ponds, power/water/sewer lines)

Location

Inside/outside of fence

repository.

Hipping Waste Option

Treated HLW calcine.

New

Inside/outside of building

Candidate for privatization?

$7,287 \mathrm{~m}^{2}$

None

Inside ICPP fence

Inside Vitrification Facility

\section{Construction Information}

Cost (\$): Pre-Operations (Other Project Costs) with escalation an

Conceptual Design

Management for Project Development

Permitting and Documentation

SO Test \& Start-Up

Contingency

Total Preconstruction

Cost (\$): Construction with escalation and contingency.

\section{ED\&!}

Management (PM/CM)

Construction

G\&APIF

Procurement Fees, Management Reserve and Contingency

Total Construction (TEC)

Schedule start/end: Preconstruction

Schedule start/end: Construction

Schedule start/end: SO Test \& Start-up

Number of workers each year of construction (new/existing)

Nonradiation

Number of radiation workers (construction)

Average annual worker radiation dose (rem/yr)

\section{Heavy equipment}

Equipment used

Trips (construction materials delivery)

Hours of operation

Acres disturbed and duration of disturbance

New

Previous
$\$ 5,000,000$
$\$ 4,000,000$
$\$ 2,728,000$
$\$ 4,500,000$
$\$ 4,072,000$
$\$ 20,300,000$

$$
\begin{array}{r}
\$ 33,085,000 \\
\$ 49,305,000 \\
\$ 208,097,000 \\
\$ 12,729,000 \\
\$ 65,384,000 \\
\$ 368,600,000
\end{array}
$$

January 2003 through December 2007

January 2006 through December 2011

January 2012 through December 2012

\author{
92 New workers/yr \\ None \\ None
}

Excavator, dump trucks, cranes, cement trucks,grader, material delivery

1,349

8,140 hours (total)

January 2006 through December 2011

None

2.5 acres 
Table 3. (continuedid).

Revegetated

Air emissions

Dust

Major gases $\left(\mathrm{CO}_{2}, \mathrm{H}_{2} \mathrm{O}, \mathrm{O}_{2}, \mathrm{~N}_{2}\right)$ from diesel exhaust

Contaminants (Particulates, $\mathrm{CO}, \mathrm{NO}_{\mathrm{x}}, \mathrm{SO}_{2}$, hydrocarbons) from diesel exhaust.

Total air emissions

Effluents

Sanitary wastewater

Lube Oil

Solid wastes

Type - construction trash

Radioactive wastes

Hazardous/toxic chemicals and wastes (type)

Hazardous waste (construction)

Pits/Ponds created $\left(\mathrm{m}^{2}\right)$

Water usage:

Dust control

Domestic water

Energy requirements

Electrical (MWh/yr)

Fossil fuel (liters)

Permits needed for construction
Storage/inventory

\section{Project Data Sheet for Interim Storage Facility for HIP \\ None}

108 tons (total)

4,017 tons (total)

24 tons (total)

4,149 tons (total)

$5,876,213$ liters (total)

1,541 liters (total)

$3,272 \mathrm{~m}^{3}$ (total)

None

$$
\begin{aligned}
& 13 \mathrm{~m}^{3} \\
& 217 \mathrm{~m}^{3} \text { (total) } \\
& \text { None }
\end{aligned}
$$

771,012 liters (total)

$5,876,213$ liters (total)

\section{$156 \mathrm{MWh} / \mathrm{yr}$ \\ 310,806 liters (total)}

NEPA documentation (prior to start of Title II construction); New stationary sources/PTC/NOC/PSD for non-rad air emissions; HAP's and TAP's and RCRA (part AA,BB, and CC for air) for hazardous air emissions; air operating permit; NESHAP's, NPDES, NESHAP's subpart $H$ for rad air emissions; approval of Engineering Plans; Cross Connection Control Plans; report and specifications for drinking water supply;RCRA Part A and Part B permits.

\section{Operational Information}

Cost (\$): Operations (unescalated)

Facility/Administration

Operations/Process Facility

Procurement, materials, utilities, maintenance

Total operations

Schedule start/end

$\$ 7,293,000$

$\$ 18,299,000$

$\$ 70,097,000$

$\$ 95,689,000$

January 2013 through December 2068

Number of workers each year of operation (new/existing) 
Table 3. (continued).

Operations

Maintenance

Support

Total

Number of radiation workers

Average annual work radiation dose (rem/yr)

Heavy Equipment

Air Emissions

Radioactive off-gas

Effluents

Sanitary Wastewater

Solid Wastes

Sanitary/Industrial Trash

Radioactive wastes (canisters)

Hazardous/toxic chemicals and wastes

Pits/Ponds used $\left(\mathrm{m}^{2}\right)$

Water usage:

Domestic water

Energy Requirements:

Electrical (MWh/yr)

Fossil fuel (liters/yr)

Permits needed (for facility operations)

\section{Project Data Sheet for Interim Storage Facility for HIP}

0.5

3

6.5

2.5 (included in above total)

$0.19 \mathrm{rem} / \mathrm{yr}$ perworker

None

None

224,498 liters/yr

$36 \mathrm{~m}^{3} / \mathrm{yr}$
$4,309 \mathrm{~m}^{3} / \mathrm{yr}$

None

224,498 liters/yr

4,368 MWh/yr

None

HAP's and TAP's and RCRA (part AA,BB, and CC for air) for hazardous air emissions; air operating permit; NESHAP's, NPDES, NESHAP's subpart H for rad air emissions; approval of Engineering Plans; Cross Connection Control Plans; report and specifications for drinking water supply;RCRA Part $A$ and Part $B$ permits.

Decontamination \& Decommissioning (D\&D) Information

Cost (\$): D\&D (unescalated)

Decommission

Decontamination

Demolition

Total D\&D

Schedule start/end: D\&D

Number of workers each year of D\&D (new/existing)

Number of radiation workers (D\&D)

Average annual worker radiation dose (rem/yr)

Heavy equipment:

Equipment used

Mobile Cranes, Roll-off trucks, Dozers, Loaders 
Table 3. (continued):-

Project Data Sheet for Interim Storage Facility for HIP

Trips Roll-off trucks

Hours of operation (all heavy equipment)

35,640 Hours

Acres disturbed and duration of disturbance

New

Previous

Revegetated

January 2069 through December 2071
None
2.5 acres
None

Air emissions

non-radioactive Fuel combustion gases $\left(\mathrm{CO}_{2}, \mathrm{H}_{2} \mathrm{O}, \mathrm{O}_{2}, \mathrm{~N}_{2}\right)$

20,715 tons (total)

non-radioactive Fuel combustion contaminants (CO,

121 tons (total)

radioactive HEPA filtered off-gas

None tons (total)

Effluents

radioactive Spent decontamination solution

non-radioactive Sanitary wastewater

non-radioactive Lube oil

None liters (total)

$9,818,650$ liters (total)

6,745 liters (total)

Solid wastes:

radioactive

Non-radioactive (industrial)

Hazardous

None $\mathrm{m}^{3}$

$4 \mathrm{~m}^{3}$

Hazardous/toxic chemicals and wastes (type)

Storage/inventory

Pits/Ponds created $\left(\mathrm{m}^{2}\right)$

Hazardous wastes

None $\mathrm{m}^{3}$ (total)

None

$909 \mathrm{~m}^{3}$ (total)

Water usage:

Domestic water

Source of water

$9,818,650$ liters (total)

ICPP site wells

Energy requirements:

Electrical (MWh/yr)

Fossil fuel (liters)

Permits needed (e.g. for facility closures, physical characteristics and quantities of radioactive and hazardous materials remaining after closure)

$156 \mathrm{MWh} / \mathrm{yr}$
809,384 liters (total)

Work will be done under closure provisions of existing permits. 
Table 3. (continue ब).

\section{Project Data Sheet for Storage at FPR for WFS}

Generic Information

Description/function

EIS Altematives ( $A, B$, etc.)

Project type or waste stream

Action type

Structure type

Size $\left(\mathrm{m}^{2}\right)$

Other features (e.g. pits, ponds, power/water/sewer lines)

Location

Inside/outside of fence

Inside/outside of building

Candidate for privatization?
Provide Long-term storage for roadready containers awaiting shipment to a permanent repository.

Full Separations Waste Option

Treated HLW calcine.

New

$5,853 \mathrm{~m}^{2}$

None

Inside ICPP fence

FPR Facility

\section{Construction Information}

Cost (\$): Pre-Operations (Other Project Costs) with escalation and contingency.

\section{Conceptual Design}

Management for Project Development

Permitting and Documentation

SO Test \& Start-Up

Contingency

Total Preconstruction

Cost (\$): Construction with escalation and contingency.

\section{ED\&l}

Management (PM/CM)

Construction

G\&A/PIF

Procurement Fees, Management Reserve and Contingency

Total Construction (TEC)

Schedule start/end: Preconstruction

Schedule start/end: Construction

Schedule start/end: SO Test \& Start-up

Number of workers each year of construction (new/existing)

Nonradiation

Number of radiation workers (construction)

Average annual worker radiation dose (rem/yr)

Heavy equipment

Equipment used

Trips (construction materials delivery)

Hours of operation

\section{Acres disturbed and duration of disturbance}

New

Previous
$\$ 3,170,000$

$\$ 4,501,000$

$\$ 3,629,000$

$\$ 7,110,000$

$\$ 4,590,000$

$\$ 23,000,000$

$\$ 20,965,000$

$\$ 20,566,000$

$\$ 85,690,000$

$\$ 5,332,000$

$\$ 29,947,000$

$\$ 162,500,000$

January 1999 through December 2001 January 2011 through December 2014 January 2015 through December 2015

161 New workers/yr

None

None

Excavator, dump

trucks, cranes,

\section{2}

14,769 hours (total)

January 2011 through December 2014

None

155 acres 
Table 3. (continued).

\section{Project Data Sheet for Storage at FPR for WFS}

Revegetated

None

\section{Air emissions}

Dust

Major gases $\left(\mathrm{CO}_{2}, \mathrm{H}_{2} \mathrm{O}, \mathrm{O}_{2}, \mathrm{~N}_{2}\right)$ from diesel exhaust

4,464 tons (total)

10,261 tons (total)

Contaminants (Particulates, $\mathrm{CO}, \mathrm{NO}_{x}, \mathrm{SO}_{2}$, hydrocarbons) from

diesel exhaust.

60 tons (total)

Total air emissions

14,785 tons (total)

\section{Effluents}

Sanitary wastewater

Lube Oil

\section{Solid wastes}

Type - construction trash

Radioactive wastes

$$
\begin{aligned}
& 3,817 \mathrm{~m}^{3} \text { (total) } \\
& \text { None }
\end{aligned}
$$

Hazardous/toxic chemicals and wastes (type)

\section{Storage/inventory}

Hazardous waste (construction)

Pits/Ponds created $\left(\mathrm{m}^{2}\right)$

$$
\begin{aligned}
& 13 \mathrm{~m}^{3} \\
& 217 \mathrm{~m}^{3} \text { (total) } \\
& \text { None }
\end{aligned}
$$

Water usage:

Dust control

$31,868,496$ liters (total)

Domestic water

Energy requirements

Electrical (MWh/yr)

Fossil fuel (liters)

\section{$150 \mathrm{MWh} / \mathrm{yr}$}

0 liters (total)

NEPA documentation (prior to start of Title II construction); New stationary

sources/PTC/NOC/PSD for non-rad air emissions; HAP's and TAP's and RCRA (part AA,BB, and CC

Permits needed for construction for air) for hazardous air emissions; air operating permit; NESHAP'S, NPDES, NESHAP's subpart $H$ for rad air emissions; approval of Engineering Plans; Cross Connection Control Plans; report and specifications for drinking water supply;RCRA Part A and Part B permits.

Operational Information
Cost (\$): Operations (unescalated)
Facility/Administration
Operations/Process Facility
Procurement, materials, utilities, maintenance
Total operations

Schedule start/end

$$
\begin{array}{r}
\$ 6,721,000 \\
\$ 18,940,000 \\
\$ 19,864,000 \\
\$ 45,525,000
\end{array}
$$

January 2015 through December 2068

Number of workers each year of operation (new/existing) 
Table 3. (continued).

\section{Project Data Sheet for Storage at FPR for WFS}

Operations

Maintenance

Support

Total

Number of radiation workers

Average annual work radiation dose (rem/yr)

Heavy Equipment

Air Emissions

Radioactive off-gas

Effluents

Sanitary Wastewater

Solid Wastes

Sanitary/Industrial Trash

Radioactive wastes (canisters)

Hazardous/toxic chemicals and wastes

Pits/Ponds used $\left(\mathrm{m}^{2}\right)$

Water usage:

Domestic water

Energy Requirements:

Electrical (MWh/yr)

Steam

Permits needed (for facility operations)

\section{3}

1

3

7

2.5 (included in above total)

$0.19 \mathrm{rem} / \mathrm{yr}$ perworker

None

None

241,767 liters/yr

$$
\begin{array}{r}
39 \mathrm{~m}^{3} / \mathrm{yr} \\
147 \mathrm{~m}^{3} / \mathrm{yr}
\end{array}
$$

None

241,767 liters/yr

\begin{abstract}
4,368 MWh/yr

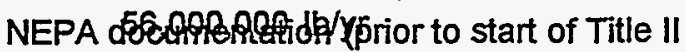
construction); New stationary sources/PTC/NOC/PSD for non-rad air emissions; HAP's and TAP's and RCRA (part AA,BB, and CC for air) for hazardous air emissions; air operating permit; NESHAP's, NPDES, NESHAP's subpart $H$ for rad air emissions; approval of Engineering Plans; Cross Connection Control Plans; report and
\end{abstract}

Decontamination \& Decommissioning (D\&D) Information

Cost (\$): D\&D (unescalated)

Decommission

Decontamination

Demolition

Total D\&D

Schedule start/end: D\&D

Number of workers each year of D\&D (new/existing)

Number of radiation workers (D\&D)

Average annual worker radiation dose (rem/yr)

Heavy equipment:

Equipment used

\section{$\$ 4,284,000$}

$\$ 0$

$\$ 24,785,000$

$\$ 29,069,000$

January 2069 through December 2071 68 New workers/yr

7 New workers/yr

$0.19 \mathrm{rem} / \mathrm{yr}$ perworker

Mobile Cranes, Roll-off trucks, Dozers, Loaders 
Table 3. (continued).

Project Data Sheet for Storage at FPR for WFS

Trips

Roll-off trucks

Hours of operation (all heavy equipment)

6 per day

Acres disturbed and duration of disturbance

New

Previous

Revegetated

14,280 Hours

January 2074 through December 2076
None
165 acres
None

Air emissions

non-radioactive Fuel combustion gases $\left(\mathrm{CO}_{2}, \mathrm{H}_{2} \mathrm{O}, \mathrm{O}_{2}, \mathrm{~N}_{2}\right)$

8,300 tons (total)

non-radioactive

Fuel combustion contaminants ( $\mathrm{CO}$, particulates, $\mathrm{NO}_{x}, \mathrm{SO}_{2}$, hydrocarbons)

radioactive HEPA filtered off-gas

48 tons (total)

None tons (total)

Effluents

radioactive Spent decontamination solution

non-radioactive Sanitary wastewater

non-radioactive Lube oil

None liters (total)

$4,364,496$ liters (total)

2,702 liters (total)

Solid wastes:

radioactive

Non-radioactive (industrial)

Hazardous

$18,628 \mathrm{~m}^{3}$

$4 \mathrm{~m}^{3}$

$40 \mathrm{~m}^{3}$

Hazardous/toxic chemicals and wastes (type)

Storage/inventory

Pits/Ponds created $\left(\mathrm{m}^{2}\right)$

Hazardous waste

None $\mathrm{m}^{3}$ (total)

None

$909 \mathrm{~m}^{3}$ (total)

Water usage:

Domestic water

Source of water

$4,364,496$ liters (total)

ICPP site wells

Energy requirements:

Electrical (MWh/yr)

$156 \mathrm{MWh} / \mathrm{yr}$

Fossil fuel (liters)

Permits needed (e.g. for facility closures, physical characteristics 324,299 liters (total)

and quantities of radioactive and hazardous materials remaining after closure)

Work will be done under closure provisions of existing permits. 
Table 3. (continued).

\section{Project Data Sheet for Storage at FPR for TRU}

Generic Information

Description/function

EIS Alternatives (A, B, etc.)

Project type or waste stream

Action type

Structure type

Size $\left(\mathrm{m}^{2}\right)$

Other features (e.g. pits, ponds, power/water/sewer lines)

Location

Inside/outside of fence

Inside/outside of building

Candidate for privatization?

Provide Long-term storage for road-ready containers awaiting shipment to a permanent repository.

TRU Separations Waste Option

Treated HLW calcine.

New

$5,853 \mathrm{~m}^{2}$

None

Inside ICPP fence

FPR Facility

\section{Construction Information}

Cost (\$): Pre-Operations (Other Project Costs) with escalation an

Conceptual Design

Management for Project Development

Permitting and Documentation

SO Test \& Start-Up

Contingency

Total Preconstruction

$\$ 2,470,000$

$\$ 3,470,000$

$\$ 2,728,000$

$\$ 4,500,000$

$\$ 3,332,000$

$\$ 16,500,000$

Cost (\$): Construction with escalation and contingency.

ED\&l

Management (PM/CM)

Construction

G\&A/PIF

Procurement Fees, Management Reserve and Contingency

Total Construction (TEC)

Schedule start/end: Preconstruction

Schedule start/end: Construction

Schedule start/end: SO Test \& Start-up

Number of workers each year of construction (new/existing)

Nonradiation

Number of radiation workers (construction)

Average annual worker radiation dose (rem/yr)

Heavy equipment

Equipment used

Trips (construction materials delivery)

Hours of operation

Acres disturbed and duration of disturbance

New

Previous
$\$ 12,352,000$

$\$ 11,858,000$

$\$ 49,408,000$

$\$ 3,107,000$

$\$ 18,075,000$

$\$ 94,800,000$

January 1999 through December 2001

January 2010 through December 2013

January 2014 through December 2014

155 New workers/yr
None
None

Excavator, dump trucks, cranes, cement trucks,grader, material delivery 702

14,740 hours (total)

January 2010 through December 2013

None

155 acres 
Table 3. (continued).

\section{Project Data Sheet for Storage at FPR for TRU}

Revegetated

None

Air emissions

Dust

Major gases $\left(\mathrm{CO}_{2}, \mathrm{H}_{2} \mathrm{O}, \mathrm{O}_{2}, \mathrm{~N}_{2}\right)$ from diesel exhaust

Contaminants (Particulates, $\mathrm{CO}, \mathrm{NO}_{\mathrm{x}}, \mathrm{SO}_{2}$, hydrocarbons) from

diesel exhaust.

Total air emissions

4,464 tons (total)

5,174 tons (total)

30 tons (total)

9,668 tons (total)

Effluents

Sanitary wastewater

Lube Oil

$6,600,094$ liters (total)

2,790 liters (total)

Solid wastes

Type - construction trash

Radioactive wastes

$3,675 \mathrm{~m}^{3}$ (total)

None

Hazardous/toxic chemicals and wastes (type)

Storage/inventory

Hazardous waste (construction)

Pits/Ponds created $\left(\mathrm{m}^{2}\right)$

$$
\begin{aligned}
& 13 \mathrm{~m}^{3} \\
& 217 \mathrm{~m}^{3} \text { (total) } \\
& \text { None }
\end{aligned}
$$

Water usage:

Dust control

Domestic water

$31,868,496$ liters (total)

$6,600,094$ liters (total)

$150 \mathrm{MWh} / \mathrm{yr}$

0 liters (total)

NEPA documentation (prior to start of Title II construction); New stationary

sources/PTC/NOC/PSD for non-rad air emissions; HAP's and TAP's and RCRA (part AA,BB, and CC for air) for hazardous air emisșions; air operating permit; NESHAP's, NPDES, NESHAP's subpart H for rad air emissions; approval of Engineering Plans; Cross Connection Control Plans; report and specifications for drinking water supply;RCRA Part A and Part $B$ permits.

Operational Information

Cost (\$): Operations (unescalated)

Facility/Administration

Operations/Process Facility

Procurement, materials, utilities, maintenance

Total operations

Schedule start/end

$\$ 7,007,000$

$\$ 19,746,000$

$\$ 18,677,000$

$\$ 45,430,000$

January 2014 through December 2068

Number of workers each year of operation (new/existing) 
Table 3. (continued).

\section{Project Data Sheet for Storage at FPR for TRU}

Operations

Maintenance

Support

Total

Number of radiation workers

Average annual work radiation dose (rem/yr)

Heavy Equipment

3
1

3

7

2.5 (included in above total)

$0.19 \mathrm{rem} / \mathrm{yr}$ perworker

None

Air Emissions

Radioactive off-gas

None

Effluents

Sanitary Wastewater

241,767 liters/yr

\section{Solid Wastes}

Sanitary/Industrial Trash

Radioactive wastes (canisters)

$$
\begin{array}{r}
39 \mathrm{~m}^{3} / \mathrm{yr} \\
141 \mathrm{~m}^{3} / \mathrm{yr}
\end{array}
$$

Hazardous/toxic chemicals and wastes

Pits/Ponds used $\left(\mathrm{m}^{2}\right)$

None

Water usage:

Domestic water

241,767 liters/yr

Energy Requirements:

Electrical (MWh/yr)

Steam

$$
\begin{aligned}
& \text { 4,368 MWh/yr } \\
& 56,000,000 \mathrm{lb} / \mathrm{yr}
\end{aligned}
$$

HAP's and TAP's and RCRA (part $A A, B B$, and $C C$ for air) for hazardous air emissions; air operating permit; NESHAP's, NPDES, NESHAP's subpart $H$

Permits needed (for facility operations) for rad air emissions; approval of Engineering Plans; Cross Connection Control Plans; report and specifications for drinking water supply:RCRA Part A and Part B permits.

Decontamination \& Decommissioning (D\&D) Information Cost (\$): D\&D (unescalated)

Decommission

Decontamination

Demolition

Total D\&D

Schedule start/end: D\&D

Number of workers each year of D\&D (new/existing)

Number of radiation workers (D\&D)

Average annual worker radiation dose (rem/yr)

$$
\$ 3,706,000
$$

$\$ 0$

$\$ 21,693,000$

$\$ 25,399,000$

January 2069 through December 2071

60 New workers/yr

6 New workers/yr

$0.19 \mathrm{rem} / \mathrm{yr}$ perworker

Mobile Cranes, Roll-off trucks, Dozers, Loaders

Heavy equipment:

Equipment used 
Table 3. (continued)-

Trips

Roll-off trucks

Hours of operation (all heavy equipment)

Acres disturbed and duration of disturbance

New

Previous

Revegetated

Air emissions

non-radioactive Fuel combustion gases $\left(\mathrm{CO}_{2}, \mathrm{H}_{2} \mathrm{O}, \mathrm{O}_{2}, \mathrm{~N}_{2}\right)$

non-radioactive Fuel combustion contaminants ( $\mathrm{CO}$,

radioactive HEPA filtered off-gas

Effluents

radioactive Spent decontamination solution

non-radioactive Sanitary wastewater

non-radioactive Lube oil

Solid wastes:

radioactive

Non-radioactive (industrial)

Hazardous

Hazardous/toxic chemicals and wastes (type)

Storage/inventory

Pits/Ponds created $\left(\mathrm{m}^{2}\right)$

Hazardous waste

Water usage:

Domestic water

Source of water

Energy requirements:

Electrical (MWh/yr)

Fossil fuel (liters)

Permits needed (e.g. for facility closures, physical characteristics and quantities of radioactive and hazardous materials remaining after closure)
January 2069 through December 2071

None

155.0 acres

None

7,672 tons (total)

45 tons (total)

None tons (total)

None liters (total)

$3,813,258$ liters (total)

2,498 liters (total)

$18,628 \mathrm{~m}^{3}$

$4 \mathrm{~m}^{3}$

$40 \mathrm{~m}^{3}$

None $\mathrm{m}^{3}$ (total)

None

$909 \mathrm{~m}^{3}$ (total)

$3,813,258$ liters (total)

ICPP site wells

$156 \mathrm{MWh} / \mathrm{yr}$

299,772 liters (total)

Work will be done under closure provisions of existing permits. 
Table 3. (continued).

\section{Project Data Sheet for Storage at FPR for HWO}

\section{Generic Information}

Description/function

ElS Alternatives (A, $B$, etc.)

Project type or waste stream

Action type

Structure type

Size $\left(\mathrm{m}^{2}\right)$

Other features (e.g. pits, ponds, power/water/sewer lines)

Location

Inside/outside of fence

Inside/outside of building

Candidate for privatization?
Provide Long-term storage for road-ready containers awaiting shipment to a permanent repository. Hot Isostatic Pressing Waste Option Treated HLW calcine. New $5,853 \mathrm{~m}^{2}$ None

Inside ICPP fence FPR Facility

\section{Construction Information}

Cost (\$): Pre-Operations (Other Project Costs) with escalation an

Conceptual Design

Management for Project Development

Permitting and Documentation

SO Test \& Start-Up

Contingency

Total Preconstruction

Cost (\$): Construction with escalation and contingency.

\section{ED\&I}

Management (PM/CM)

Construction

G\&AVIF

Procurement Fees, Management Reserve and Contingency

Total Construction (TEC)

Schedule start/end: Preconstruction

Schedule start/end: Construction

Schedule start/end: SO Test \& Start-up

Number of workers each year of construction (new/existing) Nonradiation

Number of radiation workers (construction)

Average annual worker radiation dose (rem/yr)

\section{Heavy equipment}

Equipment used

Trips (construction materials delivery)

Hours of operation

Acres disturbed and duration of disturbance

New

Previous
$\$ 5,000,000$
$\$ 4,000,000$
$\$ 2,728,000$
$\$ 4,500,000$
$\$ 4,072,000$
$\$ 20,300,000$

$\$ 24,604,000$

$\$ 31,699,000$

$\$ 132,077,000$

$\$ 8,021,000$

$\$ 43,999,000$

$\$ 240,400,000$

January 2004 through December 2005

January 2007 through December 2011

January 2012 through December 2012

\author{
152 New workers/yr \\ None \\ None
}

Excavator, dump trucks, cranes, cement trucks,grader, material delivery

702

27,414 hours (total)

January 2007 through December 2011

None

155 acres 
Table 3. (continued).

Revegetated

Air emissions

Dust

Major gases $\left(\mathrm{CO}_{2}, \mathrm{H}_{2} \mathrm{O}, \mathrm{O}_{2}, \mathrm{~N}_{2}\right)$ from diesel exhaust

Contaminants (Particulates, $\mathrm{CO}, \mathrm{NO}_{\mathrm{x}_{1}} \mathrm{SO}_{2}$, hydrocarbons) from diesel exhaust.

Total air emissions

Effluents

Sanitary wastewater

Lube Oil

Solid wastes

Type - construction trash

Radioactive wastes

Hazardous/toxic chemicals and wastes (type)

Storage/inventory

Hazardous waste (construction)

Pits/Ponds created $\left(\mathrm{m}^{2}\right)$

Water usage:

Dust control

Domestic water

Energy requirements

Electrical (MWh/yr)

Fossil fuel (liters)

Permits needed for construction

\section{Project Data Shëet for Storage at FPR for HWO \\ None}

6,696 tons (total)

17,610 tons (total)

103 tons (total)

24,409 tons (total)

$9,708,525$ liters (total)

5,188 liters (total)

$5,406 \mathrm{~m}^{3}$ (total)

None

$$
\begin{aligned}
& 13 \mathrm{~m}^{3} \\
& 217 \mathrm{~m}^{3} \text { (total) } \\
& \text { None }
\end{aligned}
$$

$47,802,744$ liters (total)

$9,708,525$ liters (total)

$150 \mathrm{MWh} / \mathrm{yr}$

0 liters (total)

NEPA documentation (prior to start of Title II construction); New stationary sources/PTC/NOC/PSD for non-rad air emissions; HAP's and TAP's and RCRA (part AA,BB, and CC for air) for hazardous air emissions; air operating permit; NESHAP'S, NPDES, NESHAP's subpart H for rad air emissions; approval of Engineering Plans; Cross Connection Control Plans; report and specifications for drinking water supply;RCRA Part A and Part B permits.

Operational Information

Cost (\$): Operations (unescalated)

Facility/Administration

Operations/Process Facility

Procurement, materials, utilities, maintenance

Total operations

Schedule start/end

$\$ 5,039,000$

$\$ 18,299,000$

$\$ 63,902,000$

$\$ 87,240,000$

January 2012 through December 2068

Number of workers each year of operation (new/existing) 
Table 3. (continued).

\section{Project Data Sheet for Storage at FPR for HWO}

Operations

Maintenance

Support

Total

Number of radiation workers

Average annual work radiation dose (rem/yr)

Heavy Equipment

Air Emissions

Radioactive off-gas

Effluents

Sanitary Wastewater

Solid Wastes

Sanitary/Industrial Trash

Radioactive wastes (canisters)

Hazardous/toxic chemicals and wastes

Pits/Ponds used $\left(\mathrm{m}^{2}\right)$

Water usage:

Domestic water

Energy Requirements:

Electrical (MWh/yr)

Steam

Permits needed (for facility operations)
3

1

3

7

2.5 (included in above total)

$0.19 \mathrm{rem} / \mathrm{yr}$ perworker

None

None

241,767 liters/yr

$39 \mathrm{~m}^{3} / \mathrm{yr}$

$4,309 \mathrm{~m}^{3} / \mathrm{yr}$

None

241,767 liters/yr

$$
\begin{aligned}
& \text { 4,368 MWh/yr } \\
& 56,000,000 \mathrm{lb} / \mathrm{yr}
\end{aligned}
$$

HAP's and TAP's and RCRA (part AA,BB, and CC for air) for hazardous air emissions; air operating permit; NESHAP's, NPDES, NESHAP's subpart $H$ for rad air emissions; approval of Engineering Plans; Cross Connection Control Plans; report and specifications for drinking water supply;RCRA Part A and Part B permits.

Decontamination \& Decommissioning (D\&D) Information

Cost (\$): D\&D (unescalated)

Decommission

$\$ 7,381,000$

Decontamination

$\$ 0$

Demolition

$\$ 51,348,000$

Total D\&D

$\$ 58,729,000$

Schedule start/end: D\&D

Number of workers each year of D\&D (new/existing)

Number of radiation workers (D\&D)

Average annual worker radiation dose (rem/yr)

January 2069 through December 2071

138 New workers/yr

14 New workers/yr

$0.19 \mathrm{rem} / \mathrm{yr}$ perworker

Heavy equipment:

Equipment used

Mobile Cranes, Roll-off trucks, Dozers, Loaders 
Table 3. (continued).

Trips Roll-off trucks

Hours of operation (all heavy equipment)

Acres disturbed and duration of disturbance

New

Previous

Revegetated

Air emissions

non-radioactive Fuel combustion gases $\left(\mathrm{CO}_{2}, \mathrm{H}_{2} \mathrm{O}, \mathrm{O}_{2}, \mathrm{~N}_{2}\right)$

non-radioactive Fuel combustion contaminants ( $\mathrm{CO}$,

radioactive HEPA filtered off-gas

Effluents

radioactive Spent decontamination solution

non-radioactive Sanitary wastewater

non-radioactive Lube oil

Solid wastes:

radioactive

Non-radioactive (industrial)

Hazardous

Hazardous/toxic chemicals and wastes (type)

Storage/inventory

Pits/Ponds created $\left(\mathrm{m}^{2}\right)$

radioactive (mixed waste)

Water usage:

Domestic water

Source of water

Energy requirements:

Electrical (MWh/yr)

Fossil fuel (liters)

Permits needed (e.g. for facility closures, physical characteristics

and quantities of radioactive and hazardous materials remaining after closure)
$-$

perday

29,700 Hours

January 2069 through December 2071

None

155 acres

None

17,263 tons (total)

101 tons (total)

None tons (total)

None liters (total)

$8,806,174$ liters (total)

5,621 liters (total)

$$
\begin{array}{r}
18,628 \mathrm{~m}^{3} \\
4 \mathrm{~m}^{3} \\
40 \mathrm{~m}^{3}
\end{array}
$$

Ci

$$
\begin{aligned}
& \text { None } \mathrm{m}^{3} \text { (total) } \\
& \text { None } \\
& \text { None } \mathrm{m}^{3} \text { (total) }
\end{aligned}
$$

ICPP site wells

$8,806,174$ liters (total)

$156 \mathrm{MWh} / \mathrm{yr}$

674,487 liters (total)

Work will be done under closure provisions of existing permits. 


\section{PROJECT-SPECIFIC OPTIONS}

No project-specific options were assigned, identified, or evaluated.

\section{POTENTIAL IMPACTS OF NRC LICENSING}

Licensing of an ISF by the NRC under 10 CFR 72 is assumed, however the cost impact for this licensing is not included in the cost estimates in Appendix 1 of the respective EDFs (EDF-IS-001 and EDF-IS-002). These EDFs are in Appendix $A$ of this report. Licensing a nuclear facility requires preparing and submitting an application and supporting documents to the NRC, such as Safety Analysis Reports, Environmental Reports, quality assurance documents, training plans, monitoring plans, and safeguards and security plans. The NRC licensing process is divided into four stages: preapplication stage, application review stage, construction and operating license stage, and decontamination and site closure stage. The licensing duration from submitting the application to receiving of the license is expected to take 3 to 5 more years, or longer. The benefits of NRC licensing are enhanced operating safety, strengthened relationships with stakeholders, and license-holder participation in future regulation development.

According to data developed in the Fluor Daniel Corporation, Waste Treatment Facilities Feasibility Study Report, ${ }^{2}$ the estimated cost for NRC licensing is $26 \%$ of the Total Estimated Cost. This additional cost is required to comply with the NRC requirements that exceed the current DOE requirements for ISFs. An NRC licensed facility will have greater cost during the operating period as well. These additional costs are discussed in the reports for the separate processing options ${ }^{6-10}$ and the cost is identified in the section titled "Potential Impacts of NRC Licensing" in those reports.

Some of the potential major impacts associated with NRC licensing of ISF facilities, other than cost, are:

- Increased oversight, including more public involvement and input in all decision processes

- More restrictive physical limits on some parameters, including exposure limits, seismic, and tornado

- More strict radiation monitoring

- Restrictions on sharing utilities between facilities

- More stringent evaluations of the impact from off-Site hazards.

- Full testing required for emergency utilities

- Physical changes to the plant and equipment

- More elapsed schedule time required

- Methods to comply with some other codes and standards may be complicated and require more time 
- Although the NRC may license the treatment facilities, it may not automatically inherit or adopt the same agreements and obligations with the State of Idaho and EPA Region 10 that are in place for DOE and INEEL.

\section{RECOMMENDATIONS}

The impact for NRC licensing of a waste ISF, particularly an existing facility, should be investigated further. Licensing of an ISF has not been done and licensing of an existing facility that was not built initially to the current requirements raises questions that have not been studied in this work.

Consideration should be given to the passive cooling capability in a new ISF. The modified FPR would require forced ventilation that adds safety considerations to the operation of the facility.

A future study should be conducted to determine if a standard 4.6-m (15-ft) long "Hanford" canister should be substituted for the 3-m (10-ft) SRS canister assumed in this study. Use of a 4.6-m (15-ft) canister would increase the interim storage construction costs but would reduce the operation (handling) costs, and greatly reduce offsite transportation and disposal costs. A large net cost saving, primarily as a result of reduced transportation and disposal costs, could be realized by using the $4.6-\mathrm{m}(15-\mathrm{ft})$ canister. 


\section{CONCLUSIONS}

This study has investigated the design of a new ISF and modification of FPR for storage of HLW that results from processing the waste at ICPP. The processing options are discussed in separate reports. It is concluded that a new facility can be designed, built, tested, and placed into operation that can store the waste in canisters for the period from processing until the time a permanent repository is available for receipt of this waste. This study determined that FPR can hold a maximum of 9,344 canisters and only those processing options that produce fewer canisters can be accommodated in the FPR. It is concluded that FPR can be modified and placed into operation to store the waste in canisters for the Full and TRU/Class A Separations Option and the HIP Option for the period from processing until the time a permanent repository is available for receipt of this waste. The schedules and costs associated with interim storage for the processing options are presented in this report. 


\section{REFERENCES}

1. October 17, 1995 Consent Order and Settlement Agreement between DOE and the State of Idaho regarding spent fuel and nuclear waste issues.

2. Idaho Chemical Processing, Plant Waste Treatment Facilities, Feasibility Study Report, December 12, 1997, Fluor Daniel Corporation.

3. DPC Loading Feasibility Study Report, INEEL/EXT-97-01251, November 1997.

4. U.S. Department of Energy, Office of Civilian Radioactive Waste Management, Quality Assurance Requirements and Description (QARD), DOE/RW-0333P.

5. Utilities and Infrastructure Report, INEEU/EXT-98-01398, February 1998.

6. TRU Separations Option Study Report, INEEL/EXT-01428, February 1998.

7. Vitrified Waste Option Study Report, INEEL/EXT-01389, February 1998.

8. Direct Cementitious Waste Option Study Report, INEEL/EXT-01399, February 1998.

9. Cementitious Waste Option Study Report, INEEL/EXT-01389, February 1998.

10. Hot Isostatic Press Waste Option Report, INEEL/EXT-01392, February 1998. 


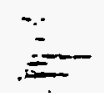

Appendix A

Engineering Design Files 


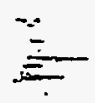

\section{CONTENTS}

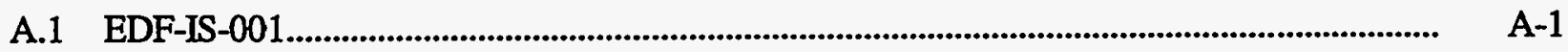

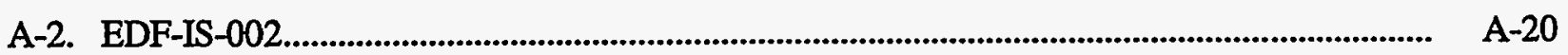


Project File Number

02BJ0

Project/Task Waste Treatment Project Feasibility Studies

Subtask Interim Storage Study

Title: New Facility for Interim Storage of HLW

Summary: This summary briefly defines the problem or activity to be addressed in the Engineering Design File (EDF), gives a summary of the activities performed in addressing the problem, and states the conclusions, recommendations, or results arrived at from this task.

High-level radioactive waste (HLW) stored at the Idaho Chemical Processing Plant (ICPP) in the form of calcine and liquid will be processed to provide a stable waste form and prepare the waste to be transported to a permanent repository. Because a permanent repository will not be available when the waste is processed, the waste must be stored at ICPP in an Interim Storage Facility (ISF). This EDF documents consideration of a new ISF for each of the processing options.

Distribution (complete package): Included in Appendix A of Interim Storage Study Report, INEEL/EXT-97-01393, February 1998.

Distribution (summary package only):

\begin{tabular}{|c|c|c|c|c|c|}
\hline Author & Dept. & Reviewed & Date & Approved & Date \\
\hline Qterewh. & 4130 & $\begin{array}{l}\text { LMITCO } \\
\text { Review } \\
\text { DJEkelln }\end{array}$ & $\begin{array}{l}\text { Date } \\
2 / 10 / 98\end{array}$ & $\begin{array}{l}\text { LMTTCO Approval } \\
\text { K.C. G. Llllarias }\end{array}$ & $\begin{array}{l}\text { Date } \\
2 / 10 / 99\end{array}$ \\
\hline
\end{tabular}

See Management Control Procedure (MCP) 6 for instructions on use of this form. 


\title{
New Facility for Interim Storage of HLW
}

\begin{abstract}
High-level radioactive waste (HLW) stored at ICPP in the form of calcine and liquid and liquid sodiumbearing waste (SBW) will be processed to provide a stable waste form and prepare the waste to be transported to a permanent repository. Because a permanent repository will not be available when the waste is processed, the waste must be stored at ICPP in an ISF. This EDF documents consideration of a new ISF for each of the processing options.
\end{abstract}

\section{SUMMARY}

Disposal of radioactive wastes now stored at the ICPP at the Idaho National Engineering and Environmental Laboratory (INEEL) is mandated under a "Settlement Agreement" between the Department of Energy (DOE) and the State of Idaho. Among other things, the Settlement Agreement requires that existing inventory of liquid high-level radioactive waste (HLW) be calcined by June 30 , 1998 , and SBW by the end of 2012, and the target date for all waste to be ready to ship from the INEEL is 2035.

The Department of Energy Idaho Operations Office (DOE-ID) is preparing the High-Level Waste and Facilities Disposition Environmental Impact Statement (HLW\&FD EIS), in accordance with the National Environmental Policy Act, to support the decision-making process regarding $\mathrm{HL} W$ management at the INEEL. Fluor Daniel Corporation and Lockheed Martin Idaho Technologies Company (LMITCO) are studying waste treatment options. Because a repository for the waste will not be available as processing is performed, an ISF is required for storage for the period of time between processing and when a permanent repository is available.

This EDF documents a study that investigated the design, costs, and environmental impacts for a new ISF for each of the processing options.

\section{Conclusions}

It is concluded that a new facility can be designed, built, tested, and placed into operation that can store the waste in canisters for the period from processing until the time a permanent repository is available for receipt of this waste. The schedules and costs associated for the various processing options are presented in this EDF.

\section{Recommendations}

The complexity that Nuclear Regulatory Commission (NRC) licensing adds to the permitting of an ISF has not been estimated in this study. The ramifications should be investigated in greater detail before the design of an ISF is started.

A future study should be performed to determine if a standard 4.6-m (15-ft) long "Hanford" canister should be substituted for the 3-meters (10-ft) SRS Defense Waste Processing Facility (DWPF) canister assumed in this study. Use of a $4.6-\mathrm{m}(15-\mathrm{ft})$ canister would increase the interim storage 
construction costs but would reduce the operation (handling) costs, and greatly reduce offsite transportation and disposal costs. A large net cost saving, primarily as a result of reduced transportation and disposal costs, could be realized by using the $4.6-\mathrm{m}(15-\mathrm{ft})$ canister.

\section{ACKNOWLEDGMENTS}

The assistance of Byron L. Blakely who designed the new ISF presented in this EDF is greatly appreciated. Rick D. Adams prepared the project cost estimates and Robert J. Turk prepared the Life-Cycle Costs. Jennifer Quigley and Rod R. Kimmitt prepared the Project Data Sheets. Karen Williams was the Project Lead. Stephen S. Lortie performed technical editing.

\section{ACRONYMS}

\begin{tabular}{|c|c|}
\hline CFR & Code of Federal Regulations \\
\hline CWO & Cementitious Waste Option \\
\hline DCWO & Direct Cementitious Waste Option \\
\hline DOE & Department of Energy \\
\hline DOE-ID & DOE Idaho Operations Office \\
\hline DOT & Department of Transportation \\
\hline DPC & Dual Purpose Canister \\
\hline DWPF & Defense Waste Processing Facility \\
\hline EDF & Engineering Design File \\
\hline EIS & Environmental Impact Statement \\
\hline HAW & high-activity waste \\
\hline HIP & Hot Isostatic Press \\
\hline HILW & high-level radioactive waste \\
\hline HLW\&FD & High-Level Waste and Facilities Disposition \\
\hline HWO & HIIP Waste Option \\
\hline INEEL & Idaho National Engineering and Environmental Laboratory \\
\hline ICPP & Idaho Chemical Processing Plant \\
\hline ISF & Interim Storage Facility \\
\hline LAW & low-activity waste \\
\hline LMITCO & Lockheed Martin Idaho Technologies Company \\
\hline NEPA & National Environmental Policy Act \\
\hline NRC & Nuclear Regulatory Commission \\
\hline NUHOMS & $\begin{array}{l}\text { Trade mark for concrete storage modules commercially available through } \\
\text { VECTRA Technologies, soon to be purchased by Chem Nuclear, Inc. }\end{array}$ \\
\hline RCRA & Resource Conservation and Recovery Act \\
\hline SBW & sodium-bearing waste \\
\hline SNF & spent nuclear fuel \\
\hline SRS & Savannah River Site \\
\hline TRU & Transuranic \\
\hline VWO & Vitrified Waste Option \\
\hline WAC & Waste Acceptance Criteria \\
\hline WIPP & Waste Isolation Pilot Plant \\
\hline
\end{tabular}




\subsection{INTRODUCTION}

\subsection{Background}

The Idaho Chemical Processing Plant (ICPP) located at the Idaho National Engineering and Environmental Laboratory (INEEL) has been used for reprocessing spent nuclear fuel (SNF) since 1953. SNF was reprocessed to recover fissile uranium; however, changing world events have diminished the need to recover and recycle this material. In April 1992, the Department of Energy (DOE) discontinued reprocessing SNF and shifted its focus toward continued management and disposal of waste accumulated from previous reprocessing activities. ICPP waste treatment activities produce a granular solid waste (calcine) that contains dissolved fission products and fuel cladding material. Decontamination activities at the ICPP produce liquid radioactive, sodium-bearing waste (SBW) and ongoing operations at the ICPP produce liquid waste that contains low levels of radioactivity. The treatment of radioactive wastes for transfer out of Idaho is mandated under a "Settlement Agreement" between DOE and the State of Idaho." The Settlement Agreement requires that the existing inventory of liquid high-level radioactive waste (HLW) be calcined by June 30,1998, and SBW by the end of 2012, and the target date for all waste to be ready to ship from the INEEL is 2035.

The Department of Energy Idaho Operations Office (DOE-ID) is preparing the High-Level Waste and Facilities Disposition Environmental Impact Statement (HLW\&FD EIS), in accordance with the National Environmental Policy Act, to support the decision-making process regarding HLW management at the INEEL. The Fluor Daniel Corporation and the INEEL operating contractor, Lockheed Martin Idaho Technologies Company (LMITCO), have been tasked with performing studies that provide the basis for portions of the EIS. Waste treatment options include dissolving the calcine; both the dissolved calcine and the liquid SBW would then be separated into high-activity waste (HAW) and low-activity waste (LAW). The HAW would be mixed with a glass matrix and poured into canisters. This is termed the "Separations Option." Alternative options that do not involve a separation step are termed the "Nonseparations Option." The Waste Treatment Studies ${ }^{2-9}$ consider the several alternative processes for preparing the calcine and liquid waste for ultimate removal from the INEEL: Separations [(Full and Transuranic (TRU)] and Nonseparations (Vitrified, Hot Isostatic Press, Cementitious, and Direct Cementitious). Fluor Daniel Corporation is studying the Full Separations alternative and the others are being studied by LMITCO. The product of each of the processes will be waste in a storage canister that is ready for shipment to a permanent repository. Because the repositories will not be capable to receive the canisters at the time they are produced, interim storage will be required at the INEEL. This EDF documents cost, schedule, and environmental impact for storage of the waste generated by the above processes in a new Interim Storage Facility (ISF). A new ISF for the Full Separations alternative is included in the Fluor Daniel Corporation Study. This study includes a modified existing facility study for interim storage for the Full Separations Option. Interim storage in the FPR at the ICPP is documented in EDF-IS-002.

\subsection{Objective and Scope of Work}

The objective of this study is to explore alternatives for new interim storage of the waste in canisters that result from HLW processing of the calcine and liquid waste at ICPP. Table 1 gives the processing options and associated data that are included in this study: 
Table 1. Waste processing options and study data.

\begin{tabular}{|c|c|c|c|c|c|}
\hline & 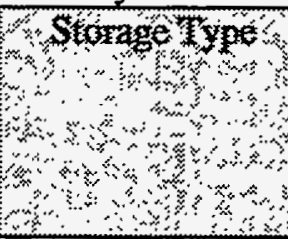 & 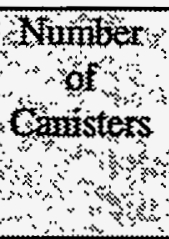 & 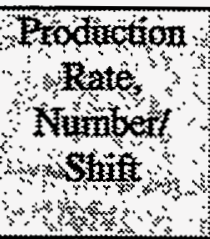 & 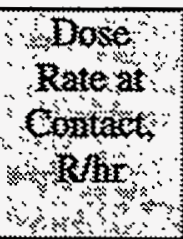 & 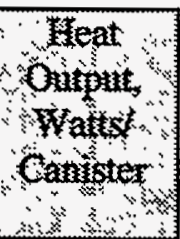 \\
\hline \multicolumn{6}{|l|}{ Separations Options } \\
\hline \multirow[t]{2}{*}{ TRU/Class A Separations } & New Facility & 170 & 1/month & 160,000 & 12,350 \\
\hline & NUHOMSTM & 170 & 1/month & 160,000 & 12,350 \\
\hline \multicolumn{6}{|l|}{ Nonseparations Options: } \\
\hline Vitrified & New Facility & 14,000 & 6 & $6.1-172$ & $0.6-14.7$ \\
\hline Direct Cementitious & New Facility & 18,000 & 35 & $6.9-287$ & $0.4-16.4$ \\
\hline \multirow[t]{2}{*}{ Cementitious } & New Facility & 16,000 & 32 & 140 & 8.9 \\
\hline & $\begin{array}{l}\text { HIP Preliminary } \\
\text { Plan }\end{array}$ & $\begin{array}{l}31,000 \\
\text { drums }\end{array}$ & 64 & 140 & 8.9 \\
\hline Hot Isostatic Press & New Facility & 5,700 & 9 & $14-580$ & $1.7-61$ \\
\hline
\end{tabular}

The objective of this study was to determine the cost, schedule, and environmental impacts for the storage of the waste canisters that result from each of the processing options in Table 1. A new facility was studied that incorporates appropriate design features from the Fluor Daniel Corporation design for interim storage presented in their document, Waste Treatment Facilities, Feasibility Study Report, December 12, 1997 (see Reference 2). The storage of canisters resulting from the Full Separations Option in NUHOMS ${ }^{\mathrm{M}}$ modules was the subject of report, DPC Loading Feasibility Study Report, INEEL/EXT-97-01251, November 1997 (see Reference 3). This study considers storage of the canisters that result from the TRU/Class A Separations Option in the NUHOMSTM module. The TRU/Class A Separations option would generate three waste streams designated as LLW: LAW, HAW, and TRU waste. All of these waste streams are actually considered HLW. It is expected that the LAW and TRU meet the definition of NRC LLW Class A and TRU, respectively. However, these wastes do not conform to the existing classification scheme for radioactive waste. Therefore, they will need the evaluation and concurrence of appropriate government authorities (NRC and DOE) for reclassification. Only the HAW will require interim storage. The LAW is assumed to go directly to a Class A waste disposal site and the TRU waste to go directly to the Waste Isolation Pilot Plant (WIPP). The TRU/Class C Separations Option does not require interim storage.

A preliminary plan for the Cementitious Option is under consideration. The resulting grout would be placed into drums within a 5-year period and placed into the ISF. Over a 20-year period, the drums would be subjected to a Hot Isostatic Press (HIP) process that would further reduce the volume. This alternative was included in this study.

\subsection{DESIGN BASIS, KEY ASSUMPTIONS, AND REQUIREMENTS}

\subsection{Design Basis}

A facility is required near the processing facility to store the HLW from the time it leaves the processing facility until it can be moved to a permanent waste repository. The quantities of canisters and 
their characteristics given in Table 1 result from the studies for the processing alternatives. The design life for the facilities is from 40 to 60 years.

The Savanna River Site (SRS) HLW canister and the West Valley Demonstration Project HLW canister meet the repository Waste Acceptance Criteria (WAC) for dimensions, although there are significant differences in these canisters' physical makeup. The SRS canister is used as the baseline for this study because it was selected in the ICPP Waste Treatment Facilities Feasibility Study Report. ${ }^{2}$ However, the DOE Hanford Facility has adopted a 4.6-m (15-ft) long canister for containing immobilized HLW. This longer canister is apparently acceptable for co-disposal at the repository, and therefore should be considered before finalizing the canister size. Consideration of the 4.6-m (15-ft) canister is addressed in the recommendations in Section 9. Some cost increase would result because the head height for handling the longer canisters is greater, and depth of excavation, equipment capacities, etc., increase. Cost increases would also occur because of the increase in the ISF transport tunnel height, canister handling machine size and capacity, and the ceiling height. However, smaller interim storage facilities may be adequate with fewer canisters, and handling, shipping and final disposal operations with fewer canisters would offset the cost increases if the longer canisters were adopted. The net result of the cost changes is unknown.

Transport of the waste to the interim storage facility is included, however transport from the facility is not included in this study. Equipment for handling the canisters for movement into and from the facility is included.

A passive cooling system is desired. The additional safety issues and operating costs of an active system would thus be avoided.

\subsection{Key Assumptions}

The following assumptions formed the framework, i.e., and the directed charter, for this study. Therefore, although some of these assumptions are likely to change, and such changes may have a significant impact on the results of this study, it is beyond the scope of this study to predict such changes or evaluate the associated risk.

1. The quantities and characteristics of waste to be stored are as tabulated in Table 1.

2. The waste will be stored in the SRS canister described in EDF-DPC-001, Appendix E of DPC Loading Feasibility Study Report, INEEL/EXT-97-01251, November 1997. All canisters will be sealed and radiologically clean.

3. The processed waste is not Resource Conservation and Recovery Act (RCRA) hazardous waste as a result of delisting before processing and by virtue of processing when all "listed" hazardous and characteristic constituents are treated to the Land Disposal Regulation standards, then the waste is no longer classified as RCRA waste.

4. The LAW and TRU waste will be reclassified as LLW Class A and TRU, respectively.

5. The NRC will license the facility in accordance with the requirements of $10 \mathrm{CFR}$ Part 72 . However, the cost for the licensing is not included. 
6. The processing facilities will not have lag storage. If the ISF cannot handle the canisters at the rate produced by the facility as given in Table 1, the ISF must have the capability to store them in a lag storage area. This lag storage would only be used if the processing facility releases the canisters in a batch or for maintenance of the canister handling equipment in the ISF.

7. The handling equipment will interface with an MP-187 cask for canister shipment from the ISF.

8. Physical security requirements are enveloped by those for SNF.

9. Dual Purpose Canisters (DPCs) will be acceptable for storage under 10 CFR 72.

10. The maximum weight of a canister is $5,300 \mathrm{lb}$.

11. Others will provide the proposed rail spur.

12. The transfer cart systems are described in the process facility options.

13. Shipments from the facility will be on a licensed rail shipping cask at grade and shipments to the facility are on a transfer cart in a belowgrade shielded transfer tunnel.

14. Storage of canisters shall be stacked as deep as three high inside sealed carbon steel tubes supported by support stools on the vault floor, both spaced for convection cooling and optimizing storage inside shielded concrete vaults.

15. A charged face floor system shall be used to maximize storage capacity, shielding, and strength while minimizing floor weight and contractibility concerns.

16. Convection air-cooling shall be used for cooling the canisters.

17. The same low profile gantry crane used in the proposed Interim Storage Facility Study at ICPP by Fluor Daniel Corporation ${ }^{2}$ shall be used in the Charge House for placing the canisters and transferring them for future shipments.

18. The Administrative Support building shall be like the one that supports the proposed Interim Storage Facility Study at ICPP by Fluor Daniel Corporation. ${ }^{2}$

19. The north and south end walls shall be designed so as to allow expansion in both directions.

20. Assumed concrete shielding thickness to be .9 meters ( 3 feet) thick for all storage alternatives except for the TRU/Class A Separations option that will have 1.5 meters ( 5 feet) thick concrete.

21. The assumed transfer cask on the gantry crane is to be similar to that of a bottom loading Peach Bottom cask. 


\subsection{Requirements}

\subsubsection{Regulations}

The NRC in accordance with 10 CFR Part 72 will license the storage facility, however the cost for licensing is not included in this report. It is assumed that the canisters, as received from the processing facility, will meet Department of Transportation (DOT), NRC, and/or HLW Repository WAC requirements and require no further conditioning, testing, treatment, marking, etc. This requires that the waste will be excluded from RCRA hazardous waste management requirements at the INEEL due to successful delisting and/or treatment.

The ISF will be licensed to meet 10 CFR Part 72 for HLW; therefore the receipt, handling, packaging, and storage of canisters of waste must also be governed under 10 CFR Part 72. Changes required to the facility license must be evaluated through a 10 CFR 72.48 evaluation, issuance of a Safety Analysis Report, and licensing documentation amendments. It is assumed that the Department of Energy Idaho Operations Office (DOE-ID) will continue to implement the quality assurance program described in DOE-RW-0333P, Quality Assurance Requirements and Description ${ }^{4}$ and will apply it to this effort as well. The impact of implementing DOE-RW-0333P is reflected in the capital and operating cost estimates generated by this feasibility study.

\subsubsection{HLW Repository WAC}

The requirements of the potential future repository waste acceptance will be satisfied by the processing facility before the canisters being released to the ISF. The ISF will not provide any capability to process, modify, or change the waste or canisters.

\subsubsection{Applicable Codes and Standards}

The NRC licenses and regulates waste storage facilities in accordance with 10 CFR Part 72. DOE requirements that are included in 10 CFR Part 72 will be superseded by them, however, any DOE requirements that are not covered by 10 CFR Part 72 will also apply.

\subsection{INTERIM STORAGE FACILITY DESIGNS}

\subsection{Basic Design Concepts}

Each alternative in the study will be designed to meet all applicable codes and standards. Because of the minimal physical access requirements for the job descriptions of the ISF to maintain safety, to control operations and to evacuate all personnel of a potentially dangerous work environment in an emergency, the alternative floor plans proposed in these studies do not reflect accommodations for all the National Statutes (such as Americans with Disabilities Act). This is also due to the minimal number of employees involved for staffing and feasibility of such in a remote nuclear facility of this type. Detailed information on other applicable and relevant codes and standards shall be further defined in the Conceptual Design. In summary, the following codes and standards shall apply to the ISF: 
- United States Nuclear Regulatory Commission

- Code of Federal Regulations (such as 10 CFR Part 72)

- Department of Energy Orders, Standards, and Manuals

- National and International Standards (such as ANSI, ASHRAE, UBC, ISO, etc.)

- State of Idaho Requirements

- LMTTCO Manuals and Procedures.

The same Administrative Support building and the same gantry crane used in the Fluor Daniel Corporation study ISF is used as a baseline in these ISF alternatives.

Convection air cooling of the vault storage is used requiring no heating, ventilating, and cooling equipment. Heating, ventilating, and air conditioning is provided in the Administrative Support Structure.

The elevation of the ISF was determined by the railroad-shipping bay being fixed at existing grade to allow rail-car shipments to be more convenient and to avoid flood plain concerns.

The ISF as shown in the drawings in Appendix 2 can be modular in design by varying the number of vaults depending on which alternative is applicable. The ISF is designed to hold SRS canisters resulting from HLW processing options of the calcined and liquid waste at ICPP in individual vertically sealed storage tubes ( $66 \mathrm{~cm} / 26$ inch diameter) retained within a concrete structure forming the storage vault. The storage tube provides structural support for the stacked canisters with cushion blocks and a mechanical barrier between the canisters and the environmental cooling circuit.

The typical vault has a concrete floor and walls with inlet and outlet air cooling ducts. The roof of the vault is a composite steel and concrete structure called the Charge Face Structure, which has holes to receive the storage tubes that each hold three canisters and their cushion blocks. The Charge Face Structure combined with support stools in the vault floor maintains a fixed array of storage tubes optimized for maximum density and efficient cooling.

Removable shield plugs in the Charge Face Structure allow loading of the storage tubes, multiple canisters, and their cushion blocks. In this configuration a vault can store 1,890 canisters.

Cooling air enters through a protective screen mesh, flows through a labyrinth that prevents radiation streaming, and enters the vault through distribution louvers. The air picks up heat from the HLW before passing through the exit louvers, up the concrete chase, and out into the atmosphere via protective screen mesh and weather protected stack.

The cooling system is passive, self-regulating and driven by the natural buoyancy of warm air. The louvers are designed to direct airflow as well as regulate the volume of airflow during extreme weather conditions. The size of the inlets and outlets are anticipated to be oversized for partial and entire blockages even for extended time periods, however, these conditions are to be further evaluated in conceptual design for actual conditions and when design heat loads are known.

Supplementary Lag Storage locations are provided at the end of the transfer cart tunnel to provide more immediate storage in the event of failure of a transfer crane so that the other transfer crane can keep up and avoid a bottleneck in storage from the production line. The Supplementary Lag Storage also offers an additional degree of independence during off-normal event storage should HLW canisters need additional isolation and for fluctuations during future ISF shipping operations. 
Transfer Cart Tunnel and Reception Bay is located belowgrade approximately 37 feet below the Charge Face Floor. The enclosed reception area is shielded with 5 feet thick concrete construction for transfer of HLW canisters arriving in a transfer cart from the process facility. There will be camera monitoring required in the Reception Bay and access for manned entry when operation procedure allows such to occur. A 5-ton overhead crane will be provided for remote maintenance should conditions require it.

The Shipping Cask Bay is an enclosed area located at the same grade as the rail spur with 3.7-m (12-ft) X 5.5-m (18-ft) overhead doors located at each end. The Shipping Cask Bay is located at finish grade, 6.4 meters (21 feet) below the Transfer Bay. A specialized Cask Maneuvering Hydraulic Platform will be provided to upright and recline the shipping cask for loading while on the rail-car and also be capable of lifting the shipping cask and rail-car (up to 180 tons) to receive transfer of HLW from the Transfer Bay above with restraints to prevent movement in the event of a seismic disturbance.

The Transfer Bay is an approximate $13.7-\mathrm{m}(45-\mathrm{ft}) \times 36.6-\mathrm{m}(120-\mathrm{ft})$ bay of the Charge House in which all the receiving, shipping, transfer, and lag storage of the HLW canisters take place.

The enclosed area above the Charge Face Structure and the Transfer Bay is the Charge Hall, which provides a weatherproof enclosure for the loading and unloading operations of the HLW canisters, crane maintenance areas, and two facility gantry cranes (in most storage alternatives). The Charge Hall slopes up from $14.6 \mathrm{~m}$ (48 ft) at the lowest eave leaving a clear height inside of approximately $11 \mathrm{~m}$ $(36 \mathrm{ft})$. The enclosure is an unconditioned space with a concrete and uninsulated metal skin. The top of the stack where the cooling air outlet is located is $19.5-\mathrm{m}(64-\mathrm{ft})$ above the Charge Face Floor.

\subsection{Modular Expansion to Satisfy Processing Concept Requirements}

The proposed new ISF is designed to be modular construction to accommodate a variety of alternative process requirements, quantities, and time durations.

There are five design alternatives supported for the IFS using the generic modular designs with slight variations in number of modules and equipment, but with one exception. The TRU/Class A Separations Option with 170 canisters has a much higher gamma field due to the $\mathrm{HL} W$ being more concentrated and therefore requires 5-foot thick instead of 3-foot thick concrete shielding in vault construction. The alternatives considered for the ISF are listed below. See the drawings in Appendix 2.

1. One vault facility for the TRU/Class A Separations Option, requiring storage for $\mathbf{1 7 0}$ canisters over a 20-year period.

2. Four vault facility for the HIP Waste Option, requiring storage for 5,700 canisters over a 20 -year period.

3. Eight vault facility for the Vitrified Waste Option, requiring storage for 14,000 canisters over a 20-year period.

4. Nine vault facility for the Cementitious Waste Option and the preliminary plan, requiring storage for 16,000 canisters over a 5-year period.

5. Ten vault facility for the Direct Cementitious Waste Option, requiring storage for 18,000 canisters over a 5-year period. 


\subsection{Cementitious Waste Option (CWO) and Direct Cementitious Waste Option (DCWO) HIP Preliminary Plan}

A preliminary plan for subjecting the CWO waste to the HIP process is under consideration. The preliminary plan is to perform the initial processing to place cementitious waste in drums over a 5-year processing period. The drums selected are those shown in EDF-CWO-001 in Appendix E of the report Cementitious Waste Option Study Report, INEEL/EXT-01389 (see Reference 8). The waste in the drums will then be subjected to the HIP process that will reduce the waste volume. Interim storage of the drums is required for the period of time from initial processing to the time the HIP process is performed. The CWO process will take place over the first 5 years of the 20 years that the HIP process takes and the HIP process over the full 20 years. For storage, the drums will be stored in the $66-\mathrm{cm}$ (26-inch) diameter sleeves that will ultimately store the waste in the canisters. As the waste in drums is removed from storage for the HIP process, storage sleeves will be emptied. The drums can be stored in the same storage sleeves that are planned for the canisters. A special handling grapple would be required to handle the drums in addition to the handling equipment for canisters. The grapple must be capable of lowering the drums into the storage sleeves and releasing from the drum. The grapple must also be capable of the reverse operation to remove the drums for processing. The unprocessed drums are 142-cm (56 inches) long, therefore, six can be stored in each storage sleeve. The maximum storage requirement will be at the end of 5 years of operation when 7,750 drums have been HIPed and placed into 2,583 canisters. The facility will be required, at the end of the $5^{\text {th }}$ year of operation, to also store 23,250 drums. The canisters and drums will require 4,736 storage sleeves or eight modules, the same size as is needed for the VWO Option, which is one module smaller than required for the CWO option in Section 3.6.1. The cost for the facility is, therefore, the same as for the VWO Option with the additional cost for the handling equipment for the drums.

The preliminary plan for HIPing with the DCWO produces waste in drums that are HIPed and placed into canisters directly. Instead of 18,000 canisters, only 11,700 require storage. A seven-module storage facility would be required. Because the drums are not handled in the interim storage facility, no additional handling equipment is required. 


\subsection{TOTAL PROJECT, LIFE-CYCLIE, AND D\&D COSTS}

Cost estimates for the new interim storage alternatives are in Appendix 1. The costs are summarized in Table 2.

The costs shown for the DCWO and CWO Preliminary Plan have been arrived at as follows: the cost estimate for Vitrified Waste Option (VWO) with eight modules has been scaled to get the cost for the ISF for the DCWO Preliminary Plan with seven modules. The cost for the CWO Preliminary Plan was arrived at by adding the estimated cost for the drum handling equipment, $\$ 100,000$, to the estimate for the VWO facility, because a facility that is the same size as the VWO facility would be required. 
Table 2. Project Costs

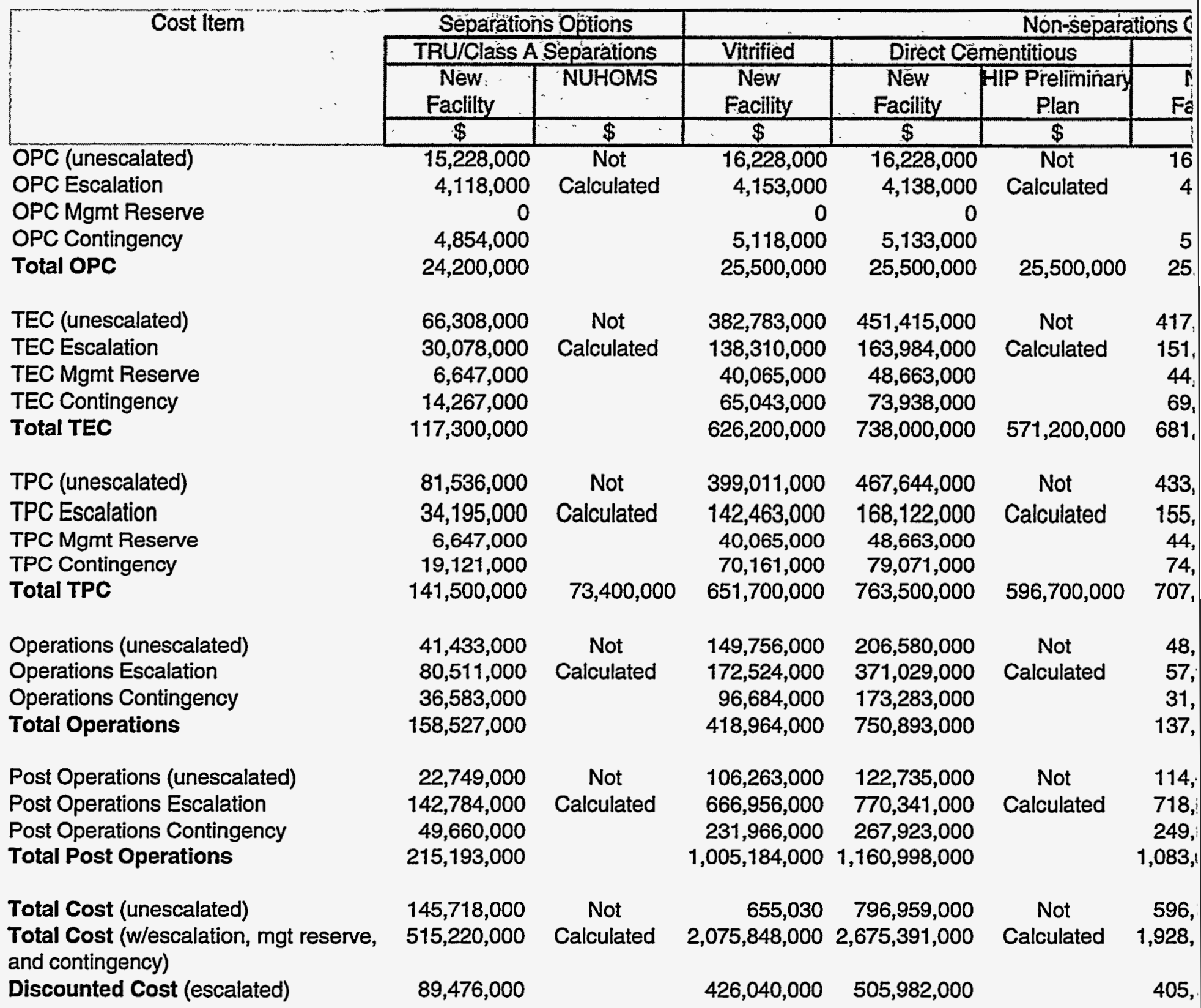


\begin{tabular}{l|l}
\hline Cementitious - & Hot lso. Pressed \\
\hline
\end{tabular}

\begin{tabular}{|c|c|c|}
\hline $\begin{array}{l}N \\
\text { ity }\end{array}$ & $\begin{array}{c}\text { HIP Preliminary } \\
\text { Plan }\end{array}$ & $\begin{array}{l}\text { New } \\
\text { Facility }\end{array}$ \\
\hline & $\$$ & $\$$ \\
\hline 28,000 & Not & $16,228,000$ \\
\hline$\lceil 3,000$ & Calculated & $4,310,000$ \\
\hline 0 & & 0 \\
\hline 18,000 & & $5,161,000$ \\
\hline 10,000 & $25,500,000$ & $25,700,000$ \\
\hline 13,000 & Not & $224,756,000$ \\
\hline 14,000 & Calculated & $80,542,000$ \\
\hline$i 3,000$ & & $22,291,000$ \\
\hline 0,000 & & $41,012,000$ \\
\hline 10,000 & $626,300,000$ & $368,600,000$ \\
\hline :1,000 & Not & $240,984,000$ \\
\hline 8,000 & Calculated & $84,852,000$ \\
\hline 3,000 & & $22,291,000$ \\
\hline 8,000 & & $46,173,000$ \\
\hline 0,000 & $651,800,000$ & $394,300,000$ \\
\hline 5,000 & Not & $95,689,000$ \\
\hline 4,000 & Calculated & $119,204,000$ \\
\hline 0,000 & & $64,468,000$ \\
\hline 8,000 & & $279,361,000$ \\
\hline 7,000 & Not & $65,515,000$ \\
\hline 9,000 & Calculated & $411,201,000$ \\
\hline 1,000 & & $143,015,000$ \\
\hline 7,000 & & $619,730,000$ \\
\hline 3,000 & Not & $402,188,000$ \\
\hline 5,000 & Calculated & $1,293,391,000$ \\
\hline 4,000 & & $255,814,000$ \\
\hline
\end{tabular}




\subsection{PROJECT SCHEDULE}

Figure 2 for each processing option and interim storage alternatives show the schedules for the installation or modification of the storage facilities.

\subsection{RISK ASSESSMENT}

High-level risks identified in this study are categorized below. The assumptions in Section 2.2 provide the framework for this list. The highest risk is rated at "3" (out of "9"). Data Sheets for all the identified risks are included in Appendix 3, along with an explanation of the Risk Rating calculation method.

1. The quantities and characteristics of the waste are different than assumed in the study. Risk $=3$ (Probability $=3$, Impact $=1$ ).

2. Waste will not be stored in the SRS canister. Risk $=2$ (Probability $=2$, Impact $=1$ ).

3. The facility would have to be redesigned if the waste is classified as a RCRA waste after processing. Risk $=3$ (Probability $=1$, Impact $=3$ ).

4. A storage canister will be mishandled or dropped during waste transfer to, from, or in the facility. Risk = 2 (Probability = 1, Impact $=2$ ).

5. The facility might not be operational when needed. Risk $=2$ (Probability $=1$, Impact $=2$ ).

Many risks including environmental, safety, and health risks were not identified in this study. 
Figure 2. (continued).

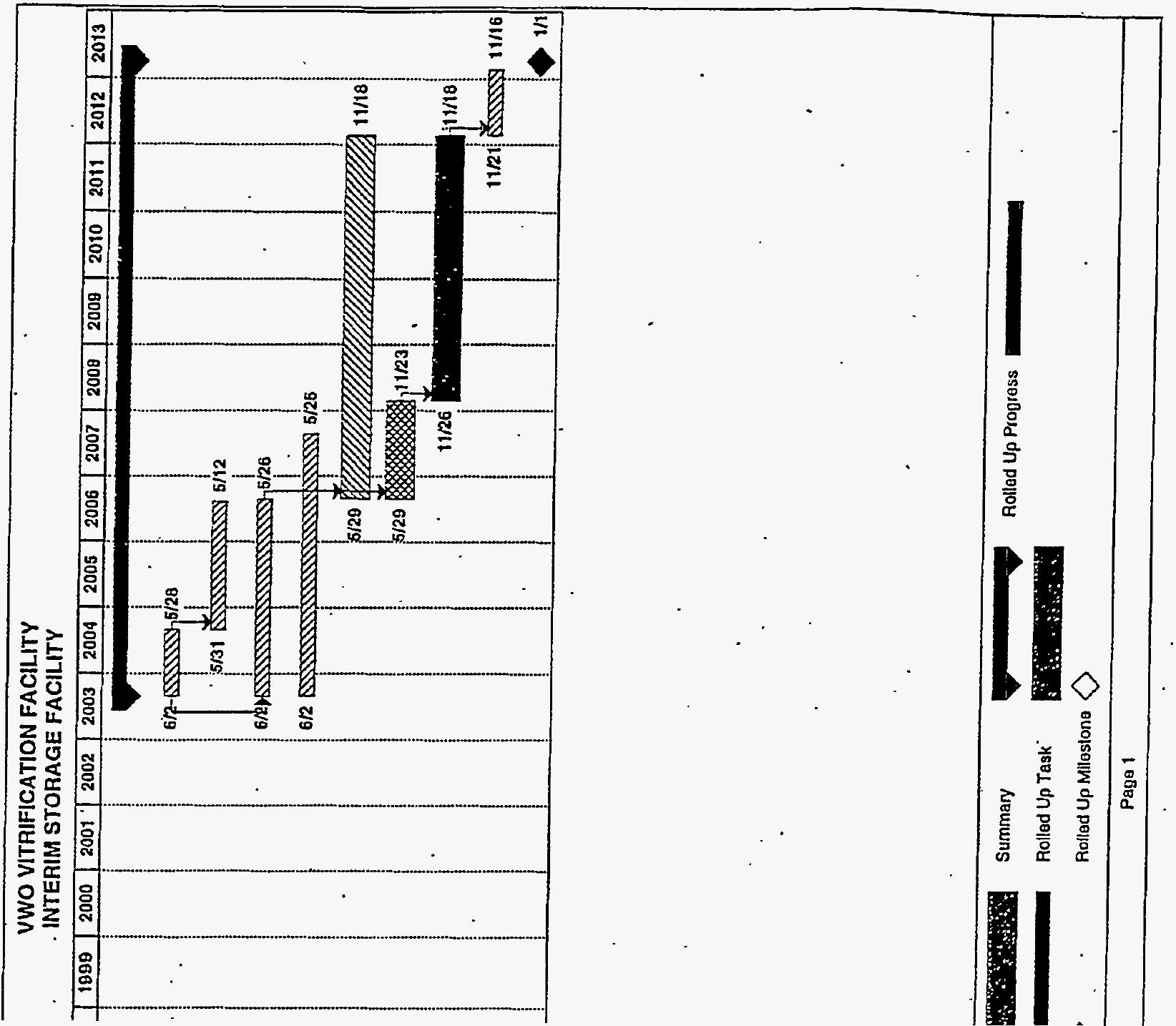




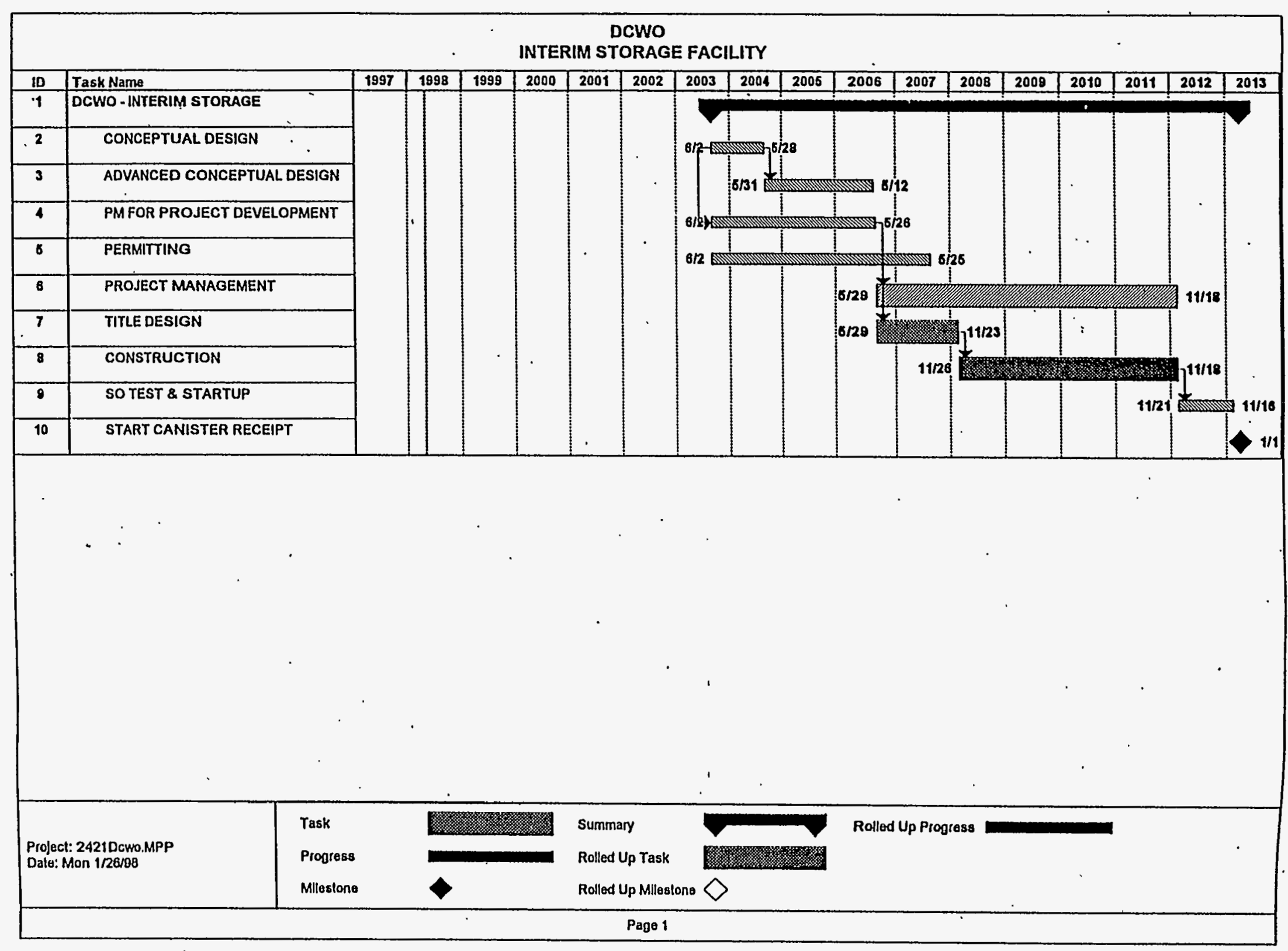




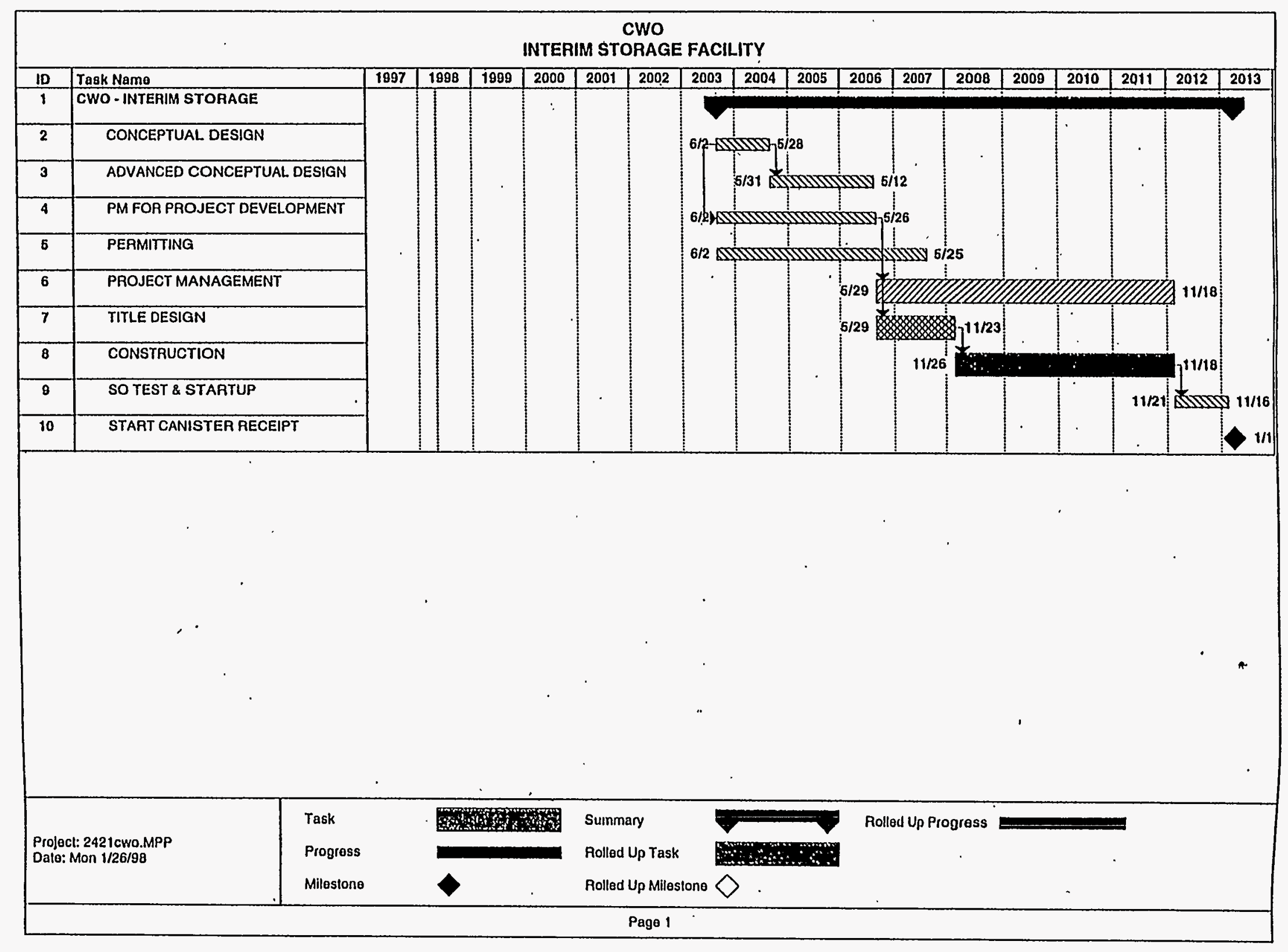


Figure 2. (continued).

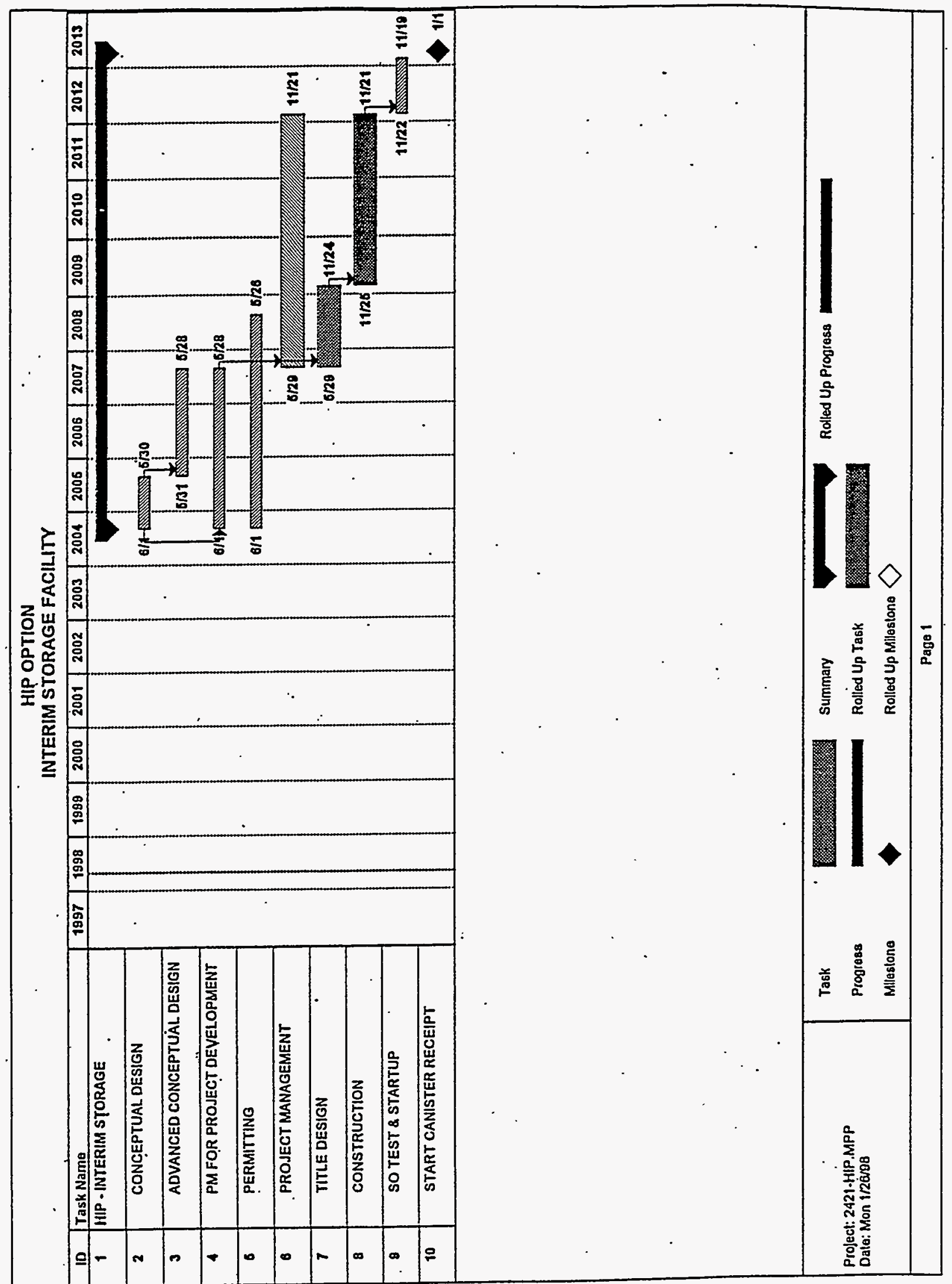




\subsection{CONSTRUCTION AND OPERATIONAL ENVIRONMENTAL IMPACTS}

The Product Data Sheets that summarize the construction and operational environmental impacts for the interim storage facilities that support the waste processing options are in Table 3. Data are presented for the construction, operation, and decommissioning of the project. Estimates are included for the following parameters:

- Cost

- Schedule

- Air emissions

- Liquid effluents

- Solid wastes

- Utilities used

- Manpower requirements

- Regulatory requirements.

Because this project is at a very preliminary stage of design, much of the information presented in the Project Data Sheets is based on representative literature values, existing laboratory data, and engineering judgment. The cost data in Table 3 were obtained from the cost estimates in Appendix 1 and the supporting information for the remainder of the data is in Appendix 4. The Product Data Sheets are presented in the following order in Table 3:

\begin{tabular}{|l|l|}
\hline TRU/Class A Separations - New ISF & PDS for Interim Storage Facility for TRU \\
\hline Vitrified & PDS for Interim Storage Facility for VWO \\
\hline Direct Cementitious & PDS for Interim Storage Facility for DCWO - 20 Yr \\
\hline Cementitious & PDS for Interim Storage Facility for CWO \\
\hline Hot Isostatic Press - New ISF & PDS for Interim Storage Facility for HIP \\
\hline
\end{tabular}




\section{Project Data Sheet for Interim Storage Facility for TRU}

\section{Generic Information}

Description/function

EIS Altematives ( $A, B$, etc.)

Project type or waste stream

Action type

Structure type

Size $\left(\mathrm{m}^{2}\right)$

Other features (e.g. pits, ponds, power/water/sewer lines)

Location

Inside/outside of fence

Inside/outside of building

Candidate for privatization?
Provide Long-term storage for road-ready containers awaiting shipment to a permanent repository.

TRU Separations Waste Option

Treated HLW calcine.

New

$$
\begin{aligned}
& 3,350 \mathrm{~m}^{2} \\
& \text { None }
\end{aligned}
$$

Inside ICPP' fence

Inside TRU Separation Facility

Yes

\section{Construction Information}

Cost (\$): Pre-Operations (Other Project Costs) with escalation an

Conceptual Design

Management for Project Development

Permitting and Documentation

SO Test \& Start-Up

Contingency

Total Preconstruction

Cost $(\$):$ Construction with escalation and contingency.

ED\&l

Management (PM/CM)

Construction

G\&A/PIF

Procurement Fees, Management Reserve and Contingency

Total Construction (TEC)

Schedule start/end: Preconstruction

Schedule start/end: Construction

Schedule start/end: SO Test \& Start-up

Number of workers each year of construction (new/existing)

Nonradiation

Number of radiation workers (construction)

Average annual worker radiation dose (rem/yr)

\section{Heavy equipment}

Equipment used

Trips (construction materials delivery)

Hours of operation

Acres disturbed and duration of disturbance

New

Previous
$\$ 4,240,000$
$\$ 4,520,000$
$\$ 3,656,000$
$\$ 6,930,000$
$\$ 4,854,000$
$\$ 24,200,000$

$\$ 10,601,000$

$\$ 10,177,000$

$\$ 42,405,000$

$\$ 2,701,000$

$\$ 15,116,000$

$\$ 81,000,000$

January 1999 through December 2001

January 2010 through December 2013

January 2014 through December 2014

\author{
58 New workers/yr \\ None \\ None
}

Excavator, dump trucks, cranes, cement trucks,grader, material delivery

$$
\begin{aligned}
& 1,156 \\
& 5,287 \text { hours (total) }
\end{aligned}
$$

January 2010 through December 2013

None

3.0 acres 
431.02\#

06/17/97

Rev. $\# 04$

Revegetated

\section{Air emissions}

Dust

Major gases $\left(\mathrm{CO}_{2}, \mathrm{H}_{2} \mathrm{O}, \mathrm{O}_{2}, \mathrm{~N}_{2}\right)$ from diesel exhaust

Contaminants (Particulates, $\mathrm{CO}, \mathrm{NO}_{x}, \mathrm{SO}_{2}$, hydrocarbons) from diesel exhaust.

Total air emissions

\section{Effluents}

Sanitary wastewater

Lube Oil

\section{Solid wastes}

Type - construction trash

Radioactive wastes

Hazardous/toxic chemicals and wastes (type)

Storage/inventory

Hazardous waste (construction)

Pits/Ponds created $\left(\mathrm{m}^{2}\right)$

Water usage:

Dust control

Domestic water

Energy requirements

Electrical (MWh/yr)

Fossil fuel (liters)

Permits needed for construction
Function File Number - SPR-01

EDF Serial Number - EDF-IS-001

Page 22 of 42

\section{Project Data Sheet for Interim Storage Facility for TRU \\ None}

86 tons (total)

4,814 tons (total)

28 tons (total)

4,928 tons (total)

$2,469,713$ liters (total) 1,001 liters (total)

$1,375 \mathrm{~m}^{3}$ (total)

None

$$
\begin{aligned}
& 13 \mathrm{~m}^{3} \\
& 217 \mathrm{~m}^{3} \text { (total) } \\
& \text { None }
\end{aligned}
$$

616,810 liters (total)

$2,469,713$ liters (total)

$156 \mathrm{MWh} / \mathrm{yr}$

0 liters (total)

NEPA documentation (prior to start of Title II construction); New stationary

sources/PTC/NOC/PSD for non-rad air emissions; HAP's and TAP's and RCRA (part $A A, B B$, and $C C$ for air) for hazardous air emissions; air operating permit; NESHAP's, NPDES, NESHAP's subpart $H$ for rad air emissions; approval of Engineering Plans; Cross Connection Control Plans; report and specifications for drinking water supply;RCRA Part A and Part B permits.

\section{Operational Information}

Cost (\$): Operations (unescalated)

Facility/Administration

$\$ 7,007,000$

Operations/Process Facility

$\$ 17,581,000$

$\$ 16,845,000$

Procurement, materials, utilities, maintenance

$\$ 41,433,000$

Total operations

January 2014 through December 2068

Number of workers each year of operation (new/existing) 
Operations

Maintenance

Support

Total

Number of radiation workers

Average annual work radiation dose (rem/yr)

Heavy Equiipment

Air Emissions

Radioactive off-gas

Effluents

Sanitary Wastewater

\section{Solid Wastes}

Sanitary/Industrial Trash

Radioactive wastes (canisters)

Hazardous/toxic chemicals and wastes

Pits/Ponds used $\left(\mathrm{m}^{2}\right)$

Water usage:

Domestic water

Energy Requirements:

Electrical (MWh/yr)

Fossil fuel (liters/yr)

Permits needed (for facility operations)
Function File Number - SPR-01

EDF Serial Number - EDF-IS-001

Page 23 of 42

\section{Project Data Sheet for Interim Storage Facility for TRU}

\author{
3 \\ 0.5 \\ 3 \\ 6.5 \\ 2.5 (included in above total) \\ $0.19 \mathrm{rem} / \mathrm{yr}$ perworker
}

None

None

224,498 liters/yr

$$
\begin{array}{r}
36 \mathrm{~m}^{3} / \mathrm{yr} \\
122 \mathrm{~m}^{3} / \mathrm{yr}
\end{array}
$$

None

224,498 liters/yr

\section{2,533 MWh/yŕ \\ None}

HAP's and TAP's and RCRA (part AA,BB, and CC for air) for hazardous air emissions; air operating permit; NESHAP's, NPDES, NESHAP's subpart $H$ for rad air emissions; approval of Engineering Plans; Cross Connection Control Plans; report and specifications for drinking water supply;RCRA Part A and Part $B$ permits.
Decontamination \& Decommissioning (D\&D) Information

\section{Cost (\$): D\&D (unescalated)}

Decommission

Decontamination

Demolition

Total D\&D

Schedule start/end: D\&D

Number of workers each year of D\&D (new/existing)

Number of radiation workers (D\&D)

Average annual worker radiation dose (rem/yr)
$\$ 3,180,000$

$\$ 0$

$\$ 19,569,000$

$\$ 22,749,000$

January 2069 through December 2071

53 New workers/yr

5 New workers/yr

$0.19 \mathrm{rem} / \mathrm{yr}$ perworker

Mobile Cranes, Roll-off trucks, Dozers, Loaders

Heavy equipment:

Equipment used 
$431.02 \#$

$06 / 17 / 97$

Rev. \#04

\section{ENGINEERING DESIGN FILE}

Table 3. (continued).
Function File Number - SPR-01

EDF Serial Number - EDF-IS-001

Page 24 of 42

\section{Project Data Sheet for Interim Storage Facility for TRU}

Trips

Roll-off trucks

Hours of operation (all heavy equipment)

9,480 Hours

Acres disturbed and duration of disturbance

New

Previous

Revegetated

3 per day

January 2069 through December 2071

None

3.0 acres

None

Air emissions

non-radioactive Fuel combustion gases $\left(\mathrm{CO}_{2}, \mathrm{H}_{2} \mathrm{O}, \mathrm{O}_{2}, \mathrm{~N}_{2}\right)$

5,510 tons (total)

non-radioactive

Fuel combustion contaminants ( $\mathrm{CO}$,

particulates, $\mathrm{NO}_{x}, \mathrm{SO}_{2}$, hydrocarbons)

32 tons (total)

radioactive HEPA filtered off-gas

None tons (total)

Effluents

radioactive Spent decontamination solution .

None liters (total)

non-radioactive Sanitary wastewater

non-radioactive Lube oil

$3,414,175$ liters (total)

1,794 liters (total)

Solid wastes:

radioactive

Non-radioactive (industrial)

Hazardous

$$
\begin{array}{r}
10,663 \mathrm{~m}^{3} \\
2 \mathrm{~m}^{3} \\
23 \mathrm{~m}^{3}
\end{array}
$$

Hazardous/toxic chemicals and wastes (type)

Storage/inventory

Pits/Ponds created $\left(\mathrm{m}^{2}\right)$

Hazardous Waste

$$
\begin{aligned}
& \text { None } \mathrm{m}^{3} \text { (total) } \\
& \text { None } \\
& 909 \mathrm{~m}^{3} \text { (total) }
\end{aligned}
$$

Water usage:

Domestic water

$3,414,175$ liters (total)

Source of water

ICPP site wells

Energy requirements:

Electrical (MWh/yr)

$156 \mathrm{MWh} / \mathrm{yr}$

Fossil fuel (liters)

Permits needed (e.g. for facility closures, physical characteristics

and quantities of radioactive and hazardous materials remaining

after closure)

Work will be done under closure provisions of existing.permits. 
431.02\#

$06 / 17 / 97$

Rev. \#04
ENGINEERING DESIGN FILE

Table 3. (continued).
Function File Number - SPR-01

EDF Serial Number - EDF-IS-001

Page 25 of 42

\section{Project Data Sheet for Interim Storage Facility for WWO}

Generic Information

Description/function

EIS Alternatives (A, $B$, etc.)

Project type or waste stream

Action type

Structure type

Size $\left(m^{2}\right)$

Other features (e.g. pits, ponds, power/water/sewer lines)

Location

Inside/outside of fence

Inside/outside of building

Candidate for privatization?
- Provide Long-term storage for road-ready containers awaiting shipment to a permanent repository.

Vitrified Waste Option

Treated HLW calcine.

New

$13,493 \mathrm{~m}^{2}$

None

Inside ICPP fence

Inside Vitrification Facility

Yes

Construction Information

Cost (\$): Pre-Operations (Other Project Costs) with escalation and contingency.

Conceptual Design

Management for Project Development

Permitting and Documentation

SO Test \& Start-Up

Contingency

Total Preconstruction

Cost (\$): Construction with escalation and contingency.

ED\&l

Management (PM/CM)

Construction

G\&AJPIF

Procurement Fees, Management Reserve and Contingency

Total Construction (TEC)

Schedule start/end: Preconstruction

Schedule start/end: Construction

Schedule start/end: SO Test \& Start-up

Number of workers each year of construction (new/existing)

Nonradiation

Number of radiation workers (construction)

Average annual worker radiation dose (rem/yr)

\section{Heavy equipment}

Equipment used

Trips (construction materials delivery)

Hours of operation

Acres disturbed and duration of disturbance

New

Previous
$\$ 5,000,000$

$\$ 4,000,000$

$\$ 2,728,432$

$\$ 4,500,000$

$\$ 7,571,568$

$\$ 23,800,000$

$\$ 35,000,000$

$\$ 55,337,700$

$\$ 273,376,540$

$\$ 16,334,715$

$\$ 79,451,045$

$\$ 459,500,000$

January 2003 through December 2007

January 2006 through December 2011

January 2012 through December 2012

114 New workers/yr

None

None

Excavator, dump trucks, cranes, cement trucks, grader, material delivery

2,191

13,683 hours (total)

January 2006 through December 2011

None

5 acres 
431.02\#

$06 / 17 / 97$

Rev. \#04

Revegetated

Air emissions

Dust

Major gases $\left(\mathrm{CO}_{2}, \mathrm{H}_{2} \mathrm{O}, \mathrm{O}_{2}, \mathrm{~N}_{2}\right)$ from diesel exhaust

Contaminants (Particulates, $\mathrm{CO}, \mathrm{NO}_{x}, \mathrm{SO}_{2}$, hydrocarbons) from

diesel exhaust.

Total air emissions

Effluents

Sanitary wastewater

Lube Oil

Solid wastes

Type - construction trash

Radioactive wastes

Hazardous/toxic chemicals and wastes (type)

Storage/inventory

Hazardous waste (construction)

Pits/Ponds created $\left(\mathrm{m}^{2}\right)$

Water usage:

Dust control

Domestic water

Energy requirements

Electrical (MWh/yr)

Fossil fuel (liters)

Permits needed for construction
Function File Number - SPR-01

EDF Serial Number - EDF-IS-001

Page 26 of 42

\section{Project Data Sheet for Interim Storage Facility for WWO \\ None}

288 tons (total)

13,189 tons (total)

77 tons (total)

13,554 tons (total)

$9,708,525$ liters (total)

2,590 liters (total)

$5,406 \mathrm{~m}^{3}$ (total)

None

$$
\begin{aligned}
& 13 \mathrm{~m}^{3} \\
& 217 \mathrm{~m}^{3} \text { (total) } \\
& \text { None }
\end{aligned}
$$

$2,056,032$ liters (total)

$9,708,525$ liters (total)

NEPA documentation (prior to start of Title II construction); New stationary sources/PTC/NOC/PSD for non-rad air emissions; HAP's and TAP's and RCRA (part $A A, B B$, and $C C$ for air) for hazardous air emissions; air operating permit; NESHAP's, NPDES, NESHAP's subpart $H$ for rad air emissions; approval of Engineering Plans; Cross Connection Control Plans; report and specifications for drinking water supply;RCRA Part A and Part $B$ permits.

Operational Information

Cost (\$): Operations (unescalated)

Facility/Administration

$\$ 7,293,000$

Operations/Process Facility

Procurement, materials, utilities, maintenance

Total operations

Schedule start/end

$\$ 18,299,000$

$\$ 124,165,000$

$\$ 149,757,000$

January 2012 through December 2068

Number of workers each year of operation (new/existing) 
431.02\#

$06 / 17 / 97$

Rev. \#04
ENGINEERING DESIGN FILE

Table 3. (continued).
Function File Number - SPR-01

EDF Serial Number - EDF-IS-001

Page 27 of 42

\section{Project Data Sheet for Interim Storage Facility for WWO}

Operations

Maintenance

Support

Total

Number of radiation workers

Average annual work radiation dose (rem/yr)

Heavy Equipment

Air Emissions

Radioactive off-gas

Effluents

Sanitary Wastewater

Solid Wastes

Sanitary/Industrial Trash

Radioactive wastes (canisters)

Hazardous/toxic chemicals and wastes

Pits/Ponds used $\left(\mathrm{m}^{2}\right)$

Water usage:

Domestic water

Energy Requirements:

Electrical (MWh/yr)

Fossil fuel (liters/yr)

Permits needed (for facility operations)

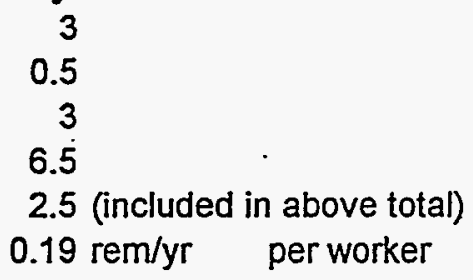

0.5

3

6.5

2.5 (included in above total)

$0.19 \mathrm{rem} / \mathrm{yr}$ perworker

None

None

224,498 liters/yr

$$
\begin{array}{r}
36 \mathrm{~m}^{3} / \mathrm{yr} \\
10,584 \mathrm{~m}^{3} / \mathrm{yr}
\end{array}
$$

None

224,498 liters/yr

4,368 MWh/yr
None

HAP'S and TAP's and RCRA (part AA,BB, and CC for air) for hazardous air emissions; air operating permit; NESHAP's, NPDES, NESHAP's subpart $H$ for rad air emissions; approval of Engineering Plans; Cross Connection Control Plans; report and specifications for drinking water supply;RCRA Part A and Part B permits.

\section{Decontamination \& Decommissioning (D\&D) Information}

Cost (\$): D\&D (unescalated)

Decommission $\$ 10,500,000$

Decontamination

Demolition

$\$ 95,783,000$

Total D\&D

Schedule start/end: D\&D

Number of workers each year of D\&D (new/existing)

$\$ 106,283,000$

Number of radiation workers (D\&D)

Average annual worker radiation dose (rem/yr)

January 2069 through December 2071

249 New workers/yr

25 New workers/yr

$0.19 \mathrm{rem} / \mathrm{yr} \quad$ per worker

Heavy equipment:

Equipment used

Mobile Cranes, Roll-off trucks, Dozers, Loaders 
Trips

\section{Project}

Roll-off trucks

Hours of operation (all heavy equipment)

Acres disturbed and duration of disturbance

New

Previous

Revegetated

\section{Air emissions}

non-radioactive Fuel combustion gases $\left(\mathrm{CO}_{2}, \mathrm{H}_{2} \mathrm{O}, \mathrm{O}_{2}, \mathrm{~N}_{2}\right)$

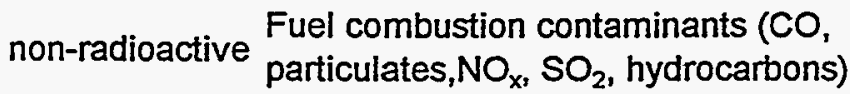

radioactive HEPA filtered off-gas

\section{Effluents}

radioactive Spent decontamination solution

non-radioactive Sanitary wastewater

non-radioactive Lube oil

Solid wastes:

radioactive

Non-radioactive (industrial)

Hazardous

Hazardous/toxic chemicals and wastes (type)

Storage/inventory

Pits/Ponds created $\left(\mathrm{m}^{2}\right)$

Hazardous waste

Water usage:

Domestic water

Source of water

\section{Energy requirements:}

Electrical (MWh/yr)

Fossil fuel (liters)

Permits needed (e.g. for facility closures, physical characteristics

and quantities of radioactive and hazardous materials remaining after closure)
29,189 tons (total)

170 tons (total)

None tons (total)

None liters (total)

$15,898,593$ liters (total)

9,504 liters (total)

$$
\begin{aligned}
\text { None } \mathrm{m}^{3} \\
42,946 \mathrm{~m}^{3} \\
8 \mathrm{~m}^{3}
\end{aligned}
$$

$$
\begin{aligned}
& \text { None } \mathrm{m}^{3} \text { (total) } \\
& \text { None } \\
& 909 \mathrm{~m}^{3} \text { (total) }
\end{aligned}
$$

$15,898,593$ liters (total)

ICPP site wells

$$
\begin{gathered}
156 \mathrm{MWh} / \mathrm{yr} \\
1,140,496 \text { liters (total) }
\end{gathered}
$$

Work will be done under closure provisions of existing permits. 
431.02\#

$06 / 17 / 97$

Rev. \#04
ENGINEERING DESIGN FILE

Table 3. (continued).

Project Data Sheet for Interim Storage Facility for DCWO - 20 yr
Function File Number - SPR-01

EDF Serial Number - EDF-IS-001

Page 29 of 42

\section{Generic Information}

Description/function

EIS Alternatives ( $A, B$, etc.)

Project type or waste stream

Action type

Structure type

Size $\left(\mathrm{m}^{2}\right)$

Other features (e.g. pits, ponds, power/water/sewer lines)

Location

Inside/outside of fence

Inside/outside of building

Candidate for privatization?

Provide Long-term storage for road-ready containers awaiting shipment to a permanent repository.

Direct Cementitious Waste Option

Treated HLW calcine.

New

$$
\begin{aligned}
& 15,967 \mathrm{~m}^{2} \\
& \text { None }
\end{aligned}
$$

Inside ICPP fence

Inside Grouting Facility

Yes

\section{Construction Information}

Cost (\$): Pre-Operations (Other Project Costs) with escalation an

Conceptual Design

Management for Project Development

Permitting and Documentation

SO Test \& Start-Up

Contingency

Total Preconstruction

Cost (\$): Construction with escalation and contingency.

\section{ED\&I}

Management (PM/CM)

Construction

G\&APIF

Procurement Fees, Management Reserve and Contingency

Total Construction (TEC)

Schedule start/end: Preconstruction

Schedule start/end: Construction

Schedule start/end: SO Test \& Start-up

Number of workers each year of construction (new/existing)

Nonradiation

Number of radiation workers (construction)

Average annual worker radiation dose (rem/yr)

Heavy equipment

Equipment used

Trips (construction materials delivery)

Hours of operation

Acres disturbed and duration of disturbance

New

Previous
$\$ 5,850,000$

$\$ 4,700,000$

$\$ 3,247,000$

$\$ 6,570,000$

$\$ 5,133,000$

$\$ 25,500,000$

$$
\begin{array}{r}
\$ 45,750,000 \\
\$ 83,023,000 \\
\$ 455,030,000 \\
\$ 27,046,000 \\
\$ 127,151,000 \\
\$ 738,000,000
\end{array}
$$

January 2003 through December 2007

January 2006 through December 2011

January 2012 through December 2013

134 New workers/yr

None

None

Excavator, dump trucks, cranes, cement trucks,grader, material delivery

2,482

15,642 hours (total)

January 2006 through December 2011

None

9 acres 
431.02\#

$06 / 17 / 97$

Rev. \#04
ENGINEERING DESIGN FILE

Table 3. (continued).
Function File Number - SPR-01

EDF Serial Number - EDF-IS-001

Page 30 of 42
Revegetated

\section{Project Data Sheet for Interim Storage Facility for DCWO - 20 yr}

None

Air emissions

Dust

Major gases $\left(\mathrm{CO}_{2}, \mathrm{H}_{2} \mathrm{O}, \mathrm{O}_{2}, \mathrm{~N}_{2}\right)$ from diesel exhaust

Contaminants (Particulates, $\mathrm{CO}, \mathrm{NO}_{\mathrm{x}}, \mathrm{SO}_{2}$, hydrocarbons) from

diesel exhaust.

Total air emissions

Effluents

Sanitary wastewater

Lube Oil

\section{Solid wastes}

Type - construction trash

Radioactive wastes

Hazardous/toxic chemicals and wastes (type)

Storage/inventory

Hazardous waste (construction)

Pits/Ponds created $\left(\mathrm{m}^{2}\right)$

Water usage:

Dust control

Domestic water

Energy requirements

Electrical (MWh/yr)

Fossil fuel (liters)

Permits needed for construction
518 tons (total)

15,023 tons (total)

88 tons (total)

15,629 tons (total)

$11,411,775$ liters (total)

2,960 liters (total)

$6,355 \mathrm{~m}^{3}$ (total)

None

$$
\begin{aligned}
& 13 \mathrm{~m}^{3} \\
& 217 \mathrm{~m}^{3} \text { (total) } \\
& \text { None }
\end{aligned}
$$

$3,700,858$ liters (total) $11,411,775$ liters (total)

\author{
$156 \mathrm{MWh} / \mathrm{yr}$ \\ 586,966 liters (total)
}

NEPA documentation (prior to start of Title II construction); New stationary sources/PTC/NOC/PSD for non-rad air emissions; HAP's and TAP's and RCRA (part AA,BB, and CC for air) for hazardous air emissions; air operating permit; NESHAP's, NPDES, NESHAP's subpart $H$ for rad air emissions; approval of Engineering Plans; Cross Connection Control Plans; report and specifications for drinking water supply;RCRA Part A and Part B permits.

\section{Operational Information}

Cost (\$): Operations (unescalated)

Facility/Administration

Operations/Process Facility

Procurement, materials, utilities, maintenance

Total operations

Schedule start/end

$\$ 7,293,000$

$\$ 18,299,000$

$\$ 180,989,000$

$\$ 206,581,000$

January 2012 through December 2068

Number of workers each year of operation (new/existing) 
431.02\#

$06 / 17 / 97$

Rev. \#04
ENGINEERING DESIGN FILE

Table 3. (continued).
Function File Number - SPR-01

EDF Serial Number - EDF-IS-001

Page 31 of 42
Operations

Maintenance

Support

Total

Number of radiation workers

Average annual work radiation dose (rem/yr)

Heavy Equipment

Air Emissions

Radioactive off-gas

Effluents

Sanitary Wastewater

Solid Wastes

Sanitary/Industrial Trash

Radioactive wastes (canisters)

Hazardous/toxic chemicals and wastes

Pits/Ponds used $\left(\mathrm{m}^{2}\right)$

Water usage:

Domestic water

Energy Requirements:

Electrical (MWh/yr)

Fossil fuel (liters/yr)

Permits needed (for facility operations)
0.5

4

8.5

2.5 (included in above total)

$0.19 \mathrm{rem} / \mathrm{yr}$ perworker

None

None

293,574 liters/yr

\author{
$47 \mathrm{~m}^{3} / \mathrm{yr}$ \\ $12,096 \mathrm{~m}^{3} / \mathrm{yr}$
}

None

293,574 liters/yr

4,586 MWh/yr
None

HAP's and TAP's and RCRA (part AA,BB, and CC for air) for hazardous air emissions; air operating permit; NESHAP's, NPDES, NESHAP's subpart $H$ for rad air emissions; approval of Engineering Plans; Cross Connection Control Plans; report and specifications for drinking water supply:RCRA Part A and Part $B$ permits.
Decontamination \& Decommissioning (D\&D) Information

Cost (\$): D\&D

Decommission

Decontamination

Demolition

Total D\&D

Schedule start/end: D\&D

Number of workers each year of D\&D (new/existing)

Number of radiation workers (D\&D)

Average annual worker radiation dose (rem/yr)
$\$ 10,500,000$

$\$ 0$

$\$ 112,235,000$

$\$ 122,735,000$

January 2069 through December 2071

287 New workers/yr

29 New workers/yr

$0.19 \mathrm{rem} / \mathrm{yr} \quad$ perworker

Mobile Cranes, Roll-off trucks, Dozers, Loaders

Heavy equipment:

Equipment used 
431.02\#

$06 / 17 / 97$

Rev. \#04
ENGINEERING DESIGN FILE

Table 3. (continued).
Function File Number - SPR-01 EDF Serial Number - EDF-IS-001

Page 32 of 42

Trips

Project Data Sheet for Interim Storage Facility for DCWO - $20 \mathrm{yr}$

Hours of operation (all heavy equipment)

12 per day

Roll-off trucks

Acres disturbed and duration of disturbance

New

Previous

Revegetated

62,100 Hours

January 2073 through December 2075

None

9.0 acres

None

Air emissions

non-radioactive Fuel combustion gases $\left(\mathrm{CO}_{2}, \mathrm{H}_{2} \mathrm{O}, \mathrm{O}_{2}, \mathrm{~N}_{2}\right)$

36,094 tons (total)

non-radioactive

Fuel combustion contaminants ( $\mathrm{CO}$,

particulates, $\mathrm{NO}_{x}, \mathrm{SO}_{2}$, hydrocarbons)

radioactive HEPA filtered off-gas

211 tons (total)

None tons (total)

Effluents

radioactive Spent decontamination solution

$5,109,750$ liters (total)

non-radioactive Sanitary wastewater

non-radioactive Lube oil

$18,346,756$ liters (total)

11,752 liters (total)

Solid wastes:

radioactive

Non-radioactive (industrial)

Hazardous

Hazardous/toxic chemicals and wastes (type)

Storage/inventory

Pits/Ponds created $\left(\mathrm{m}^{2}\right)$

radioactive (mixed waste)

Water usage:

Domestic water

Source of water

\section{Energy requirements:}

Electrical (MWh/yr).

Fossil fuel (liters)

Permits needed (e.g. for facility closures, physical characteristics

and quantities of radioactive and hazardous materials remaining after closure)

$$
\begin{array}{r}
50,817 \mathrm{~m}^{3} \\
10 \mathrm{~m}^{3} \\
108 \mathrm{~m}^{3}
\end{array}
$$

None $\mathrm{m}^{3}$ (total)

None

$909 \mathrm{~m}^{3}$ (total)

$18,346,756$ liters (total)

ICPP site wells

$156 \mathrm{MWh} / \mathrm{yr}$

$\mathrm{Ci}$

$1,410,291$ liters (total)

Work will be done under closure provisions of existing permits. 
431.02\#

$06 / 17 / 97$

Rev. \#04
ENGINEERING DESIGN FILE

Table 3. (continued).
Function File Number - SPR-01

EDF Serial Number - EDF-IS-001

Page 33 of 42

\section{Project Data Sheet for Interim Storage Facility for CWO}

\section{Generic Information}

Description/function

EIS Alternatives ( $A, B$, etc.)

Project type or waste stream

Action type

Structure type

Size $\left(\mathrm{m}^{2}\right)$

Other features (e.g. pits, ponds, power/water/sewer lines)

Location

Inside/outside of fence

Inside/outside of building

Candidate for privatization?
Provide Long-term storage for road-ready containers awaiting shipment to a permanent repository.

Calcined Waste Option

Treated HLW calcine.

New

$$
\begin{aligned}
& 14,725 \mathrm{~m}^{2} \\
& \text { None }
\end{aligned}
$$

Inside ICPP fence

Inside Grouting Facility

Yes

\section{Construction Information}

Cost (\$): Pre-Operations (Other Project Costs) with escalation an

Conceptual Design

Management for Project Development

Permitting and Documentation

SO Test \& Start-Up

Contingency

Total Preconstruction

Cost (\$): Construction with escalation and contingency.

\section{ED\&l}

Management (PM/CM)

Construction

G\&A/PIF

Procurement Fees, Management Reserve and Contingency

Total Construction (TEC)

Schedule start/end: Preconstruction

Schedule start/end: Construction

Schedule start/end: SO Test \& Start-up

Number of workers each year of construction (new/existing)

Nonradiation

Number of radiation workers (construction)

Average annual worker radiation dose (rem/yr)

\section{Heavy equipment}

Equipment used

Trips (construction materials delivery)

Hours of operation

Acres disturbed and duration of disturbance

New

Previous
$\$ 5,000,000$

$\$ 4,000,000$

$\$ 2,728,000$

$\$ 4,500,000$

$\$ 4,072,000$

$\$ 20,300,000$

$\$ 35,000,000$

$\$ 58,275,000$

$\$ 302,752,000$

$\$ 18,038,000$

$\$ 85,935,000$

$\$ 500,000,000$

January 2003 through December 2006

January 2006 through December 2011

January 2012 through December 2013

124 New workers/yr

None

None

Excavator, dump trucks, cranes, cement trucks, grader, material delivery

2,337

14,637 hours (total)

January 2006 through December 2011

None

9 acres 
431.02\#

06/17/97

Rev. \#04
ENGINEERING DESIGN FILE

Table 3. (continued).
Function File Number - SPR-01

EDF Serial Number - EDF-IS-001

Page 34 of 42

\section{Project Data Sheet for Interim Storage Facility for CWO}

Revegetated

Air emissions

Dust

Major gases $\left(\mathrm{CO}_{2}, \mathrm{H}_{2} \mathrm{O}, \mathrm{O}_{2}, \mathrm{~N}_{2}\right)$ from diesel exhaust

Contaminants (Particulates, $\mathrm{CO}, \mathrm{NO}_{x}, \mathrm{SO}_{2}$, hydrocarbons) from diesel exhaust.

Total air emissions

Effluents

Sanitary wastewater

Lube Oil

Solid wastes

Type - construction trash

Radioactive wastes

\section{Hazardous/toxic chemicals and wastes (type)}

Storage/inventory

Hazardous waste (construction)

Pits/Ponds created $\left(\mathrm{m}^{2}\right)$

Water usage:

Dust control

Domestic water

Energy requirements

Electrical (MWh/yr)

Fossil fuel (liters)

Permits needed for construction
None

518 tons (total)

14,091 tons (total)

83 tons (total)

14,692 tons (total)

$10,560,150$ liters (total)

2,770 liters (total)

$$
5,880 \mathrm{~m}^{3} \text { (total) }
$$

None

$$
\begin{aligned}
& 13 \mathrm{~m}^{3} \\
& 217 \mathrm{~m}^{3} \text { (total) } \\
& \text { None }
\end{aligned}
$$

$3,700,858$ liters (total)

$10,560,150$ liters (total)

\author{
$156 \mathrm{MWh} / \mathrm{yr}$ \\ 0 liters (total)
}

NEPA documentation (prior to start of Title II construction); New stationary sources/PTC/NOC/PSD for non-rad air emissions; HAP's and TAP's and RCRA (part AA,BB, and CC for air) for hazardous air emissions; air operating permit; NESHAP's, NPDES, NESHAP's subpart H for rad air emissions; approval of. Engineering Plans; Cross Connection Control Plans; report and specifications for drinking water supply;RCRA Part A and Part B permits.

Operational Information

Cost (\$): Operations (unescalated)

Facility/Administration

$\$ 3,003,000$

$\$ 7,535,000$

Operations/Process Facility

Procurement, materials, utilities, maintenance

Total operations

Schedule start/end

$\$ 37,708,000$

$\$ 48,246,000$

January 2012 through December 2068

Number of workers each year of operation (new/existing) 
Operations

Maintenance

Support

Total

Number of radiation workers

Average annual work radiation dose (rem/yr)

Heavy Equipment

Air Emissions

Radioactive off-gàs

Effiuents

Sanitary Wastewater

Solid Wastes

Sanitary/Industrial Trash

Radioactive wastes (canisters)

Hazardous/toxic chemicals and wastes

Pits/Ponds used $\left(\mathrm{m}^{2}\right)$

Water usage:

Domestic water

Energy Requirements:

Electrical (MWh/yr)

Fossil fuel (liters/yr)

Permits needed (for facility operations)

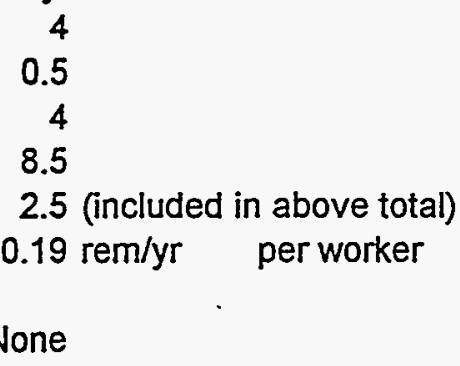

None

293,574 liters/yr

\author{
$47 \mathrm{~m}^{3} / \mathrm{yr}$ \\ $3,456 \mathrm{~m}^{3} / \mathrm{yr}$
}

None

293,574 liters/yr

4,368 MWh/yr
None

HAP's and TAP's and RCRA (part AA,BB, and CC for air) for hazardous air emissions; air operating permit; NESHAP's, NPDES, NESHAP's subpart $H$ for rad air emissions; approval of Engineering Plans; Cross Connection Control Plans; report and specifications for drinking water supply;RCRA Part A and Part B permits.
Decontamination \& Decommissioning (D\&D) Information

Cost (\$): D\&D (unescalated)

Decommission

Decontamination

Demolition

Total D\&D

Schedule start/end: D\&D .

Number of workers each year of D\&D (new/existing)

Number of radiation workers (D\&D)

Average annual worker radiation dose (rem/yr)

$$
\$ 10,500,000
$$

$\$ 0$

$\$ 103,997,000$

$\$ 114,497 ; 000$

January 2069 through December 2071

268 New workers/yr

14 New workers/yr

$0.19 \mathrm{rem} / \mathrm{yr} \quad$ per worker

Mobile Cranes, Roll-off trucks, Dozers, Loaders

Heavy equipment:

Equipment used 
Trips - Roll-off trucks

Hours of operation (all heavy equipment)

Acres disturbed and duration of disturbance

New

Previous

Revegetated

\section{Air emissions}

non-radioactive Fuel combustion gases $\left(\mathrm{CO}_{2}, \mathrm{H}_{2} \mathrm{O}, \mathrm{O}_{2}, \mathrm{~N}_{2}\right)$

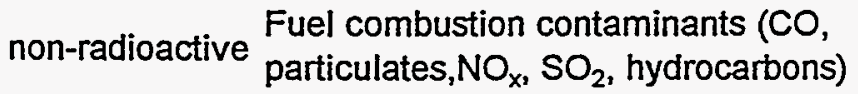

radioactive HEPA filtered off-gas

\section{Effluents}

radioactive Spent decontamination solution

non-radioactive Sanitary wastewater

non-radioactive Lube oil

Solid wastes:

radioactive

Non-radioactive (industrial)

Hazardous

Hazardous/toxic chemicals and wastes (type)

Storage/inventory

Pits/Ponds created $\left(\mathrm{m}^{2}\right)$

radioactive (mixed waste)

Water usage:

Domestic water

Source of water

Energy requirements:

Electrical (MWh/yr)

Fossil fuel (liters)

Permits needed (e.g. for facility closures, physical characteristics and quantities of radioactive and hazardous materials remaining after closure)
$=$

\section{Storage Facility for CWO}

12 per day

67,500 Hours

January 2069 through December 2071

None

9.0 acres

None

39,233 tons (total)

229 tons (total)

None tons (total)

None liters (total)

$17,111,456$ liters (total)

12,774 liters (total)

$$
\begin{array}{r}
46,864 \mathrm{~m}^{3} \\
9 \cdot \mathrm{m}^{3} \\
100 \mathrm{~m}^{3}
\end{array}
$$

$$
\text { None } \mathrm{m}^{3} \text { (total) }
$$

None

None $\mathrm{m}^{3}$ (total)

$17,111,456$ liters (total)

ICPP site wells

$156 \mathrm{MWh} / \mathrm{yr}$

$1,532,925$ liters (total)

Work will be done under closure provisions of existing permits. 


\section{Project Data Sheet for Interim Storage Facility for HIP}

\section{Generic Information}

Description/function

Els Alternatives ( $A, B$, etc.)

Project type or waste stream

Action type

Structure type

Size $\left(\mathrm{m}^{2}\right)$

Other features (e.g. pits, ponds, power/water/sewer lines)

Location

Inside/outside of fence

Inside/outside of building

Candidate for privatization?
Provide Long-term storage for road-ready containers awaiting shipment to a permanent repository.

Hipping Waste Option

Treated HLW calcine.

New

$7,287 \mathrm{~m}^{2}$

None

Inside ICPP fence

Inside Vitrification Facility .

\section{Construction Information}

Cost (\$): Pre-Operations (Other Project Costs) with escalation an

Conceptual Design

Management for Project Development

Permitting and Documentation

SO Test \& Start-Up

Contingency

Total Preconstruction

Cost (\$): Construction with escalation and contingency.

\section{ED\&l}

Management (PM/CM)

Construction

G\&APIF

Procurement Fees, Management Reserve and Contingency

Total Construction (TEC)

Schedule start/end: Preconstruction

Schedule start/end: Construction

Schedule start/end: SO Test \& Start-up

Number of workers each year of construction (new/existing)

Nonradiation

Number of radiation workers (construction)

Average annual worker radiation dose (rem/yr)

\section{Heavy equipment}

\section{Equipment used}

Trips (construction materials delivery)

Hours of operation

\section{Acres disturbed and duration of disturbance}

New

Previous

$$
\begin{array}{r}
\$ 5,000,000 \\
\$ 4,000,000 \\
\$ 2,728,000 \\
\$ 4,500,000 \\
\$ 4,072,000 \\
\$ 20,300,000
\end{array}
$$

$$
\begin{array}{r}
\$ 33,085,000 \\
\$ 49,305,000 \\
\$ 208,097,000 \\
\$ 12,729,000 \\
\$ 65,384,000 \\
\$ 368,600,000
\end{array}
$$

January 2003 through December 2007 January 2006 through December 2011 January 2012 through December 2012

\author{
92 New workers/yr \\ None \\ None
}

Excavator, dump trucks, cranes, cement trucks,grader, material delivery

$$
\begin{aligned}
& 1,349 \\
& 8,140 \text { hours (total) }
\end{aligned}
$$

January 2006 through December 2011

None

2.5 acres 
431.02\#

$06 / 17 / 97$

Rev. $¥ 04$

Revegetated

Air emissions

Dust

Major gases $\left(\mathrm{CO}_{2}, \mathrm{H}_{2} \mathrm{O}, \mathrm{O}_{2}, \mathrm{~N}_{2}\right)$ from diesel exhaust

Contaminants (Particulates, $\mathrm{CO}, \mathrm{NO}_{x}, \mathrm{SO}_{2}$, hydrocarbons) from

diesel exhaust.

Total air emissions

Effluents

Sanitary wastewater

Lube Oil

Solid wastes

Type - construction trash

Radioactive wastes

Hazardous/toxic chemicals and wastes (type)

Storage/inventory

Hazardous waste (construction)

Pits/Ponds created $\left(\mathrm{m}^{2}\right)$

Water usage:

Dust control

Domestic water

Energy requirements

Electrical (MWh/yr)

Fossil fuel (liters)

Permits needed for construction

ENGINEERING DESIGN FILE
Table 3. (continued).

Project Data Sheet for Interim Storage Facility for HIP

None

108 tons (total)

4,017 tons (total)

24 tons (total)

4,149 tons (total)

$5,876,213$ liters (total)

1,541 liters (total)

$3,272 \mathrm{~m}^{3}$ (total)

None

\author{
$13 \mathrm{~m}^{3}$ \\ $217 \mathrm{~m}^{3}$ (total) \\ None
}

771,012 liters (total)

$5,876,213$ liters (total)

$156 \mathrm{MWh} / \mathrm{yr}$

310,806 liters (total)

NEPA documentation (prior to start of Title II construction); New stationary

sources/PTC/NOC/PSD for non-rad air emissions; HAP's and TAP's and RCRA (part AA,BB, and CC for air) for hazardous air emissions; air operating permit; NESHAP's, NPDES, NESHAP's subpart H for rad air emissions; approval of Engineering Plans; Cross Connection Control Plans; report and specifications for drinking water supply;RCRA Part A and Part B permits.

\section{Operational Information}

Cost (\$): Operations (unescalated)

Facility/Administration

Operations/Process Facility

Procurement, materials, utilities, maintenance

Total operations

Schedule startend

$\$ 7,293,000$
$\$ 18,299,000$
$\$ 70,097,000$
$\$ 95,689,000$

January 2013 through December 2068

Number of workers each year of operation (new/existing) 


\section{ENGINEERING DESIGN FILE}

Table 3. (continued).
Function File Number - SPR-01

EDF Serial Number - EDF-IS-001

Page 39 of 42
Operations

Maintenance

Support

Total

Number of radiation workers

Average annual work radiation dose (rem/yr)

Heavy Equipment

Air Emissions

Radioactive off-gas

Effluents.

Sanitary Wastewater

Solid Wastes

Sanitary/Industrial Trash

Radioactive wastes (canisters)

Hazardous/toxic chemicals and wastes

Pits/Ponds used $\left(\mathrm{m}^{2}\right)$

Water usage:

Domestic water

Energy Requirements:

Electrical (MWh/yr)

Fossil fuel (liters/yr)

Permits needed (for facility operations)
224,498 liters/yr

$$
\begin{array}{r}
36 \mathrm{~m}^{3} / \mathrm{yr} \\
4,309 \mathrm{~m}^{3} / \mathrm{yr}
\end{array}
$$

None

224,498 liters/yr

\section{4,368 MWh/yr \\ None}

HAP's and TAP's and RCRA (part AA,BB, and CC for air) for hazardous air emissions; air operating permit; NESHAP's, NPDES, NESHAP's subpart $H$ for rad air emissions; approval of Engineering Plans; Cross Connection Control Plans; report and specifications for drinking water supply;RCRA Part A and Part B permits.

\section{Decontamination \& Decommissioning (D\&D) Information}

Cost (\$): D\&D (unescalated)

Decommission

Decontamination

Demolition

Total D\&D

Schedule start/end: D\&D

Number of workers each year of D\&D (new/existing)

Number of radiation workers (D\&D)

Average annual worker radiation dose (rem/yr)

Heavy equipment:

Equipment used

$$
\begin{array}{r}
\$ 7,678,000 \\
\$ 0 \\
\$ 57,836,000 \\
\$ 65,514,000
\end{array}
$$

January 2069 through December 2071

154 New workers/yr

16 New workers/yr

$0.19 \mathrm{rem} / \mathrm{yr}$ perworker

Mobile Cranes, Roll-off trucks, Dozers, Loaders 
Acres disturbed and duration of disturbance

New

Previous

Revegetated

\section{Air emissions}

non-radioactive Fuel combustion gases $\left(\mathrm{CO}_{2}, \mathrm{H}_{2} \mathrm{O}, \mathrm{O}_{2}, \mathrm{~N}_{2}\right)$

non-radioactive Fuel combustion contaminants $(\mathrm{CO}$,

radioactive HEPA filtered off-gas

Effluents

radioactive Spent decontamination solution

non-radioactive Sanitary wastewater

non-radioactive Lube oil

Solid wastes:

radioactive

Non-radioactive (industrial)

Hazardous

Hazardous/toxic chemicals and wastes (type)

Storage/inventory

Pits/Ponds created $\left(\mathrm{m}^{2}\right)$

Hazardous wastes

Water usage:

Domestic water

Source of water

\section{Eneray requirements:}

Electrical (MWh/yr)

Fossil fuel (liters)

Permits needed (e.g. for facility closures, physical characteristics and quantities of radioactive and hazardous materials remaining after closure)
January 2069 through December 2071

None

2.5 acres

None

20,715 tons (total)

121 tons (total)

None tons (total)

None liters (total) $9,818,650$ liters (total)

6,745 liters (total)

$$
\text { None } \mathrm{m}^{3}
$$

$23,191 \mathrm{~m}^{3}$

$4 \mathrm{~m}^{3}$

$$
\begin{aligned}
& \text { None } \mathrm{m}^{3} \text { (total) } \\
& \text { None } \\
& 909 \mathrm{~m}^{3} \text { (total) }
\end{aligned}
$$

ICPP site wells

$$
9,818,650 \text { liters (total) }
$$

$156 \mathrm{MWh} / \mathrm{yr}$

809,384 liters (total)

Work will be done under closure provisions of existing permits. 


\subsection{PROJECT-SPECIFTC OPTIONS}

No project-specific options were assigned, identified, or evaluated.

\subsection{RECOMMENDATIONS}

The complexity that NRC licensing adds to the permitting of an ISF has not been estimated in this study. The ramifications should be investigated in greater detail before the design of an ISF is started.

A future study should be performed to determine if a standard 4.6-m (15-ft) long "Hanford" canister should be substituted for the 3-m (10-ft) SRS DWPF canister assumed in this study. Use of a 3-m (15-ft) canister would increase the interim storage construction costs but would reduce the operation (handling) costs, and greatly reduce offsite transportation and disposal costs. A large net cost saving, primarily as a result of reduced transportation and disposal costs, could be realized by using the $4.6-\mathrm{m}$ (15-ft) canister.

\subsection{CONCLUSIONS}

This study has investigated the design of a new interim storage facility for storage of HLW that results from processing the waste at ICPP. The processing options are discussed in separate reports. It is concluded that a new facility can be designed, built, tested, and placed into operation that can store the waste in canisters for the period from processing until the time a permanent repository is available for receipt of this waste. The schedules and costs associated with interim storage for the various processing options are presented in the body of this EDF.

\subsection{REFERENCES}

1. October 17, 1995, Consent Order and Settlement Agreement between DOE and the State of Idaho regarding spent fuel and nuclear waste issues.

2. Waste Treatment Facilities Feasibility Study Report, December 12, 1997, Fluor Daniel Corporation.

3. Ronald E. Dafoe, Daryl A. Lopez, and Karen L. Williams, DPC Loading Feasibility Study Report, INEEL/EXT-97-01251, November 1997.

4. DOE/RW-0333P, U. S. Department of Energy, Office of Civilian Radioactive Waste Management, Quality Assurance Requirements and Description (QARD).

5. William H. Landman, TRU Separations Option Study Report, INEEL/EXT-01428, February 1998.

6. Daryl A. Lopez, Vitrified Waste Option Study Report, INEEL/EXT-01389, February 1998.

7. Ronald E. Dafoe, Direct Cementitious Waste Option Study Report, INEEL/EXT-01399, February 1998. 
8. Allen E. Lee, Cementitious Waste Option Study Report, INEEL/EXT-01389, February 1998.

9. Neal E. Russell, Hot Isostatic Press Waste Option Report, INEEL/EXT-01392, February 1998.

\subsection{APPENDICES}

Appendix 1 - Cost Estimates

Appendix 2-Drawings and Sketches

Appendix 3 - Risk Assessment/Data Sheets

Appendix 4-Background Information for Project Data Sheets 


\section{Appendix 1}

\section{Cost Estimates}


The cost estimates for the new Interim Storage Facilities (ISFs) are contained in this appendix in the same order as in the following summary table (see estimates attached to the letter RDA-3-98):

Summary Table of Cost Estimates

\begin{tabular}{|l|c|c|c|c|c|c|}
\hline \multirow{3}{*}{ Waste Option } & \multicolumn{3}{|c|}{ Not Escalated } & \multicolumn{3}{c|}{ Escalated } \\
\cline { 2 - 7 } & $\begin{array}{c}\text { Total } \\
\text { Estimate } \\
\end{array}$ & $\begin{array}{c}\text { Other } \\
\text { Project }\end{array}$ & $\begin{array}{c}\text { Total } \\
\text { Project } \\
\text { Cost, } \\
\text { Cost, } \\
\text { Cost, }\end{array}$ & $\begin{array}{c}\text { Total } \\
\text { Estimate } \\
\text { d Cost, } \\
\text { K\$ } \$\end{array}$ & $\begin{array}{c}\text { Other } \\
\text { Project } \\
\text { Cost, } \\
\text { K\$ }\end{array}$ & $\begin{array}{c}\text { Total } \\
\text { Project } \\
\text { Cost, } \\
\text { K } \$\end{array}$ \\
\hline TRU/Class A Separations & 81,000 & 19,000 & 100,000 & 117,300 & 24,200 & 141,500 \\
\hline Vitrified & 440,300 & 23,800 & 464,100 & 600,100 & 29,700 & 629,800 \\
\hline Direct Cementitious & 540,400 & 20,300 & 560,700 & 738,000 & 25,500 & 763,500 \\
\hline Cementitious & 500,000 & 20,300 & 520,300 & 681,900 & 25,600 & 707,500 \\
\hline Hot Isostatic Press & 271,500 & 20,300 & 291,800 & 368,600 & 25,700 & 394,300 \\
\hline
\end{tabular}

The Life-Cycle Cost Estimate for the TRU/Class A Separations Option is attached to the letter, "Economic and Life-Cycle Analysis conducted for the Interim Storage Facility-TRU - RJT-23-98," dated February 3, 1998.

The Life-Cycle Cost Estimate for the Vitrified Option is attached to the letter "Economic and Life-Cycle Analysis conducted for the Vitrification Interim Storage Facilities - RJT-13-98," dated January 30, 1998.

The Life-Cycle Cost Estimate for the Direct Cementitious Option is attached to the letter "Economic and Life-Cycle Analysis conducted for the Direct Cementitious Waste Option (DCWO)-20 Yr Storage Facility - RJT-19-98," dated February 2, 1998.

The Life-Cycle Cost Estimate for the Cementitious Option is attached to the letter 'Economic and Life-Cycle Analysis conducted for the Cementitious Waste Option (CWO) Storage Facility - RJT-2198," dated February 2, 1998.

The Life-Cycle Cost Estimate for the Hot Isostatic Press Option is attached to the letter "Economic and Life-Cycle Analysis conducted for the Hot Isostatic Pressing (HIP) Storage Facilities RJT-16-98," dated January 30, 1998. 
Date: January 28,1998

$\begin{array}{llll}\text { To: } & \text { J. K. Rawlins } & \text { MS 3765 } & 6-4303 \\ \text { From: } & \text { R. D. Adams ROA } & \text { MS 3655 } & 6-2963\end{array}$

Subject: $\quad$ INTERIM STORAGE STUDY - RDA-3-98

Cost Estimating has prepared Planning Cost Estimates for the subject project. These estimates were based on information received from you, your design team and reviewers from November 11, 1997 through January 23, 1998.

These estimates include permitting costs, direct and indirect construction costs, G\&A, Performance Incentive Fee (PIF), procurement fee, Engineering costs, Inspection costs, Project Management costs, Construction Management costs, escalation and contingency. The estimated costs have been escalated to the midpoint of the anticipated schedule. For more detailed information, please refer to the Cost Estimate Support Data Recapitulation forms.

Attached for your review are the Summary Estimate sheets, the Cost Estimate Support Data Recapitulation form, detailed estimate sheets, Contingency Analysis sheets G\&A/PIF Adder Calculation Sheets.

Also attached are the schedules.

Please refer to the attached table for a summary of the subject estimates.

If you have any questions regarding this estimate, please contact me at 526-2963.

RDA

Attachments:

cc: Estimate File \#2421

R. D. Adams File 
Lockheed Martin Idaho Technologies

(Rev. 6/96)

\section{COST ESTIMATE SUPPORT DATA RECAPITULATION}

Project Title: HLW INTERIM STORAGE FACILITY - TRU

Type of Estimate: PROJECT SUPPORT

File No: 2421-TRU
Estimator: RDA

Date:1/26/98

Approved By:

\section{SCOPE OF FORK: Brief description of the proposed project.}

This facility will unload, store in vertical tubes and load out canisters containing High Level Waste (EILW)

from the proposed TRU Separations class A option (TRU).

II. BASIS OF THE ESTIMATE : Drawings, Design Report, Engineers notes, and/or other documentation upon which the estimate is originated.

Draft sketches, a draft EDF, and the SNF Canister Storage Building Final Design Estimate from Fluor Daniel Northwest, Inc. were used as the basis for this estimate.

III. ASSUMPTIONS : Condition statements accepted or supposed true without proof or demonstration. An assumption has a direct impact on total estimated cost.

1. The Interim Storage Facility will be located within 100 feet of the TRU Seps facility.

2. Rock will not be encountered during excavation.

3. The estimates include both present day costs summaries and contingency analysis and escalated sumaries and contingency analysis. The escalated summaries and contingency analysis reflect the attached schedule. All activities are escalated to the appropriate schedule mid-point.

4. Per the draft EDF, one storage module, containing 630 storage tubes, will accommodate the estimated 170 canisters produced at the TRU Seps facility.

5. Most quantities used for this estimate were obtained by preliminary take offs from the draft sketches.

6. The storage tube costs, gantry crane cost and associated rail system costs were used from the Fluor estimate of a facility which is approximately eighty percent complete.

7. All assumptions and information included in the draft "New Interim Storage Facility for Interim Storage of $\mathrm{HLW}^{\prime \prime}$ apply to the estimate.

8. Where quantities could not be determined from the draft sketches, factors or assumed quantities were used by the estimator.

9. Sufficient construction craft will be available to support this project.

10. Markups on direct construction costs to get the construction costs listed on the summary sheet are as follows:

Labor indirects, overheads and profit (OH\&P) $50 \%$ of labor

Material \& equipment OH\&P

Subcontract OH\&O

Construction equipment allowance

NQA-I quality requirements

DOE/RW/0333P quality requirements

subcontractor procurement

Bond
$5 \%$ of material and equipment

108 of subcontract cost

108 of labor

308 of material

108 of material

18 of material and equipment

28 of everything 
Lockheed Martin Idaho Technologies

\section{COST ESTIMATE SUPPORT DATA RECAPITULATION (CONTINUATION)}

File No: 2396

Page 2 of 2

III. ASSUMPTIONS: (Continued)

12. Only the railroad track within the storage facility is included in this estimate. The remainder of the branch line is included in the infrastructure estimate.

13. All utilities are assumed to be available at the facility wall.

IV. CONTINGENCY GUIDEIINE IMPLEMENTATION: The percentage used for contingency as determined by the contingency allowance guidelines can be altered to refiect the type of construction and conditions that may impact the total estimated cost.

A large portion of the estimated costs is based on actual costs from Fluor's SNF Canister Storage Building. The majority of the remaining costs are for standard construction activities. For these reasons, the contingency is lower than would be experienced for a processing type facility at this stage of development.

V. OTHER COMMNTS/CONCERNS SPECIEIC TO THE ESTIMATE:

1. All FY' 98 and later projects are to be assessed a Procurement Fee of $1 \%$ a G\&A Fee of $23 \%$, with a $\$ 500,000$ ceiling per year, and a Performance Incentive Fee (PIF) of $5.5 \%$. See the attached G\&A/PIF calculation sheet for the method used to calculate these fees.

2. A procurement fee of one percent of construction was used to cover the operating contractor support to DOE-ID for their contract administration. It is felt that one percent is sufficient for jobs of this size. 
Lockheed Martin Idaho Technologies Co.

PROECT NAME: TRU SEPS. FACILITY - INTERIM STORAGE FACILITY-ESCALATED

INEELACPP

J. K. RAWLINS
COST ESTIMATE SUMMARY

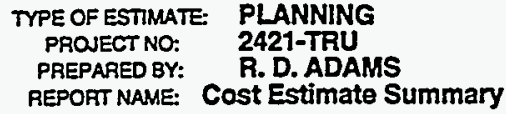

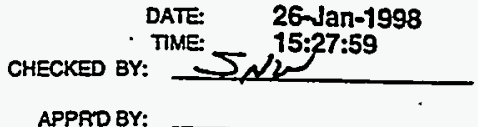

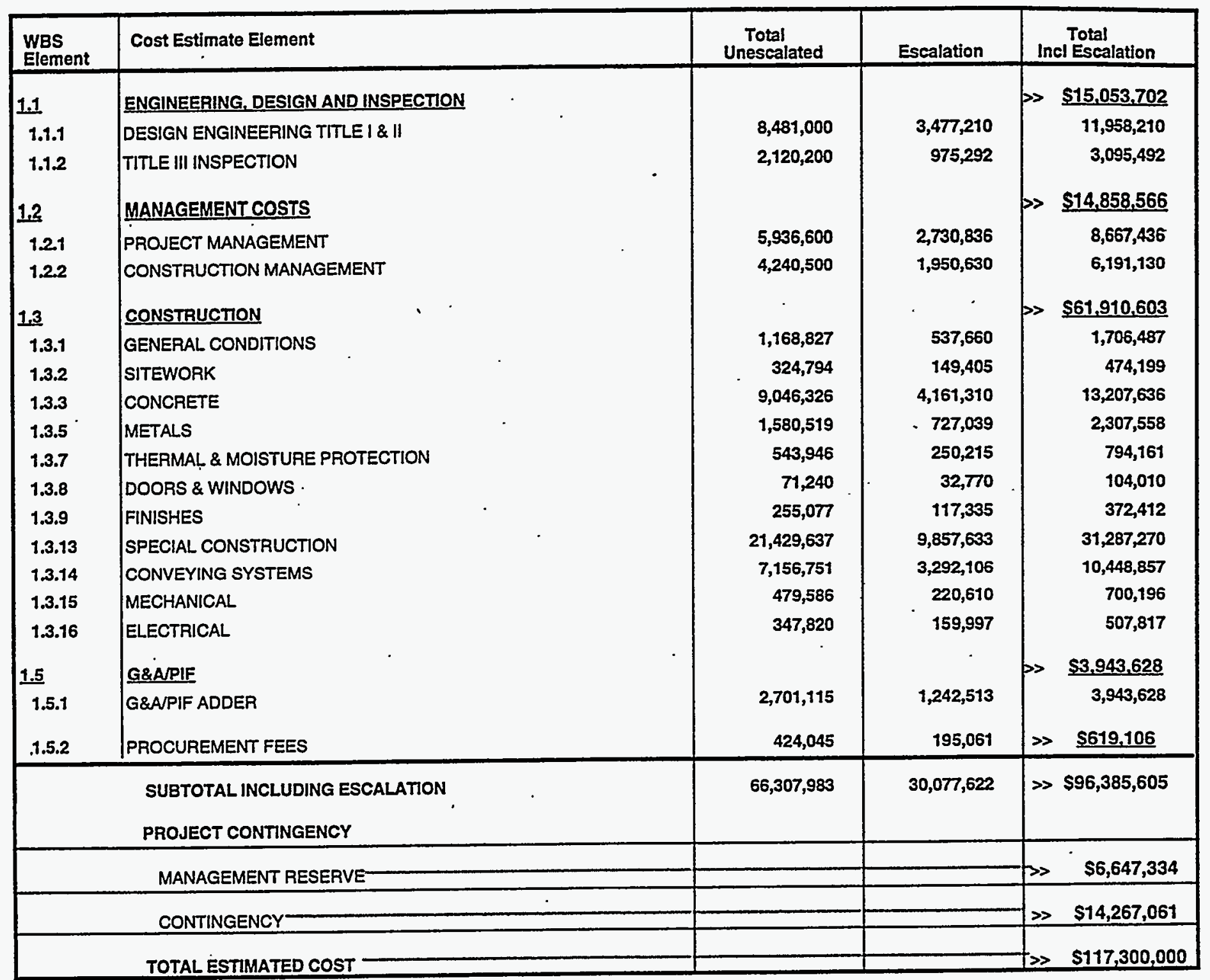

\section{PROJECT COST PARAMETERS}

EDI AS A $\%$ OF CONST. + GFE $=24.00 \%$ 
Lockheed Martin Idaho Technologies Co.

PROIECT NAME: TRU SEPS. FACILITY - INTERIM STORAGE FACILITY - UNESCALATED

LOCATION $1:$

INEELICPP

REQUESTOR:

J. K. RAWLINS
COST ESTIMATE SUMMARY

TYPE OFESTIMATE: PLANNING PROSECT NO: 2421-TRU

PREPARED BY: R.D.ADAMS

REPORT NAME: Cost Estimate Summary

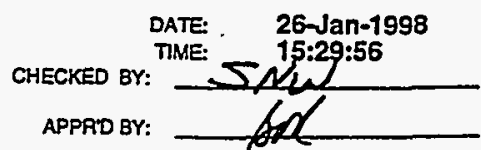

APPRD BY:

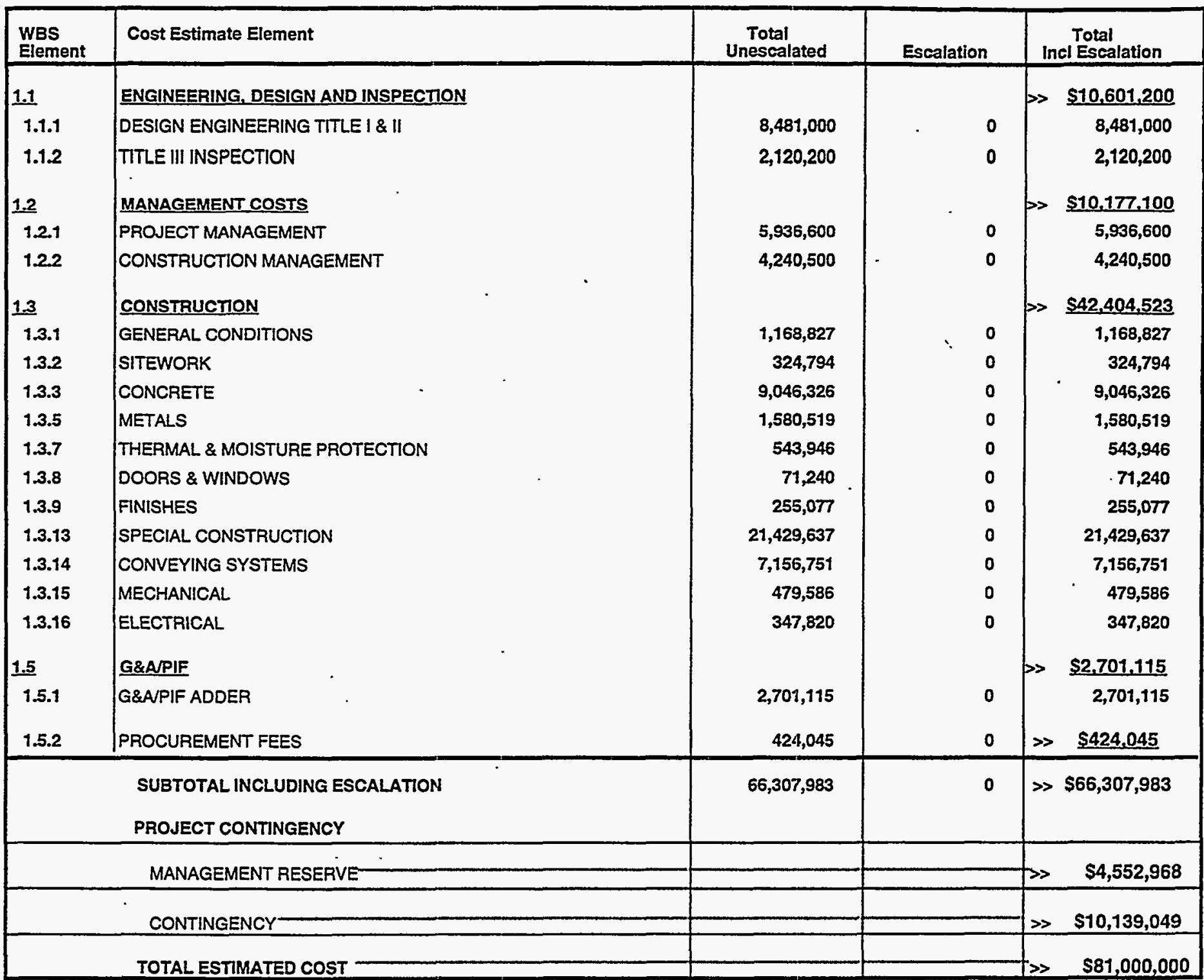

PROJECT COST PARAMETERS

EDI AS A \% OF CONST. + GFE $=25.00 \%$ 
Lockheed Martin Idaho Technologles Co.

Rov. 6/96

PROIECT NAME: TRU SEPS. FACILITY - INTERIM STORAGE

FACILITY

LOCATION $1:$ INEELICPP

REQUESTOR: J. K. RAWLINS

\section{DETAILED COST ESTIMATE SHEET}

TYPE OF ESTIMATE: PLANNING

PROJECT NO:: 2421-TRU

PREPARED BY: R. D. ADAMS
PAGE 1

DATE 26-Jan்-1998.

TIME: 15:25:49

REPORT NAME: Detall Cost Estimate Sheot

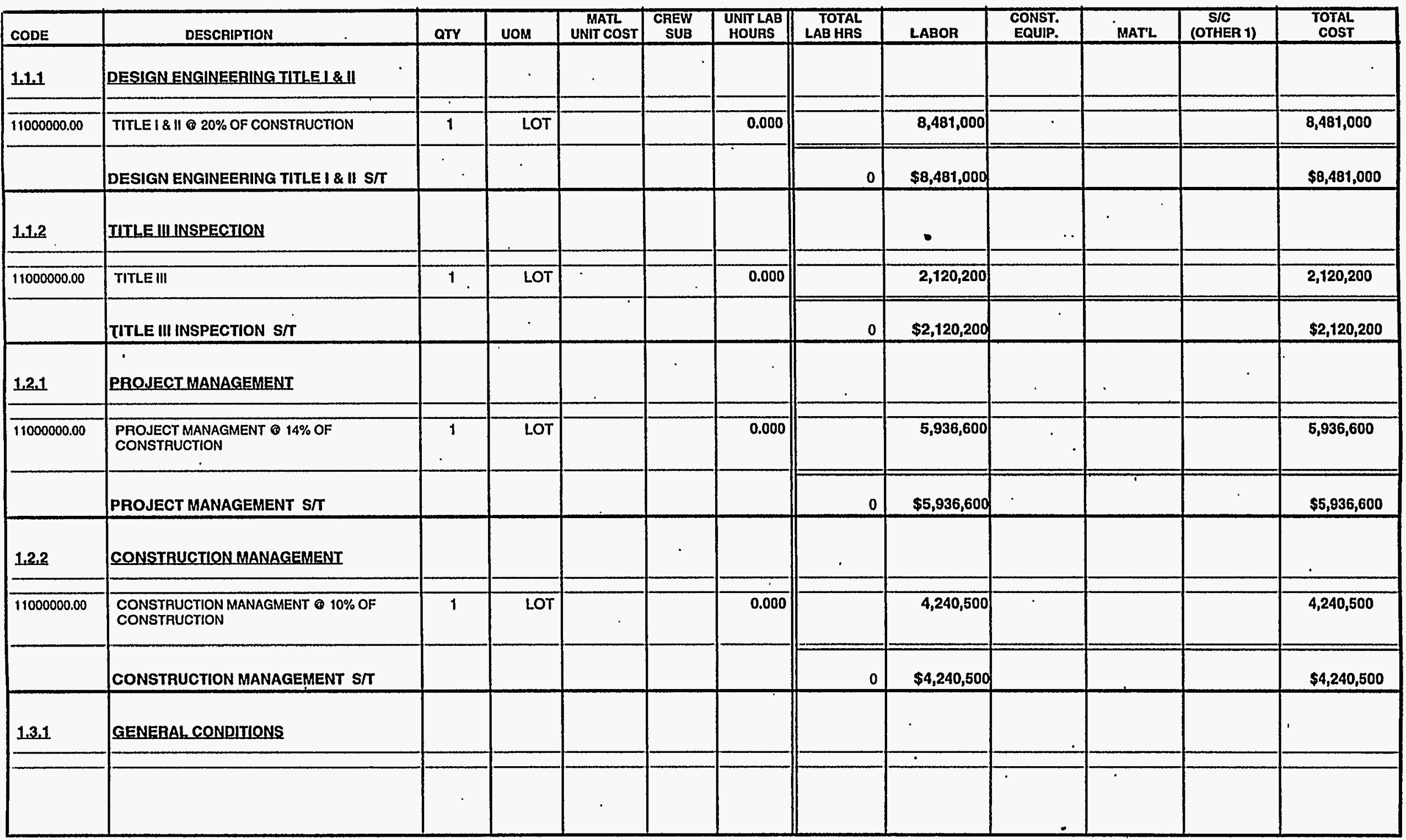


Lockheed Martin Idaho Technologles Co. Rev. 6/98

PROJECT NAME: TRU SEPS. FACILITY - INTERIM STORAGE FACILITY

LOCATION 1: INEELICPP

REQUESTOR: J. K. RAWLINS

\section{DETAILED COST ESTIMATE SHEET}

TYPE OF ESTIMATE: PLANNING

PROJECT NO: 2421-TRU

PREPARED BY: R. D. ADAMS
PAge* 2

DATE 26-Jan-1998

TIME: $15: 25: 49$

RePort NAME: Detall Cost EstImato Sheo

\begin{tabular}{|c|c|c|c|c|c|c|c|c|c|c|c|c|}
\hline CODE & DESCAIPTION & QTY & UOM & $\begin{array}{l}\text { MATL } \\
\text { UNIT COST }\end{array}$ & $\begin{array}{l}\text { CAEW } \\
\text { SUB }\end{array}$ & $\begin{array}{l}\text { UNITLAB } \\
\text { HOURS }\end{array}$ & $\begin{array}{l}\text { TOTAL } \\
\text { LAB HAS }\end{array}$ & LABOR & $\begin{array}{l}\text { CONST. } \\
\text { EQUIP. }\end{array}$ & MAT'L & $\begin{array}{c}\text { S/C } \\
\text { (OTHER 1) }\end{array}$ & $\begin{array}{l}\text { TOTAL } \\
\text { COST }\end{array}$ \\
\hline \multirow[t]{4}{*}{1.3 .1} & $\begin{array}{l}\text { GENERALCONDITIONS } \\
\text { SUPERVISION } \odot 5 \% \text { OF LABOR HOURS }\end{array}$ & 1 & LOT & & CARF & 8400.00 & 8,400 & 289,716 & & & & 289,716 \\
\hline & TRAINING $\bigcirc \%$ OF LABOR HOURS & 1 & LOT & & SSWK & 5045.00 & 5,045 & 185,706 & & & & 185,706 \\
\hline & $\begin{array}{l}\text { SMALL TOOLS \& CONSUMABLES } \odot 4 \% \text { OF } \\
\text { LABOR DOLLARS }\end{array}$ & 1 & LOT & $227,000.00$ & GEN & 0.000 & & & & 227,000 & & 227,000 \\
\hline & GENEAAL CONDITIONS $S / T$ & & & & & . & 13,445 & $\$ 475,422$ & & $\$ 227,000$ & & $\$ 702,422$ \\
\hline \multirow[t]{6}{*}{1.3 .2} & SITEWORK & & & & & , & & & & & & $\cdot$ \\
\hline & BUILDING EXCAVATION & 11,040 & CY & & ENGR & 0.050 & 552 & 17,416 & 55,200 & & & 72,616 \\
\hline & BUILDING BACKFILL & 5,300 & CY & & ENGR & 0.100 & 530 & 16,722 & 15,900 & & & 32,622 \\
\hline & BUILDING BERM FILL & 16,150 & $\mathrm{Cr}$ & & ENGR & 0.100 & 1,615 & 50,953 & 48,450 & & & 99,403 \\
\hline & IMPORT MATERIAL & 10,850 & $\mathrm{CY}$ & & $\begin{array}{c}\text { TRHV } \\
\text { GES }\end{array}$ & 0.030 & 326 & 10,136 & 32,550 & & & 42,686 \\
\hline & SITEWORK S/T & & & & & & 3,023 & $\$ 95,226$ & $\$ 152,100$ & & & $\$ 247,326$ \\
\hline \multirow[t]{6}{*}{1.3 .3} & CONCRETE & & & & & & & & & . & & \\
\hline & & & & & & & $\cdot$ & & & & & \\
\hline & BUILDING CONCAETE & 9,260 & $\mathrm{CY}$ & 126.10 & $\begin{array}{c}\text { SKWK } \\
\text { GEN }\end{array}$ & 12.300 & 113,898 & $3,771,163$ & 233,815 & $1,167,686$ & & $5,172,664$ \\
\hline & CHARGE FACE SLAB & 460 & $\overline{c Y}$ & 121.70 & SKWK & 8.000 & 3,680 & 121,845 & 1,440 & 65,982 & & 179,267 \\
\hline & HATCH PLUGS - ALLOW & 3 & EA & & GEN & 0.000 & . & & & . & 225,000 & 225,000 \\
\hline & CONCRETE S/T & & & & & & 117,578 & $\$ 3,893,008$ & $\$ 235,255$ & $\$ 1,223,668$ & $\$ 225,000$ & $\$ 5,576,930$ \\
\hline 1.3 .5 & METALS & & & & & & & & & & & \\
\hline & & & & & & & & & & & & \\
\hline & & & & & & & & & & & & \\
\hline
\end{tabular}


Lockheed Martin Idaho Technologles Co.

ReV. G/96
PROJECT NAME: TAU SEPS. FACILITY - INTERIM STORAGE FACILITY

LOCATION 1: INEELICPP

REQUESTOR: • J. K. RAWLINS

\section{DETAILED COST ESTIMATE SHEET}

TYPE OF Estimate: PLANNING

PROJECT NO: 2421-TRU

PREPARED QY: R. D. ADAMS
PAGE" 3

DATE 26-Jan-1998

TIME: 15:25:49

REPORT NAME: Dotail Cost Estlmate Sheot

\begin{tabular}{|c|c|c|c|c|c|c|c|c|c|c|c|c|}
\hline CODE & DESCRIPTION & QTY & uom & \begin{tabular}{c|} 
MATL \\
UNIT COST \\
\end{tabular} & $\begin{array}{l}\text { CAEW } \\
\text { SUB }\end{array}$ & $\begin{array}{l}\text { UNIT LAB } \\
\text { HOURS }\end{array}$ & $\begin{array}{l}\text { TOTAL } \\
\text { LAB HAS } \\
\end{array}$ & LABOR & $\begin{array}{l}\text { CONST. } \\
\text { EQUIP. }\end{array}$ & MAT'L & $\begin{array}{c}\text { S/C } \\
\text { (OTHER 1) } \\
\end{array}$ & $\begin{array}{r}\text { TOTAL } \\
\text { COST }\end{array}$ \\
\hline \multirow[t]{8}{*}{1.3 .5} & $\begin{array}{l}\text { METALS } \\
\text { CHARGE FACE SLAB FRAME }\end{array}$ & 33 & TON & $1,100.00$ & IRON & 6.000 & 198 & 7,288 & * & 36,300 & & 43,588 \\
\hline & BUILDING STRUCTURAL STEEL & 433 & TON & $1,200.00$ & IRON & 10.000 & 4,330 & 159,387 & 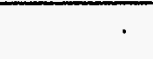 & 519,600 & . & 678,987 \\
\hline & GANTAY CRANE RAILS, EMBEDS, ETC. & 200 & LF & 161.00 & $\begin{array}{r}\text { IRON } \\
\text { GEN }\end{array}$ & 3.850 & 770 & 28,344 & & 32,200 & & 60,544 \\
\hline & RAILROAD TRACKS - WITHIN BUILDING & 300 & LF & 92.00 & $\begin{array}{c}\text { IRON } \\
\text { GEN }\end{array}$ & 2.000 & 600 & 22,086 & & 27,600 & & 49,686 \\
\hline & TAANSFER CART RAILS & 350 & LF & 92.00 & $\begin{array}{c}\text { IRON } \\
\text { GEN }\end{array}$ & 2.000 & 700 & 25,767 & & 32,200 & & 57,967 \\
\hline & BIRD SCREEN AND VENT LOUVERS & 960 & SF & 40.00 & $\begin{array}{c}\text { SHEE } \\
\text { GEN }\end{array}$ & 0.250 & 240 & 8,318 & & 38,400 & ? & 46,718 \\
\hline & AIR OUTLET WALL (INSIDE) & 3,200 & $\mathbf{S F}$ & & GEN & 0.000 & . & & & & 38,400 & 38,400 \\
\hline & METALS S/T & & & & & & 6,838 & $\$ 251,191$ & & $\$ 686,300$ & $\$ 38,400$ & $\$ 975,891$ \\
\hline \multirow[t]{6}{*}{1.3 .7} & IHERMAL \& MOISTURE PROTECTION & & & & & . & & & & & & \\
\hline & & & & & & & $\therefore$ & & & & & \\
\hline & SUBGRADE WATERPROOFING & 16,000 & SF & & GEN & 0.000 & & . & & & . 24,000 & 24,000 \\
\hline & $\begin{array}{l}\text { ROOF \& WALL SYSTEMS } \\
\text { includes motal reollho or wall panolinswlation }\end{array}$ & 38,400 & sf & & GEN & 0.000 & & & & & 460,800 & 460,800 \\
\hline & $\begin{array}{l}\text { and hanging system. Assume slanding soam } \\
\text { rool. Assume all sheols protinlshed. }\end{array}$ & & & & & & & & & & & \\
\hline & THERMAL \& MOISTURE PROTECTION S/T & & & & & & 0 & & & & $\$ 484,800$ & $\$ 484,800$ \\
\hline \multirow[t]{4}{*}{$1,3.8$} & DOQRS \& WINDOWS & & & & & & & & & & & . \\
\hline & OVERHEAD DOORS & 2 & EA & $16,000.00$ & $\begin{array}{c}\text { SKWK } \\
G E N\end{array}$ & 75.000 & 150 & 4,967 & & 32,000 & & 36,967 \\
\hline & PEASONNEL DOORS & 5 & EA & $1,000.00$ & $\begin{array}{r}\text { SKWK } \\
\text { GEN }\end{array}$ & 10.000 & 50 & 1,656 & & 5,000 & & 6,656 \\
\hline & DOORS \& WINDOWS S/T & & & & & & 200 & $\$ 6,622$ & & $\$ 37,000$ & & $\$ 43,622$ \\
\hline & & & & & & : & & & & & & • \\
\hline
\end{tabular}


Lockheed Martin Idaho Technologles Co. Rov. 8/80 PRONECT NAME: TRU SEPS. FACILITY - INTERIM STORAGE FACILITY

LOCATION 1: INEEL/ICPP

REQUESTOR: J. K. RAWLINS

\section{DETAILED COST ESTIMATE SHEET}

TYPE OF Estimate: PLANNING

PROJECT NO.: 2421-TRU

PREPARED BY: R. D. ADAMS
PAGE \# 4

DATE 26-Jan-1998

TIME: 15:25:49

REPORT NAME: Detall Cost Estimate Sheot

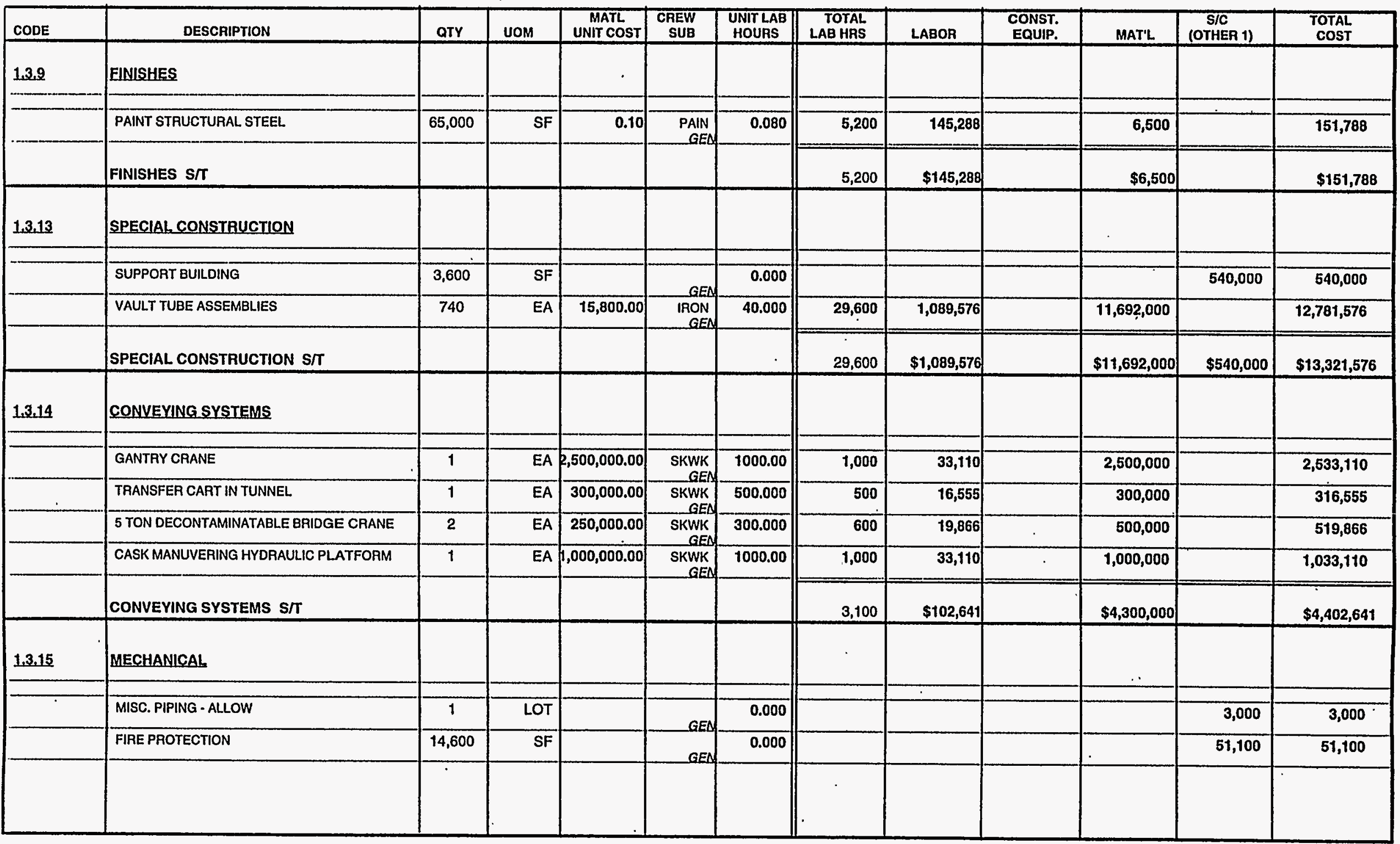


Lockheed Martin Idaho Technologles Co. Rov. 6/96

PROJECT NAME: TRU SEPS. FACILITY - INTERIM STORAGE

FACILITY

LOCATION 1: INEELICPP

REQUESTOR: J. K. RAWLINS

\section{DETAILED COST ESTIMATE SHEET}

TYPE OF ESTIMATE: PLANNING

PROJECT NO: 2421-TRU

PREPARED BY: R. D. ADAMS
PAGE 5

DATE 26-Jan-1998

TIME: 15:25:49

Report name: Detall Cost Estimate Sheet

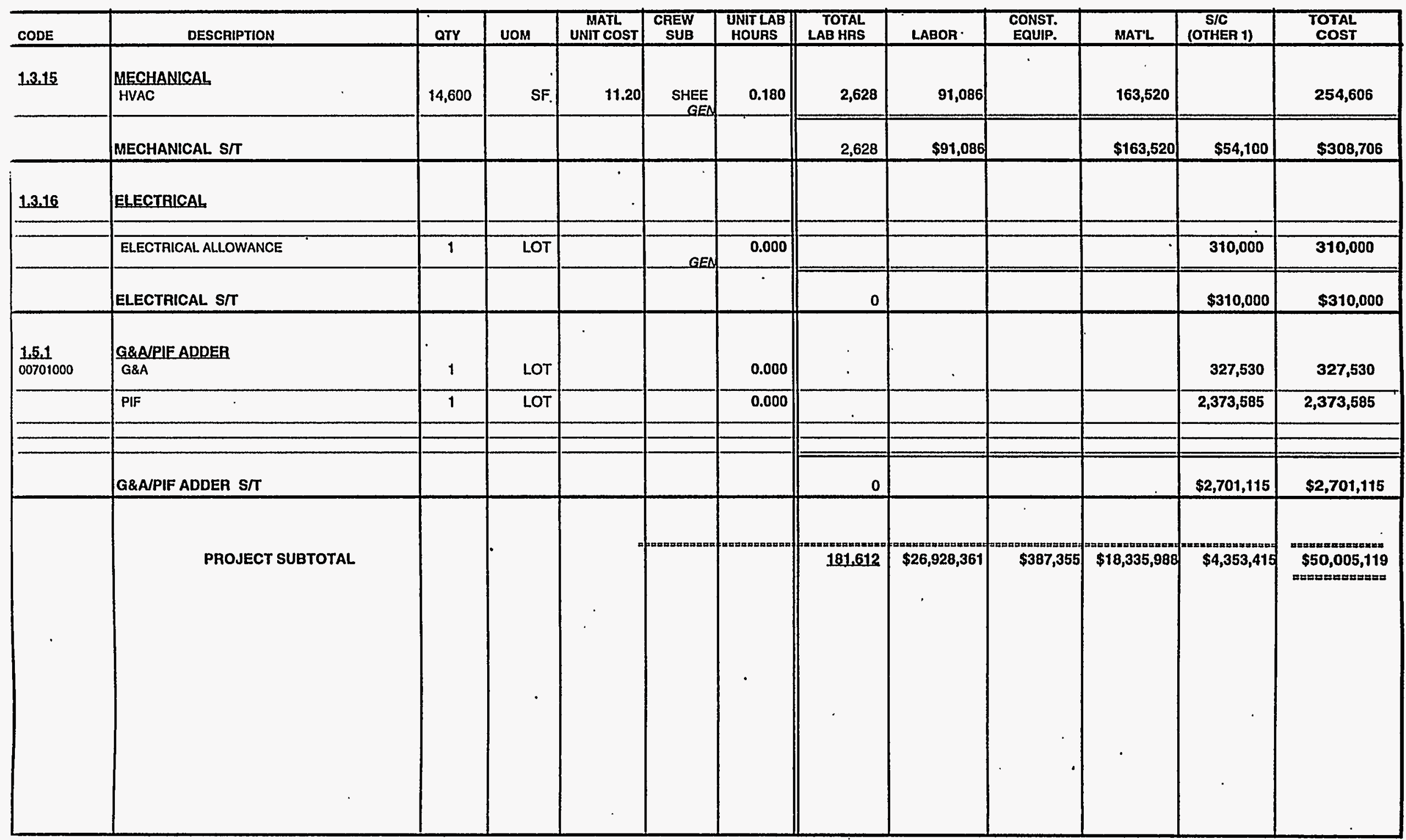


Lockheed Martin Idaho Technologies Co. PROIECT NAME: TRU SEPS. FACILITY - INTERIM STORAGE ,

LOCATION 1: REQUESTOR:
FACILITY - UNESCALATED

INEELICPP

J. K. RAWLINS

\section{CONTINGENCY ANALYSIS}

$\begin{array}{cl}\text { TYPE OF ESTIMATE: } & \text { PLANNING } \\ \text { PROJECT NO: } & \text { 2421-TRU } \\ \text { PAEPAREO BY: } & \text { R.D. ADAMS }\end{array}$

DATE: $26-$ Jan-1998

TIME: 15:30:08

REPORT NAME: Contingency Analysis

\begin{tabular}{|c|c|c|c|c|c|c|c|c|c|c|c|}
\hline & PROB & BLE \% VARIAT & ON & & & & & & $\begin{array}{l}\text { PROI } \\
\text { CONTII }\end{array}$ & $\begin{array}{l}\text { CT } \\
\text { iENCY }\end{array}$ & SUMMARY \\
\hline \begin{tabular}{|l|} 
WBS \\
Element
\end{tabular} & Cost Estimate Element & Total Cost w/o & $\begin{array}{c}\% \text { Total } \\
\text { Cost }\end{array}$ & & $\begin{array}{l}\% \text { Var. } \\
\text { n Est. }\end{array}$ & Wt. \% & Prob. & Contingency & $\%$ & Cost & Total Cost \\
\hline & & Contingency & & - & + & - & + & & & & by Element \\
\hline 1.1 .1 & DESIGN ENGINEERING TITLE I \&\| & $8,481,000$ & 12.79 & 20 & 35 & 2.56 & 4.48 & $3.773 \%$ & $16.99 \%$ & $2,496,401$ & $10,977,401$ \\
\hline 1.12 & TITLEIII INSPECTION & $2,120,200$ & 3.20 & 20 & 30 & 0.64 & 0.96 & $0.799 \%$ & $3.60 \%$ & 528,886 & $2,649,086$ \\
\hline 1.2 .1 & PROJECT MANAGEMENT & $5,936,600$ & 8.95 & 20 & 35 & 1.79 & 3.13 & $2.641 \%$ & $11.89 \%$ & $1,747,451$ & $7,684,051$ \\
\hline 1.22 & CONSTRUCTION MANAGEMENT & $4,240,500$ & 6.40 & 20 & 30 & 1.28 & 1.92 & $1.599 \%$ & $7.20 \%$ & $1,057,797$ & $5,298,297$ \\
\hline 1.3 .1 & GENERAL CONDITIONS & $1,168,827$ & 1.76 & 20 & 35 & 0.35 & 0.62 & $0.520 \%$ & $2.34 \%$ & 344,047 & $1,512,874$ \\
\hline 1.3 .2 & SITEWORK & 324,794 & 0.49 & 20 & 30 & 0.10 & 0.15 & $0.122 \%$ & $0.55 \%$ & 81,020 & 405,814 \\
\hline 1.3 .3 & CONCRETE & $9,046,326$ & 13.64 & 20 & 30 & 2.73 & 4.09 & $3.411 \%$ & $15.36 \%$ & $2,256,615$ & $11,302,941$ \\
\hline 1.3 .5 & METALS & $1,580,519$ & 2.38 & 20 & 25 & 0.48 & 0.60 & $0.489 \%$ & $2.20 \%$ & 323,295 & $1,903,814$ \\
\hline 1.3 .7 & THERMAL \& MOISTURE PROTECTION & 543,946 & 0.82 & 20 & 30 & 0.16 & 0.25 & $0.205 \%$ & $0.92 \%$ & 135,688 & 679,634 \\
\hline 1.3 .8 & DOORS \& WINDOWS & 71,240 & 0.11 & 20 & 20 & 0.02 & 0.02 & $0.017 \%$ & $0.08 \%$ & 11,373 & 82,613 \\
\hline 1.3 .9 & FINISHES & 255,077 & 0.38 & 20 & 35 & 0.08 & 0.13 & $0.113 \%$ & $0.51 \%$ & 75,082 & 330,159 \\
\hline 1.3.13 & SPECIAL CONSTRUCTION & $21,429,637$ & 32.32 & 20 & 20 & 6.46 & 6.46 & $5.171 \%$ & $23.29 \%$ & $3,421,213$ & $24,850,850$ \\
\hline 1.3 .14 & CONVEYING SYSTEMS & $7,156,751$ & 10.79 & 20 & 25 & 2.16 & 2.70 & $2.213 \%$ & $9.96 \%$ & $1,463,912$ & $8,620,663$ \\
\hline 1.3 .15 & MECHANICAL & 479,586 & 0.72 & 20 & 30 & 0.14 & 0.22 & $0.181 \%$ & $0.81 \%$ & 119,633 & 599,219 \\
\hline 1.3.16 & ELECTRICAL & 347,820 & 0.52 & 20 & 25 & 0.10 & 0.13 & $0.108 \%$ & $0.48 \%$ & 71,147 & 418,967 \\
\hline 1.5 .1 & G\&APIF ADDER & $2,701,115$ & 4.07 & 5 & 20 & 0.20 & 0.81 & $0.713 \%$ & $3.21 \%$ & 471,657 & $3,172,772$ \\
\hline 1.5 .2 & PROCUREMENT FEES & 424,045 & 0.64 & 20 & 25 & 0.13 & 0.16 & $0.131 \%$ & $0.59 \%$ & 86,738 & 510,783 \\
\hline & ESCALATION & 0 & 0.00 & 20 & 25 & 0.00 & 0.00 & $0.000 \%$ & $0.00 \%$ & 62 & 62 \\
\hline & SUBTOTAL & $66,307,983$ & 100.00 & & & & & $22.206 \%$ & & & \\
\hline & CALCULATED CONTINGENCY & $14,724,290$ & & & & & & & & & \\
\hline & RESULTANT TEC & $81,032,273$ & & & & & & & & & \\
\hline & ROUNDED TEC & $81,000,000$ & & & & & & & & & \\
\hline & PROJECT CONTINGENCY & $14,692,017$ & & & & & & $22.16 \%$ & & & \\
\hline & MANAGEMENT RESERVE & $4,552,968$ & & & & & & & & & \\
\hline & CONTINGENCY & $10,139,049$ & & & & & & & & & \\
\hline & TOTAL ESTIMATED COST & $81,000,000$ & & & & & & & & $14,692,017$ & $81,000,000$ \\
\hline
\end{tabular}

\section{CONFIDENCE LEVEL AND ASSUMED RISKS:}

The Lockheed Idaho Technologies Co. Cost Estimate Contingency Analysis Model is based on the applied contingency and the assumptions upon which the estimate was predicated. The model is applied with a suggested risk level of $18 \%$ and a level of confidence of $90 \%$ the estimate will fall within the bid range. The Contingency Analysis is based on a weighted average to provide a

$90 \%$ probability of underrun and a $10 \%$ probability of overrun.
CONTINGENCY ANALYSIS GUIDE BY TYPE OF ESTIMATE

Guidelines established by DOE/FM 50, Cost Estimating Guide, Vol. 6 , Cost Guide, and as presented in the INEL Cost Estimating Guide.

PLANNING $20 \%-30 \%$ Experimental/Special Conditions.............Up to 50\% Conceptual $15 \%-25 \%$. Experimental/Special Conditions............. Up to $40 \%$ TITLE!

TITLE II

TITLE II/AFC
$10 \%-20 \%$

$5 \%-15 \%$ Market Conditions 
Lockheed Martin Idaho Technologies Co.

$$
\text { Rov. } 6196
$$

PROIECT NAME: TRU SEPS. FACILITY - INTERIM STORAGE

LOCATON 1: FACILITY-ESCALATED

REQUESTOR: J.K. RAWLINS
CONTINGENCY ANALYSIS

$\begin{array}{cl}\text { TYPE OF ESTIMATE: } & \text { PLANNING } \\ \text { PROJECT NO: } & \text { 2421-TRU } \\ \text { PREPARED BY: } & \text { R.D. ADAMS }\end{array}$

DATE: 26-Jan-1998

TIME: 15:28:05

REPORT NAME: Contingency Analysis

\begin{tabular}{|c|c|c|c|c|c|c|c|c|c|c|c|}
\hline \multicolumn{9}{|c|}{ PROBABLE \% VARIATION } & \multicolumn{2}{|c|}{$\begin{array}{l}\text { PROJECT } \\
\text { CONTINGENCY }\end{array}$} & \multirow{3}{*}{$\begin{array}{l}\text { SUMMARY } \\
\text { Total Cost } \\
\text { by Element }\end{array}$} \\
\hline \multirow{2}{*}{$\begin{array}{l}\text { WBS } \\
\text { Element }\end{array}$} & \multirow{2}{*}{ Cost Estimate Element } & \multirow{2}{*}{$\begin{array}{c}\text { Total Cost w/o } \\
\text { Contingency }\end{array}$} & \multirow[t]{2}{*}{$\begin{array}{l}\text { \% Total } \\
\text { Cost }\end{array}$} & \multicolumn{2}{|c|}{$\begin{array}{l}\text { Prob. \% Var. } \\
\text { From Est. }\end{array}$} & \multicolumn{2}{|c|}{ Wt. \% of Prob. } & \multirow{2}{*}{ Contingency } & \multirow{2}{*}{$\%$} & \multirow{2}{*}{ Cost } & \\
\hline & & & & - & + & - & + & & & & \\
\hline 1.1 .1 & DESIGN ENGINEERING TILE I \&\| & $8,481,000$ & 8.80 & 20 & 35 & 1.76 & 3.08 & $2596 \%$ & $11.98 \%$ & $2,504,743$ & $10,985,743$ \\
\hline 1.12 & TITLE III INSPECTION & $2,120,200$ & 2.20 & 20 & 30 & 0.44 & 0.66 & $0.550 \%$ & $254 \%$ & 530,653 & $2,650,853$ \\
\hline 1.2 .1 & PROJECT MANAGEMENT & $5,936,600$ & 6.16 & 20 & 35 & 1.23 & 216 & $1.817 \%$ & $8.38 \%$ & $1,753,290$ & $7,689,890$ \\
\hline 1.2 .2 & CONSTRUCTION MANAGEMENT & $4,240,500$ & 4.40 & 20 & 30 & 0.88 & 1.32 & $1.100 \%$ & $5.07 \%$ & $1,061,332$ & $5,301,832$ \\
\hline 1.3 .1 & GENERAL CONDITIONS & $1,168,827$ & 1.21 & 20 & 35 & 0.24 & 0.42 & $0.358 \%$ & $1.65 \%$ & 345,196 & $1,514,023$ \\
\hline 1.3 .2 & SITEWORK & 324,794 & 0.34 & 20 & 30 & 0.07 & 0.10 & $0.084 \%$ & $0.39 \%$ & 81,291 & 406,085 \\
\hline 1.3 .3 & CONCRETE & $9,046,326$ & 9.39 & 20 & 30 & 1.88 & 2.82 & $2346 \%$ & $10.83 \%$ & $2,264,156$ & $11,310,482$ \\
\hline 1.3 .5 & METALS & $1,580,519$ & 1.64 & 20 & 25 & 0.33 & 0.41 & $0.336 \%$ & $1.55 \%$ & 324,375 & $1,904,894$ \\
\hline 1.3 .7 & THERMAL \& MOISTURE PROTECTION & 543,946 & 0.56 & 20 & 30 & 0.11 & 0.17 & $0.141 \%$ & $0.65 \%$ & 136,141 & 680,087 \\
\hline 1.3 .8 & DOORS \& WINDOWS & 71,240 & 0.07 & 20 & 20 & 0.01 & 0.01 & $0.012 \%$ & $0.05 \%$ & 11,411 & 82,651 \\
\hline 1.3 .9 & FINISHES & 255,077 & 0.26 & 20 & 35 & 0.05 & 0.09 & $0.078 \%$ & $0.36 \%$ & 75,333 & 330,410 \\
\hline 1.3.13 & SPECIAL CONSTRUCTION & $21,429,637$ & 2223 & 20 & 20 & 4.45 & 4.45 & $3.557 \%$ & $16.41 \%$ & $3,432,645$ & $24,862,282$ \\
\hline 1.3.14 & CONVEYING SYSTEMS & $7,156,751$ & 7.43 & 20 & 25 & 1.49 & 1.86 & $1.522 \%$ & $7.02 \%$ & $1,468,804$ & $8,625,555$ \\
\hline 1.3 .15 & MECHANICAL & 479,586 & 0.50 & 20 & 30 & 0.10 & 0.15 & $0.124 \%$ & $0.57 \%$ & 120,033 & 599,619 \\
\hline 1.3 .16 & ELECTRICAL & 347,820 & 0.36 & 20 & 25 & 0.07 & 0.09 & $0.074 \%$ & $0.34 \%$ & 71,384 & 419,204 \\
\hline 1.5 .1 & G\&AVIF ADDEA & $2,701,115$ & 2.80 & 5 & 20 & 0.14 & 0.56 & $0.490 \%$ & $2.26 \%$ & 473,233 & $3,174,348$ \\
\hline \multirow[t]{2}{*}{1.5 .2} & PROCUREMENT FEES & 424,045 & 0.44 & 20 & 25 & 0.09 & 0.11 & $0.090 \%$ & $0.42 \%$ & 87,028 & 511,073 \\
\hline & ESCALATION & $30,077,622$ & 31.21 & 20 & 25 & 6.24 & 7.80 & $6.397 \%$ & $29.52 \%$ & 6.173 .347 & $36,250,969$ \\
\hline & SUBTOTAL & $96,385,605$ & 100.00 & & & & & $21.674 \%$ & & & \\
\hline & CALCULATED CONTINGENCY & $20,890,202$ & & & & & & & & & \\
\hline & PESULTANT TEC & $117,275,807$ & & & & & & & & & \\
\hline & ROUNDED TEC & $117,300,000$ & & & & & & & & & \\
\hline & PROJECT CONTINGENCY & $20,914,395$ & & & & & & $21.70 \%$ & & & \\
\hline & MANAGEMENT RESERVE & $6,647,334$ & & & & & & & & & \\
\hline & CONTINGENCY & $14,267,061$ & & & & & & & & & \\
\hline & TOTAL ESTIMATED COST & $117,300,000$ & & & & & & & & $20,914,395$ & $117,300,000$ \\
\hline
\end{tabular}

\section{CONFIDENCE LEVEL AND ASSUMED RISKS:}

The Lockheed Idaho Technologies Co. Cost Estimate Contingency Analysis Model is based on the applied contingency and the assumptions upon which the estimate was predicated. The model is applied with a suggested risk level of $18 \%$ and a level of confidence of $90 \%$ the estimate will fall within the bid range.

The Contingency Analysis is based on a weighted average to provide a

$90 \%$ probability of underrun and a $10 \%$ probability of overrun.
CONTINGENCY ANALYSIS GUIDE BY TYPE OF ESTIMATE

Guidelines established by DOE/FM 50, Cost Estimating Guide, Vol. 6 , Cost Guide, and as presented in the INEL Cost Estimating Guide. PLANNING Experimental/Special Conditions. Conceptual Experimental/Special Conditions.............Up to $40 \%$ TITLE I TITLE II/AFC ......Up to $50 \%$ $10 \%=20 \%$ Market Conditions 


\section{G\&APIF ADDER CALCULATION SHEET}

PROCUREMENT FEE:

\begin{tabular}{l} 
CONSTRUCTION $=$ \\
GFE $=$ \\
$\quad$ Subtotal \\
\cline { 2 - 2 }
\end{tabular}

FEE (1\% $1 \%$

$\$ 42,404,523 * 0.01 \boxminus \quad \$ 424,045.23$

G\&A @ 23\% (with a ceiling of $\$ 500,000$ imposed per year

CONSTRUCTION OR

CEILING * 2 YEARS =

GFE =

$\$ 1,000,000$

PROCUREMENT FEE =

$\$ 424,045$

Subtotal $\$ 1,424,045$

FEE @ $23 \%=$

$\$ 1,424,045 \cdot 0.23=$

$\$ 327,530$

PIF @ 5.5\%

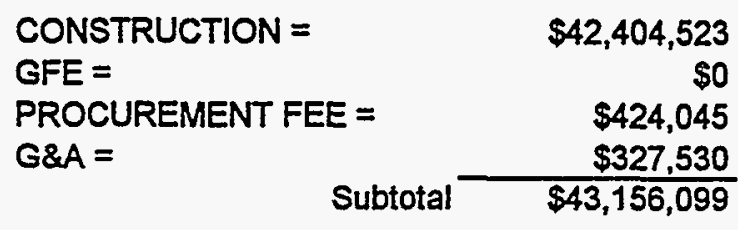

FEE @ $5.5 \%=$

$\$ 43,156,099 \cdot 0.055=$

$\$ 2,373,585$

TOTAL PROCUREMENT FEE:

$\$ 424,045$

TOTAL G\&A FEE:

$\$ 327,530$

TOTAL PIF:

$\$ 2,373,585$ 
Lockheed Martin Idaho Technologies Co. Rev. 696

PROAECT NAME: TRU - INTERIM STORAGE

FACILITY - OPC - ESCALATED

INEELICPP

LOCATION I:

AEQUESTOR

J.K. RAWLINS
COST ESTIMATE SUMMARY

TYPE OF ESTIMATE: PLANNING PROIECT NO: 242106

PREPARED BY: R. D. ADAMS

REPORT NAME: Cost Estimate Summary

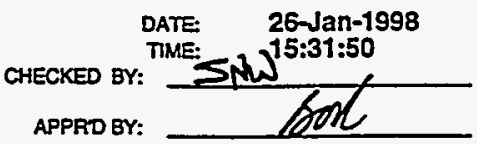

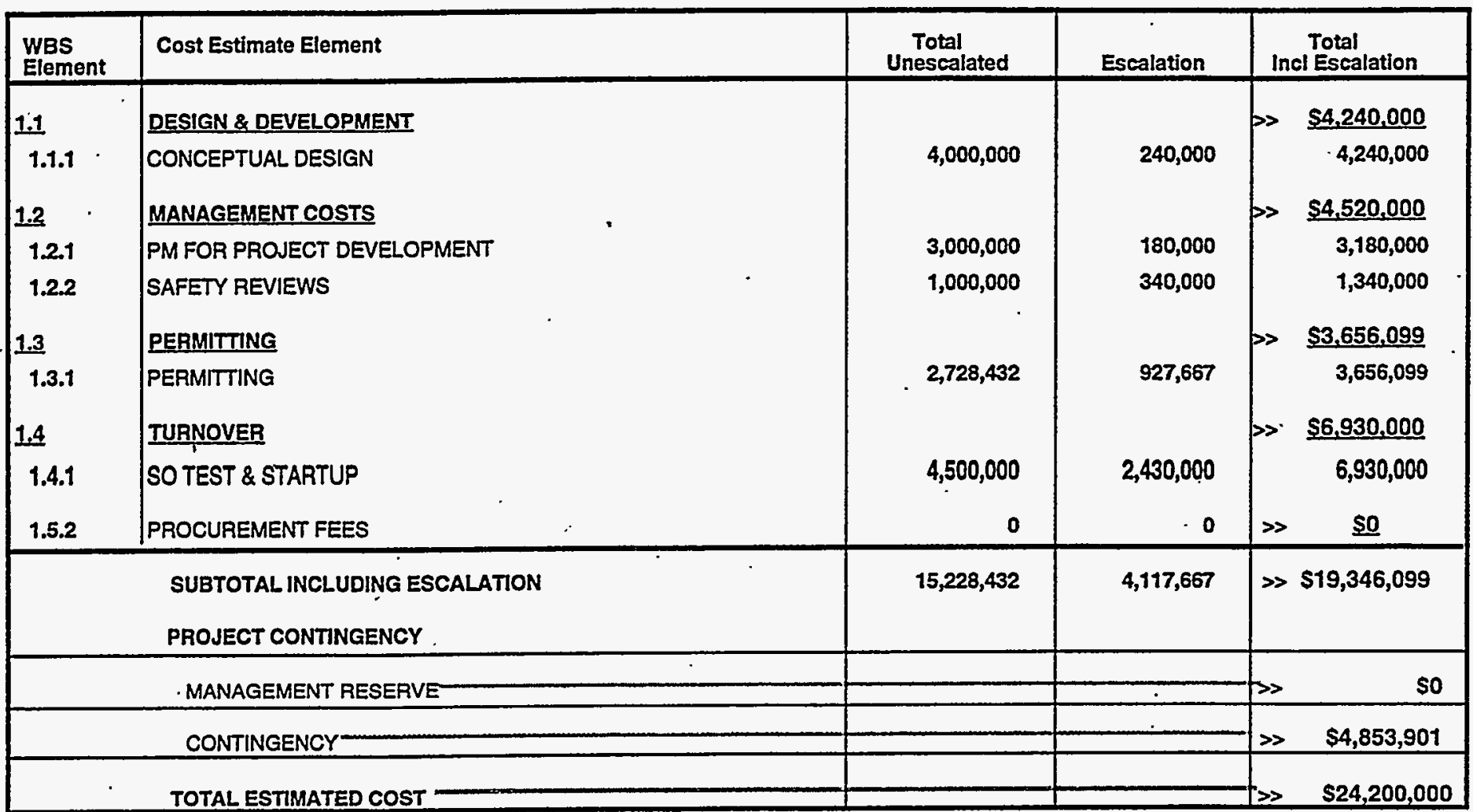

\section{PROJECT COST PARAMETERS}

EDI AS A $\%$ OF CONST. + GFE $=40.00 \%$ 
Lockheed Martin Idaho Technologies Co. PROSECT NAME

LOCATION 1:

TRU - INTERIM STORAGE

FACILITY - OPC - UNESCALATED

INEELICPP

J. K. RAWLINS
COST ESTIMATE SUMMARY

TYPE OFESTMMATE: PLANNING

PRONECT NO: 242106

PREPARED BY: R.D. ADAMS

REPORT NAME: Cost Estimate Summary

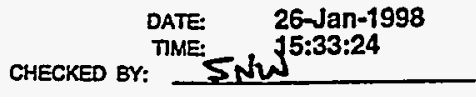

APPRO BY:

\begin{tabular}{|c|c|c|c|c|}
\hline $\begin{array}{l}\text { WBS } \\
\text { Element }\end{array}$ & Cost Estimate Element & $\begin{array}{c}\text { Total } \\
\text { Unescalated }\end{array}$ & Escalation & $\begin{array}{l}\text { Total } \\
\text { Incl Escalation }\end{array}$ \\
\hline $\begin{array}{l}\frac{1.1}{1.1 .1} \\
\frac{1.2}{1.2 .1} \\
1.2 .2 \\
\frac{1.3}{1.3 .1} \\
\frac{1.4}{1.4 .1} \\
1.5 .2\end{array}$ & $\begin{array}{l}\text { DESIGN \& DEVELOPMENT } \\
\text { CONCEPTUAL DESIGN } \\
\text { MANAGEMENT COSTS } \\
\text { PM FOR PROJECT DEVELOPMENT } \\
\text { SAFETY REVIEWS } \\
\text { PERMITTING } \\
\text { PERMITTING } \\
\text { TURNOVER } \\
\text { SO TEST \& STARTUP } \\
\text { PROCUREMENT FEES }\end{array}$ & $\begin{array}{r}4,000,000 \\
3,000,000 \\
1,000,000 \\
2,728,432 \\
4,500,000 \\
0\end{array}$ & $\begin{array}{l}0 \\
0\end{array}$ & $\begin{array}{r}\gg \frac{\$ 4,000,000}{4,000,000} \\
\gg \quad \begin{array}{r}\$ 4,000,000 \\
3,000,000 \\
1,000,000\end{array} \\
\gg \quad \frac{\$ 2,728,432}{2,728,432} \\
\gg \quad \frac{\$ 4,500,000}{4,500,000} \\
>\quad \text { \$2. }\end{array}$ \\
\hline & $\begin{array}{l}\text { SUBTOTAL INCLUDING ESCALATION } \\
\text { PROJECT CONTINGENCY }\end{array}$ & $15,228,432$ & $\mathbf{0}$ & $\gg \$ 15,228,432$ \\
\hline & MANAGEMENT RESERVE & & & $\gg$ \\
\hline & CONTINGENCY & & & $\$ 3,771,568$ \\
\hline & TOTAL ESTIMATED COST - & & & $\$ 19,000,000$ \\
\hline
\end{tabular}


Lockheed Martin Idaho Technologies Co.

Rev. 6198

PROJECT NAME: TRU - INTERIM STORAGE

FACILITY - OPC

LOCATION 1: INEELICPP

REQUESTOR: J. K. RAWLINS

\section{DETAILED COST ESTIMATE SHEET}

TYPE OF ESTIMATE: PLANNING

PROJECT NO:: 242106

PREPARED BY: R.D. ADAMS
PAGE: 1

DATE 26-Jan-1998

TIME: 15:30:58

REPORT NAME: Detall Cost Estimate Sheot

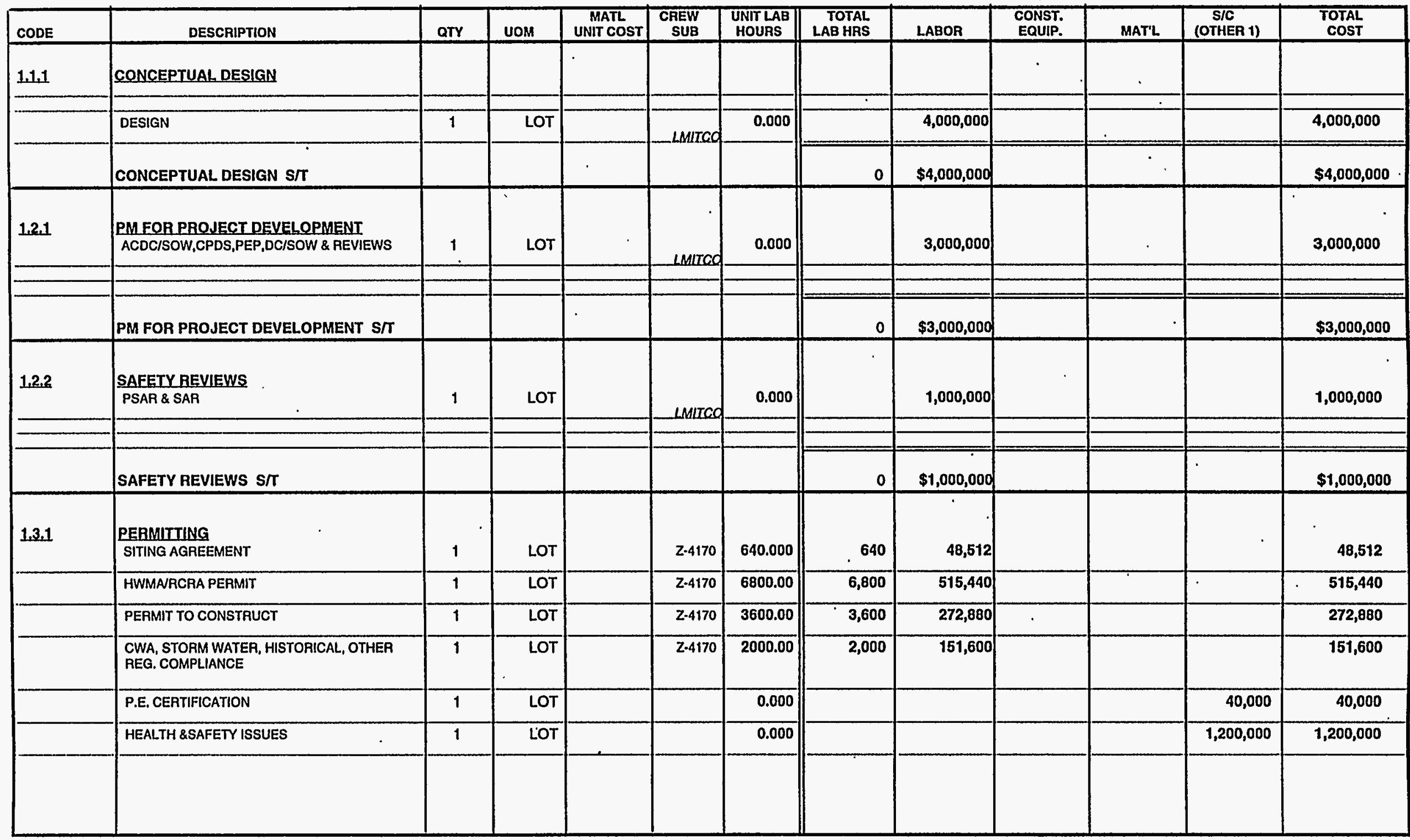




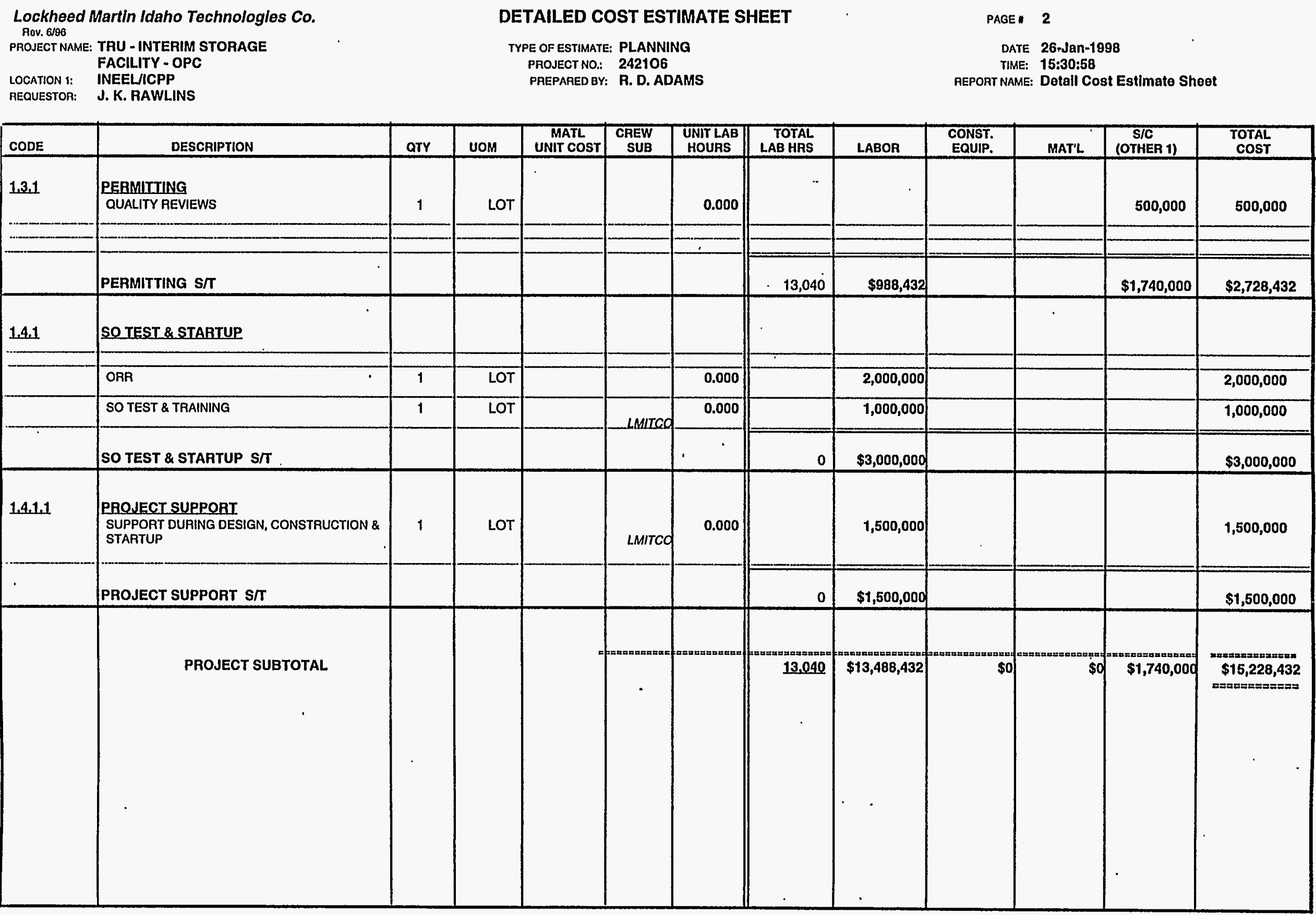



Lockheed Martin Idaho Technologies Co. PROAECT NAME: TRU - INTERIM STORAGE

LOCATION 1: FACILITY - OPC - UNESCALATED INEEU ICPP REQUESTOR
CONTINGENCY ANALYSIS

TYPE OF ESTIMATE PROSECT NO:

PLANNING PREPARED BY:
DATE: 26 Jan-1998

TIME: 15:33:29

\begin{tabular}{|c|c|c|c|c|c|c|c|c|c|c|c|}
\hline & PROB & BLE \% VARIAT & ON & & & & & & $\begin{array}{l}\text { PROJ } \\
\text { CONTIN }\end{array}$ & $\begin{array}{l}\text { ET } \\
\text { ENCY }\end{array}$ & SUMMARY \\
\hline $\begin{array}{l}\text { WBS } \\
\text { Element }\end{array}$ & Cost Estimate Element & Total Cost w/o & $\begin{array}{c}\% \text { Total } \\
\text { Cost }\end{array}$ & & $\begin{array}{l}\% \text { Var. } \\
\text { m Est. }\end{array}$ & Wt. \% & Prob. & Contingeney & $\%$ & Cost & Total Cost \\
\hline & & Contingency & & - & + & - & \pm & & & & by Element \\
\hline 1.1 .1 & CONCEPTUAL DESIGN & $4,000,000$ & 26.27 & 20 & 30 & 5.25 & 7.88 & $6.567 \%$ & $2627 \%$ & 990,665 & $4,990,665$ \\
\hline 1.21 & PM FOR PROJECT DEVELOPMENT & $3,000,000$ & 19.70 & 20 & 30 & 3.94 & 5.91 & 4.925\% & $19.70 \%$ & 742,999 & $3,742,999$ \\
\hline 1.22 & SAFETY REVIEWS & $1,000,000$ & 6.57 & 20 & 30 & 1.31 & 1.97 & 7.642\% & 6.57\% & 247,666 & $1,247,666$ \\
\hline 1.3.1 & PERMITTING & $2,728,432$ & 17.92 & 20 & 30 & 3.58 & 5.38 & 4.479\% & $17.92 \%$ & 675,740 & $3,404,172$ \\
\hline 1.4 .1 & SO TEST \& STARTUP & $4,500,000$ & 29.55 & 20 & 30 & 5.91 & 8.86 & $7.387 \%$ & $29.55 \%$ & $1,114,498$ & $5,614,498$ \\
\hline 1.5.2 & PROCUREMENT FEES & 0 & 0.00 & 5 & 20 & 0.00 & 0.00 & $0.000 \%$ & $0.00 \%$ & 0 & 0 \\
\hline & ESCALATION & 0 & 0.00 & 20 & 30 & 0.00 & 0.00 & $0.000 \%$ & $0.00 \%$ & 0 & 0 \\
\hline & SUBTOTAL & $15,228,432$ & 100.00 & & & & & $25.000 \%$ & & & \\
\hline & CALCULATED CONTINGENCY & $3,807,108$ & & & & & & & & & \\
\hline & RESULTANT TEC & $19,035,540$ & & & & & & & & & \\
\hline & ROUNDED TEC & $19,000,000$ & & & & & & & & & \\
\hline & PROJECT CONTINGENCY & $3,771,568$ & & & & & & $24.77 \%$ & & & \\
\hline & MANAGEMENT RESERVE & 0 & & & & & & & & & \\
\hline & CONTINGENCY & $3,771,568$ & & & & & & & & & \\
\hline & TOTAL ESTIMATED COST & $19,000,000$ & & & & & & & & $3,771,568$ & $19,000,000$ \\
\hline
\end{tabular}

CONFIDENCE LEVEL AND ASSUMED RISKS:

The Lockheed Idaho Technologies Co. Cost Estimate Contingency Analysis Model is based on the applied contingency and the assumptions upon which the estimate was predicated. The model is applied with a suggested risk level of $18 \%$ and a level of confidence of $90 \%$ the estimate will fall within the bid range. The Contingency Analysis is based on a weighted average to provide a $90 \%$ probability of underrun and a $10 \%$ probability of overrun.
CONTINGENCY ANALYSIS GUIDE BY TYPE OF ESTIMATE

Guidelines established by DOE/FM 50, Cost Estimating Guide, Vol. 6, Cost Guide, and as presented in the INEL Cost Estimating Guide. PLANNING Conceptual TITLEI TITLE \| TITLE II/AFC $15 \%-25 \%$ $\ldots$.... to $40 \%$ $10 \%-20 \%$ $5 \%-15 \%$ Market Conditions 
Lockheed Martin Idaho Technologies Co.

PROSECT NAME: TRU - INTERIM STORAGE

LOCATON 1: FACILITY - OPC-ESCALATED

REQUESTOR:

\section{CONTINGENCY ANALYSIS}

TYPE OF ESTIMATE: PROJECT NO: PREPAFED BY:
PLANNING
242106

R. D. ADAMS
DATE: 26-Jan-1998

TIME: 15:31:55

REPORT NAME: Contingency Analysis

\begin{tabular}{|c|c|c|c|c|c|c|c|c|c|c|c|}
\hline & PROB & BLE \% VARIA & ON & & & & & & $\begin{array}{l}\text { PROJ } \\
\text { CONTII }\end{array}$ & $\begin{array}{l}\text { CT } \\
\text { ENCY. }\end{array}$ & SUMMARY \\
\hline $\begin{array}{l}\text { WBS } \\
\text { Element }\end{array}$ & Cost Estimate Element & Total Cost w/o & $\begin{array}{c}\text { \% Total } \\
\text { Cost }\end{array}$ & & $\begin{array}{l}\% \text { Var. } \\
\text { m Est. }\end{array}$ & Wt. \% & Prob. & Contingency & $\%$ & Cost & Total Cost \\
\hline & & Contingency & & - & + & - & + & & & & by Elament \\
\hline 1.1.1 & CONCEPTUAL DESIGN & $4,000,000$ & 20.68 & 20 & 30 & 4.14 & 6.20 & $5.169 \%$ & $20.68 \%$ & $1,003,593$ & $5,003,593$ \\
\hline 1.2 .1 & PM FOR PROJECT DEVELOPMENT & $3,000,000$ & 15.51 & 20 & 30 & 3.10 & 4.65 & $3.877 \%$ & $15.51 \%$ & 752,695 & $3,752,695$ \\
\hline 1.22 & SAFEIY REVIEWS & $1,000,000$ & 5.17 & 20 & 30 & 1.03 & 1.55 & $1292 \%$ & $5.17 \%$ & 250,898 & $1,250,898$ \\
\hline 1.3 .1 & PERMITIING & $2,728,432$ & 14.10 & 20 & 30 & 282 & 4.23 & $3.526 \%$ & $14.10 \%$ & 684,559 & $3,412,991$ \\
\hline 1.4 .1 & SO TEST \& STARTUP & $4,500,000$ & 23.26 & 20 & 30 & 4.65 & 6.98 & $5.815 \%$ & $23.26 \%$ & $1,129,042$ & $5,629,042$ \\
\hline 1.5 .2 & PROCUREMENT FEES & 0 & 0.00 & 5 & 20 & 0.00 & 0.00 & $0.000 \%$ & $0.00 \%$ & 0 & ○ \\
\hline & ESCALATION & $4,117,667$ & 21.28 & 20 & 30 & 4.26 & 6.39 & $5.321 \%$ & $21.28 \%$ & $1,033,114$ & $5,150,781$ \\
\hline & SUBTOTAL & $19,346,099$ & 100.00 & & & & & $25.000 \%$ & & & \\
\hline & CALCULATED CONTINGENCY & $4,836,525$ & & & & & & & & & \\
\hline & RESULTANT TEC & $24,182,624$ & & & & & & & & & \\
\hline & ROUNDED TEC & $24,200,000$ & & & & & & & & & \\
\hline & PROJECT CONTINGENCY & $4,853,901$ & & & & & & $25.09 \%$ & & & \\
\hline & MANAGEMENT RESERVE & 0 & & & & & & & & & \\
\hline & CONTINGENCY & $4,853,901$ & & & & & & & & & \\
\hline & TOTAL ESTIMATED COST & $24,200,000$ & & & & & & & & $4,853,901$ & $24,200,000$ \\
\hline
\end{tabular}

\section{CONFIDENCE LEVEL AND ASSUMED RISKS:}

The Lockheed Idaho Technologies Co. Cost Estimate Contingency Analysis

Model is based on the applied contingency and the assumptions upon which

the estimate was predicated. The model is applied with a suggested risk level

of $18 \%$ and a level of confidence of $90 \%$ the estimate will fall within the bid range.

The Contingency Analysis is based on a weighted average to provide a

$90 \%$ probability of undernu and a $10 \%$ probability of overun.
CONTINGENCY ANALYSIS GUIDE BY TYPE OF ESTIMATE Guidelines established by DOE/FM 50, Cost Estimating Guide, Vol. 6, Cost Guide, and as presented in the INEL Cost Estimating Guide. PLANNING Experimental/Special Conditions $20 \%-30 \%$ Conceptual

ExperimentalSpecial Conditions.............Up to $40 \%$ TITLEI TITLE II

TITLE IVAFC $10 \%-20 \%$ $5 \%-15 \%$ Market Conditions 


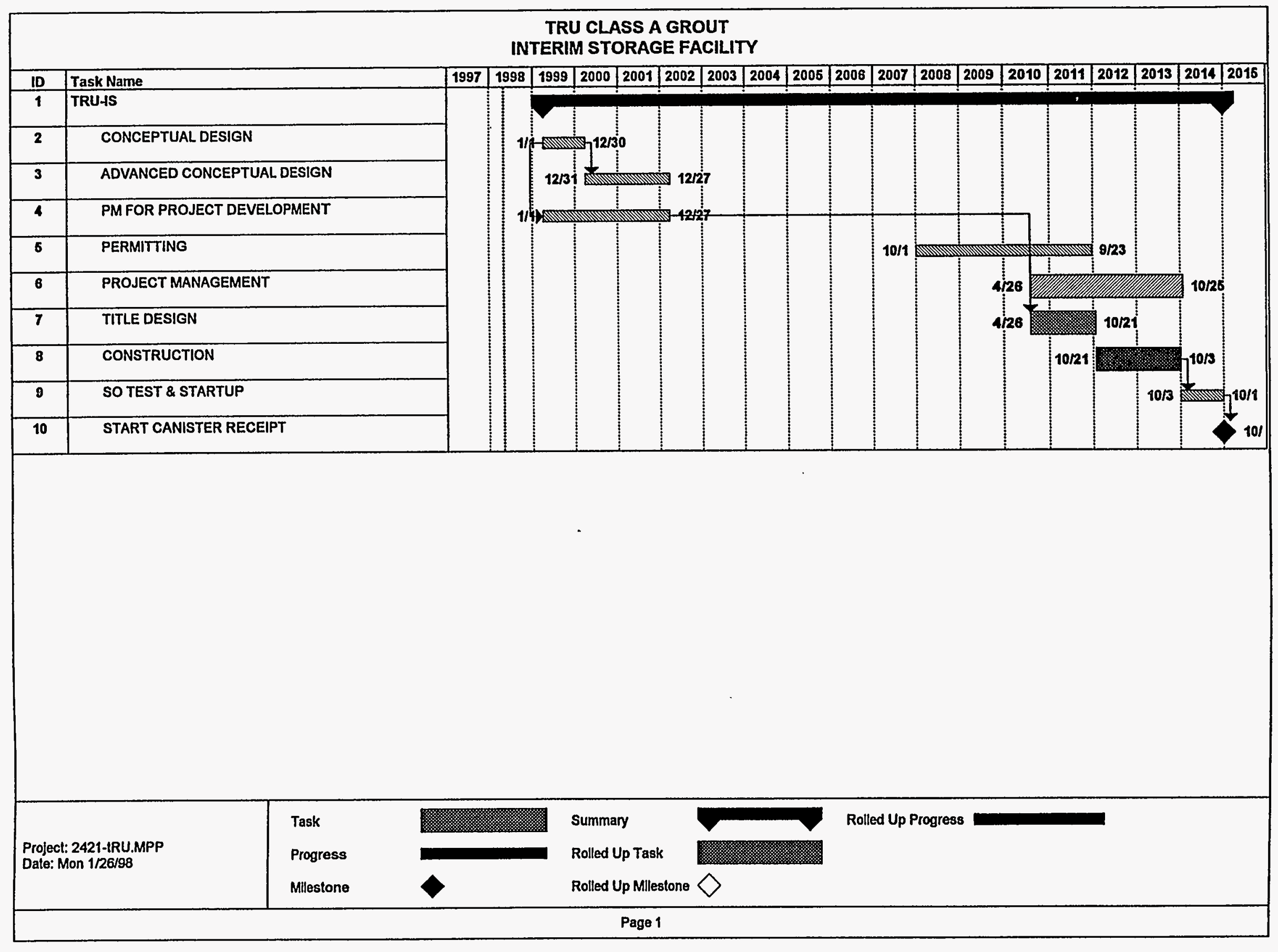


COST ESTIMATE SUPPORT DATA RECAPITULATION

Project Title: HLW ISF - DCWO

Date: January 26, 1998

Approved By:
Estimator: R. D. Adams

File: 2421-DCWO

I. SCOPE OF WORK: Brief description of the proposed project.

This facility will unload, store in vertical tubes and load out canisters containing High Level Waste (HIW) from the proposed Direct Cementious Waste Option (DCWO).

II. BASIS OF THE ESTIMATE: Drawings, Design Report, Engineers Notes andlor other documentation upon which the estimate is originated.

Draft sketches, a draft EDF, and the SNF Canister Storage Building Final Design Estimate from Fluor Daniel Northwest, Inc. were used as the basis for this estimate. 


\section{COST ESTIMATE SUPPORT DATA RECAPITULATION}

\begin{tabular}{lr} 
Project Title: 1 HLW ISF - DCWO & Estimator: R D. Adams \\
Date: January 26, 1998 & File: $2421-D C W O$ \\
Approved By: & \\
\hline
\end{tabular}

III. ASSUMPTIONS: Conditions statements accepted or supposed true without proof of demonstration. An assumption has a direct impact on total estimated cost.

1. The Interim Storage Facility will be located within 100 feet of the VWO facility.

2. Rock will not be encountered during excavation.

3. The estimates include both present day costs summaries and contingency analysis and escalated summaries and contingency analysis. The escalated summaries and contingency analysis reflect the attached schedule. All activities are escalated to the appropriate schedule mid-point.

4. Per the draft EDF, ten storage modules, containing 630 storage tubes each, will accommodate the estimated 18,000 canisters produced at the Cementious Waste Facility.

5. Most quantities used for this estimate were obtained by preliminary take offs from the draft sketches.

6. The storage tube costs, gantry crane cost and associated rail system costs were used from the Fluor estimate of a facility which is approximately eighty percent complete.

7. All assumptions and information included in the draft "New Interim Storage Facility for Interim Storage of HLW" apply to the estimate.

8. Where quantities could not be determined from the draft sketches, factors or assumed quantities were used by the estimator.

9. Since the storage tubes and conveying equipment represent such a large percentage of this facility's cost, lower design, inspection, Project Management and turnover costs are used in the estimate.

10. Sufficient construction craft will be available to support this project.

11. Markups on direct construction costs to get the construction costs listed on the summary sheet are as follows:

Labor indirects, overheads and profit(OH\&P) $\quad 50 \%$ of labor

Material \& equipment OH\&P 5\% of material and equipment

- Subcontract OH\&O $10 \%$ of subcontract cost

Construction equipment allowance $10 \%$ of labor

NQA-1 quality requirements $30 \%$ of material

DOE/RW/0333P quality requirements $\quad 10 \%$ of material

Subcontractor procurement $1 \%$ of material and equipment

Bond $2 \%$ of everything

12. Only the railroad track within the storage facility is included in this estimate. The remainder of the branch line is included in the infrastructure estimate.

13. All utilities are assumed to be available at the facility wall. 


\section{COST ESTIMATE SUPPORT DATA RECAPITULATION}

Project Title: HLW ISF - DCWO

Date: January 26, 1998

Approved By:
Estimator: R. D. Adams

File: 2421-DCWO

IV. CONTINGENCY GUIDELINE IMPLEMENTATION: The percentage used for contingency as determined by the contingency allowance guidelines can be altered to reflect the type of construction and conditions that may impact the total estimated cost.

A large portion of the estimated costs is based on actual costs from Fluor's SNF Canister Storage Building. The majority of the remaining costs are for standard construction activities. For these reasons, the contingency is lower than would be experienced for a processing type facility at this stage of development.

\section{OTHER COMMENTS/CONCERNS SPECIFIC TO THE ESTIMATE:}

1. All FY'98 and later projects are to be assessed a Procurement Fee of 1\%, a G\&A Fee of 23\%, with a $\$ 500,000$ ceiling per year, and a Performance Incentive Fee (PIF) of 5.5\%. See the attached G\&A/PIF calculation sheet for the method used to calculate these fees.

2. A procurement fee of one percent of construction was used to cover the operating contractor support to DOE-ID for their contract administration. It is felt that one percent is sufficient for jobs of this size. 
Lockheed Martin Idaho Technologies Co. PROIECT NAME DCWO FACILTY - INTERIM STORAGE LOCATON 1: AEQUESTOR: FACILITY - ESCALATED INEELICPP

J. K. RAWLINS

\section{COST ESTIMATE SUMMARY}

TYPE OF ESTIMATE: PLANNING PROJECT NO: 2421-DCWO PREPARED BY: R. D. ADAMIS REPORT NAME: Cost Estimate Summary

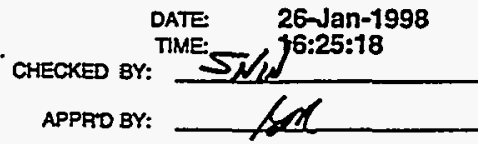

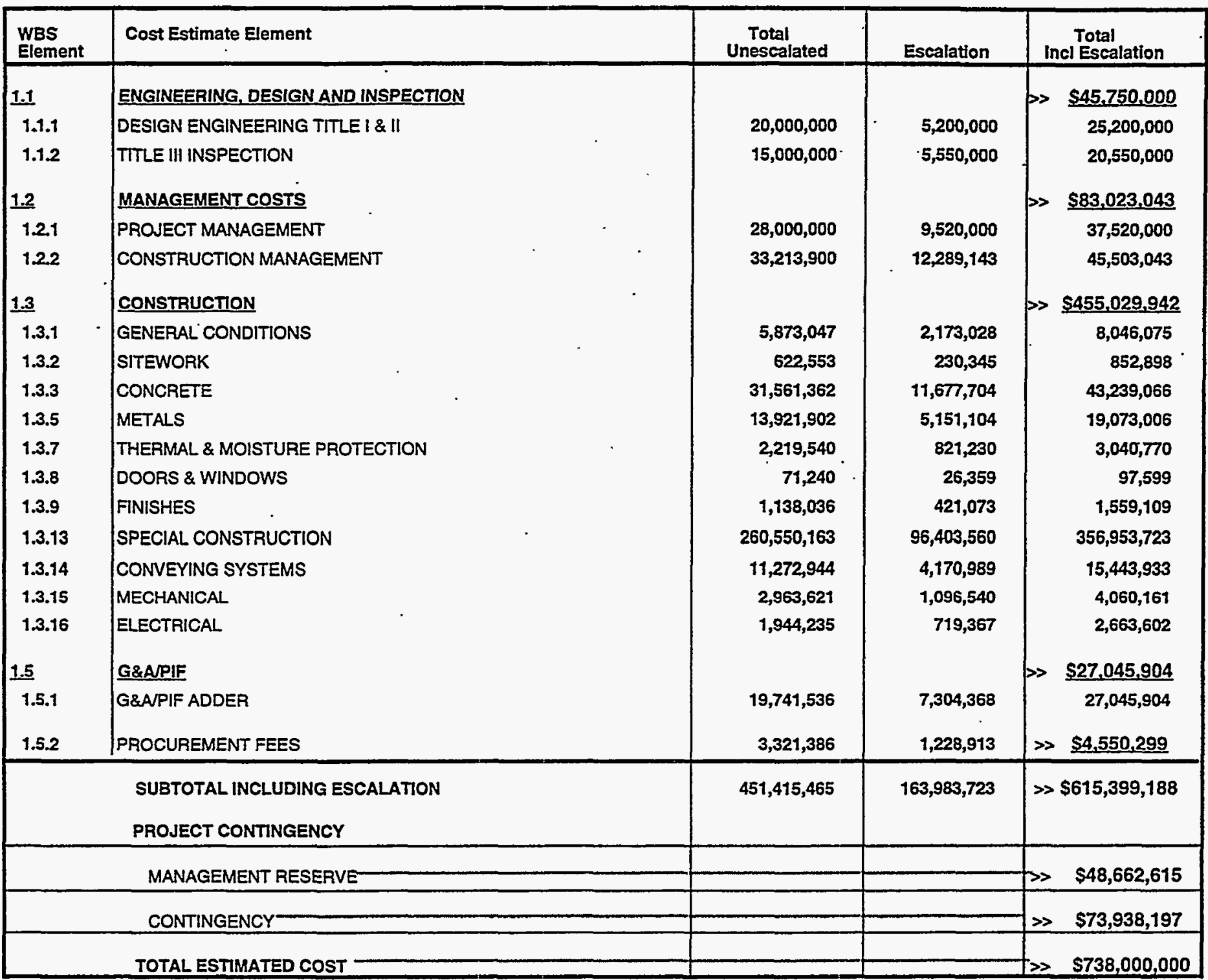

\section{PROJECT COST PARAMETERS}

EDI AS A $\%$ OF CONST. + GFE $=10.00 \%$ 
Lockheed Martin Idaho Technologies Co. PROSECT NAME: DCWO FACILITY - INTERIM STORAGE FACILITY - UNESCALATED

INEELICPP

LOCATON 1: REQUESTOR:
COST ESTIMATE SUMMARY

TYPEOF ESTIMATE: PLANNING PROSECT NO: 2421-DCWO REPORT NAME: Cost Estimate Summary
CHECKED

APPRD BY:

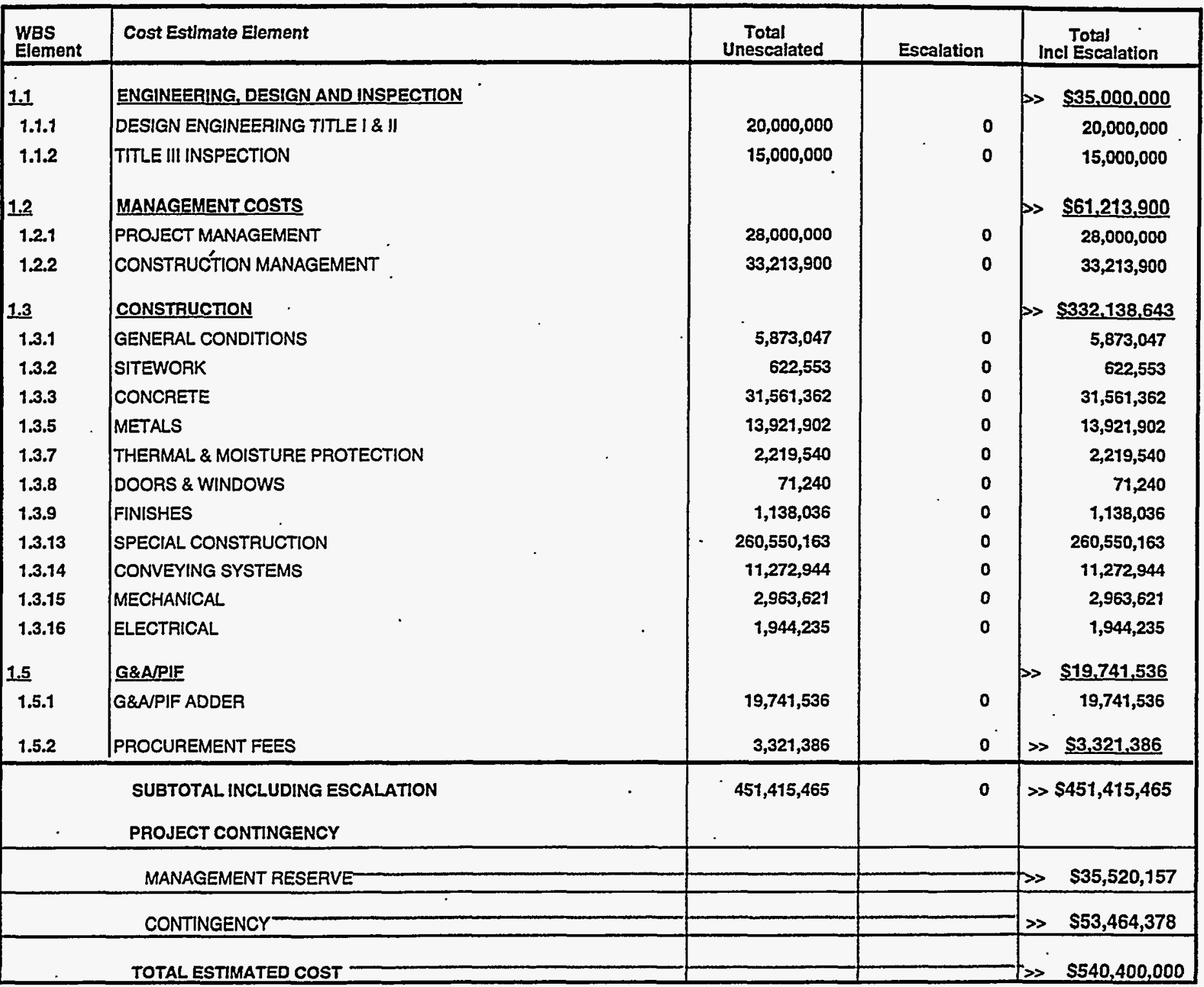

\section{PROJECT COST PARAMETERS}

EDI AS A \% OF CONST. + GFE $=11.00 \%$ CONTINGENCY $=19.71 \%$ 
Lockheed Martin Idaho Tochnologies Co. Rev. 6108 PROJECT NAME: DCWO FACILITY - INTERIM STORAGE FACILITY

LOCATION 1: INEELACPP REQUESTOR: J. K. RAWLINS
DETAILED COST ESTIMATE SHEET

TYPE OF ESTIMATE: PLANNING

PROJECT NO: 2421-DCWO

PREPARED BY: R. D. ADAMS
PAGE " 1

DATE 26-Jan-1998

TIME: 16:24:00

REPORT NAME: Detall Cost Estimate Sheot

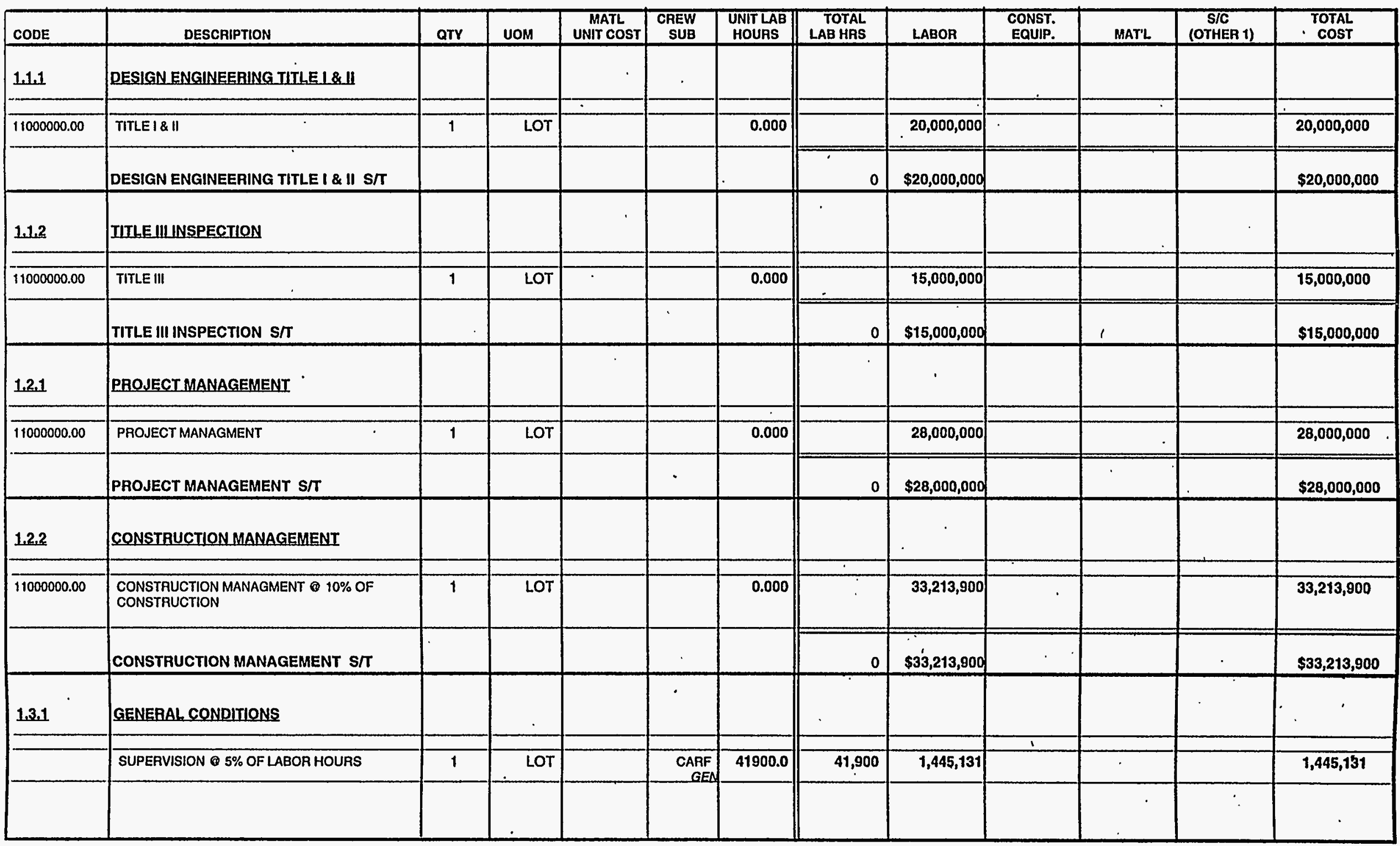


Lockheed Martin Idaho Technologles Co.

Rov. 6/96

PROJECT NAME: DCWO FACILITY - INTERIM STORAGE

FACILITY

LOCATION 1: INEELACPP

REQUESTOR: - J. K. RAWLINS

\section{DETAILED COST ESTIMATE SHEET}

TYPE OF ESTIMATE: PLANNING

PROJECT NO: 2421-DCWO

PREPARED BY: R. D. ADAMS
PAGE: 2

DATE 26-Jan-1998

TIME: 16:24:00

REPORT NAME: Detall Cost Estlmate Sheot

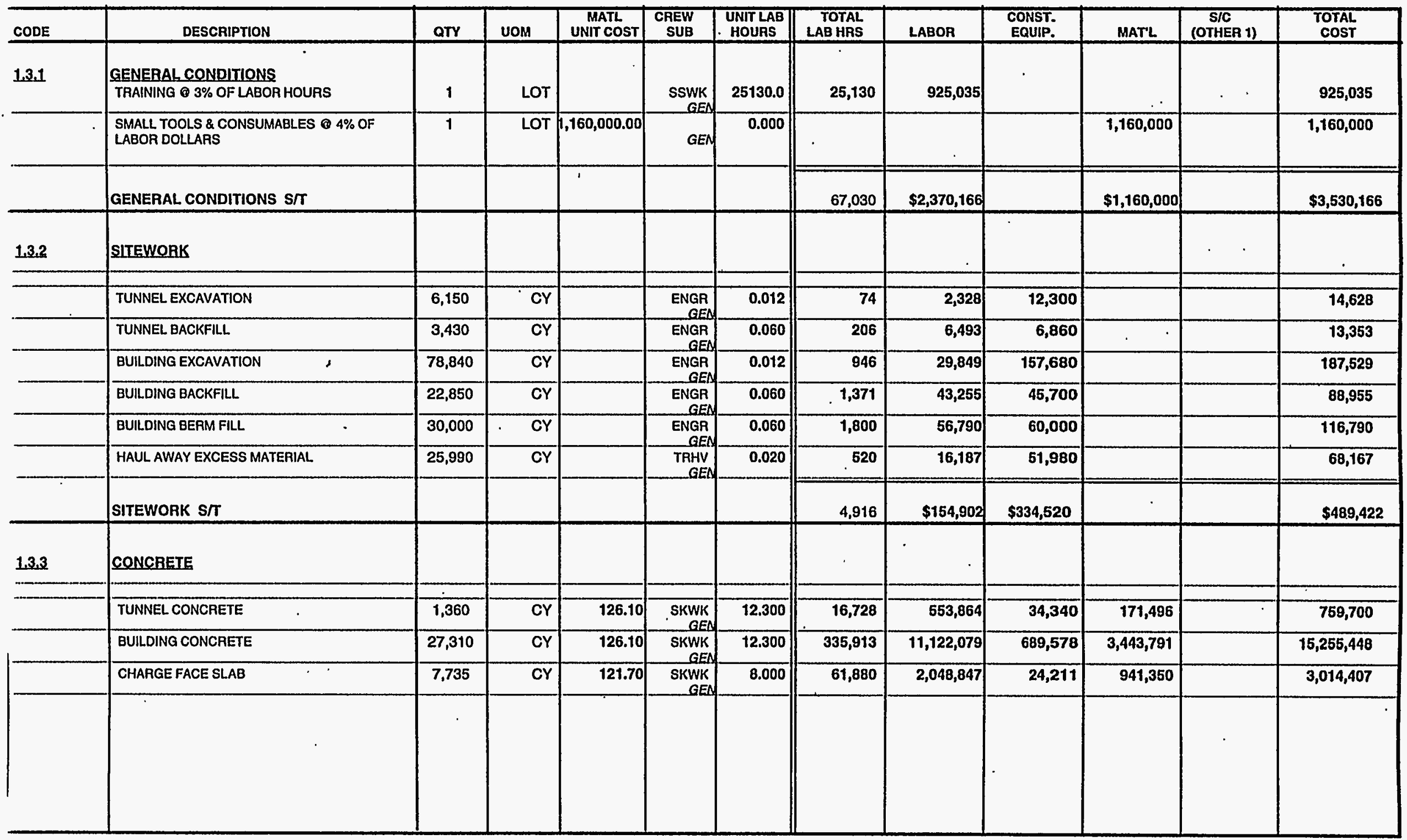


Lockheed Martin Idaho Technologles Co.$$
\text { Rov. 6/96 }
$$

PROJECT NAME: DCWO FACILITY - INTERIM STORAGE

FACILITY

LOCATION 1: INEELICPP

REQUESTOR: J. K. RAWLINS
DETAILED COST ESTIMATE SHEET

TYPE OF ESTIMATE: PLANNING

PROJECT NO:: 2421-DCWO

PREPARED BY: R. D. ADAMS
PAGE" 3

DATE 26-Jan-1998

TIME: 16:24:00

report name: Detall Cost Estimate Sheot

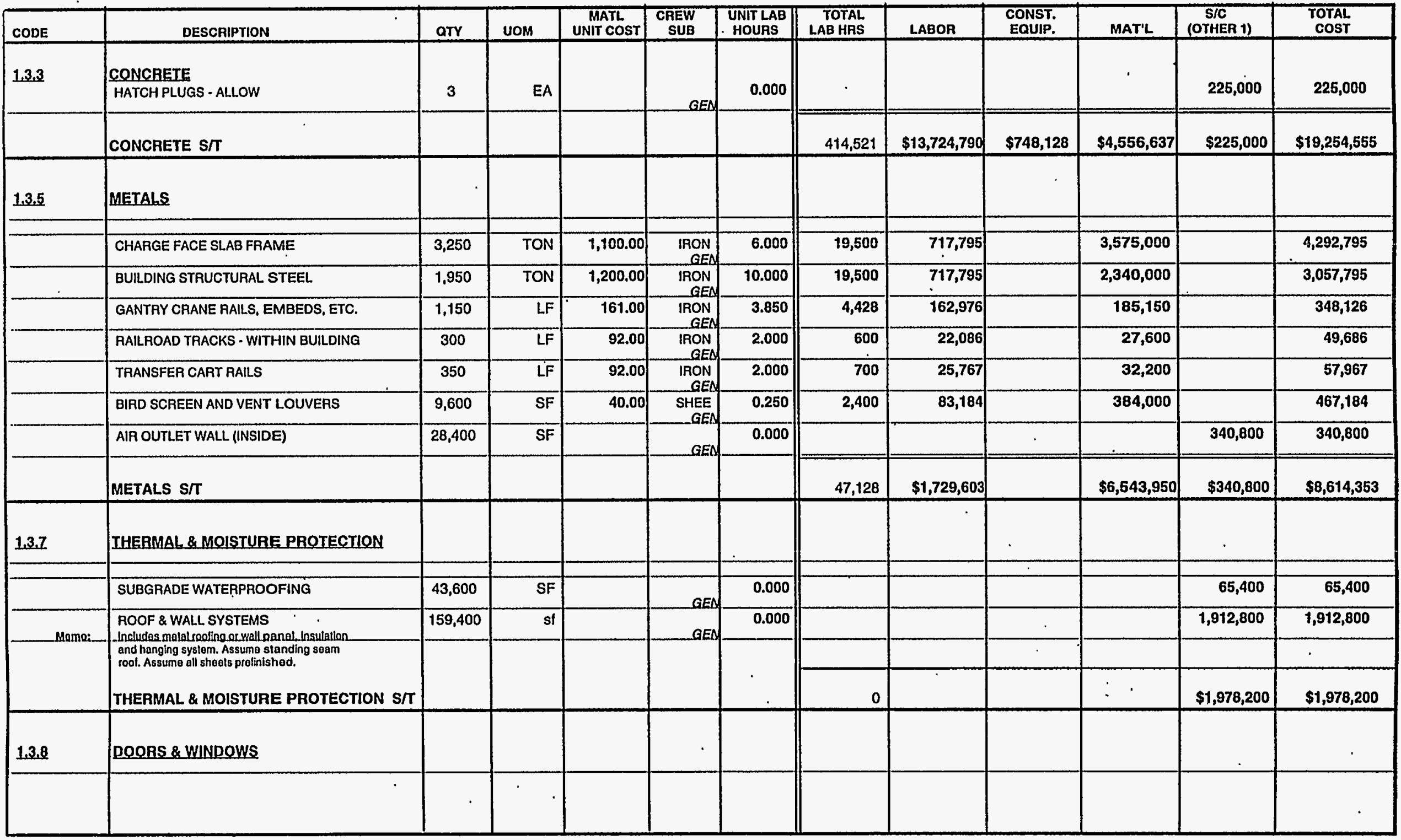


Lockheed Martin Idaho Technologles Co. Rev. 6/96

PRONECT NAME: DCWO FACILITY - INTERIM STORAGE FACILITY

LOCATION 1: INEELACPP

REQUESTOR: J.K. RAWLINS

\section{DETAILED COST ESTIMATE SHEET}

TYPE OF ESTIMATE: PLANNING

PROJECT NO:: 2421-DCWO

PREPARED BY: R. D. ADAMS
PAGE 4

DATE 26-Jan-1998

TIME: 16:24:00

REPort nAME: Detall Cost Estimate Sheet

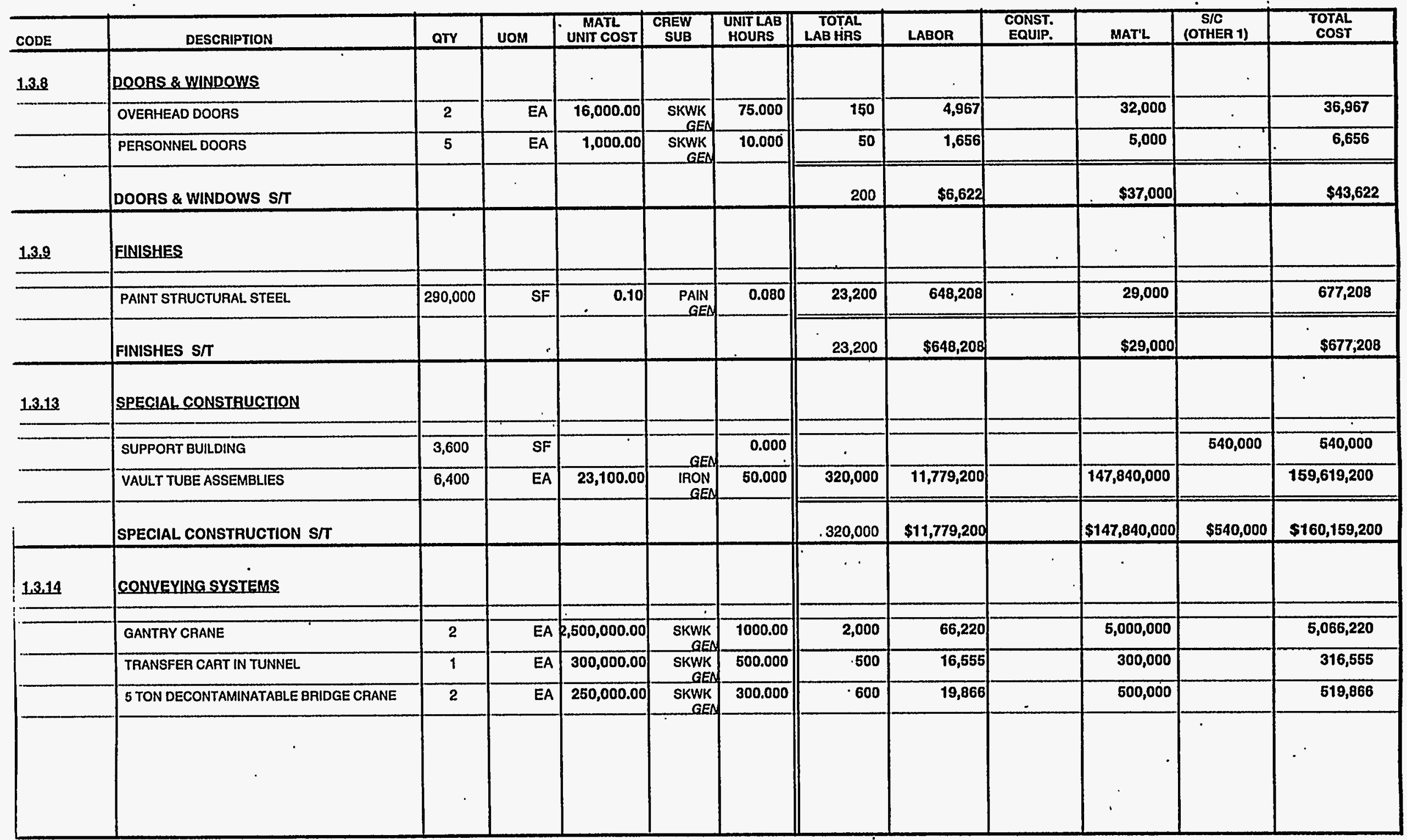


Lockheed Martin Idaho Technologles. Co. Rov. 6/96

PRONECT NAME: DCWO FACILITY - INTERIM STORAGE FACILITY

LOCATION 1: INEELICPP

REQUESTOR: J. K. RAWLINS

\section{DETAILED COST ESTIMATE SHEET}

TYPE Of estimate: PLANNING

PROJECT NO:: 2421-DCWO

PAEPARED BY: R. D. ADAMS
PAGE 5

DATE 26-Jan-1998

TIME: 16:24:00

REPORT NAME: Detall Cost Estlmate Shoet

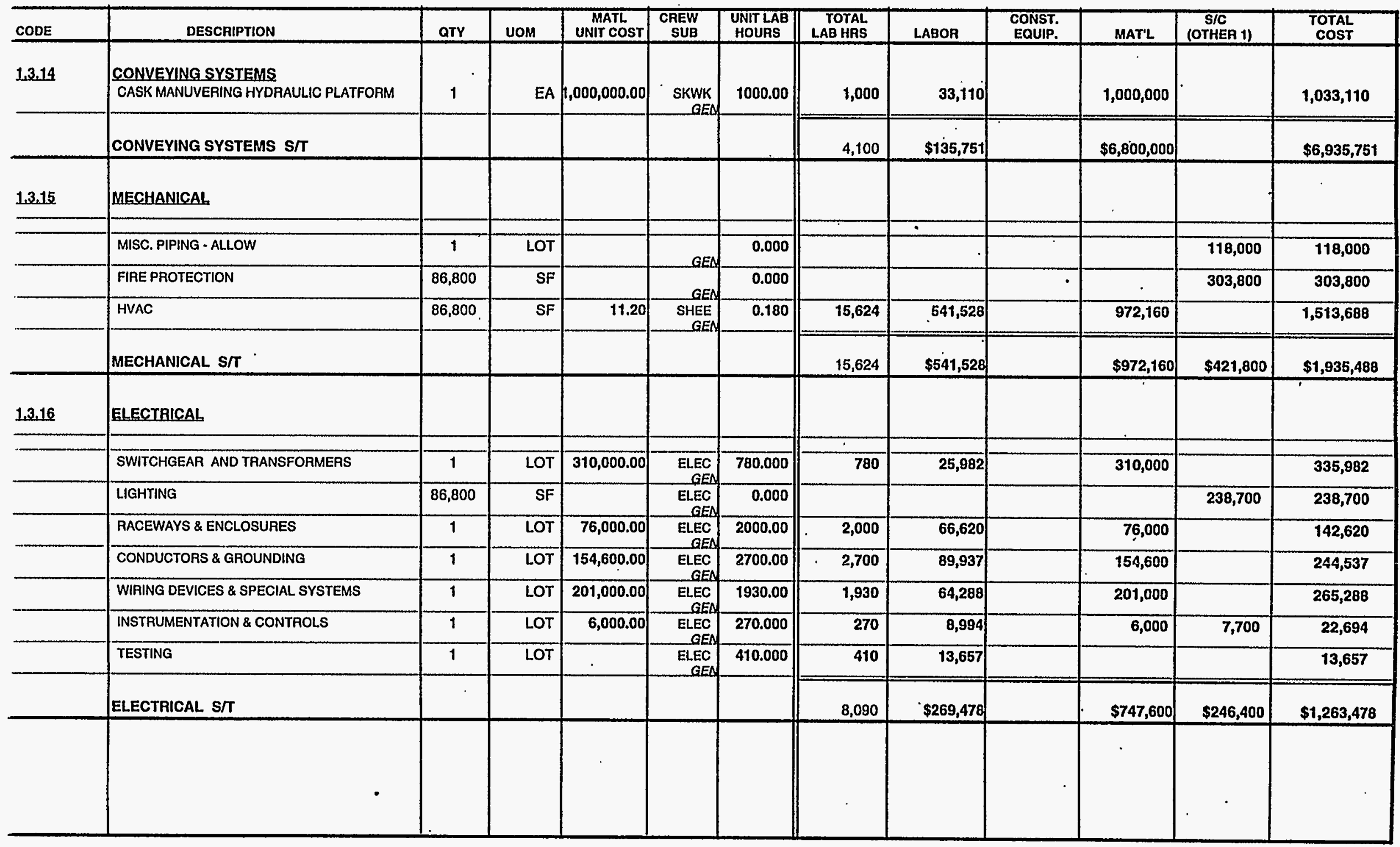




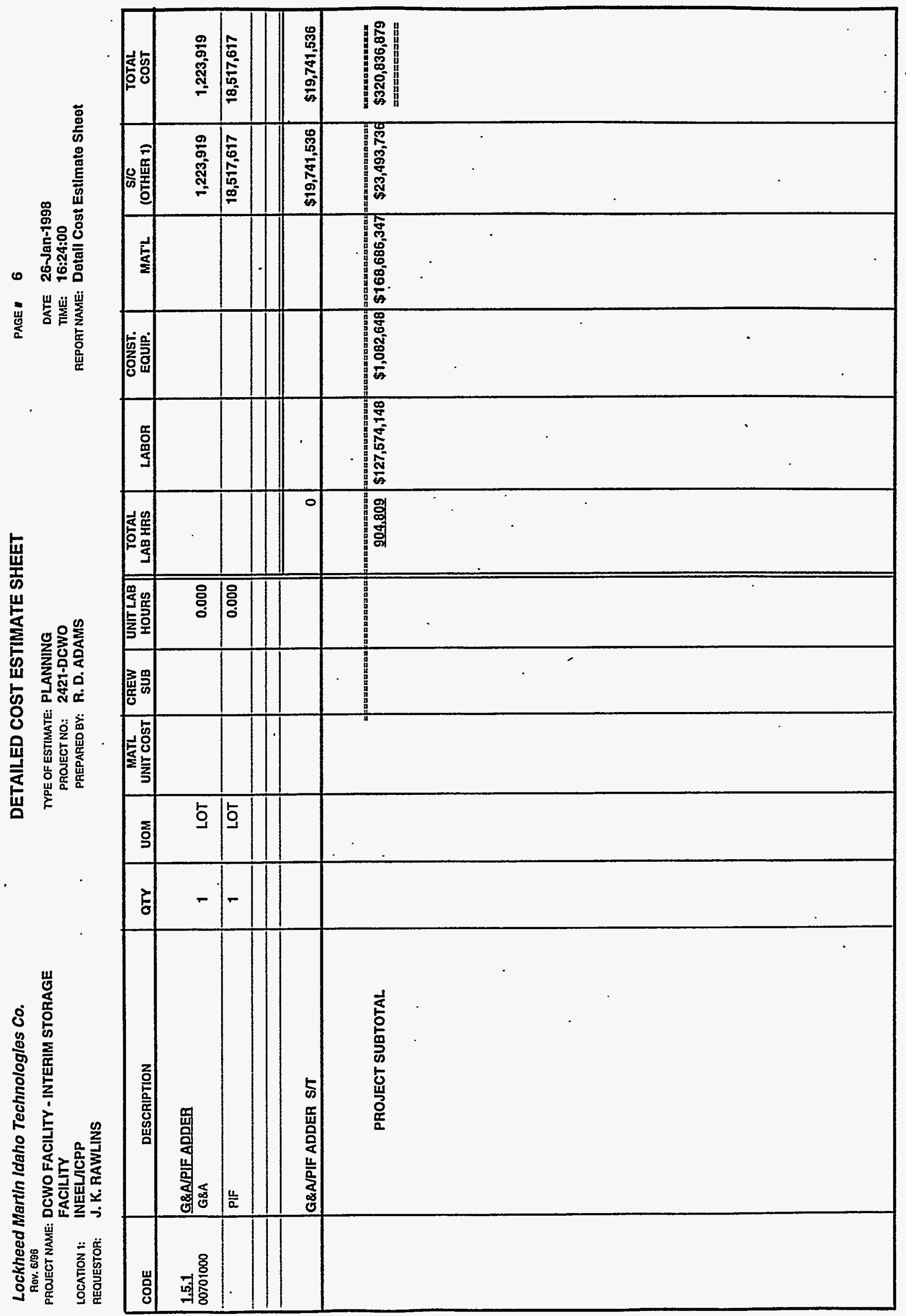


Lockheed Martin Idaho Technologies Co. PROSECT NAME: DCWO FACILITY - INTERIM STORAGE FACILITY - UNESCALATED LOCATION 1: INEELICPP REQUESTOR: J.K. RAWLINS
CONTINGENCY ANALYSIS

TYPE OF ESTIMATE: PLANNING
PROAECT NO: $\begin{array}{ll}\text { PRONECT NO: } & \text { 2421-DCWO } \\ \text { PREPARED BY: } & \text { R. D. ADAMS }\end{array}$
DATE: $26-$ Jan-1998

TIME: 16:27:32

REPORT NAME: Contingency Analysis

\begin{tabular}{|c|c|c|c|c|c|c|c|c|c|c|c|}
\hline & PROB. & BLE \% VARIA & ON & & & & & & $\begin{array}{l}\text { PROJ } \\
\text { CONTII }\end{array}$ & ET & SUMMARY \\
\hline \begin{tabular}{|l|} 
WBS \\
Element
\end{tabular} & Cost Estimate Ejement & Total Cost w/o & $\begin{array}{l}\text { \% Total } \\
\text { Cost }\end{array}$ & & $\begin{array}{l}\% \text { Var. } \\
\text { m Est. }\end{array}$ & Wt \% & Prob. & Contingency & $\%$ & Cost & Total Cost \\
\hline & & Contingency & & - & + & - & + & & & & by Element \\
\hline 1.1.1 & DESIGN ENGINEERING TILE I \& $\|$ & $20,000,000$ & 4.43 & 20 & 35 & 0.89 & $i .55$ & $1.307 \%$ & $6.53 \%$ & $5,897,408$ & $25,897,408$ \\
\hline 0.1 .2 & TITLE III INSPECTION & $15,000,000$ & 3.32 & 20 & 30 & 0.66 & 1.00 & $0.831 \%$ & $4.21 \%$ & $3,748,353$ & $18,748,353$ \\
\hline 1.2 .1 & PROJECT MANAGEMENT & $28,000,000$ & 6.20 & 20 & 35 & 1.24 & 2.17 & $1.830 \%$ & $9.28 \%$ & $8,256,371$ & $36,256,371$ \\
\hline 122 & CONSTRUCTION MANAGEMENT & $33,213,900$ & 7.36 & 20 & 30 & 1.47 & 221 & $1.839 \%$ & $9.33 \%$ & $8,299,827$ & $41,513,727$ \\
\hline 1.3 .1 & GENERAL CONDITIONS & $5,873,047$ & 1.30 & 20 & 35 & 0.26 & 0.46 & $0.384 \%$ & $1.95 \%$ & $1,731,788$ & $7,604,835$ \\
\hline 1.3 .2 & SITEWOAK & 622,553 & 0.14 & 20 & 30 & 0.03 & 0.04 & $0.034 \%$ & $0.17 \%$ & 155,570 & 778,123 \\
\hline 1.3 .3 & CONCRETE & $31,561,362$ & 6.99 & 20 & 30 & 1.40 & 210 & $1.748 \%$ & $8.86 \%$ & $7,886,874$ & $39,448,236$ \\
\hline 1.3 .5 & METALS & $13,921,902$ & 3.08 & 20 & 25 & 0.62 & 0.77 & $0.632 \%$ & $3.21 \%$ & $2,852,736$ & $16,774,638$ \\
\hline 1.3.7 & THERMAL \& MOISTURE PROTECTION & $2,219,540$ & 0.49 & 20 & 30 & 0.10 & 0.15 & $0.123 \%$ & $0.62 \%$ & 554,641 & $2,774,181$ \\
\hline 1.3 .8 & DOORS \& WINDOWS & 71,240 & 0.02 & 20 & 20 & 0.00 & 0.00 & $0.003 \%$ & $0.01 \%$ & 11,393 & 82,633 \\
\hline 1.3 .9 & FINISHES & $1,138,036$ & 0.25 & 20 & 35 & 0.05 & 0.09 & $0.074 \%$ & $0.38 \%$ & 335,573 & $1,473,609$ \\
\hline 1.3 .13 & SPECIAL CONSTRUCTION & $260,550,163$ & 57.72 & 20 & 20 & 11.54 & 11.54 & $9.235 \%$ & $46.83 \%$ & $41,669,712$ & $302,219,875$ \\
\hline 1.3.14 & CONVEYING SYSTEMS & $11,272,944$ & 2.50 & 20 & 25 & 0.50 & 0.62 & $0.512 \%$ & $2.60 \%$ & $2,309,938$ & $13,582,882$ \\
\hline 1.3 .15 & MECHANICAL & $2,963,621$ & 0.66 & 20 & 30 & 0.13 & 0.20 & $0.164 \%$ & $0.83 \%$ & 740,580 & $3,704,201$ \\
\hline 1.3 .16 & ELECTRICAL & $1,944,235$ & 0.43 & 20 & 25 & 0.09 & 0.11 & $0.088 \%$ & $0.45 \%$ & 398,393 & $2,342,628$ \\
\hline 9.5 .1 & G\&APIF ADDER & $19,741,536$ & 4.37 & 5 & 20 & 0.22 & 0.87 & $0.765 \%$ & $3.28 \%$ & $3,453,251$ & $23,194,787$ \\
\hline 1.5 .2 & PROCUREMENT FEES & $3,321,386$ & 0.74 & 20 & 25 & 0.15 & 0.18 & $0.151 \%$ & $0.76 \%$ & 680,585 & $4,001,971$ \\
\hline & ESCALATION & 0 & 0.00 & 20 & 25 & 0.00 & 0.00 & $0.000 \%$ & $0.00 \%$ & 1.542 & 1,542 \\
\hline & SUBTOTAL & $451,415,465$ & 100.00 & & & & & $19.721 \%$ & & & \\
\hline & CALCULATED CONTINGENCY & $89,022,102$ & & & & & & & & & \\
\hline & RESULTANT TEC & $540,437,567$ & & & & & & & & & \\
\hline & ROUNDED TEC & $540,400,000$ & & & & & & & & & \\
\hline & PROJECT CONTINGENCY & $88,984,535$ & & & & & & $19.71 \%$ & & & \\
\hline & MANAGEMENT RESERVE & $35,520,157$ & & & & & & & & & \\
\hline & CONTINGENCY & $53,464,378$ & & & & & & & & & \\
\hline & TOTAL ESTIMATED COST & $540,400,000$ & & & & & & & & $88,984,535$ & $540,400,000$ \\
\hline
\end{tabular}

CONFIDENCE LEVEL AND ASSUMED RISKS:

The Lockheed Idaho Technologies Co. Cost Estimate Contingency Analysis

Model is based on the applied contingency and the assumptions upon which

the estimate was predicated. The model is applied with a suggested risk level

of $18 \%$ and a level of confidence of $90 \%$ the estimate will fall within the bid range.

The Contingency Analysis is based on a weighted average to provide a

$90 \%$ probability of underrun and a $10 \%$ probability of overnun.
CONTINGENCY ANALYSIS GUIDE BY TYPE OF ESTIMATE

Guidelines established by DOE/FM 50, Cost Estimating Guide, Vol. 6 ,

Cost Guide, and as presented in the INEL Cost Estimating Guide. PLANNING
Experimental/Special Conditions............. $20 \%$ to $50 \%$ Conceptual

$15 \%-25 \%$

Experimental/Special Conditions.............Up to $40 \%$

TITLE I 10\% - $20 \%$

TITLE II $\quad 5 \%-15 \%$

TITLE II/AFC $\quad$ Market Conditions 
Lockheed Martin Idaho Technologies Co. PROIECT NAME: DCWO FACILITY - INTERIM STORAGE FACILITY - ESCALATED

LOCATION :: INEELICPP REQUESTOR: J. K. RAWLINS

\section{CONTINGENCY ANALYSIS}

$\begin{array}{cl}\text { TYPE OF ESTIMATE: } & \text { PLANNING } \\ \text { PROJECT NO: } & \text { 2421-DCWO } \\ \text { PREPARED BY: } & \text { R. D. ADAMS }\end{array}$

DATE: 26-Jan-1998

TME: 16:25:25

REPORT NAME: Contingency Analysis

\begin{tabular}{|c|c|c|c|c|c|c|c|c|c|c|c|}
\hline \multicolumn{9}{|c|}{ PROBABLE \% VARIATION } & \multicolumn{2}{|c|}{$\begin{array}{l}\text { PROJECT } \\
\text { CONTINGENCY }\end{array}$} & \multirow{3}{*}{$\begin{array}{l}\text { SUMMARY } \\
\begin{array}{l}\text { Total Cost } \\
\text { by Element }\end{array}\end{array}$} \\
\hline \multirow[t]{2}{*}{$\begin{array}{l}\text { WBS } \\
\text { Element }\end{array}$} & \multirow[t]{2}{*}{ Cost Estimate Element } & \multirow{2}{*}{$\begin{array}{c}\text { Total Cost w/o } \\
\text { Contingency }\end{array}$} & \multirow[t]{2}{*}{$\begin{array}{l}\text { \% Total } \\
\text { Cost }\end{array}$} & \multicolumn{2}{|c|}{$\begin{array}{c}\text { Prob. \% Var. } \\
\text { Frpm Est. }\end{array}$} & \multicolumn{2}{|c|}{ Wt. \% of Prob. } & \multirow[t]{2}{*}{ Contingeney } & \multirow[t]{2}{*}{$\%$} & \multirow[t]{2}{*}{ Cost } & \\
\hline & & & & - & + & - & + & & & & \\
\hline 1.1 .1 & DESIGN ENGINEERING TTLE I \& II & $20,000,000$ & 3.25 & 20 & 35 & 0.65 & 1.14 & $0.959 \%$ & $4.81 \%$ & $5,898,271$ & $25,898,271$ \\
\hline 1.1 .2 & TITLE III INSPECTION & $15,000,000$ & 2.44 & 20 & 30 & 0.49 & 0.73 & $0.609 \%$ & $3.06 \%$ & $3,748,901$ & $18,748,901$ \\
\hline 1.2 .1 & PROJECT MANAGEMENT & $28,000,000$ & 4.55 & 20 & 35 & 0.91 & 1.59 & $1.342 \%$ & $6.74 \%$ & $8,257,579$ & $36,257,579$ \\
\hline 1.22 & CONSTRUCTION MANAGEMENT & $33,213,900$ & 5.40 & 20 & 30 & 1.08 & 1.62 & $1.349 \%$ & $6.77 \%$ & $8,301,042$ & $41,514,942$ \\
\hline 1.3 .1 & GENERAL CONDITIONS & $5,873,047$ & 0.95 & 20 & 35 & 0.19 & 0.33 & $0.282 \%$ & $1.41 \%$ & $1,732,041$ & $7,605,088$ \\
\hline 1.3 .2 & SITEWORK & 622,553 & 0.10 & 20 & 30 & 0.02 & 0.03 & $0.025 \%$ & $0.13 \%$ & 155,593 & 778,146 \\
\hline 1.3 .3 & CONCREIE & $31,561,362$ & 5.13 & 20 & 30 & 1.03 & 1.54 & $1.282 \%$ & $6.43 \%$ & $7,888,028$ & $39,449,390$ \\
\hline 1.3 .5 & METALS & $13,921,902$ & 2.26 & 20 & 25 & 0.45 & 0.57 & $0.464 \%$ & $2.33 \%$ & $2,853,154$ & $16,775,056$ \\
\hline 1.3 .7 & THERMAL \& MOISTURE PROTECTION & $2,219,540$ & 0.36 & 20 & 30 & 0.07 & 0.11 & $0.090 \%$ & $0.45 \%$ & 554,722 & $2,774,262$ \\
\hline 1.3 .8 & DOORS \& WINDOWS & 71,240 & 0.01 & 20 & 20 & 0.00 & 0.00 & $0.002 \%$ & $0.01 \%$ & 11,395 & 82,635 \\
\hline 1.3 .9 & FINISHES & $1,138,036$ & 0.18 & 20 & 35 & 0.04 & 0.06 & $0.055 \%$ & $0.27 \%$ & 335,622 & $1,473,658$ \\
\hline 1.3 .13 & SPECIAL CONSTRUCTION & $260,550,163$ & 42.34 & 20 & 20 & 8.47 & 8.47 & $6.774 \%$ & $33.99 \%$ & $41,675,810$ & $302,225,973$ \\
\hline 1.3 .14 & CONVEYING SYSTEMS & $11,272,944$ & 1.83 & 20 & 25 & 0.37 & 0.46 & $0.376 \%$ & $1.88 \%$ & $2,310,276$ & $13,583,220$ \\
\hline 1.3 .15 & MECHANICAL & $2,963,621$ & 0.48 & 20 & 30 & 0.10 & 0.14 . & $0.120 \%$ & $0.60 \%$ & 740,688 & $3,704,309$ \\
\hline 1.3 .16 & ELECTRICAL & $1,944,235$ & 0.32 & 20 & 25 & 0.06 & 0.08 & $0.065 \%$ & $0.32 \%$ & 398,451 & $2,342,686$ \\
\hline 1.5.1 & G\&APIF ADDER & $19,741,536$ & 3.21 & 5 & 20 & 0.16 & 0.64 & $0.561 \%$ & $2.82 \%$ & $3,453,756$ & $23,195,292$ \\
\hline \multirow[t]{2}{*}{1.5 .2} & PROCUREMENT FEES & $3,321,386$ & 0.54 & 20 & 25 & 0.11 & 0.13 & $0.111 \%$ & $0.56 \%$ & 680,685 & $4,002,071$ \\
\hline & ESCALATION & $163,983,723$ & 26.65 & 20 & 25 & 5.33 & 6.66 & $5.463 \%$ & $27.41 \%$ & $33,504,798$ & $197,588,521$ \\
\hline & SUBTOTAL & $615,399,188$ & 900.00 & & & & & $19.928 \%$ & & & \\
\hline & CALCULATED CONTINGENCY & $122,638,766$ & & & & & & & & & \\
\hline & RESULTANT TEC & $738,037,954$ & & & & & & & & & \\
\hline & ROUNDED TEC & $738,000,000$ & & & & & & & & & \\
\hline & PROJECT CONTINGENCY & $122,600,812$ & & & & & & $19.92 \%$ & & & \\
\hline & MANAGEMENT RESERVE & $48,662,615$ & & & & & & & & & \\
\hline & CONTINGENCY & $73,938,197$ & & & & & & & & & \\
\hline & TOTAL ESTIMATED COST & $738,000,000$ & & & & & & & & $122,600,812$ & $738,000,000$ \\
\hline
\end{tabular}

CONFIDENCE LEVEL AND ASSUMED RISKS:

The Lockheed Idaho Technologies Co. Cost Estimate Contingency Analysis Model is based on the applied contingency and the assumptions upon which the estimate was predicated. The model is applied with a suggested risk level of $18 \%$ and a level of confidence of $90 \%$ the estimate will fall within the bid range. The Contingency Analysis is based on a weighted average to provide a

$90 \%$ probability of underrun and a $10 \%$ probability of ovemun.
CONTINGENCY ANALYSIS GUIDE BY TYPE OF ESTIMATE

Guidelines established by DOE/FM 50, Cost Estimating Guide, Vol. 6. Cost Guide, and as presented in the INEL Cost Estimating Guide.

PLANNING Experimental/Special Conditions............. Up to $50 \%$ Conceptual $15 \%-25 \%$

Experimental/Special Conditions.............Up to $40 \%$ TITLE I $10 \%-20 \%$

TITLE II

TITLE II/AFC Market Conditions 


\section{G\&APIF ADDER CALCULATION SHEET}

PROCUREMENT FEE:

\begin{tabular}{|c|c|c|c|c|}
\hline $\begin{array}{l}\text { CONSTRUCTION = } \\
\text { GFE = }\end{array}$ & Subtotal & $\begin{array}{r}\$ 332,138,643 \\
\$ 0 \\
\$ 332,138,643\end{array}$ & & \\
\hline FEE @ $1 \%=$ & & $\$ 332,138,643$ & $\bullet 0.01=$ & $\$ 3,321,386.43$ \\
\hline
\end{tabular}

G\&A @ 23\% (with a ceiling of $\$ 500,000$ imposed per year

CONSTRUCTION OR

CEILING $\bullet 4$ YEARS $=$

GFE =

$\$ 2,000,000$

PROCUREMENT FEE =

Subtotal $\$ \frac{\$ 5,321,386}{}$

FEE @ $23 \%=$

$\$ 5,321,386 \cdot 0.23=$

$\$ 1,223,919$

PIF @ 5.5\%

\begin{tabular}{|c|c|c|}
\hline $\begin{array}{l}\text { CONSTRUCTION = } \\
\text { GFE = } \\
\text { PROCUREMENT FEE = } \\
\text { G\&A }=\quad \text { Subtotal }\end{array}$ & $\begin{array}{r}\$ 332,138,643 \\
\$ 0 \\
\$ 3,321,386 \\
\$ 1,223,919 \\
\$ 336,683,948\end{array}$ & \\
\hline FEE @ $5.5 \%=$ & $\$ 336,683,948 \cdot 0.055=$ & $\$ 18,517,617$ \\
\hline
\end{tabular}

TOTAL PROCUREMENT FEE:

$\$ 3,321,386$

TOTAL G\&A FEE:

$\$ 1,223,919$

TOTAL PIF:

$\$ 18,517,617$ 
Lockheed Martin Idaho Technologies Co.

Rov. $6 / 98$

PROJECT NAME: DCWO FACILITY - INTERIM STORAGE

FACILITY - OPC

LOCATION 1: INEELICPP

REQUESTOR: J. K. RAWLINS
DETAILED COST ESTIMATE SHEET

TYPE OF ESTIMATE: PLANNING

PRONECTNO:: 242104

PREPARED BY: R. D. ADAMS
PAGE" 1

DATE 26-Jan-1998

TIME: 16:28:27

REPORT NAME: Detall Cost Estlmate Sheot

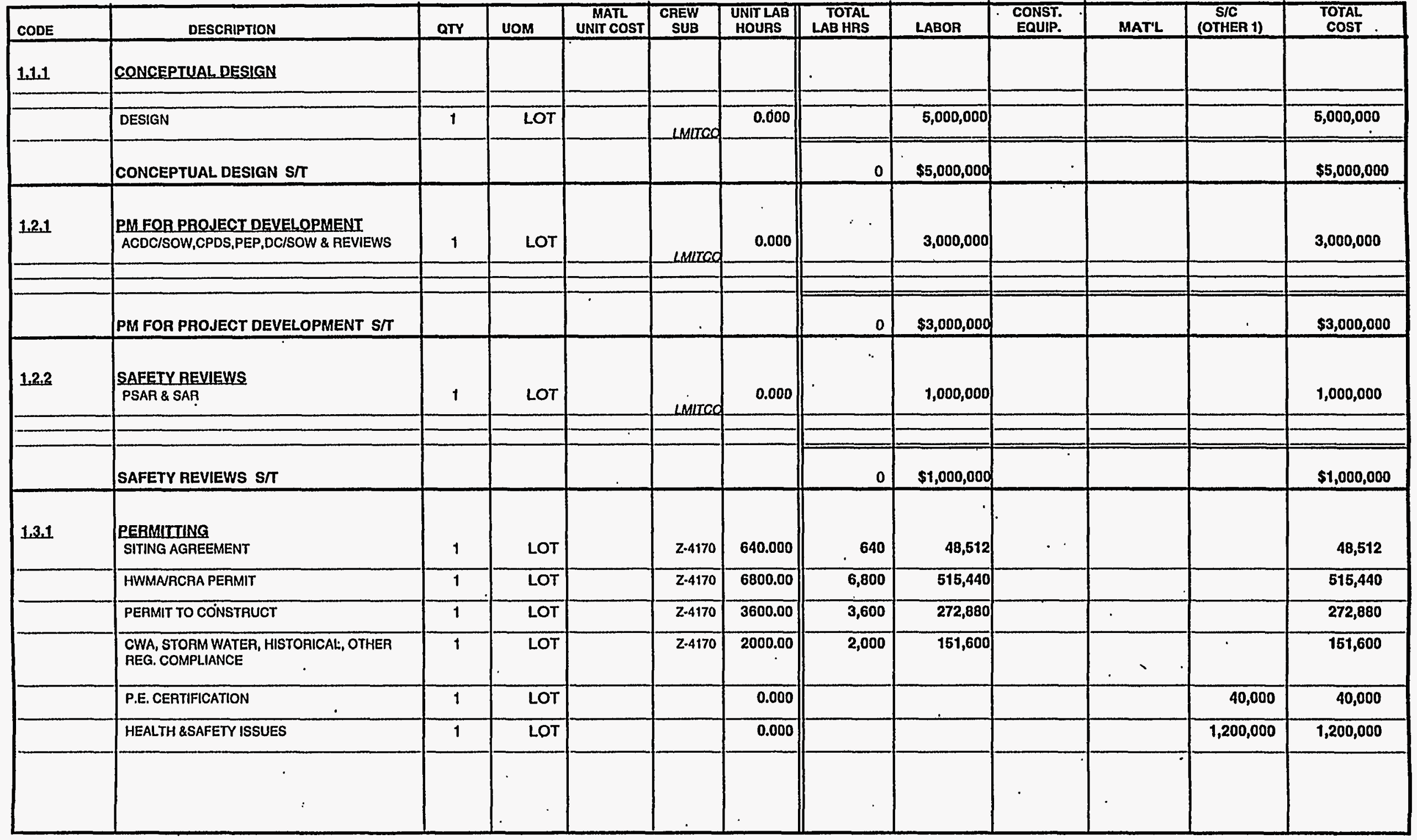




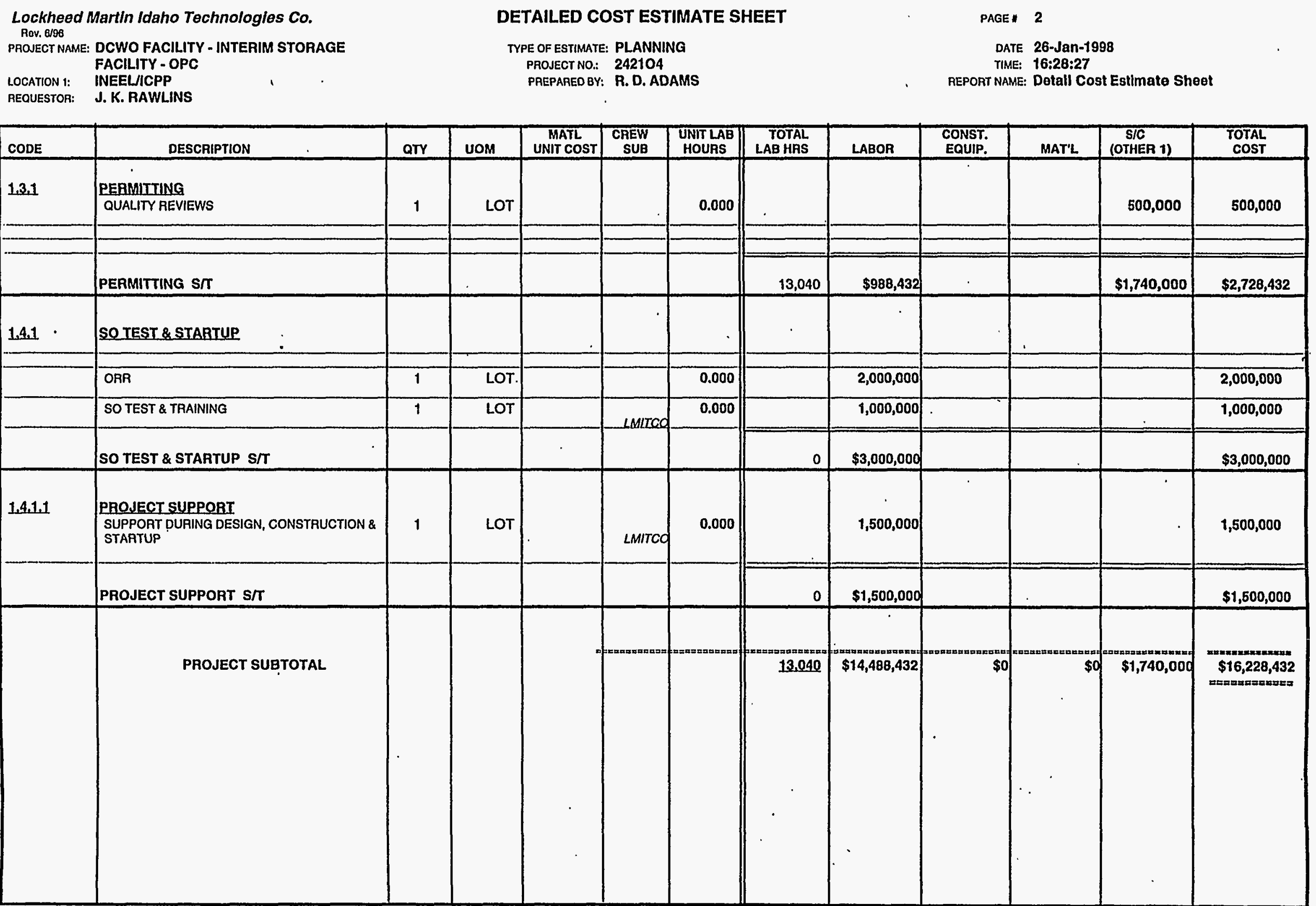


Lockheed Martin Idaho Technologies Co. PROUECT NAME: DCWO FACILITY - INTERIM STORAGE

LOCATION 1: REQUESTOR: FACILTY - OPC - UNESCALATED INEEL/CPP

J. K. RAWLINS
CONTINGENCY ANALYSIS

TYPE OF ESTIMATE: PROSECT NO: PREPARED BY:

PLANNING
242104
R. D. ADAMS

DATE: 26-Jan-1998

TIME: 16:31:09

REPORT NAME: Contingency Analysis

\begin{tabular}{|c|c|c|c|c|c|c|c|c|c|c|c|}
\hline \multicolumn{9}{|c|}{ PROBABLE \% VARIATION } & \multicolumn{2}{|c|}{$\begin{array}{l}\text { PROJECT } \\
\text { CONTINGENCY }\end{array}$} & \multirow{3}{*}{$\begin{array}{l}\text { SUMMARY } \\
\begin{array}{l}\text { Total Cost } \\
\text { by Element }\end{array}\end{array}$} \\
\hline \multirow[t]{2}{*}{$\begin{array}{l}\text { WBS } \\
\text { Element }\end{array}$} & \multirow{2}{*}{ Cost Estimate Element } & \multirow{2}{*}{$\begin{array}{l}\text { Total cost w/o } \\
\text { Contingency }\end{array}$} & \multirow[t]{2}{*}{$\begin{array}{l}\text { \% Total } \\
\text { Cost }\end{array}$} & \multicolumn{2}{|c|}{$\begin{array}{l}\text { Prob. \% Var. } \\
\text { From Est. }\end{array}$} & \multicolumn{2}{|c|}{ Wh. \% of Prob. } & \multirow[t]{2}{*}{ Contingency } & \multirow[t]{2}{*}{$\%$} & \multirow[t]{2}{*}{ Cost } & \\
\hline & & & & - & + & - & + & & & & \\
\hline 1.1 .1 & CONCEPTUAL DESIGN & $5,000,000$ & 30.81 & 20 & 30 & 6.16 & 9.24 & $7.703 \%$ & $30.81 \%$ & $1,254,455$ & $6,254,455$ \\
\hline 1.2 .1 & PM FOR PROJECT DEVELOPMENT & $3,000,000$ & 18.49 & 20 & 30 & 3.70 & 5.55 & $4.622 \%$ & $18.49 \%$ & 752,673 & $3,752,573$ \\
\hline 122 & SAFETY REVIEWS & $1,000,000$ & 6.16 & 20 & 30 & 1.23 & 1.85 & $1.541 \%$ & $6.16 \%$ & 250,891 & $1,250,891$ \\
\hline 1.3 .1 & PERMITTING & $2,728,432$ & 16.81 & 20 & 30 & 3.36 & 5.04 & $4.203 \%$ & $16.81 \%$ & 684,539 & $3,412,971$ \\
\hline 1.4 .1 & SO TEST \& STARTUP & $4,500,000$ & 27.73 & 20 & 30 & 5.55 & 8.32 & $6.932 \%$ & $27.73 \%$ & $1,129,010$ & $5,629,010$ \\
\hline 1.5 .2 & PROCUREMENT FEES & 0 & 0.00 & 5 & 20 & 0.00 & 0.00 & $0.000 \%$ & $0.00 \%$ & 0 & 0 \\
\hline & ESCALATION & 0 & 0.00 & 20 & 30 & 0.00 & 0.00 & $0.000 \%$ & $0.00 \%$ & 0 & 0 \\
\hline & SUBTOTAL & $16,228,432$ & 100.00 & & & & & $25.000 \%$ & & & \\
\hline & CALCULATED CONTINGENCY & $4,057,108$ & & & & & & & & & \\
\hline & RESULTANT TEC & $20,285,540^{\circ}$ & & & & & & & & & \\
\hline & ROUNDED TEC & $20,300,000$ & & & & & & & & & \\
\hline & PROJECT CONTINGENCY & $4,071,568$ & & & & & & $25.09 \%$ & & & \\
\hline & MANAGEMENT RESERVE & 0 & & & & & & & & & \\
\hline & CONTINGENCY & $4,071,568$ & & & & & & & & & \\
\hline & TOTAL ESTIMATED COST & $20,300,000$ & & & & & . & & & $4,071,568$ & $20,300,000$ \\
\hline
\end{tabular}

\section{CONFIDENCE LEVEL AND ASSUMED RISKS:}

The Lockheed Idaho Technologies Co. Cost Estimate Contingency Analysis Model is based on the applied contingency and the assumptions upon which the estimate was predicated. The model is applied with a suggested risk level of $18 \%$ and a level of confidence of $90 \%$ the estimate will fall within the bid range. The Contingency Analysis is based on a weighted average to provide a $90 \%$ probability of underrun and a $10 \%$ probability of overrun.
CONTINGENCY ANALYSIS GUIDE BY TYPE OF ESTIMATE Guidelines established by DOE/FM 50, Cost Estimating Guide, Vol. 6 , Cost Guide, and as presented in the INEL Cost Estimating Guide.

$$
\text { PLANNING }
$$
Conceptual inal Conditions....... Experimental/Special Conditions............. TITLE TITLE II TITLE II/AFC $10 \%-20 \%$
$5 \%-15 \%$ Market Conditions 
Lockheed Martin Idaho Technologies Co. PROMECT NAME: DCWO FACILITY - INTERIM STORAGE

LOCATION 1: INEELICPP

LOCATION

REQUESTOR:
J. K. RAWLINS
CONTINGENCY ANALYSIS

$\begin{array}{cl}\text { TYPE OF ESTIMATE: } & \text { PLANNING } \\ \text { PRONECT NO: } & \text { 2421O4 } \\ \text { PREPARED BY: } & \text { R. D. ADAMS }\end{array}$

DATE: 26-Jan-1998

TIME: 16:29:30

REPORT NAME: Contingency Analysis

\begin{tabular}{|c|c|c|c|c|c|c|c|c|c|c|c|}
\hline \multicolumn{9}{|c|}{ PROBABLE \% VARIATION } & \multicolumn{2}{|c|}{$\begin{array}{l}\text { PROJECT } \\
\text { CONTINGENCY }\end{array}$} & \multirow{3}{*}{$\begin{array}{l}\text { SUMMARY } \\
\text { Total Cost } \\
\text { by Element }\end{array}$} \\
\hline \multirow[t]{2}{*}{$\begin{array}{l}\text { WBS } \\
\text { Element }\end{array}$} & \multirow[t]{2}{*}{ Cost Estimate Element } & \multirow{2}{*}{$\begin{array}{l}\text { Total Cost w/o } \\
\text { Contingency }\end{array}$} & \multirow[t]{2}{*}{$\begin{array}{c}\text { \% Total } \\
\text { Cost }\end{array}$} & \multicolumn{2}{|c|}{$\begin{array}{c}\text { Prob. \% Var. } \\
\text { From Est. }\end{array}$} & \multicolumn{2}{|c|}{ Wt. \% of Prob. } & \multirow[t]{2}{*}{ Contingency } & \multirow[t]{2}{*}{$\%$} & \multirow[t]{2}{*}{ Cost } & \\
\hline & & & & - & + & - & + & & & & \\
\hline 1.1 .1 & CONCEPTUAL DESIGN & $5,000,000$ & 24.55 & 20 & 30 & 4.91 & 7.36 & $6.137 \%$ & $24.55 \%$ & $1,260,178$ & $6,260,178$ \\
\hline 121 & PM FOR PROJECT DEVELOPMENT & $3,000,000$ & 14.73 & 20 & 30 & 2.95 & 4.42 & $3.682 \%$ & $14.73 \%$ & 756,107 & $3,756,107$ \\
\hline 122 & SAFETY REVIEWS & $1,000,000$ & 4.91 & 20 & 30 & 0.98 & 1.47 & $1.227 \%$ & $4.91 \%$ & 252,036 & $1,252,036$ \\
\hline 1.3 .1 & PERMITTING & $2,728,432$ & 13.40 & 20 & 30 & 2.68 & 4.02 & $3.349 \%$ & $13.40 \%$ & 687,662 & $3,416,094$ \\
\hline 1.4 .1 & SO TEST \& STARTUP & $4,500,000$ & 22.09 & 20 & 30 & 4.42 & 6.63 & $5.524 \%$ & $22.09 \%$ & $1,134,160$ & $5,634,160$ \\
\hline \multirow[t]{2}{*}{1.52} & PROCUREMENT FEES & 0 & 0.00 & 5 & 20 & 0.00 & 0.00 & $0.000 \%$ & $0.00 \%$ & 0 & 0 \\
\hline & ESCALATION & $4,138,402$ & 20.32 & 20 & 30 & 4.06 & 6.10 & $5.080 \%$ & $20.32 \%$ & $1,043,023$ & $5,181,425$ \\
\hline & SUBTOTAL & $20,366,834$ & 100.00 & & & & & $25.000 \%$ & & & \\
\hline & CALCULATED CONTINGENCY & - $5,091,709$ & & & & & & & & & \\
\hline & RESULTANT TEC & $25,458,543$ & & & & & & & & & \\
\hline & ROUNDED TEC & $25,500,000$ & & & & & & & & & \\
\hline & PROJECT CONTINGENCY & $5,133,166$ & & & & & & $25.20 \%$ & & & \\
\hline & MANAGEMENT RESERVE & 0 & & & & & & & & & \\
\hline & CONTINGENCY & $5,133,166$ & & & & & & & & & \\
\hline & TOTAL ESTIMATED COST & $25,500,000$ & & & & & & & & $5,133,166$ & $25,500,000$ \\
\hline
\end{tabular}

CONFIDENCE LEVEL AND ASSUMED RISKS:

The Lockheed Idaho Technologies Co. Cost Estimate Contingency Analysis Model is based on the applied contingency and the assumptions upon which

the estimate was predicated. The model is applied with a suggested risk level of $18 \%$ and a level of confidence of $90 \%$ the estimate will fall within the bid range. The Contingency Analysis is based on a weighted average to provide a

$90 \%$ probability of underrun and a $10 \%$ probability of overrun.
CONTINGENCY ANALYYSIS GUIDE BY TYPE OF ESTIMATE

Guidelines established by DOE/FM 50, Cost Estimating Guide, Vol. 6 , Cost Guide, and as presented in the INEL Cost Estimating Guide.

PLANNING $20 \%-30 \%$ Experimental/Special Conditions............Up to $50 \%$ Conceptual $15 \%-25 \%$ Experimental/Special Conditions.............Up to $40 \%$ TITLE I TITLE II TITLE II/AFC
$10 \%-20 \%$ $15 \%$ Market Conditions 
DCWO

INTERIM STORAGE FACILITY

\begin{tabular}{|c|c|c|c|c|c|c|c|c|c|c|c|c|c|c|c|c|c|c|}
\hline ID & Task Name & 1997 & 1998 & 1999 & 2000 & 2001 & 2002 & 2003 & 2004 & 2006 & 2006 & 2007 & 2008 & 2009 & 2010 & 2011 & 2012 & 2013 \\
\hline 1 & DCWO - INTERIM STORAGE & & & & & & & & & & & & & & & & & \\
\hline 2 & CONCEPTUAL DESIGN & & & & & & & & & & & & & & & & & \\
\hline 3 & ADVANCED CONCEPTUAL DESIGN & & & & & & & & $\delta / 5$ & & & & & & & & & \\
\hline 4 & PM FOR PROJECT DEVELOPMENT & & & & & & & & & & & & & & & & & \\
\hline 6 & PERMITTING & & & & & & & $6 / 2$ & & & & & & & & & & \\
\hline 6 & PROJECT MANAGEMENT & & & & & & & & & & $6 / 28$ & & & & & & $11 / 18$ & \\
\hline 7 & TITLEDESIGN & & & & & & & & & & $6 / 28$ & & & & & & & \\
\hline 8 & CONSTRUCTION & & & & & & & & & & & $11 / 2$ & & & & & & \\
\hline 9 & SO TEST \& STARTUP & & & & & & & & & & & & & & & $1 / 2$ & & \\
\hline 10 & START CANISTER RECEIPT & & & & & & & & & & & & & & & & & \\
\hline
\end{tabular}

\begin{tabular}{|l|lll|}
\hline & Task & Summary \\
Project: 2421Dcwo.MPP & Rolled Up Progress \\
Dale: Mon 1/26/98 & Rolled Up Task & Rolled Up Milestone & Page 1 \\
\hline
\end{tabular}


Iockheed Martin Idaho Technologies

\section{COST ESTIMATE SUPPORT DATA RECAPITULATION (CONTINUATION)}

File No: 2396

Page 2 of 2

\section{ASSUMPTIONS: (Continued)}

12. Only the railroad track within the storage facility is included in this estimate. The remainder of the branch line is included in the infrastructure estimate.

13. All utilities are assumed to be available at the facility wall.

IV. CONTINGENCY GUIDELINE IMPLEMENTATION: The percentage used for contingency as determined by the contingency allowance guidelines can be altered to refiect the type of construction and conditions that may impact the total estimated cost.

A large portion of the estimated costs is based on actual costs from Fluor's SNF Canister Storage Building. The majority of the remaining costs are for standard construction activities. For these reasons, the contingency is lower than would be experienced for a processing type facility at this stage of development.

\section{OTHER COMMENTS/CONCERNS SPECIFIC TO THE ESTIMATE:}

1. All FY' 98 and later projects are to be assessed a Procurement Fee of $1 \%$, a G\&A Fee of $23 \%$, with a $\$ 500,000$ ceiling per year, and a Performance Incentive Fee (PIF) of 5.5\%. See the attached G\&A/PIF calculation sheet for the method used to calculate these fees.

2. A procurement fee of one percent of construction was used to cover the operating contractor support to DOE-ID for their contract administration. It is felt that one percent is sufficient for jobs of this size. 
Lockheed Martin Idaho Technologies Co. Rev, 6/96 PROIECT NAME: CWO FACILITY - INTERIM STORAGE FACILITY-ESCALATED

LOCATION I: REQUESTOP

J. K. RAWLINS
COST ESTIMATE SUMMARY

TYPE OFESTIMATE: PLANNING $\begin{array}{ll}\text { PRONECT NO: } & \text { 2421-CWOA } \\ \text { PREPARED BY: R.D.ADAMS }\end{array}$ PREPARED BY:
REPORT NAME: Cost Estimate Summary

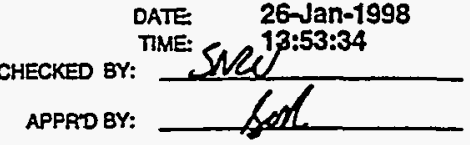

\begin{tabular}{|c|c|c|c|c|}
\hline $\begin{array}{l}\text { WBS } \\
\text { Element }\end{array}$ & Cost Estimate Element & $\begin{array}{c}\text { Total } \\
\text { Unescalated }\end{array}$ & Escalation & $\begin{array}{c}\text { Total } \\
\text { Inci Escalation }\end{array}$ \\
\hline $\begin{array}{l}1.1 \\
1.1 .1 \\
1.1 .2 \\
1.2 \\
1.2 .1 \\
1.2 .2 \\
1.3 \\
1.3 .1 \\
1.3 .2 \\
1.3 .3 \\
1.3 .5 \\
1.3 .7 \\
1.3 .8 \\
1.3 .9 \\
1.3 .13 \\
1.3 .14 \\
1.3 .15 \\
1.3 .16 \\
1.5 \\
1.5 .1 \\
1.5 .2 \\
\end{array}$ & $\begin{array}{l}\text { ENGINEERING, DESIGN AND INSPECTION } \\
\text { DESIGN ENGINEERING TITLE I \& } \\
\text { TITLE III INSPECTION } \\
\text { MANAGEMENT COSTS } \\
\text { PROJECT MANAGEMENT } \\
\text { CONSTRUCTION MANAGEMENT } \\
\text { CONSTRUCTION } \\
\text { GENERAL CONDITIONS } \\
\text { SITEWORK } \\
\text { CONCRETE } \\
\text { METALS } \\
\text { THERMAL \& MOISTURE PROTECTION } \\
\text { DOORS \& WINDOWS } \\
\text { FINISHES } \\
\text { SPECIAL CONSTRUCTION } \\
\text { CONVEYING SYSTEMS } \\
\text { MECHANICAL } \\
\text { ELECTRICAL } \\
\text { G\&AIPIF } \\
\text { G\&APIF ADDER } \\
\text { PROCUREMENT FEES }\end{array}$ & $\begin{array}{r}20,000,000 \\
15,000,000 \\
\\
28,000,000 \\
30,275,200 \\
\\
5,423,282 \\
589,840 \\
29,671,461 \\
12,679,179 \\
2,049,726 \\
71,240 \\
1,059,550 \\
235,368,061 \\
11,272,944 \\
2,762,200 \\
1,804,688 \\
\end{array}$ & $\begin{array}{r}5,200,000 \\
5,550,000 \\
\\
9,240,000 \\
11,201,824 \\
\\
2,006,614 \\
218,241 \\
10,978,440 \\
4,691,296 \\
758,399 \\
26,359 \\
392,034 \\
87,086,182 \\
4,170,989 \\
1,022,014 \\
667,735 \\
\end{array}$ & $\begin{array}{r}\$ 45,750,000 \\
25,200,000 \\
20,550,000 \\
>\quad \$ 78,717,024 \\
37,240,000 \\
41,477,024 \\
\$ \$ \$ 414,770,474 \\
7,429,896 \\
808,081 \\
40,649,901 \\
17,370,475 \\
2,808,125 \\
97,599 \\
1,451,584 \\
322,454,243 \\
15,443,933 \\
3,784,214 \\
2,472,423 \\
\$ \$ 24,711,801 \\
24,711,801 \\
\$ \$ 4,147,705 \\
\end{array}$ \\
\hline & $\begin{array}{l}\text { SUBTOTAL INCLUDING ESCALATION } \\
\text { PROJECT CONTINGENCY }\end{array}$ & $417,092,704$ & $151,004,300$ & $\gg \$ 568,097,004$ \\
\hline & MANAGEMENT RESERVE & & & $\gg \quad \$ 44,362,998$ \\
\hline & CONTINGENCY- & & & $\gg \quad \$ 69,439,998$ \\
\hline & TOTAL ESTIMATED COST & & & $\gg \quad \$ 681,900,000$ \\
\hline
\end{tabular}

\section{PROJECT COST PARAMETERS}

EDI AS A $\%$ OF CONST. + GFE= $11.00 \%$

CONTINGENCY $=20.03 \%$ 
Lockheed Martin Idaho Technologies Co.

PROSECT NAME: CWO FACILITY - INTERIM STORAGE FACILITY-UNESCALATED

INEELMCPP

LOCATION

J. K. RAWLINS
COST ESTIMATE SUMMARY

TYPE OF ESTIMATE: PLANNING PROJECT NO: 2421-CWOA PREPARED BY: R.D. ADAMS REPORT NAME: Cost Estimate Summary

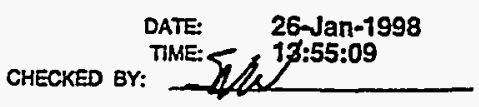

APPRD BY:

\begin{tabular}{|c|c|c|c|c|}
\hline $\begin{array}{l}\text { WBS } \\
\text { Element }\end{array}$ & Cost Estimate Element & $\begin{array}{c}\text { Total } \\
\text { Unescalated }\end{array}$ & Escalation & $\begin{array}{c}\text { Total } \\
\text { Incl Escalation }\end{array}$ \\
\hline $\begin{array}{l}1.1 \\
1.1 .1 \\
1.1 .2 \\
1.2 \\
1.2 .1 \\
1.2 .2 \\
1.3 \\
1.3 .1 \\
1.3 .2 \\
1.3 .3 \\
1.3 .5 \\
1.3 .7 \\
1.3 .8 \\
1.3 .9 \\
1.3 .13 \\
1.3 .14 \\
1.3 .15 \\
1.3 .16 \\
1.5 \\
1.5 .1 \\
1.5 .2 \\
\end{array}$ & $\begin{array}{l}\text { ENGINEERING, DESIGN AND INSPECTION } \\
\text { DESIGN ENGINEERING TITLE I \& } \| \\
\text { TITLE III INSPECTION } \\
\text { MANAGEMENT COSTS } \\
\text { PROJECT MANAGEMENT } \\
\text { CONSTRUCTION MANAGEMENT } \\
\text { CONSTRUCTION } \\
\text { GENERAL CONDITIONS } \\
\text { SITEWORK } \\
\text { CONCRETE } \\
\text { METALS } \\
\text { THERMAL \& MOISTURE PROTECTION } \\
\text { DOORS \& WINDOWS } \\
\text { FINISHES } \\
\text { SPECIAL CONSTRUCTION } \\
\text { CONVEYING SYSTEMS } \\
\text { MECHANICAL } \\
\text { ELECTRICAL } \\
\text { G\&AIPIF } \\
\text { G\&APIF ADDER } \\
\text { PROCUREMENT FEES }\end{array}$ & $\begin{array}{r}20,000,000 \\
15,000,000 \\
28,000,000 \\
30,275,200 \\
\\
5,423,282 \\
589,840 \\
29,571,461 \\
12,679,179 \\
2,049,726 \\
71,240 \\
1,059,550 \\
235,368,061 \\
11,272,944 \\
2,762,200 \\
1,804,688 \\
\end{array}$ & $\begin{array}{l}0 \\
0 \\
0 \\
0 \\
0 \\
0 \\
0 \\
0 \\
0 \\
0 \\
0\end{array}$ & $\begin{array}{r}\$ 35,000,000 \\
20,000,000 \\
15,000,000 \\
\$ \$ 58,275,200 \\
28,000,000 \\
30,275,200 \\
\$ \$ \$ 302,752,171 \\
5,423,282 \\
589,840 \\
29,671,461 \\
12,679,179 \\
2,049,726 \\
71,240 \\
1,059,550 \\
235,368,061 \\
11,272,944 \\
2,762,200 \\
1,804,688 \\
\\
>>\$ 3,027,522\end{array}$ \\
\hline & $\begin{array}{l}\text { SUBTOTALINCLUDING ESCALATION } \\
\text { PROJECT CONTINGENCY }\end{array}$ & $417,092,704$ & 0 & $\gg \$ 417,092,704$ \\
\hline & MANAGEMENT RESERVE & & & $\gg \quad \$ 32,381,750$ \\
\hline & CONTINGENCY - & & & $\gg \quad \$ 50,525,546$ \\
\hline & TOTAL ESTIMATED COST - & & & $\gg \quad \$ 500,000,000$ \\
\hline
\end{tabular}

\section{PROJECT COST PARAMETERS}

EDI AS A \% OF CONST. + GFE $=12.00 \%$ 
Lockheed Martin Idaho Technologles Co.

Rev. 6196

PROJECT NAME: CWO FACILITY - INTERIM STORAGE FACILITY

LOCATION 1: INEELACPP

REQUESTOR: J.K. RAWLINS

\section{DETAILED COST ESTIMATE SHEET}

TYPE OF ESTIMATE: PLANNING

PROJECT NO: 2421-CWO

PREPARED BY: R. D. ADAMS
PAge: 1

DATE 26-Jan-1998

TIME: 10:13:34

REPORT NAME: Detall Cost Estimate Sheo

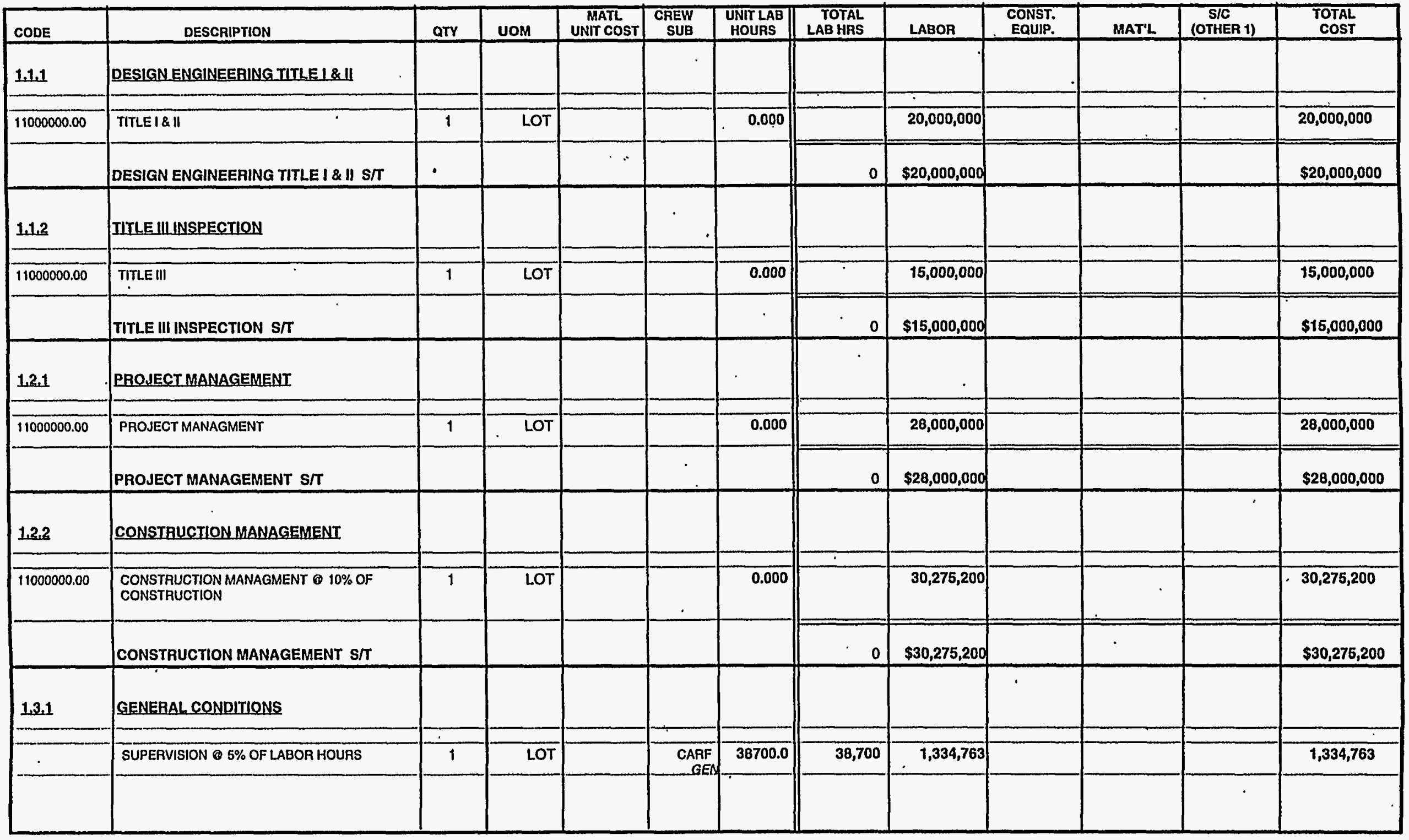


Lockheed Martin Idaho Technologies Co.

Rov. $6 / 96$

PROJECT NAME: CWO FACILITY - INTERIM STORAGE

FACILITY

LOCATION 1: INEELICPP

REQUESTOR: J.K. RAWLINS
DETAILED COST ESTIMATE SHEET

TYPE OF ESTIMATE: PLANNING

PROJECT NO: 2421-CWO

PREPARED BY: R. D. ADAMS
PAGE 2

DATE 26-Jan-1998

TIME: 10:13:34

Aeport NAME: Detall Cost Estimate Sheet

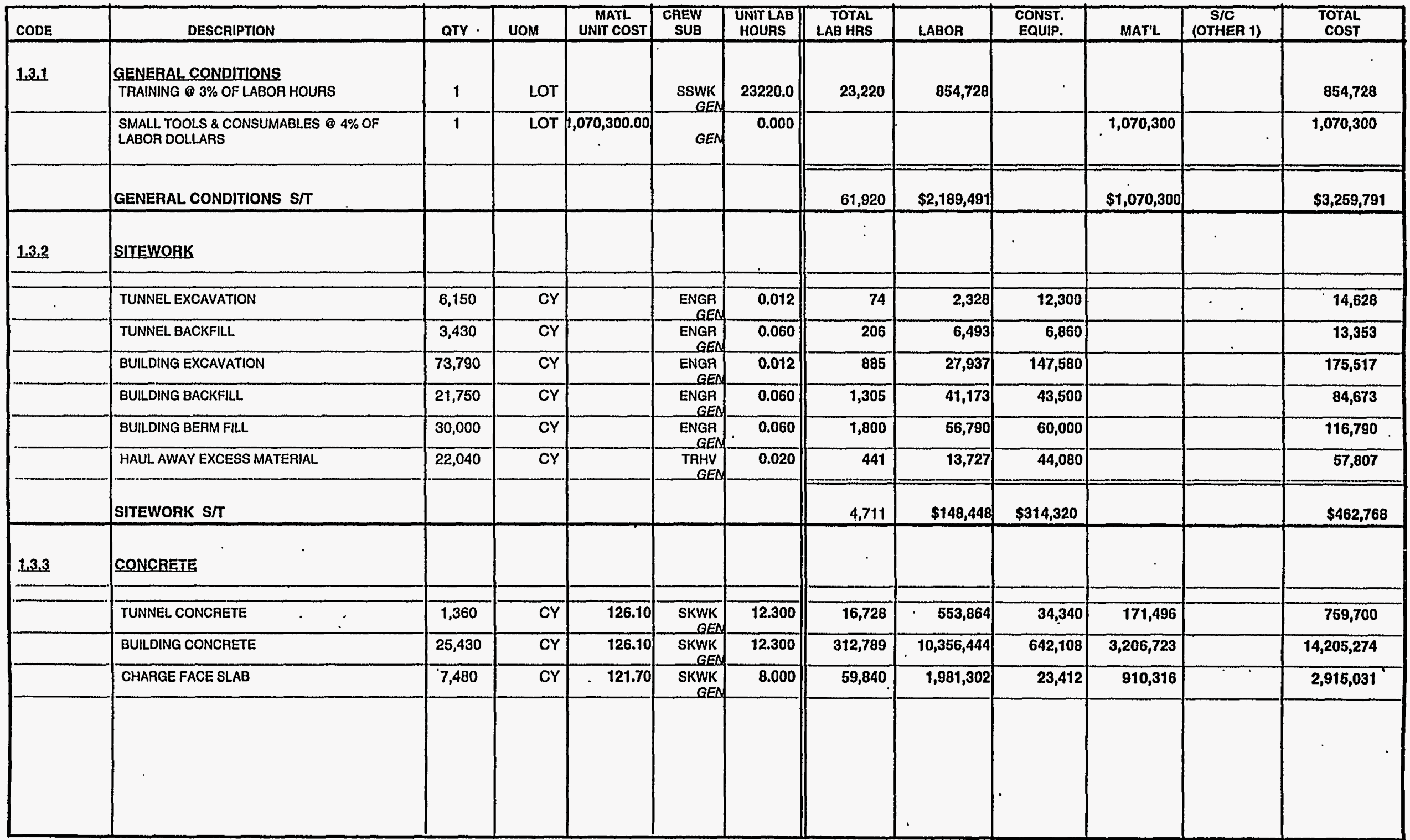


Lockheed Martin Idaho Technologles Co. Rov. 6/95 PROJECT NAME: CWO FACILITY - INTERIM STORAGE FACILITY

LOCATION 1: INEELICPP

REQUESTOR: J.K. RAWLINS
DETAILED COST ESTIMATE SHEET

TYPE OF ESTIMATE: PLANNING

PROJECT NO: 2421-CWO

PREPARED BY: R. D. ADAMS
PAGE 3 .

DATE 26-Jan-1998

TIME: 10:13:34

REPORT NAME: Detal! Cost Estlmate Sheot

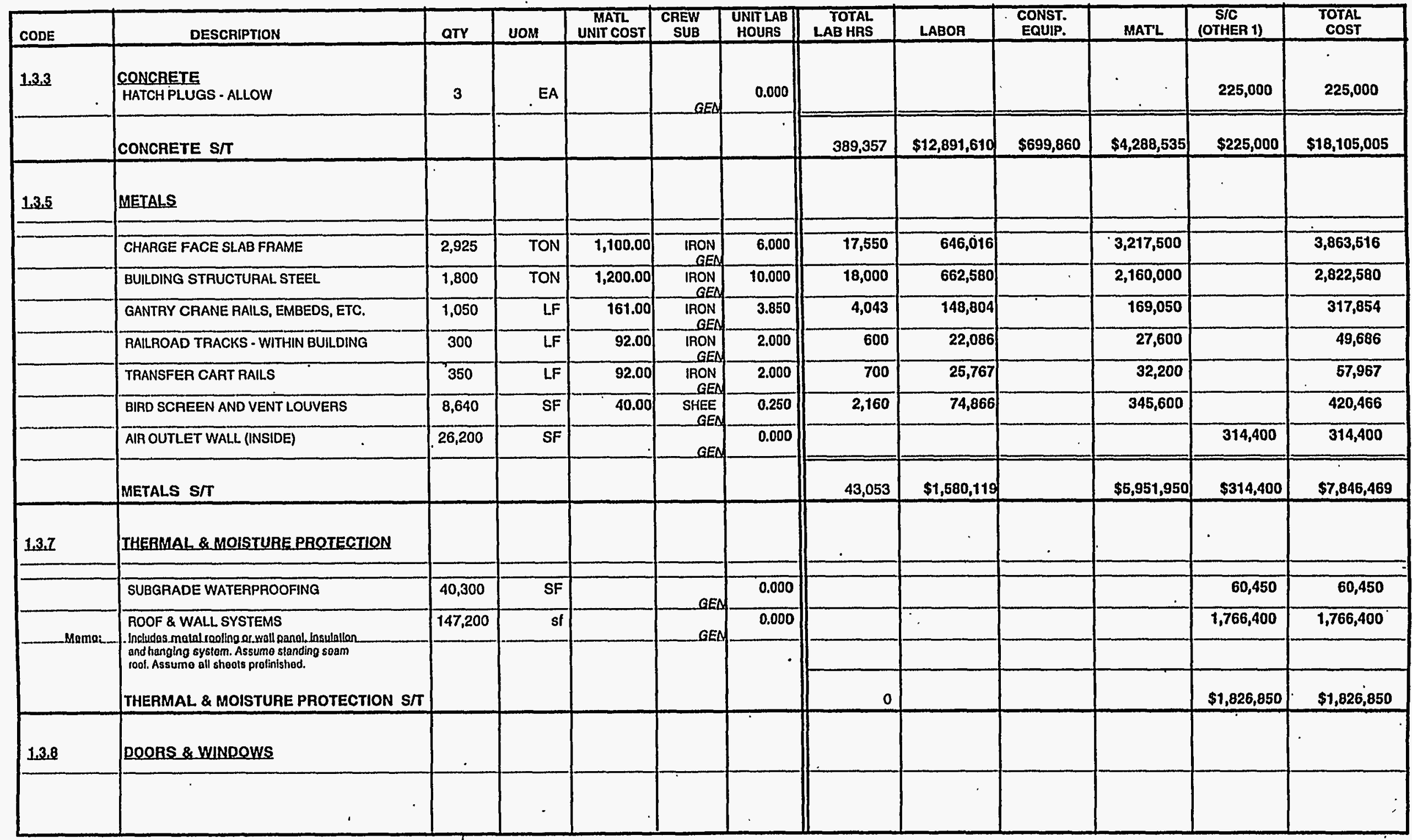


Lockheed Martin Idaho Technologies Co.

PROSECT NAME: CWO FACILITY - INTERIM STORAGE FACILITY

LOCATION 1: INEELICPP

REQUESTOR: .J. K. RAWLINS

\section{DETAILED COST ESTIMATE SHEET}

TYPE OF ESTIMATE: PLANNING

PROJECT NO.: 2421-CWO

PREPARED BY: R. D. ADAMS
PAgE: 4

DATE 26-Jan-1998

TIME: 10:13:34

REPORT NAME: Dotal| Cost Estimate Shoot

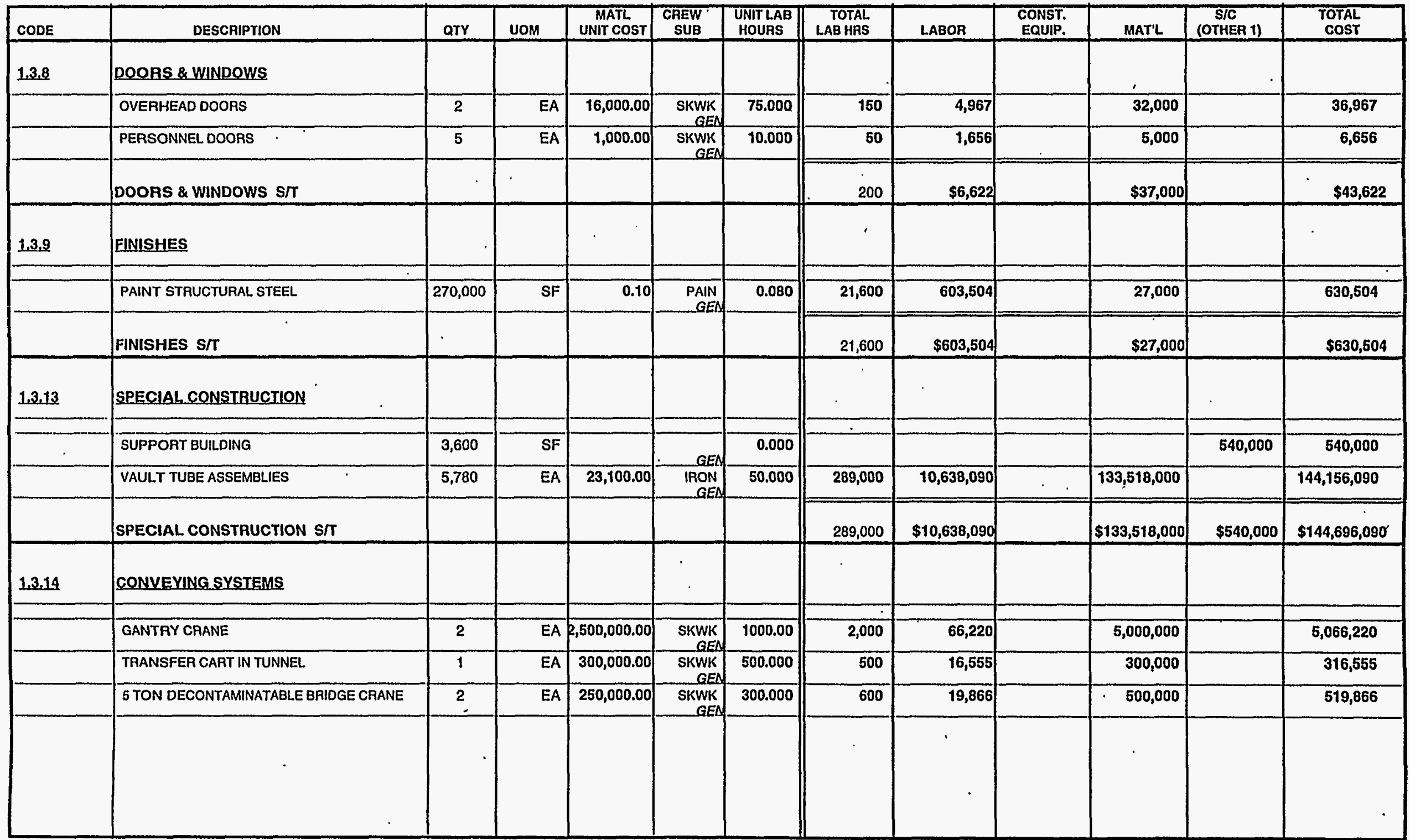


Lockheed Martin Idaho Technologies Co.

Rov. 6/96
PROJECT NAME: CWO FACILITY - INTERIM STORAGE

FACILITY

LOCATION 1: INEELICPP
DETAILED COST ESTIMATE SHEET

TYPE OF ESTMMATE: PLANNING

PROJECT NO: 2421-CWO

PREPARED BY: R. D. ADAMS
PAGE" 5

DATE 26-Jan-1998

TIME: 10:13:34

REPORT NAME: Detall Cost Estimate Shoot

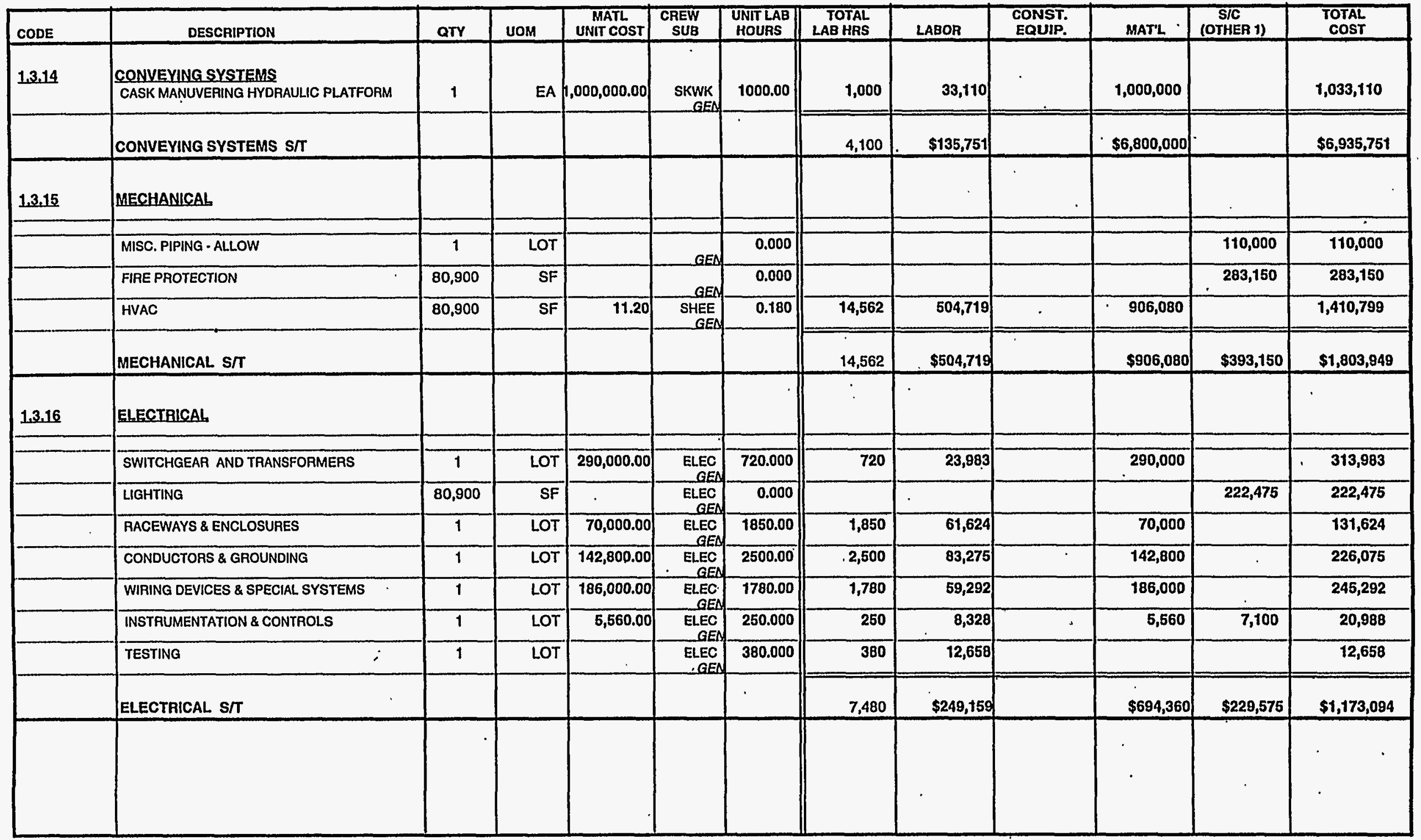


Lockheed Martin Idaho Technologies Co. Rov. 6/98

PROJECT NAME: CWO FACILITY - INTERIM STORAGE

$$
\text { FACILITY }
$$

LOCATION 1: INEELICPP

REQUESTOR: J. K. RAWLINS
DETAILED COST ESTIMATE SHEET

TYPE OF ESTIMATE: PLANNING

PROJECT NO: 2421-CWO

PREPARED BY: R. D. ADAMS
PAGE 6

DATE 26-Jan-1998

TIME: 10:13:34

AEPORT NAME: Detail Cost Estimate Sheet

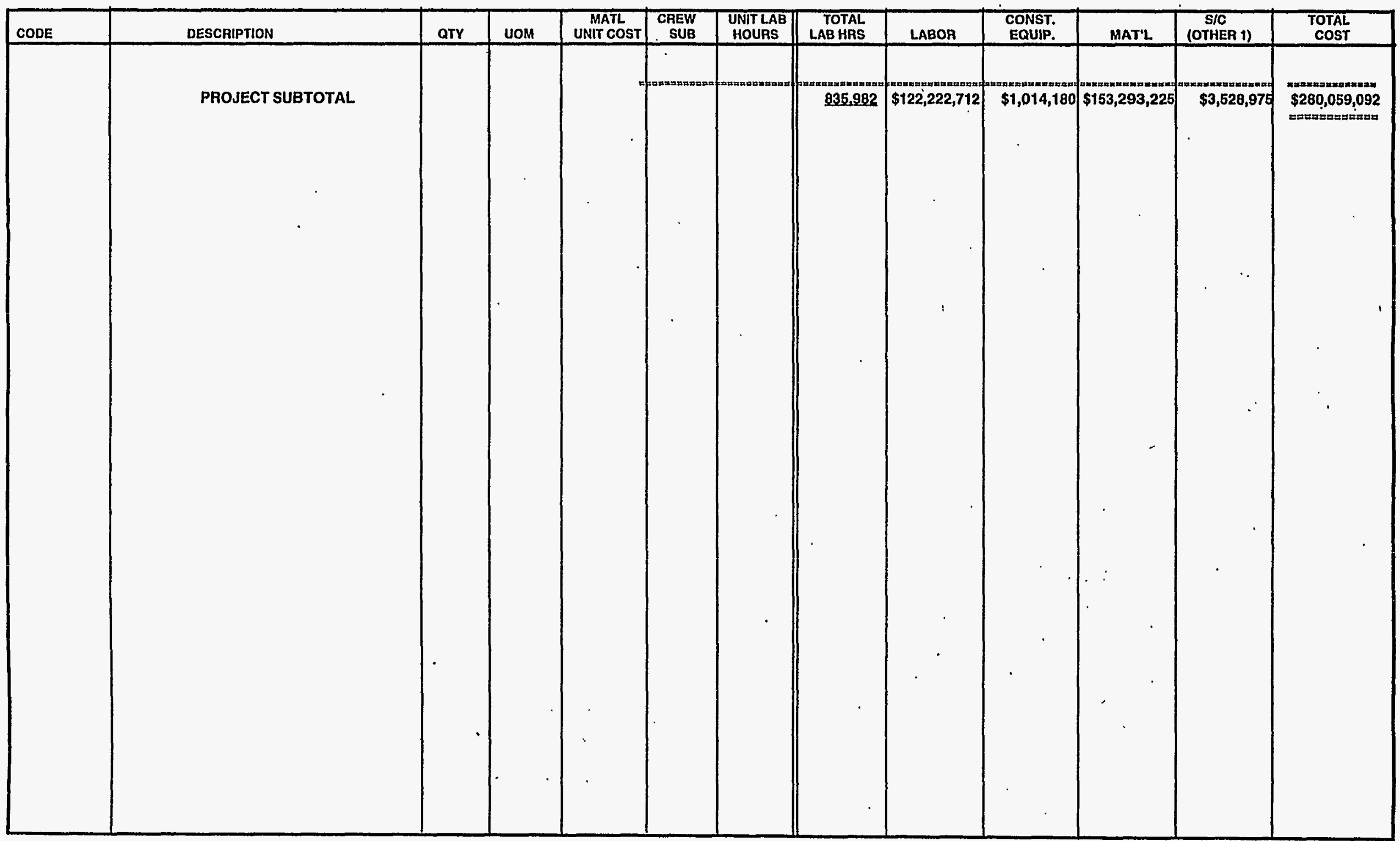


Lockheed Martin Jdaho Technologies Co. PROSECT NAME: CWO FACILITY - INTERIM STORAGE

LOCATION 1: FACILITY-UNESCALATED

INEELICPP

REQUESTOR: J. K. RAWLINS

\section{CONTINGENCY ANALYSIS}

TYPE OFESTIMATE: PLANNING
PROJECT NO: $\begin{array}{ll}\text { PROJECT NO: } & \text { 2421-CWOA } \\ \text { PREPARED BY: } & \text { R.D. ADAMS }\end{array}$
DATE: 26-Jan-1998

TIME: $13: 55: 14$

REPORT NAME: Contingency Analysis

\begin{tabular}{|c|c|c|c|c|c|c|c|c|c|c|c|}
\hline \multicolumn{9}{|c|}{ PROBABLE \% VARIATION } & \multicolumn{2}{|c|}{$\begin{array}{l}\text { PROJECT } \\
\text { CONTINGENCY }\end{array}$} & \multirow{3}{*}{$\begin{array}{l}\text { SUMMARY } \\
\begin{array}{l}\text { Total Cost } \\
\text { by Element }\end{array}\end{array}$} \\
\hline \multirow[t]{2}{*}{$\begin{array}{l}\text { WBS } \\
\text { Element }\end{array}$} & \multirow[t]{2}{*}{ Cost Estimate Element } & \multirow{2}{*}{$\begin{array}{c}\text { Total Cost w/o } \\
\text { Contingency }\end{array}$} & \multirow[t]{2}{*}{$\begin{array}{l}\text { \% Total } \\
\text { Cost }\end{array}$} & \multicolumn{2}{|c|}{$\begin{array}{c}\text { Prob. \% Var. } \\
\text { From Est. }\end{array}$} & \multicolumn{2}{|c|}{ Wt. $\%$ of Prob. } & \multirow[t]{2}{*}{ Contingency } & \multirow[t]{2}{*}{$\%$} & \multirow[t]{2}{*}{ Cost } & \\
\hline & & & & - & + & - & $t$ & & & & \\
\hline 1.1 .1 & DESIGN ENGINEERING TITLE \& \& $\|$ & $20,000,000$ & 4.80 & 20 & 35 & 0.96 & 1.68 & $1.415 \%$ & $7.12 \%$ & $5,901,314$ & $25,901,314$ \\
\hline 1.1 .2 & TITLE III INSPECTTON & $15,000,000$ & 3.60 & 20 & 30 & 0.72 & 1.08 & $0.899 \%$ & $4.52 \%$ & $3,750,835$ & $18,750,835$ \\
\hline 1.2 .1 & PROJECT MANAGEMENT & $28,000,000$ & 6.71 & 20 & 35 & 1.34 & 2.35 & $1.980 \%$ & $9.97 \%$ & $8,261,840$ & $36,261,840$ \\
\hline 1.2 .2 & CONSTRUCTION MANAGEMENT & $30,275,200$ & 7.26 & 20 & 30 & 1.45 & 218 & 1.815\% & $9.13 \%$ & $7,570,486$ & $37,845,686$ \\
\hline 1.3 .1 & GENERALCONDITIONS & $5,423,282$ & 1.30 & 20 & 35 & 0.26 & 0.45 & $0.384 \%$ & $1.93 \%$ & $1,600,225$ & $7,023,507$ \\
\hline 1.3 .2 & SITEWORK & 589,840 & 0.14 & 20 & 30 & 0.03 & 0.04 & $0.035 \%$ & $0.18 \%$ & 147,493 & 737,333 \\
\hline 1.3 .3 & CONCAETE & $29,671,461$ & 7.11 & 20 & 30 & 1.42 & 2.13 & $1.778 \%$ & $8.95 \%$ & $7,419,518$ & $37,090,979$ \\
\hline 1.3 .5 & METALS & $12,679,179$ & 3.04 & 20 & 25 & 0.61 & 0.76 & $0.623 \%$ & $3.14 \%$ & $2,599,811$ & $15,278,990$ \\
\hline 1.3 .7 & THERMAL \& MOISTURE PROTECTION & $2,049,726$ & 0.49 & 20 & 30 & 0.10 & 0.15 & $0.123 \%$ & $0.62 \%$ & 512,546 & $2,562,272$ \\
\hline 1.3 .8 & DOOAS \& WINDOWS & 71,240 & 0.02 & 20 & 20 & 0.00 & 0.00 & $0.003 \%$ & $0.01 \%$ & 11,401 & 82,641 \\
\hline 1.3 .9 & FINISHES & $1,059,550$ & 0.25 & 20 & 35 & 0.05 & 0.09 & $0.075 \%$ & $0.38 \%$ & 312,637 & $1,372,187$ \\
\hline 1.3 .13 & SPECIAL CONSTRUCTON & $235,368,061$ & 56.43 & 20 & 20 & 11.29 & 11.29 & $9.029 \%$ & 45.43\% & $37,667,278$ & $273,035,339$ \\
\hline 1.3 .14 & CONVEYING SYSTEMS & $11,272,944$ & 2.70 & 20 & 25 & 0.54 & 0.68 & $0.554 \%$ & $279 \%$ & $2,311,468$ & $13,584,412$ \\
\hline 1.3 .15 & MECHANICAL & $2,762,200$ & 0.66 & 20 & 30 & 0.13 & 0.20 & $0.166 \%$ & $0.83 \%$ & 690,704 & $3,452,904$ \\
\hline 1.3 .16 & ELECTAICAL & $1,804,688$ & 0.43 & 20 & 25 & 0.09 & 0.11 & $0.089 \%$ & $0.45 \%$ & 370,043 & $2,174,731$ \\
\hline 1.5 .1 & G\&APIF ADDER & $18,037,811$ & 4.32 & 5 & 20 & 0.22 & 0.86 & $0.757 \%$ & $3.81 \%$ & $3,157,320$ & $21,195,131$ \\
\hline \multirow[t]{2}{*}{1.5 .2} & PROCUREMENT FEES & $3,027,522$ & 0.73 & 20 & 25 & 0.15 & 0.18 & $0.149 \%$ & $0.75 \%$ & 620,780 & $3,648,302$ \\
\hline & ESCALATION & 0 & 0.00 & 20 & 25 & 0.00 & 0.00 & $0.000 \%$ & $0.00 \%$ & 1.597 & 1,597 \\
\hline & SUBTOTAL & $417,092,704$ & 100.00 & & & & & $19.873 \%$ & & & \\
\hline & CALCULATED CONTINGENCY & $82,887,236$ & & & & & & & & & \\
\hline & RESULTANT TEC & $499,979,940$ & & & & & & & & & \\
\hline & ROUNDED TEC & $500,000,000$ & & & & & & & & & \\
\hline & PROJECT CONTINGENCY & $82,907,296$ & & & & & & $19.88 \%$ & & & \\
\hline & MANAGEMENT RESERVE & $32,381,750$ & & & & & & & & & \\
\hline & CONTINGENCY & $50,525,546$ & & & & & & & & & \\
\hline & TOTAL ESTIMATED COST & $500,000,000$ & & & & & & & & $82,907,296$ & $500,000,000$ \\
\hline
\end{tabular}

CONFIDENCE LEVEL AND ASSUMED RISKS:

The Lockheed Idaho Technologies Co. Cost Estimate Contingency Analysis Model is based on the applied contingency and the assumptions upon which the estimate was predicated. The model is applied with a suggested risk level of $18 \%$ and a level of confidence of $90 \%$ the estimate will fall within the bid range. The Contingency Analysis is based on a weighted average to provide a

$90 \%$ probability of underrun and a $10 \%$ probability of overrun.
CONTINGENCY ANALYSIS GUIDE BY TYPE OF ESTIMATE

Guidelines established by DOEJFM 50, Cost Estimating Guide, Vol. 6, Cost Guide, and as presented in the INEL Cost Estimating Guide. PLANNING $20 \%-30 \%$ Experimental/Special Conditions.............. Up to 50\% Conceptual

Experimental/Special Conditions TITLE I $10 \%-20 \%$

TITLE II

TITLE U/AFC 
Lockheed Martin Idaho Technologies Co. PROUECT NAME: CWO FACILITY - INTERIM STORAGE FACILITY-ESCALATED

LOCATION 1: INEELICPP

REQUESTOR: J.K. RAWLINS

\section{CONTINGENCY ANALYSIS}

TYPE OF ESTIMATE: PROJECT NO: PREPAAED BY:

\author{
PLANNING \\ 2421-CWOA \\ R. D. ADAMS
}

DATE: 26-Jan-1998

TME: 13:53:39

REPORT NAME: Contingency Analysis

\begin{tabular}{|c|c|c|c|c|c|c|c|c|c|c|c|}
\hline \multicolumn{9}{|c|}{ PROBABLE \% VARIATION } & \multicolumn{2}{|c|}{$\begin{array}{c}\text { PROJECT } \\
\text { CONTINGENCY }\end{array}$} & \multirow{3}{*}{$\begin{array}{l}\text { SUMMARY } \\
\begin{array}{l}\text { Total Cost } \\
\text { by Element }\end{array}\end{array}$} \\
\hline $\begin{array}{l}\text { WBS } \\
\text { Element }\end{array}$ & \multirow[t]{2}{*}{ Cost Estimate Element } & \multirow{2}{*}{$\begin{array}{c}\text { Total Cost w/o } \\
\text { Contingency }\end{array}$} & \multirow[t]{2}{*}{$\begin{array}{l}\text { \% Total } \\
\text { Cost }\end{array}$} & \multicolumn{2}{|c|}{$\begin{array}{c}\text { Prob. \% Var. } \\
\text { From Est. }\end{array}$} & \multicolumn{2}{|c|}{ Wt \% of Prob. } & \multirow[t]{2}{*}{ Contingency } & \multirow[t]{2}{*}{$\%$} & \multirow[t]{2}{*}{ Cost } & \\
\hline & & & & - & + & - & + & & & & \\
\hline 1.1 .1 & DESIGN ENGINEERING TITLE $\mid \& \|$ & $20,000,000$ & 3.52 & 20 & 35 & 0.70 & 1.23 & $1.039 \%$ & $5.18 \%$ & $5,898,033$ & $25,898,033$ \\
\hline 1.1 .2 & TITLE III INSPECTION & $15,000,000$ & 264 & 20 & 30 & 0.53 & 0.79 & $0.660 \%$ & $3229 \%$ & $3,748,749$ & $18,748,749$ \\
\hline 1.21 & PROJECT MANAGEMENT & $28,000,000$ & 4.93 & 20 & 35 & 0.99 & 1.73 & $1.454 \%$ & $7.26 \%$ & $8,257,246$ & $36,257,246$ \\
\hline 1.22 & CONSTRUCTION MANAGEMENT & $30,275,200$ & 5.33 & 20 & 30 & 1.07 & 1.60 & $1.332 \%$ & $6.65 \%$ & $7,566,276$ & $37,841,476$ \\
\hline 1.3 .1 & GENERAL CONDITIONS & $5,423,282$ & 0.95 & 20 & 35 & 0.19 & 0.33 & $0.282 \%$ & $1.41 \%$ & $1,599,335$ & $7,022,617$ \\
\hline 1.3 .2 & SITEWORK & 589,840 & 0.10 & 20 & 30 & 0.02 & 0.03 & $0.026 \%$ & $0.13 \%$ & 147,411 & 737,251 \\
\hline 1.3 .3 & CONCRETE & $29,671,461$ & 5.22 & 20 & 30 & 1.04 & 1.57 & $1.306 \%$ & $6.52 \%$ & $7,415,392$ & $37,086,853$ \\
\hline 1.3 .5 & METALS & $12,679,179$ & 2.23 & 20 & 25 & 0.45 & 0.56 & $0.458 \%$ & $228 \%$ & $2,598,365$ & $15,277,544$ \\
\hline 1.3 .7 & THERMAL \& MOISTURE PROTECTION & $2,049,726$ & 0.36 & 20 & 30 & 0.07 & 0.11 & $0.090 \%$ & $0.45 \%$ & 512,261 & $2,561,987$ \\
\hline 1.3 .8 & DCORS \& WINDOWS & 71,240 & 0.01 & 20 & 20 & 0.00 & 0.00 & $0.002 \%$ & $0.01 \%$ & 11,395 & 82,635 \\
\hline 1.3 .9 & FINISHES & $1,059,550$ & 0.19 & 20 & 35 & 0.04 & 0.07 & $0.055 \%$ & $0.27 \%$ & 312,463 & $1,372,013$ \\
\hline 1.3 .13 & SPECIAL CONSTRUCTION & $235,368,061$ & 41.43 & 20 & 20 & 8.29 & 8.29 & $6.629 \%$ & $33.08 \%$ & $37,646,332$ & $273,014,393$ \\
\hline 1.3 .14 & CONVEYING SYSTEMS & $11,272,944$ & 1.98 & 20 & 25 & 0.40 & 0.50 & $0.407 \%$ & $2.03 \%$ & $2,310,183$ & $13,583,127$ \\
\hline 1.3 .15 & MECHANICAL & $2,762,200$ & 0.49 & 20 & 30 & 0.10 & 0.15 & $0.122 \%$ & $0.61 \%$ & 690,320 & $3,452,520$ \\
\hline 1.3 .16 & ELECTRICAL & $1,804,688$ & 0.32 & 20 & 25 & 0.06 & 0.08 & $0.065 \%$ & $0.32 \%$ & 369,838 & $2,174,526$ \\
\hline 1.5 .1 & G\&APIF ADDER & $18,037,811$ & 3.18 & 5 & 20 & 0.16 & 0.64 & $0.556 \%$ & $2.7 \%$ & $3,155,564$ & $21,193,375$ \\
\hline \multirow[t]{2}{*}{1.5 .2} & PROCUREMENT FEES & $3,027,522$ & 0.53 & 20 & 25 & 0.11 & 0.13 & $0.109 \%$ & $0.55 \%$ & 620,435 & $3,647,957$ \\
\hline & ESCALATION & $151,004,300$ & 26.58 & 20 & 25 & 5.32 & 6.65 & $5.449 \%$ & $27.19 \%$ & $30,943,398$ & $181,947,698$ \\
\hline & SUBTOTAL & $568,097,004$ & 100.00 & & & & & $20.039 \%$ & & & \\
\hline & CALCULATED CONTINGENCY & $113,843,117$ & & & & & & & & & \\
\hline & RESULTANT TEC & $681,940,121$ & & & & & & & & & \\
\hline & ROUNDED TEC & $681,900,000$ & & & & & & & & & \\
\hline & PROJECT CONTINGENCY & $113,802,996$ & & & & & & $20.03 \%$ & & & \\
\hline & MANAGEMENT RESERVE & $44,362,998$ & & & & & & & & & \\
\hline & CONTINGENCY & $69,439,998$ & & & & & & & & & \\
\hline & TOTAL ESTIMATED COST & $681,900,000$ & & & & & & & & $113,802,996$ & $681,900,000$ \\
\hline
\end{tabular}

CONFIDENCE LEVEL AND ASSUMED RISKS:

The Lockheed Idaho Technologies Co. Cost Estimate Contingency Analysis Model is based on the applied contingency and the assumptions upon which

the estimate was predicated. The model is applied with a suggested risk level

of $18 \%$ and a level of confidence of $90 \%$ the estimate will fall within the bid range.

The Contingency Analysis is based on a weighted average to provide a

$90 \%$ probability of underrun and a $10 \%$ probability of overrun.
"CONTINGENCY ANALYSIS GUIDE BY TYPE OF ESTIMATE

Guidelines established by DOE/FM 50, Cost Estimating Guide, Vol. 6.

Cost Guide, and as presented in the INEL Cost Estimating Guide.

PLANNING $20 \%-30 \%$ Experimental/Special Conditions............Up to 50\% Conceptual $15 \%-25 \%$ Experimental/Special Conditions............Up to $40 \%$ TITLE! $10 \%-20 \%$ TITLE II $\quad 5 \%-15 \%$ TITLE II/AFC Market Conditions 


\section{G\&A/PIF ADDER CALCULATION SHEET}

PROCUREMENT FEE:

\begin{tabular}{|c|c|c|c|}
\hline $\begin{array}{l}\text { CONSTRUCTION = } \\
\text { GFE = }\end{array}$ & Subtotal & $\begin{array}{r}\$ 302,752,171 \\
\$ 0 \\
\$ 302,752,171\end{array}$ & \\
\hline FEE @ $1 \%=$ & & $\$ 302,752,171 \cdot 0.01=$ & $\$ 3,027,521.71$ \\
\hline
\end{tabular}

G\&A @ 23\% (with a ceiling of $\$ 500,000$ imposed per year

CONSTRUCTION OR

CEILING $\bullet 4$ YEARS $=\quad . \quad \$ 2,000,000$

GFE =

$\$ 0$

PROCUREMENT FEE = $\$ 3,027,522$

Subtotal $\$ 5,027,522$

FEE @ $23 \%=$

$\$ 5,027,522 \cdot 0.23=$

$\$ 1,156,330$

PIF @ 5.5\% CONSTRUCTION =
GFE =
PROCUREMENT FEE =
G\&A $=$

FEE @ 5.5\% =
$\$ 302,752,171$

$\$ 3,027,522$

$\$ 1,156,330$

$\$ 306,936,023$

$\$ 306,936,023 \cdot 0.055=\quad \$ 16,881,481$

TOTAL PROCUREMENT FEE:

$\$ 3,027,522$

TOTAL G\&A FEE:

$\$ 1,156,330$

TOTAL PIF:

$\$ 16,881,481$ 
Lockheed Martin Idaho Technologies Co. PRONECT NAME: CWO FACILITY - INTERIM STORAGE FACILTY - OPC -ESCALATED INEELICPP

LOCATION 1 J. K. RAWLINS

\section{COST ESTIMATE SUMMARY}

TYPE OFESTMATE: PLANNING PROSECT NO: 242105

PREPARED BY: R. D. ADAMS

REPORT NAME: Cost Estimate Summary

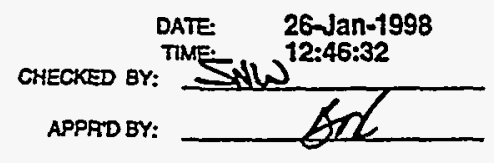

\begin{tabular}{|c|c|c|c|c|}
\hline $\begin{array}{l}\text { WBS } \\
\text { Element }\end{array}$ & Cost Estimate Element & $\begin{array}{c}\text { Total } \\
\text { Unescalated }\end{array}$ & Escalation & $\begin{array}{l}\text { Total } \\
\text { Inel Escalation }\end{array}$ \\
\hline 1.1 & DESIGN \& DEVELOPMENT & - & & $\$ 5,950,000$ \\
\hline 1.1.1 & CONCEPTUAL DESIGN & $5,000,000$ & 950,000 & $5,950,000$ \\
\hline 1.2 & MANAGEMENT COSTS & & & $\gg \quad \$ 4,760,000$ \\
\hline 1.2 .1 & PM FOR PROJECT DEVELOPMENT & $3,000,000$ & 570,000 & $3,570,000$ \\
\hline 1.2 .2 & SAFETY REVIEWS. & $1,000,000$ & 190,000 & $1,190,000$ \\
\hline 1.3 & PERMITTING & & & $\gg \quad \$ 3,246,834$ \\
\hline 1.3 .1 & PERMITTING & $2,728,432$ & 518,402 & $3,246,834$ \\
\hline 1.4 & TURNOVER & & & $\gg \quad \$ 6,525,000$ \\
\hline 1.4.1 & SO TEST \& STARTUP & $4,500,000$ & $2,025,000$ & $6,525,000$ \\
\hline 1.5 .2 & PROCUREMENT FEES & 0 & $\mathbf{0}$ & $\gg$ \\
\hline & $\begin{array}{l}\text { SUBTOTAL INCLUDING ESCALATION } \\
\text { PROJECT CONTINGENCY }\end{array}$ & $16,228,432$ & $4,253,402$ & $\gg>\$ 20,481,834$ \\
\hline & MANAGEMENT RESERVE- & & & so \\
\hline & CONTINGENCY - & & & $\$ 5,118,166$ \\
\hline & TOTAL ESTMATED COST: & & & $\gg \quad \$ 25,600,000$ \\
\hline
\end{tabular}

\section{PROJECT COST PARAMETERS}

EDI AS A \% OF CONST. + GFE $=61.00 \%$ 
Lockheed Martin Idaho Technologies Co.

PROIECT NAYE. CWO FACILITY - INTERIM STORAGE

LOCATION 1:

RECUESTOR:

FACILITY - OPC - UNESCALATED

INEELICPP

J. K. RAWLINS
COST ESTIMATE SUMMARY

TYPE OFESTIMATE: PLANNING PROJECT NO: 242105 PREPARED BY: R.D.ADAMS REPOAT NAME: Cost Estimate Summary

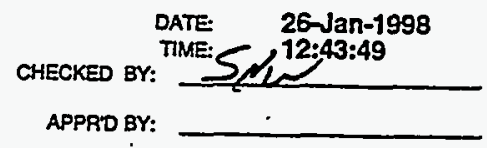

\begin{tabular}{|c|c|c|c|c|}
\hline $\begin{array}{l}\text { WBS } \\
\text { Element }\end{array}$ & Cost Estimate Element & $\begin{array}{c}\text { Total } \\
\text { Unescalated } \\
\end{array}$ & Escalation & $\begin{array}{l}\text { Total } \\
\text { Incl Escalation }\end{array}$ \\
\hline 1.1 & DESIGN \& DEVELOPMENT & & & $\gg \quad \$ 5,000,000$ \\
\hline 1.1 .1 & CONCEPTUAL DESIGN & $5,000,000$ & D & $5,000,000$ \\
\hline 1.2 & MANAGEMENT COSTS & & & $\gg \$ 4,000,000$ \\
\hline 1.2 .1 & PM FOR PROJECT DEVELOPMENT & $3,000,000$ & 0 & $3,000,000$ \\
\hline 1.2 .2 & SAFETY REVIEWS & $1,000,000$ & 0 & $1,000,000$ \\
\hline 1.3 & PERMITTING & & & $\gg \quad \$ 2,728,432$ \\
\hline 1.3 .1 & PERMITTING & $2,728,432$ & 0 & $2,728,432$ \\
\hline 1.4 & TURNOVER & & & $\Rightarrow \$ 4,500,000$ \\
\hline 1.4.1 & SO TEST \& STARTUP & $4,500,000$ & 0 & $4,500,000$ \\
\hline \multirow[t]{2}{*}{1.5 .2} & PROCUREMENT FEES & 0 & 0 & $\gg$ \\
\hline & $\begin{array}{l}\text { SUBTOTAL INCLUDING ESCALATION } \\
\text { PROJECT CONTINGENCY }\end{array}$ & $16,228,432$ & 0 & $\gg \$ 16,228,432$ \\
\hline \multicolumn{4}{|c|}{ MANAGEMENT RESERVE } & $\gg$ \\
\hline \multicolumn{4}{|c|}{ CONTINGENCY- } & $\$ 4,071,568$ \\
\hline & TOTAL ESTIMATED COST - & & & $\$ 20,300,000$ \\
\hline
\end{tabular}

\section{PROJECT COST PARAMETERS}

EDI AS A $\%$ OF CONST. + GFE $=69.00 \%$ 
Lockheed Martin Idaho Technologles Co. Rov. 6/96

PROJECT NAME: CWO FACILITY - INTERIM STORAGE FACILITY - OPC

LOCATION 1: INEELICPP

REQUESTOR: J. K. RAWLINS

\section{DETAILED COST ESTIMATE SHEET}

TYPE OF ESTIMATE: PLANNING

PROJECT NO.: 242105

PREPARED BY: R. D. ADAMS
PAGE * 1

DATE 26-Jan-1998

TIME: 12:42:44

REPORT NAME: Dotall Cost Estimate Sheet

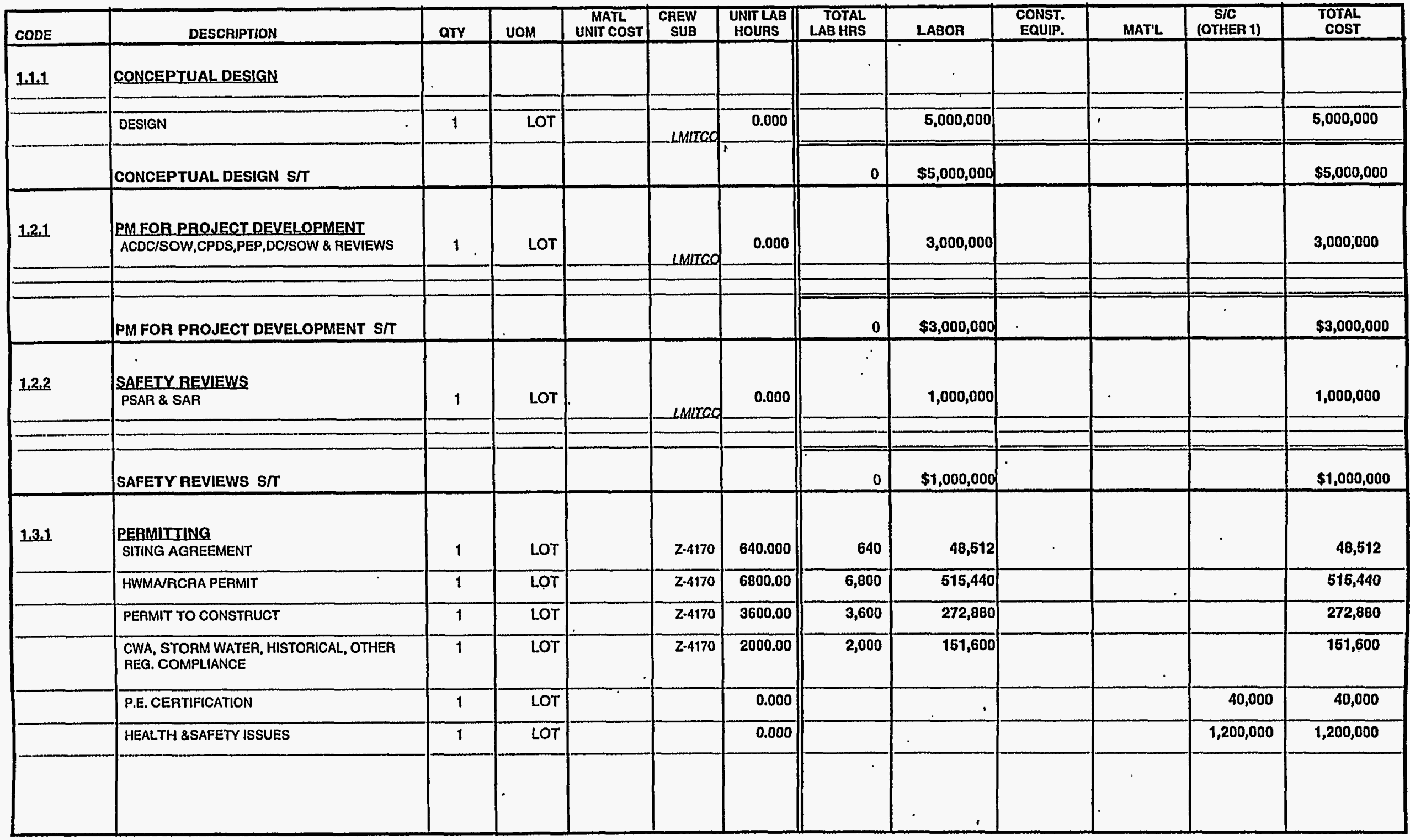


Lockheed Martln Idaho Technologies Co. Rov. B/86 PROJECT NAME: CWO FACILITY - INTERIM STORAGE FACILITY - OPC

LOCATION 1: INEEL/ICPP REQUESTOR: J. K. RAWLINS

\section{DETAILED COST ESTIMATE SHEET}

TYPE OF ESTIMATE: PLANNING

PROJECT NO.: 242105

PREPARED BY: R. D. ADAMS
PAGE " 2

DATE 26-Jan-1998

TIME: 12:42:44

AEPORT NAME: Detall Cost Estimate Sheot

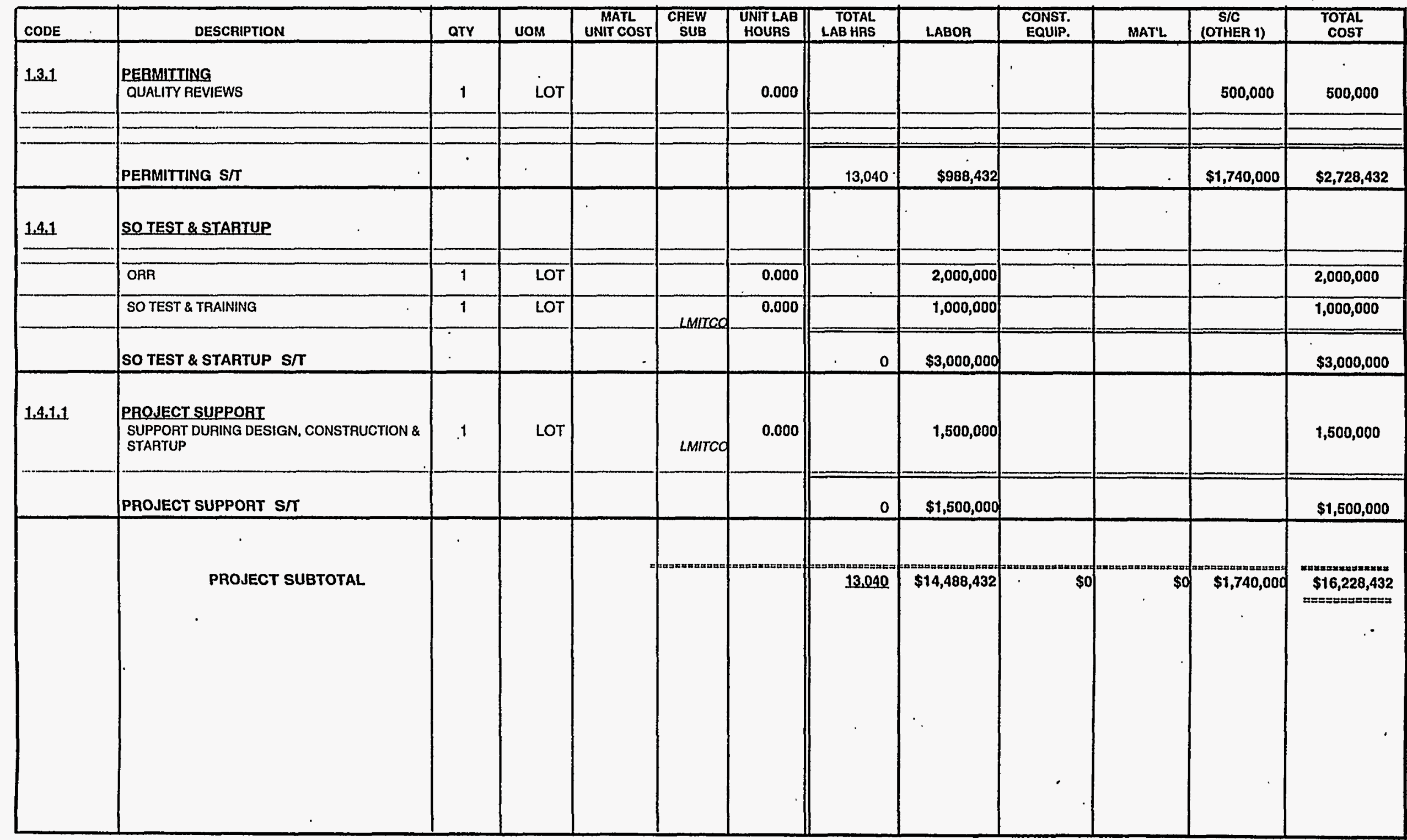


Lockheed Martin Idaho Technologies Co.$$
\text { Rov, } 8960
$$

PROIECT NAME: CWO FACILITY - INTERIM STORAGE FACILITY - OPC - UNESCALATED
INEELICPP

LOCATION 1:

REQUESTOR:

\section{CONTINGENCY ANALYSIS}

$\begin{array}{cl}\text { TYPE OF ESTIMATE: } & \text { PLANNING } \\ \text { PROAECT NO: } & 242105 \\ \text { PREPARED BY: } & \text { R. D. ADAMS }\end{array}$

DATE: 26-Jan-1998

TME: 12:43:53

REPORT NAME: Contingency Analysis

\begin{tabular}{|c|c|c|c|c|c|c|c|c|c|c|c|}
\hline \multirow{3}{*}{$\begin{array}{l}\text { WBS } \\
\text { Element }\end{array}$} & \multicolumn{8}{|c|}{ PROBABLE \% VARIATION } & \multicolumn{2}{|c|}{$\begin{array}{l}\text { PROJECT } \\
\text { CONTINGENCY }\end{array}$} & \multirow{3}{*}{$\begin{array}{l}\text { SUMMARY } \\
\text { Total Cost } \\
\text { by Element }\end{array}$} \\
\hline & \multirow{2}{*}{ Cost Estimate Eement } & \multirow{2}{*}{$\begin{array}{c}\text { Total Cost w/o } \\
\text { Contingeney }\end{array}$} & \multirow[t]{2}{*}{$\begin{array}{l}\text { \% Total } \\
\text { Cost }\end{array}$} & \multicolumn{2}{|c|}{$\begin{array}{c}\text { Prob. \% Var. } \\
\text { From Est. }\end{array}$} & \multicolumn{2}{|c|}{ Wt. \% of Prob. } & \multirow[t]{2}{*}{ Contingency } & \multirow[t]{2}{*}{$\%$} & \multirow[t]{2}{*}{ Cost } & \\
\hline & & & & - & + & - & + & & & & \\
\hline 1.1 .1 & CONCEPTUAL DESIGN & $5,000,000$ & 30.81 & 20 & 30 & 6.16 & 9.24 & $7.703 \%$ & $30.81 \%$ & $1,254,455$ & $6,254,455$ \\
\hline 1.21 & PM FOR PROSECT DEVELOPMENT. & $3,000,000$ & 18.49 & 20 & 30 & 3.70 & 5.55 & $4.622 \%$ & 18.49\% & 752,673 & $3,752,673$ \\
\hline 1.22 & SAFETY REVIEWS & $1,000,000$ & 6.16 & 20 & 30 & 123 & 1.85 & $1.541 \%$ & $6.16 \%$ & 250,891 & $1,250,891$ \\
\hline 1.3 .1 & PERMITTING & $2,728,432$ & 16.81 & 20 & 30 & 3.35 & 5.04 & $4.203 \%$ & $16.81 \%$ & 684,539 & $3,412,971$ \\
\hline 1.4 .1 & SO TEST \& STARTUP & $4,500,000$ & 27.73 & 20 & 30 & 5.55 & 8.32 & $6.932 \%$ & $27.73 \%$ & $1,129,010$ & $5,629,010$ \\
\hline \multirow[t]{2}{*}{1.52} & PROCUREMENT FEES & 0 & 0.00 & 5 & 20 & 0.00 & 0.00 & $0.000 \%$ & $0.00 \%$ & o) & 0 \\
\hline & ESCALATION & 0 & 0.00 & 20 & 30 & 0.00 & $0.00^{\circ}$ & $0.000 \%$ & $0.00 \%$ & 0 & $\overline{0}$ \\
\hline & SUBTOTAL & $16,228,432$ & 100.00 & & & . & & $25.000 \%$ & & & \\
\hline & CALCULATED CONTINGENCY & $4,057,108$ & & & & & & & & & \\
\hline & RESULTANT TEC & $20,285,540$ & & & & & & & & & \\
\hline & ROUNDED TEC & $20,300,000$ & & & & & & & & & \\
\hline & PROJECT CONTINGENCY & $4,071,568$ & & & & & & $25.09 \%$ & & & \\
\hline & MANAGEMENT RESERVE & 0 & & & & & & & & & \\
\hline & CONTINGENCY & $4,071,568$ & & & & & & & & & \\
\hline & TOTAL ESTIMATED COST & $20,300,000$ & & & & & & & & $4,071,568$ & $20,300,000$ \\
\hline
\end{tabular}

\section{CONFIDENCE LEVEL AND ASSUMED RISKS:}

The Lockheed Idaho Technologies Co. Cost Estimate Contingency Analysis Model is based on the applied contingency and the assumptions upon which the estimate was predicated. The model is applied with a suggested risk level of $18 \%$ and a level of confidence of $90 \%$ the estimate will fall within the bid range. The Contingency Analysis is based on a weighted average to provide a

$90 \%$ probability of underrun and a $10 \%$ probability of overrun.
CONTINGENCY ANALYSIS GUIDE BY TYPE OF ESTIMATE

Guidelines established by DOE/FM 50, Cost Estimating Guide, Vol. 6 , Cost Guide, and as presented in the INEL Cost Estimating Guide.
PLANNING
Experimental/Special Conditions............Up to 50\%
Conceptual
... Experi
TITLE II
...Up to $40 \%$
$10 \%-20 \%$
$5 \%-15 \%$

TITLE II/AFC
Market Conditions 
Lockheed Martin Idaho Technologies Co.

PROSECT NAME: CWO FACILITY - INTERIM STORAGE FACILTYY -OPC - ESCALATED

LOCATION INEELACPP

REOUESTOR:

J.K. RAWLINS
CONTINGENCY ANALYSIS

$\begin{array}{cl}\text { TYPE OF ESTIMATE: } & \text { PLANNING } \\ \text { PROECT NO: } & 242105 \\ \text { PREPARED BY: } & \text { R. D. ADAMS }\end{array}$

DATE: 26-1an-1998

TIME: 12:46:36

REPORT NAME: Contingency Analysis

\begin{tabular}{|c|c|c|c|c|c|c|c|c|c|c|c|}
\hline \multirow{3}{*}{$\begin{array}{l}\text { WBS } \\
\text { Element }\end{array}$} & \multicolumn{8}{|c|}{ PROBABLE \% VARIATION } & \multicolumn{2}{|c|}{$\begin{array}{l}\text { PROJECT } \\
\text { CONTINGENCY }\end{array}$} & \multirow{3}{*}{$\begin{array}{l}\text { SUMMARY } \\
\begin{array}{l}\text { Total Cost } \\
\text { by Element }\end{array}\end{array}$} \\
\hline & \multirow[t]{2}{*}{ Cost Estimate Element } & \multirow{2}{*}{$\begin{array}{c}\text { Total Cost w/o } \\
\text { Contingency }\end{array}$} & \multirow[t]{2}{*}{$\begin{array}{l}\text { \% Total } \\
\text { Cost }\end{array}$} & \multicolumn{2}{|c|}{$\begin{array}{c}\text { Prob. \% Var. } \\
\text { Frpm Est. }\end{array}$} & \multicolumn{2}{|c|}{ Wt. \% of Prob. } & \multirow[t]{2}{*}{ Contingency } & \multirow[t]{2}{*}{$\%$} & \multirow[t]{2}{*}{ Cost } & \\
\hline & & & & - & \pm & - & + & & & & \\
\hline 1.1 .1 & CONCEPTUAL DESIGN & $5,000,000$ & 24.41 & 20 & 30 & 4.88 & 7.32 & $6.103 \%$ & $24.41 \%$ & $1,249,440$ & $6,249,440$ \\
\hline 1.2 .1 & PM FOR PROJECT DEVELOPMENT & $3,000,000$ & 14.65 & 20 & 30 & 2.93 & 4.39 & $3.662 \%$ & $14.65 \%$ & 749,664 & $3,749,664$ \\
\hline 1.2 .2 & SAFETY REVIEWS & $1,000,000$ & 4.88 & 20 & 30 & 0.98 & 1.46 & $1.221 \%$ & $4.88 \%$ & 249,888 & $1,249,888$ \\
\hline 1.3 .1 & PERMITTING & $2,728,432$ & 13.32 & 20 & 30 & 266 & 4.00 & $3.330 \%$ & $13.32 \%$ & 681,803 & $3,410,235$ \\
\hline 1.4 .1 & SO TEST \& STARTUP & $4,500,000$ & 21.97 & 20 & 30 & 4.39 & 6.59 & $5.493 \%$ & $21.97 \%$ & $1,124,496$ & $5,624,496$ \\
\hline \multirow[t]{2}{*}{1.5 .2} & PROCUREMENT FEES & 0 & 0.00 & 5 & 20 & 0.00 & 0.00 & $0.000 \%$ & $0.00 \%$ & 0 & 0 \\
\hline & ESCALATTON & $4,253,402$ & 20.77 & 20 & 30 & 4.15 & $6.233^{2}$ & $5.192 \%$ & $20.77 \%$ & $1,062,875$ & $5,316,277$ \\
\hline \multicolumn{2}{|r|}{ SUBTOTAL } & $20,481,234$ & 100.00 & & & & & $25.000 \%$ & & & \\
\hline \multicolumn{2}{|r|}{ CALCULATED CONTINGENCY } & $5,120,459$ & & & & & & & & & \\
\hline \multicolumn{2}{|r|}{ RESULTANT TEC } & $25,602,293$ & & & & & & & & & \\
\hline \multicolumn{2}{|r|}{ ROUNDED TEC } & $25,600,000$ & & & & & & & & & \\
\hline \multicolumn{2}{|r|}{ PROJECT CONTINGENCY } & $5,118,166$ & & & & & & $24.99 \%$ & & & \\
\hline \multicolumn{2}{|r|}{ MANAGEMENT RESERVE } & 0 & & & & & & & & & \\
\hline \multicolumn{2}{|r|}{ CONTINGENCY } & $5,118,166$ & & & & & & & & & \\
\hline \multicolumn{2}{|r|}{ TOTAL ESTIMATED COST } & $25,600,000$ & & & & & & & & $5,118,166$ & $25,600,000$ \\
\hline
\end{tabular}

CONFIDENCE LEVEL AND ASSUMED RISKS:

The Lockheed Idaho Technologies Co. Cost Estimate Contingency Analysis Model is based on the applied contingency and the assumptions upon which

the estimate was predicated. The model is applied with a suggested risk level

of $18 \%$ and a level of confidence of $90 \%$ the estimate will fall within the bid range.

The Contingency Analysis is based on a weighted average to provide a

$90 \%$ probability of underrun and a $10 \%$ probability of overnun.
CONTINGENCY ANALYSIS GUIDE BY TYPE OF ESTIMATE

Guidelines established by DOE/FM 50, Cost Estimating Guide, Vol. 6 , Cost Guide, and as presented in the INEL Cost Estimating Guide. PLANNING

$20 \%-30 \%$

ditions...........Up to $50 \%$

Experimental/Special Conditions............Up to $40 \%$

TITLE I

TITLE ॥

TITLE II/AFC

$5 \%-15 \%$

Market Conditions 


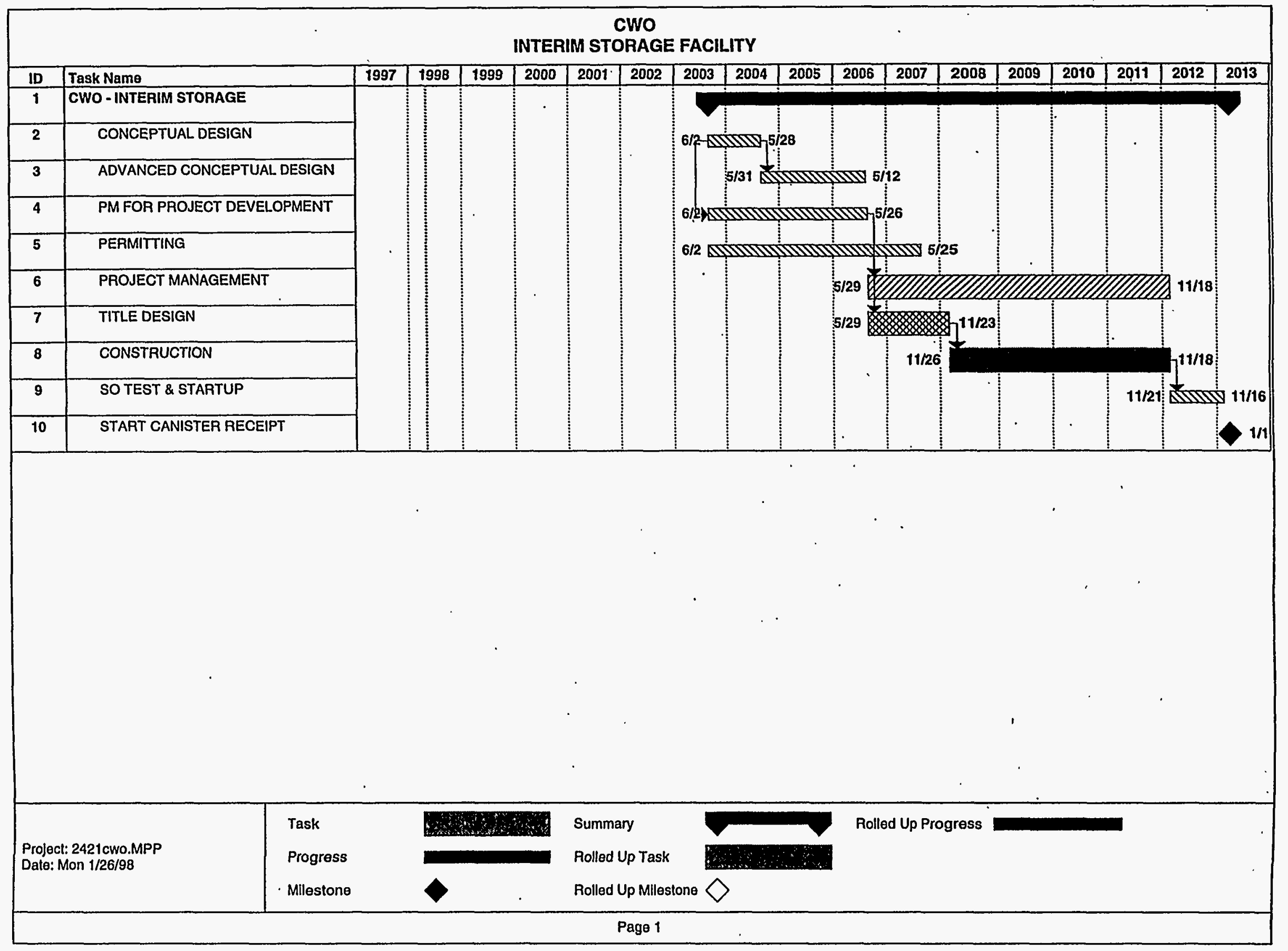


Lockheed Martin Idaho Technologies

(Rev. 6/96)

\section{COST ESTIMATE SUPPORT DATA RECAPITULATION}

Project Title: HLW INTERIM STORAGE FACILITY - HWO

Type of Estimate: PROJECT SUPPORT

File No: 2421-HWO

\author{
Estimator: RDA \\ Date:1/26/98 \\ Approved By:
}

\section{SCOPE OF WORK: Brief description of the proposed project.}

This facility will unload, store in vertical tubes and load out canisters containing High Level Waste (HLW)

from the proposed Hot Isokinetic Press Waste Option (VWO).

II. BASIS OF THE ESTIMATE : Drawings, Design Report, Engineers notes, and/or other documentation upon which the estimate is originated.

Draft sketches, a draft EDF, and the SNF Canister Storage Building Final Design Estimate from Fluor Daniel Northwest, Inc. were used as the basis for this estimate.

III. ASSUMPTIONS : Condition statements accepted or supposed true without proof or demonstration. An assumption has a direct impact on total estimated cost.

1. The Interim Storage Facility will be located within 100 feet of the HWO facility.

2. Rock will not be encountered during excavation.

3. The estimates include both present day costs sumaries and contingency analysis and escalated sumaries and contingency analysis. The escalated summaries and contingency analysis reflect the attached schedule. AlI activities are escalated to the appropriate schedule mid-point.

4. Per the draft EDF, four storage modules, containing 630 storage tubes each, will accomodate the estimated 5,700 canisters produced at the HIP Facility.

5. Most quantities used for this estimate were obtained by preliminary take offs from the draft sketches.

6. The storage tube costs, gantry crane cost and associated rail system costs were used from the Fluor estimate of a facility which is approximately eighty percent complete.

7. All assumptions and information included in the draft "New Interim Storage Facility for Interim Storage of $\mathrm{HLW}^{\prime \prime}$ apply to the estimate.

8. Where quantities could not be determined from the draft sketches, factors or assumed quantities were used by the estimator.

9. Since the storage tubes and conveying equipment represent such a large percentage of this facility's cost, lower design, inspection, Project Management and turnover costs are used in the estimate.

10. Sufficient construction craft will be available to support this project.

11. Markups on direct construction costs to get the construction costs listed on the sumary sheet are as follows:

Labor indirects, overheads and profit (OH\&P) 508 of labor

Material \& equipment OH\&P 58 of material and equipment

Subcontract OH\&O

108 of subcontract cost

Construction equipment allowance $10 \%$ of labor

NQA-1 quality requirements $30 \%$ of material

DOE/RW/0333P quality requirements $10 \%$ of material

Subcontractor procurement 18 of material and equipment Bond

$2 \%$ of everything 
Iockheed Martin Idaho Technologies

\section{COST ESTIMATE SUPPORT DATA RECAPITULATION} (CONTINUATION)

File No: 2396

Page 2 of 2

\section{ASSUMPTIONS: (Continued)}

12. Only the railroad track within the storage facility is included in this estimate. The remainder of the branch line is included in the infrastructure estimate.

13. All utilities are assumed to be available at the facility wall.

IV. CONTINGENCY GUIDEIINE IMPLEMENTATION: The percentage used for contingency as determined by the contingency allowance guidelines can be aftered to refiect the type of construction and conditions that may impact the total estimated cost.

A large portion of the estimated costs is based on actual costs from Fluor's SNF Canister Storage Building. The majority of the remaining costs are for standard construction activities. For these reasons, the contingency is lower than would be experienced for a processing type facility at this stage of development.

V. OTHER COMMENTS/CONCERNS SPECIFIC TO THE ESTIMATE:

1. All FY' 98 and later projects are to be assessed a Procurement Fee of $1 \%$, a G\&A Fee of $23 \%$, with a $\$ 500,000$ ceiling per year, and a Performance Incentive Fee (PIF) of $5.5 \%$. See the attached G\&A/PIF calculation sheet for the method used to calculate these fees.

2. A procurement fee of one percent of construction was used to cover the operating contractor support to DOE-ID for their contract administration. It is felt that one percent is sufficient for jobs of this size. 
Lockheed Martin Idaho Technologies Co. PRONECT NAME: HIP FACILITY - INTERIM STORAGE FACILITY -ESCALATED

LOCATION :: REOUESTOR:
COST ESTIMATE SUMMARY

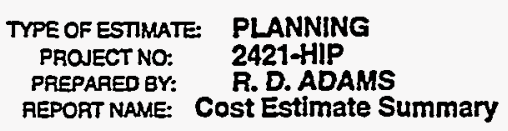

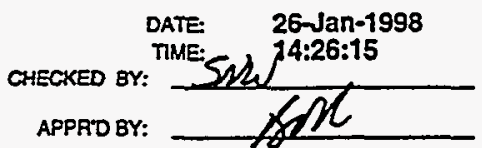

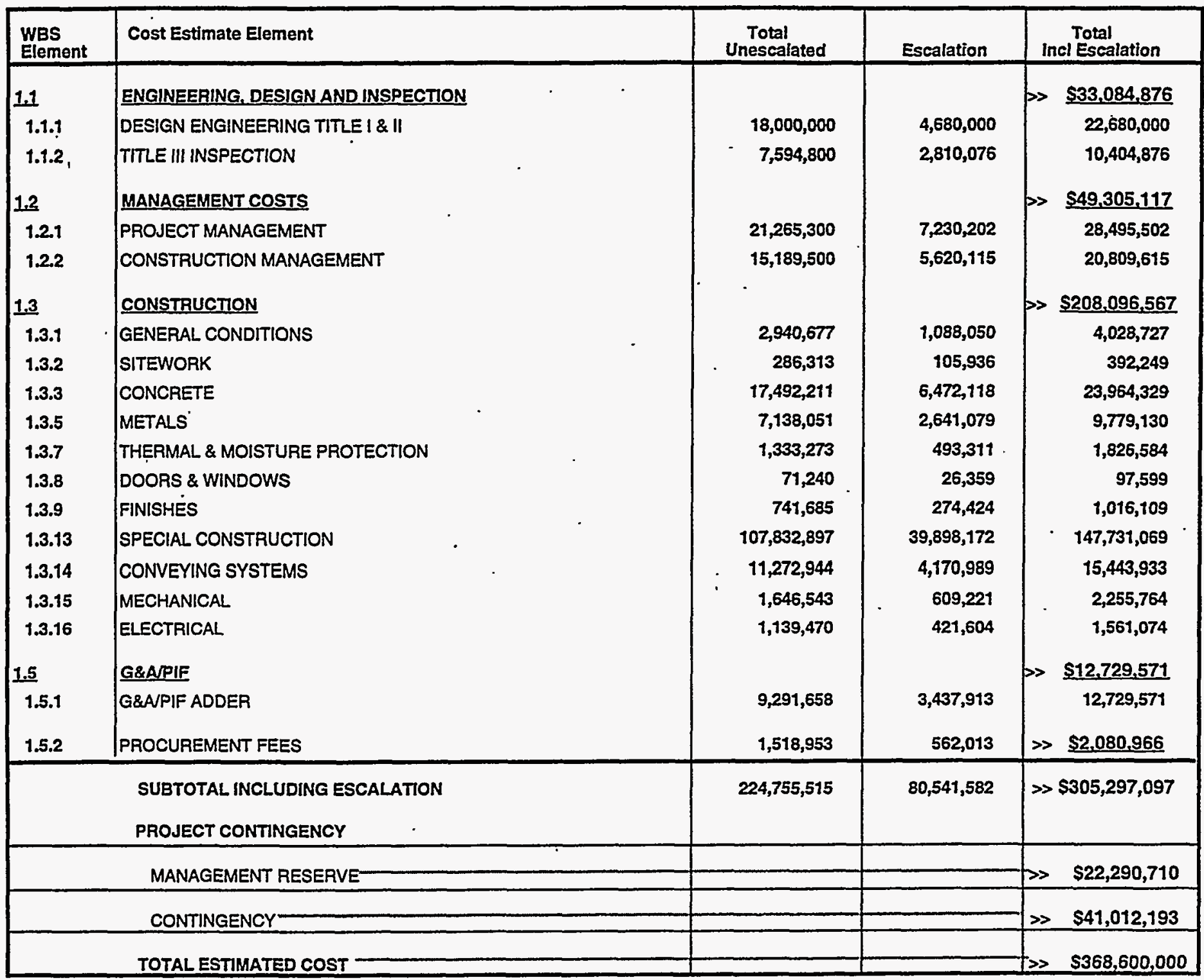

PROJECT COST PARAMETERS

EDI AS A $\%$ OF CONST. + GFE $=16.00 \%$ 
Lockheed Martin Idaho Technologies Co. PROJECT NAME: HIP FACILITY - INTERIM STORAGE FACILITY - UNESCALATED

INEELICPP

LOCATION ::

REQUESTOR:

J. K. RAWLINS
COST ESTIMATE SUMMARY

TYPE OF ESTIMATE: PLANNING PROSECT NO: 2421-HIP PREPARED BY: R. D.ADAMS REPORT NAME: Cost Estimate Summary

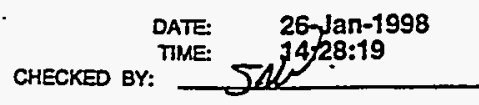

APPRD BY:

\begin{tabular}{|c|c|c|c|c|}
\hline $\begin{array}{l}\text { WBS } \\
\text { Element }\end{array}$ & Cost Estimate Element & $\begin{array}{c}\text { Total } \\
\text { Unescalated }\end{array}$ & Escalation & $\begin{array}{c}\text { Total } \\
\text { Incl Escalation }\end{array}$ \\
\hline 1.1 & ENGINEERING, DESIGN AND INSPECTION & & 4 & $\gg \$ 25,594,800$ \\
\hline 1.1.1 & DESIGN ENGINEERING TITLE I \& \| & $18,000,000$ & 0 & $18,000,000$ \\
\hline 1.1 .2 & TITLE III INSPECTION & $7,594,800$ & 0 & $7,594,800$ \\
\hline 1.2 & MANAGEMENT COSTS & & & $\gg \$ \$ 36,454,800$ \\
\hline 1.2 .1 & PROJECT MANAGEMENT & $21,265,300$ & 0 & $21,265,300$ \\
\hline 1.2 .2 & CONSTRUCTION MANAGEMENT & $15,189,500$ & 0 & $15,189,500$ \\
\hline 1.3 & CONSTRUCTION & & & $\gg \$ 151,895,304$ \\
\hline 1.3.1 & GENERAL CONDITIONS & $2,940,677$ & 0 & $2,940,677$ \\
\hline 1.3 .2 & SITEWORK & 286,313 & 0 & 286,313 \\
\hline 1.3.3 & CONCRETE & $17,492,211$ & $\mathbf{0}$ & $17,492,211$ \\
\hline 1.3.5 & METALS & $7,138,051$ & 0 & $7,138,051$ \\
\hline 1.3.7 & THERMAL \& MOISTURE PROTECTION & $1,333,273$ & $\mathbf{0}$ & $1,333,273$ \\
\hline 1.3.8 & DOORS \& WINDOWS & $71,240^{\circ}$ & 0 & 71,240 \\
\hline 1.3 .9 & FINISHES & 741,685 & 0 & 741,685 \\
\hline 1.3.13 & SPECIAL CONSTRUCTION & $107,832,897$ & 0 & $107,832,897$ \\
\hline 1.3 .14 & CONVEYING SYSTEMS & $11,272,944$ & 0 & $11,272,944$ \\
\hline 1.3.15 & MECHANICAL & $1,646,543$ & 0 & $1,646,543$ \\
\hline 1.3 .16 & ELECTRICAL & $1,139,470$ & 0 & $1,139,470$ \\
\hline 1.5 & G\&AMPIF & & & $\$ 9.291,658$ \\
\hline 1.5.1 & G\&AVIF ADDER & $9,291,658$ & 0 & $9,291,658$ \\
\hline 1.5 .2 & PROCUREMENT FEES & $1,518,953$ & 0 & $\gg \$ 1,518,953$ \\
\hline • & $\begin{array}{l}\text { SUBTOTAL INCLUDING ESCALATION } \\
\text { PROJECT CONTINGENCY }\end{array}$ & $224,755,515$ & 0 & >> $\$ 224,755,515$ \\
\hline & MANAGEMENT RESERVE- & & & $\gg \quad \$ 16,270,591$ \\
\hline & CONTINGENCY - & & & $\gg \quad \$ 30,473,894$ \\
\hline & TOTAL ESTIMATED COST - & & & $\gg \quad \$ 271,500,000$ \\
\hline
\end{tabular}

PROJECT COST PARAMETERS

EDI AS A $\%$ OF CONST. + GFE $=17.00 \%$ 
Lockheed Martin Idaho Technologles Co. Rov. 696

PROJECT NAME: HIP FACILITY - INTERIM STORAGE FACILITY

LOCATION 1: INEELICPP

REQUESTOR: J. K. RAWLINS
DETAILED COST ESTIMATE SHEET

TYPE OF ESTIMATE: PLANNING

PROJECT NO:: 2421-HIP

PAEPARED BY: R. D.ADAMS
PAgeः 1

DATE 26-Jan-1998

TIME: 14:25:04

Report name: Detall Cost Estlmate Sheot

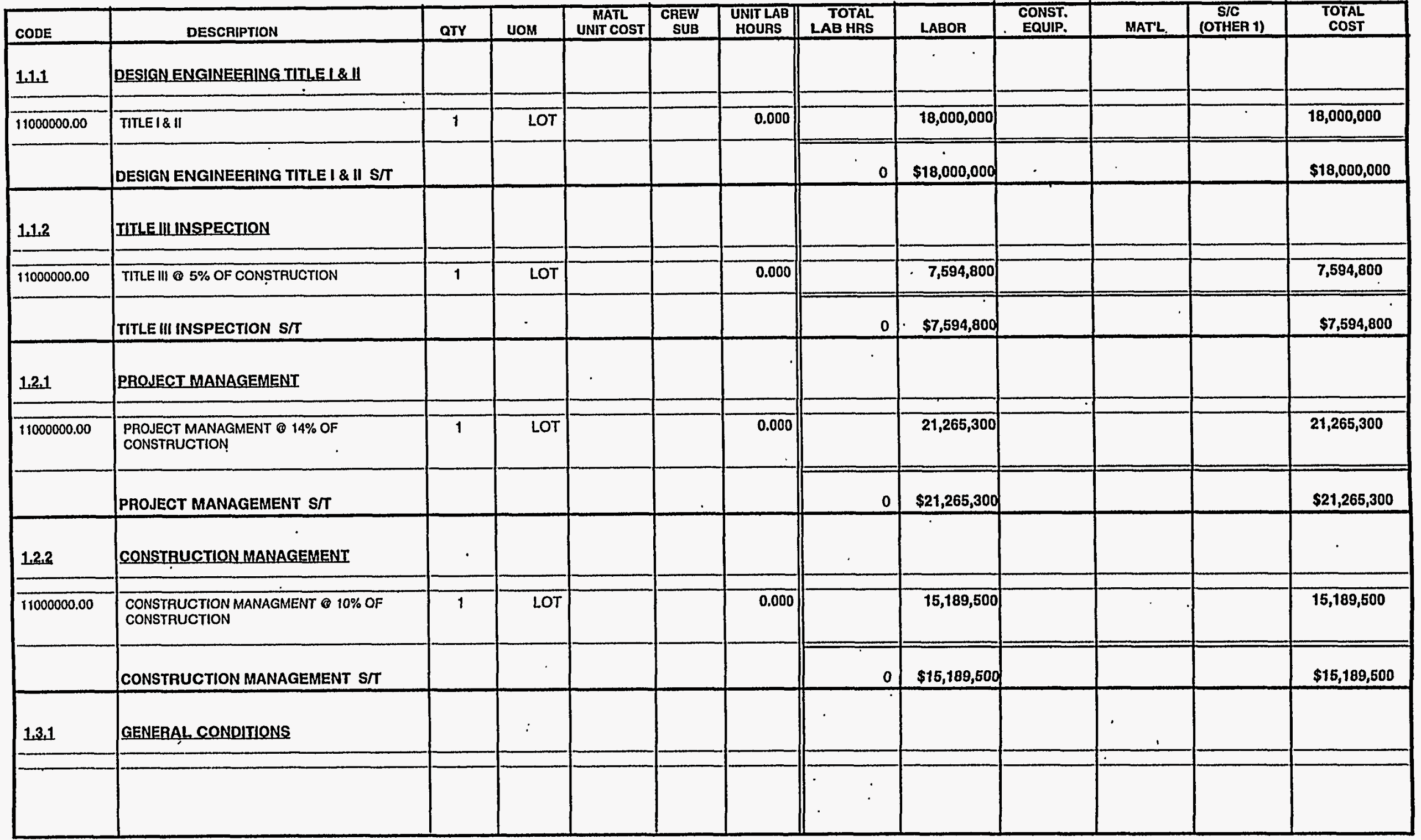


Lockheed Martin Idaho Technologles Co.

$$
\text { Rov. 6/90 }
$$

PROJECT NAME: HIP FACILITY - INTERIM STORAGE

FACILITY

LOCATION : INEEL/ICPP

REQUESTOR: J. K. RAWLINS

\section{DETAILED COST ESTIMATE SHEET}

TYPE OF ESTIMATE: PLANNING

PROJECT NO:: 2421-HIP

PREPARED BY: R. D. ADAMS
PAgE 2

DATE 26-Jan-1998

TIME: 14:25:04

REPORT NAME: Detall Cost Estimate Sheet

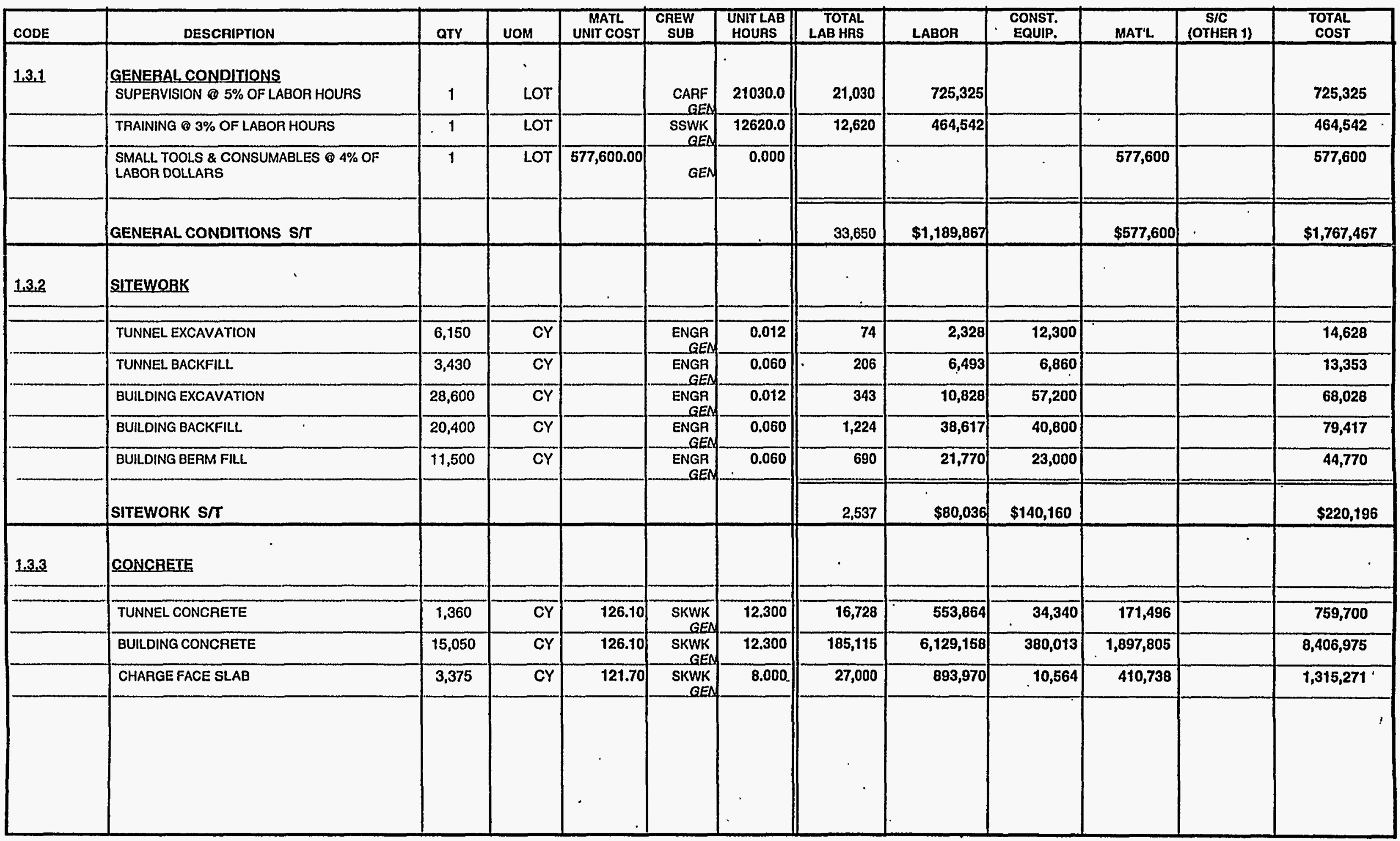


Lockheed Martin Idaho Technologles Co.

Revi. 6/96

PROJECT NAME: HIP FACILITY - INTERIM STORAGE

FACILITY

LOCATION 1: INEELICPP

REQUESTOR: J. K. RAWLINS
DETAILED COST ESTIMATE SHEET

TYPE OF ESTIMATE: PLANNING

PROJECT NO.: 2421-HIP

PREPARED BY: R. D. ADAMS
PAGE 3

DATE 26-Jan-1998

TIME: 14:25:04

AEPORT NAME: Dotall Cost Estimate Sheot

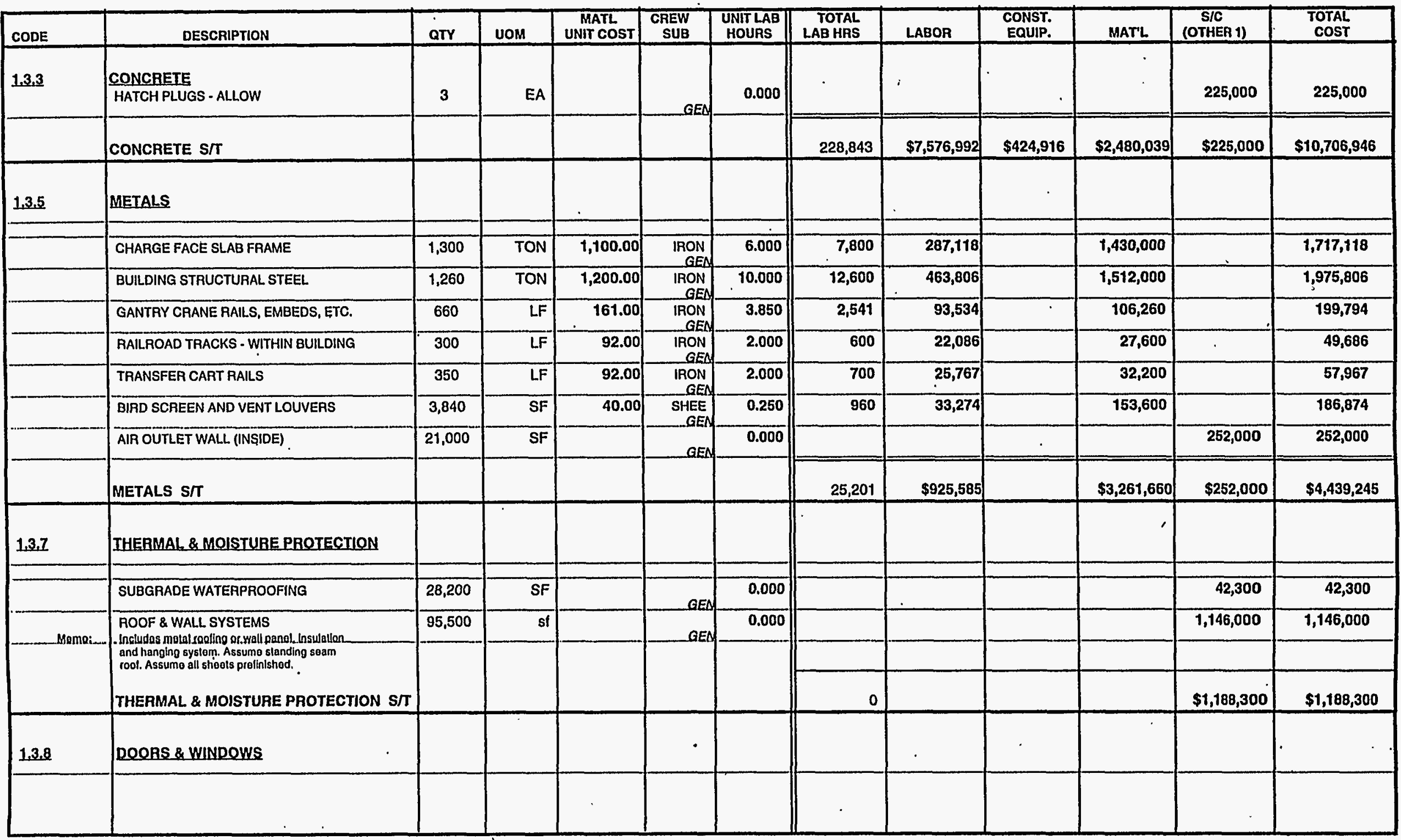


Lockheed Martin Idaho Technologies Co.

Rov. 6/86

PROIECT NAME HIP FACILITY - INTERIM STORAGE

FACILITY

LOCATION 1: INEELICPP

REQUESTOR: J. K. RAWLINS
DETAILED COST ESTIMATE SHEET

TYPE OF ESTIMATE: PLANNING

PROJECT NO: 2421-HIP

PREPARED BY: R. D. ADAMS
PAGE * 4

DATE 26-Jan-1998

TIME: 14:25:04

Report NaMe: Detall Cost Estimate Sheet

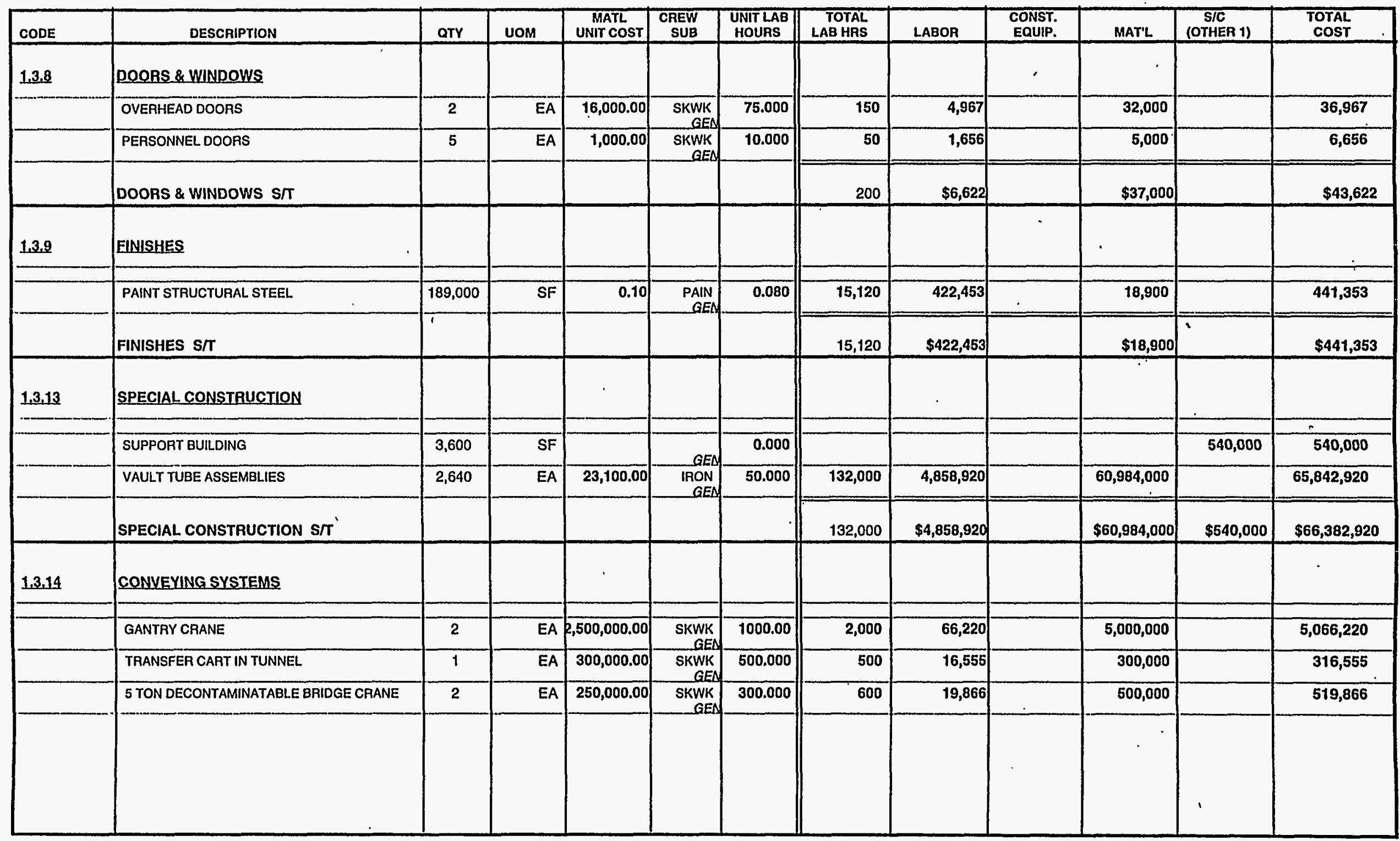


Lockheed Martin Idaho Technologies Co.

Rov. 6/96

PROJECT NAME: HIP FACILITY - INTERIM STORAGE

FACILITY

REQUESTOR: J.K. RAWLINS

\section{DETAILED COST ESTIMATE SHEET}

TYPE OF ESTIMATE: PLANNING

PROJECT NO: 2421-HIP

PREPARED BY: R. D. ADAMS
PAGE 5

DATE 26-Jan-1998

TIME: 14:25:04

REPORT NAME: Detall Cost Estlmate Sheot

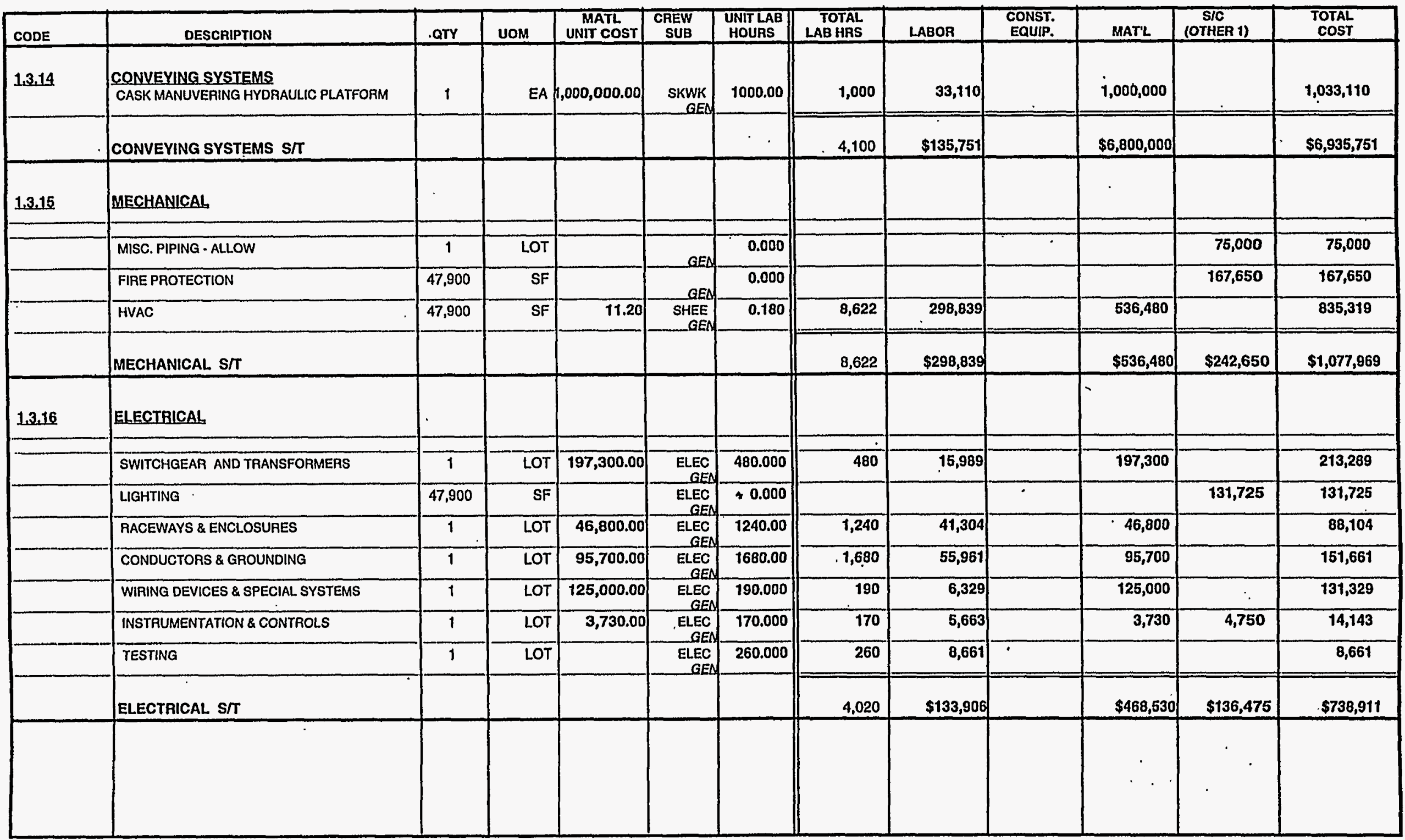


Lockheed Martin Idaho Technologies Co. Rov. 6/88

PROJECT NAME: HIP FACILITY - INTERIM STORAGE FACILITY

LOCATION 1: INEELICPP

REQUESTOR: J. K. RAWLINS

\section{DETAILED COST ESTIMATE SHEET}

TYPE OF ESTIMATE: PLANNING

PROJECT NO: 2421-HIP

PAEPARED BY: R. D. ADAMS
PAGE 6

DATE 26-Jan-1998

TIME: 14:26:04

RePORT NAME: Detall Cost Estlmate Sheet

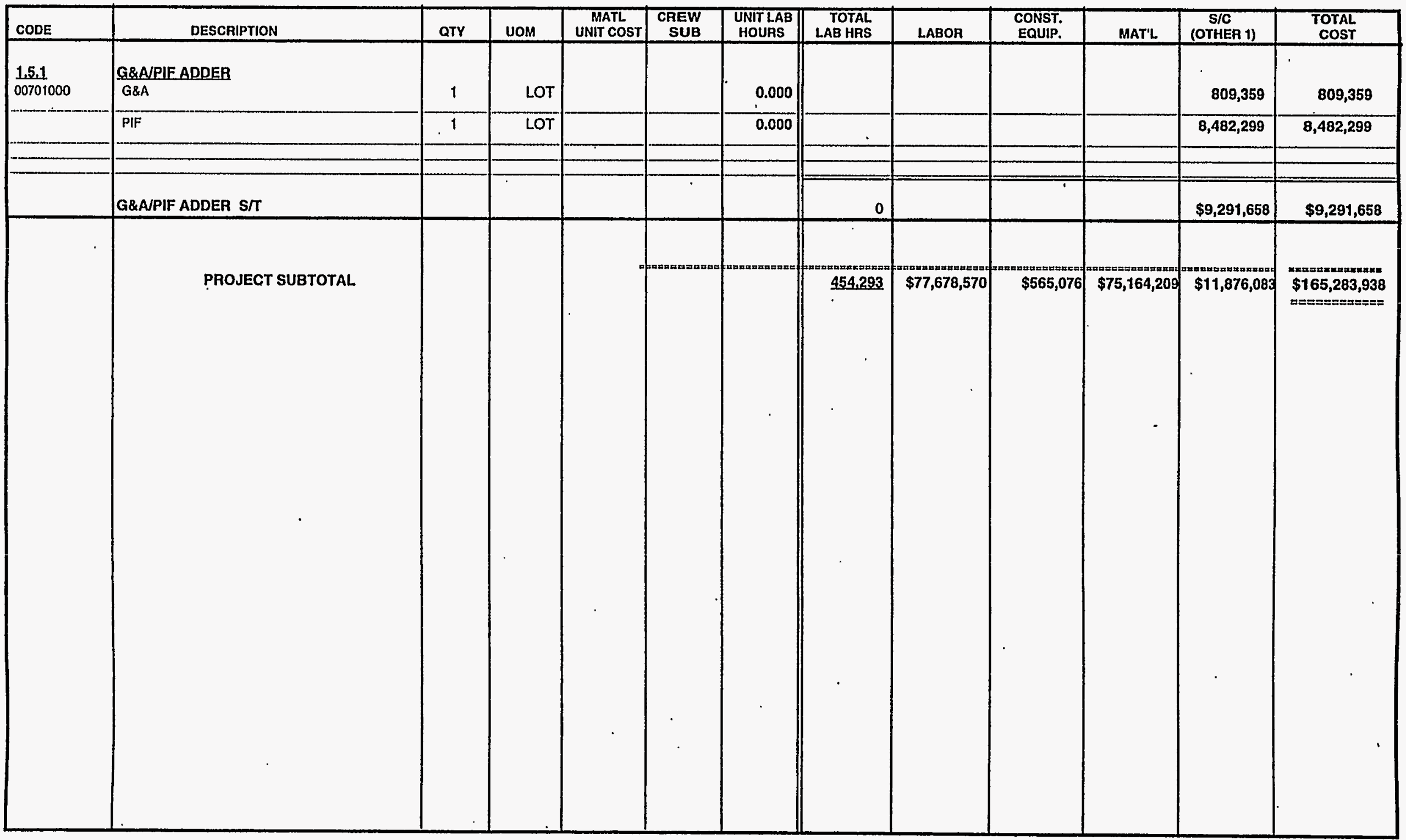


Lockheed Martin Idaho Technologies Co. PROIECT NAME: HIP FACILITY - INTERIM STORAGE FACILITY - UNESCALATED

LOCATION t: REQUESTOR:
CONTINGENCY ANALYSIS

$\begin{array}{cl}\text { TYPE OF ESTMATE: } & \text { PLANNING } \\ \text { PROJECT NO: } & \text { 2421-HIP } \\ \text { PREPARED BY: } & \text { R. D. ADAMS }\end{array}$

DATE: 26-Jan-1998

TME: 14:28:24

REPORT NAME: Contingency Analysis

\begin{tabular}{|c|c|c|c|c|c|c|c|c|c|c|c|}
\hline & PROBA & BLE \% VARIA & ON & & & & & & $\begin{array}{l}\text { PROJ } \\
\text { CONTI }\end{array}$ & $\begin{array}{l}\text { CT } \\
\text { IENCY }\end{array}$ & SUMMARY \\
\hline $\begin{array}{l}\text { WBS } \\
\text { Element }\end{array}$ & Cost Estimate Element & Total Cost w/o & $\begin{array}{c}\text { \% Total } \\
\text { Cost }\end{array}$ & & $\begin{array}{l}\text { \% Var. } \\
\text { m Est. }\end{array}$ & Wt \% & Prob. & Contingency & $\%$ & Cost & Total Cost \\
\hline & & Contingency & & - & + & - & + & & & & by Element \\
\hline 1.1.1 & DESIGN ENGINEERING TITLE $\mid \& \|$ & $18,000,000$ & 8.01 & 20 & 35 & 1.60 & 2.80 & $2.363 \%$ & $11.35 \%$ & $5,307,684$ & $23,307,624$ \\
\hline 1.1 .2 & TIILE III INSPECTION & $7,594,800$ & 3.38 & 20 & 30 & 0.68 & 1.01 & $0.845 \%$ & $4.06 \%$ & $1,897,872$ & $9,492,672$ \\
\hline 1.21 & PROJECT MANAGEMENT & $21,265,300$ & 9.46 & 20 & 35 & 1.89 & 3.31 & $2.791 \%$ & $13.41 \%$ & $6,270,528$ & $27,535,828$ \\
\hline 1.2 .2 & CONSTRUCTION MANAGEMENT & $15,189,500$ & 6.76 & 20 & 30 & 1.35 & 2.03 & $1.690 \%$ & $8.12 \%$ & $3,795,719$ & $18,985,219$ \\
\hline 1.3 .1 & GENERAL CONDITIONS & $2,940,677$ & 1.31 & 20 & 35 & 0.26 & 0.46 & $0.386 \%$ & $1.86 \%$ & 867,121 & $3,807,798$ \\
\hline 1.3 .2 & SITEWORK & 286,313 & 0.13 & 20 & 30 & 0.03 & 0.04 & $0.032 \%$ & $0.15 \%$ & 71,547 & 357,860 \\
\hline 1.3 .3 & CONCRETE & $17,492,211$ & 7.78 & 20 & 30 & 1.56 & 2.33 & $1.946 \%$ & $9.35 \%$ & $4,371,146$ & $21,863,357$ \\
\hline 1.3 .5 & METALS & $7,138,051$ & 3.18 & 20 & 25 & 0.64 & 0.79 & $0.651 \%$ & $3.13 \%$ & $1,462,662$ & $8,600,713$ \\
\hline 1.3 .7 & THERMAL \& MOISTURE PROTECTION & $1,333,273$ & 0.59 & 20 & 30 & 0.12 & 0.18 & $0.148 \%$ & $0.71 \%$ & 333,173 & $1,666,446$ \\
\hline 1.3 .8 & DOORS \& WINDOWS & 71,240 & 0.03 & 20 & 20 & 0.01 & 0.01 & $0.005 \%$ & $0.02 \%$ & 11,393 & 82,633 \\
\hline 1.3 .9 & FINISHES & 741,685 & 0.33 & 20 & 35 & 0.07 & 0.12 & $0.097 \%$ & $0.47 \%$ & 218,702 & 960,387 \\
\hline 1.3 .13 & SPECIAL CONSTRUCTION & $107,832,897$ & 47.98 & 20 & 20 & 9.60 & 9.60 & $7.676 \%$ & $36.89 \%$ & $17,245,739$ & $125,078,636$ \\
\hline 1.3 .14 & CONVEYING SYSTEMS & $11,272,944$ & 5.02 & 20 & 25 & 1.00 & 1.25 & $1.028 \%$ & $4.94 \%$ & $2,309,946$ & $13,582,890$ \\
\hline 1.3 .15 & MECHANICAL & $1,646,543$ & 0.73 & 20 & 30 & 0.15 & 0.22 & $0.183 \%$ & $0.88 \%$ & 411,456 & $2,057,999$ \\
\hline 1.3 .16 & ELECTRICAL & $1,139,470$ & 0.51 & 20 & 25 & 0.10 & 0.13 & $0.104 \%$ & $0.50 \%$ & 233,489 & $1,372,959$ \\
\hline 1.5 .1 & G\&AIPIF ADDER & $9,291,658$ & 4.13 & 5 & 20 & 0.21 & 0.83 & $0.723 \%$ & $3.48 \%$ & $1,625,331$ & $10,916,989$ \\
\hline 1.52 & PROCUREMENT FEES & $1,518,953$ & 0.68 & 20 & 25 & 0.14 & 0.17 & $0.139 \%$ & $0.67 \%$ & 311,250 & $1,830,203$ \\
\hline & ESCALATION & 0 & 0.00 & 20 & 25 & 0.00 & 0.00 & $0.000 \%$ & $0.00 \%$ & (273) & (273) \\
\hline & SUBTOTAL. & $224,755,515$ & 100.00 & & & & & $20.807 \%$ & & & \\
\hline & CALCULATED CONTINGENCY & $46,765,153$ & & & & & & & & & \\
\hline & RESULTANT TEC & $271,520,668$ & & & & & & & & & \\
\hline & ROUNDED TEC & $271,500,000$ & & & & & & & & & \\
\hline & PROJECT CONTINGENCY & $46,744,485$ & & & & & & $20.80 \%$ & & & \\
\hline & MANAGEMENT RESERVE & $16,270,591$ & & & & & & & & & \\
\hline & CONTINGENCY & $30,473,894$ & & & & & & & & & \\
\hline & TOTAL ESTIMATED COST & $271,500,000$ & & & & & & & & $46,744,485$ & $271,500,000$ \\
\hline
\end{tabular}

\section{CONFIDENCE LEVEL AND ASSUMED RISKS:}

The Lockheed Idaho Technologies Co. Cost Estimate Contingency Analysis Model is based on the applied contingency and the assumptions upon which the estimate was predicated. The model is applied with a suggested risk level of $18 \%$ and a level of confidence of $90 \%$ the estimate will fall within the bid range. The Contingency Analysis is based on a weighted average to provide a

$90 \%$ probability of underrun and a $10 \%$ probability of overrun.
CONTINGENCY ANALYSIS GUIDE BY TYPE OF ESTIMATE Guidelines established by DOE/FM 50, Cost Estimating Guide, Vol. 6 , Cost Guide, and as presented in the INEL Cost Estimating Guide. PLANNING $20 \%-30 \%$ Experimental/Special Conditions............Up to 50\% Conceptual $15 \%-25 \%$ Experimental/Special Conditions............ TITLE

TITLE II TITLE II/AFC 
Lockheed Martin Idaho Technologies Co.

PROIECT NAME: HIP FACILITY - INTERIM STORAGE FACILITY - ESCALATED

LOCATION 1: INEELICPP

REOUESTOR: J.K. RAWLINS

\section{CONTINGENCY ANALYSIS}

$\begin{array}{cl}\text { TYPE OF ESTIMATE: } & \text { PLANNING } \\ \text { PROSECT NO: } & \text { 2421-HIP } \\ \text { PREPARED BY: } & \text { R. D. ADAMS }\end{array}$

DATE: $26-1 a n-1998$

TIME: 14:26:21

REPORT NAME: Contingency Analysis

\begin{tabular}{|c|c|c|c|c|c|c|c|c|c|c|c|}
\hline \multicolumn{9}{|c|}{ PROBABLE \% VARIATION } & \multicolumn{2}{|c|}{$\begin{array}{l}\text { PROJECT } \\
\text { CONTINGENCY }\end{array}$} & \multirow{3}{*}{$\begin{array}{l}\text { SUMMARY } \\
\begin{array}{l}\text { Total Cost } \\
\text { by Element }\end{array}\end{array}$} \\
\hline \multirow[t]{2}{*}{$\begin{array}{l}\text { WBS } \\
\text { Element }\end{array}$} & \multirow[t]{2}{*}{ Cost Estimate Element } & \multirow{2}{*}{$\begin{array}{c}\text { Total Cost w/o } \\
\text { Contingency }\end{array}$} & \multirow[t]{2}{*}{$\begin{array}{l}\text { \% Total } \\
\text { Cost }\end{array}$} & \multicolumn{2}{|c|}{$\begin{array}{c}\text { Prob. \% Var. } \\
\text { From Est. }\end{array}$} & \multicolumn{2}{|c|}{ Wt. $\%$ of Prob. } & \multirow[t]{2}{*}{ Contingency } & \multirow[t]{2}{*}{$\%$} & \multirow[t]{2}{*}{ Cost } & \\
\hline & & & & - & + & $=$ & + & & & & \\
\hline 1.1.1 & DESIGN ENGINEERING TILE I \& II & $18,000,000$ & 5.90 & 20 & 35 & 1.18 & 2.06 & $1.739 \%$ & $8.39 \%$ & $5,312,268$ & $23,312,268$ \\
\hline 1.1 .2 & TITLE III INSPECTION & $7,594,800$ & 2.49 & 20 & 30 & 0.50 & 0.75 & $0.622 \%$ & $3.00 \%$ & $1,899,511$ & $9,494,311$ \\
\hline 12.1 & PROJECT MANAGEMENT & $21,265,300$ & 6.97 & 20 & 35 & 1.39 & 2.44 & $2.055 \%$ & $9.91 \%$ & $6,275,943$ & $27,541,243$ \\
\hline 1.22 & CONSTRUCTION MANAGEMENT & $15,189,500$ & 4.98 & 20 & 30 & 1.00 & 1.49 & $1.244 \%$ & $6.00 \%$ & $3,798,997$ & $18,988,497$ \\
\hline 1.3 .1 & GENERAL CONDITIONS & $2,940,677$ & 0.96 & 20 & 35 & 0.19 & 0.34 & $0.284 \%$ & $1.37 \%$ & 867,870 & $3,808,547$ \\
\hline 1.3 .2 & SITEWORK & 286,313 & 0.09 & 20 & 30 & 0.02 & 0.03 & $0.023 \%$ & $0.11 \%$ & 71,609 & 357,922 \\
\hline 1.3 .3 & CONCRETE & $17,492,211$ & 5.73 & 20 & 30 & 1.15 & 1.72 & $1.432 \%$ & $6.91 \%$ & $4,374,921$ & $21,867,132$ \\
\hline 1.3 .5 & METALS & $7,138,051$ & 2.34 & 20 & 25 & 0.47 & 0.58 & $0.479 \%$ & $2.31 \%$ & $1,463,925$ & $8,601,976$ \\
\hline 1.3 .7 & THERMAL \& MOISTURE PROTECTION & $1,333,273$ & 0.44 & 20 & 30 & 0.09 & 0.13 & $0.109 \%$ & $0.53 \%$ & 333,461 & $1,656,734$ \\
\hline 1.3 .8 & DOORS \& WINDOWS & 71,240 & 0.02 & 20 & 20 & 0.00 & 0.00 & $0.004 \%$ & $0.02 \%$ & 11,403 & 82,643 \\
\hline 1.3 .9 & FINISHES & 741,685 & 0.24 & 20 & 35 & 0.05 & 0.09 & $0.072 \%$ & $0.35 \%$ & 218,891 & 960,576 \\
\hline 1.3 .13 & SPECIAL CONSTRUCTION & $107,832,897$ & 35.32 & 20 & 20 & 7.06 & 7.06 & $5.651 \%$ & $27.27 \%$ & $17,260,633$ & $125,093,530$ \\
\hline 1.3 .14 & CONVEYING SYSTEMS & $11,272,944$ & 3.69 & 20 & 25 & 0.74 & 0.92 & $0.757 \%$ & $3.65 \%$ & $2,311,941$ & $13,584,885$ \\
\hline 1.3 .15 & MECHANICAL & $1,646,543$ & 0.54 & 20 & 30 & 0.11 & 0.16 & $0.135 \%$ & $0.65 \%$ & 411,812 & $2,058,355$ \\
\hline 1.3 .16 & ELECTRICAL & $1,139,470$ & 0.37 & 20 & 25 & 0.07 & 0.09 & $0.077 \%$ & $0.37 \%$ & 233,691 & $1,373,161$ \\
\hline 1.5 .1 & G\&AVPIF ADDER & $9,291,658$ & 3.04 & 5 & 20 & 0.15 & 0.61 & $0.533 \%$ & $2.57 \%$ & $1,626,735$ & $10,918,393$ \\
\hline \multirow[t]{2}{*}{1.5 .2} & PROCUREMENT FEES & $1,518,953$ & 0.50 & 20 & 25 & 0.10 & 0.12 & $0.102 \%$ & $0.49 \%$ & 311,518 & $1,830,471$ \\
\hline & ESCALATION & $80,541,582$ & 26.38 & 20 & 25 & 5.28 & 6.60 & $5.408 \%$ & $26.09 \%$ & $16,517,774$ & $97,059,356$ \\
\hline & SUBTOTAL & $305,297,097$ & 100.00 & & & & & $20.726 \%$ & & & \\
\hline & CALCULATED CONTINGENCY & $63,276,177$ & & & & & & & & & \\
\hline & RESULTANT TEC & $368,573,274$ & & & & & & & & & \\
\hline & ROUNDED TEC & $368,600,000$ & & & & & & & & & \\
\hline & PROJECT CONTINGENCY & $63,302,903$ & & & & & & $20.73 \%$ & & & \\
\hline & MANAGEMENT RESERVE & $22,290,710$ & & & & & & & & & \\
\hline & CONTINGENCY & $41,012,193$ & & & & & & & & & \\
\hline & TOTAL ESTIMATED COST & $368,600,000$ & & & & & & & & $63,302,903$ & $368,600,000$ \\
\hline
\end{tabular}

\section{CONFIDENCE LEVEL AND ASSUMED RISKS:}

The Lockheed Idaho Technologies Co. Cost Estimate Contingency Analysis Model is based on the applied contingency and the assumptions upon which the estimate was predicated. The model is applied with a suggested risk level of $18 \%$ and a level of confidence of $90 \%$ the estimate will fall within the bid range. The Contingency Analysis is based on a weighted average to provide a

$90 \%$ probability of underrun and a $10 \%$ probability of overrun.
CONTINGENCY ANALYYSIS GUIDE BY TYPE OF ESTIMATE

Guidelines established by DOE/FM 50, Cost Estimating Guide, Vol. 6,

Cost Guide, and as presented in the INEL Cost Estimating Guide. PLANNING $20 \%-30 \%$ Experimental/Special Conditions.............. to to 50\% Conceptual $\quad 15 \%-25 \%$ Experimental/Special Conditions.............. Up to $40 \%$ TITLE I

TITLE II

TITLE IIIAFC
$10 \%-20 \%$
$5 \%-15 \%$ Market Conditions 


\section{G\&APIF ADDER CALCULATION SHEET}

PROCUREMENT FEE:

\begin{tabular}{|c|c|c|c|c|}
\hline $\begin{array}{l}\text { CONSTRUCTION = } \\
\text { GFE = }\end{array}$ & Subtotal & $\begin{array}{r}\$ 151,895,303 \\
\$ 0 \\
\$ 151,895,303\end{array}$ & & \\
\hline FEE @ $1 \%=$ & & $\$ 151,895,303$ & $\cdot 0.01=$ & $\$ 1,518,953.03$ \\
\hline
\end{tabular}

G\&A @ 23\% (with a ceiling of $\$ 500,000$ imposed per year

CONSTRUCTION OR CEILING $* 4$ YEARS $=\quad \$ 2,000,000$

GFE = $\$ 0$

PROCUREMENT FEE $=$

$\$ 1,518,953$

Subtotal $\$ 3,518,953$

FEE @ 23\% =

$\$ 3,518,953 \cdot 0.23=$

$\$ 809,359$

PIF @ 5.5\%

\begin{tabular}{|c|c|}
\hline $\begin{array}{l}\text { CONSTRUCTION = } \\
\text { GFE = } \\
\text { PROCUREMENT FEE }= \\
\text { G\&A }=\quad \text { Subtotal }\end{array}$ & $\begin{array}{r}\$ 151,895,303 \\
\$ 0 \\
\$ 1,518,953 \\
\$ 809,359 \\
\$ 154,223,615\end{array}$ \\
\hline FEE @ $5.5 \%=$ & $\$ 154,223,615 \cdot 0.055=$ \\
\hline
\end{tabular}

TOTAL PROCUREMENT FEE:

$\$ 1,518,953$

TOTAL G\&A FEE:

$\$ 809,359$

TOTAL PIF:

$\$ 8,482,299$ 

Lockheed Martin Idaho Technologies Co.

$$
\text { Rev. } 6 / 96
$$

PROIECT NAME: HIP FACILITY - INTERIM STORAGE

OCATION FACILITY - OPC - ESCALATED

REQUESTOR: J.K. RAWLINS
COST ESTIMATE SUMMARY

TYPE OF ESTIMATE: PLANNING PRONECT NO: 242102 PREPARED BY: R.D. ADAMS REPORT NAME: Cost Estimate Summary

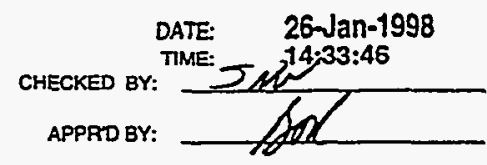

\begin{tabular}{|c|c|c|c|c|}
\hline $\begin{array}{l}\text { WBS } \\
\text { Element }\end{array}$ & Cost Estimate Element & $\begin{array}{c}\text { Total } \\
\text { Unescalated }\end{array}$ & Escalation & $\begin{array}{c}\text { Total } \\
\text { Inci Escalation }\end{array}$ \\
\hline 1.1 & DESIGN \& DEVELOPMENT. & & & $>\$ 5,850,000$ \\
\hline 1.1.1 & CONCEPTUAL DESIGN & $5,000,000$ & 850,000 & $5,850,000$ \\
\hline 1.2 & MANAGEMENT COSTS & & & $\gg \quad \$ 4,790,000$ \\
\hline 1.21 & PM FOR PROJECT DEVELOPMENT & $3,000,000$ & 570,000 & $3,570,000$ \\
\hline 1.2 .2 & SAFETY REVIEWS & $1,000,000$ & 220,000 & $1,220,000$ \\
\hline 1.3 & PERMITTING & & & $\gg \quad \$ 3,328,687$ \\
\hline 1.3.1 & PERMITTING & $2 ; 728,432$ & 600,255 & $3,328,687$ \\
\hline 1.4 & TURNOVER & & & $>\quad \$ 6,570,000$ \\
\hline 1.4.1 & SO TEST \& STARTUP & $4,500,000$ & $2,070,000$ & $6,570,000$ \\
\hline \multirow[t]{2}{*}{ 1.5.2 } & PROCUREMENT FEES & 0 & 0 & $\gg$ \\
\hline & $\begin{array}{l}\text { SUBTOTAL INCLUDING ESCALATION } \\
\text { PROJECT CONTINGENCY }\end{array}$ & $16,228,432$ & $4,310,255$ & >> $\$ 20,538,687$ \\
\hline & MANAGEMENT RESERVE- & & & so \\
\hline & CONTINGENCY- & & & $\gg \quad \$ 5,161,313$ \\
\hline & TOTAL ESTIMATED COST - & & & $\gg \quad \$ 25,700,000$ \\
\hline
\end{tabular}


Lockheed Martin Idaho Technologies Co.' PROSECT NAME: HIP FACILITY - INTERIM STORAGE FACILITY - OPC - UNESCALATED

LOCATION 1:

REOUESTOR:

NEELICPP

J. K. RAWLINS
COST ESTIMATE SUMMARY

TYPE OF ESTIMATE: PLANNING

PROJECT NO: 242102

PREPARED BY: R. D. ADAMS

REPORT NAME: Cost Estimate Summary

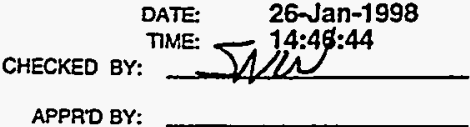

APPRD BY:

Total

Incl Escalation

Unescalated

Escalation

DESIGN \& DEVELOPMENT

CONCEPTUAL DESIGN

MANAGEMENT COSTS

PM FOR PROJECT DEVELOPMENT

SAFETY REVIEWS

PERMITTING

PERMITTING

TURNOVER

SO TEST \& STARTUP

1.4 .1

1.5 .2

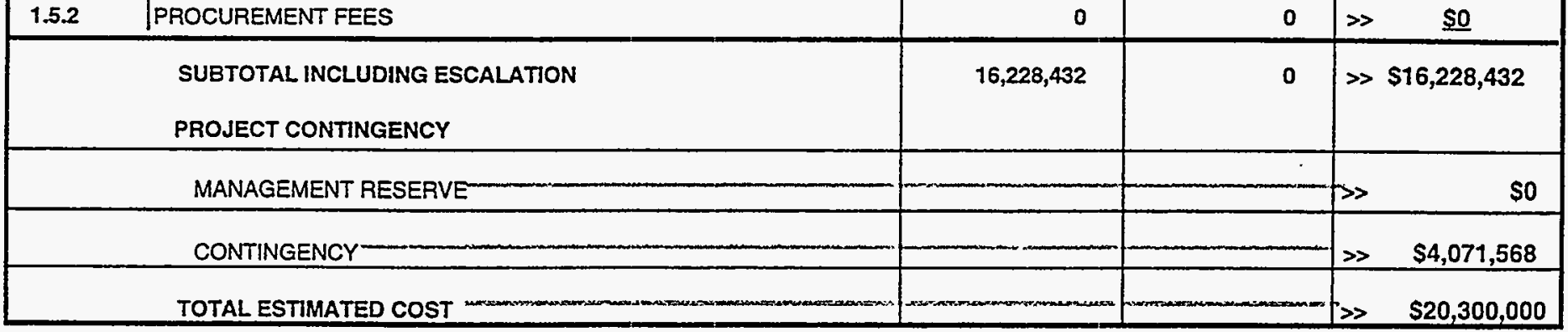

\section{PROJECT COST PARAMETERS}

EDI AS A \% OF CONST. + GFE $=69.00 \%$ 
Lockheed Martin Idaho Technologies Co.

PROJECT NAME: HIP FACILITY - INTERIM STORAGE FACILITY , OPC

LOCATION 1: INEELICPP

REOUESTOR: J. K. RAWLINS
DETAILED COST ESTIMATE SHEET

TYPE OF ESTIMATE: PLANNING

PROJECT NO: 242102

PREPAREOBY: R. D. ADAMS
PAGE : 1

DATE 26-Jan-1998

TIME: 14:31:05 REPORT NAME: Detall Cost Estimate Sheet

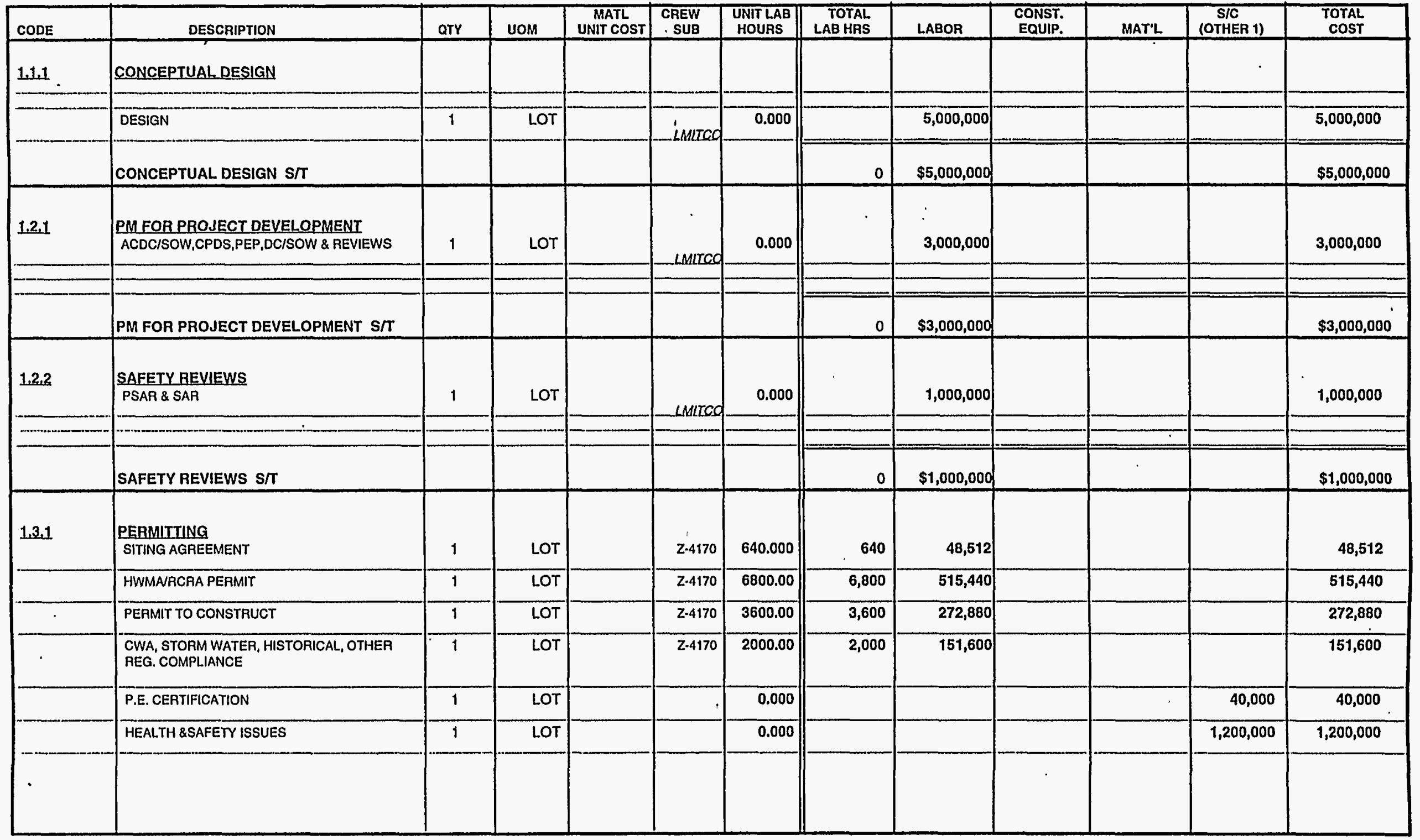


Lockheed Martin Idaho Technologles Co.

PROJECT NAME: HIP FACILITY - INTERIM STORAGE FACILITY - OPC

LOCATION 1: INEELICPP

REQUESTOR: J. K. RAWLINS

\section{DETAILED COST ESTIMATE SHEET}

TYPE OF Estimate: PLANNING

PROJECT NO:: 242102

PAEPARED BY: R. D. ADAMS
PAGE " 2

DATE 26-Jan-1998

TIME: 14:31:05

REPORT NAME: Detail Cost Estimate Sheet

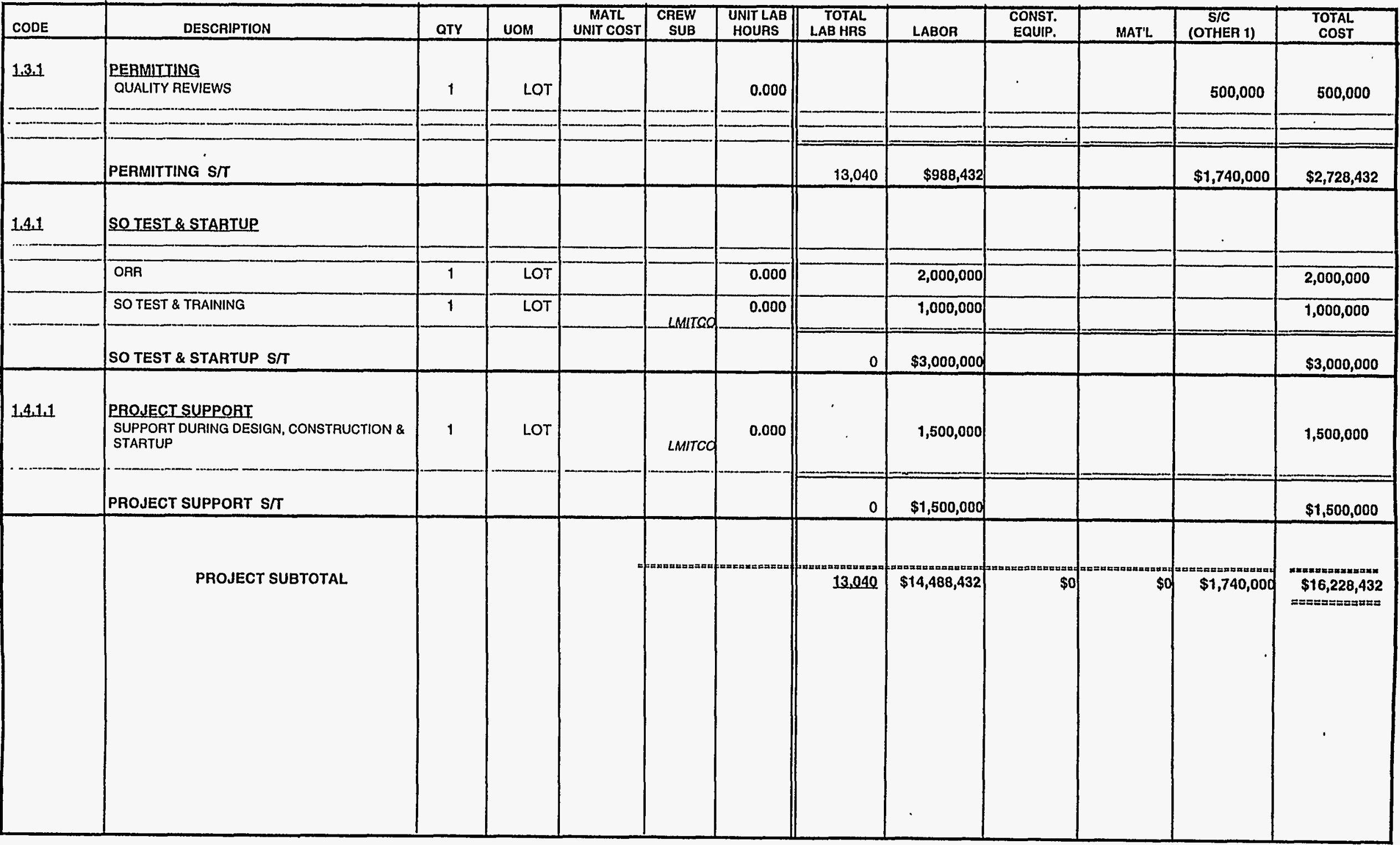


Lockheed Martin Idaho Technologies Co. PROJECT NAME: HIP FACILITY - INTERIM STORAGE FACILITY - OPC - UNESCALATED LOCATION 1 INEELITCP

REQUESTOR:
CONTINGENCY ANALYSIS

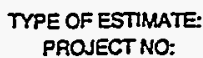
PREPARED BY:
PLANNING
242102
R.D. ADAMS
DATE: $26-$ Jan-1998

TIME: 14:46:50

REPORT NAME: Contingency Analysis

\begin{tabular}{|c|c|c|c|c|c|c|c|c|c|c|c|}
\hline \multirow{3}{*}{$\begin{array}{l}\text { WBS } \\
\text { Element }\end{array}$} & \multicolumn{8}{|c|}{ PROBABLE \% VARIATION } & \multicolumn{2}{|c|}{$\begin{array}{c}\text { PROJECT } \\
\text { CONTINGENCY }\end{array}$} & \multirow{3}{*}{$\begin{array}{l}\text { SUMMARY } \\
\text { Total Cost } \\
\text { by Element }\end{array}$} \\
\hline & \multirow{2}{*}{ Cost Estimate Element } & \multirow{2}{*}{$\begin{array}{c}\text { Total Cost w/o } \\
\text { Contingency }\end{array}$} & \multirow[t]{2}{*}{$\begin{array}{l}\text { \% Total } \\
\text { Cost }\end{array}$} & \multicolumn{2}{|c|}{$\begin{array}{l}\text { Prob. \% Var. } \\
\text { From Est. }\end{array}$} & \multicolumn{2}{|c|}{ Wt \% of Prob. } & \multirow[t]{2}{*}{ Contingency } & \multirow[t]{2}{*}{$\%$} & \multirow[t]{2}{*}{ Cost } & \\
\hline & & & & - & + & - & + & & & & \\
\hline 1.1 .1 & CONCEPTUAL DESIGN & $5,000,000$ & 30.81 & 20 & 30 & 6.16 & 9.24 & $7.703 \%$ & $30.81 \%$ & $1,254,455$ & $6,254,455$ \\
\hline 1.2 .1 & PM FOR PROJECT DEVELOPMENT & $3,000,000$ & 18.49 & 20 & 30 & 3.70 & 5.55 & $4.622 \%$ & $18.49 \%$ & 752,673 & $3,752,573$ \\
\hline 1.22 & SAFETY REVIEWS & $1,000,000$ & 6.16 & 20 & 30 & 1.23 & 1.85 & $1.541 \%$ & $6.16 \%$ & 250,891 & $1,250,891$ \\
\hline 1.3 .1 & PERMITTING & $2,728,432$ & 16.81 & 20 & 30 & 3.36 & 5.04 & $4203 \%$ & $16.81 \%$ & 684,539 & $3,412,971$ \\
\hline 1.4 .1 & SO TEST \& STARTUP & $4,500,000$ & 27.73 & 20 & 30 & 5.55 & 8.32 & $6.932 \%$ & $27.73 \%$ & $1,129,010$ & $5,629,010$ \\
\hline \multirow[t]{2}{*}{1.5 .2} & PROCUREMENT FEES & 0 & 0.00 & 5 & 20 & 0.00 & 0.00 & $0.000 \%$ & $0.00 \%$ & 0 & 0 \\
\hline & ESCALATION & 0 & 0.00 & 20 & 30 & 0.00 & 0.00 & $0.000 \%$ & $0.00 \%$ & 0 & 0 \\
\hline & SUBTOTAL & $16,228,432$ & 100.00 & & & & & $25.000 \%$ & & & \\
\hline & CALCULATED CONTINGENCY & $4,057,108$ & & & & & & & & & \\
\hline & RESULTANT TEC & $20,285,540$ & & & & & & - & & & \\
\hline & ROUNDED TEC & $20,300,000$ & & & & & & & & & \\
\hline & PROJECT CONTINGENCY & $4,071,568$ & & & & & & $25.09 \%$ & & . & \\
\hline & MANAGEMENT RESERVE & 0 & & & & & & & & & \\
\hline & CONTINGENCY & $4,071,568$ & & & & & & & & & \\
\hline & TOTAL ESTIMATED COST & $20,300,000$ & & & & & & & & $4,077,568$ & $20,300,000$ \\
\hline
\end{tabular}

\section{CONFIDENCE LEVEL AND ASSUMED RISKS}

The Lockheed Idaho Technologies Co. Cost Estimate Contingency Analysis Model is based on the applied contingency and the assumptions upon which

the estimate was predicated. The model is applied with a suggested risk level

of $18 \%$ and a level of confidence of $90 \%$ the estimate will fall within the bid range.

The Contingency Analysis is based on a weighted average to provide a

$90 \%$ probability of underriun and a $10 \%$ probability of ovemun.
CONTINGENCY ANALYSIS GUIDE BY TYPE OF ESTIMATE

Gujdelines established by DOE/FM 50, Cost Estimating Guide, Vol. 6 ,

Cost Guide, and as presented in the INEL Cost Estimating Guide.

PLANNING $20 \%-30 \%$

Experimental/Special Conditions............. Up to $50 \%$ Conceptual

Experimental/Special Conditions............ Up to $40 \%$

TITLE I

TITLE II

TITLE II/AFC
$10 \%-20 \%$

$5 \%-15 \%$ Market Conditions 
Lockheed Martin Idaho Technologies Co. PROIECT NAME: HIP FACILITY - INTERIM STORAGE LOCATION T: REQUESTOR: FACILUTY - OPC - ESCALATED INEELICPP

J. K. RAWLINS
CONTINGENCY ANALYSIS TYPE OF ESTIMATE: PLANNING
PROJECT NO: PREPARED BY:
R. D. ADAMS
DATE: 26-Jan-1998

TIME: 14:33:51

REPORT NAME: Contingency Analysis

\begin{tabular}{|c|c|c|c|c|c|c|c|c|c|c|c|}
\hline \multicolumn{9}{|c|}{ PROBABLE \% VARIATION } & \multicolumn{2}{|c|}{$\begin{array}{c}\text { PROJECT } \\
\text { CONTINGENCY }\end{array}$} & \multirow{3}{*}{$\begin{array}{l}\text { SUMMARY } \\
\text { Total Cost } \\
\text { by Element }\end{array}$} \\
\hline \multirow[t]{2}{*}{$\begin{array}{l}\text { WBS } \\
\text { Element }\end{array}$} & \multirow[t]{2}{*}{ Cost Estimate Element } & \multirow{2}{*}{$\begin{array}{c}\text { Total Cost w/o } \\
\text { Contingency }\end{array}$} & \multirow[t]{2}{*}{$\begin{array}{l}\text { \% Total } \\
\text { Cost }\end{array}$} & \multicolumn{2}{|c|}{$\begin{array}{c}\text { Prob. \% Var. } \\
\text { From Est. }\end{array}$} & \multicolumn{2}{|c|}{ Wt. \% of Prob. } & \multirow[t]{2}{*}{ Contingency } & \multirow[t]{2}{*}{$\%$} & \multirow[t]{2}{*}{ Cost } & \\
\hline & & & & - & + & $\cdots$ & + & & & & \\
\hline 1.1 .1 & CONCEPTUAL DESIGN & $5,000,000$ & 24.34 & 20 & 30 & 4.87 & 7.30 & $6.086 \%$ & $24.34 \%$ & $1,256,486$ & $6,256,486$ \\
\hline 1.21 & PM FOA PROJECT DEVELOPMENT & $3,000,000$ & 14.61 & 20 & 30 & 292 & 4.38 & $3.652 \%$ & $14.61 \%$ & 753,891 & $3,753,891$ \\
\hline 1.2 .2 & SAFETY REVIEWS & $1,000,000$ & 4.87 & 20 & 30 & 0.97 & 1.46 & $1.217 \%$ & $4.87 \%$ & 251,297 & $1,251,297$ \\
\hline 1.3 .1 & PERMITTING & $2,728,432$ & 13.28 & 20 & 30 & 2.66 & 3.99 & $3.321 \%$ & $13.28 \%$ & 685,647 & $3,414,079$ \\
\hline 1.4 .1 & SO TEST \& STARTUP & $4,500,000$ & 21.91 & 20 & 30 & 4.38 & 6.57 & $5.477 \%$ & $21.91 \%$ & $1,130,837$ & $5,630,837$ \\
\hline \multirow[t]{2}{*}{1.5 .2} & PROCUREMENT FEES & 0 & 0.00 & 5 & 20 & 0.00 & 0.00 & $0.000 \%$ & $0.00 \%$ & of & 0 \\
\hline & ESCALATION & $4,310,255$ & 20.99 & 20 & 30 & 4.20 & 6.30 & $5.247 \%$ & $20.99 \%$ & 1.083 .155 & $5,393,410$ \\
\hline & SUBTOTAL & $20,538,687$ & 100.00 & & & & & $25.000 \%$ & & & \\
\hline & CALCULATED CONTINGENCY & $5,134,672$ & & & & & & & & & \\
\hline & RESULTANT TEC & $25,673,359$ & & & & & & & & & \\
\hline & ROUNDED TEC & $25,700,000$ & & & & & & & & & \\
\hline & PROJECT CONTINGENCY & $5,161,313$ & & & & & & $25.13 \%$ & & & \\
\hline & MANAGEMENT RESERVE & 0 & & & & & & & & & \\
\hline & CONTINGENCY & $5,161,313$ & & & & & $\dot{-}$ & & & & \\
\hline & TOTAL ESTIMATED COST & $25,700,000$ & & & & & & & & $5,161,313$ & $25,700,000$ \\
\hline
\end{tabular}

CONFIDENCE LEVEL AND ASSUMED RISKS:

The Lockheed Idaho Technologies Co. Cost Estimate Contingency Analysis Model is based on the applied contingency and the assumptions upon which

the estimate was predicated. The model is applied with a suggested risk level

of $18 \%$ and a level of confidence of $90 \%$ the estimate will fall within the bid range.

The Contingency Analysis is based on a weighted average to provide a

$90 \%$ probability of underun and a $10 \%$ probability of overrun.
CONTINGENCY ANALYSIS GUIDE BY TYPE OF ESTIMATE

Guidelines established by DOE/FM 50, Cost Estimating Guide, Vol. 6 ,

Cost Guide, and as presented in the INEL Cost Estimating Guide.

PLANNING $20 \%-30 \%$ Experimental/Special Conditions............Up to $50 \%$ Conceptual $15 \%-25 \%$ Experimental/Special Conditions............Up to $40 \%$ TITLE I

TITLE II

TITLE H/AFC
$10 \%-20 \%$

$5 \%-15 \%$ Market Conditions 


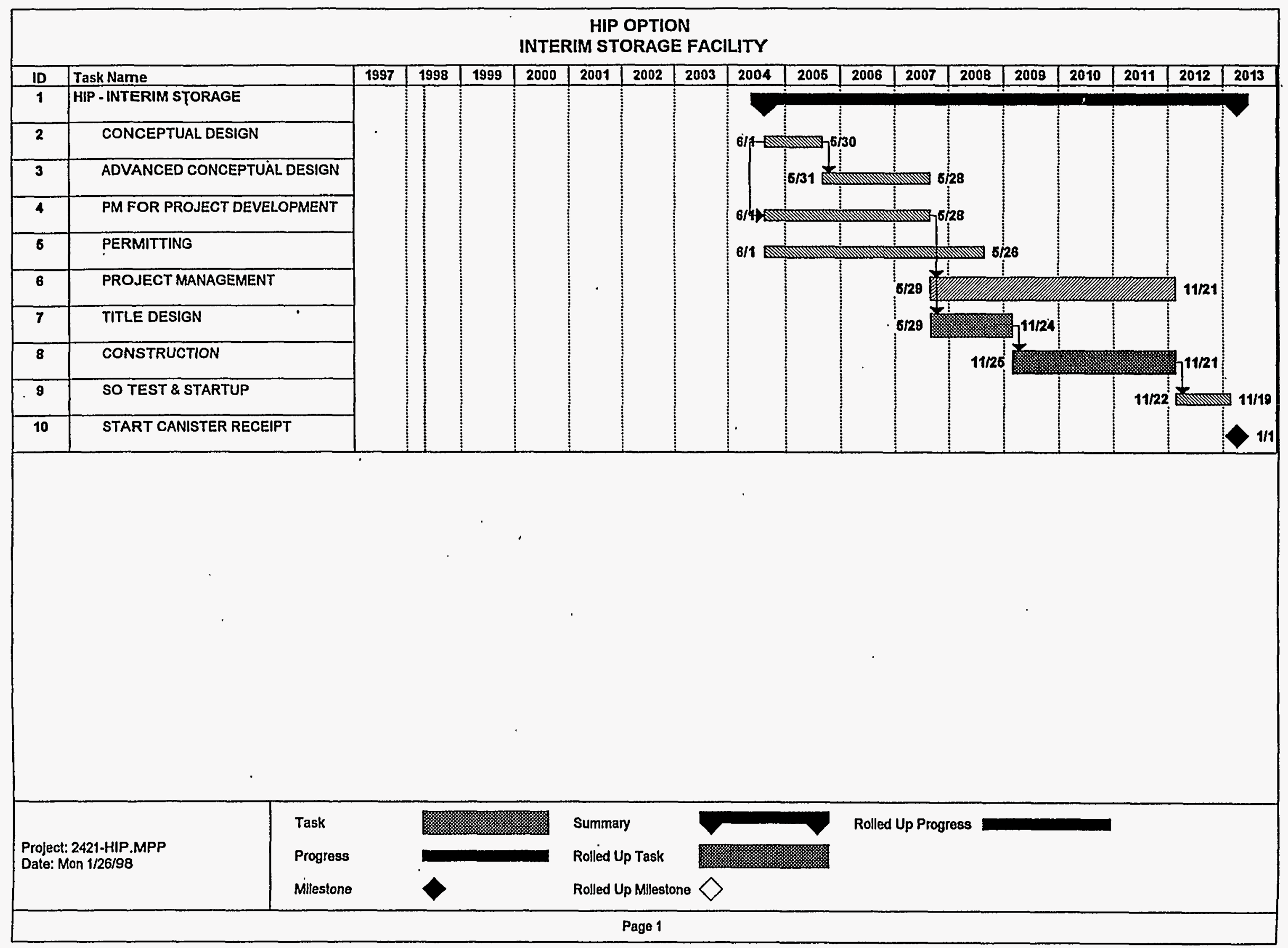


Lockheed Martin Idaho Technologies

(Rev. 6/96)

\section{COST ESTIMATE SUPPORT DATA RECAPITULATION}

Project Title: HLW INTERIM STORAGE FACILITY - VWO

Type of Estimate: PROJECT SUPPORT

File No: 2421-VWO
Estimator:RDA

Date: $1 / 26 / 98$

Approved By:

\section{SCOPE OF FORK: Brief description of the proposed project.}

This facility will unload, store in vertical tubes and load out canisters containing High Level Waste (HLW) from the proposed Vitrified Waste Option (VWO).

II. BASIS OF THE ESTIMATE : Drawings, Design Report, Engineers notes, and/or other documentation upon which the estimate is originated.

Draft sketches, a draft EDF, and the SNF Canister Storage Building Final Design Estimate from Fluor Daniel Northwest, Inc. were used as the basis for this estimate.

\section{ASSUMPTIONS : Condition statements accepted or supposed true without proof or demonstration.} An assumption has a direct impact on total estimated cost.

1. The Interim Storage Facility will be located within 100 feet of the CWO facility.

2. Rock will not be encountered during excavation.

3. The estimates include both present day costs summaries and contingency analysis and escalated summaries and contingency analysis. The escalated summaries and contingency analysis reflect the attached schedule. All activities are escalated to the appropriate schedule mid-point.

4. Per the draft EDF, nine storage modules, containing 630 storage tubes each, will accommodate the estimated 16,000 canisters produced at the Cementious Waste Facility.

5. Most quantities used for this estimate were obtained by preliminary take offs from the draft sketches.

6. The storage tube costs, gantry crane cost and associated rail system costs were used from the Fluor estimate of a facility which is approximately eighty percent complete.

7. All assumptions and information included in the draft "New Interim Storage Facility for Interim Storage of HLW" apply to the estimate.

8. Where quantities could not be determined from the draft sketches, factors or assumed quantities were used by the estimator.

9. Since the storage tubes and conveying equipment represent such a large percentage of this facility's cost, lower design, inspection, Project Management and turnover costs are used in the estimate.

10. Sufficient construction craft will be available to support this project.

11. Markups on direct construction costs to get the construction costs listed on the summary sheet are as follows:

Labor indirects, overheads and profit(OH\&P)

Material \& equipment OH\&P

Subcontract OH\&O

Construction equipment allowance

NQA-1 quality requirements

DOE/RW/0333P quality requirements

Subcontractor procurement

Bond
$50 \%$ of labor

$5 \%$ of material and equipment $10 \%$ of subcontract cost

$10 \%$ of labor

$30 \%$ of material

$10 \%$ of material

$1 \%$ of material and

equipment

$2 \%$ of everything 
Lockheed Martin Idaho Technologies

\section{COST ESTIMATE SUPPORT DATA RECAPITULATION (CONTINUATION)}

\section{ASSUMPTIONS: (Continued)}

12. Only the railroad track within the storage facility is included in this estimate. The remainder of the branch line is included in the infrastructure estimate.

13. All utilities are assumed to be available at the facility wall.

\section{CONTINGENCY GUIDELINE IMPIEMENTATION: The percentage used for contingency as determined by the contingency allowance guidelines can be altered to refiect the type of construction and conditions that may impact the total estimated cost.}

A large portion of the estimated costs is based on actual costs from Fluor's SNF Canister Storage Building. The majority of the remaining costs are for standard construction activities. For these reasons, the contingency is lower than would be experienced for a processing type facility at this stage of development.

\section{OTHER COMMENTS/CONCERNS SPECIEIC TO THE ESTIMATE:}

1. All FY' 98 and later projects are to be assessed a Procurement Fee of $1 \%$, a G\&A Fee of $23 \%$, with a $\$ 500,000$ ceiling per year, and a Performance Incentive Fee (PIF) of $5.5 \%$. See the attached G\&A/PIF calculation sheet for the method used to calculate these fees.

2. A procurement fee of one percent of construction was used to cover the operating contractor support to DOE-ID for their contract administration. It is felt that one percent is sufficient for jobs of this size. 
Lockheed Martin Idaho Technologies Co. PROIECT NAME: VIT FACILITY - INTERIM STORAGE FACILITY - ESCALATED

LOCATION 1: REQUESTOR:
INEELICPP

J.K. RAWLINS
COST ESTIMATE SUMMARY

TYPE OF ESTIMATE: PLANNING PROSECT NO: 2421-VIT

PREPARED BY: R. D. ADAMS

PEPORT NAME: Cost Estimate Summary

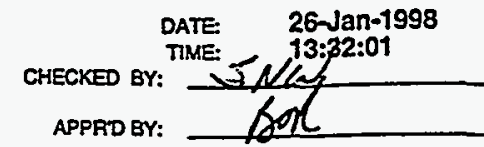

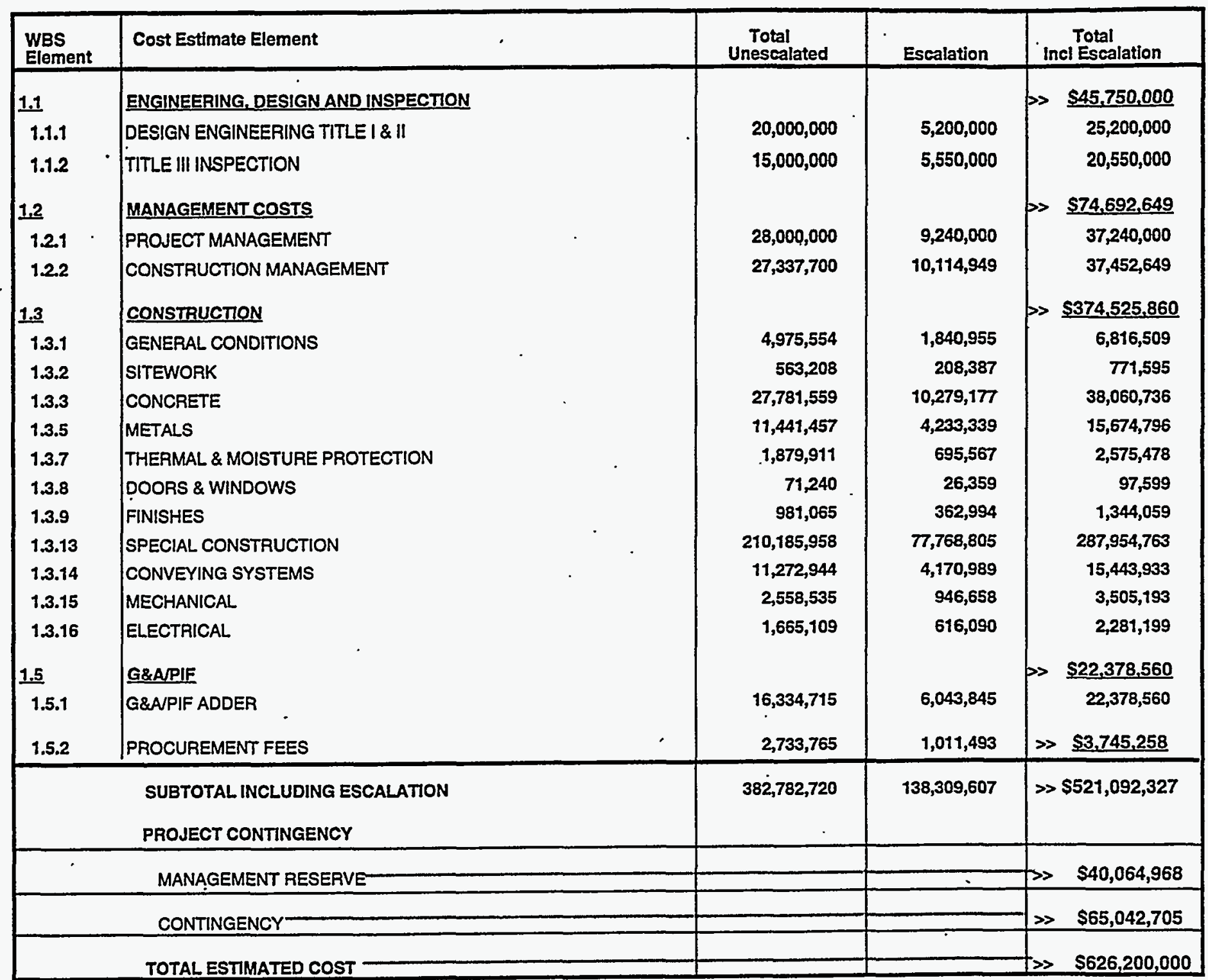

PROJECT COST PARAMETERS

EDI AS A $\%$ OF CONST. + GFE $=12.00 \%$

CONTINGENCY $=20.17 \%$ 
Lockheed Martin Idaho Technologies Co.

PROIECT NAME: VIT FACILITY - INTERIM STORAGE FACILITY - UNESCALATED

INEELICPP

LOCATION 1:

REOUESTOR:

J. K. RAWLINS
COST ESTIMATE SUMMARY

TYPE OFESTIMATE: PLANNING PROJECT NO: 2421-VIT PREPARED BY: R.D.ADAMS REPORT NAME: Cost Estimate Summary

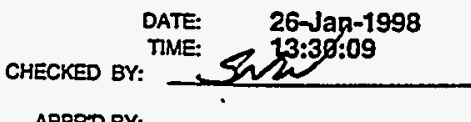

\begin{tabular}{|c|c|c|c|c|}
\hline $\begin{array}{l}\text { WBS } \\
\text { Element }\end{array}$ & Cost Estimate Element & $\begin{array}{c}\text { Total } \\
\text { Unescalated }\end{array}$ & Escalation & $\begin{array}{l}\text { Total } \\
\text { Incl Escalation }\end{array}$ \\
\hline 1.1 & ENGINEERING, DESIGN AND INSPECTION & & & $\gg \$ 35,000,000$ \\
\hline t.1.1 & DESIGN ENGINEERING TITLE | \& \| & $20,000,000$ & 0 & $20,000,000$ \\
\hline 1.1 .2 & TITLE III INSPECTION & $15,000,000$ & 0 & $15,000,000$ \\
\hline 1.2 & MANAGEMENT COSTS & & & $\gg \$ \$ 55,337,700$ \\
\hline 1.2 .1 & PROJECT MANAGEMENT & $28,000,000$ & 0 & $28,000,000$ \\
\hline 1.2 .2 & CONSTRUCTION MANAGEMENT & $27,337,700$ & 0 & $27,337,700$ \\
\hline 1.3 & CONSTRUCTION & & & $\gg>\$ 273,376,540$ \\
\hline 1.3.1 & GENERAL CONDITIONS & $4,975,554$ & 0 & $4,975,554$ \\
\hline 1.3 .2 & SITEWORK & 563,208 & 0 & 563,208 \\
\hline 1.3 .3 & CONCRETE & $27,781,559$ & 0 & $27,781,559$ \\
\hline 1.3 .5 & METALS & $11,441,457$ & 0 & $11,441,457$ \\
\hline 1.3.7 & THERMAL \& MOISTURE PROTECTION & $1,879,911$ & 0 & $1,879,911$ \\
\hline 1.3 .8 & DOORS \& WINDOWS & 71,240 & $\mathbf{0}$ & 71,240 \\
\hline 1.3 .9 & FINISHES & 981,065 & 0 & 981,065 \\
\hline 1.3 .13 & SPECIAL CONSTRUCTION & $210,185,958$ & 0 & $210,185,9588^{\prime}$ \\
\hline 1.3.14 & CONVEYING SYSTEMS & $11,272,944$ & 0 & $11,272,944$ \\
\hline 1.3 .15 & MECHANICAL & $2,558,535$ & 0 & $2,558,535$ \\
\hline 1.3.16 & ELECTRICAL & $1,665,109$ & 0 & $1,665,109$ \\
\hline 1.5 & G\&APIF & & & $\gg>\$ 16,334,715$ \\
\hline 1.5.1 & G\&AMPIF ADDER & $16,334,715$ & 0 & $16,334,715$ \\
\hline 1.5 .2 & PROCUREMENT FEES & $2,733,765$ & 0 & $\gg \$ 2,733,765$ \\
\hline & $\begin{array}{l}\text { SUBTOTAL INCLUDING ESCALATION } \\
\text { PROJECT CONTINGENCY }\end{array}$ & $382,782,720$ & 0 & >> \$382,782,720 \\
\hline & MANAGEMENT RESERVE- & & & $\gg \quad \$ 29,244,502$ \\
\hline & CONTINGENCY- & & & $\gg \$ 47,472,778$ \\
\hline & TOTAL ESTIMATED COST - & & & $\gg>\$ 459,500,000$ \\
\hline
\end{tabular}

PROJECT COST PARAMETERS

EDI AS A \% OF CONST. + GFE= $13.00 \%$ 
Lockheed Martin Idaho Technologles Co. Rev. G/96

PROJECT NAME: VIT FACILITY - INTERIM STORAGE FACILITY

LOCATION 1: INEELICPP

REQUESTOR: J. K. RAWLINS

\section{DETAILED COST ESTIMATE SHEET}

TyPe Of estimate: PLANNING

PROJECT NO: 2421-VIT

PREPARED BY: R. D. ADAMS
PAgen 1

DATE 26-Jan-1998

TIME: 13:28:34

REPORT NAME: Detall Cost Estimate Sheet

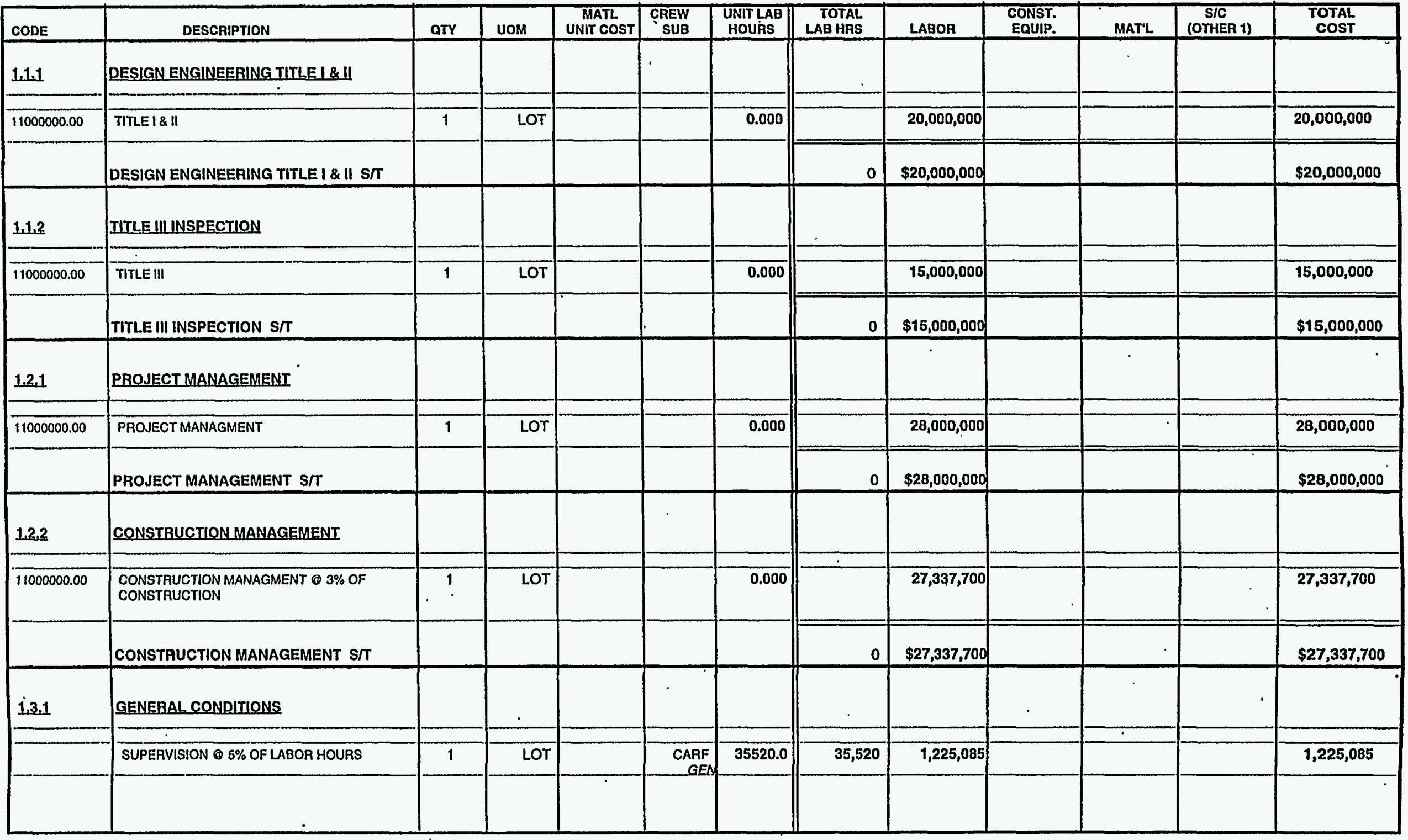


Lockheed Martin Idaho Technologies Co.
Rov. $6 / 96$

PROJECT NAME: VIT FACILITY - INTERIM STORAGE FACILITY

LOCATION I: INEELICPP

REQUESTOR: J. K. RAWLINS

\section{DETAILED COST ESTIMATE SHEET}

TYPE OF Estimate: PLANNING

PROJECT NO: 2421-VIT

PREPARED BY: R. D. ADAMS
PAgE 2

DATE 26-Jan-1998

TIME: 13:28:34

REPORT NAME: Detall Cost Estimate Sheot

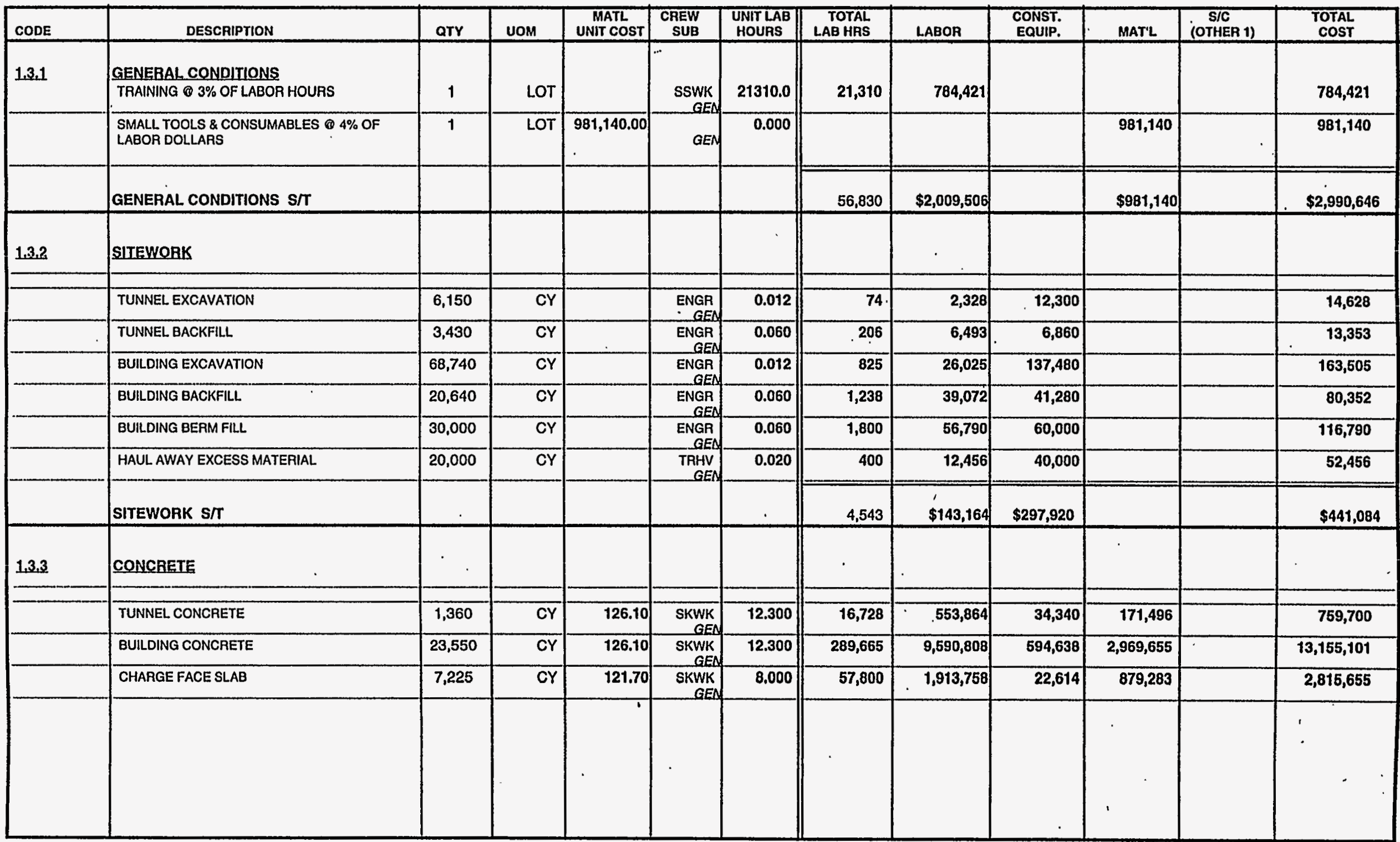


Lockheed Martin Idaho Technologies Co. Pov. 6/96

PROJECT NAME: VIT FACILITY - INTERIM STORAGE FACILITY

LOCATION I: INEELACPP

REQUESTOR: J. K. RAWLINS

\section{DETAILED COST ESTIMATE SHEET}

TYPE OF ESTIMATE: PLANNING

PROJECT NO:: 2421-VIT

PREPARED BY: R.D. ADAMS
PAGE 3

DATE 26-Jan-1998

TIME: 13:28:34

REPORT NAME: Detall Cost Estimate Shoot

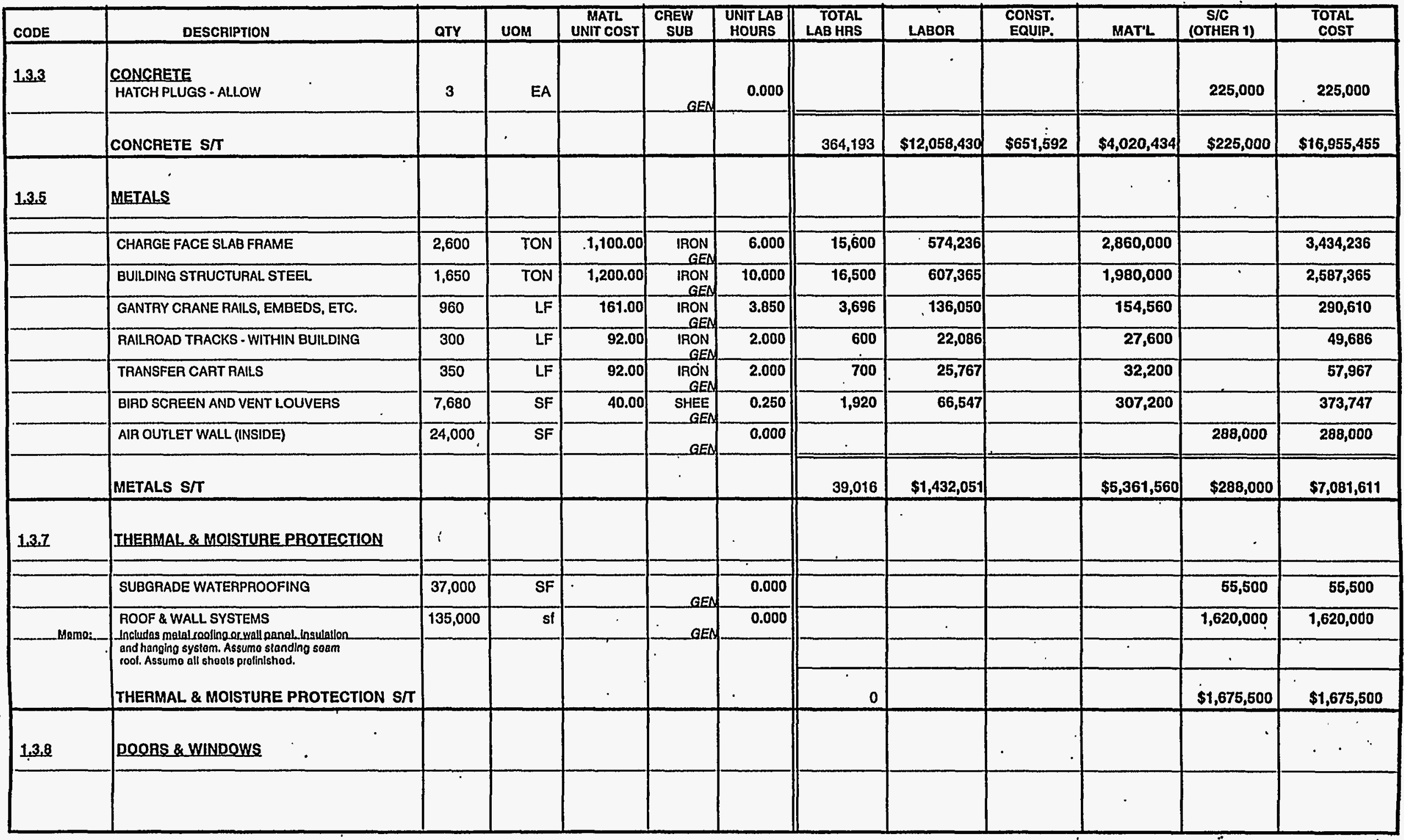


Lockheed Martin Idaho Technologies Co. Rov. 6/96

PROJECT NAME: VIT FACILITY - INTERIM STORAGE FACILITY

LOCATION 1: INEELICPP

REQUESTOR: J.K. RAWLINS

\section{DETAILED COST ESTIMATE SHEET}

TYPE Of estimate: PLANNING

PROJECT NO:: 2421-VIT

PREPARED BY: R. D. ADAMS
PAgE " 4

DATE 26-Jan-1998

TIME: 13:28:34

REPORT NAME: Detail Cost Estimate Sheet

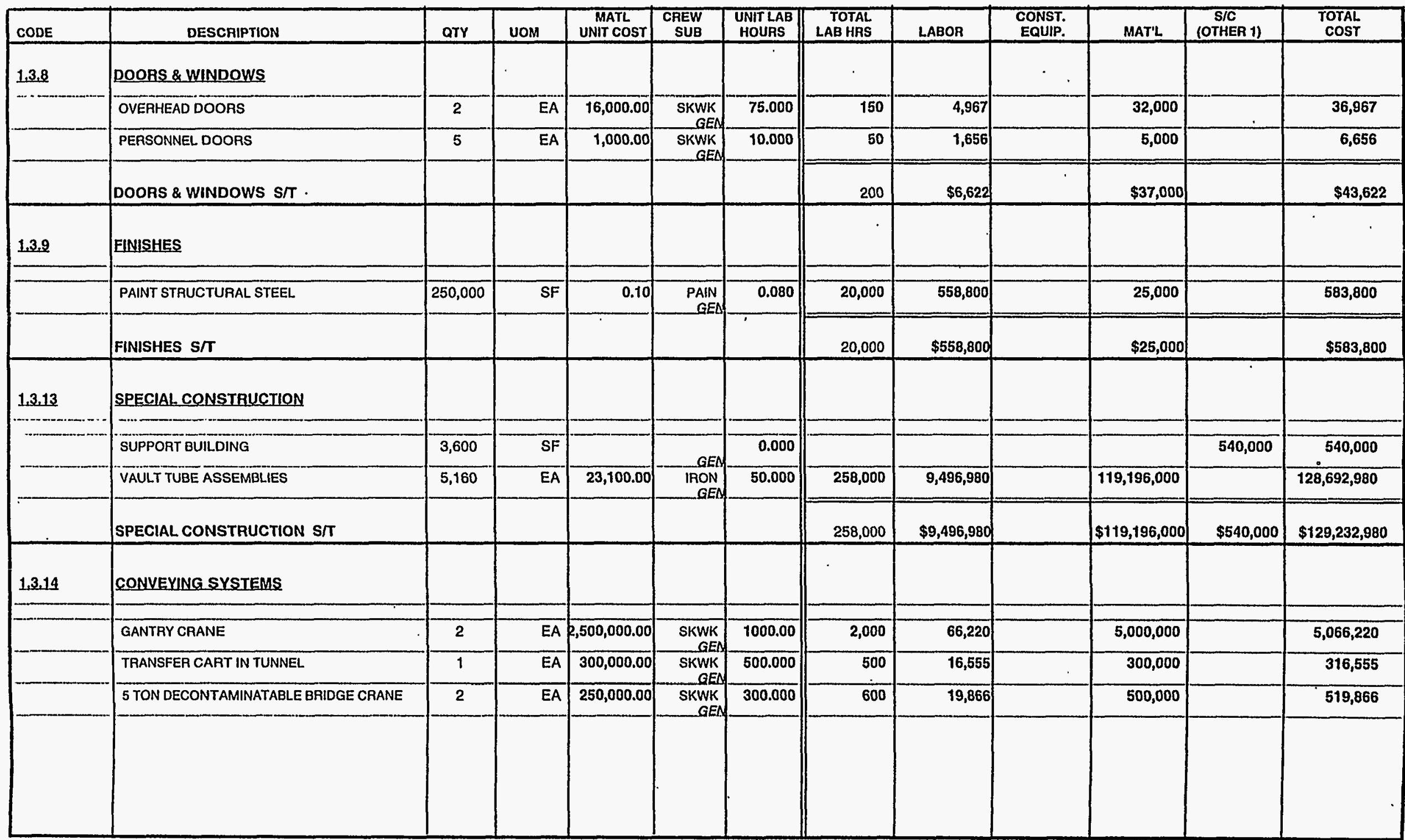


Lockheed Martin Idaho Technologles Co. Rov. 6/96 PROJECT NAME: VIT FACILITY - INTERIM STORAGE FACILITY

LOCATION 1: INEELACPP

REQUESTOR: J. K. RAWLINS

\section{DETAILED COST ESTIMATE SHEET}

TYPE OF ESTIMATE: PLANNING

PAOJECT NO.: 2421-VIT

PREPARED BY: R. D. ADAMS
PAgE 5

DATE 26-Jan-1998

TME: 13:28:34

AEPORT NAME: Detall Cost Estlmate Sheot

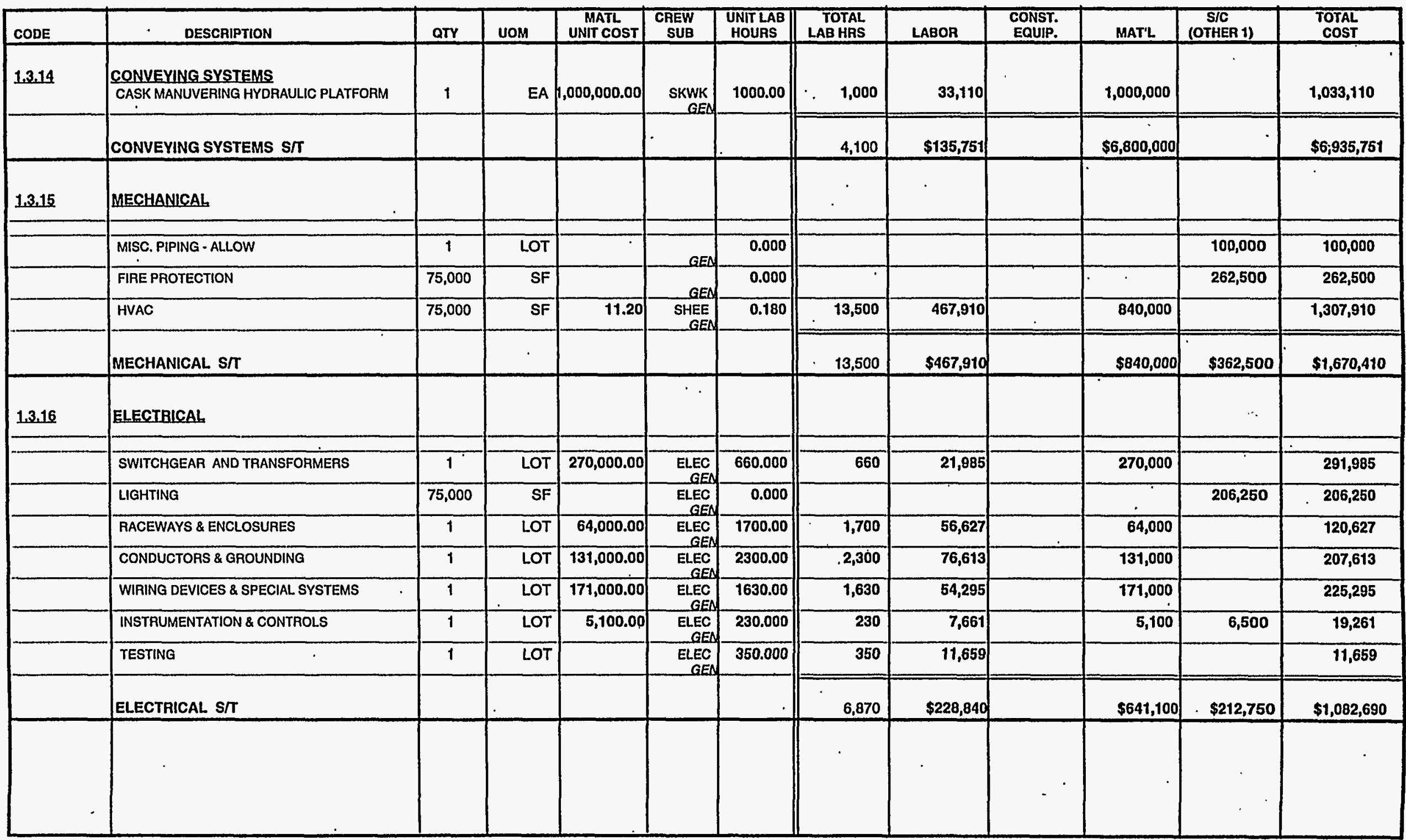


Lockheed Martin Idaho Technologies Co. Rov. 8/90

PROJECT NAME: VIT FACILITY - INTERIM STORAGE FACILITY

LOCATION 1: INEELICPP

REQUESTOA: J. K. RAWLINS

\section{DETAILED COST ESTIMATE SHEET}

TYPE OF ESTIMATE: PLANNING

PROJECT NO: 2421-VIT

PAEPARED BY: R. D. ADAMS
PAGE" 6

DATE 26-Jan-1998

TIME: 13:28:34

heport naMe: Detall Cost Estimate Sheot

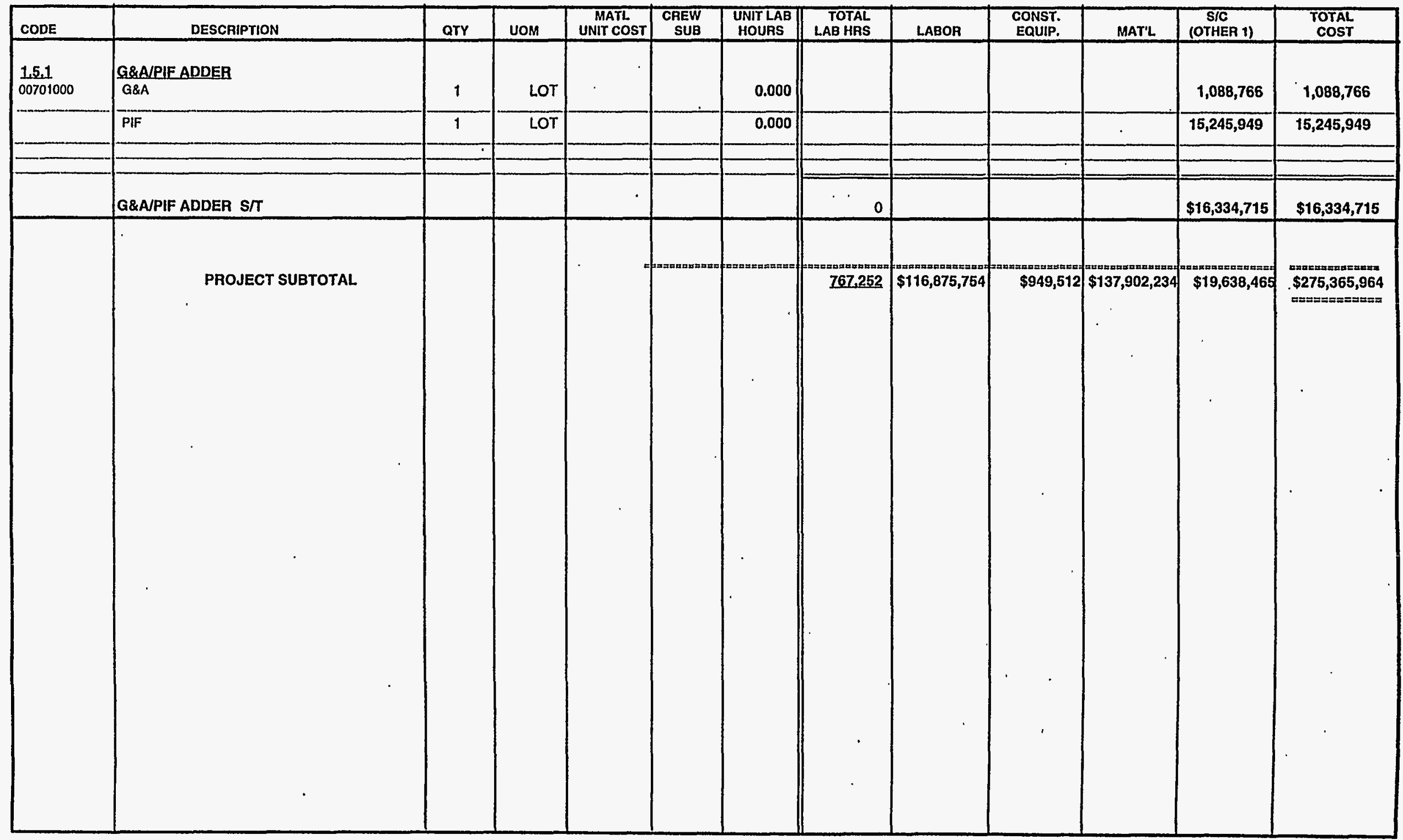


Lockheed Martin Idaho Technologies Co. PROIECT NAME: VIT FACILITY - INTERIM STORAGE FACILITY - UNESCALATED

LOCATION 1: REQUESTOR:
J. K. RAWLINS
INEELICPP
CONTINGENCY ANALYSIS

\begin{tabular}{|c|c|}
\hline $\begin{array}{l}\text { IPE OF ESTIMATE: } \\
\text { PROJECT NO: }\end{array}$ & $\begin{array}{l}\text { PLANNING } \\
2421 \text {-VIT }\end{array}$ \\
\hline
\end{tabular}

REPOAT NAME: Contingency Analysis

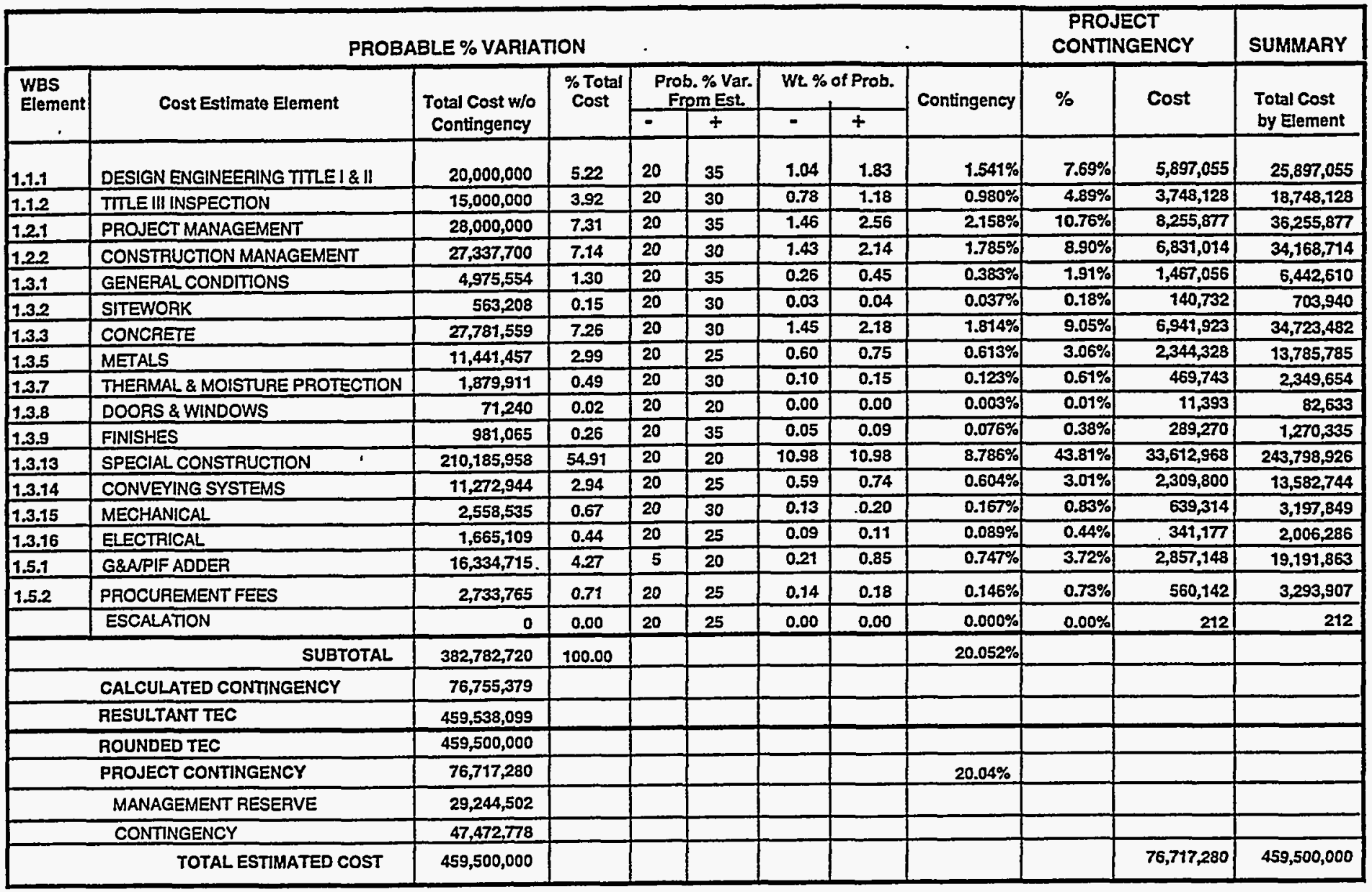

CONFIDENCE LEVEL AND ASSUMED RISKS:

The Lockheed Idaho Technologies Co. Cost Estimate Contingency Analysis Model is based on the applied contingency and the assumptions upon which the estimate was predicated. The model is applied with a suggested risk level of $18 \%$ and a level of confidence of $90 \%$ the estimate will fall within the bid range. The Contingency Analysis is based on a weighted average to provide a $90 \%$ probability of underrun and a $10 \%$ probability of overrun.
CONTINGENCY ANALYSIS GUIDE BY TYPE OF ESTIMATE

Guidelines established by DOE/FM 50, Cost Estimating Guide, Vol. 6, Cost Guide, and as presented in the INEL Cost Estimating Guide. PLANNING Experimental/Special Conditions Conceptual TITLE I TITLE II TITLE II/AFC
$15 \%-25 \%$ ....Up to $40 \%$ $10 \%-20 \%$ $5 \%-15 \%$ Market Conditions 
Lockheed Martin Idaho Technologies Co. REv. 696

LOCATION I: REQUESTOR:
VIT FACILITY - INTERIM STORAGE FACILITY - ESCALATED INEELICPP J. K. RAWLINS
CONTINGENCY ANALYSIS

$\begin{array}{cl}\text { TYPE OF ESTIMATE: } & \text { PLANNING } \\ \text { PROAECT NO: } & \text { 2421-VIT } \\ \text { PAEPARED BY: } & \text { R. D. ADAMS }\end{array}$

DATE: 26-Jan-1998

TME: 13:32:06

REPOAT NAME: Contingency Analysis

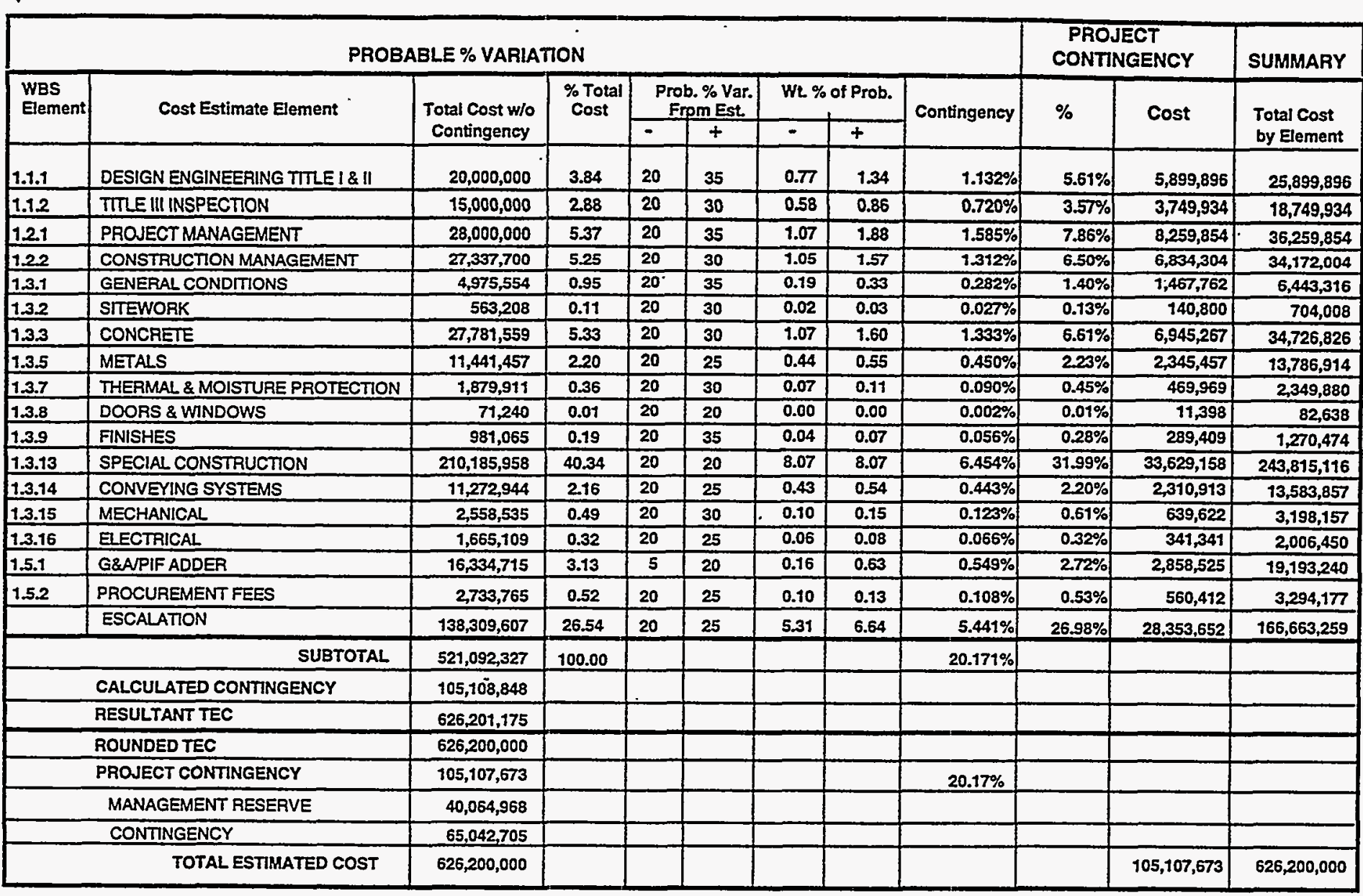

CONFIDENCE LEVEL AND ASSUMED RISKS:

The Lockheed Idaho Technologies Co. Cost Estimate Contingency Analysis Model is based on the applied contingency and the assumptions upon which

the estimate was predicated. The model is applied with a suggested risk level

of $18 \%$ and a level of confidence of $90 \%$ the estimate will fall within the bid range.

The Contingency Analysis is based on a weighted average to provide a

$90 \%$ probability of underrun and a $10 \%$ probability of overrun.
CONTINGENCY ANALYSIS GUIDE BY TYPE OF ESTIMATE

Guidelines established by DOE/FM 50, Cost Estimating Guide, Vol. 6 ,

Cost Guide, and as presented in the INEL, Cost Estimating Guide.

PLANNING $20 \%-30 \%$

Experimental/Special Conditions.............Up to $50 \%$

Conceptual

Experimental/Special Conditions.............Up to $40 \%$

TITLE I

TITLE II

TITLE IIIAFC
$10 \%-20 \%$

$5 \%=15 \%$
Market Conditions 


\section{G\&AIPIF ADDER CALCULATION SHEET}

PROCUREMENT FEE:

CONSTRUCTION $=\quad \begin{array}{r}\$ 273,376,541 \\ \text { GFE }= \\ \quad \text { Subtotal } \\ \quad \$ 273,376,541\end{array}$

FEE @ $1 \%=$

$\$ 273,376,541 * 0.01=\$ 2,733,765.41$

G\&A @ 23\% (with a ceiling of $\$ 500,000$ imposed per year

CONSTRUCTION OR

CEILING * 4 YEARS $=\quad \$ 2,000,000$

GFE = $\$ 0$

PROCUREMENT FEE =

Subtotal

$\$ 2,733,765$

$\$ 4,733,765$

FEE @ $23 \%=$

$\$ 4,733,765 * 0.23=$

$\$ 1,088,766$

PIF @ 5.5\%

\begin{tabular}{lr} 
CONSTRUCTION $=$ & $\begin{array}{r}\$ 273,376,541 \\
\text { GFE }=\end{array}$ \\
PROCUREMENT FEE $=$ & $\$ 0$ \\
G\&A $=\quad \begin{array}{r}\$ 2,733,765 \\
\end{array} \quad$ Subtotal & $\$ 1,088,766$ \\
\cline { 2 - 2 } & $\$ 277,199,072$
\end{tabular}

FEE @ $5.5 \%=$

$\$ 277,199,072 \cdot 0.055=$

$\$ 15,245,949$

TOTAL PROCUREMENT FEE:

$\$ 2,733,765$

TOTAL G\&A FEE:

$\$ 1,088,766$

TOTAL PIF:

$\$ 15,245,949$ 
Lockheed Martin Idaho Technologies Co. PROSECT NAME: VIT FACILITY - INTERIM STORAGE FACILTY - OPC-ESCALATED

LOCATION 1: REQUESTOR:
FACILITY-OP

J. K. RAWLINS

\section{COST ESTIMATE SUMMARY}

TYPE OF ESTIMATE: PLANNING PROUECT NO: 242101 PAEPARED BY: R.D. ADAMS REPORT NAME Cost Estimate Summary

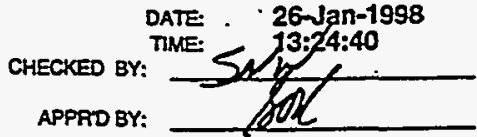

\begin{tabular}{|c|c|c|c|c|}
\hline $\begin{array}{l}\text { WBS } \\
\text { Element }\end{array}$ & Cost Estimate Element & $\begin{array}{c}\text { Total } \\
\text { Unescalated }\end{array}$ & Escalation & $\begin{array}{l}\text { Total } \\
\text { Incl Escalation }\end{array}$ \\
\hline 1.1 & DESIGN \& DEVELOPMENT & & & $\gg \$ 5,850,000$ \\
\hline 1.1.1 & CONCEPTUAL DESIGN & $5,000,000$ & 850,000 & $5,850,000$ \\
\hline 1,2 & MANAGEMENT COSTS & & & $\gg \quad \$ 4,760,000$ \\
\hline 1.2 .1 & PM FOR PROJECT DEVELOPMENT & $3,000,000$ & 570,000 & $3,570,000$ \\
\hline 1.22 & SAFETY REVIEWS & $1,000,000$ & 190,000 & $1,190,000$ \\
\hline 1,3 & PERMITTING & & & $>\$ 3,246,834$ \\
\hline 1.3.1 & PERMITTING & $2,728,432$ & 518,402 & $3,246,834$ \\
\hline 1.4 & TURNOVER & & & $\gg \$ 6,525,000$ \\
\hline 1.4.1 & SO TEST \& STARTUP & $4,500,000$ & $2,025,000$ & $6,525,000$ \\
\hline $\mathbf{1 . 5 . 2}$ & PROCUREMENT FEES & 0 & 0 & șo \\
\hline & $\begin{array}{l}\text { SUBTOTAL INCLUUDING ESCALATION } \\
\text { PROJECT CONTINGENCY }\end{array}$ & $16,228,432$ & $4,153,402$ & $\gg \$ 20,381,834$ \\
\hline & MANAGEMENT RESERVE- & & & so \\
\hline & CONTINGENCY- & & & $\$ 5,118,166$ \\
\hline & TOTAL ESTIMATED COST - & & & $\$ 25,500,000$ \\
\hline
\end{tabular}

\section{PROJECT COST PARAMETERS}

EDI AS A \% OF CONST. + GFE $=60.00 \%$ 
Lockheed Martin Idaho Technologies Co. PROJECT NAME: VIT FACILITY - INTERIM STORAGE FACILITY-OPC-UNESCALATED

LOCATION 1 REQUESTOR 
Lockheed Martin Idaho Technologies Co.

PROECT NAME: VIT FACILITY -INTERIM STORAGE FACILITY - OPC.

LOCATION 1: INEELICPP

REQUESTOR: J. K. RAWLINS
DETAILED COST ESTIMATE SHEET

TYPE OF ESTIMATE: PLANNING

PRONECT NO:: 242101

PREPARED BY: R. D. ADAMS
PAgE 1

DATE 26-Jan-1998

TIME: 13:17:46

RePORT NAME: Detall Cost Estimate Sheot

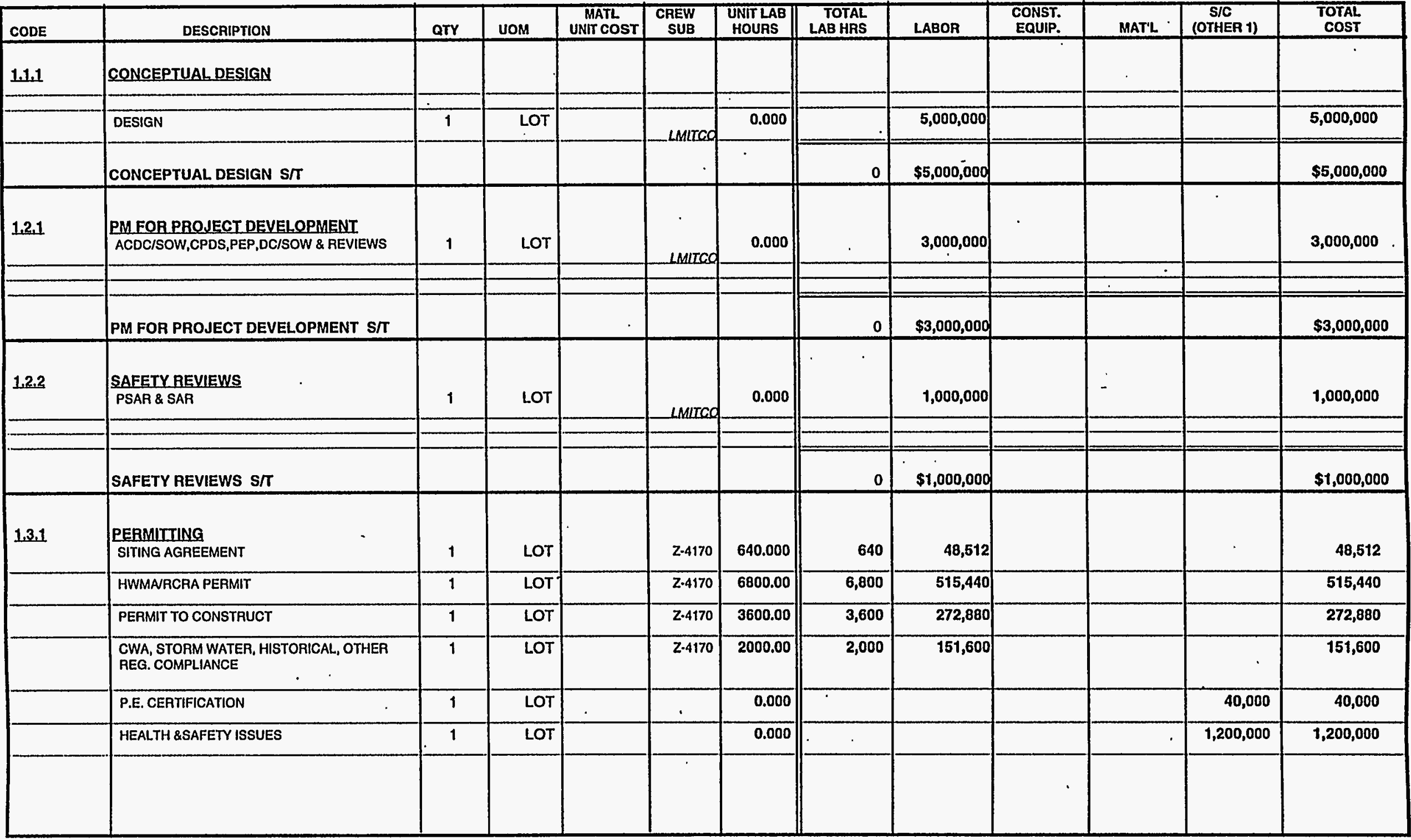




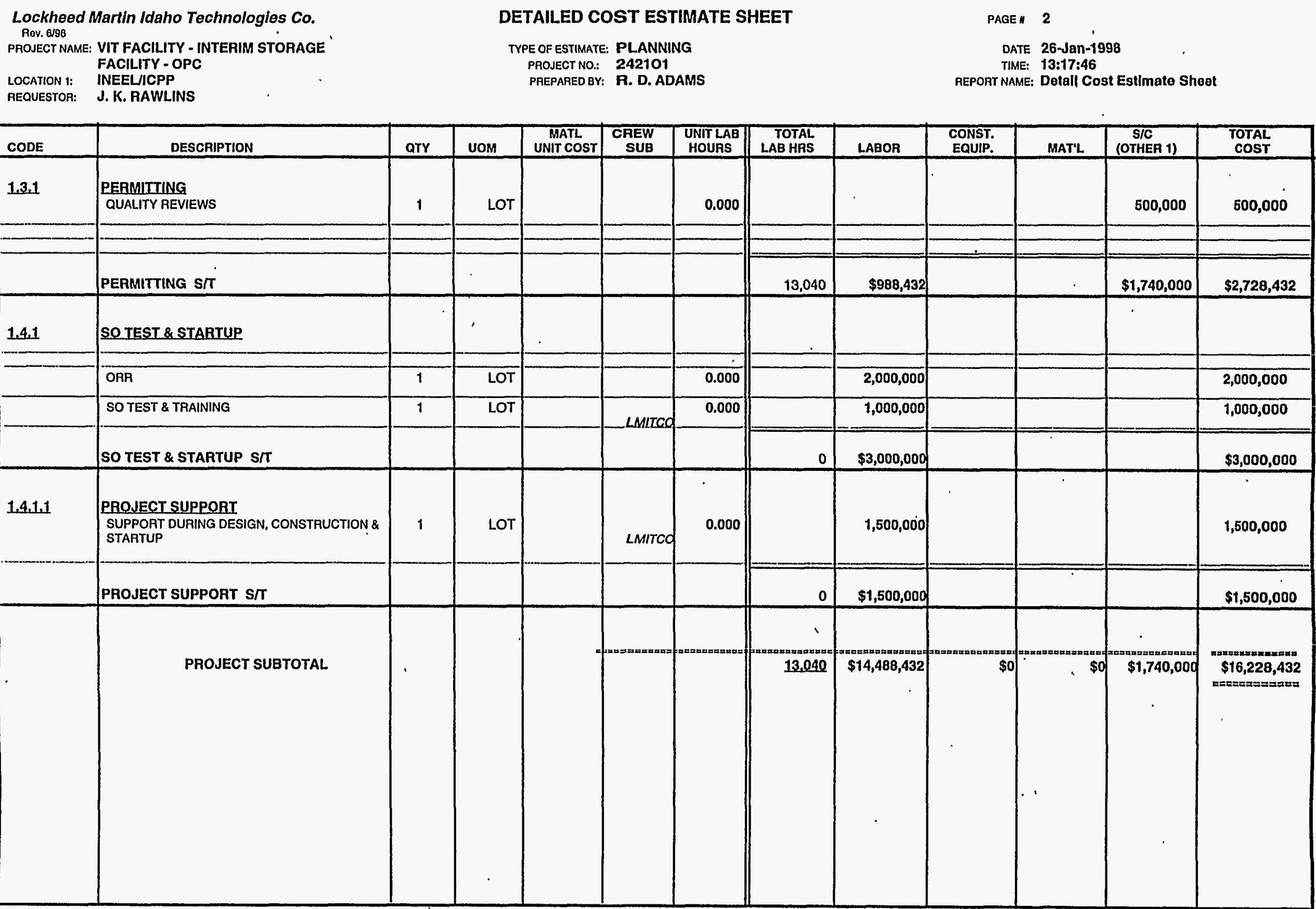


Lockheed Martin Idaho Technologies Co. PROUECT NAME: VIT FACILITY - INTERIM STORAGE FACILITY - OPC - UNESCALATED INEELICPP

LOCATION 1: REQUESTOF:
CONTINGENCY ANALYSIS

TYPE OF ESTIMATE

PROJECT NO

PLANNING

242101

PREPARED BY:

R. D. ADAMS
DATE. 26-Jan-1998

TIME: 13:18:21

REPORT NAME: Contingency Analysis

\begin{tabular}{|c|c|c|c|c|c|c|c|c|c|c|c|}
\hline \multicolumn{9}{|c|}{ PROBABLE \% VARIATION } & \multicolumn{2}{|c|}{$\begin{array}{l}\text { PROJECT } \\
\text { CONTINGENCY }\end{array}$} & \multirow{3}{*}{$\begin{array}{l}\text { SUMMARY } \\
\text { Total Cost } \\
\text { by Element }\end{array}$} \\
\hline $\begin{array}{l}\text { WBS } \\
\text { Element }\end{array}$ & \multirow[t]{2}{*}{ Cost Estimate Element } & \multirow{2}{*}{$\begin{array}{l}\text { Total Cost w/o } \\
\text { Contingency }\end{array}$} & \multirow[t]{2}{*}{$\begin{array}{l}\text { \% Total } \\
\text { Cost }\end{array}$} & \multicolumn{2}{|c|}{$\begin{array}{l}\text { Prob. \% Var. } \\
\text { Frpm Est. }\end{array}$} & \multicolumn{2}{|c|}{ Wt. \% of Prob. } & \multirow[t]{2}{*}{ Contingeney } & \multirow[t]{2}{*}{$\%$} & \multirow[t]{2}{*}{ Cost } & \\
\hline & & & & - & + & - & + & & & & \\
\hline 1.1 .1 & CONCEPTUAL DESIGN & $5,000,000$ & 30.81 & 20 & 30 & 6.16 & 9.24 & $7.703 \%$ & $30.81 \%$ & $2,332,809$ & $7,332,809$ \\
\hline 1.2 .1 & PM FOR PROJECT DEVELOPMENT & $3,000,000$ & 18.49 & 20 & 30 & 3.70 & 5.55 & $4.622 \%$ & 18.49\% & $1,399,686$ & $4,399,686$ \\
\hline 1.22 & SAFETY REVIEWS & $1,000,000$ & 6.16 & 20 & 30 & 1.23 & 1.85 & $1.541 \%$ & $6.16 \%$ & 466,562 & $1,466,562$ \\
\hline 1.3 .1 & PEAMITTING & $2,728,432$ & 16.81 & 20 & 30 & 3.36 & 5.04 & 4.203\% & $16.81 \%$ & $1,272,982$ & $4,001,414$ \\
\hline 1.4 .1 & SO TEST \& STARTUP & $4,500,000$ & 27.73 & 20 & 30 & 5.55 & 8.32 & $6.932 \%$ & $27.73 \%$ & $2,099,529$ & $6,599,529$ \\
\hline \multirow[t]{2}{*}{1.5 .2} & PROCUREMENT FEES & 0 & 0.00 & 5 & 20 & 0.00 & 0.00 & $0.000 \%$ & $0.00 \%$ & o) & 0 \\
\hline & ESCALATION & 0 & 0.00 & 20 & 30 & 0.00 & 0.00 & $0.000 \%$ & $0.00 \%$ & o] & 0 \\
\hline & SUBTOTAL & $16,228,432$ & 100.00 & & & & & $25.000 \%$ & & & \\
\hline & CALCULATED CONMNGENCY & $4,057,108$ & & & & & & & & & \\
\hline & RESULTANT TEC & $20,285,540$ & & & & & & & & & \\
\hline & ROUNOED TEC & $23,800,000$ & & & & & & & & & \\
\hline & PROJECT CONTINGENCY & $7,571,568$ & & & & & & $46.66 \%$ & & - & \\
\hline & MANAGEMENT RESERVE & 0 & & & & . & & & & & \\
\hline & CONTINGENCY & $7,571,568$ & & & & & & & & & \\
\hline & TOTAL ESTIMATED COST & $23,800,000$ & & & & & & & & $7,571,568$ & $23,800,000$ \\
\hline
\end{tabular}

CONFIDENCE LEVEL AND ASSUMED RISKS:

The Lockheed Idaho Technologies Co. Cost Estimate Contingency Analysis Model is based on the applied contingency and the assumptions upon which the estimate was predicated. The model is applied with a suggested risk leve of $18 \%$ and a level of confidence of $90 \%$ the estimate will fall within the bid range. The Contingency Analysis is based on a weighted average to provide a $90 \%$ probability of underun and a $10 \%$ probability of overrun.
CONTINGENCY ANALYSIS GUIDE BY TYPE OF ESTIMATE

Guidelines established by DOERFM 50, Cost Estimating Guide, Vol. 6 , Cost Guide, and as presented in the INEL Cost Estimating Guide.
PLANNING
Experimental/Special Conditions......... Up to $50 \%$
Conceptual
TITLE!
U... Up to $40 \%$
TITLE UI
$10 \%-20 \%$

TITLE II/AFC 

Lockheed Martin Idaho Technologies Co. PROECT NAME: VIT FACILITY - INTERIM STORAGE FACILTY - OPC - ESCALATED

LOCATION 1: REQUESTOR:
CONTINGENCY ANALYSIS

TYPE OFESTIMATE: PLANNING
PROUECT NO:

DATE: 26-Jan-1998

TIME: 13:24:44

REPORT NAME: Contingency Analysis

\begin{tabular}{|c|c|c|c|c|c|c|c|c|c|c|c|}
\hline & PROB & BLE \% VARIA & ON & & & & & & $\begin{array}{l}\text { PRO } \\
\text { CONTI }\end{array}$ & $\begin{array}{l}\text { CT } \\
\text { IENCY }\end{array}$ & SUMMARY \\
\hline \begin{tabular}{|l|} 
WBS \\
Element
\end{tabular} & Cost Estimate Eement & Total Cost w/o & $\begin{array}{l}\text { \% Total } \\
\text { Cost }\end{array}$ & & $\begin{array}{l}\text { \% Var. } \\
\text { m Est. }\end{array}$ & Wt. \% & Prob. & Contingency & $\%$ & Cost & Total Cost \\
\hline & & Contingency & & - & + & - & $t$ & & & & by Element \\
\hline 1.1.1 & CONCEPTUAL DESIGN & $5,000,000$ & 24.53 & 20 & 30 & 4.91 & 7.36 & $6.133 \%$ & $24.53 \%$ & $1,255,571$ & $6,255,571$ \\
\hline 1.21 & PM FOR PROJECT DEVELOPMENT & $3,000,000$ & 14.72 & 20 & 30 & 2.94 & 4.42 & $3.680 \%$ & $14.72 \%$ & 753,342 & $3,753,342$ \\
\hline 1.22 & SAFETY REVIEWS & $1,000,000$ & 4.91 . & 20 & 30 & 0.98 & 1.47 & $1.227 \%$ & $4.91 \%$ & 251,114 & $1,251,114$ \\
\hline 1.3 .1 & PERMITTING & $2,728,432$ & 13.39 & 20 & 30 & 2.68 & 4.02 & $3.347 \%$ & $13.39 \%$ & 685,148 & $3,413,580$ \\
\hline 1.4 .1 & SO TEST \& STARTUP & $4,500,000$ & 22.08 & 20 & 30 & 4.42 & 6.62 & $5.520 \%$ & $22.08 \%$ & $1,130,013$ & $5,630,013$ \\
\hline 1.5 .2 & PROCUREMENT FEES & 0 & 0.00 & 5 & 20 & 0.00 & 0.00 & $0.000 \%$ & $0.00 \%$ & 0 & $\overline{0}$ \\
\hline & ESCALATION & $4,153,402$ & 20.38 & 20 & 30 & 4.08 & 6.11 & $5.094 \%$ & $20.38 \%$ & 1.042 .978 & $5,196,380$ \\
\hline & SUBTOTAL & $20,381,834$ & 100.00 & & & & & $25.000 \%$ & & & \\
\hline & CALCULATED CONTINGENCY & $5,095,459$ & & & & & & & & & \\
\hline & RESULTANT TEC & $25,477,293$ & & & & & & & & & \\
\hline & ROUNDED TEC & $25,500,000$ & & & & & & & & & \\
\hline & PROJECT CONTINGENCY & $5,118,166$ & & & & & & $25.11 \%$ & & & \\
\hline & MANAGEMENT RESERVE & 0 & & & & & & & & & \\
\hline & CONTINGENCY & $5,118,166$ & & & & & & & & & \\
\hline & TOTAL ESTIMATED COST & $25,500,000$ & & & & & & & & $5,118,166$ & $25,500,000$ \\
\hline
\end{tabular}

\section{CONFIDENCE LEVEL AND ASSUMED RISKS:}

The Lockheed Idaho Technologies Co. Cost Estimate Contingency Analysis Model is based on the applied contingency and the assumptions upon which the estimate was predicated. The model is applied with a suggested risk level of $18 \%$ and a level of confidence of $90 \%$ the estimate will fall within the bid range. The Contingency Analysis is based on a weighted average to provide a

$90 \%$ probability of underrun and a $10 \%$ probability of overrun.
CONTINGENCY ANALYSIS GUIDE BY TYPE OF ESTIMATE

Guidelines established by DOEJFM 50, Cost Estimating Guide, Vol. 6 , Cost Guide, and as presented in the INEL Cost Estimating Guide. PLANNING Experimental/Special Conditions $20 \%-30 \%$ Conceptual

Experimental/Special Conditions............. 15\% to $25 \%$ TITLE 1

TITLE II

TITLE II/AFC
.... Up to $40 \%$

$10 \%-20 \%$
$5 \%-15 \%$

Market Conditions 


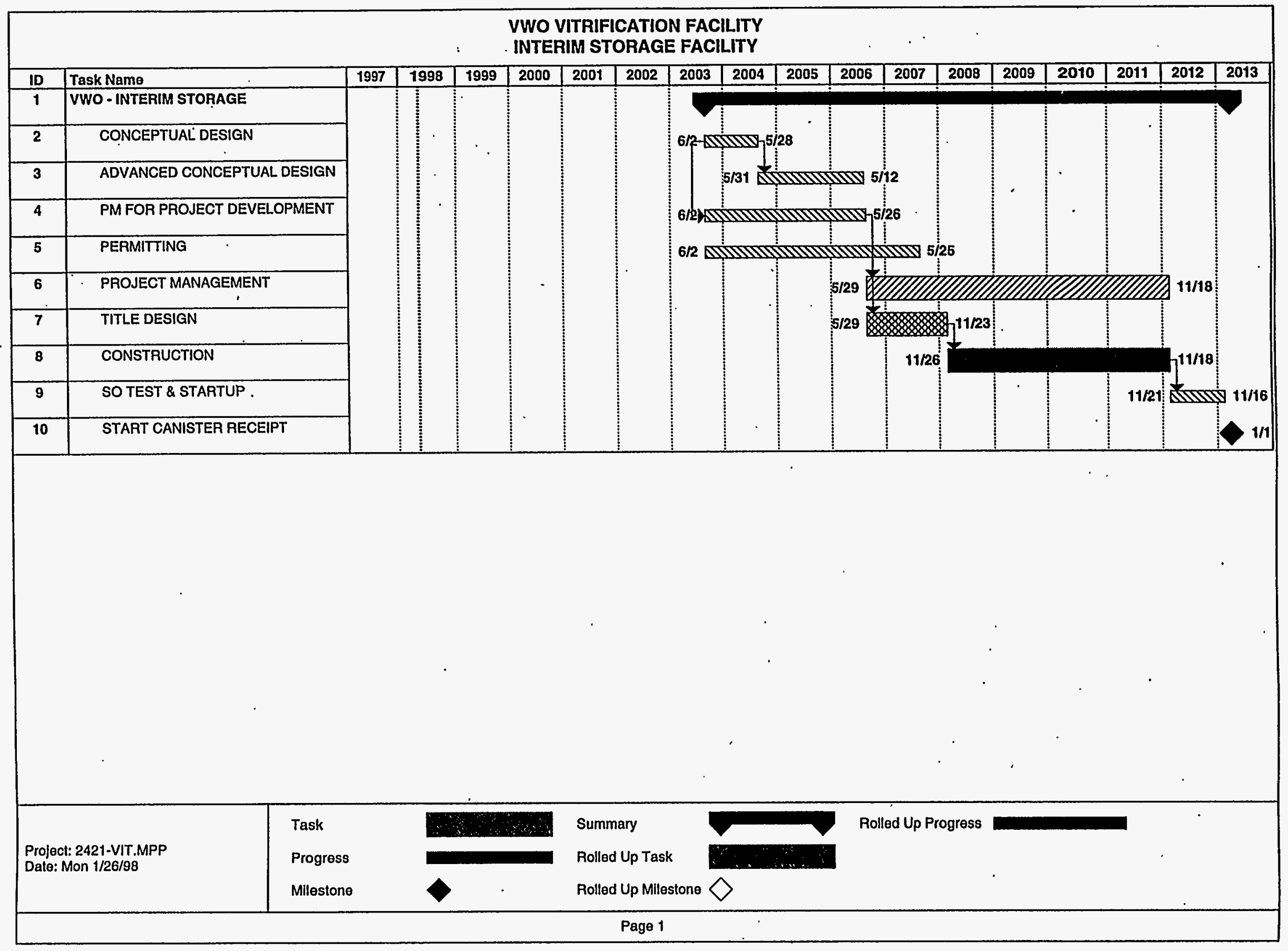




\section{Lockheed Martin Idaho Technologies Company INTERDEPARTMENTAL COMMUNICATION}

Date: $\quad$ February 3, 1998

From: $\quad$ R. J. Turk 4 6-3611

Subject: $\quad$ ECONOMIC AND LIFE CYCLE ANALYSIS CONDUCTED for the HIGH LEVEL WASTE (HLW) INTERIM STORAGE FACILITY-TRU -RJT-23-98

\section{Purpose:}

As requested an Economic and Life-Cycle Cost (LCC) has been conducted to evaluate for the HLW-TRU INTERIM STORAGE FACILITY. The storage facility was evaluated based on a scoping study that incorporate appropriate design features from the Flour Daniel Corp. design for interim storage. This storage facility will be used to house canisters resulting from the HLW production option until final repository disposition.

This economic analysis is based on information provided by J. K. Rawlins, and K. L. Williams. R. D. Adams provided cost estimates. B.L. Blakely and D.E. Keller provided process personnel modeling.

\section{Methodology:}

The Economic Evaluation assumed a 73-year period (1999-2071) since this is the estimated time required to complete all of the anticipated remediation activities. The LCC identified and evaluated the initial development, construction, operating and post operating costs over the lifecycle. A discounted LCC analysis assumes a 1998-dollar basis, discounted $6.3 \%$ annually per the Office of Management and Budget (OMB) Circular A-94 for escalated costs. All costs are conservatively discounted assuming the end-of-year convention. 
John K. Rawlins

February 3, 1998

RTT 23-98

Page 2

\section{Assumptions:}

The scope of work and requirements of all related activities are vague at this time. Facility and processing costs were developed from historical experience associated with DD\&D work at the INEEL. The LCC analysis was generated to match cost estimating cost structure. These costs include Permitting, Direct and Indirect Construction, G\&A, Procurement Fee, Engineering, Inspection, Project Management, Construction Management, Escalation and Contingency costs.

The design period is assumed to be accomplished in five-years with construction completed in two-years. Labor rates were assumed as follows: Managers, $\$ 125 / \mathrm{hr}$; Engineers, $\$ 108 \$ / \mathrm{hr}$; Other Technicians $\$ 85 / \mathrm{hr}$; Administration/support staff $\$ 65 / \mathrm{hr}$; Operators and Maintenance personnel $\$ 65 / \mathrm{hr}$. The operational period for this facility was assumed to be twenty years, to match the appropriate operating facility. This period is followed by five years for shipping to the repository, followed by three years of post-operations activities. Due to this project's lack of complexity and non-radioactive contaminated environment, this analysis assumed a decommissioning cost equal to $10 \%$ of the non-escalated engineering design cost, decontamination costs equal to $0 \%$ of non-escalated total pre-operation cost, and demolition costs equal to $8 \%$ of the non-escalated total pre-operation cost. Utilities were calculated assuming a $\$ .0824$ per $\mathrm{kWh}$. Surveillance and Monitoring costs were calculated at $\$ 65,000$ per year for manned security during operations then reduced to $\$ 10,000$ for remote surveillance during non-operation periods.

\section{Results:}

The HLW-TRU INTERIM STORAGE FACILITY has a discounted LCC of $\$ 89.5$ million. Attachments:

cc: R. J. Turk File 


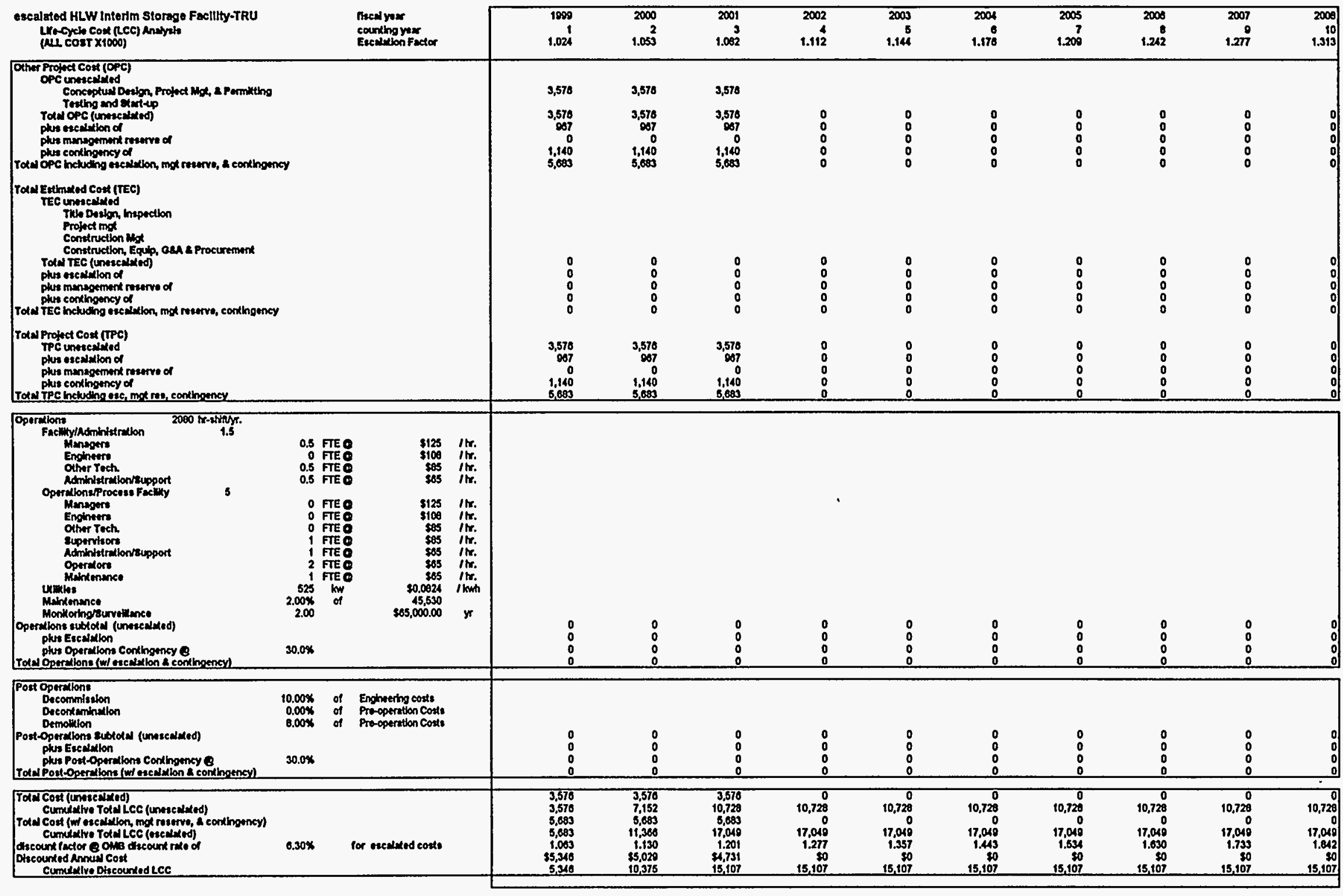




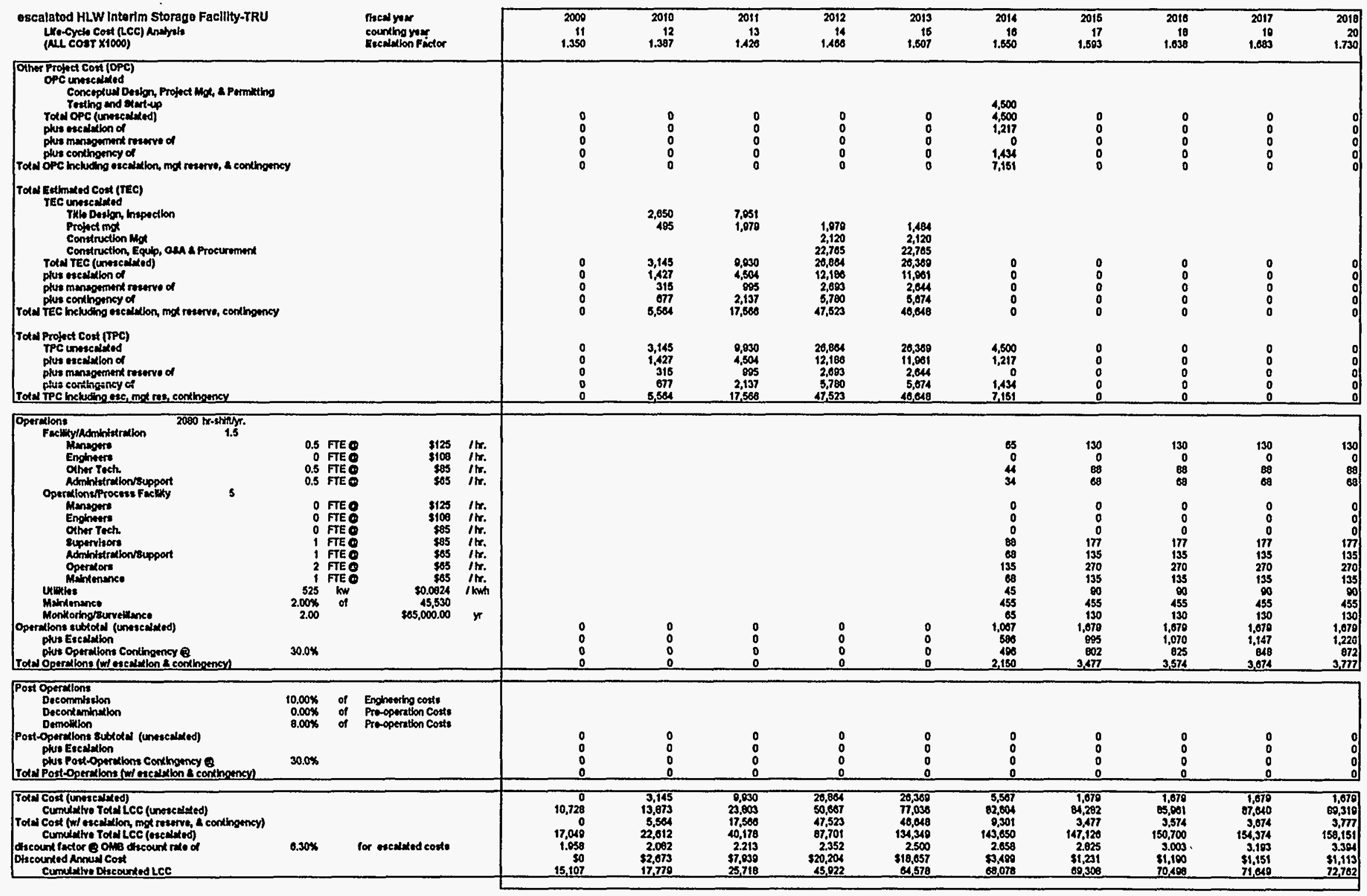




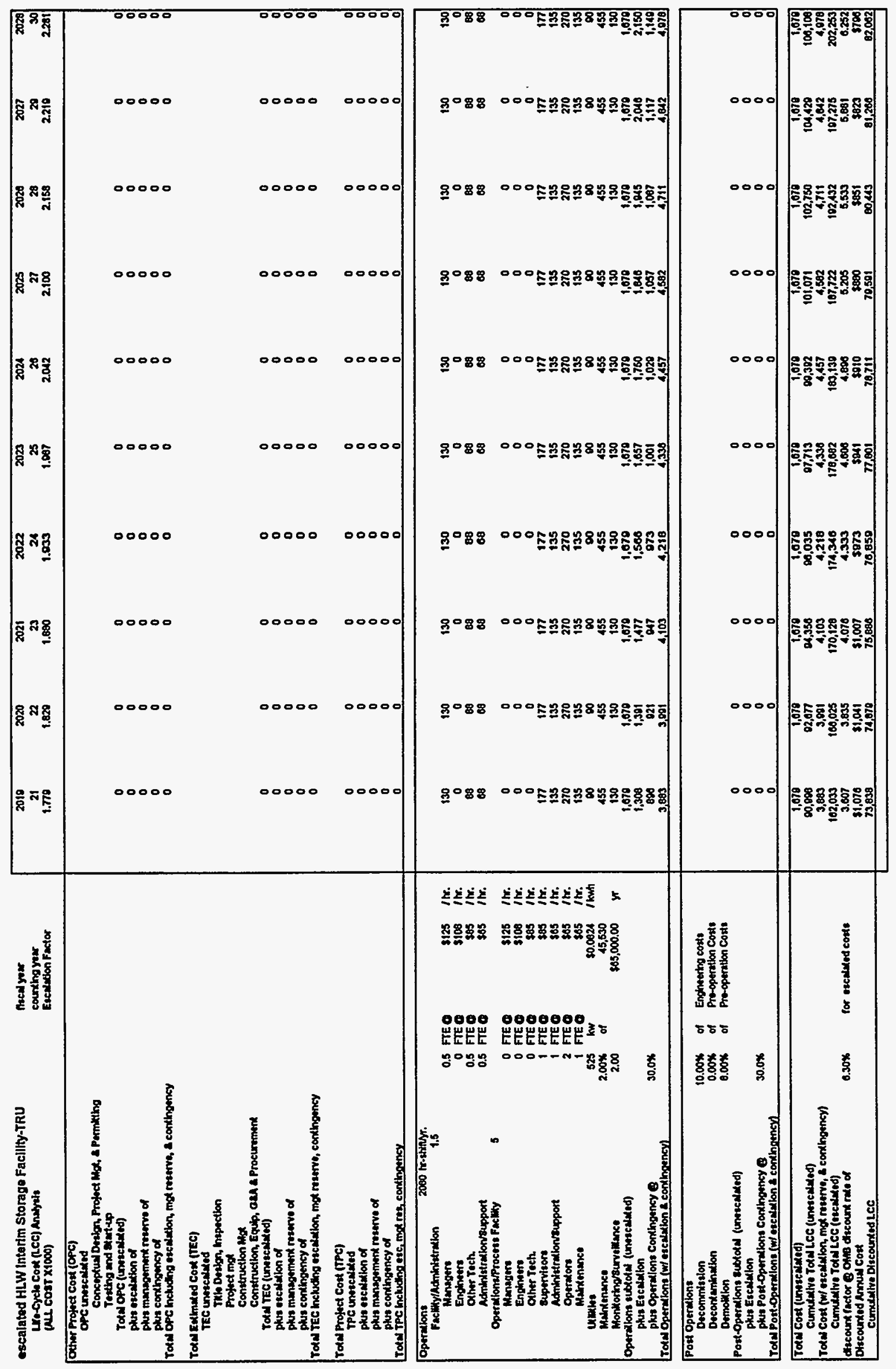




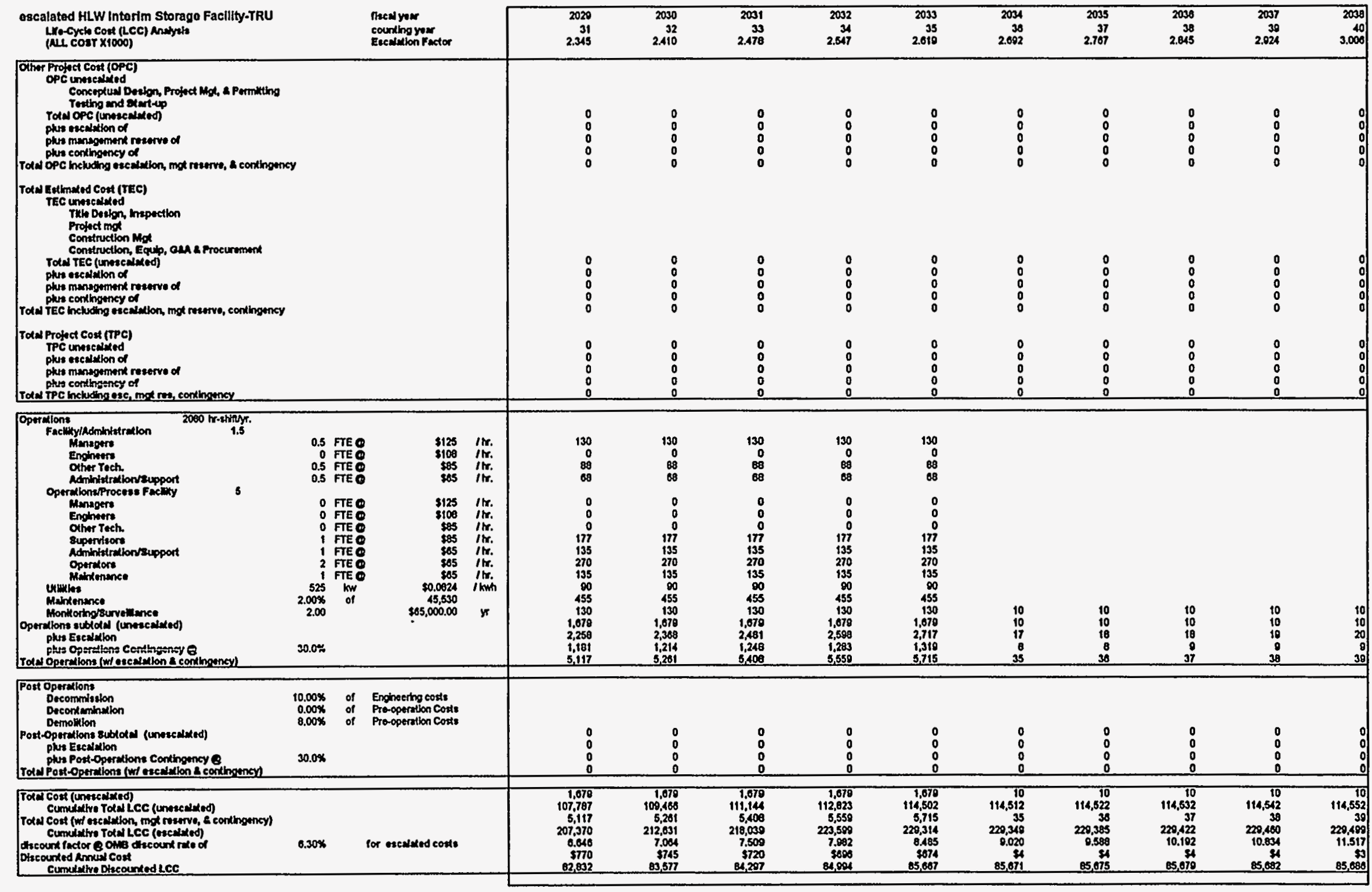




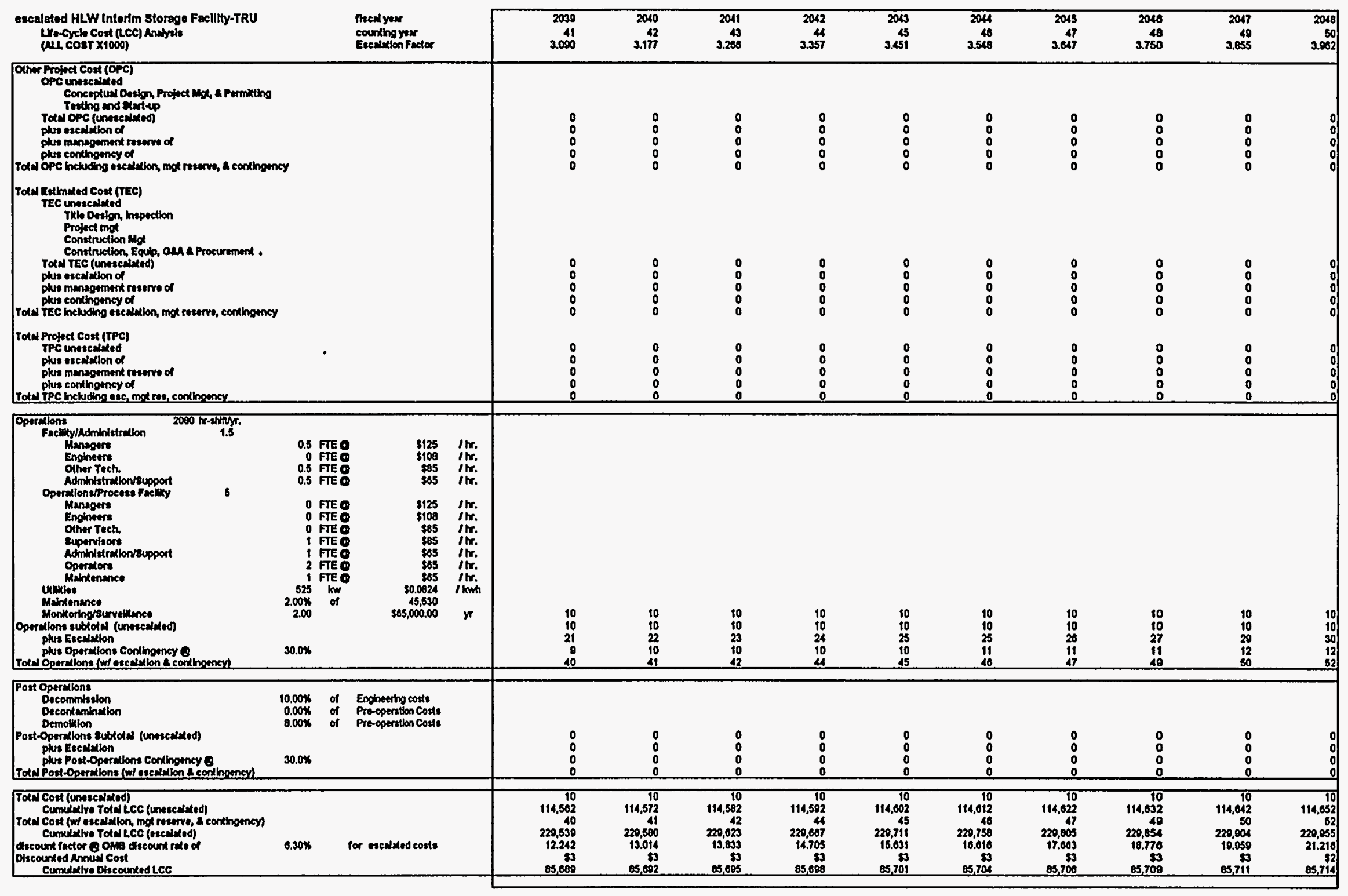




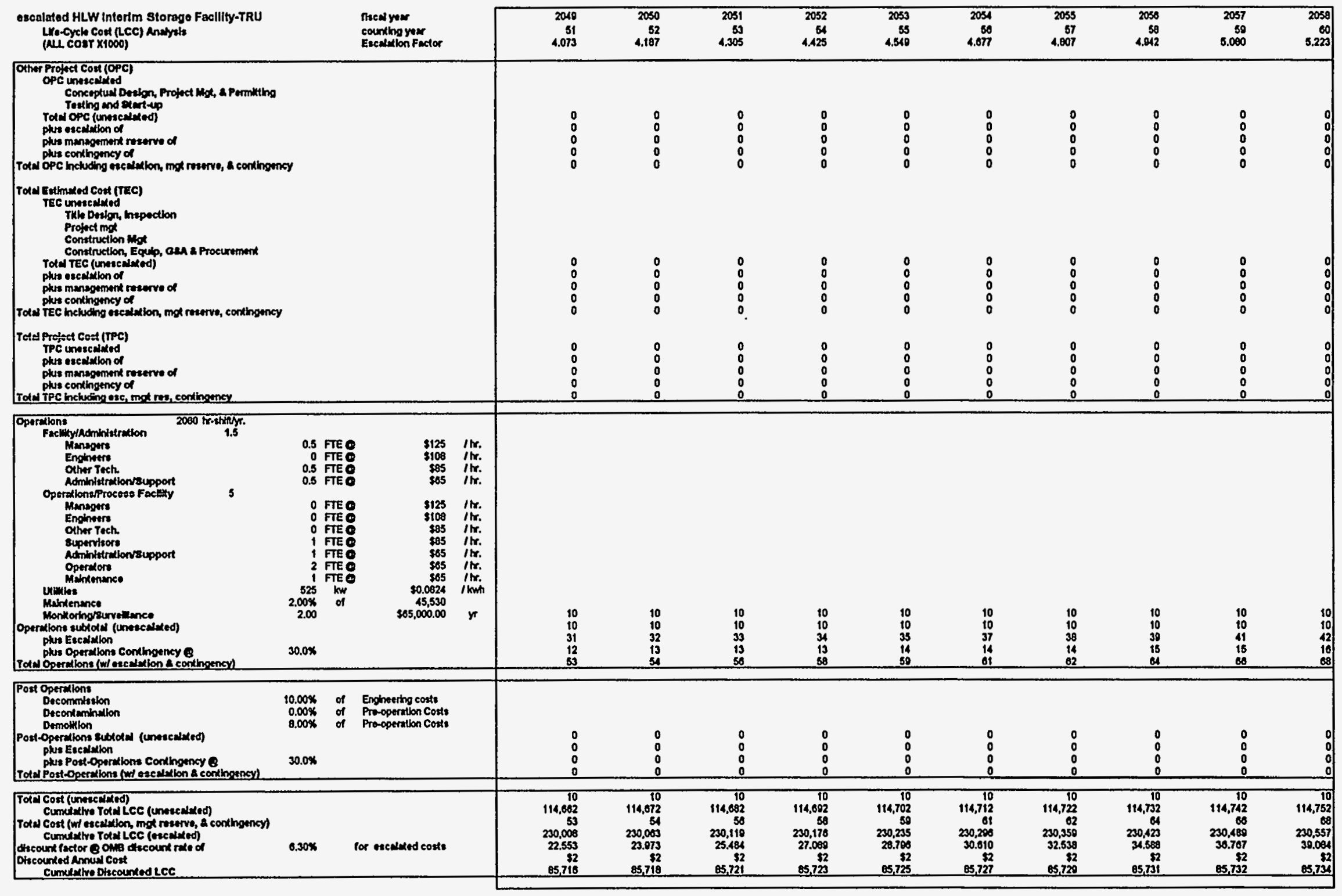




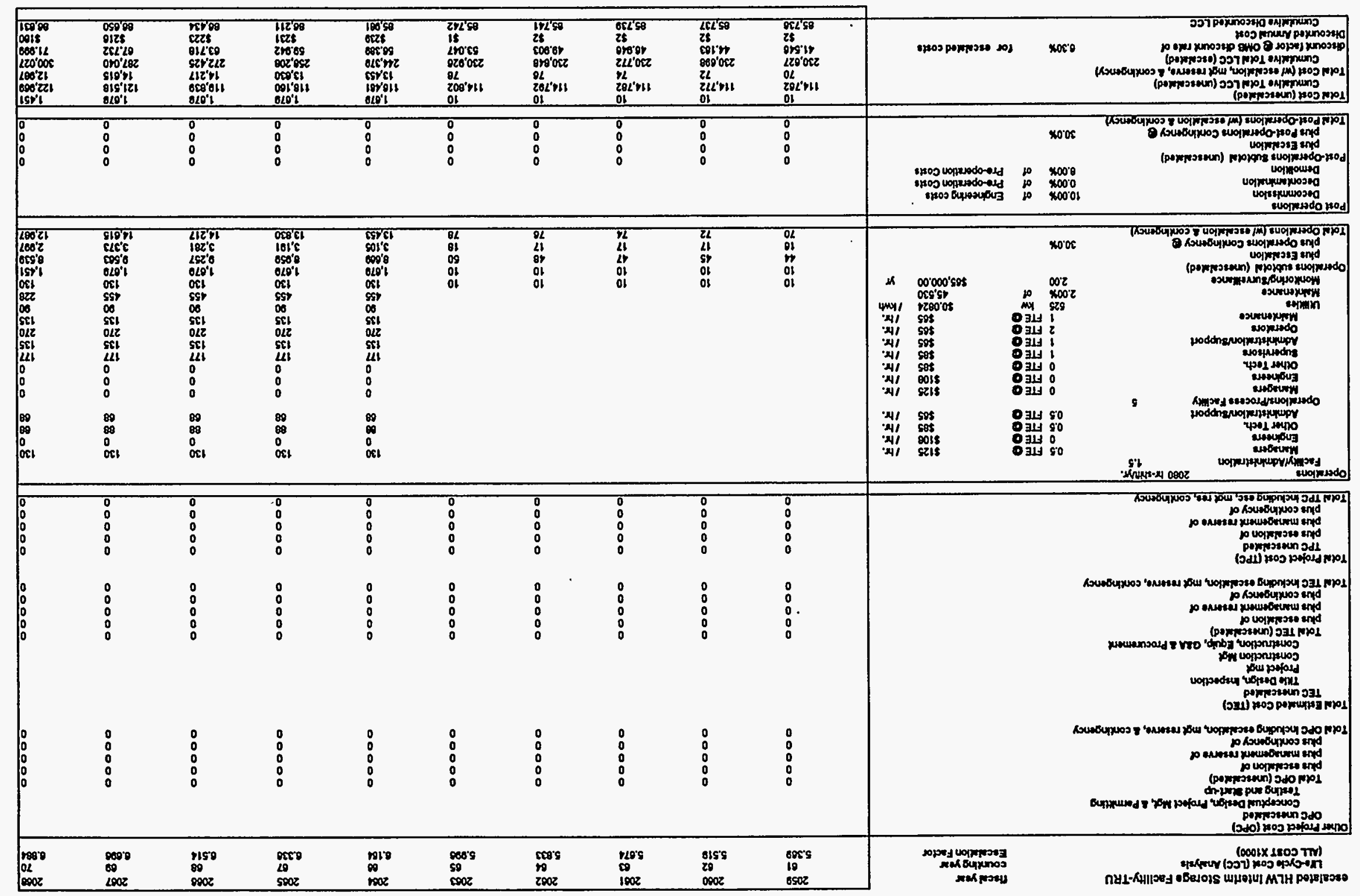




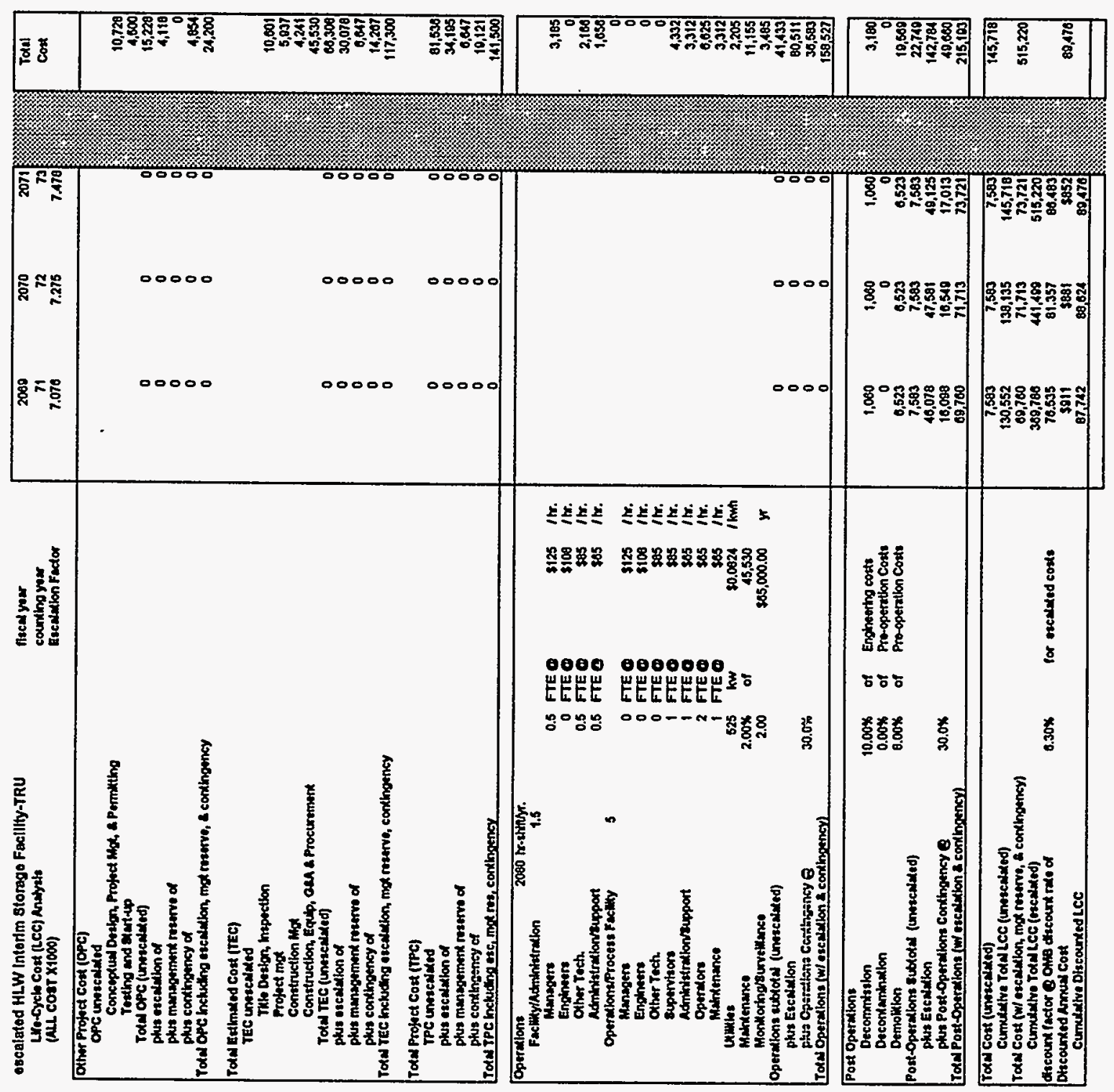




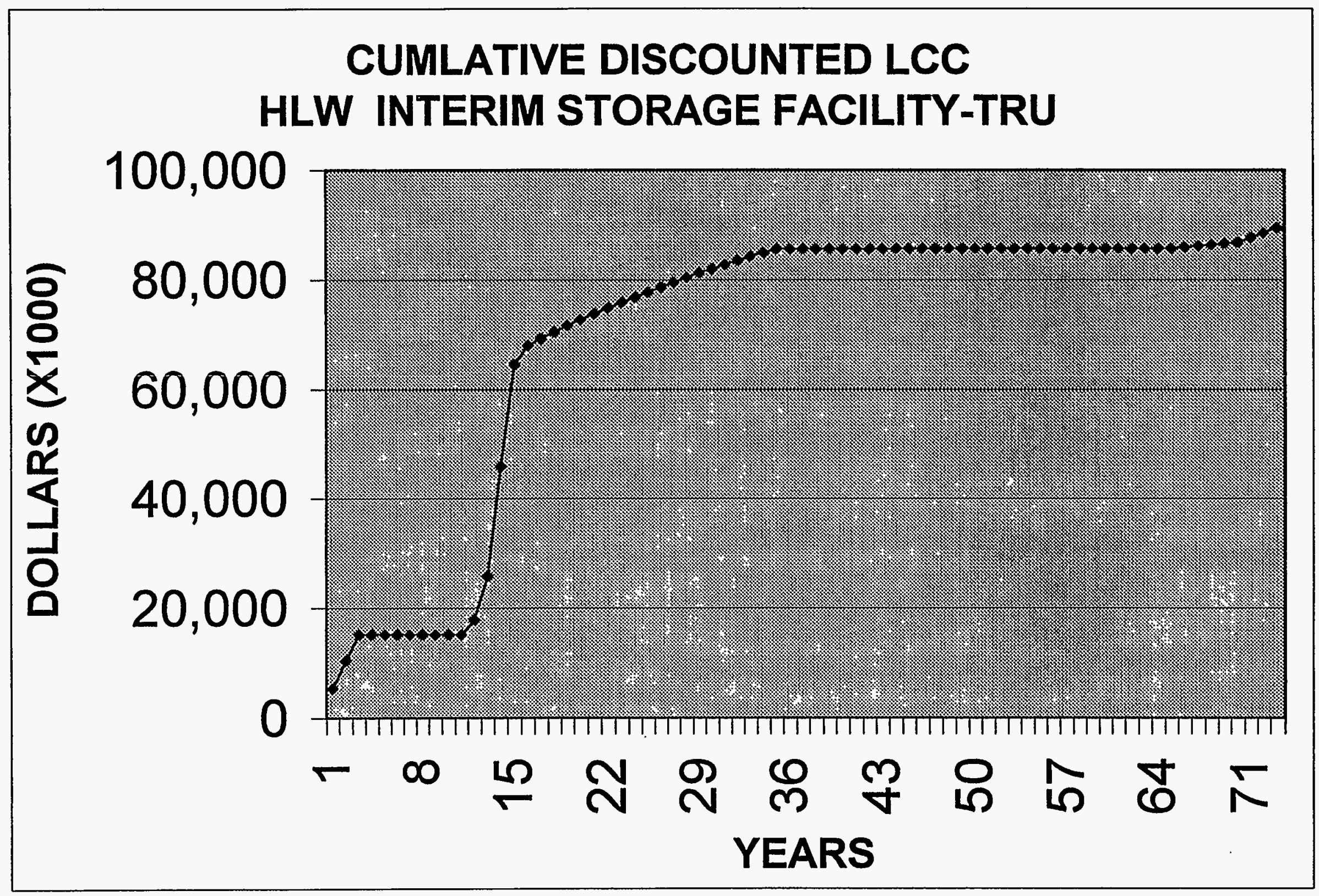




\section{TOTAL ANNUAL COST (escalated) HLW INTERIM STORAGE FACILTY-TRU}

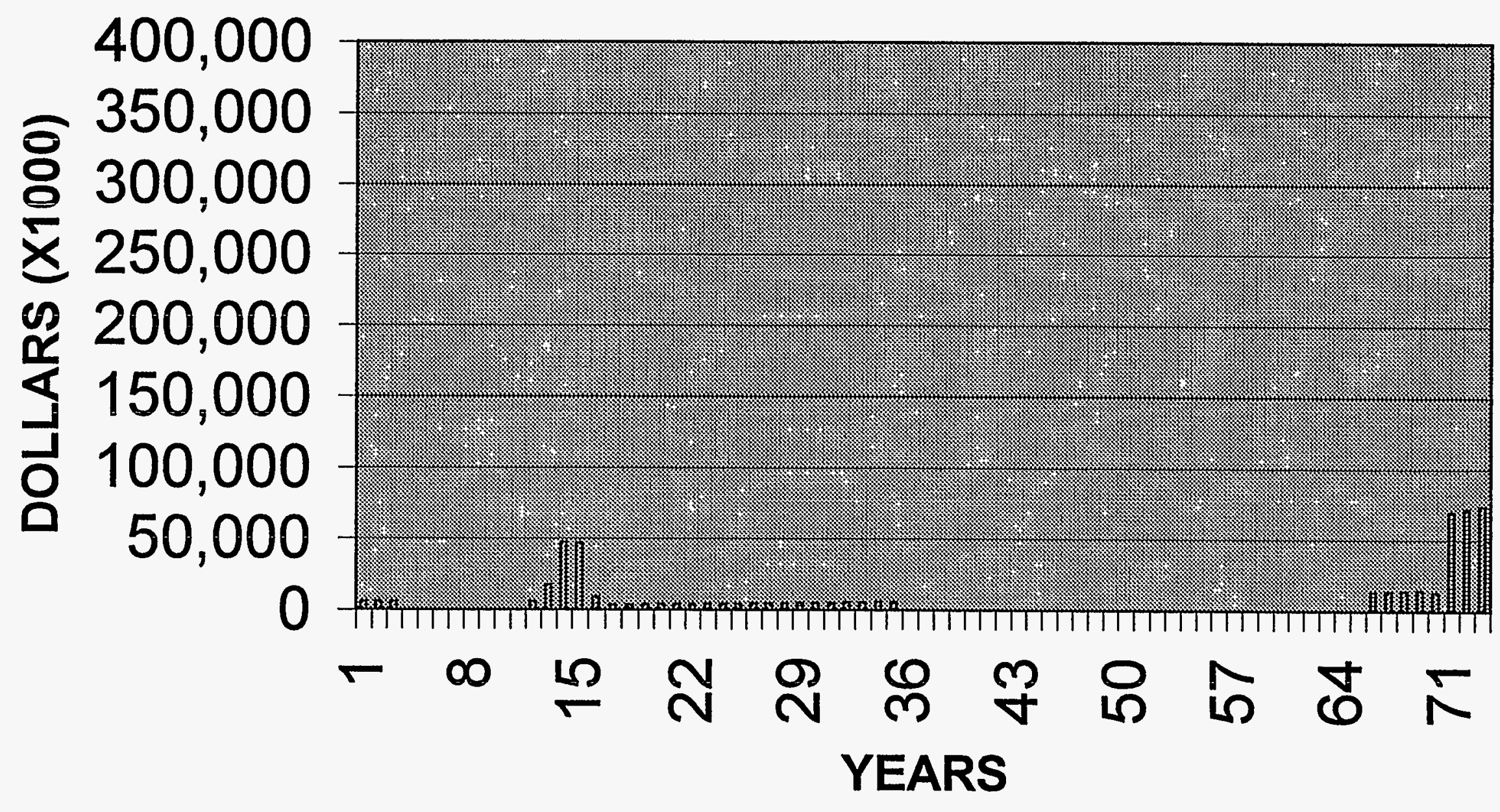


Date: January 30, 1998

To: $\quad$ John K. Rawlins $\quad$ MS 3765 6-4304

From:

R. J. Turk Rflede MS 3875

6-3611

Subject: $\quad$ ECONOMIC AND LIFE CYCLE ANALYSIS CONDUCTED for the VITRIFICATION INTERIM STORAGE FACILITIES- RJT-13-98

\section{Purpose:}

As requested an Economic and Life-Cycle Cost (LCC) has been conducted to evaluate for the INTERIM STORAGE FACILITIES. The storage facility was evaluated based on a scoping study that incorporate appropriate design features from the Flour Daniel Corp. design for interim storage. This storage facility will be used to house canisters resulting from the Vitrification production option until final repository disposition.

This economic analysis is based on information provided by J. K. Rawlins, and K. L. Williams. R. D. Adams provided cost estimates. B.L. Blakely provided process personnel modeling.

\section{Methodology:}

The Economic Evaluation assumed a 69-year period (2003-2071) since this is the estimated time required to complete all of the anticipated remediation activities. The LCC identified and evaluated the initial development, construction, operating and post operating costs over the lifecycle. A discounted LCC analysis assumes a 1998-dollar basis, discounted $6.3 \%$ annually per the Office of Management and Budget (OMB) Circular A-94 for escalated costs. All costs are conservatively discounted assuming the end-of-year convention. 


\section{Assumptions:}

The scope of work and requirements of all related activities are vague at this time. Facility and processing costs were developed from historical experience associated with DD\&D work at the INEEL. The LCC analysis was generated to match cost estimating cost structure. These costs include Permitting, Direct and Indirect Construction, G\&A, Procurement Fee, Engineering, Inspection, Project Management, Construction Management, Escalation and Contingency costs.

The design period is assumed to be accomplished in five-years with construction completed in six-years. Labor rates were assumed as follows: Managers, \$125/hr; Engineers, \$108 \$/hr; Other Technicians \$ 85/hr; Administration/support staff \$ 65/hr; Operators and Maintenance personnel $\$ 65 / \mathrm{hr}$. The operational period for this facility was assumed to be twenty years, to match the appropriate operating facility. This period is followed by five years for shipping to the repository, followed by three years of post-operations activities. Due to this project's lack of complexity and non-radioactive contaminated environment, this analysis assumed a decommissioning cost equal to $10 \%$ of the non-escalated engineering design cost, decontamination costs equal to $0 \%$ of non-escalated total pre-operation cost, and demolition costs equal to $8 \%$ of the non-escalated total pre-operation cost. Utilities were calculated assuming a $\$ .0824$ per $\mathrm{kWh}$. Surveillance and Monitoring costs were calculated at $\$ 65,000$ per year for manned security during operations then reduced to $\$ 10,000$ for remote surveillance during non-operation periods.

\section{Results:}

The VITRIFICATION FACILITY INTERIM STORAGE has a discounted LCC of $\$ 426$ million.

Attachments:

cc: R. J. Turk File 


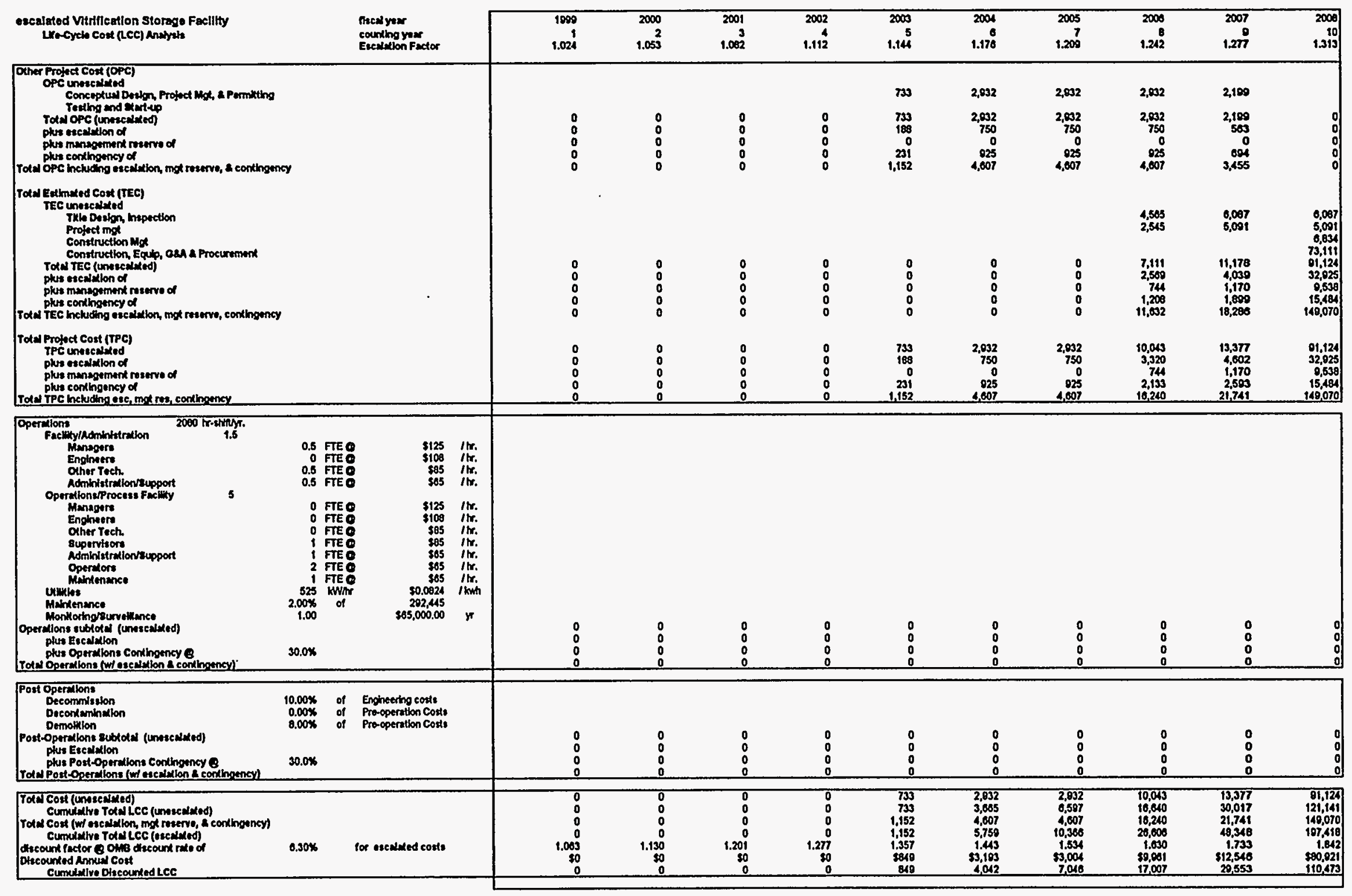




\begin{tabular}{|c|c|c|c|c|c|c|c|c|c|c|c|c|}
\hline 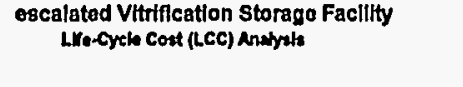 & & $\begin{array}{l}\text { Then yos } \\
\text { coundhg yew } \\
\text { Enctulion Futor }\end{array}$ & $\begin{array}{r}2009 \\
11 \\
1.350\end{array}$ & $\begin{array}{r}2010 \\
12 \\
1.387\end{array}$ & $\begin{array}{r}2011 \\
13 \\
1.428\end{array}$ & $\begin{array}{r}2012 \\
14 \\
1,480\end{array}$ & $\begin{array}{r}2013 \\
15 \\
1.507\end{array}$ & $\begin{array}{l}2014 \\
10 \\
1.350\end{array}$ & $\begin{array}{r}2015 \\
17 \\
1.593\end{array}$ & $\begin{array}{l}2010 \\
18 \\
1.038\end{array}$ & $\begin{array}{r}2017 \\
10 \\
1.603\end{array}$ & $\begin{array}{r}2010 \\
20 \\
1.730\end{array}$ \\
\hline \multicolumn{3}{|l|}{ 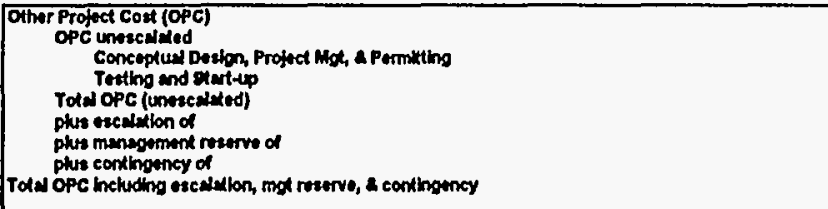 } & $\begin{array}{l}0 \\
0 \\
0 \\
0 \\
0\end{array}$ & $\begin{array}{l}0 \\
0 \\
0 \\
0\end{array}$ & $\begin{array}{l}0 \\
0 \\
0 \\
0 \\
0\end{array}$ & $\begin{array}{l}4,500 \\
4,400 \\
1,152 \\
0 \\
0 \\
7,4,18 \\
7,071\end{array}$ & $\begin{array}{l}0 \\
0 \\
0 \\
0 \\
0\end{array}$ & $\begin{array}{l}0 \\
0 \\
0 \\
0 \\
0\end{array}$ & $\begin{array}{l}0 \\
0 \\
0 \\
0 \\
0\end{array}$ & $\begin{array}{l}0 \\
0 \\
0 \\
0 \\
0\end{array}$ & $\begin{array}{l}0 \\
0 \\
0 \\
0 \\
0\end{array}$ & $\begin{array}{l}0 \\
0 \\
0 \\
0 \\
0\end{array}$ \\
\hline \multicolumn{3}{|l|}{ 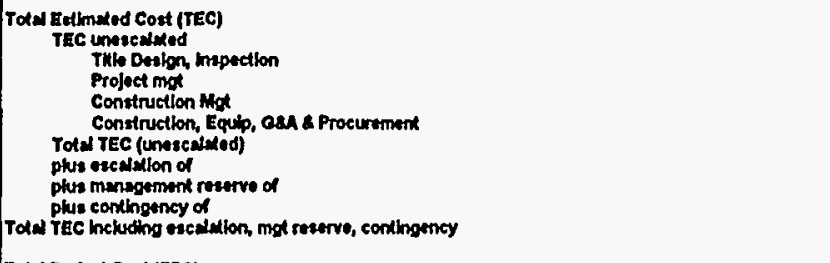 } & 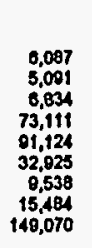 & 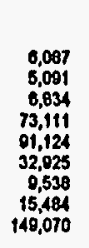 & $\begin{array}{r}0,007 \\
5,091 \\
8,034 \\
73,111 \\
01,124 \\
32,225 \\
0,538 \\
15,44 \\
149,070\end{array}$ & $\begin{array}{l}0 \\
0 \\
0 \\
0 \\
0\end{array}$ & $\begin{array}{l}0 \\
0 \\
0 \\
0 \\
0\end{array}$ & $\begin{array}{l}0 \\
0 \\
0 \\
0 \\
0\end{array}$ & $\begin{array}{l}0 \\
0 \\
0 \\
0 \\
0\end{array}$ & $\begin{array}{l}0 \\
0 \\
0 \\
0 \\
0\end{array}$ & $\begin{array}{l}0 \\
0 \\
0 \\
0 \\
0\end{array}$ & $\begin{array}{l}0 \\
0 \\
0 \\
0 \\
0\end{array}$ \\
\hline \multicolumn{3}{|l|}{ 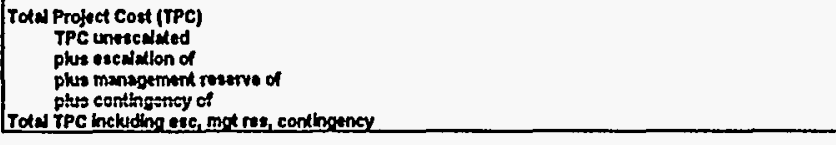 } & $\begin{array}{r}91,124 \\
32,025 \\
9,539 \\
95,404 \\
169,070 \\
\end{array}$ & $\begin{array}{r}91,121 \\
32,025 \\
0,393 \\
15,404 \\
149,070 \\
\end{array}$ & $\begin{array}{r}91,124 \\
32,025 \\
9,538 \\
15,484 \\
149,070 \\
\end{array}$ & $\begin{array}{r}4,500 \\
1,152 \\
0 \\
1,419 \\
7,071 \\
\end{array}$ & $\begin{array}{l}0 \\
0 \\
0 \\
0 \\
0 \\
\end{array}$ & $\begin{array}{l}0 \\
0 \\
0 \\
0 \\
0 \\
\end{array}$ & $\begin{array}{l}0 \\
0 \\
0 \\
0 \\
0 \\
\end{array}$ & $\begin{array}{l}0 \\
0 \\
0 \\
0 \\
0 \\
\end{array}$ & $\begin{array}{l}0 \\
0 \\
0 \\
0 \\
0 \\
\end{array}$ & \begin{tabular}{l|l}
0 \\
0 \\
0 \\
0 \\
0
\end{tabular} \\
\hline 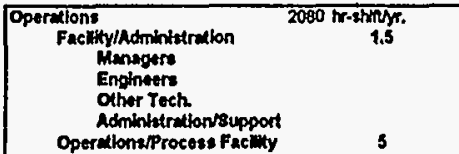 & $\begin{array}{l}0.5 \text { FTE } \\
0 \text { FTE } \\
0.5 \mathrm{FTE} \\
0.5 \text { FTE }\end{array}$ & 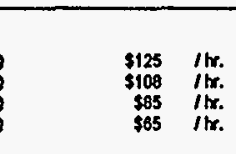 & & & & $\begin{array}{r}65 \\
0 \\
4 \\
34\end{array}$ & $\begin{array}{r}130 \\
0 \\
69 \\
69\end{array}$ & $\begin{array}{r}130 \\
0 \\
68 \\
68\end{array}$ & $\begin{array}{r}130 \\
0 \\
88 \\
88\end{array}$ & $\begin{array}{r}130 \\
0 \\
88 \\
69\end{array}$ & $\begin{array}{r}130 \\
0 \\
88 \\
89\end{array}$ & \begin{tabular}{r|}
130 \\
0 \\
89 \\
60
\end{tabular} \\
\hline 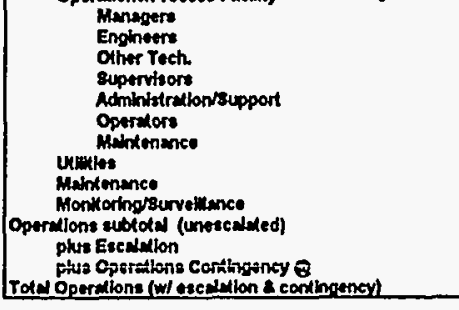 & 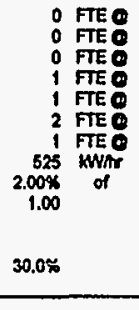 & 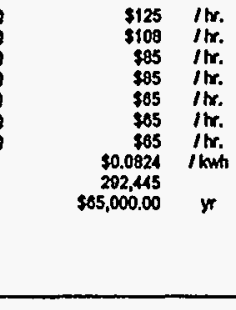 & $\begin{array}{l}0 \\
0 \\
0 \\
0 \\
\end{array}$ & $\begin{array}{l}0 \\
0 \\
0 \\
0 \\
\end{array}$ & $\begin{array}{l}0 \\
0 \\
0 \\
0 \\
\end{array}$ & $\begin{array}{r}0 \\
0 \\
0 \\
69 \\
68 \\
135 \\
68 \\
65 \\
1,024 \\
33 \\
3,504 \\
1,644 \\
1,441 \\
0,078 \\
\end{array}$ & $\begin{array}{r}0 \\
0 \\
170 \\
177 \\
135 \\
270 \\
135 \\
00 \\
5,019 \\
65 \\
7,007 \\
3,555 \\
3,168 \\
13,731 \\
\end{array}$ & $\begin{array}{r}0 \\
0 \\
177 \\
175 \\
135 \\
270 \\
135 \\
000 \\
5,840 \\
05 \\
7,007 \\
3,051 \\
3,257 \\
14,118 \\
\end{array}$ & $\begin{array}{r}0 \\
0 \\
0 \\
177 \\
135 \\
270 \\
135 \\
00 \\
5,819 \\
65 \\
7,007 \\
4.155 \\
3,349 \\
14,511 \\
\end{array}$ & $\begin{array}{r}0 \\
0 \\
177 \\
135 \\
270 \\
135 \\
80 \\
5,810 \\
05 \\
7,007 \\
4,407 \\
3,42 \\
14,017 \\
\end{array}$ & $\begin{array}{r}0 \\
0 \\
177 \\
135 \\
270 \\
135 \\
00 \\
5,648 \\
655 \\
7,007 \\
4,769 \\
3,539 \\
15,333 \\
\end{array}$ & \begin{tabular}{r|}
0 \\
0 \\
170 \\
135 \\
270 \\
135 \\
80 \\
3,840 \\
7,05 \\
7.007 \\
8,119 \\
3,038 \\
15,704 \\
\end{tabular} \\
\hline 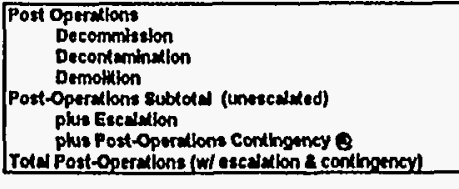 & $\begin{array}{l}10.00 \% \text { of } \\
0.00 \% \\
8.00 \% \text { of } \\
30.0 \%\end{array}$ & $\begin{array}{l}\text { Engheering costs } \\
\text { Prosopertiton Costs } \\
\text { Pro-operation Costs }\end{array}$ & $\begin{array}{l}0 \\
0 \\
0 \\
0 \\
\end{array}$ & $\begin{array}{l}0 \\
0 \\
0 \\
0 \\
\end{array}$ & $\begin{array}{l}0 \\
0 \\
0 \\
0 \\
\end{array}$ & $\begin{array}{l}0 \\
0 \\
0 \\
0\end{array}$ & $\begin{array}{l}0 \\
0 \\
0 \\
0\end{array}$ & $\begin{array}{l}0 \\
0 \\
0 \\
0 \\
\end{array}$ & $\begin{array}{l}0 \\
0 \\
0 \\
0 \\
\end{array}$ & $\begin{array}{l}0 \\
0 \\
0 \\
0 \\
\end{array}$ & $\begin{array}{l}0 \\
0 \\
0 \\
0 \\
\end{array}$ & $\begin{array}{l}0 \\
0 \\
0 \\
0\end{array}$ \\
\hline 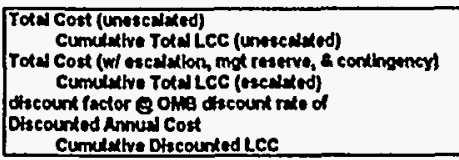 & $0.30 \%$ & for oscanded coste & 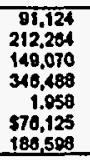 & 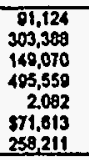 & $\begin{array}{l}91,124 \\
304,511 \\
119,01070 \\
64,029 \\
2.213 \\
507,389 \\
325,580 \\
\end{array}$ & 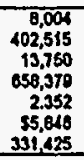 & 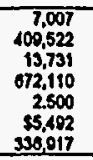 & $\begin{array}{r}7.007 \\
416,530 \\
14,110 \\
380,220 \\
2.658 \\
35,311 \\
342,228 \\
\end{array}$ & 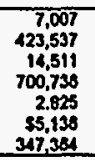 & 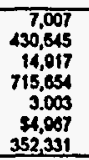 & 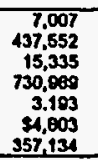 & $\begin{array}{r}7,000 \\
44,580 \\
15,740 \\
740,753 \\
3.39 \\
4,945 \\
301,780 \\
\end{array}$ \\
\hline
\end{tabular}




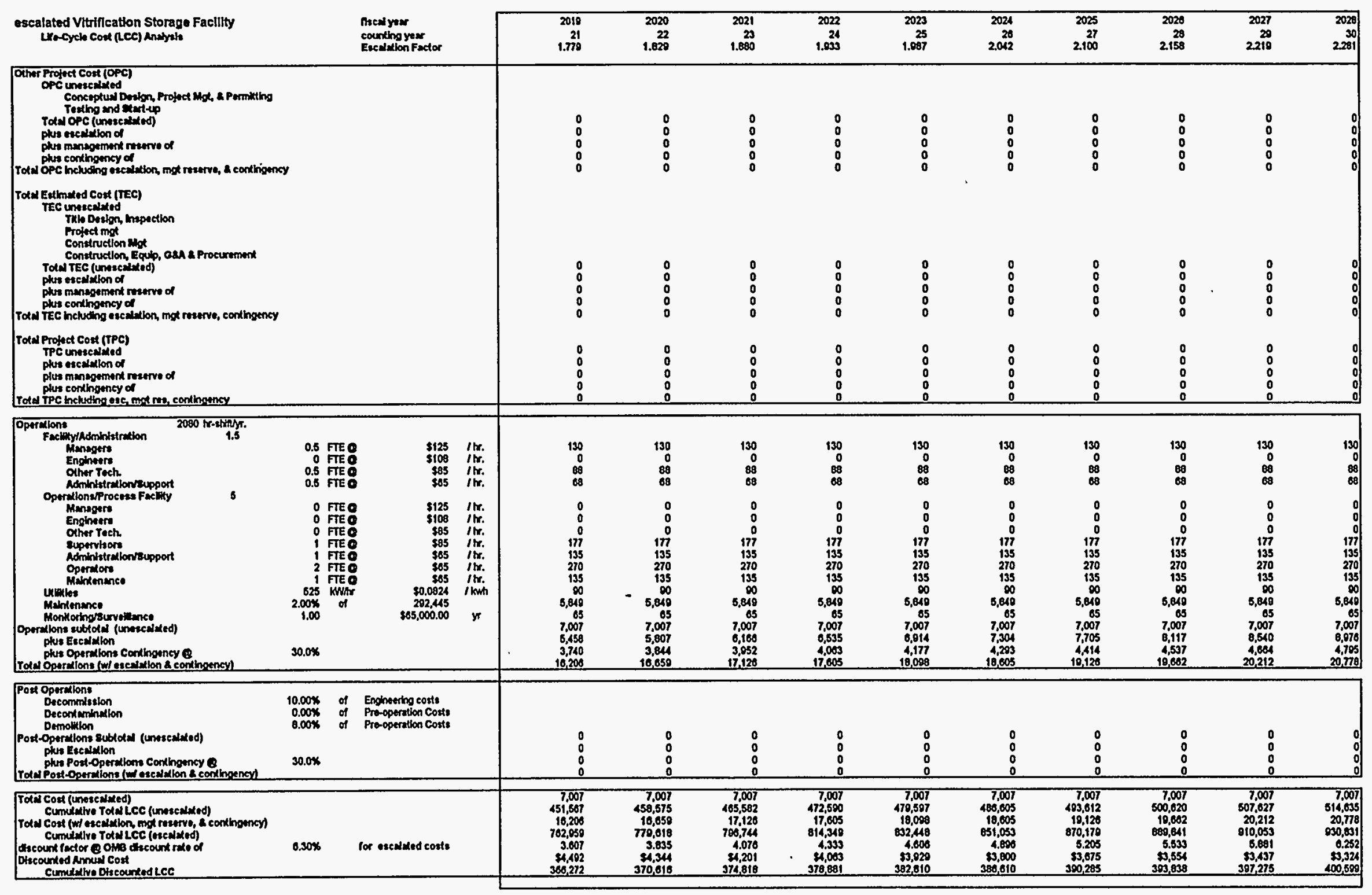




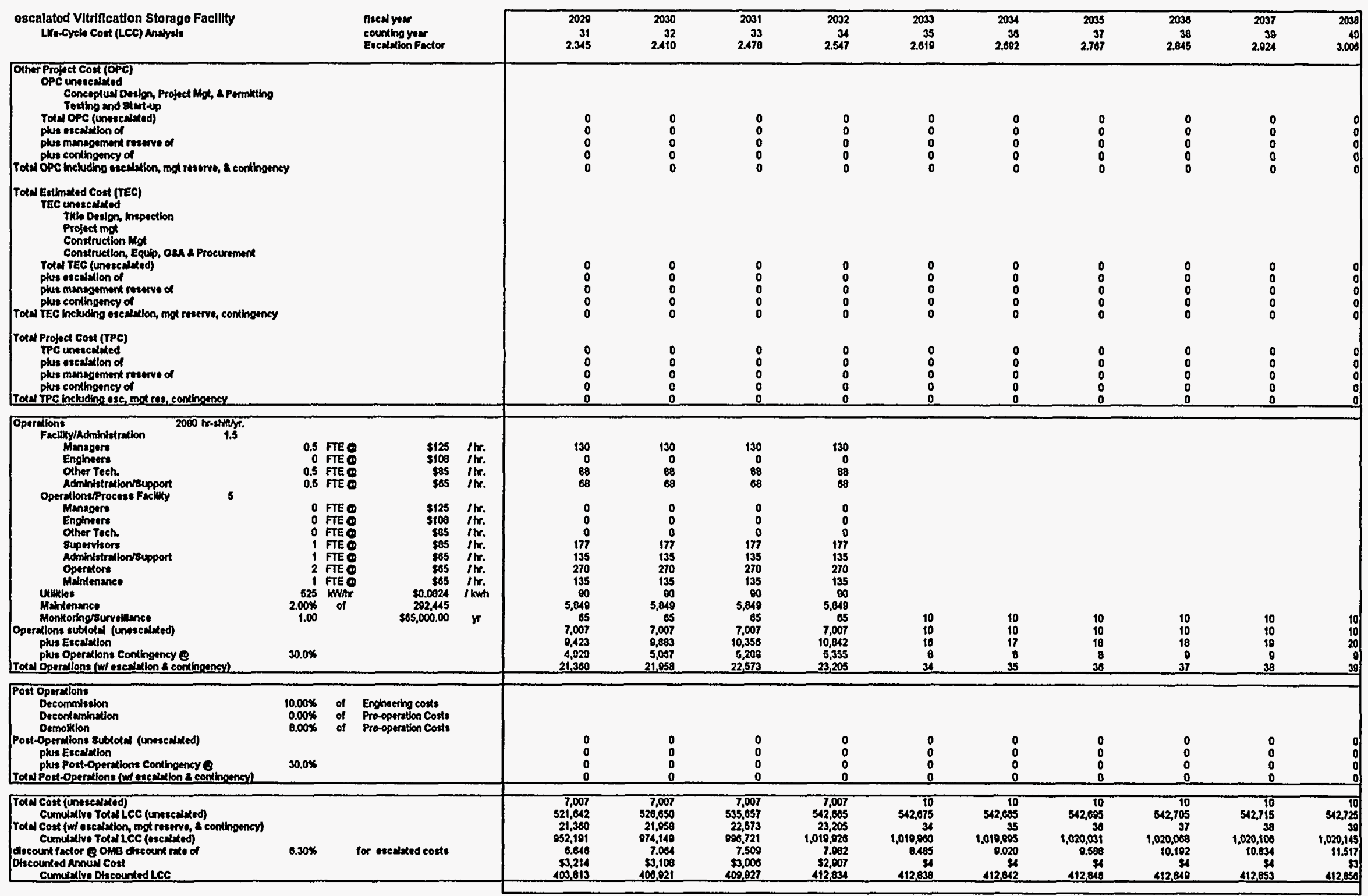




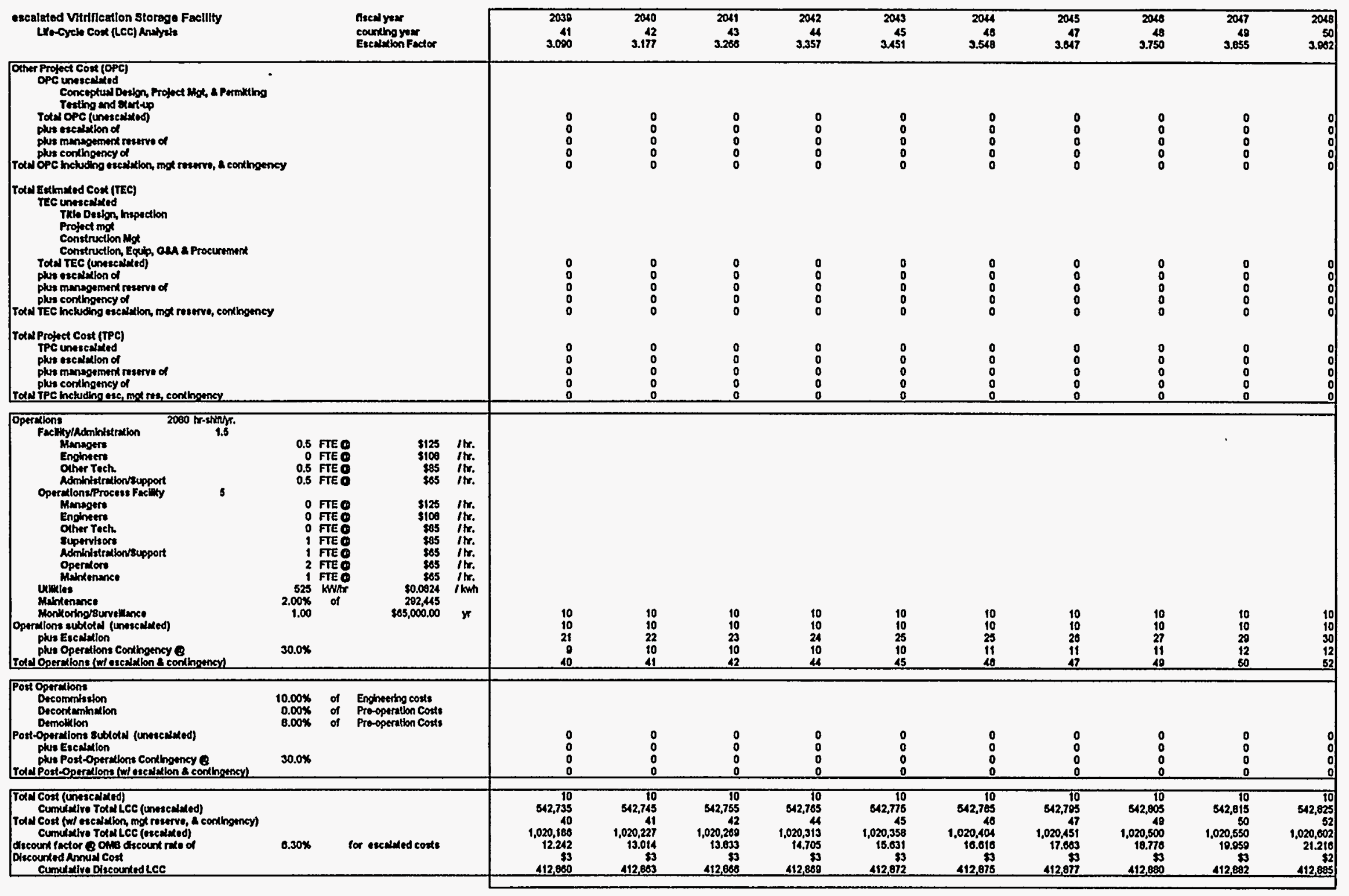




\begin{tabular}{|c|c|c|c|c|c|c|c|c|c|c|c|c|}
\hline 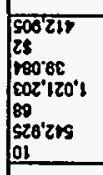 & 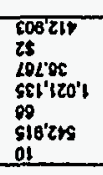 & 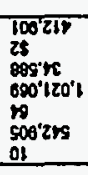 & 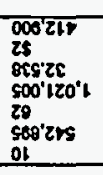 & 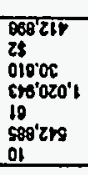 & 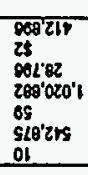 & 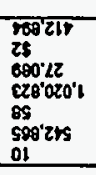 & 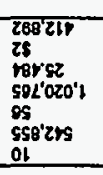 & 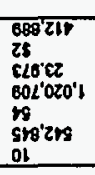 & 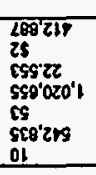 & \multicolumn{3}{|c|}{ 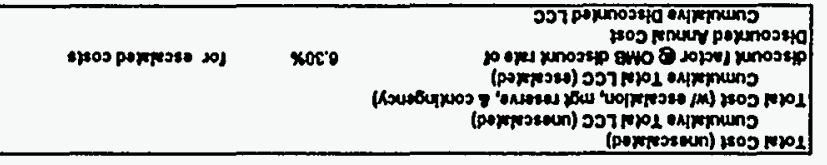 } \\
\hline $\begin{array}{l}0 \\
0 \\
0 \\
0 \\
0\end{array}$ & $\begin{array}{l}0 \\
0 \\
0 \\
0 \\
0\end{array}$ & $\begin{array}{c}0 \\
0 \\
0 \\
0 \\
0\end{array}$ & $\begin{array}{l}0 \\
\vdots \\
0\end{array}$ & $\begin{array}{l}0 \\
\vdots \\
0 \\
0\end{array}$ & $\begin{array}{l}0 \\
\vdots \\
0\end{array}$ & $\begin{array}{l}0 \\
\\
0 \\
0\end{array}$ & $\begin{array}{l}0 \\
\vdots \\
0 \\
0\end{array}$ & $\begin{array}{l}0 \\
\vdots \\
0 \\
0\end{array}$ & $\begin{array}{l}0 \\
\vdots \\
0\end{array}$ & 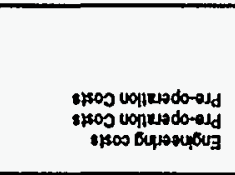 & 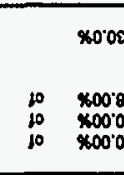 & 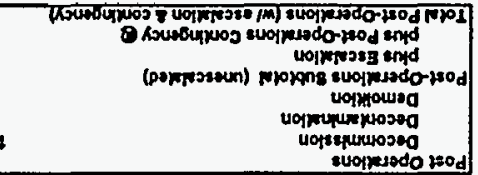 \\
\hline $\begin{array}{l}09 \\
01 \\
2 p \\
01 \\
01 \\
01\end{array}$ & $\begin{array}{l}\text { po } \\
\text { gi } \\
17 \\
01 \\
\text { ol }\end{array}$ & 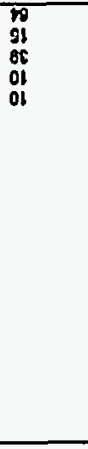 & 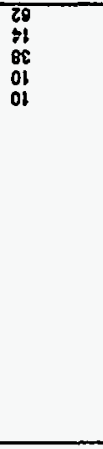 & 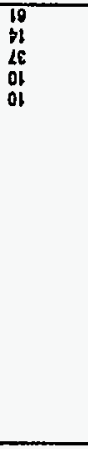 & 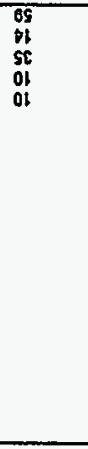 & 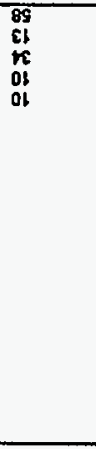 & 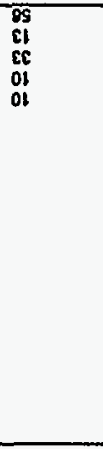 & 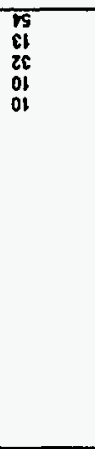 & 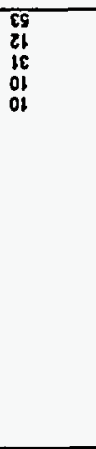 & 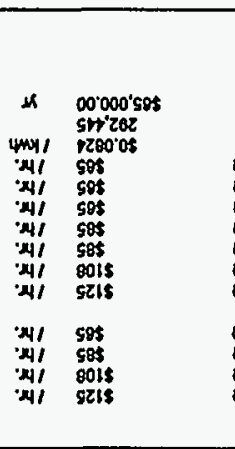 & 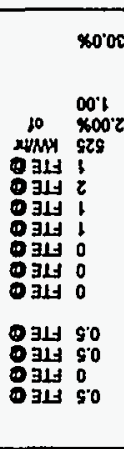 & 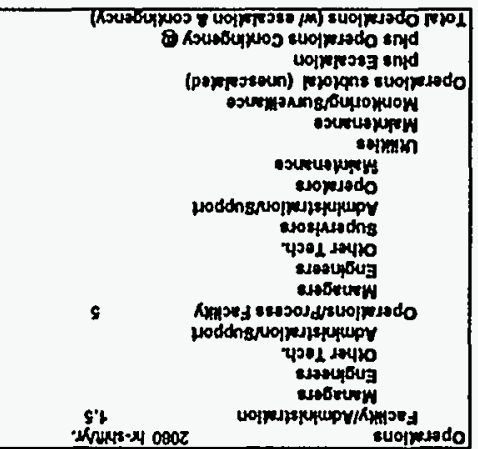 \\
\hline $\begin{array}{l}0 \\
0 \\
0 \\
0 \\
0 \\
0\end{array}$ & $\begin{array}{l} \\
0 \\
0 \\
0 \\
0\end{array}$ & $\begin{array}{l}0 \\
\vdots \\
0 \\
0 \\
0\end{array}$ & $\begin{array}{l} \\
0 \\
0 \\
0 \\
0\end{array}$ & $\begin{array}{l} \\
0 \\
0 \\
0 \\
0\end{array}$ & $\begin{array}{l} \\
0 \\
0 \\
0 \\
0\end{array}$ & $\begin{array}{l} \\
0 \\
0 \\
0 \\
0\end{array}$ & $\begin{array}{l} \\
0 \\
0 \\
0 \\
0\end{array}$ & $\begin{array}{l}0 \\
0 \\
0 \\
0 \\
0\end{array}$ & $\begin{array}{l}0 \\
0 \\
0 \\
0 \\
0\end{array}$ & & & 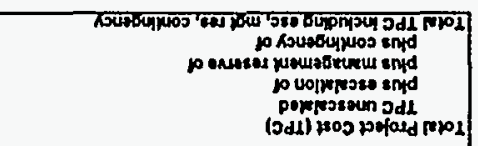 \\
\hline $\mid \begin{array}{l}0 \\
0 \\
0 \\
0 \\
0 \\
0\end{array}$ & $\begin{array}{l}0 \\
0 \\
0 \\
0 \\
0\end{array}$ & $\begin{array}{l}0 \\
0 \\
0 \\
0 \\
0\end{array}$ & $\begin{array}{l}0 \\
0 \\
0 \\
0 \\
0\end{array}$ & $\begin{array}{l}0 \\
0 \\
0 \\
0 \\
0\end{array}$ & $\begin{array}{l}0 \\
0 \\
0 \\
0 \\
0\end{array}$ & $\begin{array}{l}0 \\
0 \\
0 \\
0 \\
0\end{array}$ & $\begin{array}{l}0 \\
0 \\
0 \\
0 \\
0 \\
0\end{array}$ & $\begin{array}{l}0 \\
0 \\
0 \\
0 \\
0\end{array}$ & $\begin{array}{l}0 \\
0 \\
0 \\
0 \\
0\end{array}$ & & & 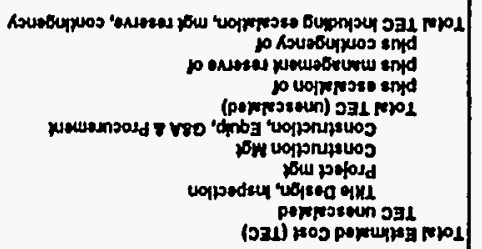 \\
\hline $\mid \begin{array}{l}0 \\
0 \\
0 \\
0 \\
0 \\
0\end{array}$ & $\begin{array}{l}0 \\
0 \\
0 \\
0\end{array}$ & $\begin{array}{l}0 \\
\vdots \\
0 \\
0 \\
0\end{array}$ & $\begin{array}{l}0 \\
0 \\
0 \\
0 \\
0\end{array}$ & $\begin{array}{l}0 \\
0 \\
0 \\
0 \\
0\end{array}$ & $\begin{array}{l}0 \\
0 \\
0 \\
0 \\
0\end{array}$ & $\begin{array}{l}0 \\
0 \\
0 \\
0 \\
0\end{array}$ & $\begin{array}{l}0 \\
\vdots \\
\vdots \\
0\end{array}$ & $\begin{array}{l}0 \\
0 \\
0 \\
0 \\
0\end{array}$ & $\begin{array}{l}0 \\
0 \\
0 \\
0 \\
0\end{array}$ & & & 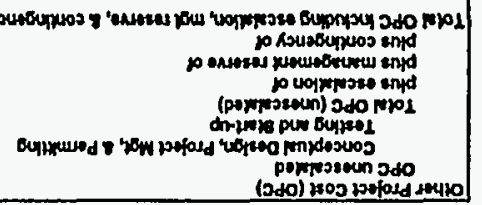 \\
\hline 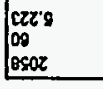 & 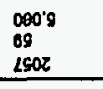 & 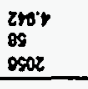 & 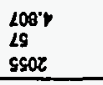 & 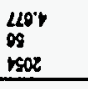 & $\begin{array}{l}\text { ersy } \\
\text { ss } \\
\text { cssoz }\end{array}$ & 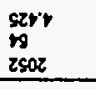 & $\begin{array}{l}\text { soct } \\
c_{c 0} \\
1502\end{array}$ & $\begin{array}{l}281 \cdot 7 \\
28 \\
0.02 z\end{array}$ & 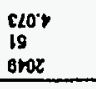 & 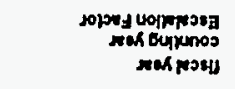 & & 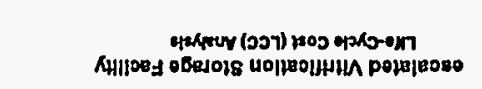 \\
\hline
\end{tabular}




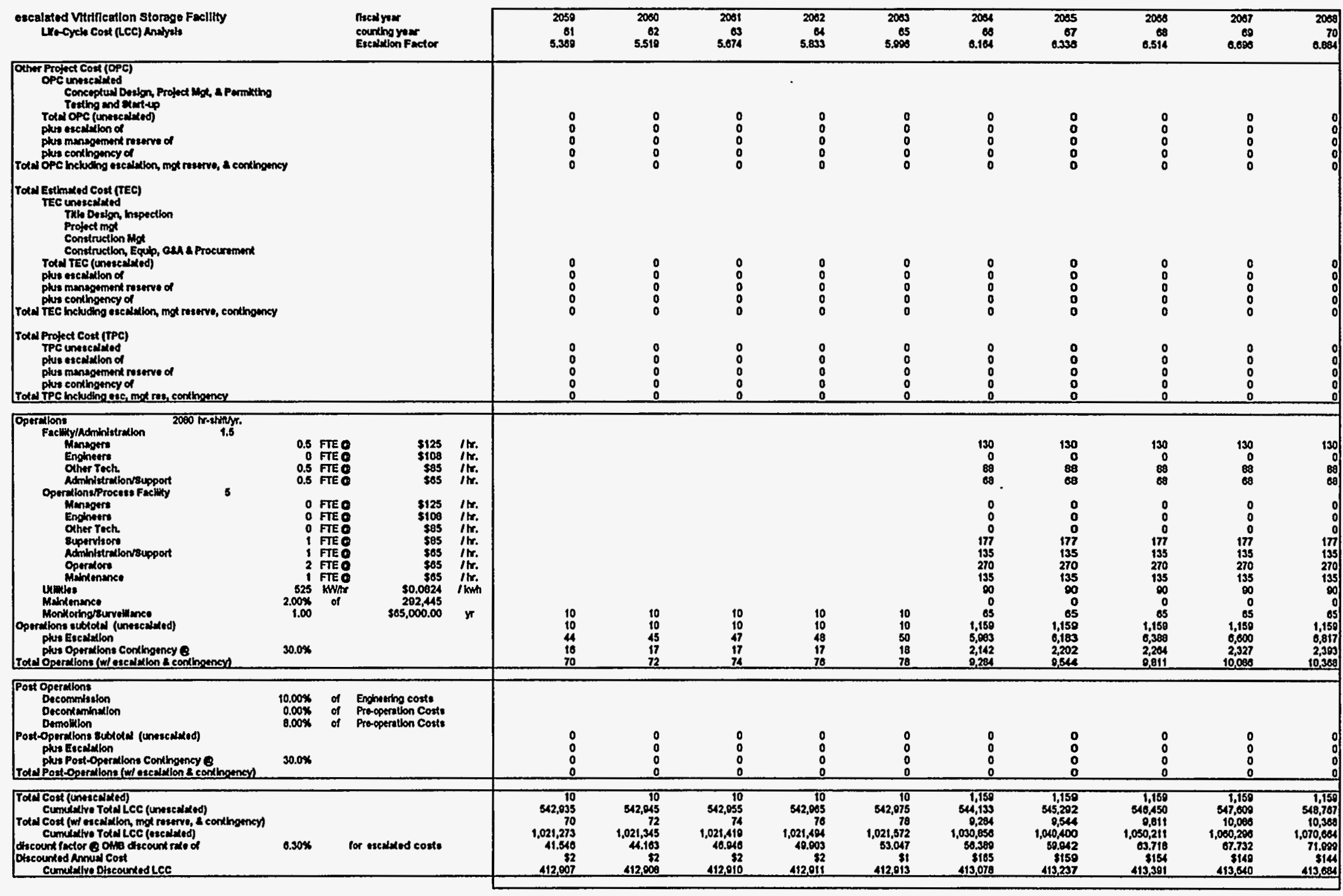




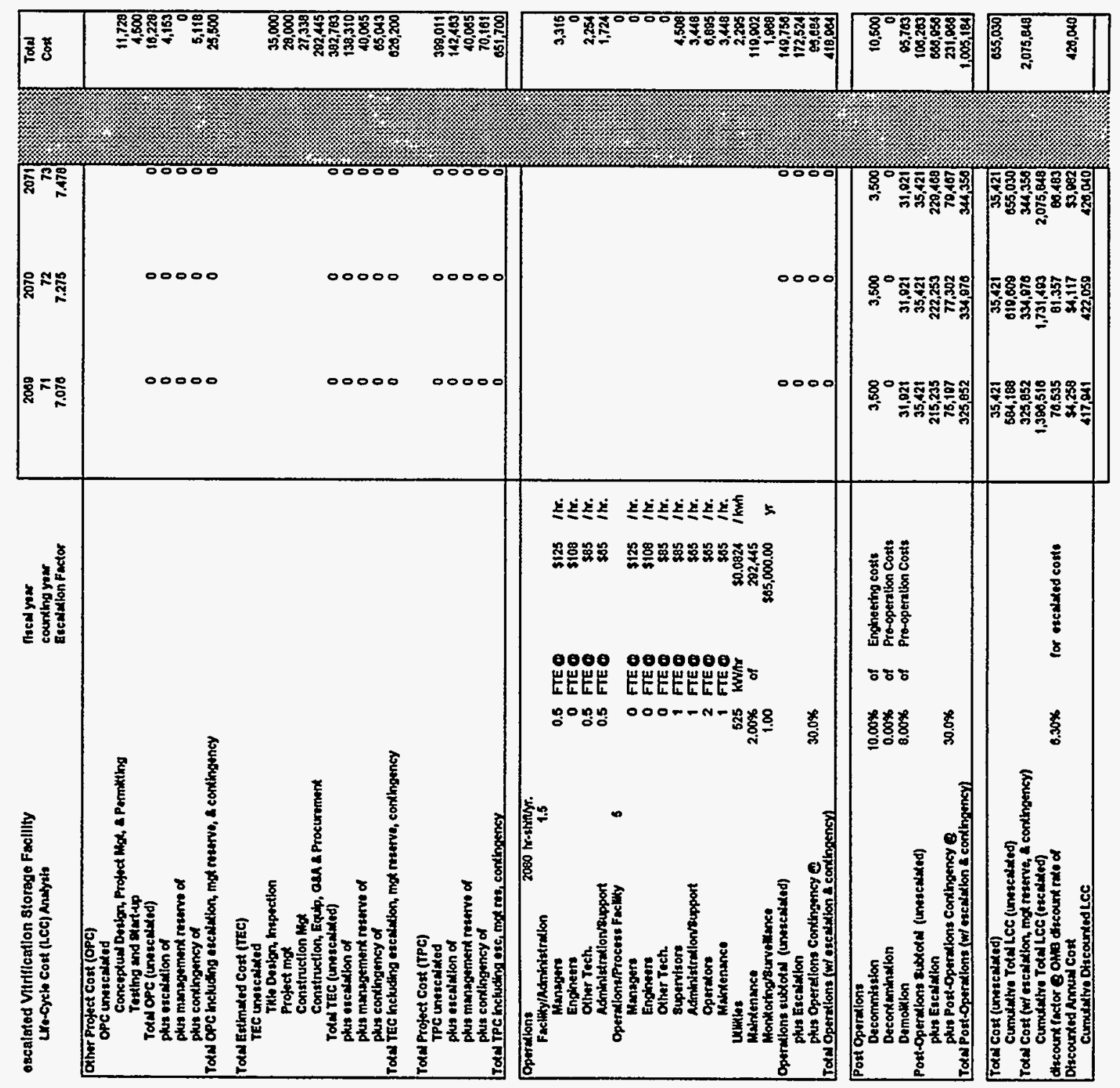




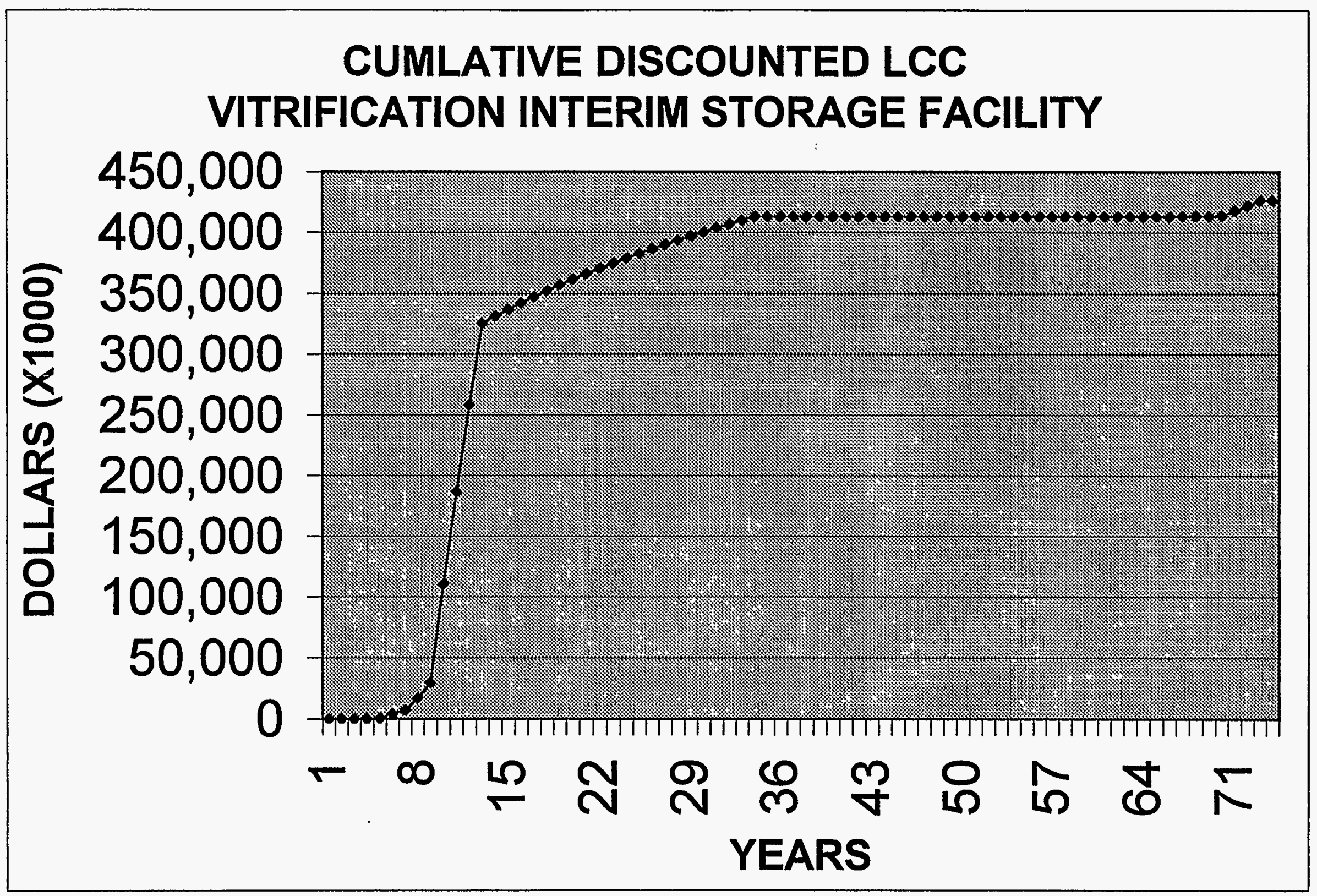




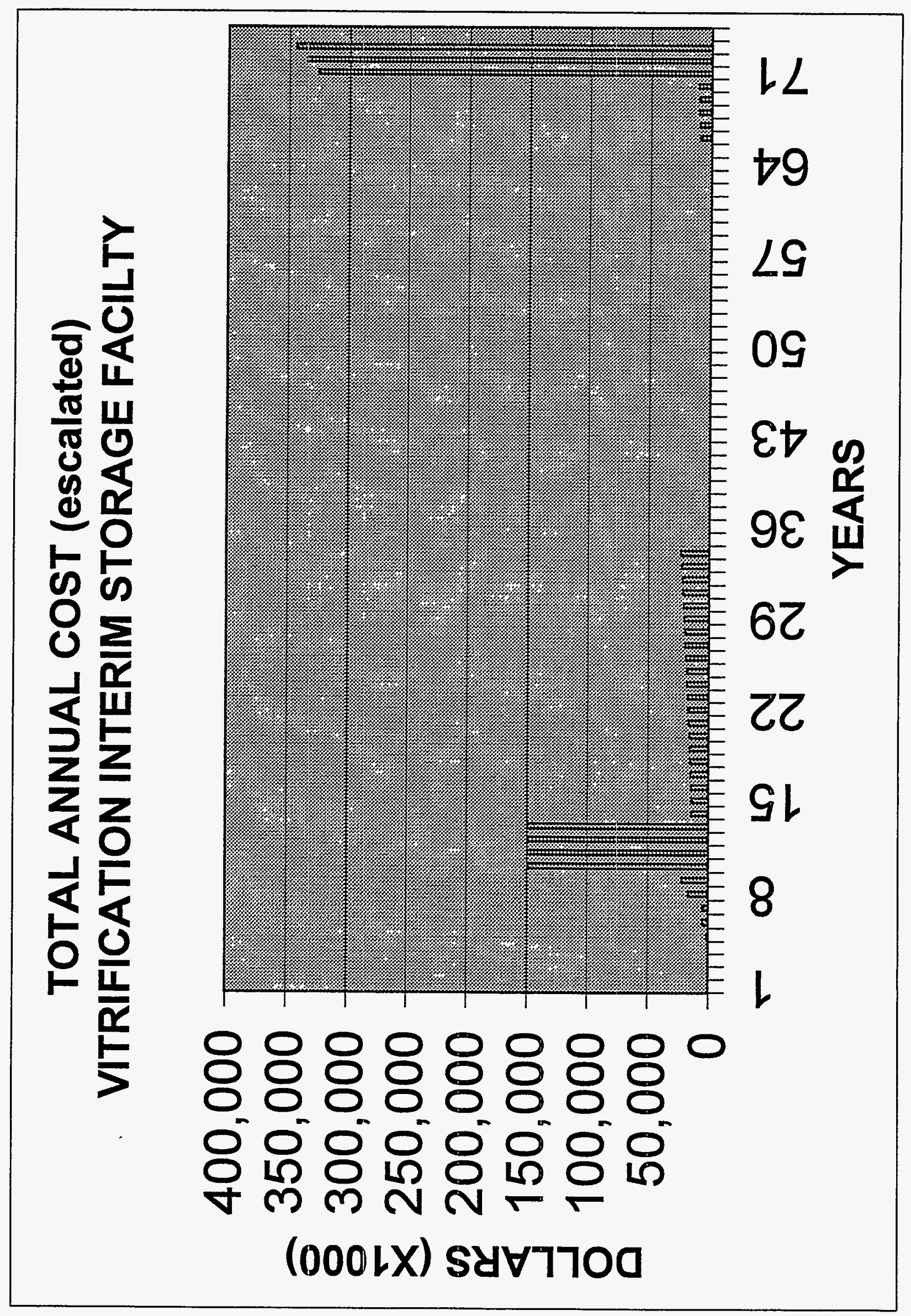


Date: $\quad$ February 2, 1998

$\begin{array}{llll}\text { To: } & \text { John K. Rawlins } & \text { MS 3765 } & \text { 6-4304 } \\ \text { From: } & \text { R. J. Turk y tued } & \text { MS 3875 } & \text { 6-3611 }\end{array}$

Subject: ECONOMIC AND LIFE CYCLE ANALYSIS CONDUCTED for the DIRECT CEMENTITIOUS WASTE OPTION (DCWO)-20YR STORAGE FACILITY RJT-19-98

\section{Purpose:}

As requested an Economic and Life-Cycle Cost (LCC) has been conducted to evaluate for the DCWO 20-yr. INTERIM STORAGE FACILITIES. The storage facility was evaluated based on a scoping study that incorporate appropriate design features from the Flour Daniel Corp. design for interim storage. This storage facility will be used to house canisters resulting from the DCWO production option until final repository disposition.

This economic analysis is based on information provided by J. K. Rawlins, and K. L. Williams. R. D. Adams provided cost estimates. B.L. Blakely provided process personnel modeling.

\section{Methodology:}

The Economic Evaluation assumed a 69-year period (2003-2071) since this is the estimated time required to complete all of the anticipated remediation activities. The LCC identified and evaluated the initial development, construction, operating and post operating costs over the lifecycle. A discounted LCC analysis assumes a 1998-dollar basis, discounted $6.3 \%$ annually per the Office of Management and Budget (OMB) Circular A-94 for escalated costs. All costs are conservatively discounted assuming the end-of-year convention. 
Page 2

\section{Assumptions:}

The scope of work and requirements of all related activities are vague at this time. Facility and processing costs were developed from historical experience associated with DD\&D work at the INEEL. The LCC analysis was generated to match cost estimating cost structure. These costs include Permitting, Direct and Indirect Construction, G\&A, Procurement Fee, Engineering, Inspection, Project Management, Construction Management, Escalation and Contingency costs.

The design period is assumed to be accomplished in five-years with construction completed in six-years. Labor rates were assumed as follows: Managers, $\$ 125 / \mathrm{hr}$; Engineers, $\$ 108 \$ / \mathrm{hr}$; Other Technicians $\$ 85 / \mathrm{hr}$; Administration/support staff $\$ 65 / \mathrm{hr}$; Operators and Maintenance personnel $\$ 65 / \mathrm{hr}$. The operational period for this facility was assumed to be twenty years, to match the appropriate operating facility. This period is followed by five years for shipping to the repository, followed by three years of post-operations activities. Due to this project's lack of complexity and non-radioactive contaminated environment, this analysis assumed a decommissioning cost equal to $10 \%$ of the non-escalated engineering design cost, decontamination costs equal to $0 \%$ of non-escalated total pre-operation cost, and demolition costs equal to $8 \%$ of the non-escalated total pre-operation cost. Utilities were calculated assuming a $\$ .0824$ per $\mathrm{kWh}$. Surveillance and Monitoring costs were calculated at $\$ 65,000$ per year for manned security during operations then reduced to $\$ 10,000$ for remote surveillance during non-operation periods.

\section{Results:}

The DCWO 20-yr. INTERIM STORAGE FACILITY has a discounted LCC of \$ 506 million.

Attachments:

cc: R. J. Turk File 


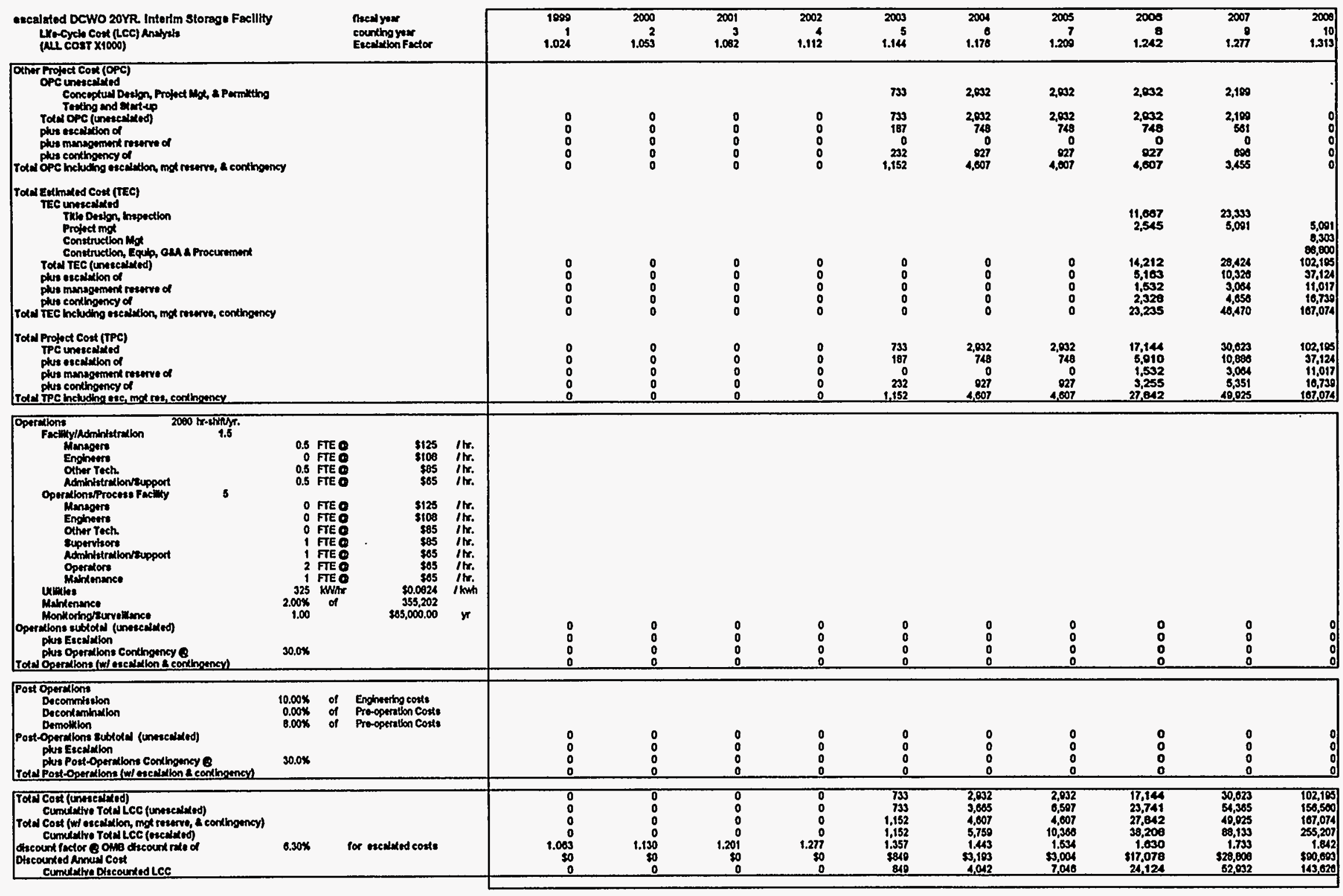




\begin{tabular}{|c|c|c|c|c|c|c|c|c|c|c|c|c|c|}
\hline 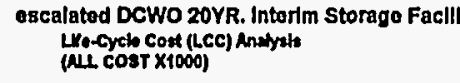 & & 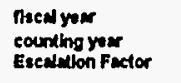 & & $\begin{array}{l}2009 \\
11 \\
1.350\end{array}$ & $\begin{array}{l}2010 \\
12 \\
1.397\end{array}$ & $\begin{array}{r}2011 \\
1.13 \\
1.420\end{array}$ & $\begin{array}{l}2012 \\
14 \\
1,406\end{array}$ & $\begin{array}{r}2013 \\
15 \\
1.607\end{array}$ & $\begin{array}{l}2014 \\
10 \\
1.550\end{array}$ & $\begin{array}{r}2015 \\
177 \\
1.583\end{array}$ & $\begin{array}{l}2016 \\
1.03 \\
1.230\end{array}$ & $\begin{array}{r}2017 \\
10 \\
1.803\end{array}$ & $\begin{array}{r}2018 \\
20 \\
1.730\end{array}$ \\
\hline \multicolumn{4}{|l|}{ 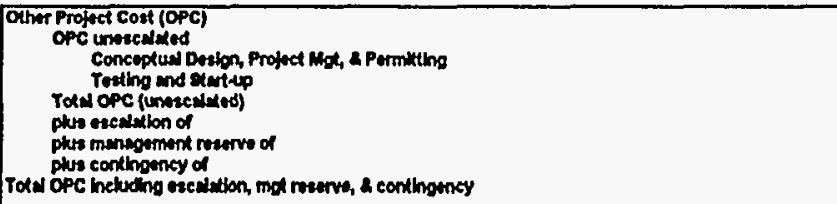 } & $\begin{array}{l}0 \\
0 \\
0 \\
0 \\
0\end{array}$ & $\begin{array}{l}0 \\
0 \\
0 \\
0 \\
0\end{array}$ & $\begin{array}{l}0 \\
0 \\
0 \\
0 \\
0\end{array}$ & $\begin{array}{r}3,375 \\
3,375 \\
861 \\
00 \\
1,008 \\
5,303\end{array}$ & $\begin{array}{r}1125 \\
1,125 \\
287 \\
0 \\
350 \\
1,708\end{array}$ & $\begin{array}{l}0 \\
: \\
0 \\
0 \\
0\end{array}$ & $\begin{array}{l}0 \\
0 \\
0 \\
0 \\
0\end{array}$ & $\begin{array}{l}0 \\
0 \\
0 \\
0 \\
0\end{array}$ & $\begin{array}{l}0 \\
0 \\
0 \\
0 \\
0\end{array}$ & $\begin{array}{l}0 \\
0 \\
0 \\
0 \\
0\end{array}$ \\
\hline \multicolumn{4}{|l|}{ 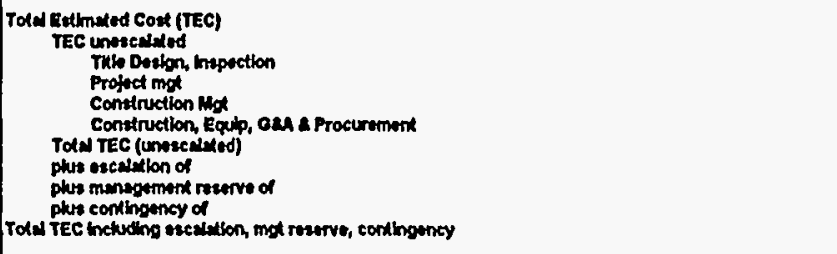 } & $\begin{array}{r}5,091 \\
8,303 \\
68,000 \\
102,185 \\
37,124 \\
11,017 \\
16,739 \\
167,074\end{array}$ & $\begin{array}{r}8,091 \\
8,303 \\
08,000 \\
102,195 \\
37,124 \\
11,017 \\
18,739 \\
107,074\end{array}$ & $\begin{array}{r}8,091 \\
8,903 \\
8,000 \\
102,105 \\
37,124 \\
11,017 \\
10,739 \\
107,074\end{array}$ & $\begin{array}{l}0 \\
0 \\
0 \\
0 \\
0\end{array}$ & $\begin{array}{l}0 \\
0 \\
0 \\
0 \\
0\end{array}$ & $\begin{array}{l}0 \\
0 \\
0 \\
0 \\
0\end{array}$ & $\begin{array}{l}0 \\
0 \\
0 \\
0 \\
0\end{array}$ & $\begin{array}{l}0 \\
0 \\
0 \\
0 \\
0\end{array}$ & $\begin{array}{l}0 \\
0 \\
0 \\
0 \\
0\end{array}$ & $\begin{array}{l}0 \\
0 \\
0 \\
0 \\
0\end{array}$ \\
\hline \multicolumn{4}{|l|}{ 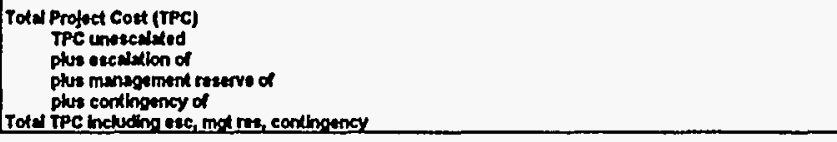 } & $\begin{array}{r}102,185 \\
37,124 \\
11,017 \\
16,739 \\
107,074 \\
\end{array}$ & $\begin{array}{r}102,105 \\
37,124 \\
11,017 \\
16,739 \\
107,074 \\
\end{array}$ & $\begin{array}{r}102,105 \\
37,124 \\
11,017 \\
16,720 \\
107,074 \\
\end{array}$ & $\begin{array}{r}3,375 \\
081 \\
0 \\
1,050 \\
3,303 \\
\end{array}$ & $\begin{array}{r}1,125 \\
287 \\
0 \\
350 \\
1,768 \\
\end{array}$ & $\begin{array}{l}0 \\
0 \\
0 \\
0 \\
0\end{array}$ & $\begin{array}{l}0 \\
0 \\
0 \\
0 \\
0 \\
\end{array}$ & $\begin{array}{l}0 \\
0 \\
0 \\
0 \\
0 \\
\end{array}$ & $\begin{array}{l}0 \\
0 \\
0 \\
0 \\
0 \\
\end{array}$ & $\begin{array}{l}0 \\
0 \\
0 \\
0 \\
0\end{array}$ \\
\hline \multirow{2}{*}{ 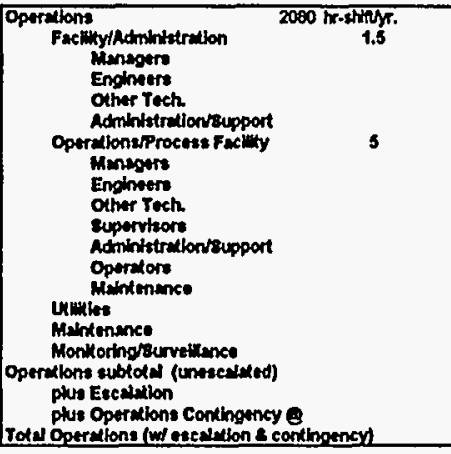 } & $\begin{array}{l}0.5 \text { FTE: } \\
0 \text { TTE } \\
0.5 \text { TTE: } \\
0.5 \text { FTE }\end{array}$ & $\begin{array}{l}\$ 125 \\
\$ 100 \\
\$ 85 \\
\$ 85\end{array}$ & 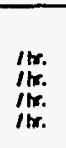 & & & & $\begin{array}{l}\text { os } \\
0 \\
4 \\
34\end{array}$ & $\begin{array}{r}130 \\
0 \\
68 \\
68\end{array}$ & $\begin{array}{r}130 \\
0 \\
89 \\
69\end{array}$ & $\begin{array}{r}130 \\
0 \\
68 \\
68\end{array}$ & $\begin{array}{r}130 \\
0 \\
09 \\
08\end{array}$ & $\begin{array}{r}130 \\
0 \\
88 \\
68\end{array}$ & $\begin{array}{r}130 \\
6 \\
68 \\
68\end{array}$ \\
\hline & 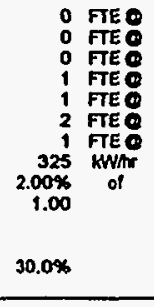 & 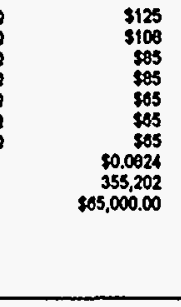 & 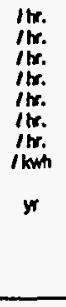 & $\begin{array}{l}0 \\
0 \\
0 \\
0\end{array}$ & $\begin{array}{l}0 \\
0 \\
0 \\
0\end{array}$ & $\begin{array}{l}0 \\
0 \\
0 \\
0\end{array}$ & 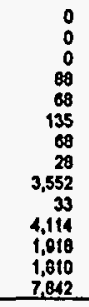 & $\begin{array}{r}0 \\
0 \\
0 \\
177 \\
135 \\
270 \\
135 \\
56 \\
7,104 \\
05 \\
0,228 \\
4,174 \\
3.721 \\
16,123 \\
\end{array}$ & $\begin{array}{r}0 \\
0 \\
0 \\
177 \\
135 \\
270 \\
135 \\
50 \\
7,104 \\
65 \\
8,228 \\
4,522 \\
3,925 \\
10,575 \\
\end{array}$ & $\begin{array}{r}0 \\
0 \\
0 \\
177 \\
135 \\
270 \\
135 \\
50 \\
8.104 \\
05 \\
8,228 \\
4,079 \\
3,932 \\
17,039 \\
\end{array}$ & $\begin{array}{r}0 \\
0 \\
0 \\
177 \\
135 \\
270 \\
135 \\
56 \\
7,104 \\
05 \\
0,228 \\
5,246 \\
4,042 \\
17,516 \\
\end{array}$ & $\begin{array}{r}0 \\
0 \\
0 \\
777 \\
133 \\
270 \\
1135 \\
58 \\
7,104 \\
65 \\
8,228 \\
38,03 \\
4,1,153 \\
18,007 \\
\end{array}$ & $\begin{array}{r}0 \\
0 \\
0 \\
173 \\
135 \\
2700 \\
135 \\
550 \\
7,104 \\
655 \\
0,228 \\
0,0141 \\
4,272 \\
18,511 \\
\end{array}$ \\
\hline 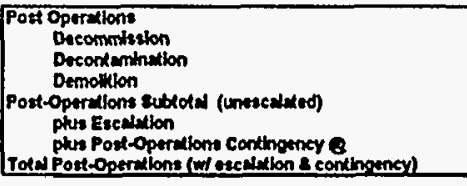 & $\begin{array}{ll}10.00 \% & \text { of } \\
0.00 \% & \text { of } \\
0.00 \% & \text { of } \\
& \\
30.0 \% & \end{array}$ & 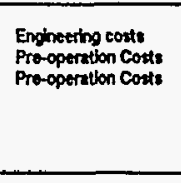 & & $\begin{array}{l}0 \\
0 \\
0 \\
0 \\
\end{array}$ & $\begin{array}{l}0 \\
0 \\
0 \\
0\end{array}$ & $\begin{array}{l}0 \\
0 \\
0 \\
0 \\
\end{array}$ & $\begin{array}{l}0 \\
0 \\
0 \\
0 \\
\end{array}$ & $\begin{array}{l}0 \\
0 \\
0 \\
0 \\
\end{array}$ & $\begin{array}{l}0 \\
0 \\
0 \\
0 \\
\end{array}$ & $\begin{array}{l}0 \\
0 \\
0 \\
0 \\
\end{array}$ & $\begin{array}{l}0 \\
: \\
0 \\
0\end{array}$ & $\begin{array}{l}0 \\
0 \\
0 \\
0\end{array}$ & $\begin{array}{l}0 \\
0 \\
0 \\
0\end{array}$ \\
\hline 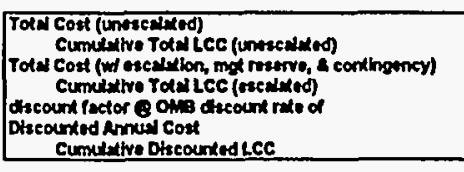 & $0.30 \%$ & for ereduned costs & & 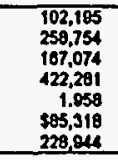 & 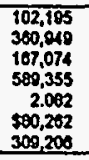 & 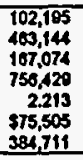 & $\begin{array}{r}7 ., 469 \\
470,633 \\
13,145 \\
769,514 \\
22.352 \\
35,509 \\
380,300 \\
\end{array}$ & 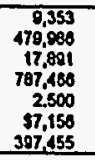 & 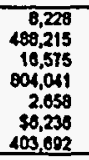 & 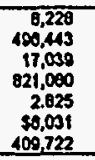 & 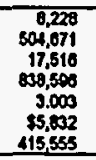 & 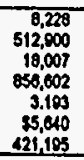 & 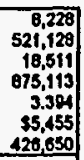 \\
\hline
\end{tabular}




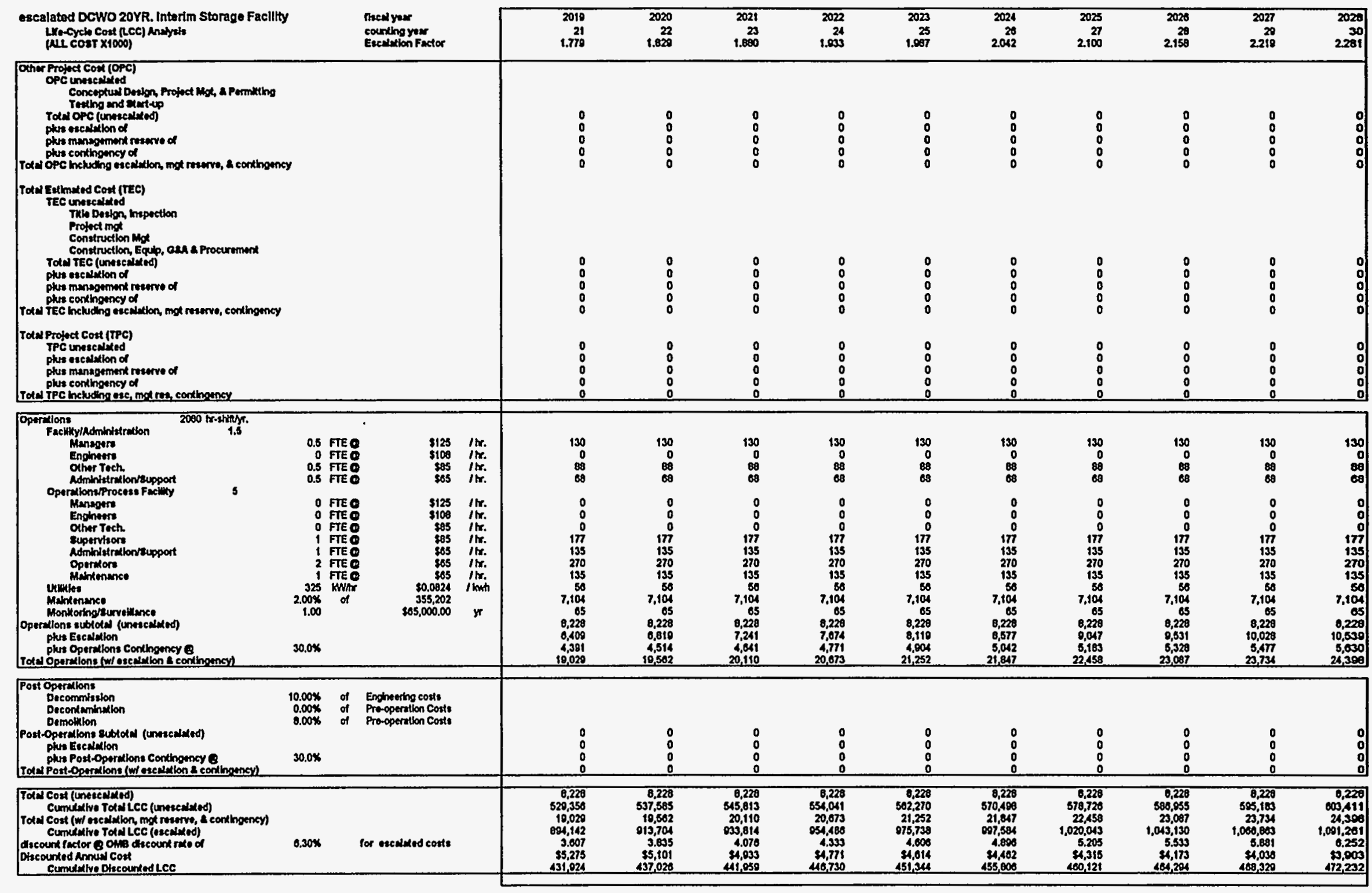




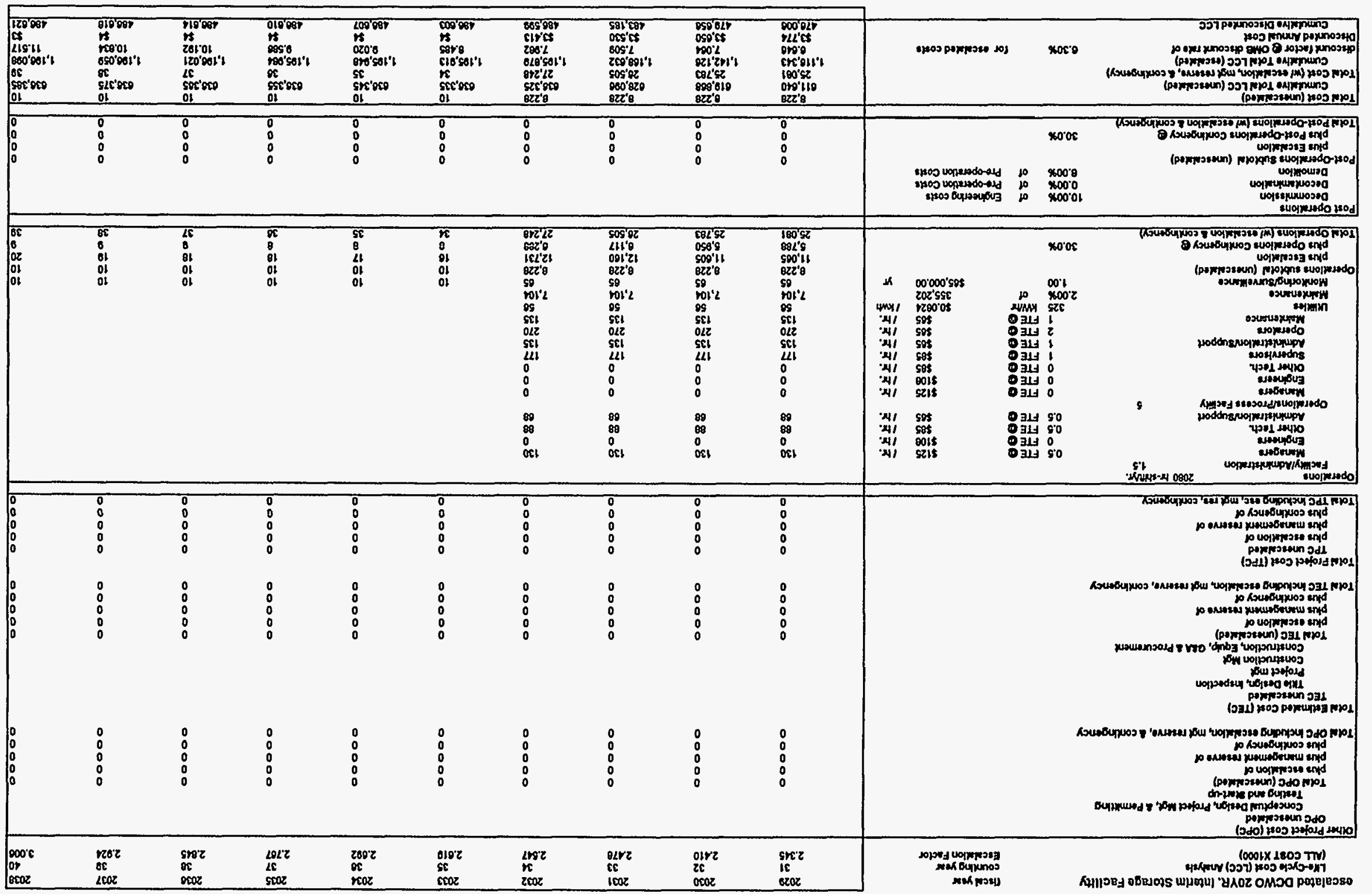




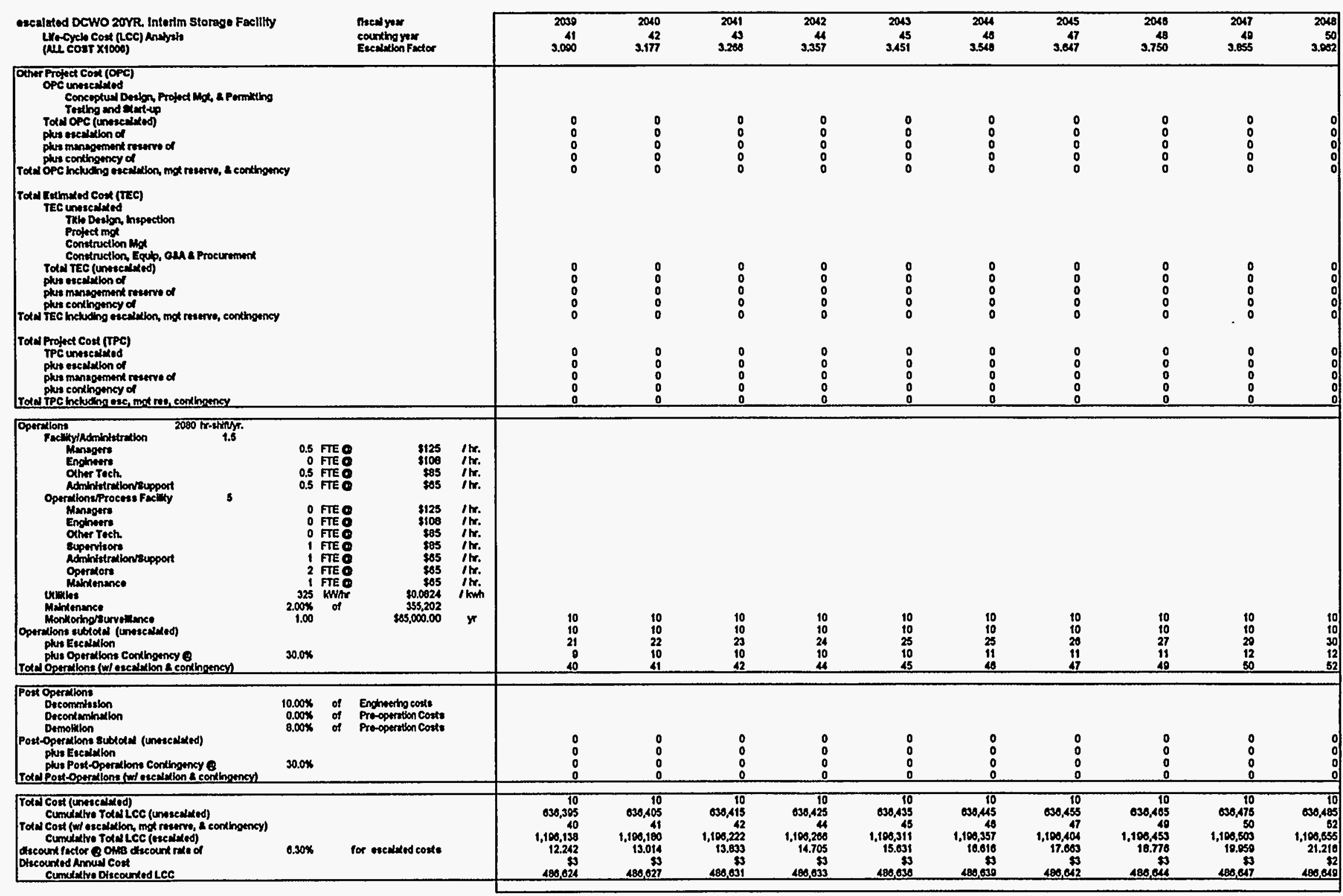




\begin{tabular}{|c|c|c|c|c|c|c|c|c|c|c|c|c|c|}
\hline \multicolumn{2}{|l|}{ 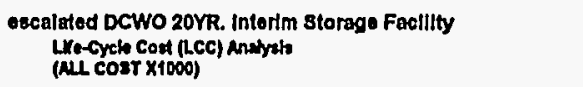 } & \multicolumn{2}{|l|}{ 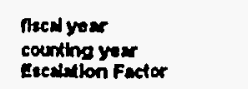 } & $\begin{array}{r}2049 \\
51 \\
4.073\end{array}$ & $\begin{array}{r}2050 \\
52 \\
4.187\end{array}$ & $\begin{array}{r}2051 \\
53 \\
4.305\end{array}$ & $\begin{array}{r}2052 \\
84 \\
4125\end{array}$ & $\begin{array}{r}2053 \\
55 \\
4.540\end{array}$ & $\begin{array}{r}2054 \\
56 \\
4.677\end{array}$ & $\begin{array}{l}2053 \\
57 \\
4.807\end{array}$ & $\begin{array}{l}2050 \\
50 \\
4.42\end{array}$ & $\begin{array}{r}2057 \\
59 \\
6.000\end{array}$ & \begin{tabular}{r|}
2058 \\
60 \\
5.223
\end{tabular} \\
\hline \multicolumn{4}{|l|}{ 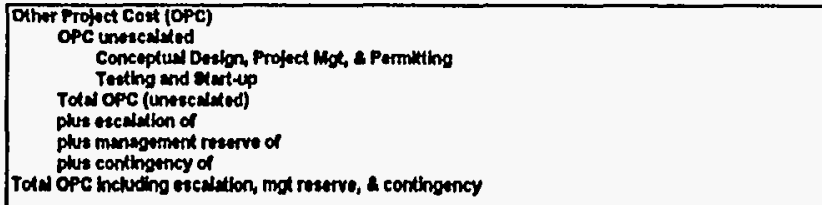 } & $\begin{array}{l}0 \\
0 \\
0 \\
0 \\
0\end{array}$ & $\begin{array}{l}0 \\
0 \\
0 \\
0 \\
0\end{array}$ & $\begin{array}{l}0 \\
0 \\
0 \\
0 \\
0\end{array}$ & $\begin{array}{l}0 \\
0 \\
0 \\
0 \\
0\end{array}$ & $\begin{array}{l}0 \\
0 \\
0 \\
0 \\
0\end{array}$ & $\begin{array}{l}0 \\
0 \\
0 \\
0 \\
0\end{array}$ & $\begin{array}{l}0 \\
0 \\
0 \\
0 \\
0\end{array}$ & $\begin{array}{l}0 \\
0 \\
0 \\
0 \\
0\end{array}$ & $\begin{array}{l}0 \\
0 \\
0 \\
0 \\
0\end{array}$ & 8 \\
\hline \multicolumn{4}{|l|}{ 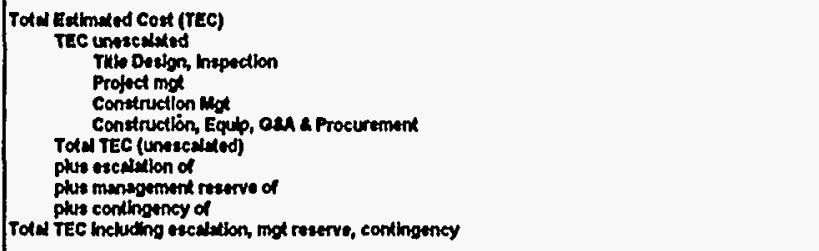 } & $\begin{array}{l}0 \\
0 \\
0 \\
0 \\
0\end{array}$ & $\begin{array}{l}0 \\
0 \\
0 \\
0 \\
0\end{array}$ & $\begin{array}{l}0 \\
0 \\
0 \\
0 \\
0\end{array}$ & $\begin{array}{l}0 \\
0 \\
0 \\
0 \\
0\end{array}$ & $\begin{array}{l}0 \\
0 \\
0 \\
0 \\
0\end{array}$ & $\begin{array}{l}0 \\
0 \\
0 \\
0 \\
0\end{array}$ & $\begin{array}{l}0 \\
0 \\
0 \\
0\end{array}$ & $\begin{array}{l}0 \\
: \\
0 \\
0\end{array}$ & $\begin{array}{l}0 \\
0 \\
0 \\
0 \\
0\end{array}$ & $\begin{array}{l}0 \\
0 \\
0 \\
0 \\
0\end{array}$ \\
\hline \multicolumn{4}{|l|}{ 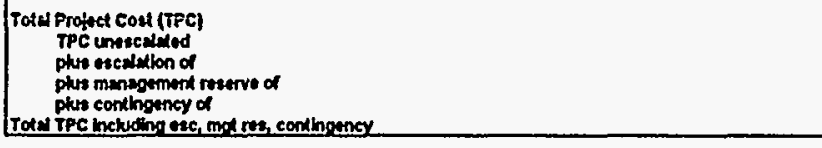 } & $\begin{array}{l}0 \\
0 \\
0 \\
0 \\
0 \\
\end{array}$ & $\begin{array}{l}0 \\
0 \\
0 \\
0 \\
0 \\
\end{array}$ & $\begin{array}{l}0 \\
0 \\
0 \\
0 \\
0 \\
\end{array}$ & $\begin{array}{l}0 \\
0 \\
0 \\
0 \\
0 \\
\end{array}$ & $\begin{array}{l}0 \\
0 \\
0 \\
0 \\
0 \\
\end{array}$ & $\begin{array}{l}0 \\
\vdots \\
0 \\
0 \\
\end{array}$ & $\begin{array}{l}0 \\
0 \\
0 \\
0 \\
\end{array}$ & $\begin{array}{l}0 \\
0 \\
0 \\
0 \\
0 \\
\end{array}$ & $\begin{array}{l}0 \\
0 \\
0 \\
0 \\
0 \\
\end{array}$ & $\begin{array}{l}0 \\
0\end{array}$ \\
\hline 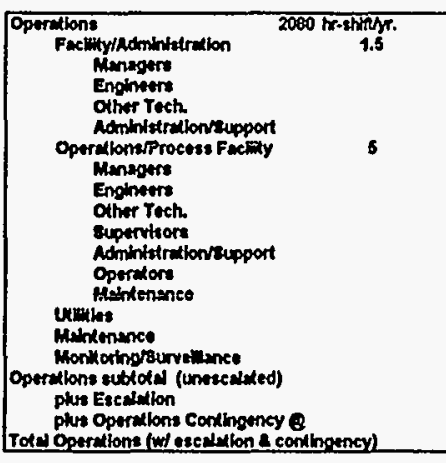 & 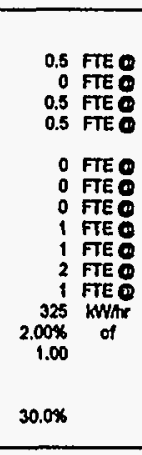 & $\begin{array}{r}\$ 125 \\
\$ 100 \\
\$ 85 \\
\$ 05 \\
\$ 1125 \\
\$ \$ 106 \\
\$ 85 \\
\$ 85 \\
\$ 65 \\
\$ 65 \\
\$ 65 \\
\$ 0.024 \\
355,202 \\
\$ 85,000.00\end{array}$ & 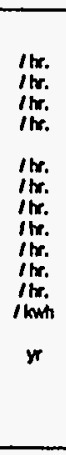 & $\begin{array}{l}10 \\
10 \\
31 \\
12 \\
33 \\
3\end{array}$ & $\begin{array}{l}10 \\
10 \\
32 \\
13 \\
51 \\
\end{array}$ & $\begin{array}{l}10 \\
10 \\
10 \\
13 \\
13 \\
58 \\
\end{array}$ & $\begin{array}{l}10 \\
10 \\
34 \\
13 \\
38 \\
\end{array}$ & $\begin{array}{l}10 \\
10 \\
35 \\
14 \\
39 \\
39\end{array}$ & $\begin{array}{l}10 \\
10 \\
37 \\
14 \\
81\end{array}$ & $\begin{array}{l}10 \\
10 \\
30 \\
14 \\
02 \\
02\end{array}$ & $\begin{array}{l}10 \\
10 \\
30 \\
10 \\
64\end{array}$ & $\begin{array}{l}10 \\
10 \\
11 \\
15 \\
06 \\
\end{array}$ & $\begin{array}{l}10 \\
10 \\
42 \\
18 \\
69\end{array}$ \\
\hline 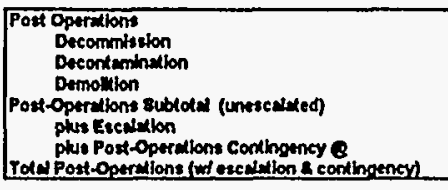 & $\begin{array}{ll}10.00 \% & \text { of } \\
0.00 \% & \text { of } \\
8.00 \% & \text { of } \\
30.0 \% & \end{array}$ & $\begin{array}{l}\text { Engheertirg costs } \\
\text { Pro-operation costs } \\
\text { Pro-operation Costs }\end{array}$ & & $\begin{array}{l}0 \\
0 \\
0 \\
0 \\
\end{array}$ & $\begin{array}{l}0 \\
0 \\
0 \\
0 \\
\end{array}$ & $\begin{array}{l}0 \\
0 \\
0 \\
0 \\
\end{array}$ & $\begin{array}{l}0 \\
0 \\
0 \\
0 \\
\end{array}$ & $\begin{array}{l}0 \\
0 \\
0 \\
0 \\
\end{array}$ & $\begin{array}{l}0 \\
0 \\
0 \\
0 \\
\end{array}$ & $\begin{array}{l}0 \\
0 \\
0 \\
0\end{array}$ & $\begin{array}{l}0 \\
0 \\
0 \\
0\end{array}$ & $\begin{array}{l}0 \\
0 \\
0 \\
0\end{array}$ & \\
\hline 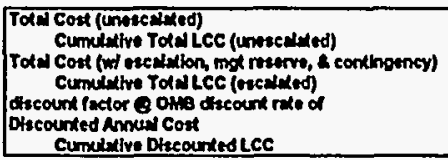 & $0.20 \%$ & for oseculited costs & & $\begin{array}{r}10 \\
030,485 \\
53 \\
1,100,608 \\
22.553 \\
\$ 2 \\
188,052 \\
\end{array}$ & 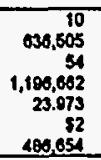 & $\begin{array}{r}10 \\
030,515 \\
50 \\
1,108,718 \\
25,684 \\
92 \\
180,650 \\
\end{array}$ & $\begin{array}{r}10 \\
038,525 \\
58 \\
1,100,775 \\
27.069 \\
\$ 2 \\
480,050 \\
\end{array}$ & 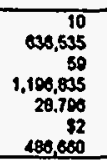 & $\begin{array}{r}10 \\
038,545 \\
61 \\
1,100,6985 \\
30.610 \\
92 \\
480,062 \\
\end{array}$ & $\begin{array}{r}10 \\
036,555 \\
02 \\
1.100,058 \\
32.538 \\
52 \\
488,004\end{array}$ & 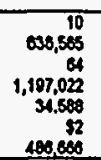 & $\begin{array}{r}10 \\
030,575 \\
66 \\
1,197,060 \\
30.767 \\
\$ 2 \\
480,668\end{array}$ & $\begin{array}{r}10 \\
638,505 \\
689 \\
1,197,1190 \\
30,000 \\
\$ 2 \\
488,670\end{array}$ \\
\hline
\end{tabular}




\begin{tabular}{|c|c|c|c|c|c|c|c|c|c|c|c|c|c|}
\hline 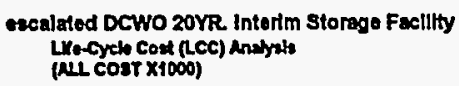 & & 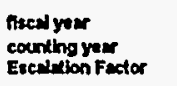 & & $\begin{array}{r}2059 \\
81 \\
5.389\end{array}$ & $\begin{array}{l}2000 \\
6.62 \\
5.510\end{array}$ & $\begin{array}{l}2001 \\
603 \\
5.674\end{array}$ & $\begin{array}{l}2002 \\
44 \\
5.833\end{array}$ & $\begin{array}{l}2000 \\
65 \\
5.000\end{array}$ & $\begin{array}{l}2004 \\
60 \\
0.194\end{array}$ & $\begin{array}{l}2005 \\
07 \\
0.336\end{array}$ & $\begin{array}{r}2086 \\
68 \\
0.514\end{array}$ & $\begin{array}{l}2007 \\
0.009 \\
0.008\end{array}$ & $\begin{array}{r}2008 \\
700 \\
0.084\end{array}$ \\
\hline \multicolumn{4}{|l|}{ 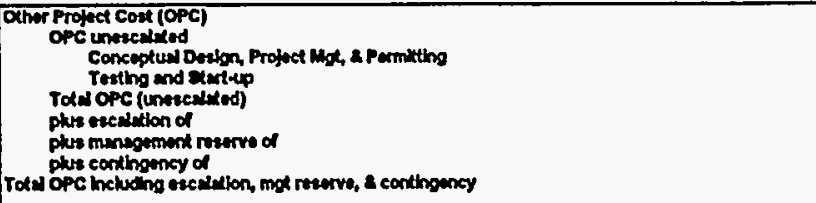 } & $\begin{array}{l}0 \\
0 \\
0 \\
0 \\
0\end{array}$ & $\begin{array}{l}0 \\
0 \\
0 \\
0 \\
0\end{array}$ & $\begin{array}{l}0 \\
0 \\
0 \\
0 \\
0\end{array}$ & $\begin{array}{l}0 \\
0 \\
0 \\
0 \\
0\end{array}$ & $\begin{array}{l}0 \\
0 \\
0 \\
0 \\
0\end{array}$ & $\begin{array}{l}0 \\
0 \\
0 \\
0 \\
0\end{array}$ & $\begin{array}{l}0 \\
0 \\
0 \\
0 \\
0\end{array}$ & $\begin{array}{l}0 \\
0 \\
0 \\
0 \\
0\end{array}$ & $\begin{array}{l}0 \\
0 \\
0 \\
0 \\
0\end{array}$ & $\begin{array}{l}0 \\
0 \\
0 \\
0 \\
0\end{array}$ \\
\hline \multicolumn{4}{|l|}{ 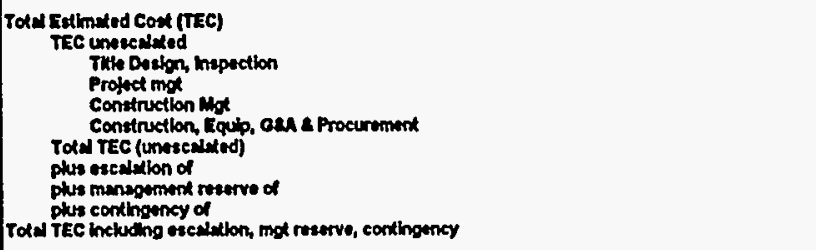 } & $\begin{array}{l}0 \\
0 \\
0 \\
0 \\
0\end{array}$ & $\begin{array}{l}0 \\
0 \\
0 \\
0 \\
0\end{array}$ & $\begin{array}{l}0 \\
0 \\
0 \\
0 \\
0\end{array}$ & $\begin{array}{l}0 \\
0 \\
0 \\
0 \\
0\end{array}$ & $\begin{array}{l}0 \\
0 \\
0 \\
0 \\
0\end{array}$ & $\begin{array}{l}0 \\
0 \\
0 \\
0 \\
0\end{array}$ & $\begin{array}{l}0 \\
0 \\
0 \\
0 \\
0\end{array}$ & $\begin{array}{l}0 \\
0 \\
0 \\
0 \\
0\end{array}$ & $\begin{array}{l}0 \\
0 \\
0 \\
0 \\
0\end{array}$ & $\begin{array}{l}0 \\
0 \\
0 \\
0 \\
0\end{array}$ \\
\hline 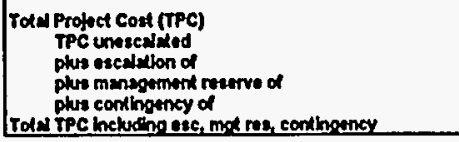 & & & & $\begin{array}{l}0 \\
0 \\
0 \\
0 \\
0 \\
\end{array}$ & $\begin{array}{l}0 \\
0 \\
0 \\
0 \\
0 \\
\end{array}$ & $\begin{array}{l}0 \\
0 \\
0 \\
0 \\
0 \\
\end{array}$ & $\begin{array}{l}0 \\
0 \\
0 \\
0 \\
0 \\
\end{array}$ & $\begin{array}{l}0 \\
0 \\
0 \\
0 \\
0 \\
\end{array}$ & $\begin{array}{l}0 \\
0 \\
0 \\
0 \\
0 \\
\end{array}$ & $\begin{array}{l}0 \\
0 \\
0 \\
0 \\
0\end{array}$ & $\begin{array}{l}0 \\
0 \\
0 \\
0 \\
0 \\
\end{array}$ & $\begin{array}{l}0 \\
: \\
: \\
\end{array}$ & $\begin{array}{l}0 \\
0 \\
0 \\
0 \\
0\end{array}$ \\
\hline 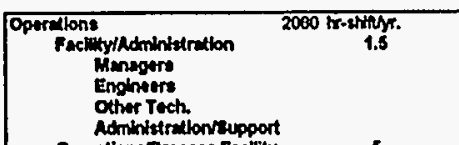 & $\begin{array}{l}0.5 \text { TTE } \\
0 \text { FTE } \\
0.5 \text { FTE } \\
0.5 \text { FTE }\end{array}$ & $\begin{array}{c}\$ 123 \\
\$ 100 \\
\$ 285 \\
865\end{array}$ & 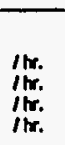 & & & & & & $\begin{array}{r}130 \\
0 \\
60 \\
60\end{array}$ & $\begin{array}{r}130 \\
0 \\
69 \\
68\end{array}$ & $\begin{array}{r}130 \\
0 \\
89 \\
88\end{array}$ & $\begin{array}{r}130 \\
0 \\
08 \\
8 B\end{array}$ & $\begin{array}{r}130 \\
0 \\
60 \\
69\end{array}$ \\
\hline 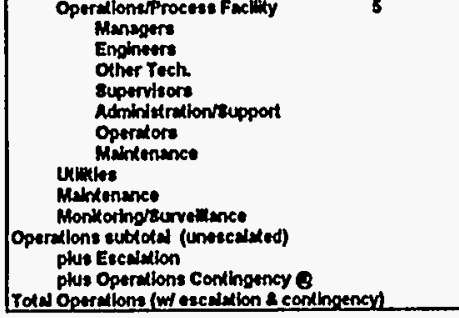 & $\begin{array}{l}0 \text { FTE } \\
\text { O FTE } \\
0 \text { FTE } \\
1 \text { FTE } \\
1 \text { FTE } \\
2 \text { FrE } \\
1 \text { FTE } \\
325 \\
2.00 \% \\
1.00\end{array}$ & $\begin{array}{r}\$ 125 \\
\$ 108 \\
\$ 85 \\
\$ 85 \\
\$ 65 \\
\$ 85 \\
\$ 65 \\
\$ 65 \\
\$ 0.032 \\
355,202 \\
\$ 05,000.00\end{array}$ & 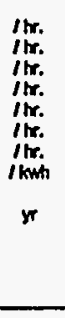 & $\begin{array}{l}10 \\
10 \\
41 \\
10 \\
70 \\
\end{array}$ & $\begin{array}{l}10 \\
10 \\
15 \\
17 \\
72 \\
\end{array}$ & $\begin{array}{l}10 \\
10 \\
17 \\
17 \\
74 \\
\end{array}$ & $\begin{array}{l}10 \\
10 \\
40 \\
17 \\
70 \\
\end{array}$ & $\begin{array}{l}10 \\
10 \\
50 \\
18 \\
78 \\
\end{array}$ & $\begin{array}{r}0 \\
0 \\
177 \\
175 \\
270 \\
135 \\
50 \\
5,104 \\
0.5 \\
8,220 \\
12,400 \\
15,215 \\
05,031 \\
\end{array}$ & $\begin{array}{r}0 \\
0 \\
177 \\
175 \\
135 \\
270 \\
135 \\
50 \\
7,104 \\
05 \\
0.229 \\
43,010 \\
13,912 \\
67,780 \\
\end{array}$ & $\begin{array}{r}0 \\
0 \\
177 \\
135 \\
270 \\
135 \\
58 \\
7,304 \\
65 \\
0.228 \\
15,370 \\
10,078 \\
09.078 \\
\end{array}$ & $\begin{array}{r}0 \\
0 \\
17 \\
135 \\
270 \\
135 \\
50 \\
7,104 \\
05 \\
0.228 \\
40.251 \\
10.530 \\
71.020 \\
\end{array}$ & $\begin{array}{r}0 \\
0 \\
177 \\
135 \\
270 \\
135 \\
30 \\
3,552 \\
65 \\
4,070 \\
27,514 \\
0.057 \\
11,84 \\
\end{array}$ \\
\hline 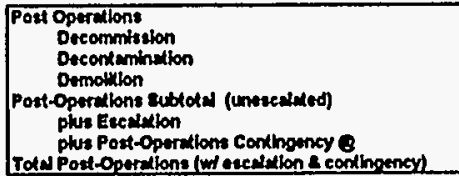 & $\begin{array}{ll}10.00 \% & \text { of } \\
0.00 \% & \text { of } \\
8.00 \% & \text { of } \\
30.0 \% & \end{array}$ & 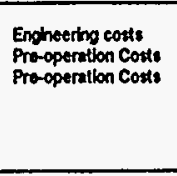 & & $\begin{array}{l}0 \\
0 \\
0 \\
0\end{array}$ & $\begin{array}{l}0 \\
0 \\
0 \\
0 \\
\end{array}$ & $\begin{array}{l}0 \\
\vdots \\
0\end{array}$ & $\begin{array}{l}0 \\
0 \\
0 \\
0 \\
\end{array}$ & $\begin{array}{l}0 \\
0 \\
0 \\
0\end{array}$ & $\begin{array}{l}0 \\
0 \\
0 \\
0 \\
\end{array}$ & $\begin{array}{l}0 \\
0 \\
0 \\
0 \\
\end{array}$ & $\begin{array}{l}0 \\
0 \\
0 \\
0\end{array}$ & $\begin{array}{l}0 \\
0 \\
0 \\
0 \\
\end{array}$ & $\begin{array}{l}0 \\
0 \\
0 \\
0\end{array}$ \\
\hline 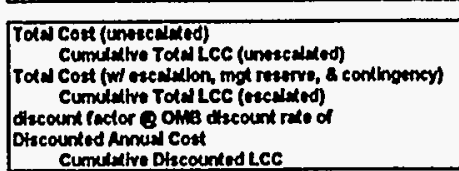 & $0.30 x$ & for menduled couts & & $\begin{array}{r}10 \\
030,595 \\
70 \\
1,107,220 \\
41.546 \\
486 \\
486,671 \\
\end{array}$ & 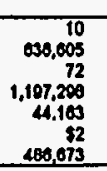 & $\begin{array}{r}10 \\
030,0015 \\
74 \\
1,197,37 \\
48.046 \\
48 \\
480,075 \\
\end{array}$ & $\begin{array}{r}10 \\
030,025 \\
76 \\
1.197,477 \\
40.003 \\
82 \\
468,076 \\
\end{array}$ & 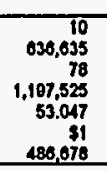 & 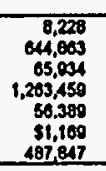 & 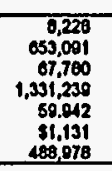 & 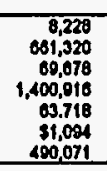 & 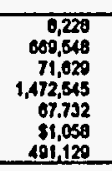 & 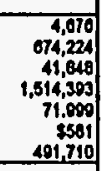 \\
\hline
\end{tabular}




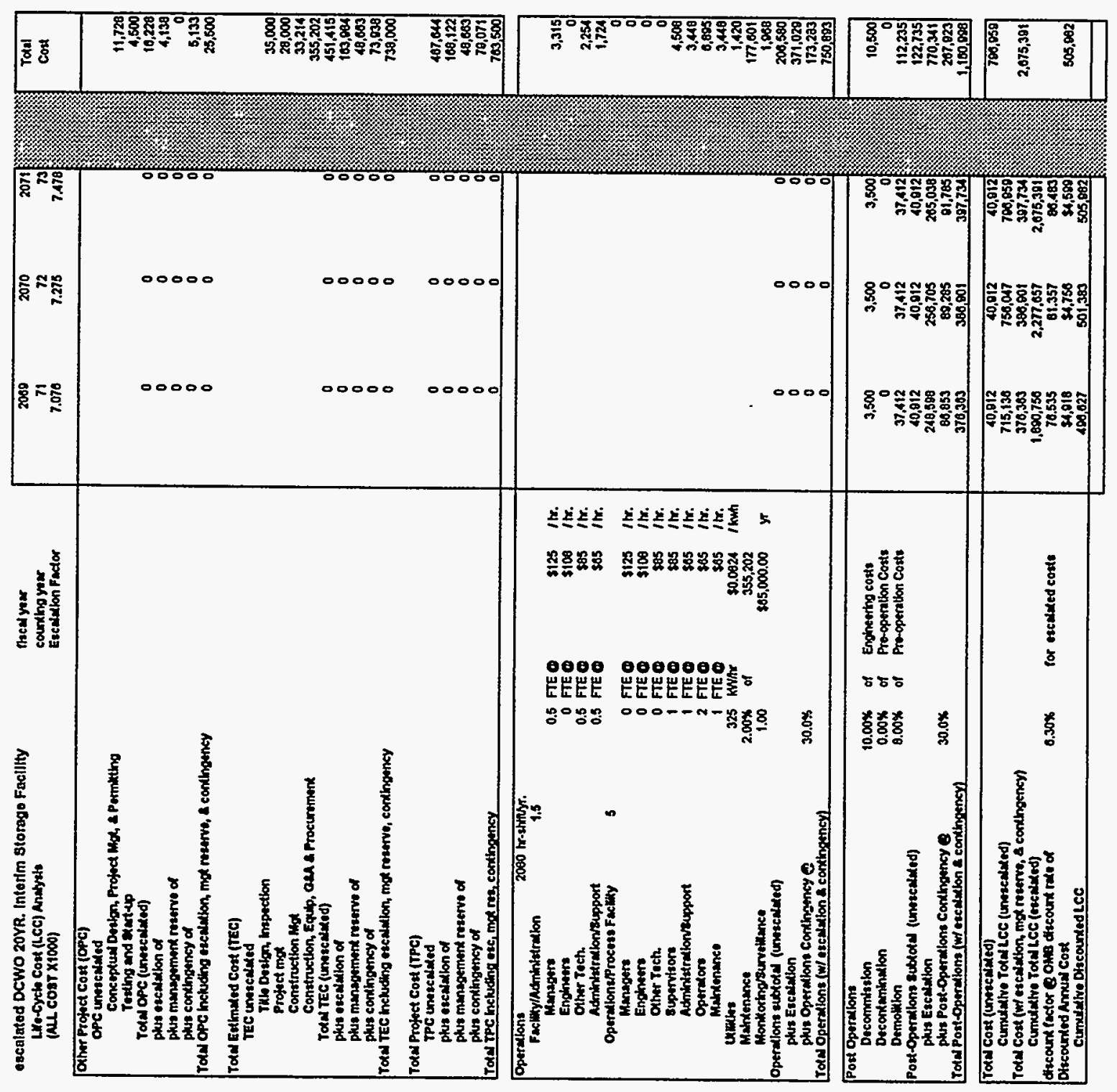




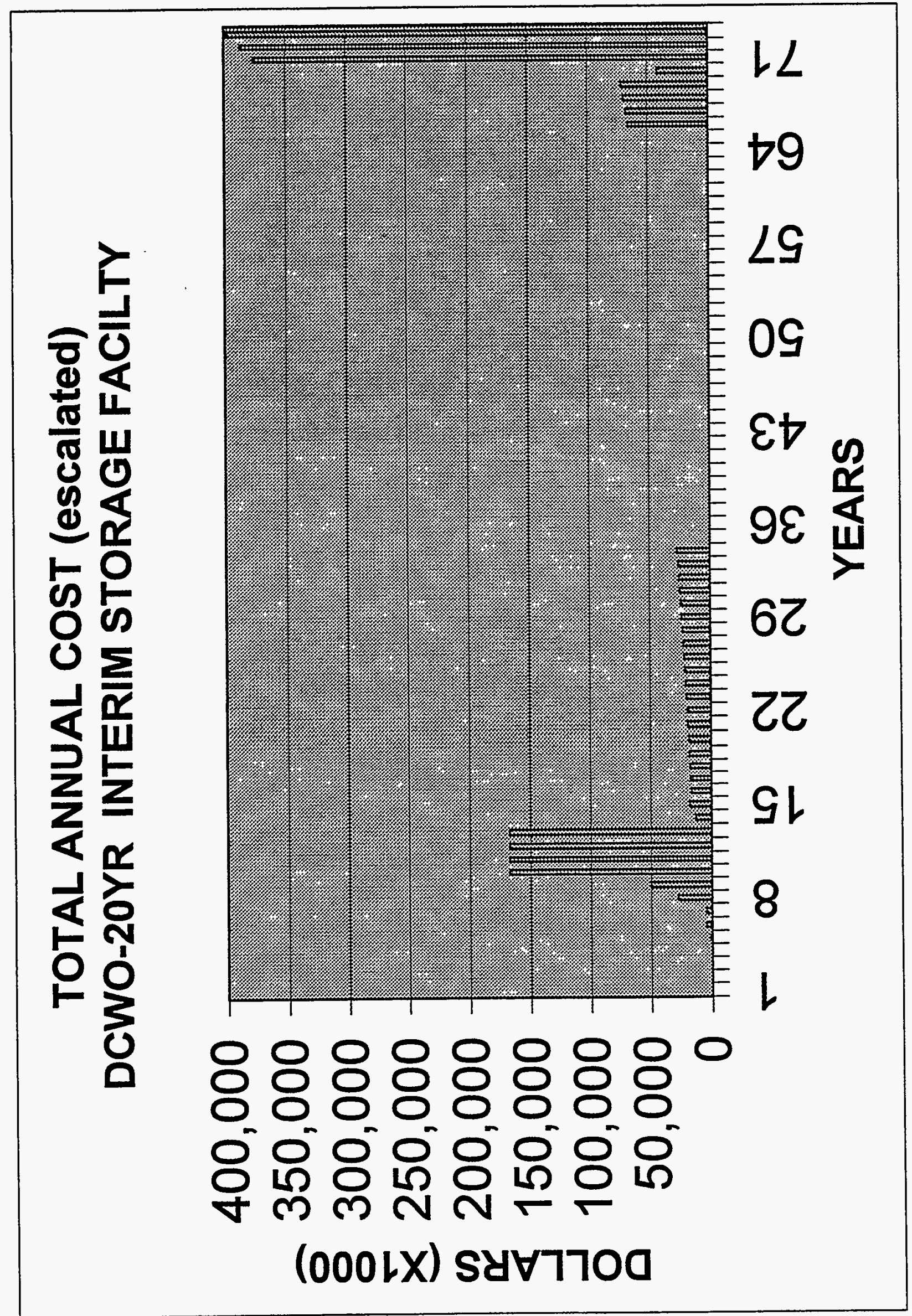




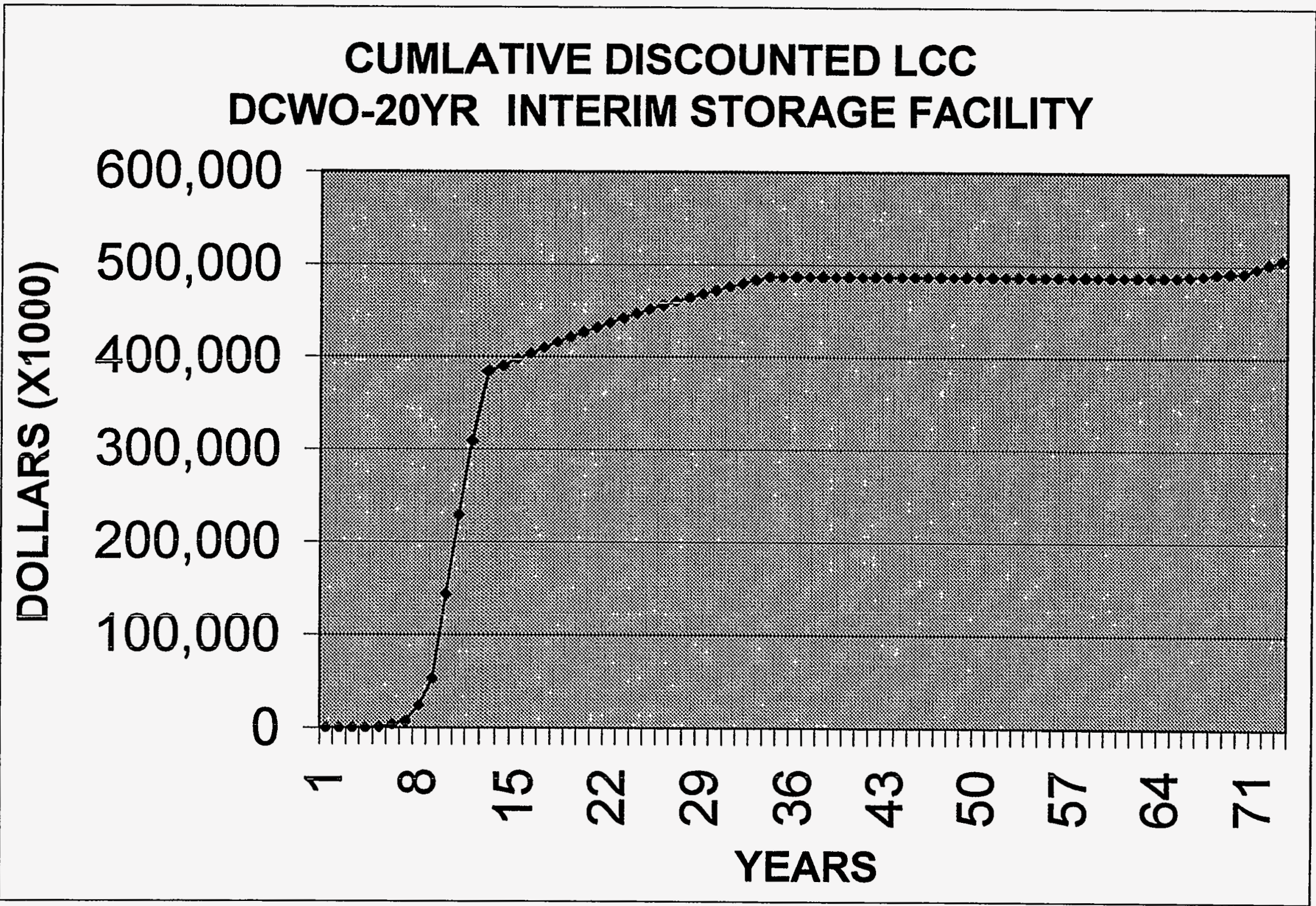


Date: February 2, 1998

To: John K. Rawlins $\quad$ MS 3765 6-4304

From: R. J. Turk Oflach 3875

Subject: $\quad$ ECONOMIC AND LIFE CYCLE ANALYSIS CONDUCTED for the CEMENTITIOUS WASTE OPTION (CWO) STORAGE FACILITY RJT-21-98

\section{Purpose:}

As requested an Economic and Life-Cycle Cost (LCC) has been conducted to evaluate for the CWO. INTERIM STORAGE FACILITY. The storage facility was evaluated based on a scoping study that incorporate appropriate design features from the Flour Daniel Corp. design for interim storage. This storage facility will be used to house canisters resulting from the CWO production option until final repository disposition.

This economic analysis is based on information provided by J. K. Rawlins, and K. L. Williams. R. D. Adams provided cost estimates. B.L. Blakely provided process personnel modeling.

\section{Methodology:}

The Economic Evaluation assumed a 69-year period (2003-2071) since this is the estimated time required to complete all of the anticipated remediation activities. The LCC identified and evaluated the initial development, construction, operating and post operating costs over the lifecycle. A discounted LCC analysis assumes a 1998-dollar basis, discounted $6.3 \%$ annually per the Office of Management and Budget (OMB) Circular A-94 for escalated costs. All costs are conservatively discounted assuming the end-of-year convention. 


\section{Assumptions:}

The scope of work and requirements of all related activities are vague at this time. Facility and processing costs were developed from historical experience associated with DD\&D work at the INEEL. The LCC analysis was generated to match cost estimating cost structure. These costs include Permitting, Direct and Indirect Construction, G\&A, Procurement Fee, Engineering, Inspection, Project Management, Construction Management, Escalation and Contingency costs.

The design period is assumed to be accomplished in five-years with construction completed in six-years. Labor rates were assumed as follows: Managers, $\$ 125 / \mathrm{hr}$; Engineers, $\$ 108 \$ / \mathrm{hr}$; Other Technicians $\$ 85 / \mathrm{hr}$; Administration/support staff $\$ 65 / \mathrm{hr}$; Operators and Maintenance personnel $\$ 65 / \mathrm{hr}$. The operational period for this facility was assumed to be five years, to match the appropriate operating facility. This period is followed by five years for shipping to the repository, followed by three years of post-operations activities. Due to this project's lack of complexity and non-radioactive contaminated environment, this analysis assumed a decommissioning cost equal to $10 \%$ of the non-escalated engineering design cost, decontamination costs equal to $0 \%$ of non-escalated total pre-operation cost, and demolition costs equal to $8 \%$ of the non-escalated total pre-operation cost. Utilities were calculated assuming a $\$ .0824$ per $\mathrm{kWh}$. Surveillance and Monitoring costs were calculated at $\$ 65,000$ per year for manned security during operations then reduced to $\$ 10,000$ for remote surveillance during non-operation periods.

\section{Results:}

The CWO INTERIM STORAGE FACILITY has a discounted LCC of $\$ 406$ million.

Attachments:

cc: R. J. Turk File 


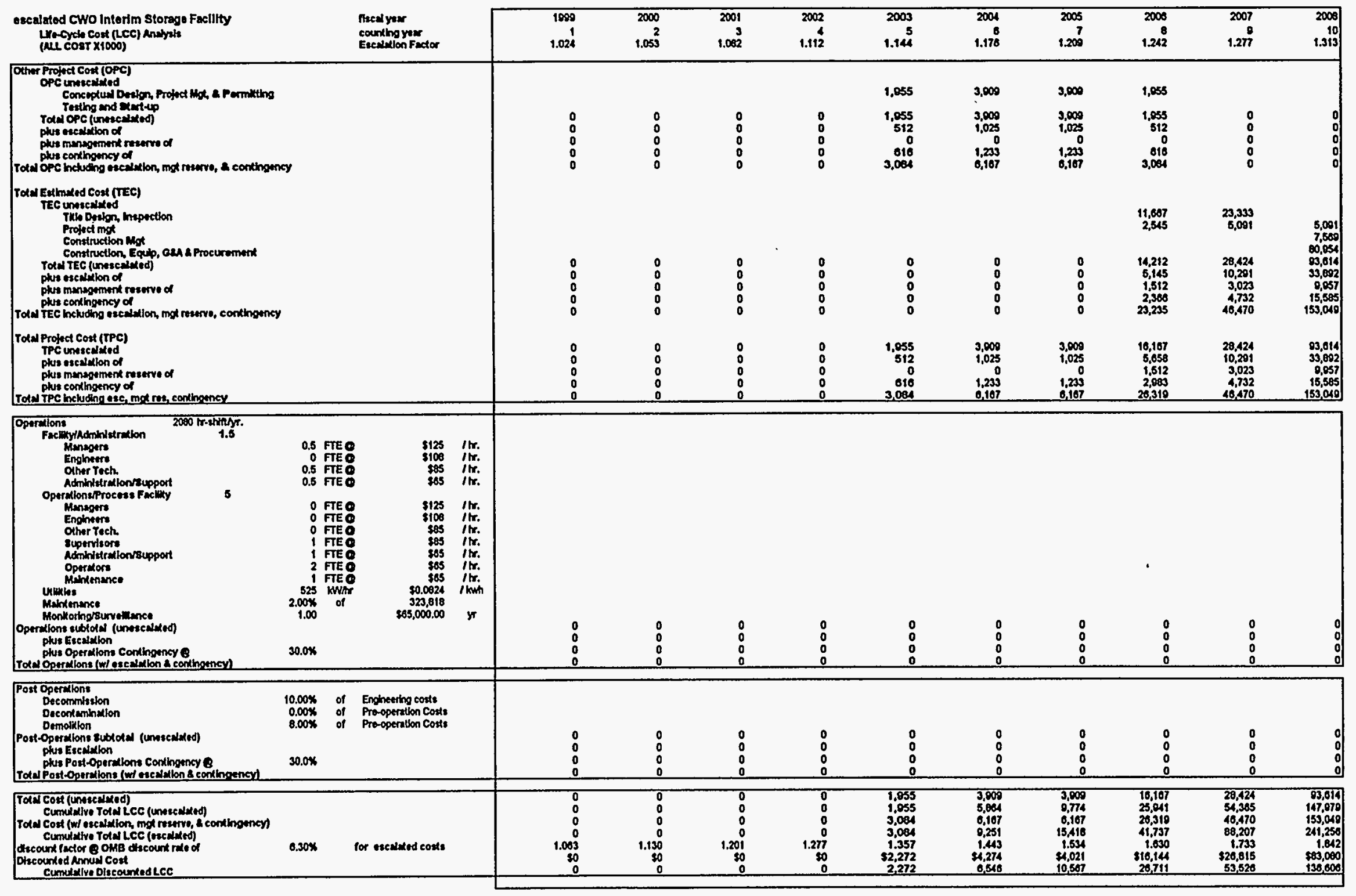




\begin{tabular}{|c|c|c|c|c|c|c|c|c|c|c|c|c|c|}
\hline 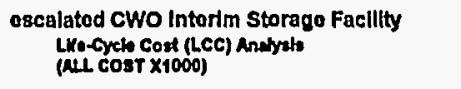 & & $\begin{array}{l}\text { Decel your } \\
\text { counding your } \\
\text { Becalation factor }\end{array}$ & & $\begin{array}{r}2008 \\
11 \\
1.350\end{array}$ & $\begin{array}{r}2010 \\
12 \\
1.387\end{array}$ & $\begin{array}{r}2011 \\
13 \\
1.426\end{array}$ & $\begin{array}{r}2012 \\
14 \\
1.486\end{array}$ & $\begin{array}{r}2013 \\
15 \\
1.607\end{array}$ & $\begin{array}{r}2014 \\
10 \\
1.550\end{array}$ & $\begin{array}{r}2015 \\
17 \\
1.593\end{array}$ & $\begin{array}{r}2016 \\
10 \\
1.638\end{array}$ & $\begin{array}{r}2017 \\
10 \\
1.603\end{array}$ & $\begin{array}{r}2018 \\
20 \\
1.730\end{array}$ \\
\hline 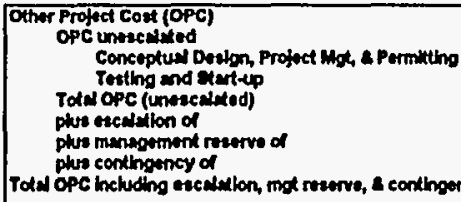 & & & & $\begin{array}{l}0 \\
0 \\
0 \\
0 \\
0\end{array}$ & $\begin{array}{l}0 \\
0 \\
0 \\
0 \\
0\end{array}$ & $\begin{array}{l}0 \\
0 \\
0 \\
0 \\
0\end{array}$ & $\begin{array}{r}3,375 \\
3,375 \\
005 \\
0 \\
1,004 \\
5,321\end{array}$ & $\begin{array}{r}1125 \\
1,125 \\
205 \\
0 \\
355 \\
1.775\end{array}$ & $\begin{array}{l}0 \\
0 \\
0 \\
0 \\
0\end{array}$ & $\begin{array}{l}0 \\
0 \\
0 \\
0 \\
0\end{array}$ & $\begin{array}{l}0 \\
0 \\
0 \\
0 \\
0\end{array}$ & $\begin{array}{l}0 \\
: \\
0 \\
0\end{array}$ & $\begin{array}{l}0 \\
0 \\
0 \\
0 \\
0\end{array}$ \\
\hline 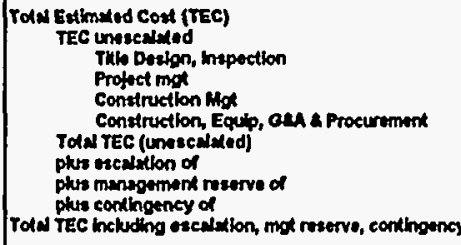 & & & & 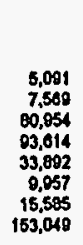 & $\begin{array}{r}5,091 \\
7,509 \\
60,054 \\
03,614 \\
33,892 \\
9,057 \\
15,505 \\
153,019\end{array}$ & $\begin{array}{r}5,091 \\
7,5609 \\
80,051 \\
93,014 \\
33,092 \\
0,097 \\
15,505 \\
153,019\end{array}$ & $\begin{array}{l}0 \\
\vdots \\
0 \\
0\end{array}$ & $\begin{array}{l}0 \\
0 \\
0 \\
0\end{array}$ & $\begin{array}{l}0 \\
0 \\
0 \\
0 \\
0\end{array}$ & $\begin{array}{l}0 \\
0 \\
0 \\
0 \\
0\end{array}$ & $\begin{array}{l}0 \\
0 \\
0 \\
0\end{array}$ & $\begin{array}{l}0 \\
0 \\
0 \\
0\end{array}$ & $\begin{array}{l}0 \\
0 \\
0 \\
0 \\
0\end{array}$ \\
\hline 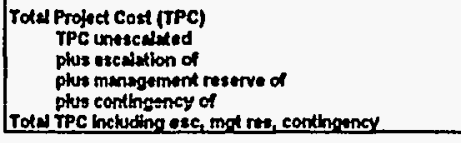 & & & & $\begin{array}{r}93,014 \\
33,002 \\
0,007 \\
15,565 \\
153,049 \\
\end{array}$ & $\begin{array}{r}93,614 \\
33,892 \\
9,857 \\
15,585 \\
153,019 \\
\end{array}$ & $\begin{array}{r}93,014 \\
33,892 \\
9,097 \\
15,595 \\
133,049 \\
\end{array}$ & $\begin{array}{r}3,375 \\
005 \\
0 \\
1,084 \\
5,324 \\
\end{array}$ & $\begin{array}{r}1,125 \\
285 \\
0 \\
355 \\
1,776 \\
\end{array}$ & $\begin{array}{l}0 \\
0 \\
0 \\
0 \\
0 \\
\end{array}$ & $\begin{array}{l}0 \\
0 \\
0 \\
0 \\
0 \\
\end{array}$ & $\begin{array}{l}0 \\
0 \\
0 \\
0 \\
0 \\
\end{array}$ & $\begin{array}{l}0 \\
0 \\
0 \\
0 \\
0 \\
\end{array}$ & $\begin{array}{l}0 \\
0 \\
0 \\
0\end{array}$ \\
\hline 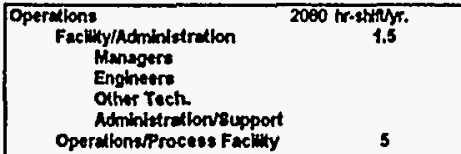 & $\begin{array}{l}0.5 \text { FTE } \\
0 \text { FTE } \\
0.5 \text { FTE } \\
0.5 \text { FTE } 8\end{array}$ & $\begin{array}{c}\$ 125 \\
\$ 100 \\
\$ 95 \\
\$ 85\end{array}$ & $\begin{array}{l}\text { itr. } \\
\text { inr. } \\
\text { int. } \\
\text { itr. }\end{array}$ & & & & $\begin{array}{r}65 \\
0 \\
4 \\
3\end{array}$ & $\begin{array}{r}130 \\
0 \\
68 \\
60\end{array}$ & $\begin{array}{r}130 \\
0 \\
60 \\
60\end{array}$ & $\begin{array}{r}130 \\
0 \\
89 \\
68\end{array}$ & $\begin{array}{r}130 \\
0 \\
69 \\
88\end{array}$ & $\begin{array}{r}130 \\
0 \\
88 \\
68\end{array}$ & \\
\hline 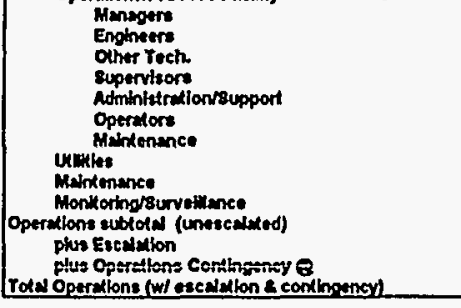 & 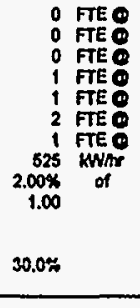 & 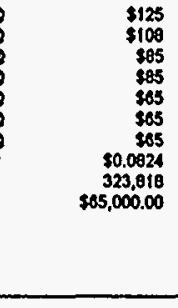 & 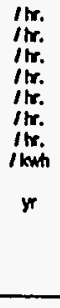 & $\begin{array}{l}0 \\
0 \\
0 \\
0 \\
\end{array}$ & $\begin{array}{l}0 \\
0 \\
0 \\
0 \\
\end{array}$ & $\begin{array}{l}0 \\
0 \\
0 \\
0\end{array}$ & $\begin{array}{r}0 \\
0 \\
0 \\
69 \\
69 \\
135 \\
69 \\
45 \\
0,238 \\
33 \\
3,917 \\
1,780 \\
1,679 \\
7,277 \\
\end{array}$ & $\begin{array}{r}0 \\
0 \\
0 \\
177 \\
135 \\
270 \\
135 \\
00 \\
0,476 \\
05 \\
7,035 \\
3,073 \\
3,452 \\
14,901 \\
\end{array}$ & $\begin{array}{r}0 \\
0 \\
0 \\
17 \\
135 \\
270 \\
135 \\
80 \\
0,470 \\
05 \\
7,035 \\
7,190 \\
j, 196 \\
15,380 \\
\end{array}$ & 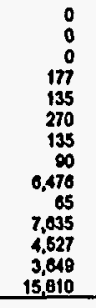 & $\begin{array}{r}0 \\
0 \\
0 \\
177 \\
135 \\
270 \\
135 \\
80 \\
0,470 \\
05 \\
7,035 \\
4,667 \\
3,751 \\
10,253 \\
\end{array}$ & $\begin{array}{r}0 \\
0 \\
0 \\
177 \\
135 \\
270 \\
135 \\
00 \\
0,478 \\
0.5 \\
7.035 \\
5.217 \\
3.050 \\
10,708 \\
\end{array}$ & $\begin{array}{r}10 \\
10 \\
7 \\
5 \\
22\end{array}$ \\
\hline 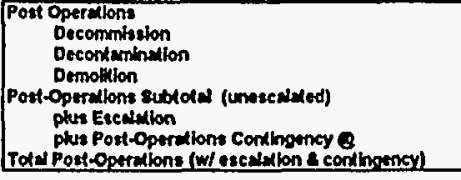 & $\begin{array}{ll}10.00 \% & \text { of } \\
0.00 \% & \text { of } \\
8.00 \% & \text { of } \\
30.0 \% & \end{array}$ & 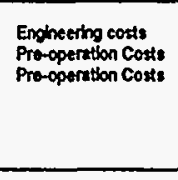 & & $\begin{array}{l}0 \\
0 \\
0 \\
0 \\
\end{array}$ & $\begin{array}{l}0 \\
0 \\
0 \\
0 \\
\end{array}$ & $\begin{array}{l}0 \\
0 \\
0 \\
0\end{array}$ & $\begin{array}{l}0 \\
0 \\
0 \\
0\end{array}$ & $\begin{array}{l}0 \\
0 \\
0 \\
\end{array}$ & $\begin{array}{l}0 \\
0 \\
0 \\
0 \\
\end{array}$ & $\begin{array}{l}0 \\
0 \\
0 \\
0 \\
\end{array}$ & $\begin{array}{l}0 \\
0 \\
0 \\
0\end{array}$ & $\begin{array}{l}0 \\
0 \\
0 \\
0 \\
\end{array}$ & $\begin{array}{l}0 \\
0 \\
0 \\
0\end{array}$ \\
\hline 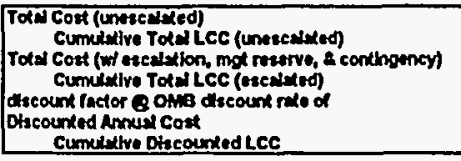 & $0.30 x$ & for oceseswlod conts & & 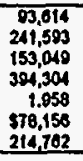 & 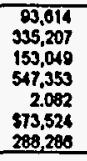 & 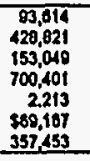 & $\begin{array}{r}7,102 \\
430,014 \\
12,001 \\
713,002 \\
2 ., 352 \\
\$ 5,237 \\
362,010 \\
\end{array}$ & $\begin{array}{r}8,760 \\
44,74 \\
10,745 \\
729,737 \\
2.500 \\
36,693 \\
369,500 \\
\end{array}$ & $\begin{array}{r}7 ., 035 \\
452,406 \\
15,300 \\
745,117 \\
22.068 \\
55,760 \\
375,200 \\
\end{array}$ & 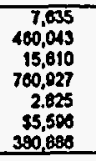 & $\begin{array}{r}7,635 \\
467,070 \\
11.253 \\
77,100 \\
3.000 \\
55,412 \\
388,297 \\
\end{array}$ & 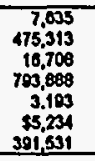 & $\begin{array}{r}10 \\
475,323 \\
22 \\
703,911 \\
3.34 \\
397 \\
301,539 \\
\end{array}$ \\
\hline
\end{tabular}




\begin{tabular}{|c|c|c|c|c|c|c|c|c|c|c|c|c|c|}
\hline 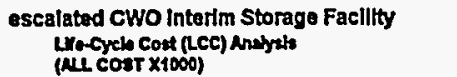 & & 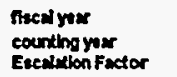 & & $\begin{array}{l}2010 \\
21 \\
1.770\end{array}$ & $\begin{array}{r}2020 \\
22 \\
1.820\end{array}$ & $\begin{array}{r}2021 \\
23 \\
1.800\end{array}$ & $\begin{array}{r}2022 \\
24 \\
1.233\end{array}$ & $\begin{array}{r}2023 \\
25 \\
1.087\end{array}$ & $\begin{array}{r}2021 \\
28 \\
2.012\end{array}$ & $\begin{array}{l}2025 \\
27 \\
2100\end{array}$ & $\begin{array}{r}2028 \\
28 \\
2.158\end{array}$ & $\begin{array}{r}2027 \\
2.29 \\
2.29\end{array}$ & $\begin{aligned} 2020 \\
300 \\
2.231\end{aligned}$ \\
\hline 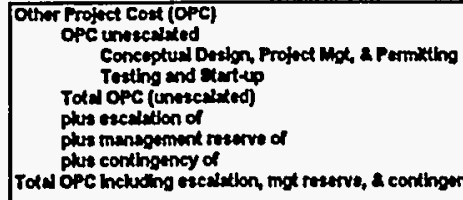 & & & & $\begin{array}{l}0 \\
0 \\
0 \\
0 \\
0\end{array}$ & $\begin{array}{l}0 \\
0 \\
0 \\
0 \\
0\end{array}$ & $\begin{array}{l}0 \\
0 \\
0 \\
0 \\
0\end{array}$ & $\begin{array}{l}0 \\
0 \\
0 \\
0 \\
0\end{array}$ & $\begin{array}{l}0 \\
0 \\
0 \\
0 \\
0\end{array}$ & $\begin{array}{l}0 \\
0 \\
0 \\
0 \\
0\end{array}$ & $\begin{array}{l}0 \\
0 \\
0 \\
0 \\
0\end{array}$ & $\begin{array}{l}0 \\
0 \\
0 \\
0 \\
0\end{array}$ & $\begin{array}{l}0 \\
0 \\
0 \\
0 \\
0\end{array}$ & $\begin{array}{l}0 \\
0 \\
0 \\
0 \\
0\end{array}$ \\
\hline 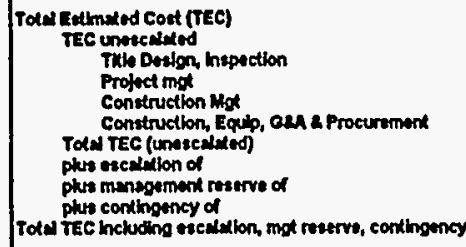 & & & & $\begin{array}{l}0 \\
0 \\
0 \\
0 \\
0\end{array}$ & $\begin{array}{l}0 \\
: \\
0 \\
0 \\
0\end{array}$ & $\begin{array}{l}0 \\
0 \\
0 \\
0 \\
0\end{array}$ & $\begin{array}{l}0 \\
0 \\
0 \\
0 \\
0\end{array}$ & $\begin{array}{l}0 \\
0 \\
0 \\
0 \\
0\end{array}$ & $\begin{array}{l}0 \\
0 \\
0 \\
0 \\
0\end{array}$ & $\begin{array}{l}0 \\
0 \\
0 \\
0 \\
0\end{array}$ & $\begin{array}{l}0 \\
0 \\
0 \\
0 \\
0\end{array}$ & $\begin{array}{l}0 \\
0 \\
0 \\
0 \\
0\end{array}$ & $\begin{array}{l}0 \\
0 \\
0 \\
0 \\
0\end{array}$ \\
\hline 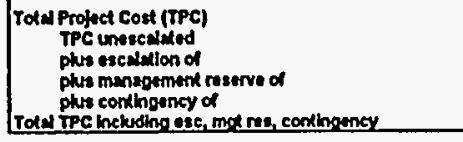 & & & & $\begin{array}{l}0 \\
0 \\
0 \\
0 \\
0 \\
\end{array}$ & $\begin{array}{l}0 \\
0 \\
0 \\
0 \\
\end{array}$ & $\begin{array}{l}0 \\
0 \\
0 \\
0 \\
0 \\
\end{array}$ & $\begin{array}{l}0 \\
0 \\
0 \\
0 \\
0 \\
\end{array}$ & $\begin{array}{l}0 \\
0 \\
0 \\
0 \\
0 \\
\end{array}$ & $\begin{array}{l}0 \\
0 \\
0 \\
0 \\
0 \\
\end{array}$ & $\begin{array}{l}0 \\
0 \\
0 \\
0 \\
0 \\
\end{array}$ & $\begin{array}{l}0 \\
0 \\
0 \\
0 \\
0 \\
\end{array}$ & $\begin{array}{l}0 \\
0 \\
0 \\
0 \\
0 \\
\end{array}$ & $\begin{array}{l}0 \\
0 \\
0 \\
0 \\
0 \\
\end{array}$ \\
\hline 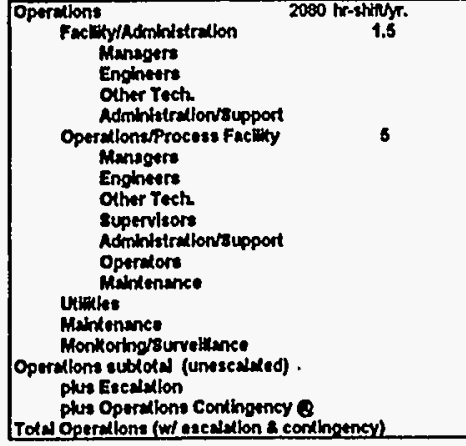 & 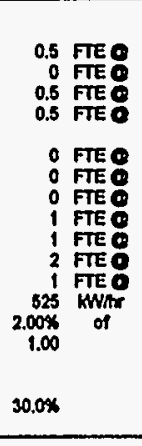 & 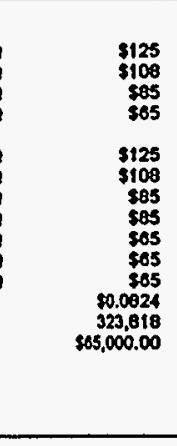 & 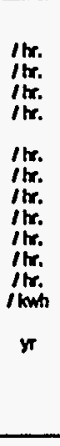 & $\begin{array}{r}10 \\
10 \\
8 \\
5 \\
23 \\
\end{array}$ & $\begin{array}{r}10 \\
10 \\
8 \\
5 \\
21 \\
\end{array}$ & $\begin{array}{r}10 \\
10 \\
8 \\
8 \\
24 \\
2\end{array}$ & $\begin{array}{r}10 \\
10 \\
8 \\
8 \\
25 \\
\end{array}$ & $\begin{array}{r}10 \\
10 \\
10 \\
10 \\
20 \\
0\end{array}$ & $\begin{array}{r}10 \\
10 \\
10 \\
8 \\
27 \\
\end{array}$ & $\begin{array}{r}10 \\
10 \\
11 \\
6 \\
27 \\
\end{array}$ & $\begin{array}{r}10 \\
10 \\
12 \\
8 \\
28 \\
\end{array}$ & $\begin{array}{r}10 \\
10 \\
12 \\
7 \\
29 \\
\end{array}$ & $\begin{array}{r}10 \\
10 \\
13 \\
7 \\
30 \\
\end{array}$ \\
\hline 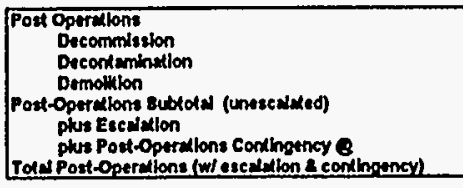 & $\begin{array}{ll}10.00 \% & \text { of } \\
0.00 \% & \text { of } \\
0.00 \% & \text { of } \\
& \\
30.0 \% & \end{array}$ & 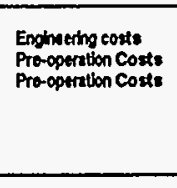 & & $\begin{array}{l}0 \\
0 \\
0 \\
0 \\
\end{array}$ & $\begin{array}{l}0 \\
0 \\
0 \\
0 \\
\end{array}$ & $\begin{array}{l}0 \\
0 \\
0 \\
0 \\
\end{array}$ & $\begin{array}{l}0 \\
0 \\
0 \\
0 \\
\end{array}$ & $\begin{array}{l}0 \\
0 \\
0 \\
0 \\
\end{array}$ & $\begin{array}{l}0 \\
0 \\
0 \\
0 \\
\end{array}$ & $\begin{array}{l}0 \\
0 \\
0 \\
0 \\
\end{array}$ & $\begin{array}{l}0 \\
0 \\
0 \\
0 \\
\end{array}$ & $\begin{array}{l}0 \\
0 \\
0 \\
0 \\
\end{array}$ & $\begin{array}{l}0 \\
0 \\
0 \\
0\end{array}$ \\
\hline 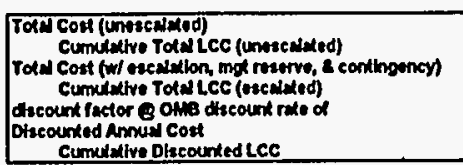 & $0.00 x$ & for esculded conts & & $\begin{array}{r}10 \\
475,333 \\
23 \\
703,034 \\
3,6007 \\
3091,54 \\
\end{array}$ & $\begin{array}{r}10 \\
475,343 \\
24 \\
703,057 \\
3.063 \\
58 \\
301,550 \\
30\end{array}$ & $\begin{array}{r}10 \\
475,333 \\
24 \\
703,082 \\
4.0010 \\
46 \\
391,556 \\
\end{array}$ & $\begin{array}{r}10 \\
475,303 \\
25 \\
70,007 \\
4 ., 333 \\
56 \\
391,502 \\
\end{array}$ & 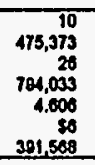 & 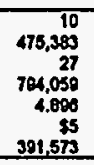 & $\begin{array}{r}100 \\
475,303 \\
27 \\
794,007 \\
5.2003 \\
55 \\
301,578 \\
\end{array}$ & $\begin{array}{r}10 \\
475,400 \\
28 \\
794,115 \\
5.533 \\
35 \\
391,583 \\
901\end{array}$ & $\begin{array}{r}10 \\
473,413 \\
29 \\
794,14 \\
3.841 \\
359 \\
301,588 \\
\end{array}$ & $\begin{array}{r}10 \\
475,423 \\
30 \\
794,173 \\
0.252 \\
453 \\
391,593 \\
\end{array}$ \\
\hline
\end{tabular}




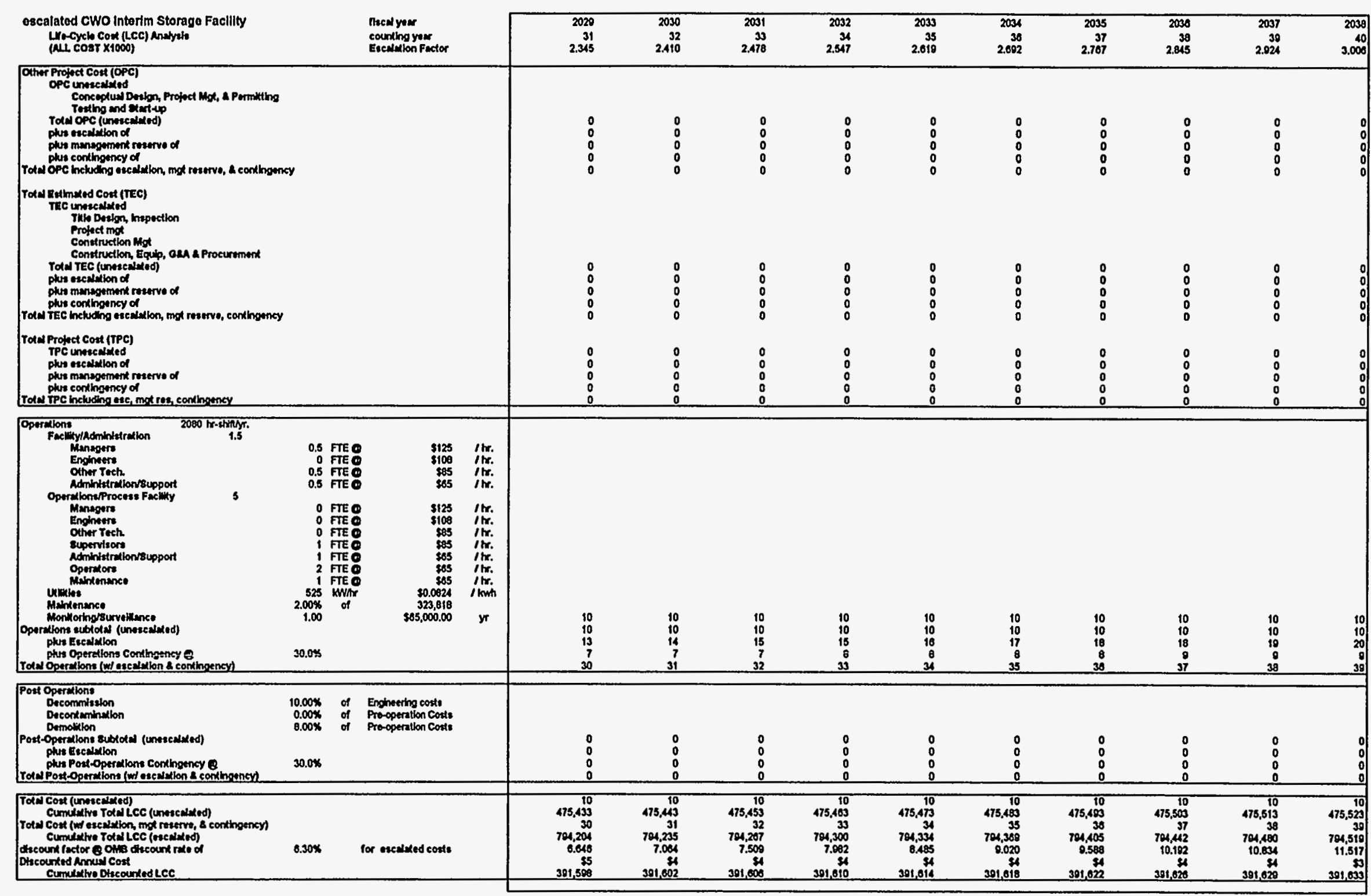




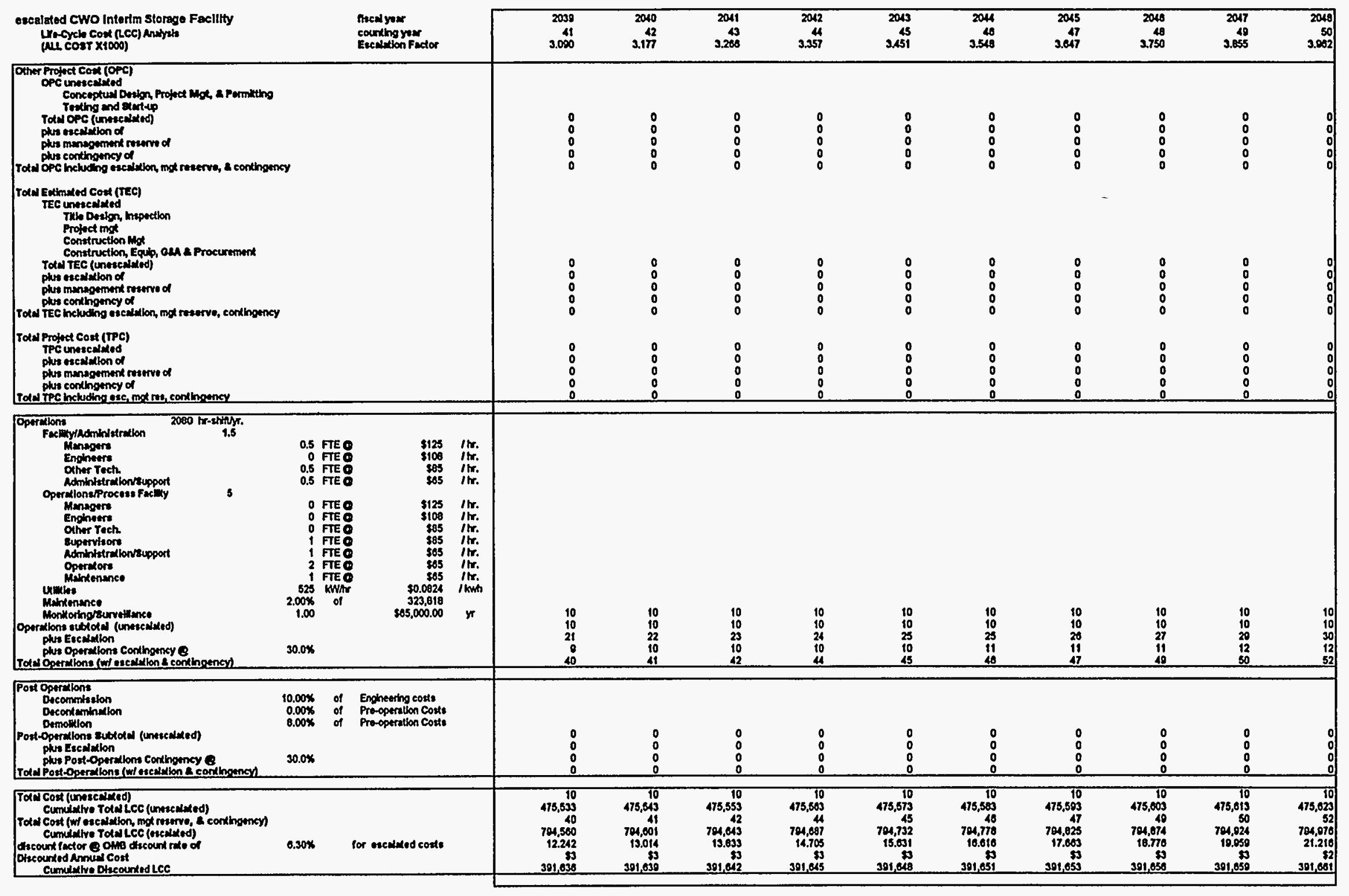




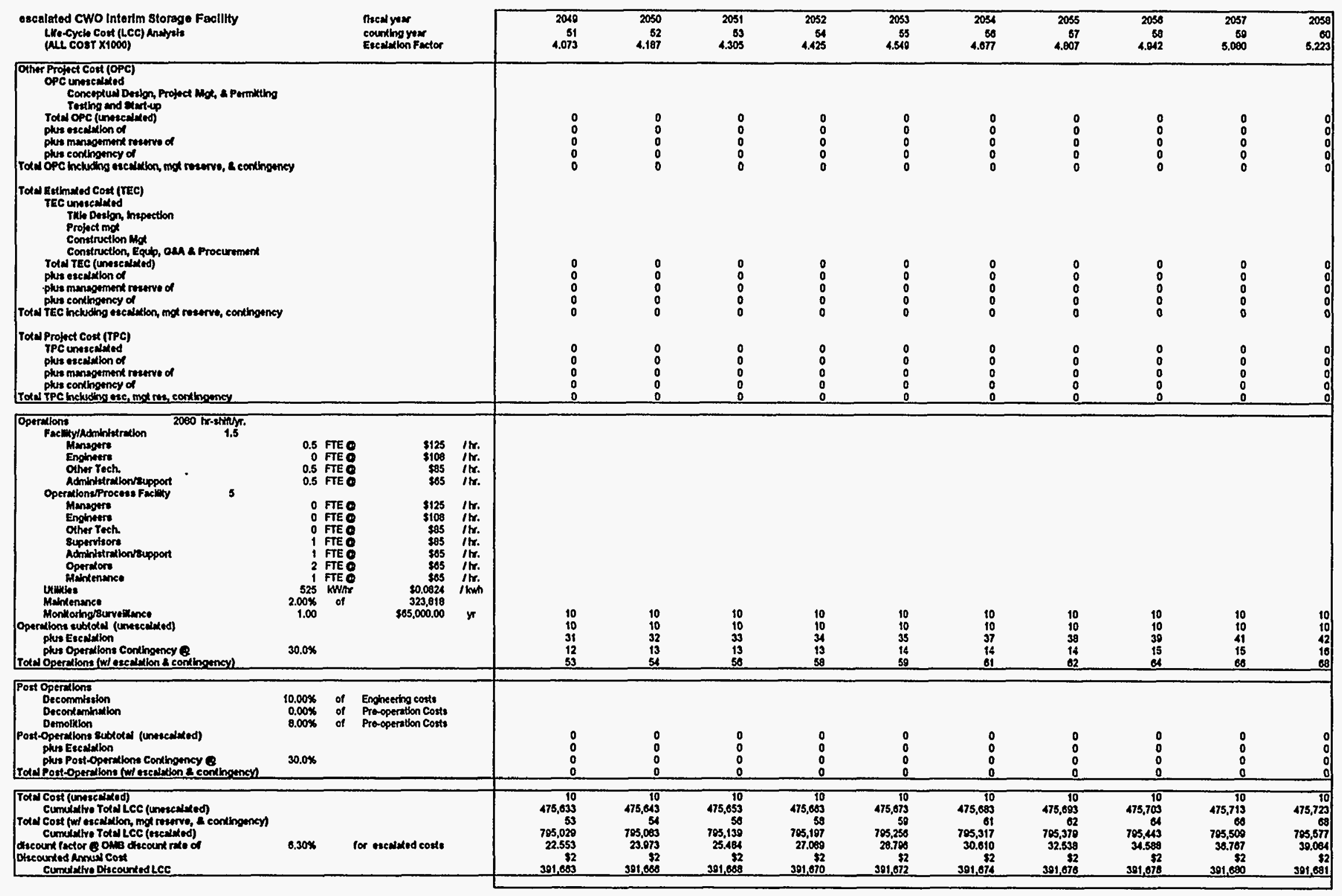




\begin{tabular}{|c|c|c|c|c|c|c|c|c|c|c|c|c|c|}
\hline 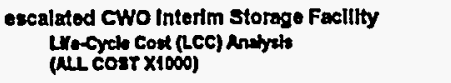 & & 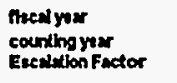 & & $\begin{array}{l}2059 \\
6.1 \\
5.300\end{array}$ & $\begin{array}{l}2000 \\
62 \\
5.510\end{array}$ & $\begin{array}{l}2001 \\
60 \\
5.074\end{array}$ & $\begin{array}{l}2002 \\
44 \\
5.633\end{array}$ & $\begin{array}{l}2003 \\
05 \\
5.096\end{array}$ & $\begin{array}{l}2004 \\
0.04 \\
0.104\end{array}$ & $\begin{array}{l}2005 \\
67 \\
0.330\end{array}$ & $\begin{array}{l}2006 \\
0.58 \\
0.514\end{array}$ & $\begin{array}{l}2007 \\
0.9 \\
0.680\end{array}$ & $\begin{array}{r}2008 \\
70 \\
0.884\end{array}$ \\
\hline \multicolumn{4}{|l|}{ 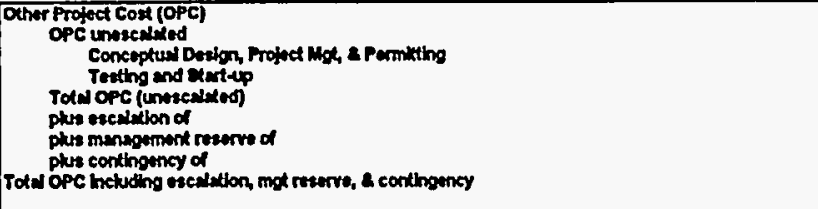 } & $\begin{array}{l}0 \\
0 \\
0 \\
0 \\
0\end{array}$ & $\begin{array}{l}0 \\
0 \\
0 \\
0 \\
0\end{array}$ & $\begin{array}{l}0 \\
0 \\
0 \\
0 \\
0\end{array}$ & $\begin{array}{l}0 \\
0 \\
0 \\
0 \\
0\end{array}$ & $\begin{array}{l}0 \\
0 \\
0 \\
0\end{array}$ & $\begin{array}{l}0 \\
0 \\
0 \\
0 \\
0\end{array}$ & $\begin{array}{l}0 \\
0 \\
0 \\
0 \\
0\end{array}$ & $\begin{array}{l}0 \\
0 \\
0 \\
0 \\
0\end{array}$ & $\begin{array}{l}0 \\
0 \\
0 \\
0 \\
0\end{array}$ & \begin{tabular}{l|l}
0 \\
0 \\
0 \\
0 \\
0
\end{tabular} \\
\hline \multicolumn{4}{|l|}{ 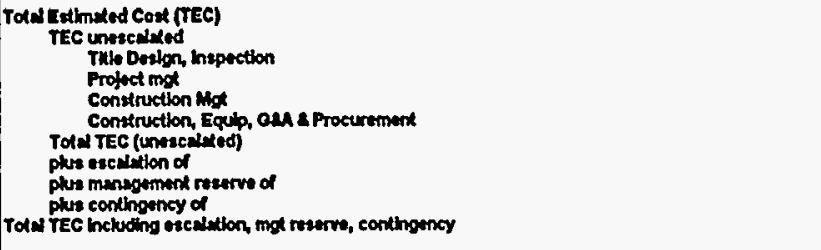 } & $\begin{array}{l}0 \\
0 \\
0 \\
0 \\
0\end{array}$ & $\begin{array}{l}0 \\
0 \\
0 \\
0 \\
0\end{array}$ & $\begin{array}{l}0 \\
0 \\
0 \\
0 \\
0\end{array}$ & $\begin{array}{l}0 \\
0 \\
0 \\
0 \\
0\end{array}$ & $\begin{array}{l}0 \\
0 \\
0 \\
0 \\
0\end{array}$ & $\begin{array}{l}0 \\
0 \\
0 \\
0 \\
0\end{array}$ & $\begin{array}{l}0 \\
0 \\
0 \\
0 \\
0\end{array}$ & $\begin{array}{l}0 \\
0 \\
0 \\
0 \\
0\end{array}$ & $\begin{array}{l}0 \\
0 \\
0 \\
0 \\
0\end{array}$ & $\begin{array}{l}0 \\
0 \\
0 \\
0 \\
0\end{array}$ \\
\hline 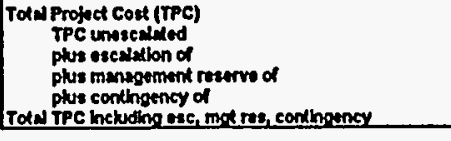 & & & & $\begin{array}{l}0 \\
0 \\
0 \\
0 \\
0 \\
\end{array}$ & $\begin{array}{l}0 \\
0 \\
0 \\
0 \\
0 \\
\end{array}$ & $\begin{array}{l}0 \\
0 \\
0 \\
0 \\
0\end{array}$ & $\begin{array}{l}0 \\
0 \\
0 \\
0 \\
0 \\
\end{array}$ & $\begin{array}{l}0 \\
0 \\
0 \\
0 \\
\end{array}$ & $\begin{array}{l}0 \\
0 \\
0 \\
0 \\
0 \\
\end{array}$ & $\begin{array}{l}0 \\
0 \\
0 \\
0 \\
0 \\
\end{array}$ & $\begin{array}{l}0 \\
0 \\
0 \\
0 \\
0 \\
\end{array}$ & $\begin{array}{l}0 \\
0 \\
0 \\
0 \\
0\end{array}$ & $\begin{array}{l}0 \\
0 \\
0 \\
0 \\
0\end{array}$ \\
\hline 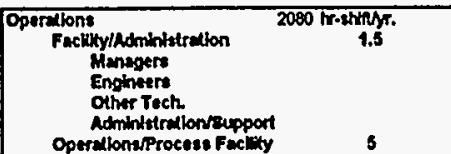 & $\begin{array}{l}0.5 \text { FTE } \\
0 \text { FTE } \\
0.5 \text { FTE: } \\
0.5 \text { FTE }\end{array}$ & $\begin{array}{c}\$ 125 \\
\$ 1106 \\
\$ 35 \\
\$ 85\end{array}$ & $\begin{array}{l}\text { Ihr. } \\
\text { lmr. } \\
\text { lir. } \\
\text { lir. }\end{array}$ & & & & & & $\begin{array}{r}130 \\
0 \\
60 \\
00\end{array}$ & $\begin{array}{r}130 \\
0 \\
89 \\
69\end{array}$ & $\begin{array}{r}130 \\
0 \\
69 \\
60\end{array}$ & $\begin{array}{r}130 \\
0 \\
89 \\
68\end{array}$ & $\begin{array}{r}130 \\
09 \\
69 \\
69\end{array}$ \\
\hline 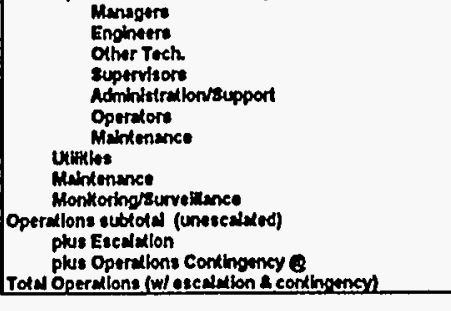 & 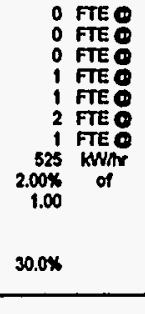 & $\begin{array}{r}\$ 125 \\
\$ 108 \\
\$ 85 \\
\$ 85 \\
\$ 85 \\
\$ 85 \\
\$ 65 \\
\$ 0.05 \\
\$ 0.0924 \\
\$ 03,018 \\
\$ 05,000.00\end{array}$ & 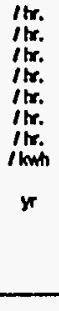 & $\begin{array}{l}10 \\
10 \\
14 \\
10 \\
70 \\
\end{array}$ & $\begin{array}{l}10 \\
10 \\
45 \\
17 \\
72 \\
\end{array}$ & $\begin{array}{l}10 \\
10 \\
17 \\
17 \\
17 \\
\end{array}$ & $\begin{array}{l}10 \\
10 \\
48 \\
17 \\
76 \\
\end{array}$ & $\begin{array}{l}10 \\
10 \\
10 \\
10 \\
70 \\
70\end{array}$ & $\begin{array}{r}0 \\
0 \\
171 \\
135 \\
270 \\
135 \\
00 \\
0 \\
05 \\
1,159 \\
5,090 \\
2,112 \\
0.284 \\
\end{array}$ & $\begin{array}{r}0 \\
0 \\
0 \\
177 \\
135 \\
270 \\
135 \\
00 \\
0 \\
05 \\
1,150 \\
0,163 \\
2,202 \\
0,344 \\
\end{array}$ & $\begin{array}{r}0 \\
0 \\
0 \\
177 \\
135 \\
270 \\
135 \\
00 \\
0 \\
05 \\
1.159 \\
6,389 \\
2,244 \\
0,811 \\
\end{array}$ & $\begin{array}{r}0 \\
0 \\
0 \\
177 \\
135 \\
270 \\
135 \\
\infty 0 \\
0 \\
05 \\
1,159 \\
0,600 \\
2,327 \\
10,068 \\
\end{array}$ & $\begin{array}{r}0 \\
0 \\
17 \\
13, \\
270 \\
135 \\
\infty \\
0 \\
05 \\
1,150 \\
0,297 \\
2,393 \\
10,390 \\
\end{array}$ \\
\hline 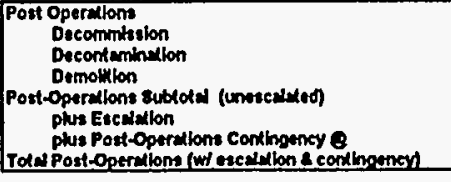 & $\begin{array}{ll}10.00 \% & \text { of } \\
0.00 \% & \text { of } \\
8.00 \% & \text { of } \\
& \\
30.0 \% & \end{array}$ & $\begin{array}{l}\text { Enghwering costs } \\
\text { Preoperntion Costs } \\
\text { Pro-opention Costs }\end{array}$ & & $\begin{array}{l}0 \\
0 \\
0 \\
0\end{array}$ & $\begin{array}{l}0 \\
0 \\
0 \\
0 \\
\end{array}$ & $\begin{array}{l}0 \\
0 \\
0 \\
0 \\
\end{array}$ & $\begin{array}{l}0 \\
0 \\
0 \\
0 \\
\end{array}$ & $\begin{array}{l}0 \\
0 \\
0 \\
0 \\
\end{array}$ & $\begin{array}{l}0 \\
0 \\
0 \\
0 \\
\end{array}$ & $\begin{array}{l}0 \\
0 \\
0 \\
0 \\
\end{array}$ & $\begin{array}{l}0 \\
0 \\
0 \\
0 \\
\end{array}$ & $\begin{array}{l}0 \\
0 \\
0 \\
0 \\
\end{array}$ & $\begin{array}{l}0 \\
0 \\
0 \\
0 \\
\end{array}$ \\
\hline 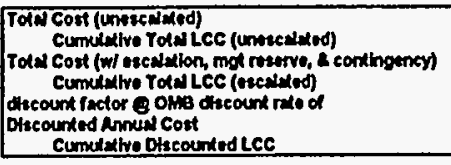 & $0.30 x$ & for osedurid costs & & $\begin{array}{r}100 \\
475,730 \\
70 \\
705,647 \\
41,460 \\
92 \\
391,803 \\
\end{array}$ & $\begin{array}{r}10 \\
475,743 \\
72 \\
785,710 \\
4.1603 \\
\$ 2 \\
391,085 \\
\end{array}$ & $\begin{array}{r}100 \\
475,753 \\
74 \\
705,703 \\
46.46 \\
\$ 2 \\
391, .886 \\
\end{array}$ & $\begin{array}{r}170 \\
475,703 \\
78 \\
795,860 \\
49.003 \\
52 \\
391,680 \\
\end{array}$ & $\begin{array}{r}10 \\
475,773 \\
70 \\
705,06 \\
53,0.047 \\
51 \\
391,600 \\
\end{array}$ & 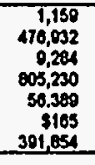 & 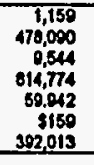 & 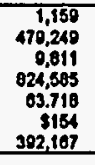 & 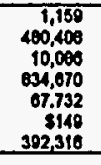 & 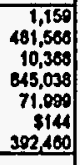 \\
\hline
\end{tabular}




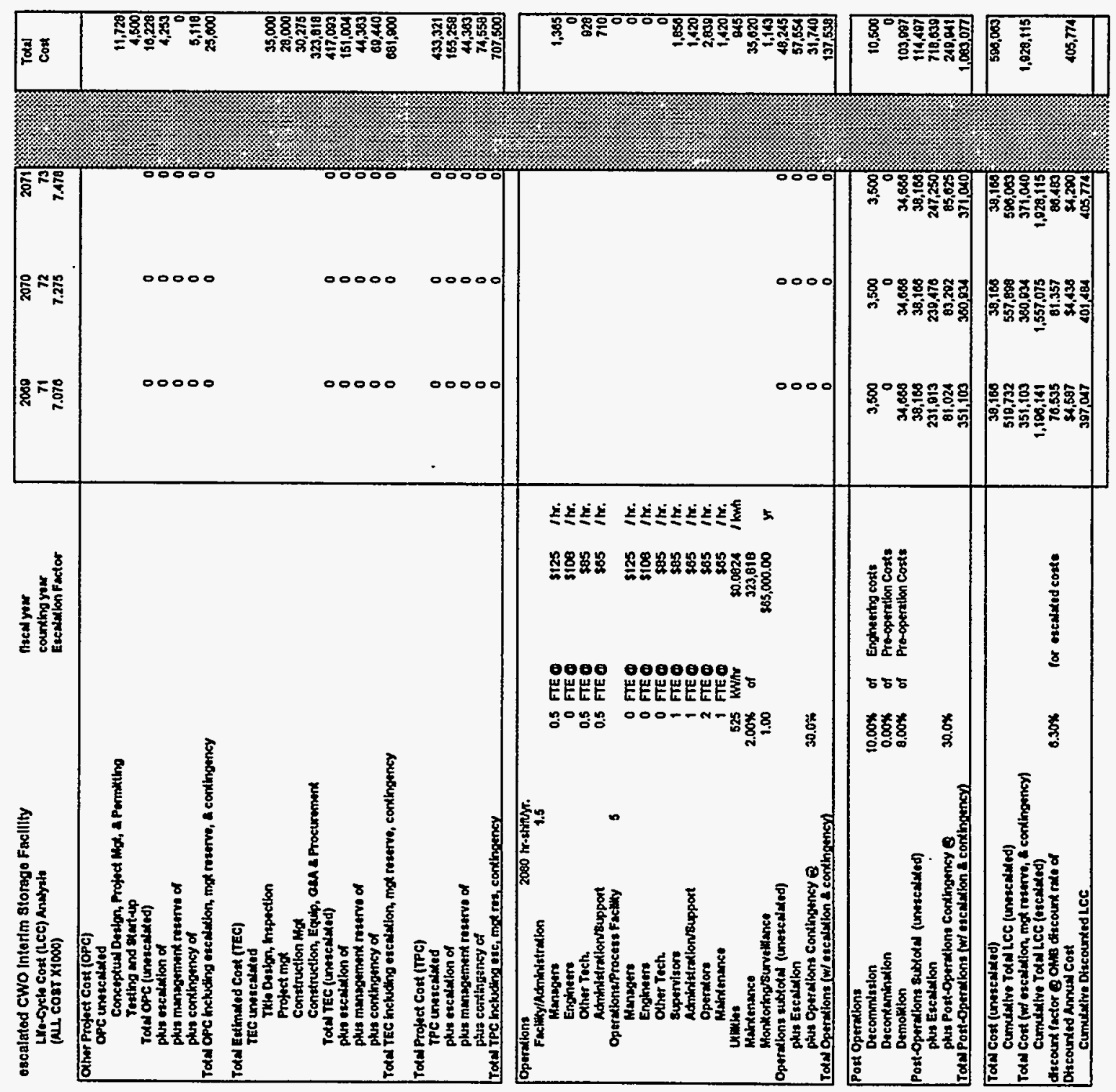




\section{TOTAL ANNUAL COST (escalated)}

CWO INTERIM STORAGE FACILTY

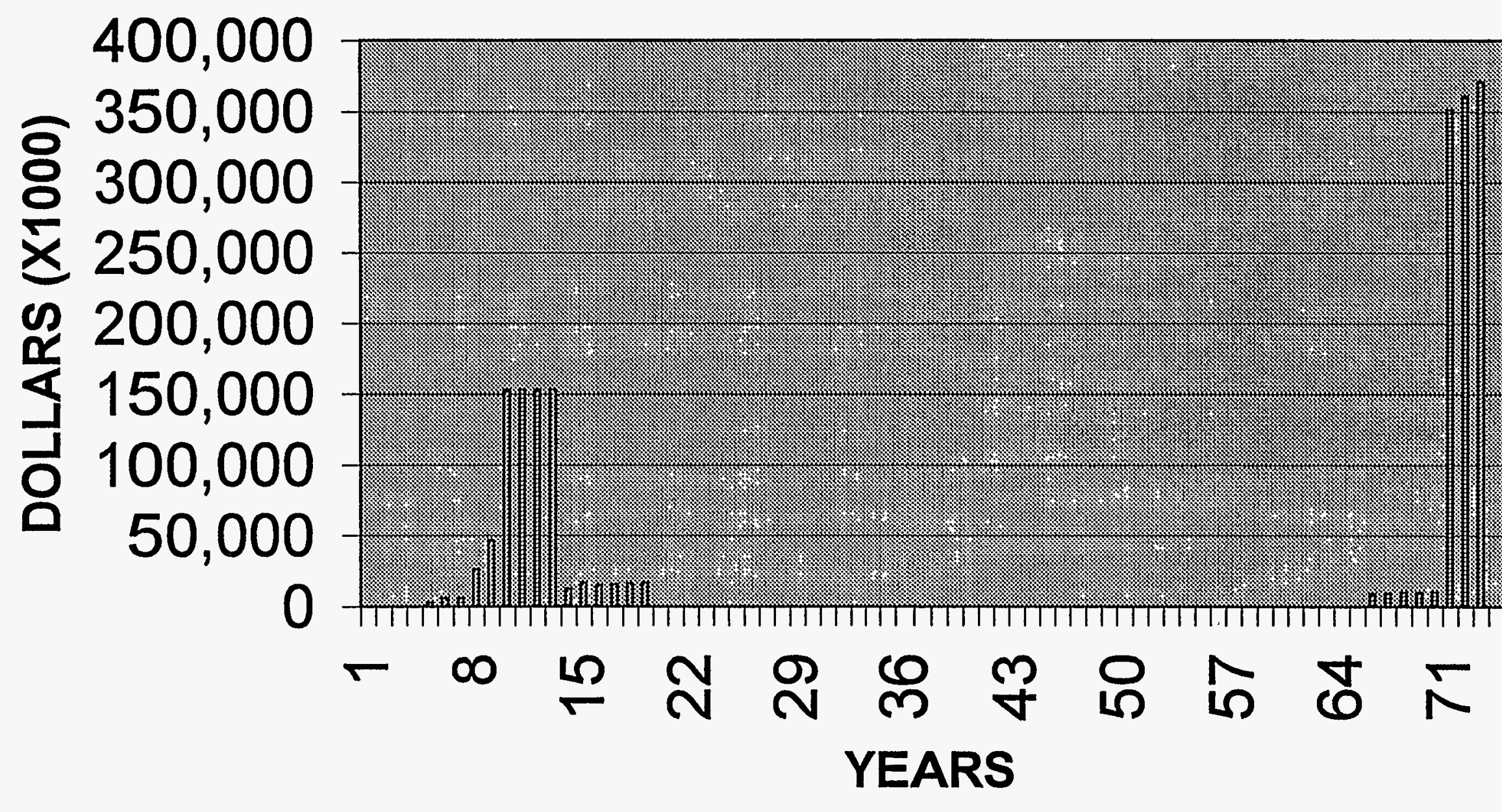




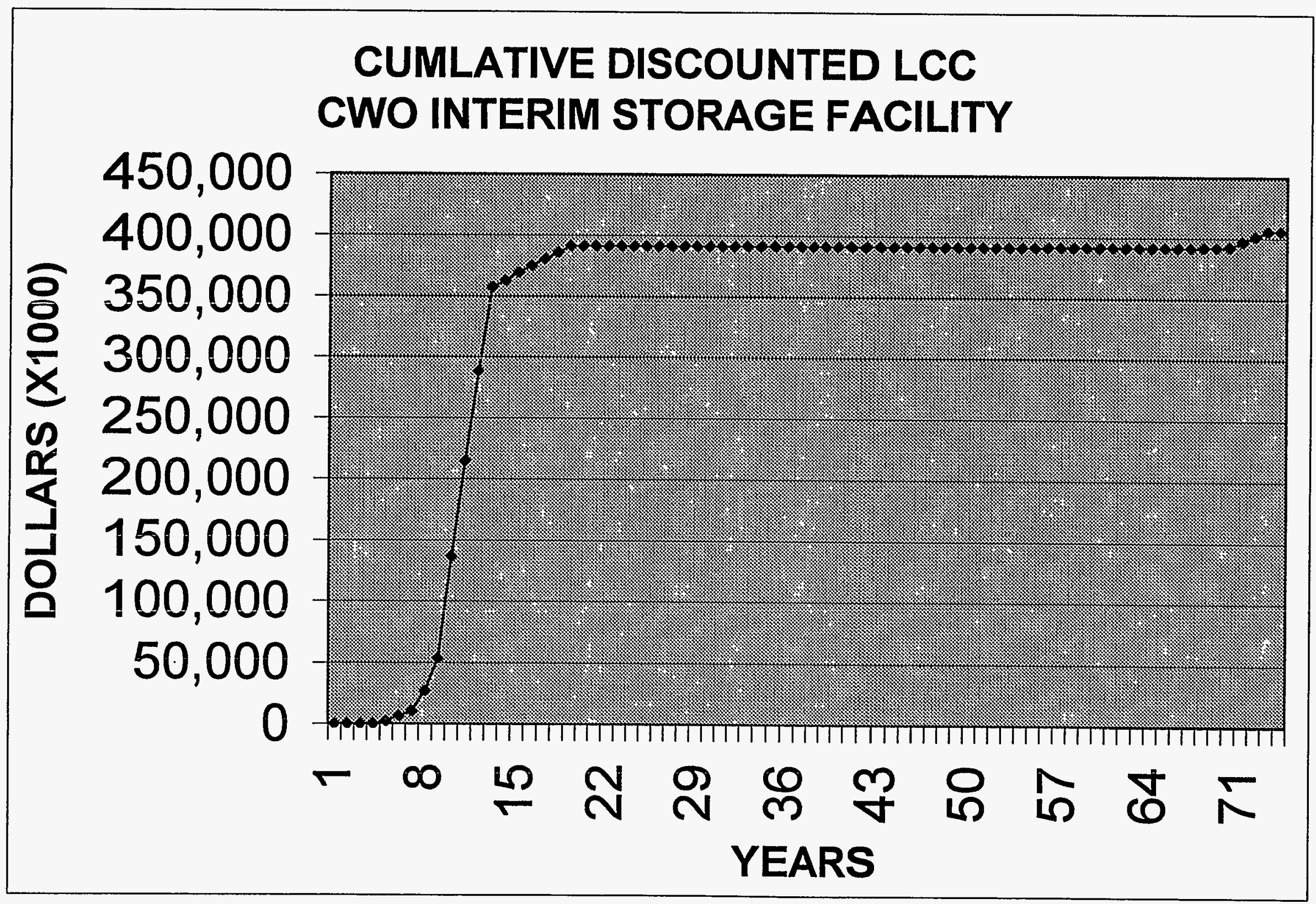




\section{Lockheed Martin Idaho Technologies Company INTERDEPARTMENTAL COMMUNICATION}

Date: January 30, 1998

To: John K. Rawlins $\quad$ MS $3765 \quad 6-4304$

From: $\quad$ R. J. Turk<smiles>[R15][AsH3]</smiles>

$6-3611$

Subject: $\quad$ ECONOMIC AND LIFE CYCLE ANALYSIS CONDUCTED for the HOT ISOSTATIC PRESSING (HIP) STORAGE FACILITIES- RJT-16-98

\section{Purpose:}

As requested an Economic and Life-Cycle Cost (LCC) has been conducted to evaluate for the INTERIM STORAGE FACILITIES. The storage facility was evaluated based on a scoping study that incorporate appropriate design features from the Flour Daniel Corp. design for interim storage. This storage facility will be used to house canisters resulting from the HIP production option until final repository disposition.

This economic analysis is based on information provided by J. K. Rawlins, and K. L. Williams. R. D. Adams provided cost estimates. B.L. Blakely provided process personnel modeling.

\section{Methodology:}

The Economic Evaluation assumed a 69-year period (2003-2071) since this is the estimated time required to complete all of the anticipated remediation activities. The LCC identified and evaluated the initial development, construction, operating and post operating costs over the lifecycle. A discounted LCC analysis assumes a 1998-dollar basis, discounted $6.3 \%$ annually per the Office of Management and Budget (OMB) Circular A-94 for escalated costs. All costs are conservatively discounted assuming the end-of-year convention. 
John K Rawlins

January 30, 1998

RJT 16-98

Page 2

\section{Assumptions:}

The scope of work and requirements of all related activities are vague at this time. Facility and processing costs were developed from historical experience associated with DD\&D work at the INEEL. The LCC analysis was generated to match cost estimating cost structure. These costs include Permitting, Direct and Indirect Construction, G\&A, Procurement Fee, Engineering, Inspection, Project Management, Construction Management, Escalation and Contingency costs.

The design period is assumed to be accomplished in five-years with construction completed in six-years. Labor rates were assumed as follows: Managers, $\$ 125 / \mathrm{hr}$; Engineers, $\$ 108$ \$/hr; Other Technicians $\$ 85 / \mathrm{hr}$; Administration/support staff $\$ 65 / \mathrm{hr}$; Operators and Maintenance personnel $\$ 65 / \mathrm{hr}$. The operational period for this facility was assumed to be twenty years, to match the appropriate operating facility. This period is followed by five years for shipping to the repository, followed by three years of post-operations activities. Due to this project's lack of complexity and non-radioactive contaminated environment, this analysis assumed a decommissioning cost equal to $10 \%$ of the non-escalated engineering design cost, decontamination costs equal to $0 \%$ of non-escalated total pre-operation cost, and demolition costs equal to $8 \%$ of the non-escalated total pre-operation cost. Utilities were calculated assuming a $\$ .0824$ per $\mathrm{kWh}$. Surveillance and Monitoring costs were calculated at $\$ 65,000$ per year for manned security during operations then reduced to $\$ 10,000$ for remote surveillance during non-operation periods.

\section{Results:}

The HOT ISOSTATIC PRESSING FACILITY INTERIM STORAGE has a discounted LCC of $\$ 255.8$ million.

Attachments:

cc: R. J. Turk File 


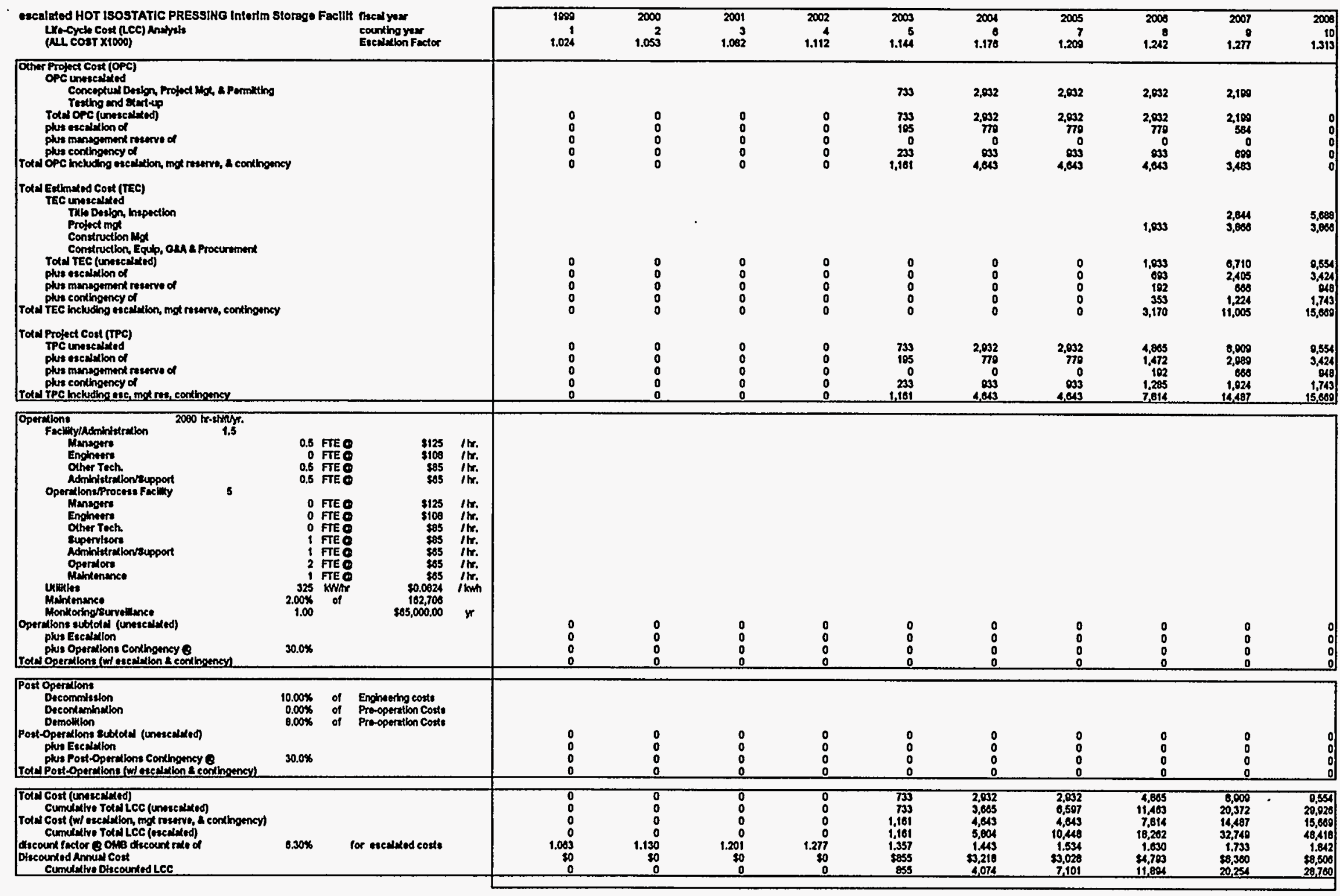




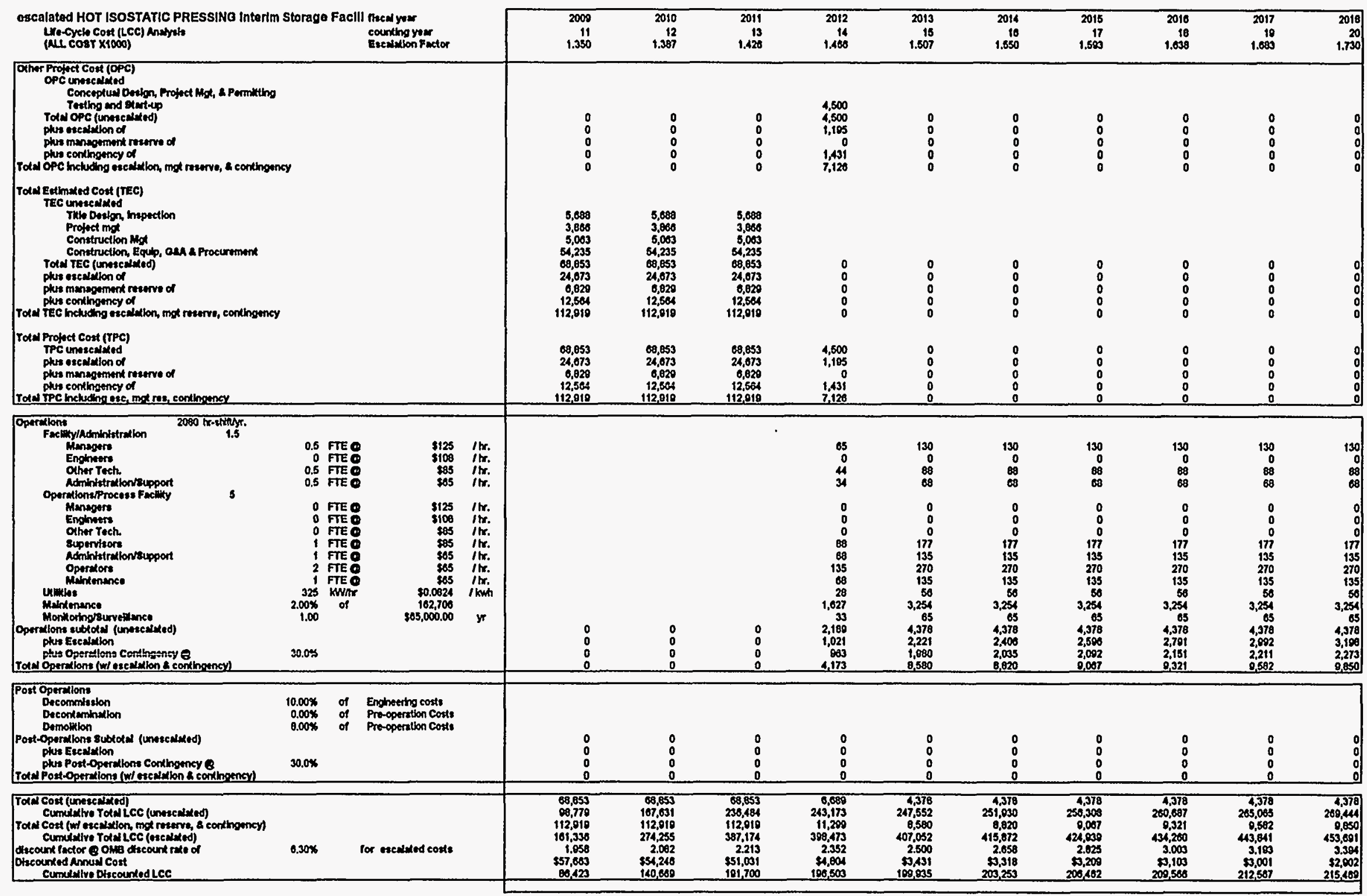




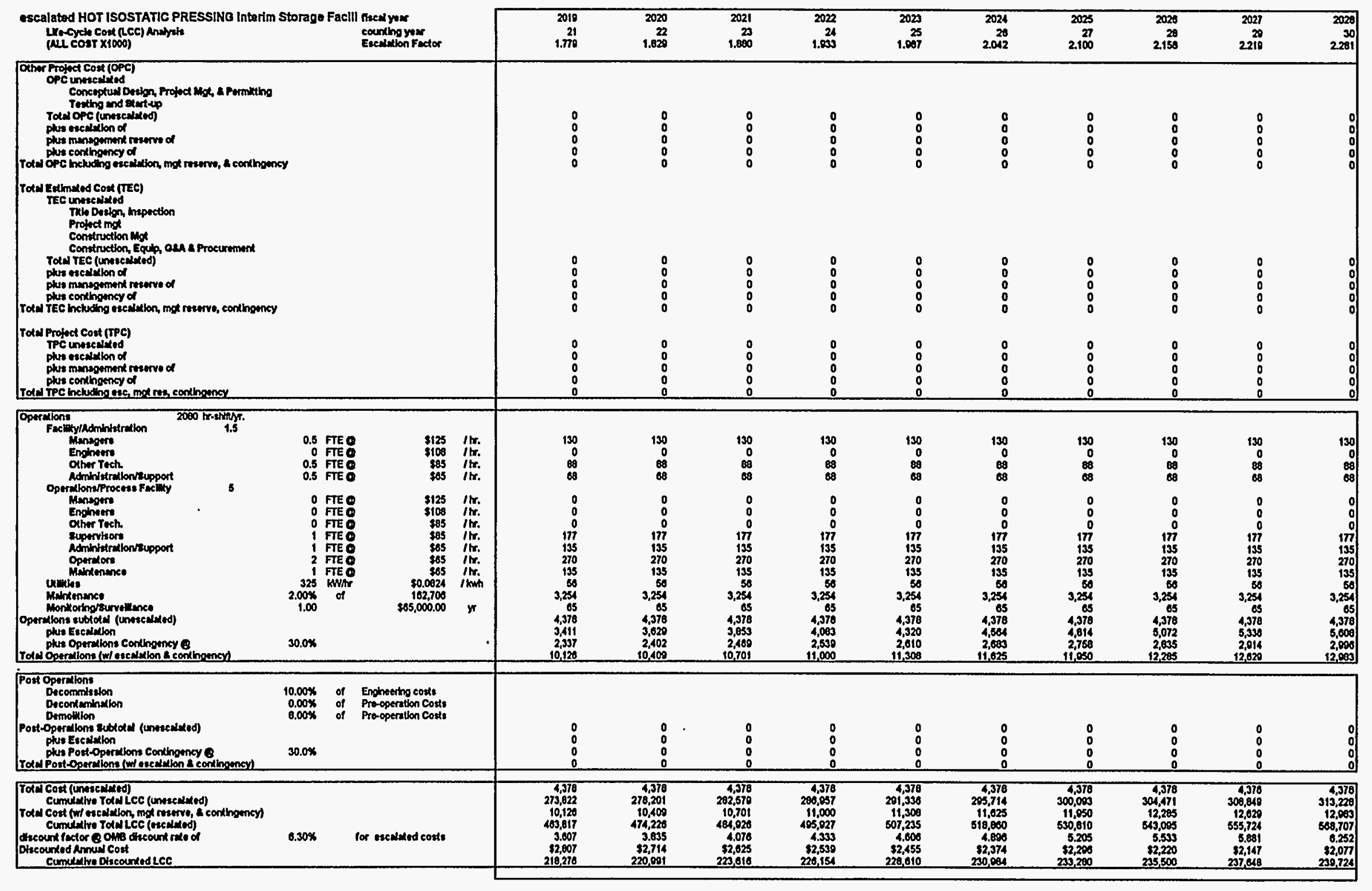




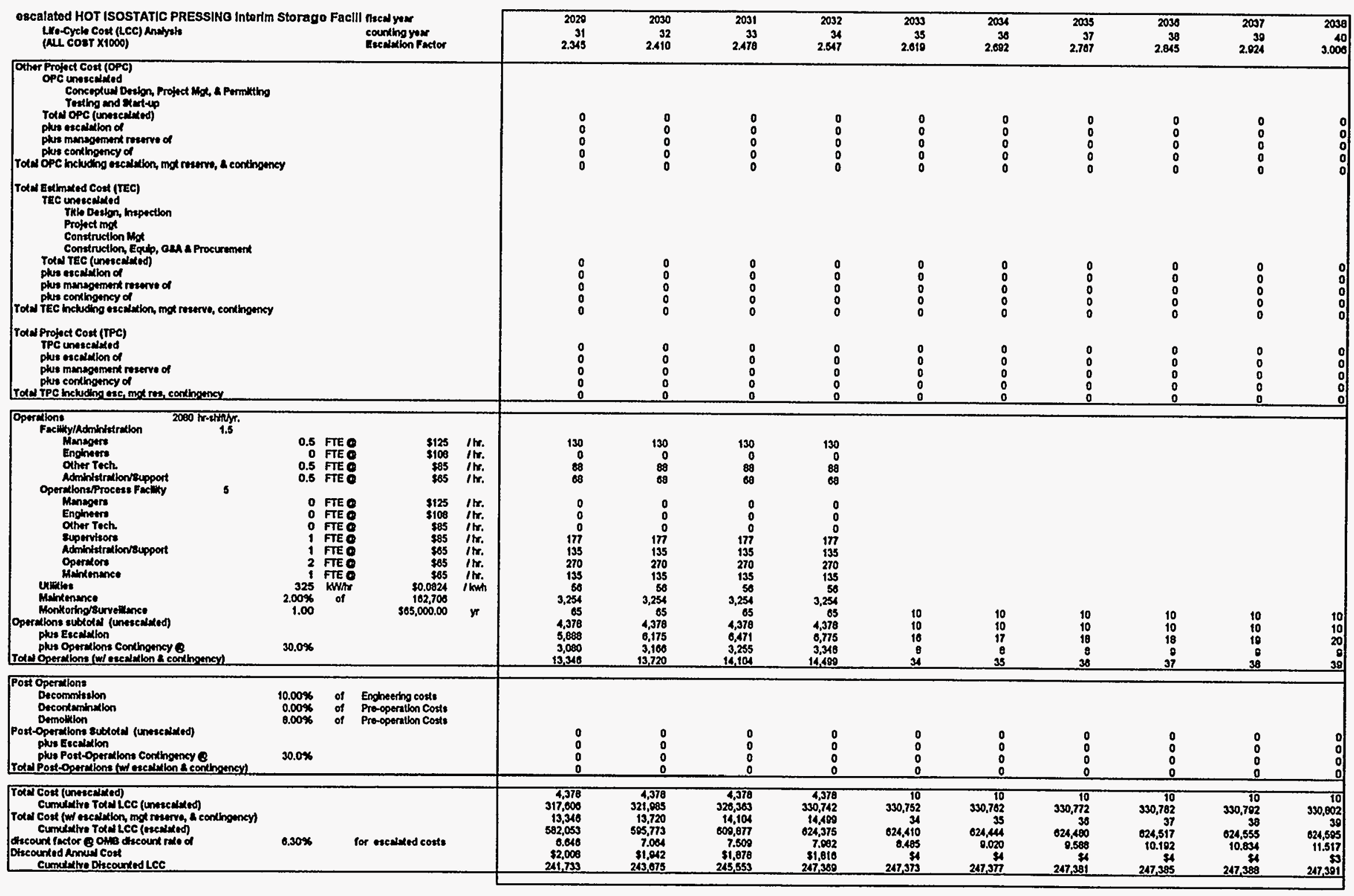




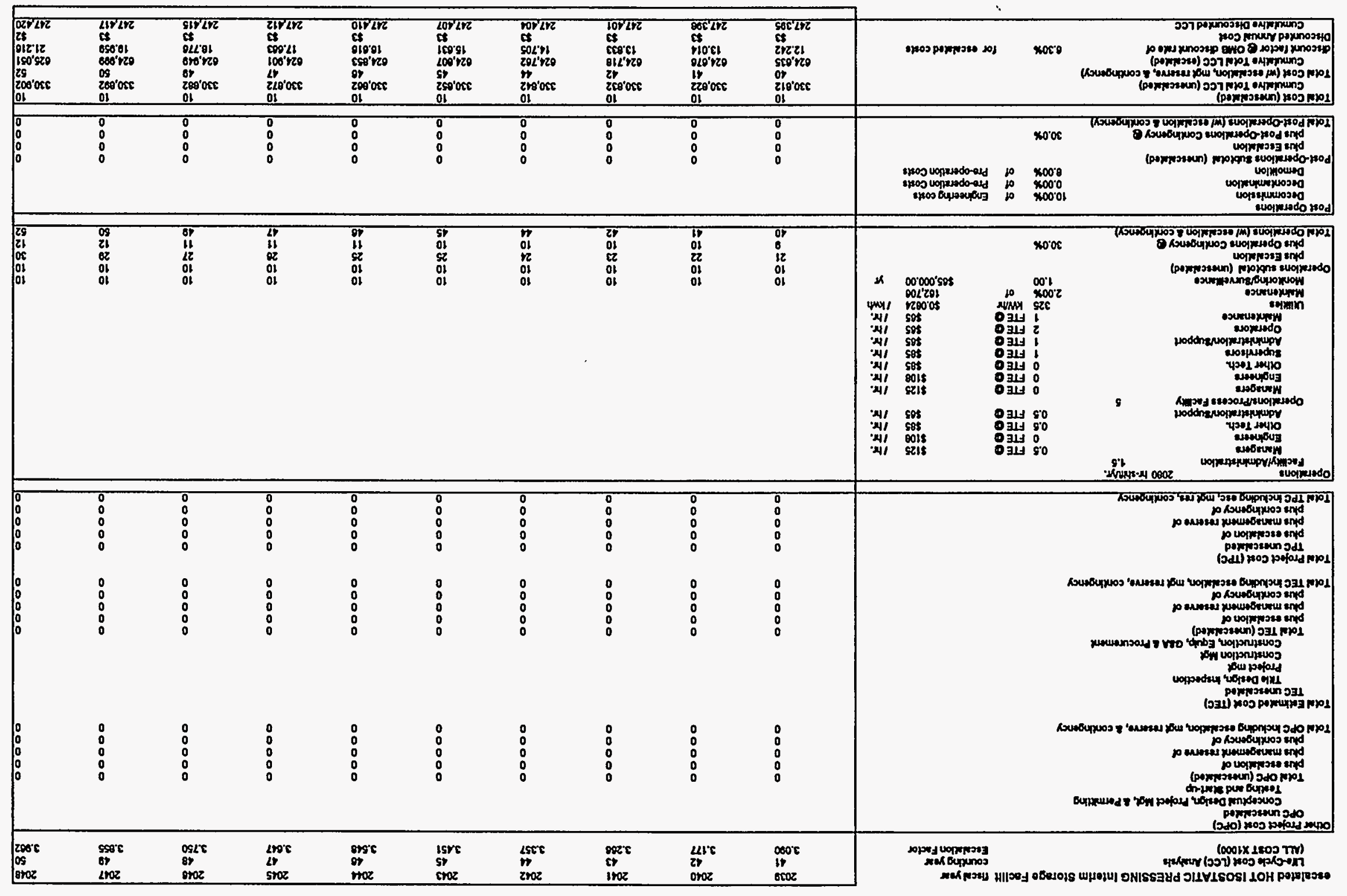




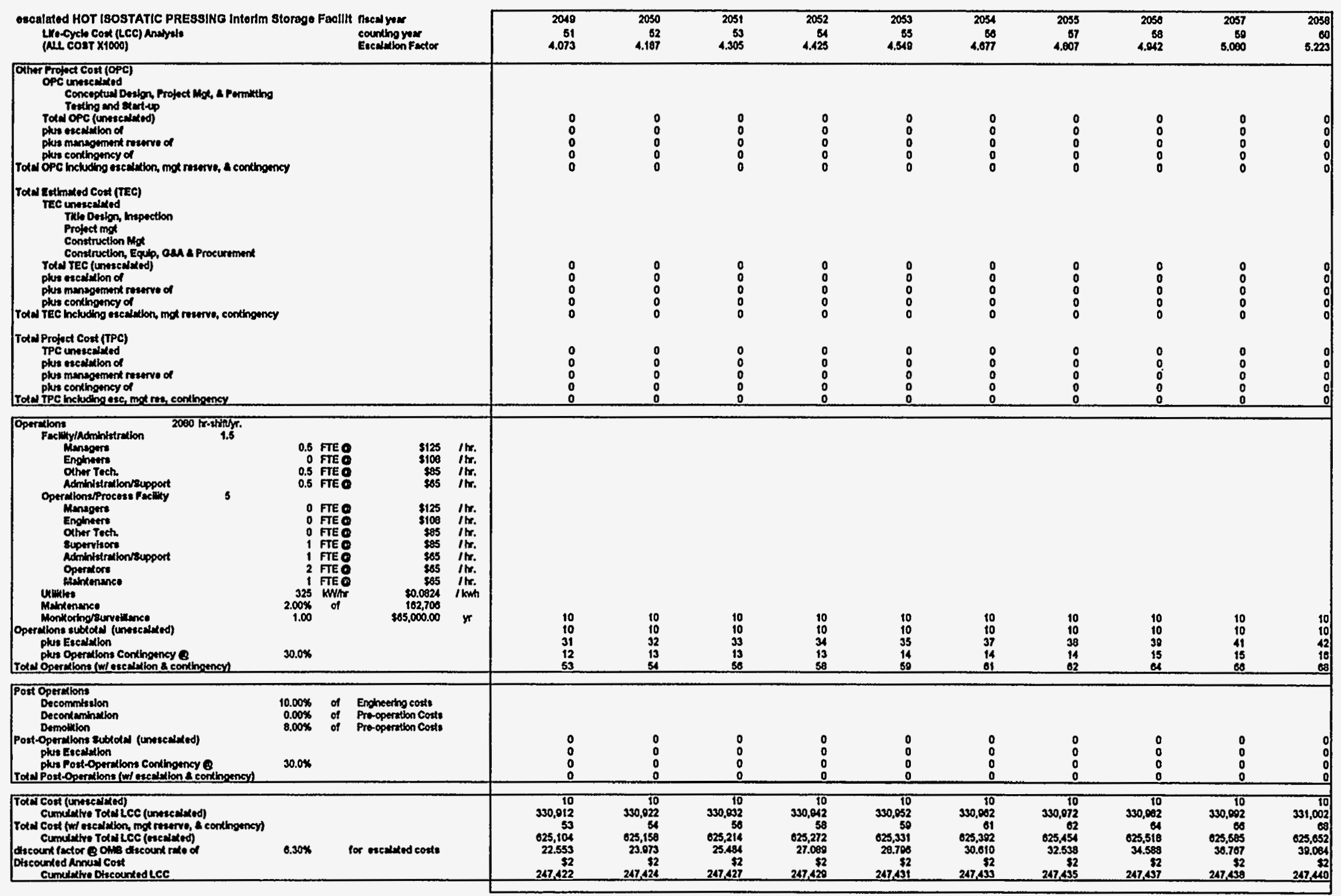




\begin{tabular}{|c|c|c|c|c|c|c|c|c|c|c|c|c|c|}
\hline 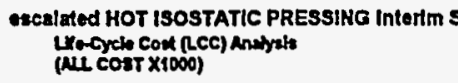 & 3torage Facillt r & 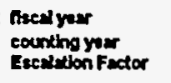 & & $\begin{array}{r}2059 \\
6.31 \\
6.309\end{array}$ & $\begin{array}{r}2030 \\
5.52\end{array}$ & $\begin{array}{r}2001 \\
6.3 \\
5.874\end{array}$ & $\begin{array}{l}2002 \\
64 \\
5.643\end{array}$ & $\begin{array}{l}2003 \\
5.05 \\
5.200\end{array}$ & $\begin{array}{l}2001 \\
680 \\
0.184\end{array}$ & $\begin{array}{l}2005 \\
67 \\
6.336\end{array}$ & $\begin{array}{l}2006 \\
6.514\end{array}$ & $\begin{array}{l}2007 \\
6.098\end{array}$ & $\begin{array}{r}2006 \\
70 \\
0.834\end{array}$ \\
\hline \multicolumn{4}{|l|}{ 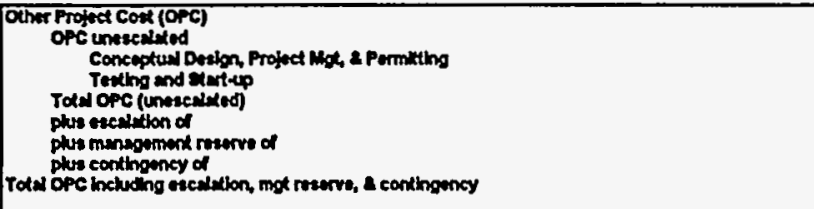 } & $\begin{array}{l}0 \\
0 \\
0 \\
0 \\
0\end{array}$ & $\begin{array}{l}0 \\
0 \\
: \\
0\end{array}$ & $\begin{array}{l}0 \\
0 \\
0 \\
0 \\
0\end{array}$ & $\begin{array}{l}0 \\
0 \\
0 \\
0 \\
0\end{array}$ & $\begin{array}{l}0 \\
: \\
0 \\
0\end{array}$ & $\begin{array}{l}0 \\
0 \\
0 \\
0 \\
0\end{array}$ & $\begin{array}{l}0 \\
0 \\
0 \\
0 \\
0\end{array}$ & $\begin{array}{l}0 \\
0 \\
0 \\
0 \\
0\end{array}$ & $\begin{array}{l}0 \\
0 \\
0 \\
0\end{array}$ & $\begin{array}{l}0 \\
0 \\
0 \\
0 \\
0\end{array}$ \\
\hline \multicolumn{4}{|l|}{ 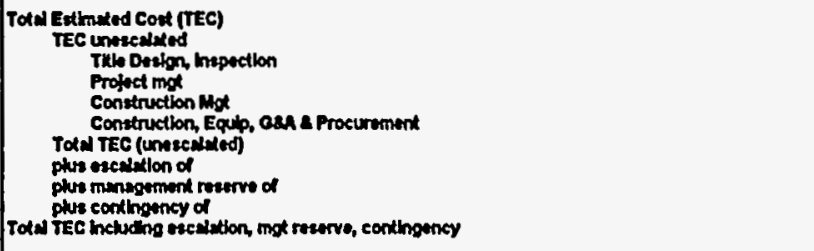 } & $\begin{array}{l}0 \\
0 \\
0 \\
0\end{array}$ & $\begin{array}{l}0 \\
0 \\
0 \\
0\end{array}$ & $\begin{array}{l}0 \\
0 \\
0 \\
0 \\
0\end{array}$ & $\begin{array}{l}0 \\
0 \\
0 \\
0 \\
0\end{array}$ & $\begin{array}{l}0 \\
0 \\
0 \\
0 \\
0\end{array}$ & $\begin{array}{l}0 \\
: \\
: \\
0\end{array}$ & $\begin{array}{l}0 \\
: \\
0\end{array}$ & $\begin{array}{l}0 \\
0 \\
0 \\
0 \\
0\end{array}$ & $\begin{array}{l}0 \\
0 \\
0 \\
0 \\
0\end{array}$ & $\begin{array}{l}0 \\
0 \\
0 \\
0 \\
0\end{array}$ \\
\hline \multicolumn{4}{|l|}{ 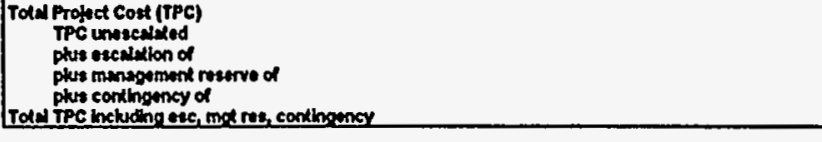 } & $\begin{array}{l}0 \\
0 \\
0 \\
0 \\
0\end{array}$ & $\begin{array}{l}0 \\
0 \\
0 \\
0 \\
0 \\
\end{array}$ & $\begin{array}{l}0 \\
0 \\
0 \\
0 \\
0\end{array}$ & $\begin{array}{l}0 \\
0 \\
0 \\
0 \\
0 \\
\end{array}$ & $\begin{array}{l}0 \\
0 \\
0 \\
0 \\
0\end{array}$ & $\begin{array}{l}0 \\
0 \\
0 \\
0 \\
0 \\
\end{array}$ & $\begin{array}{l}0 \\
0 \\
0 \\
0 \\
\end{array}$ & $\begin{array}{l}0 \\
0 \\
0 \\
0 \\
0\end{array}$ & $\begin{array}{l}0 \\
0 \\
0 \\
0 \\
0 \\
\end{array}$ & $\begin{array}{l}0 \\
0 \\
0 \\
0 \\
0 \\
\end{array}$ \\
\hline \multirow{2}{*}{ 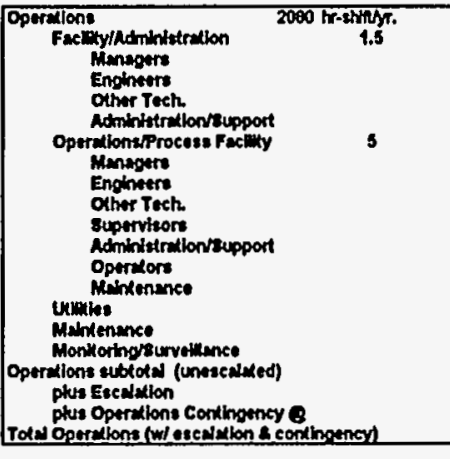 } & $\begin{array}{l}0.5 \text { FTE: } \\
0.5 \mathrm{FTE} \\
0.5 \mathrm{FTE} \\
0.5 \text { FTE }\end{array}$ & $\begin{array}{l}\$ 123 \\
\$ 108 \\
\$ 85 \\
\$ 05\end{array}$ & $\begin{array}{l}\text { Itr. } \\
\text { Itr. } \\
\text { Itr. } \\
\text { itr. }\end{array}$ & & & & & & $\begin{array}{r}130 \\
0 \\
60 \\
60\end{array}$ & $\begin{array}{r}130 \\
0 \\
83 \\
60\end{array}$ & $\begin{array}{r}130 \\
0 \\
69 \\
80\end{array}$ & $\begin{array}{r}130 \\
0 \\
80 \\
68\end{array}$ & $\begin{array}{r}130 \\
0 \\
80 \\
60\end{array}$ \\
\hline & 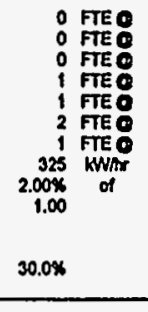 & 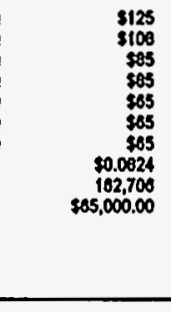 & 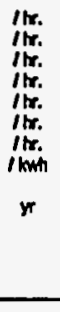 & $\begin{array}{l}10 \\
10 \\
44 \\
16 \\
70 \\
\end{array}$ & $\begin{array}{l}10 \\
10 \\
45 \\
17 \\
72 \\
\end{array}$ & $\begin{array}{l}10 \\
10 \\
17 \\
17 \\
74 \\
\end{array}$ & $\begin{array}{l}10 \\
10 \\
10 \\
17 \\
70 \\
\end{array}$ & $\begin{array}{l}10 \\
10 \\
50 \\
18 \\
76 \\
\end{array}$ & $\begin{array}{r}0 \\
0 \\
0 \\
177 \\
135 \\
270 \\
135 \\
56 \\
0 \\
85 \\
1,124 \\
5,006 \\
2,0070 \\
0.000 \\
\end{array}$ & $\begin{array}{r}0 \\
0 \\
0 \\
177 \\
135 \\
270 \\
135 \\
80 \\
0 \\
63 \\
1,121 \\
6.000 \\
2,137 \\
9261\end{array}$ & $\begin{array}{r}0 \\
0 \\
0 \\
177 \\
135 \\
270 \\
135 \\
50 \\
0 \\
05 \\
1.124 \\
0.199 \\
2,197 \\
0.521 \\
\end{array}$ & $\begin{array}{r}0 \\
0 \\
0 \\
177 \\
135 \\
270 \\
135 \\
50 \\
0 \\
05 \\
85 \\
1.124 \\
0.404 \\
2,2,250 \\
0,0707 \\
\end{array}$ & 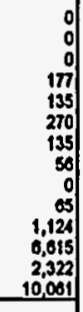 \\
\hline 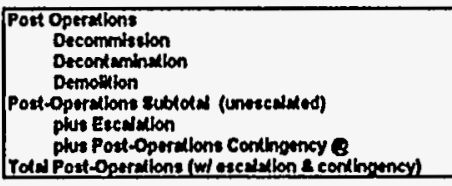 & $\begin{array}{l}10.00 \% \text { of } \\
0.00 \% \text { of } \\
8.00 \% \text { of } \\
30.0 x\end{array}$ & 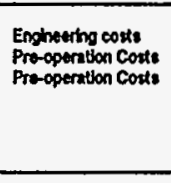 & & $\begin{array}{l}0 \\
0 \\
0 \\
0\end{array}$ & $\begin{array}{l}0 \\
0 \\
0 \\
0 \\
\end{array}$ & $\begin{array}{l}0 \\
0 \\
0 \\
0\end{array}$ & $\begin{array}{l}0 \\
0 \\
0 \\
0\end{array}$ & $\begin{array}{l}0 \\
0 \\
0 \\
0 \\
\end{array}$ & $\begin{array}{l}0 \\
0 \\
0 \\
0 \\
\end{array}$ & $\begin{array}{l}0 \\
0 \\
0 \\
0 \\
\end{array}$ & $\begin{array}{l}0 \\
0 \\
0 \\
0 \\
\end{array}$ & $\begin{array}{l}0 \\
0 \\
0 \\
0 \\
\end{array}$ & $\begin{array}{l}0 \\
0 \\
0 \\
0\end{array}$ \\
\hline 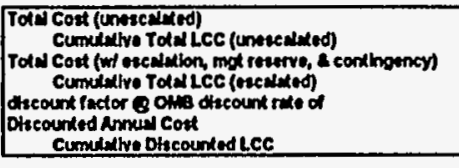 & $0.30 x$ & for mecelextd coste & & $\begin{array}{r}10 \\
331,012 \\
70 \\
025,722 \\
41.568 \\
\$ 2 \\
247,42 \\
\end{array}$ & $\begin{array}{r}10 \\
331,022 \\
72 \\
625,794 \\
44.143 \\
\$ 22 \\
247,443 \\
\end{array}$ & $\begin{array}{r}10 \\
331,032 \\
74 \\
025,069 \\
48,96 \\
\$ 22 \\
247,45 \\
\end{array}$ & $\begin{array}{r}10 \\
231,042 \\
70 \\
025,04 \\
48,003 \\
\$ 28 \\
247,47\end{array}$ & $\begin{array}{r}10 \\
331,052 \\
78 \\
020,021 \\
53,0.07 \\
411 \\
247,48 \\
\end{array}$ & $\begin{array}{r}1,121 \\
332,170 \\
0.000 \\
035,01 \\
6,0,390 \\
3100 \\
247,006 \\
\end{array}$ & $\begin{array}{r}31,1,124 \\
30,300 \\
0.201 \\
04,202 \\
50,42 \\
5135 \\
247,782 \\
\end{array}$ & 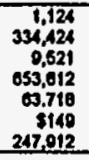 & 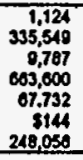 & 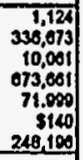 \\
\hline
\end{tabular}




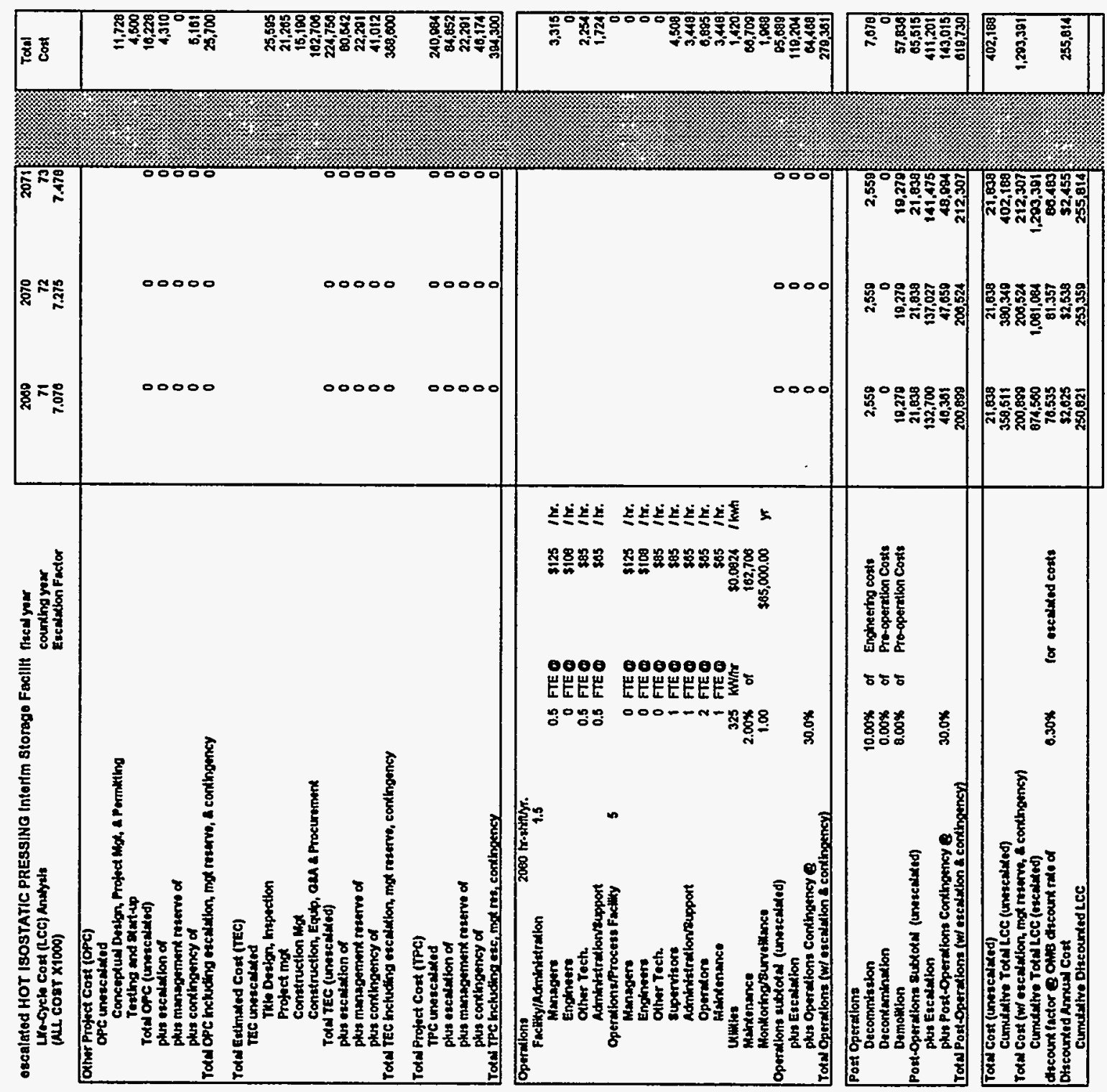




\section{TOTAL ANNUAL COST (escalated) HIP INTERIM STORAGE FACILTY}

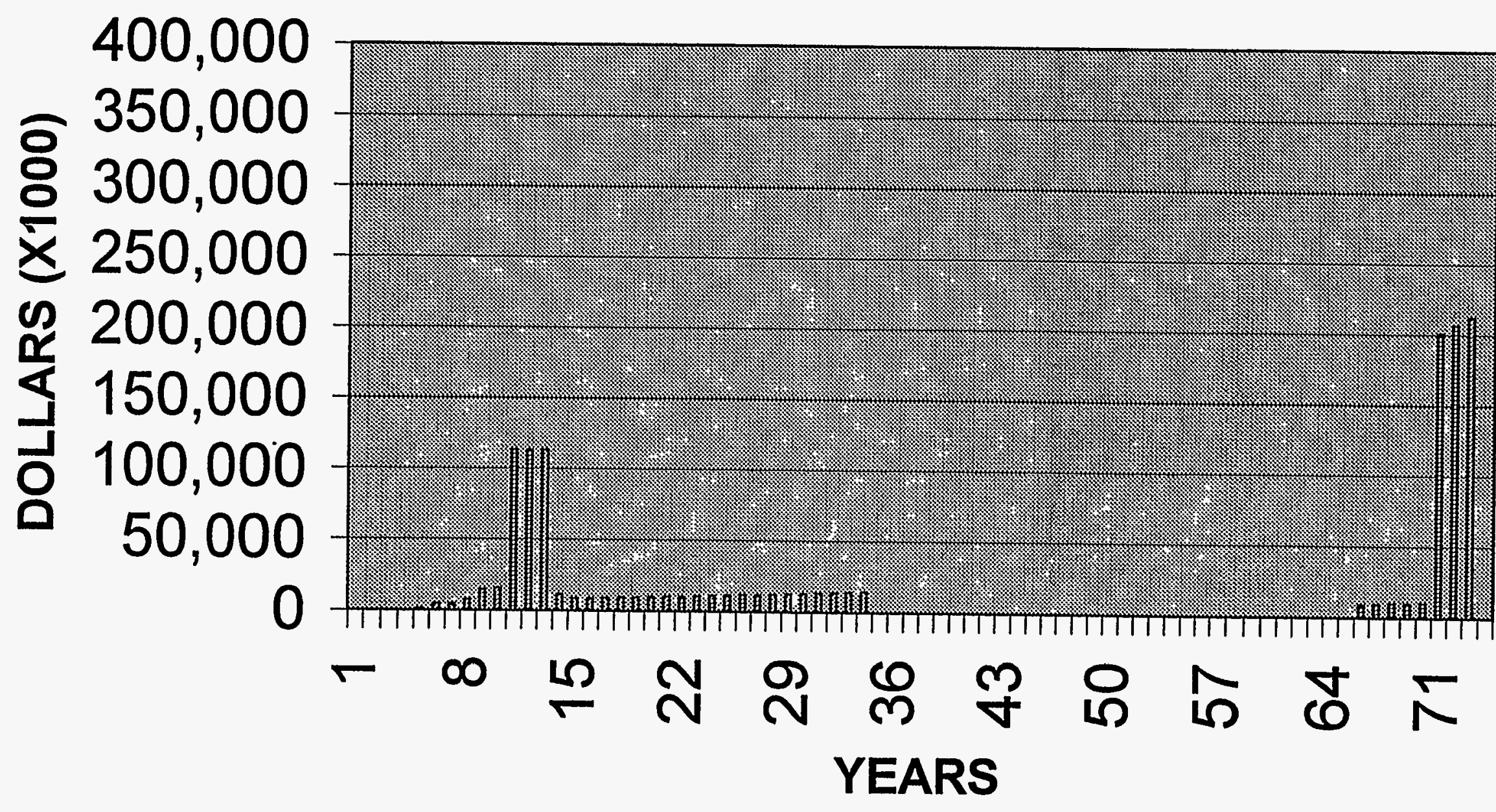




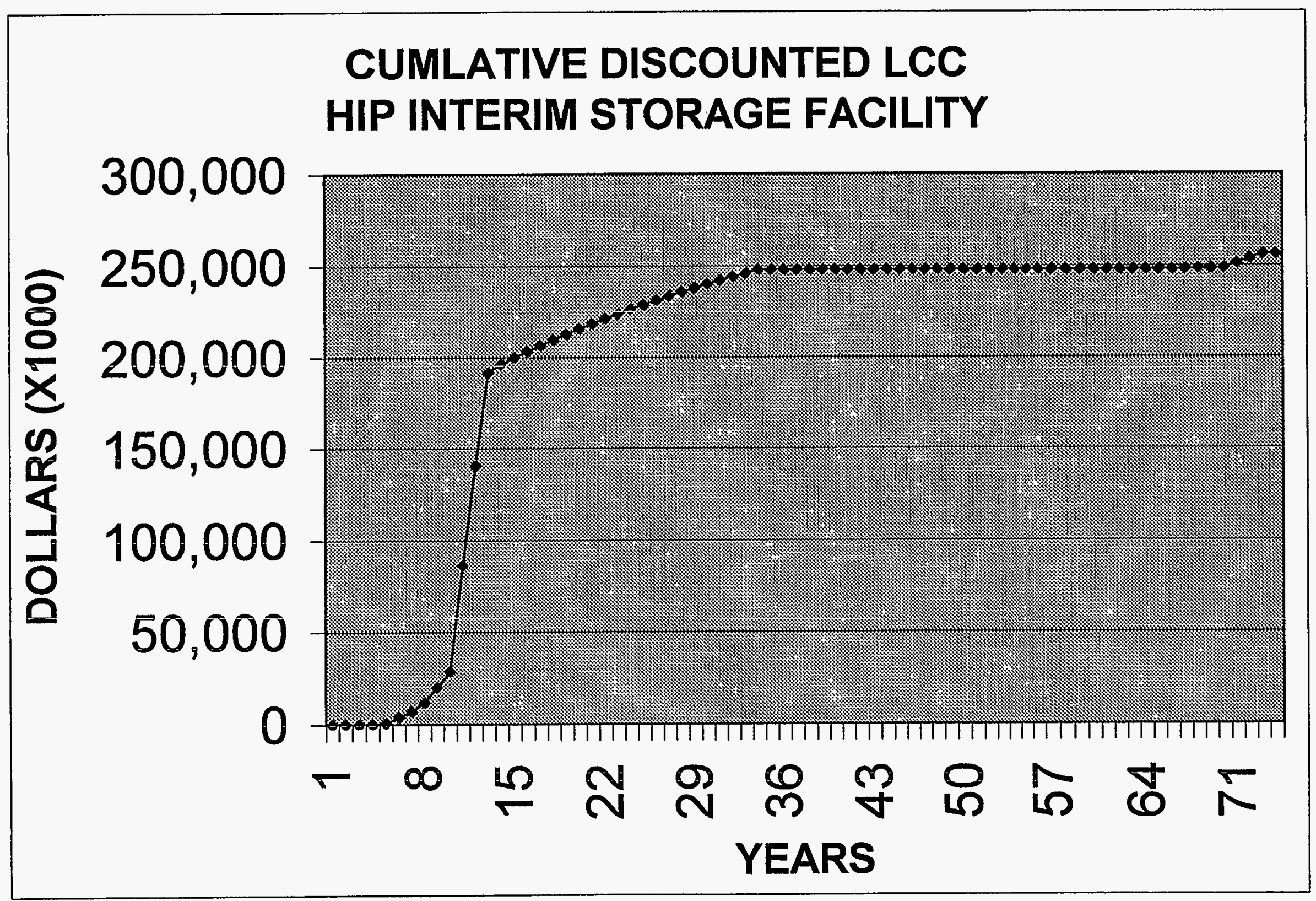


i

\section{Appendix 2}

\section{Drawings}




\section{CONTENTS}

The following drawings are included in this appendix:

TRU/Class A Separations:

Vitrified Waste Option:

Direct Cementitious Waste Option:

Cementitious Waste Option:

Hot Isostatic Press Waste Option:

\begin{tabular}{|c|c|}
\hline Site Map & TRUA-C-1 \\
\hline Floor Plan Elevation - 0'- $0^{\prime \prime}$ & TRUA-A-7 \\
\hline an Elevation $-21^{\prime}-0$ & TRUA-A-8 \\
\hline Floor $\mathrm{F}$ & TRUA-A- \\
\hline Section & TRUA- \\
\hline Section and Detail & TRUA \\
\hline Sect & TRUA \\
\hline Sect & TRL \\
\hline Site & vW \\
\hline 'lan Elevation - 0'-0" & VW \\
\hline Floor Plan Elevation - 21'-0" & VW \\
\hline Floor Plan Elev & $\mathrm{VV}$ \\
\hline Secti & VW \\
\hline and Detail & Vh \\
\hline & \\
\hline & \\
\hline
\end{tabular}

Site Plan

DCWO-C-1

Floor Plan Elevation - 0'-0" DCWO-A-14

Floor Plan Elevation - 21'-0" DCWO-A-15

Floor Plan Elevation - 37'-0"DCWO-A-16

Section

DCWO-A-17

Section and Detail

DCWO-A-18

Section

DCWO-A-19

Section

DCWO-A-20

$\begin{array}{lr}\text { Site Plan } & \text { CWO-C-1 } \\ \text { Floor Plan Elevation - 0'-0" } & \text { CWO-A-6 } \\ \text { Floor Plan Elevation - 21'-0" CWO-A-7 } \\ \text { Floor Plan Elevation - 37'-0" CWO-A-8 } \\ \text { Section } & \text { CWO-A-9 } \\ \text { Section and Detail } & \text { CWO-A-10 } \\ \text { Section } & \text { CWO-A-11 } \\ \text { Section } & \text { CWO-A-12 }\end{array}$

Site Plan

HWO-C-1

Floor Plan Elevation - 0'-0" HWO-A-7

Floor Plan Elevation - 21'-0" HWO-A-8

Floor Plan Elevation - 37' - 0"HWO-A-9

Section

HWO-A-10

Section and Detail

HWO-A-11

Section

HWO-A-12

Section 


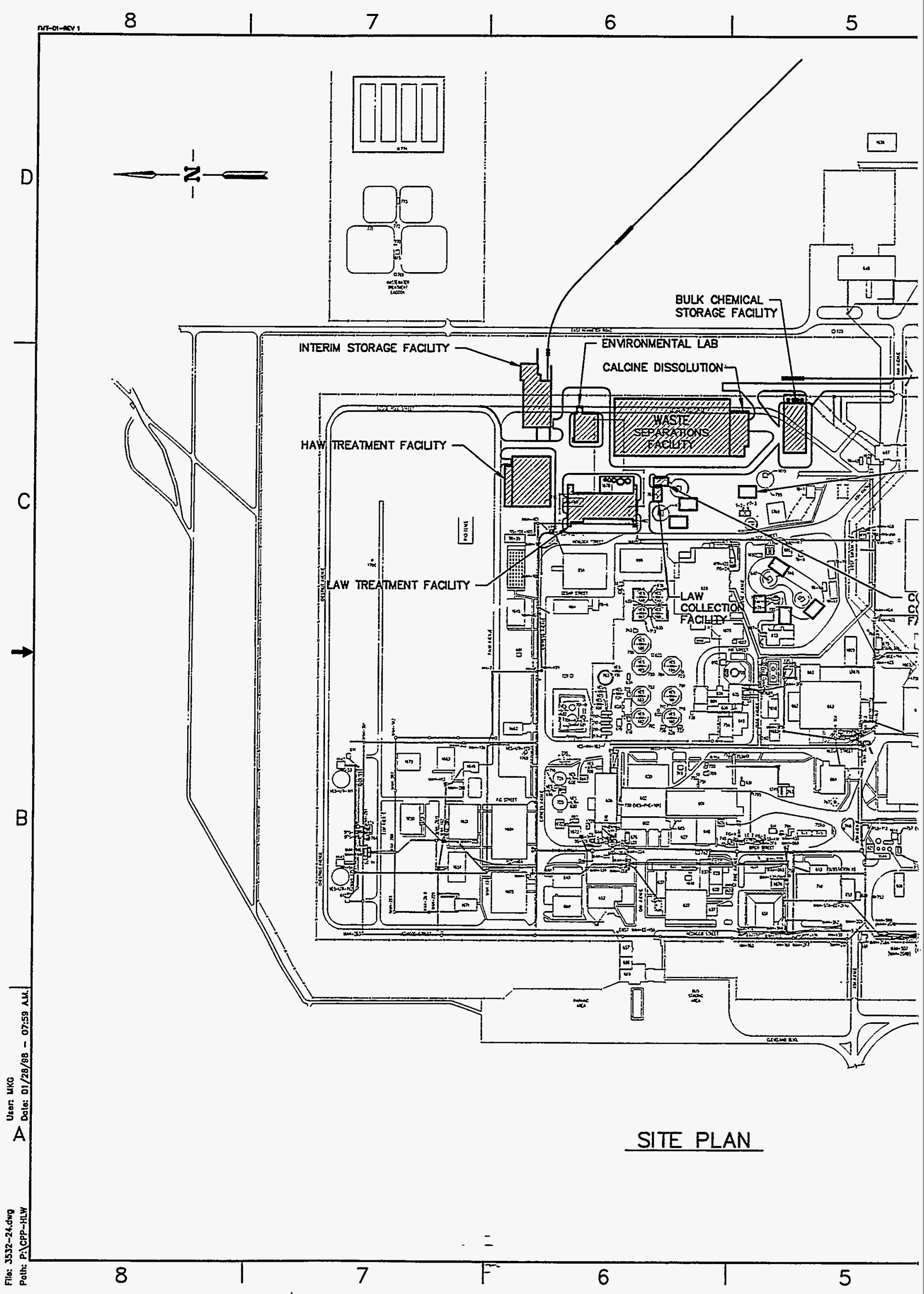




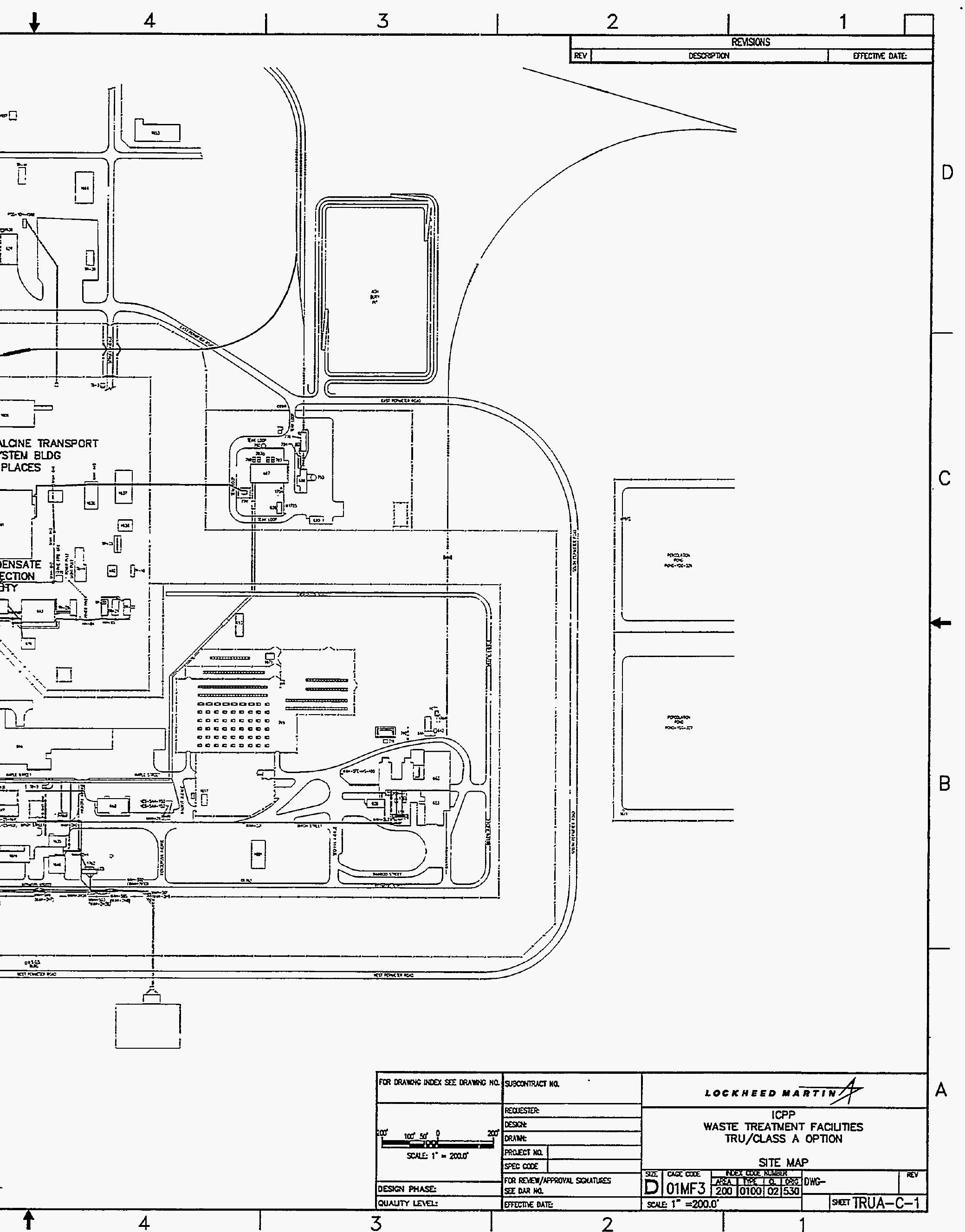




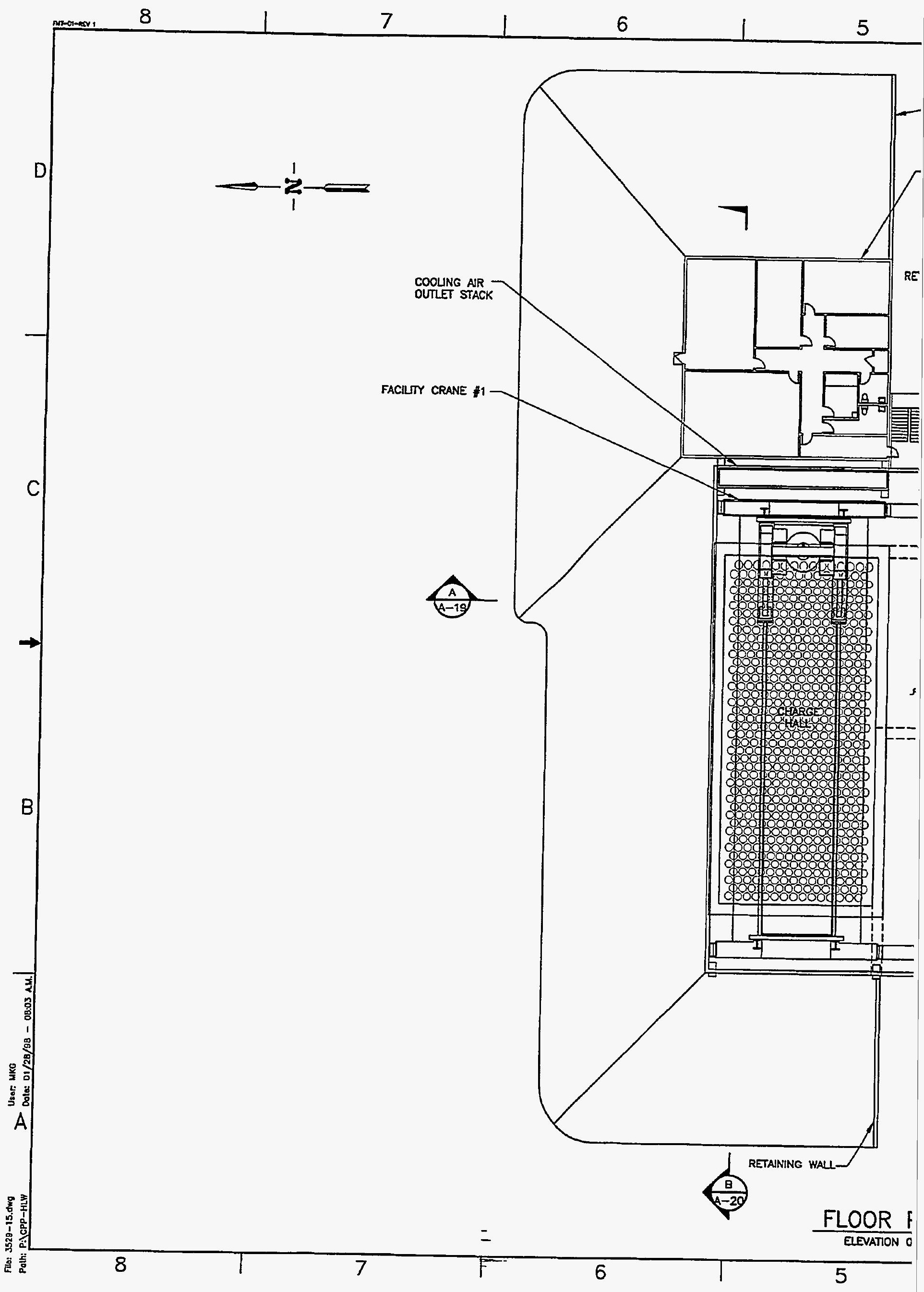




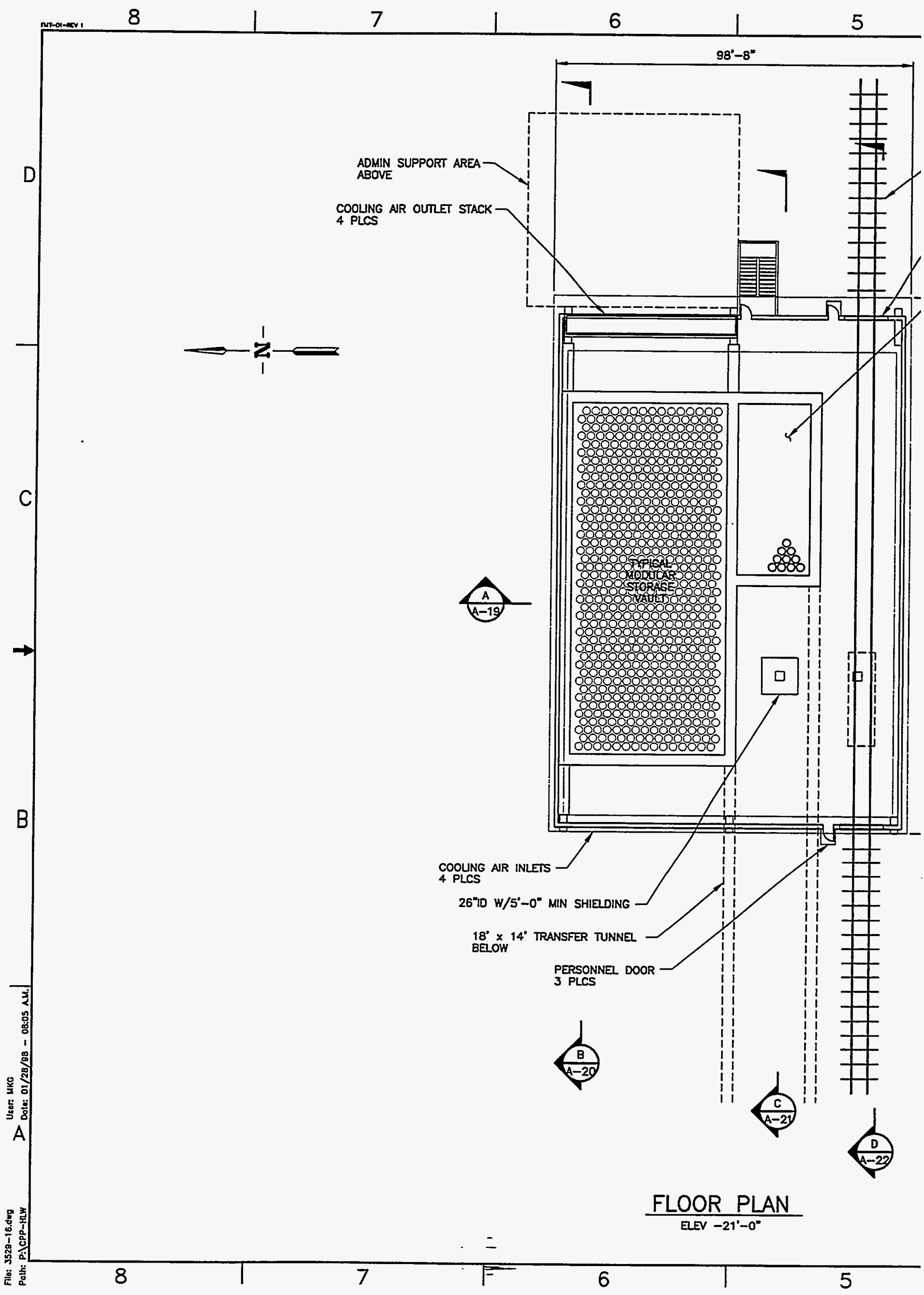




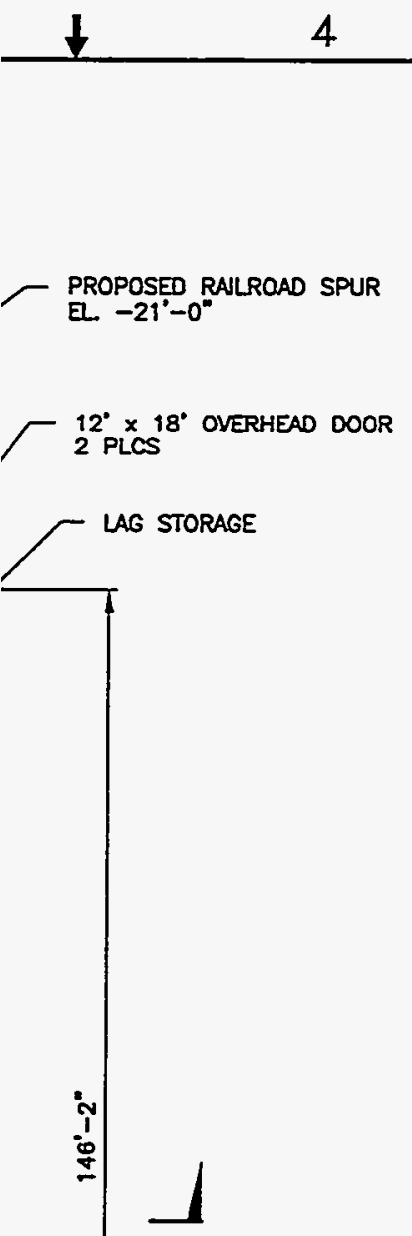

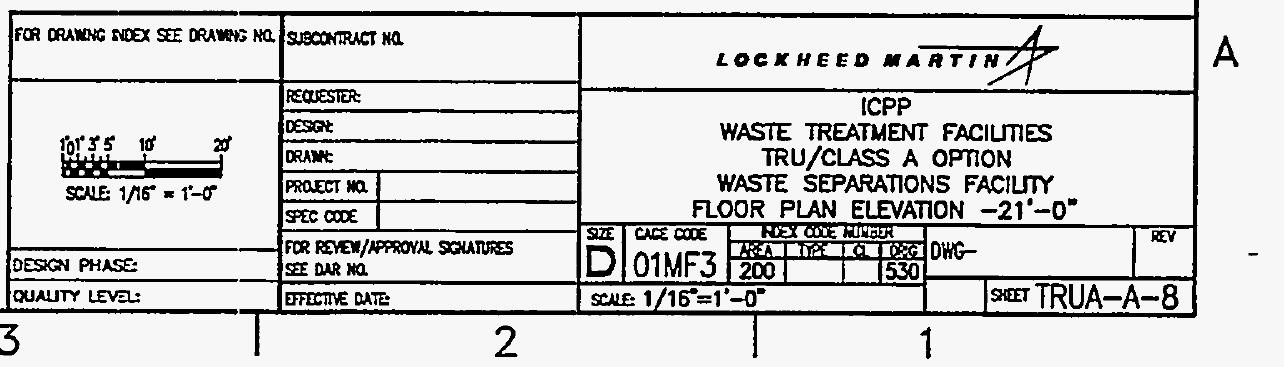




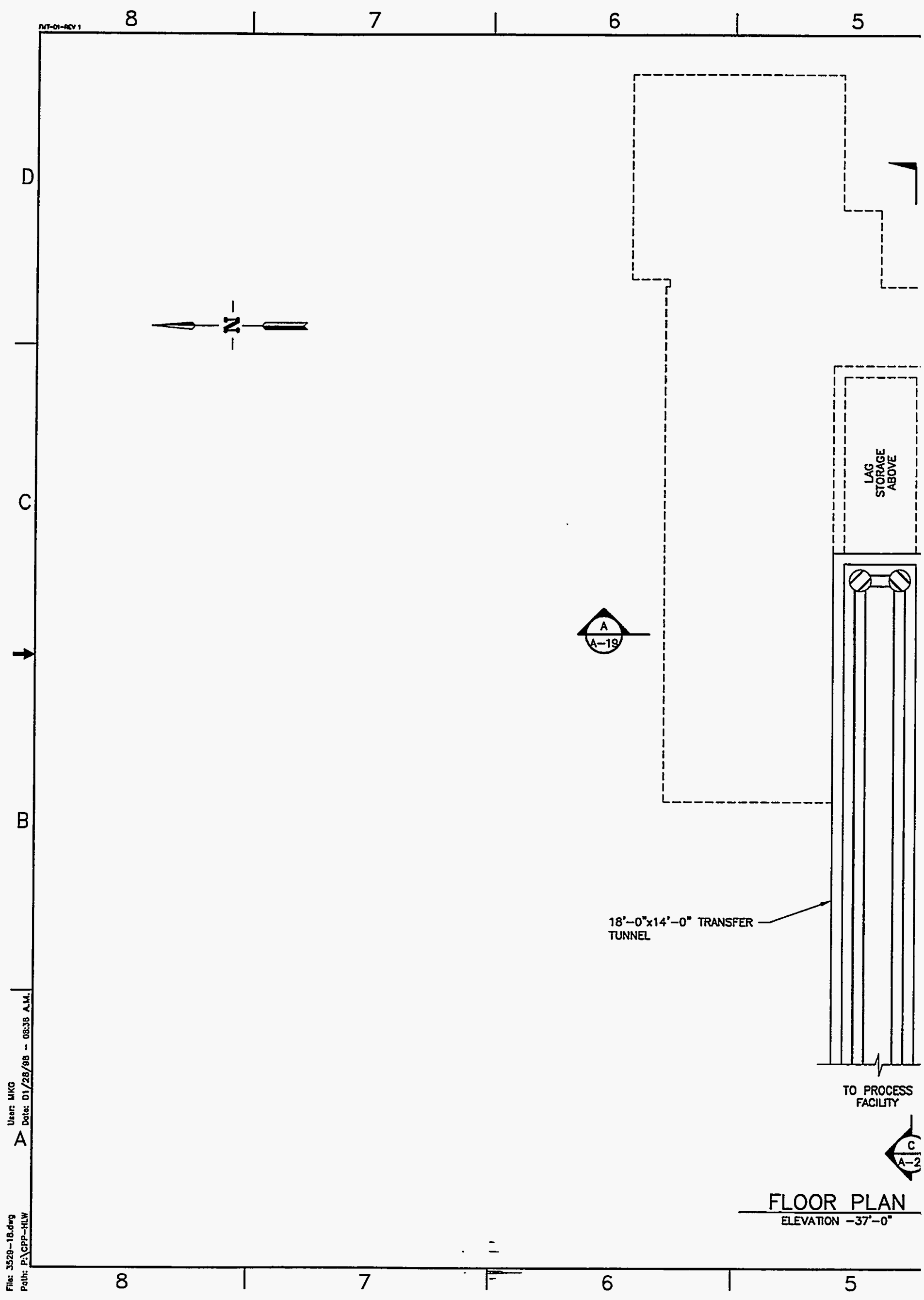




\begin{tabular}{|c|c|c|}
\hline 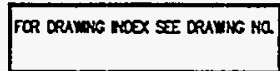 & Srecantruct ка & LOCKHEED MATTIN \\
\hline 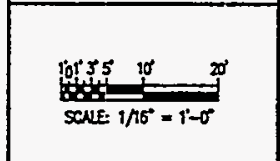 & 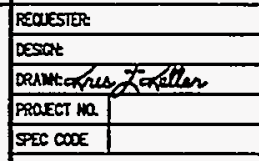 & 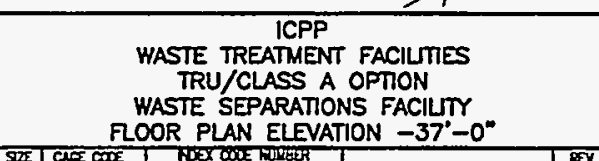 \\
\hline DESSCN PHASE: & 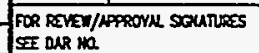 & Did \\
\hline OUNUT LEVE: & 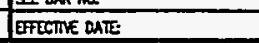 & ser TRUA-A-9 \\
\hline 3 & 2 & 1 \\
\hline
\end{tabular}




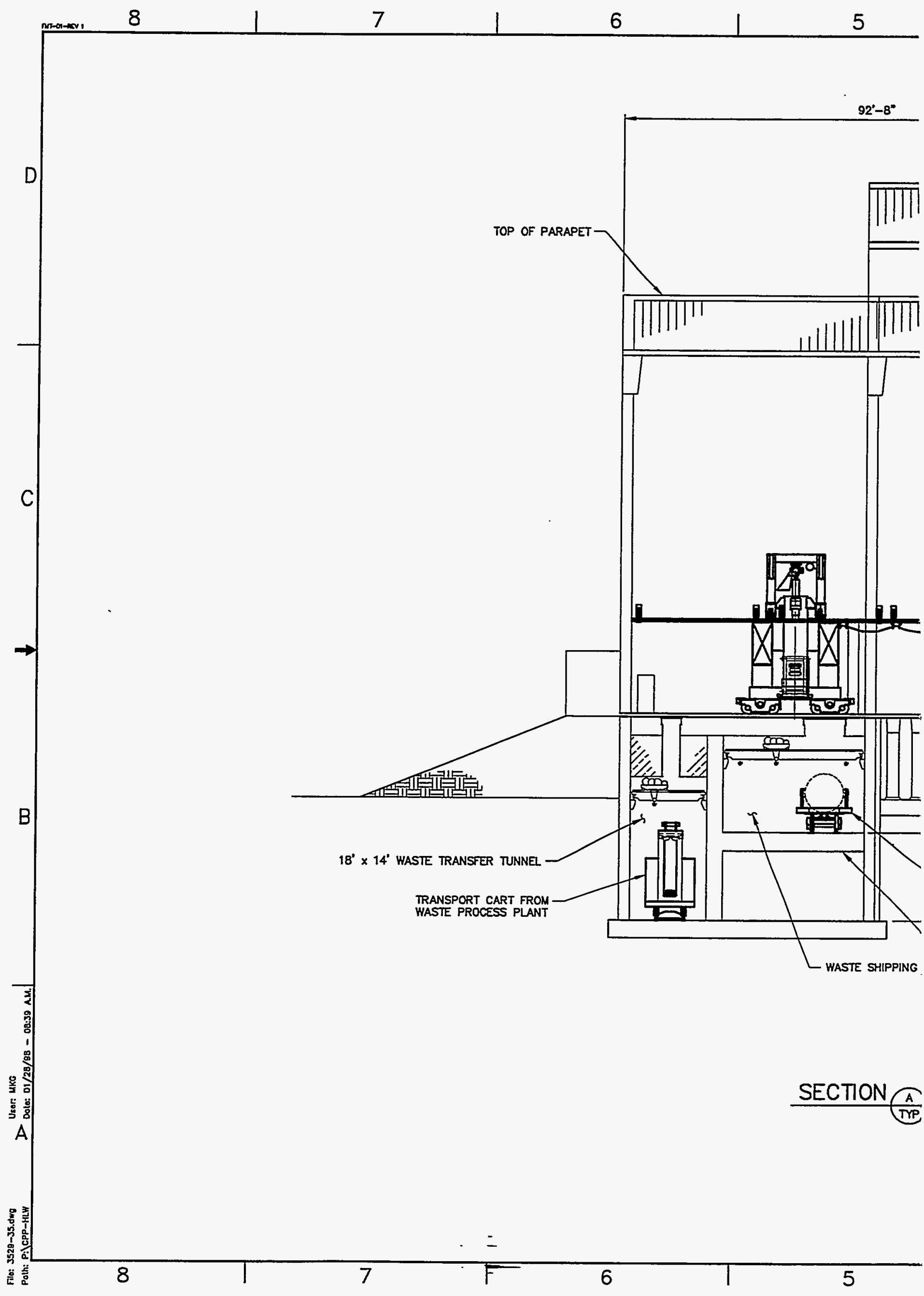




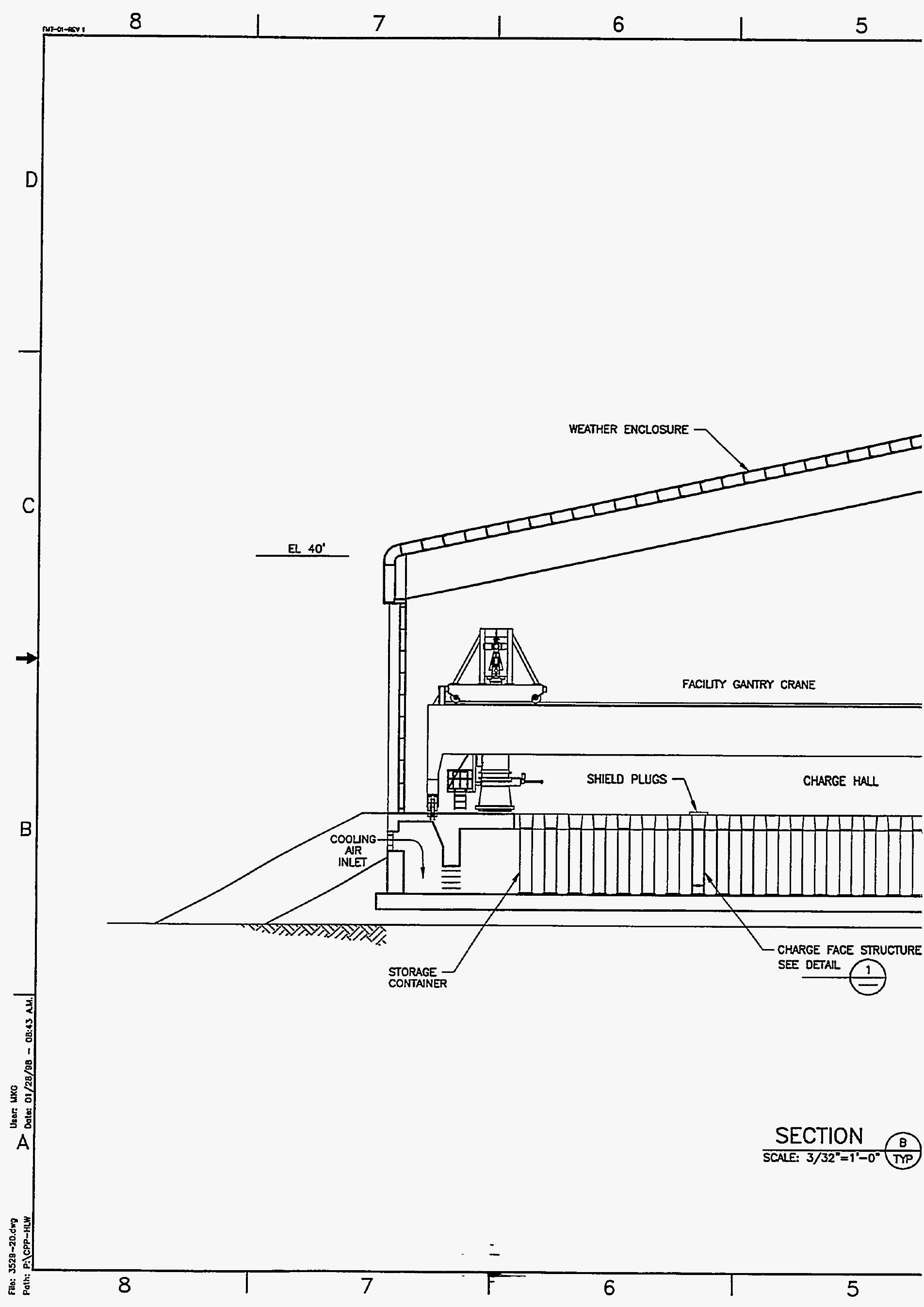




\begin{tabular}{llll|lll}
4 & 3 & 2 & 1 & 1 \\
\hline
\end{tabular}

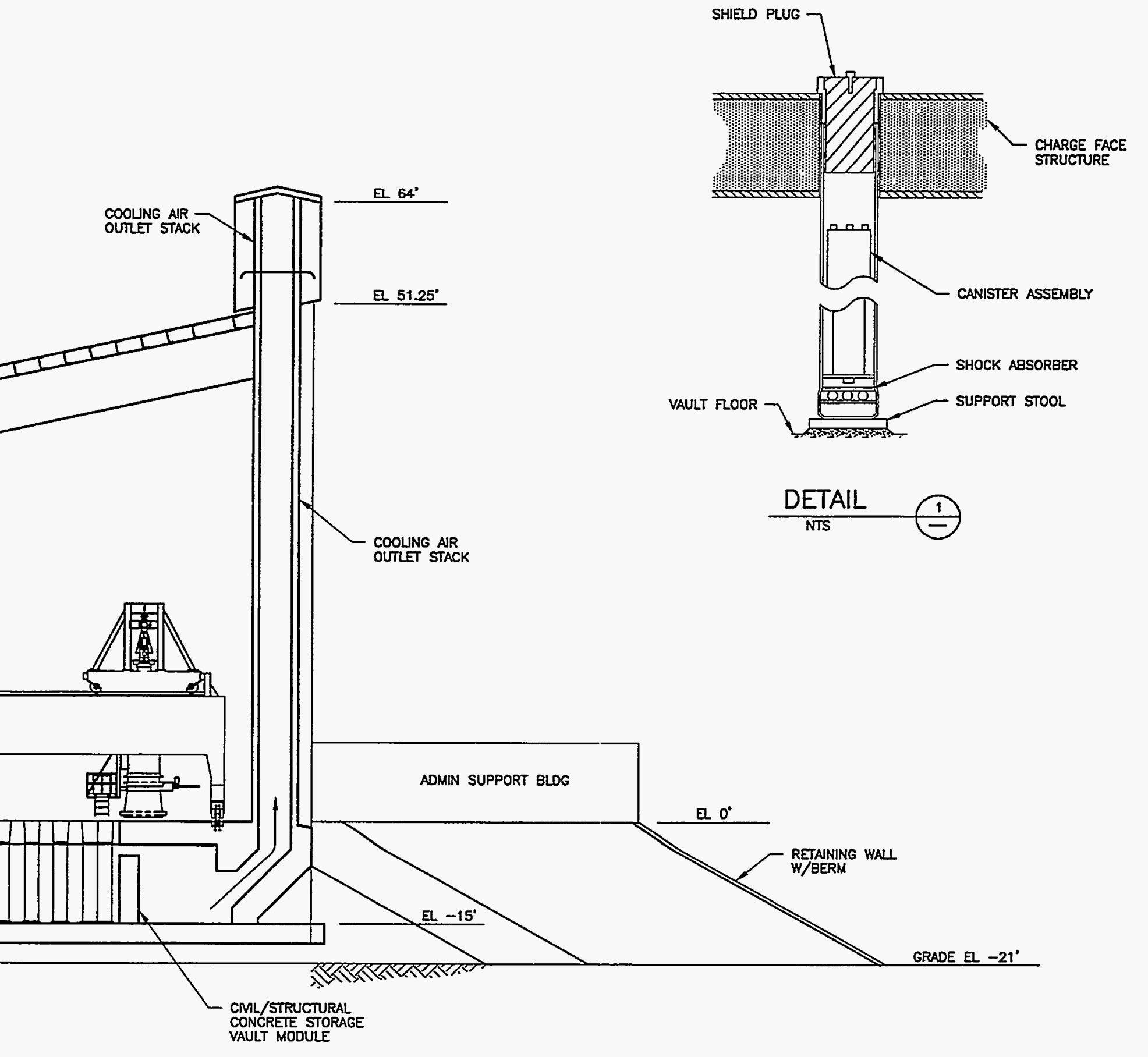

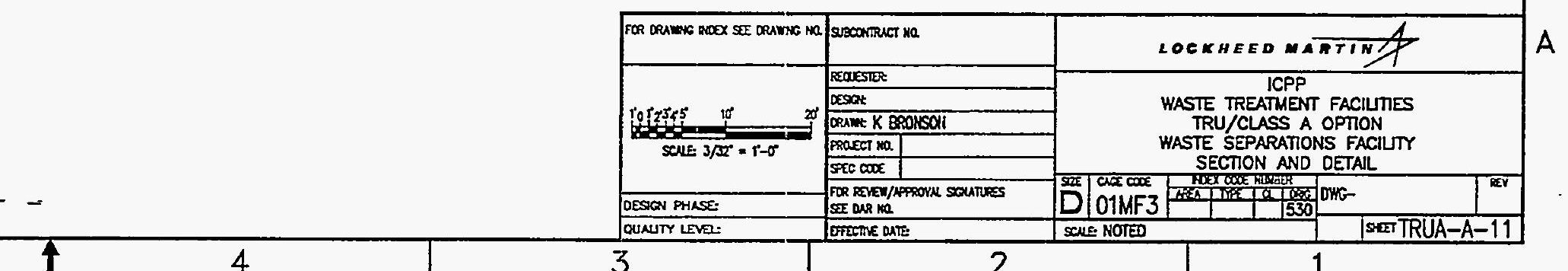




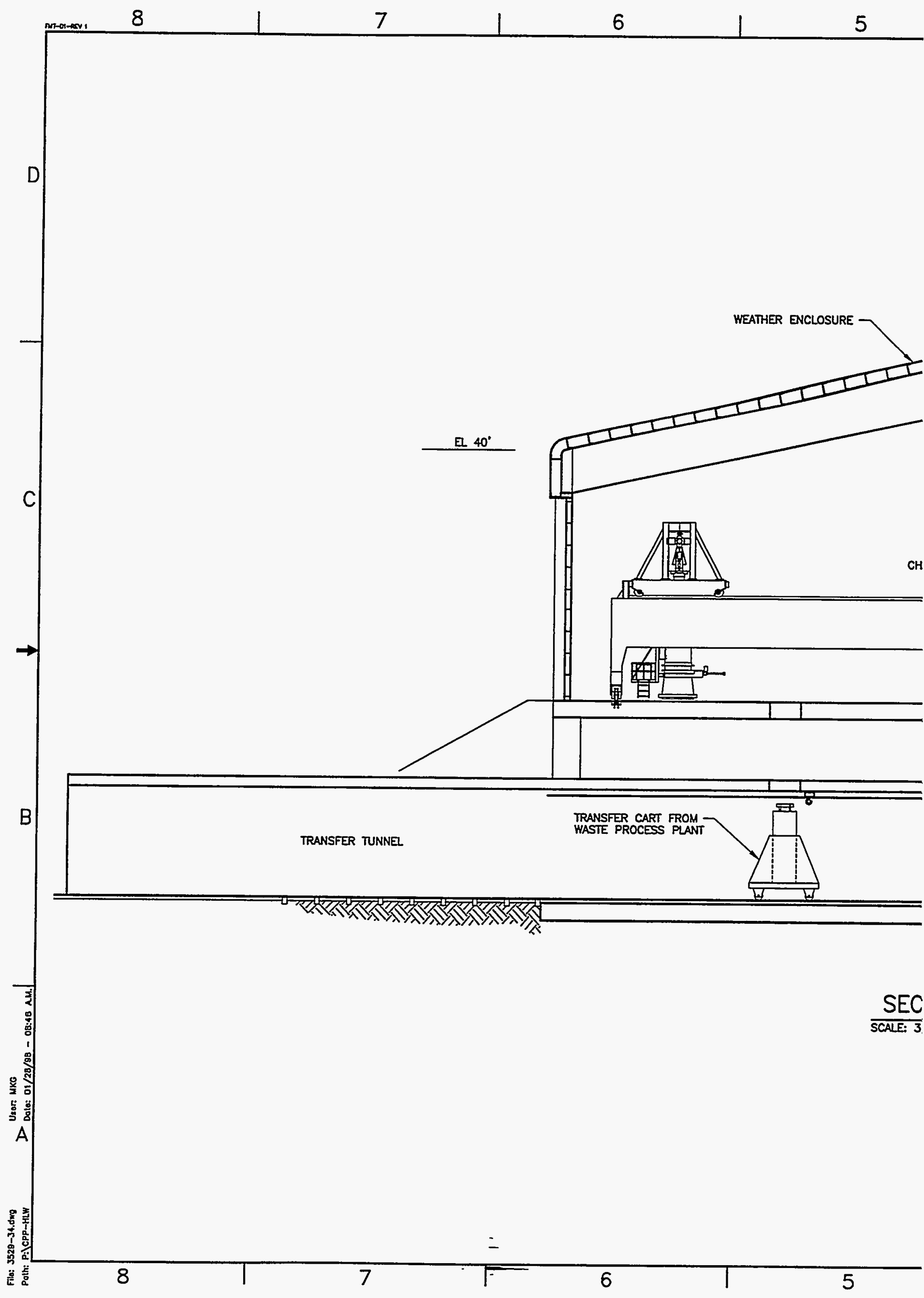




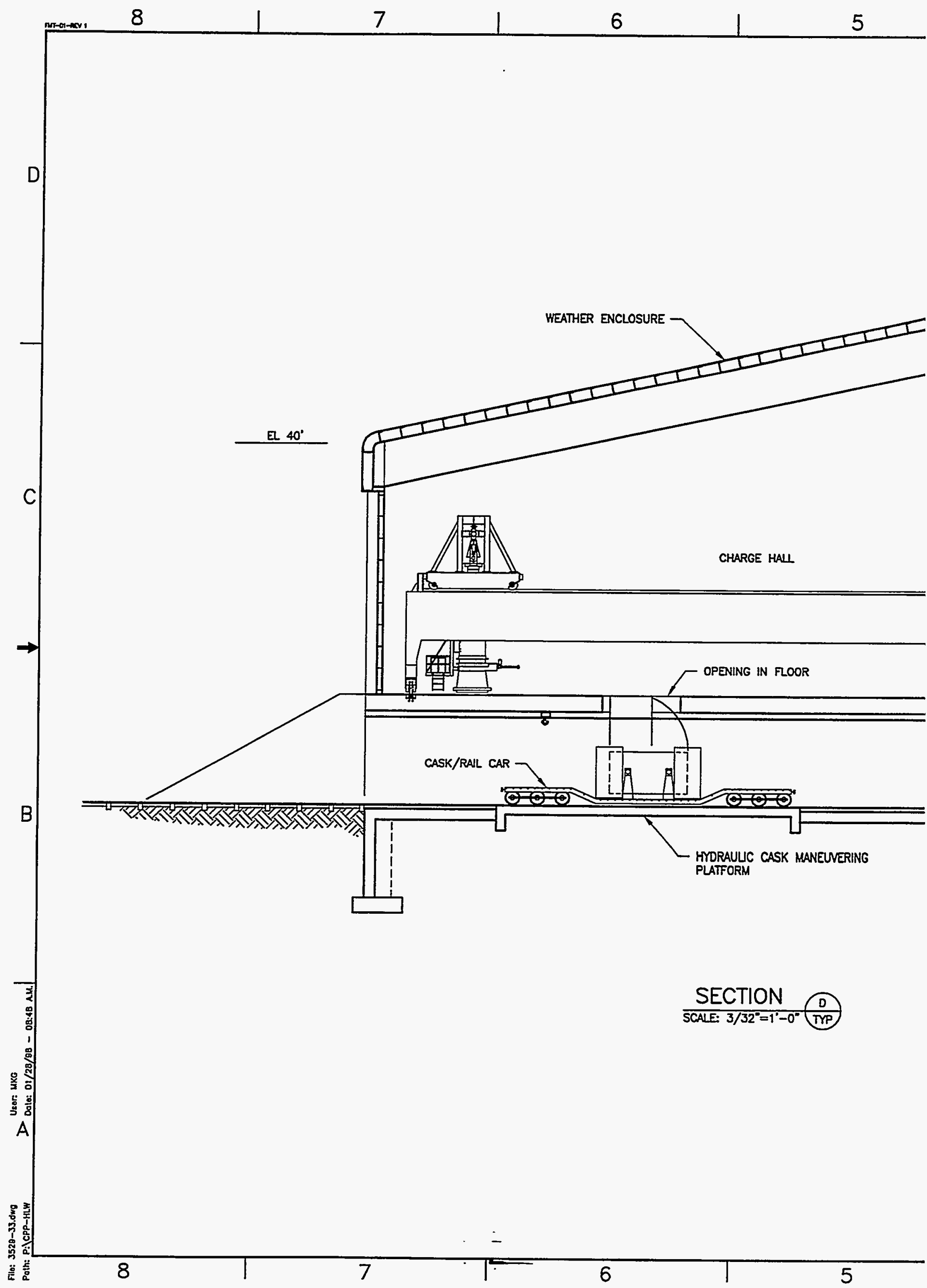




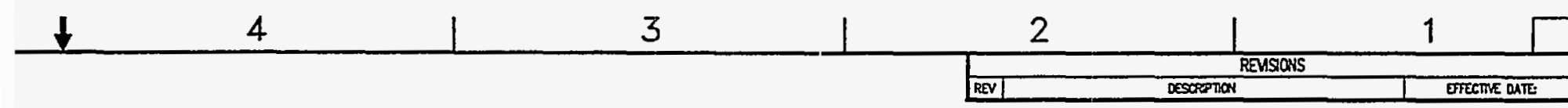

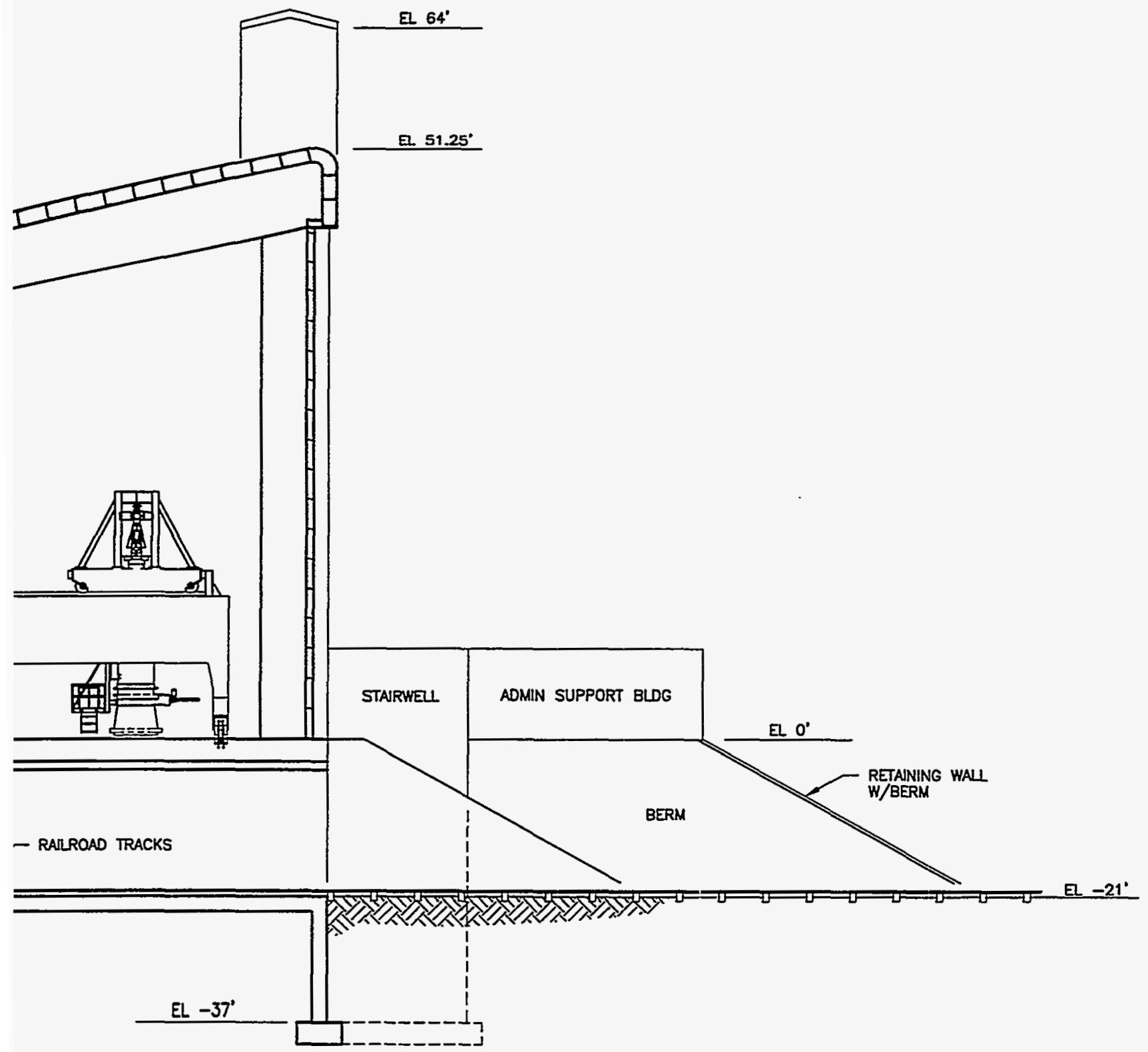

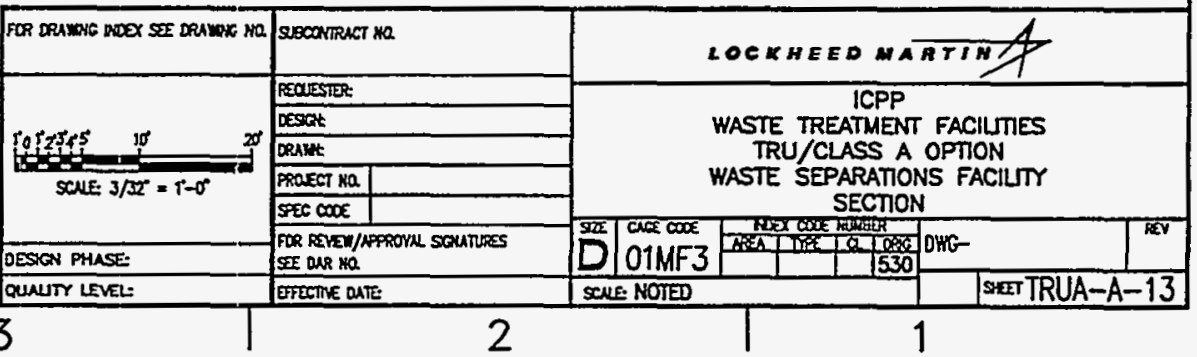




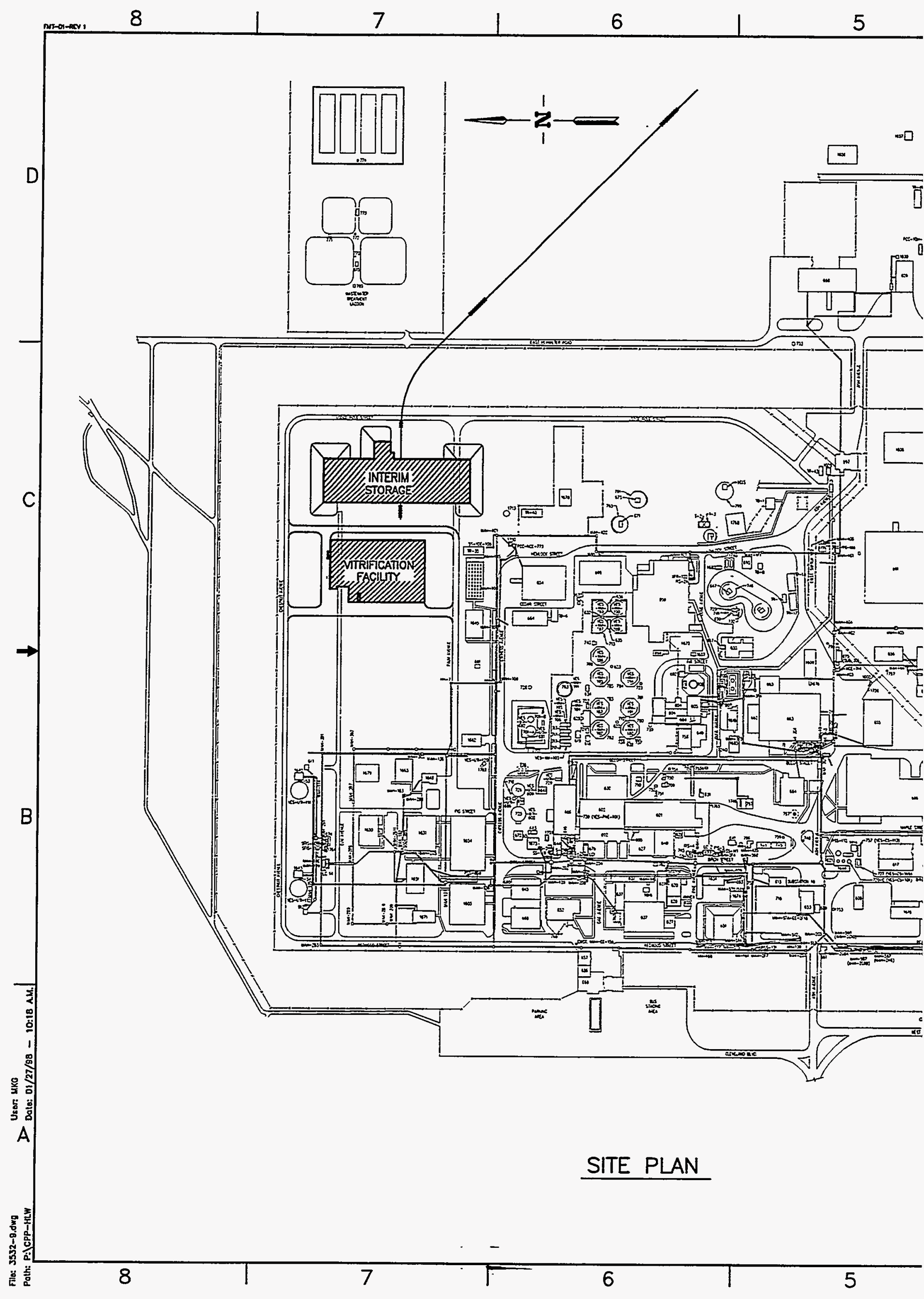




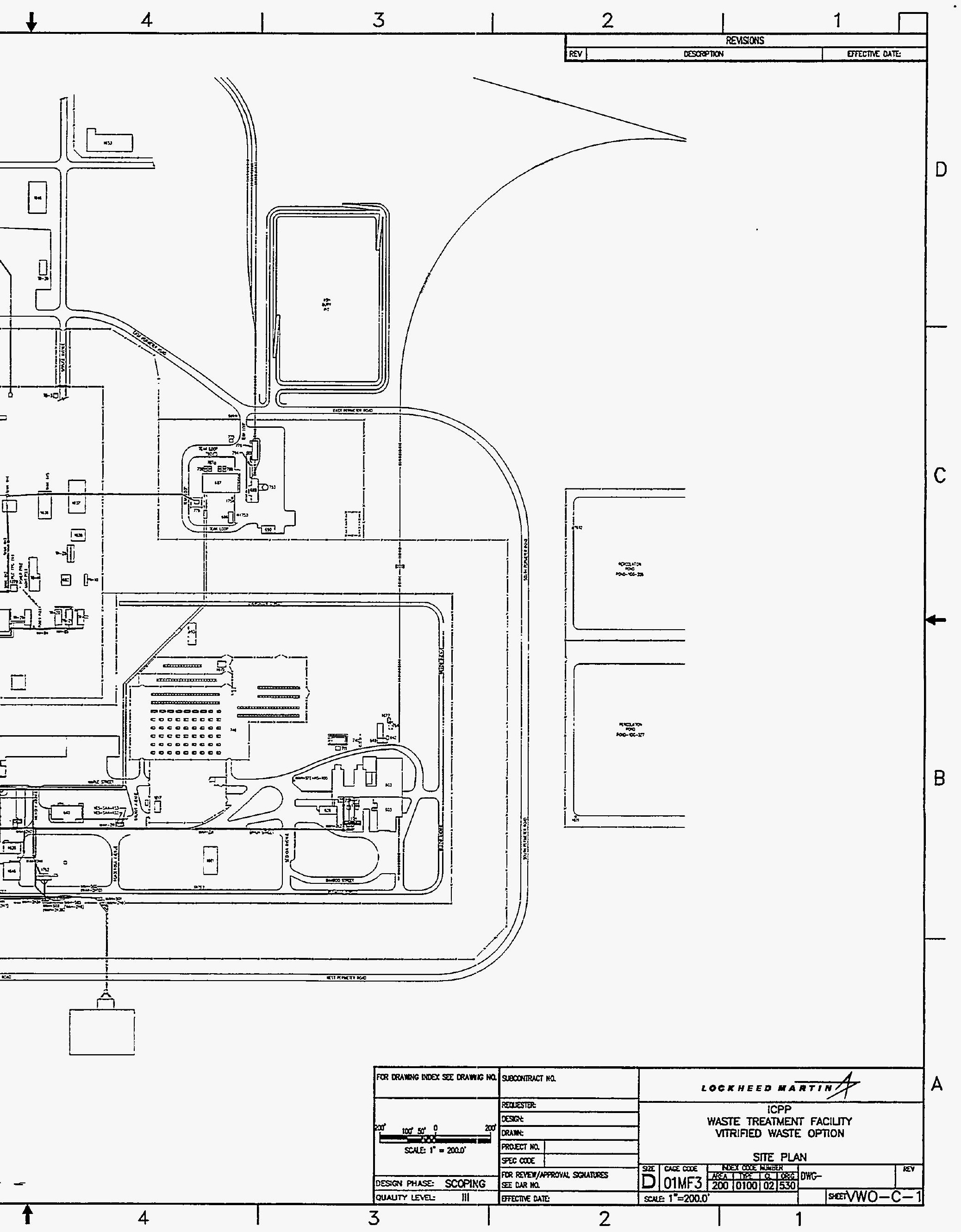




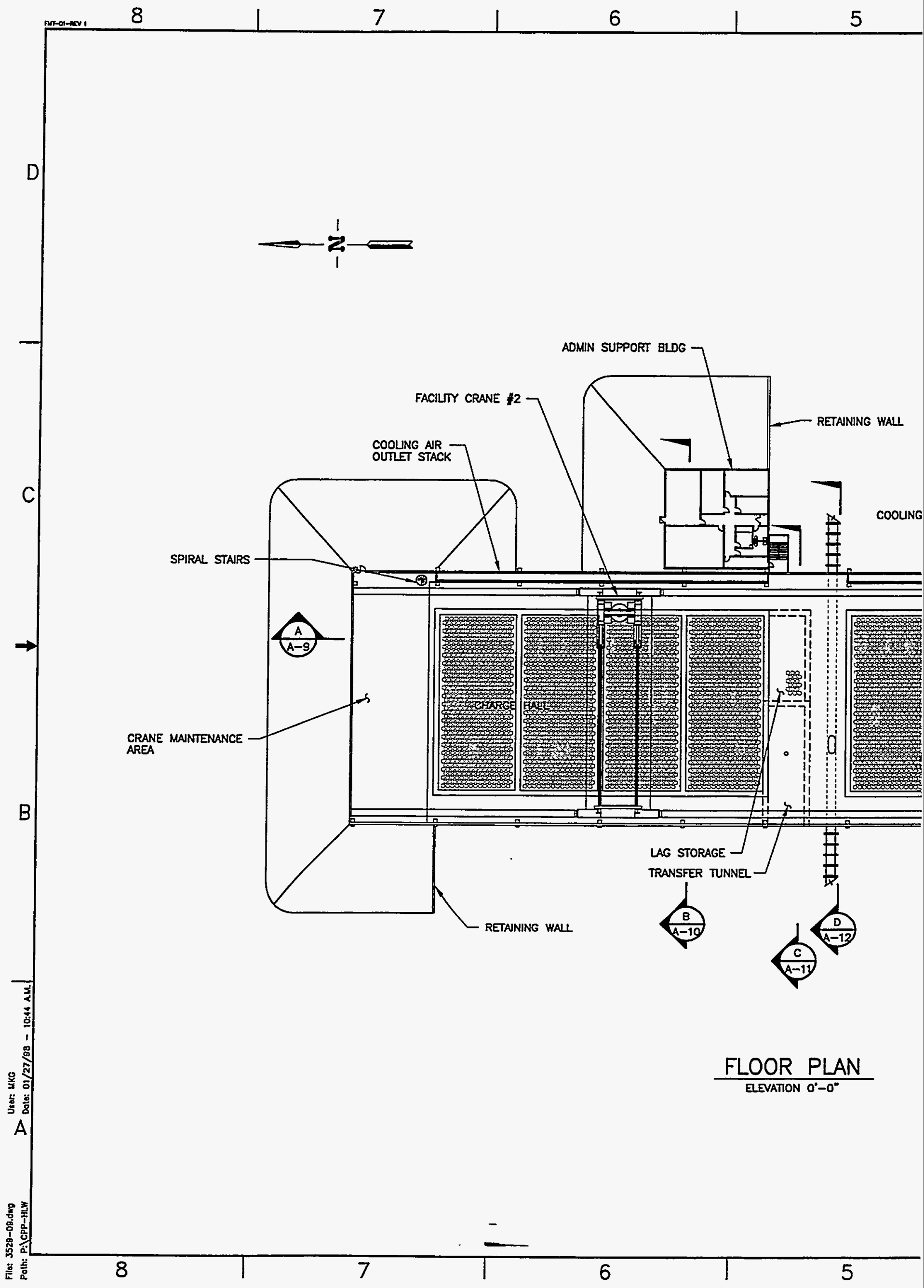




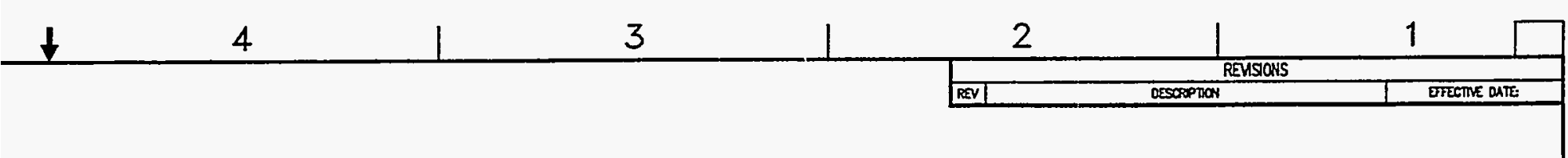

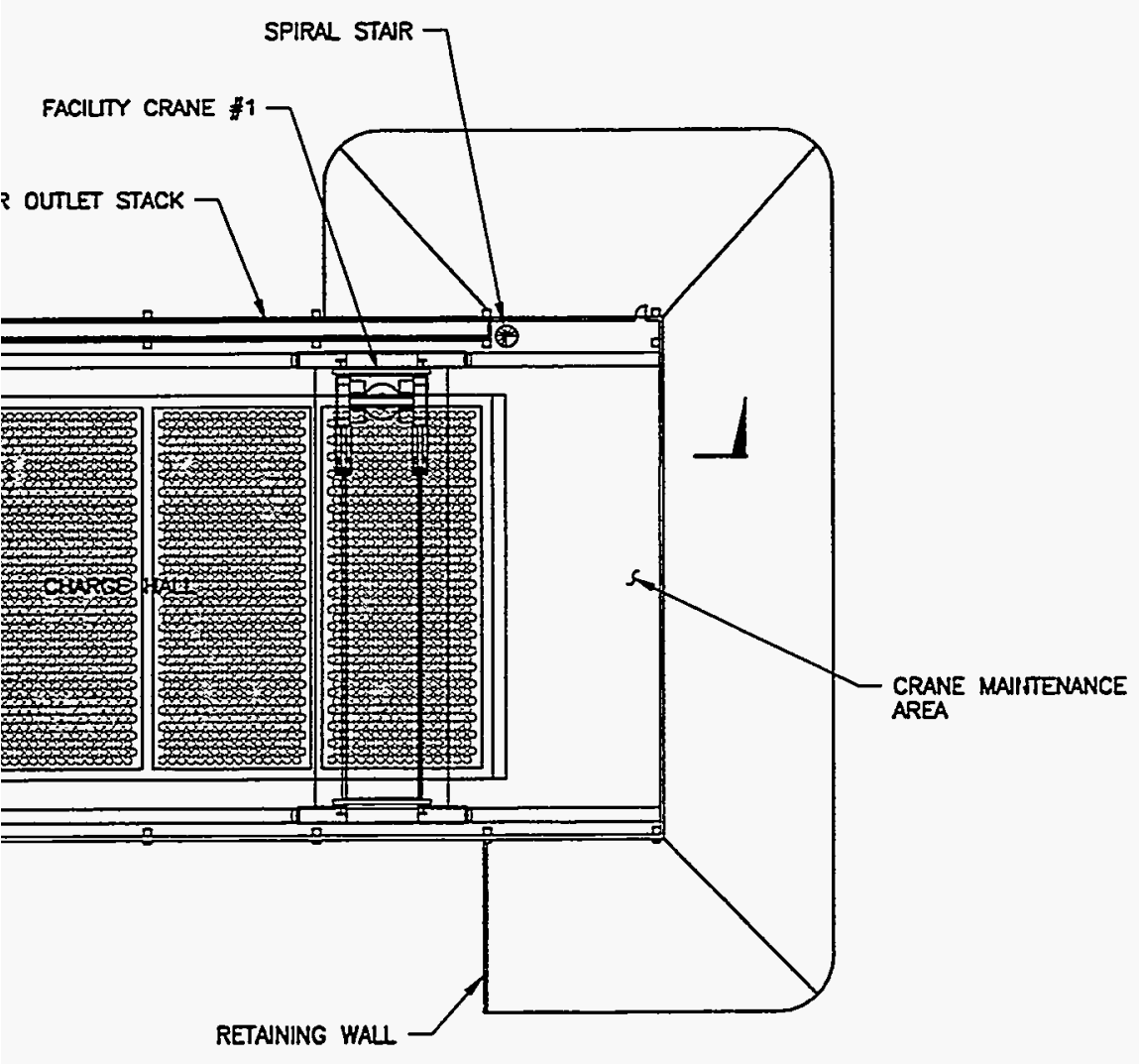

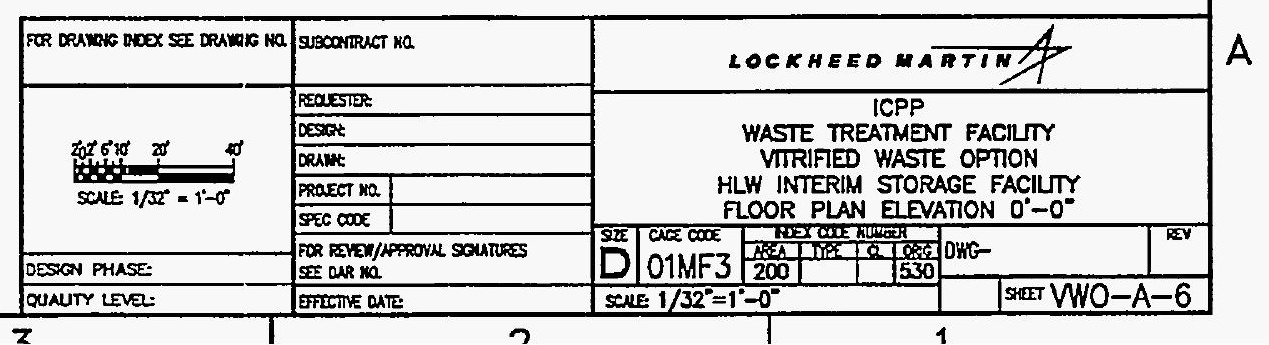




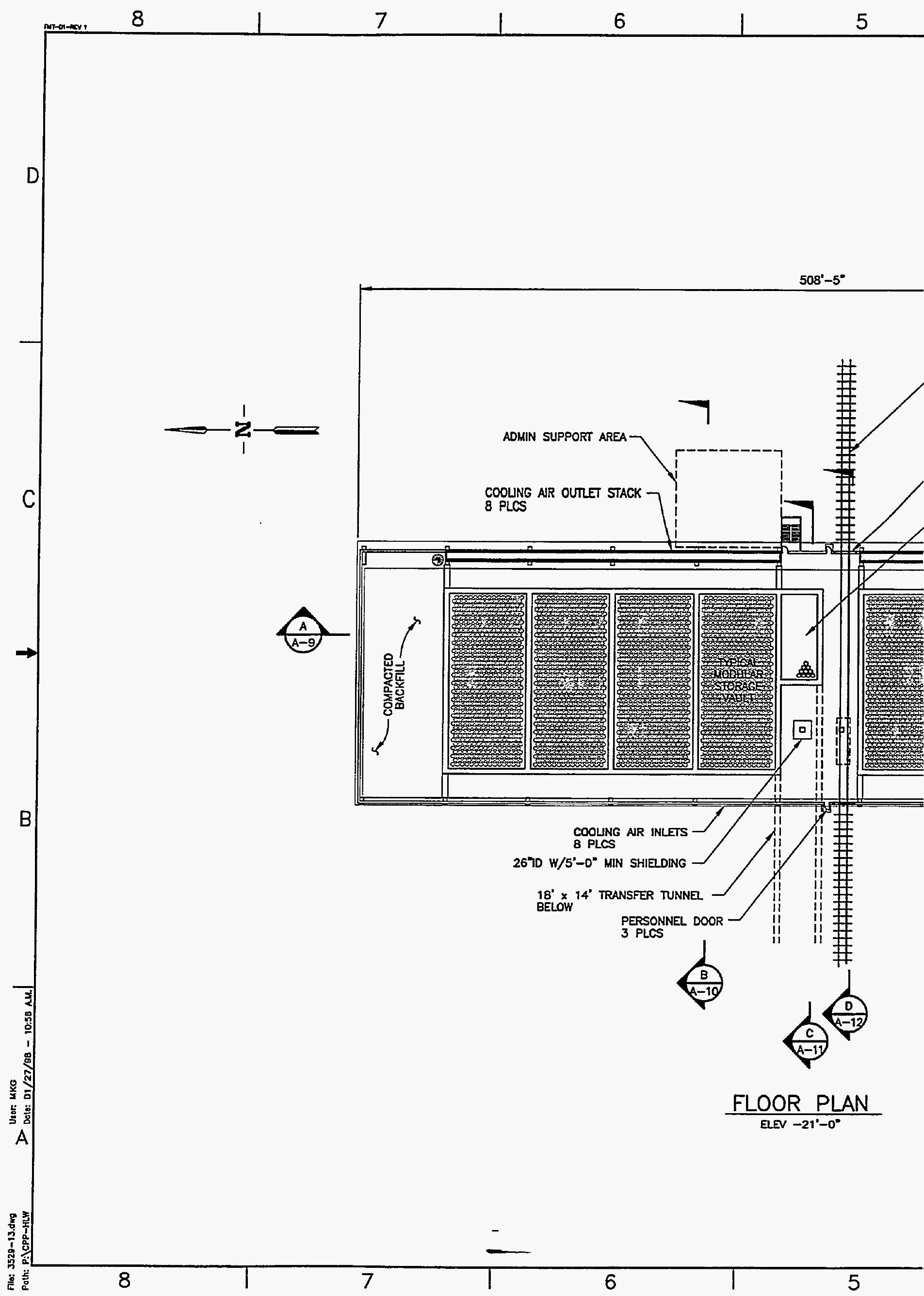




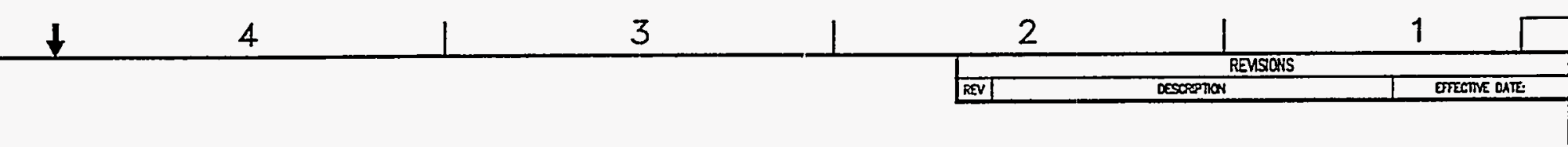

PROPOSED RAILROAD SPUR

EL. $-21^{\circ}-0^{*}$

$12^{\prime} \times 18^{\prime}$ OVERHEAD DOOR

2 PLCS

lag storage

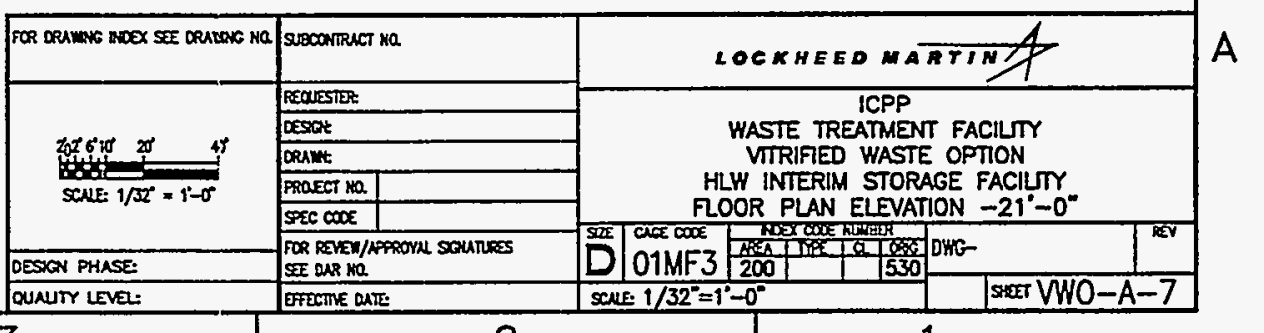




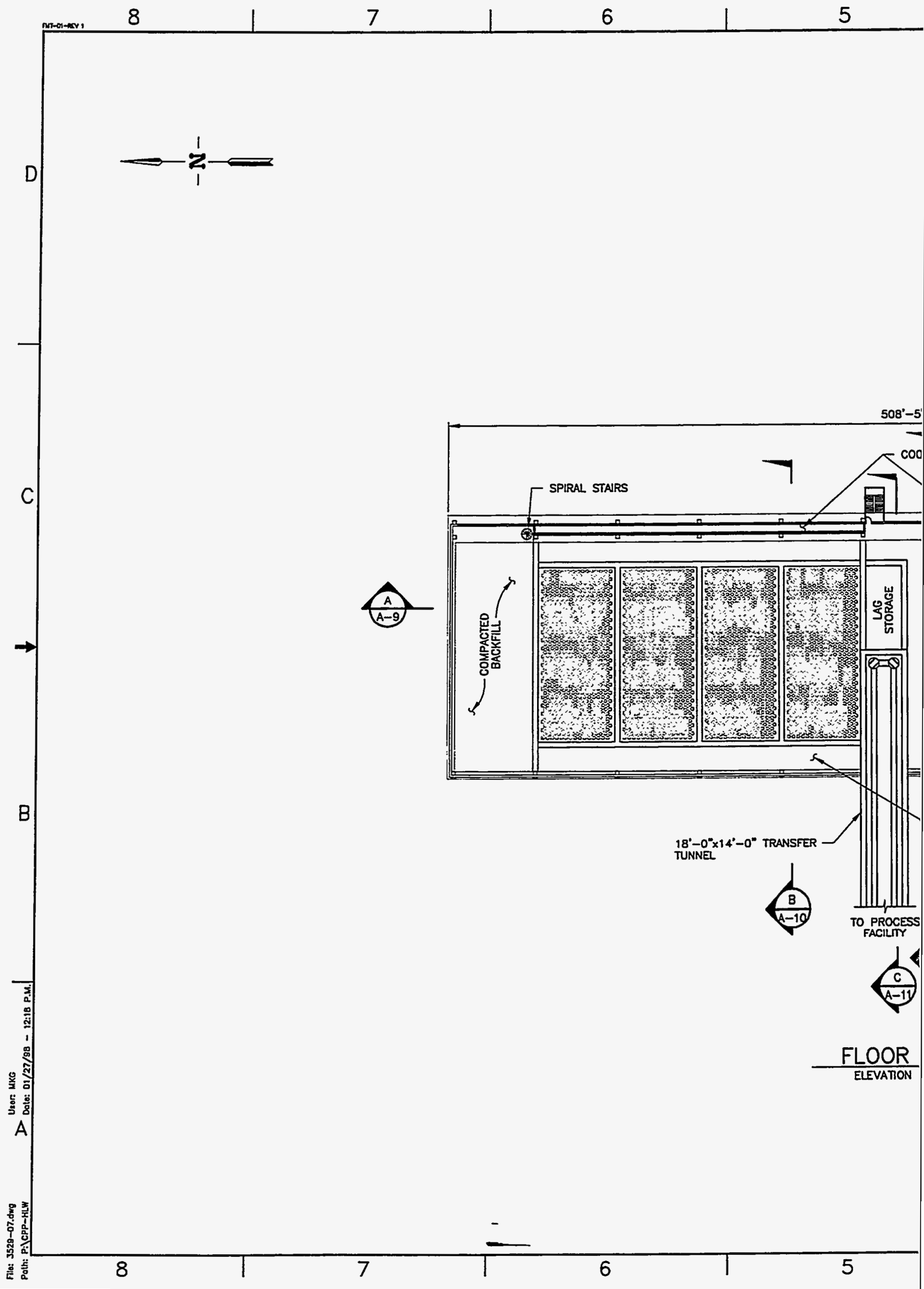




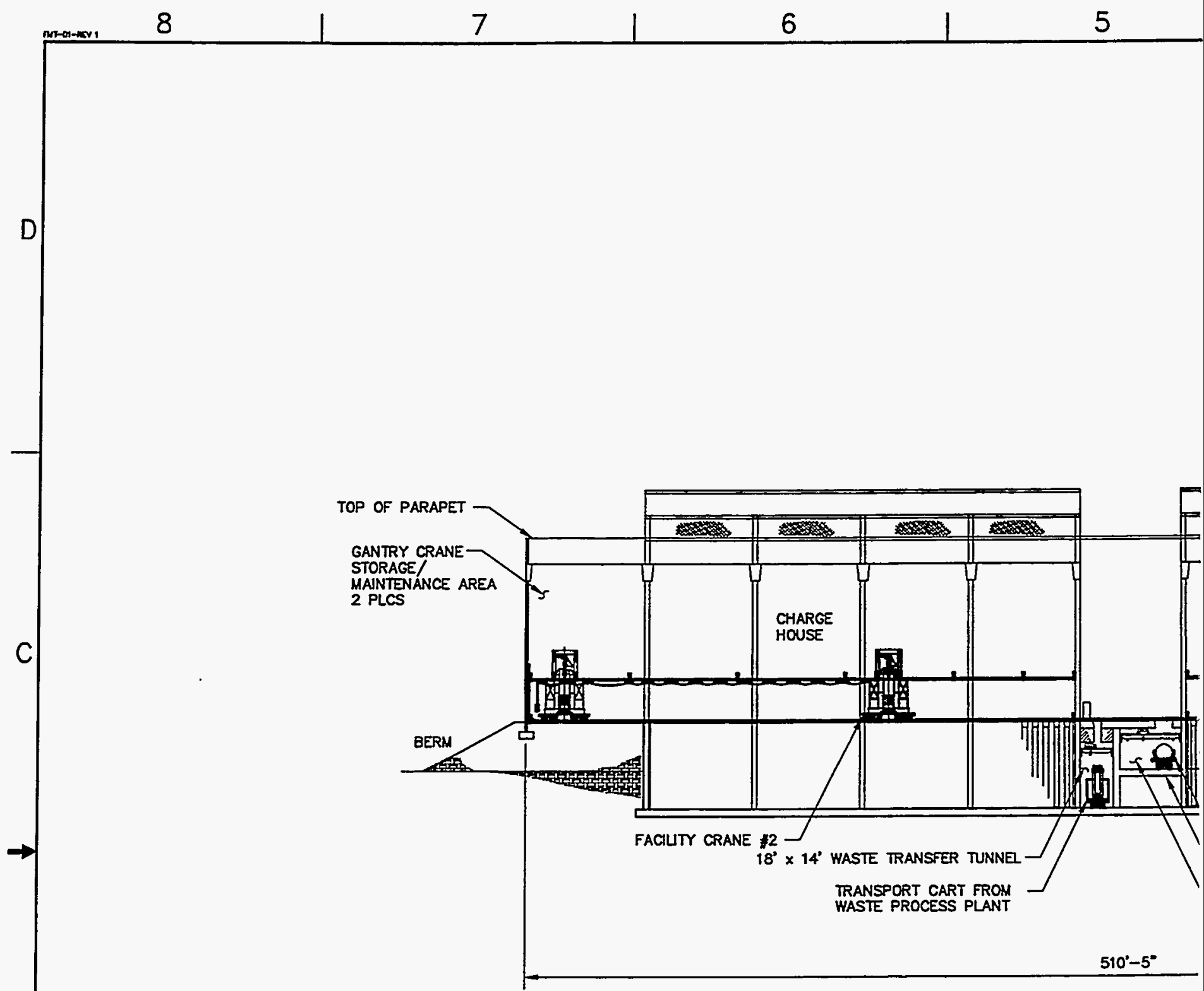

B

SECTION $\frac{A}{\pi F}$ 


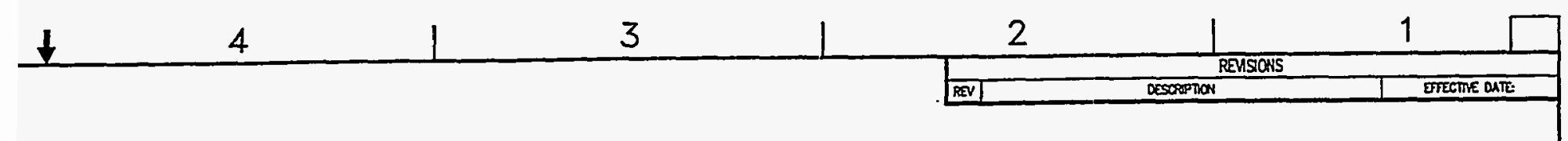

COOUNG AIR OUTET STACK

MP 8 PLCS

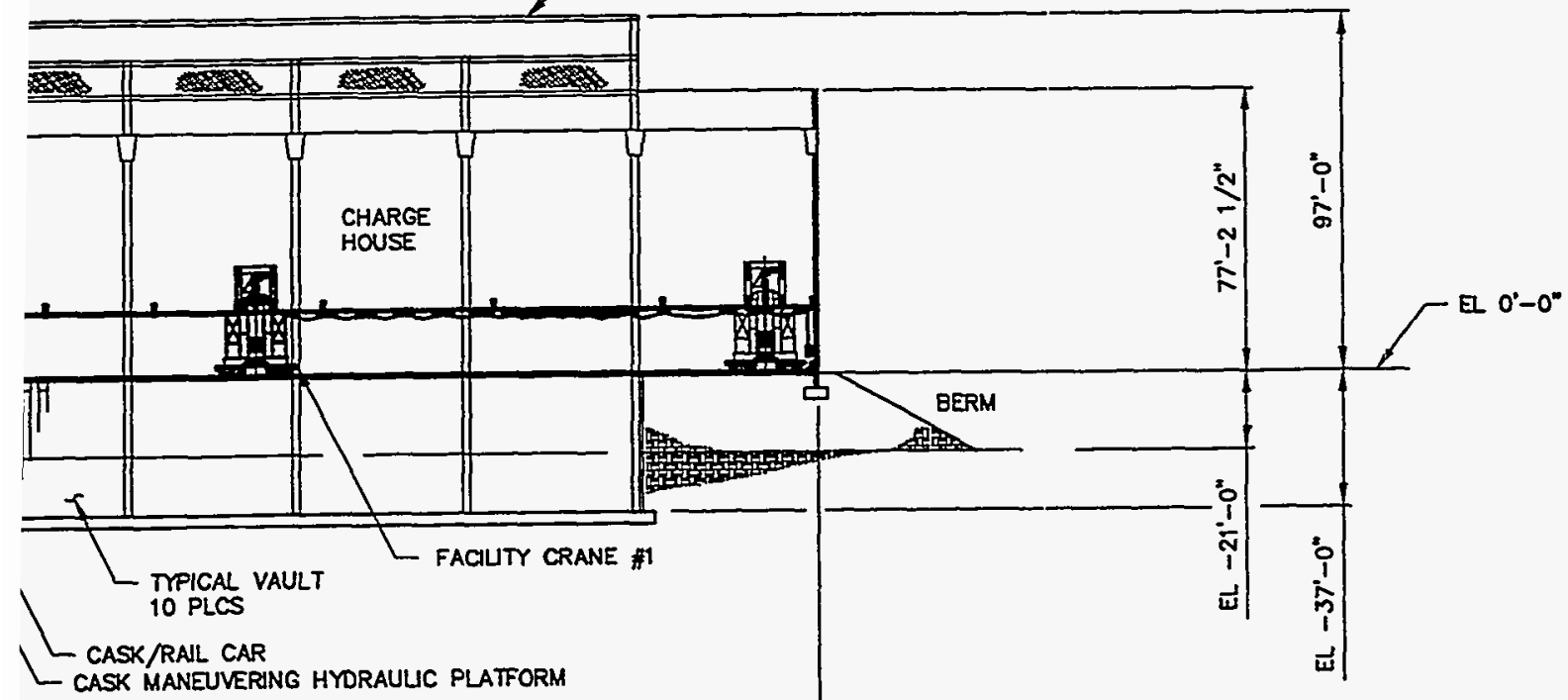

WASTE SHPPING TUNNEL

\begin{tabular}{|c|c|c|c|c|}
\hline 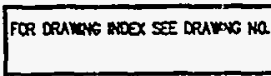 & suowimuct ke & \multicolumn{3}{|c|}{ LOCKHEEOMARTIN/ } \\
\hline \multirow{5}{*}{ 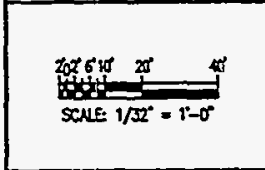 } & Egarsist: & \multirow{4}{*}{\multicolumn{2}{|c|}{$\begin{array}{l}\text { ICPP } \\
\text { WASTE TREATMENT FACIUTY } \\
\text { VTRIFED WASTE OPTON } \\
\text { HLW INTERIM STORAGE FACIUTY } \\
\text { SECTON }\end{array}$}} & \\
\hline & Dessat & & & \\
\hline & DRAMt R. S EVMAS & & & \\
\hline & 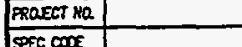 & & & \\
\hline & \multirow{2}{*}{ 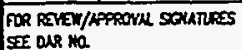 } & 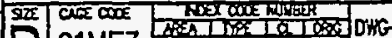 & & 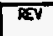 \\
\hline DESIGN PHASE: & & [D/01MF3 & & \\
\hline QUNUTY LEVEL: & EFECTIE OATE & secis $1 / 32^{\circ}=1^{\circ}-0^{\circ}$ & \multicolumn{2}{|c|}{ SArT VWO-A-9 } \\
\hline
\end{tabular}




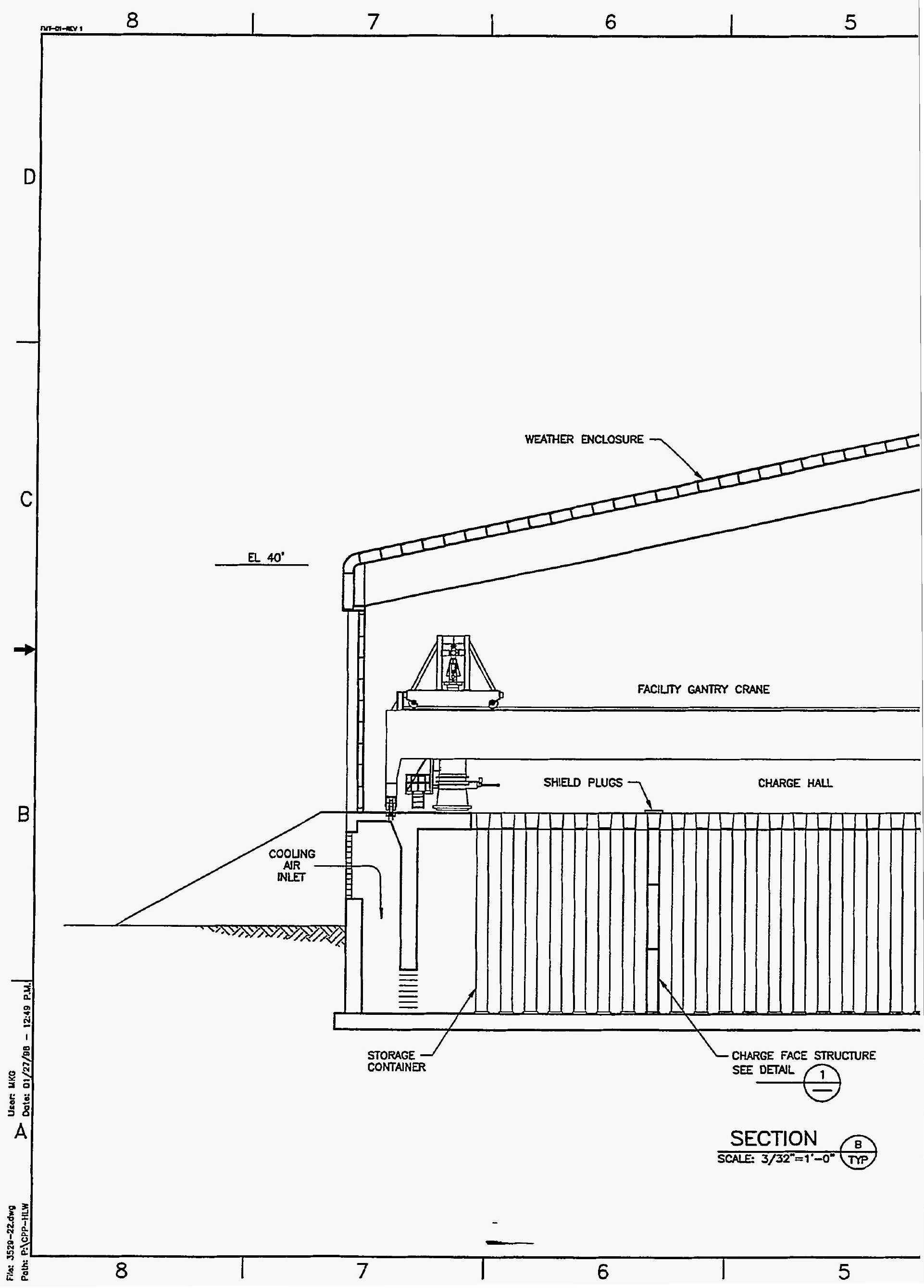




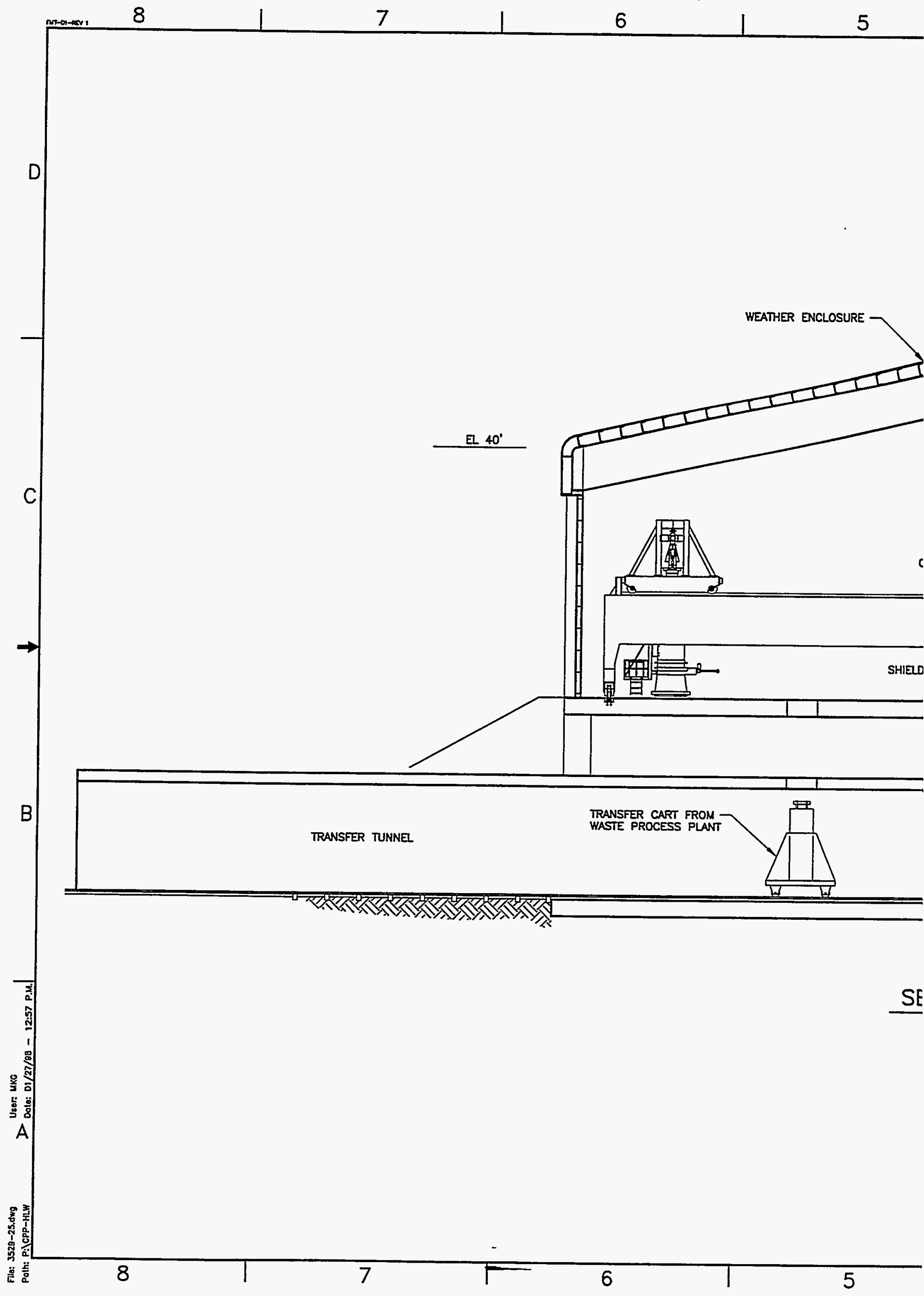




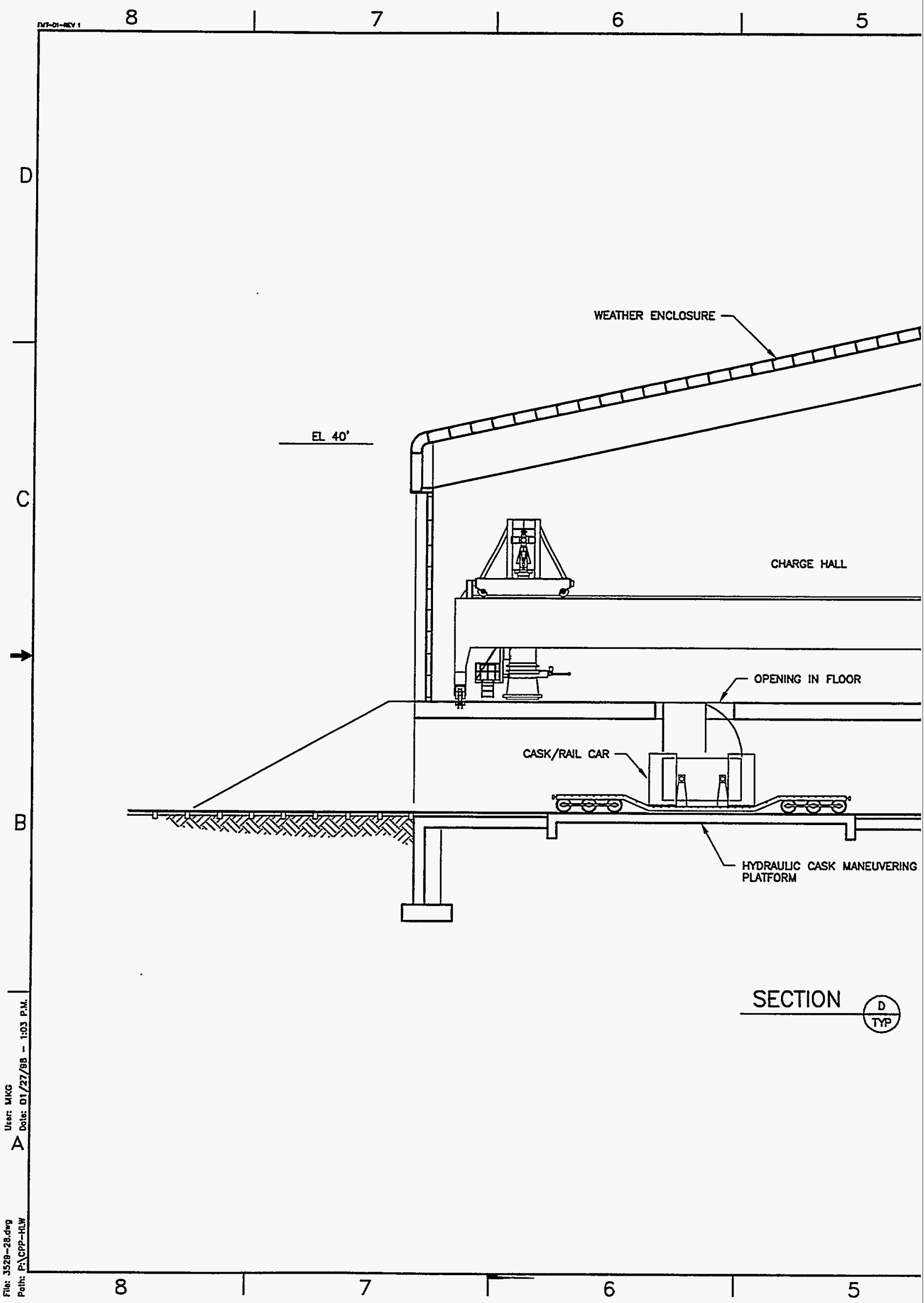




\begin{tabular}{|c|c|c|c|}
\hline$t$ & 4 & 3 & 2 \\
\hline
\end{tabular}

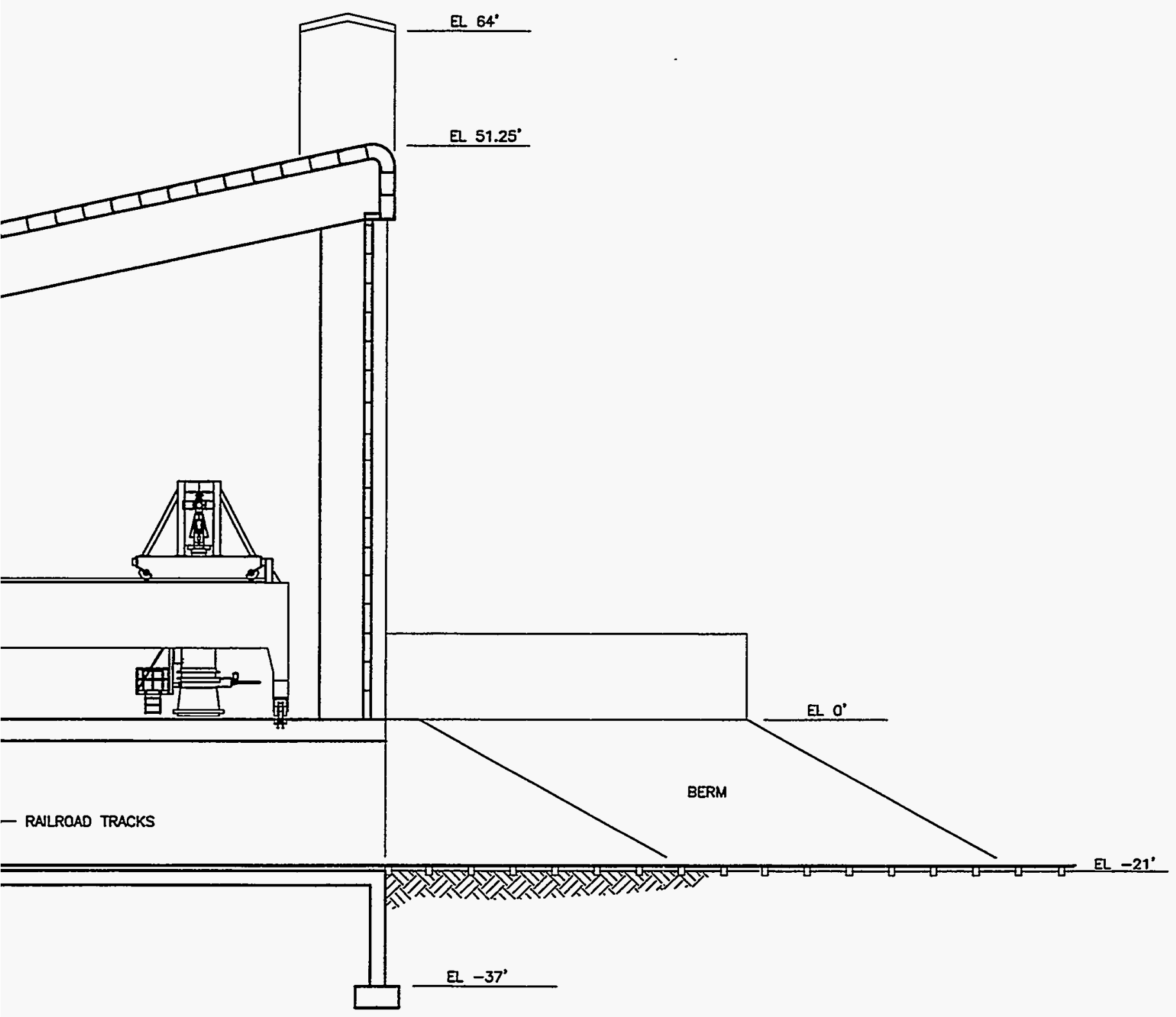

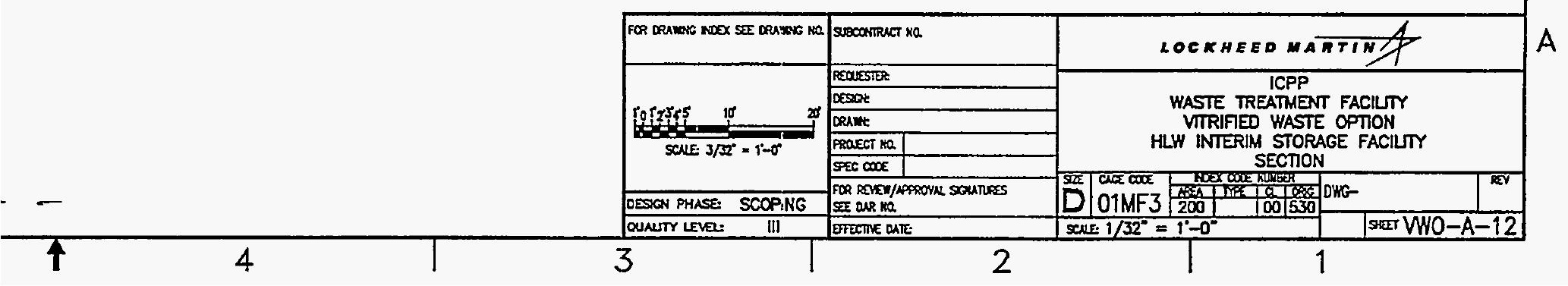




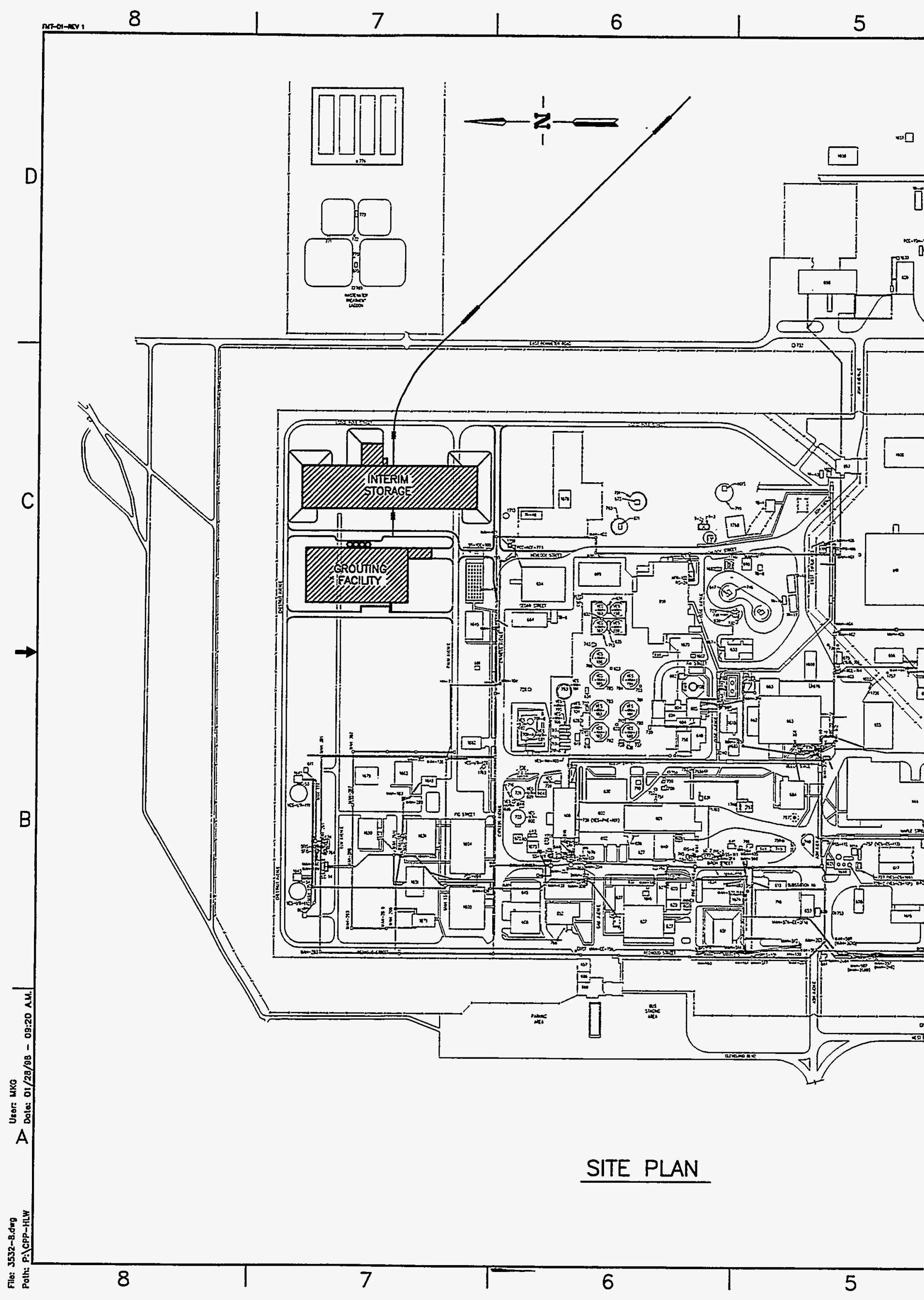




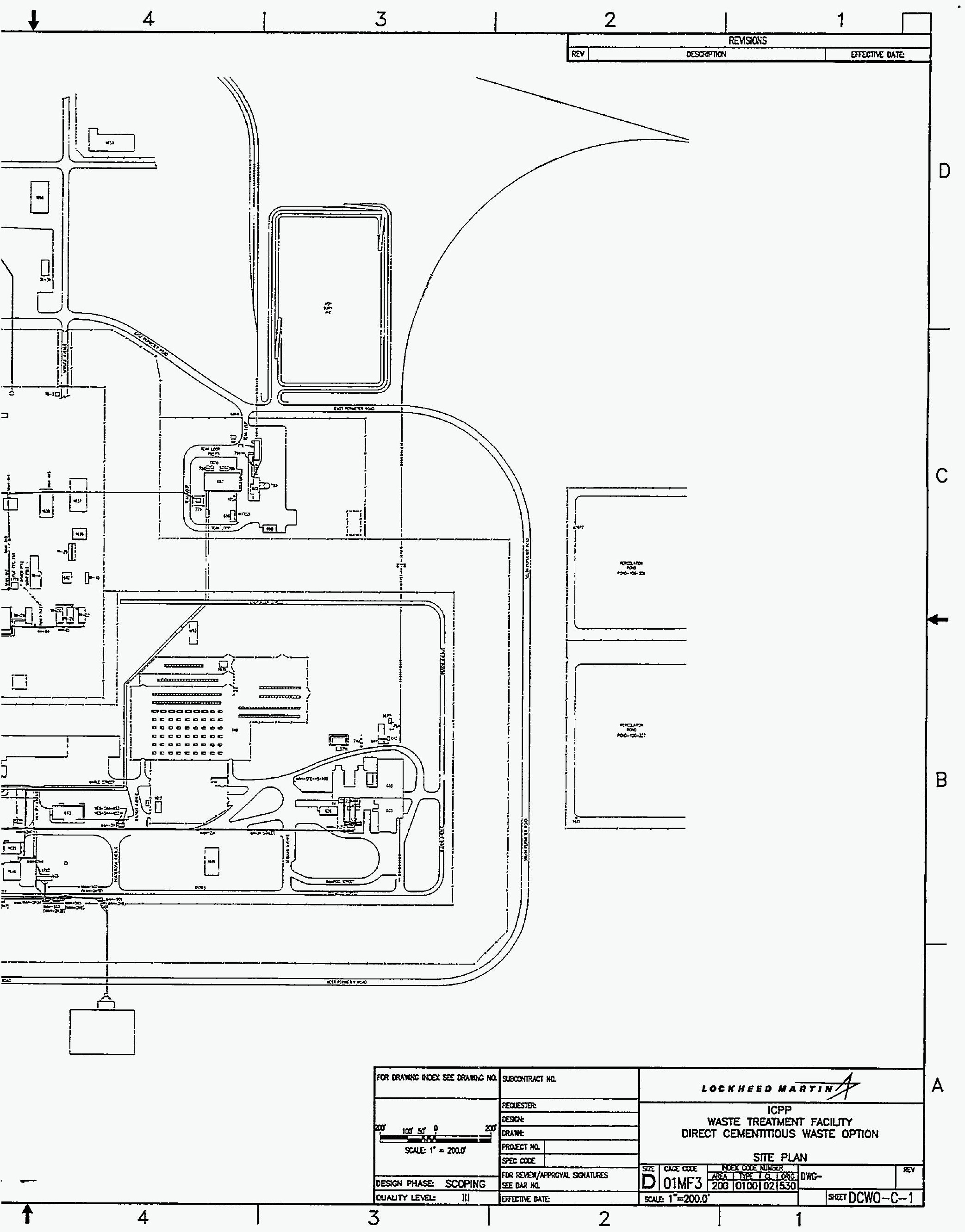




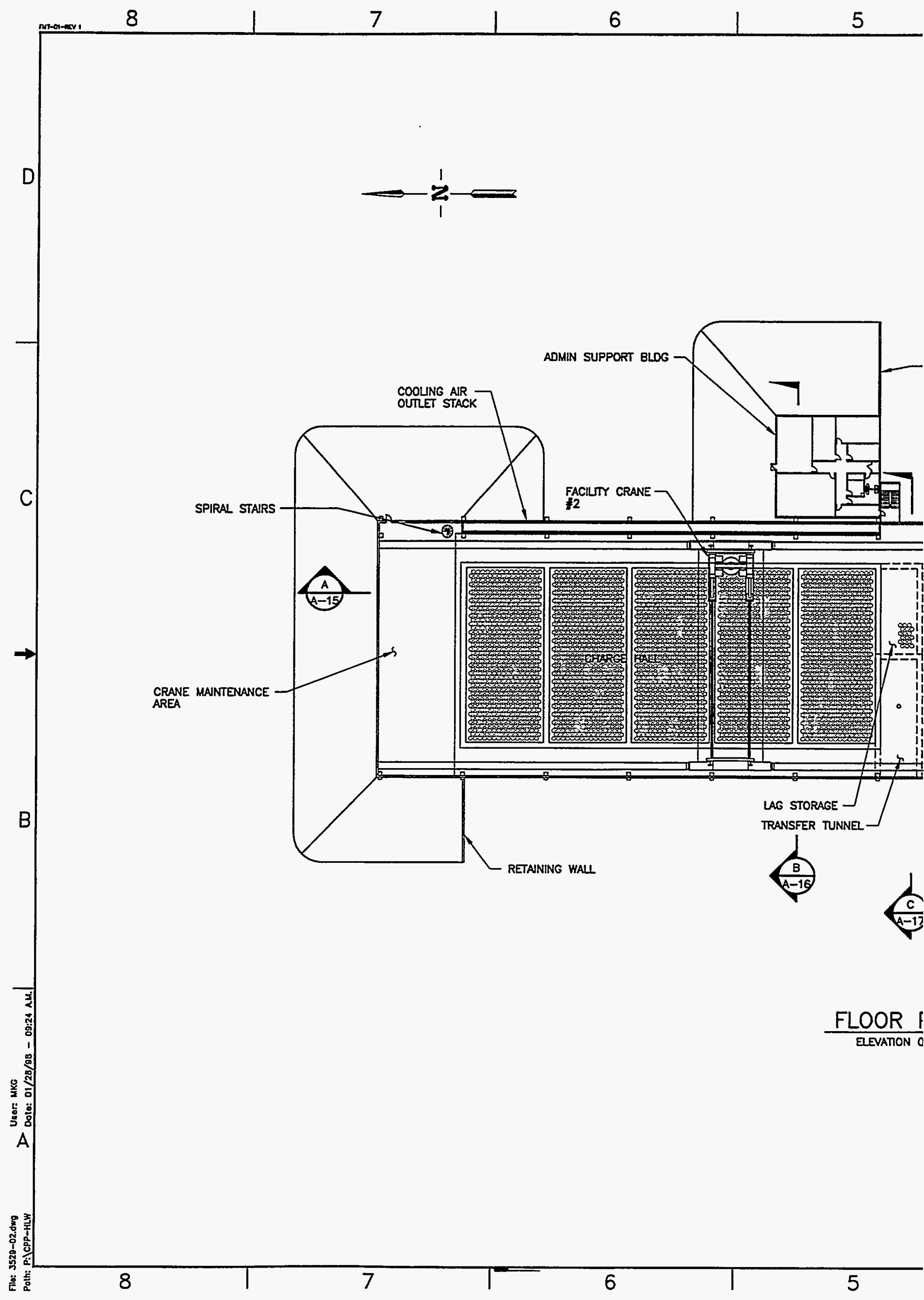




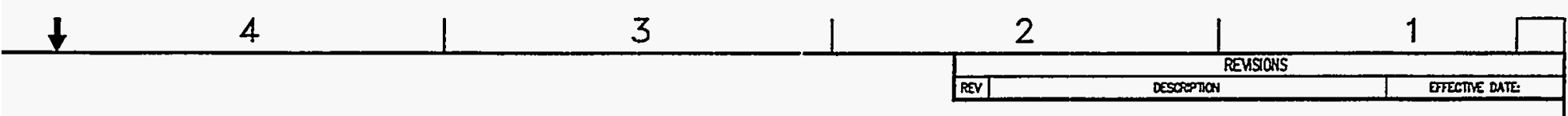

TAINING WALL

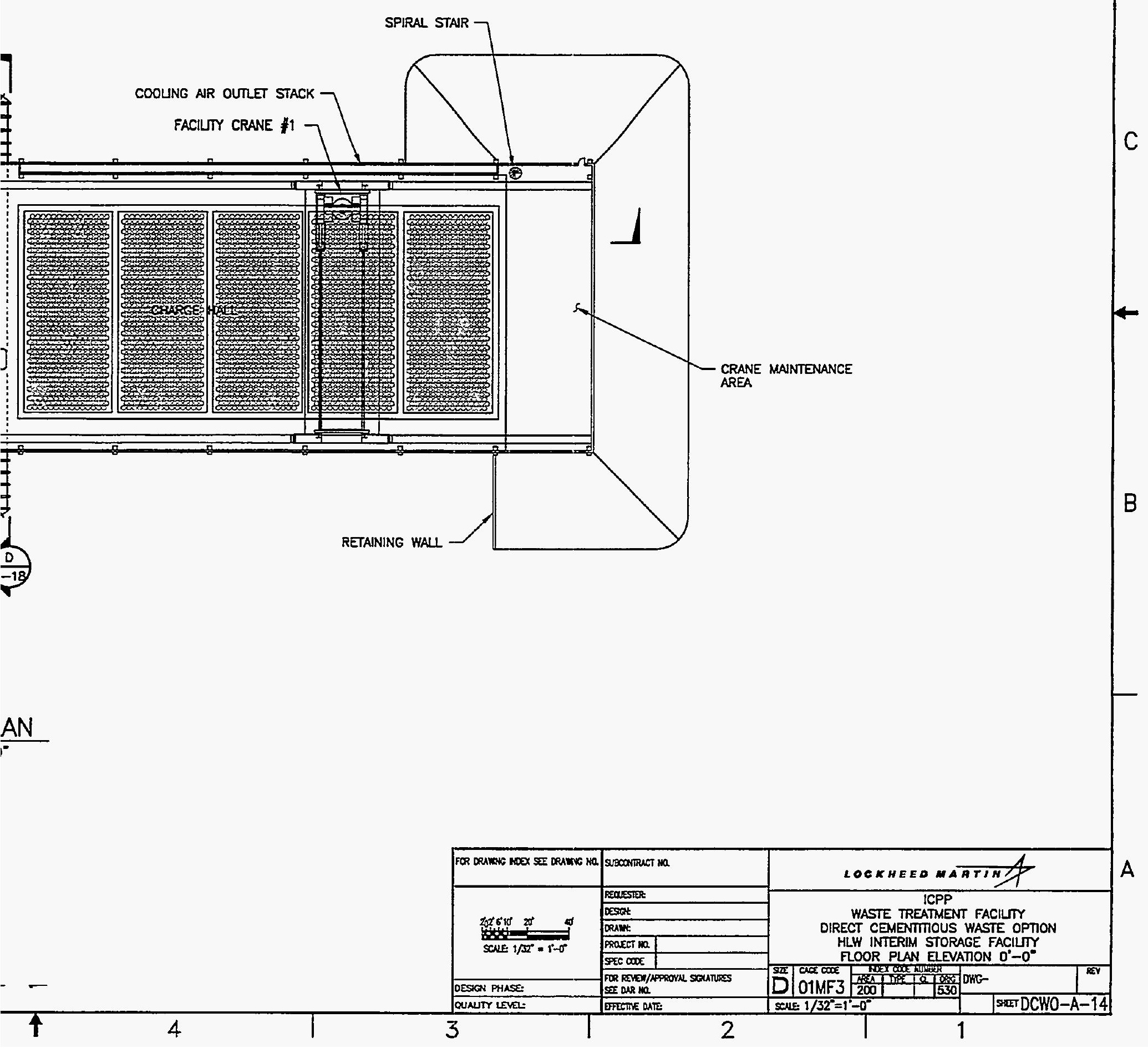




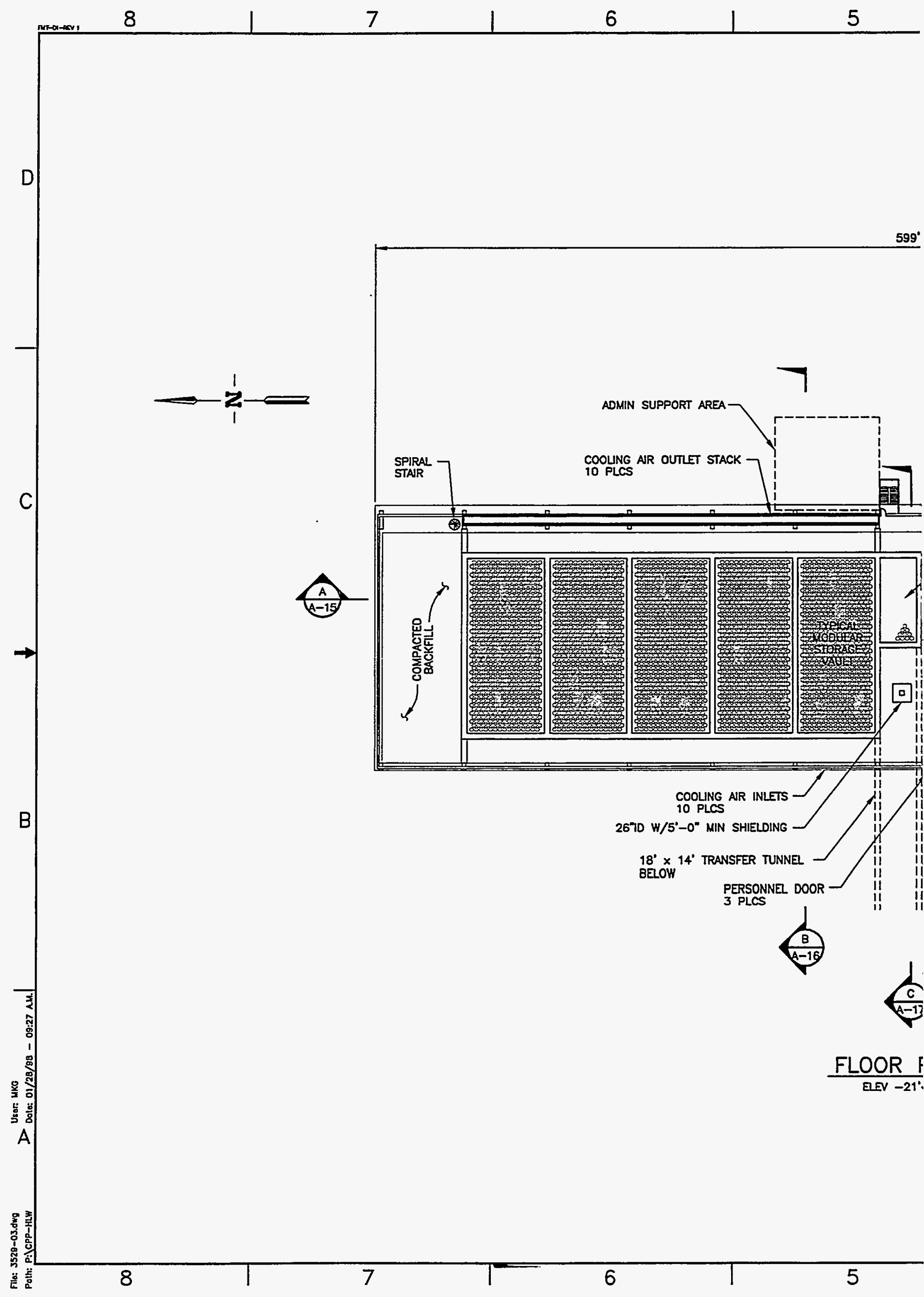




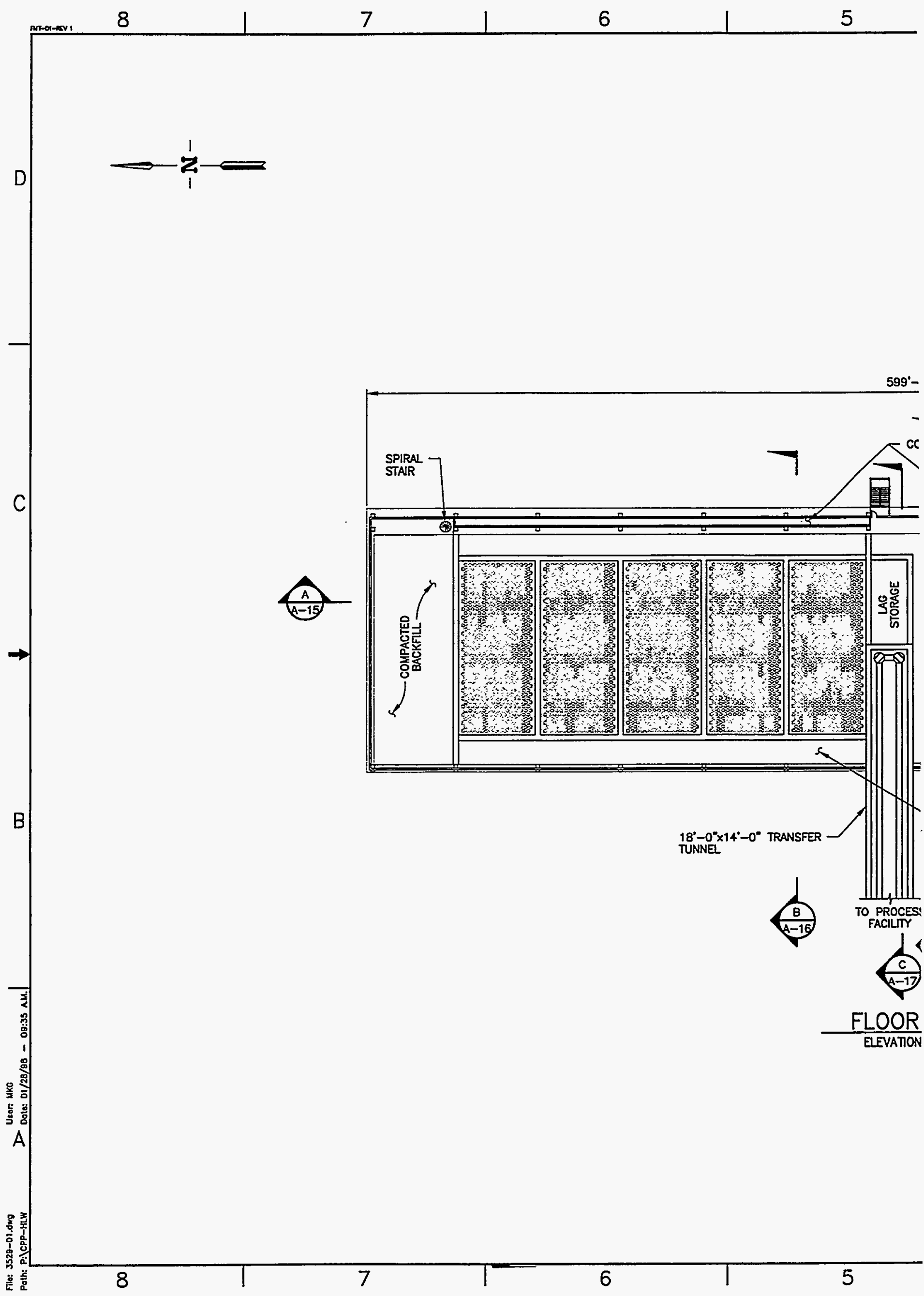




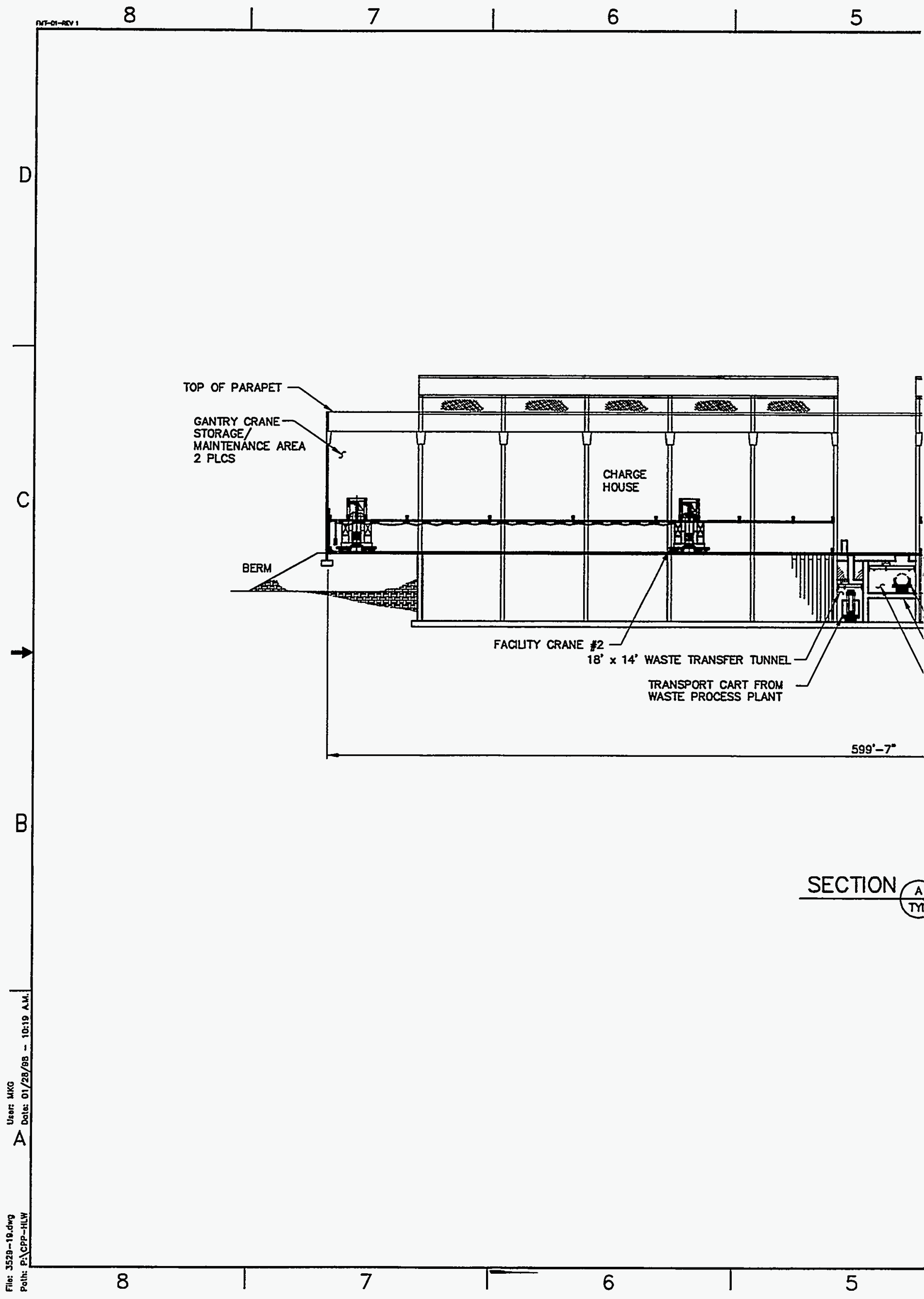




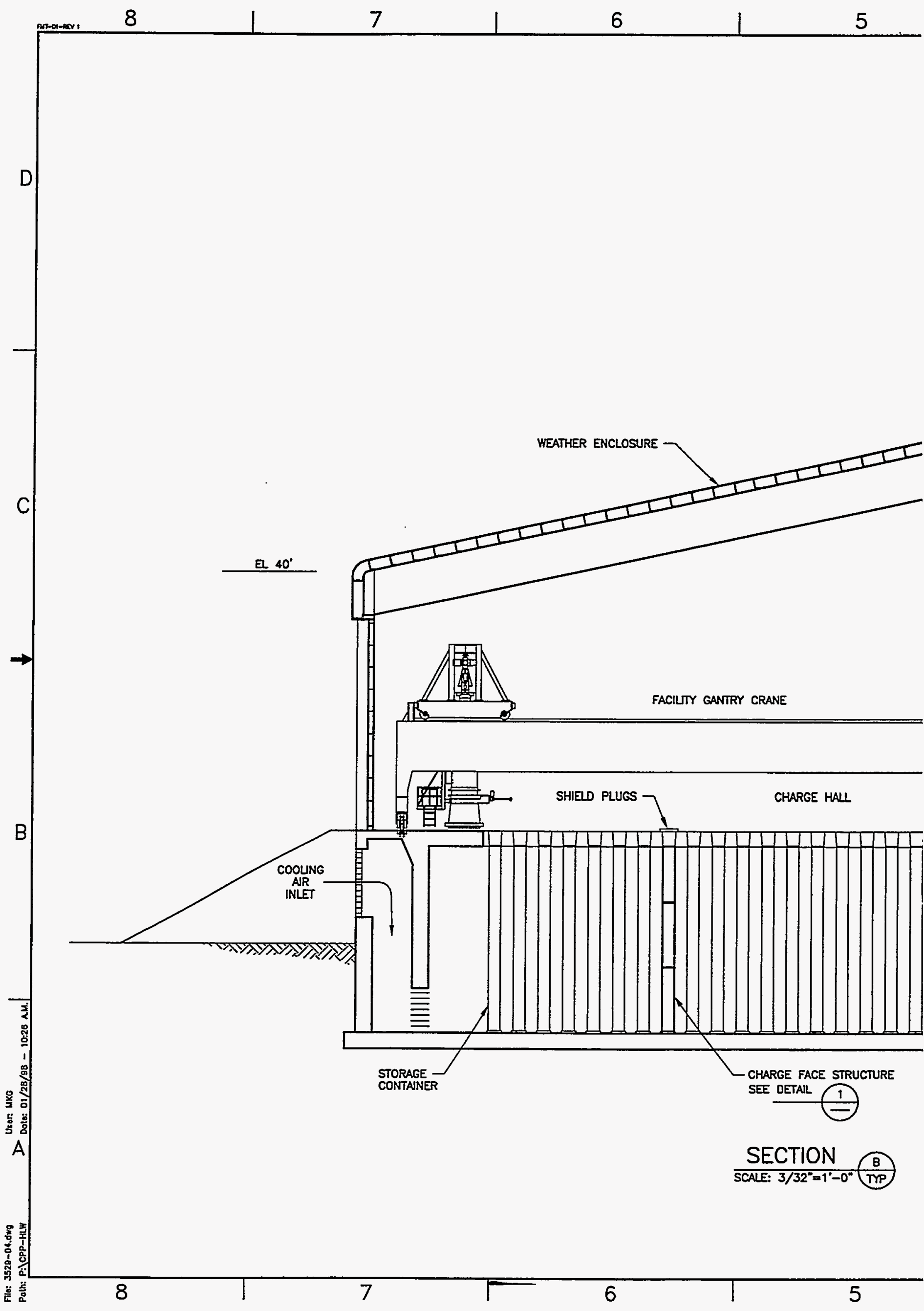




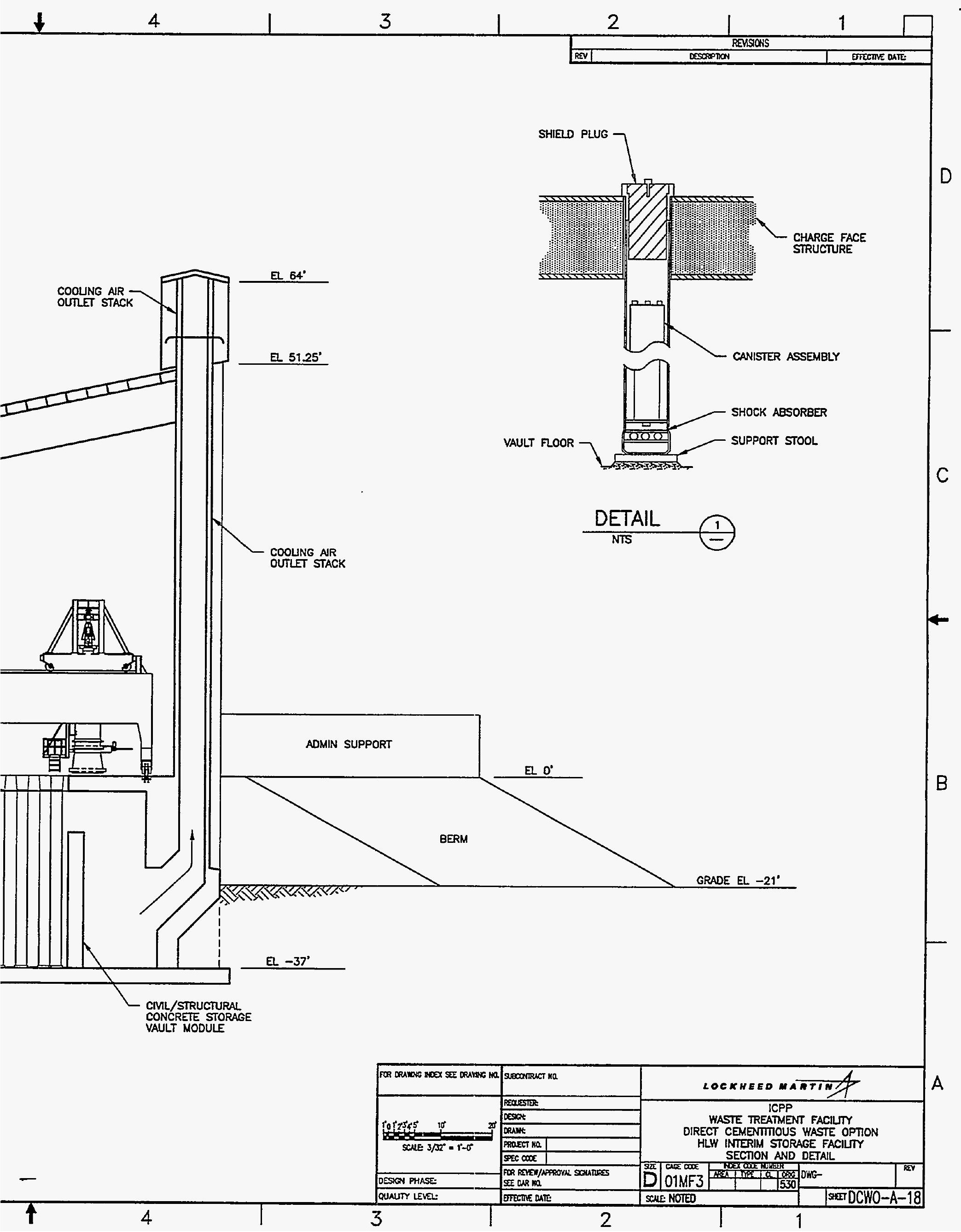




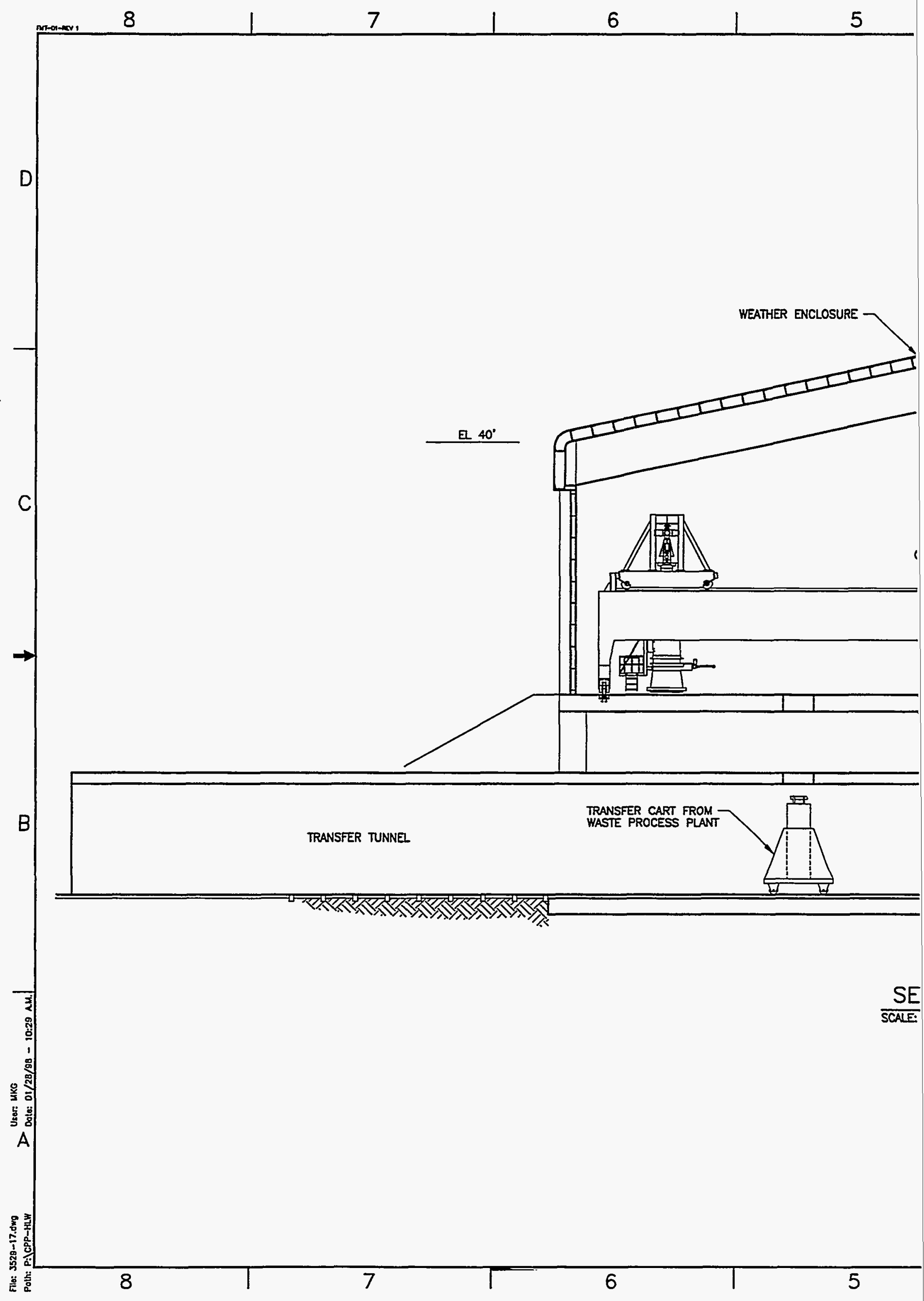




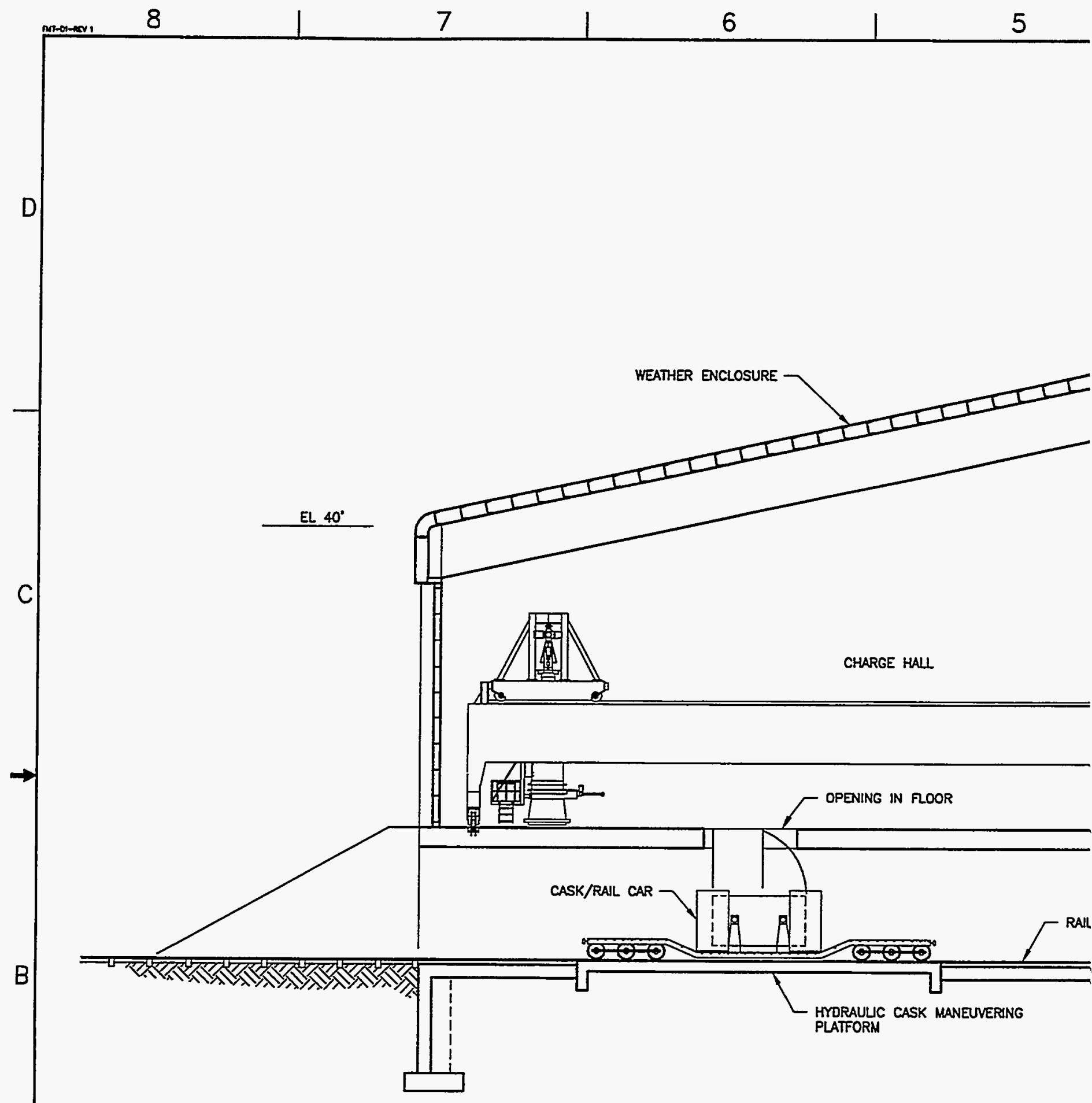

\section{$\frac{\text { SECTION D }}{\text { SCALE: } 3 / 32^{\circ}=1^{\circ}-0^{\circ} \text { TPP }}$}




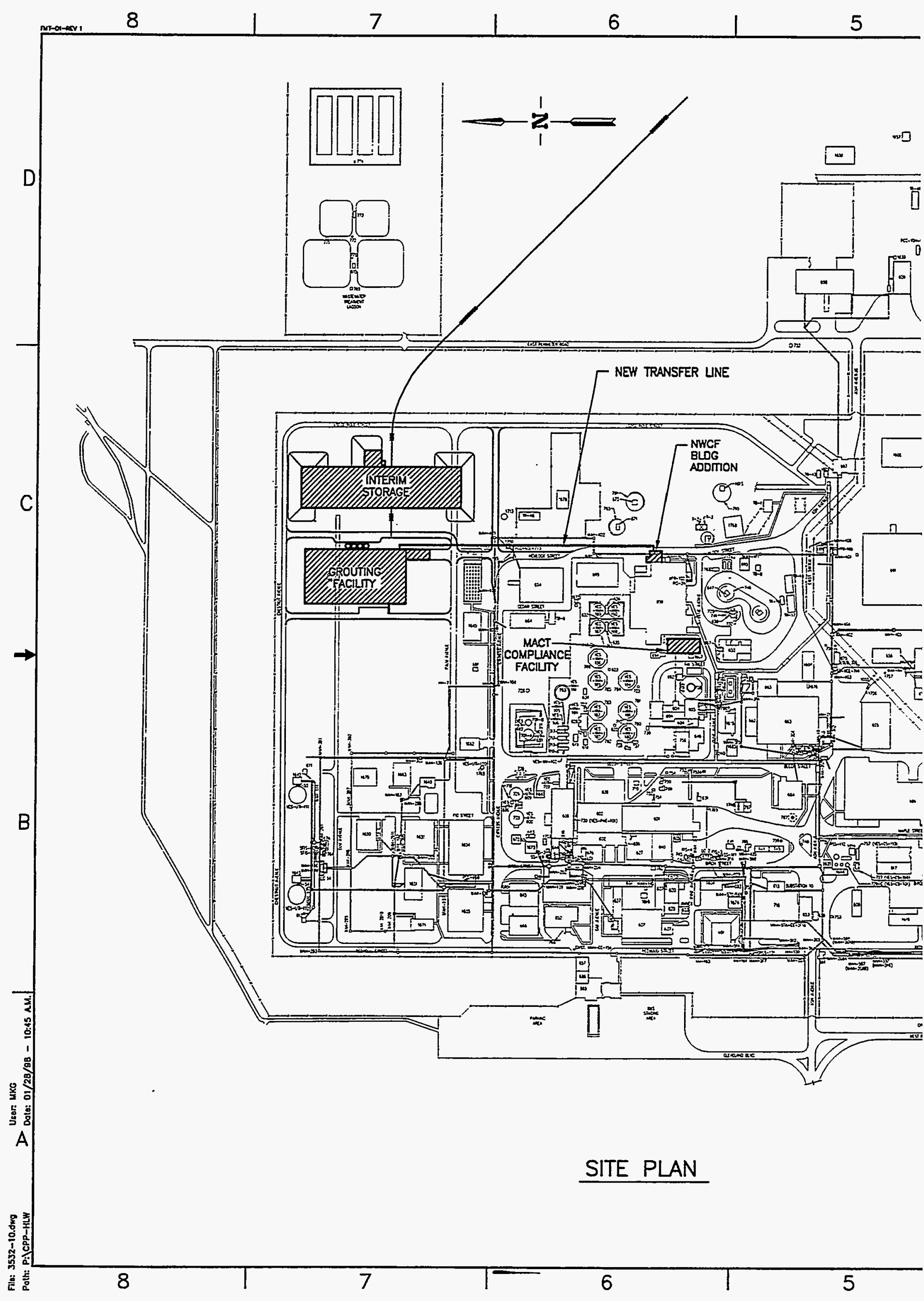




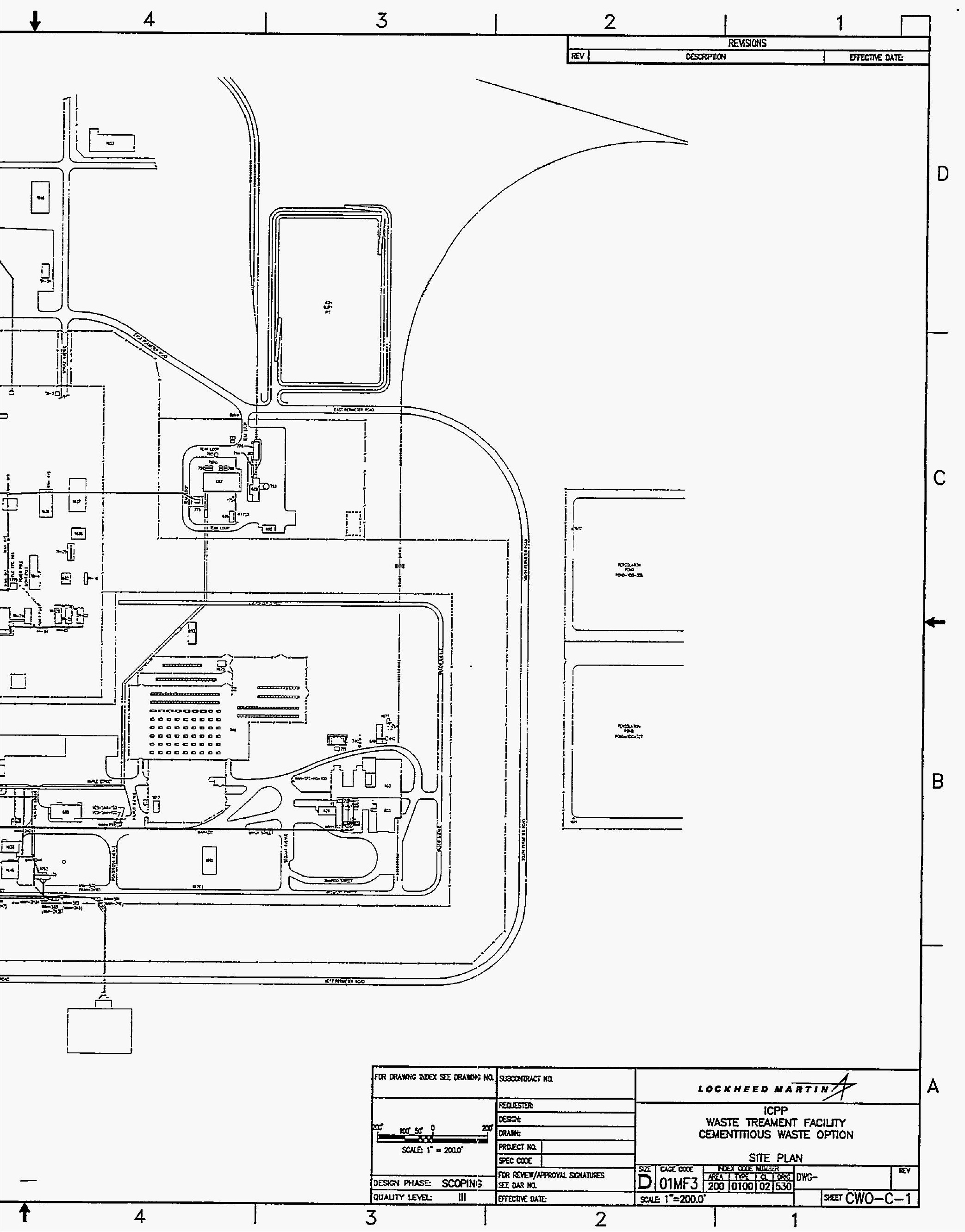




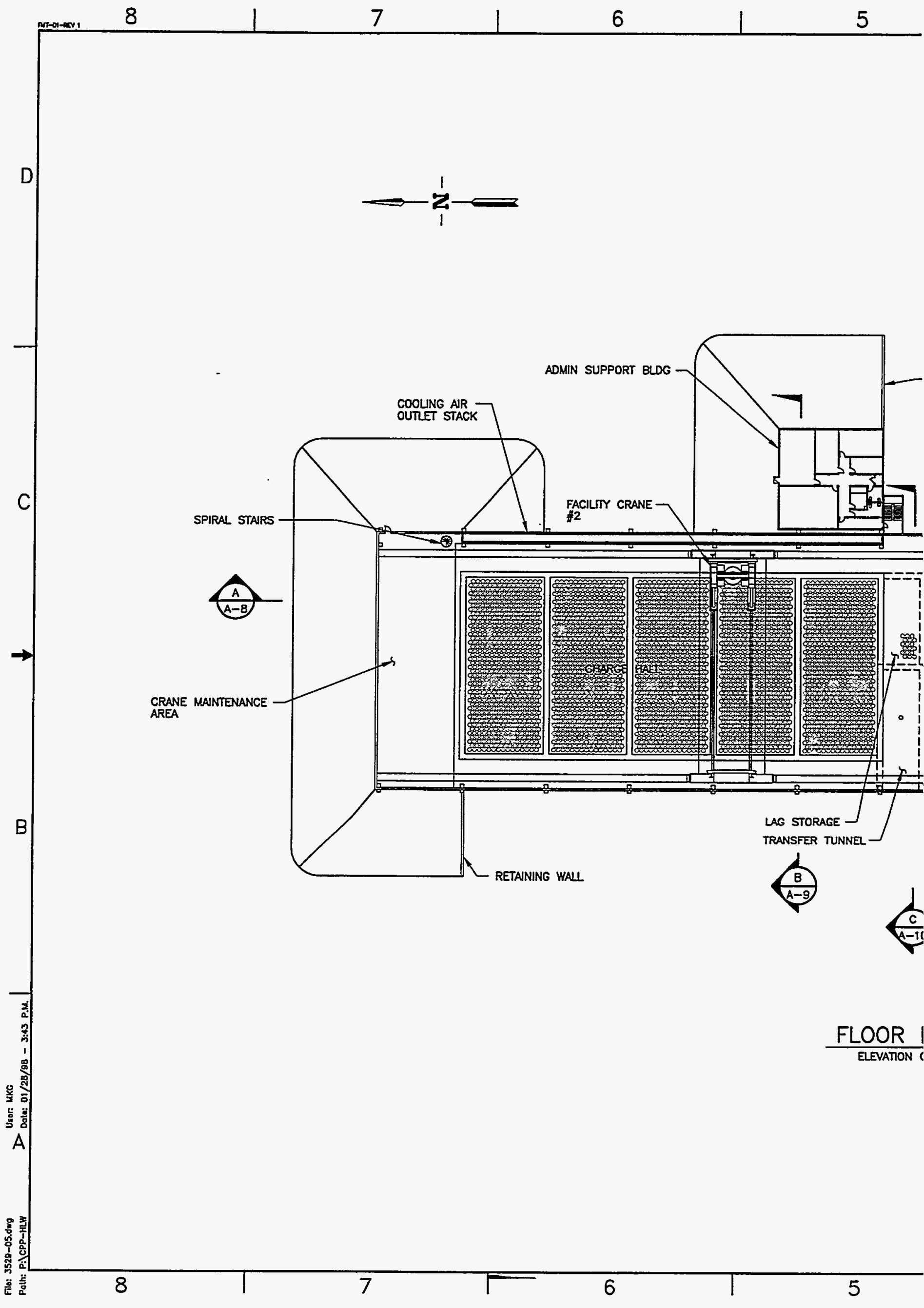




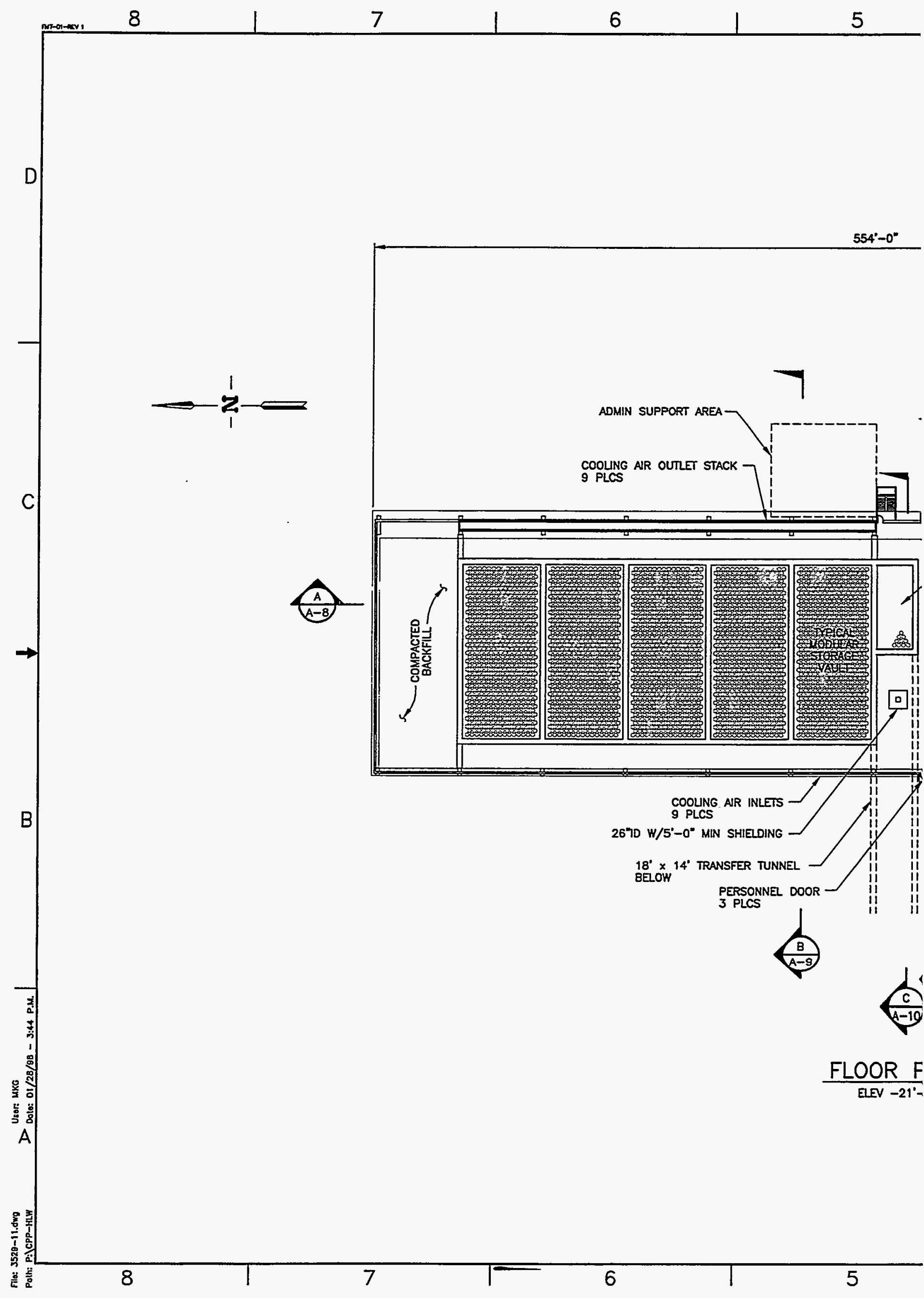




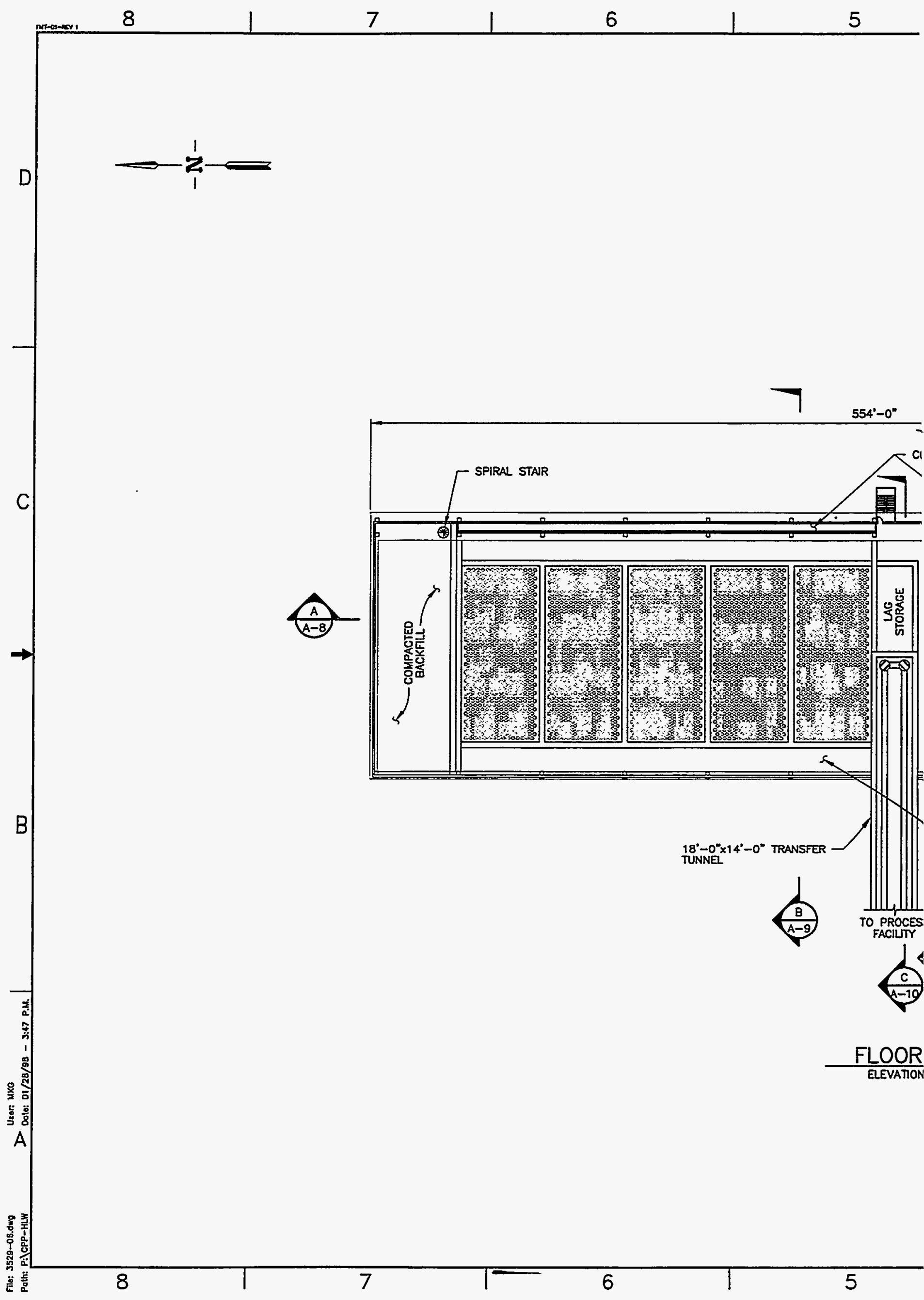




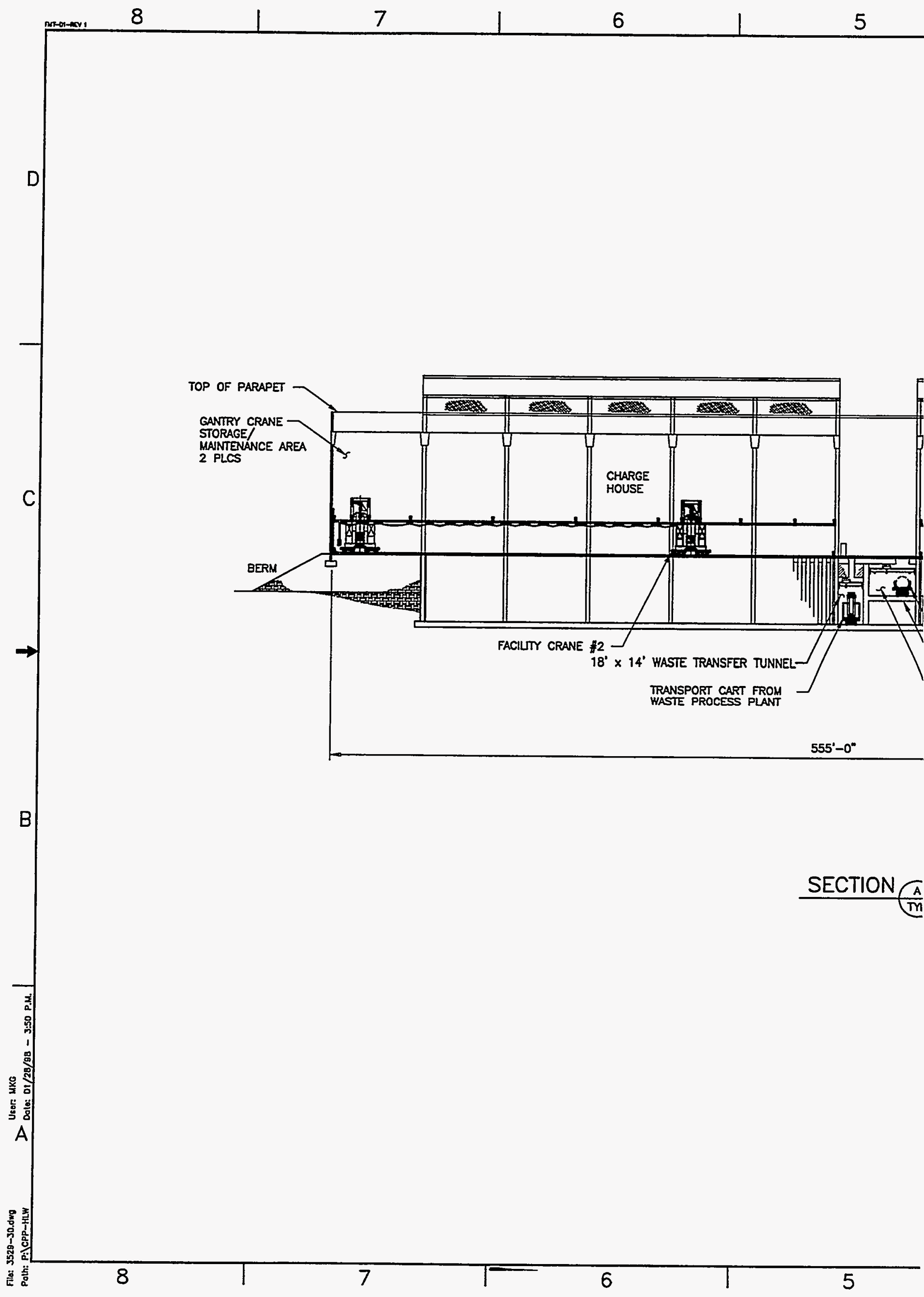




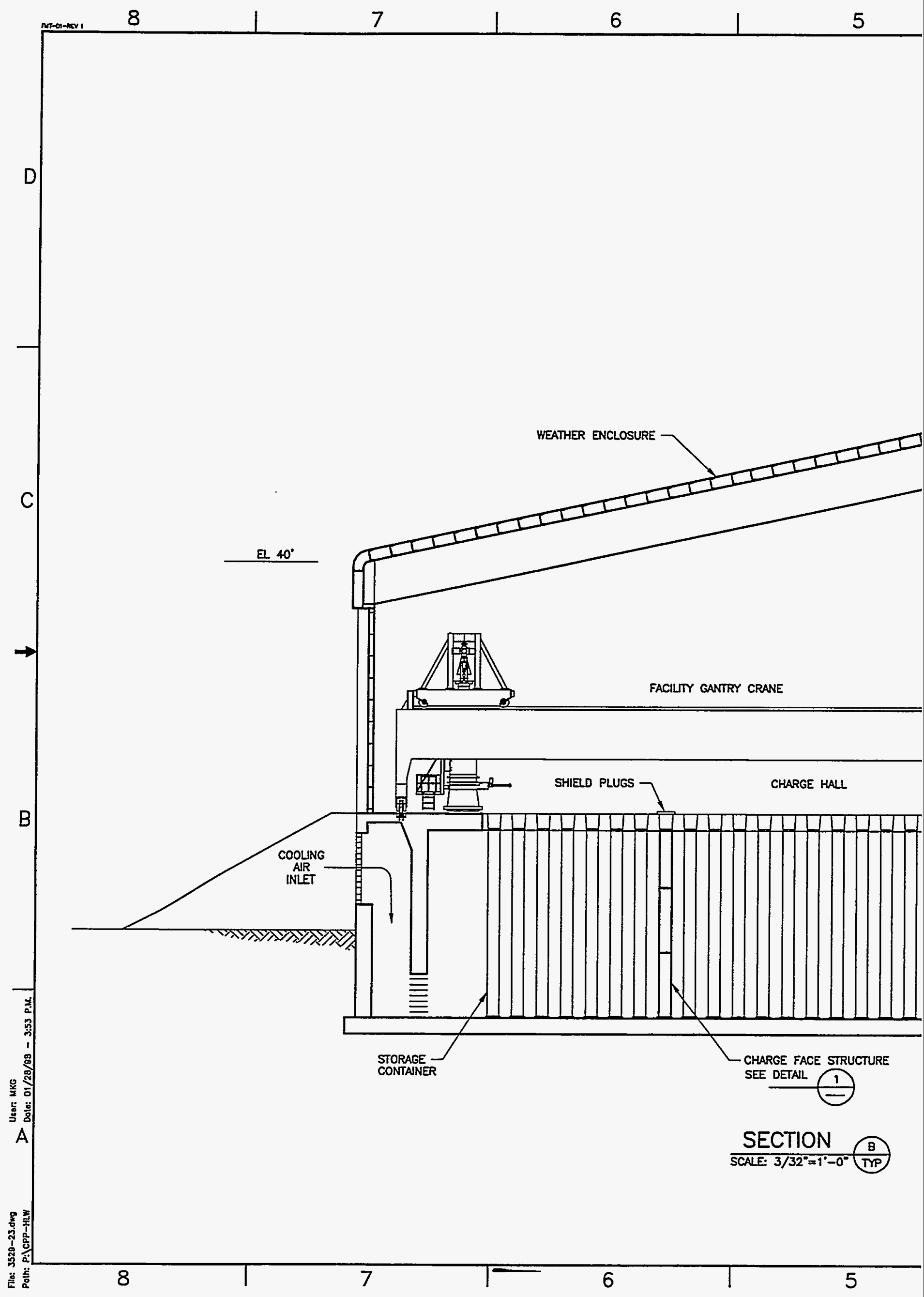




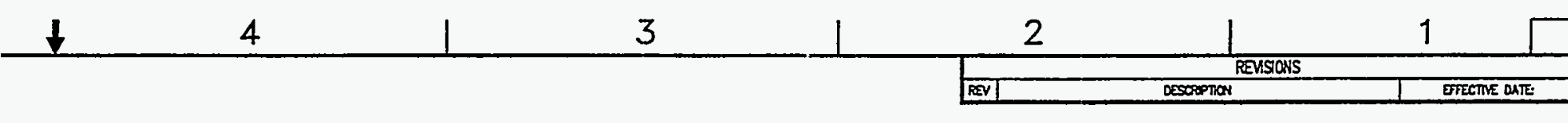
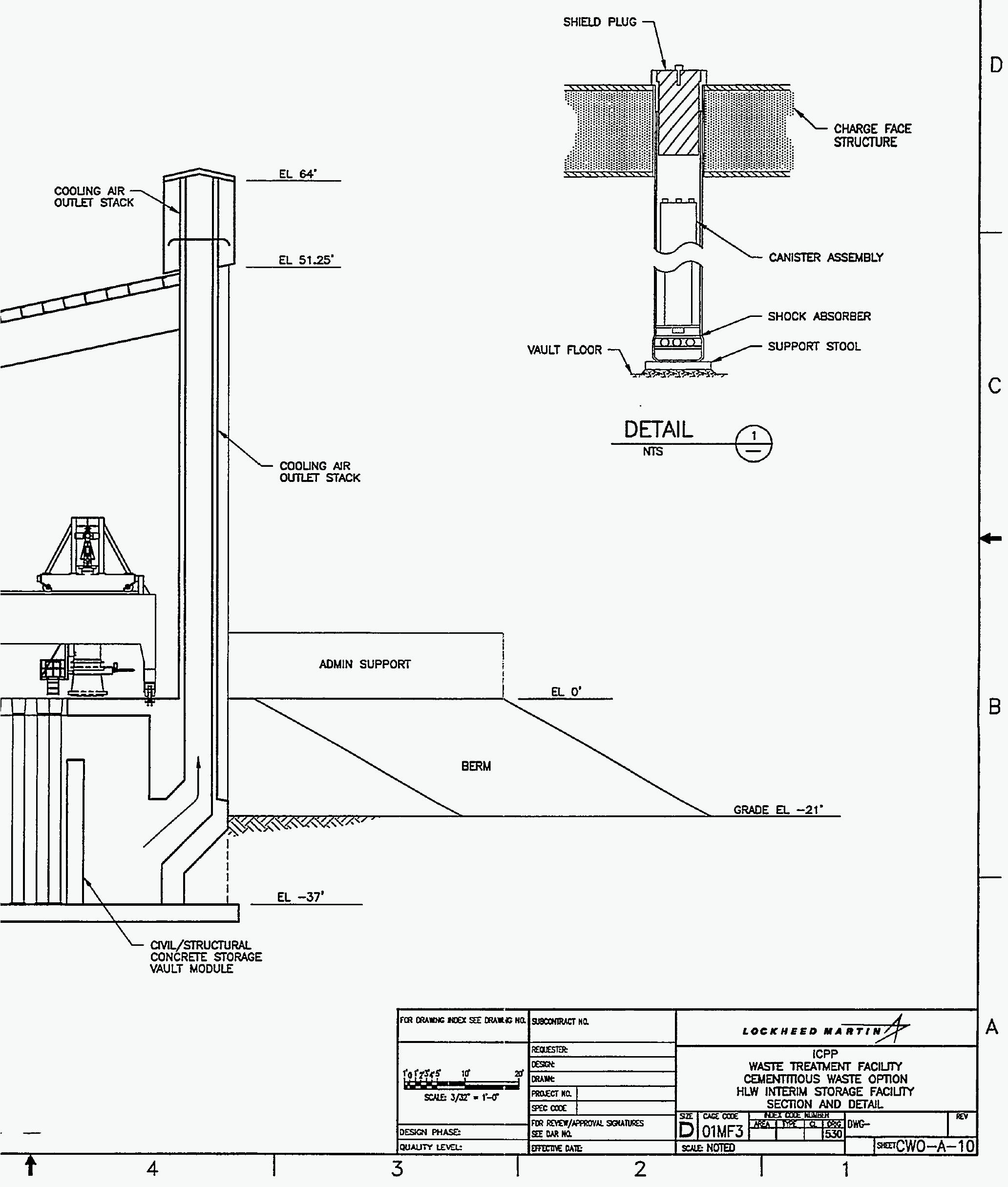


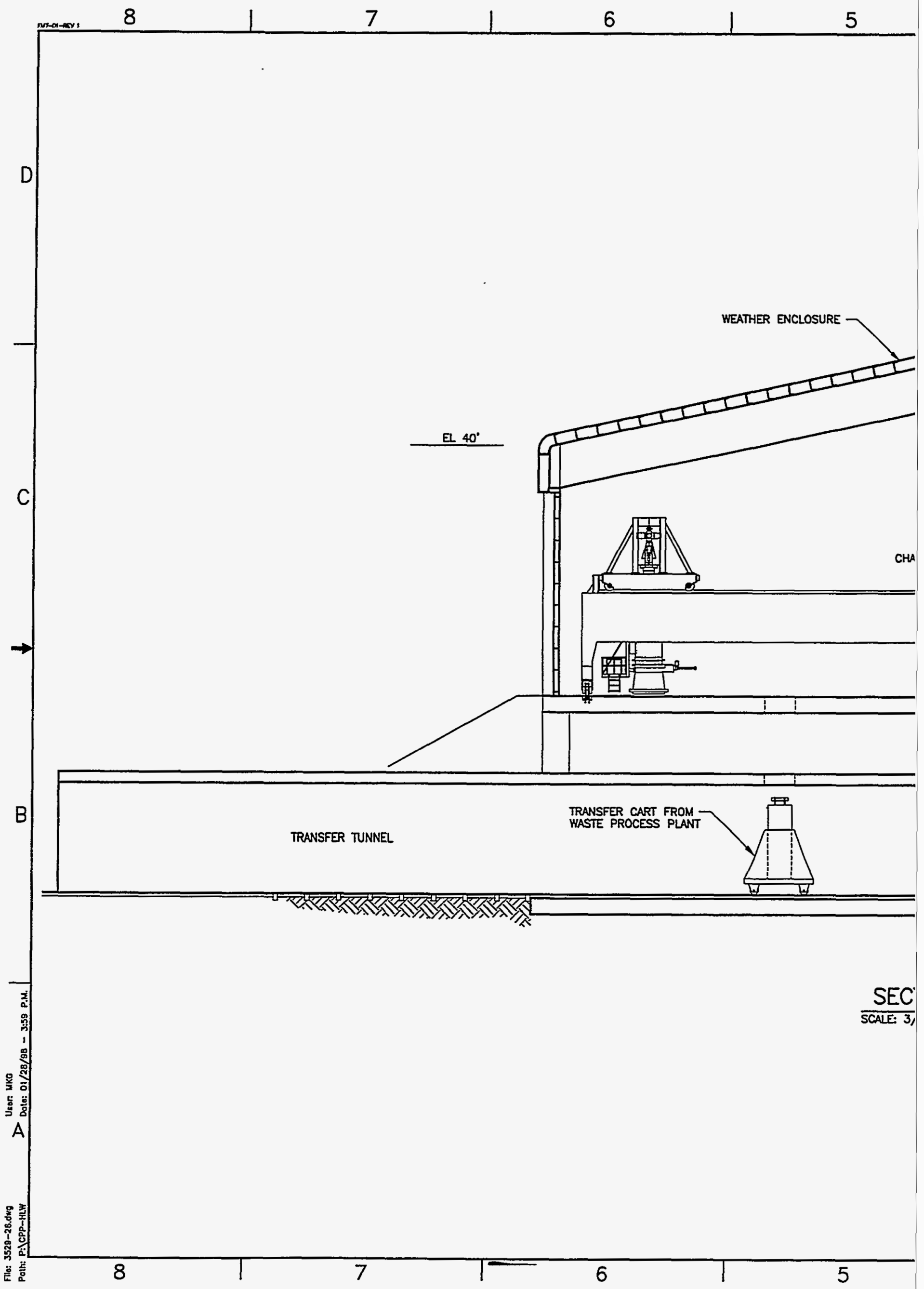




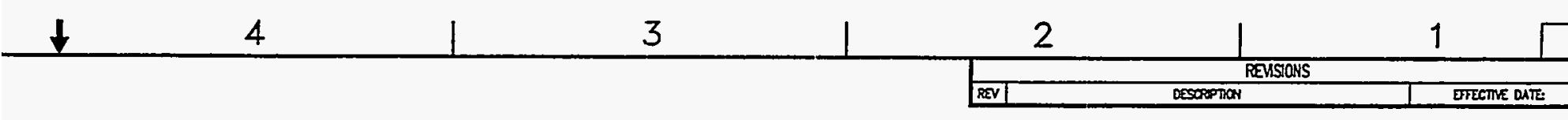

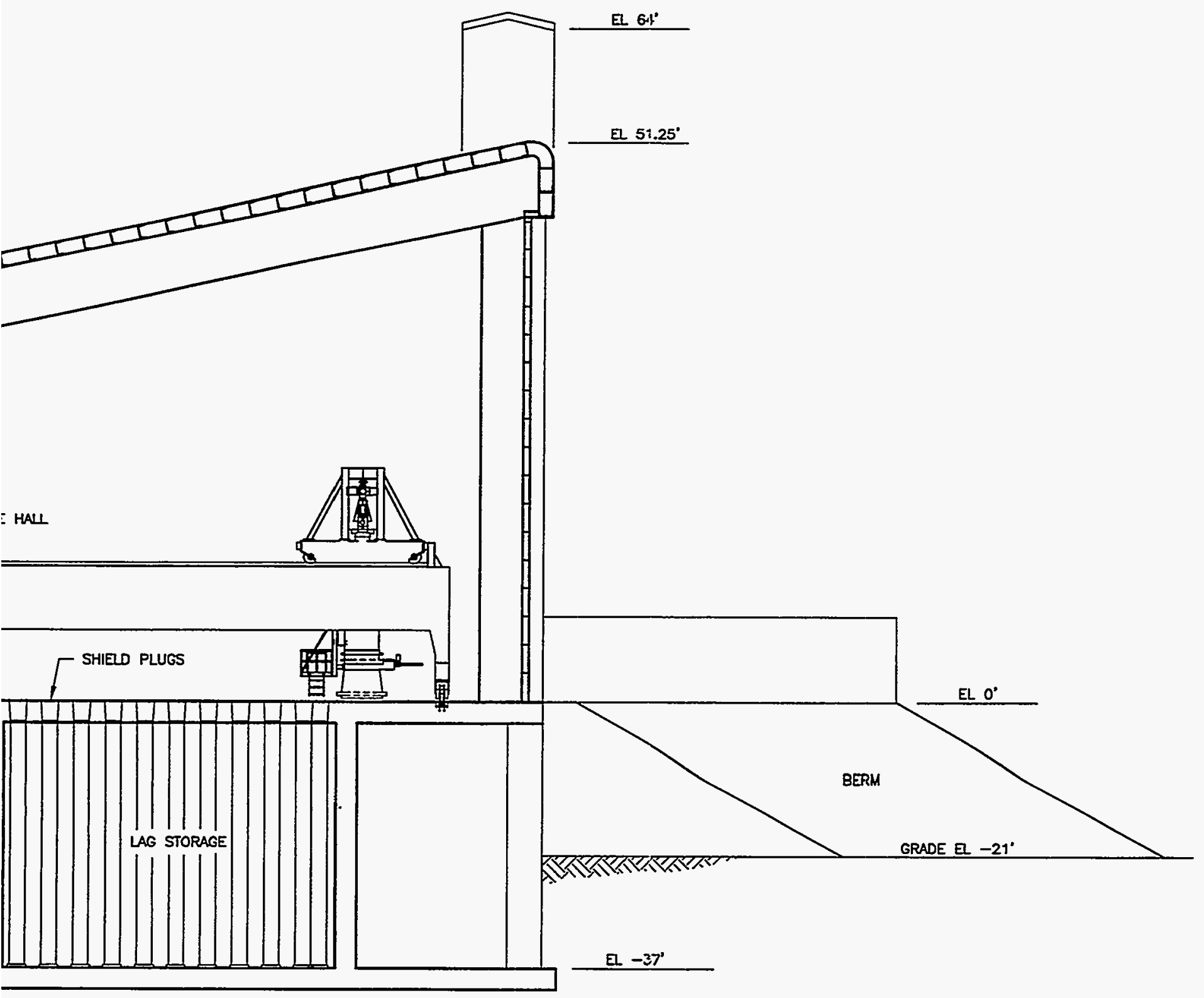

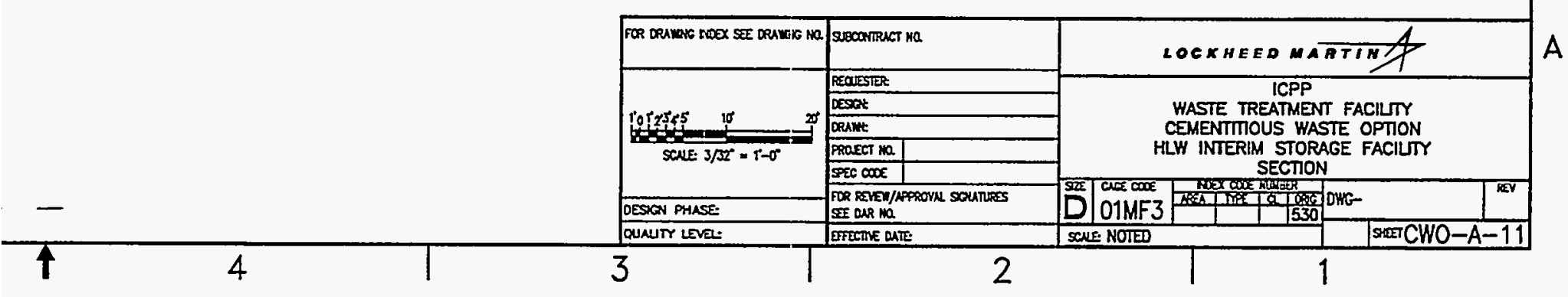




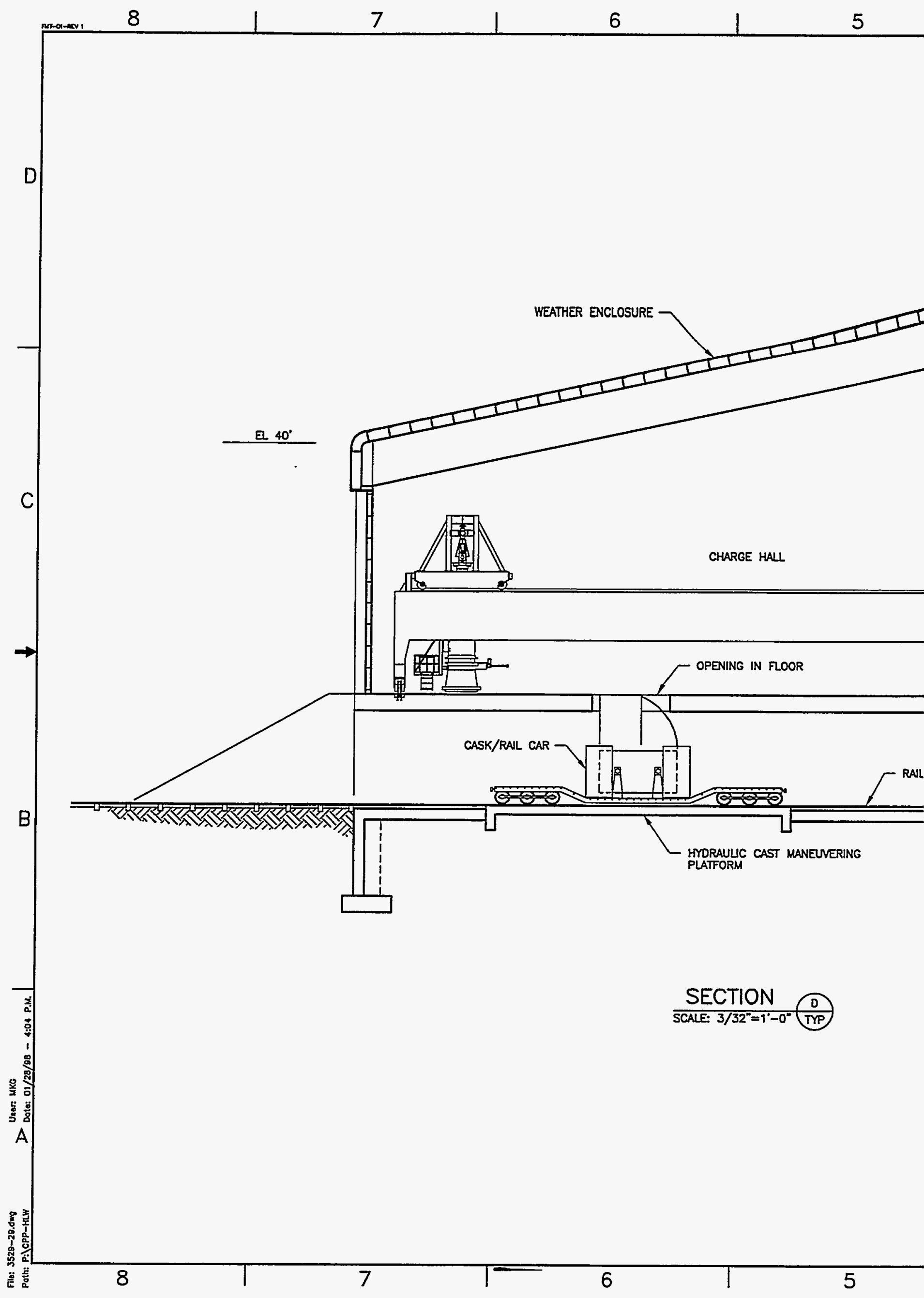




\begin{tabular}{|c|c|}
\hline$\sqrt{ }$ & $A$ \\
\hline
\end{tabular}

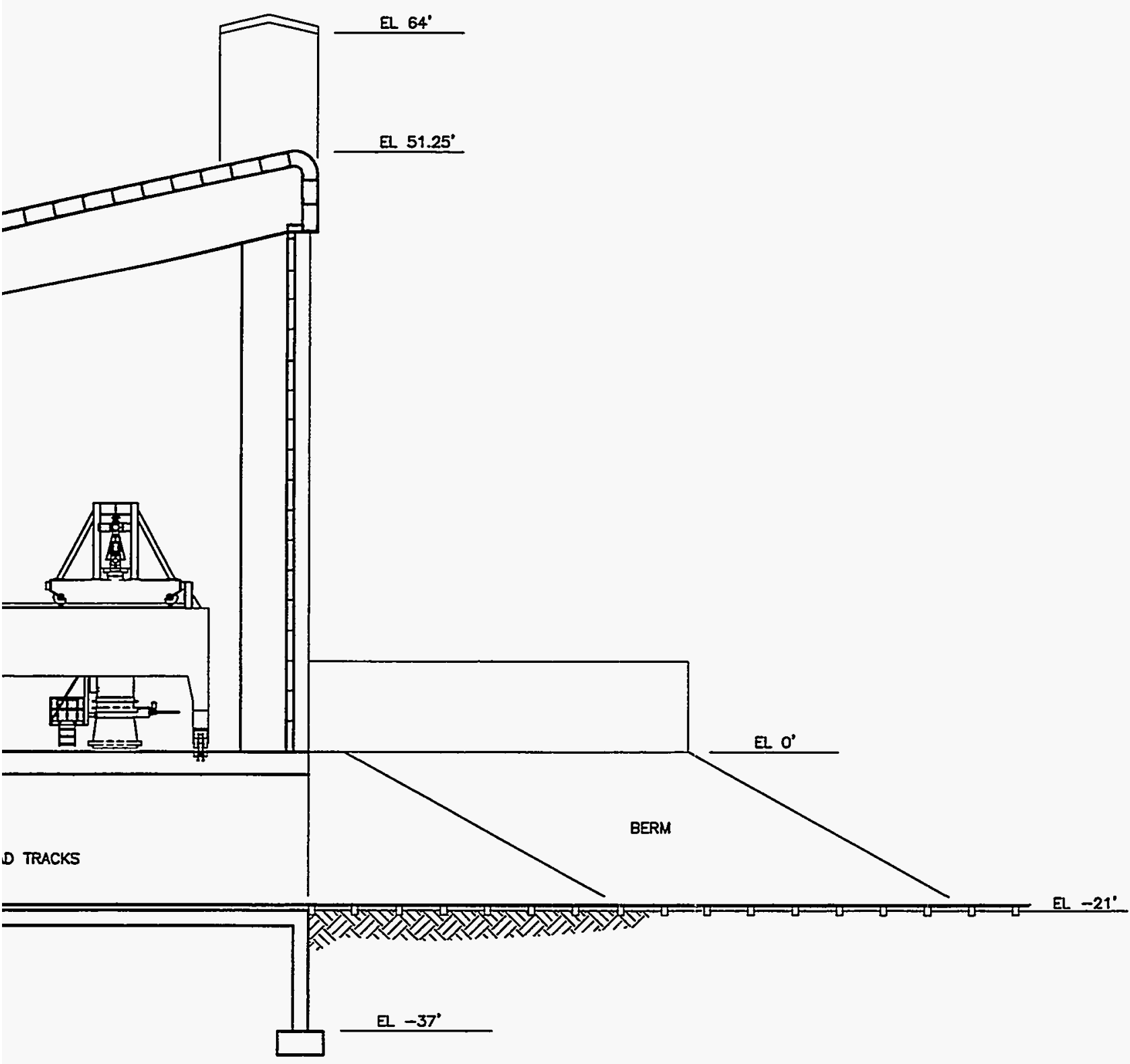

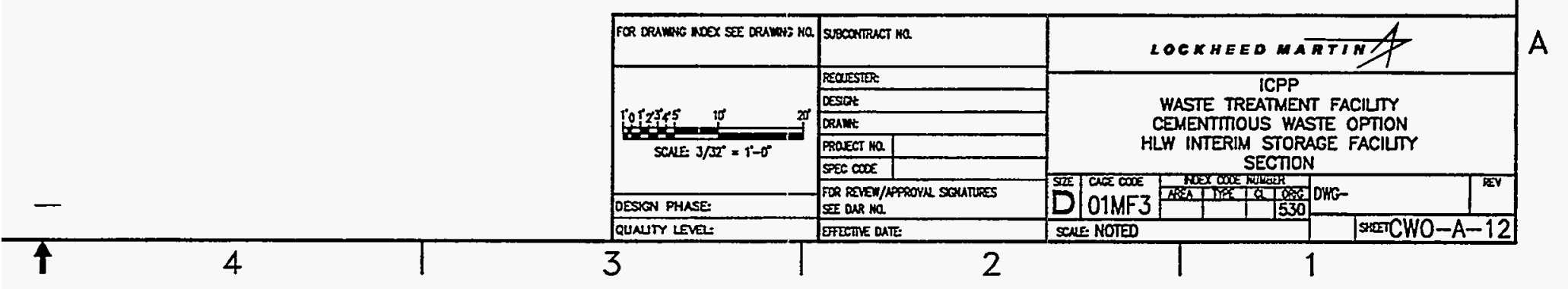




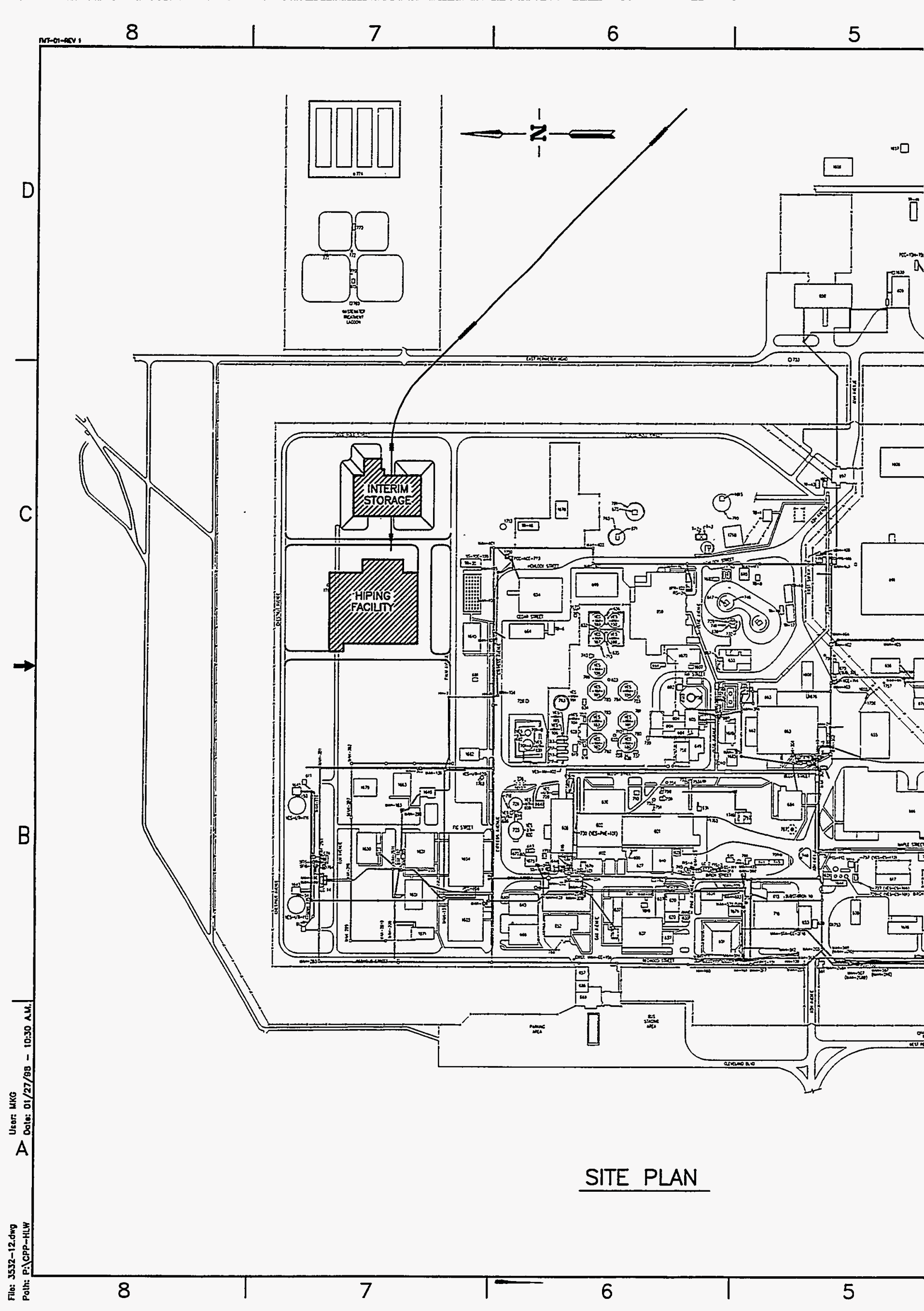




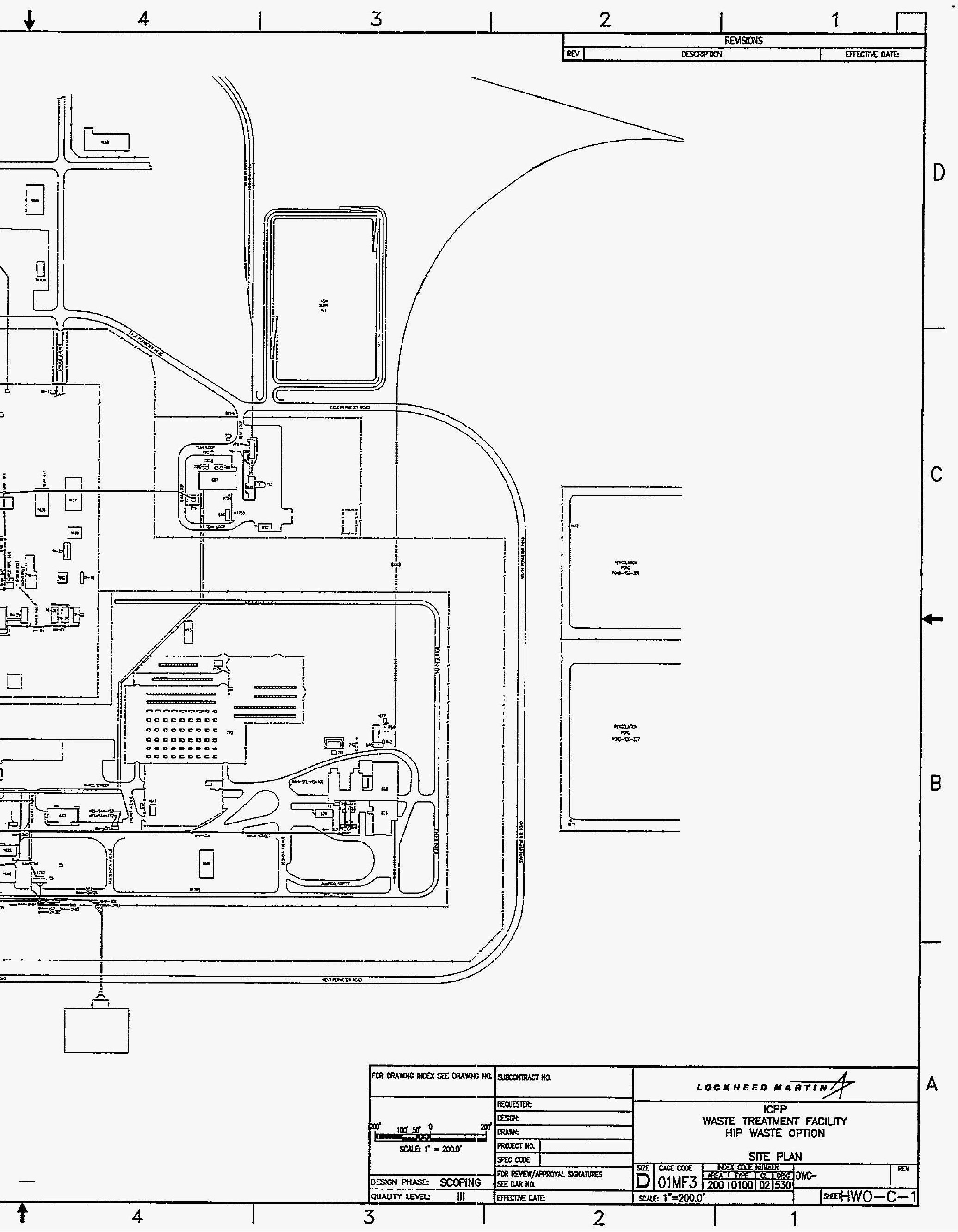




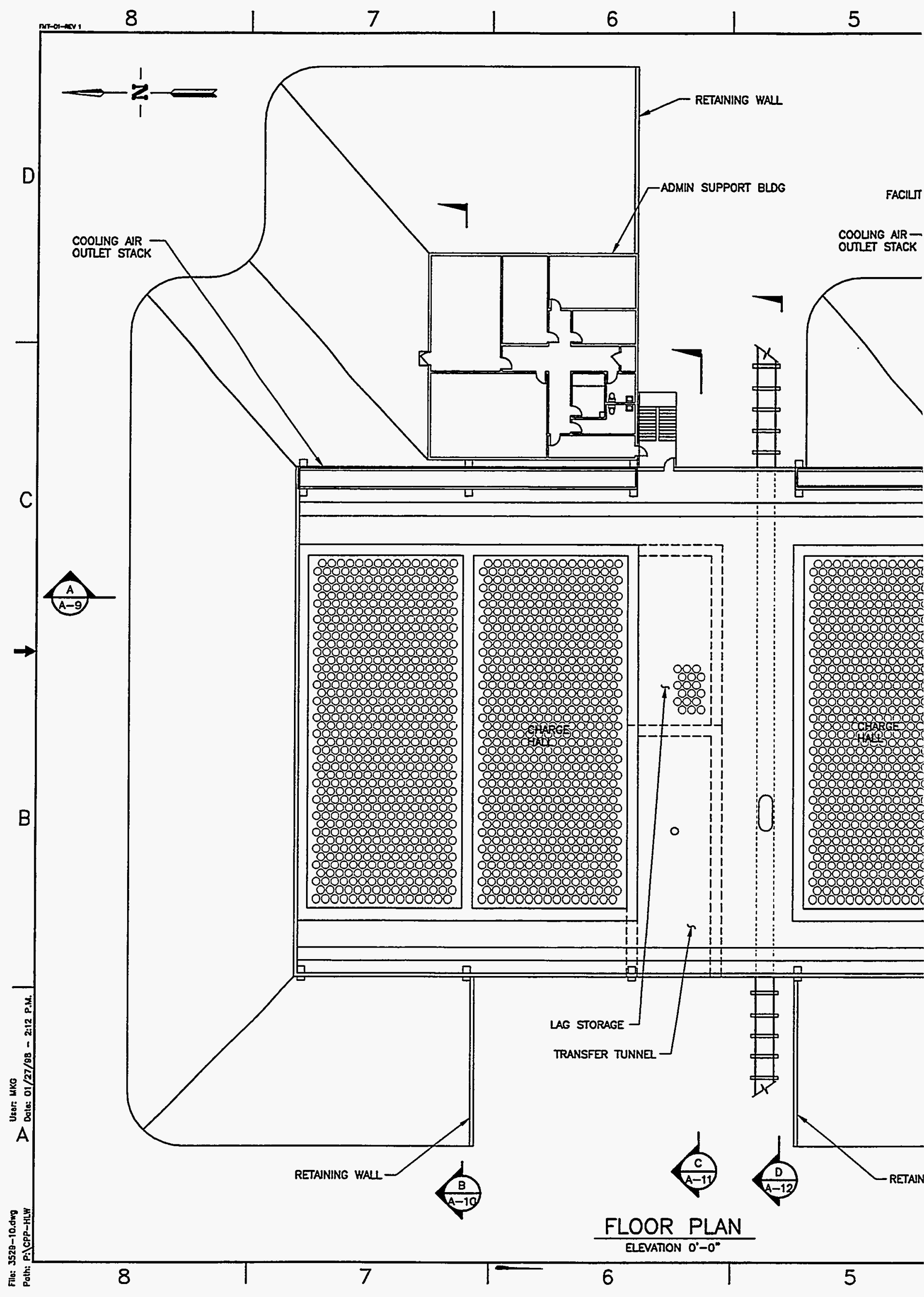




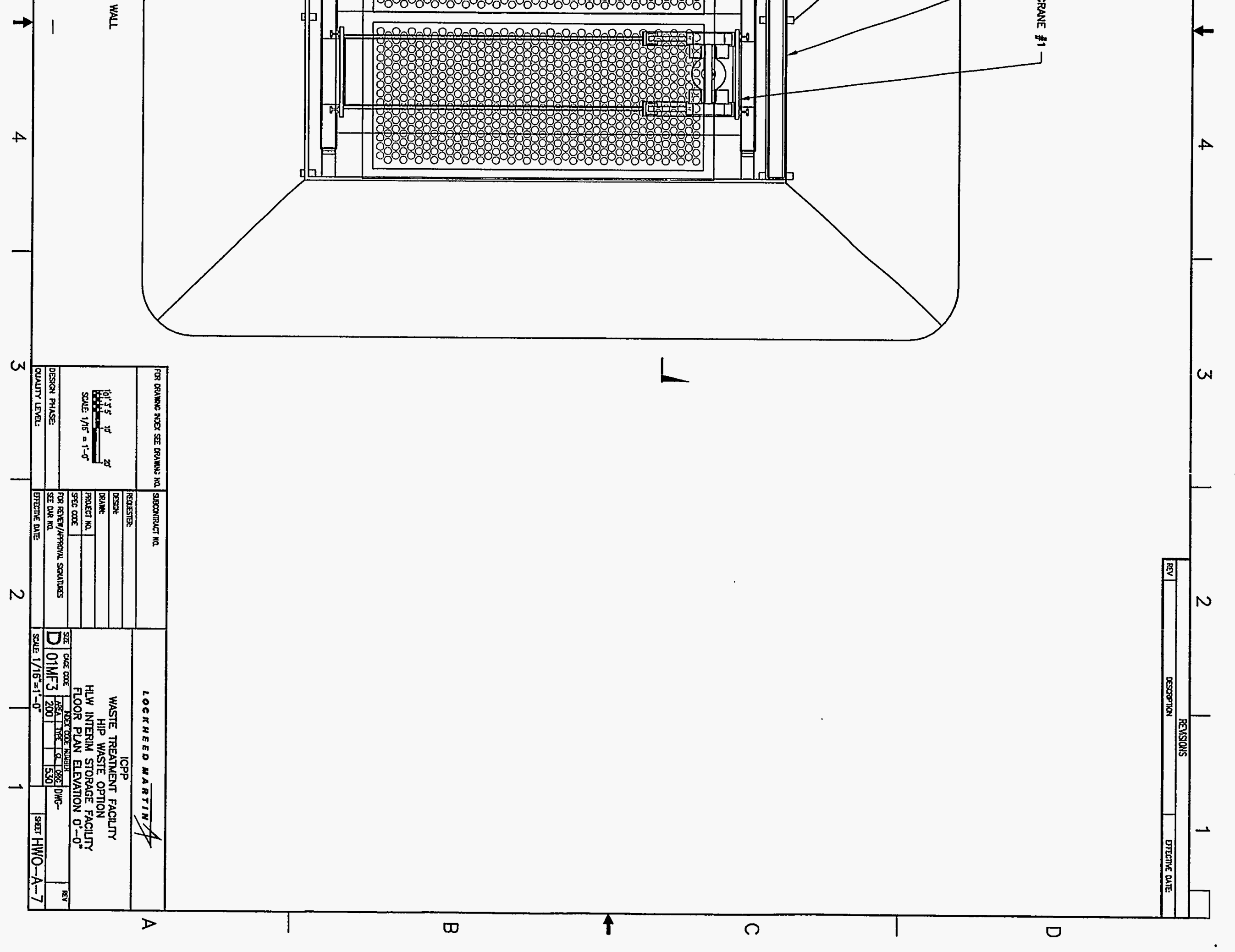




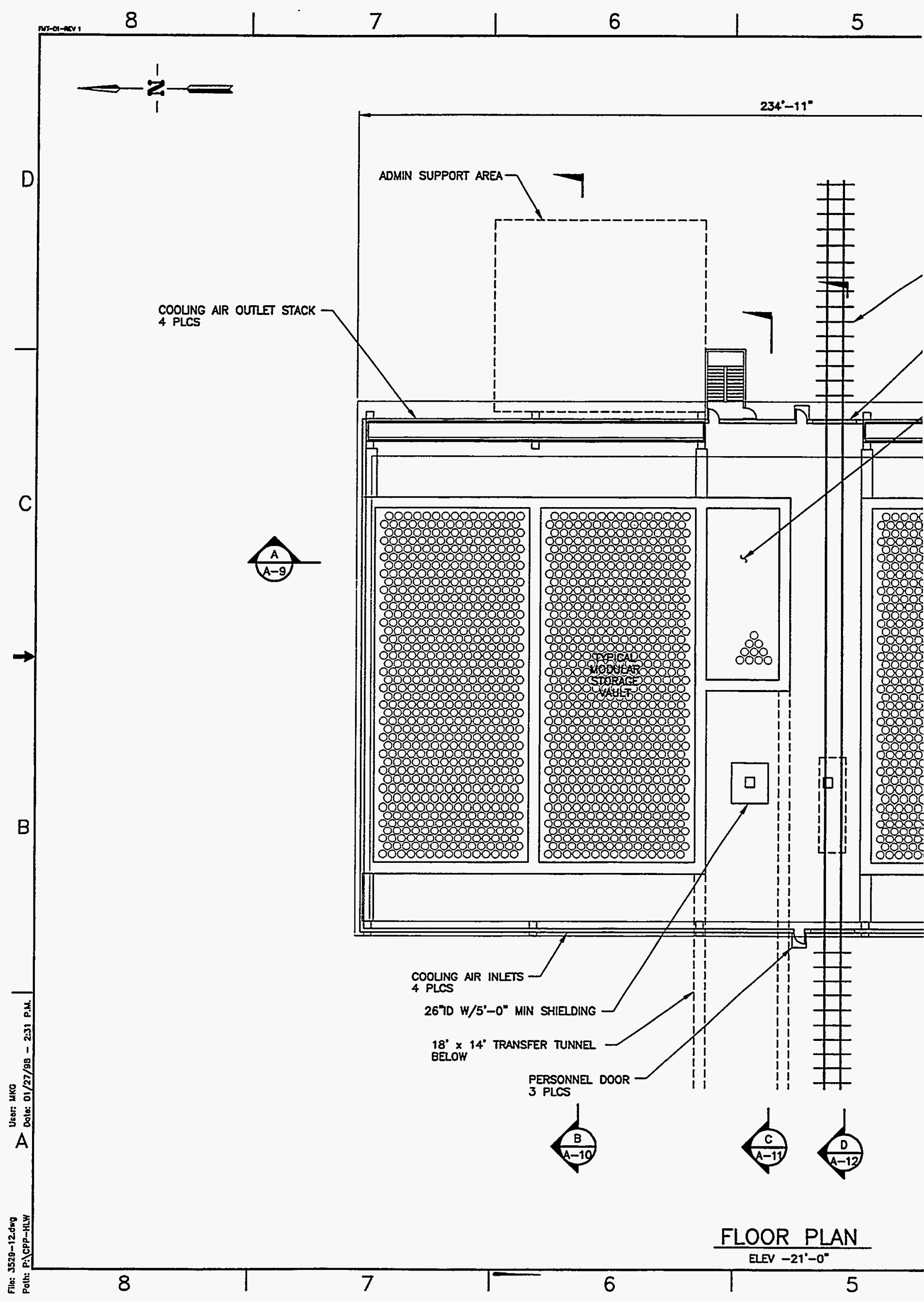




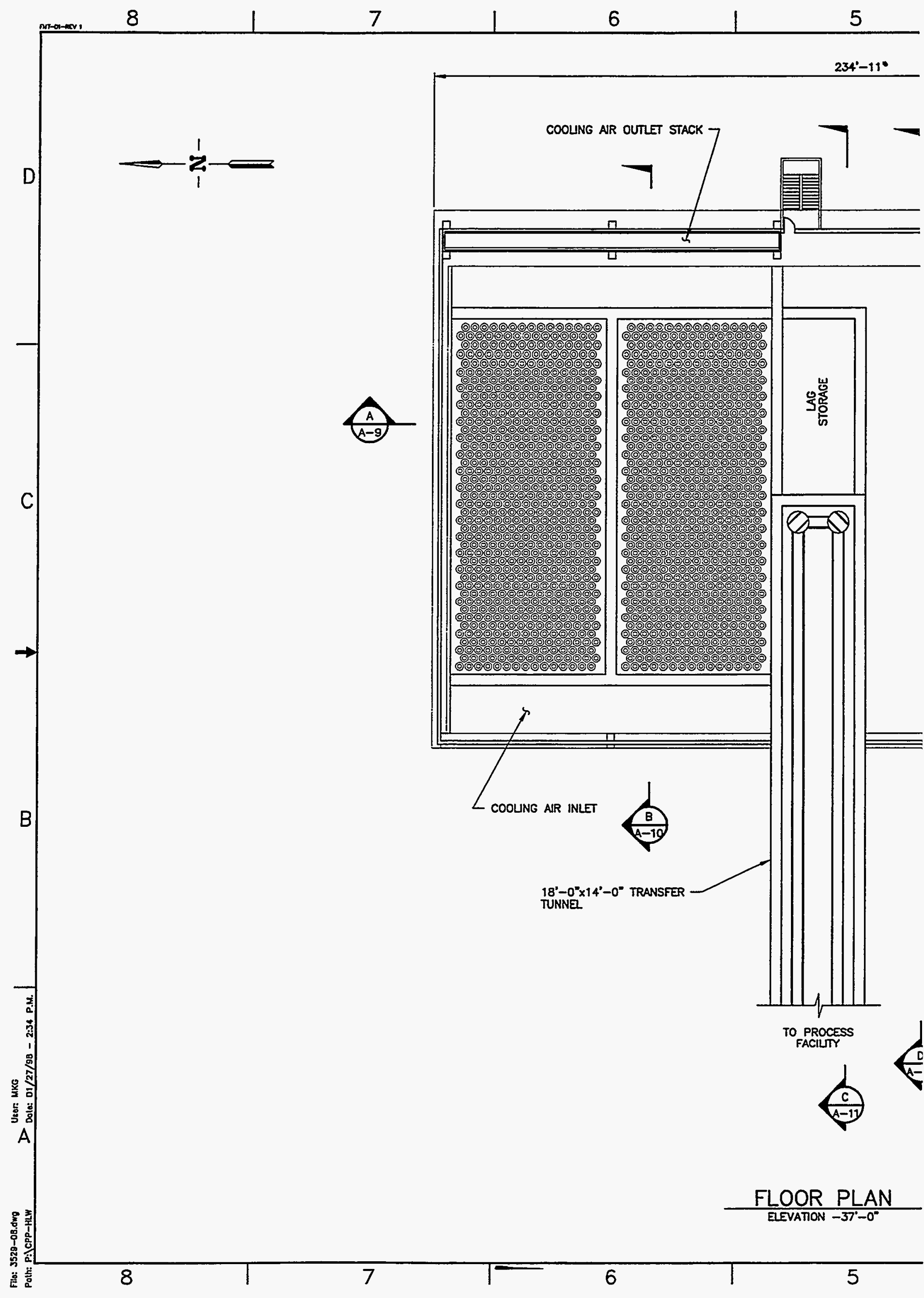




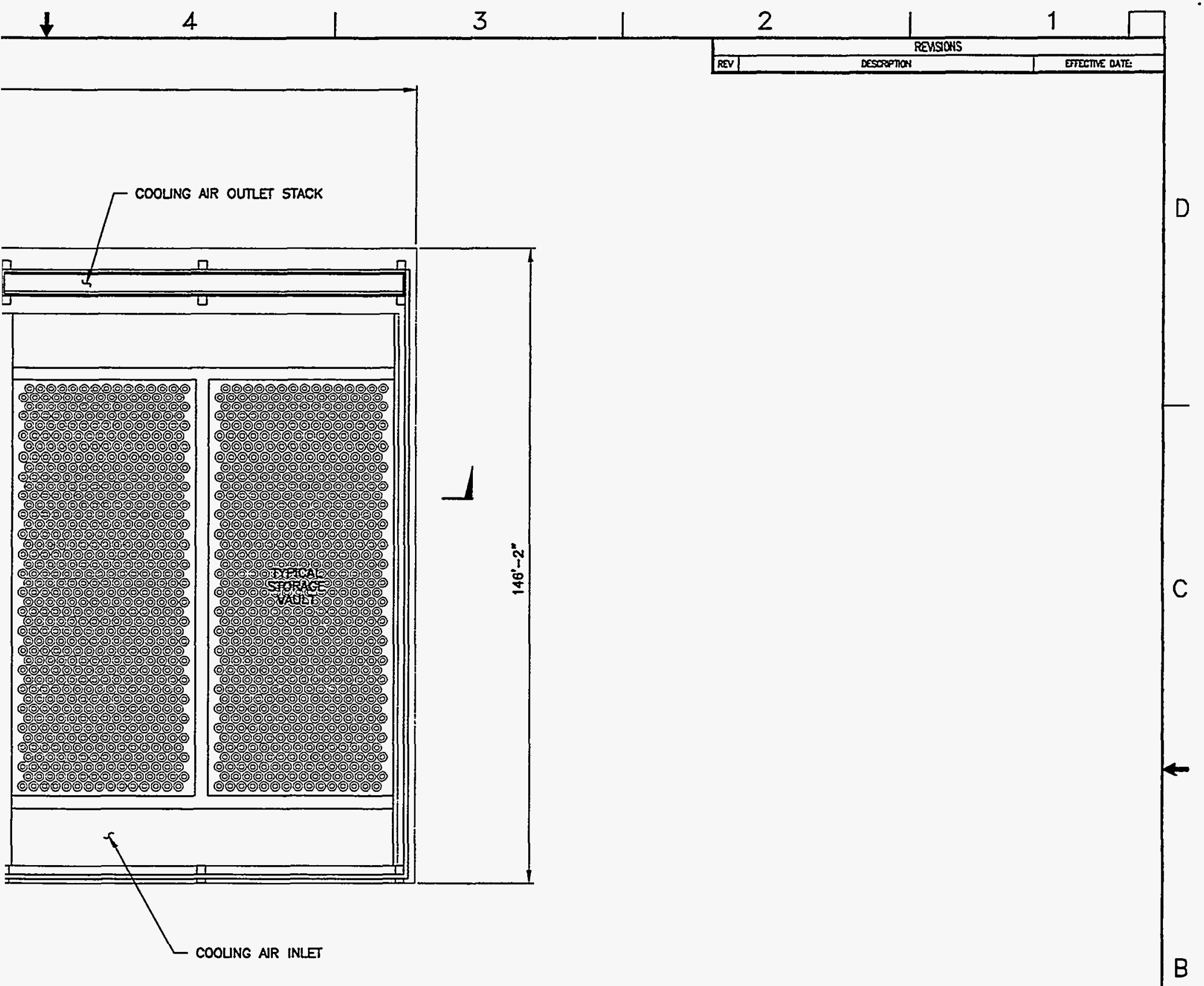




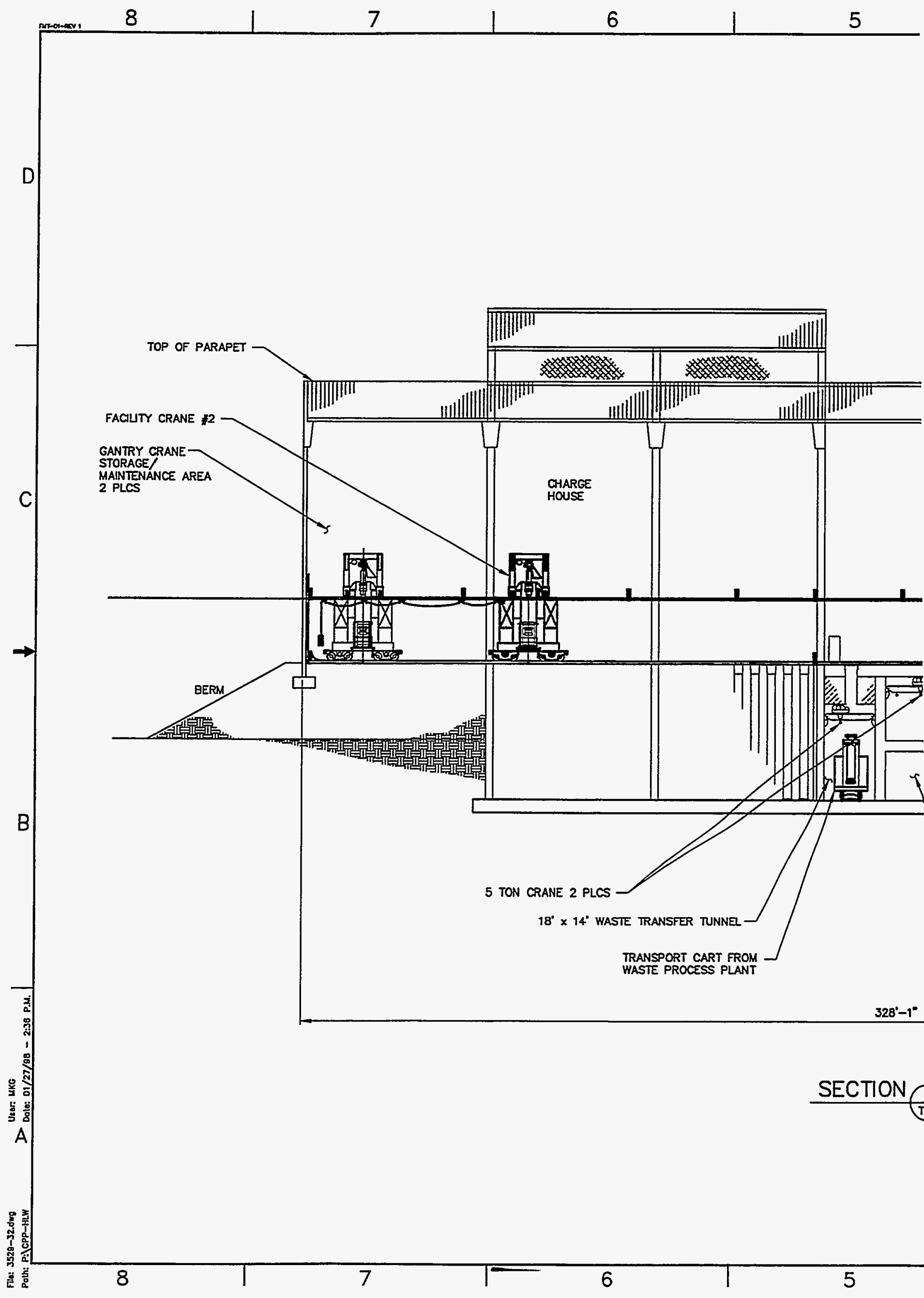




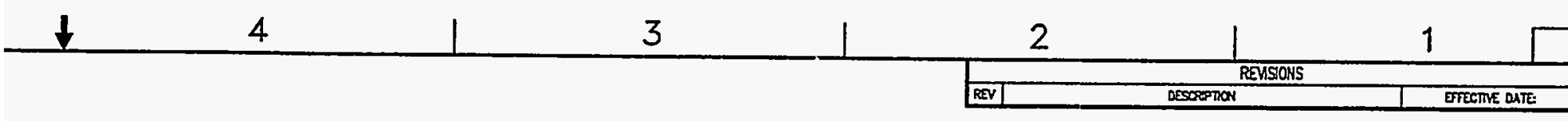

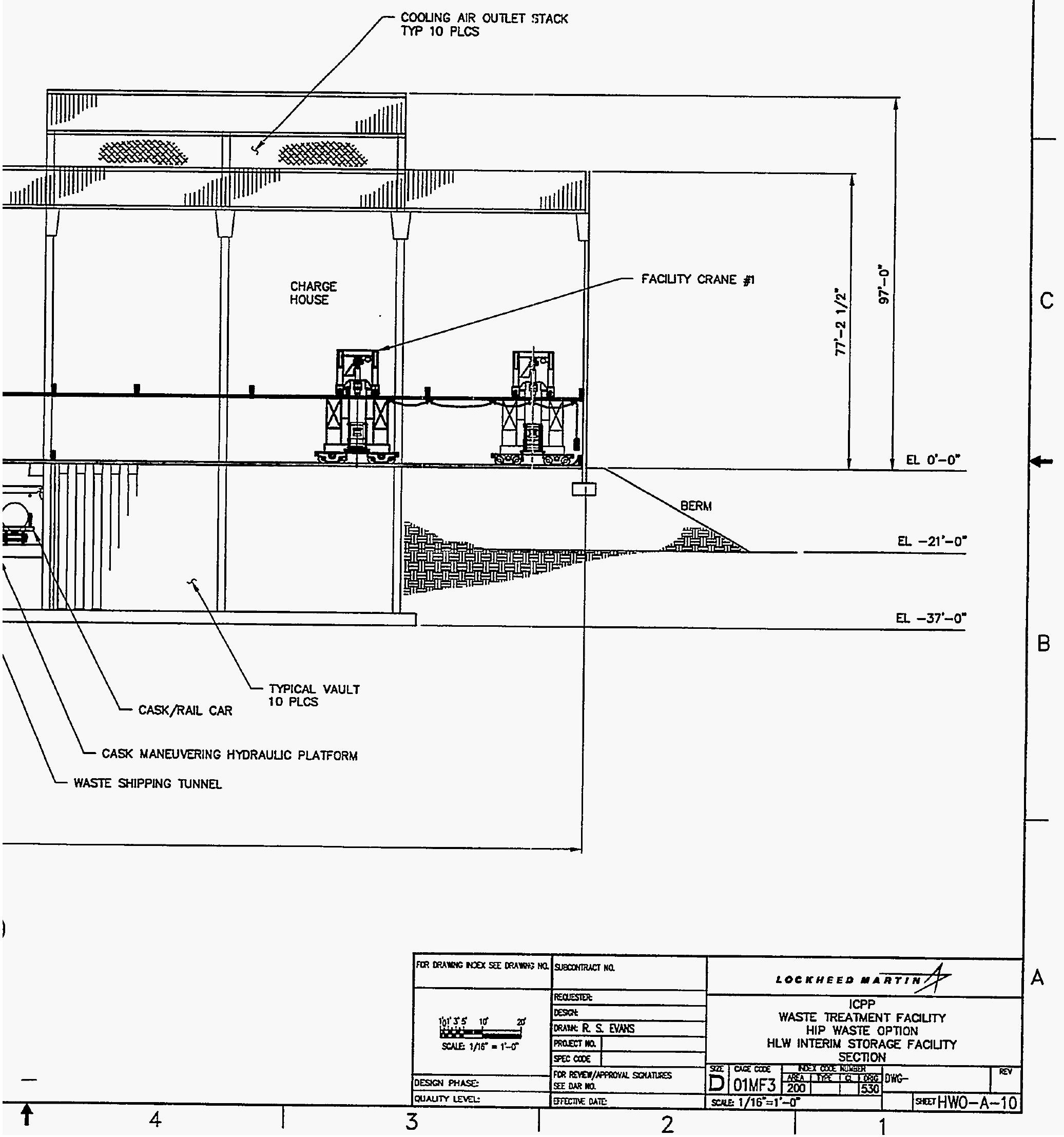




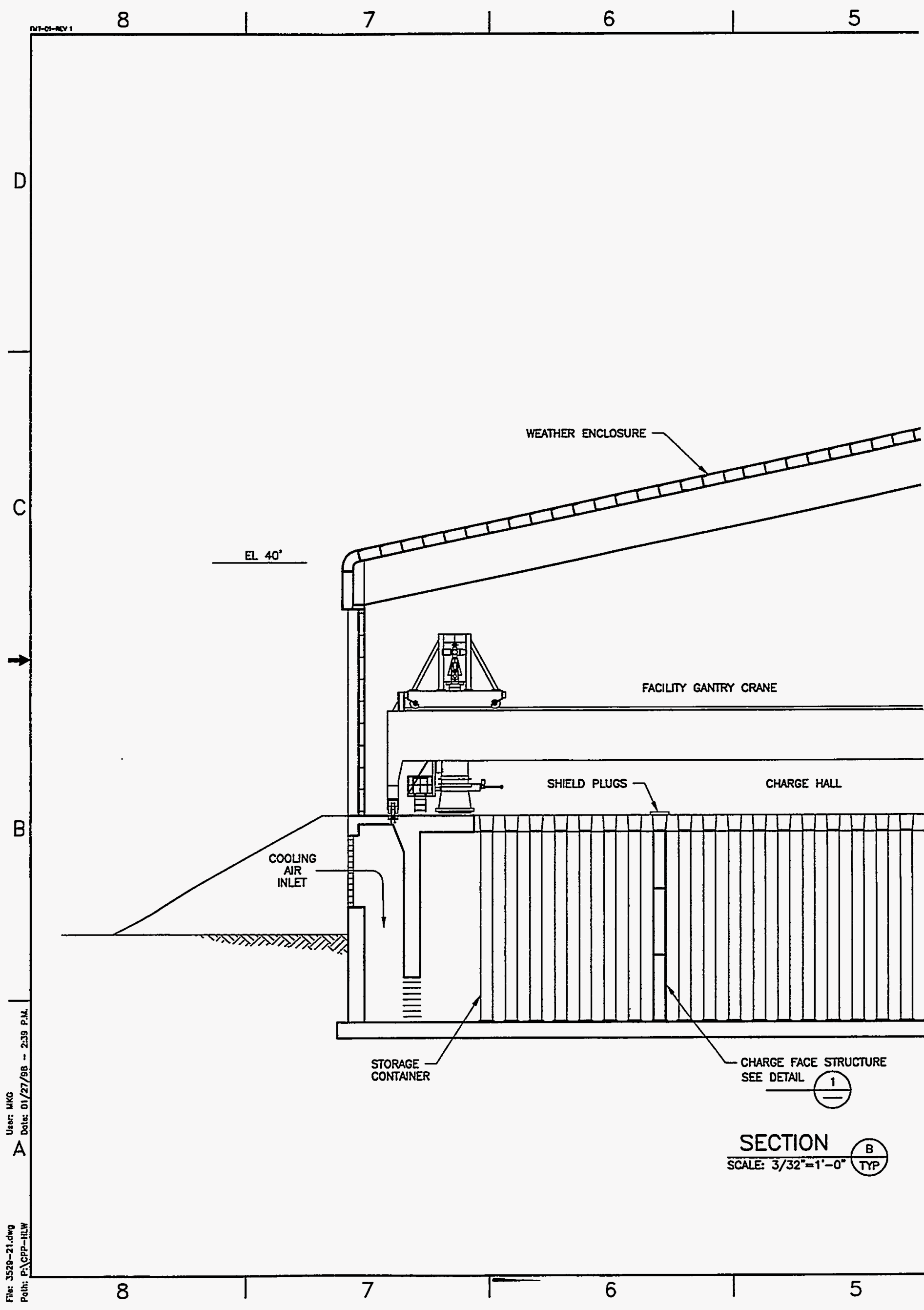




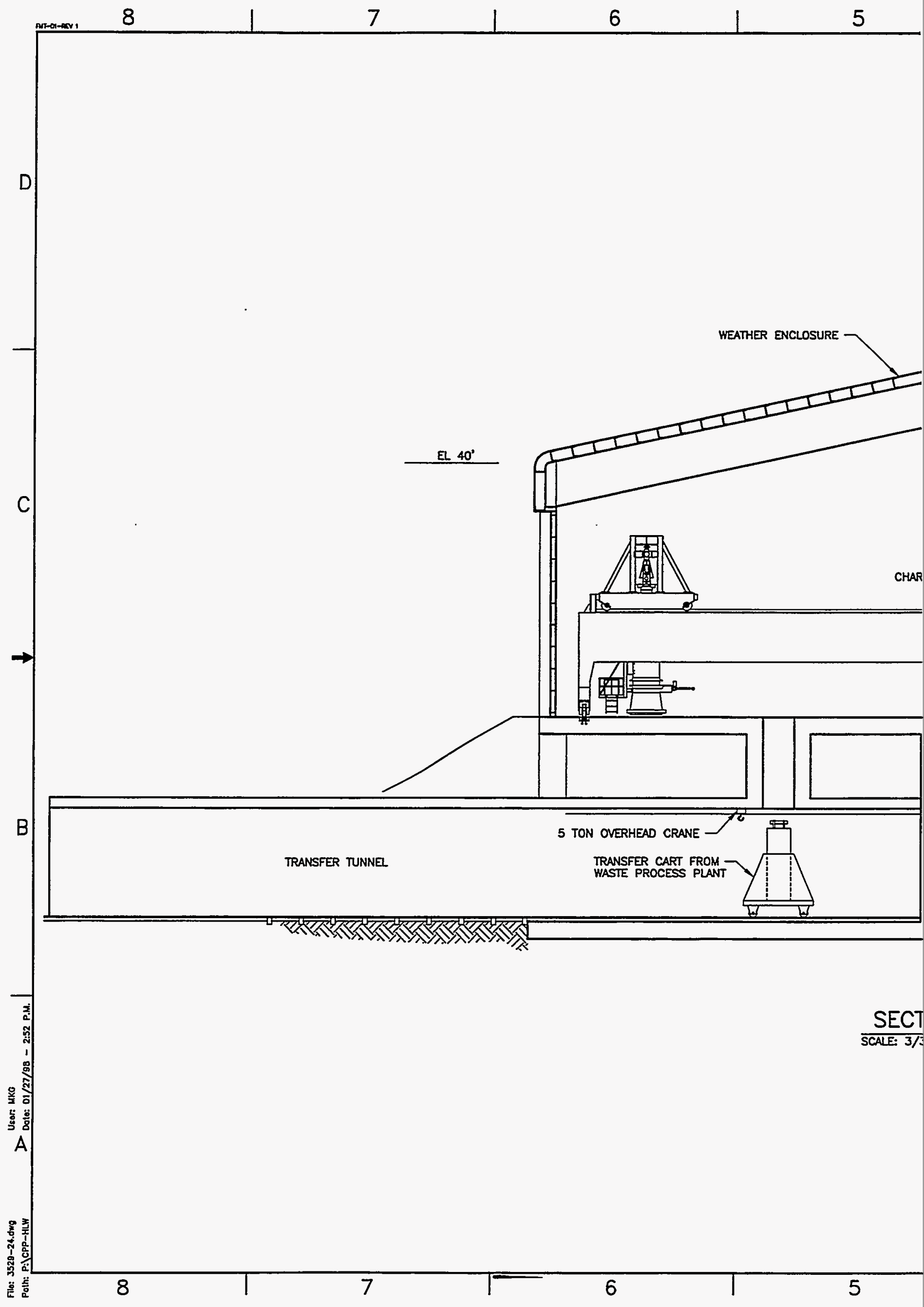




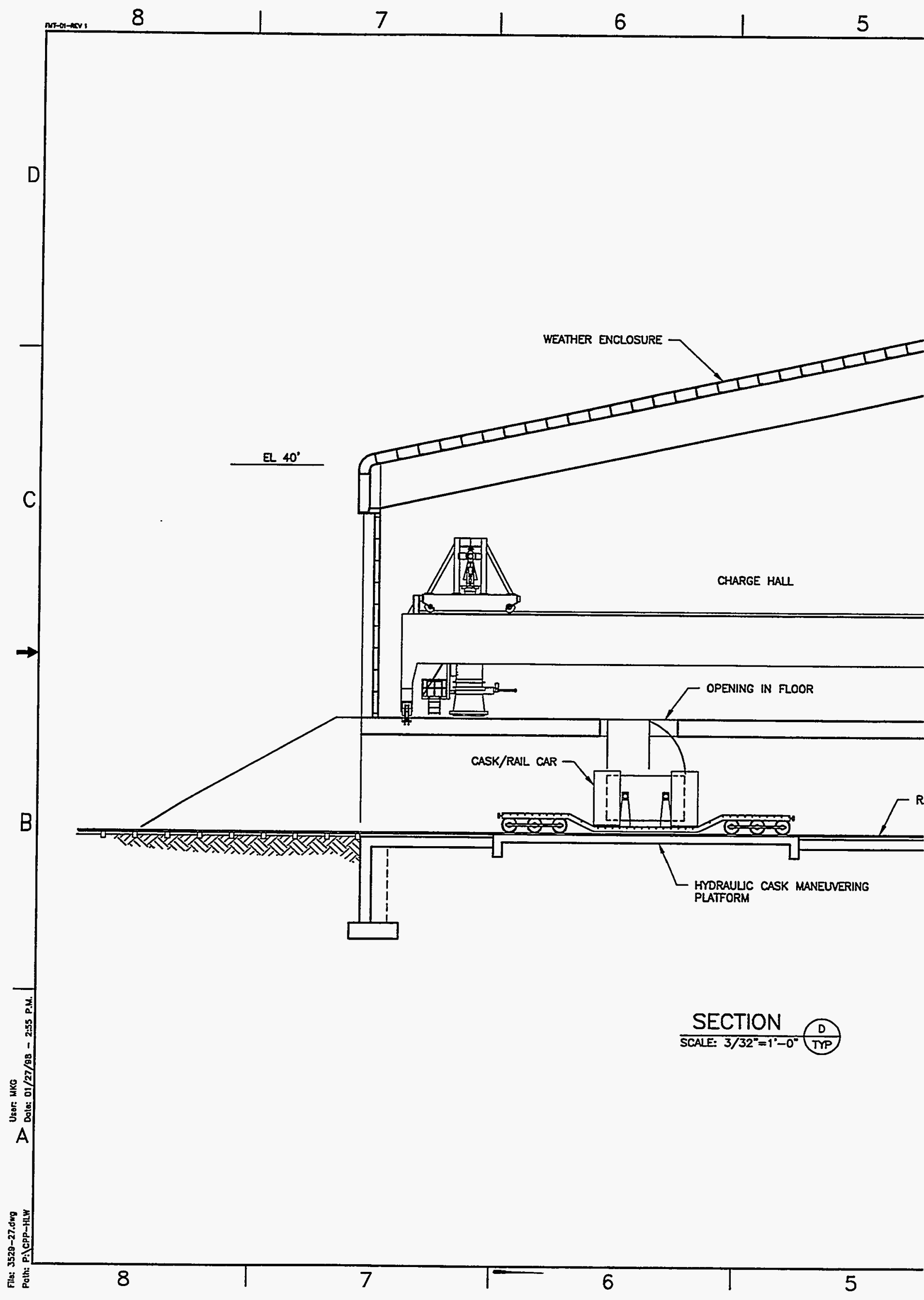




\section{Appendix 3}

\section{Risk Assessment/Data Sheets}




\section{RISK ASSESSMENT SUMMARY}

This appendix contains a data sheet for each of the following significant risk identified for the Interim Storage Facilities (ISFs):

1. The quantities and characteristics of the waste are different than assumed in the study. Risk $=3$.

2. Waste will not be stored in the Savanna River Site (SRS) canister. Risk $=2$.

3. The facility would have to be redesigned if the waste is classified as a RCRA waste after processing. Risk $=3$.

4. A storage canister will be mishandled or dropped during waste transfer to, from, or in the facility. Risk $=2$.

5. The facility might not be operational when needed. Risk $=2$.

As risks were evaluated, the probability of occurrence and the impact if the risk is realized were rated. The probability and impact were assigned numerical values of 3 for high, 2 for medium, and 1 for low, as shown on the data sheets. The equation for risk calculation is Risk = Probability $x$ Impact. The maximum risk rating is therefore 3 . The risk matrix in Figure 3-1 shows what action to take as a function of the risk rating; these actions are defined in Table 31.

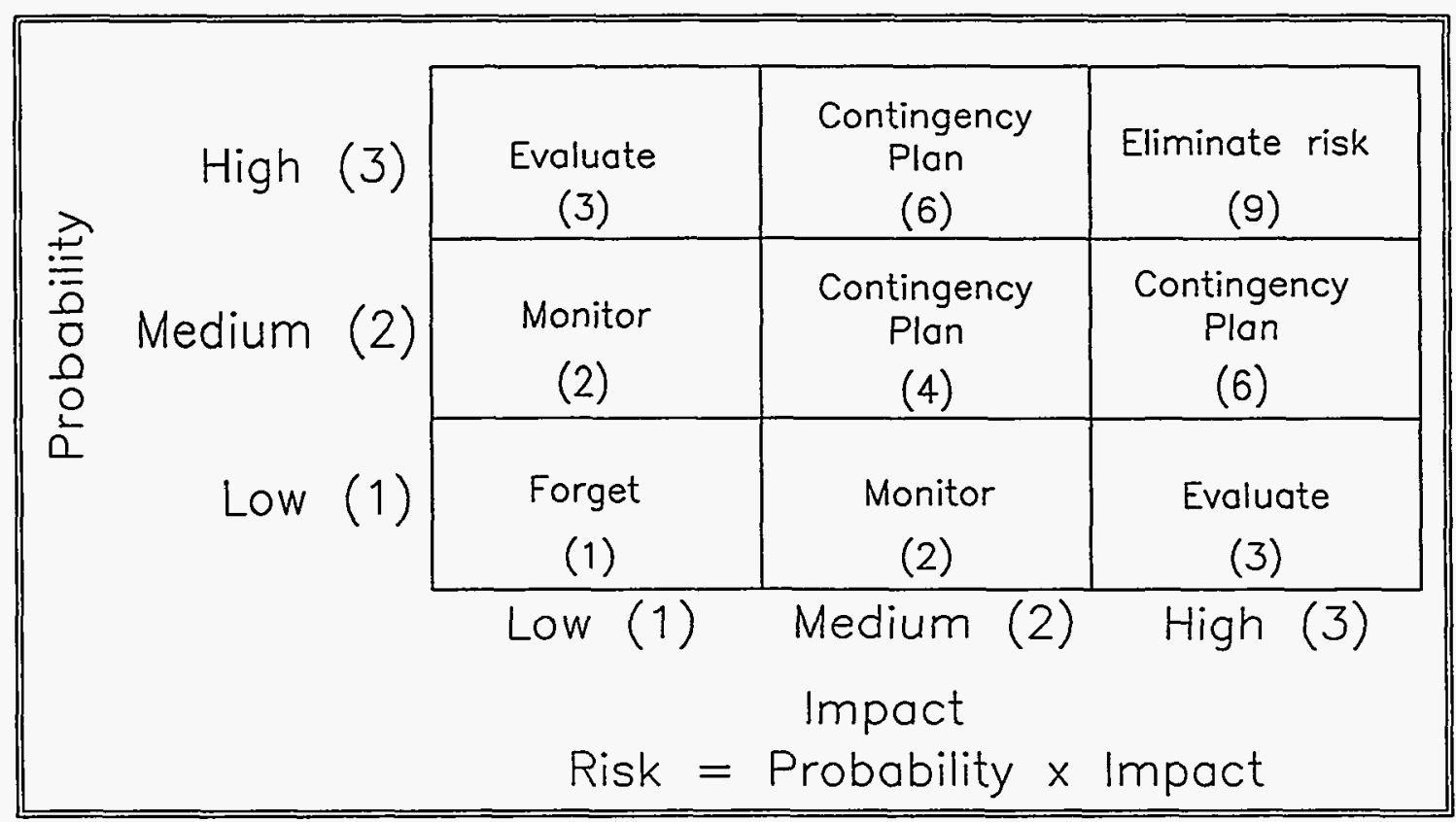

Figure 3-1. Risk Matrix. 
Table 3-1. Risk Action Definition.

Risk

Rating

Action

9 Any risk determined to be in this category will be mitigated through additional design or analysis until the risk is no longer in this category.

6 and 4 Risks in this category will be mitigated to the extent feasible within cost and schedule guidelines. For those risks that cannot be mitigated, contingency plans have been made to deal with the risk if it occurs.

3 Risks in this category will be evaluated during the project to identify if they are becoming problems.

2 These risks will be monitored, with no specific action identified.

1 These risks are noted for interest only. 
Risk Assessment - Data Sheet

Risk Type OProject O Technical OESH

RISK:

PREVENTIVE PLANS

assumed in the study

Interim storage facility sized to most current referenced information

The new interim storage facility is based on a modular design

concept allowing for additional storage to be readily incorporated

\section{PROBABLE CAUSE(S):}

Scoping study level of preliminary information

\section{CONSEQUENCES IF RISK NOT RESOLVED}

The facility design might have to be adjusted

\section{PROBABILITY}
(-) High (3)
Medium (2)
$O$ Low (1)

\section{IMPACT}

\begin{tabular}{|ll|}
\hline$\bigcirc$ & High (3) \\
$\bigcirc$ & Medium (2) \\
$\odot$ & Low (1) \\
\hline
\end{tabular}

\section{CONTINGENCY PLAN(S) IF RISK IS REALIZED:}

Redesign of facility during conceptual and title phases

\section{Risk $=3$}

Risk $=$ Probability $x \operatorname{lmpact}$

\section{Probability Definition}

High - Likely to occur during the project.

Medium - Has the potential to occur during the project.

Low - Has little potential to occur during the project.

\section{Impact Definition}

High - Likely to cause significant disruption of schedule, increase in cost, or degradation of performance.

Medium - Has the potential to cause some disrutpion to schedule, increase in cost, or degradation of performance.

Low - Has little potential to cause disruption to schedule, increase in cost, or degradation of performance.

\section{TRIGGER POINT(S) FOR EARLY RISK} IDENTIFICATION:

Evaluate continuing studies for facility design adequacy 
Risk Assessment - Data Sheet

Risk Type OProject ○Technical OESH

\begin{tabular}{l|l} 
RISK: & PREVENTIVE PLANS \\
\hline
\end{tabular}

Waste will not be stored in the Savannah River Canister

The facility is designed to be flexible. The new facility design would be adjusted to accept the new canister

PROBABLE CAUSE(S):

CONSEQUENCES IF RISK NOT RESOLVED

Different canister identified due to additional study

\begin{tabular}{|l|l|}
\hline PROBABILITY & IMPACT \\
\hline$\bigcirc$ & High (3) \\
$\bigcirc$ & Medium (2) \\
$\bigcirc$ & Low (1) \\
\hline
\end{tabular}

CONTINGENCY PLAN(S) IF RISK IS REALIZED:

Facility redesign during conceptual and title phases.

\section{Risk $=2$}

Risk $=$ Probability $\times$ Impact

Probability Definition

High - Likely to occur during the project.

Medium - Has the polential to occur during the project.

Low - Has little potential to occur during the project.

Impact Definition

High - Likely to cause significant disruption of schedule, increase in cost, or degradation of performance.

Medium - Has the potential to cause some disrutpion to schedule. increase in cost, or degradation of performance.

Low - Has little potential to cause disruption to schedule, increase in cost, or degradation of performance.

\section{TRIGGER POINT(S) FOR EARLY RISK} IDENTIFICATION:

Monitor continuing studies to identify the canister to be selected 


\section{Risk Assessment - Data Sheet}

Risk Type OProject OTechnical OESH

\section{RISK:}

The facility would have to be redesigned if the waste is classified as a RCRA waste after processing

\section{PREVENTIVE PLANS}

None

\section{PROBABLE CAUSE(S):}

The delisting of the waste after processing is not approved

Re-evaluation of the waste determines that it should be classified as RCRA waste after processing

\section{CONSEQUENCES IF RISK NOT RESOLVED}

The facility would have to be redesigned. The costs for the facility would increase.

\section{PROBABILITY}

High (3)

Medium (2)

○ Low (1)
IMPACT

$\begin{array}{ll}\odot & \text { High (3) } \\ \bigcirc & \text { Medium (2) } \\ \bigcirc & \text { Low (1) }\end{array}$

CONTINGENCY PLAN(S) IF RISK IS REALIZED:

None

\section{Risk $=3$}

Risk $=$ Probability $\times$ Impact

\section{Probability Definition}

High - Likely to occur during the project.

Medium - Has the potential to occur during the project.

Low - Has little potential to occur during the project.

\section{Impact Definition}

High - Likely to cause significant disruption of schedule, increase in cost, or degradation of performance.

Medium - Has the potential to cause some disrutpion to schedule, increase in cost, or degradation of performance.

Low - Has little potential to cause disruption to schedule, increase in cost, or degradation of performance.

\section{TRIGGER POINT(S) FOR EARLY RISK} IDENTIFICATION:

Continue to monitor the status of the option to delist the waste 
Risk Assessment - Data Sheet

Risk Type OProject ○ Technical OESH

RISK:

A storage canister will be mishandled or dropped during waste transfer to, from, or in the facility.
PREVENTIVE PLANS

Facility will be designed to be able to recover from these accidents

\section{PROBABLE CAUSE(S):}

Possible scenario include

1. Canister falls from the transfer cart in the tunnel

2. Canister is dropped during transfer from the transfer cart

\section{CONSEQUENCES IF RISK NOT RESOLVED}

Additional design for new equipment

\section{PROBABILITY}

High (3)

$\bigcirc$ Medium (2)

(-) Low (1)

\section{IMPACT}

High (3)

(-) Medium (2)

$\bigcirc \operatorname{Low}(1)$
CONTINGENCY PLAN(S) IF RISK IS REALIZED:

None

Risk $=2$

Risk $=$ Probability $\times 1$ mpact

Probability Definition

High - Likely to occur during the project.

Medium - Has the potential to occur during the project.

Low - Has little potential to occur during the project.

Impact Definition

High - Likely to cause significant disruption of schedule, increase in cost, or degradation of performance.

Medium - Has the potential to cause some disrutpion to schedule, increase in cost, or degradation of performance.

Low - Has little potential to cause disruption to schedule, increase in cost, or degradation of performance.

\section{TRIGGER POINT(S) FOR EARLY RISK}

\section{IDENTIFICATION:}

Ongoing review of the facility during design reviews, SAR preparation, etc. 
Risk Assessment - Data Sheet

Risk Type OProject O Technical OESH

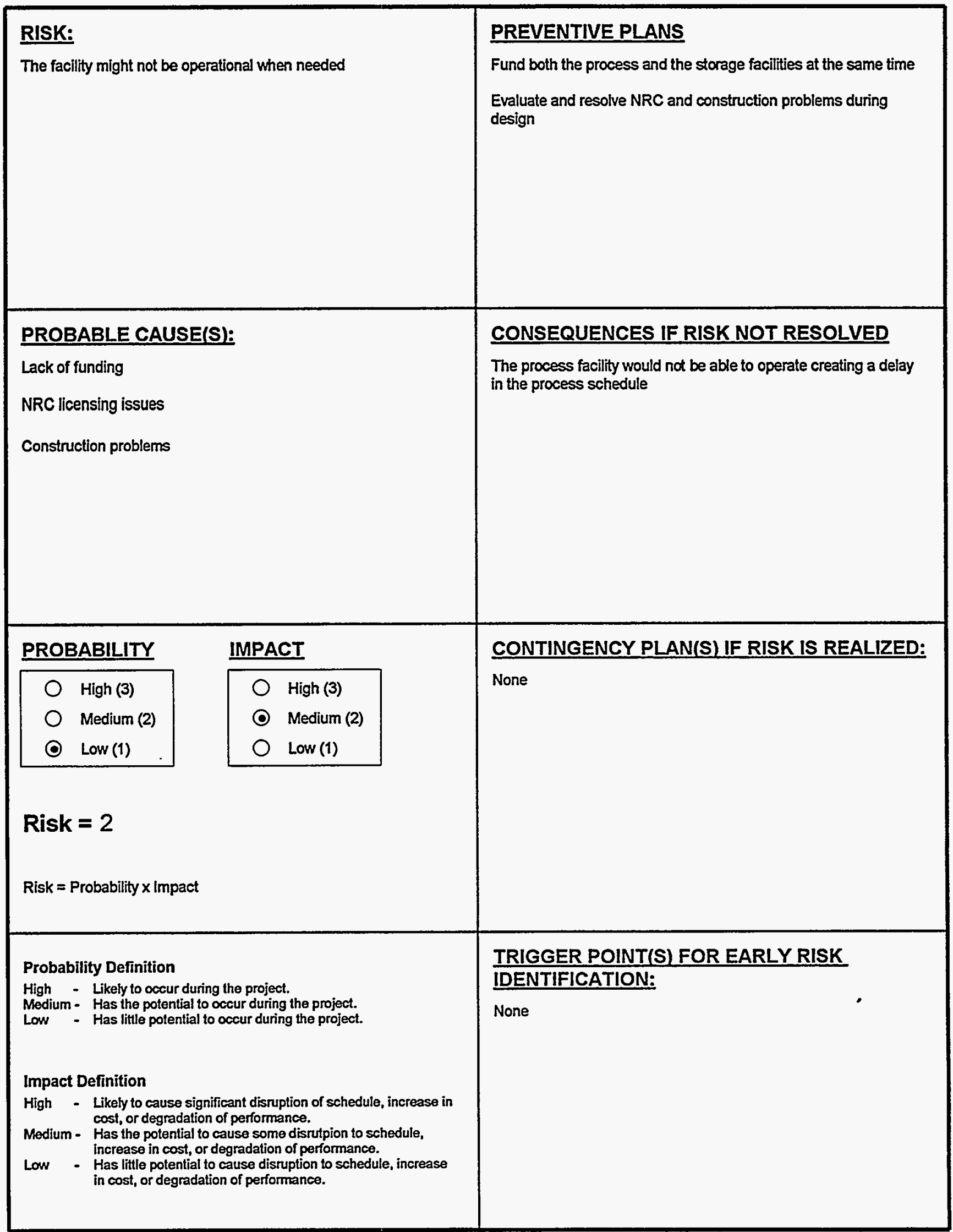




\section{Appendix 4}

\section{Background Information for Project Data Sheets}




\section{CONTENTS}

Background information for the Project Data Sheets are included in this appendix in the following order:

\section{TRU/Class A Option}

2. Vitrified Waste Option

3. Direct Cementitious Waste Option

4. Cementitious Waste Option

5. Hot Isostatic Press Waste Option.

The background information includes:

- Construction Assumptions

- Operations Assumptions

- D\&D Labor

- D\&D Assumptions

- Estimate of Diesel Engine Emissions. 


\section{TRU Class A Option}


Construction costs are from the detailed cost estimate (R. Adams)

Interim Storage Facility Option, Prepared by Byron L. Blackley, R.A., December 24, 1997.

Construction duration $=2$ years

Labor $=$

1 man-year of labor $=$

58 new workers/yr

Square footage $=$

1800 manhours

Acres disturbed $=$

36,063 sq. $\mathrm{ft}=$

$3,350 \mathrm{~m}^{2}$

3.0 acres (previous)

Excavation: Material needs to be imported.

Imported material $=$

$10,850 y d^{3}$

(Detailed Cost Estimate)

Air Emissions:

Dust during construction $=1.2$ tons/month-acre =

(from USEPA Office of Ar Quality Planning and Standards)

86 tons (total)

Air emissions from fuel usage are based on the diesel emissions spreadsheet.

Effluents:

Sanitary Wastewater $=\quad 326,250 \mathrm{gal} / \mathrm{yr}=$

(based on 25 gal/person-day and 225 days/year of construction)

$2,469,713$ liters (total)

(Benefield, LD and C.W. Randall, Biological Process Design for Wastewater Treatment, InPrint, Inc., 1987, p. 104 wastewater generation $=15-30$ gal/day-person)

Assume 3 gallons of lubricating oil and hydraulic

fluid generated for every 60 hours of operation of

heavy equipment $=$

(Heavy equipment hrs are calculated below.)

1,001 liter (total)

\section{Solid Wastes:}

Construction trash $=\quad 1,798$ yd3 (total) $=\quad 1,375 \mathrm{~m}^{3}$ (total)

(Use $15.5 \mathrm{yd} 3 / \mathrm{yr}$ per capita. This is twice the generation rate of trash from site operations)

Hazardous/toxic chemicals and wastes (type)

Hazardous waste generation $=\quad 275 \mathrm{gal} /$ week $=\quad 217 \mathrm{~m}^{3}$ (total)

(based on an assumed generation rate of 555 -gallon drums of waste per week)

Hazardous waste storage $=\quad 3300 \mathrm{gal}=\quad 13 \mathrm{~m}^{3}$

(Assume waste is accumulated for 12 weeks [ 84 days] in a 90-day accumulation area, then

\section{Water Usage:}

Water used for dust control $=$

Area

assume $0.05^{\prime \prime}$ water per week to control dust

$$
\begin{gathered}
3 \text { acres (previous) } \\
43,560 \text { sq ft/acre } \\
0.05 \text { in water/wk } \\
0.08333 \mathrm{ft} / \mathrm{in} \\
28.320 \mathrm{liter} / \mathrm{ft}^{3} \\
20 \mathrm{wk} / \mathrm{yr} \\
2 \mathrm{yr} \\
\hline 616,810 \text { liter }
\end{gathered}
$$

assume dust control required 20 weeks Length of Construction

Domestic water use is assumed to be the same as sanitary waste. 
Energy Requirements:

Electrical usage assumed to be $3,000 \mathrm{kWh}$ (from John Duggan)

$156 \mathrm{MWh} / \mathrm{yr}$

\section{Fossil Fuel Use}

Concrete:

Amount used $=$

(Detailed Cost Estimate)

\begin{aligned} $\begin{array}{r}9,260 y d^{3} \\ 460 y d^{3}\end{array} & \begin{array}{l}\text { Building } \\ \text { Charge Face Slab }\end{array} \\$\hline $9,720 y d^{3} &$ Total Concrete \end{aligned}

Number of truckloads $=$

442 (based on $22 \mathrm{yd}^{3} /$ load... tandem trailers)

Fuel usage $=$

$9,720 \mathrm{gal}$ (assumes $5 \mathrm{mpg}$ and round trip of 110 miles)

Materials delivery:

Number of truckloads =

221 (assume $1 / 2$ the loads of concrete)

Fuel usage $=$

$6,627 \mathrm{gal}$ (assumes $5 \mathrm{mpg}$ and round trip of 150 miles)

Imported Material Delivery:

Material

Number of truckloads =

Fuel usage $=$

$10,850 \mathrm{yd}^{3}$

493 (based on $22 \mathrm{yd}^{3} / \mathrm{load}$... tandem trailers)

$10,850 \mathrm{gal}$ (assumes $5 \mathrm{mpg}$ and round trip of 110 miles)

\section{Construction Equipment:}

Construction Equipment Cost

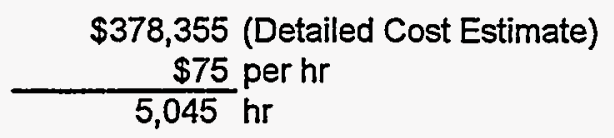

Labor Hours

181,612 (Detailed Cost Estimate) 750 labor hr/equipment hr

\begin{tabular}{c}
181,612 (Detailed Cost Estimate) \\
750 labor hr/equipment $\mathrm{hr}$ \\
\hline $242 \mathrm{hr}$
\end{tabular}

Total Equipment Hours $=$

$5,287 \mathrm{hr}$

Equipment fuel usage (see $h$ ttp://uww/deere.com/ind) = Total heavy equipment fuel usage $=$
$6 \mathrm{gal} / \mathrm{hr}$
$31,721 \mathrm{gal}$
or 120,065 liters (total)

Total heavy equipment and materials delivery fuel used $=$

Concrete Delivery

Materials Delivery

Imported Materials Deliver

Heavy Equipment
$6,627 \mathrm{gal}$

$493 \mathrm{gal}$

$10,850 \mathrm{gal}$

$31,721 \mathrm{gal}$

49,692 gal

3.785 liter/gal

188,083 liter 
Operating costs are taken from the life cycle cost estimate.

Operation

Loading $20 \mathrm{yr}$

Holding $\quad 30 \mathrm{yr}$

Shipping $5 \mathrm{yr}$

Interim Storage Facility Option, Prepared by Byron L. Blackley, R.A., December 24, 1997.

Labor: 6.5

Cannisters $\quad 170$

Power $290 \mathrm{~kW}$

Radiation worker annual dose is based on the average annual dose received at ICCP during the last three years (see attached memorandum)

\section{Effluents:}

Sanitary wastewater $=\quad 59,313 \mathrm{gal} / \mathrm{yr}=\quad 224,498 \mathrm{liters} / \mathrm{yr}$

(based on $25 \mathrm{gal} /$ day per worker, facility occupied year-round)

\section{Solid Wastes:}

Sanitary/Industrial trash $=\quad 47 \mathrm{yd}^{3} / \mathrm{yr} \quad 36 \mathrm{~m}^{3} / \mathrm{yr}$

(based on 7.25 yd3/person-year... Bob Skinner [cuber facility])

Product canisters $=$

$6 \mathrm{~m}^{3} / \mathrm{yr}$

(based on $0.72 \mathrm{~m}^{3} /$ canister)

Total rad waste $=$

$122 \mathrm{~m}^{3}$

Water Usage:

Domestic water usage $=$ same as sanitary wastewater rate.

\section{Energy Requirements}

Electric power usage =

(based on $290 \mathrm{~kW}$ ) 
D\&D Labor

D\&D Labor

Crew \# Crew Function

D Documentation

1 Characterization

2 Rad Demolition-Systems

$2 A$ Rad Demolition-Building

3 Demolition-Systems

3 A Demolition-Building

4 Asbestos Abatement

5 Decontamination

6 Prep/Fabrication

7 RADCON Surveys

Total

vailable

Notes:

1 Crew functions and daily estimates are from the D\&D program (Dave Haycraft)

2 Total costs are based on life cycle estimate by R. Turk

3 Assume all workers in crews 2, 2A, 5, and 7 are rad workers

4 Assume a man-year is 1800 hours.

\begin{tabular}{|c|c|c|}
\hline $\begin{array}{l}\text { Material } \\
\$ / \text { day }\end{array}$ & $\begin{array}{l}\text { Equipment } \\
\text { \$/day }\end{array}$ & Total \$/day \\
\hline$\$ 114$ & $\$$ & $\$ 1,250$ \\
\hline$\$ 460$ & $\$ 691$ & $\$ 3,453$ \\
\hline$\$ 818$ & $\$ 1,023$ & $\$ 5,932$ \\
\hline$\$ 1,064$ & $\$ 1,596$ & $\$ 7,979$ \\
\hline$\$ 752$ & $\$ 941$ & $\$ 5,455$ \\
\hline$\$ 962$ & $\$ 1,442$ & $\$ 7,212$ \\
\hline$\$ 375$ & $\$ 188$ & $\$ 4,316$ \\
\hline$\$ 751$ & $\$ 1,126$ & $\$ 5,630$ \\
\hline$\$ 643$ & $\$ 965$ & $\$ 4,826$ \\
\hline$\$ 519$ & $\$ 779$ & $\$ 3,894$ \\
\hline
\end{tabular}

$\$ 4,091$

$\$ 5,319$

$\$ 3,762$

$\$ 4,808$

$\$ 3,753$

$\$ 3,753$

$\$ 3,217$

$\$ 2,596$

$\$ 3,894$

$22,749,000$
D\&D Cost

Allocated

$1,150,000$

$1,720,000$

Years

From D\&D

3 Assumptions

$\$$

$\$ 4,900,000$

$9,800,000$

$\$ \quad-$

$2,900,000$

$\$ 2,279,000$

$\$ 22,749,000$

\section{Total MH Man-hours/yr}

$16,560 \quad 5,520$

$21,917 \quad 7,306$

$-$

64,675

119,578

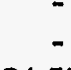

$21,558 \$ 845,261$

$39,859 \$ 1,959,456$

- $\$ 2,804,718$

36,656

$12,219 \$$

3,740

29,263

9,754

288,649

96,216 
Duration of $D \& D=\quad 3$ years

No decontamination of the storage facility will be required.

$D$ \& $D$ labor requirements are taken from $D$ \& $D$ labor and equipment spreadsheet.

$D \& D$ costs come from the life cycle cost estimate.

1 manyear of labor $=\quad 1800$ manhours

\begin{tabular}{|c|c|c|c|c|c|}
\hline Heavy Equipment & \# Used & Hours/day & Days/wk & Wks/yr & $\begin{array}{r}\text { Hours/yr } \\
300\end{array}$ \\
\hline Mobile Crane & 1 & 3 & 5 & 20 & 300 \\
\hline Roll-Off Truck & 1 & 8 & 5 & 20 & 800 \\
\hline Dozer & 1 & 6 & 5 & 20 & 600 \\
\hline Loader & 1 & 8 & 5 & 20 & 800 \\
\hline Scabbler (w/ Vacuum System) ${ }^{\star \star}$ & 1 & 6 & 3. & 20 & 360 \\
\hline Pneumatic Ram & 1 & 3 & 5 & 20 & 300 \\
\hline Demolition Machine (Remote & & & & & \\
\hline Control) & 0 & 4 & 4 & 45 & - \\
\hline \multicolumn{6}{|c|}{ Not needed, contamination not expected } \\
\hline Total hours/yr & & & & & 3,160 \\
\hline Total heavy equipment hours $=$ & & & & & 9,480 \\
\hline
\end{tabular}

Assume each piece of equipment uses 6 gallon of diesel fuel per hour. Consumption rate from John Deere Web Site (Construction Equipment - http://www.deere.com/ind/product/product.html)

No. of gallons of fuel used during $D \& D=\quad 56,880 \mathrm{gal}=$

215,291 liters (total)

Assume each roll-off truck makes 3 trips per day to RWMC

No. of trips $=$

Miles traveled @ 12 miles/round trip=

Acres disturbed and duration of disturbance

Acreage disturbed is the same as for construction =

3.0 acres

\section{Air emissions}

HEPA filter ventilation is not required during D\&D.

Air emissions from fuel are based on the diesel emissions spreadsheet.

\section{Effluents}

No decontamination solution required during $D \& D$.
Sanitary wastewater $=$
$300,676 \mathrm{gal} / \mathrm{yr}=$
$3,414,175$ liters (total)
(based on $25 \mathrm{gal} /$ day per worker, 225 day/yr)
Lube oil $=$
1,794 liters (total)
(based on $3 \mathrm{gal}$ for every 60 hours of operation)

\section{Solid wastes:}


The following factors from Dave Kenoyer are based on a contaminated building. The storage facility is not expected to be contaminated. Therefore, all items identified as LLW will be included with the non-contaminated waste. These factors are used for consistency in waste volumes with facilities which are contaminated.

Solid Waste Generation (factors from Dave Kenoyer - D\&D Program)

\begin{tabular}{|c|c|c|c|c|}
\hline Waste Type & $\begin{array}{l}\text { Factor } \\
\text { (cu.ft./sq.ft.) }\end{array}$ & $\begin{array}{l}\text { Sq.Ft. in } \\
\text { Facility }\end{array}$ & $\begin{array}{l}\text { Cu.Ft. of } \\
\text { Waste }\end{array}$ & Cu. Meters \\
\hline WERF-LLW Combustible PPEs & 0.167 & 36,063 & 6,023 & 172 \\
\hline WERF-LLW Combustible Building & 0.128 & 36,063 & 4,616 & 132 \\
\hline $\begin{array}{l}\text { WERF-LLW Compactable Building } \\
\text { RWMC-LLW Non-Compactable }\end{array}$ & 0.195 & 36,063 & 7,032 & 201 \\
\hline $\begin{array}{l}\text { Equipment } \\
\text { RWMC-LLW Non-Compt Building }\end{array}$ & 0.513 & 36,063 & 18,500 & 529 \\
\hline $\begin{array}{l}\text { Debris } \\
\text { RWMC-LLW Non-Compt Concrete }\end{array}$ & 0.684 & 36,063 & 24,667 & 705 \\
\hline Rubble * & 3.44 & 36,063 & 124,057 & 3,544 \\
\hline $\begin{array}{l}\text { RWMC-LLW Non-Compt Scrap Metal } \\
\text { RWMC-LLW Asbestos/ACM Covered }\end{array}$ & 0.778 & 36,063 & 28,057 & 802 \\
\hline $\begin{array}{l}\text { Pipe } \\
\text { CFA Landfill Non-Compt Building }\end{array}$ & 0 & 36,063 & - & \\
\hline $\begin{array}{l}\text { Debris } \\
\text { CFA Landfill Non-Compt Concrete }\end{array}$ & 1.99 & 36,063 & 71,765 & 2,050 \\
\hline Rubble * & 2.454 & 36,063 & 88,499 & 2,529 \\
\hline CFA Landfill Asbestos & 0 & 36,063 & - & \\
\hline HWSF Hazardous Mtrls (Hg/PCBs/etc) & 0.002 & 36,063 & 72 & 2 \\
\hline Metal Recycle & 0.022 & 36,063 & 793 & 23 \\
\hline $\begin{array}{l}\text { Non-Rad = } \\
\text { Hazardous = } \\
\text { Metal = }\end{array}$ & & & $\begin{array}{r}373,216 \\
72 \\
793\end{array}$ & $\begin{array}{r}10,663 \\
2 \\
23\end{array}$ \\
\hline
\end{tabular}

* (Factor used is twice that given by Dave Kenoyer due to use of concrete for shielding)

Hazardous/toxic chemicals and wastes (type)

Hazardous waste $=\quad 26,445$ gal (total) $=\quad 909 \mathrm{~m} 3$ (total)

(based on an assumed 5 55-gallon drums generated per week... work only 225 days/yr)

Water Usage

Domestic water use is assumed to be the same as sanitary waste.

Energy requirements:

Electric power usage $=\quad 156,000 \mathrm{kWh} / \mathrm{yr} \quad 156 \mathrm{MWh} / \mathrm{yr}$

(based on 3,000 kWh/wk - John Duggan)

Fossil Fuels $=\quad 215,291$ liters 
Bases \& Assumptions:

1. Air to fuel ratio $=25: 1$ (Mass Basis)

2. Diesel fuel density $=7.5 \mathrm{lbs} / \mathrm{gal}$.

3. Air is $21 \% \mathrm{O}_{2}, 79 \% \mathrm{~N}_{2}$, with a pseudomolecular weight of 29 .

4. Combustion is simulated as: $\mathrm{C}_{9} \mathrm{H}_{18}+13.5 \mathrm{O}_{2} \rightarrow 9 \mathrm{CO}_{2}+9 \mathrm{H}_{2} \mathrm{O}$

5. Particulates $=5 \mathrm{mg} / \mathrm{scf}$

6. $\mathrm{CO}=2,500 \mathrm{ppmv}$

7. $\mathrm{NO}_{\mathrm{x}}=2,000 \mathrm{ppmv}$

8. Unburned hydrocarbons $=100 \mathrm{ppmv}$

9. Diesel fuel (\#2 fuel oil) contains 1 wt. \% sulfur

10. Combustion is about $99 \%$ efficient.

Lbs. Of Construction Fuel

Lbs. Of Operations Fuel

Lbs. Of D\&D Fuel

Total Lbs. of Fuel Used

Lb-Moles of Construction Fuel

Lb-Moles of Operations Fuel

Lb-Moles of D\&D Fuel

Total Lb-Moles of Fuel (as C9H18)

Lbs of Air for Construction Fuel (based on air-to-fuel ratio)

Lbs.of Air for Operations Fuel (based on air-to-fuel ratio)

Lbs.of Air for D\&D Fuel (based on air-to-fuel ratio)

Total Lbs. of Air Added

Lb-Moles of Air for Combustion Fuel

Lb-Moles of Air for Operations Fuel

Lb-Moles of Air for D\&D Fuel

Total Lb-Moles of Air from Wark, K. and C.F. Wamer, Air Pollution, Its Origin and Control, IEP, New York, 1976, p. 446, 423

Wark and Warner, p. 446 Wark and Warner, p. 446 Wark and Wamer, p. 446 Wark and Wamer, p. 446 Wark and Wamer, p. 336

Grand Total of Materials Fed, Lbs.

372,405

426,276

798,681

2,956

3,383

6,339

$9,310,122$

$10,656,895$

$19,967,017$

321,039

367,479

688,518

$20,765,697$

\begin{tabular}{lrrrr} 
Exhaust Gases, Construction Fuel & Total Lbs. & \multicolumn{1}{c}{ Total Tons } & \multicolumn{1}{c}{ Total Moles } & \multicolumn{1}{c}{ Total SCF } \\
\hline $\mathrm{CO}_{2}$ & $1,158,711$ & 579 & 26,334 & $9,454,030$ \\
$\mathrm{H}_{2} \mathrm{O}$ & 474,018 & 237 & 26,334 & $9,454,030$ \\
$\mathrm{O}_{2}$ & 893,331 & 447 & 27,917 & $10,022,062$ \\
$\mathrm{~N}_{2}$ & $7,101,376$ & 3,551 & 253,621 & $91,049,784$ \\
\hline Subtotal of Major Gases & $\mathbf{9 , 6 2 7 , 4 3 7}$ & 4,814 & 334,206 & $119,979,906$ \\
\hline SO & 7,448 & 3.7 & & \\
Particulates & 1,321 & 0.7 & \\
$\mathrm{CO}$ & 23,394 & 11.7 & \\
NO & (assumed NO) & 10.0 & \\
Unburned Hydrocarbons & 20,052 & 2.1 & \\
Subtotal of Contaminants & 4,211 & $\mathbf{5 6 , 4 2 7}$ & 28 &
\end{tabular}




\begin{tabular}{|c|c|c|c|c|}
\hline Exhaust Gases, Operations Fuel & Total Lbs. & Total Tons & Total Moles & Total SCF \\
\hline $\mathrm{CO}_{2}$ & - & - & - & - \\
\hline $\mathrm{H}_{2} \mathrm{O}$ & - & - & - & - \\
\hline $\mathrm{O}_{2}$ & - & - & - & - \\
\hline $\mathbf{N}_{2}$ & - & - & - & - \\
\hline Subtotal of Major Gases & - & - & - & - \\
\hline $\mathrm{SO}_{2}$ & - & - & & \\
\hline Particulates & - & - & & \\
\hline $\mathrm{CO}$ & - & - & & \\
\hline $\mathrm{NO}_{x}$ (assumed $\mathrm{NO}$ ) & - & - & & \\
\hline Unburned Hydrocarbons & - & - & & \\
\hline Subtotal of Contaminants & - & - & & \\
\hline Exhaust Gases, D\&D Fuel & Total Lbs. & Total Tons & Total Moles & Total SCF \\
\hline $\mathrm{CO}_{2}$ & $1,326,327$ & 663 & 30,144 & $10,821,620$ \\
\hline $\mathrm{H}_{2} \mathrm{O}$ & 542,588 & 271 & 30,144 & $10,821,620$ \\
\hline $\mathrm{O}_{2}$ & $1,022,558$ & 511 & 31,955 & $11,471,822$ \\
\hline$N_{2}$ & $8,128,638.23$ & 4,064 & 290,309 & $104,220,754$ \\
\hline Subtotal of Major Gases & $11,020,111$ & 5,510 & 382,551 & $137,335,815$ \\
\hline $\mathrm{SO}_{2}$ & 8,259 & 4.1 & & \\
\hline $\begin{array}{l}\text { Particulates } \\
\text { CO }\end{array}$ & $\begin{array}{r}1,513 \\
26,779\end{array}$ & $\begin{array}{r}0.8 \\
13.4\end{array}$ & & \\
\hline $\mathrm{NO}_{x}$ (assumed $\mathrm{NO}$ ) & 22,953 & 11.5 & & \\
\hline Unburned Hydrocarbons & 4,820 & 2.4 & & \\
\hline Subtotal of Contaminants & 64,323 & 32 & & \\
\hline
\end{tabular}




\section{Vitrified Waste Option}


Construction costs are from the detailed cost estimate ( $R$. Adams)

\begin{tabular}{|lc|}
\hline Interim Storage Facility & Option, Prepared by Byron L. Blakely, R.A., December 24, 1997. \\
\hline Construction duration $=$ & 4 years \\
Labor $=$ & 114 new workers/yr \\
1 man-year of labor $=$ & 1800 manhours \\
Square footage $=$ & 145,242 sq.ft $=$ \\
Acres disturbed $=$ & 5 acres (previous) \\
\hline
\end{tabular}

Excavation: Excavated earth will be spread in a spoil area adjacent to ICPP, except for backfill soil.

Excess Material= (from Detailed Cost Estimate)

$20,000 \mathrm{yd}^{3}$

Air Emissions:

Dust during construction $=1.2$ tons/month-acre $=$ (from USEPA Office of Ar Quality Planning and Standards)

288 tons (total)

Air emissions from fuel usage are based on the diesel emissions spreadsheet.

\section{Effluents:}

Sanitary Wastewater $=\quad 641,250 \mathrm{gal} / \mathrm{yr}=\quad 9,708,525$ liters (total)

(based on $25 \mathrm{gal} /$ person-day and 225 days/year of construction)

(Benefield, LD and C.W. Randall, Biological Process Design for Wastewater Treatment, InPrint, Inc., 1987, p. 104 wastewater generation $=$ 15-30 gal/day-person)

Assume 3 gallons of lubricating oil and hydraulic

fluid generated for every 60 hours of operation

of heavy equipment $=$

(Heavy equipment hrs are calculated below.)

2,590 liter (total)

\section{Solid Wastes:}

Construction trash $=\quad 7,068 \mathrm{yd} 3$ (total) $=\quad 5,406 \mathrm{~m}^{3}$ (total)

(Use $15.5 \mathrm{yd} 3 / \mathrm{yr}$ per capita. This is twice the generation rate of trash from site operations)

Hazardous/toxic chemicals and wastes (type)

Hazardous waste generation $=\quad 275$ gal/week $=\quad 217 \mathrm{~m}^{3}$ (total)

(based on an assumed generation rate of 555 -gallon drums of waste per week)

Hazardous waste storage $=\quad 3300$ gal $=\quad 13 \mathrm{~m}^{3}$

(Assume waste is accumulated for 12 weeks [ 84 days] in a 90-day accumulation area, then

\section{Water Usage:}

Water used for dust control $=$

Area

assume $0.05^{\prime \prime}$ water per week to control dust

5 acres (previous)

$43,560 \mathrm{sq}$ ftacre

0.05 in water/wk

$0.08333 \mathrm{ft}$ in

assume dust control required 20 weeks

Length of Construction

28.320 liter $/ \mathrm{ft}^{3}$

$20 \mathrm{wk} / \mathrm{yr}$

$2,056,032$ liter 
Domestic water use is assumed to be the same as sanitary waste.

\section{Energy Requirements:}

Electrical usage assumed to be $3,000 \mathrm{kWh}$ (from John Duggan)

$$
156 \mathrm{MWh} / \mathrm{yr}
$$

\section{Fossil Fuel Use}

\section{Concrete:}

Amount used $=$

(Detailed Cost Estimate)

\begin{aligned} $1,360 \mathrm{yd}^{3} &$ Tunnel \\ $23,550 \mathrm{yd}^{3} &$ Building \\ $7,225 \mathrm{yd}^{3} &$ Charge Face Slab \\ \hline $32,135 \mathrm{yd}^{3} &$ Total Concrete \end{aligned}

Number of truckloads $=$

Fuel usage $=$

1,461 (based on $22 \mathrm{yd}^{3}$ hoad... tandem trailers)

$32,135 \mathrm{gal}$ (assumes $5 \mathrm{mpg}$ and round trip of 110 miles)

Materials delivery:

Number of truckloads =

730 (assume $1 / 2$ the loads of concrete)

Fuel usage $=$

$21,910 \mathrm{gal}$ (assumes $5 \mathrm{mpg}$ and round trip of 150 miles)

Construction Equipment:

Construction Equipment Cost

$\$ 949,512$ (Detailed Cost Estimate)

$\$ 75$ per hr

$12,660 \mathrm{hr}$

Labor Hours

767,252 (Detailed Cost Estimate)

750 labor hr/equipment hr

$1,023 \mathrm{hr}$

Total Equipment Hours =

$13,683 \mathrm{hr}$

Equipment fuel usage (see http://www/deere.com/ind) =

Total heavy equipment fuel usage $=$

\author{
$6 \mathrm{gal} / \mathrm{hr}$ \\ $82,099 \mathrm{gal}$
}

Total heavy equipment and materials delivery fuel used =

Concrete Delivery

Materials Delivery

Heavy Equipment
$32,135 \mathrm{gal}$

21,910 gal

$82,099 \mathrm{gal}$

136,144 gal

3.785 liter/gal

515,306 liter 
Operating costs are taken from the life cycle cost estimate.

Operation

Loading

Holding

$23 \mathrm{yr}$

Shipping

$37 \mathrm{yr}$

$5 \mathrm{yr}$

Interim Storage Facility Option, Prepared by Byron L. Blakely, R.A., December 24, 1997.

Labor.

Cannisters

6.5

Power

14000

$500 \mathrm{~kW}$

Radiation worker annual dose is based on the average annual dose received at ICCP during the last three years (see attached memorandum)

Effluents:

Sanitary wastewater $=$

$59,313 \mathrm{gallyr}=$

(based on 25 gal/day per worker, facility occupied year-round)

Solid Wastes:

Sanitary/Industrial trash $=$

(based on 7.25 yd3/person-year... Bob Skinner [cuber facility])

$47 \mathrm{yd}^{3} / \mathrm{yr} \quad 36 \mathrm{~m}^{3} / \mathrm{yr}$

Product canisters $=$ $504 \mathrm{~m}^{3} / \mathrm{yr}$

(based on 14,000 canisters @ $0.72 \mathrm{~m}^{3} /$ canister)

Total rad waste $=$

$11,592 \mathrm{~m}^{3}$

Water Usage:

Domestic water usage $=$ same as sanitary wastewater rate.

Energy Requirements

Electric power usage $=$

(based on $500 \mathrm{~kW}$ ) 
D\&D Labor

D\&D Labor

$\begin{array}{cl}\text { Crew } & \text { Crew Function } \\ \text { D } & \text { Documentation } \\ 1 & \text { Characterization } \\ 2 & \text { Rad Demolition-Systems } \\ 2 A & \text { Rad Demolition-Building } \\ 3 & \text { Demolition-Systems } \\ 3 A & \text { Demolition-Building } \\ 4 & \text { Asbestos Abatement } \\ 5 & \text { Decontamination } \\ 6 & \text { Prep/Fabrication } \\ 7 & \text { RADCON Surveys }\end{array}$

$\begin{array}{rr}\text { Total MH/day } & \text { Total } \$ / \text { day } \\ 18 & \$ 1,136 \\ 44 & \$ 2,302 \\ 77 & \$ 4,091 \\ 99 & \$ 5,319 \\ 72 & \$ 3,762 \\ 88 & \$ 4,808 \\ 77 & \$ 3,753 \\ 77 & \$ 3,753 \\ 61 & \$ 3,217 \\ 50 & \$ 2,596\end{array}$

\section{D\&D Years $=3$}

$\begin{array}{rrr}\begin{array}{c}\text { Material } \\ \$ / \text { day }\end{array} & \begin{array}{c}\text { Equipment } \\ \$ / \text { day }\end{array} & \begin{array}{r}\text { Total } \$ / \text { day } \\ \$ 114\end{array} \\ \$ & - & \$ 1,250 \\ \$ 460 & \$ 691 & \$ 3,453 \\ \$ 818 & \$ 1,023 & \$ 5,932 \\ \$ 1,064 & \$ 1,596 & \$ 7,979 \\ \$ 752 & \$ 941 & \$ 5,455 \\ \$ 962 & \$ 1,442 & \$ 7,212 \\ \$ 375 & \$ 188 & \$ 4,316 \\ \$ 751 & \$ 1,126 & \$ 5,630 \\ \$ 643 & \$ 965 & \$ 4,826 \\ \$ 519 & \$ 779 & \$ 3,894\end{array}$

$\begin{array}{lccr} & \begin{array}{c}\text { D\&D Cost } \\ \text { Allocated }\end{array} & \text { Total MH } & \text { Man-hours/yr } \\ \$ & 3,150,000 & 45,360 & 15,120 \\ \$ & 6,300,000 & 80,278 & 26,759 \\ \$ & - & - & - \\ \$ & - & - & - \\ \$ & 23,950,000 & 316,114 & 105,371 \\ \$ & 47,900,000 & 584,470 & 194,823 \\ \$ & - & - & - \\ \$ & - & - & - \\ \$ & 14,350,000 & 181,382 & 60,461 \\ \$ & 10,633,000 & 136,531 & 45,510 \\ & & & \\ \$ & 106,283,000 & 1,344,135 & 448,045 \\ & & & \end{array}$

Notes:

1 Crew functions and daily estimates are from the D\&D program (Dave Haycraft)

2 Total costs are based on life cycle estimate by $R$. Turk

3 Assume all workers in crews 2,2A, 5 , and 7 are rad workers

4 Assume a man-year is 1800 hours. 


\section{Duration of $D \& D=\quad 3$ years}

No decontamination of the storage facility will be required.

$D \& D$ labor requirements are taken from $D$ \& $D$ labor and equipment spreadsheet.

$D$ \& $D$ costs come from the life cycle cost estimate.

1 manyear of labor $=\quad 1800$ manhours

\begin{tabular}{|c|c|c|c|c|c|}
\hline Heavy Equipment & \# Used & Hours/day & Days/wk & Wks/yr & Hours/yr \\
\hline Mobile Crane & 2 & 3 & 5 & 45 & 1,350 \\
\hline Roll-Off Truck & 3 & 8 & 5 & 45 & 5,400 \\
\hline Dozer & 3 & 6 & 5 & 45 & 4,050 \\
\hline Loader & 3 & 8 & 5 & 45 & 5,400 \\
\hline Scabbler (w/ Vacuum System) $)^{\star \star}$ & 0 & 6 & 4 & 45 & - \\
\hline $\begin{array}{l}\text { Pneumatic Ram } \\
\text { Demolition Machine (Remote }\end{array}$ & 1 & 3 & 4 & 45 & 540 \\
\hline Control ${ }^{\star \star}$ & 0 & 4 & 4 & 45 & - \\
\hline \multicolumn{6}{|c|}{ Not needed, contamination not expected } \\
\hline Total hours/yr & & & & & 16,740 \\
\hline Total heavy equipment hours = & & & & & 50,220 \\
\hline
\end{tabular}

Assume each piece of equipment uses 6 gallon of diesel fuel per hour. Consumption rate from John Deere Web Site (Construction Equipment - http://www.deere.com/ind/product/product.html)

No. of gallons of fuel used during $D \& D=$

Assume each roll-off truck makes 3 trips per day to RWMC

No. of trips $=$

Miles traveled @ 12 miles/round trip=

Acres disturbed and duration of disturbance

Acreage disturbed is the same as for construction =

5.0 acres

\section{Air emissions}

HEPA filter ventilation is not required during D\&D.

Air emissions from fuel are based on the diesel emissions spreadsheet.

\section{Effluents}

No decontamination solution required during D\&D.

Sanitary wastewater $=$ (based on $25 \mathrm{gal} / \mathrm{day}$ per worker, 225 day/yr)

$$
1,400,140 \mathrm{gal} / \mathrm{yr}=
$$

$15,898,593$ liters (total)

$$
\text { Lube oil = 9,504 liters (total) }
$$

(based on 3 gal for every 60 hours of operation)

\section{Solid wastes:}


The following factors from Dave Kenoyer are based on a contaminated building. The storage facility is not expected to be contaminated. Therefore, all items identified as LLW will be included with the non-contaminated waste. These factors are used for consistency in waste volumes with facilities which are contaminated.

Solid Waste Generation (factors from Dave Kenoyer - D\&D Program)

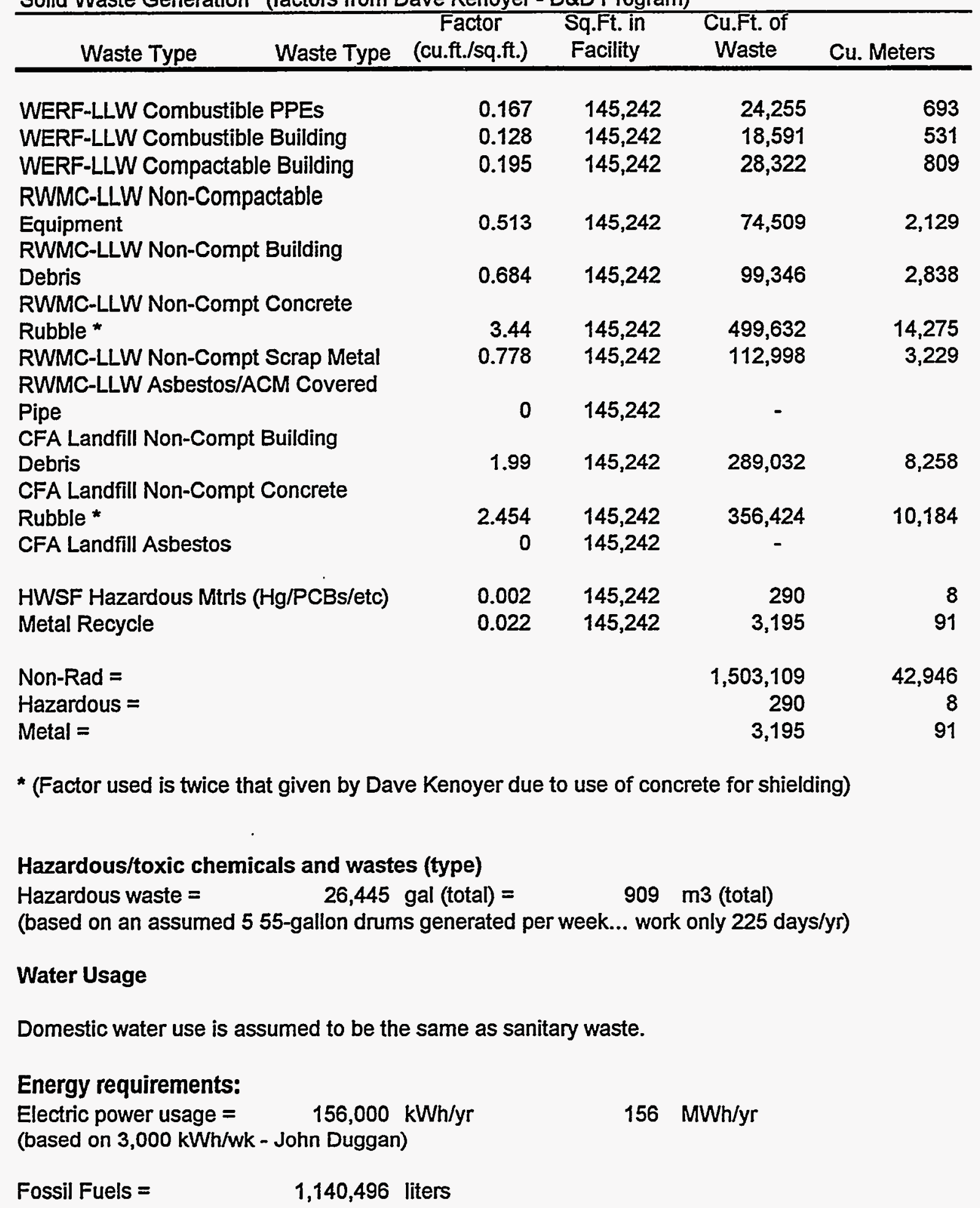




\section{Estimate of Diesel Engine Emissions}

Bases \& Assumptions:

\section{VWO}

1. Air to fuel ratio $=25: 1$ (Mass Basis)

from Wark, K. and C.F. Wamer, Air Pollution, its Origin and Control, IEP, New York, 1976, p. 446, 423

2. Diesel fuel density $=7.5 \mathrm{lbs}$.Jgal.

3. Air is $21 \% \mathrm{O}_{2}, 79 \% \mathrm{~N}_{2}$, with a pseudomolecular weight of 29 .

4. Combustion is simulated as: $\mathrm{C}_{9} \mathrm{H}_{18}+13.5 \mathrm{O}_{2} \rightarrow 9 \mathrm{CO}_{2}+9 \mathrm{H}_{2} \mathrm{O}$

5. Particulates $=5 \mathrm{mg} / \mathrm{scf}$

6. $\mathrm{CO}=2,500 \mathrm{ppmv}$

7. $\mathrm{NO}_{\mathrm{x}}=2,000 \mathrm{ppmv}$

8. Unburned hydrocarbons $=100 \mathrm{ppmv}$

9. Diesel fuel (\# 2 fuel oil) contains 1 wt. \% sulfur

10. Combustion is about $99 \%$ efficient.

Wark and Warner, p. 446

Wark and Wamer, p. 446

Wark and Warner, p. 446

Wark and Wamer, p. 446

Wark and Warner, p. 336

Lbs. Of Construction Fuel

$1,020,306$

Lbs. Of Operations Fuel

Lbs. Of D\&D Fuel

Total Lbs. of Fuel Used

Lb-Moles of Construction Fuel

8,098

Lb-Moles of Operations Fuel

Lb-Moles of D\&D Fuel

17,922

Total Lb-Moles of Fuel (as $\mathrm{C} 9 \mathrm{H} 18$ )

26,020

Lbs of Air for Construction Fuel (based on air-to-fuel ratio)

$25,507,638$

Lbs.of Air for Operations Fuel (based on air-to-fuel ratio)

$-$

Lbs.of Air for D\&D Fuel (based on air-to-fuel ratio)

Total Lbs. of Air Added

$56,454,562$

$81,962,199$

Lb-Moles of Air for Combustion Fuel

879,574

Lb-Moles of Air for Operations Fuel

Lb-Moles of Air for D\&D Fuel

$1,946,709$

$2,826,283$

Total Lb-Moles of Air

$85,240,687$

Grand Total of Materials Fed, Lbs.

\begin{tabular}{lrrrr} 
Exhaust Gases, Construction Fuel & \multicolumn{1}{c}{ Total Lbs. } & \multicolumn{1}{c}{ Total Tons } & \multicolumn{1}{c}{ Total Moles } & \multicolumn{1}{c}{ Total SCF } \\
\hline $\mathrm{CO}_{2}$ & $3,174,608$ & 1,587 & 72,150 & $25,901,913$ \\
$\mathrm{H}_{2} \mathrm{O}$ & $1,298,703$ & 649 & 72,150 & $25,901,913$ \\
$\mathrm{O}_{2}$ & $2,447,527$ & 1,224 & 76,485 & $27,458,193$ \\
$\mathrm{~N}_{2}$ & $19,456,170$ & 9,728 & 694,863 & $249,455,900$ \\
\hline Subtotal of Major Gases & $\mathbf{2 6 , 3 7 7 , 0 0 8}$ & $\mathbf{1 3 , 1 8 9}$ & $\mathbf{9 1 5 , 6 4 9}$ & $\mathbf{3 2 8 , 7 1 7 , 9 1 8}$ \\
\hline $\mathrm{SO}_{2}$ & 20,406 & 10.2 & & \\
Particulates & 3,620 & 1.8 & \\
$\mathrm{CO}$ & 64,095 & 32.0 & \\
NO (assumed NO) & 54,939 & 27.5 & \\
Unburned Hydrocarbons & 11,537 & 5.8 & \\
Subtotal of Contaminants & $\mathbf{1 5 4 , 5 9 8}$ & $\mathbf{7 7}$ & \\
\hline
\end{tabular}




\begin{tabular}{|c|c|c|c|c|}
\hline Exhaust Gases, Operations Fuel & Total Lbs. & Total Tons & Total Moles & Total SCF \\
\hline$\overline{\mathrm{CO}_{2}}$ & - & - & - & - \\
\hline $\mathrm{H}_{2} \mathrm{O}$ & - & - & - & - \\
\hline $\mathrm{O}_{2}$ & - & - & - & - \\
\hline $\mathrm{N}_{2}$ & - & - & - & - \\
\hline Subtotal of Major Gases & - & - & - & - \\
\hline $\mathrm{SO}_{2}$ & - & - & & \\
\hline Particulates & - & - & & \\
\hline co & - & - & & \\
\hline $\mathrm{NO}_{x}$ (assumed NO) & - & - & & \\
\hline Unburned Hydrocarbons & - & - & & \\
\hline Subtotal of Contaminants & - & - & & \\
\hline Exhaust Gases, D\&D Fuel & Total Lbs. & Total Tons & Total Moles & Total SCF \\
\hline$\overline{\mathrm{CO}_{2}}$ & $7,026,173$ & 3,513 & 159,686 & $57,327,188$ \\
\hline $\mathrm{H}_{2} \mathrm{O}$ & $2,874,344$ & 1,437 & 159,686 & $57,327,188$ \\
\hline $\mathrm{O}_{2}$ & $5,416,968$ & 2,708 & 169,280 & $60,771,612$ \\
\hline $\mathrm{N}_{2}$ & $43,061,203.77$ & 21,531 & $1,537,900$ & $552,106,148$ \\
\hline Subtotal of Major Gases & $58,378,689$ & 29,189 & $2,026,552$ & $727,532,136$ \\
\hline $\mathrm{SO}_{2}$ & 43,752 & 21.9 & & \\
\hline $\begin{array}{l}\text { Particulates } \\
\text { CO }\end{array}$ & $\begin{array}{r}8,012 \\
141,859\end{array}$ & $\begin{array}{r}4.0 \\
70.9\end{array}$ & & \\
\hline $\mathrm{NO}_{x}$ (assumed NO) & 121,593 & 60.8 & & \\
\hline Unbumed Hydrocarbons & 25,535 & 12.8 & & \\
\hline Subtotal of Contaminants & 340,751 & 170 & & \\
\hline
\end{tabular}




\section{Direct Cementitious Waste Option}


Construction costs are from the detailed cost estimate (R. Adams)

Interim Storage Facility Option, Prepared by Byron L. Blackley, R.A., December 24, 1997.

Construction duration $=$

Labor $=$

4 years

1 man-year of labor $=$

134 new workers/yr

Square footage $=$

1800 manhours

Acres disturbed $=$

$171,863 \mathrm{sq} . \mathrm{ft}=$

9 acres (previous)

Excavation: Excavated earth will be spread in a spoil area adjacent to ICPP, except for backfill soil.

Excess Material= $25,990 \mathrm{yd}^{3}$

(from Detailed Cost Estimate)

Air Emissions:

Dust during construction $=1.2$ tons/month-acre $\square$

(from USEPA Office of Ar Quality Planning and Standards)

518 tons (total)

Air emissions from fuel usage are based on the diesel emissions spreadsheet.

Effluents:

Sanitary Wastewater $=\quad 753,750 \mathrm{gal} / \mathrm{yr}=$

(based on 25 gallperson-day and 225 days/year of construction)

$11,411,775$ liters (total)

(Benefield, LD and C.W. Randall, Biological Process Design for Wastewater Treatment, InPrint, Inc., 1987, p. 104 wastewater generation $=15-30$ gal/day-person)

Assume 3 gallons of lubricating oil and hydraulic

fluid generated for every 60 hours of operation

of heavy equipment $=$

$2,960.20$ liter (total)

(Heavy equipment hrs are calculated below.)

\section{Solid Wastes:}

Construction trash $=\quad 8,308$ yd3 (total) $=\quad 6,355 \mathrm{~m}^{3}$ (total)

(Use $15.5 \mathrm{yd} 3 / \mathrm{yr}$ per capita. This is twice the generation rate of trash from site operations)

Hazardous/toxic chemicals and wastes (type)

Hazardous waste generation $=\quad 275$ gal/week $=\quad 217 \mathrm{~m}^{3}$ (total)

(based on an assumed generation rate of 555 -gallon drums of waste per week)

Hazardous waste storage $=\quad 3300$ gal $=\quad 13 \mathrm{~m}^{3}$

(Assume waste is accumulated for 12 weeks [ 84 days] in a 90-day accumulation area, then

\section{Water Usage:}

Water used for dust control $=$

Area

assume $0.05^{n}$ water per week to control dust

$$
9 \text { acres (previous) }
$$

$43,560 \mathrm{sq}$ ftacre

0.05 in water/wk

$0.08333 \mathrm{ft} / \mathrm{in}$

28.320 liter $/ \mathrm{ft}^{3}$

assume dust control required 20 weeks

$20 \mathrm{wk} / \mathrm{yr}$

Length of Construction

$4 \mathrm{yr}$

$3,700,858$ liter 
Domestic water use is assumed to be the same as sanitary waste.

\section{Energy Requirements:}

Electrical usage assumed to be $3,000 \mathrm{kWh}$ (from John Duggan)

$$
156 \mathrm{MWh} / \mathrm{yr}
$$

\section{Fossil Fuel Use}

Concrete:

Amount used $=$ (Detailed Cost Estimate)

\begin{aligned} $1,360 y^{3} &$ Tunnel \\ $27,310 y d^{3} &$ Building \\ $7,735 y d^{3} &$ Charge Face Slab \\ \hline $36,405 y d^{3} &$ Total Concrete \end{aligned}

Number of truckloads $=$ Fuel usage $=$

Materials delivery:

Number of truckloads $=$

Fuel usage $=$
1,655 (based on $22 \mathrm{yd}^{3} /$ load... tandem trailers) $36,405 \mathrm{gal}$ (assumes $5 \mathrm{mpg}$ and round trip of 110 miles)

827 (assume 1/2 the loads of concrete) $24,822 \mathrm{gal}$ (assumes $5 \mathrm{mpg}$ and round trip of 150 miles)

\section{Construction Equipment:}

Construction Equipment Cost

$$
\begin{gathered}
\$ 1,082,648 \text { (Detailed Cost Estimate) } \\
\$ 75 \\
\text { per hr }
\end{gathered}
$$

Labor Hours

904,809 (Detailed Cost Estimate)

750 labor hr/equipment hr

$$
1,206 \mathrm{hr}
$$

Total Equipment Hours $=$

$15,642 \mathrm{hr}$

Equipment fuel usage (see http://www/deere.com/ind) = Total heavy equipment fuel usage $=$

Total heavy equipment and materials delivery fuel used $=$
$6 \mathrm{gal} / \mathrm{hr}$
$93,850.31 \mathrm{gal}$
355,223 liters (total)
586,966 liters 


\begin{tabular}{|l|l|}
\hline Operating costs are taken from the life cycle cost estimate. \\
\hline Operation & $21 \mathrm{yr}$ \\
Loading & $31 \mathrm{yr}$ \\
Holding & $5 \mathrm{yr}$ \\
Shipping & \\
\hline
\end{tabular}

Interim Storage Facility Option, Prepared by Byron L. Blackley, R.A., December 24, 1997.

Labor: 8.5

Cannisters $\quad 16000$

Power $\quad 525 \mathrm{~kW}$

Radiation worker annual dose is based on the average annual dose received at ICCP during the last three years (see attached memorandum)

\section{Effluents:}

Sanitary wastewater $=\quad 77,563 \mathrm{gal} / \mathrm{yr}=293,574 \mathrm{liters} / \mathrm{yr}$

(based on 25 gal/day per worker, facility occupied year-round)

\section{Solid Wastes:}

Sanitary/Industrial trash =

(based on 7.25 yd3/person-year... Bob Skinner [cuber facility])

$62 \mathrm{yd}^{3} / \mathrm{yr} \quad 47 \mathrm{~m}^{3} / \mathrm{yr}$

Product canisters $=$ $576 \mathrm{~m}^{3} / \mathrm{yr}$

(based on 16,000 canisters @ $0.72 \mathrm{~m}^{3} /$ canister)

Total rad waste $=$ $12,096 \mathrm{~m}^{3}$

Water Usage:

Domestic water usage $=$ same as sanitary wastewater rate.

Energy Requirements Electric power usage $=$ (based on $525 \mathrm{~kW}$ )

\section{4,586 MWh/yr}


D\&D Labor

D\&D Labor

Crew \# Crew Function

D Documentation

1 Characterization

2 Rad Demolition-Systems

2A Rad Demolition-Building

3 Demolition-Systems

3 A Demolition-Building

4 Asbestos Abatement

5 Decontamination

6 Prep/Fabrication

7 RADCON Surveys

Total

vailable

Notes:

1 Crew functions and daily estimates are from the D\&D program (Dave Haycraft)

2 Total costs are based on life cycle estimate by $R$. Turk

3 Assume all workers in crews 2, 2A, 5, and 7 are rad workers

4 Assume a man-year is 1800 hours.
Years

From D\&D

3 Assumptions

\section{D\&D Cost}

Allocated

$\$ /$ day $\$ /$ day Total $\$ /$ day

$\$ 114 \$$

$\$ 460$

$\$ 818$

$\$ /$ day

$\$ 1,250$

$\$ 3,453$

$\$ 2,302$

$\$ 4,091$

$\$ 3,762$

$\$ 1,064$

$\$ 691$

$\$ 1,023$

$\$ 1,596$

$\$ 941$

$\$ 5,932$

$\$ 7,979$

$\$ 5,455$

$\$ 4,808$

$\$ 1,442$

$\$ 188$

$\$ 3,753$

$\$ 3,217$

$\$ 375$

$\$ 751$

$\$ 1,126$

$\$ 965$

$\$ 643$

$\$ 519$

$\$ 779$

$\$ 7,212$

$\$ 4,316$

$\$ 5,630$

$\$ 4,826$

$\$ 3,894$

$\$$

$\$ 3,150,000$

$6,300,000$

Total MH Man-hours/yr

45,360

15,120

$\$$ -

$28,059,000$

$-$

26,759

$56,117,000$

370,348

684,733

123,449

-

$16,835,000$

$\$ 12,274,000$

$-$

228,244

0,931

52,534

$\$ 122,735,000$

$1,551,113$

$\$ 122,735,000$ 
Duration of $D \& D=\quad 3$ years

No decontamination of the storage facility will be required.

$D \& D$ labor requirements are taken from $D$ \& $D$ labor and equipment spreadsheet.

$D \& D$ costs come from the life cycle cost estimate.

1 manyear of labor $=\quad 1800$ manhours

\begin{tabular}{lrrrr|r} 
Heavy Equipment & \# Used & Hours/day & Days/wk & Wks/yr & Hours/yr \\
Mobile Crane & 1 & 6 & 5 & 45 & 1,350 \\
Roll-Off Truck & 4 & 8 & 5 & 45 & 7,200 \\
Dozer & 3 & 6 & 5 & 45 & 4,050 \\
Loader & 4 & 8 & 5 & 45 & 7,200 \\
Scabbler (w/ Vacuum System) & 0 & 6 & 5 & 45 & - \\
Pneumatic Ram & 1 & 4 & 5 & 45 & 900 \\
Demolition Machine (Remote & 0 & 4 & 4 & 45 & - \\
\hline Control) ** & & & &
\end{tabular}

$\begin{array}{ll}\text { Total heavy equipment hours }= & 62,100\end{array}$

Assume each piece of equipment uses 6 gallon of diesel fuel per hour. Consumption rate from John Deere Web Site (Construction Equipment - http://www.deere.com/ind/product/product.html)

No. of gallons of fuel used during $D \& D=$ 372,600 gal $=$

$1,410,291$ liters (total)

Assume each roll-off truck makes 3 trips per day to RWMC

No. of trips =

Miles traveled @ 12 miles/round trip=

Acres disturbed and duration of disturbance

Acreage disturbed is the same as for construction =

9.0 acres

\section{Air emissions}

No decontamination solution required during D\&D.

Air emissions from fuel are based on the diesel emissions spreadsheet.

\section{Effluents}

Assume daily spent decon. solution=

(assumes 225 days/yr total)

$$
2000 \text { gal/day } \quad 5,109,750 \text { liters (total) }
$$
Sanitary wastewater $=$
(based on 25 gal/day per worker, 225 day/yr)
$1,615,742 \mathrm{gal} / \mathrm{yr}=$
$18,346,756$ liters (total)

Lube oil =

11,752 liters (total)

(based on 3 gal for every 60 hours of operation)

\section{Solid wastes:}

The following factors from Dave Kenoyer are based on a contaminated building. The storage facility is not expected to be contaminated. Therefore, all items identified as LLW will be included with the non-contaminated waste. These factors are used for consistency in waste volumes with facilities which are contaminated. 
D D Assumptions

\begin{tabular}{|c|c|c|c|c|}
\hline Waste Type & $\begin{array}{c}\text { Factor } \\
\text { (cu.ft./sq.ft.) }\end{array}$ & $\begin{array}{l}\text { Sq.Ft. in } \\
\text { Facility }\end{array}$ & $\begin{array}{l}\text { Cu.Ft. of } \\
\text { Waste }\end{array}$ & Cu. Meters \\
\hline WERF-LLW Combustible PPEs & 0.167 & 171,863 & 28,701 & 820 \\
\hline WERF-LLW Combustible Building & 0.128 & 171,863 & 21,998 & 629 \\
\hline $\begin{array}{l}\text { WERF-LLW Compactable Building } \\
\text { RWMC-LLW Non-Compactable }\end{array}$ & 0.195 & 171,863 & 33,513 & 958 \\
\hline $\begin{array}{l}\text { Equipment } \\
\text { RWMC-LLW Non-Compt Building }\end{array}$ & 0.513 & 171,863 & 88,166 & 2,519 \\
\hline $\begin{array}{l}\text { Debris } \\
\text { RWMC-LLW Non-Compt Concrete }\end{array}$ & 0.684 & 171,863 & 117,554 & 3,359 \\
\hline Rubble* & 3.44 & 171,863 & 591,209 & 16,892 \\
\hline $\begin{array}{l}\text { RWMC-LLW Non-Compt Scrap Metal } \\
\text { RWMC-LLW Asbestos/ACM Covered }\end{array}$ & 0.778 & 171,863 & 133,709 & 3,820 \\
\hline $\begin{array}{l}\text { Pipe } \\
\text { CFA Landfill Non-Compt Building }\end{array}$ & 0 & 171,863 & - & \\
\hline $\begin{array}{l}\text { Debris } \\
\text { CFA Landfill Non-Compt Concrete }\end{array}$ & 1.99 & 171,863 & 342,007 & 9,772 \\
\hline $\begin{array}{l}\text { Rubble * } \\
\text { CFA Landfill Asbestos }\end{array}$ & $\begin{array}{r}2.454 \\
0\end{array}$ & $\begin{array}{l}171,863 \\
171,863\end{array}$ & 421,752 & 12,050 \\
\hline $\begin{array}{l}\text { HWSF Hazardous Mtrls (Hg/PCBs/etc) } \\
\text { Metal Recycle }\end{array}$ & $\begin{array}{l}0.002 \\
0.022\end{array}$ & $\begin{array}{l}171,863 \\
171,863\end{array}$ & $\begin{array}{r}344 \\
3,781\end{array}$ & $\begin{array}{r}10 \\
108\end{array}$ \\
\hline $\begin{array}{l}\text { Non-Rad = } \\
\text { Hazardous = } \\
\text { Metal = }\end{array}$ & & & $\begin{array}{r}1,778,610 \\
344 \\
3,781\end{array}$ & $\begin{array}{r}50,817 \\
10 \\
108\end{array}$ \\
\hline
\end{tabular}

- (Factor used is twice that given by Dave Kenoyer due to use of concrete for shielding)

Hazardous/toxic chemicals and wastes (type)

Hazardous waste $=\quad 26,445$ gal (total) $=\quad 909 \mathrm{~m} 3$ (total)

(based on an assumed 555 -gallon drums generated per week... work only 225 days/yr)

Water Usage

Domestic water use is assumed to be the same as sanitary waste.

Energy requirements:

Electric power usage $=\quad 156,000 \mathrm{kWh} / \mathrm{yr} \quad 156 \mathrm{MWh} / \mathrm{yr}$

(based on 3,000 kWh/wk - John Duggan)

Fossil Fuels $=\quad 1,410,291$ liters 


\section{Estimate of Diesel Engine Emissions}

\section{DCWO}

Bases \& Assumptions:

1. Air to fuel ratio $=25: 1$ (Mass Basis)

2. Diesel fuel density $=7.5 \mathrm{lbs} . / \mathrm{gal}$.

3. Air is $21 \% \mathrm{O}_{2}, 79 \% \mathrm{~N}_{2}$, with a pseudomolecular weight of 29 .

4. Combustion is simulated as: $\mathrm{C}_{9} \mathrm{H}_{18}+13.5 \mathrm{O}_{2} \longrightarrow 9 \mathrm{CO}_{2}+9 \mathrm{H}_{2} \mathrm{O}$

5. Particulates $=5 \mathrm{mg} / \mathrm{scf}$

6. $\mathrm{CO}=2,500 \mathrm{ppmv}$

7. $\mathrm{NO}_{\mathrm{x}}=2,000 \mathrm{ppmv}$

8. Unburned hydrocarbons $=100 \mathrm{ppmv}$

9. Diesel fuel (\# 2 fuel oil) contains 1 wt. \% sulfur

10. Combustion is about $99 \%$ efficient.

Lbs. Of Construction Fuel

Lbs. Of Operations Fuel

Lbs. Of D\&D Fuel

Total Lbs. of Fuel Used

Lb-Moles of Construction Fuel

Lb-Moles of Operations Fuel

Lb-Moles of D\&D Fuel

Total Lb-Moles of Fuel (as C9H18)

Lbs of Air for Construction Fuel (based on air-to-fuel ratio)

Lbs.of Air for Operations Fuel (based on air-to-fuel ratio)

Lbs.of Air for D\&D Fuel (based on air-to-fuel ratio)

Total Lbs. of Air Added

Lb-Moles of Air for Combustion Fuel

Lb-Moles of Air for Operations Fuel

Lb-Moles of Air for D\&D Fuel

Total Lb-Moles of Air

Grand Total of Materials Fed, Lbs. from Wark, K. and C.F. Warner, Air Pollution, Its Origin and Control, IEP, New York, 1976, p. 446, 423

Wark and Wamer, p. 446

Wark and Warner, p. 446

Wark and Wamer, p. 446

Wark and Wamer, p. 446

Wark and Warner, p. 336

\begin{tabular}{|c|c|c|c|c|}
\hline Exhaust Gases, Construction Fuel & Total Lbs. & Total Tons & Total Moles & Total SCF \\
\hline$\overline{\mathrm{CO}_{2}}$ & $3,616,080$ & 1,808 & 82,184 & $29,503,925$ \\
\hline $\mathrm{H}_{2} \mathrm{O}$ & $1,479,305$ & 740 & 82,184 & $29,503,925$ \\
\hline $\mathrm{O}_{2}$ & $2,787,889$ & 1,394 & 87,122 & $31,276,627$ \\
\hline $\mathrm{N}_{2}$ & $22,161,815$ & 11,081 & 791,493 & $284,146,129$ \\
\hline Subtotal of Major Gases & $30,045,089$ & 15,023 & $1,042,982$ & $374,430,607$ \\
\hline $\mathrm{SO}_{2}$ & 23,244 & 11.6 & & \\
\hline $\begin{array}{l}\text { Particulates } \\
\text { co }\end{array}$ & $\begin{array}{r}4,124 \\
73,009\end{array}$ & $\begin{array}{r}2.1 \\
36.5\end{array}$ & & \\
\hline $\mathrm{NO}_{x}$ (assumed NO) & 62,579 & 31.3 & & \\
\hline Unburned Hydrocarbons & 13,142 & 6.6 & & \\
\hline Subtotal of Contaminants & 176,097 & 88 & & \\
\hline
\end{tabular}




\begin{tabular}{|c|c|c|c|c|}
\hline Exhaust Gases, Operations Fuel & Total Lbs. & Total Tons & Total Moles & Total SCF \\
\hline $\mathrm{CO}_{2}$ & - & - & - & - \\
\hline $\mathrm{H}_{2} \mathrm{O}$ & - & - & - & - \\
\hline $\mathrm{O}_{2}$ & - & - & - & - \\
\hline $\mathrm{N}_{2}$ & - & - & - & - \\
\hline Subtotal of Major Gases & - & - & - & - \\
\hline $\mathrm{SO}_{2}$ & - & - & & \\
\hline Particulates & - & - & & \\
\hline CO & - & - & & \\
\hline $\mathrm{NO}_{x}$ (assumed NO) & - & - & & \\
\hline Unbumed Hydrocarbons & - & - & & \\
\hline Subtotal of Contaminants & - & - & & \\
\hline Exhaust Gases, D\&D Fuel & Total Lbs. & Total Tons & Total Moles & Total SCF \\
\hline $\mathrm{CO}_{2}$ & $8,688,279$ & 4,344 & 197,461 & $70,888,458$ \\
\hline $\mathrm{H}_{2} \mathrm{O}$ & $3,554,296$ & 1,777 & 197,461 & $70,888,458$ \\
\hline $\mathrm{O}_{2}$ & $6,698,402$ & 3,349 & 209,325 & $75,147,692$ \\
\hline $\mathrm{N}_{2}$ & $53,247,725.09$ & 26,624 & $1,901,704$ & $682,711,904$ \\
\hline Subtotal of Major Gases & $72,188,702$ & 36,094 & $2,505,951$ & $899,636,513$ \\
\hline $\mathrm{SO}_{2}$ & 54,102 & 27.1 & & \\
\hline $\begin{array}{l}\text { Particulates } \\
\text { CO }\end{array}$ & $\begin{array}{r}9,908 \\
175,417\end{array}$ & $\begin{array}{r}5.0 \\
87.7\end{array}$ & & \\
\hline $\mathrm{NO}_{x}$ (assumed NO) & 150,357 & 75.2 & & \\
\hline Unbumed Hydrocarbons & 31,575 & 15.8 & & \\
\hline Subtotal of Contaminants & 421,359 & 211 & & \\
\hline
\end{tabular}




\section{Cementitious Waste Option}


Construction costs are from the detailed cost estimate ( $R$. Adams)

\begin{tabular}{|lc|}
\hline Interim Storage Facility Option, Prepared by & Byron L. Blackley, R.A., December 24, 1997. \\
\hline Construction duration $=$ & 4 years \\
Labor $=$ & 124 new workers/yr \\
1 man-year of labor $=$ & 1800 manhours \\
Square footage $=$ & 158,494 sq.ft $=$ \\
Acres disturbed $=$ & 9 acres (previous) \\
\hline
\end{tabular}

Excavation: Excavated earth will be spread in a spoil area adjacent to ICPP, except for backfill soil.

Excess Material $=\quad 22,040 \mathrm{yd}^{3} \quad$ (detailed cost estimate)

Air Emissions:

Dust during construction $=1.2$ tons $/$ month-acre $=$ (from USEPA Office of Ar Quality Planning and Standards)

518 tons (total)

Air emissions from fuel usage are based on the diesel emissions spreadsheet.

Effluents:

Sanitary Wastewater $=\quad 697,500 \mathrm{gal} / \mathrm{yr}=\quad 10,560,150$ liters (total)

(based on $25 \mathrm{gal} / \mathrm{person}$-day and 225 days/year of construction)

(Benefield, LD and C.W. Randall, Biological Process Design for Wastewater Treatment, InPrint, Inc., 1987, p. 104 wastewater generation $=15-30$ gal/day-person)

Assume 3 gallons of lubricating oil and

hydraulic fluid generated for every 60 hours of

operation of heavy equipment $=$

(Heavy equipment hrs are calculated below.)

2,770 liter (total)

\title{
Solid Wastes:
}

Construction trash $=\quad 7,688$ yd3 (total) $=\quad 5,880 \mathrm{~m}^{3}$ (total)

(Use $15.5 \mathrm{yd} 3 / \mathrm{yr}$ per capita. This is twice the generation rate of trash from site operations)

Hazardous/toxic chemicals and wastes (type)

Hazardous waste generation $=\quad 275$ gal/week $=\quad 217 \mathrm{~m}^{3}$ (total)

(based on an assumed generation rate of 555 -gallon drums of waste per week)

Hazardous waste storage $=\quad 3300 \mathrm{gal}=\quad 13 \mathrm{~m}^{3}$

(Assume waste is accumulated for 12 weeks [ 84 days] in a 90-day accumulation area, then

\section{Water Usage:}

Water used for dust control =

Area

assume $0.05^{\prime \prime}$ water per week to control dust

\author{
9 acres (previous) \\ $43,560 \mathrm{sq}$ ftacre \\ 0.05 in water/wk \\ $0.08333 \mathrm{ft} / \mathrm{in}$ \\ 28.320 liter $/ \mathrm{ft}^{3}$
}


assume dust control required

20 weeks

Length of Construction

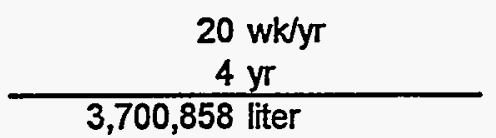

Domestic water use is assumed to be the same as sanitary waste.

Energy Requirements:

Electrical usage assumed to be $3,000 \mathrm{kWh}$ (from John Duggan)

$156 \mathrm{MWh} / \mathrm{yr}$

\section{Fossil Fuel Use}

\section{Concrete:}

Amount used $=$

(Detailed Cost Estimate)

\begin{aligned} $1,360 \mathrm{yd}^{3} &$ Tunnel \\ $25,430 \mathrm{yd}^{3} &$ Building \\ $7,480 \mathrm{yd}^{3} &$ Charge Face Slab \\ \hline $34,270 \mathrm{yd}^{3} &$ Total Concrete \end{aligned}

Number of truckloads $=$

Fuel usage $=$

1,558 (based on $22 \mathrm{yd}^{3} /$ load... tandem trailers)

$34,270 \mathrm{gal}$ (assumes $5 \mathrm{mpg}$ and round trip of 110 miles)

Materials delivery:

Number of truckloads $=$

779 (assume 1/2 the loads of concrete)

Fuel usage $=$

23,366 gal (assumes $5 \mathrm{mpg}$ and round trip of 150 miles)

Construction Equipment: (formula from Byron Blakely)

Construction Equipment Cost

$\$ 1,014,180$ (Detailed Cost Estimate)

$\$ 75$ per hr

$13,522 \mathrm{hr}$

Labor Hours

835,982 (Detailed Cost Estimate)

750 labor hr/equipment $h r$

$1,115 \mathrm{hr}$

Total Equipment Hours =

$14,637 \mathrm{hr}$

Equipment fuel usage (see http://www/deere.com/ind) = Total heavy equipment fuel usage $=$
$6 \mathrm{gal} / \mathrm{hr}$
$87,822 \mathrm{gal}$
or 332,407 liters (total)

Total heavy equipment and materials

Concrete Delivery

Materials Delivery

$34,270 \mathrm{gal}$

Heavy Equipment

$23,366 \mathrm{gal}$

87,822 gal

$145,458 \mathrm{gal}$

3.785 liter/gal

550,559 liter 


\begin{tabular}{|c|c|}
\hline Operating & $m$ the \\
\hline Operation & \\
\hline Loading & $6 \mathrm{yr}$ \\
\hline Holding & $49 \mathrm{yr}$ \\
\hline Shipping & $5 \mathrm{yr}$ \\
\hline
\end{tabular}

Interim Storage Facility Option, Prepared by Byron L. Blackley, R.A., December 24, 1997.

Labor: 8.5

Cannisters $\quad 16000$

Power $500 \mathrm{~kW}$

Radiation worker annual dose is based on the average annual dose received at ICCP during the last three years (see attached memorandum)

\section{Effluents:}

Sanitary wastewater $=$ $77,563 \mathrm{gal} / \mathrm{yr}=$

(based on 25 gal/day per worker, facility occupied year-round)

\section{Solid Wastes:}

Sanitary/Industrial trash $=$ (based on 7.25 yd3/person-year... Bob Skinner [cuber facility])
$62 \mathrm{yd}^{3} / \mathrm{yr}$
$47 \mathrm{~m}^{3} / \mathrm{yr}$

Product canisters $=$

(based on 16,000 canisters @ $0.72 \mathrm{~m}^{3} /$ canister)

Total rad waste $=$

$3,456 \mathrm{~m}^{3}$

Water Usage:

Domestic water usage $=$ same as sanitary wastewater rate.

\section{Energy Requirements}

Electric power usage $=$ (based on $500 \mathrm{~kW}$ ) 
D\&D Labor

\begin{tabular}{|c|c|c|c|c|c|c|}
\hline \multicolumn{2}{|c|}{ Crew \# Crew Function } & \multirow{2}{*}{$\begin{array}{r}\text { Total MH/day } \\
18\end{array}$} & \multirow{2}{*}{$\begin{array}{r}\text { Total \$/day } \\
\$ 1,136\end{array}$} & \multirow{2}{*}{$\begin{array}{l}\text { Material } \\
\text { \$/day } \\
\$ 114\end{array}$} & $\begin{array}{l}\text { Equipment } \\
\$ / \text { day }\end{array}$ & \multirow{2}{*}{$\begin{array}{r}\text { Total } \$ / \text { day } \\
\$ 1,250\end{array}$} \\
\hline $\mathbf{D}$ & Documentation & & & & $\$$ & \\
\hline 1 & Characterization & 44 & $\$ 2,302$ & $\$ 460$ & $\$ 691$ & $\$ 3,453$ \\
\hline 2 & Rad Demolition-Systems & 77 & $\$ 4,091$ & $\$ 818$ & $\$ 1,023$ & $\$ 5,932$ \\
\hline $2 \mathrm{~A}$ & Rad Demolition-Building & 99 & $\$ 5,319$ & $\$ 1,064$ & $\$ 1,596$ & $\$ 7,979$ \\
\hline 3 & Demolition-Systems & 72 & $\$ 3,762$ & $\$ 752$ & $\$ 941$ & $\$ 5,455$ \\
\hline $3 A$ & Demolition-Building & 88 & $\$ 4,808$ & $\$ 962$ & $\$ 1,442$ & $\$ 7,212$ \\
\hline 4 & Asbestos Abatement & 77 & $\$ 3,753$ & $\$ 375$ & $\$ 188$ & $\$ 4,316$ \\
\hline 5 & Decontamination & 77 & $\$ 3,753$ & $\$ 751$ & $\$ 1,126$ & $\$ 5,630$ \\
\hline 6 & Prep/Fabrication & 61 & $\$ 3,217$ & $\$ 643$ & $\$ 965$ & $\$ 4,826$ \\
\hline 7 & RADCON Surveys & 50 & $\$ 2,596$ & $\$ 519$ & $\$ 779$ & $\$ 3,894$ \\
\hline
\end{tabular}

\section{Total}

vallable

\begin{tabular}{cc} 
& \multicolumn{2}{c}{ D\&D Cost } \\
& Allocated \\
$\$$ & $3,990,000$ \\
$\$$ & $5,987,000$ \\
$\$$ & - \\
$\$$ & - \\
$\$$ & $29,470,000$ \\
$\$$ & $58,930,000$ \\
$\$$ & - \\
$\$$ & - \\
$\$$ & $10,400,000$ \\
$\$$ & $5,720,000$ \\
& \\
$\$$ & $114,497,000$ \\
& \\
$\$$ & $114,497,000$
\end{tabular}

Notes:

1 Crew functions and daily estimates are from the D\&D program (Dave Haycraft)

2 Total costs are based on life cycle estimate by R. Turk

3 Assume all workers in crews $2,2 A, 5$, and 7 are rad workers

4 Assume a man-year is 1800 hours.

\section{Decommission \\ Documentation \\ Characterization \\ Demolition \\ Demolition-Systems \\ Demolition-Building \\ Prep/Fabrication \\ RADCON Surveys}

Decontamination

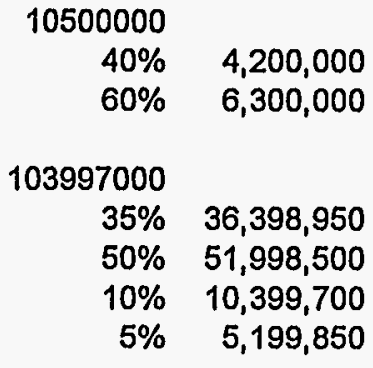

0 
No decontamination of the storage facility will be required.

$D \& D$ labor requirements are taken from $D$ \& $D$ labor and equipment spreadsheet.

$D \& D$ costs come from the life cycle cost estimate.
1 manyear of labor $=$
1800 manhours

\begin{tabular}{lrrrr|r} 
Heavy Equipment & \#Used & Hours/day & Days/wk & Wks/yr & Hours/yr \\
Mobile Crane & 2 & 5 & 5 & 45 & 2,250 \\
Roll-Off Truck & 4 & 8 & 5 & 45 & 7,200 \\
Dozer & 3 & 6 & 5 & 45 & 4,050 \\
Loader & 4 & 8 & 5 & 45 & 7,200 \\
Scabbler (w/ Vacuum System) & 0 & 6 & 5 & 45 & - \\
Pneumatic Ram & 1 & 8 & 5 & 45 & 1,800 \\
Demolition Machine (Remote & 0 & 4 & 4 & 45 & - \\
$\quad$ Control) & 0 & & & \\
\hline Not needed, contamination not expected & & & & \\
Total hours/yr & & & &
\end{tabular}

Total heavy equipment hours $=$

Assume each piece of equipment uses 6 gallon of diesel fuel per hour. Consumption rate from John Deere Web Site (Construction Equipment - http://www.deere.com/ind/product/product.html)

No. of gallons of fuel used during $D \& D=$

$$
405,000 \mathrm{gal}=
$$

Assume each roll-off truck makes 3 trips per day to RWMC

No. of trips $=$

Miles traveled @ 12 miles/round trip=

Acres disturbed and duration of disturbance

Acreage disturbed is the same as for construction =

9.0 acres

\section{Air emissions}

HEPA filter ventilation is not required during D\&D.

Air emissions from fuel are based on the diesel emissions spreadsheet.

\section{Effluents}

No decontamination solution required during D\&D.
Sanitary wastewater $=$

(based on $25 \mathrm{gal} /$ day per worker, 225 day/yr)

Lube oil = 12,774 liters (total)

(based on 3 gal for every 60 hours of operation)

\section{Solid wastes:}

The following factors from Dave Kenoyer are based on a contaminated building. The storage facility is not expected to be contaminated. Therefore, all items identified as LLW will be included with the non-contaminated waste. These factors are used for consistency in waste volumes with facilities which are contaminated.

Solid Waste Generation (factors from Dave Kenoyer - D\&D Program)

$\begin{array}{llcc}\text { Wactor } & \text { Sq.Ft. in } & \text { Cu.Ft. of } \\ \text { Waste Type } \quad \text { Waste Type (cu.ft./sq.ft.) } & \text { Facility } & \text { Waste }\end{array}$ Cu. Meters




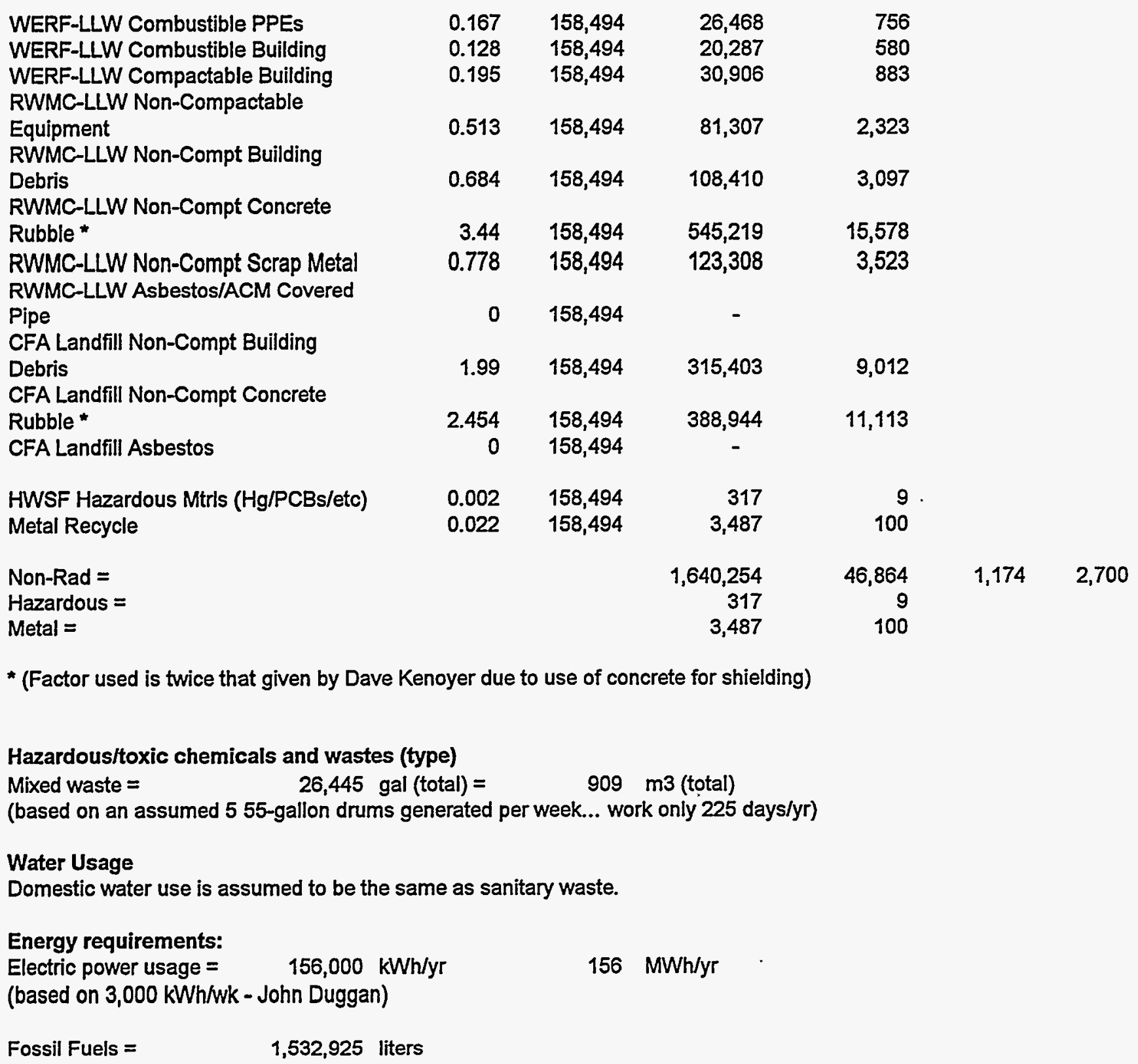




\section{Estimate of Diesel Engine Emissions}

Bases \& Assumptions:

\section{CWO}

1. Air to fuel ratio $=25: 1$ (Mass Basis)

2. Diesel fuel density $=7.5 \mathrm{lbs} / \mathrm{gal}$.

3. Air is $21 \% \mathrm{O}_{2}, 79 \% \mathrm{~N}_{2}$, with a pseudomolecular weight of 29 .

4. Combustion is simulated as: $\mathrm{C}_{9} \mathrm{H}_{18}+13.5 \mathrm{O}_{2} \longrightarrow 9 \mathrm{CO}_{2}+9 \mathrm{H}_{2} \mathrm{O}$

5. Particulates $=5 \mathrm{mg} / \mathrm{scf}$

6. $\mathrm{CO}=2,500 \mathrm{ppmv}$

7. $\mathrm{NO}_{\mathrm{x}}=2,000 \mathrm{ppmv}$

8. Unburned hydrocarbons $=100 \mathrm{ppmv}$

9. Diesel fuel (\# 2 fuel oil) contains 1 wt. \% sulfur

10. Combustion is about $99 \%$ efficient.

Lbs. Of Construction Fuel

Lbs. Of Operations Fuel

Lbs. Of D\&D Fuel

Total Lbs. of Fuel Used

Lb-Moles of Construction Fuel

Lb-Moles of Operations Fuel

Lb-Moles of D\&D Fuel

Total Lb-Moles of Fuel (as C9H18)

Lbs of Air for Construction Fuel (based on air-to-fuel ratio)

Lbs.of Air for Operations Fuel (based on air-to-fuel ratio)

Lbs.of Air for D\&D Fuel (based on air-to-fuel ratio)

Total Lbs. of Air Added

Lb-Moles of Air for Combustion Fuel

Lb-Moles of Air for Operations Fuel

Lb-Moles of Air for D\&D Fuel

Total Lb-Moles of Air from Wark, K. and C.F. Wamer, Air Pollution, Its Origin and Control, IEP, New York, 1976, p. 446, 423

Wark and Warner, p. 446 Wark and Wainer, p. 446 Wark and Wamer, p. 446 Wark and Warner, p. 446 Wark and Warner, p. 336
Grand Total of Materials Fed, Lbs.

$1,090,107$

$3,035,192$

$4,125,299$

8,652

24,089

32,740

$27,252,678$

$75,879,788$

$103,132,466$

939,748

$2,616,544$

$3,556,292$

$107,257,764$

Exhaust Gases, Construction Fuel

Total Lbs.

Total Tons

1,696

694

$1,387,551$

1,307

$\mathrm{O}_{2}$

$2,614,968$

10,394

$20,787,215$

14,091

Subtotal of Major Gases

$\mathrm{SO}_{2}$

$28,181,525$

21,802

10.9

Particulates

3,868

68,480

1.9

$\mathrm{CO}$

58,697

34.2

$\mathrm{NO}_{x}$ (assumed NO)

12,326

29.3

Unburned Hydrocarbons

165,174
Total Moles

77,086

77,086

81,718

742,401

978,291
Subtotal of Contaminants

6.2

83
Total SCF

$27,673,927$

$27,673,927$

$29,336,676$

$266,521,795$

$351,206,324$ 


\begin{tabular}{|c|c|c|c|c|}
\hline Exhaust Gases, Operations Fuel & Total Lbs. & Total Tons & Total Moles & Total SCF \\
\hline $\mathrm{CO}_{2}$ & - & - & - & - \\
\hline $\mathrm{H}_{2} \mathrm{O}$ & - & - & - & - \\
\hline $\mathrm{O}_{2}$ & - & - & - & - \\
\hline $\mathrm{N}_{2}$ & - & - & - & - \\
\hline Subtotal of Major Gases & - & - & - & - \\
\hline $\mathrm{SO}_{2}$ & - & - & & \\
\hline Particulates & - & - & & \\
\hline $\mathrm{co}$ & - & - & & \\
\hline $\mathrm{NO}_{x}$ (assumed NO) & - & - & & \\
\hline Unbumed Hydrocarbons & - & - & & \\
\hline Subtotal of Contaminants & - & - & & \\
\hline Exhaust Gases, D\&D Fuel & Total Lbs. & Total Tons & Total Moles & Total SCF \\
\hline$\overline{\mathrm{CO}_{2}}$ & $9,443,782$ & 4,722 & 214,631 & $77,052,672$ \\
\hline $\mathrm{H}_{2} \mathrm{O}$ & $3,863,365$ & 1,932 & 214,631 & $77,052,672$ \\
\hline $\mathrm{O}_{2}$ & $7,280,871$ & 3,640 & 227,527 & $81,682,274$ \\
\hline $\mathrm{N}_{2}$ & $57,877,962.05$ & 28,939 & $2,067,070$ & $742,078,156$ \\
\hline Subtotal of Major Gases & $78,465,980$ & 39,233 & $2,723,860$ & $977,865,774$ \\
\hline $\mathrm{SO}_{2}$ & 58,807 & 29.4 & & \\
\hline $\begin{array}{l}\text { Particulates } \\
\text { co }\end{array}$ & $\begin{array}{r}10,769 \\
190,670\end{array}$ & $\begin{array}{r}5.4 \\
95.3\end{array}$ & & \\
\hline $\mathrm{NO}_{x}$ (assumed NO) & 163,432 & 81.7 & & \\
\hline Unbumed Hydrocarbons & 34,321 & 17.2 & & \\
\hline Subtotal of Contaminants & 457,999 & 229 & & \\
\hline
\end{tabular}




\section{Hot Isostatic Press Waste Option}


Construction costs are from the detailed cost estimate ( $R$. Adams)

Interim Storage Facility Option, Prepared by Byron L. Blakely, R.A., December 24, 1997.

Construction duration $=$

Labor $=$

1 man-year of labor $=$

3 years

Square footage $=$

Acres disturbed $=$

92 new workers/yr

1800 manhours

$78,432 \mathrm{sq} . \mathrm{ft}=$

3 acres (previous)

$7,287 \mathrm{~m}^{2}$

Excavation: Excavated earth will be spread in a spoil area adjacent to ICPP, except for backfill soil.

Air Emissions:

Dust during construction $=1.2$ tons $/$ month-acre $=$

(from USEPA Office of Ar Quality Planning and Standards)

108 tons (total)

Air emissions from fuel usage are based on the diesel emissions spreadsheet.

\section{Effluents:}

Sanitary Wastewater $=$

$517,500 \mathrm{gal} / \mathrm{yr}=$

$5,876,213$ liters (total)

(based on 25 gal/person-day and 225 days/year of construction)

(Benefield, LD and C.W. Randall, Biological Process Design for Wastewater Treatment, InPrint, Inc., 1987, p. 104 wastewater generation $=15-30$ gal/day-person)

Assume 3 gallons of lubricating oil and hydraulic

fluid generated for every 60 hours of operation

of heavy equipment $=$

1,541 liter (total)

(Heavy equipment hrs are calculated below.)

\section{Solid Wastes:}

Construction trash $=\quad 4,278$ yd3 (total) $=\quad 3,272 \mathrm{~m}^{3}$ (total)

(Use $15.5 \mathrm{yd} 3 / \mathrm{yr}$ per capita. This is twice the generation rate of trash from site operations)

Hazardous/toxic chemicals and wastes (type)

Hazardous waste generation $=\quad 275$ gal/week $=\quad 217 \mathrm{~m}^{3}$ (total)

(based on an assumed generation rate of 555 -gallon drums of waste per week)

Hazardous waste storage $=\quad 3300 \mathrm{gal}=\quad 13 \mathrm{~m}^{3}$

(Assume waste is accumulated for 12 weeks [84 days] in a 90-day accumulation area, then

Water Usage:

Water used for dust control $=$

Area

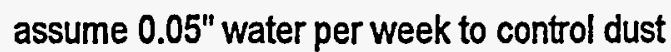

assume dust control required 20 weeks

Length of Construction

3 acres (previous)

43,560 sq fflacre

0.05 in water/wk

$0.08333 \mathrm{ft} / \mathrm{in}$

28.320 liter $/ \mathrm{ft}^{3}$

20 wk/yr

3 yr

771,012 liter 


\section{Construction Assumptions}

Domestic water use is assumed to be the same as sanitary waste.

Energy Requirements:

Electrical usage assumed to be $3,000 \mathrm{kWh}$ (from John Duggan) $156 \mathrm{MWh} / \mathrm{yr}$

\section{Fossil Fuel Use}

Concrete:

Amount used $=$

(Detailed Cost Estimate)

\begin{aligned} $1,360 \mathrm{yd}^{3} &$ Tunnel \\ $15,050 \mathrm{yd}^{3} &$ Building \\ $3,375 \mathrm{yd}^{3} &$ Charge Face Slab \\ \hline $19,785 \mathrm{yd}^{3} &$ Total Concrete \end{aligned}

Number of truckloads $=$

899 (based on $22 \mathrm{yd}^{3} /$ load... tandem trailers)

Fuel usage $=$ $19,785 \mathrm{gal}$ (assumes $5 \mathrm{mpg}$ and round trip of $110 \mathrm{miles}$ )

Materials delivery:

Number of truckloads $=$

Fuel usage $=$

450 (assume 1/2 the loads of concrete)

$13,490 \mathrm{gal}$ (assumes $5 \mathrm{mpg}$ and round trip of $150 \mathrm{miles}$ )

\section{Construction Equipment:}

Construction Equipment Cost

$\$ 565,076$ (Detailed Cost Estimate)

$\$ 75$ per hr

$7,534 \mathrm{hr}$

Labor Hours

454,293 (Detailed Cost Estimate)

750 labor hr/equipment $\mathrm{hr}$

$606 \mathrm{hr}$

Total Equipment Hours =

$8,140 \mathrm{hr}$

Equipment fuel usage (see http://www/deere.com/ind) =

Total heavy equipment fuel usage $=$

$6 \mathrm{gal} / \mathrm{hr}$
$48,840 \mathrm{gal}$

Total heavy equipment and materials

delivery fuel used =

Concrete Delivery

Materials Delivery

Heavy Equipment

\begin{tabular}{c}
$19,785 \mathrm{gal}$ \\
$13,490 \mathrm{gal}$ \\
$48,840 \mathrm{gal}$ \\
\hline $82,115 \mathrm{gal}$ \\
3.785 liter $/ \mathrm{gal}$ \\
\hline 310,806 liter
\end{tabular}




\begin{tabular}{|l|l|}
\hline Operating costs are taken from the life cycle cost estimate. \\
\hline Operation & $23 \mathrm{yr}$ \\
Loading & $37 \mathrm{yr}$ \\
Holding & $5 \mathrm{yr}$ \\
Shipping & \\
\hline
\end{tabular}

Interim Storage Facility Option, Prepared by Byron L. Blakely, R.A., December 24, 1997.

Labor: 6.5

Cannisters $\quad 5700$

Power $500 \mathrm{~kW}$

Radiation worker annual dose is based on the average annual dose received at ICCP during the last three years (see attached memorandum)

\section{Effluents:}

Sanitary wastewater $=$ (based on $\mathbf{2 5} \mathrm{gal} / \mathrm{day}$ per worker, facility occupied year-round)

\section{Solid Wastes:}

Sanitary/Industrial trash $=$ (based on 7.25 yd3/person-year... Bob Skinner [cuber facility])
$47 \mathrm{yd}^{3} / \mathrm{yr}$
$36 \mathrm{~m}^{3} / \mathrm{yr}$

Product canisters $=$

(based on 5700 canisters @ $0.72 \mathrm{~m}^{3} /$ canister)

Total rad waste $=\quad 4,720 \mathrm{~m}^{3}$

Water Usage:

Domestic water usage $\mathbf{~}$ same as sanitary wastewater rate.

\section{Energy Requirements}

Electric power usage $=$ (based on $500 \mathrm{~kW}$ ) 
D\&D Labor

Crew \# Crew Function

Documentation

Characterization

Rad Demolition-Systems

Rad Demolition-Building

Demolition-Systems

Demolition-Building

Asbestos Abatement

Decontamination

Prep/Fabrication

RADCON Surveys

$\begin{array}{rr}\text { Total MH/day } & \text { Total } \$ / \text { day } \\ 18 & \$ 1,136 \\ 44 & \$ 2,302 \\ 77 & \$ 4,091 \\ 99 & \$ 5,319 \\ 72 & \$ 3,762 \\ 88 & \$ 4,808 \\ 77 & \$ 3,753 \\ 77 & \$ 3,753 \\ 61 & \$ 3,217 \\ 50 & \$ 2,596\end{array}$

Years

From D\&D

3 Assumptions

Total

vailable

Material Equipment

$\$ /$ day

$\$ 114$

$\$ 460$

$\$ 818$

$\$ 1,064$

$\$ 752$

$\$ 962$

$\$ 375$

$\$ 751$

$\$ 643$

$\$ 519$

$\$ /$ day

$\$ 691$

$\$ 1,023$

$\$ 1,596$

$\$ 941$

$\$ 1,442$

$\$ 188$

$\$ 1,126$

$\$ 965$

$\$ 779$
Total \$/day

$\$ 1,250$

$\$ 3,453$

$\$ 5,932$

$\$ 7,979$

$\$ 5,455$

$\$ 7,212$

$\$ 4,316$

$\$ 5,630$

$\$ 4,826$

$\$ 3,894$

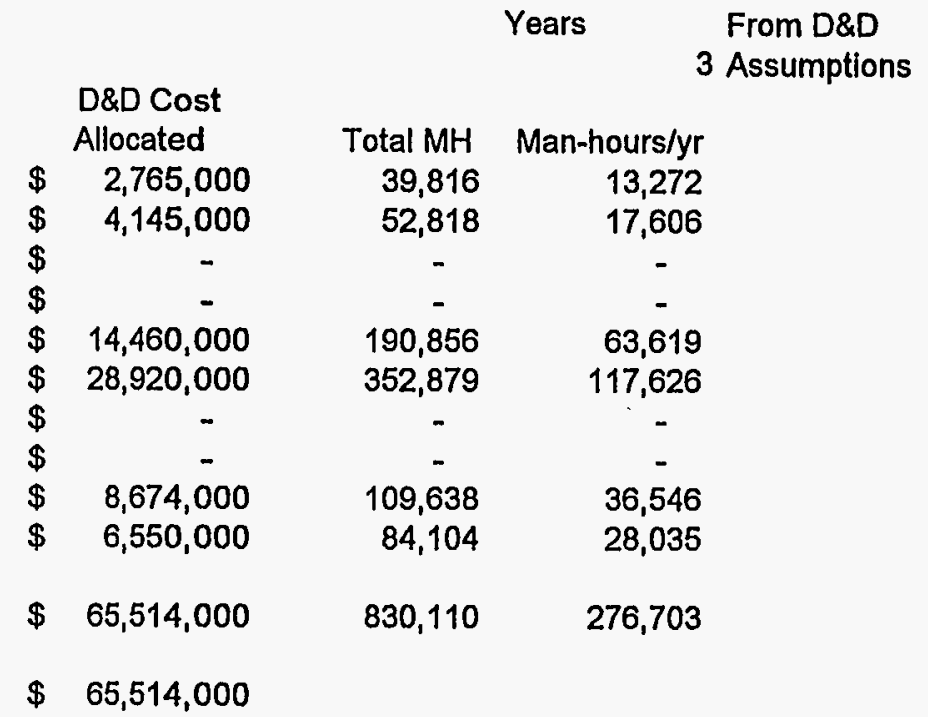

Notes:

1 Crew functions and daily estimates are from the D\&D program (Dave Haycraft)

2 Total costs are based on life cycle estimate by $R$. Turk

3 Assume all workers in crews 2,2A, 5, and 7 are rad workers

4 Assume a man-year is 1800 hours. 
No decontamination of the storage facility will be required.

$D \& D$ labor requirements are taken from $D$ \& $D$ labor and equipment spreadsheet.

$D \& D$ costs come from the life cycle cost estimate.

1 manyear of labor $=\quad 1800$ manhours

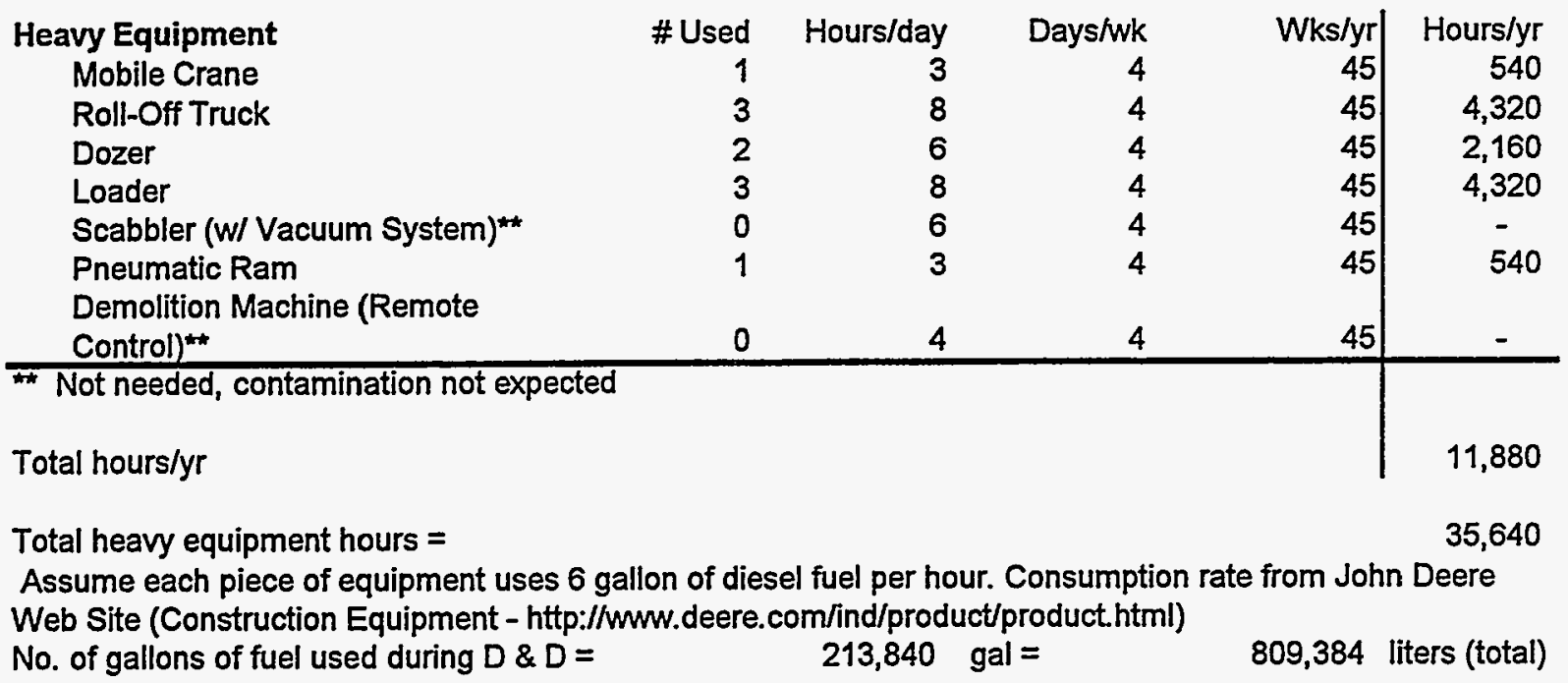

Assume each roll-off truck makes 3 trips per day to RWMC

No. of trips $=$

Miles traveled @ 12 miles/round trip= $\quad 108$

Acres disturbed and duration of disturbance

Acreage disturbed is the same as for construction = 2.5 acres

\section{Air emissions}

HEPA filter ventilation is not required during D\&D.

Air emissions from fuel are based on the diesel emissions spreadsheet.

\section{Effluents}

No decontamination solution required during D\&D.

Sanitary wastewater $=\quad 864,698$ gal/yr $=\quad 9,818,650$ liters (total)

(based on $25 \mathrm{gal} /$ day per worker, $225 \mathrm{day} / \mathrm{yr}$ )

Lube oil $=\quad 6,745$ liters (total)

(based on 3 gal for every 60 hours of operation)

\section{Solid wastes:}

The following factors from Dave Kenoyer are based on a contaminated building. The storage facility is not expected to be contaminated. Therefore, all items identified as LLW will be included with the non-contaminated waste. These factors are used for consistency in waste volumes with facilities which are contaminated.

Solid Waste Generation (factors from Dave Kenoyer - D\&D Program)

$\begin{array}{llcccc} & \text { Factor } & \text { Sq.Ft. in } & \text { Cu.Ft. of } & \\ \text { Waste Type } & \text { Waste Type } & \text { (cu.ft./sq.ft.) } & \text { Facility } & \text { Waste } & \text { Cu. Meters }\end{array}$




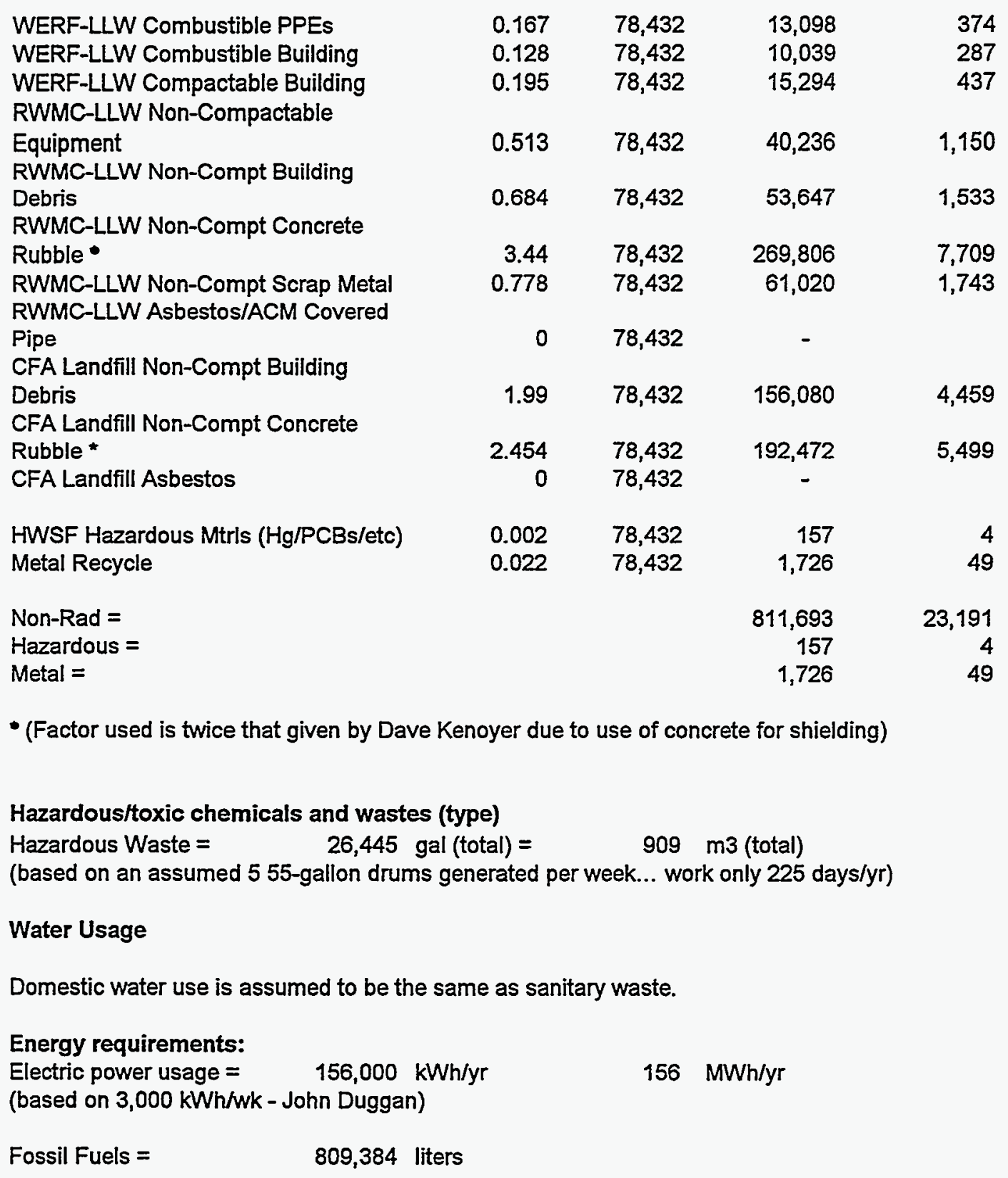


Bases \& Assumptions:

1. Air to fuel ratio $=25: 1$ (Mass Basis)

2. Diesel fuel density $=7.5 \mathrm{lbs} . \mathrm{ggal}$.

3. Air is $21 \% \mathrm{O}_{2}, 79 \% \mathrm{~N}_{2}$, with a pseudomolecular weight of 29 .

4. Combustion is simulated as: $\mathrm{C}_{9} \mathrm{H}_{18}+13.5 \mathrm{O}_{2} \rightarrow 9 \mathrm{CO}_{2}+9 \mathrm{H}_{2} \mathrm{O}$

5. Particulates $=5 \mathrm{mg} / \mathrm{scf}$

6. $\mathrm{CO}=2,500 \mathrm{ppmv}$

7. $\mathrm{NO}_{\mathrm{x}}=2,000 \mathrm{ppmv}$

8. Unburned hydrocarbons $=100 \mathrm{ppmv}$

9. Diesel fuel (\# 2 fuel oil) contains 1 wt. \% sulfur

10. Combustion is about $99 \%$ efficient.

Lbs. Of Construction Fuel

Lbs. Of Operations Fuel

Lbs. Of D\&D Fuel

Total Lbs. of Fuel Used

Lb-Moles of Construction Fuel

Lb-Moles of Operations Fuel

Lb-Moles of D\&D Fuel

Total Lb-Moles of Fuel (as C9H18)

Lbs of Air for Construction Fuel (based on air-to-fuel ratio)

Lbs.of Air for Operations Fuel (based on air-to-fuel ratio)

Lbs.of Air for D\&D Fuel (based on air-to-fuel ratio)

Total Lbs. of Air Added

Lb-Moles of Air for Combustion Fuel

Lb-Moles of Air for Operations Fuel

Lb-Moles of Air for D\&D Fuel

Total Lb-Moles of Air from Wark, K. and C.F. Warner, Air Pollution, Its Origin and Control, IEP, New York, 1976, p. 446, 423

Wark and Warner, p. 446

Wark and Warner, p. 446

Wark and Warner, p. 446

Wark and Warner, p. 446

Wark and Wamer, p. 336

Grand Total of Materials Fed, Lbs.

$$
\begin{array}{r}
310,806 \\
- \\
1,602,581 \\
1,913,387 \\
2,467 \\
- \\
12,719 \\
15,186 \\
\\
7,770,150 \\
- \\
40,064,528 \\
47,834,678 \\
\\
267,936 \\
- \\
1,381,535 \\
1,649,472
\end{array}
$$

$49,748,065$

\begin{tabular}{lrrrr} 
Exhaust Gases, Construction Fuel & \multicolumn{1}{c}{ Total Lbs. } & \multicolumn{1}{c}{ Total Tons } & \multicolumn{1}{c}{ Total Moles } & \multicolumn{1}{c}{ Total SCF } \\
\hline $\mathrm{CO}_{2}$ & 967,051 & 484 & 21,978 & $7,890,255$ \\
$\mathrm{H}_{2} \mathrm{O}$ & 395,612 & 198 & 21,978 & $7,890,255$ \\
$\mathrm{O}_{2}$ & 745,567 & 373 & 23,299 & $8,364,330$ \\
$\mathrm{~N}_{2}$ & $5,926,749$ & 2,963 & 211,670 & $75,989,392$ \\
\hline Subtotal of Major Gases & $\mathbf{8 , 0 3 4 , 9 7 9}$ & $\mathbf{4 , 0 1 7}$ & $\mathbf{2 7 8 , 9 2 5}$ & $\mathbf{1 0 0 , 1 3 4 , 2 3 2}$ \\
$\mathrm{SO}_{2}$ & 6,216 & 3.1 & \\
$\mathrm{Particulates}$ & 1,103 & 0.6 & \\
$\mathrm{CO}$ & 19,525 & 9.8 & \\
NO (assumed NO) & 16,736 & 8.4 & \\
Unburned Hydrocarbons & 3,514 & 1.8 & \\
Subtotal of Contaminants & $\mathbf{4 7 , 0 9 4}$ & $\mathbf{2 4}$ & \\
\hline
\end{tabular}




\begin{tabular}{|c|c|c|c|c|}
\hline Exhaust Gases, Operations Fuel & Total Lbs. & Total Tons & Total Moles & Total SCF \\
\hline$\overline{\mathrm{CO}_{2}}$ & - & - & $\overline{-}$ & - \\
\hline $\mathrm{H}_{2} \mathrm{O}$ & - & - & - & - \\
\hline $\mathrm{O}_{2}$ & - & - & - & - \\
\hline $\mathrm{N}_{2}$ & - & - & - & - \\
\hline Subtotal of Major Gases & - & - & - & - \\
\hline $\mathrm{SO}_{2}$ & - & - & & \\
\hline Particulates & - & - & & \\
\hline $\mathrm{co}$ & - & - & & \\
\hline $\mathrm{NO}_{x}$ (assumed $\mathrm{NO}$ ) & - & - & & \\
\hline Unburned Hydrocarbons & - & - & & \\
\hline Subtotal of Contaminants & - & - & & \\
\hline Exhaust Gases, D\&D Fuel & Total Lbs. & Total Tons & Total Moles & Total SCF \\
\hline $\mathrm{CO}_{2}$ & $4,986,317$ & 2,493 & 113,325 & $40,683,811$ \\
\hline $\mathrm{H}_{2} \mathrm{O}$ & $2,039,857$ & 1,020 & 113,325 & $40,683,811$ \\
\hline $\mathrm{O}_{2}$ & $3,844,300$ & 1,922 & 120,134 & $43,128,241$ \\
\hline $\mathrm{N}_{2}$ & $30,559,563.96$ & 15,280 & $1,091,413$ & $391,817,267$ \\
\hline Subtotal of Major Gases & $41,430,037$ & 20,715 & $1,438,198$ & $516,313,129$ \\
\hline $\mathrm{SO}_{2}$ & 31,050 & 15.5 & & \\
\hline Particulates & 5,686 & 2.8 & & \\
\hline $\mathrm{CO}$ & 100,674 & 50.3 & & \\
\hline $\mathrm{NO}_{x}$ (assumed NO) & 86,292 & 43.1 & & \\
\hline Unbumed Hydrocarbons & 18,121 & 9.1 & & \\
\hline Subtotal of Contaminants & 241,823 & 121 & & \\
\hline
\end{tabular}


Project File Number 02BJ0

Project/Task Waste Treatment Project Feasibility Studies

Subtask Interim Storage Study

\begin{tabular}{|c|c|}
\hline Summary: & $\begin{array}{l}\text { This summary briefly defines the problem or activity to be addressed in the } \\
\text { Engineering Design File (EDF), gives a summary of the activities performed in } \\
\text { addressing the problem, and states the conclusions, recommendations, or results } \\
\text { arrived at from this task. }\end{array}$ \\
\hline
\end{tabular}

High-level radioactive waste (HLW) stored at the Idaho Chemical Processing Plant (ICPP) in the form of calcine and liquid will be processed to provide a stable waste form and prepare the waste to be transported to a permanent repository. Because a permanent repository will not be available when the waste is processed, the waste must be stored at ICPP in an Interim Storage Facility (ISF). This EDF documents consideration of the Fuel Processing Restoration (FPR) facility as an ISF for each of the processing options.

Distribution (complete package): Included in Appendix A of Interim Storage Study Report, INEEL/EXT-97-01393, February 1998.

Distribution (summary package only):

\begin{tabular}{|c|c|c|c|c|c|}
\hline \begin{tabular}{|l} 
Author \\
\end{tabular} & Dept. & Reviewed & Date & Approved & Date \\
\hline Bteferverim & 4136 & $\begin{array}{l}\text { LMITCO } \\
\text { Reyiew }\end{array}$ & $\begin{array}{l}\text { Date } \\
2 / 10 / 98\end{array}$ & $\begin{array}{l}\text { LMTTCO Approval } \\
\text { W. Liteaser }\end{array}$ & $\begin{array}{l}\text { Date } \\
2 / 10 / 98\end{array}$ \\
\hline
\end{tabular}

See Management Control Procedure (MCP) 6 for instructions on use of this form. 


\section{Modification of FPR for Interim Storage of HLW}

\section{ABSTRACT}

High-level radioactive waste (HLW) stored at ICPP in the form of calcine and liquid will be processed to provide a stable waste form and prepare the waste to be transported to a permanent repository. Because a permanent repository will not be available when the waste is processed, the waste must be stored at ICPP in an ISF. This EDF documents consideration of the Fuel Processing Restoration (FPR) facility as an ISF for each of the processing options.

\section{SUMMARY}

Disposal of radioactive wastes now stored at the Idaho Chemical Processing Plant (ICPP) at the Idaho National Engineering and Environmental Laboratory (INEEL) is mandated under a "Settlement Agreement" between the Department of Energy (DOE) and the State of Idaho. Among other things, the Settlement Agreement requires that existing inventory of liquid HLW be calcined by June 30, 1998, and SBW by the end of 2012, and the target date for all waste to be ready to ship from the INEEL is 2035 .

The Department of Energy Idaho Operations Office (DOE-ID) is preparing the High-Level Waste and Facilities Disposition Environmental Impact Statement (HLW\&FD EIS), in accordance with the National Environmental Policy Act, to support the decision-making process regarding HLW management at the INEEL. Fluor Daniel Corporation and Lockheed Martin Idaho Technologies Company (LMITCO) are studying waste treatment options. Because a repository for the waste will not be available as processing is performed, an ISF is required for storage for the period of time between processing and when a permanent repository is available.

This EDF documents a study that investigated the design, costs, and environmental impacts for modification of the Fuel Processing Restoration (FPR) facility at ICPP for an ISF for each of the processing options.

\section{Conclusions}

This study has investigated the modification of the FPR for storage of HLW that results from processing the waste at ICPP. The processing options are discussed in separate reports. This study determined that FPR can hold a maximum of 9,344 canisters and only those processing options that produce fewer canisters can be accommodated in the FPR. It is concluded that FPR can be modified and placed into operation to store the waste in canisters for the Full and TRU/Class A Separations Option and the Hot Isostatic Processed (HIP) Option for the period from processing until the time a permanent repository is available for receipt of this waste. The schedules and costs associated with interim storage for these processing options are presented in the body of this EDF.

\section{Recommendations}

The potential for additional costs and a longer schedule that would result from Nuclear Regulatory Commission (NRC) licensing of the FPR for interim storage should be studied further. The NRC does not have the structure in place to review and license an existing facility for interim storage of $\mathrm{HLW}$. 
Also, the FPR has not been built to the requirements that would be required by NRC. The cost included in this study for licensing was based on a reasonable assumption of the complexities involved without the benefit of a detailed study that is recommended.

A future study should be conducted to determine if a standard 15-ft long "Hanford" canister should be substituted for the 10-ft Savannah River Site (SRS) canister assumed in this study. Use of a 15-ft canister would increase the interim storage construction costs but would reduce the operation (handling) costs, and greatly reduce offsite transportation and disposal costs. A large net cost saving, primarily as a result of reduced transportation and disposal costs, could be realized by using the 15 -ft canister.

\section{ACKNOWLEDGMENTS}

The assistance of Steven W. Hills and David E. Keller who designed the FPR Interim Storage Facility presented in this EDF is greatly appreciated. Rick $D$. Adams prepared the project cost estimates and Robert J. Turk prepared the Life-Cycle Costs. Jennifer Quigley and Rod R. Kimmitt prepared the Project Data Sheets. Karen Williams was the Project Lead. Technical editing was performed by Stephen Lortie.

\section{ACRONYMS}

$\begin{array}{ll}\text { CFR } & \text { Code of Federal Regulations } \\ \text { DOE } & \text { Department of Energy } \\ \text { DOE-ID } & \text { DOE Idaho Operations Office } \\ \text { DOT } & \text { Department of Transportation } \\ \text { EIS } & \text { Environmental Impact Statement } \\ \text { FPR } & \text { Fuel Processing Restoration } \\ \text { HAW } & \text { high-activity radioactive waste } \\ \text { HIP } & \text { Hot Isostatic Press } \\ \text { HLW\&FD } & \text { High-Level Waste and Facilities Disposition } \\ \text { HLW } & \text { high-level radioactive waste } \\ \text { ICPP } & \text { Idaho Chemical Processing Plant } \\ \text { INEEL } & \text { Idaho National Engineering and Environmental Laboratory } \\ \text { ISF } & \text { Interim Storage Facility } \\ \text { LAW } & \text { low-activity waste } \\ \text { LMTCO } & \text { Lockheed Martin Idaho Technologies Company } \\ \text { NRC } & \text { Nuclear Regulatory Commission } \\ \text { RCRA } & \text { Resource Conservation and Recovery Act } \\ \text { SBW } & \text { sodium-bearing waste } \\ \text { SNF } & \text { spent nuclear fuel } \\ \text { TRU } & \text { Transuranic } \\ \text { WAC } & \text { Waste Acceptance Criteria } \\ \text { WIPP } & \text { Waste Isolation Pilot Plant }\end{array}$




\subsection{INTRODUCTION}

\subsection{Background}

The Idaho Chemical Processing Plant (ICPP) located at the Idaho National Engineering and Environmental Laboratory (INEEL) has been used for reprocessing spent nuclear fuel (SNF) since 1953. SNF was reprocessed to recover fissile uranium; however, changing world events have diminished the need to recover and recycle this material. In April 1992, the Department of Energy (DOE) discontinued reprocessing SNF and shifted its focus toward continued management and disposing of waste accumulated from previous reprocessing activities. ICPP waste treatment activities produce a granular solid waste (calcine) that contains dissolved fission products and fuel cladding material. Decontamination activities at the ICPP produce liquid radioactive, sodium-bearing waste (SBW), and ongoing operations at the ICPP produce liquid waste that contains low levels of radioactivity. The treatment of radioactive wastes for transfer out of Idaho is mandated under a "Settlement Agreement" between DOE and the State of Idaho." The Settlement Agreement requires that existing inventory of liquid high-level radioactive waste (HLW) be calcined by June 30,1998 , and SBW by the end of 2012, and the target date for all waste to be ready to ship from the INEEL is 2035 .

The Department of Energy Idaho Operations Office (DOE-ID) is preparing the High-Level Waste and Facilities Disposition Environmental Impact Statement (HLW\&FD EIS), in accordance with the National Environmental Policy Act, to support the decision-making process regarding HLW management at the INEEL. The Fluor Daniel Corporation and the INEEL operating contractor, Lockheed Martin Idaho Technologies Company (LMITCO), have been tasked with performing studies that provide the basis for portions of the EIS. Waste treatment options include dissolving the calcine; both the dissolved calcine and the liquid SBW would then be separated into high-activity waste (HAW) and low-activity waste (LAW). The HAW would be mixed with a glass matrix and poured into canisters. This is termed the "Separations Option." Alternative options that do not involve a separation step are termed the "Nonseparations Option." The Waste Treatment Studies consider the several alternative processes for preparing the calcine and liquid waste for ultimate removal from the INEEL: Separations [Full and Transuranic (TRU)] and Nonseparations (Vitrified, Hot Isostatic Press, Cementitious, and Direct Cementitious). Fluor Daniel Corporation is studying the Full Separations alternative and the others are being studied by LMITCO. The product of each of the processes will be waste in a storage canister that is ready for shipment to a permanent repository. Because the repositories will not be capable to receive the canisters at the time they are produced, storage will be required at the INEEL. This EDF documents cost, schedule, and environmental impact for storage of the waste generated by the above processes in the existing Fuel Processing Restoration (FPR) facility after modification for an Interim Storage Facility (ISF). Interim storage in a new facility is documented in EDF-IS-001.

\subsection{Objective and Scope of Work}

The objective of this study is to explore alternatives for a modified existing facility for interim storage of the waste in canisters that result from $\mathrm{HLW}$ processing of the calcine and liquid waste at ICPP. Table 1 gives the processing options and associated data that are included in this study: 
Table 1. Waste processing options and study data.

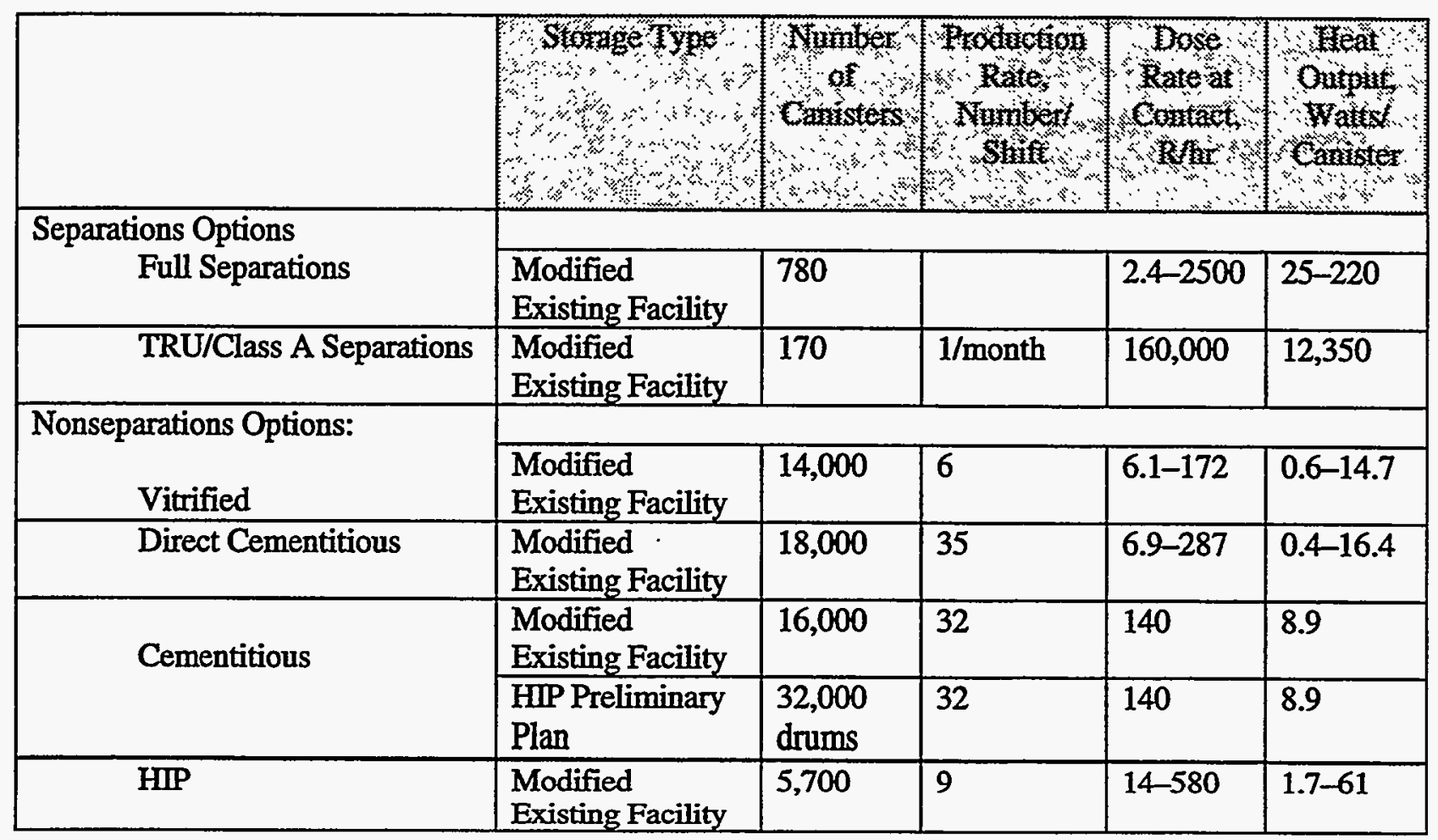

The objective of this study was to determine the cost, schedule, and environmental impacts for the storage of the waste canisters that result from each of the processing options in Table 1. A modified existing facility (FPR) was studied that incorporates appropriate design features from the Fluor Daniel Corporation design for interim storage presented in their document, Waste Treatment Facilities, Feasibility Study Report, December 12, 1997 (see Reference 2). The TRU/Class A Separations option would generate three waste streams designated as LLW: LAW, HAW, and TRU waste. All of these waste streams are actually considered HLW. It is expected that the LAW and TRU meet the definition of NRC LLW Class A and TRU, respectively. However, these wastes do not conform to the existing classification scheme for radioactive waste. Therefore, they will need the evaluation and concurrence of appropriate government authorities (NRC and DOE) for reclassification. Only the HAW will require interim storage. The LAW is assumed to go directly to a Class A waste disposal site and the TRU waste to go directly to the Waste Isolation Pilot Plant (WIPP). The TRU/Class C Separations Option does not require interim storage.

A preliminary plan for the Cementitious Option is under consideration. The resulting grout would be placed into drums within a 5-year period and placed into the ISF. Over a 20-year period, the drums would be subjected to a Hot Isostatic Press (HIP) process that would further reduce the volume. This alternative was included in this study. 


\subsection{DESIGN BASIS, KEY ASSUIMPTIONS, AND REQUIREMENTS}

\subsection{Design Basis}

A facility is required near the processing facility to store the HLW from the time it leaves the processing facility until it can be moved to a permanent waste repository. The quantities of canisters and their characteristics given in Table 1 result from the studies for the processing alternatives. The design life for the facilities is from 40 to 60 years.

The Savanna River Site (SRS) HLW canister and the West Valley Demonstration Project HLW canister meet the repository Waste Acceptance Criteria (WAC) for dimensions, although there are significant differences in these canisters' physical makeup. The SRS canister is used as the baseline for this study because it was selected in the ICPP Waste Treatment Facilities Feasibility Study Report. ${ }^{2}$ However, the DOE Hanford Facility has adopted a 4.5-m (15-ft) long canister for containing immobilized $\mathrm{HLW}$. This longer canister is apparently acceptable for co-disposal at the repository, and therefore should be considered before finalizing the canister size. Consideration of the $4.5-\mathrm{m}$ (15-ft) canister is addressed in the recommendations in Section 9. Some cost increase would result because the head height for handling the longer canisters is greater, and depth of excavation, equipment capacities, etc., increase. Cost increases would also occur because of the increase in the ISF transport tunnel height, canister handling machine size and capacity, and the ceiling height. However, smaller interim storage facilities may be adequate with fewer canisters, and handling, shipping, and final disposal operations with fewer canisters would offset the cost increases if the longer canisters were adopted. The net result of the cost changes is unknown.

Transport of the waste to the interim storage facility is included, however the transport from the facility is not included in this study. Equipment for handling the canisters for movement into and from the facility is included.

A passive cooling system is desired. The additional safety issues and operating costs of an active system would thus be avoided.

\subsection{Key Assumptions}

The following assumptions formed the framework, i.e., and the directed charter, for this study. Therefore, although some of these assumptions are likely to change, and such changes may have a significant impact on the results of this study, it is beyond the scope of this study to predict such changes or evaluate the associated risk.

1. The quantities and characteristics of waste to be stored are as tabulated in Table 1.

2. The waste will be stored in the SRS canister described in EDF-DPC-001, Appendix E of DPC Loading Feasibility Study Report, INEEL/EXT-97-01251, November 1997. All canisters will be sealed and radiologically clean.

3. The NRC will license the facility in accordance with the requirements of 10 CFR Part 72 . Existing facilities may not have been built to these requirements and replacement or upgrading of the components may not be possible. In these cases, it is assumed that NRC will consider equivalence or alternative approaches and issues the license. 
4. The processed waste is not Resource Conservation and Recovery Act (RCRA) hazardous waste as a result of delisting before processing and by virtue of processing when all "listed" hazardous and characteristic constituents are treated to the Land Disposal Regulation standards, then the waste is no longer classed as RCRA waste.

5. The LAW and TRU waste will be reclassified as LLW Class A and TRU, respectively.

6. The processing facilities will not have lag storage. If the ISF cannot handle the canisters at the rate produced by the facility as given in Table 1, the ISF must have the capability to store them in a lag storage area. This lag storage would only be used if the processing facility releases the canisters in a batch or for maintenance of the canister handling equipment in the ISF.

7. The handling equipment will interface with an MP-187 cask for canister shipment from the ISF.

8. Physical security requirements are enveloped by those for SNF.

9. The maximum weight of a canister is $5,300 \mathrm{lb}$.

10. Others will provide the proposed rail spur.

11. The transfer cart systems are described in the process facility options.

12. Shipments from the facility will be on a licensed rail shipping cask at grade and shipments to the facility are on a transfer cart in a belowgrade shielded transfer tunnel.

13. Storage of canisters shall be stacked as deep as four high inside sealed carbon steel tubes supported by support stools on the vault floor, both spaced for forced cooling and optimizing storage inside shielded concrete vaults.

14. A charged face floor system shall be used to maximize storage capacity, shielding, and strength while minimizing floor weight and contractibility concerns.

15. The same low profile gantry crane used in the proposed Interim Storage Facility Study at ICPP by Fluor Daniel Corporation ${ }^{2}$ shall be used for placing the canisters and transferring them for future shipments.

16. The assumed transfer cask on the gantry crane is to be similar to that of a bottom loading Peach Bottom cask.

\subsection{Requirements}

\subsubsection{Regulations}

The NRC in accordance with 10 CFR Part 72 will license the storage facility, however the cost for this licensing is not included in this report. It is assumed that the canisters, as received from the processing facility, will meet Department of Transportation (DOT), NRC, and/or HLW Repository WAC requirements and require no further conditioning, testing; treatment, marking, etc. This requires that the waste will be excluded from RCRA hazardous waste management requirements at the INEEL due to successful delisting and/or treatment. 
The ISF will be licensed to meet 10 CFR Part 72 for HLW; therefore the receipt, handling, packaging, and storage of canisters of waste must also be governed under 10 CFR Part 72. Changes required to the facility license must be evaluated through a 10 CFR 72.48 evaluation, issuance of a Safety Analysis Report, and licensing documentation amendments. It is assumed that DOE-ID will continue to implement the quality assurance program described in DOE-RW-0333P, Quality Assurance Requirements and Description ${ }^{3}$ and will apply it to this effort as well. The impact of implementing DOE-RW-0333P is reflected in the capital and operating cost estimates generated by this feasibility study.

\subsubsection{HLW Repository WAC}

The requirements of the potential future repository waste acceptance will be satisfied by the processing facility before the canisters being released to the ISF. The ISF will not provide any capability to process, modify, or change the waste or canisters.

\subsubsection{Applicable Codes and Standards}

The NRC licenses and regulates waste storage facilities in accordance with 10 CFR Part 72. DOE requirements that are included in 10 CFR Part 72 will be superseded by them, however, any DOE requirements that are not covered by 10 CFR Part 72 will also apply.

\subsection{INTERIM STORAGE FACILITY DESIGNS}

Waste canisters would come into the FPR facility for interim storage via an underground transport cart from the waste processing plant. The waste-handling machine in the facility would access the transport cart from above and bring the canister up into its bottom loading shielded cask. The waste handling machine would then take the canister to its designated storage sleeve in the facility, remove the sleeve plug, place the canister in the sleeve, and replace the plug. Four canisters would be stacked in each sleeve with cushioning material between them. See drawings FS-A-3, TRUA-A-16, and HWO-A-17 in Appendix 2.

When the waste canisters are removed for shipment to permanent storage, the process is reversed except that the canisters are placed in a cask on a rail car rather than the transport cart. This requires that the rail car be belowgrade, under the waste-handling machine.

\subsection{Processing Facility Siting}

The studies for the processing facilities for the various processing options are documented in the following reports:

Full Separations Option - Idaho Chemical Processing Plant, Waste Treatment Facilities, Feasibility Study Report, Prepared by Fluor Daniel, Inc., December 12, 1997 (Reference 2).

TRU Separations Option - TRU Separations Option Study Report, INEEL/EXT-01428, by William H. Landman, February 1998 (Reference ).

Hot Isostatic Press Waste Option (HIP) - Hot Isostatic Press Waste Option Report, INEEL/EXT01392, by Neal E. Russell, February 1998 (see Reference 5). 
These facilities have been sited at a location in these studies that is convenient to the calcine storage units but is away from the other facilities. Because a new interim storage facility is assumed in the studies, this siting is acceptable for the ISF. It can be located in a convenient location near the processing facility. If the FPR is used for the interim storage as documented in this study, the processing facility should be located in a different location that would be closer to the FPR and still convenient to the calcine storage units. For this reason, the processing facility has been located near the FPR in this study as shown in the drawings in Appendix 2. The siting issues have not been studied for this study and must be considered further if FPR is given further consideration as an interim storage facility for HLW.

\subsection{Modification for Transportation of Canisters}

The existing truck air lock and loading ramp on the east side of the FPR facility would be removed and replaced with a $21-\mathrm{m}$ ( $69-\mathrm{ft})$ wide by $21.3-\mathrm{m}$ ( $70-\mathrm{ft})$ long extension of the building. The abovegrade portion of the building extension would include a roof and floor at the same level as the maintenance area of the FPR facility. See drawings FS-A-1, FS-A-2, TRUA-A-14, TRUA-A-15, HWO-A-14, HWO-A-15, and HWO-A-16 in Appendix 2 for sketches showing this concept. The overhead crane rail would be extended into this new area and new rails would be installed for the waste-handling machine.

Belowgrade construction would include the waste transfer tunnel and the waste-shipping tunnel as shown in the drawings. The waste-handling machine would retrieve canisters from the cart or place canisters on the rail car through access ports in the floor of the new building extension. The waste transfer tunnel is the termination of the tunnel from the waste processing plant and the waste shipping tunnel would emerge onto a 305-m (1,000-ft) long ramp to pull the rail car up to grade on a new rail spur.

\subsection{Modification for Storage of Canisters}

Because the various processing options produce a different number of canisters, modifications to the FPR facility for storage of the canisters would vary for each option, whereas the transportation modifications would be the same for all the options.

A quick look was made to determine the maximum number of canisters that can be stored in the FPR. This was determined by placing the maximum number of 26-inch diameter storage sleeves tightly packed in the largest area that could possibly be created in the facility. If all the inner cell walls for cells 1 through 12 and the pump and valve corridor were removed to form one large cell, 2,336 sleeves could be installed. With four canisters in each sleeve, a maximum of 9,344 canisters could be stored. Therefore, only those options with fewer than 9,344 canisters were considered for storage in the FPR. The use of FPR to store part of the canisters produced by some of the options was also not pursued. A new facility would be required to store the remainder of the canisters. The duplication of facilities would result in a higher cost than building a new storage facility with a capacity for all the canisters.

\subsubsection{HIP Process}

Approximately 5,700 canisters would be produced by the HIP option. Drawing HWO-A-14 in Appendix 2 is a plan view of the facility that shows the storage sleeves in the original process cell areas. The configuration as shown would accommodate 1,474 sleeves, each of which could store four canisters. Therefore, the number of canisters that could be stored is 5,896 . This concept requires considerable modification of the facility. The concrete walls between cells 1 through 9 and the concrete floor between the hatches to the pump and valve corridor must be cut out. A steel grid arrangement would be installed on the floor of the cells to level and position the storage sleeves and steel lining would be installed on the east walls to provide the necessary shielding. The storage sleeves are 66-cm (26-inch) diameter schedule 10 carbon steel pipes that are $13.9 \mathrm{~m}$ ( $45.5 \mathrm{ft}$ ) long. The bottoms of the pipes would be sealed 
with steel plate and the tops would be closed with $30.5-\mathrm{cm}$ (12-inch) thick steel plugs. Spacers of $30.5-\mathrm{cm}$ (12-inch) thick steel would be used at the top of the pipes to position them and provide radiation shielding. The spacers and the pipes would be welded together to provide adequate air sealing so the fans can force the flow of cooling air from east to west as shown in drawing HWO-HV-2 (Appendix 2). The east-west rows of pipes are spaced about $4.3 \mathrm{~cm}(1.7 \mathrm{inch})$ apart in the north-south direction to allow the air to flow. The combinations of spacers and pipe plugs form a flat floor in the whole area.

The decay heat per canister in this option would range from 1.7 watts to 61 watts with the average at 23.2 watts. Without forced air cooling the inside temperature of the exterior concrete walls would exceed $250^{\circ} \mathrm{F}$ and the interior concrete wall between the cells and the pump and valve corridor would exceed $500^{\circ} \mathrm{F}$. Sustained temperatures greater than $200^{\circ} \mathrm{F}$ cause significant structural degradation of concrete, mainly by dehydration. Because of this, forced air-cooling would be required. An airflow rate of $21,000 \mathrm{lb} / \mathrm{hr}(4,600 \mathrm{SCFM})$ would remove this heat with an inlet to outlet temperature difference of $100^{\circ} \mathrm{F}$. A hot day at the INEEL would be $100^{\circ} \mathrm{F}$, which means the outlet temperature would be $200^{\circ} \mathrm{F}$. For $200^{\circ} \mathrm{F}$ air flowing across a storage sleeve with the hottest canisters (61 watts) inside, the centerline temperature of the waste would be approximately $220^{\circ} \mathrm{F}$. Drawing HWO-HV-2 shows six fans, three pulling and three pushing, to overcome the pressure drop across the storage area.

The waste handling machines would be similar to the design proposed by Fluor Daniel Corporation. ${ }^{2}$ Rails would be installed on the floor of the maintenance area and would extend into the new part of the building over the waste transfer and waste shipping tunnels. Since the canisters would be transferred into the interim storage at the rate of nine per shift, two waste handling machines would be used to provide redundancy of operation. When a waste-handling machine requires maintenance it would be parked at one end of the facility to allow the other machine to place the waste canisters.

\subsubsection{Separation Process Options}

The full separation option would produce 780 canisters of waste and the TRU/Class A separation option would produce 170 canisters of waste. For these options, a configuration similar to the one for the HIP option could be used except that all FPR process cell walls would be left intact. Drawings FS-A-1 and TRUA-A-14 in Appendix 2 show the layout for storage of 780 canisters and 170 canisters. The sleeve and plug design would remain the same but the pipes would be spaced at the top with two layers of steel plate with steel shot between the layers for radiation shielding. The sleeves would be spaced further apart to allow more airflow to remove the higher heat generation rate. Ventilation of the cells would be accomplished through the ducts originally designed into the process cells. Cells 5 and 6 were chosen to take advantage of the shielding provided by the thicker south wall and the additive effect of one or more adjacent cell walls. Adjacent cells and the pump and valve corridor would have restricted access. If unlimited access is required in adjacent cells, additional steel lining could be added to the cell walls. Only one waste-handling machine would be required for these storage alternatives because the production rate of one canister per month would allow any required maintenance of the machine to be done between receipt of canisters.

\subsection{TOTAL PROJECT, LIFE-CYCLE, AND D\&D COSTS}

Cost estimates for modification of FPR for the storage options are in Appendix 1. The costs are summarized in Table 2. 
Table 2. Project Costs

\begin{tabular}{|c|c|c|c|}
\hline \multirow[t]{4}{*}{ Cost item } & \multicolumn{2}{|c|}{ Separations Options } & Non-separations Options \\
\hline & Full Separations & TRU/Class A & Hot Isostatic Pressed \\
\hline & $\begin{array}{c}\text { Modified Existing } \\
\text { Facility } \\
\end{array}$ & $\begin{array}{l}\text { Modified Existing } \\
\text { Facility }\end{array}$ & $\begin{array}{l}\text { Modified Existing } \\
\text { Facility }\end{array}$ \\
\hline & $\$$ & $\$$ & $\$$ \\
\hline OPC (unescalated) & $13,941,000$ & $13,168,000$ & $16,228,000$ \\
\hline OPC Escalation & $4,469,000$ & $4,747,000$ & $4,605,000$ \\
\hline OPC Mgmt Reserve & 0 & 0 & \\
\hline OPC Contingency & $4,590,000$ & $4,484,000$ & $5,166,000$ \\
\hline Total OPC & $23,000,000$ & $22,400,000$ & $26,000,000$ \\
\hline TEC (unescalated) & $89,245,000$ & $77,219,000$ & $197,722,000$ \\
\hline TEC Escalation & $44,166,000$ & $34,353,000$ & $70,977,000$ \\
\hline TEC Mgmt Reserve & $9,188,000$ & $7,686,000$ & $19,374,000$ \\
\hline TEC Contingency & $19,901,000$ & $16,942,000$ & $37,827,000$ \\
\hline Total TEC & $162,500,000$ & $136,200,000$ & $325,900,000$ \\
\hline TPC (unescalated) & $103,186,000$ & $90,387,000$ & $213,950,000$ \\
\hline TPC Escalation & $48,634,000$ & $39,100,000$ & $75,583,000$ \\
\hline TPC Mgmt Reserve & $9,188,000$ & $7,686,000$ & $19,374,000$ \\
\hline TPC Contingency & $24,491,000$ & $21,426,000$ & $42,993,000$ \\
\hline Total TPC & $185,500,000$ & $158,600,000$ & $351,900,000$ \\
\hline Operations (unescalated) & $45,526,000$ & $45,431,000$ & $87,239,000$ \\
\hline Operations Escalation & $90,825,000$ & $88,164,000$ & $111,240,000$ \\
\hline Operations Contingency & $40,905,000$ & $40,079,000$ & $59,544,000$ \\
\hline Total Operations & $177,257,000$ & $173,674,000$ & $258,022,000$ \\
\hline Post Operations (unescalated) & $29,049,000$ & $25,399,000$ & $58,729,000$ \\
\hline Post Operations Escalation & $182,326,000$ & $159,413,000$ & $368,613,000$ \\
\hline Post Operations Contingency & $63,413,000$ & $55,444,000$ & $128,203,000$ \\
\hline Total Post Operations & $274,788,000$ & $240,256,000$ & $555,544,000$ \\
\hline Total Cost (unescalated) & $177,761,000$ & $161,217,000$ & $359,918,000$ \\
\hline $\begin{array}{l}\text { Total Cost (w/escalation, mgt reserve, } \\
\text { and contingency) }\end{array}$ & $637,545,000$ & $572,529,000$ & $1,165,466,000$ \\
\hline Discounted Cost (escalated) & $105,247,000$ & $97,808,000$ & $230,745,000$ \\
\hline
\end{tabular}




\subsection{PROJECT SCHEDULE}

Figure 2 for each processing option and interim storage alternative shows the schedules for the installation or modification of the storage facilities.

\subsection{RISK ASSESSMENT}

High-level risks identified in this study are categorized below. The assumptions in Section 2.2 provide the framework for this list. The highest risk is rated at " 4 " (out of "9"). Data Sheets for all the identified risks are included in Appendix 3, along with an explanation of the Risk Rating calculation method.

1. The quantities and characteristics of the waste are different than assumed in the study. Risk $=3$ (Probability $=3$, Impact $=1$ ).

2. Waste will not be stored in the SRS canister. Risk $=2$ (Probability $=2$, Impact $=1)$.

3. The facility would have to be redesigned if the waste is classified as a RCRA waste after processing. Risk $=3$ (Probability $=1$, Impact $=3$ ).

4. The obtaining of the NRC license for the FPR would be more difficult than assumed. Risk $=4$ (Probability =2, Impact $=2$ ).

5. A storage canister will be mishandled or dropped during waste transfer to, from, or in the facility. Risk $=2$ (Probability $=1$, Impact $=2$ ).

6. A catastrophic flood could flood the FPR facility. Risk $=2$ (Probability $=1$, Impact $=2$ ).

7. The facility might not be operational when needed. Risk $=2$ (Probability $=1$, Impact $=2$ ).

Many risks including environmental, safety, and health risks were not identified in this study. 


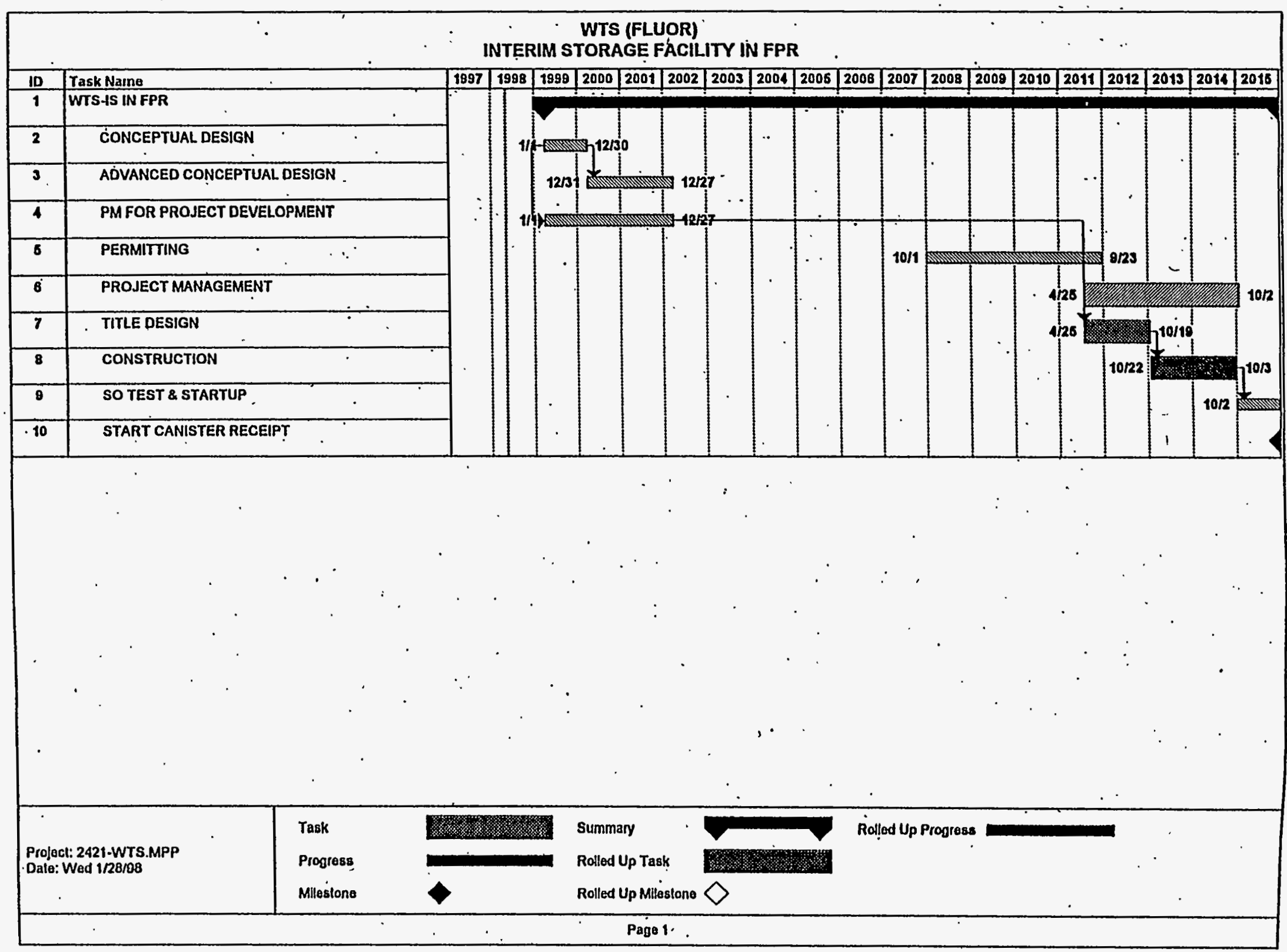




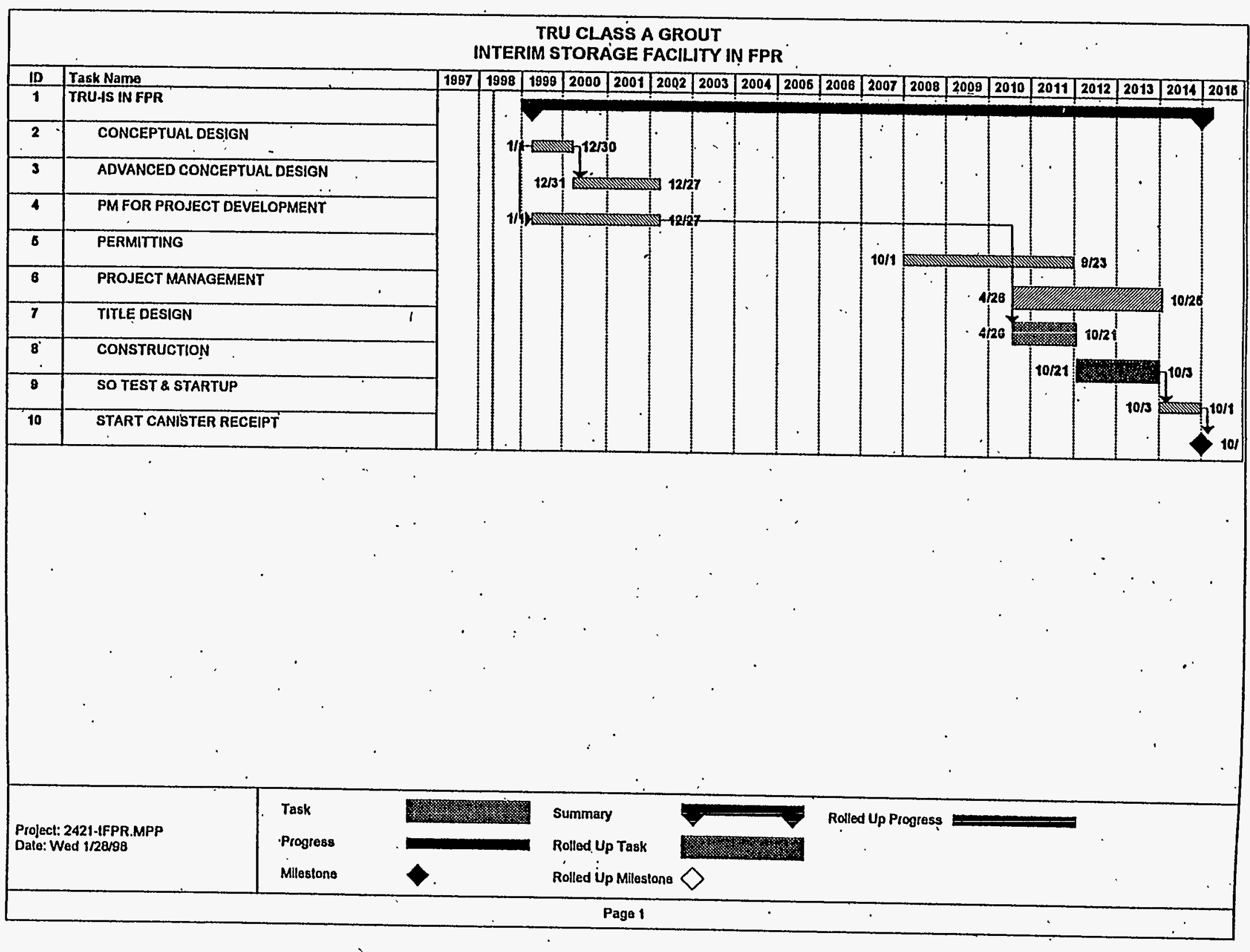




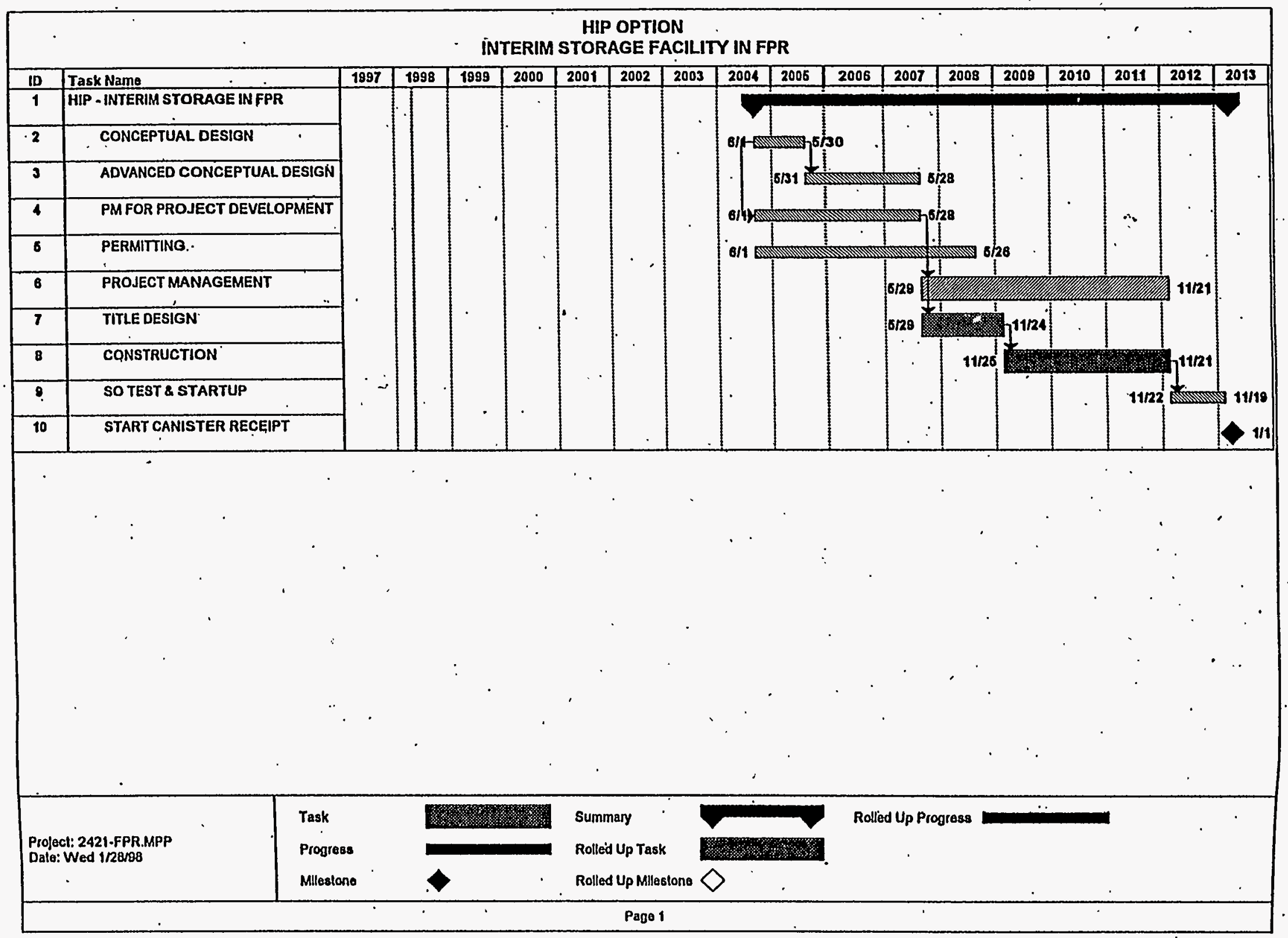

ํ.

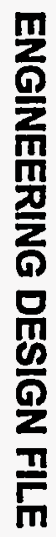

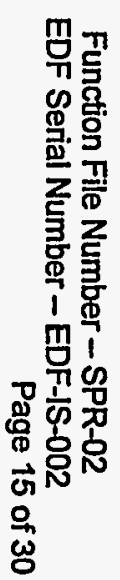




\subsection{CONSTRUCTION AND OPERATIONAL ENVIRONMENTAL IMPACTS}

The Product Data Sheets that summarize the construction and operational environmental impacts for the interim storage facilities that support the waste processing options are in Table 3. Data are presented for the construction, operation, and decommissioning of the project. Estimates are included for the following parameters:

- Cost

- Schedule

- Air emissions

- Liquid effluents

- Solid wastes

- Utilities used

- Manpower requirements

- Regulatory requirements.

Because this project is at a very preliminary stage of design, much of the information presented in the Project Data Sheets is based on representative literature values, existing laboratory data, and engineering judgment. The cost data in Table 3 were obtained from the cost estimates in Appendix 1 and the supporting information for the remainder of the data is in Appendix 4.

The Product Data Sheets are presented in the following order in Table 3:

\begin{tabular}{|l|l|}
\hline Full Separations - IS in the FPR & PDS for Storage at FPR for WFS \\
\hline TRU/Class A Separations - IS in the FPR & PDS for Storage at FPR for TRU \\
\hline Hot Isostatic Press - IS in the FPR & PDS for Storage at FPR for HWO \\
\hline
\end{tabular}




\section{Project:Data Sheet for Storage at FPR for WFS}

\section{Generic Information}

Description/function

EIS Alternatives ( $A, B$, etc.)

Project type or waste stream

Action type

Structure type

Size $\left(m^{2}\right)$

Other features (e.g. pits, ponds, power/water/sewer lines)

Location

Inside/outside of fence

Inside/outside of building

Candidate for privatization? .
Provide Long-term storage for roadready containers awaiting shipment to a permanent repository.

Full Separations Waste Option

Treated HLW calcine.

New
$5,853 \mathrm{~m}^{2}$

None

Inside ICPP fence

FPR Facility

\section{Yes}

\section{Construction Information}

Cost (\$): Pre-Operations (Other Project Costs) with escalation and contingency.

Conceptual Design

Management for Project Development

Permitting and Documentation

SO Test \& Start-Up

Contingency

Total Preconstruction

Cost (\$): Construction with escalation and contingency.

\section{ED\&I}

Management (PM/CM)

Construction

G\&A/PIF

Procurement Fees, Management Reserve and Contingency

Total Construction (TEC)

Schedule startend: Preconstruction

Schedule start/end: Construction

Schedule start/end: SO Test \& Start-up

Number of workers each year of construction (new/existing)

Nonradiation

Number of radiation workers (construction)

Average annual worker radiation dose (rem/yr)

. Heavy equipment

Equipment used

Trips (construction materials delivery)

Hours of operation

Acres disturbed and duration of disturbance

New

Previous

$$
\begin{array}{r}
\$ 3,170,000 \\
\$ 4,501,000 \\
\$ 3,629,000 \\
\$ 7,110,000 \\
\$ 4,590,000 \\
\$ 23,000,000
\end{array}
$$

$\$ 20,965,000$

$\$ 20,566,000$

$\$ 85,690,000$

$\$ 5,332,000$

$\$ 29,947,000$

$\$ 162,500,000$

January 1999 through December 2001

January 2011 through December 2014

January 2015 through December 2015

161 New workers/yr
None
None

Excavator, dump

trucks, cranes,

702

14,769 hours (total)

January 2011 through December 2014

None

155 acres 
Revegetated

Air emissions

Dust

Major gases $\left(\mathrm{CO}_{2}, \mathrm{H}_{2} \mathrm{O}, \mathrm{O}_{2}, \mathrm{~N}_{2}\right)$ from diesel exhaust

Contaminants (Particulates, $\mathrm{CO}, \mathrm{NO}_{x} ; \mathrm{SO}_{2}$, hydrocarbons) from diesel exhaust.

Total air emissions

Effluents

Sanitary wastewater

Lube Oil

Solid wastes

Type - construction trash

Radioactive wastes

Hazardous/toxic chemicals and wastes (type)

Storage/inventory

Hazardous waste (construction)

Pits/Ponds created $\left(\mathrm{m}^{2}\right)$

Water usage:

Dust control

Domestic water

Energy requirements

Electrical (MWh/yr)

Fossil fuel (liters)

Permits needed for construction
Function File Number - SPR-02

EDF Serial Number - EDF-IS-002

Page 18 of 30

\section{Project Data Sheet for Storage at FPR for WFS \\ None}

4,464 tons (total)

10,261 tons (total)

60 tons (total)

14,785 tons (total)

$6,855,581$ liters (total)

2,795 liters (total)

$3,817 \mathrm{~m}^{3}$ (total)

None

$$
\begin{aligned}
& 13 \mathrm{~m}^{3} \\
& 217 \mathrm{~m}^{3} \text { (total) } \\
& \text { None }
\end{aligned}
$$

$31,868,496$ liters (total)

$6,855,581$ liters (total)

$150 \mathrm{MWh} / \mathrm{yr}$

0 liters (total)

NEPA documentation (prior to start of Title II construction); New stationary sources/PTC/NOC/PSD for non-rad air emissions; HAP's and TAP's and RCRA (part AA,BB, and CC for air) for hazardous air emissions; air operating permit; NESHAP's, NPDES, NESHAP's subpart H for rad air emissions; approval of Engineering Plans; Cross Connection Control Plans; report and specifications for drinking water supply;RCRA Part $A$ and Part $B$ permits.

Operational Information

Cost (\$): Operations (unescalated)

Facility/Administration

$\$ 6,721,000$

Operations/Process Facility

Procurement, materials, utilities, maintenance

Total operations

Schedule start/end

$\$ 18,940,000$

$\$ 19,864,000$

$\$ 45,525,000$

January 2015 through December 2068

Number of workers each year of operation (new/existing) 
431.02\#

$06 / 17 / 97$

Rev. \#04
ENGINEERING DESIGN FILE

Table 3. (continued).
Function File Number - SPR-02

EDF Serial Number - EDF-IS-002

Page 19 of 30

\section{Operations}

Maintenance

Support

Total

Number of radiation workers

Average annual work radiation dose (rem/yr)

Heavy Equipment

Air Emissions

Radioactive off-gas

Effluents

Sanitary Wastewater

Solid Wastes

Sanitary/Industrial Trash

Radioactive wastes (canisters)

Hazardous/toxic chemicals and wastes

Pits/Ponds used $\left(\mathrm{m}^{2}\right)$

Water usage:

Domestic water

Energy Requirements:

Electrical (MWh/yr)

Steam

Permits needed (for facility operations)

\section{Project Data Sheet for Storage at FPR for WFS}

\author{
3 \\ 1 \\ 3 \\ 7 \\ 2.5 (included in above total) \\ $0.19 \mathrm{rem} / \mathrm{yr}$ perworker \\ None
}

None

241,767 liters/yr

$39 \mathrm{~m}^{3} / \mathrm{yr}$
$147 \mathrm{~m}^{3} / \mathrm{yr}$

None

241,767 liters/yr

4,368 MWh/yr

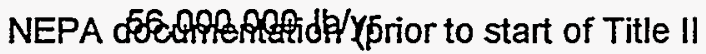

construction); New stationary

sources/PTC/NOC/PSD for non-rad air emissions;

HAP's and TAP's and RCRA (part $A A, B B$, and $C C$

for air) for hazardous air emissions; air operating permit; NESHAP's, NPDES, NESHAP's subpart H for rad air emissions; approval of Engineering Plans;

Cross Connection Control Plans; report and
Decontamination \& Decommissioning (D\&D) Information

Cost (\$): D\&D (unescalated)

Decommission

Decontamination

Demolition

Total D\&D

Schedule start/end: D\&D

Number of workers each year of D\&D (new/existing)

Number of radiation workers (D\&D)

Average annual worker radiation dose (rem/yr)
$\$ 4,284,000$

$\$ 0$

$\$ 24,785,000$

$\$ 29,069,000$

January 2069 through December 2071

68 New workers/yr

7 New workers/yr

$0.19 \mathrm{rem} / \mathrm{yr}$ perworker

Mobile Cranes, Roll-off trucks, Dozers, Loaders

Heavy equipment:

Equipment used 
431.02\#

$06 / 17 / 97$

Rev. \#04
ENGINEERING DESIGN FILE

Table 3. (continued).
Function File Number - SPR-02

EDF Serial Number - EDF-IS-002

Page 20 of 30
Trips

Roll-off trucks

Hours of operation (all heavy equipment)

Acres disturbed and duration of disturbance

New

Previous

Revegetated

Air emissions

non-radioactive Fuel combustion gases $\left(\mathrm{CO}_{2}, \mathrm{H}_{2} \mathrm{O}, \mathrm{O}_{2}, \mathrm{~N}_{2}\right)$

non-radioactive

Fuel combustion contaminants ( $\mathrm{CO}$, particulates, $\mathrm{NO}_{x}, \mathrm{SO}_{2}$, hydrocarbons)

radioactive HEPA filtered off-gas

Effluents

radioactive Spent decontamination solution

non-radioactive Sanitary wastewater

non-radioactive Lube oil

Solid wastes:

radioactive

Non-radioactive (industrial)

Hazardous

Hazardous/toxic chemicals and wastes (type)

Storage/inventory

Pits/Ponds created $\left(\mathrm{m}^{2}\right)$

Hazardous waste

Water usage:

Domestic water

Source of water

Energy requirements:

Electrical (MWh/yr)

Fossil fuel (liters)

Permits needed (e.g. for facility closures, physical characteristics

and quantities of radioactive and hazardous materials remaining after closure)
January 2074 through December 2076
None
165 acres
None

8,300 tons (total)

48 tons (total)

None tons (total)

None liters (total)

4,364,496 liters (total)

2,702 liters (total)

$$
\begin{array}{r}
18,628 \mathrm{~m}^{3} \\
4 \mathrm{~m}^{3} \\
40 \mathrm{~m}^{3}
\end{array}
$$

None $\mathrm{m}^{3}$ (total)

None

$909 \mathrm{~m}^{3}$ (total)

$4,364,496$ liters (total)

ICPP site wells

$156 \mathrm{MWh} / \mathrm{yr}$

324,299 liters (total)

Work will be done under closure provisions of existing permits. 
- 431.02\# 06/17/97

Rev. \#04
ENGINEERING DESIGN FILE

Table 3. (continued).
Function File Number - SPR-02

EDF Serial Number - EDF-IS-002

Page 21 of 30

\section{Project Data Sheet tor Storage at FPR for TRU}

\section{Generic Information}

Description/function

EIS Alternatives ( $A, B$, etc.)

Project type or waste stream

Action type

Structure type

Size $\left(\mathrm{m}^{2}\right)$

Other features (e.g. pits, ponds, power/water/sewer lines)

Location

Inside/outside of fence

Inside/outside of building

Candidate for privatization?
Provide Long-term storage for road-ready containers awaiting shipment to a permanent repository.

TRU Separations Waste Option

Treated HLW calcine.

New

$5,853 \mathrm{~m}^{2}$

None

Inside ICPP fence

FPR Facility

\section{Construction Information}

Cost (\$): Pre-Operations (Other Project Costs) with escalation an

Conceptual Design

Management for Project Development

Permitting and Documentation

SO Test \& Start-Up

Contingency

Total Preconstruction

Cost (\$): Construction with escalation and contingency.

\section{ED\&l}

Management (PM/CM)

Construction

G\&AVIF

Procurement Fees, Management Reserve and Contingency

Total Construction (TEC)

Schedule startend: Preconstruction

Schedule start/end: Construction

Schedule start/end: SO Test \& Start-up

Number of workers each year of construction (new/existing)

Nonradiation

Number of radiation workers (construction)

Average annual worker radiation dose (rem/yr)

Heavy equipment

Equipment used

Trips (construction materials delivery)

Hours of operation

Acres disturbed and duration of disturbance

New

Previous
$\$ 2,470,000$

$\$ 3,470,000$

$\$ 2,728,000$

$\$ 4,500,000$

$\$ 3,332,000$

$\$ 16,500,000$

$\$ 12,352,000$

$\$ 11,858,000$

$\$ 49,408,000$

$\$ 3,107,000$

$\$ 18,075,000$

$\$ 94,800,000$

January 1999 through December 2001

January 2010 through December 2013

January 2014 through December 2014

155 New workers/yr

None

None

Excavator, dump trucks, cranes, cement trucks,grader, material delivery

702

14,740 hours (total)

January 2010 through December 2013

None

155 acres 
431.02\#

$06 / 17 / 97$

Rev. \#04

Revegetated

Air emissions

Dust

Major gases $\left(\mathrm{CO}_{2}, \mathrm{H}_{2} \mathrm{O}, \mathrm{O}_{2}, \mathrm{~N}_{2}\right)$ from diesel exhaust

Contaminants (Particulates, $\mathrm{CO}, \mathrm{NO}_{x}, \mathrm{SO}_{2}$, hydrocarbons) from

diesel exhaust.

Total air emissions

Effluents

Sanitary wastewater

Lube Oil

Solid wastes

Type - construction trash

Radioactive wastes

Hazardous/toxic chemicals and wastes (type)

Storage/inventory

Hazardous waste (construction)

Pits/Ponds created $\left(\mathrm{m}^{2}\right)$

Water usage:

Dust control

Domestic water

Energy requirements

Electrical (MWh/yr)

Fossil fuel (liters)

Permits needed for construction
Function File Number - SPR-02

EDF Serial Number - EDF-IS-002

Page 22 of 30

\section{Project Data Sheet for Storage at FPR for TRU}

None

4,464 tons (total)

5,174 tons (total)

30 tons (total)

9,668 tons (total)

$6,600,094$ liters (total)

2,790 liters (total)

$3,675 \mathrm{~m}^{3}$ (total)

None

$13 \mathrm{~m}^{3}$

$217 \mathrm{~m}^{3}$ (total)

None

$31,868,496$ liters (total)

$6,600,094$ liters (total)

$150 \mathrm{MWh} / \mathrm{yr}$

0 liters (total)

NEPA documentation (prior to start of Title II construction); New stationary sources/PTC/NOC/PSD for non-rad air emissions; HAP's and TAP's and RCRA (part AA,BB, and CC for air) for hazardous air emissions; air operating permit; NESHAP's, NPDES, NESHAP's subpart $H$ for rad air emissions; approval of Engineering Plans; Cross Connection Control Plans; report and specifications for drinking water supply;RCRA Part $A$ and Part $B$ permits.

Operational Information

Cost (\$): Operations (unescalated)

Facility/Administration

$\$ 7,007,000$

Operations/Process Facility

Procurement, materials, utilities, maintenance

Total operations

Schedule start/end

$\$ 19,746,000$

$\$ 18,677,000$

$\$ 45,430,000$

January 2014 through December 2068

Number of workers each year of operation (new/existing) 
Table 3. (continued).

\section{Project Data Sheet for Storage at FPR for TRU}

Operations

Maintenance

Support

Total

Number of radiation workers

Average annual work radiation dose (rem/yr)

Heavy Equipment

Air Emissions

Radioactive off-gas

Effluents

Sanitary Wastewater

Solid Wastes

Sanitary/Industrial Trash

Radioactive wastes (canisters)

Hazardous/toxic chemicals and wastes

Pits/Ponds used $\left(\mathrm{m}^{2}\right)$

Water usage:

Domestic water

Energy Reguirements:

Electrical (MWh/yr)

Steam

Permits needed (for facility operations)

\author{
3 \\ 1 \\ 3 \\ 7 \\ 2.5 (included in above total) \\ $0.19 \mathrm{rem} / \mathrm{yr}$ perworker \\ None
}

None

241,767 liters/yr

$$
\begin{array}{r}
39 \mathrm{~m}^{3} / \mathrm{yr} \\
141 \mathrm{~m}^{3} / \mathrm{yr}
\end{array}
$$

None

241,767 liters/yr

$$
\begin{aligned}
& 4,368 \mathrm{MWh} / \mathrm{yr} \\
& 56,000,000 \mathrm{lb} / \mathrm{yr}
\end{aligned}
$$

HAP's and TAP's and RCRA (part AA,BB, and CC for air) for hazardous air emissions; air operating permit; NESHAP's, NPDES, NESHAP's subpart $H$ for rad air emissions; approval of Engineering Plans; Cross Connection Control Plans; report and specifications for drinking water supply;RCRA Part A and Part $B$ permits.

Decontamination \& Decommissioning (D\&D) Information

Cost (\$): D\&D (unescalated)

Decommission $\$ 3,706,000$

Decontamination $\$ 0$

Demolition

$\$ 21,693,000$

$\$ 25,399,000$

Total D\&D

Schedule start/end: $D \& D$

Number of workers each year of D\&D (new/existing)

Number of radiation workers (D\&D)

Average annual worker radiation dose (rem/yr)

Heavy equipment:

Equipment used
Page 23 of 30 
431.02\#

$06 / 17 / 97$

Rev. $\$ 04$
ENGINEERING DESIGN FILE

Table 3. (continued).
Function File Number - SPR-02

EDF Serial Number - EDF-IS-002

Page 24 of 30
Trips Roll-off trucks

Hours of operation (all heavy equipment)

Acres disturbed and duration of disturbance

New

Previous

Revegetated

\section{Air emissions}

non-radioactive Fuel combustion gases $\left(\mathrm{CO}_{2}, \mathrm{H}_{2} \mathrm{O}, \mathrm{O}_{2}, \mathrm{~N}_{2}\right)$

non-radioactive

Fuel combustion contaminants ( $\mathrm{CO}$,

particulates, $\mathrm{NO}_{x}, \mathrm{SO}_{2}$, hydrocarbons)

radioactive HEPA filtered off-gas

Effluents

radioactive Spent decontamination solution

non-radioactive Sanitary. wastewater

non-radioactive Lube oil

Solid wastes:

radioactive

Non-radioactive (industrial)

Hazardous

Hazardous/toxic chemicals and wastes (type)

Storage/inventory

Pits/Ponds created $\left(\mathrm{m}^{2}\right)$

Hazardous waste

Water usage:

Domestic water

Source of water

Energy requirements:

Electrical (MWh/yr)

Fossil fuel (liters)

Permits needed (e.g. for facility closures, physical characteristics

and quantities of radioactive and hazardous materials remaining after closure)
January 2069 through December 2071

None

155.0 acres

None

7,672 tons (total)

45 tons (total)

None tons (total)

None liters (total)

$3,813,258$ liters (total)

2,498 liters (total)

$$
\begin{array}{r}
18,628 \mathrm{~m}^{3} \\
4 \mathrm{~m}^{3} \\
40 \mathrm{~m}^{3}
\end{array}
$$

$\mathrm{Ci}$

$$
\begin{aligned}
& \text { None } \mathrm{m}^{3} \text { (total) } \\
& \text { None } \\
& 909 \mathrm{~m}^{3} \text { (total) }
\end{aligned}
$$

$3,813,258$ liters (total)

ICPP site wells

$156 \mathrm{MWh} / \mathrm{yr}$

299,772 liters (total)

Work will be done under closure provisions of existing permits. 
431.02\#

$06 / 17 / 97$

Rev. \#04
ENGINEERING DESIGN FILE

Table 3. (continued).
Function File Number - SPR-02

EDF Serial Number - EDF-IS-002

Page 25 of 30

\section{Project Data Sheet for Storage at FPR for HWO}

\section{Generic Information}

Description/function

EIS Alternatives ( $A, B$, etc.)

Project type or waste stream

Action type.

Structure type

Size $\left(\mathrm{m}^{2}\right)$

Other features (e.g. pits, ponds, power/water/sewer lines)

Location

Inside/outside of fence

Inside/outside of building

Candidate for privatization?
Provide Long-term storage for road-ready containers awaiting shipment to a permanent repository.

Hot Isostatic Pressing Waste Option

Treated HLW calcine.

New

$5,853 \cdot \mathrm{m}^{2}$

None

Inside ICPP fence

FPR Facility

\section{Construction Information}

Cost (\$): Pre-Operations (Other Project Costs) with escalation an

\section{Conceptual Design}

Management for Project Development

Permitting and Documentation

SO Test \& Start-Up

Contingency

Total Preconstruction

Cost (\$): Construction with escalation and contingency.

\section{ED\&I}

Management (PM/CM)

Construction

G\&A/PIF

Procurement Fees, Management Reserve and Contingency

Total Construction (TEC)

Schedule start/end: Preconstruction

Schedule start/end: Construction

Schedule start/end: SO Test \& Start-up

Number of workers each year of construction (new/existing)

Nonradiation .

Number of radiation workers (construction)

Average annual worker radiation dose (rem/yr)

\section{Heavy equipment}

Equipment used

Trips (construction materials delivery)

Hours of operation

Acres disturbed and duration of disturbance

New

Previous

$\$ 5,000,000$
$\$ 4,000,000$
$\$ 2,728,000$
$\$ 4,500,000$
$\$ 4,072,000$
$\$ 20,300,000$

$\$ 24,604,000$

$\$ 31,699,000$

$\$ 132,077,000$

$\$ 8,021,000$

$\$ 43,999,000$

$\$ 240,400,000$

January 2004 through December 2005

January 2007 through December 2011

January 2012 through December 2012

152 New workers/yr

None

None

Excavator, dump trucks, cranes, cement trucks,grader, material delivery

702

27,414 hours. (total)

January 2007 through December 2011

None

155 acres 
Revegetated

\section{Air emissions}

Dust

Major gases $\left(\mathrm{CO}_{2}, \mathrm{H}_{2} \mathrm{O}, \mathrm{O}_{2}, \mathrm{~N}_{2}\right)$ from diesel exhaust

Contaminants (Particulates, $\mathrm{CO}, \mathrm{NO}_{x}, \mathrm{SO}_{2}$, hydrocarbons) from diesel exhaust.

Total air emissions

Effluents

Sanitary wastewater

Lube Oil

\section{Solid wastes}

Type - construction trash

Radioactive wastes

Hazardous/toxic chemicals and wastes (type)

Storage/inventory

Hazardous waste (construction)

Pits/Ponds created $\left(\mathrm{m}^{2}\right)$

Water usage:

Dust control

Domestic water

\section{Energy requirements}

Electrical (MWh/yr)

Fossil fuel (liters)

Permits needed for construction
Function File Number - SPR-02

EDF Serial Number - EDF-IS-002

Page 26 of 30

\section{Project Data Sheet for Storage at FPR for HWO}

None

\author{
6,696 tons (total) \\ 17,610 tons (total) \\ 103 tons (total) \\ 24,409 tons (total)
}

$9,708,525$ liters (total)

5,188 liters (total)

$5,406 \mathrm{~m}^{3}$ (total)
None

$13 \mathrm{~m}^{3}$

$217 \mathrm{~m}^{3}$ (total)

None

$47,802,744$ liters (total)

$9,708,525$ liters (total)

$150 \mathrm{MWh} / \mathrm{yr}$

0 liters (total)

NEPA documentation (prior to start of Title II construction); New stationary sources/PTC/NOC/PSD for non-rad air emissions; HAP's and TAP's and RCRA (part AA,BB, and CC for air) for hazardous air emissions; air operating permit; NESHAP's, NPDES, NESHAP's subpart $H$ for rad air emissions; approval of Engineering Plans; Cross Connection Control Plans; report and specifications for drinking water supply;RCRA Part A and Part B permits.

Operational Information

Cost (\$): Operations (unescalated)

Facility/Administration

$\$ 5,039,000$

Operations/Process Facility

$\$ 18,299,000$

Procurement, materials, utilities, maintenance

$\$ 63,902,000$

Total operations

Schedule start/end

$\$ 87,240,000$

January 2012 through December 2068

Number of workers each year of operation (new/existing) 
431.02\#

$06 / 17 / 97$

Rev. \#04
ENGINEERING DESIGN FILE

Table 3. (continued).
Function File Number - SPR-02

EDF Serial Number - EDF-IS-002

Page 27 of 30

\section{Project Data Sheet for Storage at FPR for HWO}

Operations

Maintenance

Support

Total

Number of radiation workers

Average annual work radiation dose (rem/yr)

Heavy Equipment

Air Emissions

Radioactive off-gas

Effluents

Sanitary Wastewater

Solid Wastes

Sanitary/Industrial Trash

Radioactive wastes (canisters)

Hazardous/toxic chemicals and wastes

Pits/Ponds used $\left(\mathrm{m}^{2}\right)$

Water usage:

Domestic water

Energy Requirements:

Electrical (MWh/yr)

Steam

Permits needed (for facility operations)

\section{3}

1

3

7

2.5 (included in above total)

$0.19 \mathrm{rem} / \mathrm{yr}$ perworker

None

None

241,767 liters/yr

$39 \mathrm{~m}^{3} / \mathrm{yr}$

$4,309 \mathrm{~m}^{3} / \mathrm{yr}$

None

241,767 liters/yr

4,368 MWh/yr

$56,000,000 \mathrm{lb} / \mathrm{yr}$

HAP's and TAP's and RCRA (part AA,BB, and CC for air) for hazardous air emissions; air operating permit; NESHAP's, NPDES, NESHAP's subpart $H$ for rad air emissions; approval of Engineering Plans; Cross Connection Control Plans; report and specifications for drinking water supply;RCRA Part A and Part B permits.

\section{Decontamination \& Decommissioning (D\&D) Information}

Cost (\$): D\&D (unescalated)

Decommission

Decontamination

Demolition

Total D\&D

Schedule start/end: D\&D

Number of workers each year of D\&D. (new/existing)

Number of radiation workers (D\&D)

Average annual worker radiation dose (rem/yr)
$\$ 7,381,000$

\section{$\$ 0$}

$\$ 51,348,000$

$\$ 58,729,000$

January 2069 through December 2071

138 New workers/yr

14 New workers/yr

$0.19 \mathrm{rem} / \mathrm{yr}$ perworker

Mobile Cranes, Roll-off trucks, Dozers, Loaders

Heavy equipment:

Equipment used 
431.02\#

$06 / 17 / 97$

Rev. \#04
ENGINEERING DESIGN FILE

Table 3. (continued).
Function File Number - SPR-02

EDF Serial Number - EDF-IS-002

Page 28 of 30
Trips Roll-off trucks

Hours of operation (all heavy equipment)

\section{Acres disturbed and duration of disturbance}

New

Previous

Revegetated

Air emissions

non-radioactive Fuel combustion gases $\left(\mathrm{CO}_{2}, \mathrm{H}_{2} \mathrm{O}, \mathrm{O}_{2}, \mathrm{~N}_{2}\right)$

non-radioactive Fuel combustion contaminants ( $\mathrm{CO}$,

radioactive HEPA filtered off-gas

\section{Effluents}

radioactive Spent decontamination solution

non-radioactive Sanitary wastewater

non-radioactive Lube oil

\section{Solid wastes:}

radioactive

Non-radioactive (industrial)

Hazardous

Hazardous/toxic chemicals and wastes (type)

Storage/inventory

Pits/Ponds created $\left(\mathrm{m}^{2}\right)$

radioactive (mixed waste)

Water usage:

Domestic water

Source of water

Energy requirements:

Electrical (MWh/yr)

Fossil fuel (liters)

Permits needed (e.g. for facility closures, physical characteristics and quantities of radioactive and hazardous materials remaining after closure)
January 2069 through Decemiber 2071
None
155 acres
None

17,263 tons (total)

101 tons (total)

None tons (total)

None liters (total)

$8,806,174$ liters (total)

5,621 liters (total)

$$
\begin{array}{r}
18,628 \mathrm{~m}^{3} \\
4 \mathrm{~m}^{3} \\
40 \mathrm{~m}^{3}
\end{array}
$$

$\mathrm{Ci}$

$$
\begin{aligned}
& \text { None } \mathrm{m}^{3} \text { (total) } \\
& \text { None } \\
& \text { None } \mathrm{m}^{3} \text { (total) }
\end{aligned}
$$

$8,806,174$ liters (total)

ICPP site wells

$156 \mathrm{MWh} / \mathrm{yr}$

674,487 liters (total)

Work will be done under closure provisions of existing permits. 


\subsection{PROJECT-SPECIFIC OPTIONS}

No project-specific options were assigned, identified, or evaluated.

\subsection{RECOMMENDATIONS}

The additional costs and a longer schedule that would result from NRC licensing of the FPR for interim storage should be studied further. The NRC does not have the structure in place to review and license an existing facility for interim storage of $\mathrm{HLW}$. Also, the FPR has not been built to the requirements that would be required by NRC.

A future study should be conducted to determine if a standard 15-ft long "Hanford" canister should be substituted for the 10-ft SRS canister assumed in this study. Use of a 15-ft canister would increase the interim storage construction costs but would reduce the operation (handling) costs, and greatly reduce offsite transportation and disposal costs. A large net cost saving, primarily as a result of reduced transportation and disposal costs, could be realized by using the 15 - $\mathrm{ft}$ canister.

\subsection{CONCLUSIONS}

This study has investigated the modification of the FPR for storage of HLW that results from processing the waste at ICPP. The processing options are discussed in separate reports. This study determined that FPR can hold a maximum of 9,344 canisters and only those processing options that produce fewer canisters can be accommodated in the FPR. It is concluded that FPR can be modified and placed into operation to store the waste in canisters for the Full and TRU/Class A Separations Option and the HIP Option for the period from processing until the time a permanent repository is available for receipt of this waste. The schedules and costs associated with interim storage for these processing options are presented in the body of this EDF.

\subsection{REFERENCES}

1. Consent Order and Settlement Agreement between DOE and the State of Idaho Regarding Spent Fuel and Nuclear Waste Issues, October 17, 1995.

2. Waste Treatment Facilities, Feasibility Study Report, Fluor Daniel Corporation, December 12, 1997.

3. DOE/RW-0333P, U. S. Department of Energy, Office of Civilian Radioactive Waste Management, Quality Assurance Requirements and Description (QARD).

4. William H. Landman, TRU Separations Option Study Report, INEEL/EXT-01428, February 1998.

5. Neal E. Russell, Hot Isostatic Press Waste Option Report, INEEL/EXT-01392, February 1998. 


\subsection{APPENDICES}

Appendix 1-Cost Estimate

Appendix 2-Drawings

Appendix 3-Risk Assessment/Data Sheets

Appendix 4-Background Information for Project Data Sheets. 


\section{Appendix 1}

\section{Cost Estimates}


The cost estimates for modification of the FPR for Interim Storage Facilities (ISFs) are contained in this appendix in the same order as in the following summary table (see estimates attached to the letter RDA-3-98):

Summary Table of Cost Estimates.

\begin{tabular}{|c|c|c|c|c|c|c|}
\hline \multirow[b]{2}{*}{ Waste Option } & \multicolumn{3}{|c|}{ Not Escalated } & \multicolumn{3}{|c|}{ Escalated } \\
\hline & $\begin{array}{c}\text { Total } \\
\text { Estimate } \\
\text { d Cost, } \\
\text { K\$ }\end{array}$ & $\begin{array}{c}\text { Other } \\
\text { Project } \\
\text { Cost, } \\
\text { K\$ }\end{array}$ & $\begin{array}{c}\text { Total } \\
\text { Project } \\
\text { Cost, } \\
\text { K\$ }\end{array}$ & $\begin{array}{c}\text { Total } \\
\text { Estimate } \\
\text { d Cost, } \\
\text { K\$ }\end{array}$ & $\begin{array}{c}\text { Other } \\
\text { Project } \\
\text { Cost, } \\
\text { K\$ }\end{array}$ & $\begin{array}{c}\text { Total } \\
\text { Project } \\
\text { Cost, } \\
\text { K\$ }\end{array}$ \\
\hline Full Separations & 109,300 & 17,400 & 126,700 & 162,500 & 23,000 & 185,500 \\
\hline TRU/Class A Sep & 94,800 & 16,500 & 111,300 & 136,200 & 22,400 & 158,600 \\
\hline Hoc Isostatic Press & 240,400 & 20,300 & 260,700 & 325,900 & 26,000 & 351,900 \\
\hline
\end{tabular}

The Life-Cycle Cost Estimate for the Full Separations Option is attached to the letter "Economic and Life-Cycle Analysis conducted for the High-Level Waste (HLW) WTS Interim Storage Facility RJT-25-98," dated February 3, 1998.

The Life-Cycle Cost Estimate for the TRU/Class A Separations Option is attached to the letter, "Economic and Life-Cycle Analysis conducted for the High Level Waste (HLW) in FPR Interim Storage Facility-TRU - RJT-24-98," dated February 3, 1998.

The Life-Cycle Cost Estimate for the Hot Isostatic Press Option is attached to the letter "Economic and Life Cycle Analysis conducted for the Hot Isostatic Pressing (HIP) Storage Facility in FPR - RJT-17-98," dated January 30, 1998. 
Date: $\quad$ January 28,1998

To: J.K. Rawlins $\quad$ MS $3765 \quad 6-4303$

From: $\quad$ R. D. Adams ROA MS 3655 - 6-2963

Subject: $\quad$ INTERIM STORAGE STUDY - RDA-3-98

Cost Estimating has prepared Planning Cost Estimates for the subject project. These estimates were based on information received from you, your design team and reviewers from November 11, 1997 through January 23, 1998.

These estimates include permitting costs, direct and indirect construction costs, G\&A, Performance Incentive Fee (PIF), procurement fee, Engineering costs, Inspection costs, Project Management costs, Construction Management costs, escalation and contingency. The estimated costs have been escalated to the midpoint of the anticipated schedule. For more detailed information, please refer to the Cost Estimate Support Data Recapitulation forms.

Attached for your review are the Summary Estimate sheets, the Cost Estimate Support Data Recapitulation form, detailed estimate sheets, Contingency Analysis sheets G\&A/PIF Adder Calculation Sheets.

Also attached are the schedules.

Please refer to the attached table for a summary of the subject estimates.

If you have any questions regarding this estimate, please contact me at 526-2963.

RDA

Attachments:

cc: $\quad$ Estimate File \#2421

R. D. Adams File

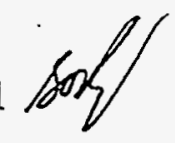




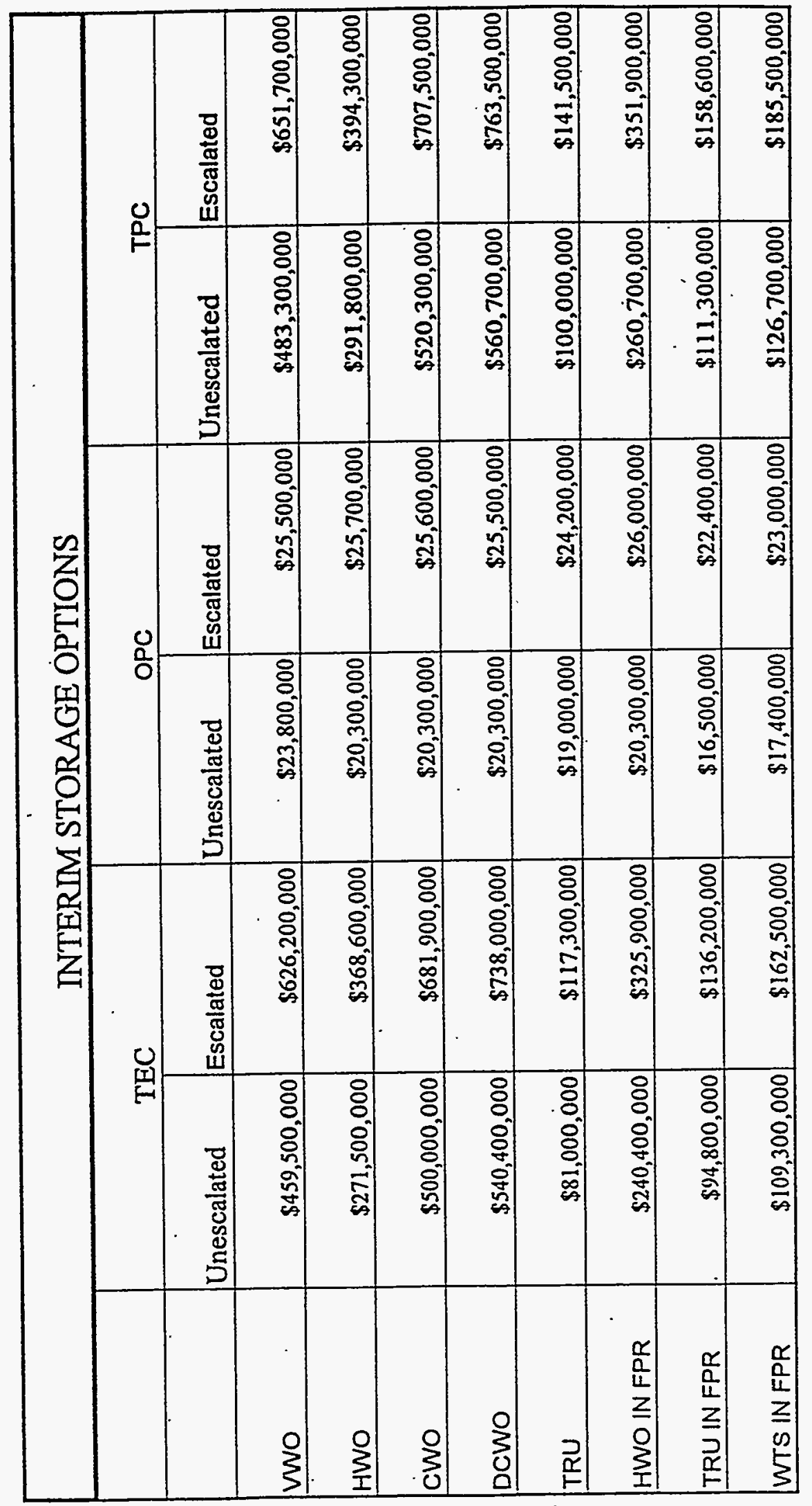




\section{COST ESTIMATE SUPPORT DATA RECAPITULATION}

Project Title: WTS-IS IN FPR

Date: $1 / 28 / 98$

Approved By:
Estimator: R D. Adams

File: 2421-FFPR PLANNING

\section{SCOPE OF WORK: Brief description of the proposed project.}

This facility will unload, store in vertical tubes and load out canisters containing High Level Waste (HLW) from the proposed Waste Treatment System(WTS) designed by Fluor Daniel Northwest Inc.

II. BASIS OF THE ESTMMATE: Drawings, Design Report, Engineers Notes and/or other documentation upon which the estimate is originated.

Draft sketches, a draft EDF, information from WINCO estimate 93108 and the SNF Canister Storage Building Final Design Estimate from Fluor Daniel Northwest, Inc. were used as the basis for this estimate.

III. ASSUMPTIONS: Conditions statements accepted or supposed true without proof of demonstration. An assumption has a direct impact on total estimated cost.

1. The Interim Storage Facility will be located within 100 feet of the WTS grouting facility.

2. Rock will not be encountered during excavation.

3. The estimates include both present day costs summaries and contingency analysis and escalated summaries and contingency analysis. The escalated summaries and contingency analysis reflect the attached schedule. All activities are escalated to the appropriate schedule mid-point.

4. FPR will have to be brought up to life safety code as reflected in estimate 93108 . It is assumed that the same materials and equipment available in storage for FPR when estimate was done are still available.

5. Most quantities used for this estimate were obtained by preliminary take offs from the draft sketches.

6. The storage tube costs, gantry crane cost and associated rail system costs were used from the Fluor estimate of a facility which is approximately eighty percent complete.

7. All assumptions and information included in the draft "New Interim Storage Facility for Interim Storage of HLW' apply to the estimate.

8. Where quantities could not be determined from the draft sketches, factors or the estimator used assumed quantities. 


\section{COST ESTIMATE SUPPORT DATA RECAPITULATÏON}

\begin{tabular}{|c|c|}
\hline Project Title: WTS-IS IN FPR & Estimator: R D. Adams \\
\hline Date: $1 / 28 / 98$ & File: 2421-FFPR PLANNING \\
\hline
\end{tabular}

9. Sufficient construction craft will be available to support this project.

10. Markups on direct construction costs to get the construction costs listed on the summary sheet are as follows:

Labor indirects, overheads and profit(OH\&P) $\quad 50 \%$ of labor

Material \& equipment OH\&P $5 \%$ of material and equipment

Subcontract OH\&O $\quad 10 \%$ of subcontract cost

Construction equipment allowance $10 \%$ of labor

NQA-1 quality requirements $30 \%$ of material

DOE/RW/0333P quality requirements $\quad 10 \%$ of material

Subcontractor procurement $1 \%$ of material and equipment

Bond $2 \%$ of everything

12. The below grade railroad track within the railroad access structure to the WTS Interim Storage Facility is included in this estimate the remainder of the branch line is included in the infrastructure estimate.

13. All utilities are assumed to be available at the facility wall.

14. The concrete that is removed from the existing FPR structure will be cut into blocks by diamond wire cutting or similar method. It will not be rubblized with the building

IV. CONTINGENCY GUIDELINE IMPLEMENTATION: The percentage used for contingency as determined by the contingency allowance guidelines can be altered to reflect the type of construction and conditions that may impact the total estimated cost.

A large portion of the estimated costs is based on actual costs from Fluor's SNF Canister Storage Building. The majority of the remaining costs are for standard construction activities. The activities involved with locating this structure inside an existing building carry a higher contingency than the standard construction tasks. For these reasons, the contingency is lower than would be experienced for a processing type facility at this stage of development.

\section{OTHER COMMENTS/CONCERNS SPECIFIC TO THE ESTIMATE:}

1. All FY'98 and later projects are to be assessed a Procurement Fee of 1\%, a G\&A Fee of $23 \%$, with a $\$ 500,000$ ceiling per year, and a Performance Incentive Fee (PIF) of 5.5\%. See the attached G\&A/PIF calculation sheet for the method used to calculate these fees. 


\section{COST ESTIMATE SUPPORT DATA RECAPITULATION}

Project Title: WTS-IS IN FPR

Date: $1 / 28 / 98$

Approved By:
Estimator: R. D. Adams

File: 2421-FFPR PLANNING

2. A procurement fee of one percent of construction was used to cover the operating contractor support to DOE-ID for their contract administration. It is felt that one percent is sufficient for jobs of this size. 
Lockheed Martin Idaho Technologies Co.

PROAECT NAME: WFS (FLUOR) - INTERIM STORAGE

FACILITY IN FPR - ESCALATED

LOCATION 1: INEELICPP

REOUESTOR: J.K. RAWLINS
COST ESTIMATE SUMMARY

TYPE OF ESTIMATE: PLANNING PROJECT NO: 2421FFPR PREPARED BY: R. D. ADAMS REPORT NAME: Cost Estimate Summary
CHECKED BY: $5 N$ N

APPR'D BY:

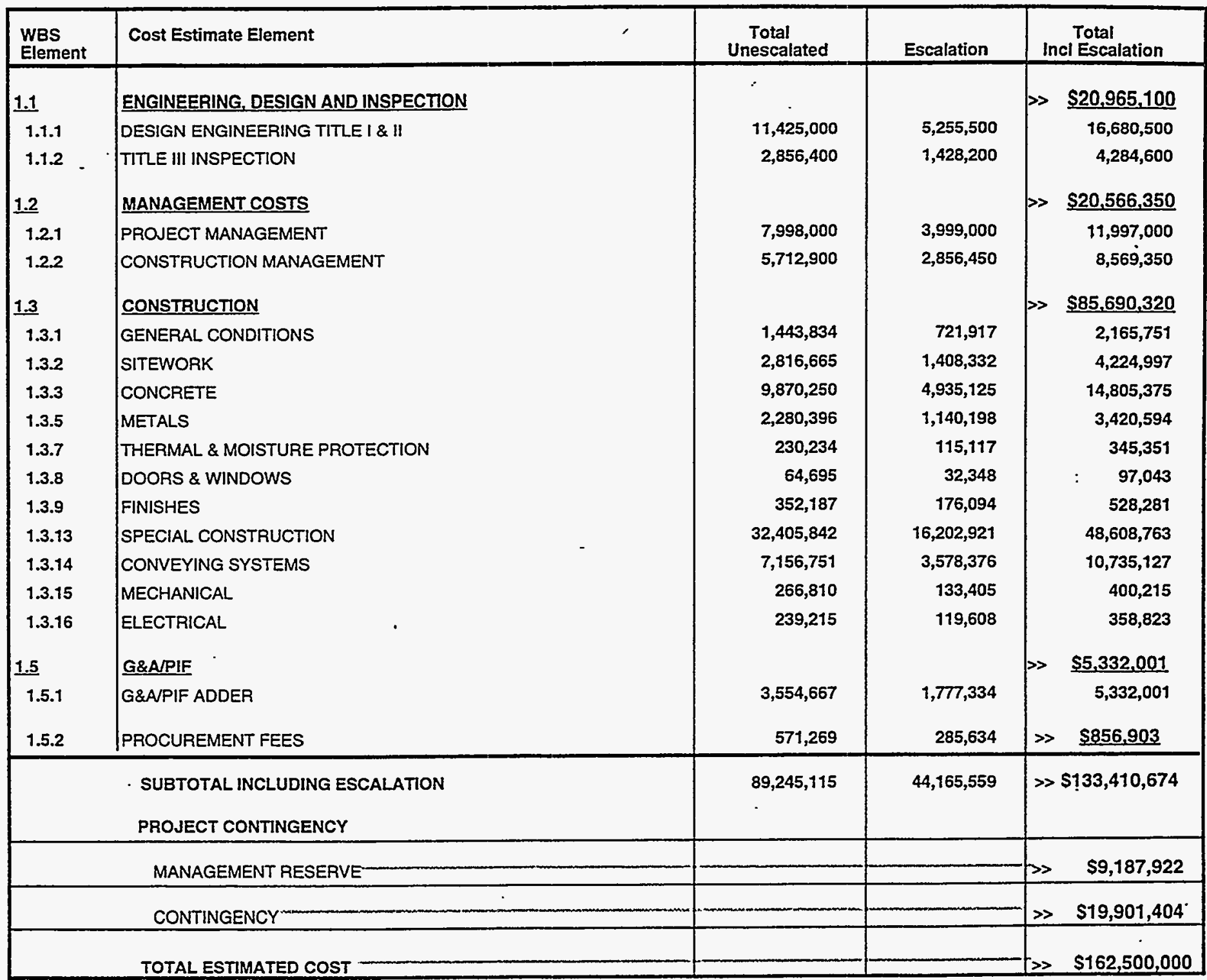

PROJECT COST PARAMETERS

EDI AS A \% OF CONST. + GFE $=24.00 \%$ 
Lockheed Martin Idaho Technologies Co. PROIECT NAME: WFS (FLUOR) - INTERIM STORAGE FACILITY IN FPR - UNESCALATED

LOCATION 1: REOUESTOR:$$
\text { J.K. RAWLINS }
$$

\section{COST ESTIMATE SUMMARY}

TYPE OF ESTIMATE: PLANNING

PROSECT NO: 2421FFPR

PREPARED BY: R.D. ADAMS

REPORT NAME: Cost Estimate Summary'

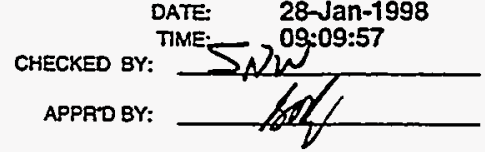

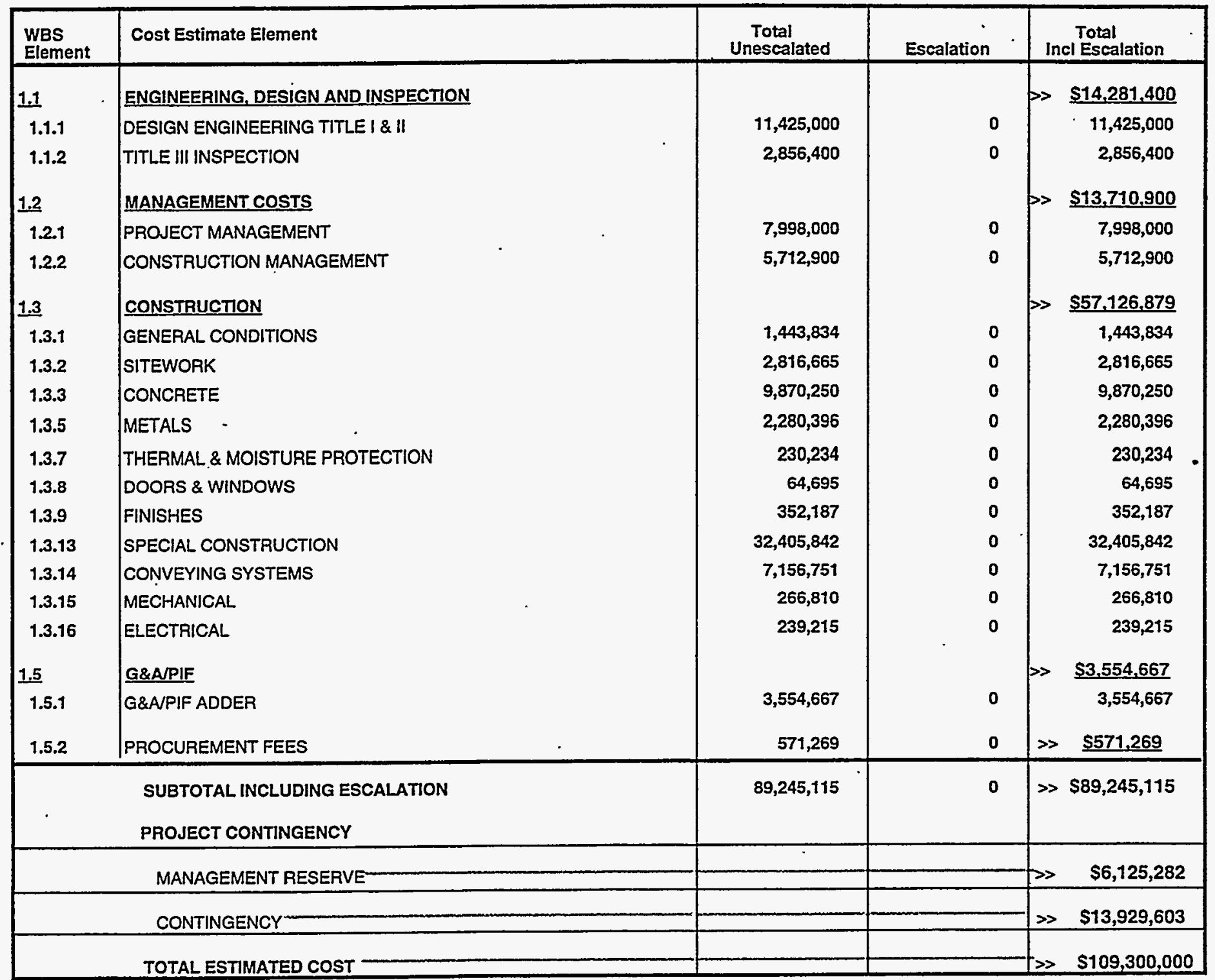

\section{PROJECT COST PARAMETERS}

EDI AS A $\%$ OF CONST. + GFE $=25.00 \%$

CONTINGENCY $=22.47 \%$ 
Lockheed Martin Idaho Technologies Co. Rov. 6/96

'ROJECT NAME: WFS (FLUOR) - INTERIM STORAGE

FACILITY IN FPR

OCATION 1: INEELICPP

IEOUESTOR: J. K. RAWLINS

\section{DETAILED COST ESTIMATE SHEET}

TYPE OF ESTIMATE: PLANNING

PAOJECT NO:: 2421FFPR

PREPARED BY: R. D. ADAMS
PAgE H 1

DATE 28-Jan-1998

TIME: 09:06:42

Report name: Detall Cost Estimate Sheet

\begin{tabular}{|c|c|c|c|c|c|c|c|c|c|c|c|c|}
\hline ODE & DESCRIPTION & QTY & UOM & $\begin{array}{c}\text { MATL } \\
\text { UNIT COST } \\
\end{array}$ & $\begin{array}{r}\text { CREW } \\
\text { SUB }\end{array}$ & $\begin{array}{c}\text { UNIT LAB } \\
\text { HOURS }\end{array}$ & $\begin{array}{r}\text { TOTAL } \\
\text { LAB HAS } \\
\end{array}$ & LABOR & $\begin{array}{l}\text { CONST. } \\
\text { EQUIP. }\end{array}$ & MAT'L & $\begin{array}{c}\text { S/C } \\
\text { (OTHER 1) } \\
\end{array}$ & $\begin{array}{r}\text { TOTAL } \\
\text { COST } \\
\end{array}$ \\
\hline 1.1 .1 & DESIGN ENGINEERING TITLE I\& $\|$ & & & & & & & & & & & \\
\hline & & & & & & & & & & & & \\
\hline 1000000.00 & TITLE I \& II & 1 & LOT & & & 0.000 & & $11,425,000$ & & & & $11,425,000$ \\
\hline & DESIGN ENGINEERING TITLE I \& $\| \mathrm{S} / \mathrm{T}$ & & & & & $\dot{-}$ & 0 & $\$ 11,425,000$ & & & & $\$ 11,425,000$ \\
\hline 1.1 .2 & IITLE III INSPECTION & & & & & & & & & & & \\
\hline$\ldots$ & & & & & & & & & & & & \\
\hline 11000000.00 & TITLE III $\bigcirc 5 \%$ OF CONSTAUCTION & 1 & LOT & & & 0.000 & & $2,856,400$ & & & & $2,856,400$ \\
\hline & TITLE III INSPECTION STT & & & & & & 0 & $\$ 2,856,400$ & & & & $\$ 2,856,400$ \\
\hline 1.2 .1 & PROJECT MANAGEMENT & & & & & & & & & & & \\
\hline 11000000.00 & $\begin{array}{l}\text { PROJECT MANAGMENT } 14 \% \text { OF } \\
\text { CONSTRUCTION }\end{array}$ & 1 & LOT & & & 0.000 & & $7,998,000$ & & & & $7,998,000$ \\
\hline & PROJECT MANAGEMENT S/T & & & & & & 0 & $\$ 7,998,000$ & & & & $\$ 7,99 \mathrm{~B}, 000$ \\
\hline $1.2,2$ & CONSTRUCTION MANAGEMENT & & & & & & & & & & & \\
\hline 11000000.00 & $\begin{array}{l}\text { CONSTRUCTION MANAGMENT } \otimes 10 \% \text { OF } \\
\text { CONSTRUCTION }\end{array}$ & 1 & LOT & & & 0.000 & & $5,712,900$ & & & & $5,712,900$ \\
\hline & CONSTRUCTION MANAGEMENT $S / T$ & & & & & & $\underline{0}$ & $\$ 5,712,900$ & & & & $\$ 5,712,900$ \\
\hline 1.3 .1 & GENERAL CONDITIONS & & & & & & & & & & & \\
\hline - & 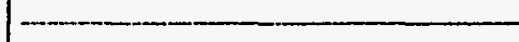 & & & & & & & & & & & \\
\hline & & & & & & & & & & & & \\
\hline
\end{tabular}


Lockheed Martin Idaho Technologles Co. Rev. 6/96

PROJECT NAME: WFS (FLUOR) - INTERIM STORAGE FACILITY IN FPR

LOCATION 1: INEELICPP

REOUESTOR: J. K. RAWLINS

\section{DETAILED COST ESTIMATE SHEET}

TYPE OF ESTIMATE: PLANNING

PROJECT NO: 2421FFPR

PREPARED BY: R. D. ADAMS
PAGE 2

DATE 28-Jan-1998

TIME: 09:06:42

REPORT NAME: Detall Cost Estimate Sheo

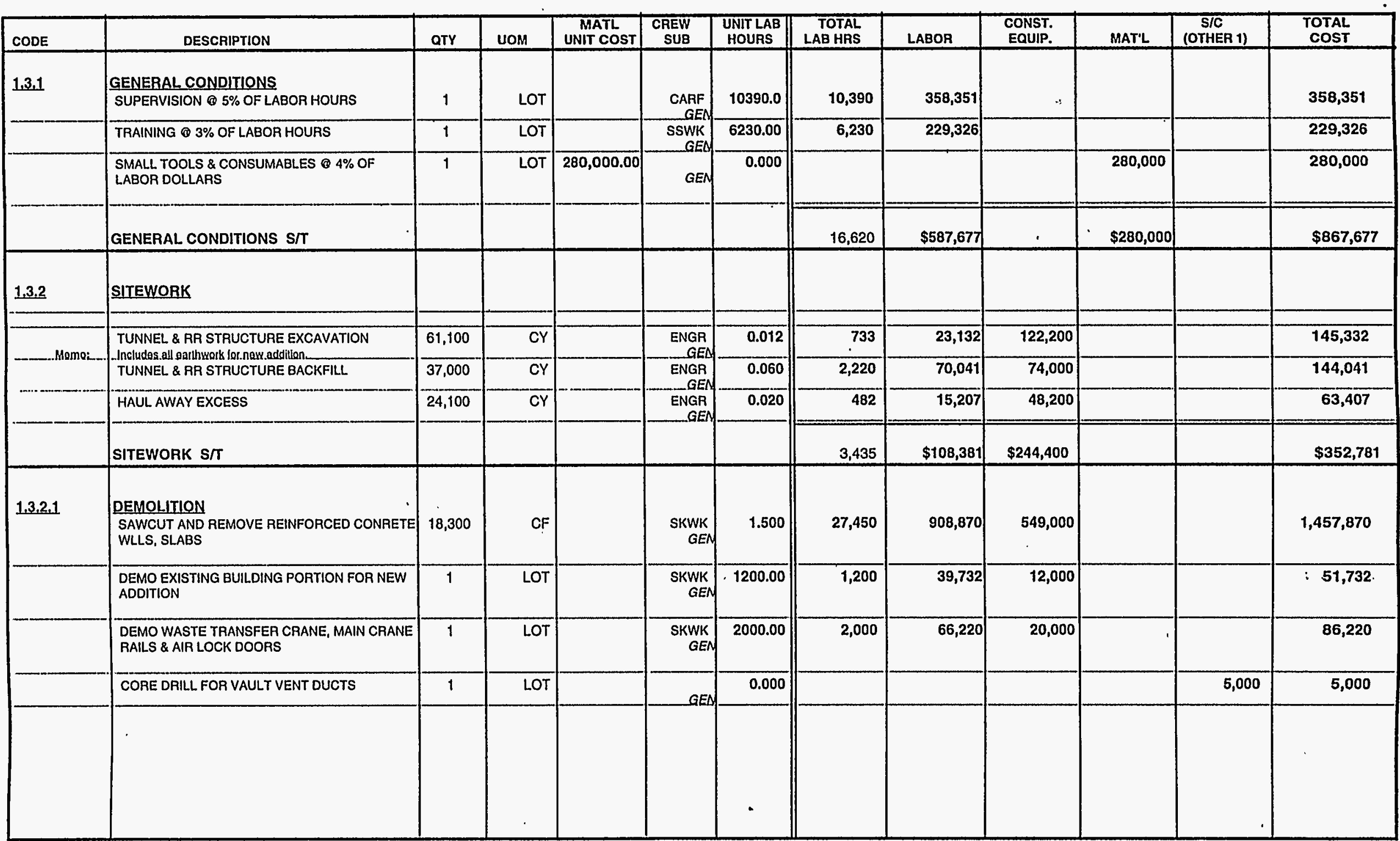


Lockheed Martin Idaho Technologies Co. Rev. $6 / 96$

PROJECT NAME: WFS (FLUOR) - INTERIM STORAGE FACILITY IN FPR

LOCATION 1: INEELICPP

REQUESTOR: J. K. RAWLINS

\section{DETAILED COST ESTIMATE SHEET}

TYPE OF ESTIMATE: PLANNING

PROJECT NO.: 2421FFPR

PREPARED BY: R. D. ADAMS
PAGE \# 4

DATE 28-Jan-1998

TIME: 09:06:42

REPORT NAME: Detall Cost Estlmate Sheet

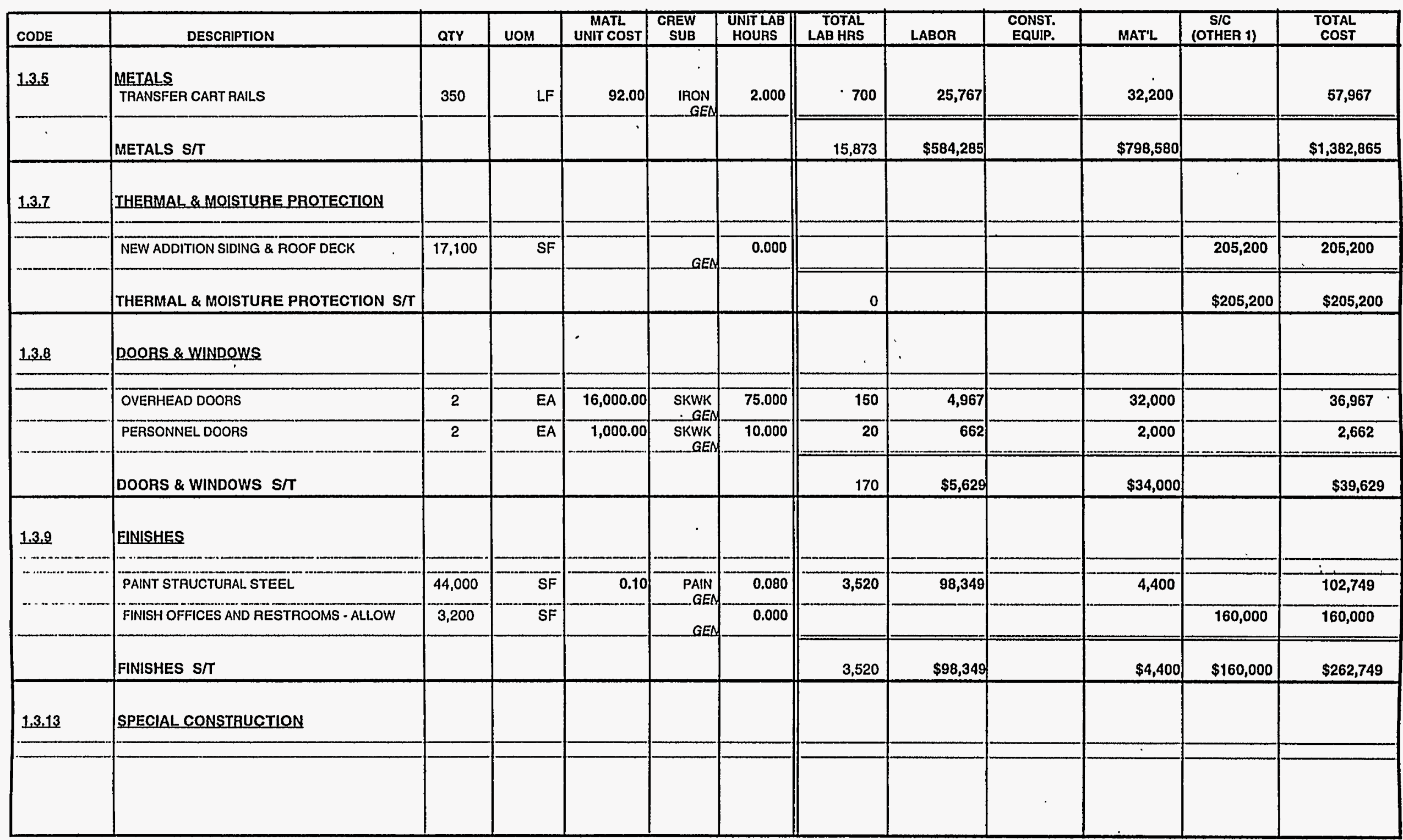


Lockheed Martln Idaho Technologles Co.

Rov. 6/96
PROJECT NAME: WFS (FLUOR) - INTERIM STORAGE FACILITY IN FPR

LOCATION 1: INEELIICPP

REQUESTOR: J. K. RAWLINS

\section{DETAILED COST ESTIMATE SHEET}

TYPE OF ESTIMATE: PLANNING

PAOJECT NO.: 2421FFPR

PREPARED BY: R. D. ADAMS
PAGE \# 5

DATE 28-Jan-1998

TIME: 09:06:42

REPORT NAME: Detail Cost Estimate Shee

\begin{tabular}{|c|c|c|c|c|c|c|c|c|c|c|c|c|}
\hline CODE & DESCRIPTION & QTY & UOM & $\begin{array}{l}\text { MATL } \\
\text { UNIT COST }\end{array}$ & $\begin{array}{l}\text { CREW } \\
\text { SUB } \\
\end{array}$ & $\begin{array}{l}\text { UNIT LAB } \\
\text { HOUAS }\end{array}$ & $\begin{array}{l}\text { TOTAL } \\
\text { LAB HRS } \\
\end{array}$ & LABOR & $\begin{array}{l}\text { CONST. } \\
\text { EQUIP. }\end{array}$ & MAT'L & $\begin{array}{c}\text { S/C } \\
\text { (OTHER 1) } \\
\end{array}$ & $\begin{array}{l}\text { TOTAL } \\
\text { COST }\end{array}$ \\
\hline $1,3,13$ & $\begin{array}{l}\text { SPECIAL CONSTRUCTION } \\
\text { SUPPORT BUILDING }\end{array}$ & 3,600 & SF & & & 0.000 & & & & & 540,000 & 540,000 \\
\hline & VAULT TUBE ASSEMBLIES & 200 & $\mathrm{EA}$ & $25,000.00$ & IRON & 100.000 & 20,000 & 736,200 & & $5,000,000$ & & $5,736,200$ \\
\hline & $\begin{array}{l}\text { BRING EXISTING BUILDING UP TO LIFE } \\
\text { SAFETY CODES - ALLLWW }\end{array}$ & 1 & LOT & & GEN & 0.000 & & 1 & & & $20,000,000$ & $20,000,000$ \\
\hline & SPECIAL CONSTRUCTION S/T & & & & & & 20,000 & $\$ 736,200$ & & $\$ 5,000,000$ & $\$ 20,540,000$ & $\$ 26,276,200$ \\
\hline $1,3,14$ & CONVEYING SYSTEMS & & & & & & & & & & & \\
\hline & GANTRY CRANE & $\overline{1}$ & $\overline{E A}$ & $2,500,000.00$ & SKWK & 1000.00 & $\overline{1,000}$ & 33,110 & & $2,500,000$ & & 2.533 .110 \\
\hline & TRANSFER CART IN TUNNEL & 1 & $E A$ & $300,000.00$ & SKWK & 500.000 & 500 & 16,555 & & 300,000 & & 316,555 \\
\hline & 5 TON DECONTAMINATABLE BRIDGE CRANE & 2 & EA & $250,000.00$ & SKWK & 300.000 & 600 & 19,866 & & 500,000 & & 519,866 \\
\hline & CASK MANUVERING HYDRAULIC PLATFORM & 1 & EA & {$[1,000,000.00 \mid$} & $\begin{array}{r}\text { SKWK } \\
\text { GEN }\end{array}$ & 1000.00 & 1,000 & 33,110 & & $1,000,000$ & & $1,033,110$ \\
\hline & CONVEYING SYSTEMS $S / T$ & & & & & & 3,100 & $\$ 102,641$ & & $\$ 4,300,000$ & & $\$ 4,402,641$ \\
\hline $1,3,15$ & MECHANICAL & & & & & & & & & & & \\
\hline & MISC: PIPING FOR NEW ADDITION-ALLOW & 1 & LOT & & & 0.000 & & & & & 75,000 & 75,000 \\
\hline & FIRE PROTECTION-NEW ADDITION & 5,600 & SF & & & 0.000 & & & & & 19,600 & 19,600 \\
\hline & HVAC - NEW ADDITION & 5,600 & SF & 11.20 & $\begin{array}{r}\text { SHEE } \\
\text { GED }\end{array}$ & 0.180 & 1,008 & 34,937 & & 62,720 & & 97,657 \\
\hline & MECHANICAL $S / T$ & & & & & & 1,008 & $\$ 34,937$ & & $\$ 62,720$ & $\$ 94,600$ & $\$ 192,257$ \\
\hline 1.3 .16 & ELECTRICAL & & & & & & & & & & & \\
\hline & & & & & & & & & & & & \\
\hline & & & & & & & & & & & & \\
\hline
\end{tabular}


Lockheed Martin Idaho Technologies Co.

PROJECT NAME: WFS (FLUOR) - INTERIM STORAGE FACILITY IN FPR

LOCATION 1: INEELICPP

REQUESTOR: J. K. RAWLINS

\section{DETAILED COST ESTIMATE SHEET}

TYPE OF ESTIMATE: PLANNING

PROJECTNO:: 2421FFPR

PREPARED BY: R. D. ADAMS
PAGE 6

ATE 28-Jan-1998

TIME: 09:06:42

REPORT NAME: Detall Cost Estimate Sheot

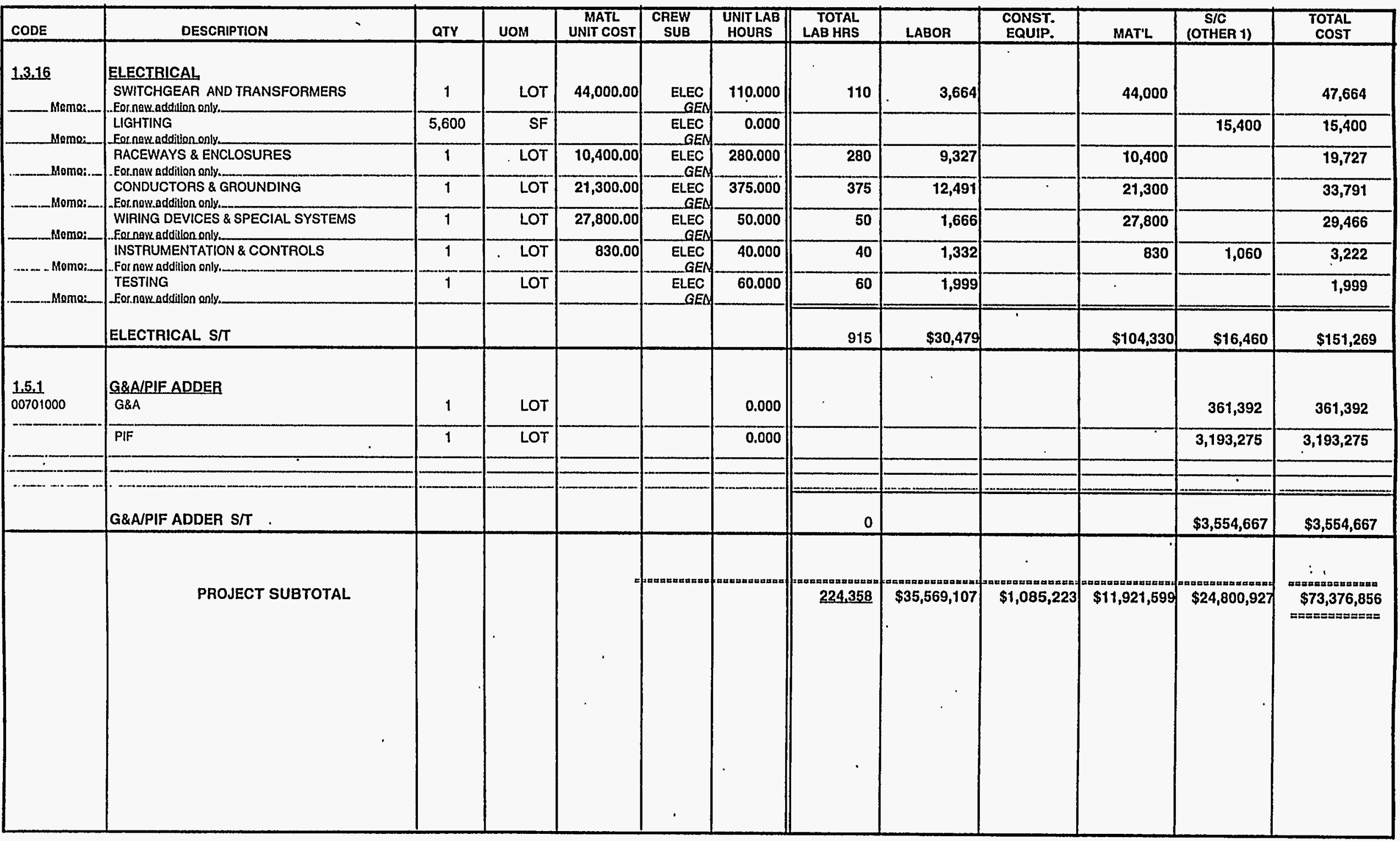


Lockheed Martin Idaho Technologies Co. PRONECT NAME: WFS (FLUOR) - INTERIM STORAGE LOCATION 1: FACILITY IN FPR - UNESCALATED INEELICPP REQUESTOR:

\section{CONTINGENCY ANALYSIS}

$\begin{array}{ll}\text { TYPE OF ESTIMATE: } & \text { PLANNING } \\ \text { PROJECT NO: } & \text { 2421FFPR }\end{array}$

PREPAREO BY:

R. D. ADAMS
DATE. 28-Jan-1998

- TIME: 09:10:03

REPORT NAME: Contingency Analysis

\begin{tabular}{|c|c|c|c|c|c|c|c|c|c|c|c|}
\hline \multicolumn{9}{|c|}{ PROBABLE \% VARIATION } & \multicolumn{2}{|c|}{$\begin{array}{c}\text { PROJECT } \\
\text { CONTINGENCY }\end{array}$} & \multirow{3}{*}{$\begin{array}{l}\text { SUMMARY } \\
\begin{array}{l}\text { Total Cost } \\
\text { by Element }\end{array}\end{array}$} \\
\hline \multirow[t]{2}{*}{$\begin{array}{l}\text { WBS } \\
\text { Element }\end{array}$} & \multirow{2}{*}{ Cost Estimate Element } & \multirow{2}{*}{$\begin{array}{l}\text { Total Cost w/o } \\
\text { Contingency }\end{array}$} & \multirow[t]{2}{*}{$\begin{array}{l}\text { \% Total } \\
\text { Cost }\end{array}$} & \multicolumn{2}{|c|}{$\begin{array}{l}\text { Prob. \% Var. } \\
\text { From Est. }\end{array}$} & \multicolumn{2}{|c|}{ Wt. \% of Prob. } & \multirow[t]{2}{*}{ Contingency } & \multirow[t]{2}{*}{$\%$} & \multirow[t]{2}{*}{ Cost } & \\
\hline & & & & - & + & - & + & & & & \\
\hline 1.1.1 & DESIGN ENGINEERING TILE $\mid \& \|$ & $11,425,000$ & 12.80 & 20 & 35 & 2.56 & 4.48 & $3.777 \%$ & $16.79 \%$ & $3,367,032$ & $14,792,032$ \\
\hline 7.1 .2 & TILE III INSPECTION & $2,856,400$ & 3.20 & 20 & 30 & 0.64 & 0.96 & $0.800 \%$ & $3.56 \%$ & 713,392 & $3,569,792$ \\
\hline 1.21 & PROJECT MANAGEMENT & $7,998,000$ & 8.96 & 20 & 35 & 1.79 & 3.14 & $2.644 \%$ & $11.75 \%$ & $2,357,070$ & $10,355,070$ \\
\hline 1.22 & CONSTRUCTION MANAGEMENT & $5,712,900$ & 6.40 & 20 & 30 & 1.28 & 1.92 & $1.600 \%$ & $7.11 \%$ & $1,426,808$ & $7,139,708$ \\
\hline 1.3 .1 & GENERAL CONDITIONS & $1,443,834$ & 1.62 & 20 & 35 & 0.32 & 0.57 & $0.477 \%$ & $2.12 \%$ & 425,509 & $1,869,343$ \\
\hline 1.3 .2 & SITEWORK & $2,816,665$ & 3.16 & 20 & 40 & 0.63 & 1.26 & $1.073 \%$ & $4.77 \%$ & 956,716 & $3,773,381$ \\
\hline 1.3 .3 & CONCRETE & $9,870,250$ & 11.06 & 20 & 30 & 2.21 & 3.32 & $2.765 \%$ & $12.29 \%$ & $2,465,115$ & $12,335,365$ \\
\hline 1.3 .5 & METALS & $2.280,396$ & 2.56 & 20 & 35 & 0.51 & 0.89 & $0.754 \%$ & $3.35 \%$ & 672,050 & $2,952,446$ \\
\hline 1.3.7 & THERMAL \& MOISTURE PROTECTION & 230,234 & 0.26 & 20 & 30 & 0.05 & 0.08 & $0.064 \%$ & $0.29 \%$ & 57,501 & 287,735 \\
\hline 1.3 .8 & DOORS \& WINDOWS & 64,695 & 0.07 & 20 & 20 & 0.01 & 0.01 & $0.012 \%$ & $0.05 \%$ & 10,341 & 75,036 \\
\hline 1.3 .9 & FINISHES & 352,187 & 0.39 & 20 & 35 & 0.08 & 0.14 & $0.116 \%$ & $0.52 \%$ & 103,792 & 455,979 \\
\hline 1.3 .13 & SPECIAL CONSTRUCTON & $32,405,842$ & 36.31 & 20 & 20 & 7.26 & 7.26 & $5.810 \%$ & $25.83 \%$ & $5,179,792$ & $37,585,634$ \\
\hline 1.3 .14 & CONVEYING SYSTEMS & $7,156,751$ & 8.02 & 20 & 25 & 1.60 & 2.00 & $1.644 \%$ & $7.31 \%$ & $1,465,679$ & $8,622,430$ \\
\hline 1.3 .15 & MECHANICAL & 266,810 & 0.30 & 20 & 30 & 0.06 & 0.09 & $0.075 \%$ & $0.33 \%$ & 66,636 & 333,446 \\
\hline 1.3.16 & ELECTRICAL & 239,215 & 0.27 & 20 & 25 & 0.05 & 0.07 & $0.055 \%$ & $0.24 \%$ & 48,990 & 288,205 \\
\hline 7.5 .1 & G\&AVPIF ADDER & $3,554,667$ & 3.98 & 5 & 20 & 0.20 & 0.80 & $0.697 \%$ & $3.10 \%$ & 621,450 & $4,176,117$ \\
\hline \multirow[t]{2}{*}{1.5 .2} & PROCUREMENTT FEES & 571,269 & 0.64 & 20 & 25 & 0.13 & 0.16 & $0.131 \%$ & $0.58 \%$ & 116,994 & 688,263 \\
\hline & ESCALATION & 0 & 0.00 & 20 & 25 & 0.00 & 0.00 & $0.000 \%$ & $0.00 \%$ & 18 & 18 \\
\hline & SUBTOTAL & $89,245,115$ & 100.00 & & & & & $22.494 \%$ & & & \\
\hline & CALCULATED CONTINGENCY & $20,074,778$ & & & & & & & & & \\
\hline & RESULTANT TEC & $109,319,893$ & & & & & & & & & \\
\hline & ROUNDED TEC & $109,300,000$ & & & & & & & & & \\
\hline & PROJECT CONTINGENCY & $20,054,885$ & & & & & & $22.47 \%$ & & & \\
\hline & MANAGEMENT RESERVE & $6,125,282$ & & & & & & & & & \\
\hline & CONTINGENCY & $13.929,603$ & & & & & & & & & \\
\hline & TOTAL ESTIMATED COST & $109,300,000$ & & & & & & & & $20,054,885$ & $109,300,000$ \\
\hline
\end{tabular}

CONFIDENCE LEVEL AND ASSUMED RISKS:

The Lockheed Idaho Technologies Co. Cost Estimate Contingency Analysis Model is based on the applied contingency and the assumptions upon which the estimate was predicated. The model is applied with a suggested risk leve

of $18 \%$ and a level of confidence of $90 \%$ the estimate will fall within the bid range. The Contingency Analysis is based on a weighted average to provide a

$90 \%$ probability of underrun and a $10 \%$ probability of overrun.
CONTINGENCY ANALYSIS GUIDE BY TYPE OF ESTIMATE

Guidelines established by DOE/FM 50 , Cost Estimating Guide, Vol. 6, Cost Guide, and as presented in the INEL Cost Estimating Guide.
PLANNING
Experimental/Special Conditions $20 \%-30 \%$
Conceptual
TITLE I
TITLE II
TITLE II/AFC
$15 \%-25 \%$
$10 \%-20 \%$
$5 \%--15 \%$
Market Conditions 
Lockheed Martin Idaho Technologies Co. PROJECT NAME: WFS (FLUOR) - INTERIM STORAGE FACILITY IN FPR - ESCALATED
INEELICPP

LOCATION 1:

REOUESTCR: J. K. RAWLINS

\section{CONTINGENCY ANALYSIS}

$\begin{array}{cl}\text { TYPE OF ESTIMATE: } & \text { PLANNING } \\ \text { PROJECT NO: } & \text { 2421FFPR } \\ \text { PREPARED BY: } & \text { R. D. ADAMS }\end{array}$

DATE: 28-Jan-1998
- TME: 09:07:54

REPORT NAME: Contingency Analysis

\begin{tabular}{|c|c|c|c|c|c|c|c|c|c|c|c|}
\hline \multirow{3}{*}{$\begin{array}{l}\text { WBS } \\
\text { Element }\end{array}$} & \multirow{3}{*}{ Cost Estimate Element } & \multirow{3}{*}{$\begin{array}{c}\text { Total Cost w/o } \\
\text { Contingency }\end{array}$} & \multirow{3}{*}{$\frac{10 N}{\begin{array}{c}\% \text { Total } \\
\text { Cost }\end{array}}$} & \multicolumn{4}{|c|}{ • } & \multirow{3}{*}{ Contingency } & \multicolumn{2}{|c|}{$\begin{array}{l}\text { PROJECT } \\
\text { CONTINGENCY }\end{array}$} & \multirow{3}{*}{$\begin{array}{l}\text { SUMMARY } \\
\begin{array}{l}\text { Total Cost } \\
\text { by Element }\end{array}\end{array}$} \\
\hline & & & & \multicolumn{2}{|c|}{$\begin{array}{c}\text { Prob. \%Var. } \\
\text { Frpm Est. }\end{array}$} & \multicolumn{2}{|c|}{ Wt. \% of Prob. } & & \multirow{2}{*}{$\%$} & \multirow{2}{*}{ Cost } & \\
\hline & & & & - & + & - & + & & & & \\
\hline 1.1 .1 & DESIGN ENGINEERING TITLE $i \& \|$ & $11,425,000$ & 8.56 & 20 & 35 & 1.71 & 3.00 & $2.526 \%$ & $11.57 \%$ & $3,365,798$ & $14,790,798$ \\
\hline 1.1 .2 & TITLE III INSPECTION & $2,856,400$ & 2.14 & 20 & 30 & 0.43 & 0.64 & $0.535 \%$ & $245 \%$ & 713,130 & $3,569,530$ \\
\hline 1.2 .1 & PROJECT MANAGEMENT & $7,998,000$ & 6.00 & 20 & 35 & 1.20 & 2.10 & $1.769 \%$ & $8.10 \%$ & $2,356,206$ & $10,354,206$ \\
\hline 1.2 .2 & CONSTRUCTION MANAGEMENT & $5,712,900$ & 4.28 & 20 & 30 & 0.86 & 1.28 & $1.071 \%$ & $4.90 \%$ & $1,426,285$ & $7,139,185$ \\
\hline 1.3 .1 & GENERAL CONDITIONS & $1,443,834$ & 1.08 & 20 & 35 & 0.22 & 0.38 & $0.319 \%$ & $1.46 \%$ & 425,353 & $1,869,187$ \\
\hline 1.3 .2 & SITEWORK & $2,816,665$ & 2.11 & 20 & 40 & 0.42 & 0.84 & $0.718 \%$ & $3.29 \%$ & 956,365 & $3,773,030$ \\
\hline 1.3 .3 & CONCRETE & $9,870,250$ & 7.40 & 20 & 30 & 1.48 & 222 & $1.850 \%$ & $8.47 \%$ & $2,464,211$ & $12,334,461$ \\
\hline 1.3 .5 & METALS & $2,280,396$ & 1.71 & 20 & 35 & 0.34 & 0.60 & $0.504 \%$ & $2.31 \%$ & 671,803 & $2,952,199$ \\
\hline 1.3 .7 & THERMAL \& MOISTURE PROTECTION & 230,234 & 0.17 & 20 & 30 & 0.03 & 0.05 & $0.043 \%$ & $0.20 \%$ & 57,480 & 287,714 \\
\hline 1.3 .8 & DOORS \& WINDOWS & 64,695 & 0.05 & 20 & 20 & 0.01 & 0.01 & $0.008 \%$ & $0.04 \%$ & 10,337 & 75,032 \\
\hline 1.3 .9 & FINISHES & 352,187 & 0.26 & 20 & 35 & 0.05 & 0.09 & $0.078 \%$ & $0.36 \%$ & 103,754 & 455,941 \\
\hline 1.3 .13 & SPECIAL CONSTRUCTION & $32,405,842$ & 24.29 & 20 & 20 & 4.86 & 4.86 & $3.886 \%$ & $17.80 \%$ & $5,177,893$ & $37,583,735$ \\
\hline 1.3 .14 & CONVEYING SYSTEMS & $7,156,751$ & 5.36 & 20 & 25 & 1.07 & 1.34 & $1.100 \%$ & $5.04 \%$ & $1,465,141$ & $8,621,892$ \\
\hline 1.3 .15 & MECHANICAL & 266,810 & 0.20 & 20 & 30 & 0.04 & 0.06 & $0.050 \%$ & $0.23 \%$ & 66,612 & 333,422 \\
\hline 1.3 .16 & ELECTRICAL & 239,215 & 0.18 & 20 & 25 & 0.04 & 0.04 & $0.037 \%$ & $0.17 \%$ & 48,972 & 288,187 \\
\hline 1.5 .1 & G\&AIPIF ADDER & $3,554,667$ & 266 & 5 & 20 & 0.13 & 0.53 & $0.466 \%$ & $2.14 \%$ & 621,222 & $4,175,889$ \\
\hline \multirow[t]{2}{*}{1.5 .2} & PROCUREMENT FEES & 571,269 & 0.43 & 20 & 25 & 0.09 & 0.11 & $0.088 \%$ & $0.40 \%$ & 116,951 & 688,220 \\
\hline & ESCALATION & $44,165,559$ & 33.10 & 20 & 25 & 6.62 & 8.28 & $6.787 \%$ & $31.08 \%$ & $9.041,813$ & $53,207,372$ \\
\hline \multicolumn{2}{|r|}{ SUBTOTAL } & $733,410,674$ & 100.00 & & & & & $21.834 \%$ & & & \\
\hline \multicolumn{2}{|r|}{ CALCULATED CONTINGENCY } & $29,128,718$ & & & & & & & & & \\
\hline \multicolumn{2}{|r|}{ RESULTANT TEC } & $162,539,392$ & & & & & & & & & \\
\hline \multicolumn{2}{|r|}{ ROUNDED TEC } & $162,500,000$ & & & & & & & & & \\
\hline \multicolumn{2}{|r|}{ PROJECT CONTINGENCY } & $29,089,326$ & & & & & & $21.80 \%$ & & & \\
\hline \multicolumn{2}{|r|}{ MANAGEMENT RESERVE } & $9,187,922$ & & & & & & & & & \\
\hline \multicolumn{2}{|r|}{ CONTINGENCY } & 19.901 .404 & & & & & & & & & \\
\hline & TOTAL ESTIMATED COST & $162,500,000$ & & & & & & & & $29,089,326$ & $162,500,000$ \\
\hline
\end{tabular}

\section{CONFIDENCE LEVEL AND ASSUMED RISKS:}

The Lockheed Idaho Technologies Co. Cost Estimate Contingency Analysis Model is based on the applied contingency and the assumptions upon which the estimate was predicated. The model is applied with a suggested risk level of $18 \%$ and a level of confidence of $90 \%$ the estimate will fall within the bid range. The Contingency Analysis is based on a weighted average to provide a

$90 \%$ probability of underrun and a $10 \%$ probability of overrun.
CONTINGENCY ANALYSIS GUIDE BY TYPE OF ESTIMATE

Guidelines established by DOEJFM 50 . Cost Estimating Guide, Vol. 6. Cost Guide, and as presented in the INEL Cost Estimating Guide.

PLANNING $20 \%-30 \%$

Experimental/Special Conditions............Up to $50 \%$ Conceptual $15 \%-25 \%$

TITLE I

TITLE II

TITLE IIJAFC
.... Up to $40 \%$

$10 \%-20 \%$

$5 \%-15 \%$

Market Conditions 


\section{G\&A/PIF ADDER CALCULATION SHEET}

PROCUREMENT FEE:

\begin{tabular}{l} 
CONSTRUCTION $=$ \\
GFE $=$ \\
\cline { 2 - 2 }
\end{tabular}

FEE @ $1 \%=$

$\$ 57,126,879 \cdot 0.01=\$ 571,268.79$

G\&A @ 23\% (with a ceiling of $\$ 500,000$ imposed per year

$\begin{array}{lr}\text { CONSTRUCTION OR } & \\ \text { CEILING } & \text { 2 YEARS }= \\ \text { GFE }= & \$ 1,000,000 \\ \text { PROCUREMENT FEE }= & \$ 0 \\ & \text { Subtotal }\end{array}$

FEE @ 23\% =

$\$ 1,571,269 * 0.23=$

$\$ 361,392$

PIF @ 5.5\%

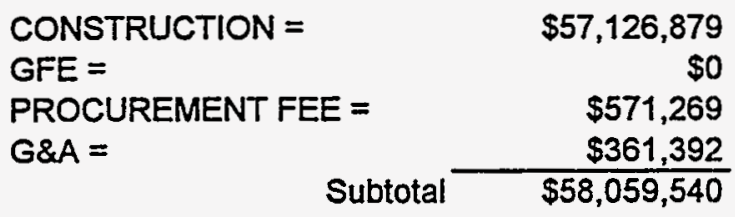

FEE @ $5.5 \%=$

$\$ 58,059,540 \cdot 0.055=$

$\$ 3,193,275$

TOTAL PROCUREMENT FEE:

$\$ 571,269$

TOTAL G\&A FEE:

$\$ 361,392$

TOTAL PIF:

$\$ 3,193,275$ 
Lockheed Martin idaho Technologies Co. PROIECT NAME: WTS(FLUOR) - INTERIM STORAGE FACILITY IN FPR - OPC - ESCALATED INEELICPP REOUESTOR: J.K. RAWLINS
COST ESTIMATE SUMMARY

TYPE OF ESTIMATE: PLANNING PROJECT NO: 242108 PREPARED BY:

R. D. ADAMS

REPORT NAME: Cost Estimate Summary

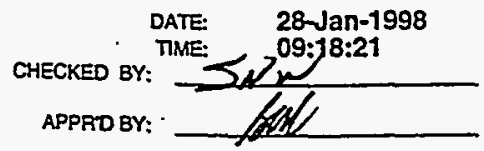

\begin{tabular}{|c|c|c|c|c|}
\hline $\begin{array}{l}\text { WBS } \\
\text { Element }\end{array}$ & Cost Estimate Element & $\begin{array}{c}\text { Total } \\
\text { Unescalated } \\
\end{array}$ & Escalation & $\begin{array}{c}\text { Total } \\
\text { Incl Escalation }\end{array}$ \\
\hline 1.1 & DESIGN \& DEVELOPMENT & & & b> $\$ 3,170,604$ \\
\hline 1.1 .1 & CONCEPTUAL DESIGN & $2,856,400$ & 314,204 & $3,170,604$ \\
\hline 1.2 & MANAGEMENT COSTS & & & $\gg \quad \$ 4,500,604$ \\
\hline 1.2 .1 & PM FOR PROJECT DEVELOPMENT & $2,856,400$ & 314,204 & $3,170,604$ \\
\hline 1.2 .2 & SAFETY REVIEWS & $1,000,000$ & 330,000 . & $1,330,000$ \\
\hline 1.3 & PERMITTING. & & & $\gg \quad \$ 3,628,815$ \\
\hline 1.3.1 & PERMITTING & $2,728,432$ & 900,383 & $3,628,815$ \\
\hline 1.4 & TURNOVER & & & $\$ 7,110,000$ \\
\hline 1.4 .1 & SO TEST \& STARTUP & $4,500,000$ & $2,610,000$ & $7,110,000$ \\
\hline \multirow[t]{2}{*}{1.5 .2} & PROCUREMENT FEES & 0 & 0 & $\gg$ \\
\hline & $\begin{array}{l}\text { SUBTOTAL INCLUDING ESCALATION } \\
\text { PROJECT CONTINGENCY }\end{array}$ & $13,941,232$ & $4,468,791$ & $\gg \$ \$ 18,410,023$ \\
\hline & MANAGEMENT RESERVE- & & & so \\
\hline & CONTINGENCY - & & & $\gg \quad \$ 4,589,977$ \\
\hline & TOTAL ESTIMATED COST & & & $\gg \quad \$ 23,000,000$ \\
\hline
\end{tabular}


Lockheed Martin Idaho Technologies Co. PROSECT NAME: WTS(FLUOR) - INTERIM STORAGE FACILITY IN FPR - OPC - UNESCALATED

J.K. RAWLINS

\section{COST ESTIMATE SUMMARY}

TYPE OFESTIMATE: PLANNING PROSECT NO: 242108 PAEPARED BY: R.D.ADAMS REPORT NAME: Cost Estimate Summary

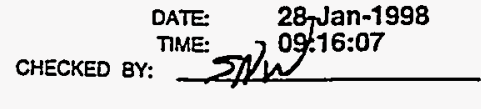

APPRO BY:

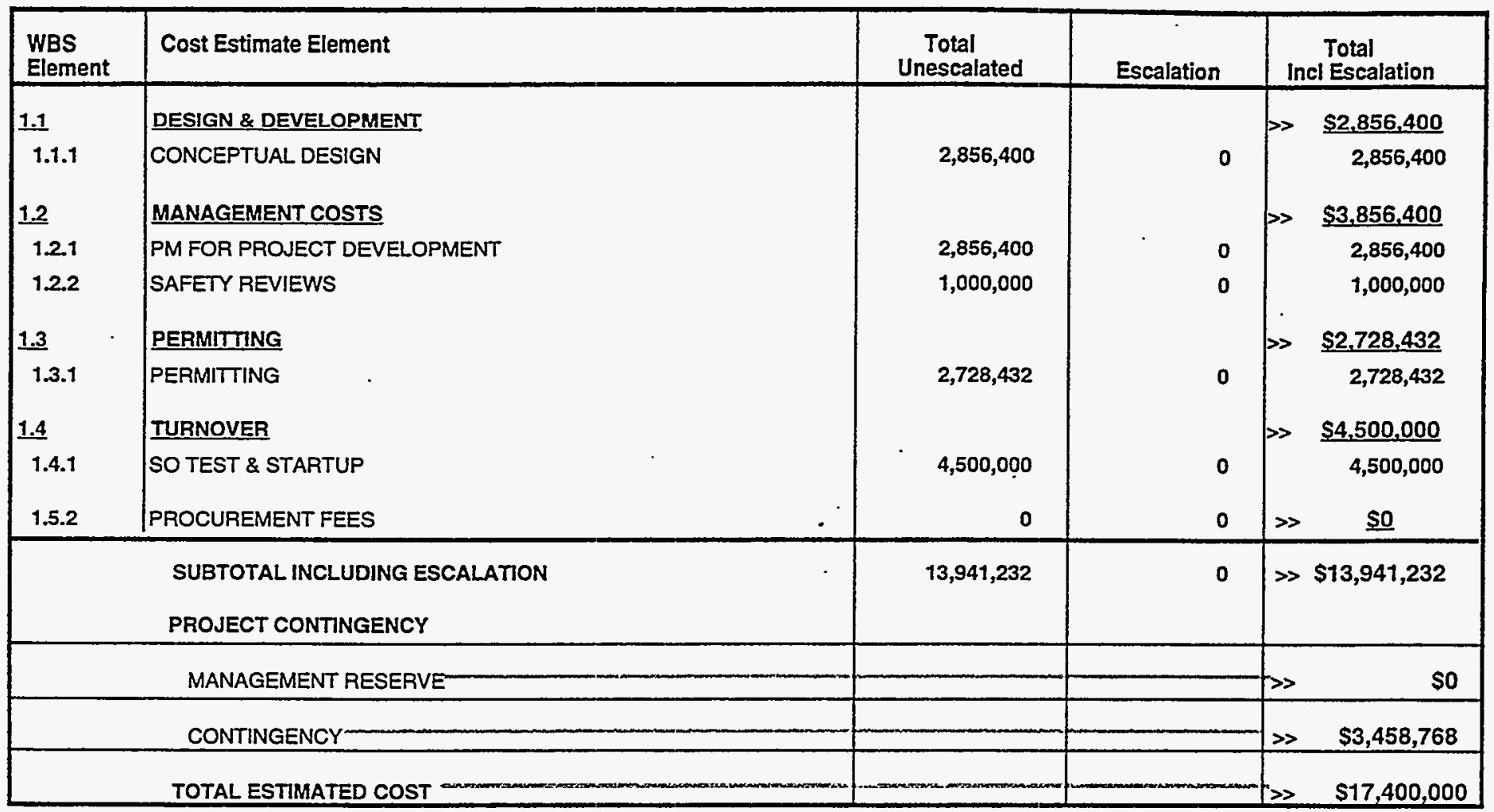

\section{PROJECT COST PARAMETERS}

EDI AS A $\%$ OF CONST. + GFE $=40.00 \%$ 
Lockheed Martin Idaho Technologies Co. PROJECT NAME: WTS(FLUOR) - INTERIM STORAGE FAOJECT NAME: WTLITY IN FPR - OPC

LOCATION I: INEEL/ICPP

REQUESTOR: J. K. RAWLINS
DETAILED COST ESTIMATE SHEET

TYPE OF ESTIMATE: PLANNING

PROJECT NO:: 242108

PREPARED BY: R. D. ADAMS
PAge : 1

DATE 28-Jan-1998

TIME: 09:15:03 REPORT NAME: Detall Cost Estimate Sheet

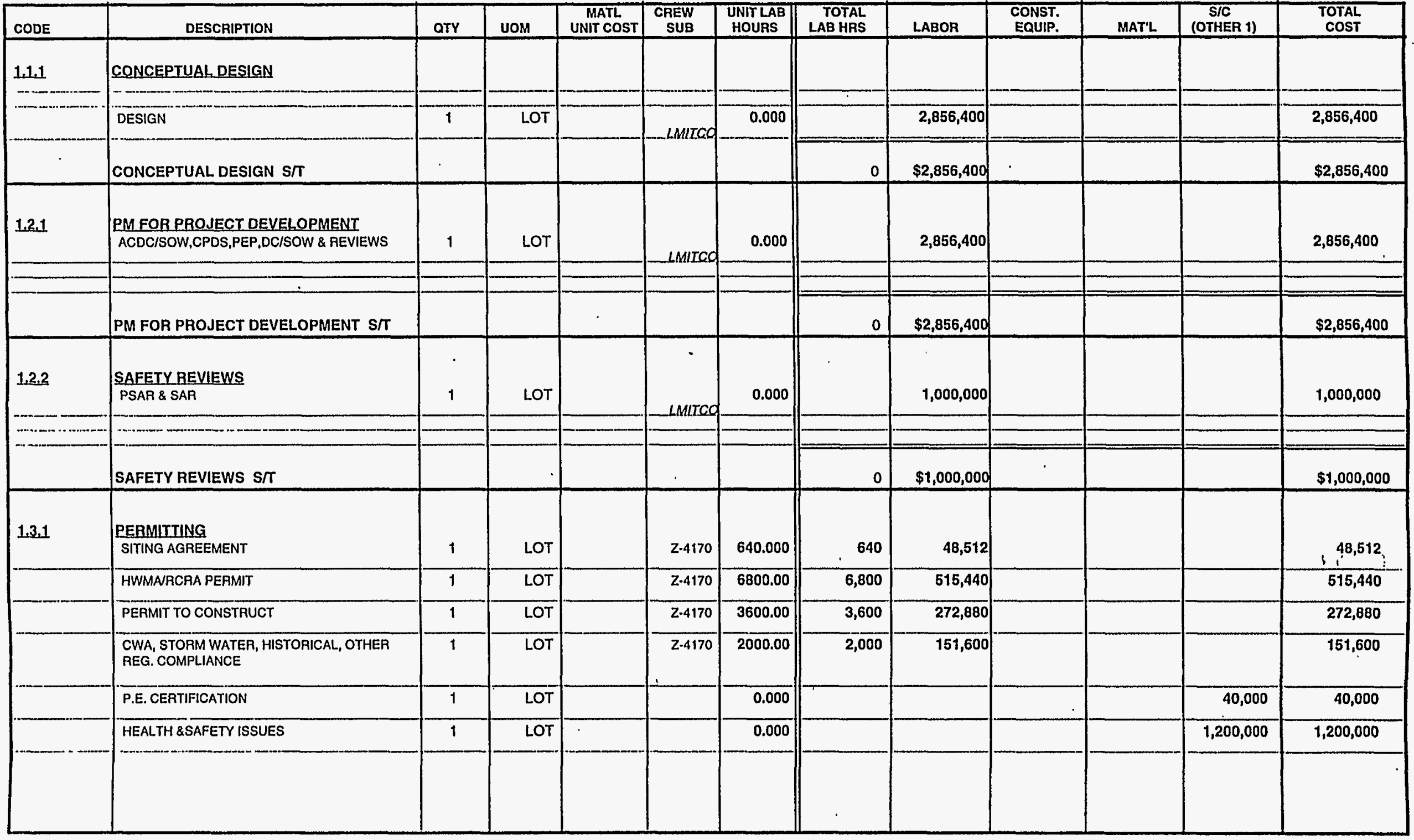


Lockheed Martin Idaho Technologies Co:

PRONECT NAME: WTS(FLUOR) - INTERIM STORAGE

LOCATION 1: FACILITY IN FPR - OPC - UNESCALATED

REQUESTOR:
CONTINGENCY ANALYSIS

TYPE OF ESTIMATE: PROSECT NO: PREPARED BY:
PLANNING

242108

R. D. ADAMS
DATE: 28-Jan-1998

- TIME: 09:16:12

REPORT nAME: Contingency Analysis

\begin{tabular}{|c|c|c|c|c|c|c|c|c|c|c|c|}
\hline \multicolumn{9}{|c|}{ PROBABLE \% VARIATION } & \multicolumn{2}{|c|}{$\begin{array}{l}\text { PROJECT } \\
\text { CONTINGENCY }\end{array}$} & \multirow{3}{*}{$\begin{array}{l}\text { SUMMARY } \\
\begin{array}{l}\text { Total Cost } \\
\text { by Element }\end{array}\end{array}$} \\
\hline \multirow[t]{2}{*}{$\begin{array}{l}\text { WBS } \\
\text { Element }\end{array}$} & \multirow{2}{*}{ Cost Estimate Element } & \multirow{2}{*}{$\begin{array}{c}\text { Total Cost w/o } \\
\text { Contingency }\end{array}$} & \multirow[t]{2}{*}{$\begin{array}{l}\text { \% Total } \\
\text { Cost }\end{array}$} & \multicolumn{2}{|c|}{$\begin{array}{c}\text { Prob. \% Var. } \\
\text { From Est. }\end{array}$} & \multicolumn{2}{|c|}{ Wt. \% of Prob. } & \multirow[t]{2}{*}{ Contingency } & \multirow[t]{2}{*}{$\%$} & \multirow[t]{2}{*}{ Cost } & \\
\hline & & & & - & + & - & + & & & & \\
\hline 1.1.1 & CONCEPTUAL DESIGN & $2,856,400$ & 20.49 & 20 & 30 & 4.10 & 6.15 & $5.122 \%$ & $20.49 \%$ & 708,652 & $3,565,062$ \\
\hline 1.2 .1 & PM FOR PROJECT DEVELOPMENT & $2,856,400$ & 20.49 & 20 & 30 & 4.10 & 6.15 & $5.122 \%$ & $20.49 \%$ & 708,662 & $3,565,062$ \\
\hline 1.22 & SAFETY REVIEWS & $1,000,000$ & 7.17 & 20 & 30 & 1.43 & 2.15 & $1.793 \%$ & $7.17 \%$ & 248,096 & $1,248,096$ \\
\hline 1.3 .1 & PERMITTING & $2,728,432$ & 19.57 & 20 & 30 & 3.91 & 5.87 & $4.893 \%$ & $19.57 \%$ & 676,914 & $3,405,346$ \\
\hline 1.4 .1 & SO TEST \& STARTUP & $4,500,000$ & 32.28 & 20 & 30 & 6.46 & 9.68 & $8.070 \%$ & $32.28 \%$ & $1,116,433$ & $5,616,433$ \\
\hline 1.5 .2 & PROCUREMENT FEES & 0 & 0.00 & 5 & 20 & 0.00 & 0.00 & $0.000 \%$ & $0.00 \%$ & 0 & $\underline{0}$ \\
\hline 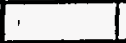 & ESCALATION & 0 & 0.00 & 20 & 30 & 0.00 & 0.00 & $0.000 \%$ & $0.00 \%$ & 1 & 1 \\
\hline & SUBTOTAL & $13,941,232$ & 100.00 & & & & & $25.000 \%$ & & & \\
\hline & CALCULATED CONTINGENCY & $3,485,308$ & & & & & & & & & \\
\hline & RESULTANT TEC & $17,426,540$ & & & & & & & & & \\
\hline & ROUNDED TEC & $17,400,000$ & & & & & & & & & \\
\hline & PROJECT CONTINGENCY & $3,458,768$ & & - & & & & $24.81 \%$ & & & \\
\hline & MANAGEMENT RESERVE & 0 & . & & & & & & & & \\
\hline & CONTINGENCY & $3,458,768$ & & & & & & & & & \\
\hline & TOTAL ESTIMATED COST & $17,400,000$ & & & & & & & & $3,458,768$ & $17,400,000$ \\
\hline
\end{tabular}

\section{CONFIDENCE LEVEL AND ASSUMED RISKS:}

The Lockheed Idaho Technologies Co. Cost Estimate Contingency Analysis Model is based on the applied contingency and the assumptions upon which the estimate was predicated. The model is applied with a suggested risk level of $18 \%$ and a level of confidence of $90 \%$ the estimate will fall within the bid range. The Contingency Analysis is based on a weighted average to provide a $90 \%$ probability of underrun and a $10 \%$ probability of ovemun.
CONTINGENCY ANALYSIS GUIDE BY TYPE OF ESTIMATE

Guidelines established by DOE/FM 50 . Cost Estimating Guide, Vol. 6 , Cost Guide, and as presented in the INEL Cost Estimating Guide. PLANNING Experimental/Special Condition $20 \%-30 \%$ Conceptual $15 \%-25 \%$ TIT Expenmentalspecial Conditions............ to $40 \%$ TITLE II TITLE II/AFC 
Lockheed Martin Idaho Technologies Co. PROIECT NAME: WTS(FLUOR) - INTERIM STORAGE LOCATION 1: FACILITY IN FPR - OPC - ESCALATED REQUESTOR: J.K. RAWLINS

\section{CONTINGENCY ANALYSIS}

TYPE OF ESTIMATE: PLANNING PROUECT NO: 242108 PREPARED BY: $\cdots$

- DATE: 28-Jan-1998

- TIME: 09:18:28

REPORT NAME: Contingency Analysis

\begin{tabular}{|c|c|c|c|c|c|c|c|c|c|c|c|}
\hline \multicolumn{9}{|c|}{ PROBABLE \% VARIATION } & \multicolumn{2}{|c|}{$\begin{array}{c}\text { PROJECT } \\
\text { CONTINGENCY } \\
\end{array}$} & \multirow{3}{*}{$\begin{array}{l}\text { SUMMARY } \\
\begin{array}{l}\text { Total Cost } \\
\text { by Element }\end{array}\end{array}$} \\
\hline \multirow[t]{2}{*}{$\begin{array}{l}\text { WBS } \\
\text { Element }\end{array}$} & \multirow[t]{2}{*}{ Cost Estimate Element } & \multirow{2}{*}{$\begin{array}{c}\text { Total Cost w/o } \\
\text { Contingency }\end{array}$} & \multirow[t]{2}{*}{$\begin{array}{c}\text { \% Total } \\
\text { Cost }\end{array}$} & \multicolumn{2}{|c|}{$\begin{array}{c}\text { Prob. \% Var. } \\
\text { Frpm Est }\end{array}$} & \multicolumn{2}{|c|}{ Wt. \% of Prob. } & \multirow[t]{2}{*}{ Contingency } & \multirow[t]{2}{*}{$\%$} & \multirow{2}{*}{ Cost } & \\
\hline & & & & - & + & - & + & & & & \\
\hline 1.1 .1 & CONCEPTUAL DESIGN & $2,856,400$ & 15.52 & 20 & 30 & 3.10 & 4.65 & $3.879 \%$ & $15.52 \%$ & 712,156 & $3,568,556$ \\
\hline 1.21 & PM FOR PROJECT DEVELOPMENT & $2,856,400$ & 15.52 & 20 & 30 & 3.10 & 4.65 & $3.879 \%$ & $15.52 \%$ & 712,156 & $3,568,556$ \\
\hline 1.2 .2 & SAFETY REVIEWS & $1,000,000$ & 5.43 & 20 & 30 & 1.09 & 1.63 & $1.358 \%$ & $5.43 \%$ & 249,319 & $1,249,319$ \\
\hline 1.3 .1 & PERMITTING & $2,728,432$ & 14.82 & 20 & 30 & 296 & 4.45 & $3.705 \%$ & $14.82 \%$ & 680,251 & $3,408,683$ \\
\hline 1.4 .1 & SO TEST \& STARTUP & $4,500,000$ & 24.44 & 20 & 30 & 4.89 & 7.33 & $6.111 \%$ & $24.44 \%$ & $1,121,938$ & $5,621,938$ \\
\hline \multirow[t]{2}{*}{1.5 .2} & PROCUREMENT FEES & 0 & 0.00 & 5 & 20 & 0.00 & 0.00 & $0.000 \%$ & $0.00 \%$ & 0 & 0 \\
\hline & ESCALATION & $4,468,791$ & 24.27 & 20 & 30 & 4.85 & 7.28 & $6.068 \%$ & $24.27 \%$ & $1,114.157$ & $5,582,948$ \\
\hline & SUBTOTAL & $18,410,023$ & 100.00 & & & & & $25.000 \%$ & & & \\
\hline & CALCULATED CONTINGENCY & $4,602,506$ & & & & & & & & & \\
\hline & RESULTANT TEC & $23,012,529$ & & & & & & & & & \\
\hline & ROUNDED TEC & $23,000,000$ & & & & & & & & & \\
\hline & PROJECT CONTINGENCY & $4,589,977$ & & & & & & $24.93 \%$ & & & \\
\hline & MANAGEMENT RESERVE & 0 & & & & & & & & & \\
\hline & CONTINGENCY & $4,589,977$ & & & & & & & & & \\
\hline & TOTAL ESTIMATED COST & $23,000,000$ & & & & & & & & $4,589,977$ & $23,000,000$ \\
\hline
\end{tabular}

CONFIDENCE LEVEL AND ASSUMED RISKS:

The Lockheed Idaho Technologies Co. Cost Estimate Contingency Analysis Model is based on the applied contingency and the assumptions upon which

the estimate was predicated. The model is applied with a suggested risk level

of $18 \%$ and a level of confidence of $90 \%$ the estimate will fall within the bid range.

The Contingency Analysis is based on a weighted average to provide a

$90 \%$ probability of underrun and a $10 \%$ probability of overrun.
CONTINGENCY ANALYSIS GUIDE BY TYPE OF ESTIMATE

Guidelines established by DOE/FM 50, Cost Estimating Guide, Vol. 6 , Cost Guide, and as presented in the INEL Cost Estimating Guide.
PLANNING Experimental/Special Conditions.............. -
Conceptual
Experimental/Special Conditions $15 \%-25 \%$
TITLE I
TITLE ॥
...
$10 \%-20 \%$

TITLE II/AFC
Market Conditions 


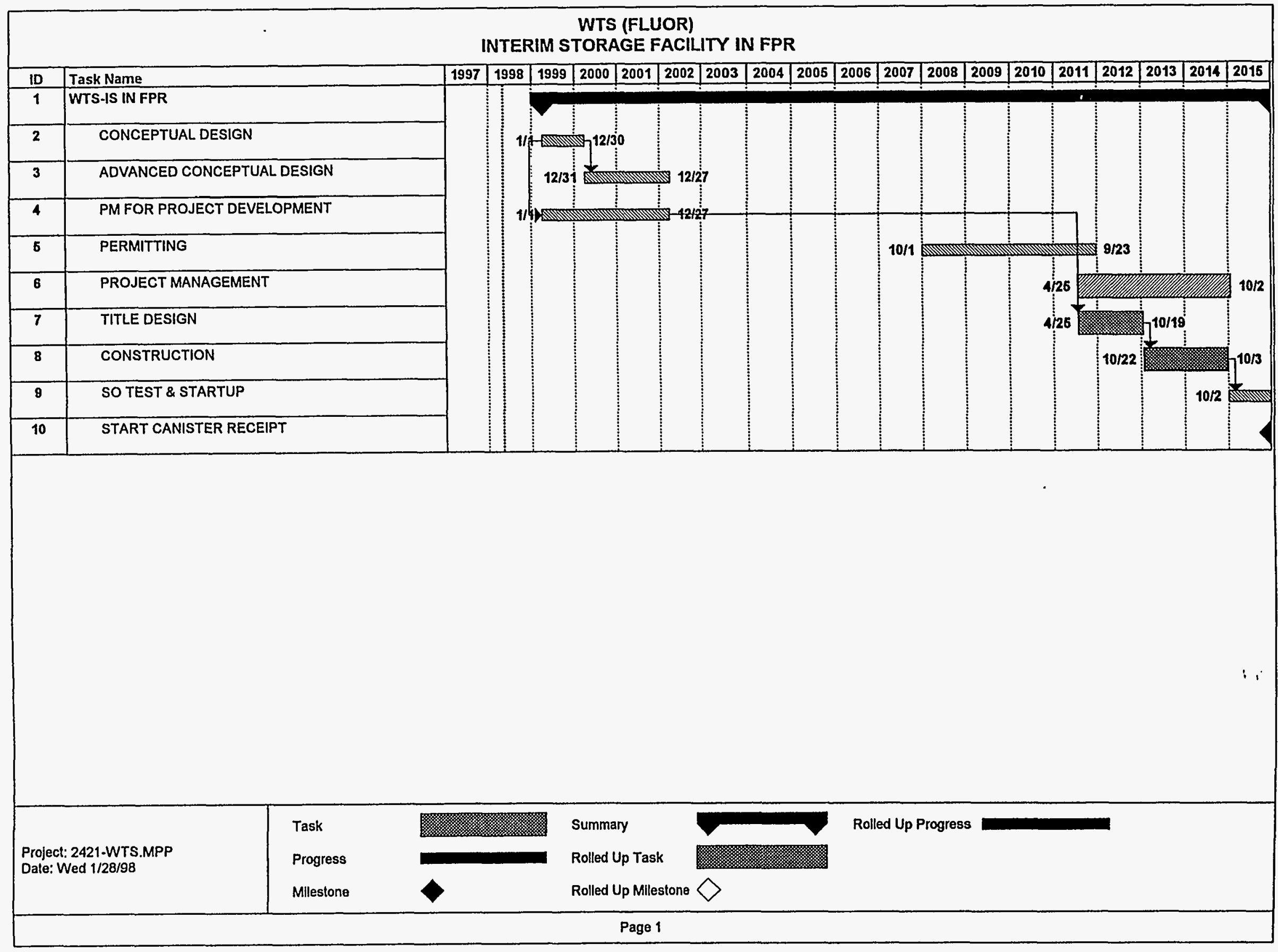




\section{COST ESTIMATE SUPPORT DATA RECAPITULATION}

Project Title: TRU-IS IN FPR

Date: $1 / 28 / 98$

Approved By:

I. SCOPE OF WORK: Brief description of the proposed project.

This facility will unload, store in vertical tubes and load out canisters containing High Level Waste (HIW) from the proposed TRU Separations - Class A Grout Option.

II. BASIS OF THE ESTIMATE: Drawings, Design Report, Engineers Notes and/or other doctmentation upon which the estimate is originated.

Draft sketches, a draft EDF, information from WINCO estimate 93108 and the SNF Canister Storage Building Final Design Estimate from Fluor Daniel Northwest, Inc. were used as the basis for this estimate.

III. ASSUMPTIONS: Conditions statements accepted or supposed true without proof of demonstration. An assumption has a direct impact on total estimated cost.

1. The Interim Storage Facility will be located within 100 feet of the TRU grouting facility.

2. Rock will not be encountered during excavation.

3. The estimates include both present day costs summaries and contingency analysis and escalated summaries and contingency analysis. The escalated summaries and contingency analysis reflect the attached schedule. All activities are escalated to the appropriate schedule mid-point.

4. FPR will have to be brought up to life safety code as reflected in estimate 93108 . It is assumed that the same materials and equipment available in storage for FPR when estimate was done are still available.

5. Most quantities used for this estimate were obtained by preliminary take offs from the draft sketches.

6. The storage tube costs, gantry crane cost and associated rail system costs were used from the Fluor estimate of a facility which is approximately eighty percent complete.

7. All assumptions and information included in the draft "New Interim Storage Facility for Interim Storage of HLW" apply. to the estimate.

8. Where quantities could not be determined from the draft sketches, factors or the estimator used assumed quantities.

9. Sufficient construction craft will be available to support this project. 


\section{COST ESTIMATE SUPPORT DATA RECAPITULATION}

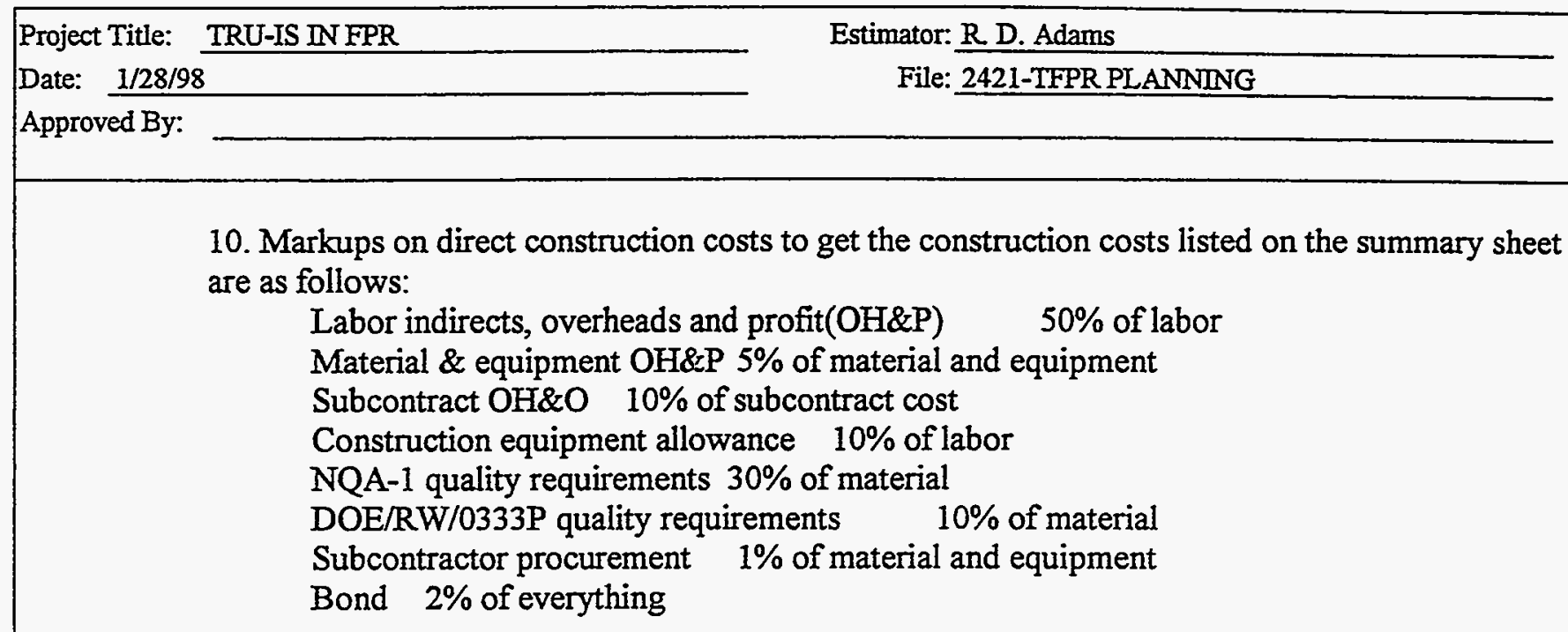

12. The below grade railroad track within the railroad access structure to the WTS Interim Storage Facility is included in this estimate the remainder of the branch line is included in the infrastructure estimate.

13. All utilities are assumed to be available at the facility wall.

14. The concrete that is removed from the existing FPR structure will be cut into blocks by diamond wire cutting or similar method. It will not be rubblized with the building

IV. CONTINGENCY GUDELINE IMPLEMENTATION: The percentage used for contingency as determined by the contingency allowance guidelines can be altered to reflect the type of construction and conditions that may impact the total estimated cost.

A large portion of the estimated costs is based on actual costs from Fluor's SNF Canister Storage Building. The majority of the remaining costs are for standard construction activities. The activities involved with locating this structure inside an existing building carry a higher contingency than the standard construction tasks. For these reasons, the contingency is lower than would be experienced for a processing type facility at this stage of development.

\section{OTHER COMMENTS/CONCERNS SPECIFIC TO THE ESTIMATE:}

1. All FY'98 and later projects are to be assessed a Procurement Fee of $1 \%$, a G\&A Fee of $23 \%$, with a $\$ 500,000$ ceiling per year, and a Performance Incentive Fee (PIF) of $5.5 \%$. See the attached $\mathrm{G} \& \mathrm{~A} / \mathrm{PIF}$ calculation sheet for the method used to calculate these fees. 
Lockheed Martin Idaho Technologies Co.

PRONECT NAME: TRU CLASS A GROUT - INTERIM STORAGE FACILITYIN FPR - ESCALATED

LOCATION 1: INEEL/CPP

REQUESTOR: J.K. RAWLINS
COST ESTIMATE SUMMARY

TYPE OF ESTIMATE: PLANNING PROAECTNO: 2421-TFPR PAEPARED BY: R.D.ADAMS REPORT NAME: Cost Estimate Summary
DATE: 28-Jan-1998

CHECKED BY: TME: $S$ WM

APPRO BY:

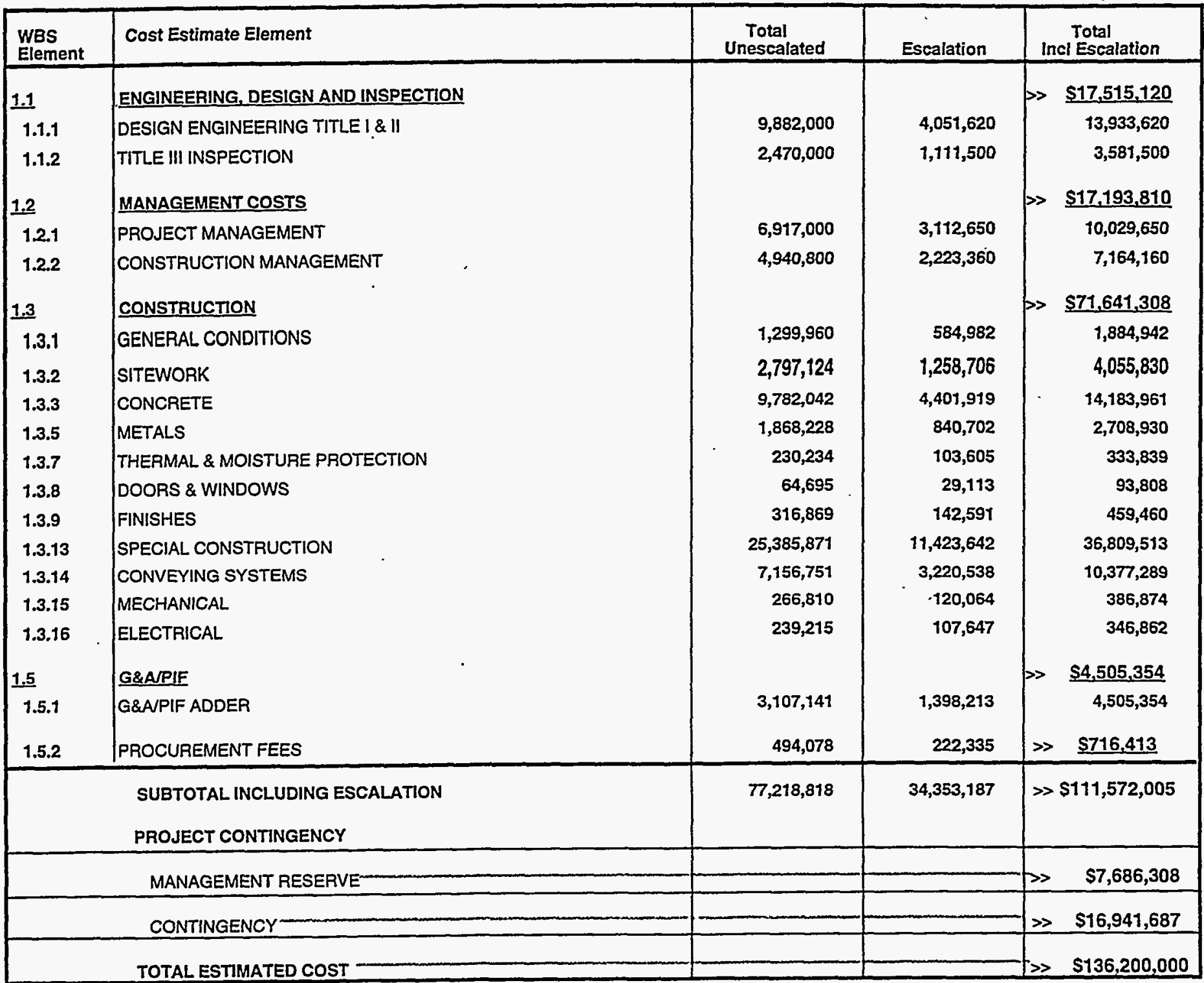

\section{PROJECT COST PARAMETERS}

EDI AS A $\%$ OF CONST. + GFE $=24.00 \%$

CONTINGENCY $=22.07 \%$ 
Lockheed Martin Idaho Technologies Co.

PROUECT NAME: TRU CLASS A GROUT - INTERIM STORAGE FACILITY IN FPR - UNESCALATED

LOCATION 1:

REQUESTOR:

INEELICPP

J. K. RAWLINS
COST ESTIMATE SUMMARY

TYPE OF ESTIMATE: PLANNING PROJECT NO: 2421-TFPR PREPARED BY:

REPORT NAME: Cost Estimate Summary

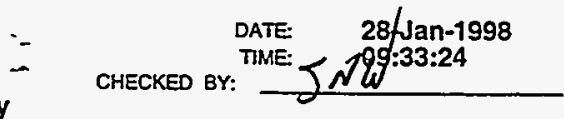

APPR'D BY:

\begin{tabular}{|c|c|c|c|c|}
\hline $\begin{array}{l}\text { WBS } \\
\text { Element }\end{array}$ & Cost Estimate Element & $\begin{array}{c}\text { Total } \\
\text { Unescalated } \\
\end{array}$ & Escalation & $\begin{array}{c}\text { Total } \\
\text { Incl Escalation }\end{array}$ \\
\hline $\begin{array}{l}\frac{1.1}{1.1 .1} \\
1.1 .2 \\
1.2 \\
1.2 .1 \\
1.2 .2 \\
1.3 \\
1.3 .1 \\
1.3 .2 \\
1.3 .3 \\
1.3 .5 \\
1.3 .7 \\
1.3 .8 \\
1.3 .9 \\
1.3 .13 \\
1.3 .14 \\
1.3 .15 \\
1.3 .16 \\
1.5 \\
1.5 .1 \\
1.5 .2\end{array}$ & $\begin{array}{l}\text { ENGINEERING, DESIGN AND INSPECTION } \\
\text { DESIGN ENGINEERING TITLE I \& II } \\
\text { TITLE III INSPECTION } \\
\text { MANAGEMENT COSTS } \\
\text { PROJECT MANAGEMENT } \\
\text { CONSTRUCTION MANAGEMENT } \\
\text { CONSTRUCTION } \\
\text { GENERAL CONDITIONS } \\
\text { SITEWORK } \\
\text { CONCRETE } \\
\text { METALS } \\
\text { THERMAL \& MOISTURE PROTECTION } \\
\text { DOORS \& WINDOWS } \\
\text { FINISHES } \\
\text { SPECIAL CONSTRUCTION } \\
\text { CONVEYING SYSTEMS } \\
\text { MECHANICAL } \\
\text { ELECTRICAL } \\
\text { G\&AIPIF } \\
\text { G\&AIPIF ADDER } \\
\text { PROCUREMENT FEES }\end{array}$ & $\begin{array}{r}9,882,000 \\
2,470,000 \\
\\
6,917,000 \\
4,940,800 \\
\\
1,299,960 \\
2,797,124 \\
9,782,042 \\
1,868,228 \\
230,234 \\
64,695 \\
316,869 \\
25,385,871 \\
7,156,751 \\
266,810 \\
239,215 \\
\end{array}$ & $\begin{array}{l}0 \\
0 \\
0 \\
0 \\
0 \\
0 \\
0 \\
0 \\
0 \\
0 \\
0 \\
0 \\
0 \\
0 \\
0\end{array}$ & 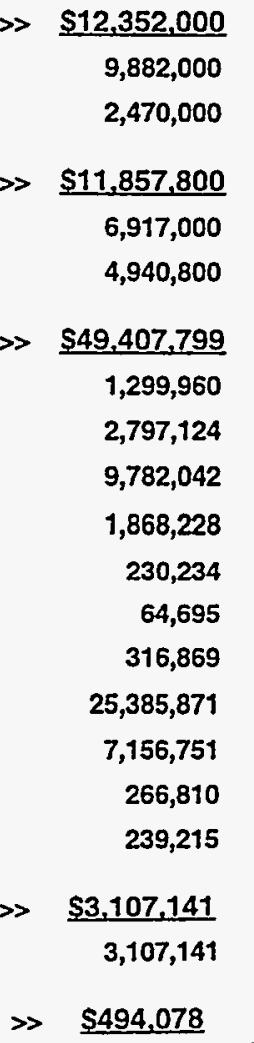 \\
\hline & $\begin{array}{l}\text { SUBTOTAL INCLUDING ESCALATION } \\
\text { PROJECT CONTINGENCY }\end{array}$ & $77,218,818$ & 0 & $>\$ 77,218,818$ \\
\hline & MANAGEMENT RESERVE & & & $\$ 5,300,902$ \\
\hline & CONTINGENCY & & & $\gg \quad \$ 12,280,280$ \\
\hline & TOTAL ESTIMATED COST & & & $\$ 94,800,000$ \\
\hline
\end{tabular}


Lockheed Martin Idaho Technologies Co. Rov. 6/96

PROJECT MAME: TRU CLASS A GROUT - INTERIM STORAGE FACILITY IN FPR

OCATION 1: INEELICPP

REQUESTOR: J. K. RAWLINS

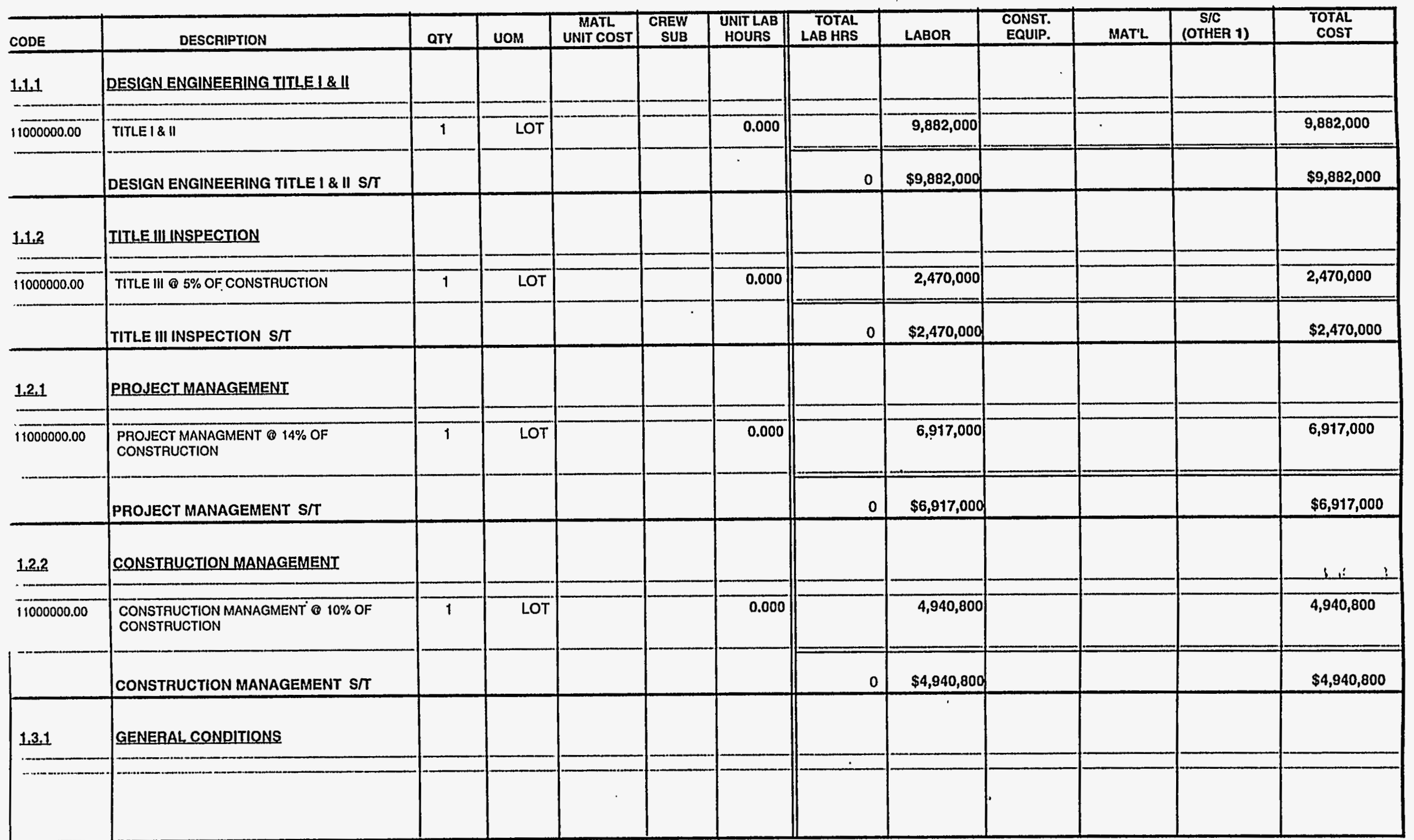

DETAILED COST ESTIMATE SHEET

TYPE OF ESTIMATE: PLANNING

PROJECT NO: 2421-TFPR
PAgE: 1

DATE 28-Jan-1998

TIME: 09:31:59

REPORT NAME: Detall Cost Estimate Sheet 
ockheed Martin Idaho Technologles Co. Rov. 6/90

IOJECT NAME: TRU CLASS A GROUT - INTERIM STORAGE FACILITY IN FPR

INEELICPP OUESTOR: J. K. RAWLINS

\section{DETAILED COST ESTIMATE SHEET}

TYPE OF ESTIMATE: PLANNING

PROJECT NO.: 2421-TFPR

PREPARED BY: R. D. ADAMS
PAGE $\| \mathbf{2}$

DATE 28-Jan-1998

TIME: 09:31:59

AEPORT NAME: Detail Cost Estimate Shoet

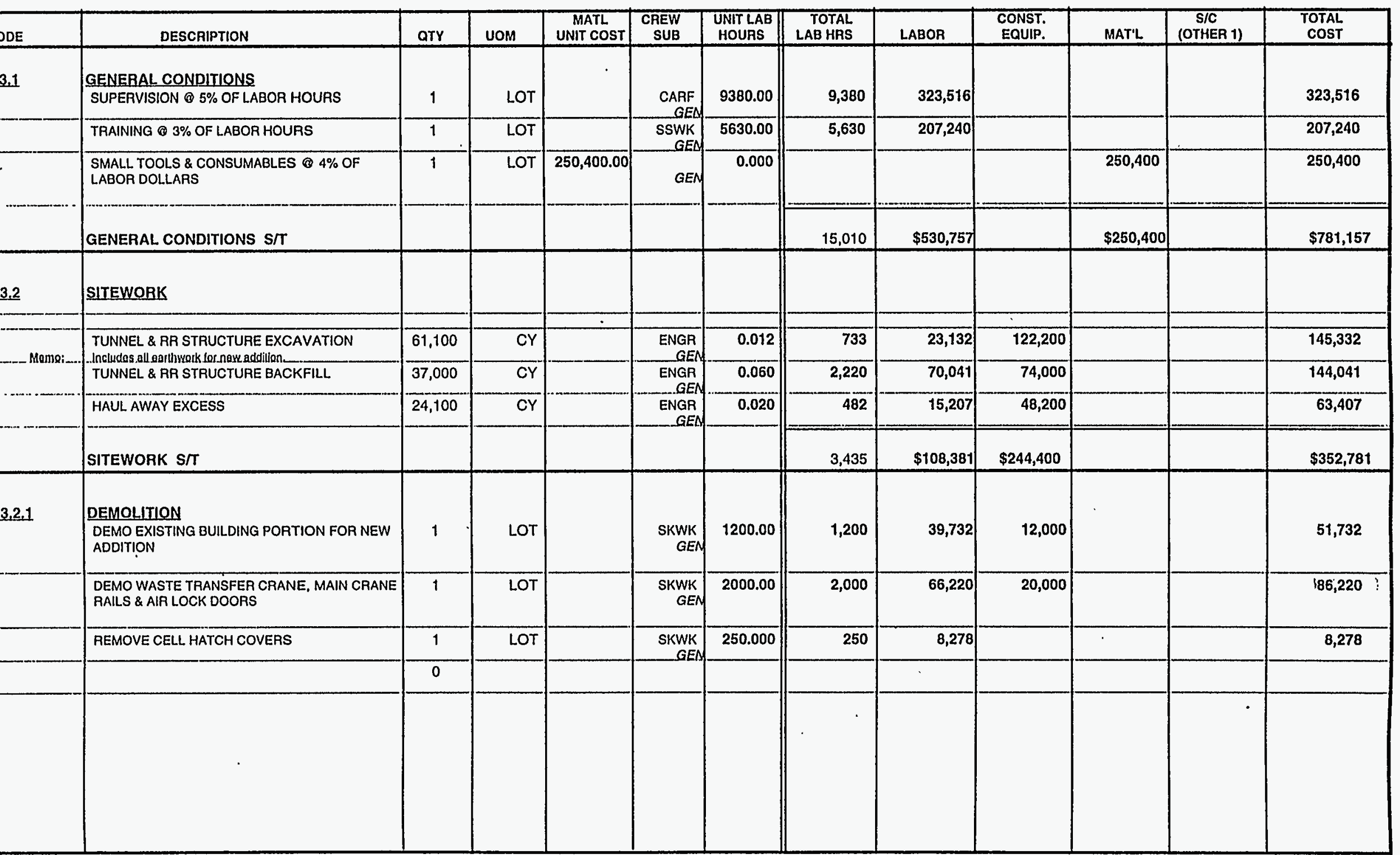


Lockheed Martin Idaho Technologles Co. Pev. 6/96

ROJECT NAME: TRU CLASS A GROUT - INTERIM STORAGE

FACILITY IN FPR

OCATION 1: INEELICPP

IEQUESTOR: J. K. RAWLINS
DETAILED COST ESTIMATE SHEET

TYPE of estimate: PLANNING

PROJECT NO: 2421-TFPR

PREPARED BY: R. D. ADAMS
PAge " 3

DATE 28-Jan-1998

TIME: 09:31:59

REPORT NAME: Detail Cost Estimate Sheet

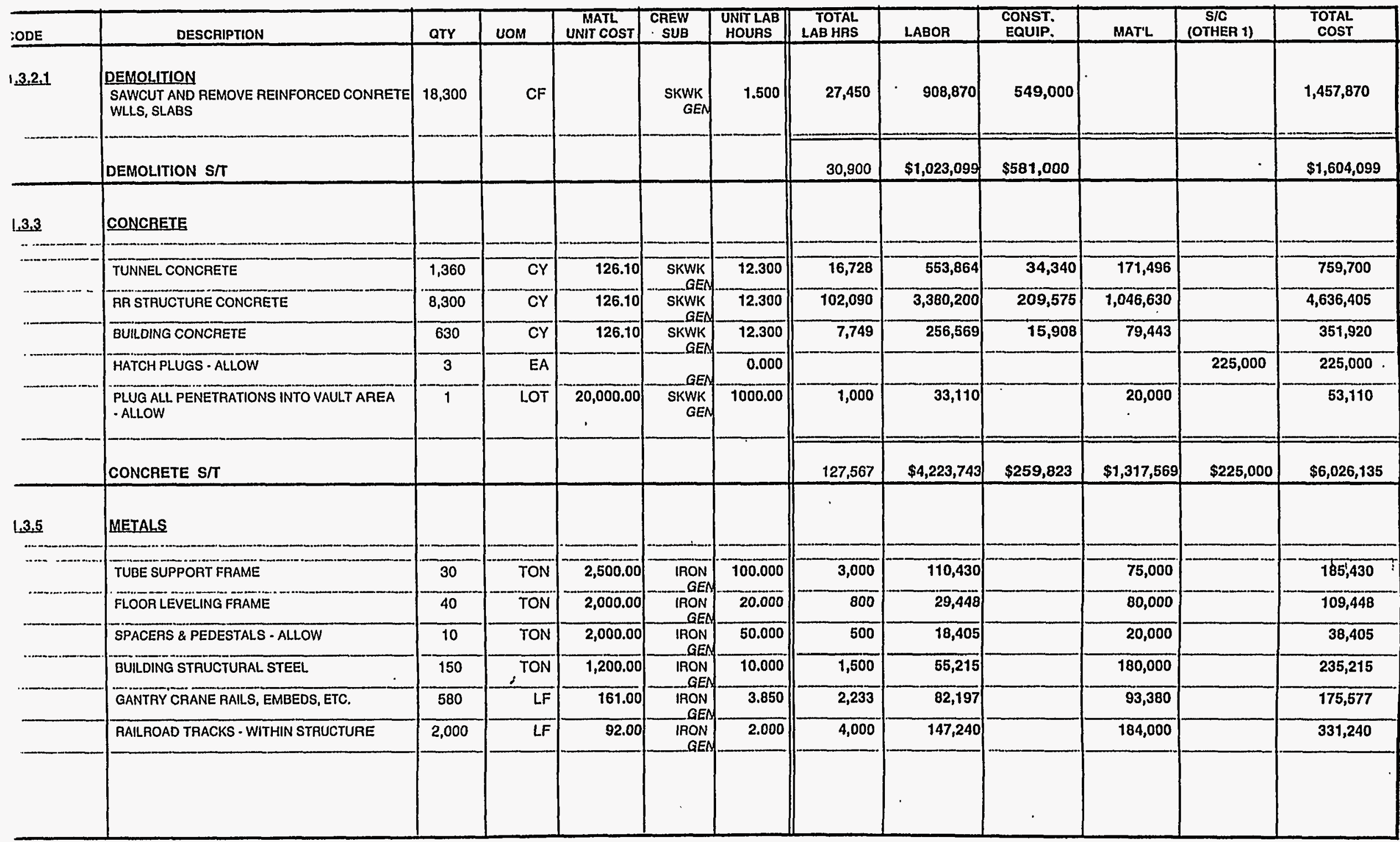


Lockheed Martin Idaho Technologies Co.

Rov. 6/96
PROJECT NAME: TRU CLASS A GROUT - INTERIM STORAGE FACILITY IN FPR

LOCATION :: INEELICPP

REQUESTOR: J. K. RAWLINS

\section{DETAILED COST ESTIMATE SHEET}

TYPE OF ESTIMATE: PLANNING

PAOJECT NO: 2421-TFPP

PREPARED BY: R. D. ADAMS
PAGE 5

DATE 28-Jan-1998

TIME: 09:31:59

REPORT NAME: Detall Cost Estimate Sheet

\begin{tabular}{|c|c|c|c|c|c|c|c|c|c|c|c|c|}
\hline CODE & DESCRIPTION & aTY & UOM & $\begin{array}{l}\text { MATL } \\
\text { UNIT COST } \\
\end{array}$ & $\begin{array}{l}\text { CREW } \\
\text { SUB } \\
\end{array}$ & $\begin{array}{l}\text { UNIT LAB } \\
\text { HOURS }\end{array}$ & $\begin{array}{l}\text { TOTAL } \\
\text { LAB HAS } \\
\end{array}$ & LABOR & $\begin{array}{l}\text { CONST. } \\
\text { EQUIP. }\end{array}$ & MAT'L & $\begin{array}{c}\text { S/C } \\
\text { (OTHER 1) } \\
\end{array}$ & $\begin{array}{l}\text { TOTAL } \\
\text { COST }\end{array}$ \\
\hline \multirow[t]{4}{*}{$1,3,13$} & $\begin{array}{l}\text { SPECIAL CONSTRUCTION } \\
\text { SUPPORT BUILDING }\end{array}$ & 3,600 & SF & & GEA $\mathrm{A}+\mathrm{C}+\mathrm{C}$ & 0.000 & & & & & 540,000 & 540,000 \\
\hline & VAULT TUBE ASSEMBLIES & 50 & $\overline{E A}$ & $25,000,00$ & IRON & 100.000 & $\overline{5,000}$ & 184,050 & & $1,250,000$ & & $1,434,050$ \\
\hline & $\begin{array}{l}\text { BRING EXISTING BUILDING UP TO LIFE } \\
\text { SAFETY CODES - ALLOW }\end{array}$ & 1 & LOT & & GEN & $\overline{0.000}$ & & & - & & $20,000,000$ & $20,000,000$ \\
\hline & SPECIAL CONSTRUCTION S/T & & & & & & 5,000 & $\$ 184,050$ & $\therefore$ & $\$ 1,250,000$ & $\$ 20,540,000$ & $\$ 21,974,050$ \\
\hline \multirow[t]{6}{*}{1.3 .14} & CONVEYING SYSTEMS & & & & & & & & & & & \\
\hline & GANTRY GRANF & 1 & FAl & 2500000000 & & 1000.00 & 1000 & 33.110 & & 2500000 & & 2533.110 \\
\hline & TRANSFER CART IN TUNNEL & 1 & EA & & $\begin{array}{l}\text { GEN } \\
\text { SKWK }\end{array}$ & 500.000 & 500 & 16,555 & & 300,000 & & 316,555 \\
\hline & 5 TON DECONTAMINATABLE BRIDGE CRANE & 2 & $\mathrm{EA}$ & $250,000.00$ & SKWEN & 300.000 & 600 & 19,866 & & 500,000 & & 519,866 \\
\hline & CASK MANUVERING HYDRAULIC PLATFORM & $\overline{1}$ & $\overline{E A}$ & $\overline{1,000,000.00}$ & SKWK & 1000.00 & 1,000 & 33,110 & & $1,000,000$ & & $1,033,110$ \\
\hline & CONVEYING SYSTEMS $S / T$ & & & & & & 3,100 & $\$ 102,641$ & & $\$ 4,300,000$ & & $\$ 4,402,641$ \\
\hline \multirow[t]{6}{*}{1.3 .15} & MECHANICAL & & & & & & & & & . & & \\
\hline & HCO OIOU & 1 & $10 \mathrm{~T}$ & & & 0.000 & & & & & 75.000 & 75000 \\
\hline & MISC. PIPING FOR NEW ADDITION-ALLOW & 1 & LOI & & GEN & & & & & & & \\
\hline & FIRE PROTECTION- NEW ADDITION & 5,600 & $\mathrm{SF}$ & & GEN & 0.000 & & & & & 19,600 & 19,600 \\
\hline & HVAC - NEW ADDITION & 5,600 & $\mathrm{SF}$ & 11.20 & $\begin{array}{c}\text { SHEE } \\
\text { GEN }\end{array}$ & 0.180 & 1,008 & 34,937 & & 62,720 & & 97,657 \\
\hline & MECHANICAL SIT & & & & & & 1,008 & $\$ 34,937$ & & $\$ 62,720$ & $\$ 94,600$ & $\$ 192,257$ \\
\hline 1.3 .16 & ELECTRICAL & & & & & & & & & & & \\
\hline & & & & & & & & & & & & \\
\hline & & & & & & & & & & & & \\
\hline
\end{tabular}


Lockheed Martin Idaho Technologies Co. Rov. 6/96

ROCT NAME: TRU CLASS A GROUT - INTERIM STORAGE FACILITY IN FPR

OCATION 1: INEEL/CPP

IEQUESTOR: J. K. RAWLINS
DETAILED COST ESTIMATE SHEET

TYPE OF ESTIMATE: PLANNING

PROJECT NO.: 2421-TFPR

PREPARED BY: R. D. ADAMS
PAGE \# 6

DATE 28-Jan-1998

TIME: 09:31:59

REPORT NAME: Detall Cost EstImate Sheet

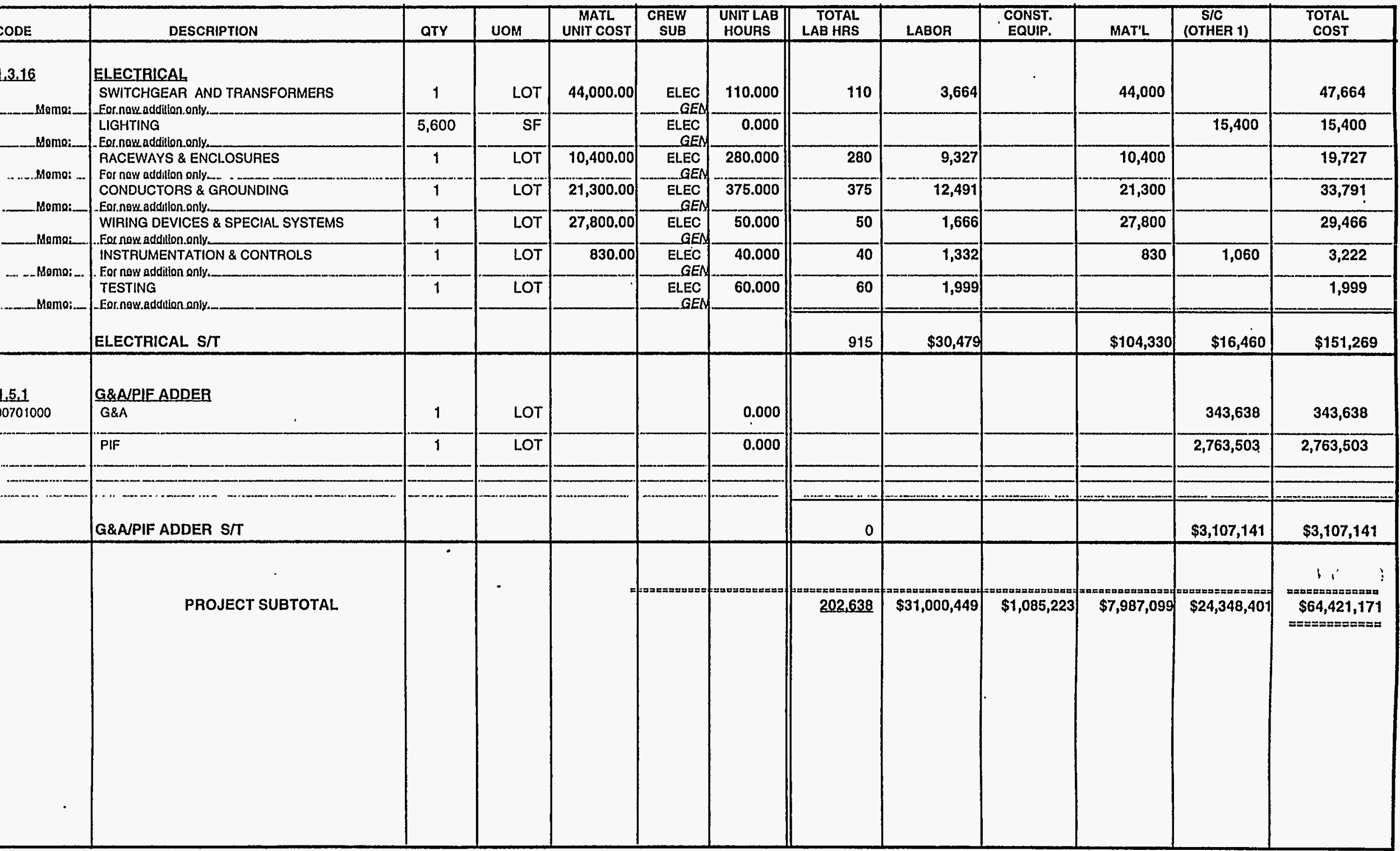


Lockheed Martin Idaho Technologies Co.

PROJECT NAME: TRU CLASS A GROUT - INTERIM STORAGE FACILITY IN FPR - UNESCALATED

LOCATON 1: INEELICPP

REOUESTOR: J. K. RAWLINS
CONTINGENCY ANALYSIS

TYPE OF ESTIMATE:
PROJECT NO: PREPARED BY:
PLANNING

2421-TFPR R. D. ADAMS
- DATE: 28-Jan-1998

- TME: 09:33:30

REPORT NAME: Contingency Analysis

\begin{tabular}{|c|c|c|c|c|c|c|c|c|c|c|c|}
\hline \multicolumn{9}{|c|}{ PROBABLE \% VARIATION } & \multicolumn{2}{|c|}{$\begin{array}{l}\text { PROJECT } \\
\text { CONTINGENCY }\end{array}$} & \multirow{3}{*}{$\begin{array}{l}\text { SUMMARY } \\
\begin{array}{l}\text { Total Cost } \\
\text { by Element }\end{array}\end{array}$} \\
\hline \multirow[t]{2}{*}{$\begin{array}{l}\text { WBS } \\
\text { Element }\end{array}$} & \multirow[t]{2}{*}{ Cost Estimate Element } & \multirow{2}{*}{$\begin{array}{c}\text { Total Cost w/o } \\
\text { Contingency }\end{array}$} & \multirow[t]{2}{*}{$\begin{array}{c}\text { \% Total } \\
\text { Cost }\end{array}$} & \multicolumn{2}{|c|}{$\begin{array}{l}\text { Prob. \% Var. } \\
\text { From Est. }\end{array}$} & \multicolumn{2}{|c|}{ Wt. \% of Prob. } & \multirow[t]{2}{*}{ Contingency } & \multirow[t]{2}{*}{$\%$} & \multirow[t]{2}{*}{ Cost } & \\
\hline & & & & - & + & - & + & & & & \\
\hline 1.1 .3 & DESIGN ENGINEERING TIRLEI\& $\|$ & $9,882,000$ & 12.80 & 20 & 35 & 2.56 & 4.48 & $3.775 \%$ & $16.57 \%$ & $2,913,655$ & $12,795,655$ \\
\hline 1.1 .2 & TIILE III INSPECTION & $2,470,000$ & 3.20 & 20 & 30 & 0.64 & 0.96 & $0.800 \%$ & $3.51 \%$ & 617,175 & $3,087,175$ \\
\hline 1.2 .1 & PROJECT MANAGEMENT & $6,917,000$ & 8.96 & 20 & 35 & 1.79 & 3.14 & $2.643 \%$ & $11.60 \%$ & $2,039,440$ & $8,956,440$ \\
\hline 1.2 .2 & CONSTRUCTION MANAGEMENT & $4,940,800$ & 6.40 & 20 & 30 & 1.28 & 1.92 & $1.600 \%$ & $7.02 \%$ & $1,234,549$ & $6,175,349$ \\
\hline 1.3 .1 & GENERAL CONDMONS & $1,299,960$ & 1.68 & 20 & 35 & 0.34 & 0.59 & $0.497 \%$ & $2.18 \%$ & 383,286 & $1,683,246$ \\
\hline 1.3 .2 & SITEWORK & $2,797,124$ & 3.62 & 20 & 40 & 0.72 & 1.45 & $1.232 \%$ & $5.41 \%$ & 950,521 & $3,747,645$ \\
\hline 1.3 .3 & CONCRETE & $9,782,042$ & 12.67 & 20 & 30 & 253 & 3.80 & $3.157 \%$ & $13.90 \%$ & $2,444,222$ & $12,226,264$ \\
\hline 1.3 .5 & METALS & $1,868,228$ & 2.42 & 20 & 35 & 0.48 & 0.85 & $0.714 \%$ & $3.13 \%$ & 550,837 & $2,419,065$ \\
\hline 1.3 .7 & THERMAL \& MOISTURE PROTECTION & 230,234 & 0.30 & 20 & 30 & 0.06 & 0.09 & $0.075 \%$ & $0.33 \%$ & 57,528 & 287,762 \\
\hline 1.3 .8 & DOORS \& WINDOWS & 64,695 & 0.08 & 20 & 20 & 0.02 & 0.02 & $0.013 \%$ & $0.06 \%$ & 10,346 & 75,041 \\
\hline 1.3 .9 & FINISHES & 316,869 & 0.41 & 20 & 35 & 0.08 & 0.14 & $0.121 \%$ & $0.53 \%$ & 93,427 & 410,296 \\
\hline 1.3 .13 & SPECIAL CONSTRUCTION & $25,385,871$ & 32.88 & 20 & 20 & 6.58 & 6.58 & $5.260 \%$ & $23.09 \%$ & $4,059,600$ & $29,445,471$ \\
\hline 1.3 .14 & CONVEYINE SYSTEMS & $7,156,751$ & 9.27 & 20 & 25 & 1.85 & 2.32 & $1.900 \%$ & $8.34 \%$ & $1,466,361$ & $8,623,112$ \\
\hline 1.3 .15 & MECHANICAL & 266,810 & 0.35 & 20 & 30 & 0.07 & 0.10 & $0.086 \%$ & $0.38 \%$ & 66,667 & 333,477 \\
\hline 1.3 .16 & ELECTRICAL & 239,215 & 0.31 & 20 & 25 & 0.06 & 0.08 & $0.064 \%$ & $0.28 \%$ & 49,013 & 288,228 \\
\hline 1.5 .1 & G\&APIF ADDER & $3,107,141$ & 4.02 & 5 & 20 & 0.20 & 0.80 & $0.704 \%$ & $3.09 \%$ & 543,463 & $3,650,604$ \\
\hline \multirow[t]{2}{*}{1.5 .2} & PROCUREMENT FEES & 494,078 & 0.64 & 20 & 25 & 0.13 & 0.16 & $0.131 \%$ & $0.58 \%$ & 101,233 & 595,311 \\
\hline & ESCALATION & 0 & 0.00 & 20 & 25 & 0.00 & 0.00 & $0.000 \%$ & $0.00 \%$ & $(741)$ & (141) \\
\hline & SUBTOTAL & $77,218,818$ & 100.00 & & & & & $22.780 \%$ & & & \\
\hline & CALCULATED CONTINGENCY & $17,590,590$ & & & & & & & & & \\
\hline & RESULTANT TEC & $94,809,408$ & & & & & & & & & \\
\hline & ROUNDED TEC & $94,800,000$ & & & & & & & & & \\
\hline & PROJECT CONTINGENCY & $17,581,182$ & & & & & & $22.77 \%$ & & & \\
\hline & MANAGEMENT RESERVE & $5,300,902$ & & & & & & & & & \\
\hline & CONTINGENCY & 12.280 .280 & & & & & & & & $\dot{-}$ & \\
\hline & TOTALESTIMATED COST & $94,800,000$ & & & & & & & & $17,581,182$ & $94,800,000$ \\
\hline
\end{tabular}

\section{CONFIDENCE LEVEL AND ASSUMED RISKS:}

The Lockheed Idaho Technologies Co. Cost Estimate Contingency Analysis Model is based on the applied contingency and the assumptions upon which the estimate was predicated. The model is applied with a suggested risk level of $18 \%$ and a level of confidence of $90 \%$ the estimate will fall within the bid range. The Contingency Analysis is based on a weighted average to provide a

$90 \%$ probability of underrun and a $10 \%$ probability of overrun.
CONTINGENCY ANALYSIS GUIDE BY TYPE OF ESTIMATE

Guidelines established by DOE/FM 50, Cost Estimating Guide, Vol. 6 ,

Cost Guide, and as presented in the INEL Cost Estimating Guide.

PLANNING $20 \%-30 \%$

Experimental/Special Conditions...........Up to 50\%

Conceptual $15 \%-25 \%$

Experimental/Special Conditions............Up to $40 \%$

TITLE!

TITLE :

TITLE II/AFC
$10 \%-20 \%$

Market Conditions 
Lockheed Martin Idaho Technologies Co. PROIECT NAME: TRU CLASS A GROUT - INTERIM STORAGE

LOCATON 1: FACILITY IN FPR - ESCALATED

REQUESTOR:
CONTINGENCY ANALYSIS

TYPE OF ESTIMATE: PROJECTNO: PREPARED BY:
PLANNING

R. D. ADAMS
- DATE: 28-Jan-1998

- TIME: 09:36:28

REPORT NAME: Contingency Analysis

\begin{tabular}{|c|c|c|c|c|c|c|c|c|c|c|c|}
\hline \multicolumn{9}{|c|}{ PROBABLE \% VARIATION } & \multicolumn{2}{|c|}{$\begin{array}{c}\text { PROJECT } \\
\text { CONTINGENCY }\end{array}$} & \multirow{3}{*}{$\begin{array}{l}\text { SUMMARY } \\
\begin{array}{l}\text { Total Cost } \\
\text { by Element }\end{array}\end{array}$} \\
\hline \multirow[t]{2}{*}{$\begin{array}{l}\text { WBS } \\
\text { Element }\end{array}$} & \multirow{2}{*}{ Cost Estimate Element } & \multirow{2}{*}{$\begin{array}{l}\text { Total Cost w/o } \\
\text { Contingency }\end{array}$} & \multirow[t]{2}{*}{$\begin{array}{c}\text { \% Total } \\
\text { Cost }\end{array}$} & \multicolumn{2}{|c|}{$\begin{array}{c}\text { Prob. \% Var. } \\
\text { From Est. }\end{array}$} & \multicolumn{2}{|c|}{ Wt. \% of Prob. } & \multirow{2}{*}{ Contingency } & \multirow{2}{*}{$\%$} & \multirow{2}{*}{ Cost } & \\
\hline & & & & - & $t$ & - & 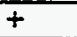 & & & & \\
\hline 1.1 .1 & DESIGN ENGINEERING TITLE I \& $\|$ & $9,882,000$ & 8.86 & 20 & 35 & 1.77 & 3.10 & $2.613 \%$ & $11.47 \%$ & $2,824,795$ & $12,706,795$ \\
\hline 1.12 & TIILE III INSPECTION & $2,470,000$ & 2.21 & 20 & 30 & 0.44 & 0.66 & $0.553 \%$ & $2.43 \%$ & 598,352 & $3,068,352$ \\
\hline 1.2 .1 & PROJECT MANAGEMENT & $6,917,000$ & 6.20 & 20 & 35 & 1.24 & 2.17 & $1.829 \%$ & $8.03 \%$ & $1,977,242$ & $8,894,242$ \\
\hline 1.2 .2 & CONSTRUCTION MANAGEMENT & $4,940,800$ & 4.43 & 20 & 30 & 0.89 & 1.33 & $1.107 \%$ & $4.86 \%$ & $1,196,899$ & $6,137,699$ \\
\hline 1.3.1 & GENERAL CONOITIONS & $1,299,960$ & 1.17 & 20 & 35 & 0.23 & 0.41 & $0.344 \%$ & $1.51 \%$ & 371,597 & $1,671,557$ \\
\hline 1.3 .2 & SITEWORK & $2,797,124$ & 2.51 & 20 & 40 & 0.50 & 1.00 & $0.852 \%$ & $3.74 \%$ & 921,533 & $3,718,657$ \\
\hline 1.3 .3 & CONCRETE & $9,782,042$ & 8.77 & 20 & 30 & 1.75 & 2.63 & $2.192 \%$ & $9.62 \%$ & $2,369,680$ & $12,151,722$ \\
\hline 1.3 .5 & METALS & $1,868,228$ & 1.67 & 20 & 35 & 0.33 & 0.59 & $0.494 \%$ & $2.17 \%$ & 534,038 & $2,402,266$ \\
\hline 1.3 .7 & THERMAL \& MOISTURE PROTECTON & 230,234 & 0.21 & 20 & 30 & 0.04 & 0.06 & $0.052 \%$ & $0.23 \%$ & 55,774 & 286,008 \\
\hline 1.3 .8 & DOORS \& WINDOWS & 64,695 & 0.06 & 20 & 20 & 0.01 & 0.01 & $0.009 \%$ & $0.04 \%$ & 10,030 & 74,725 \\
\hline 1.3 .9 & FINISHES & 316,869 & 0.28 & 20 & 35 & 0.06 & 0.10 & $0.084 \%$ & $0.37 \%$ & 90,578 & 407,447 \\
\hline 1.3 .13 & SPECIAL CONSTRUCTION & $25,385,871$ & 22.75 & 20 & 20 & 4.55 & 4.55 & $3.540 \%$ & $15.98 \%$ & $3,935,792$ & $29,321,663$ \\
\hline 1.3 .14 & CONVEYING SYSTEMS & $7,156,751$ & 6.41 & 20 & 25 & 1.28 & 1.60 & $1.315 \%$ & $5.77 \%$ & $1,421,641$ & $8,578,392$ \\
\hline 1.3 .15 & MECHANICAL & 266,810 & 0.24 & 20 & 30 & 0.05 & 0.07 & $0.060 \%$ & $0.26 \%$ & 64,634 & 331,444 \\
\hline 1.3 .16 & ELECTRICAL & 239,215 & 0.21 & 20 & 25 & 0.04 & 0.05 & $0.044 \%$ & $0.19 \%$ & 47,518 & 286,733 \\
\hline 1.5 .1 & G\&APIF ADDER & $3,107,141$ & 2.78 & 5 & 20 & 0.14 & 0.56 & $0.487 \%$ & $2.14 \%$ & 526,889 & $3,634,030$ \\
\hline \multirow[t]{2}{*}{1.5 .2} & PROCUREMENT FEES & 494,078 & 0.44 & 20 & 25 & 0.09 & 0.11 & $0.091 \%$ & $0.40 \%$ & 98,145 & 592,223 \\
\hline & ESCALATION & $34,353,187$ & 30.79 & 20 & 25 & 6.16 & 7.70 & $6.312 \%$ & $27.71 \%$ & $7,582.858$ & $41,936,045$ \\
\hline & SUBTOTAL & $111,572,005$ & 100.00 & & & & & $22.078 \%$ & & & \\
\hline & CALCULATED CONTINGENCY & $24,632,993$ & & & & & & & & & \\
\hline & RESULTANT TEC & $136,204,998$ & & & & & & & & & \\
\hline & ROUNDED TEC & $136,200,000$ & & & & & & & & & \\
\hline & PROJECT CONTINGENCY & $24,627,995$ & & & & & & $22.07 \%$ & & & \\
\hline & MANAGEMENT RESERVE & $7,686,308$ & & & & & & & & & \\
\hline & CONTINGENCY & 16.941 .687 & & & & & & & & & \\
\hline & TOTAL ESTIMATED COST & $136,200,000$ & & & & & & & & $24,627,995$ & $136,200,000$ \\
\hline
\end{tabular}

CONFIDENCE LEVEL AND ASSUMED RISKS:

The Lockheed Idaho Technologies Co. Cost Estimate Contingency Analysis Model is based on the applied contingency and the assumptions upon which

the estimate was predicated. The model is applied with a suggested risk level

of $18 \%$ and a level of confidence of $90 \%$ the estimate will fall within the bid range.

The Contingency Analysis is based on a weighted average to provide a

$90 \%$ probability of underrun and a $10 \%$ probability of overrun.
CONTINGENCY ANALYSIS GUIDE BY TYPE OF ESTIMATE

Guidelines established by DOE/FM 50 , Cost Estimating Guide, Vol. 6.

Cost Guide, and as presented in the INEL Cost Estimating Guide.
PLANNING
$20 \%-30 \%$
Experimental/Special Conditions............Up to 50\%
Conceptual
$15 \%-25 \%$
TITLE
TITLE II
TITLE II/AFC
$10 \%-20 \%$
$5 \%-15 \%$
Market Conditions 


\section{G\&AVPIF ADDER CALCULATION SHEET}

PROCUREMENT FEE:

\begin{tabular}{l} 
CONSTRUCTION $=$ \\
GFE $=$ \\
\cline { 2 - 2 }
\end{tabular}

FEE @ 1\%=

$\$ 49,407,799 * 0.01=\quad \$ 494,077.99$

G\&A@ $23 \%$ (with a ceiling of $\$ 500,000$ imposed per year

CONSTRUCTION OR

CEILING $* 2$ YEARS $=\quad \$ 1,000,000$

GFE $=\quad \$ 0$

PROCUREMENT FEE $=\quad \$ 494,078$

Subtotal $\$ 1,494,078$

FEE @ $23 \%=\quad \$ 1,494,078 \cdot 0.23=\quad \$ 343,638$

PIF @ 5.5\%

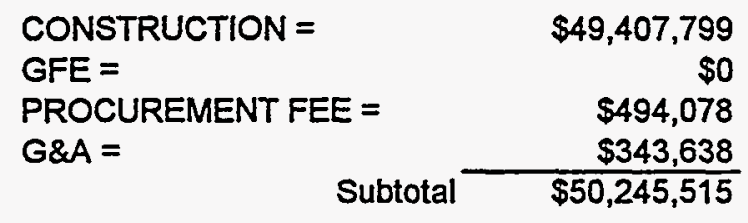

FEE @ $5.5 \%=\quad \$ 50,245,515 * 0.055=\$ 2,763,503$

TOTAL PROCUREMENT FEE:

$\$ 494,078$

TOTAL G\&A FEE:

$\$ 343,638$

TOTAL PIF:

$\$ 2,763,503$ 
Lockheed Martin Idaho Technologies Co. PROJECT NAME: TRU - INTERIM STORAGE FACILITY IN FPR - OPC - ESCALATED LOCATION 1: INEELMCPP REQUESTOR:
COST ESTIMATE SUMMARY

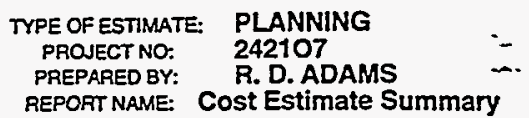

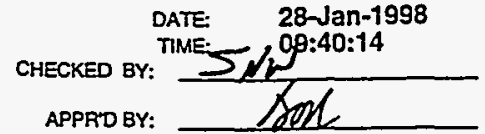

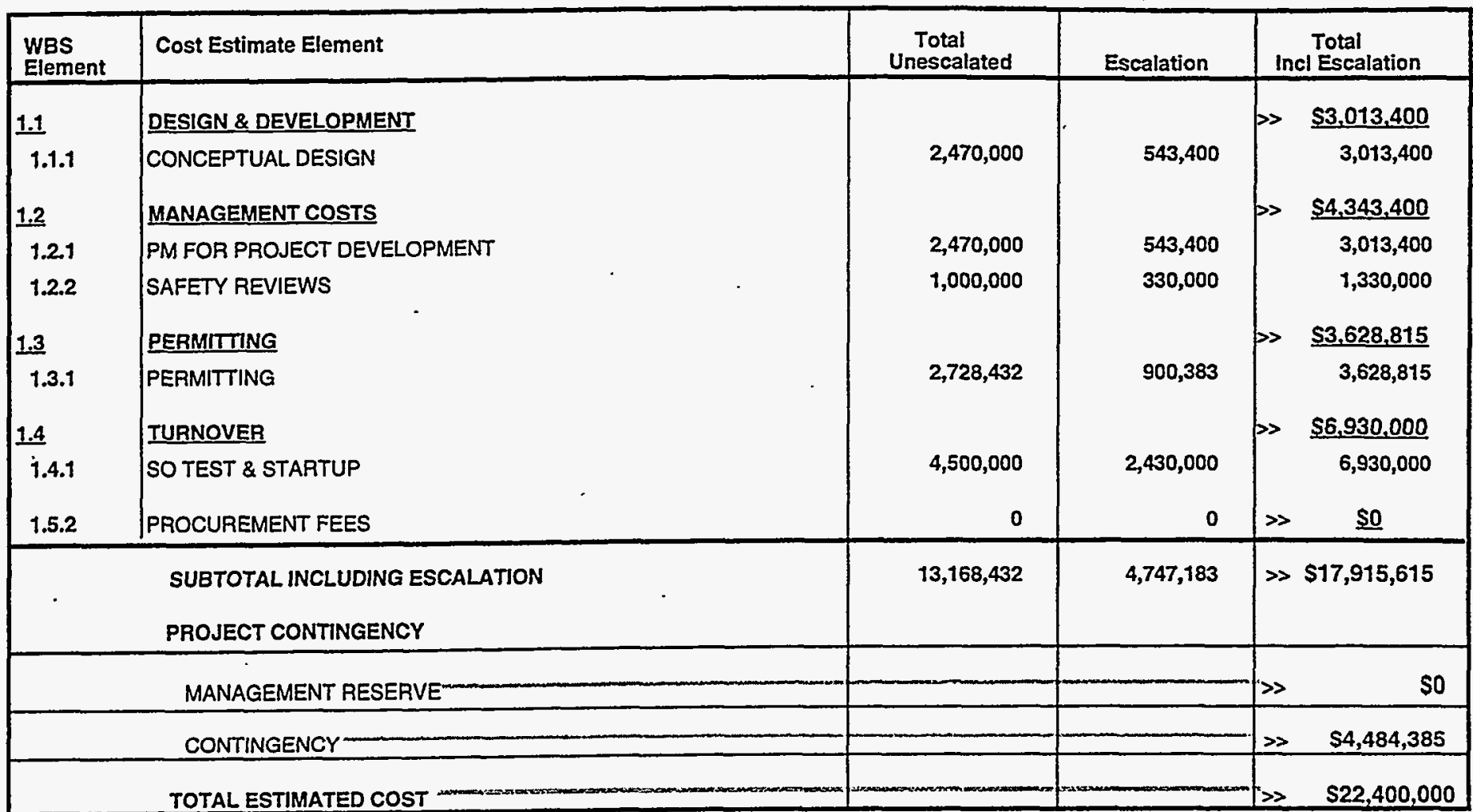

\section{PROJECT COST PARAMETERS}

EDI AS A $\%$ OF CONST + GFE $=29.00 \%$ 
Lockheed Martin Idaho Technologies Co. PROUECT NAME: TRU - INTERIM STORAGE

FACILITY IN FPR - OPC - UNESCALATED

LOCATION 1: INEELICPP

REOUESTOR: J.K. RAWLINS
COST ESTIMATE SUMMARY

TYPE OF ESTIMATE: PLANNING PRONECT NO: 242107 PREPARED BY: R. D. ADAMS REPORT NAME: Cost Estimate Summary
DATE: 28-Jan-1998

CHECKED BY: TME: $S N$ p::38:20

APPRD BY:

\begin{tabular}{|c|c|c|c|c|}
\hline $\begin{array}{l}\text { WBS } \\
\text { Element } \\
\end{array}$ & Cost Estimate Element & $\begin{array}{c}\text { Total } \\
\text { Unescalated } \\
\end{array}$ & Escalation & $\begin{array}{c}\text { Total } \\
\text { Incl Escalation }\end{array}$ \\
\hline 1.1 & DESIGN \& DEVELOPMENT & & & $\gg \quad \$ 2,470,000$. \\
\hline 1.1 .1 & CONCEPTUAL DESIGN & $2,470,000$ & 0 & $2,470,000$ \\
\hline 1.2 & MANAGEMENT COSTS & & & $\gg \quad \$ 3,470,000$ \\
\hline 1.2 .1 & PM FOR PROJECT DEVELOPMENT & $2,470,000$ & 0 & $2,470,000$ \\
\hline 1.2 .2 & SAFETY REVIEWS & $1,000,000$ & 0 & $1,000,000$ \\
\hline 1.3 & PERMITITING & & & $\gg \quad \$ 2,728,432$ \\
\hline 1.3.1 & PERMITTING & $2,728,432$ & 0 & $2,728,432$ \\
\hline 1.4 & TURNOVER & & & $\$ 4,500,000$ \\
\hline 1.4 .1 & SO TEST \& STARTUP & $4,500,000$ & 0 & $4,500,000$ \\
\hline 1.5 .2 & PROCUREMENT FEES & 0 & 0 & șo \\
\hline & SUBTOTAL INCLUDING ESCALATION & $13,168,432$. & 0 & $\gg \$ 13,168,432$ \\
\hline & PROJECT CONTINGENCY & & & \\
\hline & MANAGEMENT RESERVE & & & $\gg$ \\
\hline & CONTINGENCY- & & & $\$ 3,331,568$ \\
\hline & TOTAL ESTIMATED COST & & & $\$ 16,500,000$ \\
\hline
\end{tabular}


Lockheed Martin Idaho Technologies Co.

Rov. 6/96

PROJECT NAME: TRU - INTERIM STORAGE

FACILITY IN FPR - OPC

LOCATION 1: INEELLICPP

REOUESTOR: J. K. RAWLINS

\section{DETAILED COST ESTIMATE SHEET}

TYPE OF ESTIMATE: PLANNING

PROJECT NO: 242107

PREPARED 8Y: R. D. ADAMS
PAGE: 1

DATE 28-Jan-1998

TIME: 09:37:28

Report name: Detall Cost Estimate Sheet

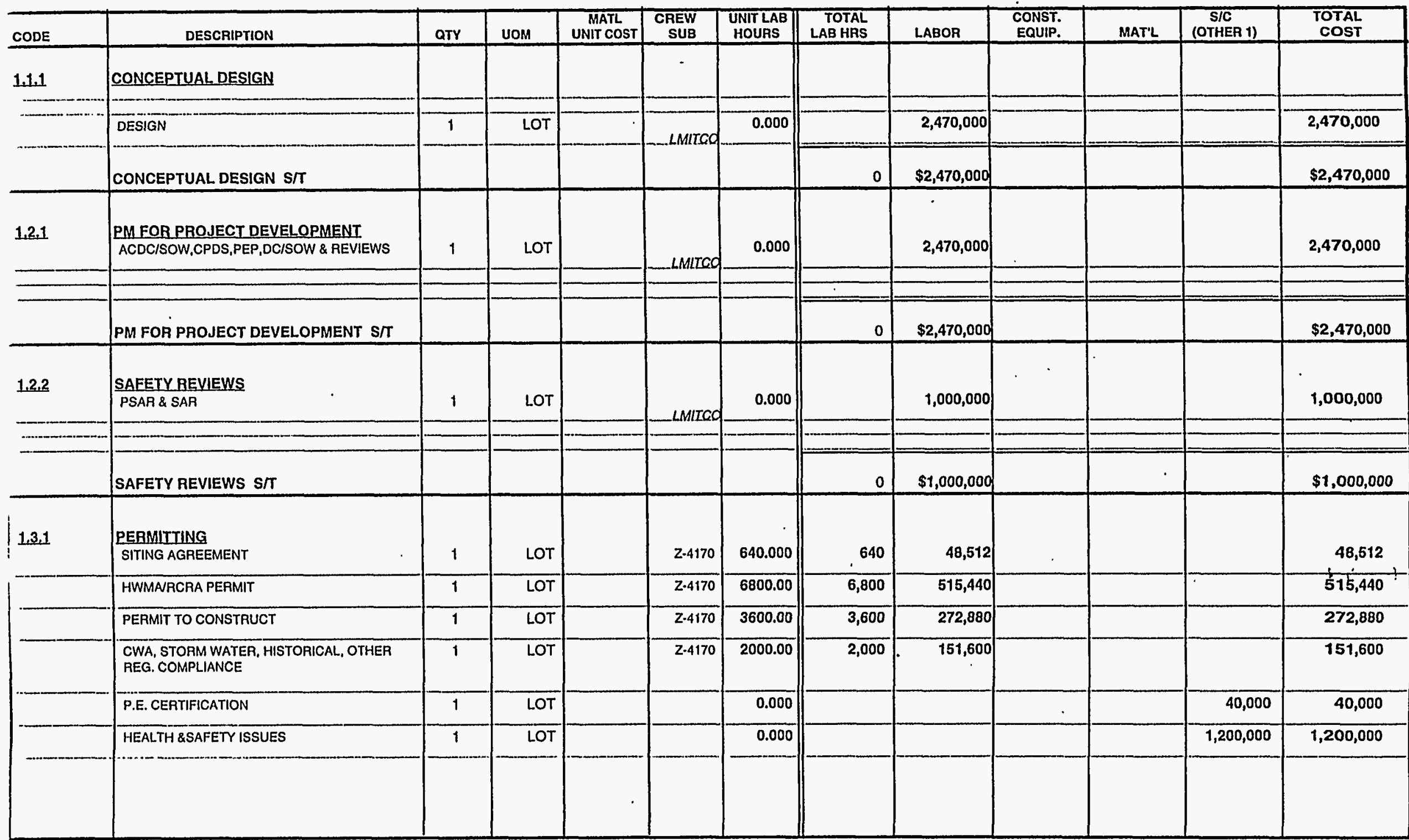


Lockheed Martin Jdaho Technologies Co.

Rev, $6 / 95$

PROEET NAME: TRU - INTERIM STORAGE

FACILITY IN FPR - OPC - UNESCALATED

OCATON 1: INEELACPP

REQUESTOR: J.K. RAWLINS
CONTINGENCY ANALYSIS

TYPE OF ESTIMATE: PROAECT NO: PREPARED BY:
PLANNING
242107

R. D. ADAMS
- DATE: 28-Jan-1998

TME: 09:38:26

REPORT NAME: Contingency Analysis

\begin{tabular}{|c|c|c|c|c|c|c|c|c|c|c|c|}
\hline \multicolumn{9}{|c|}{ PROBABLE \% VARIATION } & \multicolumn{2}{|c|}{$\begin{array}{l}\text { PROJECT } \\
\text { CONTINGENCY }\end{array}$} & \multirow{3}{*}{$\begin{array}{l}\text { SUMMARY } \\
\begin{array}{l}\text { Total Cost } \\
\text { by Element }\end{array}\end{array}$} \\
\hline $\begin{array}{l}\text { WBS } \\
\text { Element }\end{array}$ & \multirow{2}{*}{ Cost Estimate Element } & \multirow{2}{*}{$\begin{array}{c}\text { Total Cost w/o } \\
\text { Contingency }\end{array}$} & \multirow[t]{2}{*}{$\begin{array}{l}\text { \% Total } \\
\text { Cost }\end{array}$} & \multicolumn{2}{|c|}{$\begin{array}{c}\text { Prob. \% Var. } \\
\text { From Est. }\end{array}$} & \multicolumn{2}{|c|}{ Wt. \% of Prob. } & \multirow[t]{2}{*}{ Contingency } & \multirow[t]{2}{*}{$\%$} & \multirow[t]{2}{*}{ Cost } & \\
\hline & & & & $=$ & + & - & + & & & & \\
\hline 1.1 .1 & CONCEPTUAL DESIGN & $2,470,000$ & 18.76 & 20 & 30 & 3.75 & 5.63 & $4.689 \%$ & $18.76 \%$ & 624,902 & $3,094,902$ \\
\hline 1.21 & PM FOR PROJECT DEVELOPMENT & $2,470,000$ & 18.76 & 20 & 30 & 3.75 & 5.63 & $4.689 \%$ & $18.76 \%$ & 624,902 & $3,094,902$ \\
\hline 1.2 .2 & SAFETY REVIEWS & $1,000,000$ & 7.59 & 20 & 30 & 1.52 & 2.28 & $1.898 \%$ & $7.59 \%$ & 252,997 & $1,252,997$ \\
\hline 1.3 .1 & PERMITTING & $2,728,432$ & 20.72 & 20 & 30 & 4.14 & 6.22 & $5.180 \%$ & $20.72 \%$ & 690,284 & $3,418,716$ \\
\hline 1.4 .1 & SO TEST \& STARTUP & $4,500,000$ & 34.17 & 20 & 30 & 6.83 & 10.25 & $8.543 \%$ & $34.17 \%$ & $1,138,485$ & $5,638,485$ \\
\hline \multirow{2}{*}{1.5 .2} & PROCUREMENT FEES & 0 & 0.00 & 5 & 20 & 0.00 & 0.00 & $0.000 \%$ & $0.00 \%$ & o) & 0 \\
\hline & ESCALATION & 0 & 0.00 & 20 & 30 & 0.00 & 0.00 & $0.000 \%$ & $0.00 \%$ & (2) & (2) \\
\hline & SUBTOTAL & $13,168,432$ & 100.00 & & & & & $25.000 \%$ & & & \\
\hline & CALCULATED CONTINGENCY & $3,292,108$ & & & & & & & & & \\
\hline & RESULTANT TEC & $16,460,540$ & & & & & & & & & \\
\hline & ROUNDED TEC & $16,500,000$ & & & & & & & & & \\
\hline & PROJECT CONTINGENCY & $3,331,568$ & & & & & & $25.30 \%$ & & & \\
\hline & MANAGEMENT RESERVE & 0 & & & & & & & & & \\
\hline & CONTINGENCY & $3,331,568$ & & & & & & & & & \\
\hline . & TOTAL ESTIMATED COST & $16,500,000$ & & & & & & & & $3,331,568$ & $16,500,000$ \\
\hline
\end{tabular}

CONFIDENCE LEVEL AND ASSUMED RISKS:

The Lockheed Idaho Technologies Co. Cost Estimate Contingency Analysis Model is based on the applied contingency and the assumptions upon which the estimate was predicated. The model is applied with a suggested risk level

of $18 \%$ and a level of confidence of $90 \%$ the estimate will fall within the bid range.

The Contingency Analysis is based on a weighted average to provide a

$90 \%$ probability of underrun and a $10 \%$ probability of overrun.
CONTINGENCY ANALYSIS GUIDE BY TYPE OF ESTIMATE

Guidelines established by DOE/FM 50 . Cost Estimating Guide, Vol. 6 , Cost Guide, and as presented in the INEL Cost Estimating Guide.

PLANNING

Conceptual

TITLE I

TITLE II

TITLE II/AFC
$15 \%-25 \%$ ...Up to $40 \%$ $10 \%=20 \%$

$5 \%-15 \%$

Market Conditions 
Lockheed Martin Idaho Technologies Co.

PROECT NAME: TRU - INTERIM STORAGE

LOCATION 1:

FACILITY IN FPR - OPC - ESCALATED

INEELICPP

REOUESTOR:

\section{CONTINGENCY ANALYSIS}

TYPE OF ESTIMATE: PROUECT NO: PREPARED BY:
PLANNING

242107

R. D. ADAMS $\cdots$

DATE: 28-Jan-1998

- TIME: 09:40:20

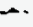

REPORT NAME: Contingency Analysis

\begin{tabular}{|c|c|c|c|c|c|c|c|c|c|c|c|}
\hline \multicolumn{9}{|c|}{ PROBABLE \% VARIATION } & \multicolumn{2}{|c|}{$\begin{array}{c}\text { PROJECT } \\
\text { CONTINGENCY }\end{array}$} & \multirow{3}{*}{$\begin{array}{l}\text { SUMMARY } \\
\text { Total Cost } \\
\text { by Element }\end{array}$} \\
\hline \multirow[t]{2}{*}{$\begin{array}{l}\text { WBS } \\
\text { Element }\end{array}$} & \multirow[t]{2}{*}{ Cost Estimate Element } & \multirow{2}{*}{$\begin{array}{c}\text { Total Cost w/o } \\
\text { Contingency }\end{array}$} & \multirow[t]{2}{*}{$\begin{array}{l}\% \text { Total } \\
\text { Cost }\end{array}$} & \multicolumn{2}{|c|}{$\begin{array}{c}\text { Prob. } \% \text { Var. } \\
\text { From Est. }\end{array}$} & \multicolumn{2}{|c|}{ Wt \% of Prob. } & \multirow[t]{2}{*}{ Contingency } & \multirow[t]{2}{*}{$\%$} & \multirow[t]{2}{*}{ Cost } & \\
\hline & & & & - & + & - & + & & & & \\
\hline 1.1 .1 & CONCEPTUAL DESIGN & $2,470,000$ & 13.79 & 20 & 30 & 2.76 & 4.14 & $3.447 \%$ & $13.79 \%$ & 618,256 & $3,088,256$ \\
\hline 1.2 .1 & PM FOR PROJECT DEVELOPMENT & $2,470,000$ & 13.79 & 20 & 30 & 276 & 4.14 & $3.447 \%$ & $13.79 \%$ & 618,256 & $3,088,256$ \\
\hline 1.2 .2 & SAFETY REVIEWS & $1,000,000$ & 5.58 & 20 & 30 & 1.12 & 1.67 & $1.395 \%$ & $5.58 \%$ & 250,306 & $1,250,306$ \\
\hline 1.3 .1 & PERMITTING & $2,728,432$ & 15.23 & 20 & 30 & 3.05 & 4.57 & $3.807 \%$ & $15.23 \%$ & 682,943 & $3,411,375$ \\
\hline 1.4 .1 & SO TEST \& STARTUP & $4,500,000$ & 25.12 & 20 & 30 & 5.02 & 7.54 & $6.279 \%$ & $25.12 \%$ & $1,126,377$ & $5,626,377$ \\
\hline \multirow[t]{2}{*}{1.5 .2} & PROCUREMENT FEES & 0 & 0.00 & 5 & 20 & 0.00 & 0.00 & $0.000 \%$ & $0.00 \%$ & 0 & 0 \\
\hline & ESCALATION & $4,747,183$ & 26.50 & 20 & 30 & 5.30 & 7.95 & $.6 .624 \%$ & $26.50 \%$ & $1,188.247$ & $5,935,430$ \\
\hline \multicolumn{2}{|r|}{ SUBTOTAL } & $17,915,615$ & 100.00 & & & & & $25.000 \%$ & & & \\
\hline \multicolumn{2}{|r|}{ CALCULATED CONTINGENCY } & $4,478,904$ & & & & & & & & & \\
\hline & RESULTANT TEC & $22,394,519$ & & & & & & & & & \\
\hline & ROUNDED TEC & $22,400,000$ & & & & & & & & & \\
\hline & PROJECT CONTINGENCY & $4,484,385$ & & & & & & $25.03 \%$ & & & \\
\hline & MANAGEMENT RESERVE & 0 & & & & & & & & & \\
\hline & CONTINGENCY & $4,484,385$ & & & & & & & & & \\
\hline & TOTAL ESTIMATED COST & $22,400,000$ & & & & & & & & $4,484,385$ & $22,400,000$ \\
\hline
\end{tabular}

CONFIDENCE LEVEL AND ASSUMED RISKS:

The Lockheed Idaho Technologies Co. Cost Estimate Contingency Analysis Model is based on the applied contingency and the assumptions upon which the estimate was predicated. The model is applied with a suggested risk level

of $18 \%$ and a level of confidence of $90 \%$ the estimate will fall within the bid range.

The Contingency Analysis is based on a weighted average to provide a

$90 \%$ probability of underrun and a $10 \%$ probability of overrun.
CONTINGENCY ANALYSIS GUIDE BY TYPE OF ESTIMATE

Guidelines established by DOE/FM 50, Cost Estimating Guide, Vol. 6, Cost Guide, and as presented in the INEL Cost Estimating Guide. PLANNING Experimental/Special Conditions. Conceptual $15 \%-25 \%$ Experimental/Special Conditions............ Up to $40 \%$ TITLE I

TITLE II

TITLE II/AFC
$10 \%-20 \%$
$5 \%-15 \%$

Market Conditions 


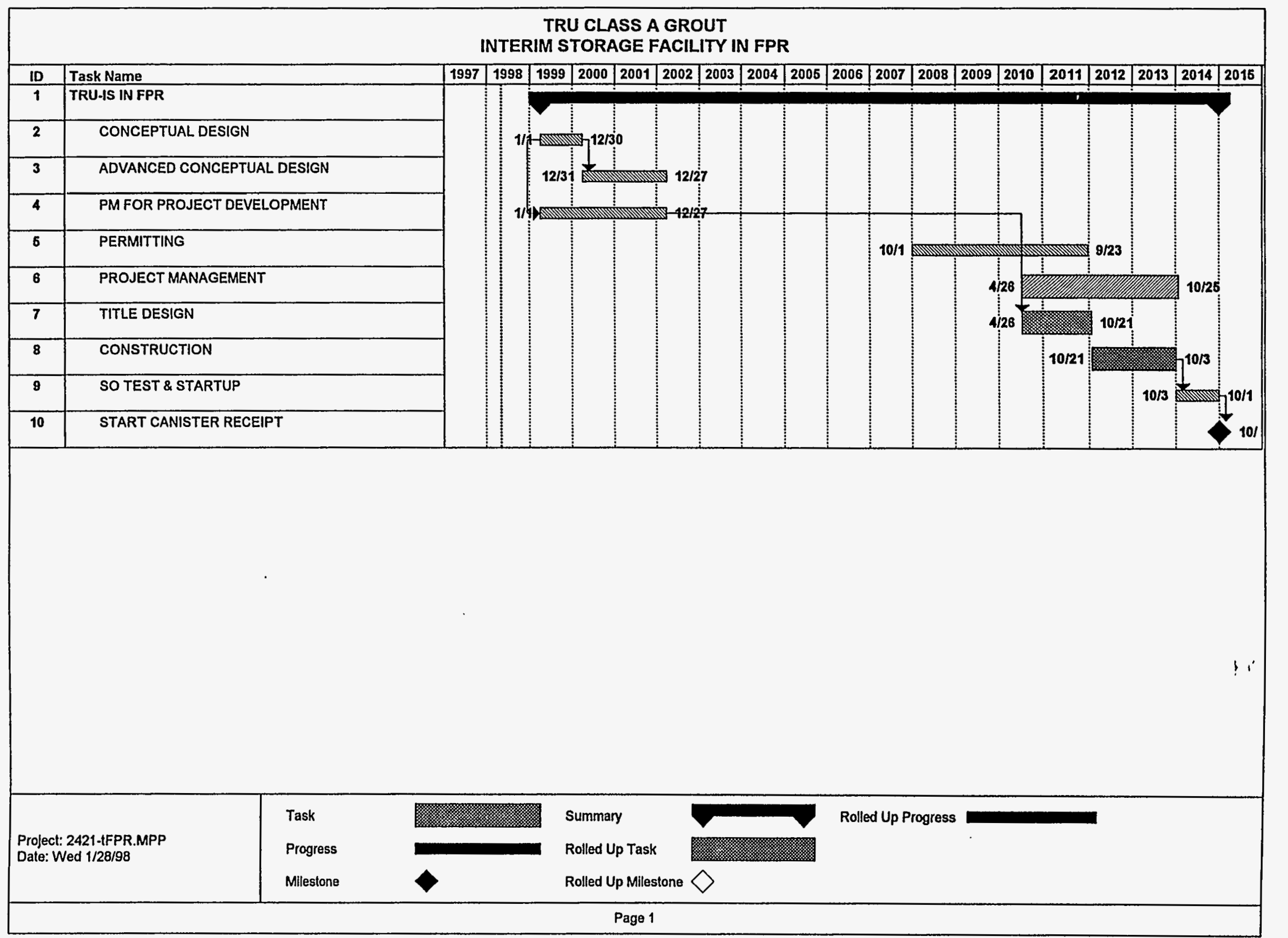


Lockheed Martin Idaho Technologies

(Rev. 6/96)

\section{COST ESTIMATE SUPPORT DATA RECAPITULATION}

Project Title: HLW INTERIM STORAGE FACILITY IN FPR - HWO

Type of Estimate: PROJECT SUPPORT

File No: 2421-FPR
Estimator:RDA

Date:1/28/98

Approved By:

\section{SCOPE OF WORK: Brief description of the proposed project.}

This facility will unload, store in vertical tubes and load out canisters containing High Level Waste (HLW) from the proposed Hot Isokinetic Press Waste Option (HWO).

II. BASIS OF THE ESTIMATE : Drawings, Design Report, Engineers notes, and/or other documentation upon which the estimate is originated.

Draft sketches, a draft EDF, and the SNF Canister Storage Building Final Design Estimate from Fluor Daniel Northwest, Inc. were used as the basis for this estimate.

III. ASSUMPTIONS : Condition statements accepted or supposed true without proof or demonstration. An assumption has a direct impact on total estimated cost.

1. The Interim Storage Facility will be located within 100 feet of the HWO facility.

2. Rock will not be encountered during excavation.

3. The estimates include both present day costs summaries and contingency analysis and escalated sumaries and contingency analysis. The escalated summaries and contingency analysis reflect the attached schedule. All activities are escalated to the appropriate schedule mid-point.

4. FPR will have to be brought up to life safety code as reflected in estimate 93108. It is assumed that the same materials and equipment available in storage for FPR when estimate was done are still available.

5. Most quantities used for this estimate were obtained by preliminary take offs from the draft sketches.

6. The storage tube costs, gantry crane cost and associated rail system costs were used from the Fluor estimate of a facility which is approximately eighty percent complete.

7. All assumptions and information included in the draft "New Interim Storage Facility for Interim storage of $\mathrm{HLW}^{\prime \prime}$ apply to the estimate.

8. Where quantities could not be determined from the draft sketches, factors or assumed quantities were used by the estimator.

9. Since the storage tubes and conveying equipment represent such a large percentage of this facility's cost, lower design, inspection, Project Management and turnover costs are used in the estimate.

10.Sufficient construction craft will be available to support this project.

11. Markups on direct construction costs to get the construction costs Iisted on the summary sheet are as follows:

Iabor indirects, overheads and profit (OH\&P) $50 \%$ of labor

Material \& equipment OH\&P 58 of material and equipment

Subcontract OH\&O

Construction equipment allowance 108 of labor

NOA-1 cuality requirements $30 \%$ of material

DOE/RW/0333P quality requirements 108 of material

Subcontractor procurement 18 of material and equipment

Bond

28 of everything 
5

Lockheed Martin Idaho Technologies

\section{COST ESTIMATE SUPPORT DATA RECAPITULATION (CONTINUATION)}

File No: 2396

Page 2 of 2

\section{ili. ASSUMPTIONS: (Continued)}

12. Only the railroad track within the storage facility is included in this estimate. The remainder of the branch line is included in the infrastructure estimate.

13. All utilities are assumed to be available at the facility wall.

14. The concrete that is removed from the existing FPR structure will be cut into blocks by diamond wire cutting or similar method. It will not be rubblized with the building.

IV. CONTINGENCY GUIDELINE IMPLEMENTATION: The percentage used for contingency as determined by the contingency allowance guidelines can be altered to reflect the type of construction and conditions that may impact the total estimated cost.

A large portion of the estimated costs is based on actual costs from Fluor's SNF Canister Storage Building. The majority of the remaining costs are for standard construction activities. For these reasons, the contingency is lower than would be experienced for a processing type facility at this stage of development.

\section{OTHER COMMENTS/CONCERNS SPECIFIC TO THE ESTIMATE :}

1. All FY' 98 and later projects are to be assessed a Procurement Fee of $1 \%$, a G\&A Fee of $23 \%$, with a $\$ 500,000$ ceiling per year, and a Performance Incentive Fee (PIF) of $5.5 \%$. See the attached G\&A/PIF calculation sheet for the method used to calculate these fees.

2. A procurement fee of one percent of construction was used to cover the operating contractor support to DOE-DD for their contract administration. It is felt that one percent is sufficient for jobs of this size. 
Lockheed Martin Idaho Technologies Co. PROSECT NAME: HIP FACILITY - INTERIM STORAGE FACILITY IN FPR - ESCALATED

LOCATION $1:$ INEELICPP

REQUESTOR
COST ESTIMATE SUMMARY

TYPE OFESTIMATE PLANNING PROIECT NO: 2421-FPR PREPARED BY: R. D. ADAMS REPORT NAME: Cost Estimate Summary

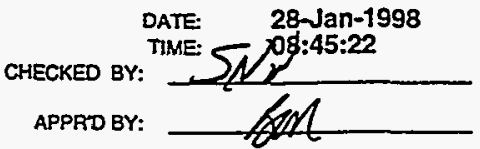

\begin{tabular}{|c|c|c|c|c|}
\hline $\begin{array}{l}\text { WBS } \\
\text { Element }\end{array}$ & Cost Estimate Element & $\begin{array}{c}\text { Total } \\
\text { Unescalated }\end{array}$ & Escalation & $\begin{array}{c}\text { Total } \\
\text { Inci Escalation }\end{array}$ \\
\hline 1.1 & ENGINEERING, DESIGN AND INSPECTION & & & $\$ 32,267,343$ \\
\hline 1.1.1 & DESIGN ENGINEERING TITLE I \& $\|$ & $18,000,000$ & $5,220,000$ & $23,220,000$ \\
\hline 1.1 .2 & TITLE III INSPECTION & $6,603,900$ & $2,443,443$ & $9,047,343$ \\
\hline 1.2 & MANAGEMENT COSTS & & & $\$ 42,687,579$ \\
\hline 1.2.1 & PROJECT MANAGEMENT & $18,491,000$ & $6,102,030$ & $24,593,030$ \\
\hline 1.2 .2 & CONSTRUCTION MANAGEMENT & $13,207,700$ & $4,886,849$ & $18,094,549$ \\
\hline 1.3 & CONSTRUCTION & & & $\gg>\$ 180,945,388^{\circ}$ \\
\hline 1.3.1 & GENERAL CONDITIONS & $3,041,928$ & $1,125,513$ & $4,167,441$ \\
\hline 1.3.2 & SITEWORK & 6,524,508 & $2,414,068$ & $8,938,576$ \\
\hline 1.3.3 & CONCRETE & $10,575,913$ & $3,913,088$ & $14,489,001$ \\
\hline 1.3.5 & METALS & $7,196,529$ & $2,662,716$ & $9,859,245$ \\
\hline 1.3.7 & THERMAL \& MOISTURE PROTECTION & 230,234 & 85,187 & 315,421 \\
\hline 1.3 .8 & DOORS \& WINDOWS & 64,695 & 23,937 & 88,632 \\
\hline 1.3.9 & FINISHES & 611,189 & 226,140 & 837,329 \\
\hline 1.3.13 & SPECIAL CONSTRUCTION & $91,982,003$ & $34,033,341$ & $126,015,344$ \\
\hline 1.3.14 & CONVEYING SYSTEMS & $11,272,944$ & $4,170,989$ & $15,443,933$ \\
\hline 1.3.15 & MECHANICAL & 337,767 & 124,974 & 462,741 \\
\hline 1.3 .16 & ELECTRICAL & 239,215 & 88,510 & 327,725 \\
\hline 1.5 & G\&A/PIF & & & $\$ 10,989,226$ \\
\hline 1.5.1 & G\&AVIF ADDER & $8,021,333$ & $2,967,893$ & $10,989,226$ \\
\hline $\mathbf{1 . 5 . 2}$ & PROCUREMENT FEES & $1,320,769$ & 488,685 & $\gg \$ 1,809,454$ \\
\hline & $\begin{array}{l}\text { SUBTOTAL INCLUDING ESCALATION } \\
\text { PROJECT CONTINGENCY }\end{array}$ & $197,721,627$ & $70,977,363$ & > $\$ 268,698,990$ \\
\hline & MANAGEMENT RESERVE- & & & $\gg \quad \$ 19,374,407$ \\
\hline & CONTINGENCY- & & & $\gg \$ 37,826,603$ \\
\hline & TOTAL ESTIMATED COST & & . & $\gg \$ 325,900,000$ \\
\hline
\end{tabular}

\section{PROJECT COST PARAMETERS}

EDI AS A \% OF CONST. + GFE $=18.00 \%$ CONTINGENCY $=21.29 \%$ 
Lockheed Martin Idaho Technologies Co. PROIECT NAME: HIP FACILITY - INTERIM STORAGE FACILITY IN FPR - UNESCALATED

LOCATION $1:$ RECUESTOR:

INEELICPP

J.K. RAWLINS
COST ESTIMATE SUMMARY

TYPE OF ESTIMATE: PROJECTNO: 2421-FPR PREPARED BY: REPORT

$2421-F P R$ R. D.ADAMS

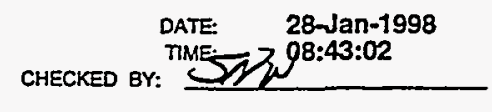

APPRD BY:

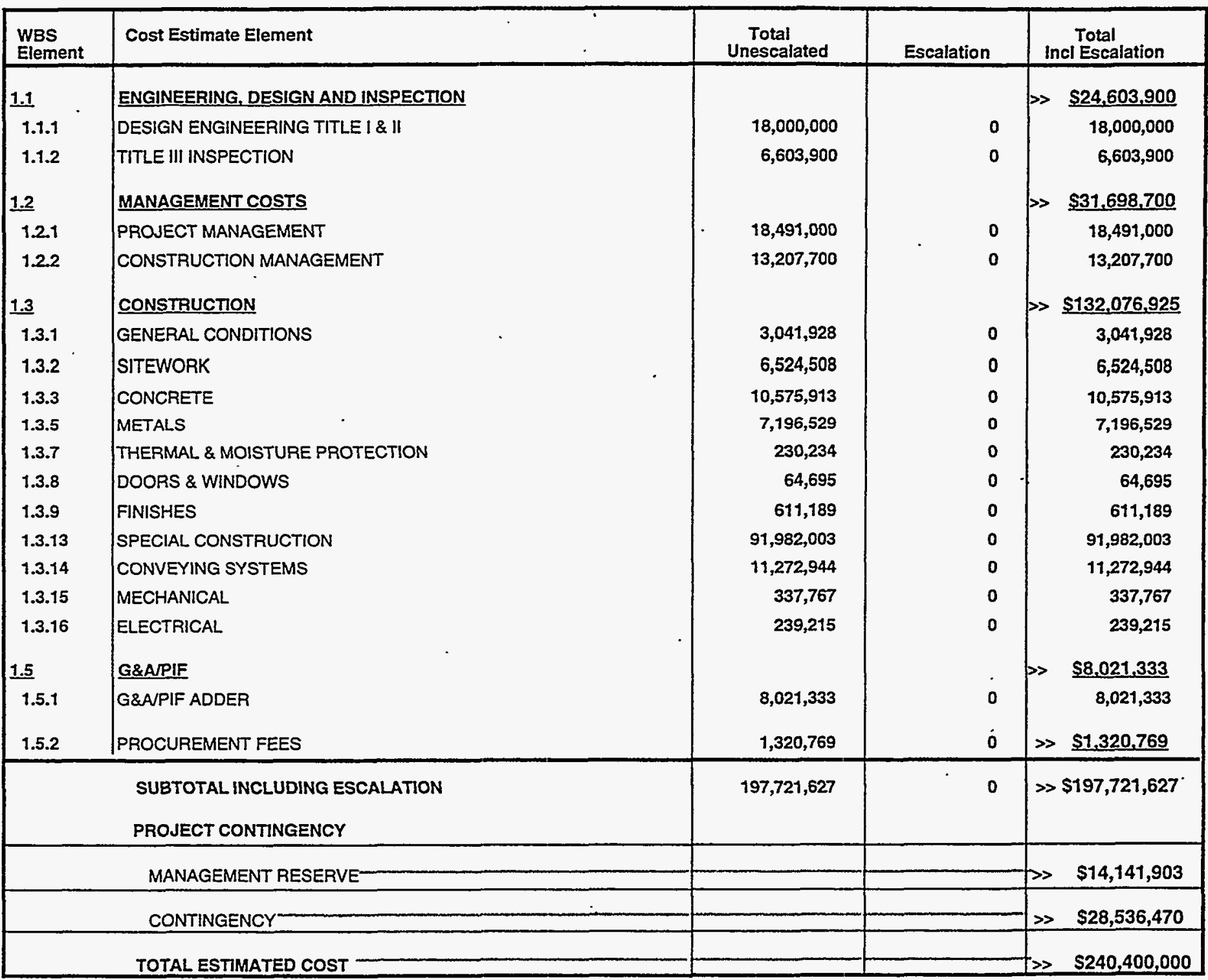


Lockheed Martin Idaho Technologies Co.

PROJECT NAME: HIP FACILITY - INTERIM STORAGE FACILITY IN FPR

LOCATION 1: INEELICPP

REQUESTOR: J. K. RAWLINS
DETAILED COST ESTIMATE SHEET

TYPE OF ESTIMATE: PLANNING

PROJECT NO.: 2421-FPR

PREPARED BY: R. D. ADAMS
PAGE 1

DATE 28-Jan-1998

TIME: 08:41:26 REPORT NAME: Detall Cost Estimate Sheot

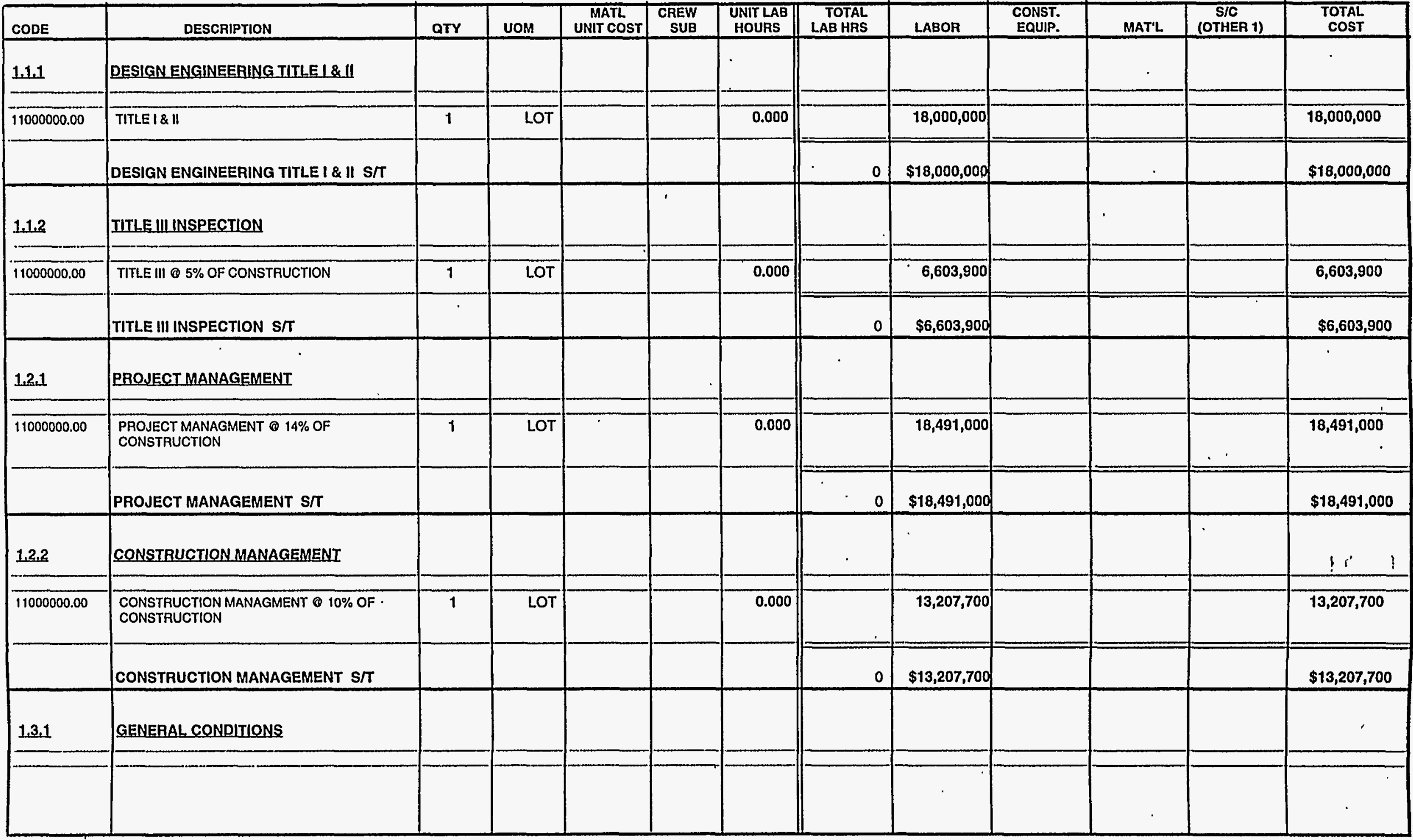


-ockheed Martln Idaho Technologies Co. Rev. 6/96

DIT FACILITY - INTERIM STORAGE

FACILITY IN FPA

JCATION 1: INEELICPP

EQUESTOR: J. K. RAWLINS
DETAILED COST, ESTIMATE SHEET

TYPE OF ESTIMATE: PLANNING

PROJECTNO: 2421-FPR

PREPARED BY: R. D. ADAMS
PAGE " 2

DATE 28-Jan-1998

TIME: 08:41:26

Report NaMe: Detall Cost Estimate Sheet

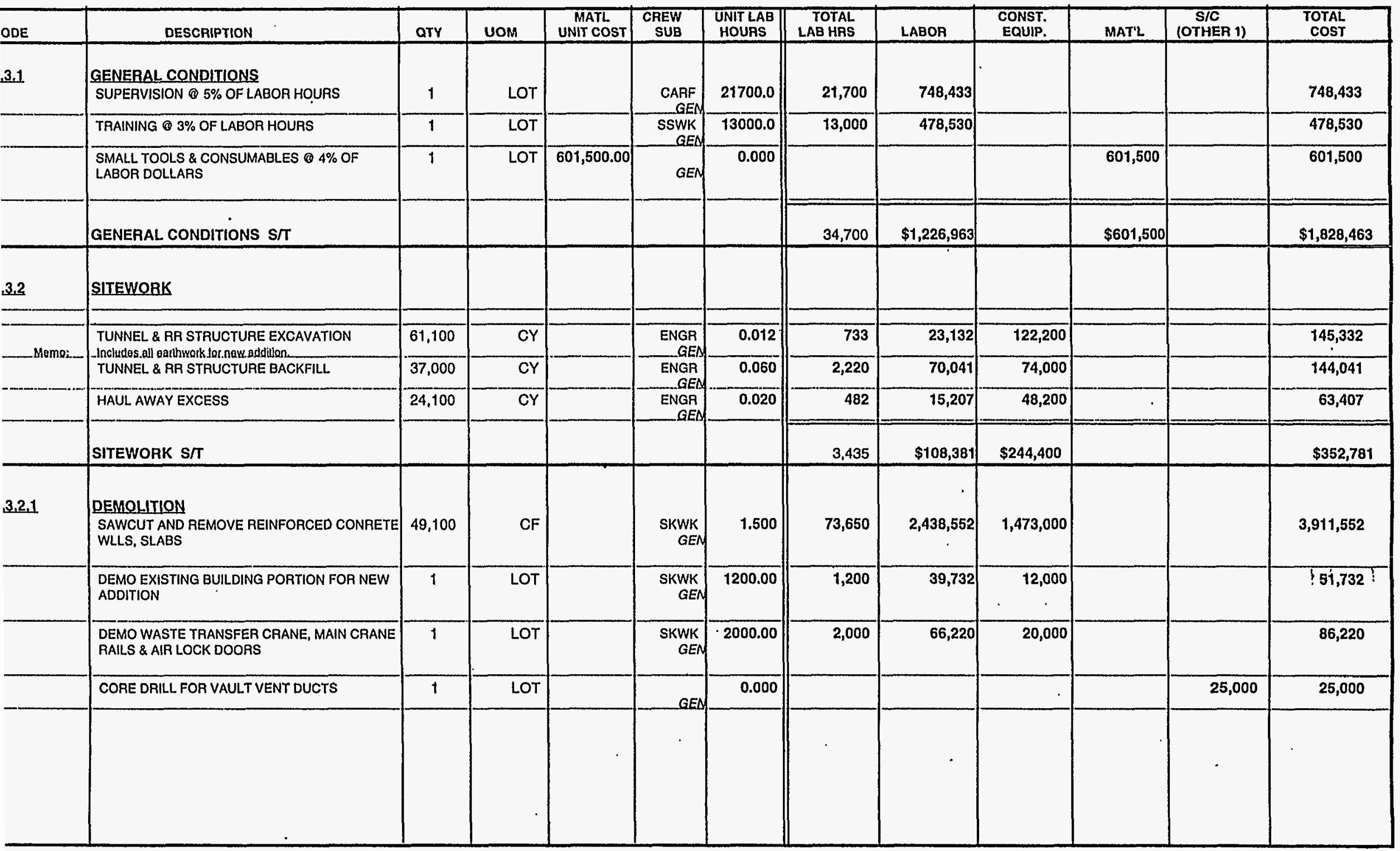


Lockheed Martin Idaho Technologies Co. Rev. 6/96 PROIECT NAME: HIP FACILITY - INTERIM STORAGE FACILITY IN FPR

LOCATION 1: INEEL/CPP REQUESTOR: J. K. RAWLINS

\section{DETAILED COST ESTIMATE SHEET}

TYPE OF ESTIMATE: PLANNING

PROJECT NO.: 2421-FPR

PAEPARED BY: R. D. ADAMS
PAgE " 3

DATE 28-Jan-1998

TIME: 08:41:26

REPORT NAME: Detall Cost Estimate Sheet

\begin{tabular}{|c|c|c|c|c|c|c|c|c|c|c|c|c|}
\hline CODE & DESCRIPTION & QTY & UOM & $\begin{array}{c}\text { MATL } \\
\text { UNIT COST } \\
\end{array}$ & $\begin{array}{r}\text { CAEW } \\
\text { SUB }\end{array}$ & $\begin{array}{l}\text { UNIT LAB } \\
\text { HOURS }\end{array}$ & $\begin{array}{r}\text { TOTAL } \\
\text { LAB HAS } \\
\end{array}$ & LABOR & $\begin{array}{l}\text { CONST. } \\
\text { EQUIP. }\end{array}$ & MAT'L & $\begin{array}{c}\text { S/C } \\
\text { (OTHER 1) }\end{array}$ & $\begin{array}{l}\text { TOTAL } \\
\text { COST }\end{array}$ \\
\hline \multirow[t]{2}{*}{$1,3,2,1$} & $\begin{array}{l}\text { DEMOLITION } \\
\text { REMOVE CELL HATCH COVERS }\end{array}$ & 1 & LOT & & $\begin{array}{c}\text { SKWK } \\
G E N\end{array}$ & 2500.00 & 2,500 & 82,775 & & & & 82,775 \\
\hline & DEMOLITION S/T & & & & & & 79,350 & $\$ 2,627,279$ & $\$ 1,505,000$ & & $\$ 25,000$ & $\$ 4,157,279$ \\
\hline \multirow[t]{7}{*}{$1,3,3$} & CONCRETE & & & & & & & & & & & \\
\hline & TUNNEL CONCRETE & 1,360 & $\overline{C Y}$ & $\overline{126.10}$ & SKWK & 12.300 & 16,728 & 553,864 & 34,340 & 171,496 & & 759,700 \\
\hline & RR STRUCTURE CONCRETE & 8,300 & CY & 126.10 & SKWK & 12.300 & 102,090 & $3,380,200$ & 209,575 & $1,046,630$ & & $4,636,405$ \\
\hline & BUILDING CONCRETE & 630 & $\overline{C Y}$ & 126.10 & SKWK & 12.300 & 7,749 & 256,569 & 15,908 & 79,443 & & 351,920 \\
\hline & HATCH PLUGS - ALLOW & 3 & EA & & & 0.000 & & & . & & 225,000 & 225,000 \\
\hline & $\begin{array}{l}\text { PLUG ALL PENETRATIONS INTO VAULT AREA } \\
\text { - ALLOW }\end{array}$ & 1 & LOT & $200,000.00$ & $\begin{array}{r}\text { SKWK } \\
\text { GEN }\end{array}$ & 10000.0 & 10,000 & 331,100 & . & 200,000 & & 531,100 \\
\hline & CONCRETE S/T & & . & & & & 136,567 & $\$ 4,521,733$ & $\$ 259,823$ & $\$ 1,497,569$ & $\$ 225,000$ & $\$ 6,504,125$ \\
\hline \multirow[t]{9}{*}{$1,3.5$} & METALS & & & & & & & & & . & & \\
\hline & TUBE SUPPORT FRAME & 250 & TON & $2,500.00$ & IRON & 100.000 & 25,000 & 920,250 & & 625,000 & & $1,545,250$ \\
\hline & FLOOR LEVELING FRAME & 360 & TON & $2,000.00$ & $\begin{array}{l}\text { IRON } \\
\text { GEN }\end{array}$ & 20.000 & 7,200 & 265,032 & & 720,000 & & 985,032 \\
\hline & SPACERS \& PEDESTALS - ALLOW & 100 & TON & $2,000.00$ & $\begin{array}{c}\text { IRON } \\
\text { GEN }\end{array}$ & 50.000 & 5,000 & 184,050 & & 200,000 & & 384,050 \\
\hline & SHIELDING PLATES & 370 & TON & $1,200.00$ & $\begin{array}{l}\text { IRON } \\
\text { GEN }\end{array}$ & 15.000 & 5,550 & 204,296 & & 444,000 & & 648,296 \\
\hline & BUILDING STRUCTURAL STEEL & 150 & TON & $1,200,00$ & $\begin{array}{c}\text { IRON } \\
\text { GEN }\end{array}$ & 10.000 & 1,500 & 55,215 & . & 180,000 & & 235,215 \\
\hline & GANTRY CRANE RAILS, EMBEDS, ETC. & 580 & LF & 161.00 & $\begin{array}{c}\text { IRON } \\
\text { GEN }\end{array}$ & 3.850 & 2,233 & 82,197 & & 93,380 & & 175,577 \\
\hline & RAILROAD TRACKS - WITHIN STRUCTURE & 2,000 & LF & 92.00 & $\begin{array}{r}\text { IRON } \\
\text { GEN }\end{array}$ & 2.000 & 4,000 & 147,240 & & 184,000 & & 331,240 \\
\hline & & & & & & & & . & & & & \\
\hline
\end{tabular}


Lockheed Martin Idaho Technologles Co.

Rov. 6/98

PROJECT NAME: HIP FACILITY - INTERIM STORAGE

FACILITY IN FPA

LOCATION 1: INEELICPP

REQUESTOR: J. K. RAWLINS

\section{DETAILED COST ESTIMATE SHEET}

TYPE OF ESTIMATE: PLANNING

PROJECT NO: 2421-FPR

PREPARED BY: R. D. ADAMS
PAGE * 5

DATE 28-Jan-1998

TIME: 08:41:26

REPORT NAME: Dotall Cost Estimate Sheot

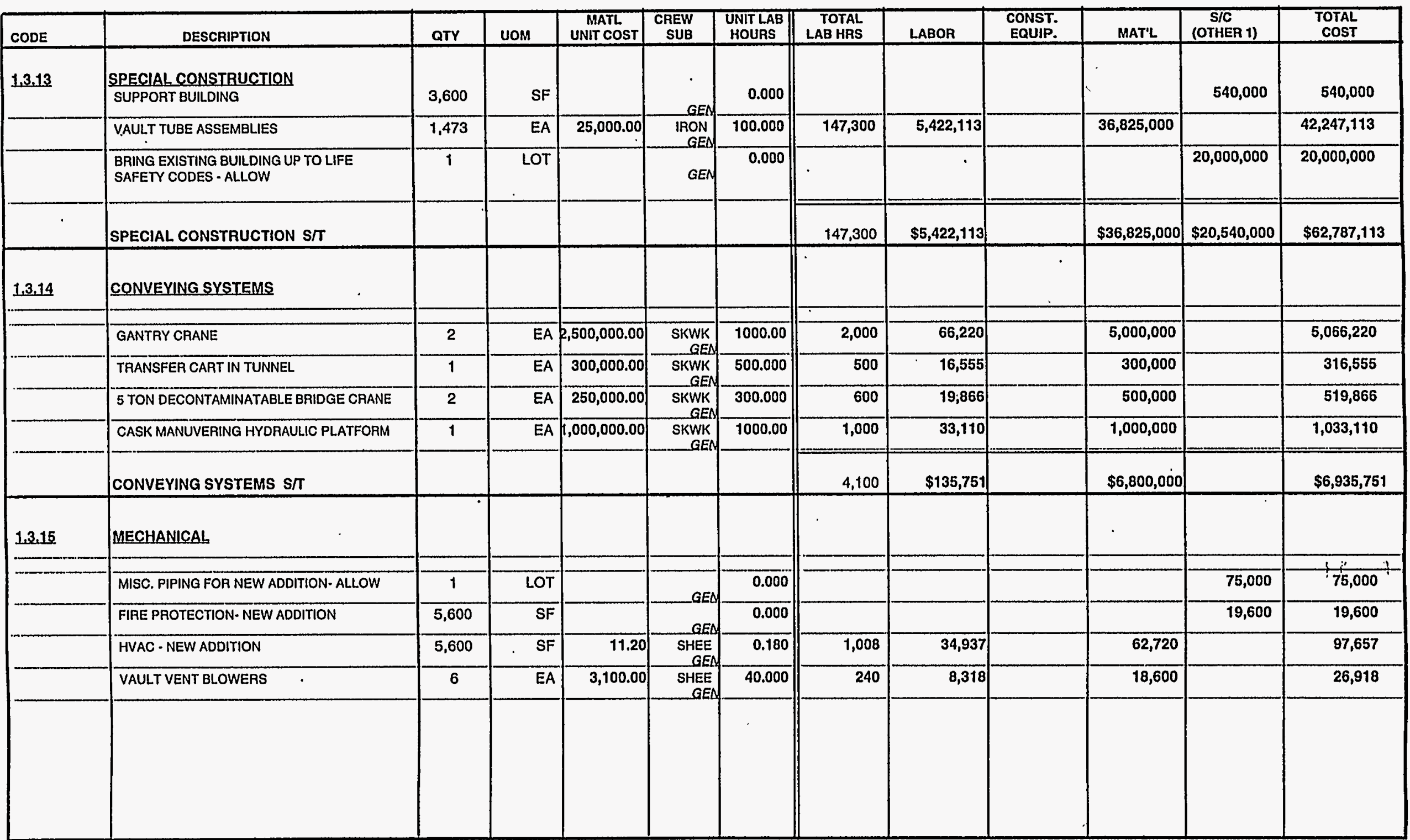


-ockheed Martin Idaho Technologies Co. Rov. 6/96

FECT NAME: HIP FACILITY - INTERIM STORAGE FACILITY IN FPA

JCATION :: INEEL/ICPP

EQUESTOR: J.K. RAWLINS

\section{DETAILED COST ESTIMATE SHEET}

TYPE OF ESTIMATE: PLANNING

PROJECT NO.: 2421-FPR

PREPAREO BY: R. D. ADAMS
PAgE 6

DATE 28-Jan-1998

TIME: 08:41:26

REPORT NAME: Detail Cost Estimate Sheet

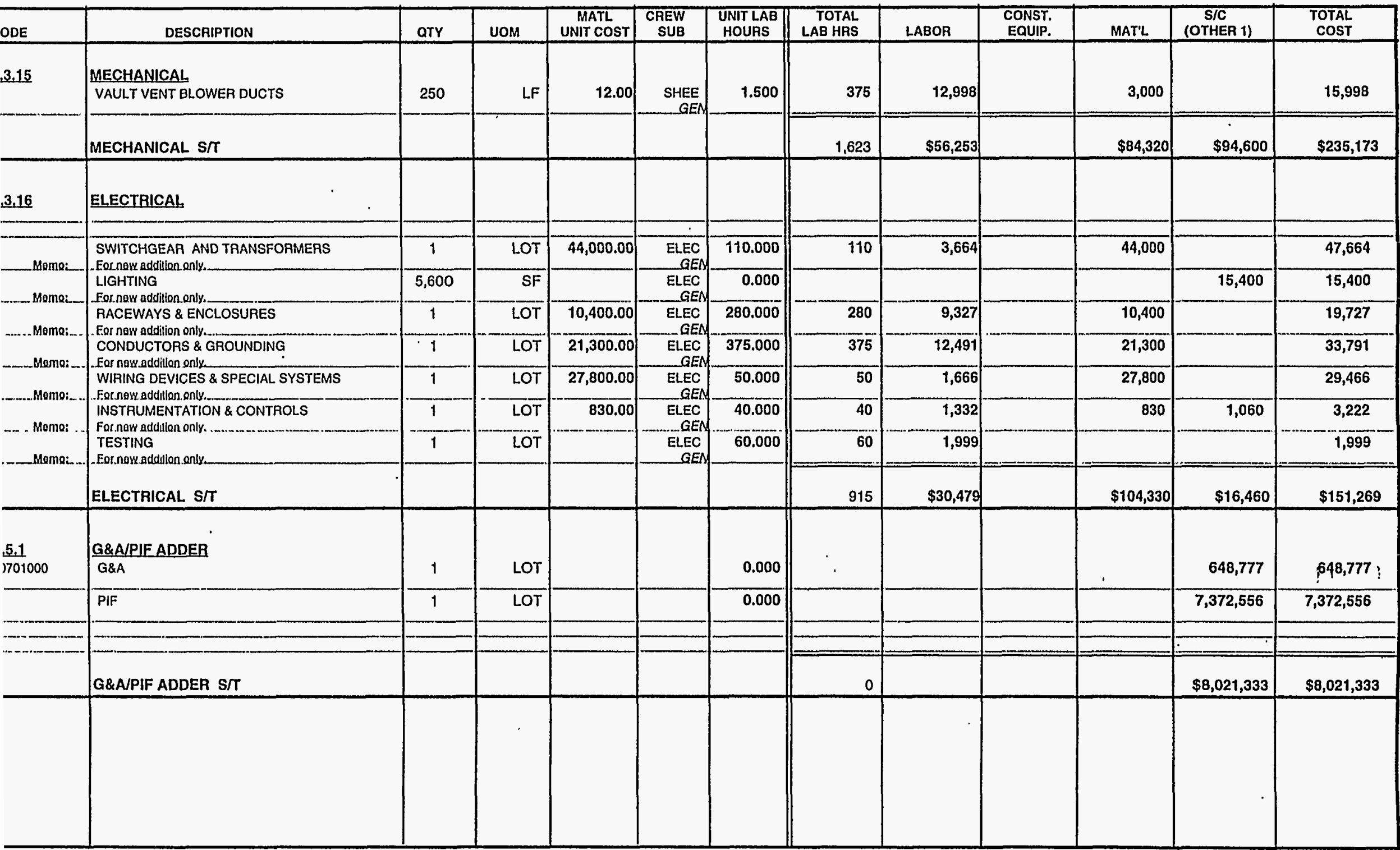


Lockheed Martin Idaho Technologies Co. nov. 6/96

PROJECT NAME: HIP FACILITY - INTERIM STORAGE FACILITY IN FP LOCATION :: INEEL/ICPP REQUESTOR: J. K. RAWLINS
DETAILED COST ESTIMATE SHEET

TYPE OF ESTIMATE: PLANNING PROJECT NO: 2421-FPR PREPARED BY: R. D. ADAMS
PAGE 7

DATE 28-Jan-1998

TIME: 08:41:26

REPORT NAME: Detail Cost Estimate Sheet

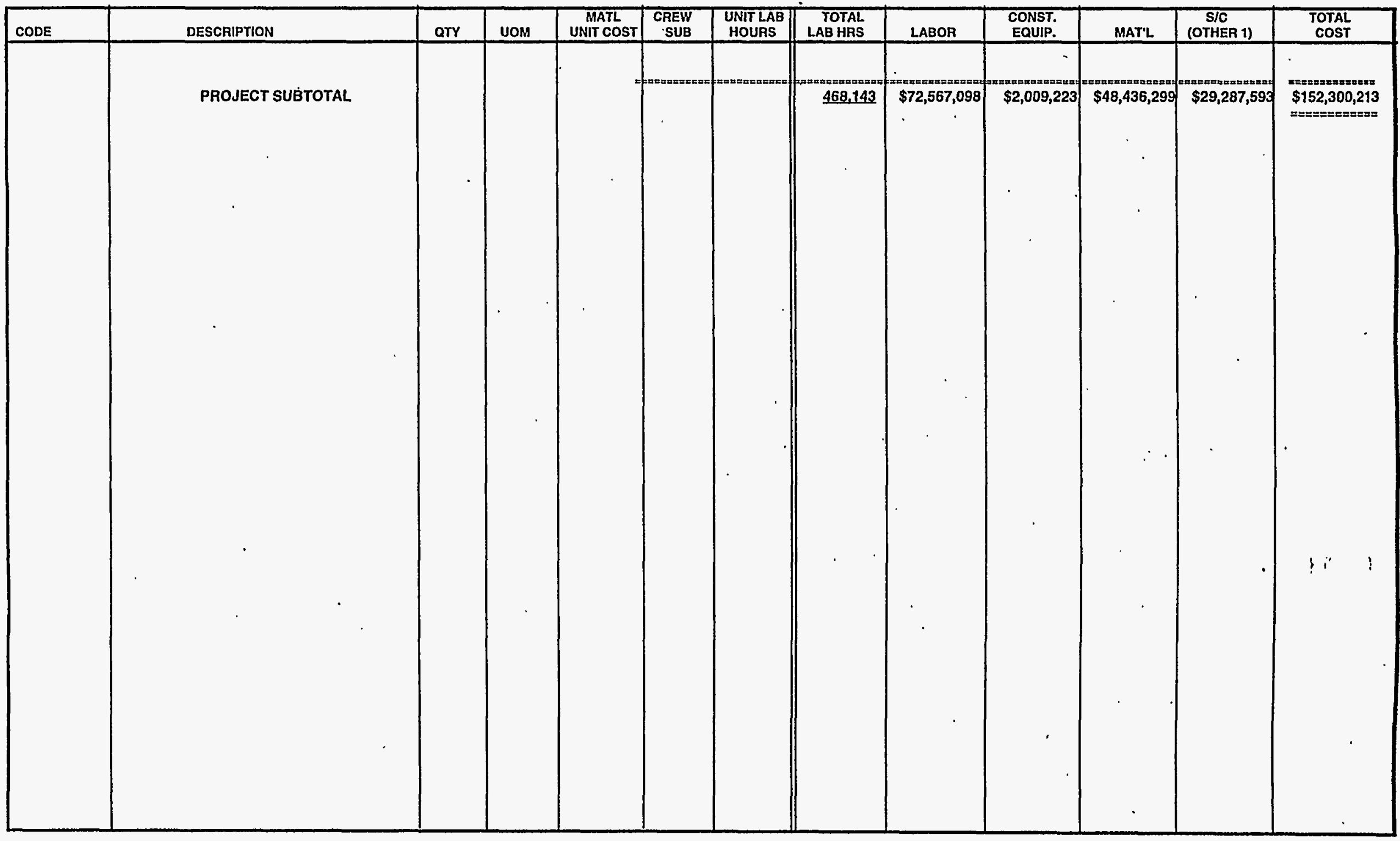


Lockheed Martin Idaho Technologies Co. Rev. 6/96 PROIECT NAME: HIP FACILITY - INTERIM STORAGE LOCATON 1: FACILITY IN FPR - UNESCALATED INEELICPP REQUESTCR:
CONTINGENCY ANALYSIS

$\begin{array}{cl}\text { TYPE OF ESTIMATE: } & \text { PLANNING } \\ \text { PROJECT NO: } & 2421 \text {-FPR }\end{array}$

PREPARED BY: R.D. ADAMS
-. DATE: 28-Jan-1998

¿- TIME: 08:43:08

REPORT NAME: Contingency Analysis

\begin{tabular}{|c|c|c|c|c|c|c|c|c|c|c|c|}
\hline \multicolumn{9}{|c|}{ PROBABLE \% VARIATION } & \multicolumn{2}{|c|}{$\begin{array}{l}\text { PROJECT } \\
\text { CONTINGENCY }\end{array}$} & \multirow{3}{*}{$\begin{array}{l}\text { SUMMARY } \\
\begin{array}{l}\text { Total Cost } \\
\text { by Element }\end{array}\end{array}$} \\
\hline \multirow[t]{2}{*}{$\begin{array}{l}\text { WBS } \\
\text { Element }\end{array}$} & \multirow[t]{2}{*}{ Cost Estimate Element } & \multirow{2}{*}{$\begin{array}{c}\text { Total Cost w/o } \\
\text { Contingency }\end{array}$} & \multirow[t]{2}{*}{$\begin{array}{c}\text { \% Total } \\
\text { Cost }\end{array}$} & \multicolumn{2}{|c|}{$\begin{array}{c}\text { Prob. \% Var. } \\
\text { From Est. }\end{array}$} & \multicolumn{2}{|c|}{ Wt. $\%$ of Prob. } & \multirow[t]{2}{*}{ Contingency } & \multirow[t]{2}{*}{$\%$} & \multirow[t]{2}{*}{ Cost } & \\
\hline & & & & - & + & - & \pm & & & & \\
\hline 1.1.1 & DESIGN ENGINEERING TILEI\&\| & $18,000,000$ & 9.10 & 20 & 35 & 1.82 & 3.19 & $2.686 \%$ & $12.44 \%$ & $5,309,282$ & $23,309,282$ \\
\hline 1.1 .2 & TITLE III INSPECTION & $6,603,900$ & 3.34 & 20 & 30 & 0.67 & 1.00 & $0.835 \%$ & $3.87 \%$ & $1,650,752$ & $8,254,652$ \\
\hline 1.2 .1 & PROJECT MANAGEMENT & $18,491,000$ & 9.35 & 20 & 35 & 1.87 & 3.27 & $2759 \%$ & $12.78 \%$ & $5,454,107$ & $23,945,107$ \\
\hline 1.2 .2 & CONSTRUCTION MANAGEMENT & $13,207,700$ & 6.68 & 20 & 30 & 1.34 & 200 & $1.670 \%$ & $7.74 \%$ & $3,301,479$ & $16,509,179$ \\
\hline 1.3 .1 & GENERAL CONDITIONS & $3,041,928$ & 1.54 & 20 & 35 & 0.37 & 0.54 & $0.454 \%$ & $2.10 \%$ & 897,247 & $3,939,175$ \\
\hline 1.3 .2 & SITEWORK & $6,524,508$ & 3.30 & 20 & 40 & 0.66 & 1.32 & $1.122 \%$ & $5.20 \%$ & $2,218,033$ & $8,742,541$ \\
\hline 1.3 .3 & CONCRETE & $10,575,913$ & 5.35 & 20 & 30 & 1.07 & 1.60 & $1.337 \%$ & $6.19 \%$ & $2,643,621$ & $13,219,534$ \\
\hline 1.3 .5 & METALS & $7,196,529$ & 3.64 & 20 & 35 & 0.73 & 1.27 & $1.074 \%$ & $4.97 \%$ & $2,122,689$ & $9,319,218$ \\
\hline 1.3 .7 & THERMAL \& MOISTURE PROTECTION & 230,234 & 0.12 & 20 & 30 & 0.02 & 0.03 & $0.029 \%$ & $0.13 \%$ & 57,551 & 287,785 \\
\hline 1.3.8 & DOORS \& WINDOWS & 64,695 & 0.03 & 20 & 20 & 0.01 & 0.01 & $0.005 \%$ & $0.02 \%$ & 10,350 & 75,045 \\
\hline 1.3 .9 & FINISHES & 611,189 & 0.31 & 20 & 35 & 0.06 & 0.11 & $0.091 \%$ & $0.42 \%$ & 180,276 & 791,465 \\
\hline 1.3.13 & SPECIAL CONSTRUCTION & $91,982,003$ & 46.52 & 20 & 20 & 9.30 & 9.30 & $7.443 \%$ & $34.48 \%$ & $14,715,130$ & $106,697,133$ \\
\hline 1.3.14 & CONVEYING SYSTEMS & $11,272,944$ & 5.70 & 20 & 25 & 1.14 & 1.43 & $1.169 \%$ & $5.41 \%$ & $2,310,641$ & $13,583,585$ \\
\hline 1.3.15 & MECHANICAL & 337,767 & 0.17 & 20 & 30 & 0.03 & 0.05 & $0.043 \%$ & $0.20 \%$ & 84,430 & 422,197 \\
\hline 1.3 .16 & ELECTRICAL & 239,215 & 0.12 & 20 & 25 & 0.02 & 0.03 & $0.025 \%$ & $0.11 \%$ & 49,032 & 288,247 \\
\hline 1.5.1 & G\&AVIF ADDER & $8,021,333$ & 4.06 & 5 & 20 & 0.20 & 0.81 & $0.710 \%$ & $3.29 \%$ & $1,403,543$ & $9,424,876$ \\
\hline \multirow[t]{2}{*}{1.5 .2} & PROCUREMENT FEES & $1,320,769$ & 0.57 & 20 & 25 & 0.13 & 0.17 & $0.137 \%$ & $0.63 \%$ & 270,721 & $1,591,490$ \\
\hline & ESCALATION & 0 & 0.00 & 20 & 25 & 0.00 & 0.00 & $0.000 \%$ & $0.00 \%$ & (511) & (511) \\
\hline & SUBTOTAL & $197,721,627$ & 100.00 & & & & & $21.588 \%$ & & & \\
\hline & CALCULATED CONTINGENCY & $42,684,657$ & & & & & & & & & \\
\hline & RESULTANT TEC & $240.406,284$ & & & & & & & & & \\
\hline & ROUNDED TEC & $240,400,000$ & & & & & & & & & \\
\hline & PROJECT CONTINGENCY & $42,678,373$ & & & & & & $21.59 \%$ & & & \\
\hline & MANAGEMENT RESERVE & $14,141,903$ & & & & & & & & & \\
\hline & CONTINGENCY & 28.536 .470 & & & & & & & & & \\
\hline & TOTAL ESTIMATED COST & $240,400,000$ & & & & & & & & $42,678,373$ & $240,400,000$ \\
\hline
\end{tabular}

CONFIDENCE LEVEL AND ASSUMED RISKS:

The Lockheed Idaho Technologies Co. Cost Estimate Contingency Analysis Model is based on the applied contingency and the assumptions upon which the estimate was predicated. The model is applied with a suggested risk level of $18 \%$ and a level of confidence of $90 \%$ the estimate will fall within the bid range. The Contingency Analysis is based on a weighted average to provide a

$90 \%$ probability of underrun and a $10 \%$ probability of overrun.
CONTINGENCY ANALYSIS GUIDE BY TYPE OF ESTIMATE

Guidelines established by DOE/FM 50, Cost Estimating Guide, Vol. 6 ,

Cost Guide, and as presented in the INEL Cost Estimating Guide.

PLANNING $20 \%-30 \%$

Experimental/Special Conditions.............. Up to $50 \%$

Conceptual

$15 \%-25 \%$

Experimental/Special Conditions.............Up to $40 \%$

TITLE I

TITLE II

TITLE II/AFC

$10 \%-20 \%$

$5 \%-15 \%$ Market Conditions 
Lockheed Martin Idaho Technologies Co. PROJECT NAME: HIP FACILITY - INTERIM STORAGE FACILITY IN FPR - ESCALATED

LOCATION 1: INEELICPP

REOUESTOR: J. K. RAWLINS
CONTINGENCY ANALYSIS

TYPE OF ESTIMATE: PROJECT NO: PREPARED BY:
PLANNING

2421-FPR

R. D. ADAMS
DATE: 28-Jan-1998

- TIME: 08:45:28

REPORT NAME: Contingency Analysis

\begin{tabular}{|c|c|c|c|c|c|c|c|c|c|c|c|}
\hline \multicolumn{9}{|c|}{ PROBABLE \% VARIATION } & \multicolumn{2}{|c|}{$\begin{array}{c}\text { PROJECT } \\
\text { CONTINGENCY }\end{array}$} & \multirow{3}{*}{$\begin{array}{l}\text { SUMMARY } \\
\text { Total Cost } \\
\text { by Element }\end{array}$} \\
\hline \multirow{2}{*}{$\begin{array}{l}\text { WBS } \\
\text { Element }\end{array}$} & \multirow{2}{*}{ Cost Estimate Element } & \multirow{2}{*}{$\begin{array}{l}\text { Total Cost w/o } \\
\text { Contingency }\end{array}$} & \multirow[t]{2}{*}{$\begin{array}{l}\text { \% Total } \\
\text { Cost }\end{array}$} & \multicolumn{2}{|c|}{$\begin{array}{l}\text { Prob. \% Var. } \\
\text { From Est. }\end{array}$} & \multicolumn{2}{|c|}{ Wt. \% of Prob. } & \multirow[t]{2}{*}{ Contingency } & \multirow[t]{2}{*}{$\%$} & \multirow[t]{2}{*}{ Cost } & \\
\hline & & & & - & + & - & + & & & & \\
\hline 1.1.1 & DESIGN ENGINEERING TITLE | \& I & $18,000,000$ & 6.70 & 20 & 35 & 1.34 & 2.34 & $1.976 \%$ & $9.28 \%$ & $5,306,794$ & $23,306,794$ \\
\hline 1.1 .2 & TITLE III INSPECTION & $6,603,900$ & 2.46 & 20 & 30 & 0.49 & 0.74 & $0.614 \%$ & $2.88 \%$ & $1,649,978$ & $8,253,878$ \\
\hline 1.2 .1 & PROJECT MANAGEMENT & $18,491,000$ & 6.88 & 20 & 35 & 1.38 & 2.41 & $2.030 \%$ & $9.53 \%$ & $5,451,551$ & $23,942,551$ \\
\hline 1.2 .2 & CONSTRUCTION MANAGEMENT & $13,207,700$ & 4.92 & 20 & 30 & 0.98 & 1.47 & $1.229 \%$ & $5.77 \%$ & $3,299,931$ & $16,507,631$ \\
\hline 1.3 .1 & GENERAL CONDITIONS & $3,041,928$ & 1.13 & 20 & 35 & 0.23 & 0.40 & $0.334 \%$ & $1.57 \%$ & 896,827 & $3,938,755$ \\
\hline 1.3 .2 & SITEWORK & $6,524,508$ & 2.43 & 20 & 40 & 0.49 & 0.97 & $0.826 \%$ & $3.88 \%$ & $2,216,993$ & $8,741,501$ \\
\hline 1.3 .3 & CONCRETE & $10,575,913$ & 3.94 & 20 & 30 & 0.79 & 1.18 & $0.984 \%$ & $4.62 \%$ & $2,642,382$ & $13,218,295$ \\
\hline 1.3 .5 & METALS & $7,196,529$ & 2.68 & 20 & 35 & 0.54 & 0.94 & $0.790 \%$ & $3.71 \%$ & $2,121,694$ & $9,318,223$ \\
\hline 1.3 .7 & THERMAL \& MOISTURE PROTECTION & 230,234 & 0.09 & 20 & 30 & 0.02 & 0.03 & $0.021 \%$ & $0.10 \%$ & 57,524 & 287,758 \\
\hline 1.3 .8 & DOORS \& WINDOWS & 64,695 & 0.02 & 20 & 20 & 0.00 & 0.00 & $0.004 \%$ & $0.02 \%$ & 10,345 & 75,040 \\
\hline 1.3.9 & FINISHES & 611,189 & 0.23 & 20 & 35 & 0.05 & 0.08 & $0.067 \%$ & $0.32 \%$ & 180,192 & 791,381 \\
\hline 1.3 .13 & SPECIAL CONSTRUCTION & $91,982,003$ & 34.23 & 20 & 20 & 6.85 & 6.85 & $5.477 \%$ & $25.71 \%$ & $14,708,234$ & $106,690,237$ \\
\hline 1.3 .14 & CONVEYING SYSTEMS & $11,272,944$ & 4.20 & 20 & 25 & 0.84 & 1.05 & $0.860 \%$ & $4.04 \%$ & $2,309,558$ & $13,582,502$ \\
\hline 1.3 .15 & MECHANICAL & 337,767 & 0.13 & 20 & 30 & 0.03 & 0.04 & $0.031 \%$ & $0.15 \%$ & 84,391 & 422,158 \\
\hline 1.3 .16 & ELECTRICAL & 239,215 & 0.09 & 20 & 25 & 0.02 & 0.02 & $0.018 \%$ & $0.09 \%$ & 49,009 & 288,224 \\
\hline 1.5 .1 & G\&APIF ADDER & $8,021,333$ & 2.99 & 5 & 20 & 0.15 & 0.60 & $0.522 \%$ & $245 \%$ & $1,402,886$ & $9,424,219$ \\
\hline \multirow[t]{2}{*}{1.5 .2} & PROCUREMENT FEES & $1,320,769$ & 0.49 & 20 & 25 & 0.10 & 0.12 & $0.101 \%$ & $0.47 \%$ & 270,594 & $1,591,363$ \\
\hline & ESCALATION & $70,977,363$ & 26.42 & 20 & 25 & 5.28 & 6.60 & $5.415 \%$ & $25.42 \%$ & 14.542 .127 & $85,519,490$ \\
\hline & SUBTOTAL & $268,698,990$ & 100.00 & & & & & $21.301 \%$ & & & \\
\hline & CALCULATED CONTINGENCY & $57,235,016$ & & & & & & & & & \\
\hline & RESULTANT TEC & $325,934,006$ & & & & & & & & & \\
\hline & ROUNDED TEC & $325,900,000$ & & & & & & & & & \\
\hline & PROJECT CONTINGENCY & $57,201,010$ & & & & & & $21.29 \%$ & & & \\
\hline & MANAGEMENT RESERVE & $19,374,407$ & & & & & & & & & \\
\hline & CONTINGENCY & 37.826 .603 & & & & & & & & & \\
\hline & TOTAL ESTIMATED COST & $325,900,000$ & & & & & & & & $57,201,010$ & $325,900,000$ \\
\hline
\end{tabular}

CONFIDENCE LEVEL AND ASSUMED RISKS:

The Lockheed Idaho Technologies Co. Cost Estimate Contingency Analysis

Model is based on the applied contingency and the assumptions upon which

the estimate was predicated. The model is applied with a suggested risk level

of $18 \%$ and a level of confidence of $90 \%$ the estimate will fall within the bid range.

The Contingency Analysis is based on a weighted average to provide a

$90 \%$ probability of underrun and a $10 \%$ probability of overrun.
CONTINGENCY ANALYSIS GUIDE BY TYPE OF ESTIMATE

Guidelines established by DOE/FM 50, Cost Estimating Guide, Vol. 6 . Cost Guide, and as presented in the INEL Cost Estimating Guide.
PLANNING
Experimental/Special Conditions 20\% -
Conceptual
$15 \%-25 \%$
TITLE I
TITLE II
.....Up to $40 \%$
$10 \%-20 \%$
$5 \%-15 \%$

TITLE II/AFC
Market Conditions 


\section{G\&A/PIF ADDER CALCULATION SHEET}

PROCUREMENT FEE:

\begin{tabular}{l} 
CONSTRUCTION $=$ \\
GFE $=$ \\
\cline { 2 - 2 } \\
FEE @ $1 \%=$
\end{tabular}

G\&A @ 23\% (with a ceiling of $\$ 500,000$ imposed per year

CONSTRUCTION OR

CEILING * 3 YEARS $=\quad \$ 1,500,000$

GFE =

$\$ 0$

PROCUREMENT FEE $=$

$\$ 1,320,769$

Subtotal $\$ 2,820,769$

FEE @ $23 \%=$

$\$ 2,820,769 * 0.23=$

$\$ 648,777$

PIF @ 5.5\%

\begin{tabular}{|c|c|}
\hline CONSTRUCTION = & $\$ 132,076,925$ \\
\hline GFE $=$ & $\$ 0$ \\
\hline PROCUREMENT FEE = & $\$ 1,320,769$ \\
\hline Subtotal & $\frac{\$ 648,777}{\$ 134,046,471}$ \\
\hline
\end{tabular}

FEE @ $5.5 \%=$

$\$ 134,046,471 * 0.055=\quad \$ 7,372,556$

TOTAL PROCUREMENT FEE:

$\$ 1,320,769$

TOTAL G\&A FEE:

$\$ 648,777$

TOTAL PIF:

$\$ 7,372,556$ 
Lockheed Martin Idaho Technologies Co. PRONECT NAME: HIP FACILITY - INTERIM STORAGE FACILITY IN FPR - OPC - ESCALATED

LOCATION 1:

INEELICPP

REQUESTOR: J.K. RAWLINS
COST ESTIMATE SUMMARY

$\begin{array}{cl}\text { TYPE OF ESTIMATE: } & \text { PLANNING } \\ \text { PROJECTNO: } & 242103 \\ \text { PREPARED BY: } & \text { R.D.ADAMS } \\ \text { REPORT NAME: } & \text { Cost Estimate Summary }\end{array}$

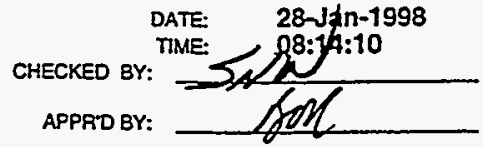

\begin{tabular}{|c|c|c|c|c|}
\hline $\begin{array}{l}\text { WBS } \\
\text { Element }\end{array}$ & Cost Estimate Element & $\begin{array}{c}\text { Total } \\
\text { Unescalated }\end{array}$ & Escalation & $\begin{array}{c}\text { Total } \\
\text { Incl Escalation }\end{array}$ \\
\hline 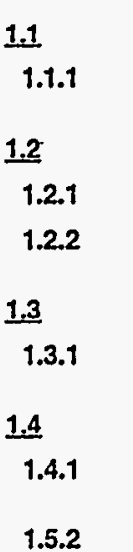 & $\begin{array}{l}\text { DESIGN \& DEVELOPMENT } \\
\text { CONCEPTUAL DESIGN } \\
\text { MANAGEMENT COSTS } \\
\text { PM FOR PROJECT DEVELOPMENT } \\
\text { SAFETY REVIEWS } \\
\text { PERMITTING } \\
\text { PERMITTING } \\
\text { TURNOVER } \\
\text { SO TEST \& STARTUP } \\
\text { PROCUREMENT FEES }\end{array}$ & $\begin{array}{r}5,000,000 \\
3,000,000 \\
1,000,000 \\
2,728,432 \\
4,500,000 \\
0\end{array}$ & $\begin{array}{r}1,100,000 \\
660,000 \\
220,000 \\
600,255 \\
2,025,000 \\
0\end{array}$ & $\begin{array}{l}\gg \frac{\$ 6,100,000}{6,100,000} \\
\gg \quad \begin{array}{r}\$ 4,880,000 \\
3,660,000 \\
1,220,000\end{array} \\
\gg \quad \frac{\$ 3,328,687}{3,328,687} \\
\gg \quad \frac{\$ 6,525,000}{6,525,000} \\
\gg \quad \underline{\$ 0}\end{array}$ \\
\hline & $\begin{array}{l}\text { SUBTOTAL INCLUDING ESCALATION } \\
\text { PRO'JECT CONTINGENCY }\end{array}$ & $16,228,432$ & $4,605,255$ & >> $\$ 20,833,687$ \\
\hline & MANAGEMENT RESERVE & & & so \\
\hline & CONTINGENCY - & & & $\$ 5,166,313$ \\
\hline & TOTAL ESTIMATED COST & & & $\$ 26,000,000$ \\
\hline
\end{tabular}

\section{PROJECT COST PARAMETERS}

EDI AS A \% OF CONST. + GFE $=62.00 \%$ 
Lockheed Martin ldaho Téchnologies Co.

PROIECT NAME: HIP FACILITY - INTERIM STORAGE FACILITY IN FPR - OPC - UNESCALATED

LOCATON 1: INEELICPP

J. K. RAWLINS
COST ESTIMATE SUMMARY

TYPE OF ESTIMATE: PLANNING PROJECT NO: 242103 PAEPARED BY: REPOAT NAME:

R. D. ADA
Cost Estimate Summary

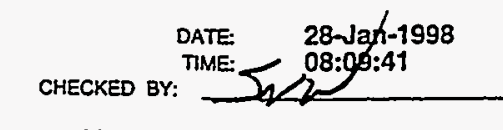

APPAD BY:

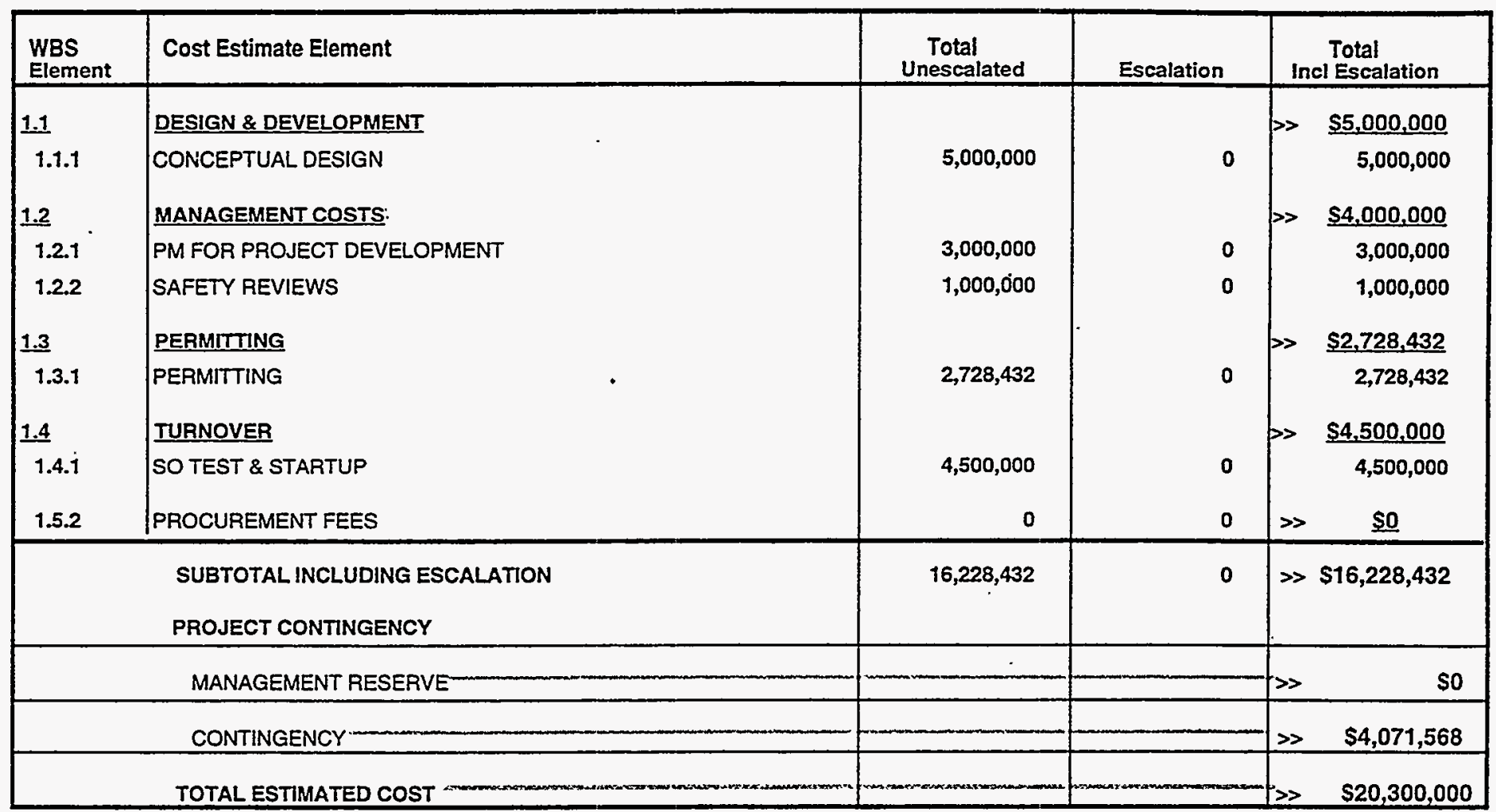


Lockheed Martin Idaho Technologies Co.

Rov. 6/9B

PROSECT NAME: HIP FACILITY - INTERIM STORAGE

FACILITY IN FPR - OPC

LOCATION 1: INEELIICPP

REQUESTOR: J. K. RAWLINS
DETAILED COST ESTIMATE SHEET

TYPE OF ESTIMATE: PLANNING

PROJECTNO.: 242103

PREPARED BY: R. D. ADAMS
PAGE \# 1

DATE 28-Jan-1998

TIME: 08:07:27

REPORT NAME: Detail Cost Estlmate Sheet

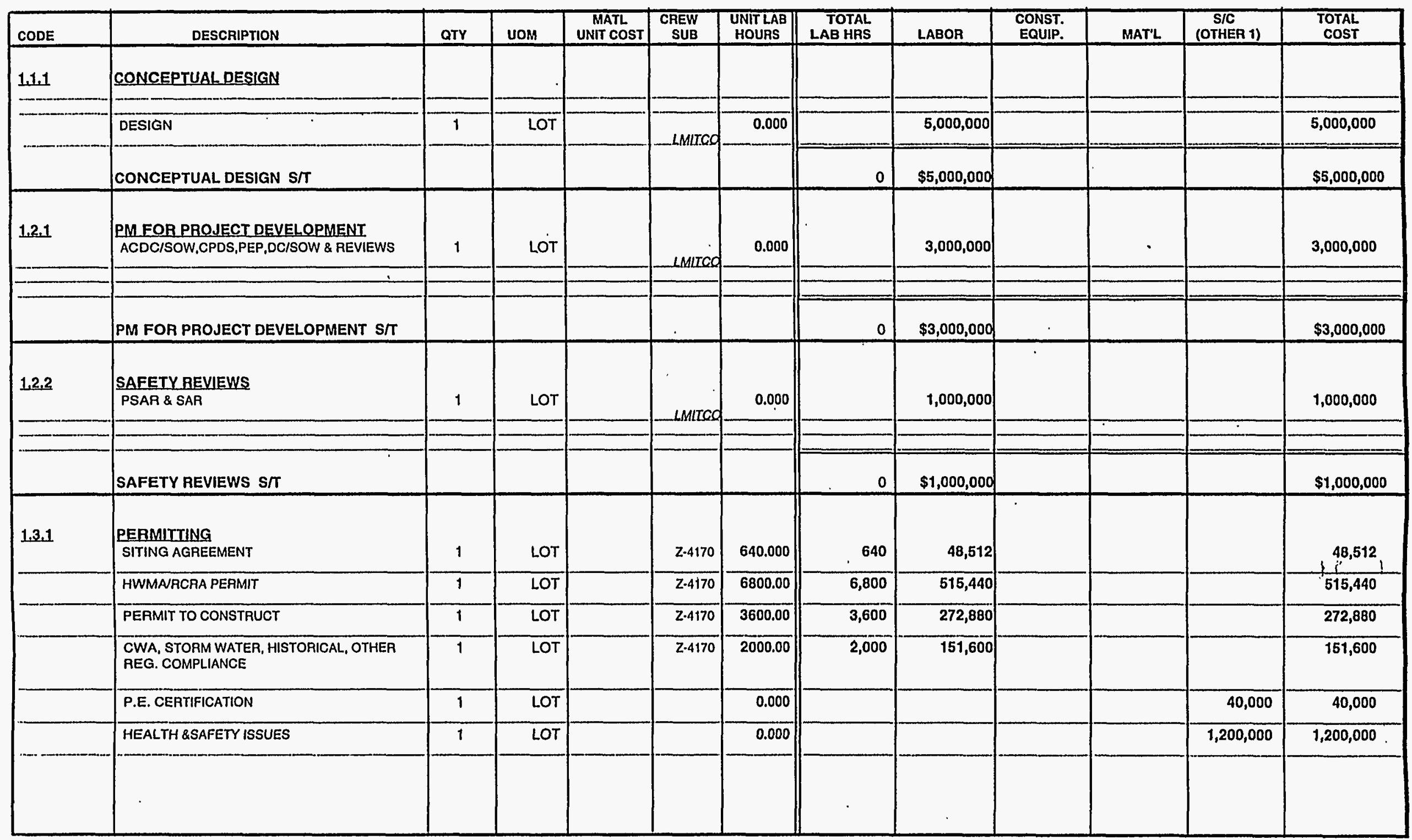


Lockheed Martin Idaho Technologies Co. ROV. 6 MS PROSECT NAME: HIP FACILITY - INTERIM STORAGE LOCATION 1: REQUESTOR: FACILITY IN FPR - OPC - UNESCALATED NEELICPP J. K. RAWLINS
CONTINGENCY ANALYSIS

TYPE OF ESTIMATE: PLANNING PROJECTNO: 2421O3 PREPARED BY: R. D. ADAMS
$-$ DATE: $28-J a n-1998$ - TIME: 08:09:47

REPORT NAME: Contingency Analysis

\begin{tabular}{|c|c|c|c|c|c|c|c|c|c|c|c|}
\hline \multicolumn{9}{|c|}{ PROBABLE \% VARIATION } & \multicolumn{2}{|c|}{$\begin{array}{c}\text { PROJECT } \\
\text { CONTINGENCY }\end{array}$} & \multirow{3}{*}{$\begin{array}{l}\text { SUMMARY } \\
\begin{array}{l}\text { Total Cost } \\
\text { by Element }\end{array}\end{array}$} \\
\hline \multirow[t]{2}{*}{$\begin{array}{l}\text { WBS } \\
\text { Element }\end{array}$} & \multirow{2}{*}{ Cost Estimate Element } & \multirow{2}{*}{$\begin{array}{l}\text { Total Cost w/o } \\
\text { Contingency }\end{array}$} & \multirow[t]{2}{*}{$\begin{array}{l}\% \text { Total } \\
\text { Cost }\end{array}$} & \multicolumn{2}{|c|}{$\begin{array}{l}\text { Prob. \% Var. } \\
\text { From Est. }\end{array}$} & \multicolumn{2}{|c|}{ Wt. \% of Prob. } & \multirow[t]{2}{*}{ Contingency } & \multirow[t]{2}{*}{$\%$} & \multirow[t]{2}{*}{ Cost } & \\
\hline & & & & $=$ & + & $=$ & + & & & & \\
\hline 1.1 .1 & CONCEPTUAL DESIGN & $5,000,000$ & 30,81 & 20 & 30 & 6.16 & 9.24 & $7.703 \%$ & $30.81 \%$ & $1,254,455$ & $6,254,455$ \\
\hline 1.2 .1 & PM FOR PROJECT DEVELOPMENT & $3,000,000$ & 18.49 & 20 & 30 & 3.70 & 5.55 & $4.622 \%$ & $18.49 \%$ & 752,573 & $3,752,673$ \\
\hline 1.2 .2 & SAFETY REVIEWS & $1,000,000$ & 6.16 & 20 & 30 & 7.23 & 1.85 & $1.541 \%$ & $6.16 \%$ & 250,891 & $1,250,891$ \\
\hline 1.3 .1 & PERMITTING & $2,728,432$ & 16.81 & 20 & 30 & 3.36 & 5.04 & $4.203 \%$ & $16.81 \%$ & 684,539 & $3,412,971$ \\
\hline 1.4 .1 & SO TEST \& STARTUP & $4,500,000$ & 27.73 & 20 & 30 & 5.55 & 8.32 & $6.932 \%$ & $27.73 \%$ & $1,129,010$ & $5,629,010$ \\
\hline 1.5 .2 & PROCUREMENT FEES & 0 & 0.00 & 5 & 20 & 0.00 & 0.00 & $0.000 \%$ & $0.00 \%$ & 0 & 0 \\
\hline & ESCALATTON & 0 & 0.00 & 20 & 30 & 0.00 & 0.00 & $0.000 \%$ & $0.00 \%$ & 0 & 0 \\
\hline & SUBTOTAL & $16,228,432$ & 100.00 & & & & & $25.000 \%$ & & & \\
\hline & CALCULATED CONTINGENCY & $4,057,108$ & & & & & & & & & \\
\hline & RESULTANT TEC & $20,285,540$ & & & & & & & & & \\
\hline & ROUNDED TEC & $20,300,000$ & & & · & & & & & & \\
\hline & PROJECT CONTINGENCY & $4,071,568$ & & & & & & $25.09 \%$ & & & \\
\hline & MANAGEMENT RESERVE & 0 & & & & & & & & & \\
\hline & CONTINGENCY & $4,071,558$ & & & & & & & & & \\
\hline & TOTAL ESTIMATED COST & $20,300,000$ & & & & & & & & $4,071,568$ & $20,300,000$ \\
\hline
\end{tabular}

CONFIDENCE LEVEL AND ASSUMED RISKS:

The Lockheed Idaho Technologies Co. Cost Estimate Contingency Analysis Model is based on the applied contingency and the assumptions upon which the estimate was predicated. The model is applied with a suggested risk level of $18 \%$ and a level of confidence of $90 \%$ the estimate will fall within the bid range. The Contingency Analysis is based on a weighted average to provide a $90 \%$ probability of underrun and a $10 \%$ probability of overrun.
CONTINGENCY ANALYSIS GUIDE BY TYPE OF ESTIMATE

Guidelines established by DOE/FM 50, Cost Estimating Guide, Vol. 6 , Cost Guide, and as presented in the INEL Cost Estimating Guide. PLANNING Experimental/Special Conditions............. Conceptual $15 \%-25 \%$ Experimental/Special Conditions............Up to $40 \%$ TITLE 1

TITLE I TITLE II/AFC 
Lockheed Martin Idaho Technologies Co.

PROSECT NAME: HIP FACILITY - INTERIM STORAGE

FACILITY IN FPR - OPC - ESCALATED

REQUESTOR: J.K. RAWLINS
CONTINGENCY ANALYSIS

TYPE OF ESTIMATE:

PROSECT NO:

PREPARED BY
PLANNING

242103

R. D. ADAMS
DATE: 28-Jan-1998

$\therefore$ TIME: 08:14:15

-

REPORT NAME: Contingency Analysis

\begin{tabular}{|c|c|c|c|c|c|c|c|c|c|c|c|}
\hline & PROE & BLE \% VARIA & ION & & & & & & $\begin{array}{l}\text { PROJ } \\
\text { CONTI }\end{array}$ & $\begin{array}{l}\text { CT } \\
\text { IENCY }\end{array}$ & SUMMARY \\
\hline \begin{tabular}{|l|} 
WBS \\
Element
\end{tabular} & Cost Estimate Element & Total Cost w/o & $\begin{array}{l}\text { \% Total } \\
\text { Cost }\end{array}$ & & $\begin{array}{l}\% \text { Var. } \\
\text { mEst. }\end{array}$ & Wt. \% & Prob. & Contingency & $\%$ & Cost & Total Cost \\
\hline & & Contingency & & - & + & - & + & & & & by Element \\
\hline 1.1 .1 & CONCEPTUAL DESIGN & $5,000,000$ & 24.00 & 20 & 30 & 4.80 & 7.20 & $6.000 \%$ & $24.00 \%$ & $1,239,894$ & $6,239,894$ \\
\hline 1.21 & PM FOR PROJECT DEVELOPMENT & $3,000,000$ & 14.40 & 20 & 30 & 2.88 & 4.32 & $3.600 \%$ & $14.40 \%$ & 743,936 & $3,743,936$ \\
\hline 1.2 .2 & SAFETY REVIEWS & $1,000,000$ & 4.80 & 20 & 30 & 0.96 & 1.44 & $1.200 \%$ & $4.80 \%$ & 247,979 & $1,247,979$ \\
\hline 1.3 .1 & PERMITTING & $2,728,432$ & 13.10 & 20 & 30 & 262 & 3.93 & $3.274 \%$ & $13.10 \%$ & 676,593 & $3,405,025$ \\
\hline 1.4 .1 & SO TEST \& STARTUP & $4,500,000$ & 21.60 & 20 & 30 & 4.32 & 6.48 & $5.400 \%$ & $21.60 \%$ & $1,+15,905$ & $5,615,905$ \\
\hline 1.5 .2 & PROCUREMENT FEES & 0 & 0.00 & 5 & 20 & 0.00 & 0.00 & $0.000 \%$ & $0.00 \%$ & 0 & 0 \\
\hline & ESCALATION & $4,605,255$ & 22.10 & 20 & 30 & 4.42 & 6.63 & $5.526 \%$ & $22.10 \%$ & 1.142 .006 & $5,747,261$ \\
\hline & SUBTOTAL & $20,833,687$ & 100.00 & & & & & $25.000 \%$ & & & \\
\hline & CALCULATED CONTINGENCY & $5,208,422$ & & & & & & & & & \\
\hline & RESULTANT TEC & $26,042,109$ & & & & & & & & & \\
\hline & ROUNDED TEC & $26,000,000$ & & & & & & & & & \\
\hline & PROJECT CONTINGENCY & $5,166,313$ & & & & & & $24.80 \%$ & & & \\
\hline & MANAGEMENT RESERVE & 0 & & & & & & & & & \\
\hline & CONTINGENCY & $5.166,313$ & & & & & & & & & \\
\hline & TOTAL ESTIMATED COST & $26,000,000$ & & & & & & & & $5,166,313$ & $26,000,000$ \\
\hline
\end{tabular}

\section{CONFIDENCE LEVEL AND ASSUMED RISKS:}

The Lockheed Idaho Technologies Co. Cost Estimate Contingency Analysis Model is based on the applied contingency and the assumptions upon which the estimate was predicated. The model is applied with a suggested risk level of $18 \%$ and a level of confidence of $90 \%$ the estimate will fall within the bid range. The Contingency Analysis is based on a weighted average to provide a $90 \%$ probability of underrun and a $10 \%$ probability of overrun.
CONTINGENCY ANALYSIS GUIDE BY TYPE OF ESTIMATE Guidelines established by DOE/FM 50, Cost Estimating Guide, Vol. 6 , Cost Guide, and as presented in the INEL Cost Estimating Guide.

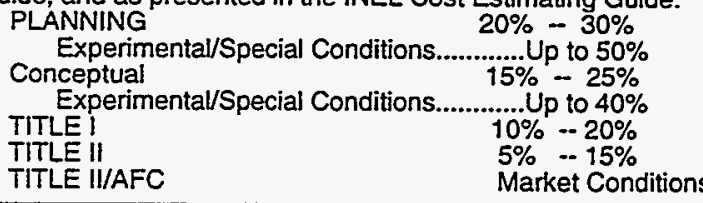


HIP OPTION

INTERIM STORAGE FACILITY IN FPR

\begin{tabular}{|c|c|c|c|c|c|c|c|c|c|c|c|c|c|c|c|c|c|c|}
\hline ID & Task Name & 1997 & 1998 & 1999 & 2000 & 2001 & 2002 & 2003 & 2004 & 2006 & 2006 & 2007 & 2008 & 2009 & 2010 & 2011 & 2012 & 2013 \\
\hline 1 & HIP - INTERIM STORAGE IN FPR & & & & & & & & & & & & & & & & & \\
\hline 2 & CONCEPTUAL DESIGN & & & & & & & & $6 / 1$ & & & & & & & & & \\
\hline 3 & ADVANCED CONCEPTUAL DESIGN & & & & & & & & & $6 / 31$ & & & & & & & & \\
\hline 4 & PM FOR PROJECT DEVELOPMENT & & & & & & & & & & & & & & & & & \\
\hline 5 & PERMITTING & & & & & & & & 6/1 & & & & & & & & & \\
\hline 6 & PROJECT MANAGEMENT & & & & & & & & & & & $6 / 20$ & & & & & $11 / 21$ & \\
\hline 7 & TITLE DESIGN & & & & & & & & & & & $6 / 29$ & & $7^{11 / 24}$ & & & & \\
\hline 8 & CONSTRUCTION & & & & & & & & & & & & $11 / 2$ & & & & 11 & \\
\hline 9 & SO TEST \& STARTUP & & & & & & & & & & & & & & & 11/22 & & \\
\hline 10 & START CANISTER RECEIPT & & & & & & & & & & & & & & & & & \\
\hline
\end{tabular}

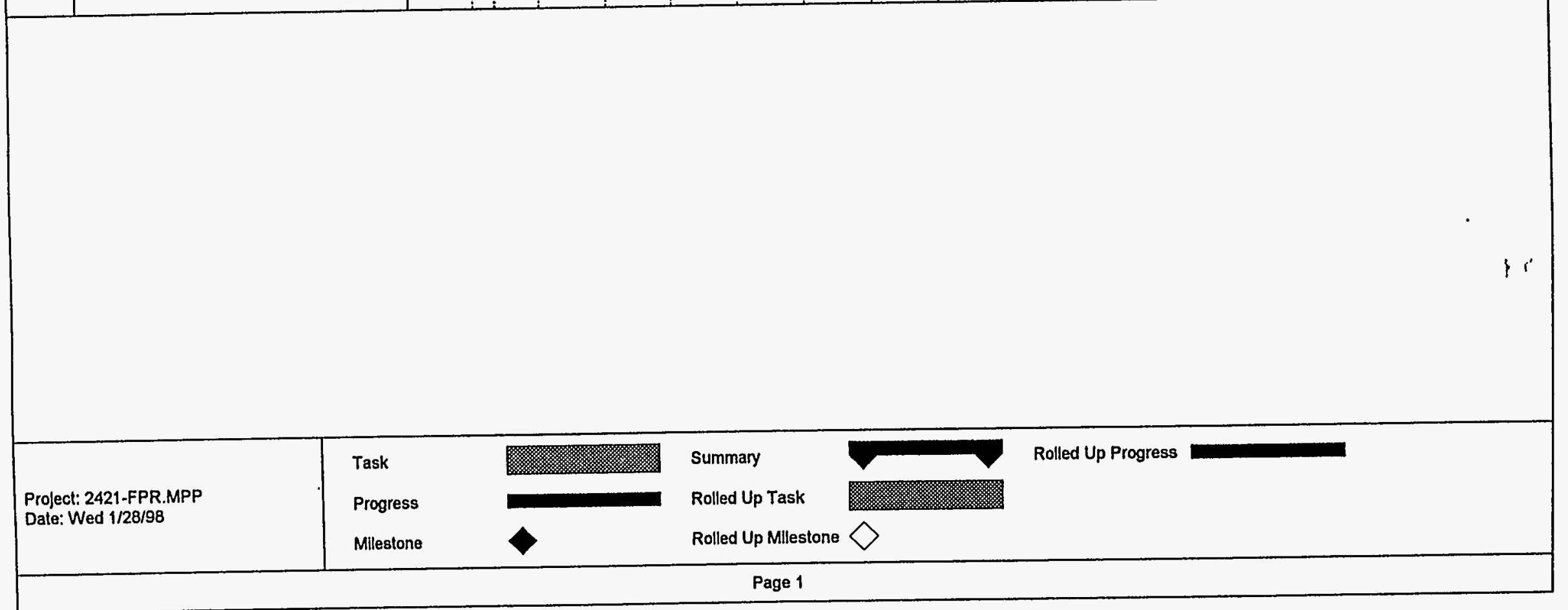


Date: $\quad$ February 3, 1998

$\begin{array}{llcc}\text { To: } & \text { John K. Rawlins } & \text { MS 3765 } & 6-4304 \\ \text { From: } & \text { R. J. Turk Lf lew }, & \text { MS } 3875 & 6-3611 \\ \text { Subject: } & \text { ECONOMIC AND LIFE CYCLE ANALYSIS CONDUCTED for the HIGH } \\ & \text { LEVEL WASTE (HLW) WTS INTERIM STORAGE FACILITY RJT-25-98 }\end{array}$

Purpose:

As requested an Economic and Life-Cycle Cost (LCC) has been conducted to evaluate for the HLW-WTS INTERIM STORAGE FACILITY. The storage facility was evaluated based on a scoping study that incorporate appropriate design features from the Flour Daniel Corp. design for interim storage. This storage facility will be used to house canisters resulting from the HLW production option until final repository disposition.

This economic analysis is based on information provided by J. K. Rawlins, and K. L. Williams. R. D. Adams provided cost estimates. B.L. Blakely and D.E. Keller provided process personnel modeling.

\section{Methodology:}

The Economic Evaluation assumed a 73-year period (1999-2071) since this is the estimated time required to complete all of the anticipated remediation activities. The LCC identified and evaluated the initial development, construction, operating and post operating costs over the lifecycle. A discounted LCC analysis assumes a 1998-dollar basis, discounted $6.3 \%$ annually per the Office of Management and Budget (OMB) Circular A-94 for escalated costs. All costs are conservatively discounted assuming the end-of-year convention. 


\section{Assumptions:}

The scope of work and requirements of all related activities are vague at this time. Facility and processing costs were developed from historical experience associated with DD\&D work at the INEEL. The LCC analysis was generated to match cost estimating cost structure. These costs include Permitting, Direct and Indirect Construction, G\&A, Procurement Fee, Engineering, Inspection, Project Management, Construction Management, Escalation and Contingency costs.

The design period is assumed to be accomplished in five-years with construction completed in two-years. Labor rates were assumed as follows: Managers, $\$ 125 / \mathrm{hr}$; Engineers, $\$ 108$ \$ $/ \mathrm{hr}$; Other Technicians $\$ 85 / \mathrm{hr}$; Administration/support staff $\$ 65 / \mathrm{hr}$; Operators and Maintenance personnel $\$ 65 / \mathrm{hr}$. The operational period for this facility was assumed to be twenty years, to match the appropriate operating facility. This period is followed by five years for shipping to the repository, followed by three years of post-operations activities. Due to this project's lack of complexity and non-radioactive contaminated environment, this analysis assumed a decommissioning cost equal to $10 \%$ of the non-escalated engineering design cost, decontamination costs equal to $0 \%$ of non-escalated total pre-operation cost, and demolition costs equal to $8 \%$ of the non-escalated total pre-operation cost. Utilities were calculated assuming a $\$ .0824$ per $\mathrm{kWh}$. Surveillance and Monitoring costs were calculated at $\$ 65,000$ per year for manned security during operations then reduced to $\$ 10,000$ for remote surveillance during non-operation periods.

\section{Results:}

The HIW-WTS INTERIM STORAGE FACILITY has a discounted LCC of $\$ 105$ million.

Attachments:

cc: R. J. Turk File 


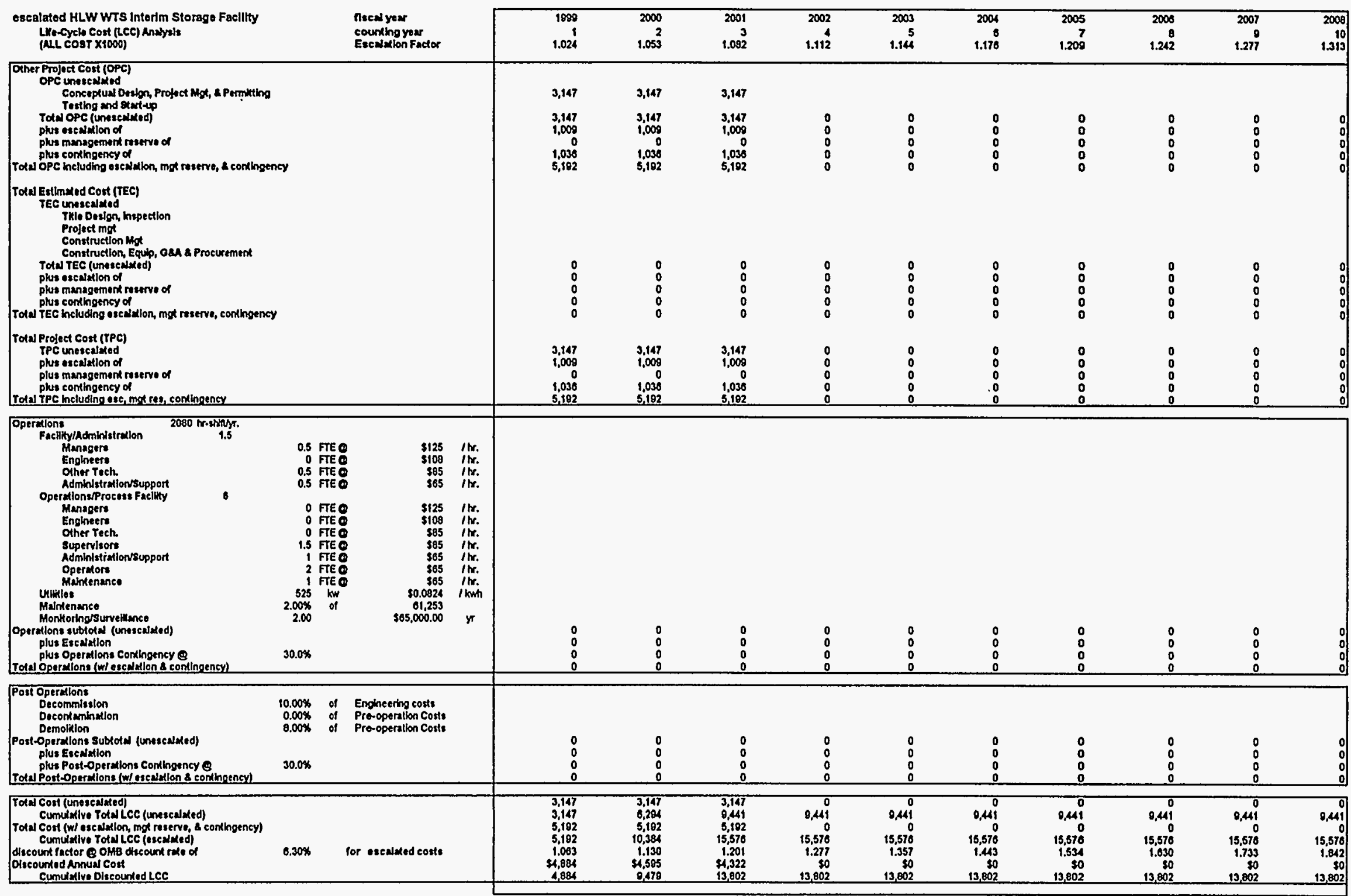




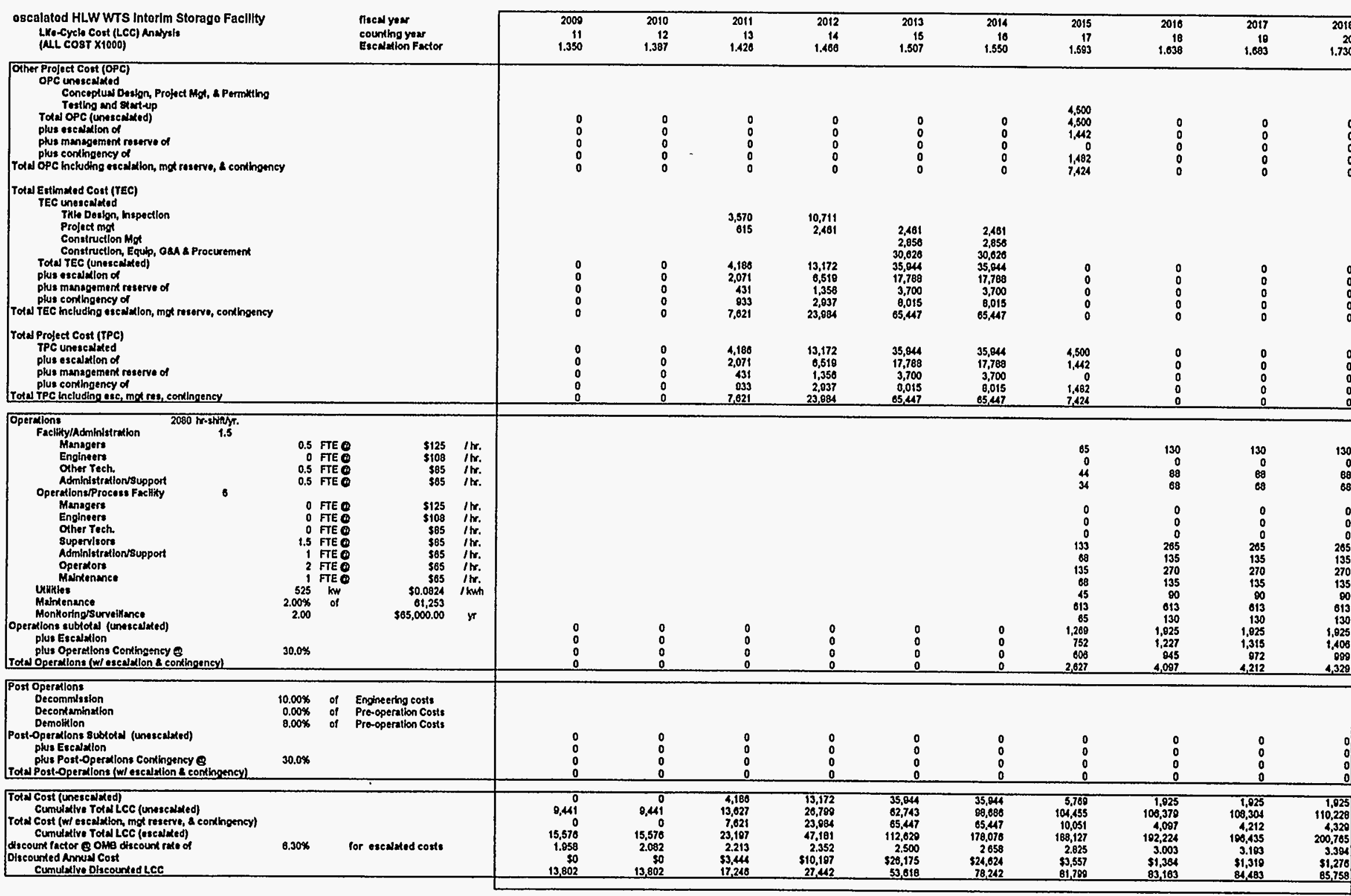




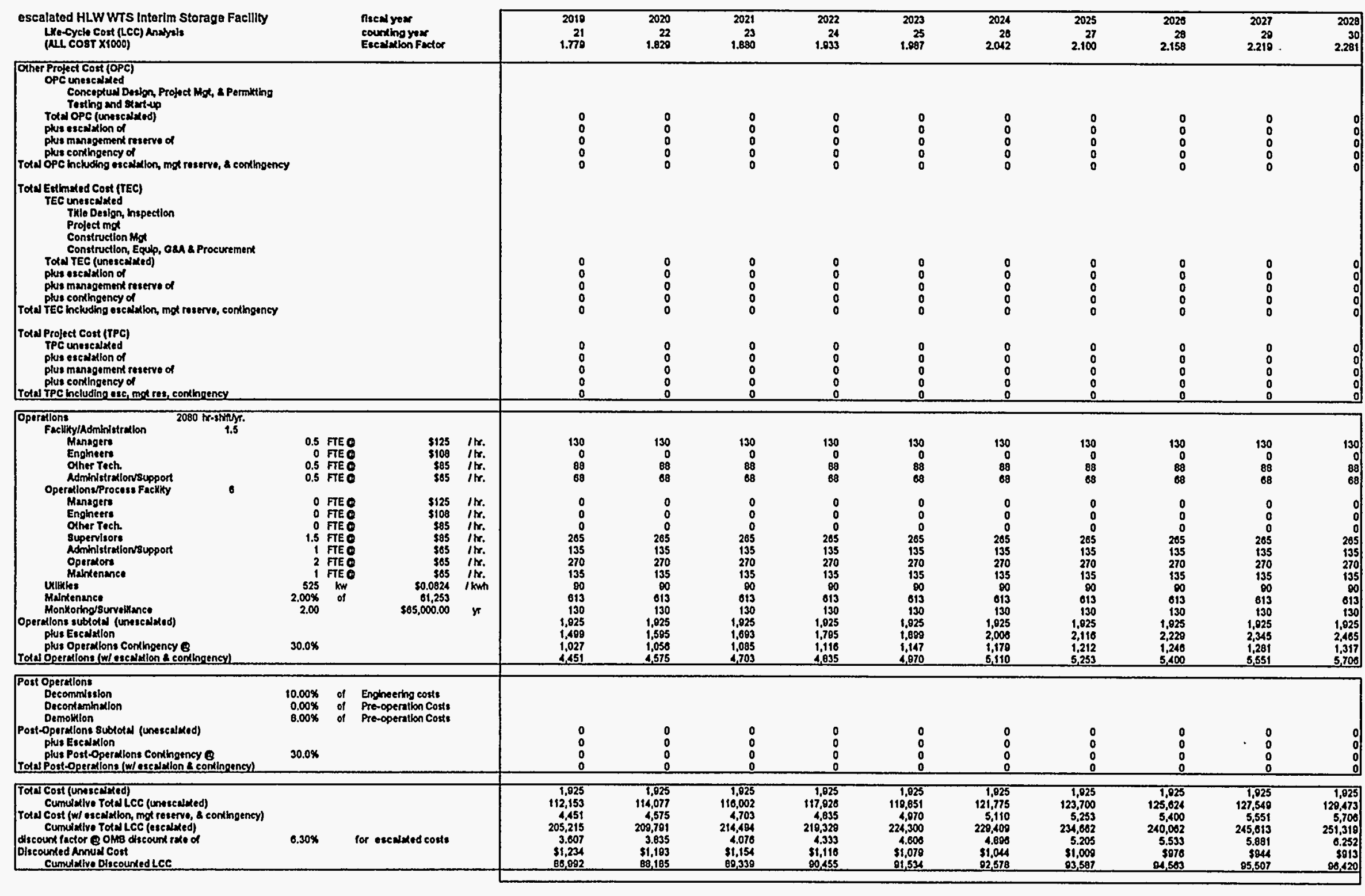




\begin{tabular}{|c|c|c|c|c|c|c|c|c|c|c|c|c|c|}
\hline \multicolumn{2}{|l|}{ 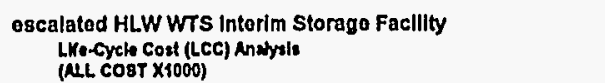 } & \multicolumn{2}{|l|}{$\begin{array}{l}\text { Riccal year } \\
\text { counting year } \\
\text { Eicalulon Factor }\end{array}$} & $\begin{array}{r}2029 \\
31 \\
2.345\end{array}$ & $\begin{array}{r}2030 \\
32 \\
2.410\end{array}$ & $\begin{array}{r}2031 \\
233 \\
2.478\end{array}$ & $\begin{array}{l}2032 \\
34 \\
2.547\end{array}$ & $\begin{array}{r}2003 \\
35 \\
2.818\end{array}$ & $\begin{array}{l}2034 \\
36 \\
2.082\end{array}$ & $\begin{array}{l}2035 \\
37 \\
2.767\end{array}$ & $\begin{array}{l}2036 \\
298 \\
2.045\end{array}$ & $\begin{array}{r}2037 \\
39 \\
2.024\end{array}$ & $\begin{array}{l}2030 \\
40 \\
3.006\end{array}$ \\
\hline \multicolumn{4}{|l|}{ 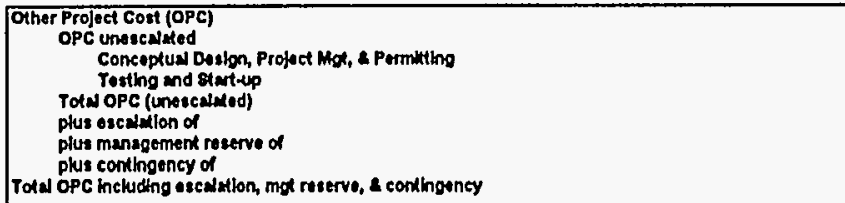 } & $\begin{array}{l}0 \\
0 \\
0 \\
0 \\
0\end{array}$ & $\begin{array}{l}0 \\
0 \\
0 \\
0 \\
0\end{array}$ & $\begin{array}{l}0 \\
0 \\
0 \\
0 \\
0\end{array}$ & $\begin{array}{l}0 \\
0 \\
0 \\
0 \\
0\end{array}$ & $\begin{array}{l}0 \\
0 \\
0 \\
0 \\
0\end{array}$ & $\begin{array}{l}0 \\
0 \\
0 \\
0 \\
0\end{array}$ & $\begin{array}{l}0 \\
0 \\
0 \\
0\end{array}$ & $\begin{array}{l}0 \\
0 \\
0 \\
0 \\
0\end{array}$ & $\begin{array}{l}0 \\
0 \\
0 \\
0 \\
0\end{array}$ & $\begin{array}{l}0 \\
0 \\
0 \\
0 \\
0\end{array}$ \\
\hline \multicolumn{4}{|l|}{ 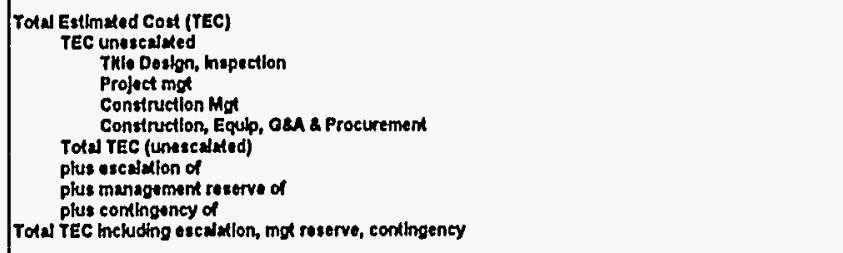 } & $\begin{array}{l}0 \\
0 \\
0 \\
0 \\
0\end{array}$ & $\begin{array}{l}0 \\
0 \\
0 \\
0 \\
0\end{array}$ & $\begin{array}{l}0 \\
0 \\
0 \\
0 \\
0\end{array}$ & $\begin{array}{l}0 \\
0 \\
0 \\
0 \\
0\end{array}$ & $\begin{array}{l}0 \\
0 \\
0 \\
0 \\
0\end{array}$ & $\begin{array}{l}0 \\
0 \\
0 \\
0 \\
0\end{array}$ & $\begin{array}{l}0 \\
0 \\
0 \\
0 \\
0\end{array}$ & $\begin{array}{l}0 \\
0 \\
0 \\
0 \\
0\end{array}$ & $\begin{array}{l}0 \\
0 \\
0 \\
0 \\
0\end{array}$ & $\begin{array}{l}0 \\
0 \\
0 \\
0 \\
0\end{array}$ \\
\hline \multicolumn{4}{|l|}{ 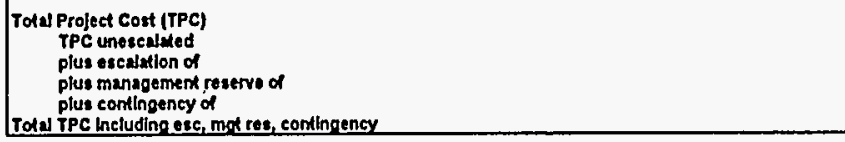 } & $\begin{array}{l}0 \\
0 \\
0 \\
0 \\
0 \\
\end{array}$ & $\begin{array}{l}0 \\
0 \\
0 \\
0 \\
0\end{array}$ & $\begin{array}{l}0 \\
0 \\
0 \\
0 \\
0 \\
\end{array}$ & $\begin{array}{l}0 \\
0 \\
0 \\
0 \\
0 \\
\end{array}$ & $\begin{array}{l}0 \\
\vdots \\
0 \\
0 \\
0\end{array}$ & $\begin{array}{l}0 \\
0 \\
0 \\
0 \\
0 \\
\end{array}$ & $\begin{array}{l}0 \\
0 \\
0 \\
0 \\
0 \\
\end{array}$ & $\begin{array}{l}0 \\
0 \\
0 \\
0 \\
0 \\
\end{array}$ & $\begin{array}{l}0 \\
0 \\
0 \\
0 \\
0 \\
\end{array}$ & $\begin{array}{l}0 \\
0 \\
0 \\
0 \\
0 \\
\end{array}$ \\
\hline \multirow{2}{*}{ 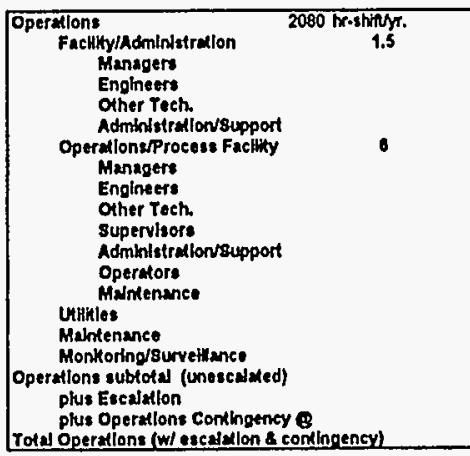 } & $\begin{array}{l}0.5 \text { FTE } \\
0 \text { FTE } \\
0.5 \text { FTE } \\
0.5 \text { FTE }\end{array}$ & $\begin{array}{c}\$ 125 \\
\$ 108 \\
\$ 85 \\
\$ 65\end{array}$ & $\begin{array}{l}\text { Ihr. } \\
\text { itr. } \\
\text { itro. } \\
\text { ine. }\end{array}$ & $\begin{array}{r}130 \\
0 \\
88 \\
88\end{array}$ & $\begin{array}{r}130 \\
80 \\
88 \\
68\end{array}$ & $\begin{array}{r}130 \\
0 \\
89 \\
80\end{array}$ & $\begin{array}{r}130 \\
0 \\
88 \\
88\end{array}$ & $\begin{array}{r}130 \\
8 \\
88 \\
80\end{array}$ & & & & & \\
\hline & $\begin{array}{rr}0 & \text { FTE } \\
0 & \text { FTE } \\
0 & \text { FTE } \\
1.5 & \text { FTE } \\
1 & \text { FTE } \\
2 & \text { FTE } \\
1 & \text { FTE } \\
525 & \mathrm{~kW} \\
2.00 \% & \text { of } \\
2.00 & \\
& \\
30.0 \% & \end{array}$ & $\begin{array}{r}\$ 125 \\
\$ 108 \\
\$ 85 \\
\$ 85 \\
\$ 85 \\
\$ 85 \\
\$ 85 \\
\$ 0.084 \\
\$ 0,253 \\
\$ 85,000.00\end{array}$ & 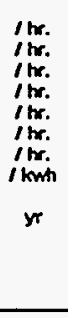 & $\begin{array}{r}0 \\
0 \\
0 \\
285 \\
135 \\
270 \\
135 \\
90 \\
813 \\
130 \\
1,925 \\
2,588 \\
1,354 \\
5,836 \\
\end{array}$ & $\begin{array}{r}0 \\
0 \\
0 \\
265 \\
135 \\
270 \\
135 \\
80 \\
613 \\
130 \\
1,925 \\
2,9714 \\
1,1932 \\
0,030 \\
\end{array}$ & $\begin{array}{r}0 \\
0 \\
0 \\
205 \\
135 \\
270 \\
135 \\
90 \\
013 \\
130 \\
1,925 \\
2,924 \\
1,431 \\
0.198 \\
\end{array}$ & $\begin{array}{r}0 \\
0 \\
0 \\
285 \\
135 \\
270 \\
135 \\
800 \\
613 \\
130 \\
1,025 \\
2,078 \\
1,471 \\
0,373 \\
\end{array}$ & $\begin{array}{r}0 \\
0 \\
0 \\
205 \\
135 \\
270 \\
135 \\
80 \\
613 \\
1130 \\
1,025 \\
3,115 \\
1.512 \\
0,551 \\
\end{array}$ & $\begin{array}{r}10 \\
10 \\
17 \\
8 \\
35 \\
\end{array}$ & $\begin{array}{r}10 \\
10 \\
18 \\
8 \\
38 \\
\end{array}$ & $\begin{array}{r}10 \\
10 \\
18 \\
8 \\
37 \\
\end{array}$ & $\begin{array}{r}10 \\
10 \\
19 \\
9 \\
30 \\
\end{array}$ & $\begin{array}{r}10 \\
10 \\
20 \\
30 \\
39 \\
\end{array}$ \\
\hline 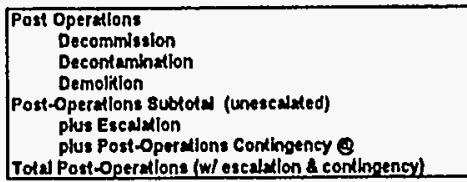 & $\begin{array}{ll}10.00 \% & \text { of } \\
0.00 \% & \text { of } \\
0.00 \% & \text { of } \\
30.0 \% & \end{array}$ & $\begin{array}{l}\text { Engheering costs } \\
\text { Pro-operatilon Costs } \\
\text { Pro-operatilon Costs }\end{array}$ & & $\begin{array}{l}0 \\
0 \\
0 \\
0 \\
\end{array}$ & $\begin{array}{l}0 \\
0 \\
0 \\
0\end{array}$ & $\begin{array}{l}0 \\
0 \\
0 \\
0 \\
\end{array}$ & $\begin{array}{l}0 \\
\vdots \\
0 \\
0\end{array}$ & $\begin{array}{l}0 \\
0 \\
0 \\
0 \\
\end{array}$ & $\begin{array}{l}0 \\
0 \\
0 \\
0 \\
\end{array}$ & $\begin{array}{l}0 \\
0 \\
0 \\
0 \\
\end{array}$ & $\begin{array}{l}0 \\
\vdots \\
0 \\
0\end{array}$ & $\begin{array}{l}0 \\
0 \\
0 \\
0 \\
\end{array}$ & $\begin{array}{l}0 \\
0 \\
0 \\
0 \\
0\end{array}$ \\
\hline 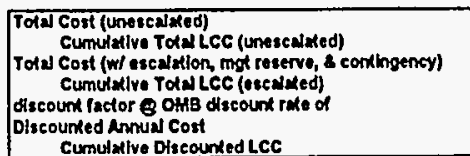 & $0.30 \%$ & lor ascalwed costs & & 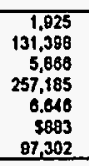 & 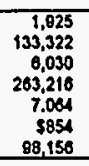 & 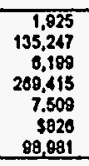 & 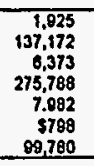 & 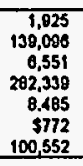 & $\begin{array}{r}10 \\
130,108 \\
35 \\
282,374 \\
0.020 \\
4 \\
100,558 \\
\end{array}$ & $\begin{array}{r}10 \\
130,118 \\
36 \\
282,410 \\
0,588 \\
84 \\
100,580 \\
100\end{array}$ & $\begin{array}{r}10 \\
130,120 \\
37 \\
282,47 \\
10,4192 \\
84 \\
100,503 \\
\end{array}$ & $\begin{array}{r}10 \\
139,138 \\
38 \\
202,485 \\
10,84 \\
4 \\
100,567 \\
\end{array}$ & $\begin{array}{r}100 \\
139,140 \\
39 \\
202,524 \\
11.517 \\
100,50 \\
100 \\
\end{array}$ \\
\hline
\end{tabular}




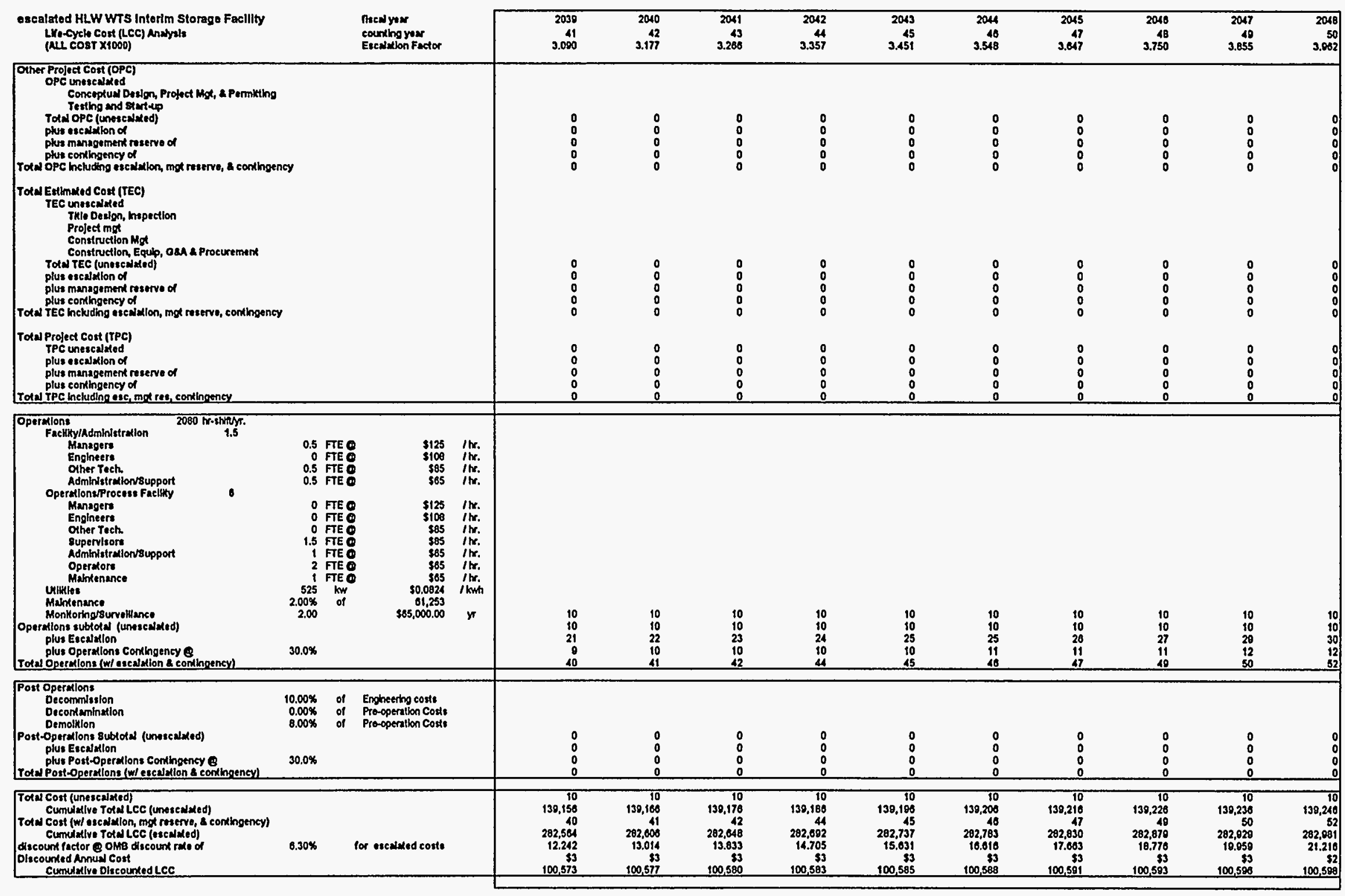




\begin{tabular}{|c|c|c|c|c|c|c|c|c|c|c|c|c|}
\hline 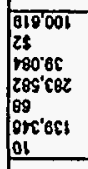 & 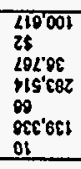 & 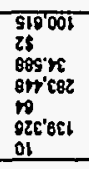 & 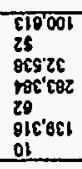 & 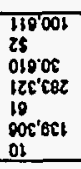 & 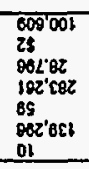 & 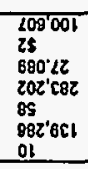 & 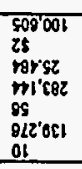 & 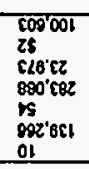 & 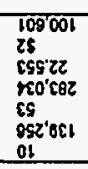 & \multicolumn{2}{|c|}{ 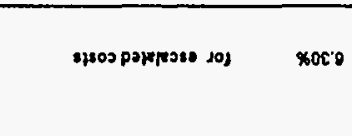 } & 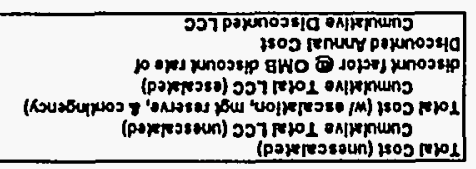 \\
\hline $\mid \begin{array}{l}0 \\
0 \\
0 \\
0\end{array}$ & $\begin{array}{l}0 \\
0 \\
0 \\
0\end{array}$ & $\begin{array}{l}0 \\
0 \\
0 \\
0\end{array}$ & $\begin{array}{l}0 \\
0 \\
0 \\
0\end{array}$ & $\begin{array}{l}0 \\
0 \\
0 \\
0\end{array}$ & $\begin{array}{l}0 \\
0 \\
0 \\
0\end{array}$ & $\begin{array}{l}0 \\
\vdots \\
\vdots \\
0\end{array}$ & $\begin{array}{l}1 \\
\vdots \\
0\end{array}$ & $\begin{array}{l}0 \\
\vdots \\
0\end{array}$ & $\begin{array}{l}0 \\
\vdots \\
\vdots \\
0\end{array}$ & 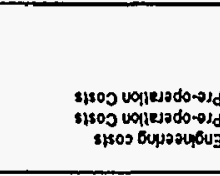 & 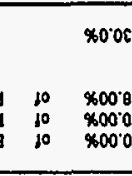 & 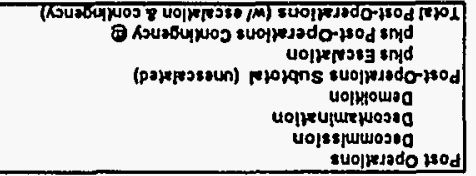 \\
\hline 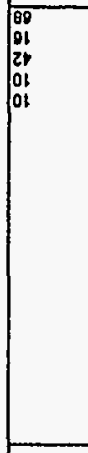 & $\begin{array}{l}89 \\
\text { s9 } \\
\text { il } \\
\text { of } \\
\text { il }\end{array}$ & 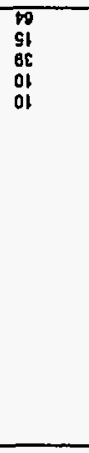 & $\begin{array}{l}78 \\
\text { po } \\
\text { of } \\
\text { of } \\
\text { of }\end{array}$ & 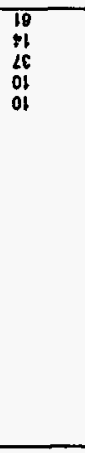 & $\begin{array}{l}855 \\
\text { sit } \\
\text { sit } \\
01 \\
01\end{array}$ & $\begin{array}{ll}85 & 51 \\
58 \\
08 \\
o f \\
o f\end{array}$ & $\begin{array}{l}95 \\
\text { ss } \\
\text { cf } \\
\text { of } \\
\text { of }\end{array}$ & 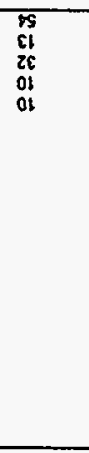 & 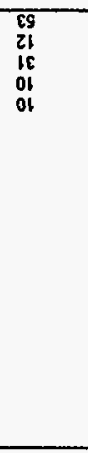 & 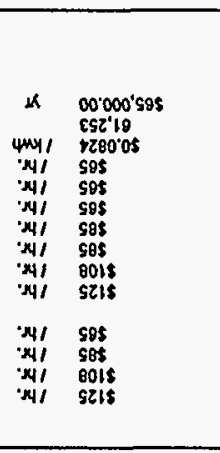 & 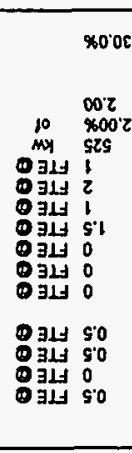 & 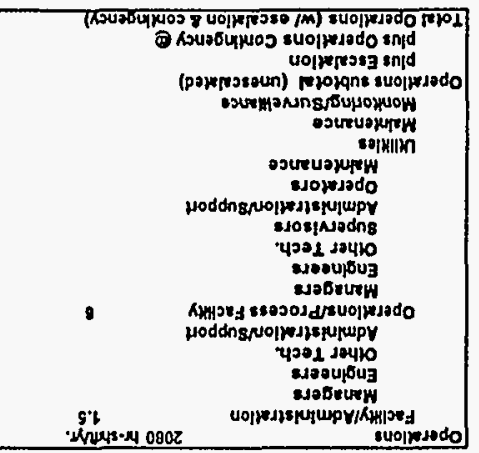 \\
\hline $\begin{array}{l}0 \\
0 \\
0 \\
0 \\
0\end{array}$ & $\begin{array}{l}0 \\
0 \\
0 \\
0 \\
0\end{array}$ & $\begin{array}{l}0 \\
0 \\
0 \\
0 \\
0\end{array}$ & $\begin{array}{l} \\
0 \\
0 \\
0 \\
0\end{array}$ & $\begin{array}{l}0 \\
0 \\
0 \\
0 \\
0\end{array}$ & $\begin{array}{l}0 \\
0 \\
0 \\
0 \\
0\end{array}$ & $\begin{array}{l}0 \\
0 \\
0 \\
0 \\
0\end{array}$ & $\begin{array}{l}0 \\
0 \\
0 \\
0 \\
0\end{array}$ & $\begin{array}{c}0 \\
0 \\
0 \\
0 \\
0\end{array}$ & $\begin{array}{l}0 \\
0 \\
0 \\
0 \\
0\end{array}$ & & & 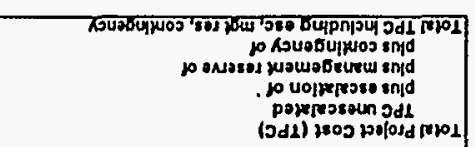 \\
\hline $\mid \begin{array}{l}0 \\
0 \\
0 \\
0 \\
0 \\
0\end{array}$ & $\begin{array}{l}0 \\
0 \\
0 \\
0 \\
0\end{array}$ & $\begin{array}{l}0 \\
0 \\
0 \\
0 \\
0\end{array}$ & $\begin{array}{l}0 \\
0 \\
0 \\
0 \\
0\end{array}$ & $\begin{array}{l}0 \\
0 \\
0 \\
0 \\
0\end{array}$ & $\begin{array}{l}0 \\
0 \\
0 \\
0 \\
0\end{array}$ & $\begin{array}{l}0 \\
0 \\
\vdots \\
0\end{array}$ & $\begin{array}{l}0 \\
0 \\
0 \\
0 \\
0\end{array}$ & $\begin{array}{l}0 \\
0 \\
0 \\
0 \\
0\end{array}$ & $\begin{array}{l}0 \\
0 \\
0 \\
0 \\
0\end{array}$ & & & 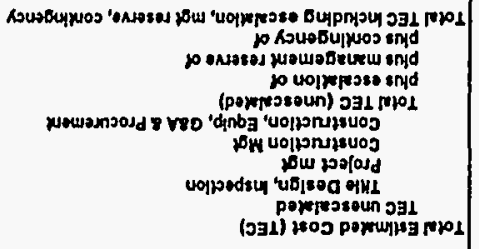 \\
\hline $\mid \begin{array}{l}0 \\
0 \\
0 \\
0 \\
0\end{array}$ & $\begin{array}{l}0 \\
0 \\
0 \\
0 \\
0\end{array}$ & $\begin{array}{l}0 \\
0 \\
0 \\
0 \\
0\end{array}$ & $\begin{array}{l}0 \\
0 \\
0 \\
0 \\
0\end{array}$ & $\begin{array}{l}0 \\
0 \\
0 \\
0 \\
0\end{array}$ & $\begin{array}{l}0 \\
0 \\
0 \\
0 \\
0\end{array}$ & $\begin{array}{l}0 \\
0 \\
0 \\
0 \\
0\end{array}$ & $\begin{array}{l}0 \\
0 \\
0 \\
0 \\
0\end{array}$ & $\begin{array}{l}0 \\
0 \\
0 \\
0 \\
0\end{array}$ & $\begin{array}{l}0 \\
0 \\
0 \\
0 \\
0\end{array}$ & & & 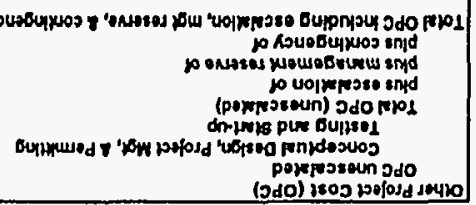 \\
\hline 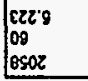 & $\begin{array}{l}\text { O80\% } \\
\text { BS } \\
2502\end{array}$ & 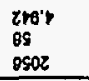 & $\begin{array}{l}l_{1008}^{20} \\
\angle 590 z\end{array}$ & $\begin{array}{l}400 \% \\
09 \\
5002\end{array}$ & 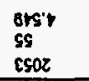 & $\begin{array}{l}\text { szry } \\
\substack{n \\
z s 0 z}\end{array}$ & $\begin{array}{c}\text { soci } \\
\text { sc } \\
1502\end{array}$ & $\begin{array}{l}\frac{18.4}{28} \\
0 \leq 528\end{array}$ & 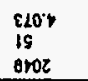 & 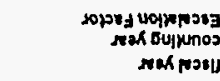 & & 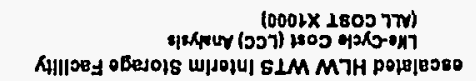 \\
\hline
\end{tabular}




\begin{tabular}{|c|c|c|c|c|c|c|c|c|c|c|c|c|c|}
\hline 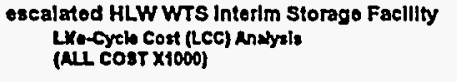 & & 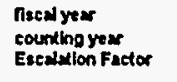 & & $\begin{array}{l}2050 \\
61 \\
5.360\end{array}$ & $\begin{array}{r}2080 \\
62 \\
5.510\end{array}$ & $\begin{array}{l}2001 \\
63 \\
5.674\end{array}$ & $\begin{array}{l}2002 \\
5.44 \\
5.033\end{array}$ & $\begin{array}{l}2083 \\
65 \\
5.820\end{array}$ & $\begin{array}{l}2004 \\
000 \\
0.194\end{array}$ & $\begin{array}{l}2005 \\
87 \\
0.336\end{array}$ & $\begin{array}{l}2005 \\
68 \\
0.514\end{array}$ & $\begin{array}{l}2007 \\
69 \\
0.098\end{array}$ & $\begin{array}{r}2008 \\
70 \\
0.834\end{array}$ \\
\hline \multicolumn{4}{|c|}{ 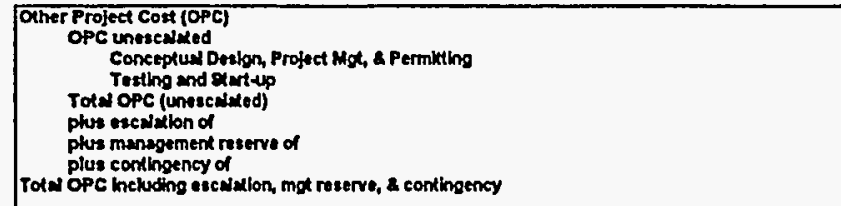 } & $\begin{array}{l}0 \\
0 \\
0 \\
0 \\
0\end{array}$ & $\begin{array}{l}0 \\
: \\
0 \\
0\end{array}$ & $\begin{array}{l}0 \\
0 \\
0 \\
0\end{array}$ & $\begin{array}{l}0 \\
0 \\
0 \\
0 \\
0\end{array}$ & $\begin{array}{l}0 \\
0 \\
0 \\
0 \\
0\end{array}$ & $\begin{array}{l}0 \\
0 \\
0 \\
0 \\
0\end{array}$ & $\begin{array}{l}0 \\
0 \\
0 \\
0 \\
0\end{array}$ & $\begin{array}{l}0 \\
0 \\
0 \\
0 \\
0\end{array}$ & $\begin{array}{l}0 \\
0 \\
0 \\
0 \\
0\end{array}$ & $\begin{array}{l}0 \\
0 \\
0 \\
0 \\
0\end{array}$ \\
\hline \multicolumn{4}{|l|}{ 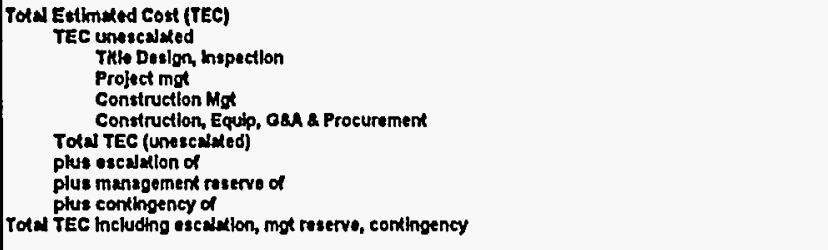 } & $\begin{array}{l}0 \\
0 \\
0 \\
0 \\
0\end{array}$ & $\begin{array}{l}0 \\
0 \\
0 \\
0 \\
0\end{array}$ & $\begin{array}{l}0 \\
0 \\
0 \\
0 \\
0\end{array}$ & $\begin{array}{l}0 \\
0 \\
0 \\
0 \\
0\end{array}$ & $\begin{array}{l}0 \\
0 \\
0 \\
0\end{array}$ & $\begin{array}{l}0 \\
0 \\
0 \\
0 \\
0\end{array}$ & $\begin{array}{l}0 \\
0 \\
0 \\
0 \\
0\end{array}$ & $\begin{array}{l}0 \\
0 \\
0 \\
0 \\
0\end{array}$ & $\begin{array}{l}0 \\
0 \\
0 \\
0 \\
0\end{array}$ & $\begin{array}{l}0 \\
0 \\
0 \\
0 \\
0\end{array}$ \\
\hline \multicolumn{4}{|l|}{ 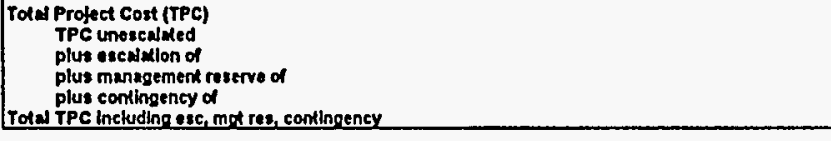 } & $\begin{array}{l}0 \\
0 \\
0 \\
0 \\
0 \\
\end{array}$ & $\begin{array}{l}0 \\
0 \\
0 \\
0 \\
0\end{array}$ & $\begin{array}{l}0 \\
0 \\
0 \\
0 \\
0 \\
\end{array}$ & $\begin{array}{l}0 \\
0 \\
0 \\
0 \\
0 \\
\end{array}$ & $\begin{array}{l}0 \\
0 \\
0 \\
0 \\
0\end{array}$ & $\begin{array}{l}0 \\
0 \\
0 \\
0 \\
0 \\
\end{array}$ & $\begin{array}{l}0 \\
0 \\
0 \\
0 \\
0 \\
\end{array}$ & $\begin{array}{l}0 \\
0 \\
0 \\
0 \\
0\end{array}$ & $\begin{array}{l}0 \\
0 \\
0 \\
0 \\
0 \\
\end{array}$ & $\begin{array}{l}0 \\
0 \\
0 \\
0 \\
0 \\
\end{array}$ \\
\hline 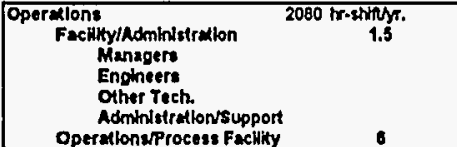 & $\begin{array}{r}0.5 \text { FTE } \\
0 \text { TTE } \\
0.5 \text { FTE } \\
0.5 \text { FTE }\end{array}$ & $\begin{array}{c}\$ 125 \\
\$ 108 \\
\$ 85 \\
\$ 65\end{array}$ & $\begin{array}{l}\text { Ihr. } \\
\text { /hr. } \\
\text { Ihr. } \\
\text { Ihr. }\end{array}$ & & & & & & $\begin{array}{r}130 \\
0 \\
88 \\
88\end{array}$ & $\begin{array}{r}130 \\
0 \\
88 \\
88\end{array}$ & $\begin{array}{r}130 \\
0 \\
80 \\
80\end{array}$ & $\begin{array}{r}130 \\
0 \\
88 \\
68\end{array}$ & $\begin{array}{r}130 \\
0 \\
89 \\
68\end{array}$ \\
\hline 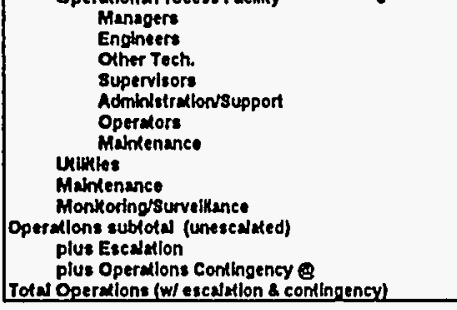 & 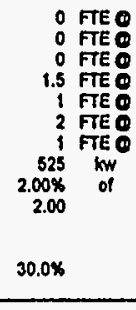 & $\begin{array}{r}\$ 125 \\
\$ \$ 108 \\
\$ 15 \\
\$ 85 \\
\$ 65 \\
\$ 65 \\
\$ 05 \\
\$ 05 \\
\$ 0.0024 \\
0.1,253 \\
\$ 05,000.00\end{array}$ & 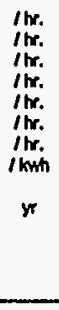 & $\begin{array}{l}10 \\
10 \\
44 \\
16 \\
70 \\
\end{array}$ & $\begin{array}{l}10 \\
10 \\
15 \\
17 \\
72 \\
\end{array}$ & $\begin{array}{l}10 \\
10 \\
47 \\
17 \\
74 \\
\end{array}$ & $\begin{array}{l}10 \\
10 \\
48 \\
17 \\
70 \\
\end{array}$ & $\begin{array}{l}10 \\
10 \\
50 \\
18 \\
78 \\
\end{array}$ & $\begin{array}{r}0 \\
0 \\
0 \\
285 \\
135 \\
270 \\
135 \\
90 \\
013 \\
013 \\
130 \\
1,025 \\
0,038 \\
3 ., 559 \\
15,421 \\
\end{array}$ & $\begin{array}{r}0 \\
0 \\
205 \\
205 \\
135 \\
270 \\
135 \\
90 \\
0913 \\
130 \\
1,025 \\
10,270 \\
3,658 \\
15,653 \\
\end{array}$ & $\begin{array}{r}0 \\
0 \\
0 \\
205 \\
135 \\
270 \\
135 \\
00 \\
013 \\
130 \\
130 \\
10,025 \\
10,011 \\
3,701 \\
10,207 \\
\end{array}$ & $\begin{array}{r}0 \\
0 \\
0 \\
205 \\
135 \\
270 \\
135 \\
90 \\
813 \\
130 \\
1,025 \\
10,802 \\
3,860 \\
10,753 \\
\end{array}$ & 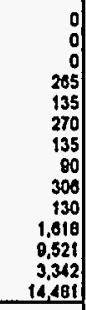 \\
\hline 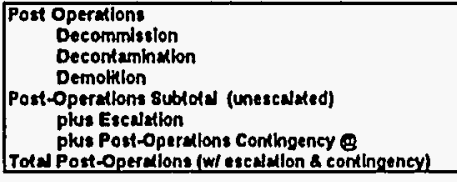 & 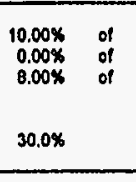 & $\begin{array}{l}\text { Engheering couts } \\
\text { Propopertin Cots } \\
\text { Pro-operation Costs }\end{array}$ & & $\begin{array}{l}0 \\
0 \\
0 \\
0 \\
\end{array}$ & $\begin{array}{l}0 \\
0 \\
0 \\
0 \\
\end{array}$ & $\begin{array}{l}0 \\
0 \\
0 \\
0 \\
\end{array}$ & $\begin{array}{l}0 \\
0 \\
0 \\
0 \\
\end{array}$ & $\begin{array}{l}0 \\
0 \\
0 \\
0 \\
\end{array}$ & $\begin{array}{l}0 \\
0 \\
0 \\
0\end{array}$ & $\begin{array}{l}0 \\
0 \\
0 \\
0 \\
\end{array}$ & $\begin{array}{l}0 \\
0 \\
0 \\
0 \\
0\end{array}$ & $\begin{array}{l}0 \\
0 \\
0 \\
0 \\
\end{array}$ & $\begin{array}{l}0 \\
0 \\
0 \\
0\end{array}$ \\
\hline 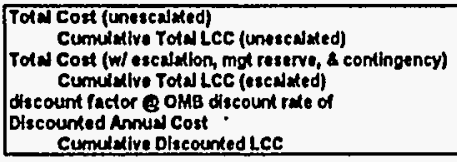 & $0.30 \%$ & for escumed coste & & $\begin{array}{r}10 \\
130,350 \\
70 \\
203,052 \\
41,540 \\
\$ 52 \\
100,020 \\
\end{array}$ & $\begin{array}{r}130 \\
130,360 \\
72 \\
283,724 \\
44,103 \\
82 \\
100,822 \\
\end{array}$ & 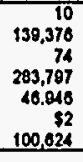 & $\begin{array}{r}10 \\
138,380 \\
2030 \\
293,073 \\
48.003 \\
52 \\
100,025 \\
\end{array}$ & $\begin{array}{r}130,308 \\
78 \\
283,051 \\
53.047 \\
\$ 41 \\
100,8227 \\
\end{array}$ & $\begin{array}{r}1,025 \\
141,321 \\
15,421 \\
209,372 \\
50.389 \\
5273 \\
100,900 \\
\end{array}$ & $\begin{array}{r}1,025 \\
143,245 \\
15,253 \\
315,225 \\
59.242 \\
5204 \\
101,165 \\
\end{array}$ & 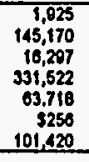 & $\begin{array}{r}1,025 \\
147,004 \\
10,753 \\
361,275 \\
67,732 \\
\$ 247 \\
101,088 \\
\end{array}$ & $\begin{array}{r}1,618 \\
148,712 \\
14,491 \\
302,757 \\
71,028 \\
55201 \\
101,609 \\
\end{array}$ \\
\hline
\end{tabular}




\begin{tabular}{|c|c|c|c|c|c|c|c|}
\hline $\begin{array}{l}\text { oscalatod HLW WTS Intorlm Storago Faclity } \\
\text { Lhe-Cycte Cost (LCC) Analyets } \\
\text { (HLL COBT X1000) }\end{array}$ & & 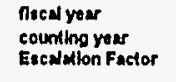 & & $\begin{array}{r}2009 \\
71 \\
7.076\end{array}$ & $\begin{array}{r}2070 \\
72 \\
7.275\end{array}$ & $\begin{array}{r}2071 \\
73 \\
7.478\end{array}$ & $\begin{array}{l}\text { Total } \\
\text { cost }\end{array}$ \\
\hline \multicolumn{4}{|l|}{ 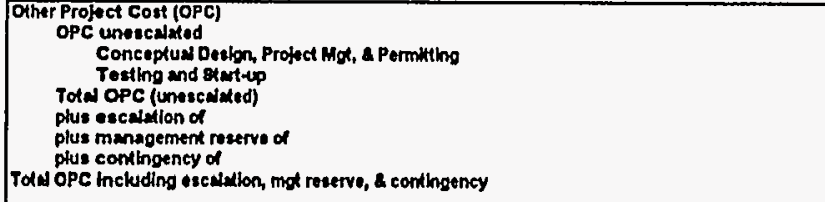 } & $\begin{array}{l}0 \\
0 \\
0 \\
0 \\
0\end{array}$ & $\begin{array}{l}0 \\
0 \\
0 \\
0 \\
0\end{array}$ & 骂 & $\begin{array}{r}0,441 \\
4,600 \\
13,411 \\
4,480 \\
1,50 \\
23,000\end{array}$ \\
\hline \multicolumn{4}{|l|}{ 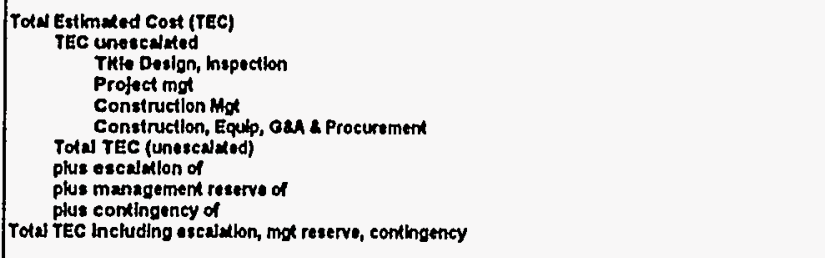 } & $\begin{array}{l}0 \\
0 \\
0 \\
0 \\
0\end{array}$ & $\begin{array}{l}0 \\
0 \\
0 \\
0 \\
0\end{array}$ & 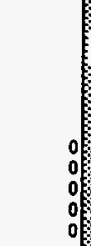 & $\begin{array}{r}14,281 \\
7,908 \\
5,713 \\
61,253 \\
89,245 \\
44,248 \\
9,168 \\
10,280 \\
102,500\end{array}$ \\
\hline \multicolumn{4}{|l|}{ 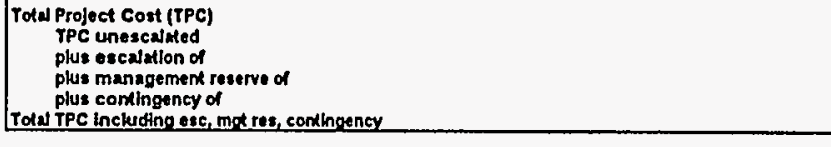 } & $\begin{array}{r}0 \\
0 \\
0 \\
0 \\
0 \\
\end{array}$ & $\begin{array}{l}0 \\
0 \\
0 \\
0 \\
0 \\
\end{array}$ & $\begin{array}{l}\text { of } \\
0 \\
0 \\
0 \\
0 \\
0\end{array}$ & $\begin{array}{r}103,188 \\
48,634 \\
8,168 \\
24,480 \\
185,300 \\
\end{array}$ \\
\hline \multirow{2}{*}{ 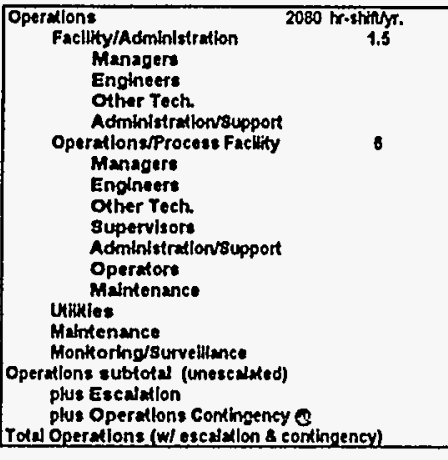 } & $\begin{array}{l}0.5 \text { FEE } \\
0 \text { FTE } \\
0.5 \text { TEE } \\
0.5 \text { FTE }\end{array}$ & $\begin{array}{l}\$ 125 \\
\$ 108 \\
\$ 85 \\
\$ 85\end{array}$ & 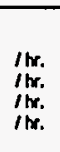 & & & & $\begin{array}{r}3,055 \\
2,077 \\
1,5689\end{array}$ \\
\hline & 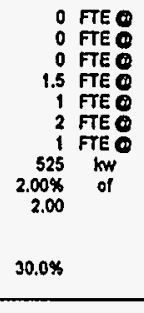 & $\begin{array}{r}\$ 125 \\
\$ 108 \\
\$ 85 \\
\$ 85 \\
\$ 65 \\
\$ 65 \\
\$ 65 \\
\$ 0.05 \\
\$ 01,253 \\
\$ 61,253 \\
\$ 65,000.00\end{array}$ & 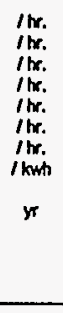 & $\begin{array}{l}0 \\
0 \\
0 \\
0 \\
\end{array}$ & $\begin{array}{l}0 \\
0 \\
0 \\
0\end{array}$ & $\begin{array}{l}0 . \\
0 \\
0\end{array}$ & 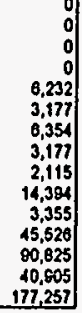 \\
\hline 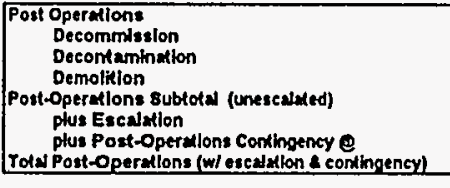 & $\begin{aligned} 10.00 \% & \text { of } \\
0.00 \% & \text { of } \\
8.00 \% & \text { of } \\
30.0 \% & \end{aligned}$ & $\begin{array}{l}\text { Engheering costs } \\
\text { Pro-operation Costs } \\
\text { Pre-operation Costs }\end{array}$ & & $\begin{array}{r}1,428 \\
0 \\
0,255 \\
0,683 \\
58,839 \\
20,557 \\
80,079 \\
\end{array}$ & $\begin{array}{r}1,428 \\
0 \\
8,255 \\
8,883 \\
60,758 \\
21,132 \\
81,573 \\
\end{array}$ & 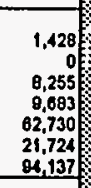 & 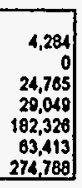 \\
\hline 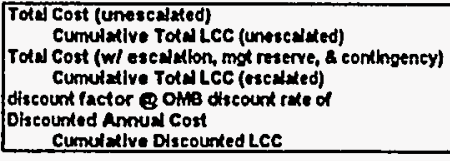 & $0.30 \%$ & lor modeted conts & & $\begin{array}{r}9 ., 883 \\
158,385 \\
89,050 \\
451,035 \\
76.535 \\
\$ 1,184 \\
103,033 \\
\end{array}$ & 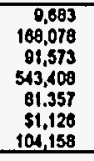 & 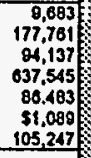 & $\begin{array}{l}177,761 \\
637,545 \\
105,247\end{array}$ \\
\hline
\end{tabular}




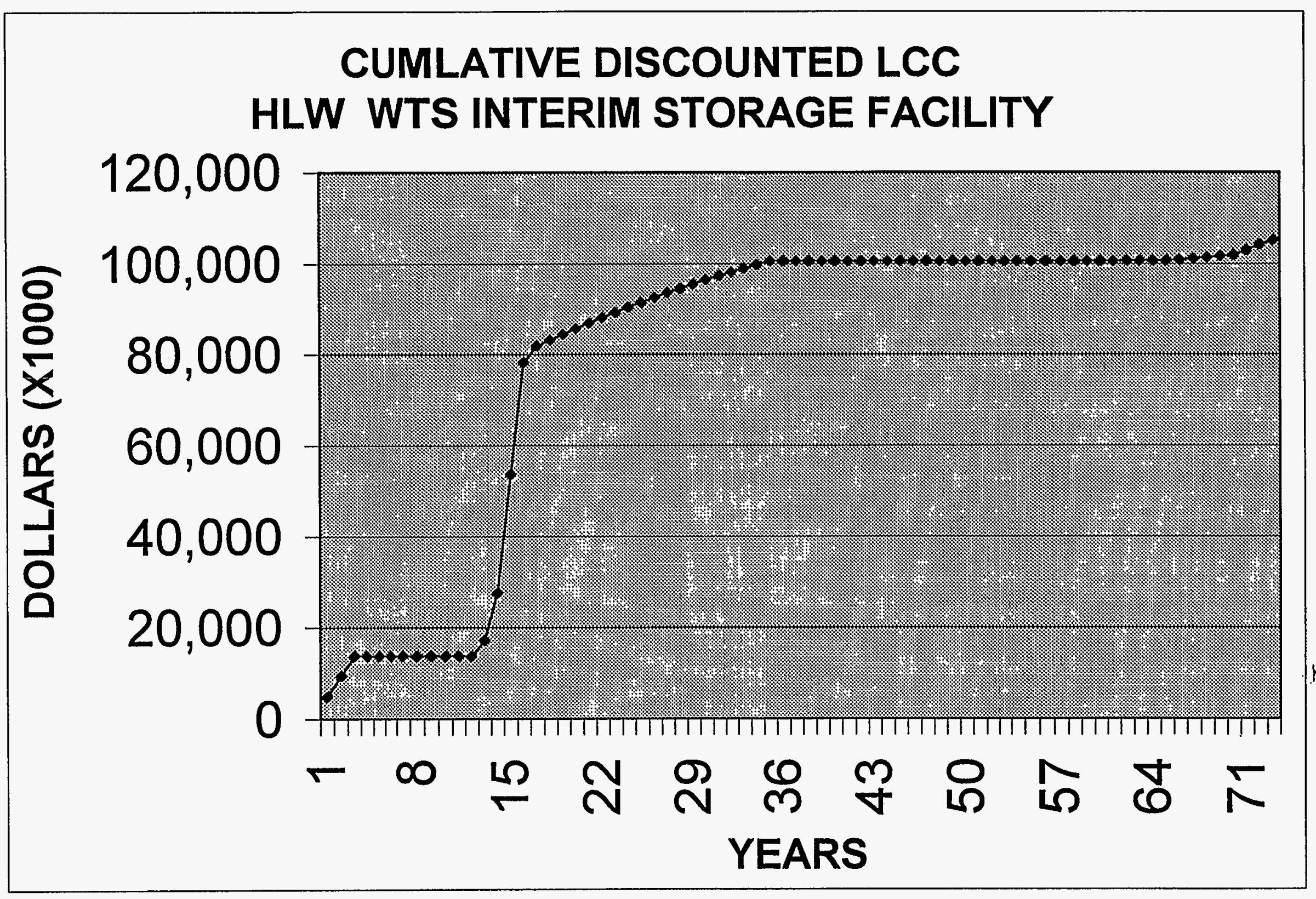




\section{TOTAL ANNUAL COST (escalated) HLW WTS INTERIM STORAGE FACILTY}

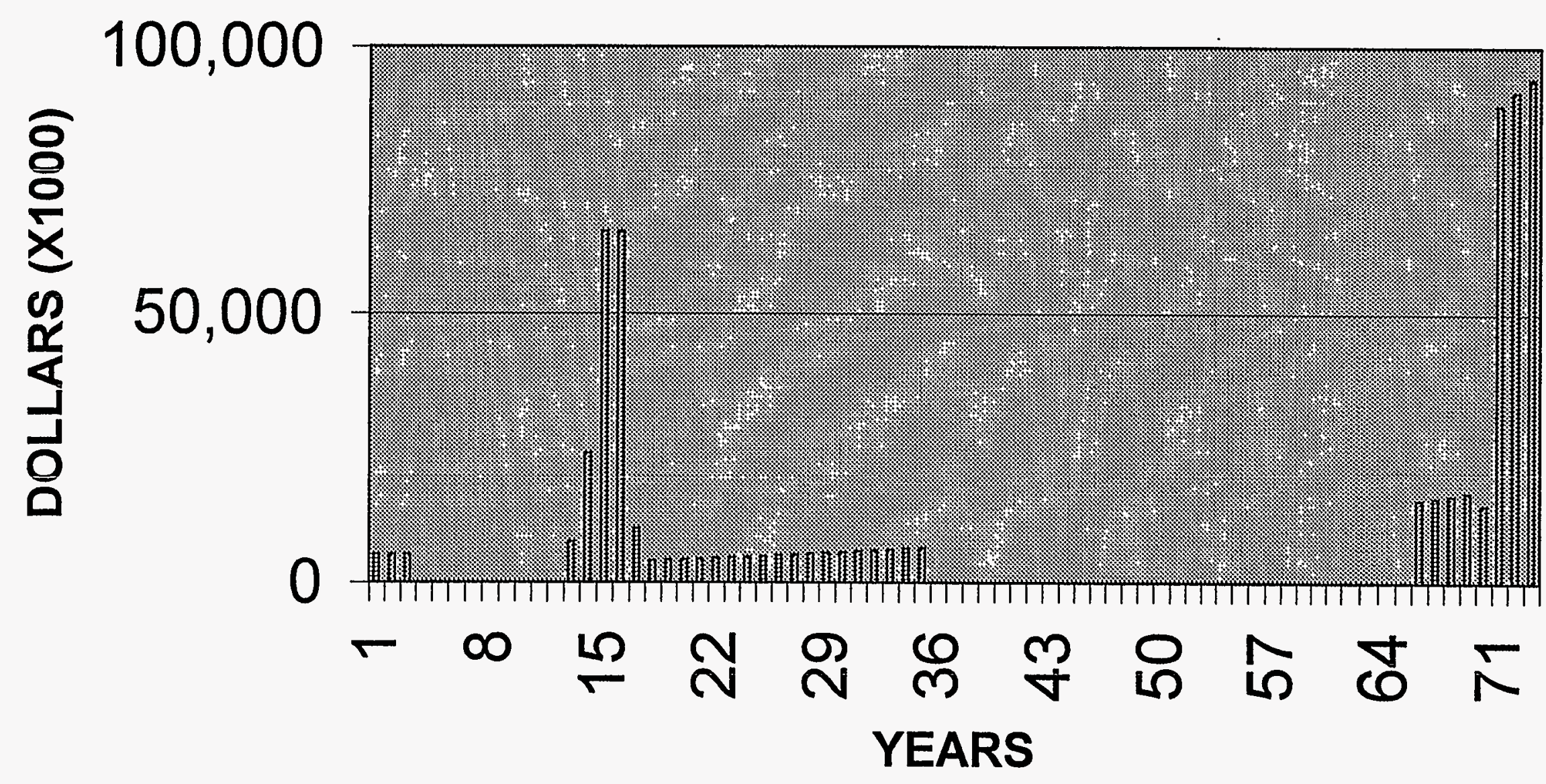




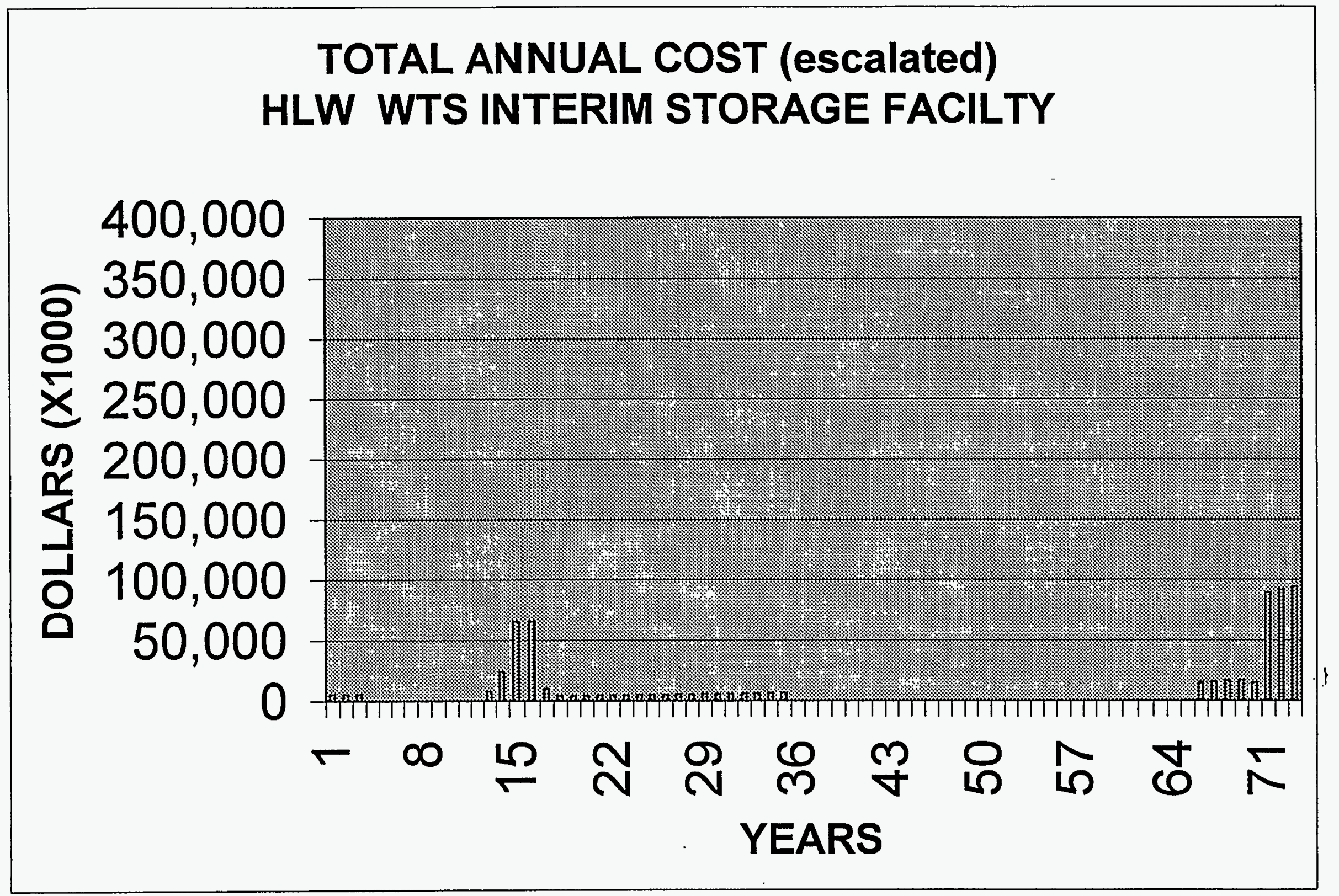


Date: $\quad$ February 3, 1998

To: $\quad$ John K. Rawlins $\quad$ MS 3765 6-4304

From: R. J.Turk 6-3611

Subject: $\quad$ ECONOMIC AND LIFE CYCLE ANALYSIS CONDUCTED for the HIGH LEVEL WASTE (HLW) in FPR INTERIM STORAGE FACIITY-TRU RJT-24-98

Purpose:

As requested an Economic and Life-Cycle Cost (LCC) has been conducted to evaluate for the HLW-TRU (in FPR) INTERIM STORAGE FACILITY. The storage facility was evaluated based on a scoping study that incorporate appropriate design features from the Flour Daniel Corp. design for interim storage. This storage facility will be used to house canisters resulting from the TRU-HLW production option until final repository disposition.

This economic analysis is based on information provided by J. K. Rawlins, and K. L. Williams. R. D. Adams provided cost estimates. B.L. Blakely and D.E. Keller provided process personnel modeling.

\section{Methodology:}

The Economic Evaluation assumed a 73-year period (1999-2071) since this is the estimated time required to complete all of the anticipated remediation activities. The LCC identified and evaluated the initial development, construction, operating and post operating costs over the lifecycle. A discounted LCC analysis assumes a 1998-dollar basis, discounted $6.3 \%$ annually per the Office of Management and Budget (OMB) Circular A-94 for escalated costs. All costs are conservatively discounted assuming the end-of-year convention. 


\section{Assumptions:}

The scope of work and requirements of all related activities are vague at this time. Facility and processing costs were developed from historical experience associated with DD\&D work at the INEEL. The LCC analysis was generated to match cost estimating cost structure. These costs include Permitting, Direct and Indirect Construction, G\&A, Procurement Fee, Engineering, Inspection, Project Management, Construction Management, Escalation and Contingency costs.

The design period is assumed to be accomplished in five-years with construction completed in two-years. Labor rates were assumed as follows: Managers, $\$ 125 / \mathrm{hr}$; Engineers, $\$ 108 \$ / \mathrm{hr}$; Other Technicians $\$ 85 / \mathrm{hr}$; Administration/support staff $\$ 65 / \mathrm{hr}$; Operators and Maintenance personnel $\$ 65 / \mathrm{hr}$. The operational period for this facility was assumed to be twenty years, to match the appropriate operating facility. This period is followed by five years for shipping to the repository, followed by three years of post-operations activities. Due to this project's lack of complexity and non-radioactive contaminated environment, this analysis assumed a decommissioning cost equal to $10 \%$ of the non-escalated engineering design cost, decontamination costs equal to $0 \%$ of non-escalated total pre-operation cost, and demolition costs equal to $8 \%$ of the non-escalated total pre-operation cost. Utilities were calculated assuming a $\$ .0824$ per $\mathrm{kWh}$. Surveillance and Monitoring costs were calculated at $\$ 65,000$ per year for manned security during operations then reduced to $\$ 10,000$ for remote surveillance during non-operation periods.

\section{Results:}

The HLW-TRU (in FPR) INTERIM STORAGE FACILITY has a discounted LCC of \$97.8 million.

Attachments:

cc: R. J. Turk File 


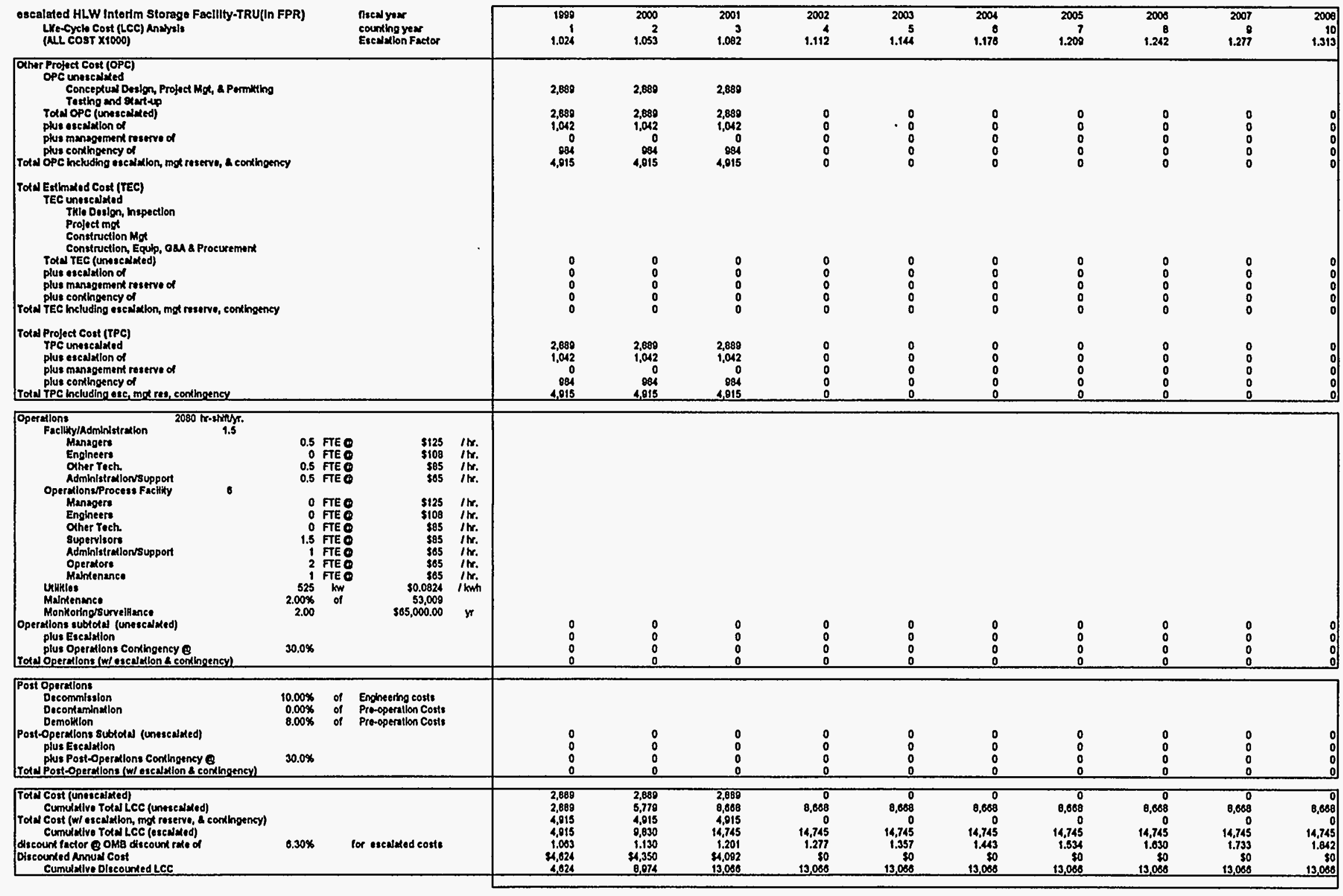




\begin{tabular}{|c|c|c|c|c|c|c|c|c|c|c|c|c|c|}
\hline $\begin{array}{l}\text { oscalated HLW Interlm Storago Facillty-TRU } \\
\text { LWe-cycto cost (LCC) Anaysts } \\
\text { (MLL cost X1000) }\end{array}$ & ( FPR) & $\begin{array}{l}\text { Riscd yea } \\
\text { countho year } \\
\text { Eeceldition Factor }\end{array}$ & & $\begin{array}{r}2009 \\
11 \\
1.350\end{array}$ & $\begin{array}{r}2010 \\
12 \\
1.387\end{array}$ & $\begin{array}{r}2011 \\
13 \\
1.426\end{array}$ & $\begin{array}{r}2012 \\
14 \\
1.468\end{array}$ & $\begin{array}{r}2013 \\
15 \\
1.507\end{array}$ & $\begin{array}{r}2014 \\
10 \\
1.550\end{array}$ & $\begin{array}{r}2015 \\
17 \\
1.593\end{array}$ & $\begin{array}{r}2018 \\
18 \\
1.038\end{array}$ & $\begin{array}{r}2017 \\
10 \\
1.003\end{array}$ & $\begin{array}{r}2018 \\
20 \\
1.730\end{array}$ \\
\hline 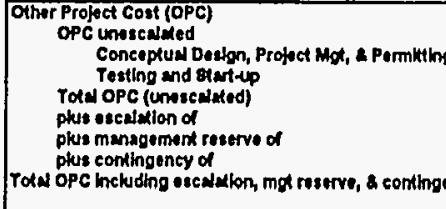 & & & & $\begin{array}{l}0 \\
0 \\
0 \\
0 \\
0\end{array}$ & $\begin{array}{l}0 \\
0 \\
0 \\
0\end{array}$ & $\begin{array}{l}0 \\
0 \\
0 \\
0\end{array}$ & $\begin{array}{l}0 \\
0 \\
0 \\
0 \\
0\end{array}$ & $\begin{array}{l}0 \\
0 \\
0 \\
0 \\
0\end{array}$ & $\begin{array}{r}4,500 \\
1,500 \\
1,022 \\
0 \\
1,532 \\
7,055\end{array}$ & $\begin{array}{l}0 \\
0 \\
0 \\
0 \\
0\end{array}$ & $\begin{array}{l}0 \\
0 \\
0 \\
0 \\
0\end{array}$ & $\begin{array}{l}0 \\
0 \\
0 \\
0 \\
0\end{array}$ & 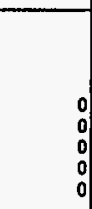 \\
\hline 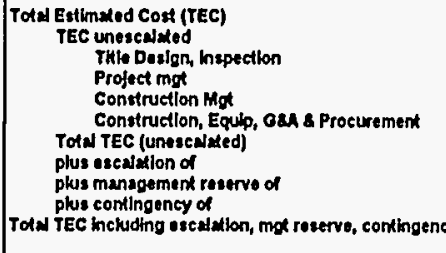 & & & & $\begin{array}{l}0 \\
0 \\
0 \\
0 \\
0\end{array}$ & $\begin{array}{r}3,088 \\
576 \\
\\
3,684 \\
1,630 \\
385 \\
604 \\
0,403\end{array}$ & $\begin{array}{r}9,264 \\
2,306 \\
\\
11,570 \\
5,147 \\
1,152 \\
2,538 \\
20,407\end{array}$ & $\begin{array}{r}2,300 \\
2,40 \\
20,503 \\
31,281 \\
13,918 \\
3,114 \\
0,863 \\
55,173\end{array}$ & $\begin{array}{r}1,729 \\
2,470 \\
28,505 \\
30,704 \\
13,600 \\
3,056 \\
8,736 \\
54,157\end{array}$ & $\begin{array}{l}0 \\
0 \\
0 \\
0 \\
0\end{array}$ & $\begin{array}{l}0 \\
0 \\
0 \\
0 \\
0\end{array}$ & $\begin{array}{l}0 \\
0 \\
0 \\
0 \\
0\end{array}$ & $\begin{array}{l}0 \\
0 \\
0 \\
0 \\
0\end{array}$ & \\
\hline 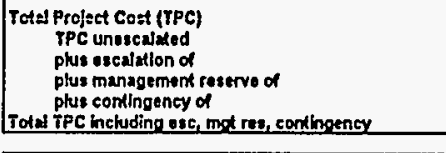 & & & & $\begin{array}{l}0 \\
0 \\
0 \\
0 \\
0 \\
\end{array}$ & $\begin{array}{r}3,604 \\
1,630 \\
305 \\
804 \\
6,403 \\
\end{array}$ & $\begin{array}{r}11,570 \\
5,147 \\
1,152 \\
2,538 \\
20,407 \\
\end{array}$ & $\begin{array}{r}31,281 \\
13,916 \\
3,114 \\
0.863 \\
55,173 \\
\end{array}$ & $\begin{array}{r}30,704 \\
13,600 \\
3,056 \\
8,736 \\
54,157 \\
\end{array}$ & $\begin{array}{r}4,500 \\
1,022 \\
0 \\
1,532 \\
7,055 \\
\end{array}$ & $\begin{array}{l}0 \\
0 \\
0 \\
0 \\
0 \\
\end{array}$ & $\begin{array}{l}0 \\
0 \\
0 \\
0 \\
0 \\
\end{array}$ & $\begin{array}{l}0 \\
0 \\
0 \\
0 \\
0 \\
\end{array}$ & \\
\hline 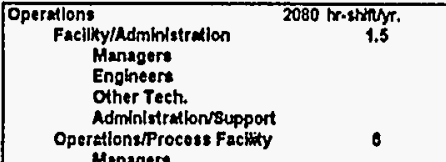 & $\begin{array}{l}0.5 \text { FTE } \\
0 \text { FTE } \\
0.5 \text { FTE } \\
0.5 \text { FTE }\end{array}$ & $\begin{array}{r}\$ 125 \\
\$ 108 \\
\$ 85 \\
\$ 85\end{array}$ & $\begin{array}{l}\text { Irr. } \\
\text { thr. } \\
\text { thr. } \\
\text { thr. } \\
\text { thr. }\end{array}$ & & & & & & $\begin{array}{l}05 \\
0 \\
4 \\
34\end{array}$ & $\begin{array}{r}130 \\
0 \\
80 \\
6 \theta\end{array}$ & $\begin{array}{r}130 \\
0 \\
68 \\
69\end{array}$ & $\begin{array}{r}130 \\
0 \\
88 \\
68\end{array}$ & $\begin{array}{r}130 \\
0 \\
88 \\
68\end{array}$ \\
\hline 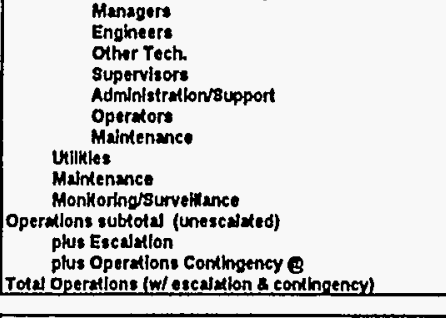 & $\begin{aligned} 0 & \text { FTE } \\
0 & \text { FTE } \\
0 & \text { FTE } \\
1.5 & \text { TFE } \\
1 & \text { TTE } \\
2 & \text { FTE } \\
1 & \text { TTE } \\
525 & \text { KW } \\
2.00 \% & \text { of } \\
2.00 & \\
& \\
30.0 \% & \end{aligned}$ & $\begin{array}{r}\$ 125 \\
\$ 108 \\
\$ 85 \\
\$ 85 \\
\$ 65 \\
\$ 65 \\
\$ 65 \\
\$ 0.05 \\
\$ 3,024 \\
\$ 65,0009 \\
\$ 65,000\end{array}$ & 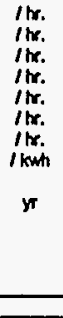 & $\begin{array}{l}0 \\
0 \\
0 \\
0 \\
\end{array}$ & $\begin{array}{l}0 \\
0 \\
0 \\
0 \\
\end{array}$ & $\begin{array}{l}0 \\
0 \\
0 \\
0\end{array}$ & $\begin{array}{l}0 \\
0 \\
0 \\
0\end{array}$ & $\begin{array}{l}0 \\
0 \\
0 \\
0 \\
\end{array}$ & $\begin{array}{r}0 \\
0 \\
0 \\
133 \\
68 \\
635 \\
135 \\
698 \\
45 \\
530 \\
65 \\
1,186 \\
6552 \\
251 \\
2,389 \\
\end{array}$ & $\begin{array}{r}0 \\
0 \\
0 \\
265 \\
135 \\
270 \\
135 \\
00 \\
530 \\
130 \\
1,042 \\
1,002 \\
880 \\
3,815 \\
\end{array}$ & $\begin{array}{r}0 \\
0 \\
0 \\
265 \\
135 \\
270 \\
135 \\
00 \\
530 \\
130 \\
1,942 \\
1,174 \\
3,005 \\
3,021 \\
\end{array}$ & $\begin{array}{r}0 \\
0 \\
0 \\
205 \\
135 \\
270 \\
135 \\
00 \\
530 \\
130 \\
1,942 \\
1,259 \\
930 \\
4,031 \\
\end{array}$ & 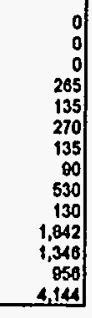 \\
\hline 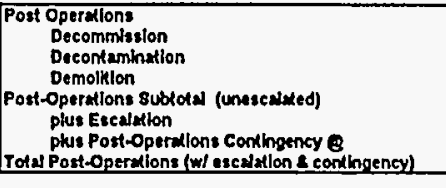 & $\begin{aligned} 10.00 \% & \text { of } \\
0.00 \% & \text { of } \\
8.00 \% & \text { of } \\
& \\
30.0 \% & \end{aligned}$ & 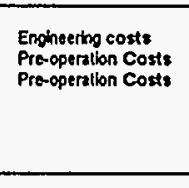 & & $\begin{array}{l}0 \\
0 \\
0 \\
0 \\
\end{array}$ & $\begin{array}{l}0 \\
0 \\
0 \\
0 \\
\end{array}$ & $\begin{array}{l}0 \\
0 \\
0 \\
0 \\
\end{array}$ & $\begin{array}{l}0 \\
0 \\
0 \\
0 \\
\end{array}$ & $\begin{array}{l}0 \\
0 \\
0 \\
0 \\
\end{array}$ & $\begin{array}{l}0 \\
0 \\
0 \\
0 \\
\end{array}$ & $\begin{array}{l}0 \\
0 \\
0 \\
0 \\
\end{array}$ & $\begin{array}{l}0 \\
0 \\
0 \\
0 \\
\end{array}$ & $\begin{array}{l}0 \\
0 \\
0 \\
0\end{array}$ & 0 \\
\hline 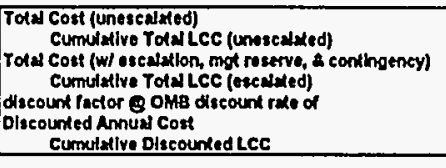 & $0.30 \%$ & for escalyed corts & & $\begin{array}{r}0.0 \\
0,068 \\
0 \\
14,745 \\
1.058 \\
50 \\
13,068 \\
\end{array}$ & $\begin{aligned} 3,604 \\
12,333 \\
0 ., 433 \\
21,209 \\
2,002 \\
53,105 \\
16,1111 \\
\end{aligned}$ & $\begin{array}{l}11,570 \\
23,003 \\
320,007 \\
41,6015 \\
2.213 \\
82,222 \\
25,393 \\
\end{array}$ & 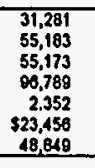 & 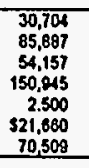 & $\begin{array}{r}5,686 \\
91,573 \\
10,0.44 \\
160,089 \\
2.058 \\
53,718 \\
76,288 \\
76,289 \\
\end{array}$ & $\begin{array}{r}1,812 \\
93,415 \\
3,4015 \\
104,804 \\
2.2825 \\
51,350 \\
75,030 \\
\end{array}$ & 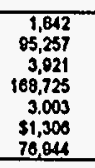 & $\begin{array}{r}1,912 \\
07,100 \\
4,031 \\
172,756 \\
31.193 \\
31,263 \\
78,207 \\
\end{array}$ & $\begin{array}{r}1,642 \\
88,022 \\
4,142 \\
176,900 \\
3 ., 32 \\
31,221 \\
70,428 \\
\end{array}$ \\
\hline
\end{tabular}




\begin{tabular}{|c|c|c|c|c|c|c|c|c|c|c|c|c|c|}
\hline \multicolumn{2}{|c|}{ 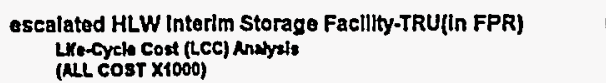 } & \multicolumn{2}{|l|}{$\begin{array}{l}\text { Mesa your } \\
\text { counxlmg your } \\
\text { Escatulion Fastor }\end{array}$} & $\begin{array}{l}2019 \\
21 \\
1.770\end{array}$ & $\begin{array}{r}2020 \\
22 \\
1.820\end{array}$ & $\begin{array}{r}2021 \\
23 \\
1.890\end{array}$ & $\begin{aligned} 2022 \\
24 \\
1.033\end{aligned}$ & $\begin{array}{l}2023 \\
25 \\
1.897\end{array}$ & $\begin{array}{r}2024 \\
2.062\end{array}$ & $\begin{array}{r}2025 \\
27 \\
2.100\end{array}$ & $\begin{array}{r}2028 \\
28 \\
2.158\end{array}$ & $\begin{array}{r}2027 \\
2.29 \\
2.210\end{array}$ & $\begin{array}{r}2028 \\
2.283 \\
203\end{array}$ \\
\hline \multicolumn{4}{|l|}{ 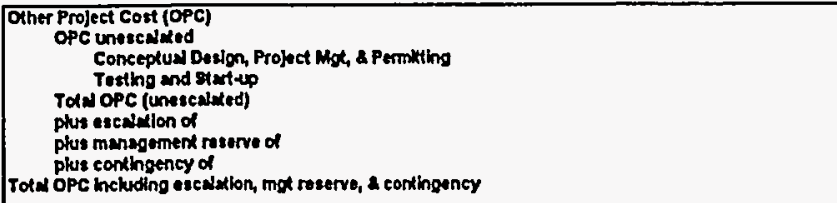 } & $\begin{array}{l}0 \\
: \\
0 \\
0 \\
0\end{array}$ & $\begin{array}{l}0 \\
0 \\
0 \\
0 \\
0\end{array}$ & $\begin{array}{l}0 \\
0 \\
0 \\
0 \\
0\end{array}$ & $\begin{array}{l}0 \\
0 \\
0 \\
0 \\
0\end{array}$ & $\begin{array}{l}0 \\
0 \\
0 \\
0 \\
0\end{array}$ & $\begin{array}{l}0 \\
0 \\
0 \\
0 \\
0\end{array}$ & $\begin{array}{l}0 \\
0 \\
0 \\
0 \\
0\end{array}$ & $\begin{array}{l}0 \\
0 \\
0 \\
0 \\
0\end{array}$ & $\begin{array}{l}0 \\
0 \\
0 \\
0 \\
0\end{array}$ & \begin{tabular}{l|l}
0 \\
0 \\
0 \\
0 \\
0
\end{tabular} \\
\hline \multicolumn{4}{|l|}{ 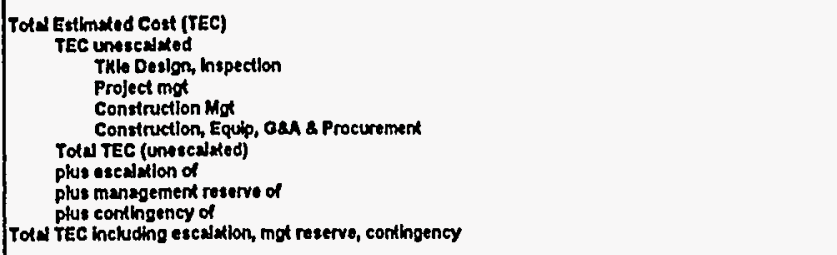 } & $\begin{array}{l}0 \\
0 \\
0 \\
0 \\
0\end{array}$ & $\begin{array}{l}0 \\
\vdots \\
0 \\
0\end{array}$ & $\begin{array}{l}0 \\
0 \\
0 \\
0 \\
0\end{array}$ & $\begin{array}{l}0 \\
0 \\
0 \\
0\end{array}$ & $\begin{array}{l}0 \\
0 \\
0 \\
0 \\
0\end{array}$ & $\begin{array}{l}0 \\
0 \\
0 \\
0 \\
0\end{array}$ & $\begin{array}{l}0 \\
0 \\
0 \\
0 \\
0\end{array}$ & $\begin{array}{l}0 \\
0 \\
0 \\
0 \\
0\end{array}$ & $\begin{array}{l}0 \\
0 \\
0 \\
0 \\
0\end{array}$ & \begin{tabular}{l|l}
0 \\
0 \\
0 \\
0 \\
0
\end{tabular} \\
\hline \multicolumn{4}{|l|}{ 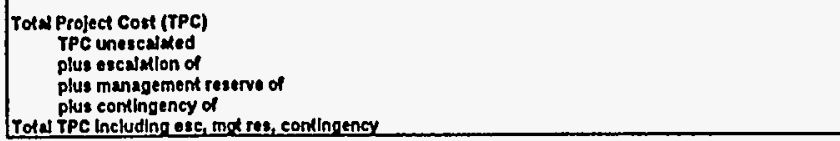 } & $\begin{array}{l}0 \\
0 \\
0 \\
0 \\
0\end{array}$ & $\begin{array}{l}0 \\
0 \\
0 \\
0 \\
0 \\
\end{array}$ & $\begin{array}{l}0 \\
0 \\
0 \\
0 \\
0 \\
\end{array}$ & $\begin{array}{l}0 \\
0 \\
0 \\
0 \\
0\end{array}$ & $\begin{array}{l}0 \\
0 \\
0 \\
0 \\
0 \\
\end{array}$ & $\begin{array}{l}0 \\
0 \\
0 \\
0 \\
0 \\
\end{array}$ & $\begin{array}{l}0 \\
0 \\
0 \\
0 \\
0 \\
\end{array}$ & $\begin{array}{l}0 \\
0 \\
0 \\
0 \\
0 \\
\end{array}$ & $\begin{array}{l}0 \\
0 \\
0 \\
0 \\
0 \\
\end{array}$ & $\begin{array}{l}0 \\
0 \\
0 \\
0 \\
0\end{array}$ \\
\hline 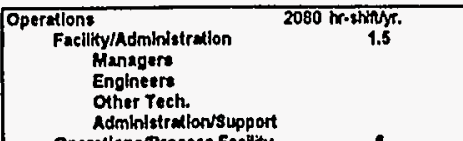 & $\begin{array}{l}0.5 \text { FEQ } \\
0 \text { TTE } \\
0.3 \text { TFE } \\
0.5 \text { FTE }\end{array}$ & $\begin{array}{c}\$ 125 \\
\$ 108 \\
\$ 95 \\
\$ 65\end{array}$ & $\begin{array}{l}\text { Itr. } \\
\text { thr. } \\
\text { thr. } \\
\text { frr. }\end{array}$ & $\begin{array}{r}130 \\
0 \\
60 \\
68\end{array}$ & $\begin{array}{r}130 \\
0 \\
89 \\
88\end{array}$ & $\begin{array}{r}130 \\
0 \\
89 \\
68\end{array}$ & $\begin{array}{r}130 \\
0 \\
89 \\
80\end{array}$ & $\begin{array}{r}130 \\
0 \\
88 \\
88\end{array}$ & $\begin{array}{r}130 \\
0 \\
89 \\
80\end{array}$ & $\begin{array}{r}130 \\
0 \\
88 \\
68\end{array}$ & $\begin{array}{r}130 \\
0 \\
88 \\
8 B\end{array}$ & $\begin{array}{r}130 \\
0 \\
88 \\
68\end{array}$ & $\begin{array}{r}130 \\
0 \\
68 \\
68\end{array}$ \\
\hline 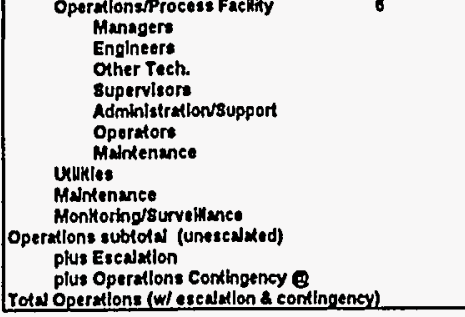 & 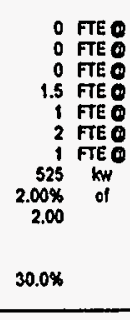 & $\begin{array}{r}\$ 125 \\
\$ 108 \\
\$ 85 \\
\$ 85 \\
\$ 85 \\
\$ 85 \\
\$ 85 \\
\$ 05 \\
\$ 0.0924 \\
50.008 \\
\$ 05,000.00\end{array}$ & 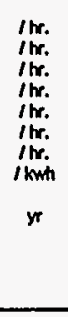 & $\begin{array}{r}0 \\
0 \\
0 \\
285 \\
135 \\
270 \\
135 \\
80 \\
530 \\
130 \\
1,30 \\
1,435 \\
1393 \\
4,200 \\
\end{array}$ & $\begin{array}{r}0 \\
0 \\
0 \\
285 \\
135 \\
270 \\
135 \\
80 \\
530 \\
110 \\
1.842 \\
1,5.527 \\
1,011 \\
4,370 \\
\end{array}$ & $\begin{array}{r}0 \\
0 \\
0 \\
205 \\
135 \\
270 \\
135 \\
80 \\
530 \\
130 \\
1,942 \\
1,621 \\
1,030 \\
4,502 \\
\end{array}$ & $\begin{array}{r}0 \\
0 \\
0 \\
205 \\
135 \\
270 \\
135 \\
80 \\
530 \\
130 \\
1,842 \\
1,718 \\
1,080 \\
4,028 \\
\end{array}$ & $\begin{array}{r}0 \\
0 \\
0 \\
205 \\
135 \\
270 \\
135 \\
00 \\
530 \\
130 \\
1,842 \\
1,818 \\
1,098 \\
2,738 \\
\end{array}$ & $\begin{array}{r}0 \\
0 \\
0 \\
285 \\
135 \\
270 \\
135 \\
00 \\
530 \\
130 \\
1,812 \\
1,020 \\
1,120 \\
4,801 \\
\end{array}$ & $\begin{array}{r}0 \\
0 \\
0 \\
205 \\
135 \\
270 \\
135 \\
00 \\
530 \\
130 \\
1,642 \\
2,025 \\
1,160 \\
5,028 \\
\end{array}$ & $\begin{array}{r}0 \\
0 \\
0 \\
285 \\
135 \\
270 \\
273 \\
000 \\
530 \\
130 \\
1.642 \\
2,134 \\
1,193 \\
5,189 \\
\end{array}$ & $\begin{array}{r}0 \\
0 \\
0 \\
285 \\
135 \\
2170 \\
135 \\
00 \\
530 \\
130 \\
1,402 \\
2,245 \\
1,220 \\
6,313 \\
\end{array}$ & 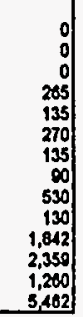 \\
\hline 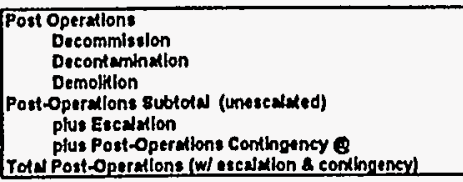 & $\begin{aligned} 10.00 \% & \text { of } \\
0.00 \% & \text { of } \\
8.00 \% & \text { of } \\
& \\
30.0 \% & \end{aligned}$ & $\begin{array}{l}\text { Engineoring costs } \\
\text { Pro-operation Costs } \\
\text { Preoperation Costs }\end{array}$ & & $\begin{array}{l}0 \\
0 \\
0 \\
0 \\
\end{array}$ & $\begin{array}{l}0 \\
0 \\
0 \\
0 \\
\end{array}$ & $\begin{array}{l}0 \\
0 \\
0 \\
0\end{array}$ & $\begin{array}{l}0 \\
0 \\
0 \\
0 \\
\end{array}$ & $\begin{array}{l}0 \\
0 \\
0 \\
0 \\
\end{array}$ & $\begin{array}{l}0 \\
0 \\
0 \\
0 \\
\end{array}$ & $\begin{array}{l}0 \\
0 \\
0 \\
0 \\
\end{array}$ & $\begin{array}{l}0 \\
0 \\
0 \\
0 \\
\end{array}$ & $\begin{array}{l}0 \\
0 \\
0 \\
0 \\
\end{array}$ & $\begin{array}{l}0 \\
0 \\
0 \\
0\end{array}$ \\
\hline 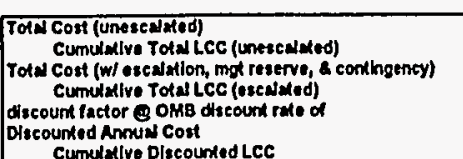 & $0.30 x$ & for escaseed conts & & 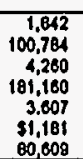 & 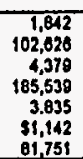 & 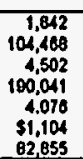 & 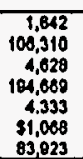 & 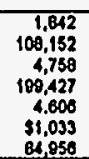 & 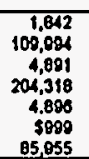 & $\begin{array}{r}11.812 \\
111,838 \\
5,029 \\
209.345 \\
5.205 \\
3806 \\
68,921\end{array}$ & 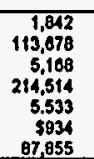 & $\begin{array}{r}1,982 \\
115,520 \\
5,313 \\
219,027 \\
5.891 \\
5803 \\
89,768\end{array}$ & 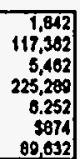 \\
\hline
\end{tabular}




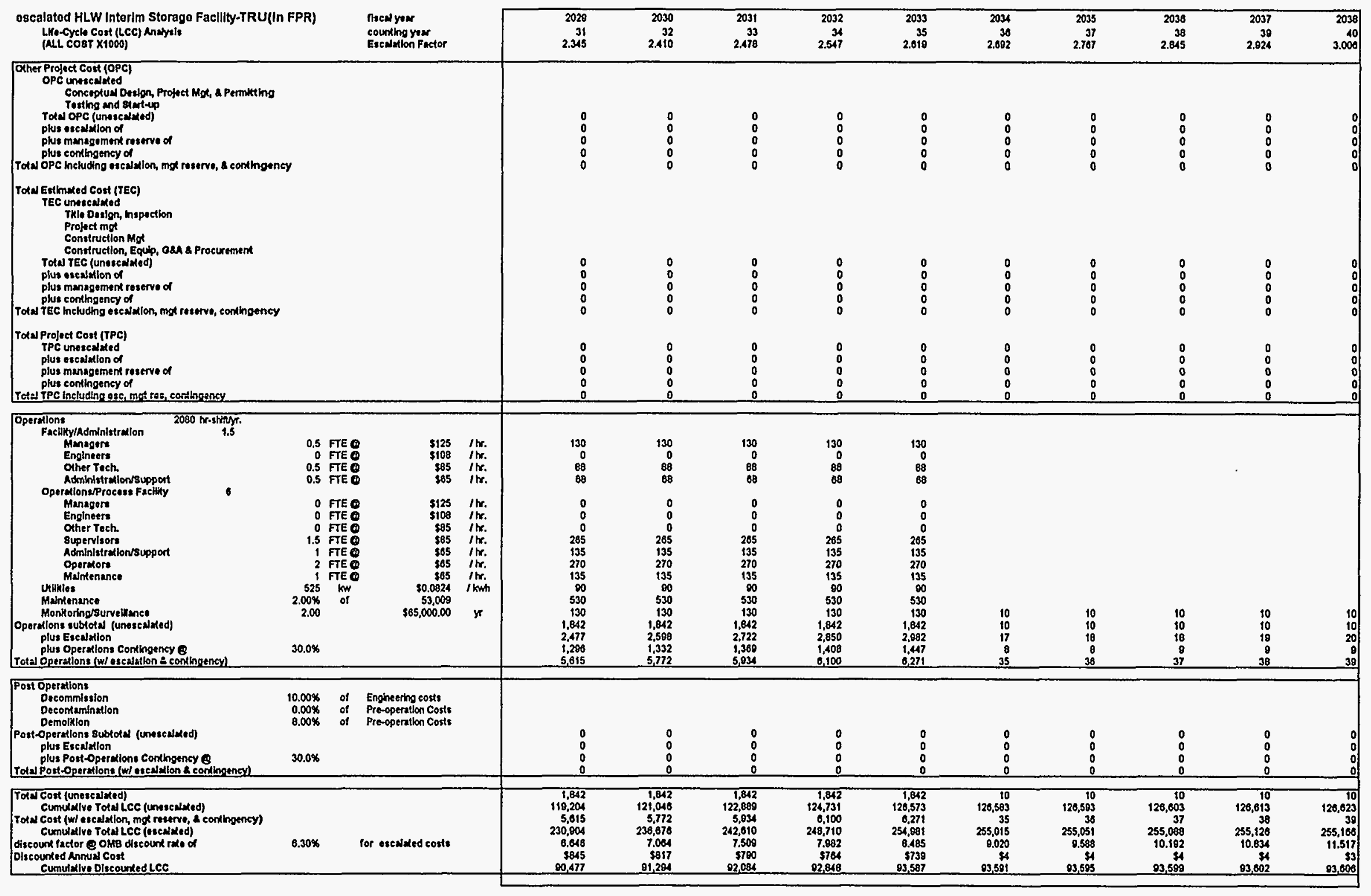




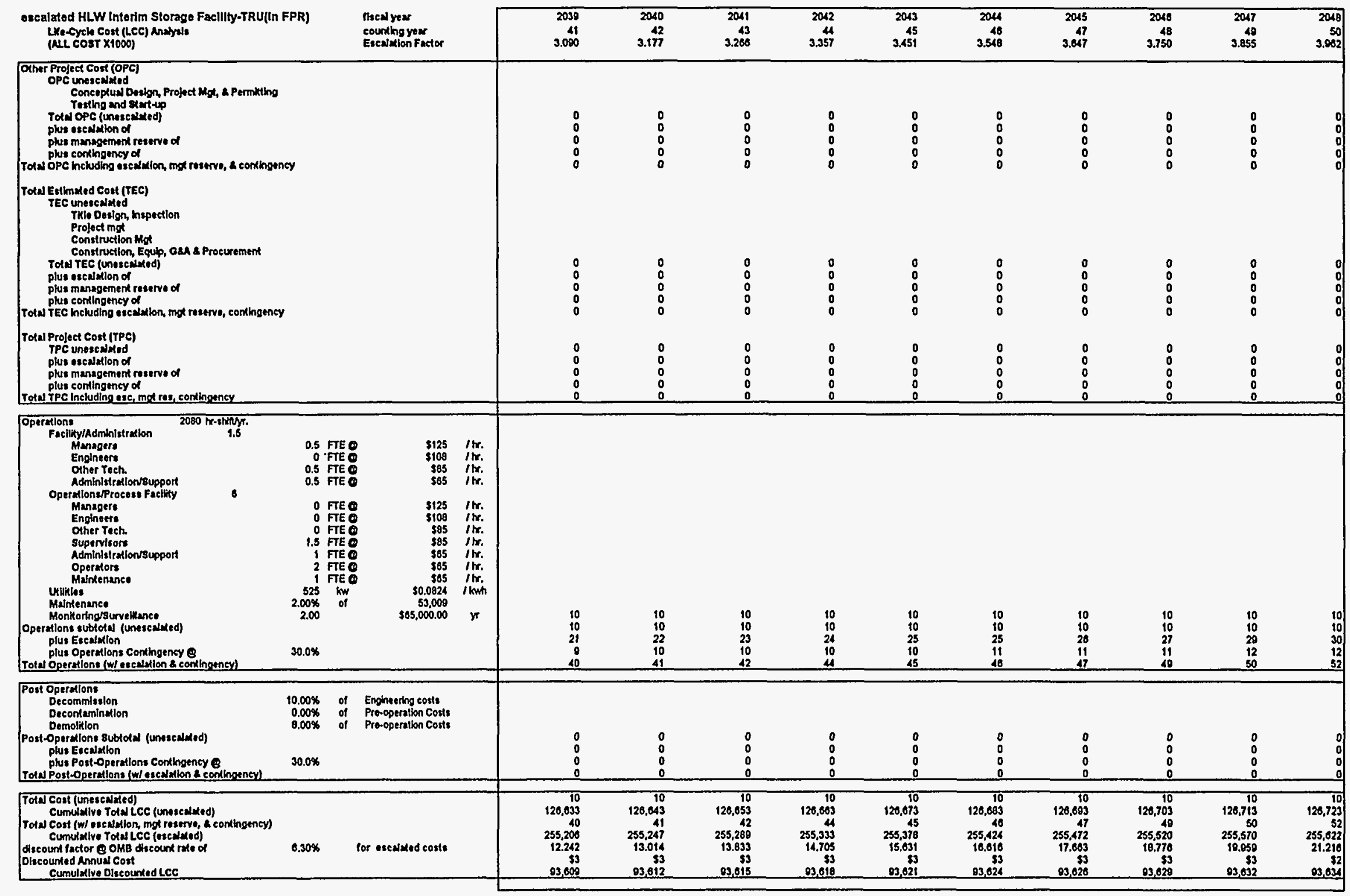




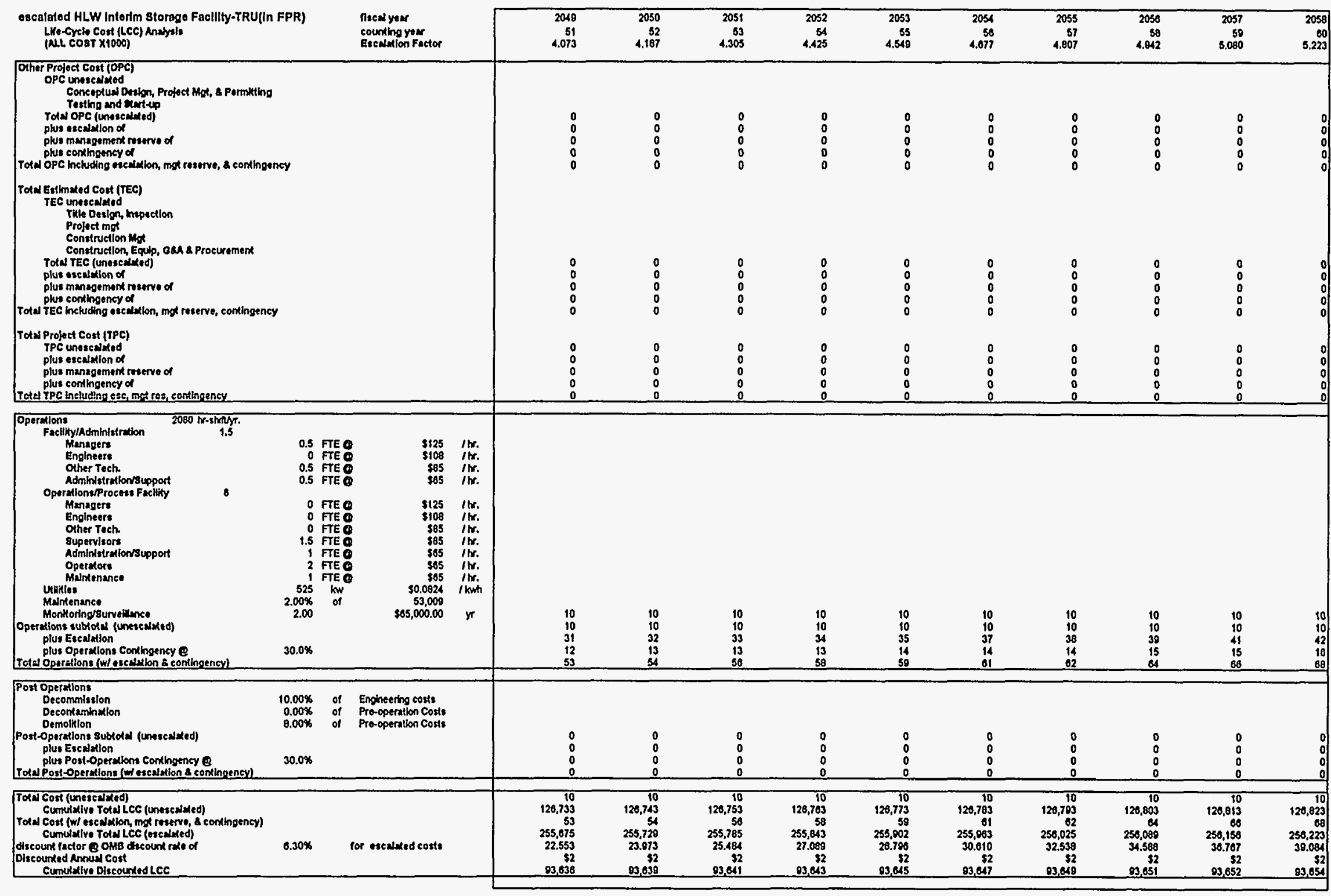




\begin{tabular}{|c|c|c|c|c|c|c|c|c|c|c|c|c|c|}
\hline 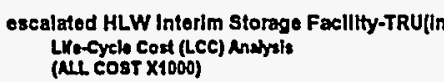 & FPR) & $\begin{array}{l}\text { Ared your } \\
\text { counthog year } \\
\text { Euctulion Factor }\end{array}$ & & $\begin{array}{r}2059 \\
61 \\
5.369\end{array}$ & $\begin{array}{r}2000 \\
62 \\
5.510\end{array}$ & $\begin{array}{r}2081 \\
63 \\
5.074\end{array}$ & $\begin{array}{r}2002 \\
64 \\
5.033\end{array}$ & $\begin{array}{r}2003 \\
655 \\
5.990\end{array}$ & $\begin{array}{l}2004 \\
0 \$ 4 \\
0.194\end{array}$ & $\begin{array}{l}2005 \\
07 \\
0.338\end{array}$ & $\begin{array}{l}2088 \\
0.58 \\
0.514\end{array}$ & $\begin{array}{r}2007 \\
0.60\end{array}$ & $\begin{array}{r}2058 \\
0.804 \\
0.884\end{array}$ \\
\hline \multicolumn{4}{|l|}{ 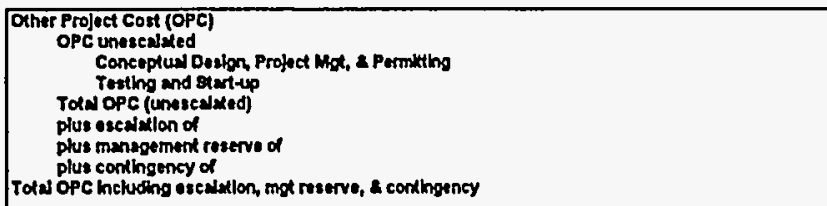 } & $\begin{array}{l}0 \\
0 \\
0 \\
0 \\
0\end{array}$ & $\begin{array}{l}0 \\
0 \\
0 \\
0 \\
0\end{array}$ & $\begin{array}{l}0 \\
0 \\
0 \\
0 \\
0\end{array}$ & $\begin{array}{l}0 \\
0 \\
0 \\
0 \\
0\end{array}$ & $\begin{array}{l}0 \\
0 \\
0 \\
0 \\
0\end{array}$ & $\begin{array}{l}0 \\
0 \\
0 \\
0 \\
0\end{array}$ & $\begin{array}{l}0 \\
0 \\
0 \\
0 \\
0\end{array}$ & $\begin{array}{l}0 \\
0 \\
0 \\
0 \\
0\end{array}$ & $\begin{array}{l}0 \\
0 \\
0 \\
0 \\
0\end{array}$ & $\begin{array}{l}0 \\
0 \\
0 \\
0 \\
0\end{array}$ \\
\hline \multicolumn{4}{|l|}{ 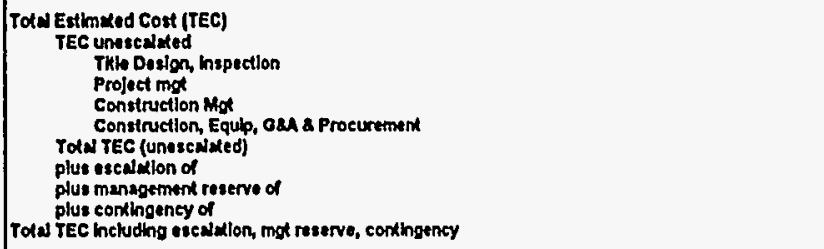 } & $\begin{array}{l}0 \\
: \\
0 \\
0\end{array}$ & $\begin{array}{l}0 \\
0 \\
0 \\
0 \\
0\end{array}$ & $\begin{array}{l}0 \\
0 \\
0 \\
0 \\
0\end{array}$ & $\begin{array}{l}0 \\
0 \\
0 \\
0 \\
0\end{array}$ & $\begin{array}{l}0 \\
: \\
0 \\
0\end{array}$ & $\begin{array}{l}0 \\
0 \\
0 \\
0 \\
0\end{array}$ & $\begin{array}{l}0 \\
: \\
0 \\
0 \\
0\end{array}$ & $\begin{array}{l}0 \\
0 \\
0 \\
0 \\
0\end{array}$ & $\begin{array}{l}0 \\
0 \\
0 \\
0 \\
0\end{array}$ & \begin{tabular}{l|l}
0 \\
0 \\
0 \\
0 \\
0
\end{tabular} \\
\hline \multicolumn{4}{|l|}{ 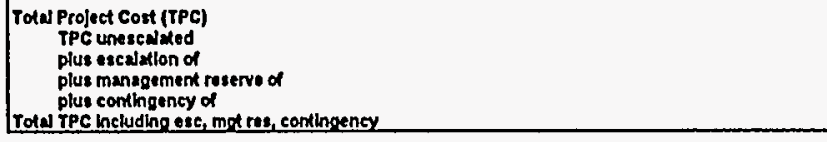 } & $\begin{array}{l}0 \\
0 \\
0 \\
0 \\
0 \\
\end{array}$ & $\begin{array}{l}0 \\
0 \\
0 \\
0 \\
0 \\
\end{array}$ & $\begin{array}{l}0 \\
0 \\
0 \\
0 \\
0 \\
\end{array}$ & $\begin{array}{l}0 \\
0 \\
0 \\
0 \\
0\end{array}$ & $\begin{array}{l}0 \\
0 \\
0 \\
0 \\
0\end{array}$ & $\begin{array}{l}0 \\
0 \\
0 \\
0 \\
0 \\
\end{array}$ & $\begin{array}{l}0 \\
0 \\
0 \\
0 \\
0 \\
\end{array}$ & $\begin{array}{l}0 \\
0 \\
0 \\
0 \\
0 \\
\end{array}$ & $\begin{array}{l}0 \\
0 \\
0 \\
0 \\
0\end{array}$ & $\begin{array}{l}0 \\
0 \\
0 \\
0 \\
\end{array}$ \\
\hline \multirow{2}{*}{ 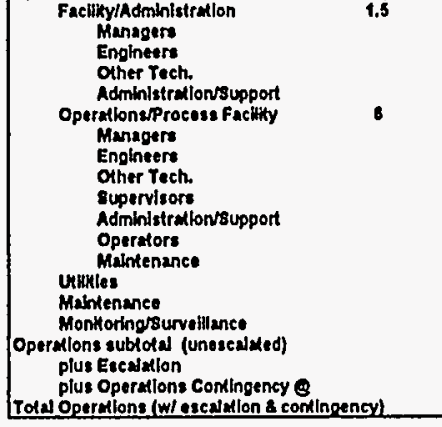 } & $\begin{array}{r}0.5 \text { FTE } \\
0 \text { FTE } \\
0.5 \text { TEQ } \\
0.5 \text { FTE }\end{array}$ & $\begin{array}{c}\$ 125 \\
\$ 100 \\
\$ 85 \\
\$ 65\end{array}$ & $\begin{array}{l}\text { Inr. } \\
\text { inr. } \\
\text { inf. } \\
\text { itr. }\end{array}$ & & & & & & $\begin{array}{r}130 \\
0 \\
88 \\
88\end{array}$ & $\begin{array}{r}130 \\
0 \\
89 \\
88\end{array}$ & $\begin{array}{r}130 \\
0 \\
68 \\
68\end{array}$ & $\begin{array}{r}130 \\
0 \\
88 \\
68\end{array}$ & $\begin{array}{r}130 \\
80 \\
86\end{array}$ \\
\hline & $\begin{array}{rr}0 & \text { FTE } \\
0 & \text { FTE } \\
0 & \text { FTE } \\
1.5 & \text { FTE } \\
1 & \text { FTE } \\
2 & \text { TFE } \\
1 & \text { FE } \\
525 & \text { WW } \\
2.0 \% \% & \text { of } \\
2.00 & \\
& \\
30.0 \% & \end{array}$ & 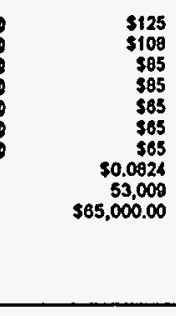 & 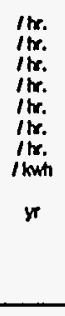 & $\begin{array}{l}10 \\
10 \\
44 \\
10 \\
70 \\
\end{array}$ & $\begin{array}{l}10 \\
10 \\
45 \\
17 \\
72 \\
\end{array}$ & $\begin{array}{l}10 \\
10 \\
47 \\
17 \\
74 \\
\end{array}$ & $\begin{array}{l}10 \\
10 \\
48 \\
17 \\
70 \\
\end{array}$ & $\begin{array}{l}10 \\
10 \\
50 \\
18 \\
78 \\
\end{array}$ & $\begin{array}{r}0 \\
0 \\
0 \\
205 \\
135 \\
270 \\
135 \\
00 \\
530 \\
130 \\
1,842 \\
0,512 \\
34,406 \\
14,701 \\
\end{array}$ & $\begin{array}{r}0 \\
0 \\
0 \\
205 \\
135 \\
270 \\
135 \\
80 \\
530 \\
130 \\
1,842 \\
0.830 \\
3,502 \\
15,174 \\
\end{array}$ & $\begin{array}{r}0 \\
0 \\
0 \\
205 \\
135 \\
270 \\
135 \\
00 \\
530 \\
130 \\
1,92 \\
10,157 \\
3.600 \\
15,509 \\
\end{array}$ & $\begin{array}{r}0 \\
0 \\
0 \\
205 \\
135 \\
270 \\
135 \\
80 \\
530 \\
130 \\
1,842 \\
10,403 \\
3,700 \\
10,035 \\
\end{array}$ & $\begin{array}{r}0 \\
0 \\
0 \\
265 \\
135 \\
270 \\
135 \\
80 \\
285 \\
130 \\
1,577 \\
0.270 \\
3,257 \\
14,113 \\
\end{array}$ \\
\hline 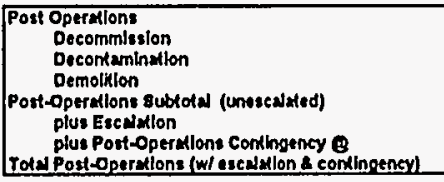 & $\begin{aligned} 10.00 x & \text { of } \\
0.00 \% & \text { of } \\
8.00 \% & \text { of } \\
& \\
30.0 \% & \end{aligned}$ & $\begin{array}{l}\text { Enghoering couts } \\
\text { Pro-operation Couts } \\
\text { Pro-operation Costs }\end{array}$ & & $\begin{array}{l}0 \\
0 \\
0 \\
0 \\
\end{array}$ & $\begin{array}{l}0 \\
0 \\
0 \\
0 \\
\end{array}$ & $\begin{array}{l}0 \\
0 \\
0 \\
0 \\
\end{array}$ & $\begin{array}{l}0 \\
0 \\
0 \\
0 \\
\end{array}$ & $\begin{array}{l}0 \\
0 \\
0 \\
0 \\
\end{array}$ & $\begin{array}{l}0 \\
0 \\
0 \\
0 \\
\end{array}$ & $\begin{array}{l}0 \\
0 \\
0 \\
0 \\
\end{array}$ & $\begin{array}{l}0 \\
0 \\
0 \\
0\end{array}$ & $\begin{array}{l}0 \\
0 \\
0 \\
0 \\
\end{array}$ & $\begin{array}{l}0 \\
0 \\
0 \\
0 \\
\end{array}$ \\
\hline 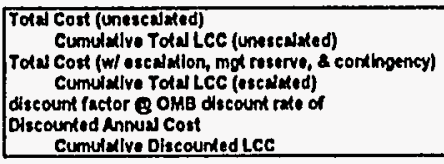 & $0.30 \%$ & for arented conts & & $\begin{array}{r}120.893 \\
70 \\
250,203 \\
41.530 \\
52 \\
93,058 \\
\end{array}$ & $\begin{array}{r}120.943 \\
72 \\
258,305 \\
4.103 \\
\$ 32 \\
93,058 \\
\end{array}$ & $\begin{array}{l}10 \\
120,053 \\
74 \\
250,430 \\
40.48 \\
52 \\
93,059 \\
\end{array}$ & $\begin{array}{r}10 \\
126,083 \\
76 \\
256,515 \\
40.003 \\
82 \\
93,681 \\
\end{array}$ & $\begin{array}{r}120,019 \\
78 \\
250,592 \\
53,0.077 \\
411 \\
03,602 \\
\end{array}$ & $\begin{array}{r}1,942 \\
128,717 \\
14,761 \\
271,353 \\
56,399 \\
\$ 3202 \\
93,024 \\
\end{array}$ & $\begin{array}{r}13,042 \\
130,557 \\
15,174 \\
280,527 \\
58.022 \\
\$ 253 \\
81,177 \\
\end{array}$ & $\begin{array}{r}1,642 \\
132,389 \\
15,599 \\
302,120 \\
63.718 \\
3245 \\
04,422 \\
\end{array}$ & 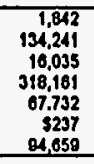 & $\begin{array}{r}1,5977 \\
135,618 \\
14,113 \\
332,274 \\
71,999 \\
31,969 \\
21,855 \\
\end{array}$ \\
\hline
\end{tabular}




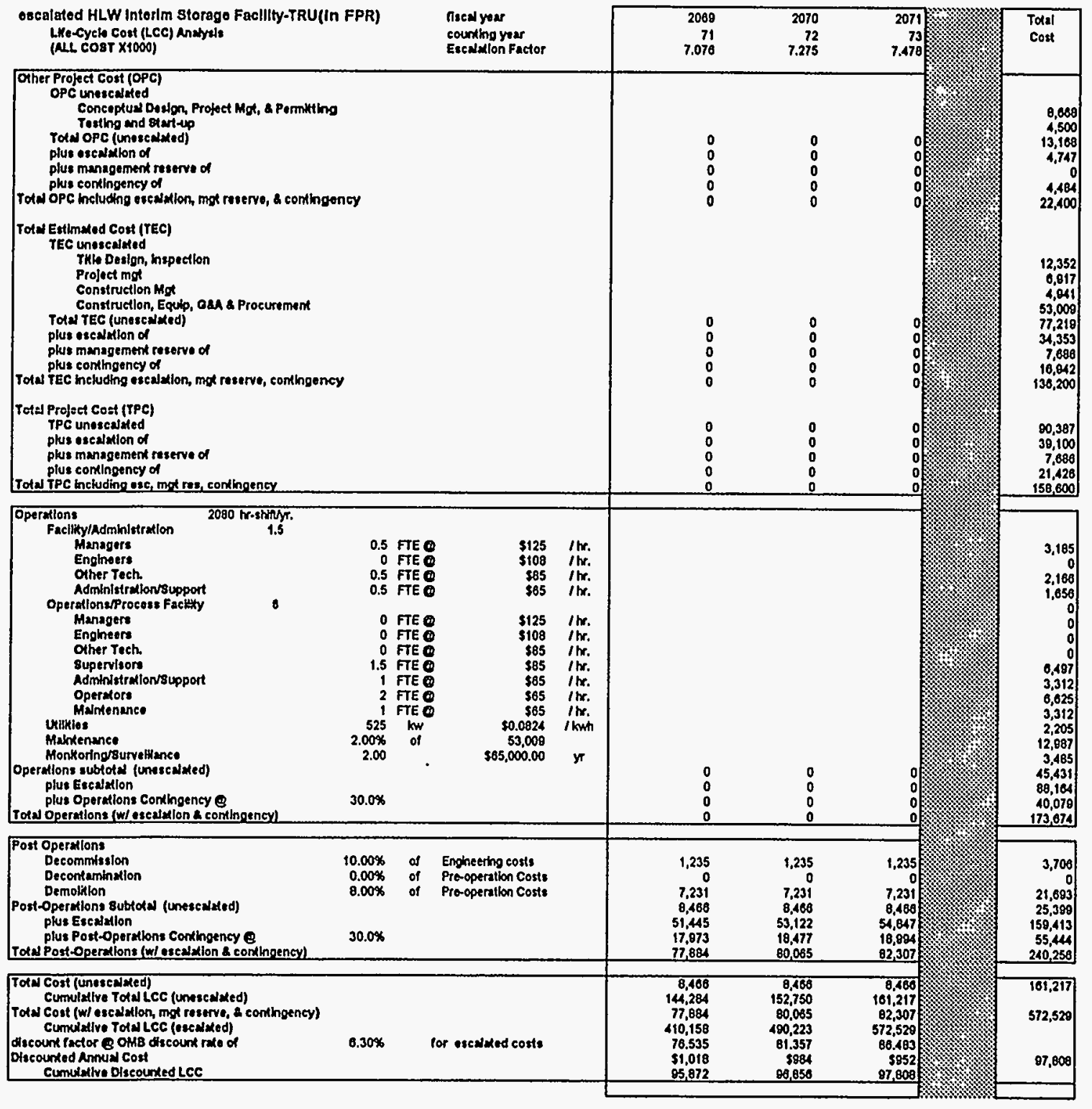




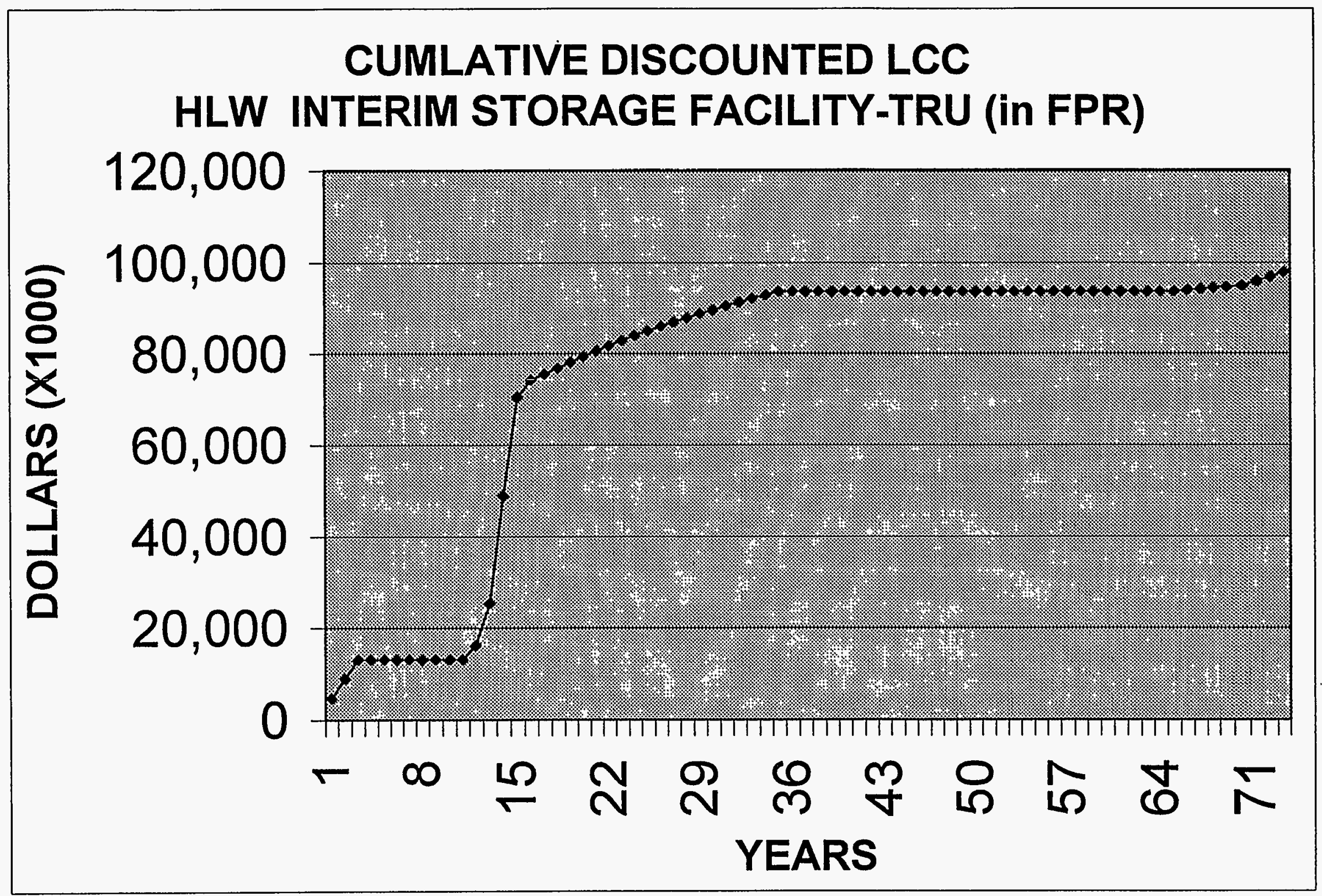




\section{TOTAL ANNUAL COST (escalated) HLW INTERIM STORAGE FACILTY-TRU (in FPR)}

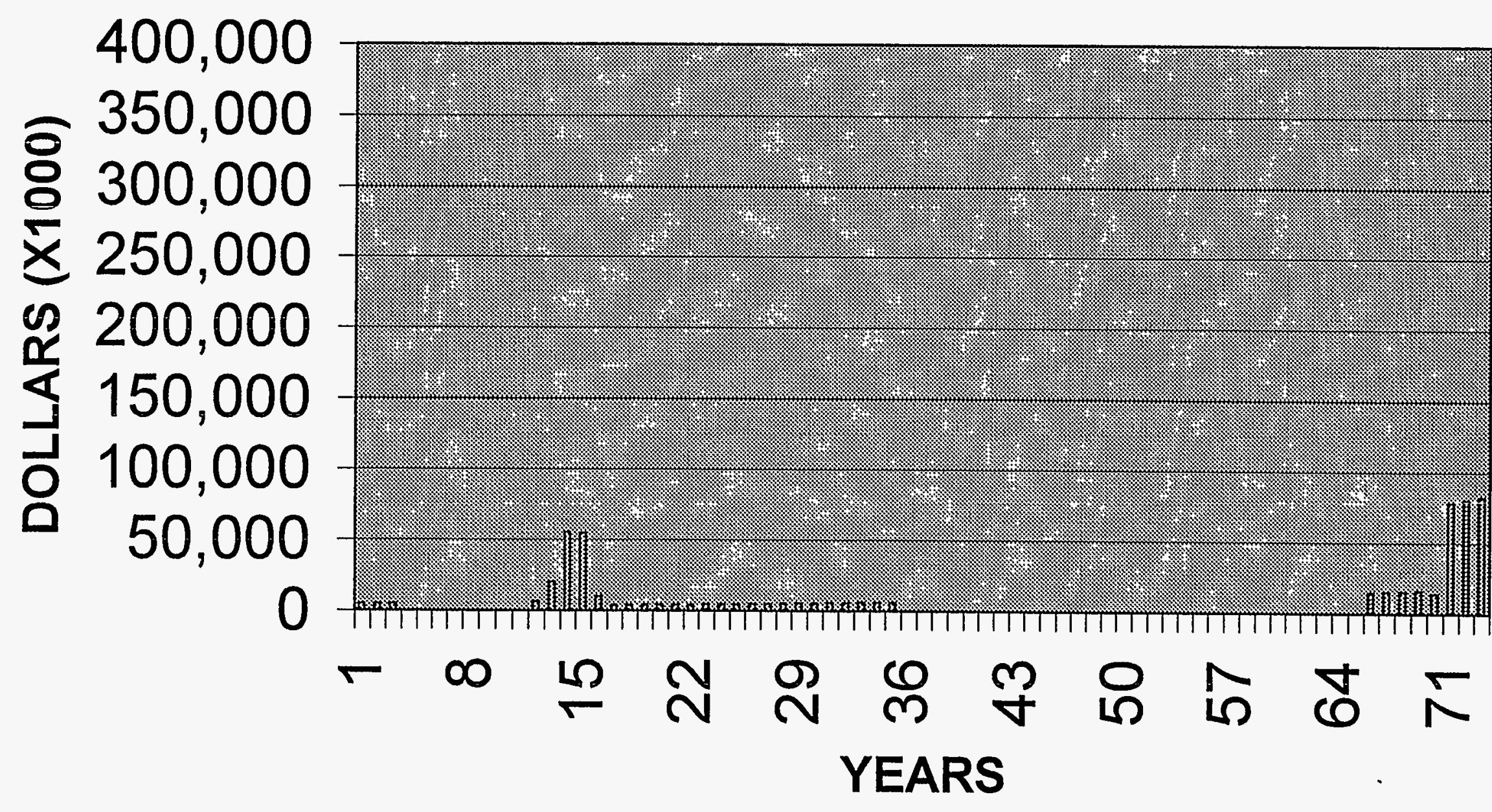


Date: January 30, 1998

To: John K. Rawlins $\quad$ MS 3765 6-4304

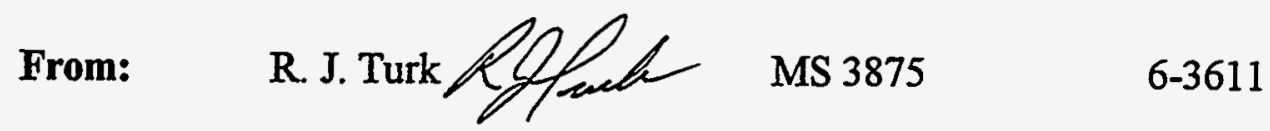

Subject: ECONOMIC AND LIFE CYCLE ANALYSIS CONDUCTED for the HOT ISOSTATIC PRESSING (HIP) STORAGE FACILITY in FPR- RJT-17-98

\section{Purpose:}

As requested an Economic and Life-Cycle Cost (LCC) has been conducted to evaluate the INTERIM STORAGE FACILY in FPR. The storage facility was evaluated based on information received by your team. This storage facility will be used to house canisters resulting from the HIIP production option until final repository disposition.

This economic analysis is based on information provided by J. K. Rawlins, and K. L. Williams. R. D. Adams provided cost estimates. D. Keller provided process personnel modeling.

\section{Methodology:}

The Economic Evaluation assumed a 69-year period (2003-2071) since this is the estimated time required to complete all of the anticipated remediation activities. The LCC identified and evaluated the initial development, construction, operating and post operating costs over the lifecycle. A discounted LCC analysis assumes a 1998-dollar basis, discounted $6.3 \%$ annually per the Office of Management and Budget (OMB) Circular A-94 for escalated costs. All costs are conservatively discounted assuming the end-of-year convention. 
Assumptions:

The scope of work and requirements of all related activities are vague at this time. Facility and processing costs were developed from historical experience associated with DD\&D work at the INEEL. The LCC analysis was generated to match cost estimating cost structure. These costs include Permitting, Direct and Indirect Construction, G\&A, Procurement Fee, Engineering, Inspection, Project Management, Construction Management, Escalation and Contingency costs.

The design period is assumed to be accomplished in five-years with construction completed in six-years. Labor rates were assumed as follows: Managers, $\$ 125 / \mathrm{hr}$; Engineers, $\$ 108 \$ / \mathrm{hr}$; Other Technicians $\$ 85 / \mathrm{hr}$; Administration/support staff $\$ 65 / \mathrm{hr}$; Operators and Maintenance personnel $\$ 65 / \mathrm{hr}$. The operational period for this facility was assumed to be twenty years, to match the appropriate operating facility. This period is followed by five years for shipping to the repository, followed by three years of post-operations activities. Due to this project's lack of complexity and non-radioactive contaminated environment, this analysis assumed a decommissioning cost equal to $10 \%$ of the non-escalated engineering design cost, decontamination costs equal to $0 \%$ of non-escalated total pre-operation cost, and demolition costs equal to $8 \%$ of the non-escalated total pre-operation cost. Utilities were calculated assuming a $\$ .0824$ per $\mathrm{kWh}$. Surveillance and Monitoring costs were calculated at $\$ 65,000$ per employee per year for manned security during operations then reduced to $\$ 10,000$ for remote surveillance during non-operation periods.

\section{Results:}

The HOT ISOSTATIC PRESSING FACILITY INTERIM STORAGE in FPR has a discounted LCC of $\$ 231$ million.

Attachments:

cc: R. J. Turk File 


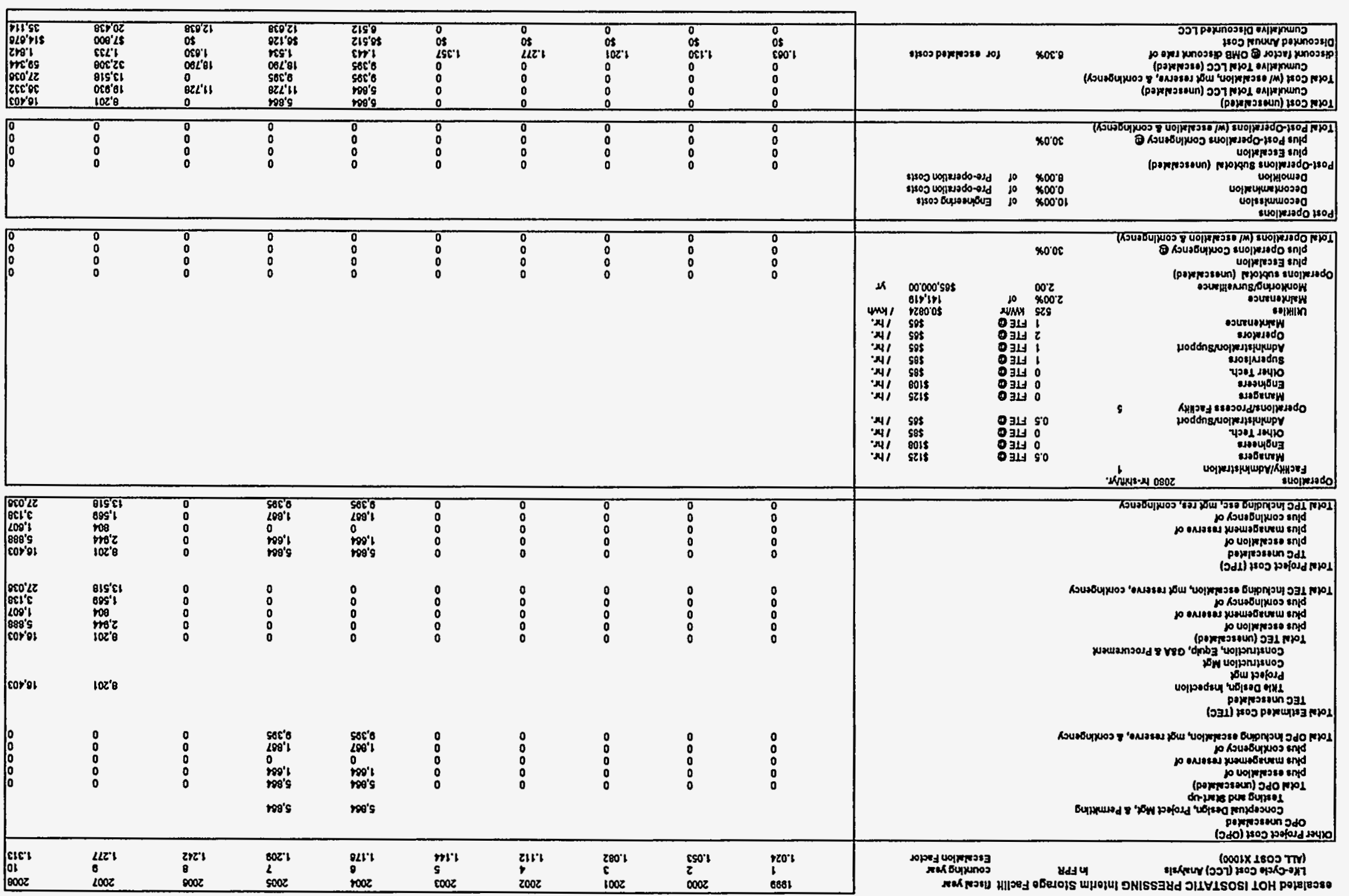




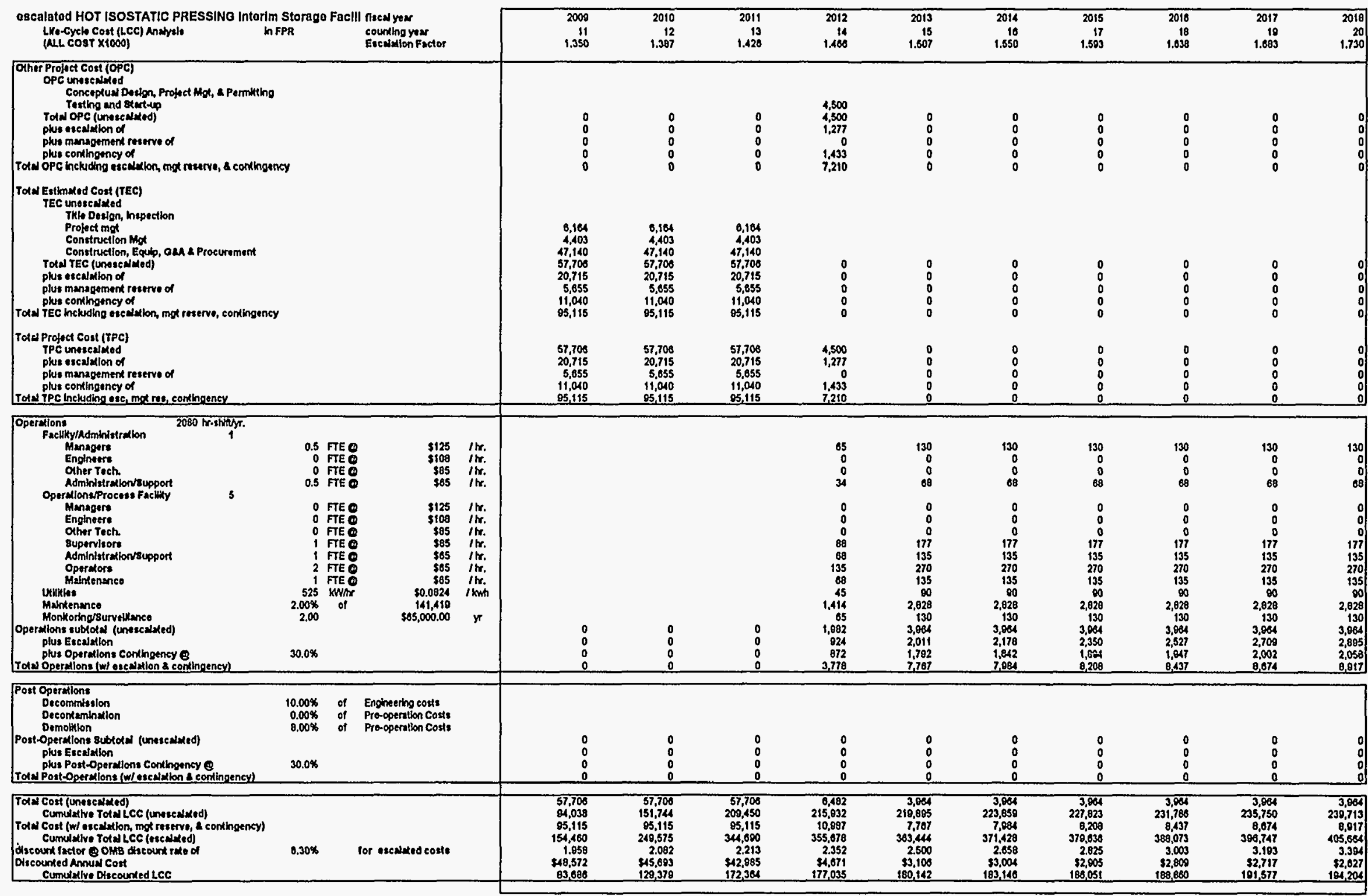




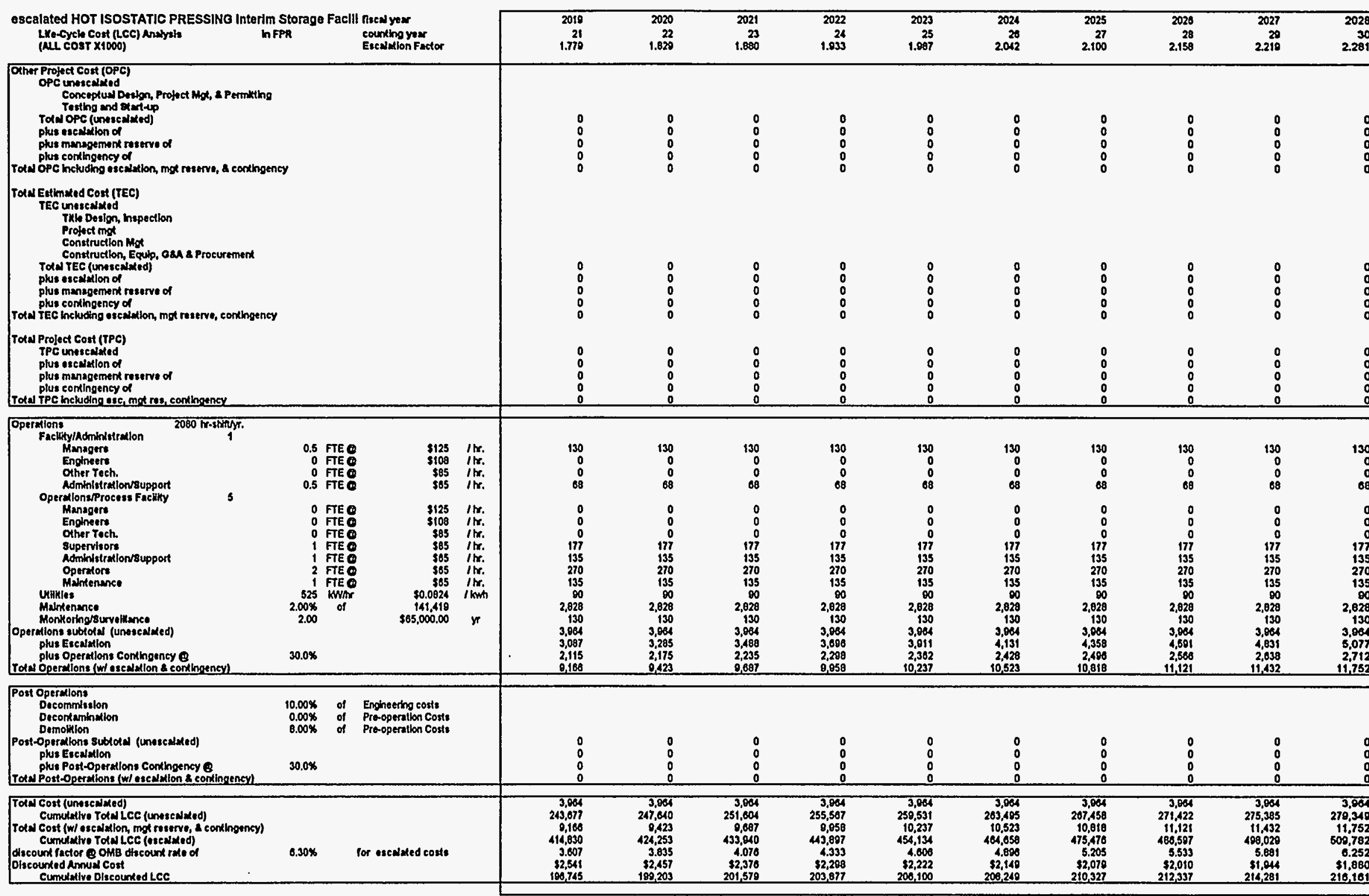




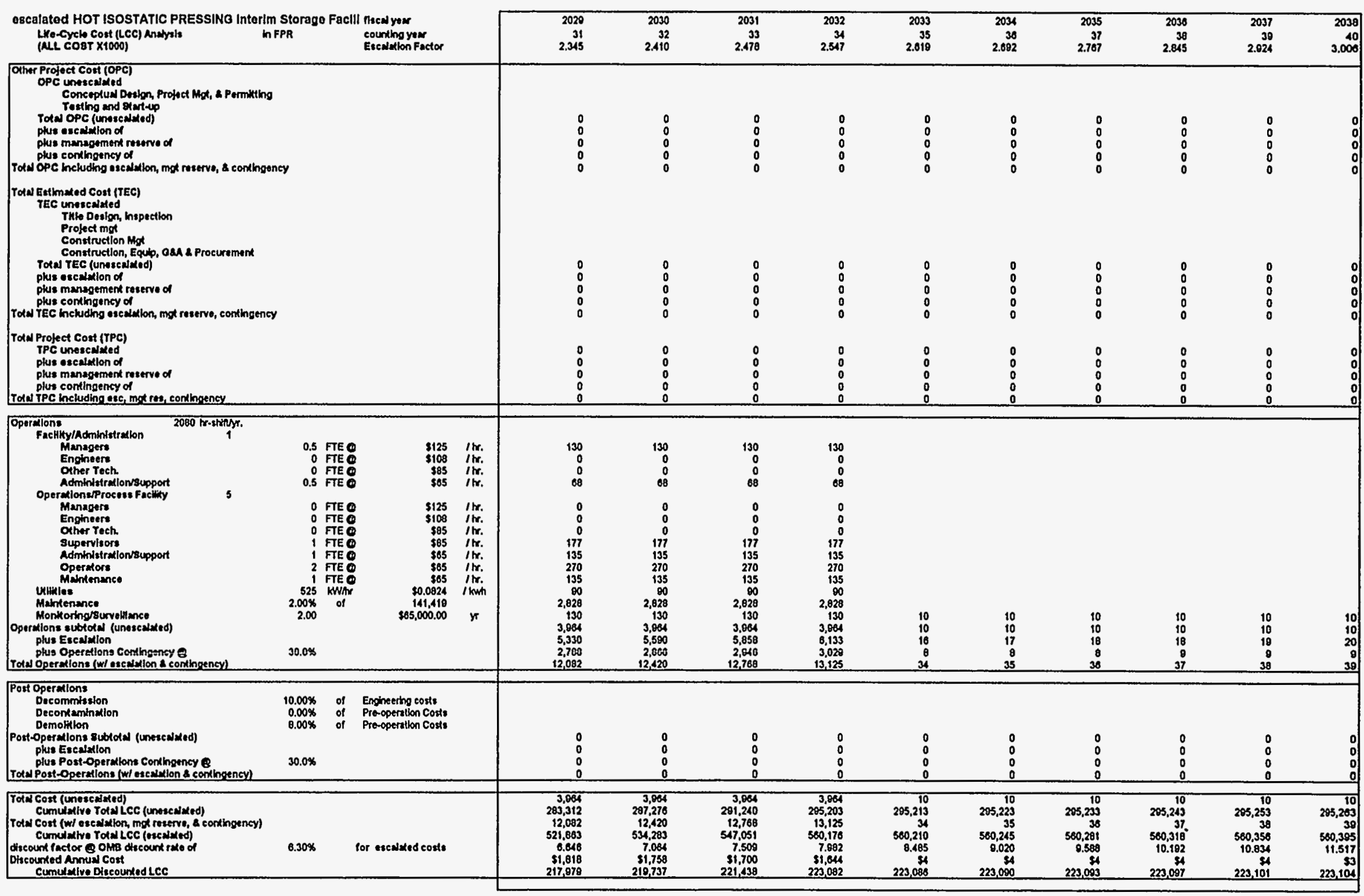




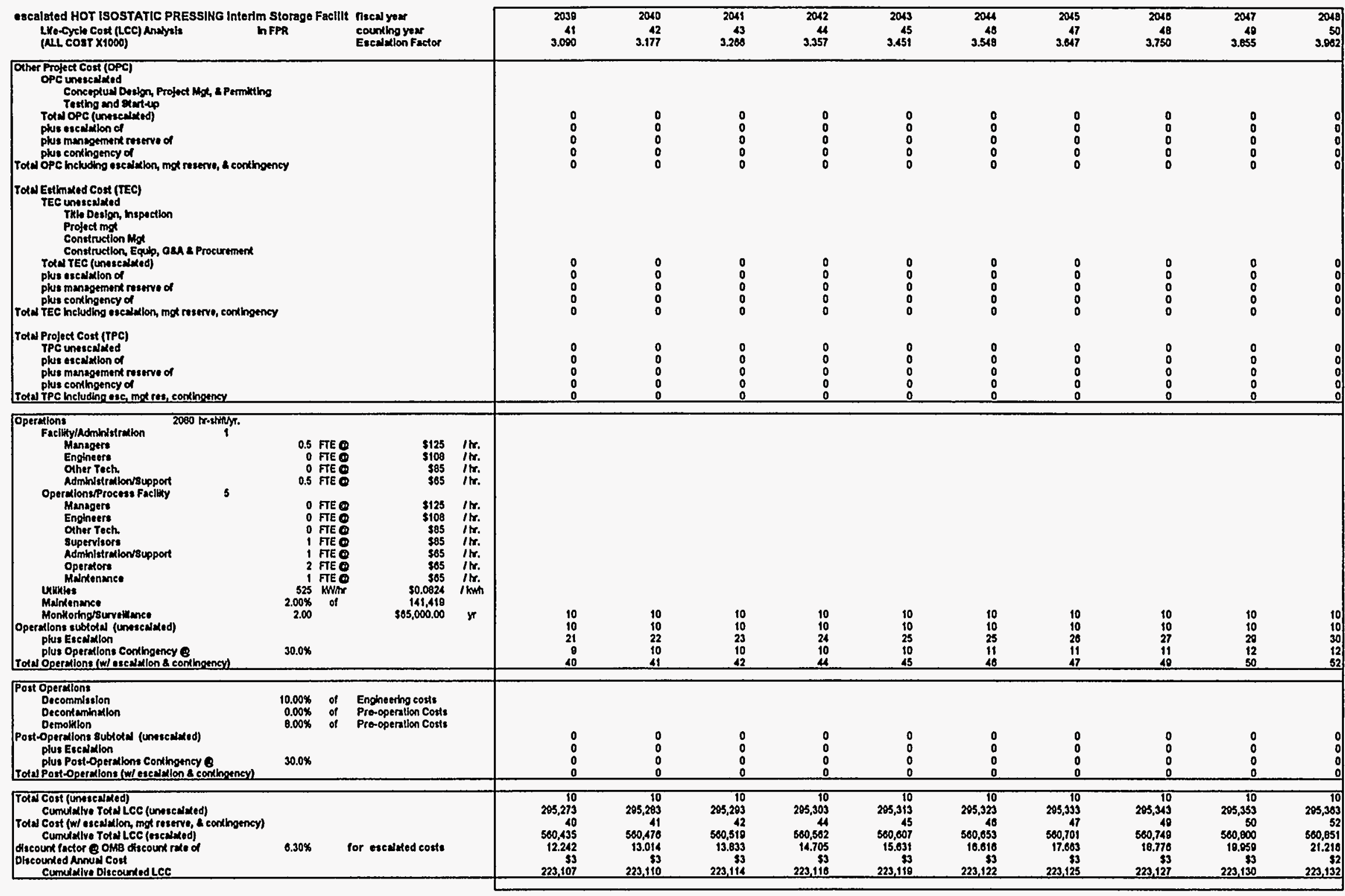




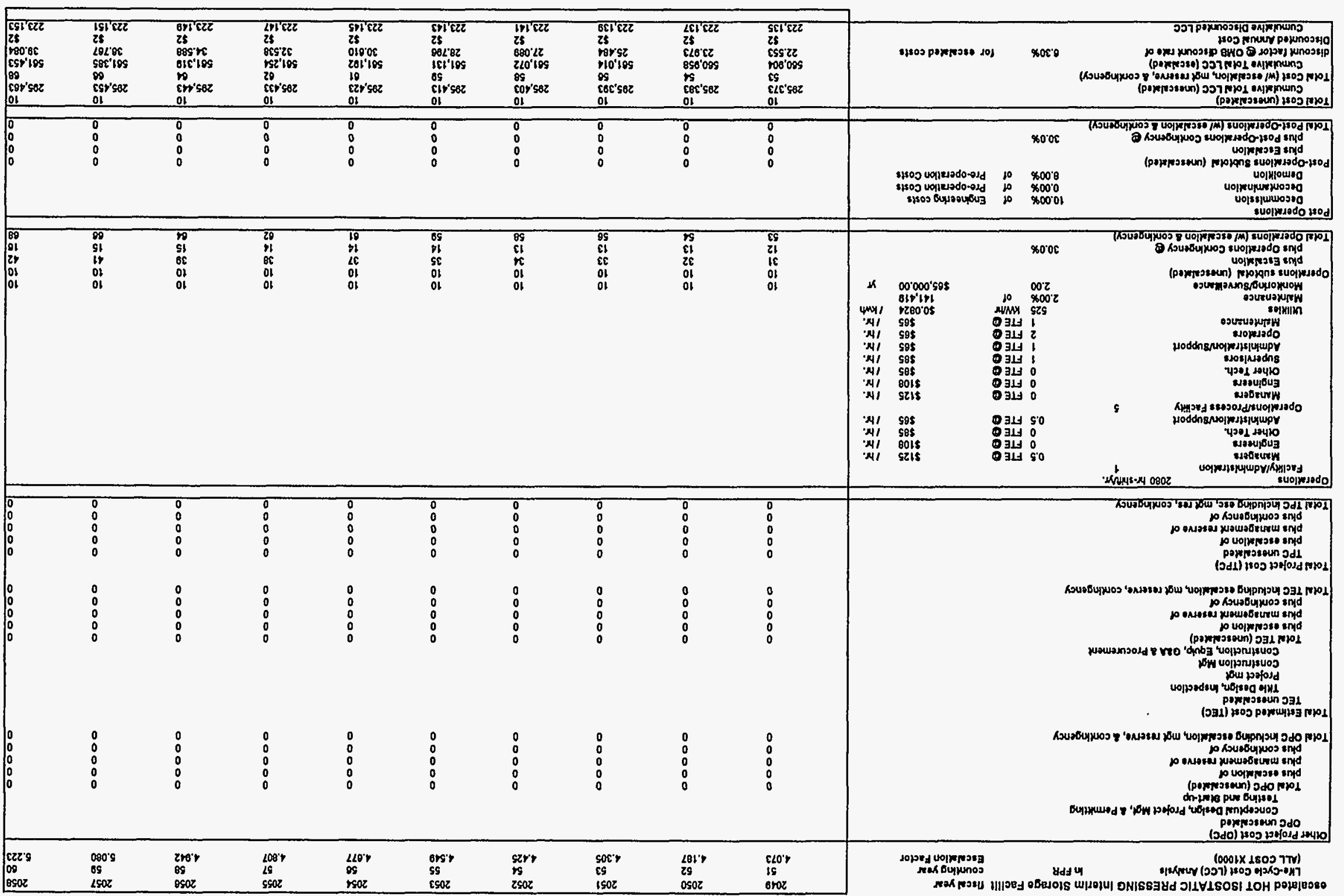




\begin{tabular}{|c|c|c|c|c|c|c|c|c|c|c|c|c|c|}
\hline 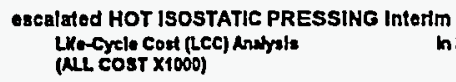 & $\begin{array}{l}\text { n Storago Facllit } \\
\text { AFPR }\end{array}$ & 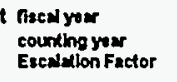 & & $\begin{array}{l}2059 \\
611 \\
5.368\end{array}$ & $\begin{array}{l}2080 \\
62 \\
5.510\end{array}$ & $\begin{array}{l}2081 \\
83 \\
5.874\end{array}$ & $\begin{array}{l}2082 \\
641 \\
5.833\end{array}$ & $\begin{array}{l}2003 \\
5.05 \\
5.808\end{array}$ & $\begin{array}{l}2004 \\
0.184\end{array}$ & $\begin{array}{l}2005 \\
67 \\
0.338\end{array}$ & $\begin{array}{l}2005 \\
6.516\end{array}$ & $\begin{array}{l}2007 \\
0.00 \\
0.008\end{array}$ & $\begin{array}{r}2068 \\
7.78 \\
0.884\end{array}$ \\
\hline \multicolumn{4}{|l|}{ 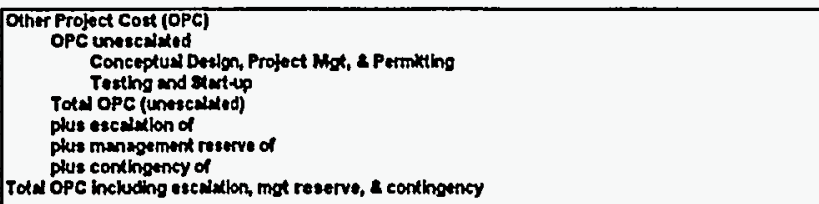 } & $\begin{array}{l}0 \\
: \\
0 \\
0\end{array}$ & $\begin{array}{l}0 \\
0 \\
0 \\
0 \\
0\end{array}$ & $\begin{array}{l}0 \\
0 \\
0 \\
0 \\
0\end{array}$ & $\begin{array}{l}0 \\
0 \\
0 \\
0\end{array}$ & $\begin{array}{l}0 \\
0 \\
0 \\
0\end{array}$ & $\begin{array}{l}0 \\
: \\
0 \\
0\end{array}$ & $\begin{array}{l}0 \\
0 \\
0 \\
0 \\
0\end{array}$ & $\begin{array}{l}0 \\
0 \\
0 \\
0 \\
0\end{array}$ & $\begin{array}{l}0 \\
0 \\
0 \\
0 \\
0\end{array}$ & $\begin{array}{l}0 \\
0 \\
0 \\
0 \\
0\end{array}$ \\
\hline \multicolumn{4}{|l|}{ 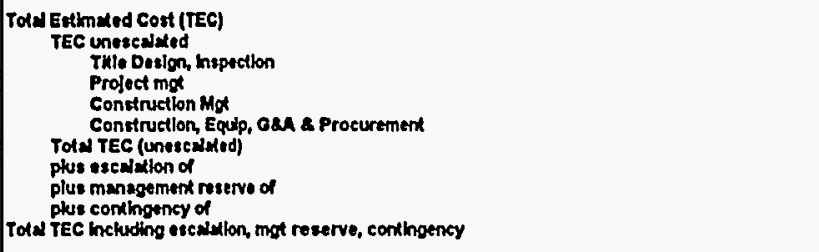 } & $\begin{array}{l}0 \\
0 \\
0 \\
0 \\
0\end{array}$ & $\begin{array}{l}0 \\
0 \\
0 \\
0 \\
0\end{array}$ & $\begin{array}{l}0 \\
0 \\
0 \\
0 \\
0\end{array}$ & $\begin{array}{l}0 \\
0 \\
0 \\
0 \\
0\end{array}$ & $\begin{array}{l}0 \\
0 \\
0 \\
0 \\
0\end{array}$ & $\begin{array}{l}0 \\
0 \\
0 \\
0 \\
0\end{array}$ & $\begin{array}{l}0 \\
0 \\
0 \\
0 \\
0\end{array}$ & $\begin{array}{l}0 \\
0 \\
0 \\
0 \\
0\end{array}$ & $\begin{array}{l}0 \\
0 \\
0 \\
0 \\
0\end{array}$ & \\
\hline \multicolumn{4}{|l|}{ 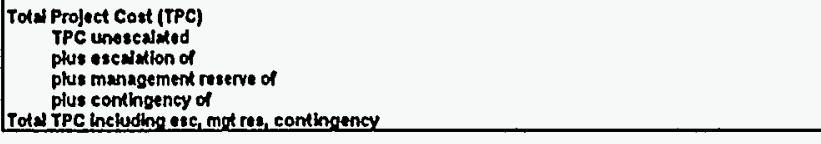 } & $\begin{array}{l}0 \\
0 \\
0 \\
0 \\
0\end{array}$ & $\begin{array}{l}0 \\
0 \\
0 \\
0 \\
0 \\
\end{array}$ & $\begin{array}{l}0 \\
0 \\
0 \\
0 \\
0 \\
\end{array}$ & $\begin{array}{l}0 \\
0 \\
0 \\
0 \\
0\end{array}$ & $\begin{array}{l}0 \\
0 \\
0 \\
0 \\
0 \\
\end{array}$ & $\begin{array}{l}0 \\
0 \\
0 \\
0 \\
0 \\
\end{array}$ & $\begin{array}{l}0 \\
0 \\
0 \\
0 \\
0 \\
\end{array}$ & $\begin{array}{l}0 \\
0 \\
0 \\
0 \\
0 \\
\end{array}$ & $\begin{array}{l}0 \\
0 \\
0 \\
0 \\
0\end{array}$ & \\
\hline \multirow{2}{*}{ 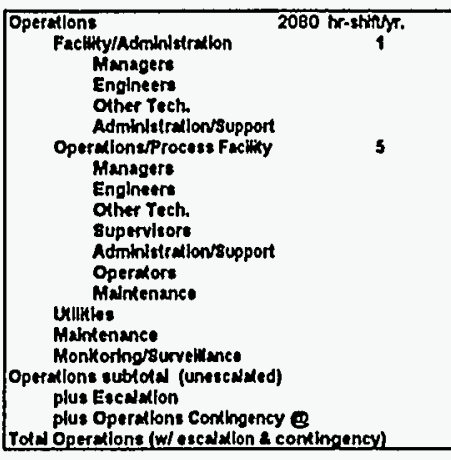 } & $\begin{array}{r}0.5 \text { FTE } \\
0 \text { TFE } \\
\text { O FTE } \\
0.5 \text { FTE }\end{array}$ & $\begin{array}{c}\$ 125 \\
\$ 108 \\
\$ 65 \\
\$ 65\end{array}$ & $\begin{array}{l}\text { Thr. } \\
\text { ihr. } \\
\text { ithr. } \\
\text { ith. }\end{array}$ & & & & & & $\begin{array}{r}130 \\
0 \\
0 \\
68\end{array}$ & $\begin{array}{r}130 \\
0 \\
0 \\
08\end{array}$ & $\begin{array}{r}130 \\
0 \\
0 \\
68\end{array}$ & $\begin{array}{r}130 \\
0 \\
0 \\
88\end{array}$ & $\begin{array}{r}130 \\
0 \\
0 \\
68\end{array}$ \\
\hline & 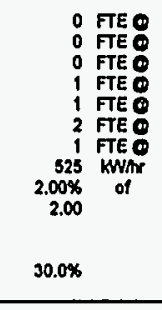 & $\begin{array}{r}\$ 125 \\
\$ 108 \\
\$ 85 \\
\$ 85 \\
\$ 65 \\
\$ 65 \\
\$ 85 \\
\$ 05 \\
\$ 10.024 \\
\$ 14,410 \\
\$ 85,000.00\end{array}$ & 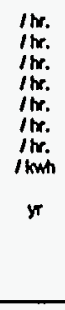 & $\begin{array}{l}10 \\
10 \\
4 \\
10 \\
70 \\
\end{array}$ & $\begin{array}{l}10 \\
10 \\
45 \\
17 \\
72 \\
\end{array}$ & $\begin{array}{l}10 \\
10 \\
47 \\
17 \\
74 \\
\end{array}$ & $\begin{array}{l}10 \\
10 \\
48 \\
17 \\
76 \\
\end{array}$ & $\begin{array}{l}10 \\
10 \\
50 \\
10 \\
78 \\
\end{array}$ & $\begin{array}{r}0 \\
0 \\
0 \\
177 \\
135 \\
270 \\
135 \\
00 \\
0 \\
130 \\
1,193 \\
5,862 \\
2,009 \\
0,000 \\
\end{array}$ & $\begin{array}{r}0 \\
0 \\
0 \\
177 \\
135 \\
270 \\
135 \\
100 \\
0 \\
0 \\
130 \\
1,135 \\
0.058 \\
2,158 \\
0,351 \\
\end{array}$ & $\begin{array}{r}0 \\
0 \\
0 \\
177 \\
135 \\
270 \\
135 \\
\infty 0 \\
0 \\
130 \\
1,135 \\
0,250 \\
2,258 \\
0,218 \\
\end{array}$ & $\begin{array}{r}0 \\
0 \\
0 \\
177 \\
135 \\
270 \\
135 \\
00 \\
0 \\
0 \\
130 \\
1,135 \\
0,460 \\
2,280 \\
0,882 \\
\end{array}$ & $\begin{array}{r}0 \\
0 \\
177 \\
135 \\
270 \\
135 \\
00 \\
0 \\
130 \\
1,135 \\
0,070 \\
2,34 \\
10,150 \\
\end{array}$ \\
\hline 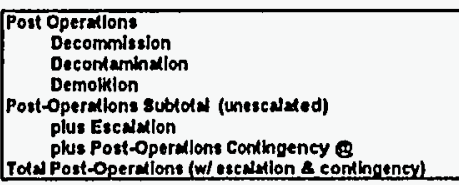 & $\begin{array}{ll}10.00 \% & \text { of } \\
0.00 \% & \text { of } \\
0.00 \% & \text { of } \\
& \\
30.0 \% & \end{array}$ & $\begin{array}{l}\text { Enoheering costs } \\
\text { Pro-opertition Costs } \\
\text { Pro-operation Costs }\end{array}$ & & $\begin{array}{l}0 \\
0 \\
0 \\
0 \\
\end{array}$ & $\begin{array}{l}0 \\
0 \\
0 \\
0 \\
\end{array}$ & $\begin{array}{l}0 \\
0 \\
0 \\
0 \\
\end{array}$ & $\begin{array}{l}0 \\
0 \\
0 \\
0 \\
\end{array}$ & $\begin{array}{l}0 \\
0 \\
0 \\
0 \\
\end{array}$ & $\begin{array}{l}0 \\
0 \\
0 \\
0 \\
\end{array}$ & $\begin{array}{l}0 \\
0 \\
0 \\
0 \\
\end{array}$ & $\begin{array}{l}0 \\
0 \\
0 \\
0 \\
\end{array}$ & $\begin{array}{l}0 \\
0 \\
0 \\
0 \\
\end{array}$ & 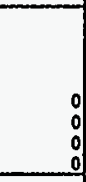 \\
\hline 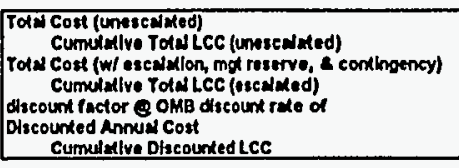 & $0.30 \%$ & for orcalued conts & & $\begin{array}{r}10 \\
205,473 \\
70 \\
561,522 \\
41,540 \\
32 \\
223,154 \\
\end{array}$ & $\begin{array}{r}10 \\
285,483 \\
72 \\
501,544 \\
44,1033 \\
32 \\
223,158 \\
\end{array}$ & $\begin{array}{r}10 \\
205,403 \\
74 \\
501,068 \\
40.916 \\
32 \\
223,158 \\
\end{array}$ & $\begin{array}{r}10 \\
205,503 \\
76 \\
501,744 \\
40,040 \\
\$ 32 \\
223,158 \\
\end{array}$ & $\begin{array}{r}10 \\
208,513 \\
78 \\
581,022 \\
53,0.07 \\
911 \\
223,161 \\
\end{array}$ & 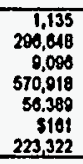 & 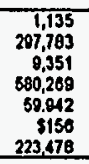 & $\begin{array}{r}1,135 \\
208,918 \\
0.013 \\
589,081 \\
03.716 \\
5151 \\
223,020 \\
\end{array}$ & 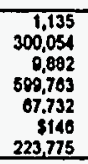 & 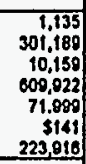 \\
\hline
\end{tabular}




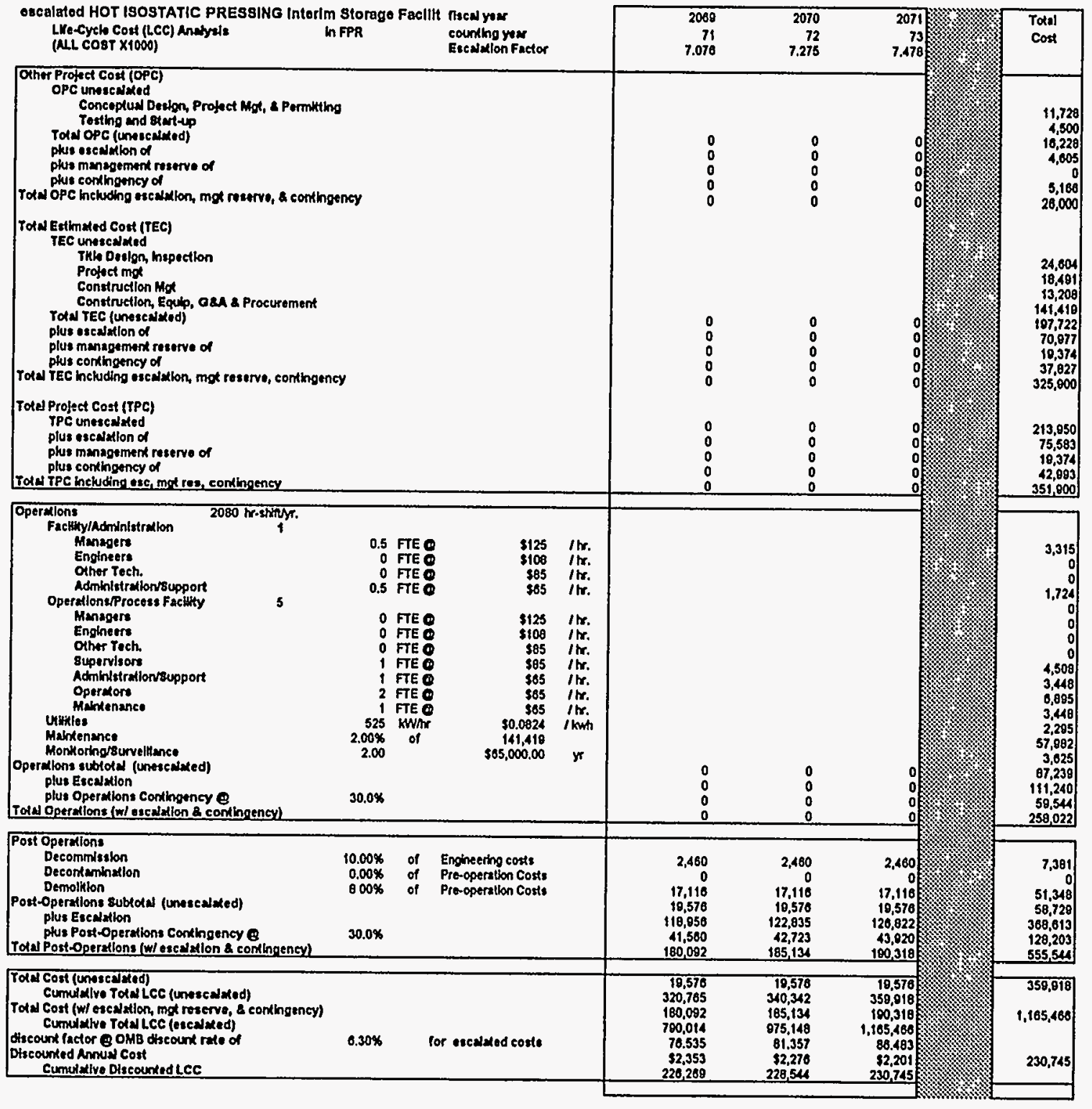




\section{TOTAL ANNUAL COST (escalated) HIP INTERIM STORAGE FACILTY IN FPR}

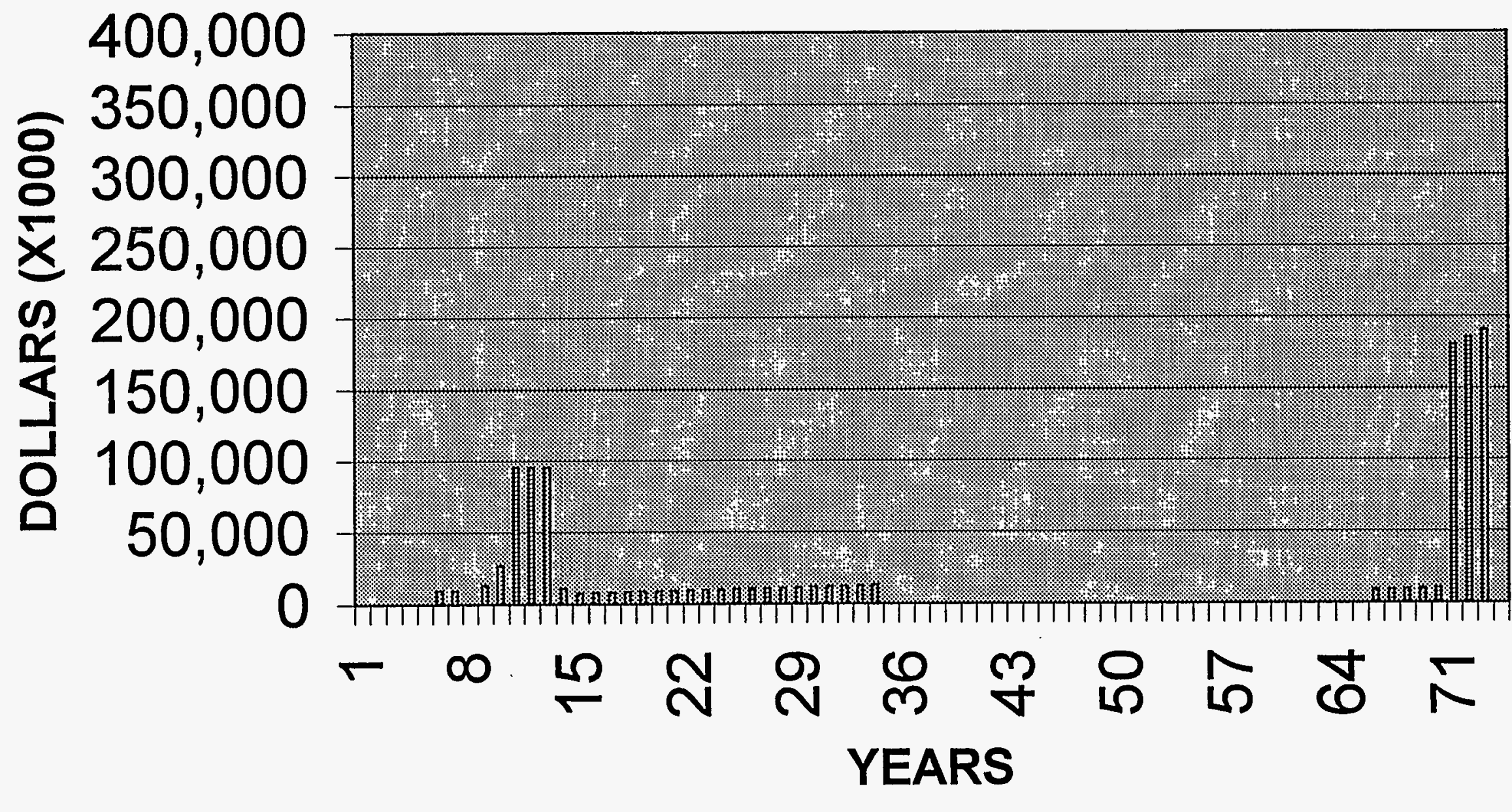




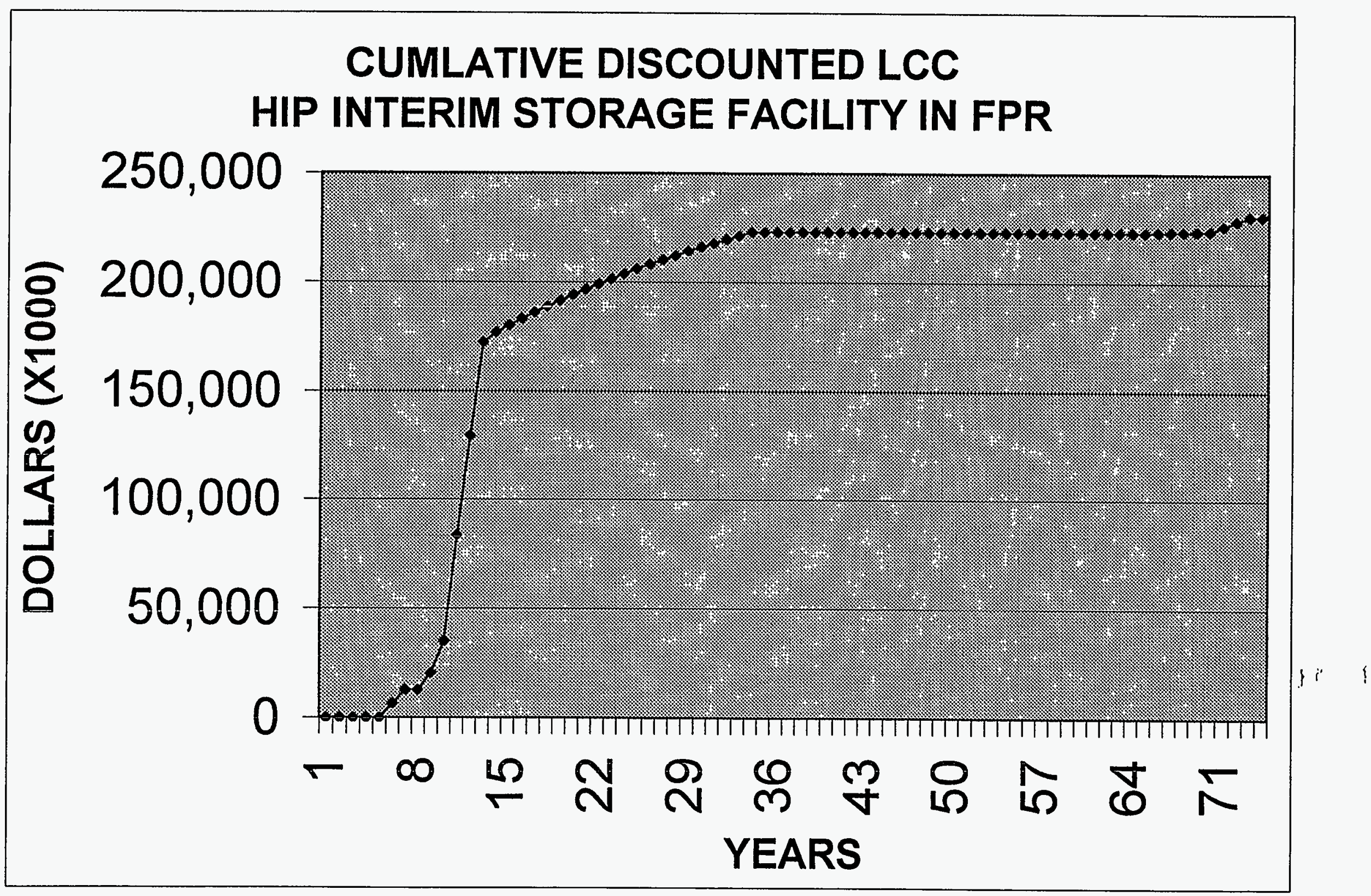


431.02\#

06/17/97

Rev. $\$ 04$
ENGINEERING DESIGN FILE

Function File Number - SPR-01

EDF Serial Number - EDF-IS-002

\section{Appendix 2}

\section{Drawings}




\section{CONTENTS}

The following drawings are included in this appendix:

Full Separations:

TRU/Class A Separations:
Site Plan

Floor Plan

Section

Storage Sleeve Detail

Site Plan

Floor Plan

Section

Storage Sleeve Detail

Hot Isostatic Press Waste Option: Site Plan

Floor Plan

Section

Section

Storage Sleeve Detail

Cooling System Plan
FS-C-1

FS-A-1

FS-A-2

FS-A-3

TRUA-C-7

TRUA-A-14

TRUA-A-15

TRUA-A-16

HWO-C-2

HWO-A-14

HWO-A-15

HWO-A-16

HWO-A-17

HWO-HV-2 


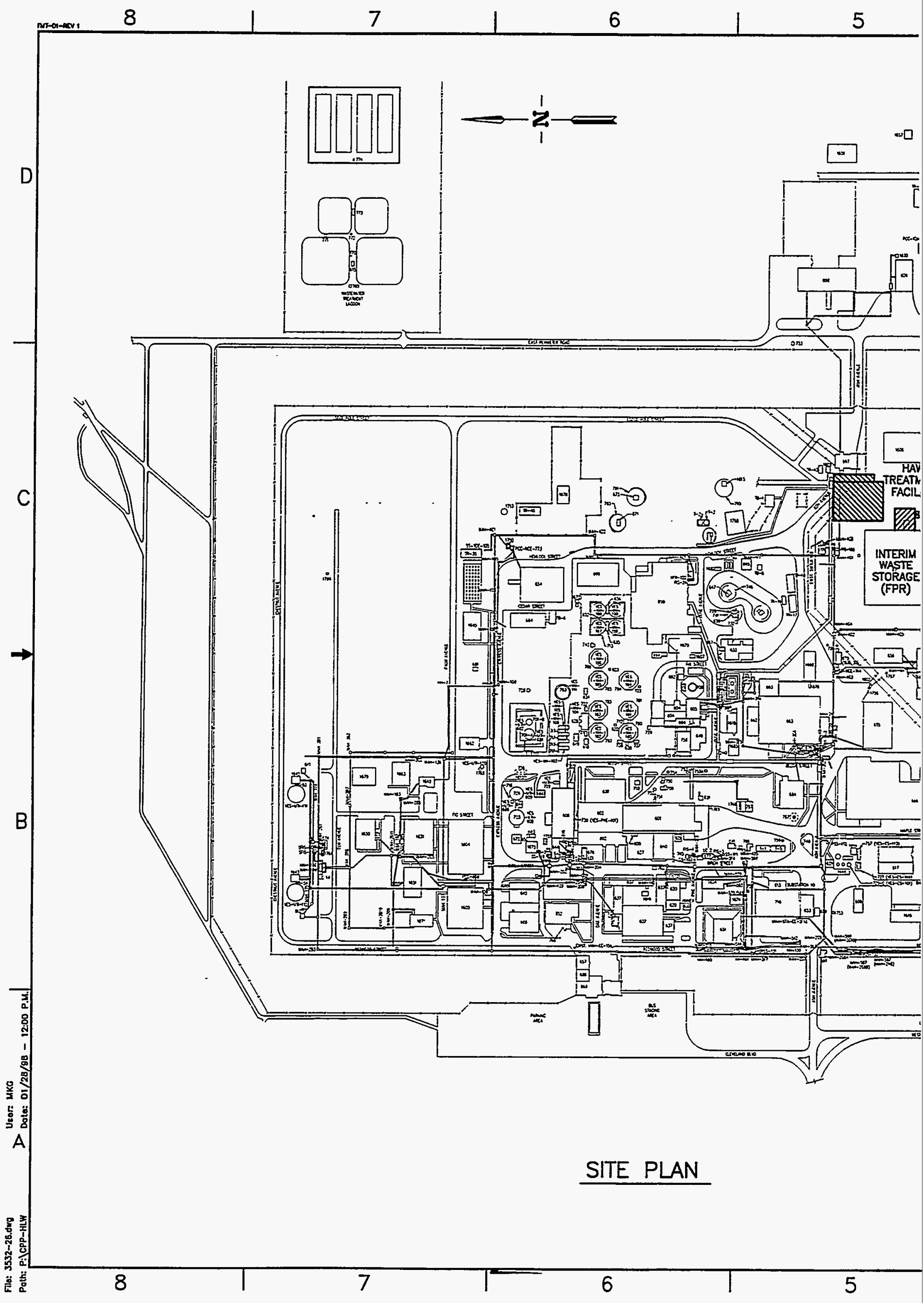




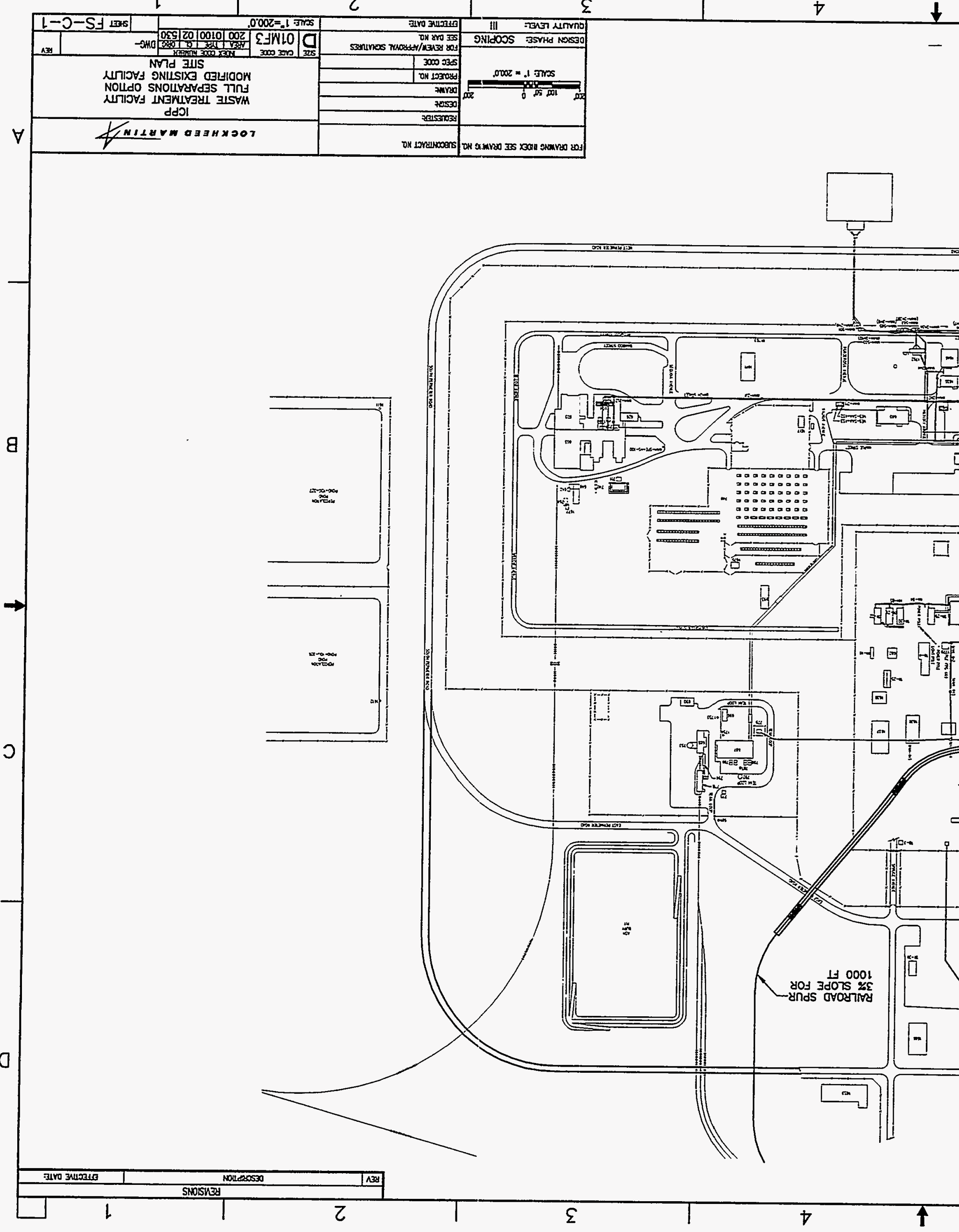




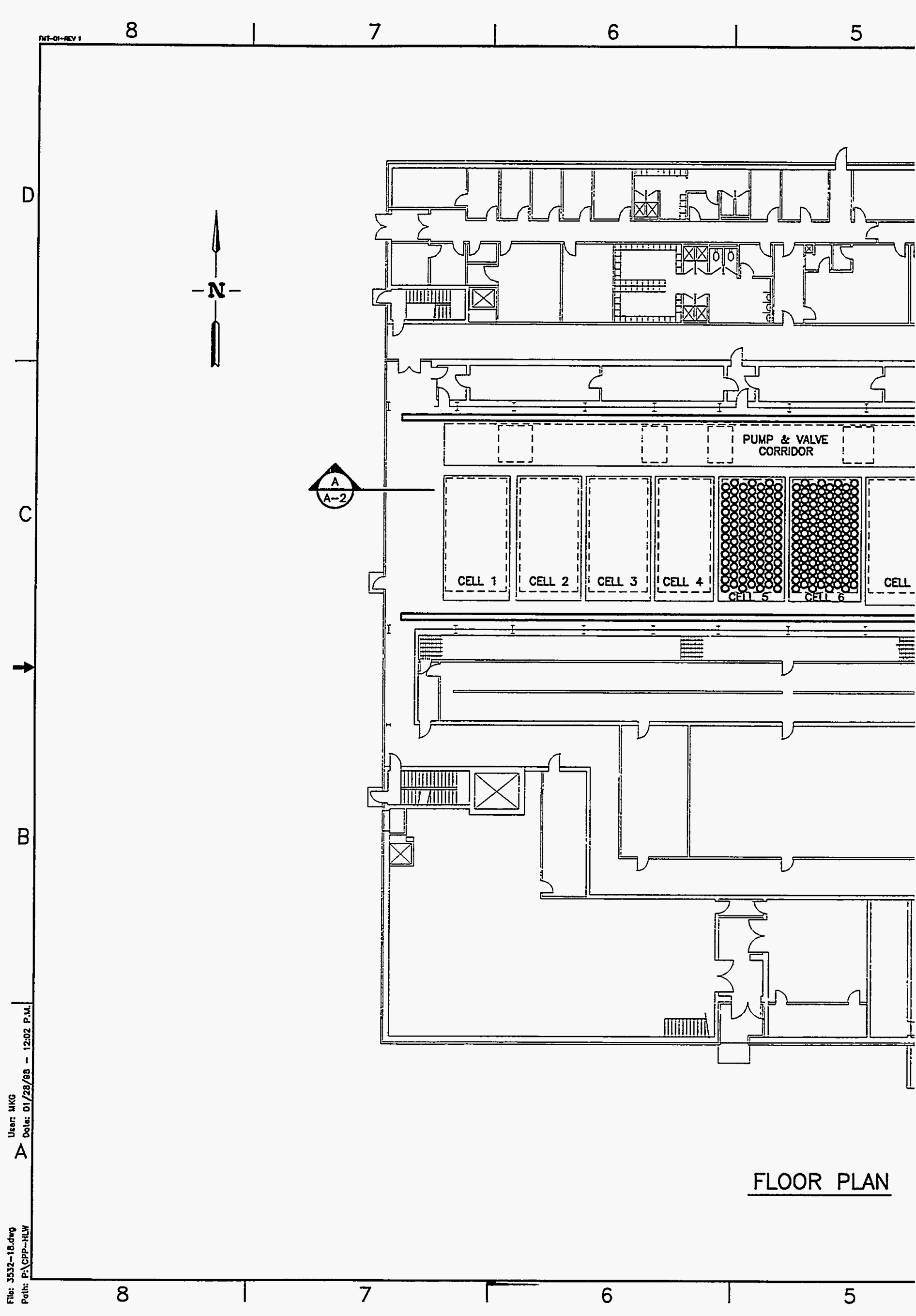




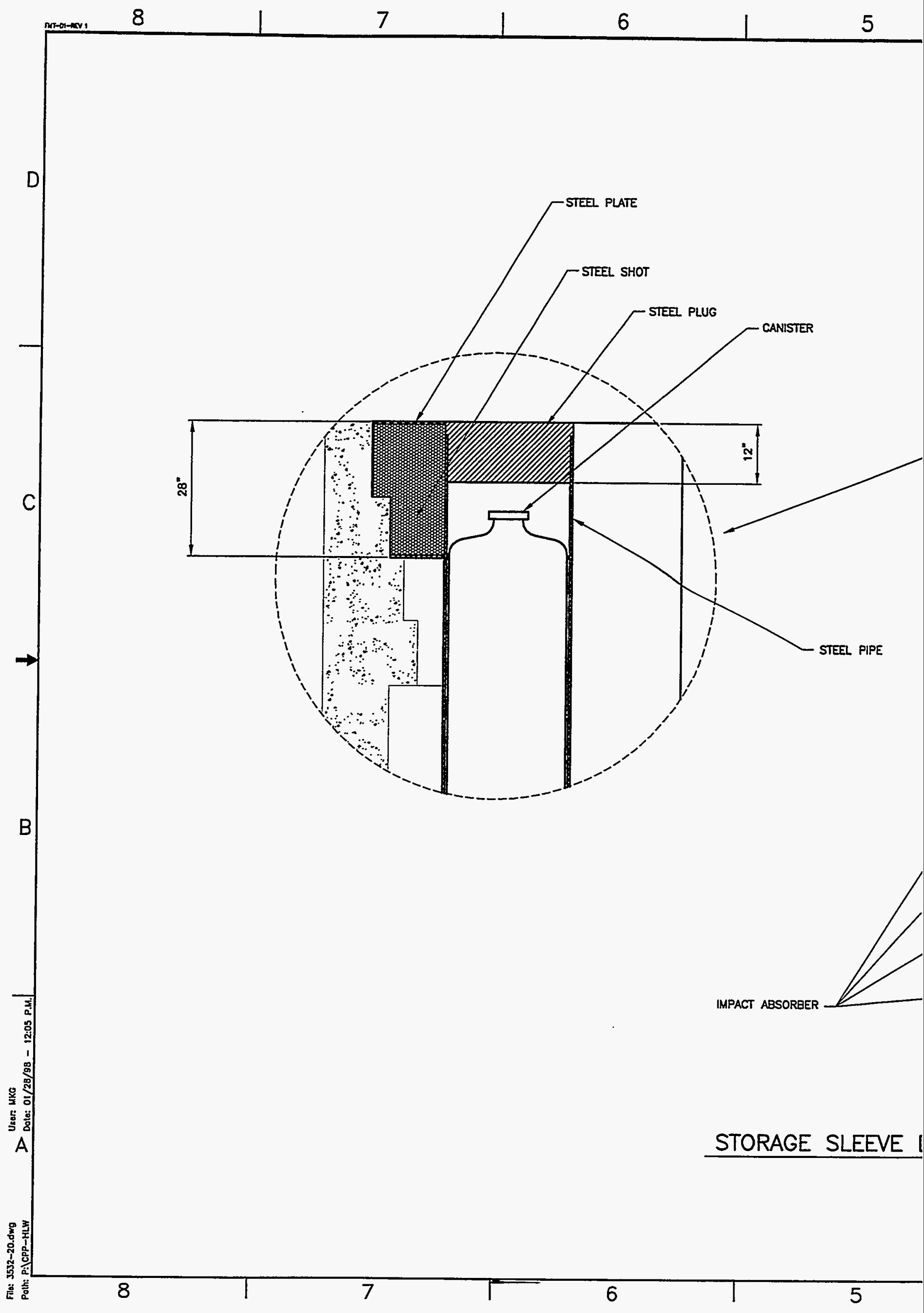




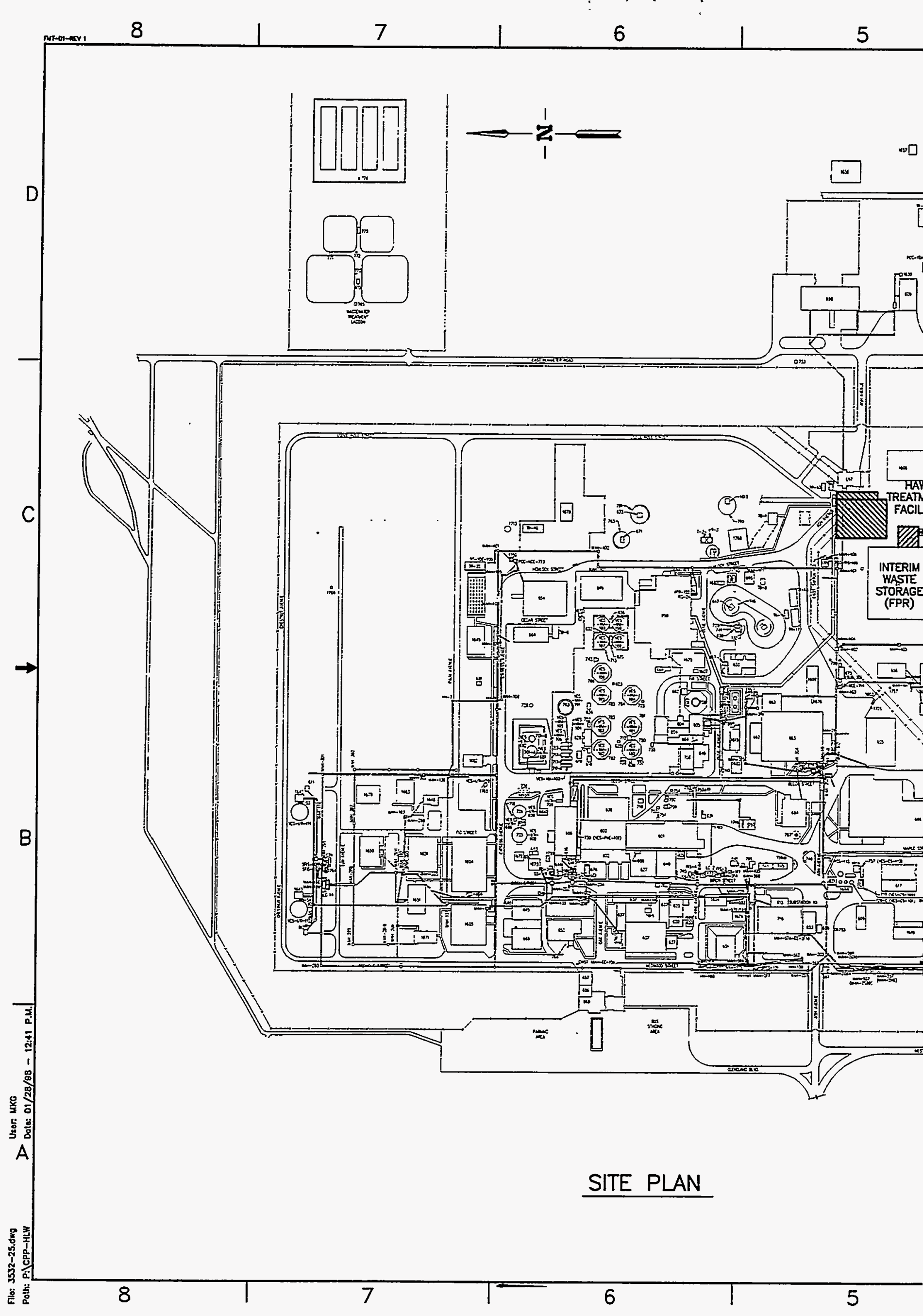




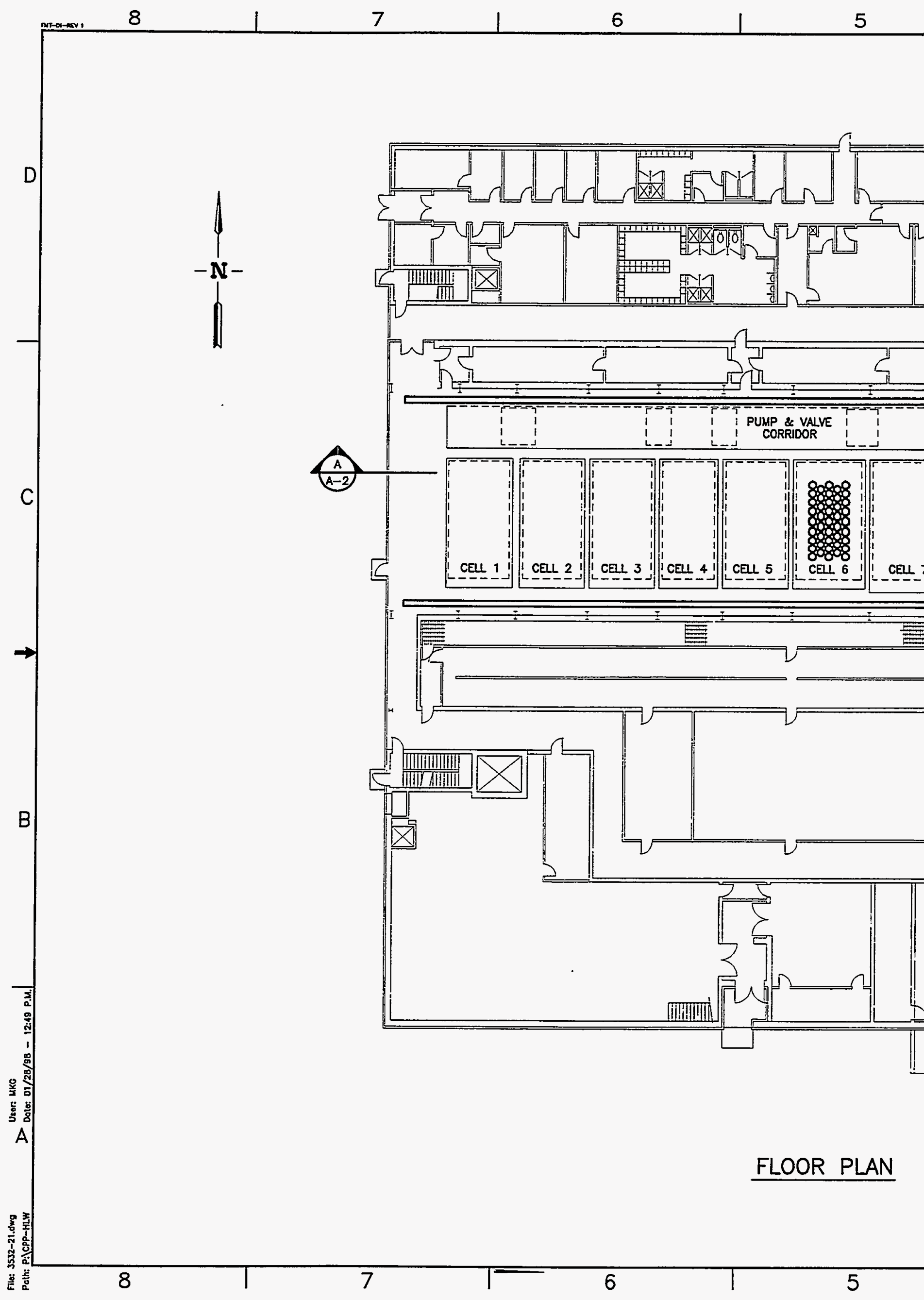




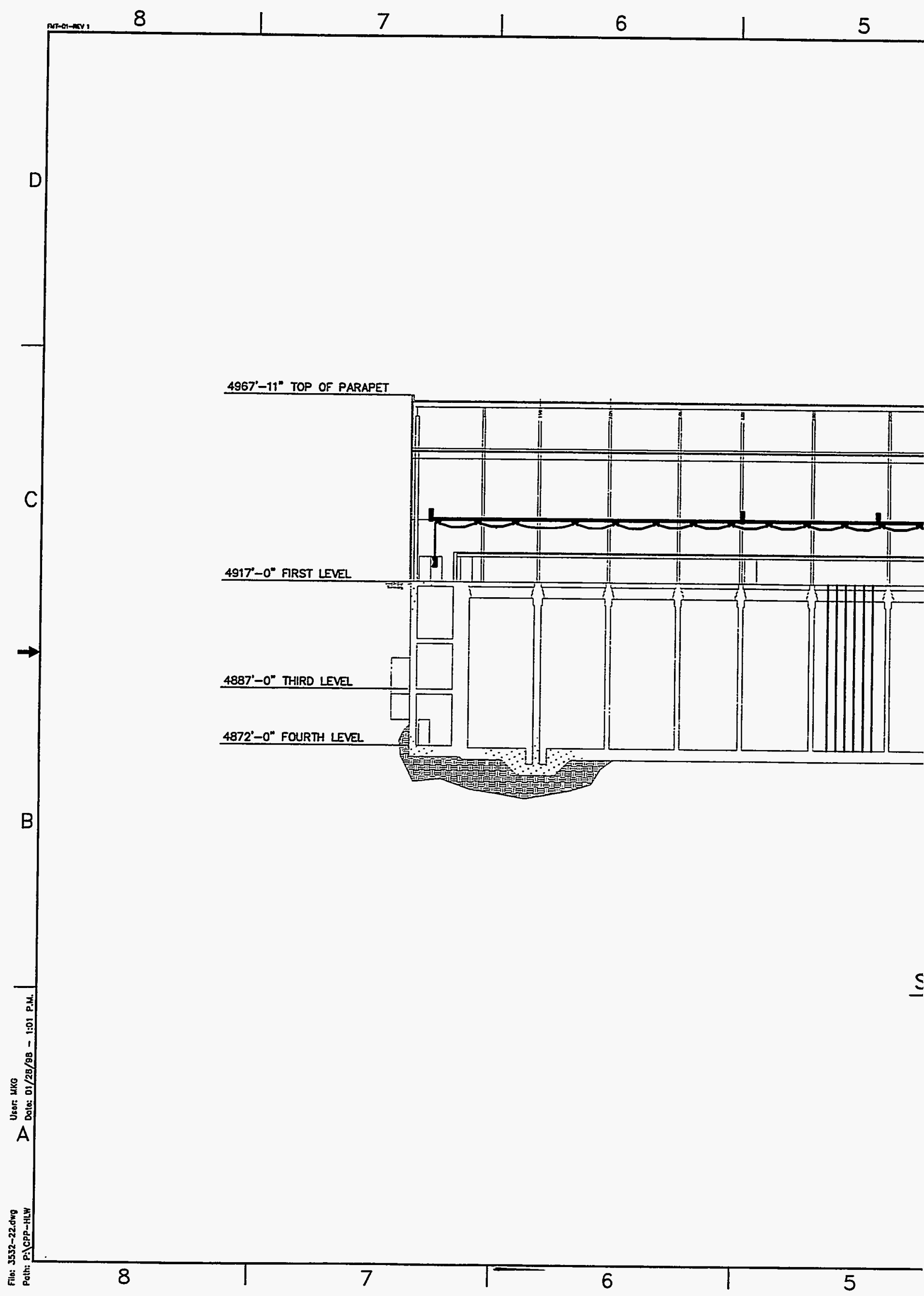




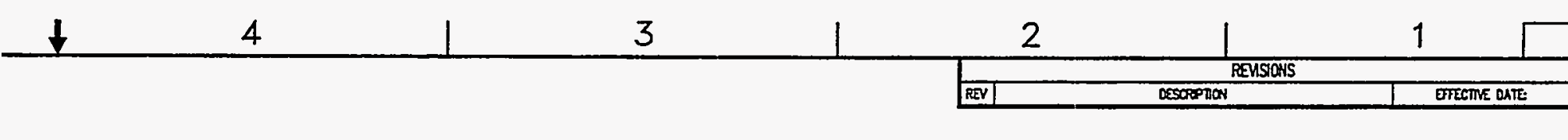

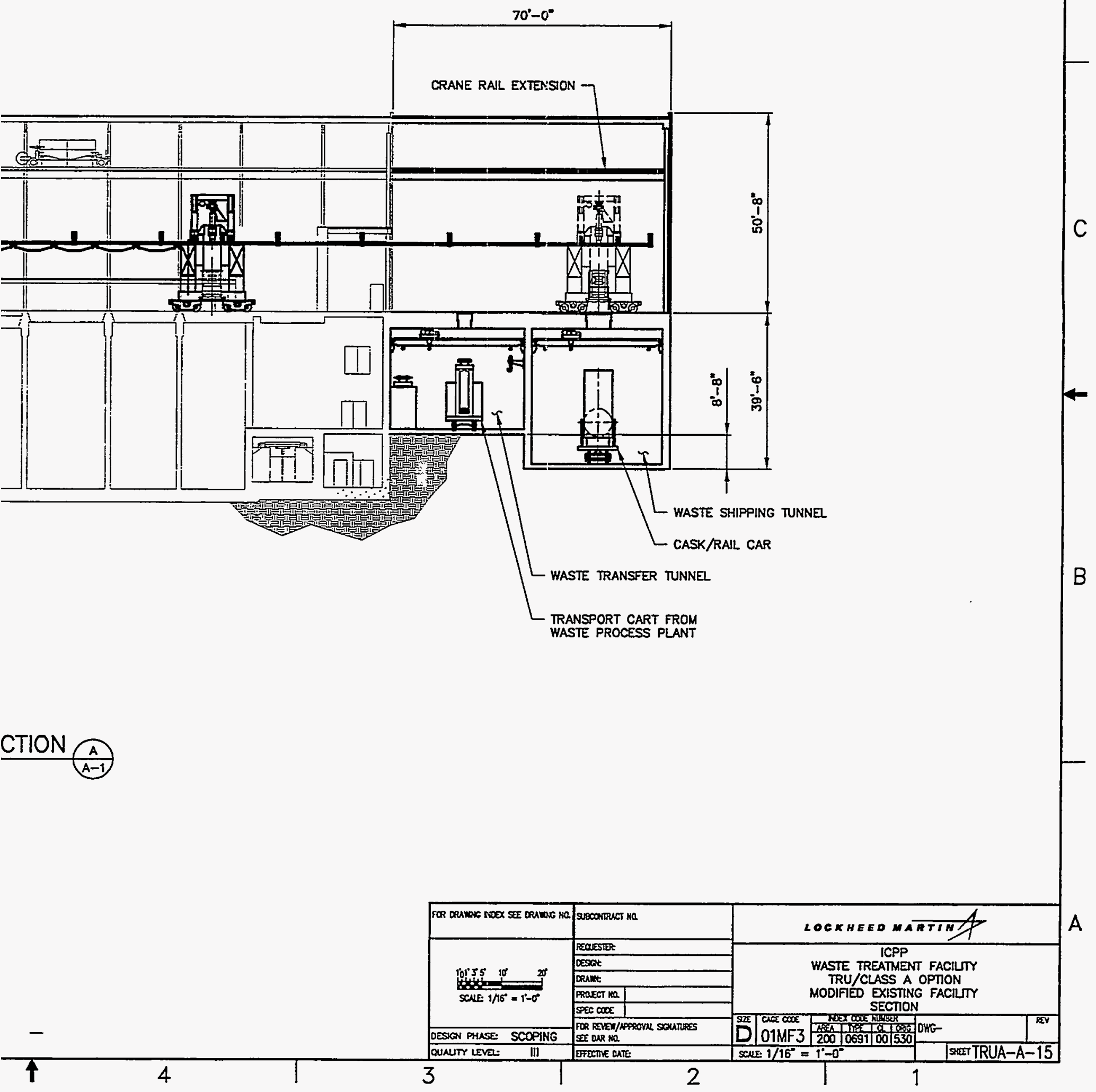




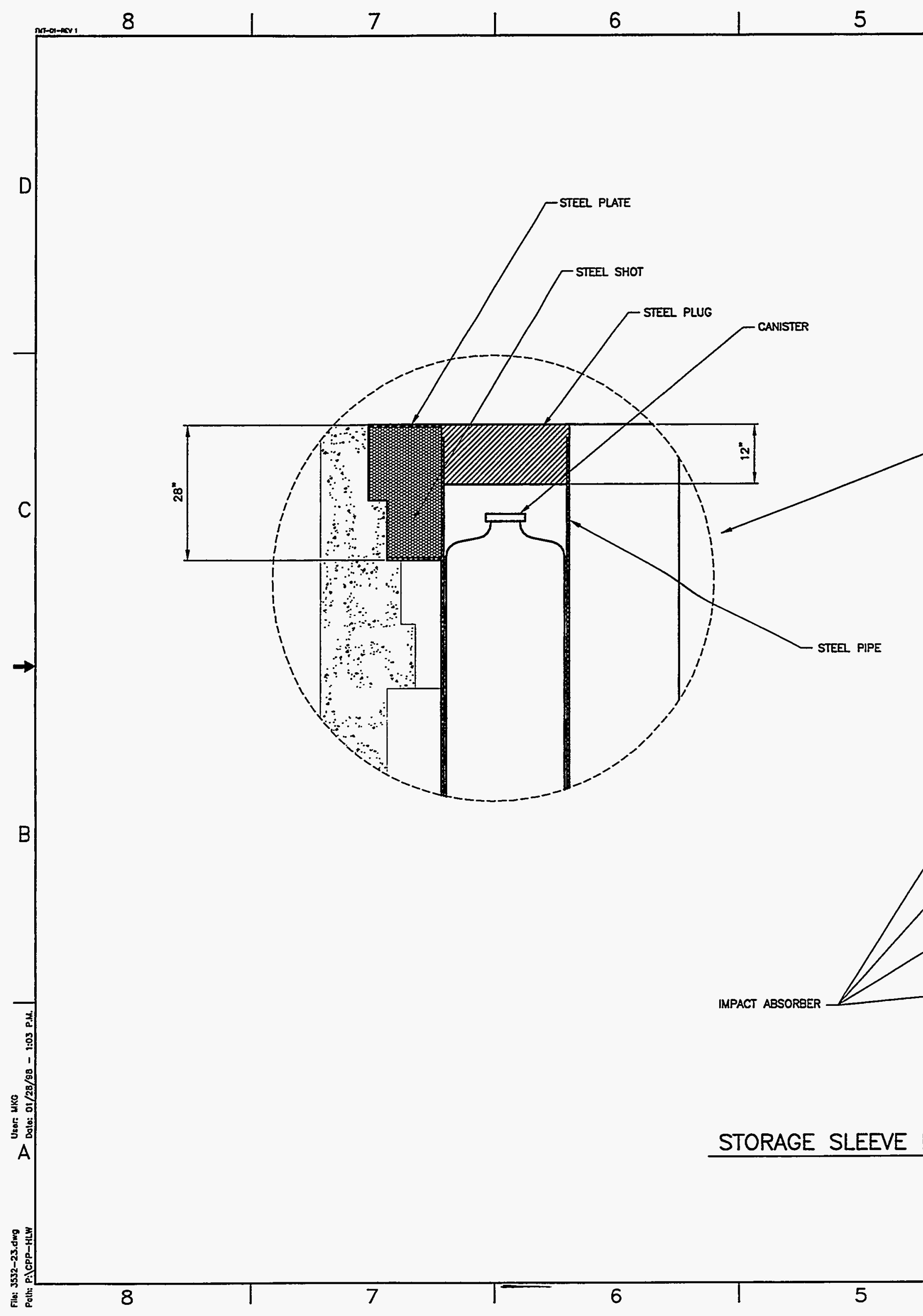




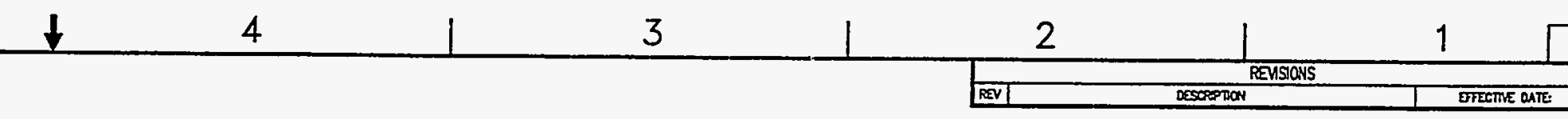

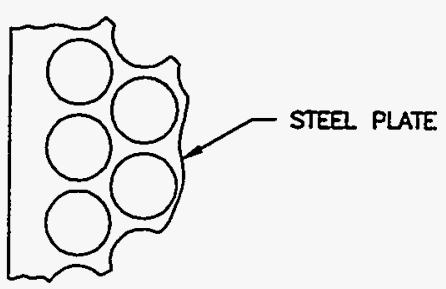

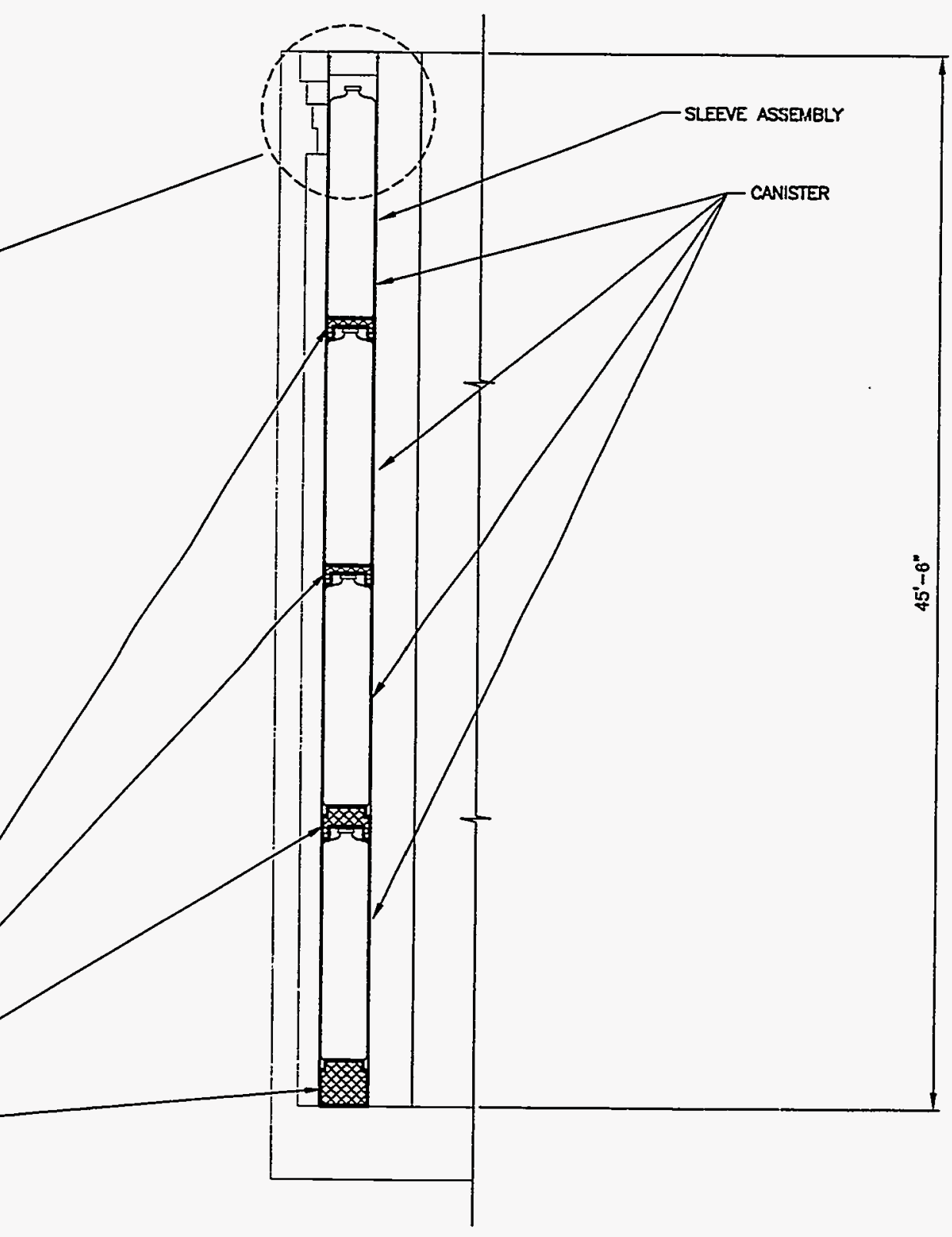

AlL

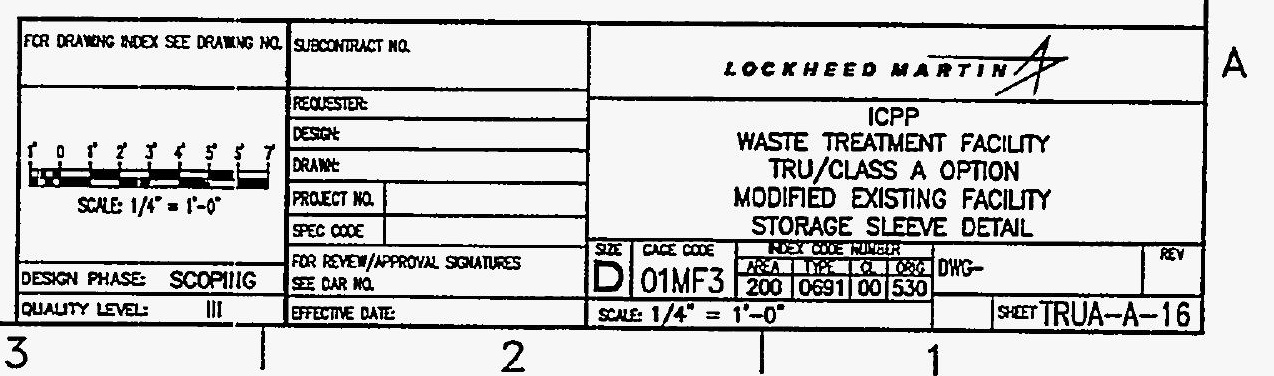




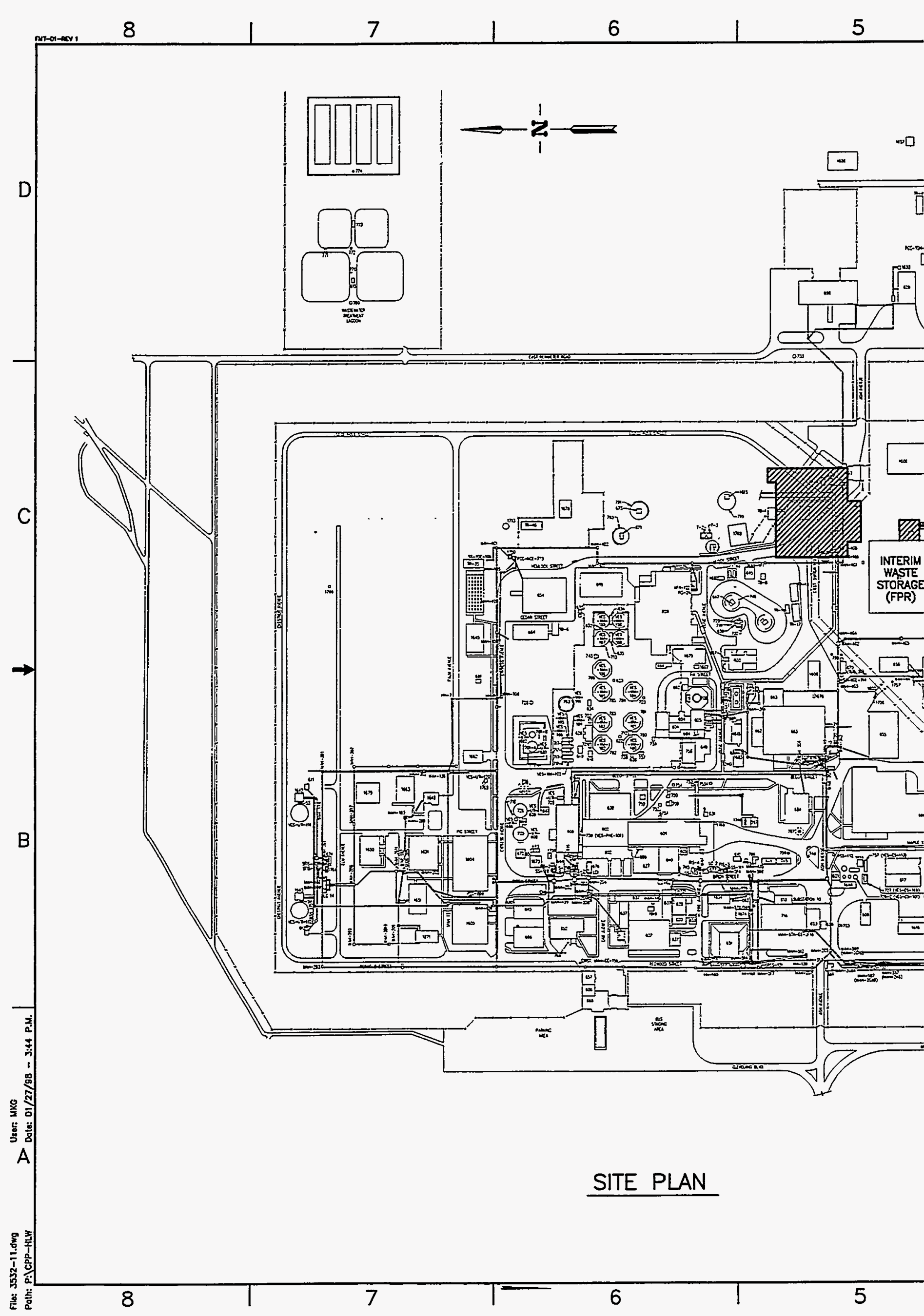




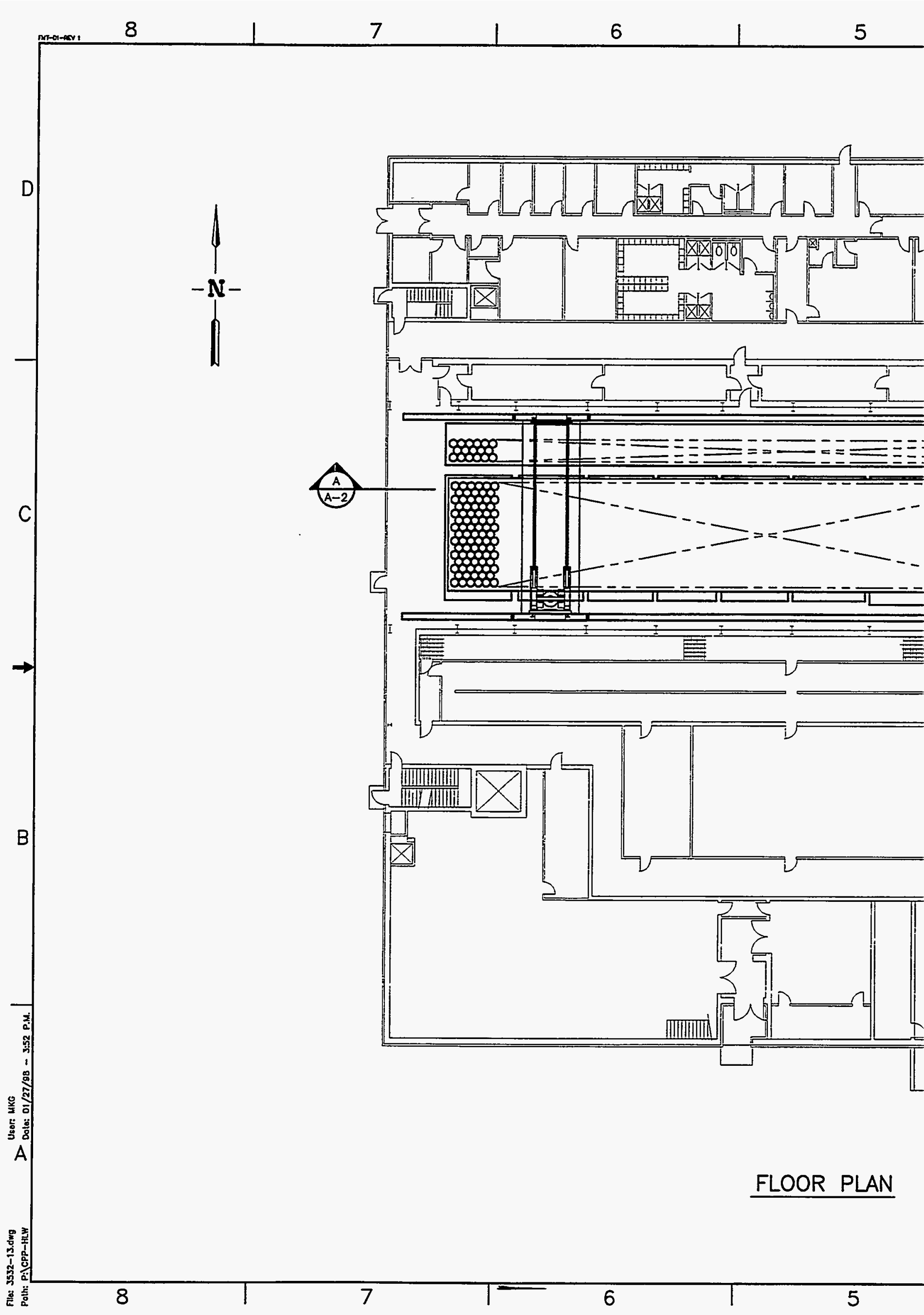




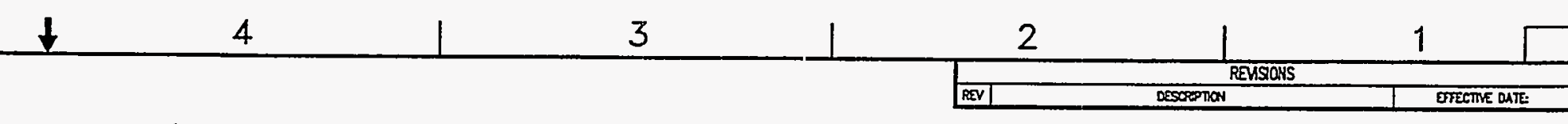

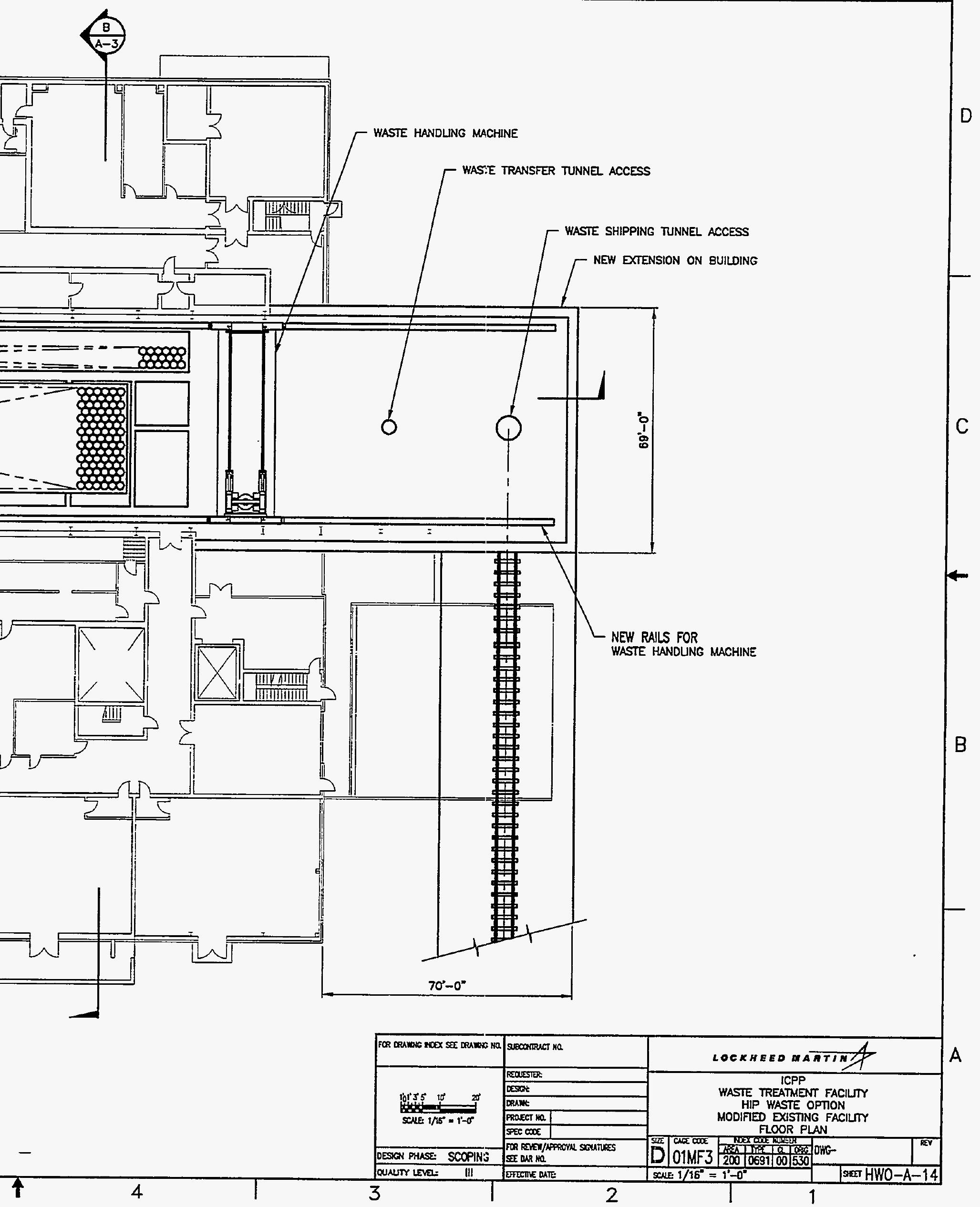




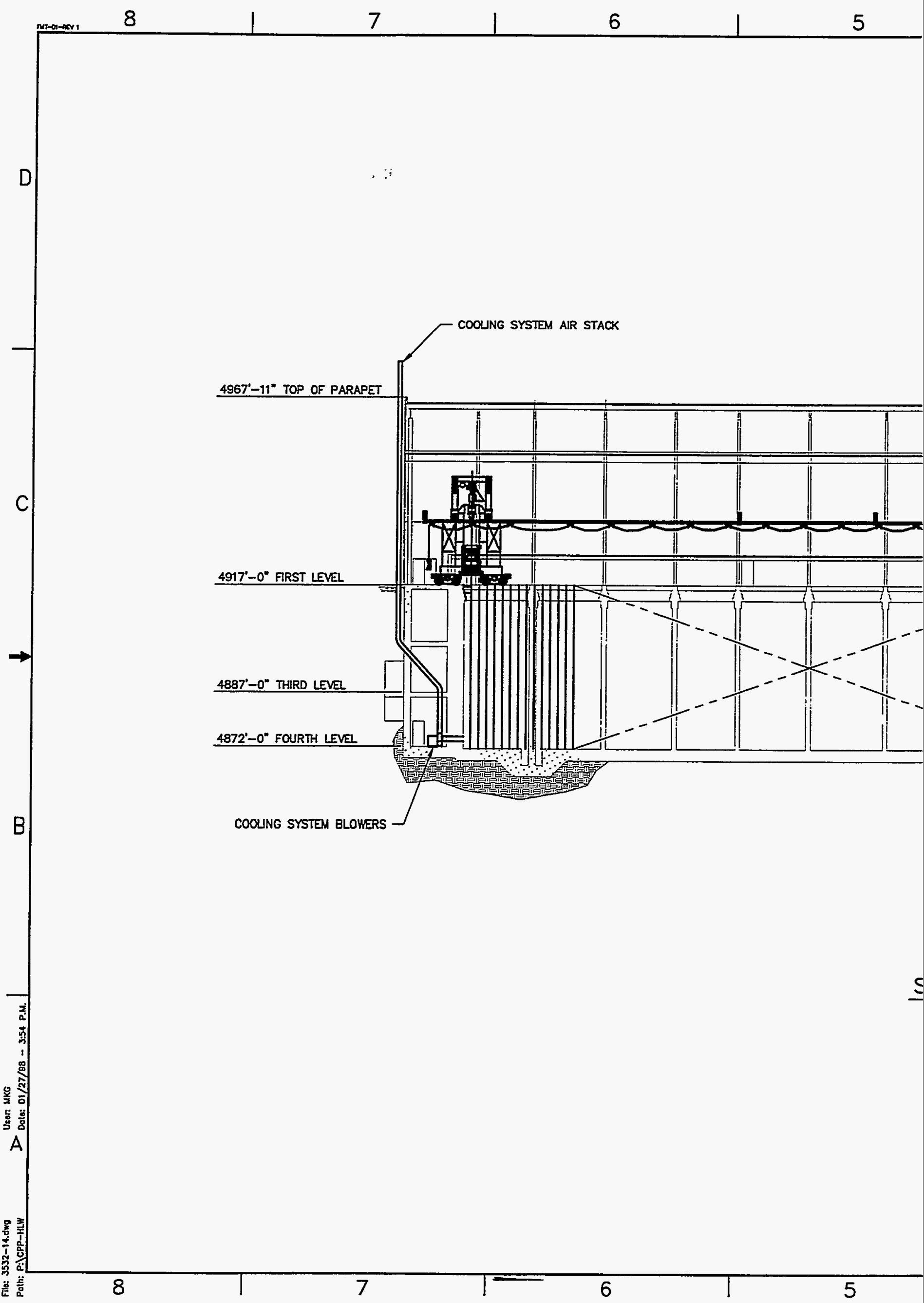




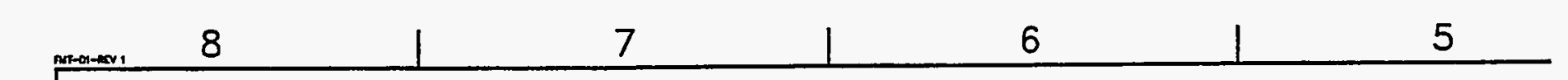

D

c

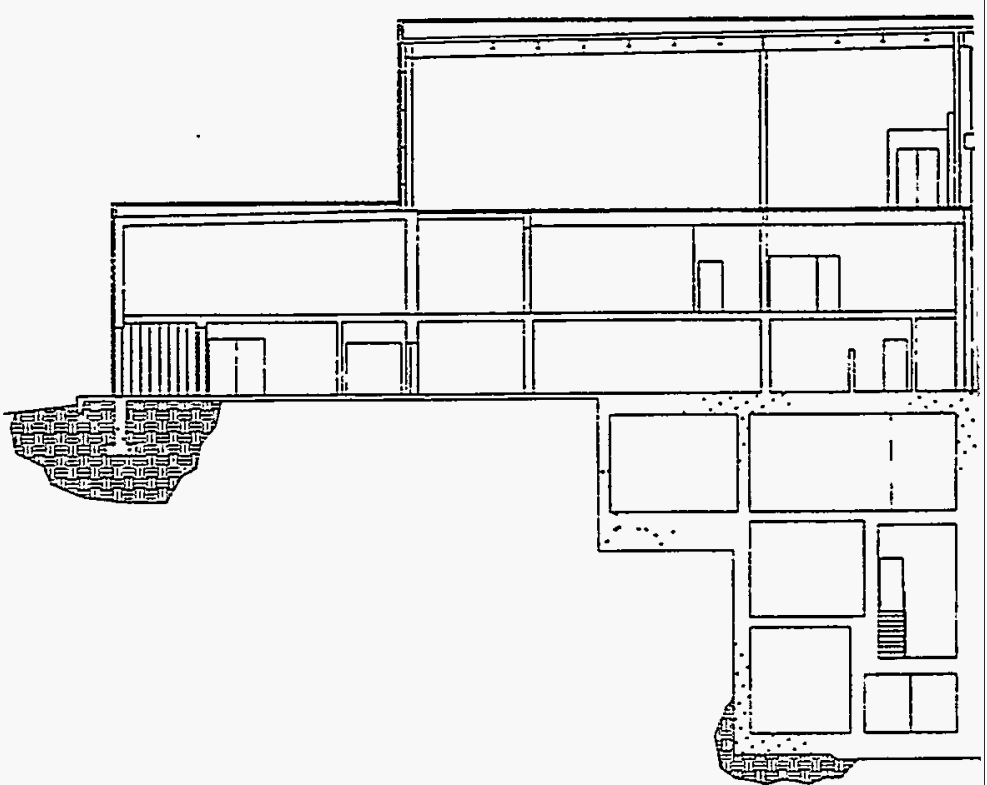

B

$\leqq$

A

琴至

苨 


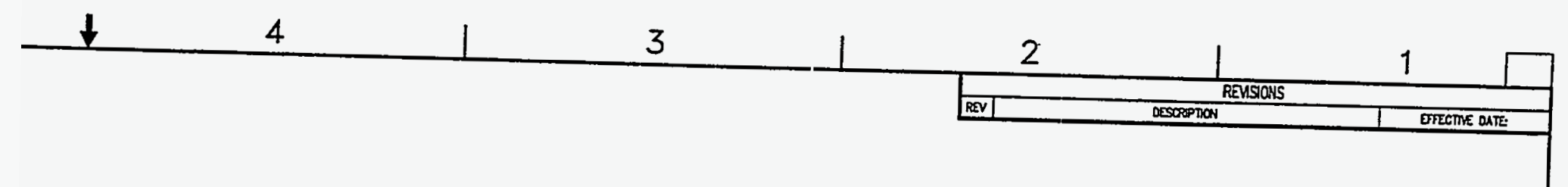

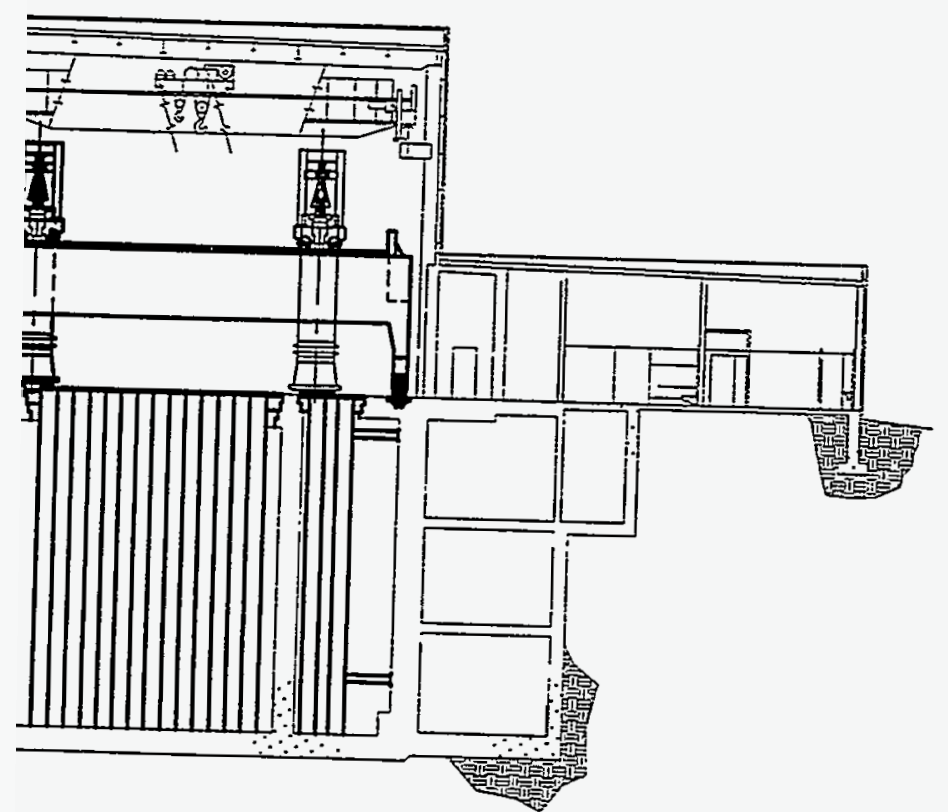

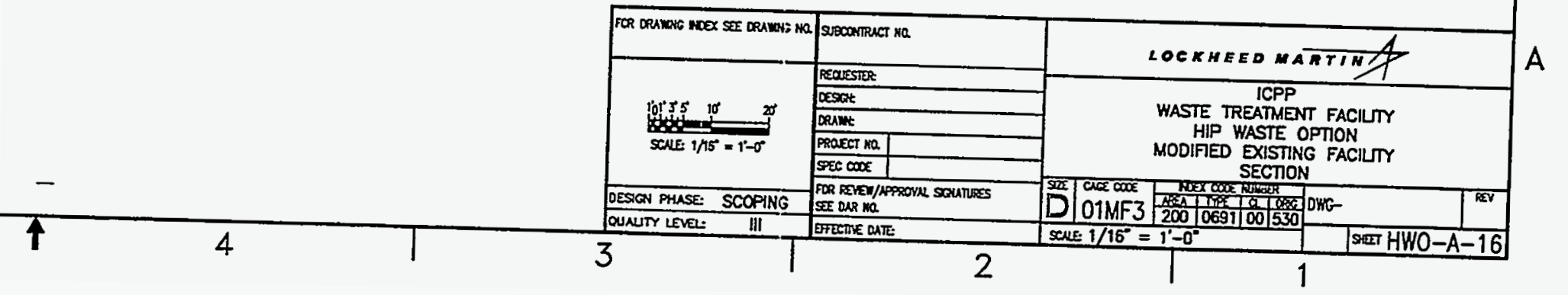




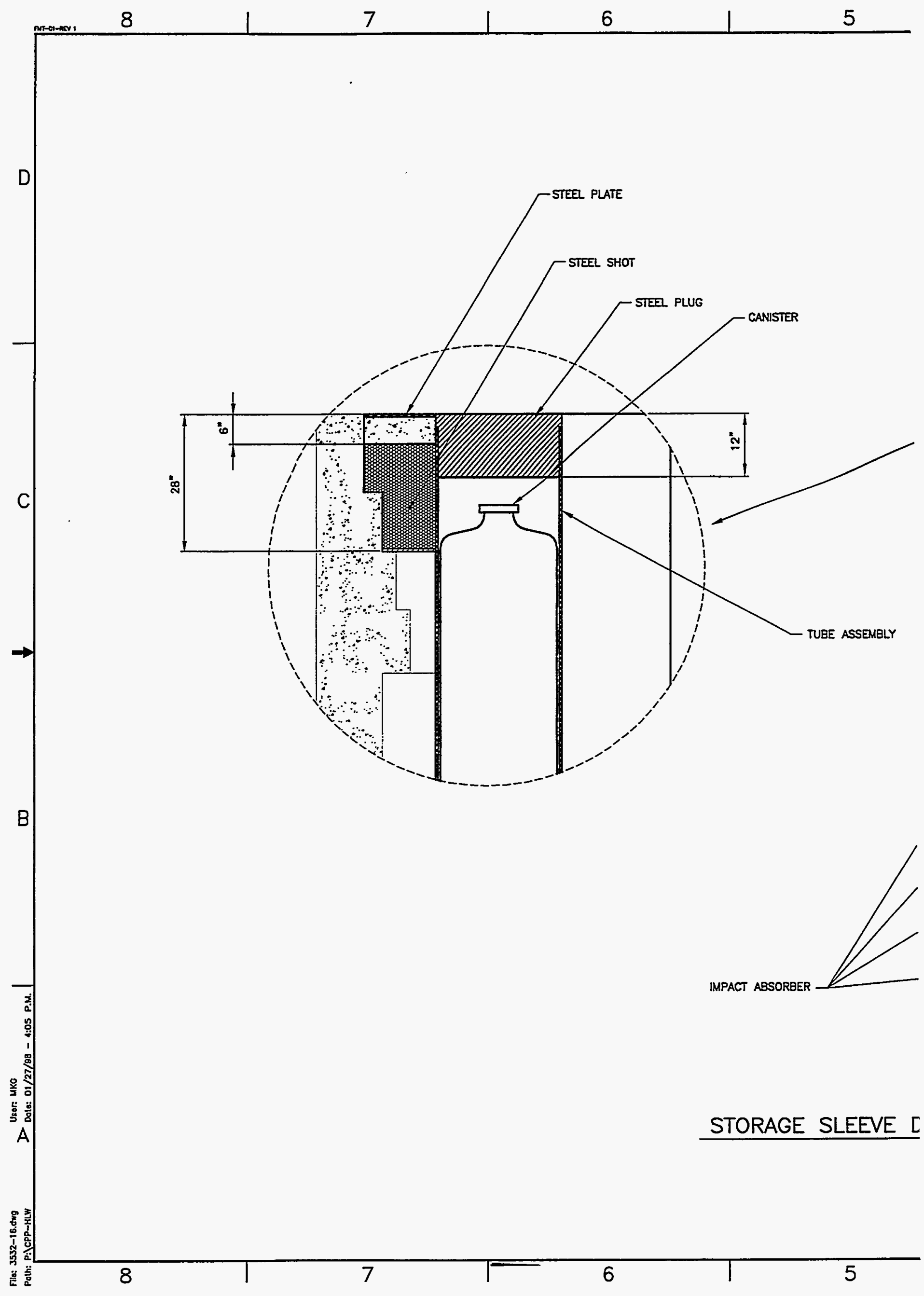




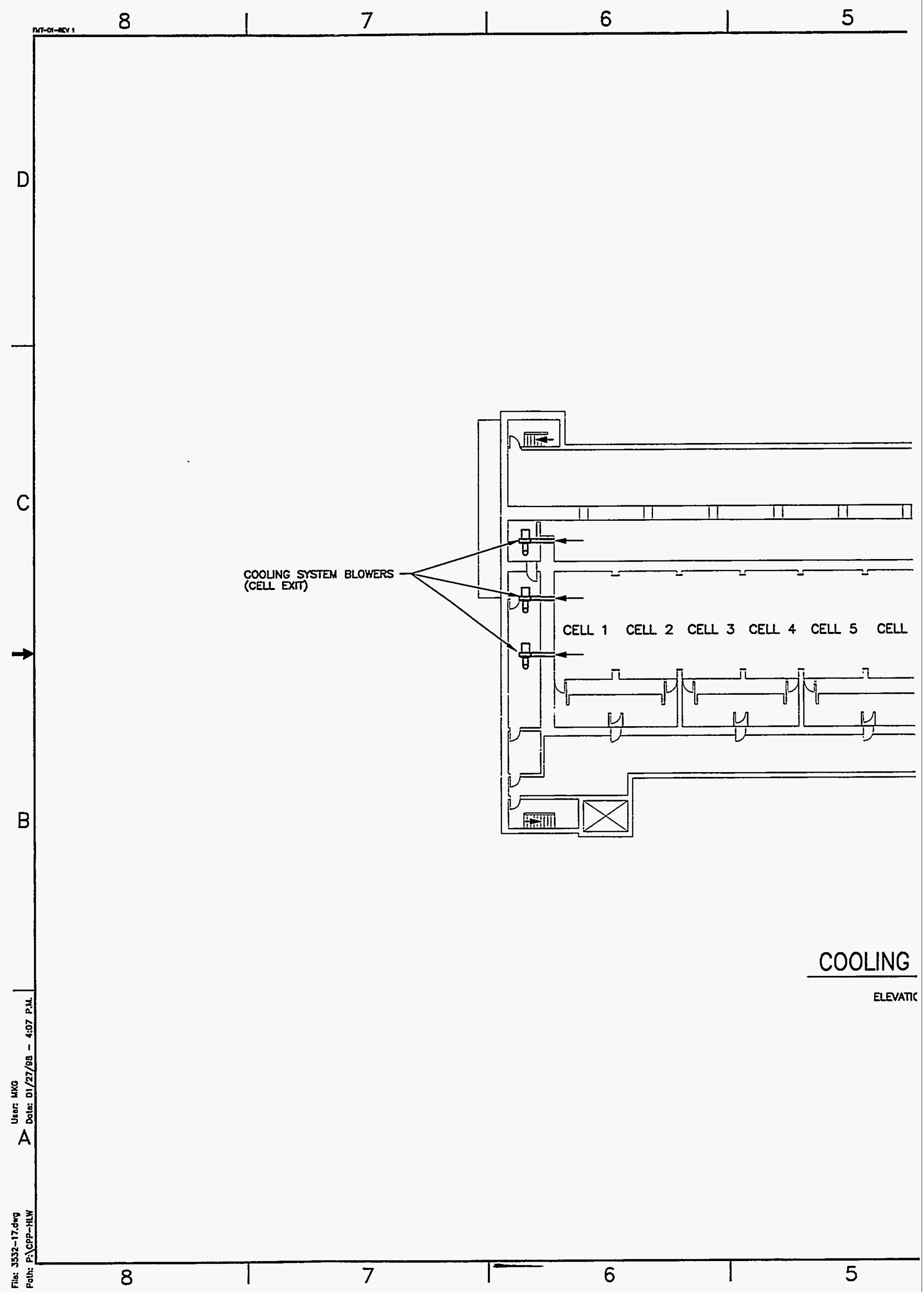




\section{Appendix 3}

\section{Risk Assessment/Data Sheets}




\section{CONTENTS}

Risk Assessment Summary

\section{FIGURES}

3-1. Risk Martix

3-1

TABLES

3-1. Risk Action Defination 


\section{RISK ASSESSMENT SUMMARY}

This appendix contains a data sheet for each of the following significant risk identified for the Interim Storage Facilities (ISFs):

1. The quantities and characteristics of the waste are different than assumed in the study. Risk $=3$.

2. Waste will not be stored in the Savanna River Canister. Risk $=2$.

3. The facility would have to be redesigned if the waste is classified as a RCRA waste after processing. Risk $=3$.

4. The obtaining of the NRC license for the FPR would be more difficult than assumed. Risk $=4$.

5. A storage canister will be mishandled or dropped during waste transfer to, from, or in the facility. Risk $=2$.

6. A catastrophic flood could flood the FPR facility. Risk $=2$.

7. The facility might not be operational when needed. Risk $=2$.

As risks were evaluated, the probability of occurrence and the impact if the risk is realized were rated. The probability and impact were assigned numerical values of 3 for high, 2 for medium, and 1 for low, as shown on the data sheets. The equation for risk calculation is Risk $=$ Probability $x$ Impact. The maximum risk rating is therefore 3 . The risk matrix in Figure 3-1 shows what action to take as a function of the risk rating; these actions are defined in Table 3-1. 


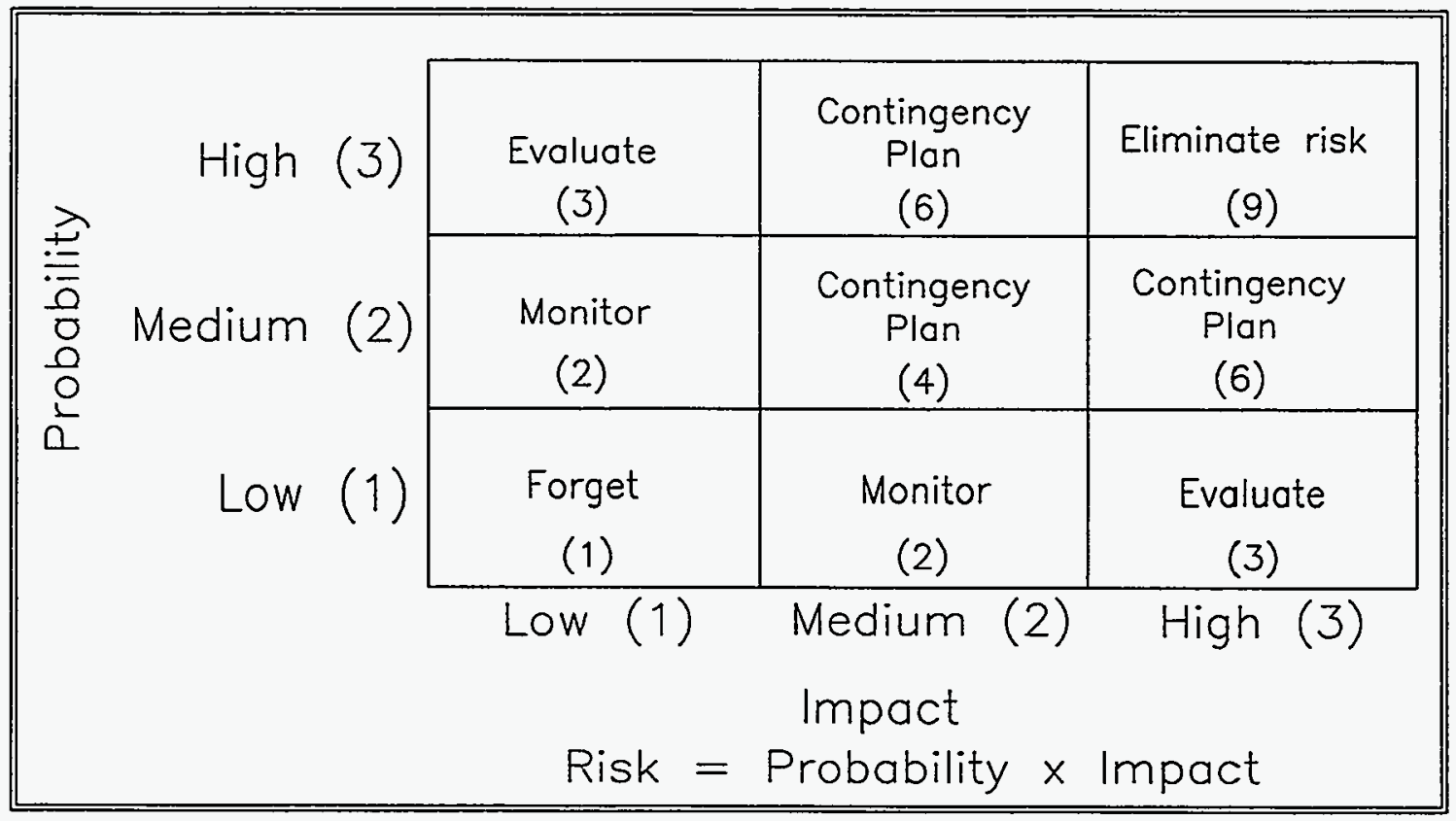

Figure 3-1. Risk Matrix.

Table 3-1. Risk Action Definition.

Risk

Rating Action

9 Any risk determined to be in this category will be mitigated through additional design or analysis until the risk is no longer in this category.

6 and 4 Risks in this category will be mitigated to the extent feasible within cost and schedule guidelines. For those risks that cannot be mitigated, contingency plans have been made to deal with the risk if it occurs.

3 Risks in this category will be evaluated during the project to identify if they are becoming problems.

2 These risks will be monitored, with no specific action identified.

1 These risks are noted for interest only. 
Risk Assessment - Data Sheet

Risk Type $\odot$ Project. OTechnical OESH

$=$

\section{RISK:}

The quantities and characteristics of the waste are different than assumed in the study

\section{PREVENTIVE PLANS}

Interim storage facility sized to most current referenced information

The new interim storage facility is based on a modular design concept allowing for additional storage to be readily incorporated

\section{PROBABLE CAUSE(S):}

Scoping study level of preliminary information

\section{CONSEQUENCES IF RISK NOT RESOLVED}

The facility design might have to be adjusted

\section{PROBABILITY}
(-) High (3)
$\bigcirc$ Medium (2)
$\bigcirc \operatorname{Low}(1)$

\section{IMPACT}

\begin{tabular}{|ll|}
\hline$\bigcirc$ & High (3) \\
$\bigcirc$ & Medium (2) \\
$\odot$ & Low (1) \\
\hline
\end{tabular}

\section{CONTINGENCY PLAN(S) IF RISK IS REALIZED:}

Redesign of facility during conceptual and title phases

\section{Risk $=3$}

Risk $=$ Probability $x$ Impact

Probability Definition

High - Likely to occur during the project.

Medium - Has the potential to occur during the project.

Low - Has little potential to occur during the project.

Impact Definition

High - Likely to cause significant disruption of schedule, increase in cost, or degradation of performance.

Medium - Has the polential to cause some disrutpion to schedule,

increase in cost, or degradation of performance.

Low - Has little potential to cause disruption to schedule, increase in cost, or degradation of performance.
TRIGGER POINT(S) FOR EARLY RISK IDENTIFICATION:

Evaluate continuing studies for facility design adequacy 
Risk Assessment - Data Sheet

Risk Type OProject @ Technical OESH

\section{RISK:}

Waste will not be stored in the Savannah River Canister

\section{PREVENTIVE PLANS}

The facility is designed to be flexible. The new facility design would be adjusted to accept the new canister

\section{PROBABLE CAUSE(S):}

Different canister identified due to additional study

\section{PROBABILITY}

High (3)

- Medium (2)

L Low (1)

\section{IMPACT}

\begin{tabular}{|ll|}
$\bigcirc$ & High (3) \\
$\bigcirc$ & Medium (2) \\
$\odot$ & Low (1) \\
\hline
\end{tabular}

\section{CONTINGENCY PLAN(S) IF RISK IS REALIZED:}

Facility redesign during conceptual and title phases.

\section{Risk $=2$}

Risk $=$ Probability $\times$ Impact

Probability Definition

High - Likely to occur during the project.

Medium - Has the potential to occur during the project.

Low - Has little potential to occur during the project.

\section{Impact Definition}

High - Likely to cause significant disruption of schedule, increase in cost, or degradation of performance.

Medium - Has the potential to cause some disrutpion to schedule. increase in cost, or degradation of performance.

Low - Has little potential to cause disruption to schedule, increase in cost, or degradation of performance.

\section{TRIGGER POINT(S) FOR EARLY RISK} IDENTIFICATION:

Monitor continuing studies to identify the canister to be selected 
Risk Assessment - Data Sheet

Risk Type
P Project

OTechnical -

OESH
$=$

.

\section{RISK:}

The facility would have to be redesigned if the waste is classified as a RCRA waste after processing

\section{PREVENTIVE PLANS}

None

\section{PROBABLE CAUSE(S):}

The delisting of the waste after processing is not approved

Re-evaluation of the waste determines that it should be classified as RCRA waste after processing

\section{CONSEQUENCES IF RISK NOT RESOLVED}

The facility would have to be redesigned. The costs for the facility would increase.

\section{PROBABILITY}

$\begin{array}{ll}O & \text { High (3) } \\ 0 & \text { Medium (2) } \\ 0 & \text { Low (1) }\end{array}$

High (3)

(-) Low (1)

\section{Risk $=3$}

Risk $=$ Probability $x$ Impact

\section{Probability Definition}

High - Likely to occur during the project.

Medium - Has the potential to occur during the project.

Low - Has little potential to occur during the project.

\section{Impact Definition}

High - Likely to cause significant disruption of schedule, increase in cost, or degradation of performance.

Medium - Has the potential to cause some distutpion to schedule. increase in cost, or degradalion of performance.

Low - Has little potential to cause disruption to schedule, increase in cost, or degradation of performance.

\section{CONTINGENCY PLAN(S) IF RISK IS REALIZED:}

None

\section{TRIGGER POINT(S) FOR EARLY RISK IDENTIFICATION:}

Continue to monitor the status of the option to delist the waste 
Risk Assessment - Data Sheet

\section{RISK:}

The obtaining of the NRC license for the FPR would be more difficult than assumed

\section{PREVENTIVE PLANS}

Identify during the conceptual design as many of the required issues and include adequate contingency to cover later issues

\section{PROBABLE CAUSE(S):}

An NRC license could be difficult to obtain for a modified existing facility that was not originally designed to licensing criteria.

\section{CONSEQUENCES IF RISK NOT RESOLVED}

The interim storage might not be operational when required
PROBABILITY
$\bigcirc \operatorname{High}(3)$
(-) Medium (2)
$\bigcirc \operatorname{Low}(1)$

\section{IMPACT}

\begin{tabular}{|ll|}
\hline & High (3) \\
$\bigcirc$ & Medium (2) \\
$\bigcirc$ & Low (1) \\
\hline
\end{tabular}

\section{CONTINGENCY PLAN(S) IF RISK IS REALIZED:}

None

\section{Risk $=4$}

Risk $=$ Probability $x$ Impact

\section{Probability Definition}

High - Likely to occur during the project.

Medium - Has the potential to occur during the project.

Low - Has little potential to occur during the project.

Impact Definition

High - Likely to cause significant disruption of schedule, increase in cost, or degradation of performance.

Medium - Has the potential to cause some disrutpion to schedule, increase in cost, or degradation of performance.

Low - Has little potential to cause disruption to schedule, increase in cost, or degradation of performance.

\section{TRIGGER POINT(S) FOR EARLY RISK} IDENTIFICATION:

Detailed study of the compatibility of the FPR to meet the NRC licensing criteria during pre-conceptual design. 
Risk Assessment - Data Sheet

Risk Type OProject OTechnical OESH

RISK:

A storage canister will be mishandled or dropped during waste transfer to, from, or in the facility.

\section{PREVENTIVE PLANS}

Facility will be designed to be able to recover from these accidents

\section{PROBABLE CAUSE(S):}

Possible scenario include

1. Canister falls from the transfer cart in the tunnel

2. Canister is dropped during transfer from the transfer cart

\section{CONSEQUENCES IF RISK NOT RESOLVED}

Additional design for new equipment

\section{PROBABILITY}

High (3)

Medium (2)

(C) Low (1)

Risk $=2$

Risk $=$ Probability $\times$ Impact

Probability Definition

High - Likely to occur during the project.

Medium - Has the potential to occur during the project.

Low - Has little potential to occur during the project.

\section{Impact Definition}

High - Likely to cause significant disruption of schedule, increase in cost, or degradation of performance.

Medium - Has the potential to cause some disrutpion to schedule, increase in cost, or degradation of performance.

Low - Has little potential to cause disruption to schedule, increase in cost, or degradation of performance.

\section{CONTINGENCY PLAN(S) IF RISK IS REALIZED:}

None

\section{TRIGGER POINT(S) FOR EARLY RISK} IDENTIFICATION:

Ongoing review of the facility during design reviews, SAR preparation, etc. 
Risk Assessment - Data Sheet

Risk Type $\odot$ Project O Technical OESH

\section{RISK:}

A catastrophic flood could flood the FPR facility

\section{PREVENTIVE PLANS}

Existing INEEL flood prevention planning

\section{PROBABLE CAUSE(S):}

Large storm

\section{CONSEQUENCES IF RISK NOT RESOLVED}

Flooding of the facility

High snow pack year

Failure of Mackay Dam

PROBABILITY

High (3)

Medium (2)

( Low (1)

\section{IMPACT}

High (3)

- Medium (2)

Low (1)

\section{CONTINGENCY PLAN(S) IF RISK IS REALIZED:}

Aggressive measures to prevent local flooding of the facility such as sandbags, berms, pumps, etc.

$$
\text { Risk }=2
$$

Risk $=$ Probability $x$ Impact

\section{Probability Definition}

High - Likely to occur during the project.

Medium - Has the potential to occur during the project.

Low - Has little polential to occur during the project.

\section{Impact Definition}

High - Likely to cause significant disruption of schedule, increase in cost, or degradation of performance.

Medium - Has the potential to cause some disrutpion to schedule. increase in cost, or degradation of performance.

Low - Has little potential to cause disruption to schedule, increase in cost, or degradation of performance.

\section{TRIGGER POINT(S) FOR EARLY RISK}

\section{IDENTIFICATION:}

Continue to monitor for potential for flooding 
Risk Assessment - Data Sheet

Risk Type $\odot$ Project O Technical OESH

$\sim$

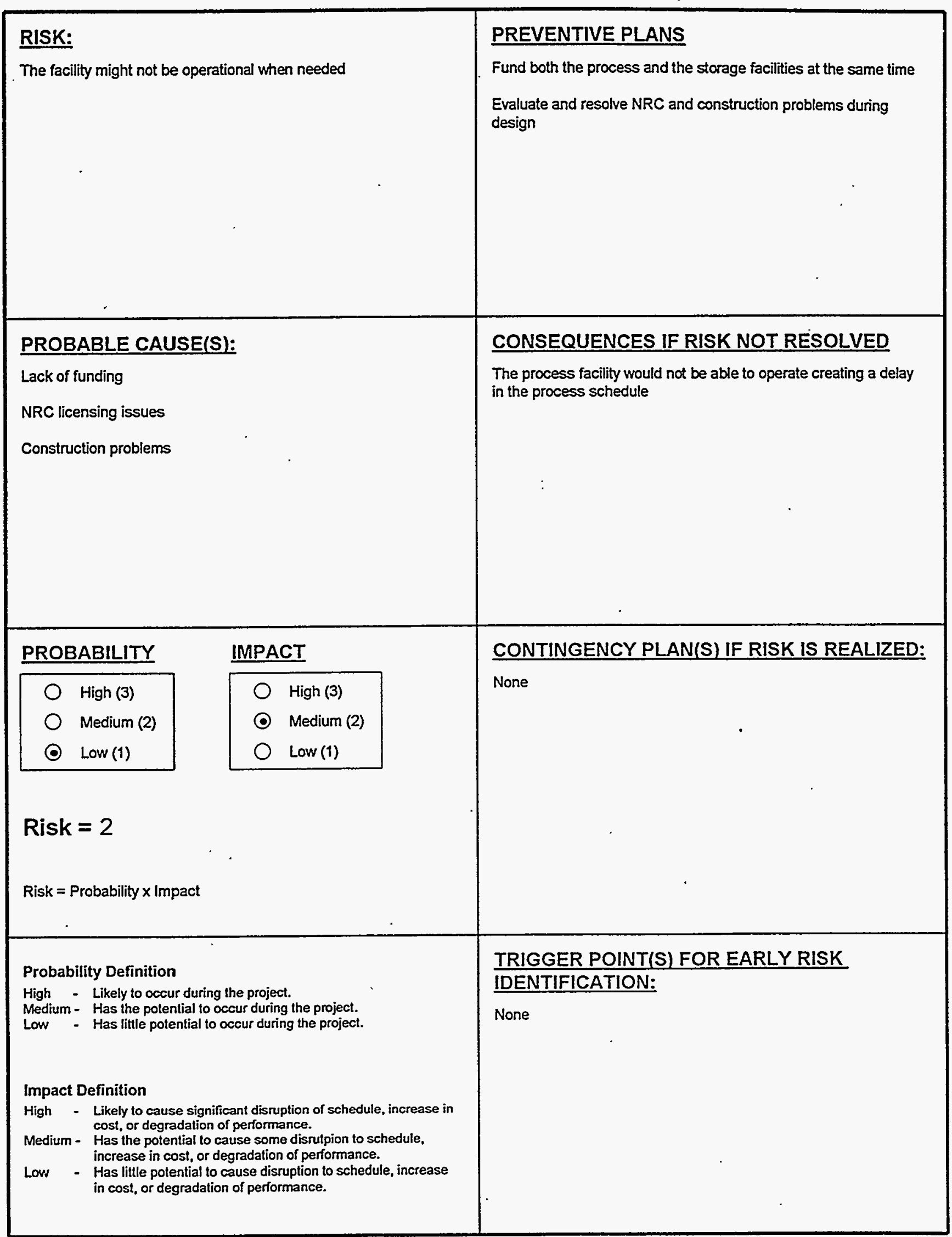




\section{Appendix 4}

\section{Background Information for Project Data Sheets}




\section{CONTENTS}

Background information for the Project Data Sheets are included in this appendix in the following order:

1. Full Separations Option

2. TRU/Class A Option

3. Hot Isostatic Press Waste Option.

The background information includes:

1. Construction Assumptions

2. Operations Assumptions

3. D\&D Labor

4. D\&D Assumptions

5. Estimate of Diesel Engine Emissions. 
Full Separations Option 
Construction costs are from the detailed cost estimate (R. Adams)

Existing Interim Storage Facility Option, Prepared by Dave Keller.

Construction duration $=$

Labor $=$

1 man-year of labor $=$

Square footage $=$

Acres disturbed $=$
2 years

161 new workers/yr

1800 manhours

63,000 sq. $\mathrm{ft}=$ 155 acres (previous)

Excavation: Excavated earth will be spread in a spoil area adjacent to ICPP, except for backfill soil.

Excess Material $=$

$24,100 \mathrm{yd}^{3}$

(from Detailed Cost Estimate)

Air Emissions:

Dust during construction $=1.2$ tons $/$ month-acre $=$

4,464 tons (total)

(from USEPA Office of Ar Quality Planning and Standards)

Air emissions from fuel usage are based on the diesel emissions spreadsheet.

\section{Effluents:}

\section{Sanitary Wastewater $=\quad 905,625 \mathrm{gal} / \mathrm{yr}=$}

(based on $\mathbf{2 5}$ gal/person-day and 225 days/year of construction)

$6,855,581$ liters (total)

(Benefield, LD and C.W. Randall, Biological Process Design for Wastewater Treatment, InPrint, Inc., 1987, p. 104 - wastewater generation = 15-30 gal/day-person)

Assume 3 gallons of lubricating oil and hydraulic fluid generated for every 60 hours of operation of 2,795 liter (total) (Heavy equipment hrs are calculated below.)

\section{Solid Wastes:}

Construction trash $=$

(Use 15.5 yd3/yr per capita. This is twice the generation rate of trash from site operations)

Hazardous/toxic chemicals and wastes (type)

$\begin{array}{lll}\text { Hazardous waste generation }= & 217 \mathrm{~m}^{3} \text { (total) } \\ \text { (based on an assumed generation rate of } 5 \text { 55-gallon drums of waste per week) }\end{array}$

Hazardous waste storage $=$ $3300 \mathrm{gal}=$ $13 m^{3}$

(Assume waste is accumulated for 12 weeks [ 84 days] in a 90 day accumulation area, then picked up for disposal.)

\section{Water Usage:}

Water used for dust control = Area

155 acres (previous)

assume $0.05^{\prime \prime}$ water per week to control dust $43,560 \mathrm{sq}$ ttacre

0.05 in water/wk $0.08333 \mathrm{ft} / \mathrm{in}$ 
assume dust control required 20

weeks

Length of Construction
28.320 liter/ft ${ }^{3}$

\begin{tabular}{c}
20 wkJyr \\
$2 \mathrm{yr}$ \\
\hline $31,868,496$ liter
\end{tabular}

Domestic water use is assumed to be the same as sanitary waste.

Energy Requirements:

Electricity

$$
150 \mathrm{MWh} / \mathrm{yr}
$$

\section{Fossil Fuel Use}

\section{Concrete:}

Amount used $=$

(Detailed Cost Estimate)

\begin{aligned} $1,360{y d^{3}}^{3} &$ Tunnel \\ $8,300 y d^{3} &$ RR Structure \\ $630 y d^{3} &$ Building \\ \hline $10,290 y d^{3} &$ Total Concrete \end{aligned}

Number of truckloads $=$

Fuel usage $=$

468 (based on $22 \mathrm{yd}^{3}$ /load... tandem trailers)

$10,290 \mathrm{gal}$ (assumes $5 \mathrm{mpg}$ and round trip of 110 miles)

Materials delivery:

Number of truckloads $=$

234 (assume 1/2 the loads of concrete)

Fuel usage $=$

$7,016 \mathrm{gal}$ (assumes $5 \mathrm{mpg}$ and round trip of 150 miles)

Construction Equipment:

Construction Equipment Cost

$\$ 1,085,223$ (Detailed Cost Estimate)

$\$ 75$ per hr

$14,470 \mathrm{hr}$

Labor Hours

224,358 (Detailed Cost Estimate)

750 labor hrlequipment hr

$299 \mathrm{hr}$

Total Equipment Hours $=$

$14,769 \mathrm{hr}$

Equipment fuel usage (see http://www/deere.com/ind) = Total heavy equipment fuel usage $=$

Total heavy equipment and materials delivery fuel used $=$ Concrete Delivery Materials Delivery $10,290 \mathrm{gal}$ $7,016 \mathrm{gal}$ Heavy Equipment

$88,613 \mathrm{gal}$ $105,919 \mathrm{gal}$

3.785 liter/gal 400,902 liter 


\begin{tabular}{|lc|}
\hline Operating costs are taken from the life cycle cost estimate. \\
\hline Operation & \\
Loading & $24 \mathrm{yr}$ \\
Holding & $27 \mathrm{yr}$ \\
Shipping & $5 \mathrm{yr}$ \\
\hline
\end{tabular}

\begin{tabular}{|lc|}
\hline Exisiting Interim Storage, Prepared by Dave Keller. \\
\hline Labor: & 7 \\
Operations & 3 \\
Maintenance & 1 \\
Support & 3 \\
Rad Workers & 2.5 Included above \\
Cannisters & 170 \\
Electrical & $4368.0 \mathrm{MWhr} / \mathrm{yr}$ \\
Steam & $5.60 \mathrm{E}+07 \mathrm{lb} / \mathrm{yr}$ \\
\hline
\end{tabular}

Radiation worker annual dose is based on the

Effluents:

Sanitary wastewater $=\quad 63,875 \mathrm{gal} / \mathrm{yr}=\quad 241,767$ liters $/ \mathrm{yr}$ (based on 25 gal/day per worker, facility occupied year-round)

\section{Solid Wastes:}

Sanitary/Industrial trash $=\quad 51 \mathrm{yd}^{3} / \mathrm{yr} \quad 39 \mathrm{~m}^{3} / \mathrm{yr}$ (based on 7.25 yd3/person-year... Bob Skinner [cuber facility])
Product canisters $=$ $6 \mathrm{~m}^{3} / \mathrm{yr}$ (based on $0.72 \mathrm{~m}^{3} /$ canister)

Total rad waste $=$ $147 \mathrm{~m}^{3}$

Water Usage:

Domestic water usage $=$ same as sanitary wastewater rate.

\section{Energy Requirements} Electric power usage $=$ Steam $=$
4368.0 MWhr/yr

$5.60 \mathrm{E}+07 \mathrm{lb} / \mathrm{yr}$ 
Years

From D\&D

Assumptions

3

D\&D Cost

Allocated

Total MH Man-hours/yr

22,176 $\quad 7,392$

29,435

9,812

81,833

$-$

27,278

$151,303 \quad 50,434$

-

46,957

37,288

15,652

12,429
$\$ 1,250$

$\$ 3,453$

$\$ 5,932$

$\$ 7,979$

$\$ 5,455$

$\$ 7,212$

$\$ 4,316$

$\$ 5,630$

$\$ 4,826$

$\$ 3,894$

\$ $1,540,000$

$2,310,000$

$\$ 3,762$

$\$ 4,808$

$\$ 3,753$

$\$ 3,217$

$\$ 2,596$

$\$ 751$

$\$ 643$

$\$ 519$

$\$$

$\$ \quad 3,715,000$

$2,904,000$

Total

vallable

\$ $29,069,000$

368,993

122,998

$\$ 29,069,000$

Notes:

1 Crew functions and daily estimates are from the D\&D program (Dave Haycraft)

2 Total costs are based on life cycle estimate by $R$. Turk

3 Assume all workers in crews $2,2 A, 5$, and 7 are rad workers

4 Assume a man-year is 1800 hours. 


\section{Duration of $D \& D=\quad 3$ years}

No decontamination of the storage facility will be required.

$D \& D$ labor requirements are taken from $D$ \& $D$ labor and equipment spreadsheet.

$D \& D$ costs come from the life cycle cost estimate.

1 manyear of labor $=\quad 1800$ manhours

Assume that the D\&D cost estimates are for the modified portion of the FPR facility. D\&D of the entire FPR is not addressed.

\begin{tabular}{lrrrr|r} 
Heavy Equipment & \#Used & Hours/day & Days/wk & Wks/yr & Hours/yr \\
Mobile Crane & 1 & 3 & 5 & 20 & 300 \\
Roll-Off Truck & 2 & 8 & 5 & 20 & 1,600 \\
Dozer & 1 & 6 & 5 & 20 & 600 \\
Loader & 2 & 8 & 5 & 20 & 1,600 \\
Scabbler (w/ Vacuum System) & 1 & 6 & 3 & 20 & 360 \\
Pneumatic Ram & 1 & 3 & 5 & 20 & 300 \\
Demolition Machine & 0 & 4 & 4 & 45 & - \\
(Remote Control) ** & 0 & & & & 4,760
\end{tabular}

Total hours/yr

Total heavy equipment hours $=$

Assume each piece of equipment uses 6 gallon of diesel fuel per hour. Consumption rate from John Deere

Web Site (Construction Equipment - http://www.deere.com/ind/product/product.html)

No. of gallons of fuel used during D \& $=\quad 85,680$ gal $=\quad 324,299$ liters (total)

Assume each roll-off truck makes 3 trips per day to RWMC

No. of trips $=$

Miles traveled @ 12 miles/round trip=

Acres disturbed and duration of disturbance

Acreage disturbed is 10 more than for construction $=$

165.0 acres

Air emissions

HEPA filter ventilation is not required during D\&D.

Air emissions from fuel are based on the diesel emissions spreadsheet.

\section{Effluents}

No decontamination solution required during D\&D.
Sanitary wastewater $=$
384,368 gallyr $=$
$4,364,496$ liters (total)
(based on 25 gal/day per worker, 225 day/yr)
Lube oil =
2,702 liters (total)
(based on 3 gal for every 60 hours of operation)

\section{Solid wastes:}

The following factors from Dave Kenoyer are based on a contaminated building. The storage facility is not expected to be contaminated. Therefore, all items identified as LLW will be included with the non-contaminated waste. These factors are used for consistency in waste volumes with facilities which are contaminated. 
Solid Waste Generation (factors from Dave Kenoyer - D\&D Program)

\begin{tabular}{|c|c|c|c|c|c|}
\hline Waste Type & Waste Type & $\begin{array}{c}\text { Factor } \\
\text { (cu.ft./sq.ft.) }\end{array}$ & $\begin{array}{l}\text { Sq.Ft. in } \\
\text { Facility }\end{array}$ & $\begin{array}{l}\text { Cu.Ft. of } \\
\text { Waste }\end{array}$ & Cu. Meters \\
\hline $\begin{array}{l}\text { WERF-LLWW } \\
\text { Combustible PPEs } \\
\text { WERF-LLW } \\
\text { Combustible Building }\end{array}$ & & 0.167 & 63,000 & 10,521 & 301 \\
\hline $\begin{array}{l}\text { Debris } \\
\text { WERF-LLW } \\
\text { Compactable Building }\end{array}$ & - & 0.128 & 63,000 & 8,064 & 230 \\
\hline $\begin{array}{l}\text { Debris } \\
\text { RWMC-LLW Non- }\end{array}$ & & 0.195 & 63,000 & 12,285 & 351 \\
\hline $\begin{array}{l}\text { Compactable } \\
\text { RWMC-LLW Non- }\end{array}$ & & 0.513 & 63,000 & 32,319 & 923 \\
\hline $\begin{array}{l}\text { Compt Building Debris } \\
\text { RWMC-LLW Non- }\end{array}$ & & 0.684 & 63,000 & 43,092 & 1,231 \\
\hline $\begin{array}{l}\text { Compt Concrete Rubble } \\
\text { RWMC-LLW Non- }\end{array}$ & & 3.44 & 63,000 & 216,720 & 6,192 \\
\hline $\begin{array}{l}\text { Compt Scrap Metal } \\
\text { RWMC-LLW }\end{array}$ & & 0.778 & 63,000 & 49,014 & 1,400 \\
\hline $\begin{array}{l}\text { Asbestos/ACM Covered } \\
\text { CFA Landfill Non-Compt }\end{array}$ & & 0 & 63,000 & - & \\
\hline $\begin{array}{l}\text { Building Debris } \\
\text { CFA Landfill Non-Compt }\end{array}$ & & 1.99 & 63,000 & 125,370 & 3,582 \\
\hline Concrete Rubble • & & 2.454 & 63,000 & 154,602 & 4,417 \\
\hline $\begin{array}{l}\text { CFA Landfill Asbestos } \\
\text { HWSF Hazardous Mtrls }\end{array}$ & & 0 & 63,000 & - & \\
\hline (Hg/PCBs/etc) & & 0.002 & 63,000 & 126 & 4 \\
\hline Metal Recycle & & 0.022 & 63,000 & 1,386 & 40 \\
\hline $\begin{array}{l}\text { Non-Rad = } \\
\text { Hazardous = } \\
\text { Metal }=\end{array}$ & & & & $\begin{array}{r}651,987 \\
126 \\
1,386\end{array}$ & $\begin{array}{r}18,628 \\
4 \\
40\end{array}$ \\
\hline
\end{tabular}

- (Factor used is twice that given by Dave Kenoyer due to use of concrete for shielding)

Hazardous/toxic chemicals and wastes (type)

Mixed waste $=\quad 26,445$ gal (total) $=\quad 909 \mathrm{~m} 3$ (total)

(based on an assumed 5 55-gallon drums generated per week... work only 225 days/yr)

Water Usage

Domestic water use is assumed to be the same as sanitary waste.

\section{Energy requirements:}

Electric power usage $=$

(based on 3,000 kWh/wk - John Duggan)

$156,000 \mathrm{kWh} / \mathrm{yr}$

Fossil Fuels $=$

324,299 liters 
Bases \& Assumptions:

1. Air to fuel ratio $=25: 1$ (Mass Basis)

2. Diesel fuel density $=7.5 \mathrm{lbs} . / \mathrm{gal}$.

3. Air is $21 \% \mathrm{O}_{2}, 79 \% \mathrm{~N}_{2}$, with a pseudomolecular weight of 29 .

4. Combustion is simulated as: $\mathrm{C}_{9} \mathrm{H}_{18}+13.5 \mathrm{O}_{2} \rightarrow 9 \mathrm{CO}_{2}+9 \mathrm{H}_{2} \mathrm{O}$

5. Particulates $=5 \mathrm{mg} / \mathrm{scf}$

6. $\mathrm{CO}=2,500 \mathrm{ppmv}$

7. $\mathrm{NO}_{\mathrm{x}}=2,000 \mathrm{ppmv}$

8. Unburned hydrocarbons $=100 \mathrm{ppmv}$

9. Diesel fuel (\# 2 fuel oil) contains 1 wt. \% sulfur

10. Combustion is about $99 \%$ efficient.

Lbs. Of Construction Fuel

Lbs. Of Operations Fuel

Lbs. Of D\&D Fuel

Total Lbs. of Fuel Used

Lb-Moles of Construction Fuel

Lb-Moles of Operations Fuel

Lb-Moles of D\&D Fuel

Total Lb-Moles of Fuel (as C9H18)

Lbs of Air for Construction Fuel (based on air-to-fuel ratio)

Lbs.of Air for Operations Fuel (based on air-to-fuel ratio)

Lbs.of Air for D\&D Fuel (based on air-to-fuel ratio)

Total Lbs. of Air Added

Lb-Moles of Air for Combustion Fuel

Lb-Moles of Air for Operations Fuel

Lb-Moles of Air for D\&D Fuel

Total Lb-Moles of Air from Wark, K. and C.F. Wamer, Air Pollution, Its Origin and Control, IEP, New York, 1976, p. 446, 423

Wark and Warner, p. 446 Wark and Warner, p. 446 Wark and Wamer, p. 446 Wark and Warner, p. 446 Wark and Warner, p. 336

Grand Total of Materials Fed, Lbs.

793,786

642,112 $1,435,897$

6,300

5,096

11,396

$19,844,647$

$16,052,791$

$35,897,437$

684,298

553,545

$1,237,843$

$37,333,335$

\begin{tabular}{lrrrr} 
Exhaust Gases, Construction Fuel & \multicolumn{1}{c}{ Total Lbs. } & \multicolumn{1}{c}{ Total Tons } & \multicolumn{1}{c}{ Total Moles } & \multicolumn{1}{c}{ Total SCF } \\
\hline $\mathrm{CO}_{2}$ & $2,469,808$ & 1,235 & 56,132 & $20,151,388$ \\
$\mathrm{H}_{2} \mathrm{O}$ & $1,010,376$ & 505 & 56,132 & $20,151,388$ \\
$\mathrm{O}_{2}$ & $1,904,148$ & 952 & 59,505 & $21,362,156$ \\
$\mathrm{~N}_{2}$ & $15,136,675$ & 7,568 & 540,596 & $194,073,800$ \\
\hline Subtotal of Major Gases & $\mathbf{2 0 , 5 2 1 , 0 0 7}$ & $\mathbf{1 0 , 2 6 1}$ & $\mathbf{7 1 2 , 3 6 4}$ & $\mathbf{2 5 5 , 7 3 8 , 7 3 2}$ \\
\hline $\mathrm{SO}_{2}$ & 15,876 & 7.9 & \\
Particulates & 2,817 & 1.4 & \\
$\mathrm{CO}$ & 49,865 & 24.9 & \\
NO (assumed NO) & 42,742 & 21.4 & \\
Unbumed Hydrocarbons & $\mathbf{8 , 9 7 6}$ & 4.5 & \\
Subtotal of Contaminants & $\mathbf{1 2 0 , 2 7 5}$ & $\mathbf{6 0}$ & \\
\hline
\end{tabular}




\begin{tabular}{|c|c|c|c|c|}
\hline Exhaust Gases, Operations Fuel & Total Lbs. & Total Tons & Total Moles & Total SCF \\
\hline $\mathrm{CO}_{2}$ & - & - & - & - \\
\hline $\mathrm{H}_{2} \mathrm{O}$ & - & - & - & - \\
\hline $\mathrm{O}_{2}$ & - & - & - & - \\
\hline $\mathrm{N}_{2}$ & - & - & - & - \\
\hline Subtotal of Major Gases & - & - & - & - \\
\hline $\mathrm{SO}_{2}$ & - & - & & \\
\hline Particulates & - & - & & \\
\hline CO & - & - & & \\
\hline $\mathrm{NO}_{x}$ (assumed NO) & - & - & & \\
\hline Unburned Hydrocarbons & - & - & & \\
\hline Subtotal of Contaminants & $=$ & - & & \\
\hline Exhaust Gases, D\&D Fuel & Total Lbs. & Total Tons & Total Moles & Total SCF \\
\hline $\mathrm{CO}_{2}$ & $1,997,884$ & 999 & 45,406 & $16,300,921$ \\
\hline $\mathrm{H}_{2} \mathrm{O}$ & 817,316 & 409 & 45,406 & $16,300,921$ \\
\hline $\mathrm{O}_{2}$ & $1,540,309$ & 770 & 48,135 & $17,280,339$ \\
\hline $\mathrm{N}_{2}$ & $12,244,404.42$ & 6,122 & 437,300 & $156,990,757$ \\
\hline Subtotal of Major Gases & $16,599,914$ & 8,300 & 576,248 & $206,872,937$ \\
\hline $\mathrm{SO}_{2}$ & 12,441 & 6.2 & & \\
\hline $\begin{array}{l}\text { Particulates } \\
\text { CO }\end{array}$ & $\begin{array}{r}2,278 \\
40,337\end{array}$ & $\begin{array}{r}1.1 \\
20.2\end{array}$ & & \\
\hline $\mathrm{NO}_{x}$ (assumed NO) & 34,575 & 17.3 & & \\
\hline Unburned Hydrocarbons & 7,261 & 3.6 & & \\
\hline Subtotal of Contaminants & 96,892 & 48 & & \\
\hline
\end{tabular}




\section{TRU Class A Option}


Construction Assumptions

-

$=$

Construction costs are from the detailed cost estimate (R. Adams)

Existing Interim Storage Facility Option, Prepared by Dave Keller.

Construction duration $=$

Labor $=$

1 man-year of labor $=$

Square footage $=$

Acres disturbed $=$
2 years

155 new workers/yr

1800 manhours

63,000 sq. $\mathrm{ft}=\quad 5,853 \mathrm{~m}^{2}$

155.0 acres (previous)

Excavation: Excavated earth will be spread in a spoil area adjacent to ICPP, except for backfill soil.

Excess Material $=$

(from Detailed Cost Estimate)

\section{Air Emissions:}

Dust during construction $=1.2$ tons $/$ month-acre $=$

(from USEPA Office of Ar Quality Planning and Standards)

$24,100 \mathrm{yd}^{3}$

Air emissions from fuel usage are based on the diesel emissions spreadsheet.

\section{Effluents:}

$\begin{array}{lcc}\text { Sanitary Wastewater }= & 871,875 \mathrm{gal} / \mathrm{yr}= & 6,600,094 \text { liters (total) } \\ \text { (based on } 25 \text { gal/person-day and } 225 \text { days/year of construction) } & \end{array}$

(Benefield, LD and C.W. Randall, Biological Process Design for Wastewater Treatment, InPrint, Inc., 1987, p. 104 wastewater generation $=15-30$ gal/day-person)

Assume 3 gallons of lubricating oil and hydraulic

fluid generated for every 60 hours of operation of

heavy equipment $=$

(Heavy equipment hrs are calculated below.)

$2,789.51$ liter (total)

\section{Solid Wastes:}

Construction trash $=$

4,805 yd3 $($ total $)=$

4,464 tons (total)

(Use $15.5 \mathrm{yd} 3 / \mathrm{yr}$ per capita. This is twice the generation rate of trash from site operations)

\section{Hazardous/toxic chemicals and wastes (type)}
Hazardous waste generation $=$
275 gal/week =

(based on an assumed generation rate of 555 -gallon drums of waste per week)

Hazardous waste storage $=$

$3300 \mathrm{gal}=$

$13 \mathrm{~m}^{3}$

(Assume waste is accumulated for 12 weeks [ 84 days] in a 90-day accumulation area, then

\section{Water Usage: \\ Water used for dust control = \\ Area}

assume $0.05^{\prime \prime}$ water per week to control dust

assume dust control required 20 weeks

Length of Construction
155 acres (previous)

43,560 sq ftacre

0.05 in water/wk

$0.08333 \mathrm{ft} /$ in

28.320 liter $/ \mathrm{ft}^{3}$

$20 \mathrm{wk} / \mathrm{yr}$

$2 \mathrm{yr}$

$31,868,496$ liter

Domestic water use is assumed to be the same as sanitary waste. 
Energy Requirements:

Electricity

$150 \mathrm{MWh} / \mathrm{yr}$

\section{Fossil Fuel Use}

Concrete:

Amount used $=$

(Detailed Cost Estimate)

\begin{tabular}{cl}
$1,360 y d^{3}$ & Tunnel \\
$8,300 y d^{3}$ & RR Structure \\
$630 y d^{3}$ & Building \\
\hline $10,290 y d^{3}$ & Total Concrete
\end{tabular}

Number of truckloads $=$ Fuel usage $=$

468 (based on $22 \mathrm{yd}^{3} / \mathrm{load} .$. tandem trailers)

$10,290 \mathrm{gal}$ (assumes $5 \mathrm{mpg}$ and round trip of 110 miles)

Materials delivery:

Number of truckloads $=$ 234 (assume $1 / 2$ the loads of concrete)

Fuel usage $=$ 7,016 gal (assumes $5 \mathrm{mpg}$ and round trip of 150 miles)

\section{Construction Equipment:}

Construction Equipment Cost

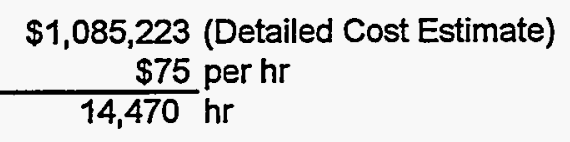

Labor Hours

\section{2,638 (Detailed Cost Estimate)}

750 labor hr/equipment hr

$$
270 \mathrm{hr}
$$

Total Equipment Hours $=$

$14,740 \mathrm{hr}$

Equipment fuel usage (see http://mww/deere.com/ind) =

Total heavy equipment fuel usage $=$

$6 \mathrm{gal} / \mathrm{hr}$
$88,439 \mathrm{gal}$
or $\quad 334,741$ liters (total)

Total heavy equipment and materials delivery fuel used = Concrete Delivery Materials Delivery

Heavy Equipment

$10,290 \mathrm{gal}$

$7,016 \mathrm{gal}$

$88,439 \mathrm{gal}$

$105,745 \mathrm{gal}$ 3.785 liter/gal

400,244 liter 


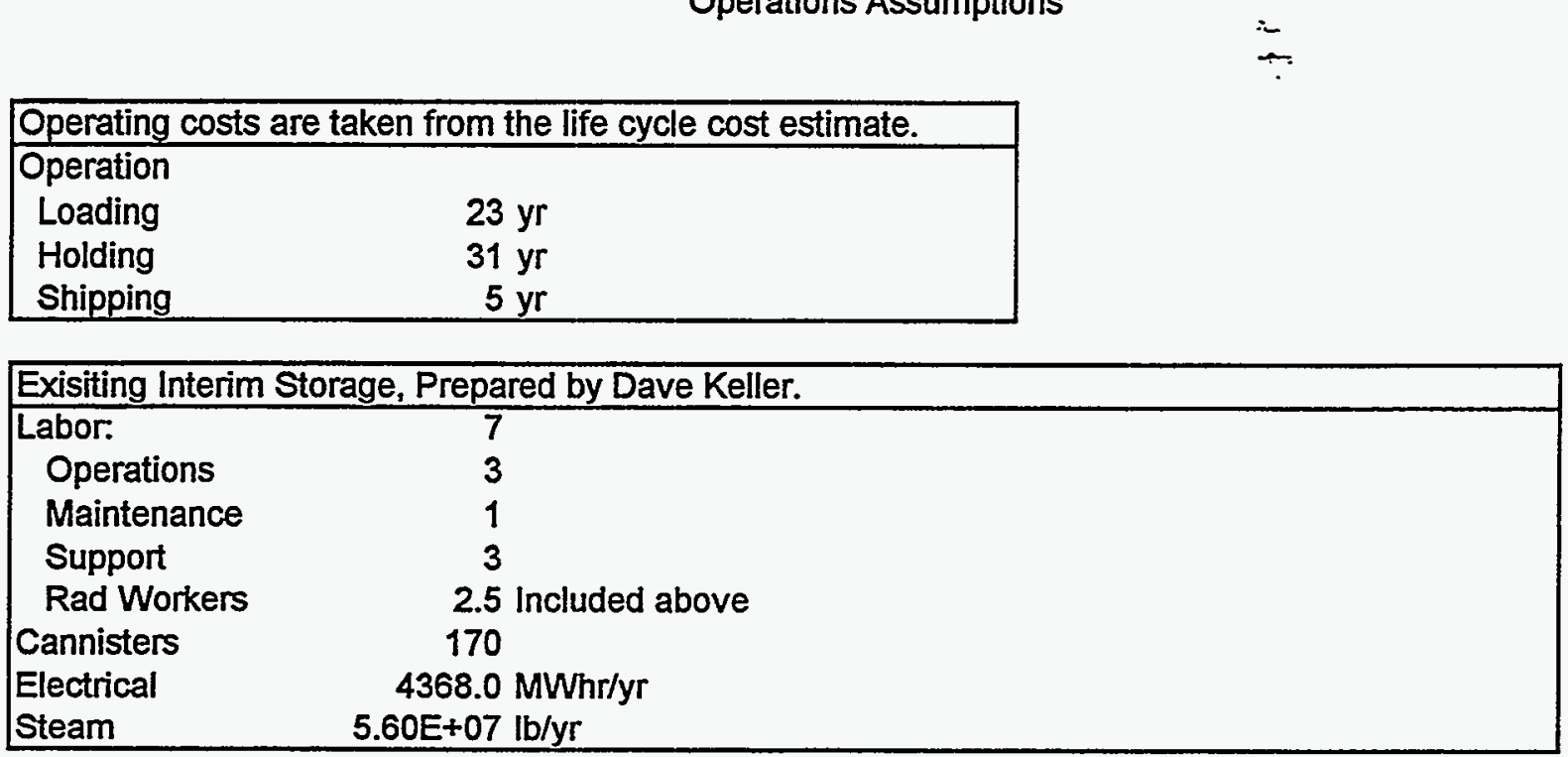

Radiation worker annual dose is based on the average annual dose received at ICCP during the last three years (see attached memorandum)

\section{Effluents:}

Sanitary wastewater $=$ (based on 25 gal/day per worker, facility occupied year-round)

$$
63,875 \mathrm{gal} / \mathrm{yr}=\quad 241,767 \mathrm{liters} / \mathrm{yr}
$$

\section{Solid Wastes:}

Sanitary/Industrial trash $=$ (based on $7.25 \mathrm{yd} 3 /$ person-year... Bob Skinner [cuber facility])

Product canisters $=$ (based on $0.72 \mathrm{~m}^{3} /$ canister)

$$
141 \mathrm{~m}^{3}
$$

\section{Water Usage:}

Domestic water usage $=$ same as sanitary wastewater rate.

\section{Energy Requirements}

Electric power usage $=$

Steam $=$

\author{
4368.0 MWhr/yr \\ $5.60 \mathrm{E}+07 \mathrm{lb} / \mathrm{yr}$
}


D\&D Labor

D\&D Labor

\section{Crew \# Crew Function}

D Documentation

1 Characterization

2 Rad Demolition-Systems

2A Rad Demolition-Building

3 Demolition-Systems

3A Demolition-Building

4 Asbestos Abatement

5 Decontamination

6 Prep/Fabrication

7 RADCON Surveys

Total

vailable

Notes:

1 Crew functions and daily estimates are from the D\&D program (Dave Haycraft)

2 Total costs are based on life cycle estimate by $R$. Turk

3 Assume all workers in crews $2,2 A, 5$, and 7 are rad workers

4 Assume a man-year is 1800 hours.
Years

From D\&D

3 Assumptions

Material Equipment

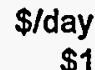

$\$ 114$

$\$ 460$

$\$ 818$

\$/day

Total \$/day

$\$ 1,250$

$\$ 3,453$

$\$ 5,932$

$\$ 7,979$

$\$ 5,455$

$\$ 7,212$

$\$ 4,316$

$\$ 5,630$

$\$ 4,826$

$\$ 3,894$

$\$ 779$
Total MH Man-hours/yr

$19,282 \quad 6,427$

$25,485 \quad 8,495$

71,538

132,390

23,846

$-$

41,080

32,614

44,130

-

13,693

10,871

$322,389 \quad 107,463$
D\&D Cost

Allocated

$\$ \quad 1,339,000$

$2,000,000$

-

$5,420,000$

$10,850,000$

-

$3,250,000$

$2,540,000$

$\$ 25,399,000$

$\$ 25,399,000$ 
No decontamination of the storage facility will be required.

$D$ \& $D$ labor requirements are taken from $D$ \& $D$ labor and equipment spreadsheet.

$D \& D$ costs come from the life cycle cost estimate.

1 manyear of labor $=$

1800 manhours

Assume that the D\&D cost estimates are for the modified portion of the FPR facility. D\&D of the entire FPR is not addressed.

$\begin{array}{lrrr}\text { Heavy Equipment } & \text { \# Used } & \text { Hours/day } & \text { Days/wk } \\ \text { Mobile Crane } & 1 & 3 & 5 \\ \text { Roll-Off Truck } & 2 & 8 & 5 \\ \text { Dozer } & 1 & 6 & 5 \\ \text { Loader } & 2 & 8 & 5 \\ \text { Scabbler (w/ Vacuum System) } & 0 & 6 & 3 \\ \text { Pneumatic Ram } & 1 & 3 & 5 \\ \text { Demolition Machine (Remote } & & & \\ \text { Control) ** } & 0 & 4 & 4 \\ \star \text { Not needed, contamination not expected } & \end{array}$

\begin{tabular}{rr|r}
5 & Wks/yr & Hours/yr \\
5 & 20 & 300 \\
5 & 20 & 1,600 \\
5 & 20 & 600 \\
5 & 20 & 1,600 \\
3 & 20 & - \\
5 & 20 & 300 \\
4 & 45 & - \\
\hline & & 4,400 \\
& &
\end{tabular}

Total hours/yr

13,200

Total heavy equipment hours $=$

Assume each piece of equipment uses 6 gallon of diesel fuel per hour. Consumption rate from John Deere Web Site (Construction Equipment - http://www.deere.com/ind/product/product.html)

No. of gallons of fuel used during $D \& D=\quad 79,200$ gal $=$

Assume each roll-off truck makes 3 trips per day to RWMC

No. of trips $=$

Miles traveled @ 12 miles/round trip=

Acres disturbed and duration of disturbance

Acreage disturbed is the same as for construction =

155.0 acres

\section{Air emissions}

HEPA filter ventilation is not required during D\&D.

Air emissions from fuel are based on the diesel emissions spreadsheet.

\section{Effluents}

No decontamination solution required during $D \& D$.

Sanitary wastewater $=$

(based on $25 \mathrm{gal} /$ day per worker, $225 \mathrm{day} / \mathrm{yr}$ )

(based on 3 gal for every 60 hours of operation) 


\section{Solid wastes:}

The following factors from Dave Kenoyer are based on a contaminated building. The storage facility is not expected to be contaminated. Therefore, all items identified as LLW will be included with the non-contaminated waste. These factors are used for consistency in waste volumes with facilities which are contaminated.

Solid Waste Generation (factors from Dave Kenoyer - D\&D Program)

\begin{tabular}{|c|c|c|c|c|}
\hline Waste Type & $\begin{array}{c}\text { Factor } \\
\text { (cu.ft./sq.ft.) }\end{array}$ & $\begin{array}{l}\text { Sq.Ft. in } \\
\text { Facility }\end{array}$ & $\begin{array}{l}\text { Cu.Ft. of } \\
\text { Waste }\end{array}$ & Cu. Meters \\
\hline WERF-LLW Combustible PPEs & 0.167 & 63,000 & 10,521 & 301 \\
\hline WERF-LLW Combustible Building & 0.128 & 63,000 & 8,064 & 230 \\
\hline $\begin{array}{l}\text { WERF-LLW Compactable Building } \\
\text { RWMC-LLW Non-Compactable }\end{array}$ & 0.195 & 63,000 & 12,285 & 351 \\
\hline $\begin{array}{l}\text { Equipment } \\
\text { RWMC-LLW Non-Compt Building }\end{array}$ & 0.513 & 63,000 & 32,319 & 923 \\
\hline $\begin{array}{l}\text { Debris } \\
\text { RWMC-LLW Non-Compt Concrete }\end{array}$ & 0.684 & 63,000 & 43,092 & 1,231 \\
\hline $\begin{array}{l}\text { Rubble " } \\
\text { RWMC-LLW Non-Compt Scrap Metal }\end{array}$ & $\begin{array}{r}3.44 \\
0.778\end{array}$ & $\begin{array}{l}63,000 \\
63,000\end{array}$ & $\begin{array}{r}216,720 \\
49,014\end{array}$ & $\begin{array}{l}6,192 \\
1,400\end{array}$ \\
\hline $\begin{array}{l}\text { RWMC-LLW Asbestos/ACM Covered } \\
\text { Pipe } \\
\text { CFA Landfill Non-Compt Building }\end{array}$ & 0 & 63,000 & - & \\
\hline $\begin{array}{l}\text { Debris } \\
\text { CFA Landfill Non-Compt Concrete }\end{array}$ & 1.99 & 63,000 & 125,370 & 3,582 \\
\hline Rubble * & 2.454 & 63,000 & 154,602 & 4,417 \\
\hline CFA Landfill Asbestos & 0 & 63,000 & - & \\
\hline HWSF Hazardous Mtrls (Hg/PCBs/etc) & 0.002 & 63,000 & 126 & 4 \\
\hline Metal Recycle & 0.022 & 63,000 & 1,386 & 40 \\
\hline $\begin{array}{l}\text { Non-Rad }= \\
\text { Hazardous = } \\
\text { Metal }=\end{array}$ & & & $\begin{array}{r}651,987 \\
126 \\
1,386\end{array}$ & $\begin{array}{r}18,628 \\
4 \\
40\end{array}$ \\
\hline
\end{tabular}

* (Factor used is twice that given by Dave Kenoyer due to use of concrete for shielding)

Hazardous/toxic chemicals and wastes (type)

Mixed waste $=\quad 26,445$ gal (total) $=\quad 909 \mathrm{~m} 3$ (total)

(based on an assumed 5 55-gallon drums generated per week... work only 225 days/yr)

\section{Water Usage}

Domestic water use is assumed to be the same as sanitary waste.

Energy requirements:

Electric power usage $=$

(based on 3,000 kWh/wk - John Duggan)

$156,000 \mathrm{kWh} / \mathrm{yr}$

$156 \mathrm{MWh} / \mathrm{yr}$

Fossil Fuels $=$

299,772 liters 
Bases \& Assumptions:

\section{Estimate of Diesel Engine Emissions \\ Storage at FPR - TRU}

1. Air to fuel ratio $=25: 1$ (Mass Basis)

2. Diesel fuel density $=7.5 \mathrm{lbs} . / \mathrm{gal}$.

3. Air is $21 \% \mathrm{O}_{2}, 79 \% \mathrm{~N}_{2}$, with a pseudomolecular weight of 29 .

4. Combustion is simulated as: $\mathrm{C}_{9} \mathrm{H}_{18}+13.5 \mathrm{O}_{2} \rightarrow 9 \mathrm{CO}_{2}+9 \mathrm{H}_{2} \mathrm{O}$

5. Particulates $=5 \mathrm{mg} / \mathrm{scf}$

6. $\mathrm{CO}=2,500 \mathrm{ppmv}$

7. $\mathrm{NO}_{\mathrm{x}}=2,000 \mathrm{ppmv}$

8. Unburned hydrocarbons $=100 \mathrm{ppmv}$

9. Diesel fuel (\# 2 fuel oil) contains 1 wt. \% sulfur

10. Combustion is about $99 \%$ efficient. from Wark, K. and C.F. Wamer, Air Pollution, its Origin and Control, IEP, New York, 1976, p. 446, 423

Wark and Wamer, p. 446 Wark and Wamer, p. 446 Wark and Warner, p. 446 Wark and Wamer, p. 446 Wark and Wamer, p. 336

Lbs. Of Construction Fuel

400,244

Lbs. Of Operations Fuel

Lbs. Of D\&D Fuel

Total Lbs. of Fuel Used

Lb-Moles of Construction Fuel

Lb-Moles of Operations Fuel

Lb-Moles of D\&D Fuel

7,887

Lbs of Air for Construction Fuel (based on air-to-fuel ratio)

$10,006,107$

Lbs.of Air for Operations Fuel (based on air-to-fuel ratio)

$14,838,714$

Lbs. of Air for D\&D Fuel (based on air-to-fuel ratio)

$24,844,821$

Lb-Moles of Air for Combustion Fuel

345,038

Lb-Moles of Air for Operations Fuel

Lb-Moles of Air for D\&D Fuel

$511, \overline{680}$

856,718

Grand Total of Materials Fed, Lbs.

$25,838,614$

\begin{tabular}{lrrrr} 
Exhaust Gases, Construction Fuel & Total Lbs. & \multicolumn{1}{c}{ Total Tons } & \multicolumn{1}{c}{ Total Moles } & \multicolumn{1}{c}{ Total SCF } \\
\hline $\mathrm{CO}_{2}$ & $1,245,331$ & 623 & 28,303 & $10,160,773$ \\
$\mathrm{H}_{2} \mathrm{O}$ & 509,454 & 255 & 28,303 & $10,160,773$ \\
$\mathrm{O}_{2}$ & 960,113 & 480 & 30,004 & $10,771,268$ \\
$\mathrm{~N}_{2}$ & $7,632,244$ & 3,816 & 272,580 & $97,856,273$ \\
\hline Subtotal of Major Gases & $10,347,142$ & 5,174 & 359,190 & $128,949,087$ \\
\hline $\mathrm{SO}_{2}$ & 8,005 & 4.0 & \\
Particulates & 1,420 & 0.7 & \\
$\mathrm{CO}$ & 25,143 & 12.6 & \\
$\mathrm{NO}_{x}$ (assumed NO) & 21,551 & 10.8 & \\
Unburned Hydrocarbons & 4,526 & 2.3 & \\
Subtotal of Contaminants & 60,645 & 30 & \\
\hline
\end{tabular}




\begin{tabular}{lcccc} 
Exhaust Gases, Operations Fuel & Total Lbs. & Total Tons & Total Moles & Total SCF \\
\hline $\mathrm{CO}_{2}$ & - & - & - & - \\
$\mathrm{H}_{2} \mathrm{O}$ & - & - & - & - \\
$\mathrm{O}_{2}$ & - & - & - & - \\
$\mathrm{N}_{2}$ & - & - & - & - \\
\hline Subtotal of Major Gases & - & - & - \\
$\mathrm{SO}_{2}$ & - & - & \\
$\mathrm{Particulates}$ & - & - & \\
$\mathrm{CO}$ & - & - & \\
$\mathrm{NO}_{\mathrm{x}}$ (assumed NO) & - & - & \\
Unbumed Hydrocarbons & - & - \\
Subtotal of Contaminants & - & - \\
\hline
\end{tabular}

\begin{tabular}{lrrrr} 
Exhaust Gases, D\&D Fuel & Total Lbs. & Total Tons & Total Moles & \multicolumn{1}{c}{ Total SCF } \\
\hline $\mathrm{CO}_{2}$ & $1,846,784$ & 923 & 41,972 & $15,068,078$ \\
$\mathrm{H}_{2} \mathrm{O}$ & 755,503 & 378 & 41,972 & $15,068,078$ \\
$\mathrm{O}_{2}$ & $1,423,815$ & 712 & 44,494 & $15,973,422$ \\
$\mathrm{~N}_{2}$ & $11,318,357.02$ & 5,659 & 404,227 & $145,117,506$ \\
\hline Subtotal of Major Gases & $\mathbf{1 5 , 3 4 4 , 4 5 8}$ & $\mathbf{7 , 6 7 2}$ & $\mathbf{5 3 2 , 6 6 6}$ & $\mathbf{1 9 1 , 2 2 7 , 0 8 5}$ \\
\hline $\mathrm{SO}_{2}$ & 11,500 & $\mathbf{5 . 8}$ & & \\
Particulates & 2,106 & 1.1 & \\
$\mathrm{CO}$ & 37,287 & 18.6 & \\
NO & 31,960 & 16.0 & \\
Unburned Hydrocarbons & 6,712 & 3.4 & \\
Subtotal of Contaminants & 89,564 & $\mathbf{4 5}$ & & \\
\hline
\end{tabular}


431.02\#

06/17/97

Rev. $\# 04$
ENGINEERING DESIGN FILE

Hot Ilsostatic Press Waste Option
Function File Number - SPR-01

EDF Serial Number - EDF-IS-002 
Construction costs are from the detailed cost estimate (R. Adams)

Existing Interim Storage Facility Option, Prepared by Dave Keller.

Construction duration $=$

Labor $=$

1 man-year of labor $=$

Square footage $=$

Acres disturbed =
3 years

152 new workers/yr

1800 manhours

63,000 sq. $f t=\quad 5,853 \mathrm{~m}^{2}$

Excavation: Excavated earth will be spread in a spoil area adjacent to ICPP, except for backfill soil.

Excess Material=

$24,100 \mathrm{yd}^{3}$

(from Detailed Cost Estimate)

Air Emissions:

Dust during construction $=1.2$ tons/month-acre $=$

(from USEPA Office of Ar Quality Planning and Standards)

6,696 tons (total)

Air emissions from fuel usage are based on the diesel emissions spreadsheet.

Effluents:

Sanitary Wastewater $=$

$855,000 \mathrm{gal} / \mathrm{yr}=$

(based on $25 \mathrm{gal} / \mathrm{person}$-day and 225 days/year of construction)

$9,708,525$ liters (total)

(Benefield, LD and C.W. Randall, Biological Process Design for Wastewater Treatment, InPrint, Inc., 1987, p. 104 wastewater generation $=15-30$ gal/day-person)

Assume 3 gallons of lubricating oil and hydraulic fluid generated for every 60 hours of operation of heavy equipment $=$

5,188 liter (total)

(Heavy equipment hrs are calculated below.)

\section{Solid Wastes:}

Construction trash $=\quad 7,068 \mathrm{yd}^{3}$ (total) $=\quad 5,406 \mathrm{~m}^{3}$ (total)

(Use $15.5 \mathrm{yd} 3 / \mathrm{yr}$ per capita. This is twice the generation rate of trash from site operations)

Hazardous/toxic chemicals and wastes (type)
Hazardous waste generation $=$
275 gal/week $=$
$217 \mathrm{~m}^{3}$ (total)

(based on an assumed generation rate of 555 -gallon drums of waste per week)

Hazardous waste storage $=$

$3300 \mathrm{gal}=$

$13 \mathrm{~m}^{3}$

(Assume waste is accumulated for 12 weeks [84 days] in a 90-day accumulation area, then

Water Usage:

Water used for dust control $=$

Area

assume $0.05^{\prime \prime}$ water per week to control dust

$$
\begin{gathered}
155 \text { acres (previous) } \\
43,560 \mathrm{sq} \mathrm{ftacre} \\
0.05 \mathrm{in} \mathrm{water/wk} \\
0.08333 \mathrm{ft} / \mathrm{in} \\
28.320 \mathrm{liter} / \mathrm{ft}^{3} \\
20 \mathrm{wk} / \mathrm{yr} \\
3 \mathrm{yr} \\
\hline 4,802,744 \text { liter }
\end{gathered}
$$

assume dust control required 20 weeks Length of Construction

Domestic water use is assumed to be the same as sanitary waste. 
Energy Requirements:

Electricity

$150 \mathrm{MWh} / \mathrm{yr}$

\section{Fossil Fuel Use}

Concrete:

Amount used $=$

(Detailed Cost Estimate)

\begin{aligned} $1,360 y d^{3} &$ Tunnel \\ $8,300 y d^{3} &$ RR Structure \\ $630 y d^{3} &$ Building \\ \hline $10,290 y d^{3} &$ Total Concrete \end{aligned}

Number of truckloads $=$

Fuel usage $=$

468 (based on $22 \mathrm{yd}^{3} /$ load... tandem trailers)

$10,290 \mathrm{gal}$ (assumes $5 \mathrm{mpg}$ and round trip of 110 miles)

Materials delivery:

Number of truckloads $=$

Fuel usage $=$

234 (assume $1 / 2$ the loads of concrete)

$7,016 \mathrm{gal}$ (assumes $5 \mathrm{mpg}$ and round trip of 150 miles)

\section{Construction Equipment:}

Construction Equipment Cost

\begin{tabular}{c}
$\$ 2,009,223$ (Detailed Cost Estimate) \\
$\$ 75$ per hr \\
\hline $26,790 \mathrm{hr}$
\end{tabular}

Labor Hours

468,143 (Detailed Cost Estimate)

750 labor hrlequipment hr

$624 \mathrm{hr}$

Total Equipment Hours $=$

$27,414 \mathrm{hr}$

Equipment fuel usage (see http://uww/deere.com/ind) =

Total heavy equipment fuel usage $=$

$\begin{array}{cl} & 6 \mathrm{gal} / \mathrm{hr} \\ & 164,483 \mathrm{gal} \\ \text { or } \quad 622,568 \text { liters (total) }\end{array}$

Total heavy equipment and materials delivery fuel used = Concrete Delivery Materials Delivery $10,290 \mathrm{gal}$ $7,016 \mathrm{gal}$ Heavy Equipment $164,483 \mathrm{gal}$ $181,789 \mathrm{gal}$ 3.785 liter/gal

688,071 liter 
Operating costs are taken from the life cycle cost estimate.

Operation

Loading $23 \mathrm{yr}$

Holding $31 \mathrm{yr}$

Shipping $5 \mathrm{yr}$

Exisiting Interim Storage, Prepared by Dave Keller.

Labor: 7

Operations 3

Maintenance 1

Support 3

Rad Workers $\quad 2.5$ Included above

Cannisters $\quad 5700$

Electrical 4,368 MWh/yr

Steam $\quad 5.60 \mathrm{E}+07 \mathrm{lb} / \mathrm{yr}$

Radiation worker annual dose is based on the average annual dose received at ICCP during the last three years (see attached memorandum)

\section{Effluents:}

Sanitary wastewater $=$ $63,875 \mathrm{gal} / \mathrm{yr}=$

(based on 25 gal/day per worker, facility occupied year-round)

\section{Solid Wastes:}

Sanitary/Industrial trash =

(based on $7.25 \mathrm{yd}^{3} /$ person-year... Bob Skinner [cuber facility])

Product canisters $=$

(based on $0.72 \mathrm{~m}^{3} /$ canister)

$$
205 \mathrm{~m}^{3} / \mathrm{yr}
$$

Total rad waste $=$

$$
4,720 \mathrm{~m}^{3}
$$

Water Usage:

Domestic water usage $=$ same as sanitary wastewater rate.

\section{Energy Requirements}

Electric power usage $=$ 
D\&D Labor

D\&D Labor

$\begin{array}{cl}\text { Crew \# } & \text { Crew Function } \\ \text { D } & \text { Documentation } \\ 1 & \text { Characterization } \\ 2 & \text { Rad Demolition-Systems } \\ 2 A & \text { Rad Demolition-Building } \\ 3 & \text { Demolition-Systems } \\ 3 A & \text { Demolition-Building } \\ 4 & \text { Asbestos Abatement } \\ 5 & \text { Decontamination } \\ 6 & \text { Prep/Fabrication } \\ 7 & \text { RADCON Surveys }\end{array}$

Total

vailable

Notes:

1 Crew functions and daily estimates are from the D\&D program (Dave Haycraft)

2 Total costs are based on life cycle estimate by $R$. Turk

3 Assume all workers in crews 2, 2A, 5, and 7 are rad workers

4 Assume a man-year is 1800 hours.
Years

From D\&D

3 Assumptions

\begin{tabular}{|c|c|c|}
\hline $\begin{array}{c}\text { Material } \\
\$ / \text { day }\end{array}$ & & $\begin{array}{l}\text { uipment } \\
\text { p/day }\end{array}$ \\
\hline$\$ 114$ & $\$$ & 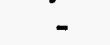 \\
\hline$\$ 460$ & & $\$ 691$ \\
\hline$\$ 818$ & & $\$ 1,023$ \\
\hline$\$ 1,064$ & & $\$ 1,596$ \\
\hline$\$ 752$ & & $\$ 941$ \\
\hline$\$ 962$ & & $\$ 1,442$ \\
\hline$\$ 375$ & & $\$ 188$ \\
\hline$\$ 751$ & & $\$ 1,126$ \\
\hline$\$ 643$ & & $\$ 965$ \\
\hline$\$ 519$ & & \\
\hline
\end{tabular}

Total $\$ /$ day

$\$ 1,250$

$\$ 3,453$

$\$ 5,932$

$\$ 7,979$

$\$ 5,455$

$\$ 7,212$

$\$ 4,316$

$\$ 5,630$

$\$ 4,826$

$\$ 3,894$

D\&D Cost

Allocated

\$ 2,650,000

\$ $3,990,000$

$\$$

$\$$ -

$\$ 12,840,000$

$\$ 25,674,000$

$\$ 7,700,000$

$\begin{array}{ll}\$ & 7,700,000 \\ \$ & 5,875,000\end{array}$

$\$ 58,729,000$

Total MH

38,160

50,843

$-$

69,474

313,271

-

97,327

75,437

12,720

16,948

-

6,491

104,424

-

32,442

25,146

$744,511 \quad 248,170$

$\$ 58,729,000$ 
Duration of $D \& D=\quad 3$ years

No decontamination of the storage facility will be required.

$D$ \& $D$ labor requirements are taken from $D$ \& $D$ labor and equipment spreadsheet.

$D \& D$ costs come from the life cycle cost estimate.

1 manyear of labor $=\quad 1800$ manhours

Assume that the D\&D cost estimates are for the modified portion of the FPR facility. D\&D of the entire FPR is not addressed.

\begin{tabular}{lrrrr|r} 
Heavy Equipment & \# Used & Hours/day & Days/wk & Wks/yr & Hours/yr \\
Mobile Crane & 1 & 3 & 5 & 45 & 675 \\
Roll-Off Truck & 2 & 8 & 5 & 45 & 3,600 \\
Dozer & 1 & 6 & 5 & 45 & 1,350 \\
Loader & 2 & 8 & 5 & 45 & 3,600 \\
Scabbler (w/ Vacuum System) *t & 0 & 6 & 3 & 45 & - \\
Pneumatic Ram & 1 & 3 & 5 & 45 & 675 \\
Demolition Machine (Remote & 0 & 4 & 4 & 45 & - \\
$\quad$ Control) ** & & & & 9,900
\end{tabular}

Total heavy equipment hours = 29,700 Assume each piece of equipment uses 6 gallon of diesel fuel per hour. Consumption rate from John Deere Web Site (Construction Equipment - http://www.deere.com/ind/product/product.html)

No. of gallons of fuel used during $D \& D=\quad 178,200 \mathrm{gal}=$

Assume each roll-off truck makes 3 trips per day to RWMC

No. of trips =

Miles traveled @ 12 miles/round trip=

Acres disturbed and duration of disturbance

Acreage disturbed is the same as for construction =

155.0 acres

\section{Air emissions}

HEPA filter ventilation is not required during D\&D.

Air emissions from fuel are based on the diesel emissions spreadsheet.

\section{Effluents}

No decontamination solution required during D\&D.

Sanitary wastewater $=\quad 775,533 \mathrm{gal} / \mathrm{yr}=\quad 8,806,174$ liters (total)

(based on $25 \mathrm{gal} /$ day per worker, $225 \mathrm{day} / \mathrm{yr}$ )

Lube oil = $\quad 5,621$ liters (total)

(based on 3 gal for every 60 hours of operation)

\section{Solid wastes:}


The following factors from Dave Kenoyer are based on a contaminated building. The storage facility is not expected to be contaminated. Therefore, all items identified as LLW will be included with the non-contaminated waste. These factors are used for consistency in waste volumes with facilities which are contaminated.

Solid Waste Generation (factors from Dave Kenoyer - D\&D Program)

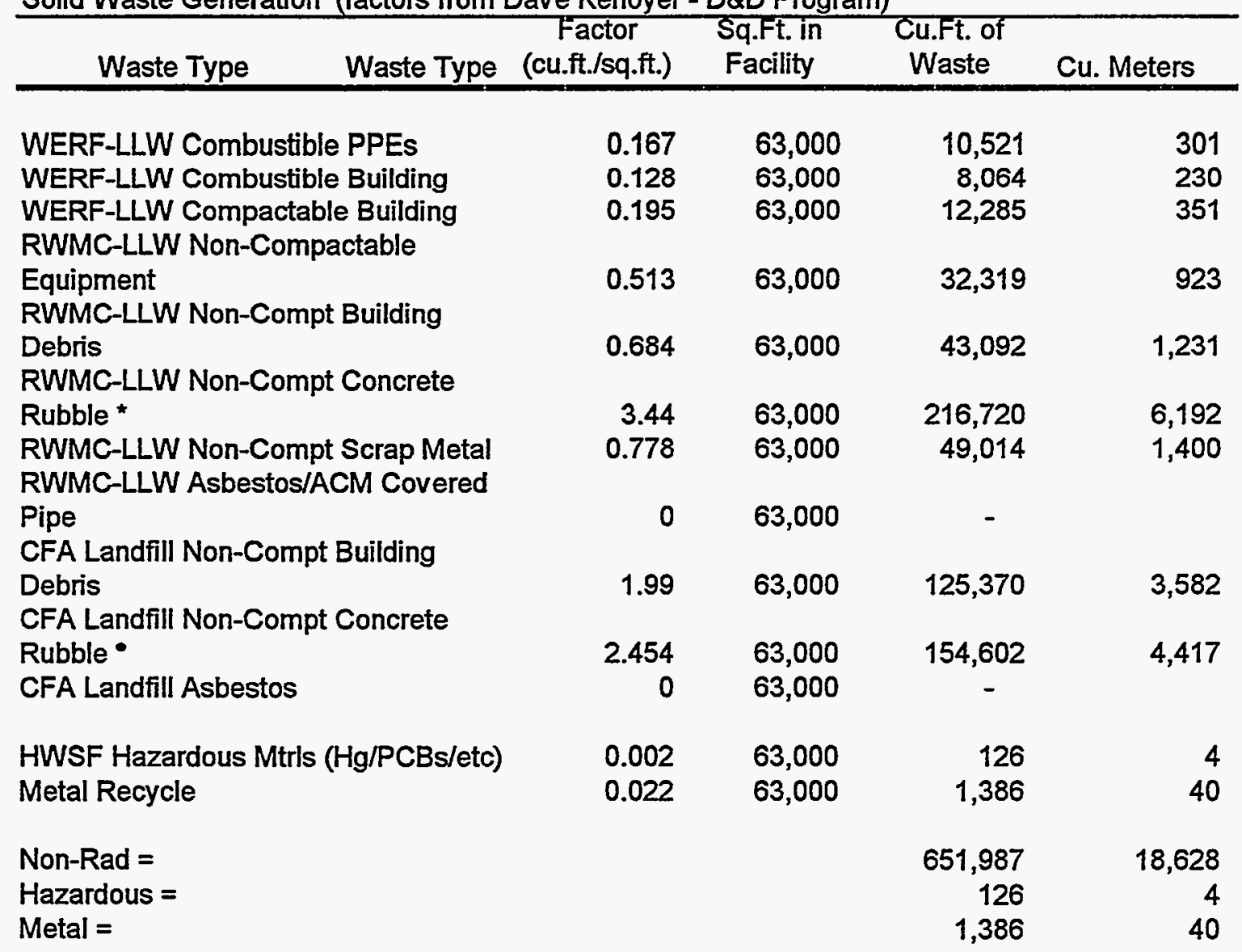

- (Factor used is twice that given by Dave Kenoyer due to use of concrete for shielding)

Hazardous/toxic chemicals and wastes (type)

Mixed waste $=\quad 26,445$ gal (total) $=\quad 909 \mathrm{~m} 3$ (total)

(based on an assumed 555 -gallon drums generated per week... work only 225 days/yr)

\section{Water Usage}

Domestic water use is assumed to be the same as sanitary waste.

Energy requirements:

Electric power usage $=\quad 156,000 \mathrm{kWh} / \mathrm{yr}$ (based on 3,000 kWh/wk - John Duggan)

Fossil Fuels $=\quad 674,487$ liters
$156 \mathrm{MWh} / \mathrm{yr}$ 
Bases \& Assumptions:

\section{Estimate of Diesel Engine Emissions \\ Storage at FPR - HWO}

1. Air to fuel ratio $=25: 1$ (Mass Basis)

2. Diesel fuel density $=7.5 \mathrm{lbs} . / \mathrm{gal}$.

3. Air is $21 \% \mathrm{O}_{2}, 79 \% \mathrm{~N}_{2}$, with a pseudomolecular weight of 29 .

4. Combustion is simulated as: $\mathrm{C}_{9} \mathrm{H}_{18}+13.5 \mathrm{O}_{2} \rightarrow 9 \mathrm{CO}_{2}+9 \mathrm{H}_{2} \mathrm{O}$

5. Particulates $=5 \mathrm{mg} / \mathrm{scf}$

6. $\mathrm{CO}=2,500 \mathrm{ppmv}$

7. $\mathrm{NO}_{\mathrm{x}}=2,000 \mathrm{ppmv}$

8. Unburned hydrocarbons $=100 \mathrm{ppmv}$

9. Diesel fuel (\# 2 fuel oil) contains 1 wt. \% sulfur

10. Combustion is about $99 \%$ efficient.

Lbs. Of Construction Fuel

Lbs. Of Operations Fuel

Lbs. Of D\&D Fuel

Total Lbs. of Fuel Used

Lb-Moles of Construction Fuel

Lb-Moles of Operations Fuel

Lb-Moles of D\&D Fuel

Total Lb-Moles of Fuel (as C9H18)

Lbs of Air for Construction Fuel (based on air-to-fuel ratio)

Lbs.of Air for Operations Fuel (based on air-to-fuel ratio)

Lbs.of Air for D\&D Fuel (based on air-to-fuel ratio)

Total Lbs. of Air Added

Lb-Moles of Air for Combustion Fuel

Lb-Moles of Air for Operations Fuel

Lb-Moles of Air for D\&D Fuel

Total Lb-Moles of Air

Grand Total of Materials Fed, Lbs. from Wark, K. and C.F. Warner, Air Pollution, Its Origin and Control, IEP, New York, 1976, p. 446, 423

Wark and Warner, p. 446 Wark and Warner, p. 446 Wark and Wamer, p. 446 Wark and Wamer, p. 446 Wark and Warner, p. 336

$$
\begin{array}{r}
1,362,381 \\
- \\
1,335,484 \\
2,697,865 \\
10,813 \\
- \\
10,599 \\
21,412 \\
\\
34,059,513 \\
- \\
33,387,107 \\
67,446,619 \\
\\
1,174,466 \\
- \\
1,151,280 \\
2,325,745
\end{array}
$$

$70,144,484$

\begin{tabular}{lrrrr} 
Exhaust Gases, Construction Fuel & \multicolumn{1}{c}{ Total Lbs. } & Total Tons & \multicolumn{1}{c}{ Total Moles } & \multicolumn{1}{c}{ Total SCF } \\
\hline $\mathrm{CO}_{2}$ & $4,238,950$ & 2,119 & 96,340 & $34,585,975$ \\
$\mathrm{H}_{2} \mathrm{O}$ & $1,734,116$ & 867 & 96,340 & $34,585,975$ \\
$\mathrm{O}_{2}$ & $3,268,103$ & 1,634 & 102,128 & $36,664,025$ \\
$\mathrm{~N}_{2}$ & $25,979,187$ & 12,990 & 927,828 & $333,090,288$ \\
\hline Subtotal of Major Gases & $35,220,355$ & 17,610 & $1,222,636$ & $438,926,264$ \\
\hline $\mathrm{SO}_{2}$ & 27,248 & 13.6 & & \\
Particulates & 4,834 & 2.4 & \\
$\mathrm{CO}$ & 85,585 & 42.8 & \\
$\mathrm{NO}_{\mathrm{x}}$ (assumed NO) & 73,358 & 36.7 & \\
Unburned Hydrocarbons & 15,405 & 7.7 & \\
Subtotal of Contaminants & $\mathbf{2 0 6 , 4 2 9}$ & $\mathbf{1 0 3}$ & & \\
\hline
\end{tabular}




\begin{tabular}{lcccc} 
Exhaust Gases, Operations Fuel & Total Lbs. & Total Tons & Total Moles & Total SCF \\
\hline $\mathrm{CO}_{2}$ & - & - & - & - \\
$\mathrm{H}_{2} \mathrm{O}$ & - & - & - & - \\
$\mathrm{O}_{2}$ & - & - & - & - \\
$\mathrm{N}_{2}$ & - & - & - & - \\
\hline Subtotal of Major Gases & - & - & \\
$\mathrm{SO}_{2}$ & - & - & \\
$\mathrm{Particulates}_{\mathrm{CO}}$ & - & - & \\
$\mathrm{NO}_{\mathrm{X}}$ (assumed NO) & - & - & \\
Unbumed Hydrocarbons & - & - & \\
Subtotal of Contaminants & - & - \\
\hline
\end{tabular}

\begin{tabular}{|c|c|c|c|c|}
\hline Exhaust Gases, D\&D Fuel & Total Lbs. & Total Tons & Total Moles & Total SCF \\
\hline $\mathrm{CO}_{2}$ & $4,155,264$ & 2,078 & 94,438 & $33,903,176$ \\
\hline $\mathrm{H}_{2} \mathrm{O}$ & $1,699,881$ & 850 & 94,438 & $33,903,176$ \\
\hline $\mathrm{O}_{2}$ & $3,203,583$ & 1,602 & 100,112 & $35,940,200$ \\
\hline $\mathrm{N}_{2}$ & $25,466,303.30$ & 12,733 & 909,511 & $326,514,389$ \\
\hline Subtotal of Major Gases & $34,525,031$ & 17,263 & $1,198,498$ & $430,260,941$ \\
\hline $\mathrm{SO}_{2}$ & 25,875 & 12.9 & & \\
\hline $\begin{array}{l}\text { Particulates } \\
\text { co }\end{array}$ & $\begin{array}{r}4,739 \\
83,895\end{array}$ & $\begin{array}{r}2.4 \\
41.9\end{array}$ & & \\
\hline $\mathrm{NO}_{x}$ (assumed NO) & 71,910 & 36.0 & & \\
\hline Unburned Hydrocarbons & 15,101 & 7.6 & & \\
\hline Subtotal of Contaminants & 201,519 & 101 & & \\
\hline
\end{tabular}

